\title{
WORK-RELATED LUNG DISEASE SURVEILLANCE REPORT 1996
}

Division of Respiratory Disease Studies

National Institute for Occupational Safety and Health

U.S. DEPARTMENT OF HEALTH AND HUMAN SERVICES

Public Health Service

Centers for Disease Control and Prevention

October 1996 


\section{DISCLAIMER}

Mention of the name of any company or product does not constitute the endorsement by the National Institute for Occupational Safety and Health.

Copies of this and other NIOSH documents are available from:

Publications Dissemination, EID

National Institute for Occupational Safety and Health

4676 Columbia Parkway

Cincinnati, OH 45226-1998

FAX (513) 533-8573

DHHS (NIOSH) Number 96-134 


\section{Preface}

This 1996 Work-Related Lung Disease (WoRLD) Surveillance Report is the fourth in a series of occupational respiratory disease surveillance reports produced by the National Institute for Occupational Safety and Health (NIOSH). The purpose of this 1996 report is to provide national and state-specific summaries of occupational respiratory disease surveillance data focusing on pneumoconiosis mortality. Selected occupational respiratory hazard sampling data relevant to pneumoconiosis are also presented.

The 1996 WoRLD Surveillance Report has three sections: 1) a highlights and limitations section that provides data highlights and data usage limitations; 2) a United States section that serves to update and expand overall national data provided in the 1994 WoRLD Surveillance Report; and 3) a state section that provides detailed profiles of pneumoconiosis data for each state in the U.S..

The United States section updates pneumoconiosis mortality surveillance data published previously in the 1994 WoRLD Surveillance Report, by including data available for 1991 and 1992. For each condition, this section presents national data such as counts, crude and age-adjusted mortality rates, and years of potential life lost to age 65 to and life expectancy. Proportionate mortality ratios by industry and occupation, are based on data from a subset of states (see state list, Appendix C) for which usual industry and occupation have been coded for decedents. Also presented are U.S. county level maps showing the geographic distribution of mortality for each pneumoconiosis condition. In addition, this section presents selected occupational exposure sampling data for asbestos, coal and coal mine dust, silica dust, cotton dust, etc. (see agent categories, Appendix D).

The State section provides more detailed pneumoconiosis mortality surveillance data for each state and for the District of Columbia. The State section is organized so that tables and graphs of data for each state are grouped together. Selected graphs, tables, and maps present pneumoconiosis mortality from 1968 to 1992 for each state, as well as for counties within each state. Surveillance data include counts, crude and age-adjusted mortality rates, and years of potential life lost to life expectancy.

Pneumoconiosis conditions highlighted in the report include asbestosis, coal workers' pneumoconiosis, silicosis, byssinosis, and pneumoconioses classified as either "unspecified" or "other," as well as all pneumoconioses aggregated. Although some experts do not consider byssinosis a typical pneumoconiosis, it is included because the International Classification of Disease (ICD) system places byssinosis (code 504) within the series of codes for the pneumoconioses (500-505) and because byssinosis is included with other pneumoconioses in a new occupational safety and health objective for the nation (\#10.17 in Healthy People 2000: Midcourse Review and 1995 Revisions).

Data contained in the report originate from publications, reports, and data provided by the National Center for Health Statistics (NCHS), the Occupational Safety and Health Administration (OSHA), the Mine Safety and Health Administration (MSHA), and the Bureau of Mines $(\mathrm{BoM})$. Details on the major data sources and on the methods used to compute specific statistics can be found in Appendices A and B, respectively. Interpreted with appropriate care, information contained in this report can help to establish priorities for investigation and intervention, as well as to track progress toward the elimination of an important subset of preventable occupational respiratory diseases.

A description of previous editions of the WoRLD Surveillance Report, along with revisions and errata can be found in Appendix E. Comments and suggestions from users of earlier editions have influenced the content and format of this 1996 edition. To increase the utility of future editions, comments on the current report and descriptions of how the information is used are invited.

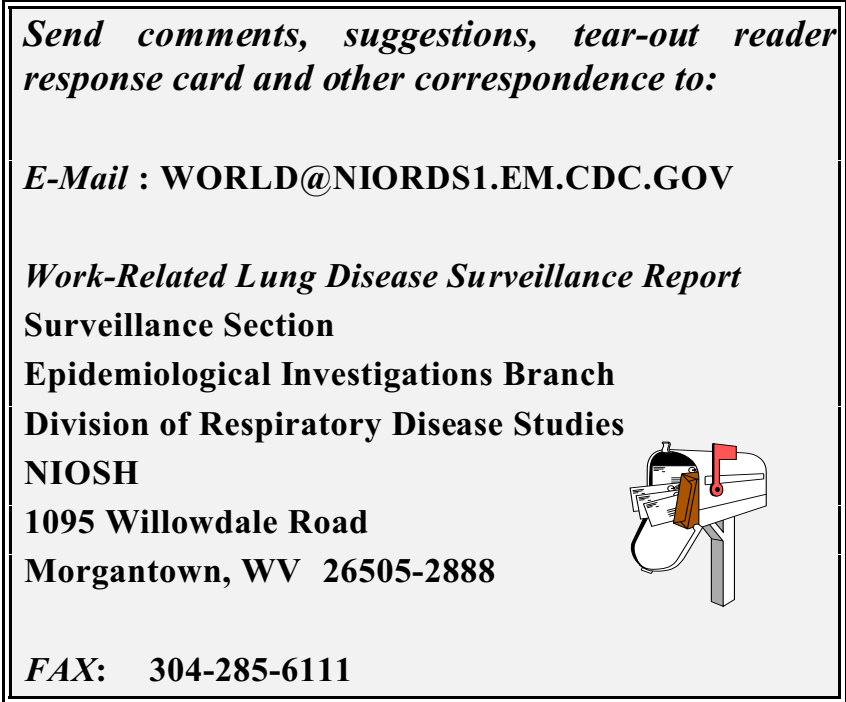




\section{Acknowledgments}

This report was prepared primarily by the staff of the Surveillance Section, Epidemiological Investigations Branch, Division of Respiratory Disease Studies (DRDS), National Institute for Occupational Safety and Health (NIOSH). Key contributors included Rochelle B. Althouse, Robert M. Castellan, Steven R. Game, Ruth Ann Romero Jajosky, Jay H. Kim, Helen S. Montagliani, Kelly L. Pryor, John M. Wood, Ki Moon Bang, Section Chief, John E. Parker, Acting Branch Chief, Nancy J. Bollinger, Deputy Division Director, and Gregory R. Wagner, Division Director.

Dennis W. Groce and Janet M. Hale, of the Environmental Investigations Branch, DRDS, contributed information relating to occupational respiratory exposure sampling data.

Draft segments of this report were provided for review and comment to many individuals affiliated with academic institutions and public health organizations, as well as to others within NIOSH. Their thoughtful comments have resulted in a number of improvements in this final report. 


\begin{tabular}{|c|c|}
\hline Avg. Sev. & average severity level \\
\hline BoM & Bureau of Mines \\
\hline $\mathrm{CDC}$ & Centers for Disease Control and Prevention \\
\hline CFR & Code of Federal Regulations \\
\hline CIC & Census Industry Code \\
\hline $\mathrm{COC}$ & Census Occupation Code \\
\hline CWP & coal workers' pneumoconiosis \\
\hline DHHS & Department of Health and Human Services \\
\hline DRDS & Division of Respiratory Disease Studies \\
\hline $\mathrm{ICD}$ & International Classification of Disease \\
\hline IMIS & Integrated Management Information System \\
\hline $\mathrm{LCL}$ & lower confidence limit \\
\hline MIDAS & Mine Inspection Data Analysis System \\
\hline MMWR & Morbidity and Mortality Weekly Report \\
\hline MRE & Mining Research Establishment \\
\hline MSHA & Mine Safety and Health Administration \\
\hline NCHS & National Center for Health Statistics \\
\hline NEC & not elsewhere classifiable \\
\hline n.e.c. & not elsewhere classified \\
\hline NIOSH & National Institute for Occupational Safety and Health \\
\hline NOS & not otherwise specified \\
\hline OSHA & Occupational Safety and Health Administration \\
\hline PEL & permissible exposure limit \\
\hline PHS & Public Health Service \\
\hline PMR & proportionate mortality ratio \\
\hline SENSOR & Sentinel Event Notification Systems for Occupational Risks \\
\hline TWA & time-weighted average \\
\hline $\mathrm{UCL}$ & upper confidence limit \\
\hline U.S. & United States \\
\hline WONDER & Wide-ranging Online Data for Epidemiologic Research \\
\hline WoRLD & Work-Related Lung Disease \\
\hline YPLL & years of potential life lost \\
\hline
\end{tabular}



Preface $\ldots \ldots \ldots \ldots \ldots \ldots \ldots \ldots \ldots \ldots \ldots \ldots \ldots \ldots$

Acknow ledgments $\ldots \ldots \ldots \ldots \ldots \ldots \ldots \ldots \ldots$ iv

Abbreviations ....................

\section{HIGHLIGHTS AND LIMITATIONS}

Selected Highlights $\ldots \ldots \ldots \ldots \ldots \ldots \ldots \ldots \ldots \ldots$

Selected Limitations $\ldots \ldots \ldots \ldots \ldots \ldots \ldots \ldots \ldots$

\section{UNITED STATES SECTION}

List of tables and figures $\ldots \ldots \ldots \ldots \ldots \ldots \ldots$

Asbestosis Mortality .................. 11

Asbestos Exposure $\ldots \ldots \ldots \ldots \ldots \ldots \ldots \ldots \ldots$

Coal Workers' Pneumoconiosis Mortality ......... 33

Coal and Coal Mine Dust Exposure ..........43

Silicosis Mortality .................55

Silica Exposure $\ldots \ldots \ldots \ldots \ldots \ldots \ldots \ldots \ldots$

Byssinosis Mortality $\ldots \ldots \ldots \ldots \ldots \ldots \ldots \ldots 77$

Cotton Dust Exposure $\ldots \ldots \ldots \ldots \ldots \ldots \ldots \ldots$

Unspecified/Other Pneumoconioses Mortality ...... 95

All Pneumoconioses Mortality $\ldots \ldots \ldots \ldots \ldots \ldots \ldots 105$

Pneumoconiotic Agents Exposure .......... 113

\section{STATE SECTION}

List of tables and figures $\ldots \ldots \ldots \ldots \ldots \ldots \ldots$

Alabama $\ldots \ldots \ldots \ldots \ldots \ldots \ldots \ldots \ldots \ldots \ldots$

Alaska $\ldots \ldots \ldots \ldots \ldots \ldots \ldots \ldots \ldots \ldots \ldots \ldots$

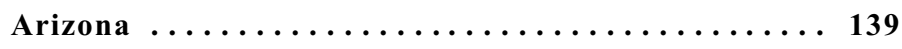

Arkansas $\ldots \ldots \ldots \ldots \ldots \ldots \ldots \ldots \ldots \ldots$

California $\ldots \ldots \ldots \ldots \ldots \ldots \ldots \ldots 151$

Colorado ................. 157

Connecticut $\ldots \ldots \ldots \ldots \ldots \ldots \ldots \ldots \ldots$

Delaware $\ldots \ldots \ldots \ldots \ldots \ldots \ldots \ldots$

District of Columbia ............. 175

Florida ................... 181

Georgia $\ldots \ldots \ldots \ldots \ldots \ldots \ldots \ldots \ldots$

Hawaii .................... 195

Idaho $\ldots \ldots \ldots \ldots \ldots \ldots \ldots \ldots \ldots \ldots$

Illinois $\ldots \ldots \ldots \ldots \ldots \ldots \ldots \ldots \ldots \ldots$

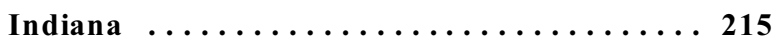

Iowa $\ldots \ldots \ldots \ldots \ldots \ldots \ldots \ldots \ldots \ldots 22$

Kansas ..................... 229

Kentucky .................... 235

Louisiana .................. 243

Maine .................... 249

Maryland $\ldots \ldots \ldots \ldots \ldots \ldots \ldots \ldots \ldots 255$

Massachusetts ................ 261

Michigan ................. 267

Minnesota $\ldots \ldots \ldots \ldots \ldots \ldots \ldots \ldots \ldots 273$

Mississippi .................. 279

Missouri ..................... 285

Montana ................... 291

Nebraska ................... 297

Nevada ................... 303

New Hampshire ................ 309 


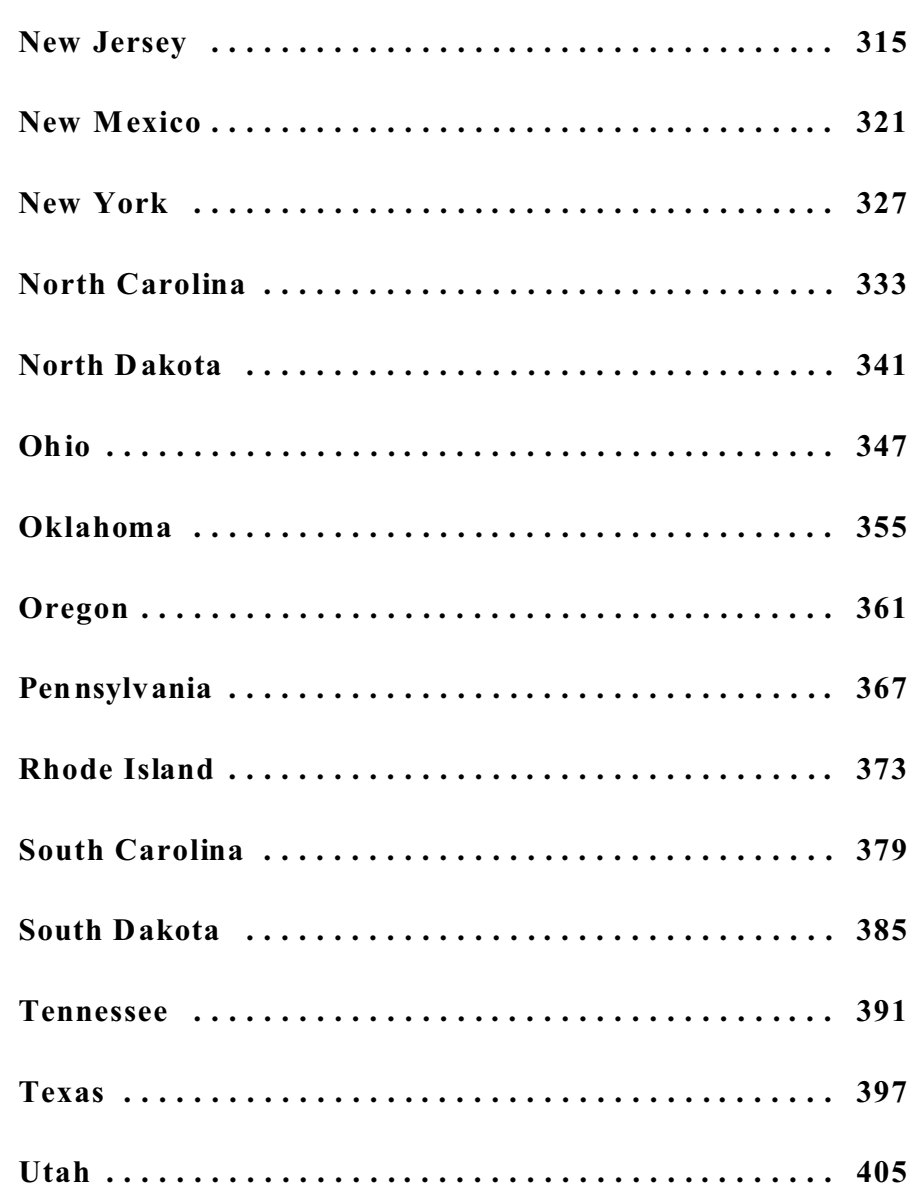

Vermont $\ldots \ldots \ldots \ldots \ldots \ldots \ldots \ldots \ldots \ldots 411$

Virginia $\ldots \ldots \ldots \ldots \ldots \ldots \ldots \ldots \ldots \ldots 417$

Washington .................. 425

West Virginia $\ldots \ldots \ldots \ldots \ldots \ldots \ldots \ldots \ldots 431$

Wisconsin $\ldots \ldots \ldots \ldots \ldots \ldots \ldots \ldots \ldots 437$

Wyoming .................... 443

APPENDICES

Appendix A. Sources of Data ......... A-1

Appendix B. Methods $\ldots \ldots \ldots \ldots \ldots \ldots$ B-1

Appendix C. Reporting States........ C-1

Appendix D. Exposure Categories ...... D-1

Appendix E. Previous Reports

Summaries .......... E-1

Revisions ............ E-2

Errata ............ E-18 
HIGHLIGHTS and LIMITATIONS 
The following paragraphs highlight data presented in the United States Section of this report. Many other highlights could have been mentioned, both from the United States Section and from the State Section.

\section{Pneumoconiosis Mortality}

During the 25-year period from 1968 to 1992 there were a total of $\mathbf{1 0 0 , 8 9 0}$ deaths with pneumoconiosis among U.S. residents, age 15 and over.

Overall pneumoconiosis mortality in the U.S. has been gradually declining over the past two decades, from a peak of more than 5,000 deaths in 1972 to 3,230 in 1992. Mining industries have the two highest (and three of the five highest) proportionate mortality ratios for all pneumoconioses by industry. Ship building and repair, various manufacturing industries, and construction also have significantly elevated PMRs.

Asbestosis deaths represent 10 percent of pneumoconiosis deaths during the 25-year period from 1968 to 1992, and have increased from fewer than 100 to nearly 1,000 annually. Over the most recent three-year period, 1990 to 1992, however, asbestosis mortality appears to have leveled off. The geographic distribution of asbestos mortality tends to be coastal, and nearly 25 percent of asbestosis decedents during the 1968-1992 period were residents of California and New Jersey. Based on a large subset of the national data, various construction occupations account for many of these deaths.

Coal workers' pneumoconiosis (CWP) deaths clearly outnumber other types of pneumoconiosis, amounting to nearly 60 percent of pneumoconiosis deaths over the 25-year period from 1968 to 1992. Over the past decade, CWP mortality has declined each year and CWP deaths number fewer than 2,000 annually for 1990, 1991, and 1992. CWP mortality has been concentrated largely in coal producing states, and about two-thirds of all CWP decedents from 1968-1992 were accounted for by residents of Pennsylvania, alone. Nearly all CWP deaths were associated with employment in the coal mining industry, for which the CWP proportionate mortality ratio of $100(95 \% \mathrm{CI}=97-104)$ was calculated based on a large subset of the national data from 1985 to 1992.

Silicosis deaths represent nearly 15 percent of all pneumoconiosis deaths in the U.S. from 1968 to 1992. Over these 25 years, mortality associated with silicosis has declined more than that associated with other types of pneumoconiosis, from well over 1,000 deaths annually before 1971 to less than 300 in 1992. Compared to asbestosis, CWP, and byssinosis, silicosis mortality is much less concentrated by geographic region or by industry.

In comparison with other pneumoconioses, byssinosis deaths (as enumerated from death certificate data) remain very few in number--less than 20 annually. Byssinosis mortality is concentrated in the textile producing areas of North and South Carolina, and only one industry ("yarn, thread, and fabric mills") was associated with high byssinosis mortality in the proportionate mortality ratio analysis $(\mathrm{PMR}=33 ; 95 \% \mathrm{CI}=23-45)$.

The pattern of deaths from unspecified/other pneumoconioses, which account for 18 percent of all pneumoconiosis deaths during the 25 -year period from 1968 to 1992, resembles CWP mortality with respect to: geographic distribution; a clear peak in 1972; and associated occupations and industries.

\section{Occupational Respiratory Hazard Sampling}

During a recent 2-year period (1993-1994), non-mining industries accounted for about 9 percent of all reported federal inspector samples for airborne pneumoconiotic agents. Within the non-mining industries, the construction industry was the most frequently sampled, but fewer than 1,000 samples were reported in this industry over these same two years. The average severity level for samples collected in the construction industry was 1.51 . This indicates that, on average, measured exposure levels in the construction industry were about one and onehalf times the applicable PEL. 
Approximately one-half of all federal inspector samples for airborne pneumoconiotic agents $(45,132$ of 83,475 samples) reported for a recent 2-year period (1993-1994) were collected in the coal mining industry. The average severity level for respirable coal mine dust samples was less than 0.5 , indicating that, on average, exposure concentrations measured by these inspectors were less than 1.0 $\mathrm{mg} / \mathrm{m}^{3}$. For comparison, the respirable coal mine dust permissible exposure limit (PEL), unadjusted for silica content, is $2.0 \mathrm{mg} / \mathrm{m}^{3}$ MRE.

The data presented in this report indicate that, in recent years, OSHA and MSHA inspectors have found overexposures to silica more frequently and in more states than overexposures to other pneumoconiotic agents. For the years 1985-1994, approximately 15 percent of the 147,000 OSHA and MSHA inspector samples for silica were in excess of the PEL. By comparison, for the same time period, approximately 7 percent of the 268,000 OSHA and MSHA inspector samples for all other pneumoconiotic agents were in excess of the PEL. The OSHA inspectors in 42 states reported at least 10 percent of their silica samples in excess of the PEL for the years 1985-1994. MSHA inspectors in 29 states reported at least 10 percent of their silica samples in excess of the PEL in the same time period.

OSHA inspectors found overexposures to cotton dust for 31 percent of the 543 samples obtained during 1985-1994; the data were reported from six states. 


\section{Selected Limitations}

\section{Mortality Data}

The focus of this report is on pneumoconiosis. Readers are therefore cautioned not to erroneously infer that the data presented are comprehensive for occupational lung diseases in general. Other types of lung disease (e.g., asthma and other obstructive airways disease) are also caused by exposure to respiratory hazards in the workplace. However, for most other work-related lung diseases, in contrast with pneumoconiosis, an assumption of an occupational etiology on the basis of death certificate information alone cannot be made without significant misclassification.

The assumption of work-relatedness of pneumoconiosis is reasonable for this surveillance report. Readers are cautioned, however, to realize that a very small proportion of those who die with pneumoconiosis may have developed disease as a result of non-occupational (e.g., avocational) exposure to pneumoconiotic agents.

The focus of this report is on pneumoconiosis mortality, since national pneumoconiosis morbidity data are not readily available. Readers are therefore cautioned not to erroneously infer that data presented are comprehensive for pneumoconiosis in general. Pneumoconiosis is typically (though not always) a chronic disease and, as reflected in median age at death data presented in this report, many affected individuals live to or past life expectancy. The fact that many individuals with pneumoconiosis do not die as a direct result of the pneumoconiosis has led to the decision to consider underlying and all contributing causes of death for each decedent in the data presented in this report. Readers are therefore cautioned to understand that for many pneumoconiosis decedents for which data are presented in this report, pneumoconiosis is a contributing cause of death, not necessarily the underlying cause.

Categorization of types of pneumoconiosis are limited by the ICD coding systems in use over the 25 -year period covered by data presented in this report. ICD-8 to ICD-9 disease rubrics differ somewhat for all types of pneumoconiosis except for asbestosis (see table in Appendix B.) This fact alone may have had an effect on the classification of cause of death, although the effect is not considered substantial (e.g., there is no indication in the yearly trend in national silicosis mortality, despite a change in the ICD disease category related to silicosis).

As with any analysis based on death certificate data, there is undoubtedly some misclassification of cause of death. A treating physician may not correctly diagnose pneumoconiosis during a patient's life or, upon death, a certifying physician may fail to list an existing diagnosis of pneumoconiosis on the death certificate, particularly if other diseases were directly responsible for the decedent's death.

As mentioned in the Preface, some occupational lung disease experts do not consider byssinosis as a pneumoconiosis. One feature that clearly distinguishes byssinosis from the more typical pneumoconioses is the absence of characteristic lung opacities on radiographic examination of the chest. In advanced stages of the disease, byssinosis is therefore clinically indistinguishable from other chronic obstructive pulmonary diseases. This may lead to more substantial underdiagnosis of byssinosis compared with that of the pneumoconioses associated with radiographic abnormalities.

Physician diagnostic practices are influenced by many factors - not only advances in medical knowledge, including awareness of jobs and industries associated with particular hazardous occupational exposures, but also changing occupational disease compensation programs (e.g., a Federal "Black Lung" compensation program was established for coal miners following the passage of the Federal Mine Heath and Safety Act of 1969) and pressure associated with private litigation (e.g., many third party torts based on injury resulting from adverse health outcomes associated with asbestos have appeared in the courts over the past 20 years). These and other factors are undoubtedly reflected in data presented in this report (e.g., the obvious peak in CWP deaths in 1972). 
County and state of residence at death may not represent the geographic location of the decedent's occupational exposure to pneumoconiotic agents. The geographic pattern of CWP mortality is illustrative in this regard, in that it highlights not only coal producing areas associated with risk of disease, but also areas in Florida and California to which many affected miners have retired.

Usual occupation and industry codes are often, but not always, those which were associated with the exposure responsible for the pneumoconiosis. Readers are therefore cautioned with regard to assuming causative inferences about all occupations and industries associated with pneumoconiosis in various tables in this report. Moreover, readers are reminded that usual occupation and industry data analyzed in this report represent only a subset of the mortality data (i.e., for selected states from 1985 to 1992, see Appendix C).

Denominators used to calculate rates presented in this report are based on general population estimates for the location (e.g., nation, state, or county) and for the years in which the deaths occurred. The resulting rates have clear public health significance, but readers are cautioned not to fail to realize, as suggested by the proportionate mortality ratios presented in this report by occupation and by industry, that rates are much higher in certain occupational groups. Apparent changes in mortality rates may reflect, in part or in whole, changes in employment patterns affecting the number of people at risk.

Over the 25-year period covered by data presented in this report, median ages at death have been generally increasing for all types of pneumoconiosis. The reader is cautioned to realize that this increase is the result of many factors, only one of which may be a general lessening of pneumoconiosis severity (e.g., due to enhanced diagnostic sensitivity in addition to fewer severe cases). Other contributing factors undoubtedly include less mortality pressure from other causes of death, in general.
Many tables and figures presented in this report contain small numbers. Those responsible for preparing this report decided to err on the side of providing all the data (i.e., not censoring on the basis of small numbers), recognizing that by doing so opportunities are being provided for overinterpretation of the data. The small-numbers issue applies especially in the State Section, where data are presented by county, but also applies in the United States Section of the report. Moreover, rates have been calculated on the basis of these small numbers and are shown, along with rankings of these rates. The reader is cautioned that rates based on small numbers can be quite unstable, so any inferences should be drawn with care. By keeping both rates and counts in mind while considering the tabled data, and/or by logically aggregating cells (e.g., county-specific data) on the basis of the location of particular industries within a particular state, knowledgeable individuals can use the data in this report to identify potential opportunities for enhancement of efforts to prevent pneumoconiosis.

\section{Exposure Data}

Industrial hygiene inspection records maintained by OSHA, MSHA, and the former Bureau of Mines were used in this report as a gauge of the range of exposures to pneumoconiotic agents in U.S. industry. These data are considered provisional and subject to revision as additional information becomes available. The inspector samples were gathered for regulatory compliance purposes, rather than for the surveillance of worker exposures. Nonetheless, the inspector data reported herein are the best available information for assessing the range of exposures encountered by U.S. workers on a national scale.

MSHA and OSHA inspection data for similar exposure agents are presented in this report in a parallel format. The reader is cautioned that MSHA and OSHA are separate agencies with separate regulatory jurisdictions. Thus, the number of samples gathered by one agency versus the other, or by one agency from year to year, is not necessarily a valid basis for comparison. A variety of factors 
(e.g., Congressional actions, regulatory policies, and changes in analytical methods) can affect the number of samples and the exposure levels being reported by the inspectors.

The list of pneumoconiotic agents used to select inspection sample records from available OSHA and MSHA data files was defined based on the agents which MSHA and OSHA have included in their computerized data systems, and is not a comprehensive list of all agents associated with pneumoconiosis. Nevertheless, the listed agents are associated with what are widely recognized as the most prevalent types of pneumoconiosis. Some agents for which an association with pneumoconiosis is less clear-cut (e.g., OSHA data for "particulates not otherwise regulated") were not included in the analyzed data.

The exposure data analyzed in this report include 69,427 MSHA inspector samples for respirable particulates not otherwise regulated, from non-coal mines. MSHA inspector sampling policies indicate that these samples were, in fact, originally obtained due to the potential for silica exposures. For that reason, the report includes those 69,427 samples as a part of the silica exposure dataset. The reader is cautioned that this inclusion marks a departure from the analytical approach used in previous WoRLD Surveillance Reports.
The severity levels for pneumoconiotic agents were generally derived by dividing the exposure concentrations by the permissible exposure limit (PEL), and can therefore generally be considered related to actual enforceable PELs. However, there are important exceptions to this general rule. Specifically, due to complexities related to determination of enforceable PELs associated with respirable coal mine dust and with respirable coal mine quartz samples, denominators used to calculate the average severity levels for respirable coal mine dust and for respirable coal mine quartz were 2.0 $\mathrm{mg} / \mathrm{m}^{3} \mathrm{MRE}$ and $0.1 \mathrm{mg} / \mathrm{m}^{3} \mathrm{MRE}$, respectively (see Appendix B.)

Although available exposure data for agents associated with each type of pneumoconiosis are presented in this report following the presentation of mortality data for that same condition, the reader is reminded that the time period over which the exposure data were collected is not expected to correspond to the time period during which most of the decedents represented in the mortality data acquired their disease. For most pneumoconiosis deaths, there is a typical latency period of at least several years between first occupational exposure and onset of disease, and subsequent death typically occurs many years after disease onset.

For the time period covered by this report (through 1994), MSHA's respirable coal mine dust inspector samples with less than $0.45 \mathrm{mg}$ net weight gain were not analyzed for quartz. 


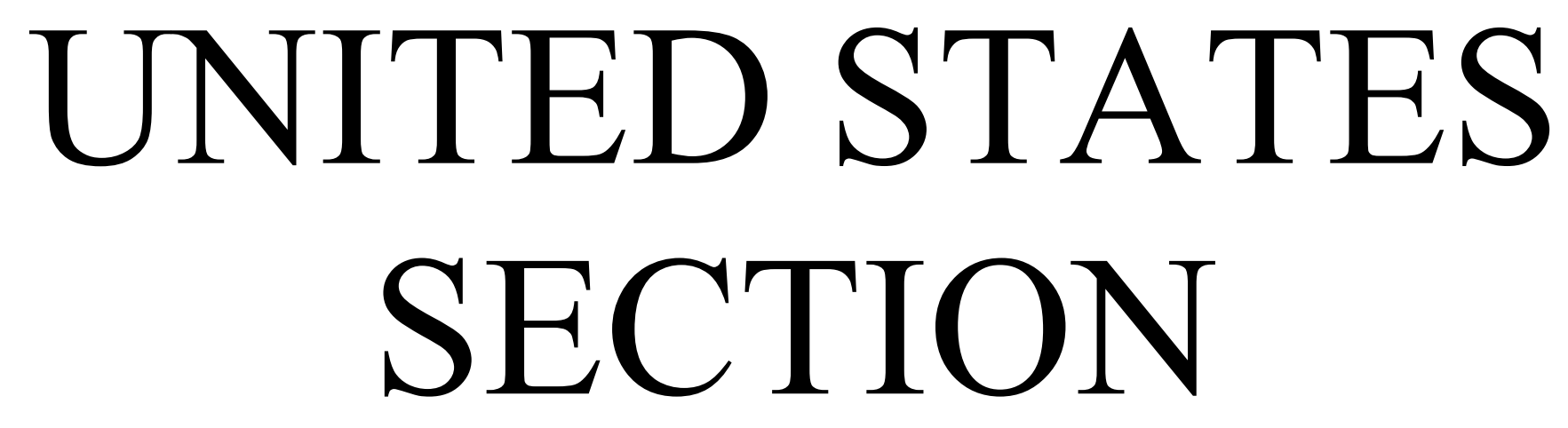




\section{United States Section}

\section{Asbestosis Mortality}

Figure 1-1. Asbestosis: Number of deaths, crude and age-adjusted mortality rates, U.S. residents age 15 and over, 1968-1992

Figure 1-2. Asbestosis: Crude mortality rates by state, U.S. residents age 15 and over, 1991-1992

Figure 1-3. Asbestosis: Median age at death, U. S. residents age 15 and over, 1968-1992

Table 1-1. Asbestosis: Number of deaths by sex, race, and age, U.S. residents age 15 and over, 1991-1992

Table 1-2. Asbestosis: Number of deaths by state, U.S. residents age 15 and over, 1968-1992

Table 1-3. Asbestosis: Mortality rates (per 1,000,000 population) by race and sex, U.S. residents age 15 and over, 1991-992

Table 1-4. Asbestosis: Years of potential life lost by race and sex, U.S. residents age 15 and over, 1991-1992

Table 1-5. Asbestosis: Total number of deaths, crude and age-adjusted mortality rates (per 1,000,000 population), and total years of potential life lost (YPLL) to life expectancy, by state, U.S. residents age 15 and over, 1988-1992

Table 1-6. Asbestosis: Most frequently recorded occupations on death certificate, selected states, U.S. residents age 15 and over, 1991-1992

Table 1-7. Asbestosis: Most frequently recorded industries on death certificate, selected states, U.S. residents age 15 and over, 1991-1992

Table 1-8. Asbestosis: Proportionate mortality ratio (PMR) by usual occupation, selected states and years, U.S. residents age 15 and over, 1985-1992

Table 1-9. Asbestosis: Proportionate mortality ratios (PMR) by usual industry, selected states and years, U.S. residents age 15 and over, 1985-1992

Figure 1-4. Asbestosis: Total number of deaths by county, U.S. residents age 15 and over, 1983-1992

Figure 1-5. Asbestosis: Age-adjusted mortality rates by county, U.S. residents age 15 and over, 1983-1992

\section{Asbestos Exposure}

Figure 1-6. Asbestos: Number of MSHA and OSHA inspector samples and percent exceeding the permissible exposure limit (PEL), 1974-1994

Figure 1-7. Asbestos: Number of MSHA and OSHA inspector samples and average severity levels, 1974-1994

Table 1-10. Asbestos: Number of MSHA and OSHA inspector samples, percent exceeding the permissible exposure limit (PEL) and average severity level, by industry, 1993-1994

Table 1-11. Asbestos: Number of MSHA and OSHA inspector samples, percent exceeding the permissible exposure limit (PEL) and average severity level, by industry, 1993-1994

Table 1-12. Asbestos: Number of MSHA inspector samples, percent exceeding the permissible exposure limit (PEL) and average severity levels (Avg. Sev.), by state, 1974-1994

Figure 1-8. Asbestos: Number of MSHA inspector samples collected by state, 1993-1994

Figure 1-9. Asbestos: Percent of MSHA inspector samples that exceeded the permissible exposure limit (PEL), by state, 1993-1994

Figure 1-10. Asbestos: Average severity level by state for MSHA inspector samples, 1993-1994

Figure 1-11. Asbestos: Average severity level by state for MSHA inspector samples that exceeded the permissible exposure limit (PEL), 1993-1994

Figure 1-12. Asbestos: Number of MSHA inspector samples collected, by county, 1993-1994 
Table 1-13. Asbestos: Number of OSHA inspector samples, percent exceeding the permissible exposure limit (PEL) and average severity levels (Avg. Sev.), by state, 1979-1994

Figure 1-13. Asbestos: Number of OSHA inspector samples collected by state, 1993-1994

Figure 1-14. Asbestos: Percent of OSHA inspector samples that exceeded the permissible exposure limit (PEL), by state, 1993-1994

Figure 1-15. Asbestos: Average severity level by state for OSHA inspector samples, 1993-1994

Figure 1-16. Asbestos: Average severity level by state for OSHA inspector samples that exceeded the permissible exposure limit (PEL), 1993-1994

Figure 1-17. Asbestos: Number of OSHA inspector samples collected by county, 1993-1994

Coal Workers' Pneumoconiosis Mortality

All tables and figures are analogous to those listed under Asbestosis Mortality.

\section{Coal and Coal Mine Dust Exposure}

All tables and figures are analogous to those listed under Asbestos Exposure.

\section{Silicosis Mortality}

All tables and figures are analogous to those listed under Asbestosis Mortality.

\section{Silica Exposure}

All tables and figures are analogous to those listed under Asbestos Exposure.

\section{Byssinosis Mortality}

All tables and figures are analogous to those listed under Asbestosis Mortality.

\section{Cotton Dust Exposure}

Figure 4-6. Cotton dust: Number of OSHA inspector samples and percent exceeding the permissible exposure limit (PEL), 1979-1994

Figure 4-7. Cotton dust: Number of OSHA inspector samples and average severity levels, 1979-1994

Table 4-10. Cotton dust: Number of OSHA inspector samples, percent exceeding the permissible exposure limit (PEL) and average severity level, by industry, 1993-1994

Table 4-11. Cotton dust: Number of OSHA inspector samples, percent exceeding the permissible exposure limit (PEL) and average severity level, by industry, 1993-1994

Table 4-12. Cotton dust: Number of OSHA inspector samples, percent exceeding the permissible exposure limit (PEL) and average severity levels (Avg. Sev.), by state, 1979-1994

Figure 4-8. Cotton dust: Number of OSHA inspector samples collected by state, 1993-1994

Figure 4-9. Cotton dust: Percent of OSHA inspector samples that exceeded the permissible exposure limit (PEL), by state, 1993-1994

Figure 4-10. Cotton dust: Average severity level by state for OSHA inspector samples, 1993-1994

Figure 4-11. Cotton dust: Average severity level by state for OSHA inspector samples that exceeded the permissible exposure limit (PEL), 1993-1994

Figure 4-12. Cotton dust: Number of OSHA inspector samples collected by county, 1993-1994

\section{Unspecified/Other Pneumoconioses Mortality}

All tables and figures are analogous to those listed under Asbestosis Mortality. 


\section{All Pneumoconioses Mortality}

Figure 6-1. All pneumoconioses: Number of deaths, crude and age-adjusted mortality rates, U.S. residents age 15 and over, 1968-1992

Figure 6-2. All pneumoconioses: Crude mortality rates by state, U.S. residents age 15 and over, 1991-1992

Table 6-1. All pneumoconioses: Number of deaths by state, U.S. residents age 15 and over, 1968-1992

Table 6-2. All pneumoconioses: Percent of deaths by condition and state, U.S. residents age 15 and over, 1968-1992

Table 6-3. All pneumoconioses: Total number of deaths, crude and age-adjusted mortality rates (per 1,000,000 population), and total years of potential life lost (YPLL) to life expectancy, by state, U.S. residents age 15 and over, 1988-1992

Table 6-4. All pneumoconioses: Proportionate mortality ratio (PMR) by usual occupation, selected states and years, U.S. residents age 15 and over, 1985-1992

Table 6-5. All pneumoconioses: Proportionate mortality ratios (PMR) by usual industry, selected states and years, U.S. residents age 15 and over, 1985-1992

Figure 6-3. All pneumoconioses: Total number of deaths by county, U.S. residents age 15 and over, 19831992

Figure 6-4. All pneumoconioses: Age-adjusted mortality rates by county, U.S. residents age 15 and over, 1983-1992

\section{Pneumoconiotic Agents Exposure}

Figure 6-5. Pneumoconiotic agents: Number of MSHA and OSHA inspector samples and percent exceeding the permissible exposure limit (PEL), 1970-1994

Figure 6-6. Pneumoconiotic agents: Number of MSHA and OSHA inspector samples and average severity levels, 1970-1994

Table 6-6. Pneumoconiotic agents: Number of MSHA and OSHA inspector samples, percent exceeding the permissible exposure limit (PEL) and average severity level, by industry, 1993-1994

Table 6-7. Pneumoconiotic agents: Number of MSHA and OSHA inspector samples, percent exceeding the permissible exposure limit (PEL) and average severity level, by industry, 1993-1994

Table 6-8. Pneumoconiotic agents: Number of MSHA inspector samples, percent exceeding the permissible exposure limit (PEL) and average severity levels (Avg. Sev.), by state, 1970 -1994

Figure 6-7. Pneumoconiotic agents: Number of MSHA inspector samples collected by state, 1993-1994

Figure 6-8. Pneumoconiotic agents: Percent of MSHA inspector samples that exceeded the permissible exposure limit (PEL), by state, 1993-1994

Figure 6-9. Pneumoconiotic agents: Average severity level by state for MSHA inspector samples, 1993-1994

Figure 6-10. Pneumoconiotic agents: Average severity level by state for MSHA inspector samples that exceeded the permissible exposure limit (PEL), 1993-1994

Figure 6-11. Pneumoconiotic agents: Number of MSHA inspector samples collected by county, 1993-1994

Table 6-9. Pneumoconiotic agents: Number of OSHA inspector samples, percent exceeding the permissible exposure limit (PEL) and average severity levels (Avg. Sev.), by state, 1979-1994

Figure 6-12. Pneumoconiotic agents: Number of OSHA inspector samples collected by state, 1993-1994

Figure 6-13. Pneumoconiotic agents: Percent of OSHA inspector samples that exceeded the permissible exposure limit (PEL), by state, 1993-1994

Figure 6-14. Pneumoconiotic agents: Average severity level by state for OSHA inspector samples, 1993-1994

Figure 6-15. Pneumoconiotic agents: Average severity level by state for OSHA inspector samples that exceeded the permissible exposure limit (PEL), 1993-1994

Figure 6-16. Pneumoconiotic agents: Number of OSHA inspector samples collected by county, 1993-1994 
Figure 1-1. Asbestosis: Number of deaths, crude and age-adjusted mortality rates, U.S. residents age 15 and over, 1968-1992

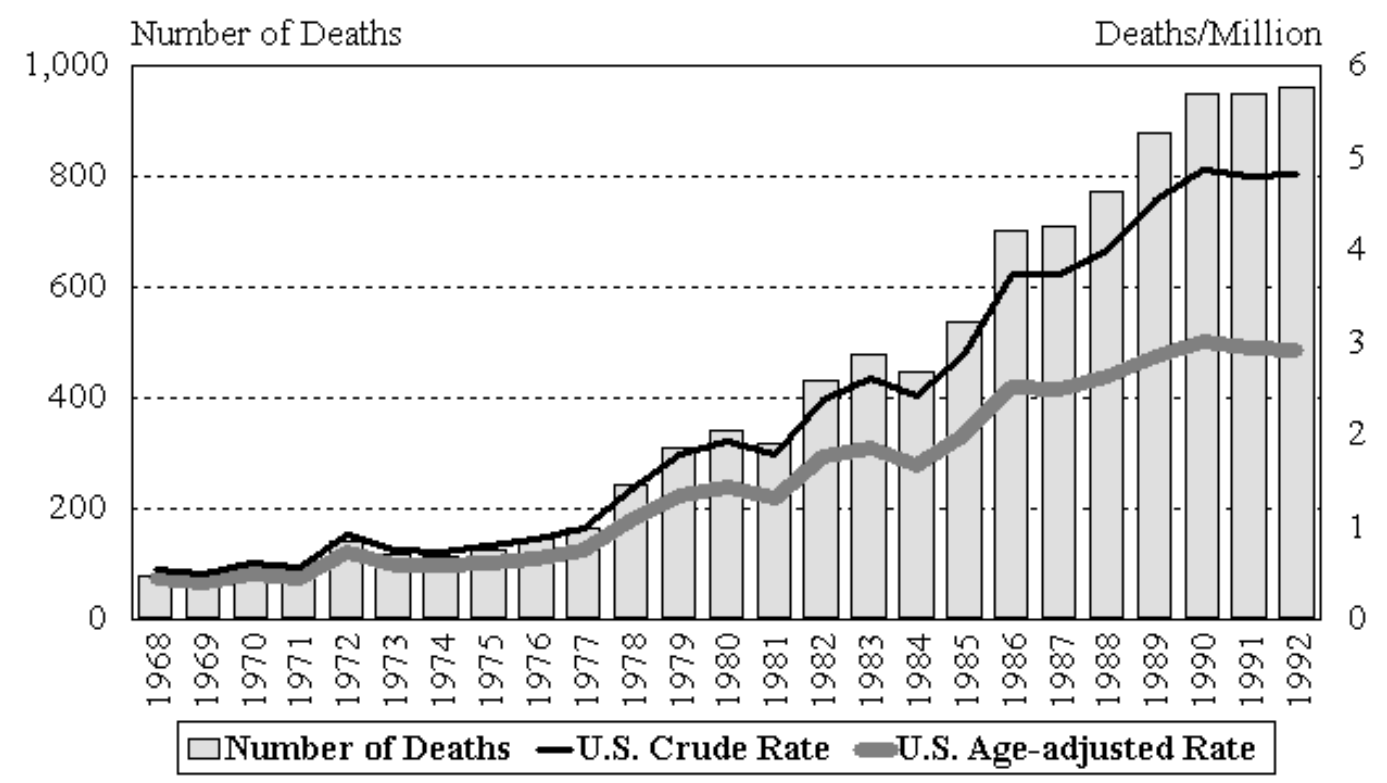

NOTE: $\quad$ See Appendix A for source description and Appendix B for methods and ICD-8 and ICD-9 codes.

SOURCE: National Center for Health Statistics multiple cause of death data. Population estimates from U.S. Bureau of the Census.

Figure 1-2. Asbestosis: Crude mortality rates by state, U.S. residents age 15 and over, 1991-1992

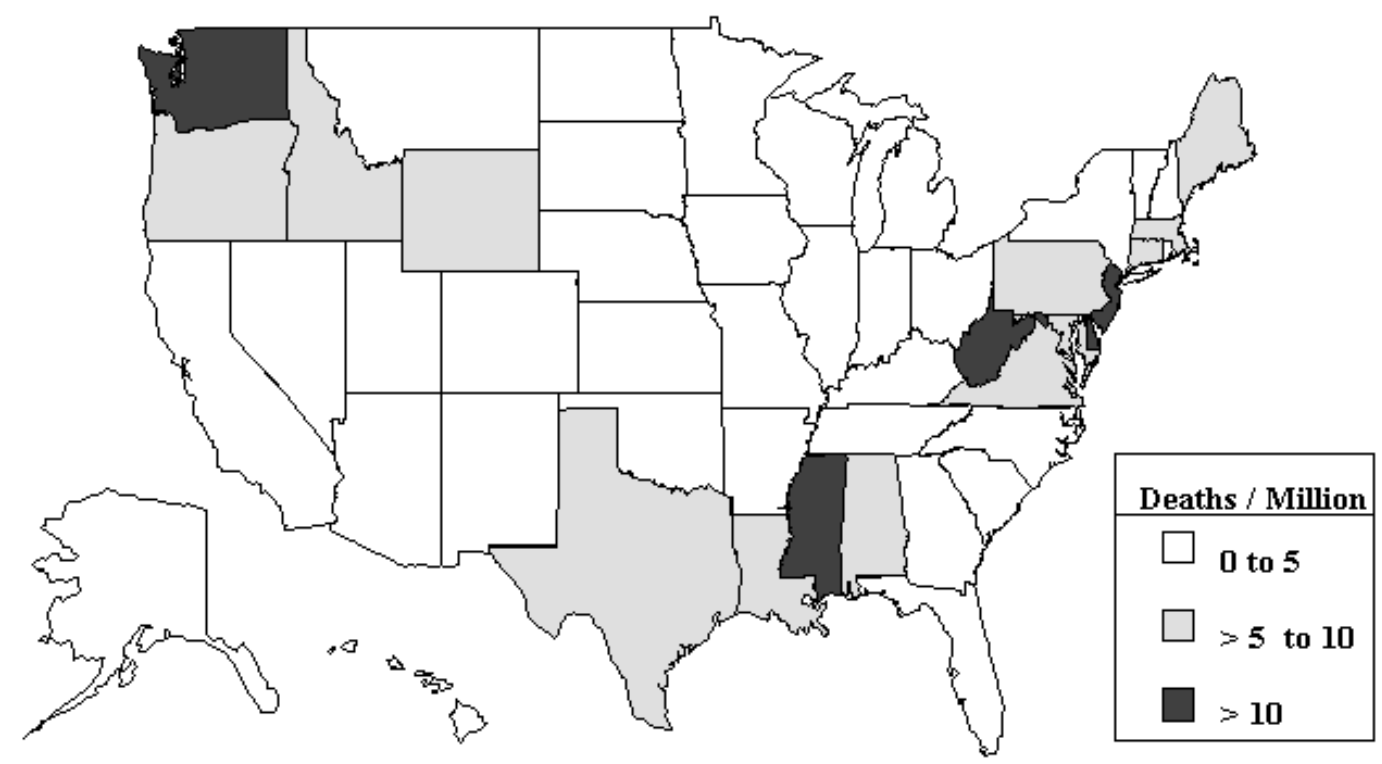

NOTE: $\quad$ See Appendix A for source description and Appendix B for methods and ICD-8 and ICD-9 codes.

SOURCE: National Center for Health Statistics multiple cause of death data. Population estimates from U.S. Bureau of the Census. 
Figure 1-3. Asbestosis: Median age at death, U.S. residents age 15 and over, 1968-1992

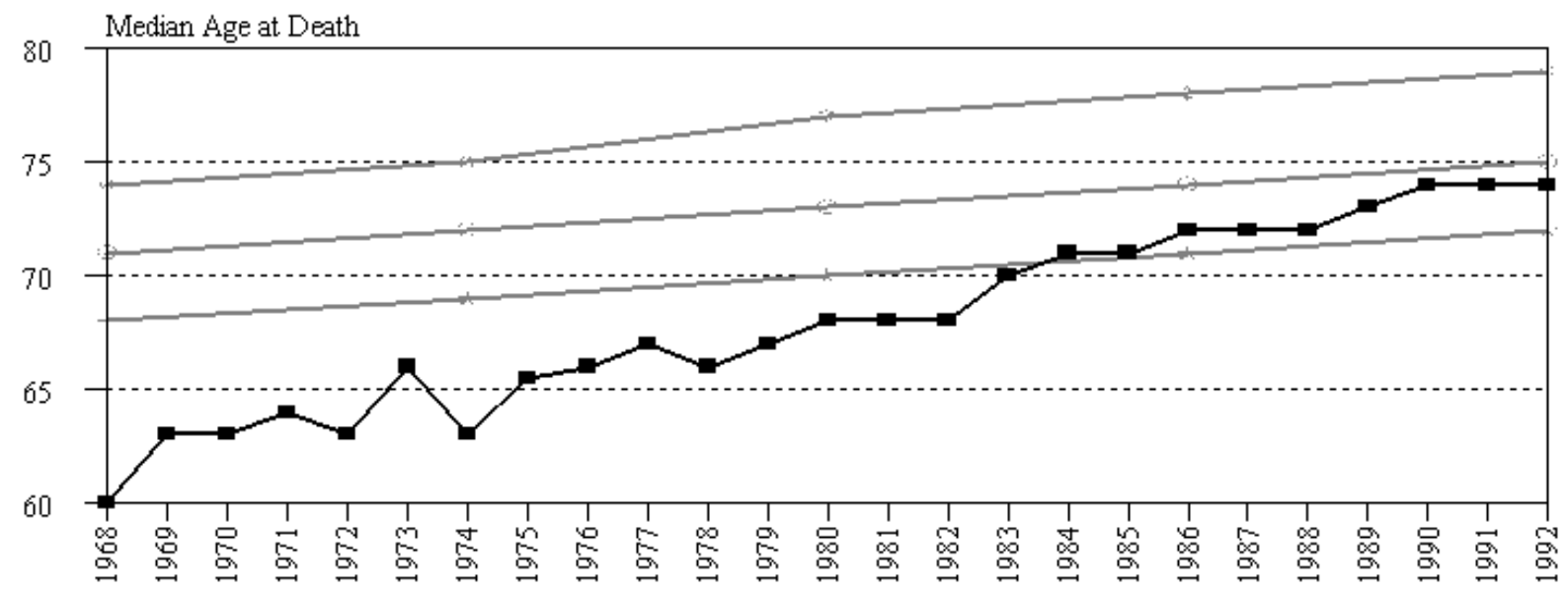

\begin{tabular}{|c|c|}
\hline - Asbestosis Deaths & U.S. Deaths \\
\hline *U.S. Male Deaths & -U.S. Female Deaths \\
\hline
\end{tabular}

NOTE: See Appendix A for source description and Appendix B for methods and ICD-8 and ICD-9 codes.

SOURCE: National Center for Health Statistics multiple cause of death data.

Table 1-1. Asbestosis: Number of deaths by sex, race, and age, U.S. residents age 15 and over, 1991-1992

\begin{tabular}{|c|c|c|c|c|c|}
\hline & & \multicolumn{2}{|c|}{1991} & \multicolumn{2}{|c|}{1992} \\
\hline & & Number & Percent & Number & Percent \\
\hline \multicolumn{2}{|c|}{ Total deaths } & 946 & 100.0 & 959 & 100.0 \\
\hline \multirow[t]{2}{*}{ Sex } & Male & 908 & 96.0 & 923 & 96.2 \\
\hline & Female & 38 & 4.0 & 36 & 3.8 \\
\hline \multirow[t]{3}{*}{ Race } & White & 877 & 92.7 & 898 & 93.6 \\
\hline & Black & 63 & 6.7 & 57 & 5.9 \\
\hline & Other & 6 & 0.6 & 4 & 0.4 \\
\hline \multirow[t]{10}{*}{ Age } & $15-24$ & 1 & 0.1 & 0 & 0.0 \\
\hline & $25-34$ & 0 & 0.0 & 0 & 0.0 \\
\hline & $35-44$ & 1 & 0.1 & 3 & 0.3 \\
\hline & $45-54$ & 25 & 2.6 & 13 & 1.4 \\
\hline & $55-64$ & 114 & 12.1 & 124 & 12.9 \\
\hline & $65-74$ & 370 & 39.1 & 371 & 38.7 \\
\hline & $75-84$ & 358 & 37.8 & 355 & 37.0 \\
\hline & 85 and over & 77 & 8.1 & 93 & 9.7 \\
\hline & Mean age & 73.1 & & 73.5 & \\
\hline & Range for age & $19-96$ & & $38-100$ & \\
\hline
\end{tabular}


Table 1-2. Asbestosis: Number of deaths by state, U.S. residents age 15 and over, 1968-1992

\begin{tabular}{|c|c|c|c|c|c|}
\hline State & 1968-1978 & 1979-1990 & 1991 & 1992 & TOTAL \\
\hline Alabama & 6 & 92 & 23 & 18 & 139 \\
\hline Alaska & 3 & 4 & 1 & 1 & 9 \\
\hline Arizona & 20 & 64 & 6 & 8 & 98 \\
\hline Arkansas & 1 & 35 & 6 & 5 & 47 \\
\hline California & 157 & 954 & 94 & 95 & 1,300 \\
\hline Colorado & 5 & 38 & 5 & 4 & 52 \\
\hline Connecticut & 22 & 94 & 14 & 17 & 147 \\
\hline Delaware & 4 & 37 & 14 & 8 & 63 \\
\hline District of Columbia & 2 & 6 & 1 & - & 9 \\
\hline Florida & 37 & 348 & 54 & 52 & 491 \\
\hline Georgia & 16 & 78 & 10 & 18 & 122 \\
\hline Hawaii & 1 & 31 & 4 & 4 & 40 \\
\hline Idaho & 3 & 33 & 6 & 3 & 45 \\
\hline Illinois & 40 & 147 & 20 & 21 & 228 \\
\hline Indiana & 9 & 49 & 4 & 4 & 66 \\
\hline Iowa & 5 & 25 & 3 & 7 & 40 \\
\hline Kansas & 1 & 23 & 3 & 7 & 34 \\
\hline Kentucky & 2 & 31 & 5 & 5 & 43 \\
\hline Louisiana & 13 & 113 & 20 & 14 & 160 \\
\hline Maine & 9 & 98 & 8 & 8 & 123 \\
\hline Maryland & 19 & 172 & 27 & 33 & 251 \\
\hline Massachusetts & 104 & 340 & 27 & 48 & 519 \\
\hline Michigan & 19 & 85 & 15 & 16 & 135 \\
\hline Minnesota & 11 & 68 & 6 & 17 & 102 \\
\hline Mississippi & 2 & 97 & 25 & 25 & 149 \\
\hline Missouri & 23 & 84 & 11 & 14 & 132 \\
\hline Montana & 3 & 30 & 2 & 4 & 39 \\
\hline Nebraska & 2 & 20 & 3 & 2 & 27 \\
\hline Nevada & 3 & 19 & 3 & 1 & 26 \\
\hline New Hampshire & 5 & 48 & 1 & 4 & 58 \\
\hline New Jersey & 232 & 800 & 93 & 80 & 1,205 \\
\hline New Mexico & 4 & 19 & 3 & 1 & 27 \\
\hline New York & 91 & 272 & 37 & 30 & 430 \\
\hline North Carolina & 24 & 140 & 21 & 25 & 210 \\
\hline North Dakota & - & 6 & 3 & - & 9 \\
\hline Ohio & 32 & 167 & 24 & 32 & 255 \\
\hline Oklahoma & 3 & 39 & 6 & 5 & 53 \\
\hline Oregon & 25 & 144 & 22 & 22 & 213 \\
\hline Pennsylvania & 165 & 640 & 83 & 100 & 988 \\
\hline Rhode Island & 5 & 48 & 1 & 5 & 59 \\
\hline South Carolina & 37 & 100 & 8 & 13 & 158 \\
\hline South Dakota & 1 & 2 & - & - & 3 \\
\hline Tennessee & 12 & 73 & 8 & 4 & 97 \\
\hline Texas & 34 & 354 & 95 & 54 & 537 \\
\hline Utah & 1 & 16 & - & 4 & 21 \\
\hline Vermont & 2 & 11 & - & 3 & 16 \\
\hline Virginia & 39 & 271 & 36 & 43 & 389 \\
\hline Washington & 81 & 342 & 50 & 40 & 513 \\
\hline West Virginia & 6 & 88 & 20 & 28 & 142 \\
\hline Wisconsin & 16 & 56 & 11 & 6 & 89 \\
\hline Wyoming & 2 & 5 & 4 & 1 & 12 \\
\hline TOTAL & 1,359 & 6,856 & 946 & 959 & 10,120 \\
\hline
\end{tabular}

- indicates no deaths listed.

NOTE: $\quad$ See Appendix A for source description and Appendix B for methods and ICD-8 and ICD-9 codes.

SOURCE: National Center for Health Statistics multiple cause of death data. 
Table 1-3. Asbestosis: Mortality rates (per 1,000,000 population) by race and sex, U.S. residents age 15 and over, 1991-1992

\begin{tabular}{|c|c|c|c|c|c|}
\hline \multirow[b]{2}{*}{ Year } & \multirow[b]{2}{*}{ Overall rate } & \multicolumn{2}{|c|}{ White } & \multicolumn{2}{|c|}{ Black } \\
\hline & & Males & Females & Males & Females \\
\hline & \multicolumn{5}{|c|}{ Crude mortality rate } \\
\hline 1991 & 4.80 & 10.42 & 0.42 & 5.86 & 0.16 \\
\hline \multirow[t]{2}{*}{1992} & 4.82 & 10.63 & 0.37 & 5.00 & 0.32 \\
\hline & \multicolumn{5}{|c|}{ Age-adjusted mortality rate } \\
\hline 1991 & 2.94 & 6.91 & 0.20 & 5.79 & 0.14 \\
\hline 1992 & 2.90 & 6.93 & 0.17 & 4.99 & 0.28 \\
\hline NOTE: & $\begin{array}{l}\text { See Appendix A for source descriptio } \\
\text { Work-Related Lung Disease Surveilla } \\
\text { See Appendix E for revised rates for } \\
\text { National Center for Health Statistics }\end{array}$ & $\begin{array}{l}\text { lix B for } \mathrm{m} \\
994, \text { see A } \\
\text { of death }\end{array}$ & ICD-8 and & es. Data ff & an be found $\mathrm{i}$ \\
\hline
\end{tabular}

\section{Table 1-4. Asbestosis: Years of potential life lost by race and sex, U.S. residents age 15 and over, 1991-1992}

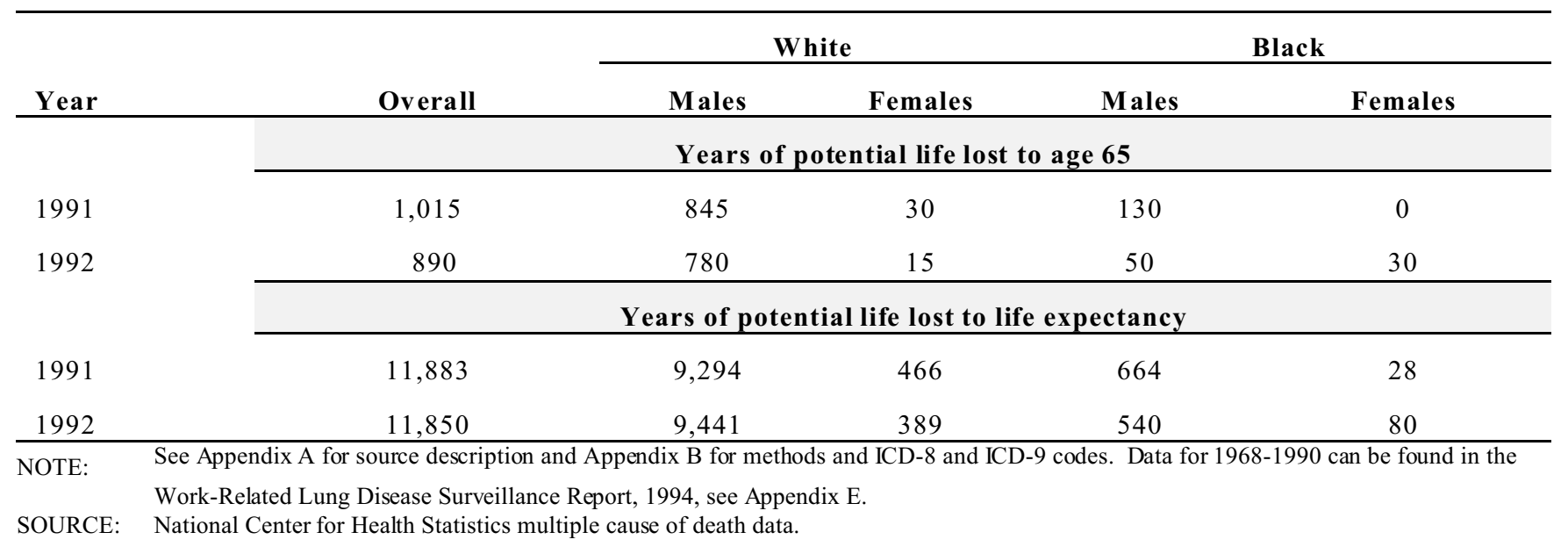


Table 1-5. Asbestosis: Total number of deaths, crude and age-adjusted mortality rates (per 1,000,000 population), and total years of potential life lost (YPLL) to life expectancy, by state, U.S. residents age 15 and over, 1988-1992

\begin{tabular}{|c|c|c|c|c|c|c|c|c|c|c|}
\hline \multirow[b]{2}{*}{ State } & \multirow{2}{*}{$\begin{array}{r}\text { Total } \\
\text { deaths }\end{array}$} & \multirow[b]{2}{*}{ Rank } & \multicolumn{2}{|c|}{ Crude mortality } & \multicolumn{2}{|c|}{ Age-adjusted mortality } & \multicolumn{4}{|c|}{ YPLL to life expectancy } \\
\hline & & & Rate & Rank & Rate & Rank & Years & Rank & Years/death & Rank \\
\hline Alabama & 94 & 15 & 5.94 & 14 & 3.79 & 15 & 1,254 & 14 & 13.3 & 17 \\
\hline Alaska & 5 & 47 & 2.49 & 29 & 3.96 & 13 & 84 & 48 & 16.8 & 2 \\
\hline Arizona & 30 & 29 & 2.11 & 37 & 1.39 & 35 & 414 & 29 & 13.8 & 13 \\
\hline Arkansas & 20 & 36 & 2.18 & 34 & 1.34 & 37 & 283 & 35 & 14.2 & 8 \\
\hline California & 459 & 2 & 3.96 & 23 & 2.75 & 23 & 5,503 & 2 & 12.0 & 40 \\
\hline Colorado & 22 & 33 & 1.71 & 43 & 1.27 & 40 & 309 & 33 & 14.0 & 9 \\
\hline Connecticut & 66 & 19 & 4.98 & 19 & 2.87 & 22 & 797 & 21 & 12.1 & 39 \\
\hline Delaware & 49 & 25 & 18.55 & 1 & 13.08 & 1 & 727 & 23 & 14.8 & 4 \\
\hline District of Columbia & 1 & 50 & 0.39 & 50 & 0.29 & 50 & 14 & 50 & 14.0 & 12 \\
\hline Florida & 264 & 5 & 5.00 & 18 & 2.35 & 26 & 3,287 & 5 & 12.5 & 32 \\
\hline Georgia & 55 & 22 & 2.18 & 34 & 1.79 & 28 & 806 & 20 & 14.7 & 5 \\
\hline Hawaii & 20 & 36 & 4.58 & 22 & 2.91 & 21 & 227 & 39 & 11.4 & 43 \\
\hline Idaho & 22 & 33 & 5.88 & 15 & 3.72 & 17 & 279 & 36 & 12.7 & 29 \\
\hline Illinois & 96 & 14 & 2.15 & 36 & 1.40 & 34 & 1,288 & 13 & 13.4 & 16 \\
\hline Indiana & 26 & 32 & 1.20 & 49 & 0.73 & 49 & 316 & 32 & 12.2 & 37 \\
\hline Iowa & 18 & 40 & 1.66 & 45 & 0.91 & 47 & 232 & 38 & 12.9 & 20 \\
\hline Kansas & 20 & 36 & 2.09 & 38 & 1.05 & 45 & 226 & 40 & 11.3 & 44 \\
\hline Kentucky & 19 & 39 & 1.31 & 48 & 0.87 & 48 & 260 & 37 & 13.7 & 14 \\
\hline Louisiana & 86 & 17 & 5.41 & 16 & 3.59 & 18 & 1,102 & 16 & 12.8 & 23 \\
\hline Maine & 53 & 23 & 10.94 & 4 & 5.96 & 6 & 610 & 25 & 11.5 & 42 \\
\hline Maryland & 136 & 10 & 7.16 & 12 & 5.30 & 8 & 1,825 & 10 & 13.4 & 15 \\
\hline Massachusetts & 184 & 8 & 7.56 & 10 & 4.05 & 12 & 2,054 & 9 & 11.2 & 47 \\
\hline Michigan & 66 & 19 & 1.83 & 41 & 1.32 & 38 & 987 & 19 & 15.0 & 3 \\
\hline Minnesota & 47 & 26 & 2.78 & 26 & 1.78 & 29 & 585 & 26 & 12.4 & 33 \\
\hline Mississippi & 98 & 13 & 10.05 & 6 & 6.22 & 4 & 1,247 & 15 & 12.7 & 27 \\
\hline Missouri & 52 & 24 & 2.59 & 28 & 1.44 & 31 & 635 & 24 & 12.2 & 36 \\
\hline Montana & 22 & 33 & 7.20 & 11 & 4.57 & 10 & 309 & 33 & 14.0 & 9 \\
\hline Nebraska & 12 & 43 & 1.98 & 39 & 0.94 & 46 & 127 & 43 & 10.6 & 49 \\
\hline Nevada & 7 & 46 & 1.46 & 47 & 1.06 & 44 & 93 & 45 & 13.3 & 18 \\
\hline New Hampshire & 17 & 41 & 3.90 & 24 & 2.74 & 24 & 214 & 41 & 12.6 & 30 \\
\hline New Jersey & 473 & 1 & 15.21 & 2 & 8.88 & 2 & 5,821 & 1 & 12.3 & 34 \\
\hline New Mexico & 13 & 42 & 2.29 & 32 & 1.57 & 30 & 167 & 42 & 12.8 & 21 \\
\hline New York & 172 & 9 & 2.39 & 31 & 1.42 & 33 & 2,196 & 7 & 12.8 & 25 \\
\hline North Carolina & 100 & 12 & 3.77 & 25 & 2.51 & 25 & 1,320 & 12 & 13.2 & 19 \\
\hline North Dakota & 4 & 49 & 1.63 & 46 & 1.36 & 36 & 87 & 47 & 21.8 & 1 \\
\hline Ohio & 118 & 11 & 2.78 & 26 & 1.88 & 27 & 1,715 & 11 & 14.5 & 6 \\
\hline Oklahoma & 30 & 29 & 2.46 & 30 & 1.43 & 32 & 376 & 30 & 12.5 & 31 \\
\hline Oregon & 94 & 15 & 8.41 & 8 & 4.32 & 11 & 1,060 & 18 & 11.3 & 45 \\
\hline Pennsylvania & 430 & 3 & 9.02 & 7 & 4.85 & 9 & 5,493 & 3 & 12.8 & 24 \\
\hline Rhode Island & 29 & 31 & 7.14 & 13 & 3.86 & 14 & 351 & 31 & 12.1 & 38 \\
\hline South Carolina & 64 & 21 & 4.70 & 21 & 3.11 & 19 & 784 & 22 & 12.3 & 35 \\
\hline South Dakota & - & - & - & - & - & - & - & - & - & - \\
\hline Tennessee & 35 & 28 & 1.81 & 42 & 1.26 & 41 & 500 & 28 & 14.3 & 7 \\
\hline Texas & 335 & 4 & 5.18 & 17 & 3.79 & 15 & 4,269 & 4 & 12.7 & 26 \\
\hline Utah & 10 & 44 & 1.68 & 44 & 1.26 & 41 & 119 & 44 & 11.9 & 41 \\
\hline Vermont & 5 & 47 & 2.26 & 33 & 1.22 & 43 & 50 & 49 & 10.0 & 50 \\
\hline Virginia & 196 & 6 & 7.95 & 9 & 5.79 & 7 & 2,516 & 6 & 12.8 & 22 \\
\hline Washington & 195 & 7 & 10.25 & 5 & 6.10 & 5 & 2,191 & 8 & 11.2 & 46 \\
\hline West Virginia & 86 & 17 & 12.03 & 3 & 6.53 & 3 & 1,091 & 17 & 12.7 & 28 \\
\hline Wisconsin & 37 & 27 & 1.95 & 40 & 1.28 & 39 & 519 & 27 & 14.0 & 11 \\
\hline Wyoming & 8 & 45 & 4.72 & 20 & 2.94 & 20 & 88 & 46 & 11.0 & 48 \\
\hline
\end{tabular}

- indicates no deaths listed.

NOTE: Ranks are based on unrounded values. See Appendix A for source description and Appendix B for methods and ICD-8 and ICD-9 codes.

SOURCE: National Center for Health Statistics multiple cause of death data. Population estimates from U.S. Bureau of the Census. 
Table 1-6. Asbestosis: Most frequently recorded occupations on death certificate, selected states, U.S. residents age 15 and over, 1991-1992

\begin{tabular}{llrr}
\hline COC & Occupation & Number & Percent \\
\hline 585 & Plumbers, pipefitters, and steamfitters & 46 & 8.0 \\
575 & Electricians & 31 & 5.4 \\
593 & Insulation workers & 29 & 5.0 \\
567 & Carpenters & 25 & 4.3 \\
889 & Laborers, except construction & 24 & 4.2 \\
633 & Supervisors, precision production occupations & 18 & 3.1 \\
643 & Boilermakers & 18 & 3.1 \\
019 & Managers and administrators, n.e.c. & 17 & 2.9 \\
783 & Welders and cutters & 17 & 2.9 \\
453 & Janitors and cleaners & 15 & 2.6 \\
& All other occupations & 322 & 55.7 \\
& Occupation not reported & 16 & 2.8 \\
& TOTAL & 578 & 100.0 \\
\hline
\end{tabular}

COC - 1980 Census Occupation Code

n.e.c. - not elsewhere classified

NOTE: $\quad$ See Appendix A for source description, Appendix B for methods and ICD -8 and ICD -9 codes, and Appendix C for list of 25 states reporting usual occupation and years reporting. Data for 1985-1990 can be found in the Work-Related Lung Disease Surveillance Report, 1994, see Appendix E.

SOURCE: National Center for Health Statistics multiple cause of death data.

Table 1-7. Asbestosis: Most frequently recorded industries on death certificate, selected states, U.S. residents age 15 and over, 1991-1992

\begin{tabular}{llrr}
\hline CIC & Industry & Number & Percent \\
\hline 060 & Construction & 149 & 25.8 \\
360 & Ship and boat building and repairing & 50 & 8.7 \\
192 & Industrial and miscellaneous chemicals & 23 & 4.0 \\
262 & Miscellaneous nonmetallic mineral stone products & 23 & 4.0 \\
400 & Railroads & 17 & 2.9 \\
901 & General government, n.e.c. & 17 & 2.9 \\
142 & Yarn, thread, and fabric mills & 11 & 1.9 \\
211 & Other rubber products, and plastic footwear and belting & 9 & 1.6 \\
392 & Not specified manufacturing industries & 9 & 1.6 \\
410 & Trucking service & 9 & 1.6 \\
& All other industries & 244 & 42.2 \\
& Industry not reported & 17 & 2.9 \\
& TOTAL & 578 & 100.0 \\
\hline
\end{tabular}

CIC - 1980 Census Industry Code

NOTE: $\quad$ See Appendix A for source description, Appendix B for methods and ICD-8 and ICD-9 codes, and Appendix C for list of 25 states reporting usual industry and years reporting. Data for 1985-1990 can be found in the Work-Related Lung Disease Surveillance Report, 1994, see Appendix E.

SOURCE: National Center for Health Statistics multiple cause of death data. 
Table 1-8. Asbestosis: Proportionate mortality ratio (PMR) by usual occupation, selected states and years, U.S. residents age 15 and over, 1985-1992

\begin{tabular}{|c|c|c|c|c|c|}
\hline \multirow[b]{2}{*}{$\mathrm{COC}$} & \multirow[b]{2}{*}{ Occupation } & \multirow{2}{*}{$\begin{array}{l}\text { Number } \\
\text { of deaths }\end{array}$} & \multirow[b]{2}{*}{ PMR } & \multicolumn{2}{|c|}{$95 \%$ confidence interval } \\
\hline & & & & $\mathrm{LCL}$ & $\mathrm{UCL}$ \\
\hline 593 & Insulation workers & 110 & 261.01 & 212.20 & 317.53 \\
\hline 643 & Boilermakers & 48 & 50.47 & 36.84 & 67.56 \\
\hline 646 & Lay-out workers & 9 & 30.60 & 14.04 & 58.06 \\
\hline 585 & Plumbers, pipefitters, and steamfitters & 153 & 19.06 & 16.03 & 22.50 \\
\hline 653 & Sheet metal workers & 39 & 14.03 & 9.74 & 19.51 \\
\hline 534 & Heating, air conditioning, and refrigeration mechanics & 11 & 11.20 & 5.60 & 20.04 \\
\hline 584 & Plasterers & 6 & 10.61 & 3.89 & 23.12 \\
\hline 575 & Electricians & 74 & 8.14 & 6.36 & 10.29 \\
\hline 759 & Painting, paint spray machine operators & 11 & 7.06 & 3.53 & 12.63 \\
\hline 829 & Sailors and deckhands & 6 & 6.41 & 2.35 & 13.97 \\
\hline 757 & Separate, filter, clarify machine operators & 8 & 6.01 & 2.59 & 11.83 \\
\hline 547 & Specified mechanics and repairers, n.e.c. & 13 & 5.29 & 2.81 & 9.04 \\
\hline 783 & Welders and cutters & 40 & 4.91 & 3.51 & 6.69 \\
\hline 363 & Production coordinators & 5 & 4.68 & 1.51 & 10.93 \\
\hline 518 & Industrial machinery repairers & 19 & 4.41 & 2.66 & 6.89 \\
\hline 563 & Brickmasons and stonemasons & 19 & 4.36 & 2.63 & 6.81 \\
\hline 544 & Millwrights & 11 & 4.21 & 2.10 & 7.53 \\
\hline 516 & Heavy equipment mechanics & 9 & 4.20 & 1.93 & 7.97 \\
\hline 056 & Industrial engineer & 6 & 3.89 & 1.42 & 8.47 \\
\hline 756 & Mixing, blending machine operators & 5 & 3.64 & 1.18 & 8.50 \\
\hline 558 & Supervisors, construction, n.e.c. & 26 & 3.30 & 2.16 & 4.84 \\
\hline 696 & Stationary engineers & 14 & 3.13 & 1.71 & 5.25 \\
\hline 549 & Not specified mechanics and repairers & 12 & 2.99 & 1.54 & 5.22 \\
\hline 579 & Painters, construction and maintenance & 22 & 2.85 & 1.78 & 4.32 \\
\hline 856 & Industrial truck, tractor equipment operators & 8 & 2.76 & 1.19 & 5.43 \\
\hline 637 & Machinists & 47 & 2.65 & 1.93 & 3.55 \\
\hline 777 & Miscellaneous machine operators, n.e.c. & 27 & 2.61 & 1.72 & 3.80 \\
\hline 779 & Machine operators, not specified & 37 & 2.47 & 1.72 & 3.44 \\
\hline 633 & Supervisors, production occupations & 47 & 2.35 & 1.72 & 3.15 \\
\hline 567 & Carpenters & 51 & 2.33 & 1.73 & 3.07 \\
\hline 869 & Construction laborers & 32 & 1.79 & 1.21 & 2.56 \\
\hline 453 & Janitors and cleaners & 43 & 1.46 & 1.04 & 1.99 \\
\hline
\end{tabular}


Table 1-9. Asbestosis: Proportionate mortality ratio (PMR) by usual industry, selected states and years, U.S. residents age 15 and over, 1985-1992

\begin{tabular}{|c|c|c|c|c|c|}
\hline \multirow[b]{2}{*}{$\mathrm{CIC}$} & \multirow[b]{2}{*}{ Industry } & \multirow{2}{*}{$\begin{array}{l}\text { Number } \\
\text { of deaths }\end{array}$} & \multirow[b]{2}{*}{ PMR } & \multicolumn{2}{|c|}{$95 \%$ confidence interval } \\
\hline & & & & LCL & $\mathrm{UCL}$ \\
\hline 360 & Ship and boat building and repairing & 164 & 43.01 & 36.60 & 50.19 \\
\hline 262 & Miscellaneous nonmetallic mineral and stone products & 54 & 29.17 & 21.61 & 38.48 \\
\hline 192 & Industrial and miscellaneous chemicals & 54 & 7.02 & 5.20 & 9.26 \\
\hline 502 & Lumber and construction materials & 7 & 6.64 & 2.67 & 13.69 \\
\hline 282 & Fabricated structural metal products & 29 & 6.22 & 4.17 & 8.94 \\
\hline 462 & Electric and gas, and other combinations & 9 & 6.13 & 2.81 & 11.63 \\
\hline 200 & Petroleum refining & 19 & 5.82 & 3.51 & 9.09 \\
\hline 521 & Hardware, plumbing and heating supplies & 8 & 4.74 & 2.04 & 9.33 \\
\hline 420 & Water transportation & 16 & 4.61 & 2.63 & 7.48 \\
\hline 211 & Other rubber products, and plastics footwear and belting & 19 & 4.49 & 2.70 & 7.02 \\
\hline 060 & Construction & 435 & 4.42 & 4.00 & 4.87 \\
\hline 181 & Drugs & 8 & 4.38 & 1.89 & 8.62 \\
\hline 881 & Membership organizations & 12 & 3.96 & 2.04 & 6.91 \\
\hline 180 & Plastics, synthetics, and resins & 6 & 3.31 & 1.21 & 7.21 \\
\hline 460 & Electric light and power & 25 & 3.29 & 2.12 & 4.85 \\
\hline 210 & Tires and inner tubes & 10 & 2.95 & 1.42 & 5.42 \\
\hline 272 & Primary aluminum industries & 7 & 2.88 & 1.16 & 5.94 \\
\hline 400 & Railroads & 46 & 2.15 & 1.57 & 2.88 \\
\hline 160 & Pulp, paper, and paperboard mills & 15 & 2.02 & 1.13 & 3.33 \\
\hline 392 & Not specified manufacturing industries & 43 & 1.66 & 1.19 & 2.26 \\
\hline
\end{tabular}

CIC - 1980 Census Industry Code LCL - lower confidence limit UCL - upper confidence limit

NOTE: See Appendix A for source description, Appendix B for methods and ICD-8 and ICD-9 codes, and Appendix C for list of 25 states reporting usual industry and years

$\begin{array}{ll} & \text { reporting. } \\ \text { SOURCE: } & \text { National Center for Health Statistics multiple cause of death data. }\end{array}$ 
Figure 1-4. Asbestosis: Total number of deaths by county,

U.S. residents age 15 and over, 1983-1992

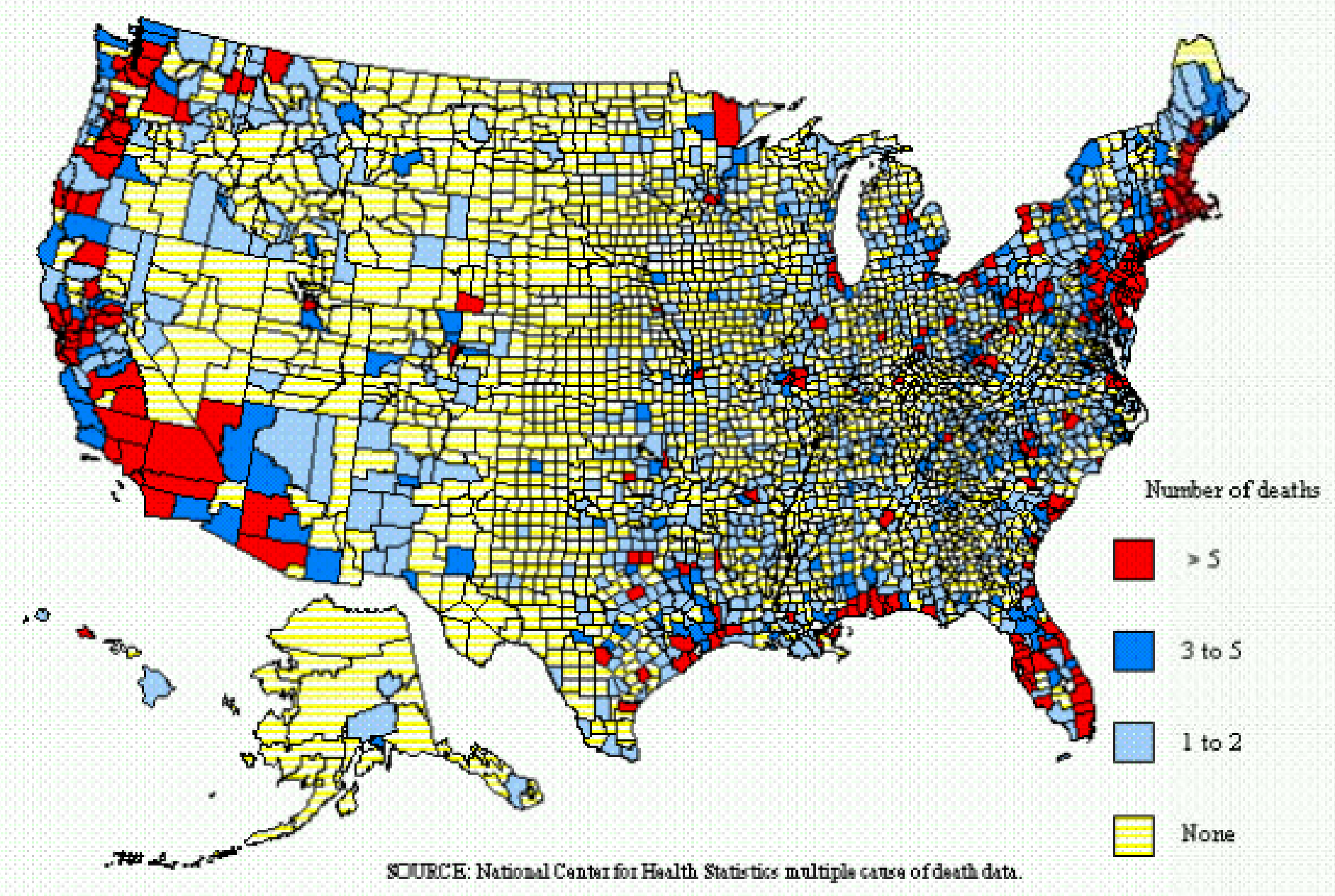


Figure 1-5. Asbestosis: Age-adjusted mortality rates by county,

U.S. residents age 15 and over, 1983-1992

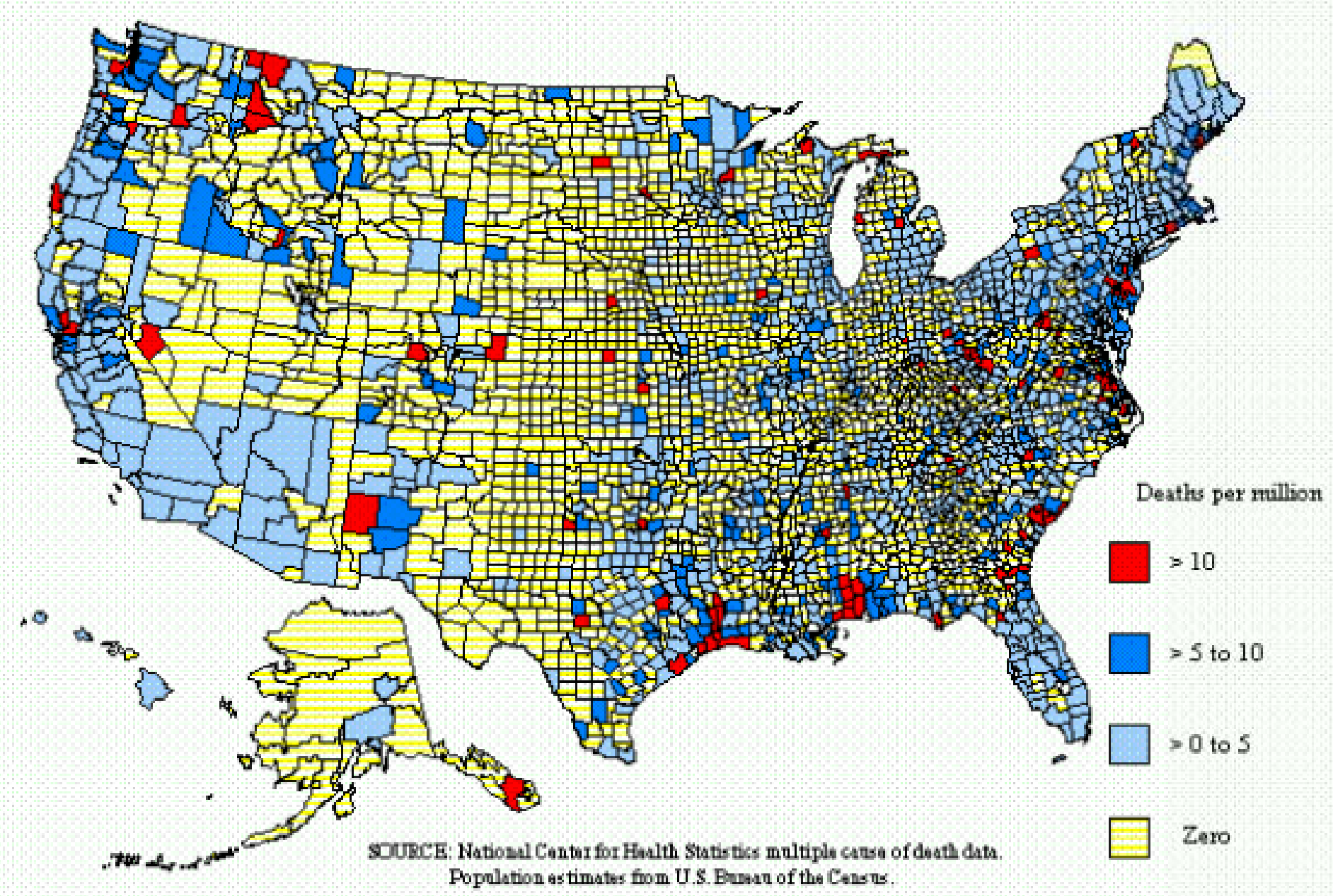


Figure 1-6. Asbestos: Number of MSHA and OSHA inspector samples and percent exceeding the permissible exposure limit (PEL), 1974-1994

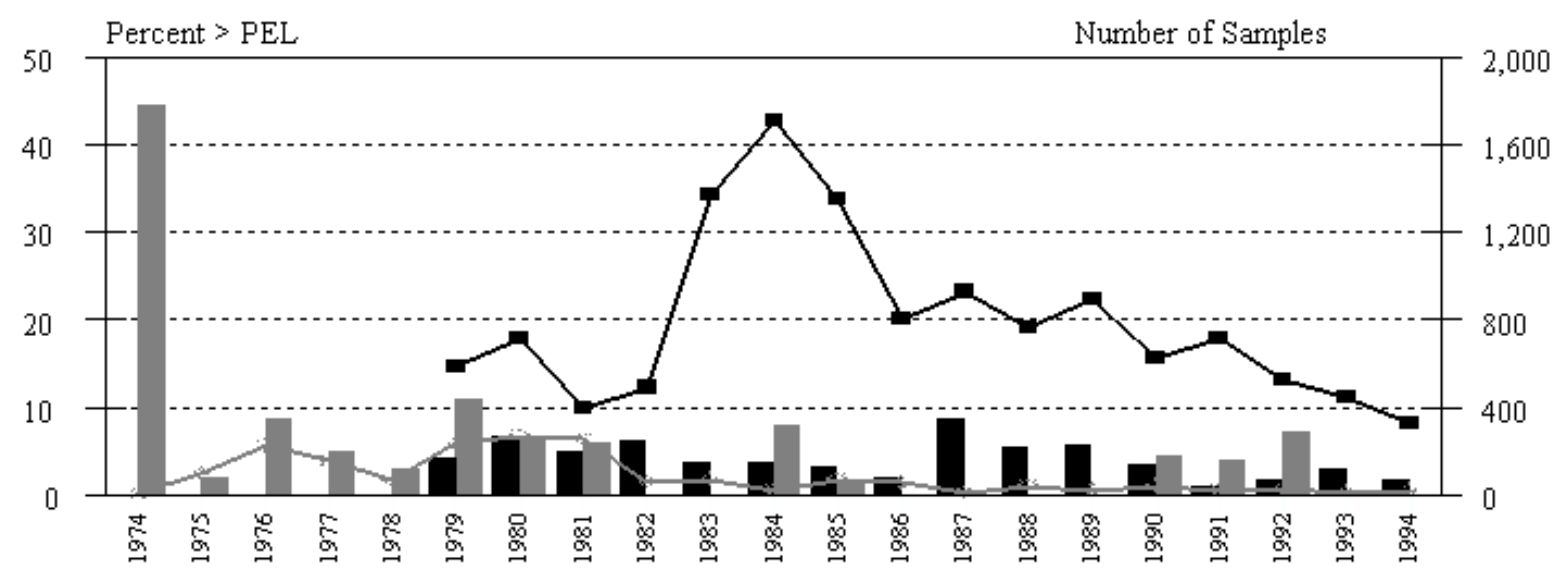

\begin{tabular}{ll|} 
- OSHA Percent > PEL & MSHA Percent > PEL \\
- \# OSHA Samples & \#MSHA Samples \\
\hline
\end{tabular}

NOTE: See Appendix A for source description, Appendix B for methods, and Appendix D for agents.

SOURCE: Bureau of Mines: Mine Inspection Data Analysis System. Occupational Safety and Health Administration: Integrated Management Information System.

Figure 1-7. Asbestos: Number of MSHA and OSHA inspector samples and average severity levels, 1974-1994

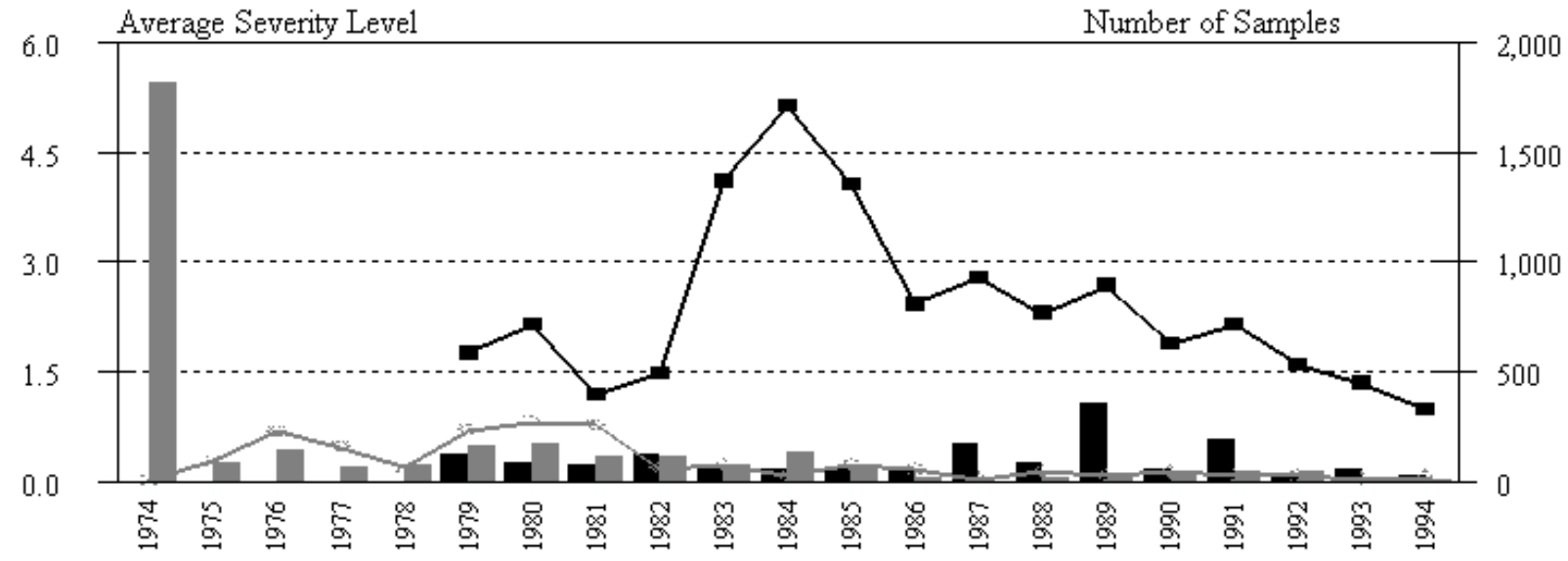

OSHA Severity Levels

\# OSHA Samples

\section{MSHA Severity Levels} $\because$ \# MSHA Samples

NOTE: See Appendix A for source description, Appendix B for methods, and Appendix D for agents.

SOURCE: Bureau of Mines: Mine Inspection Data Analysis System. Occupational Safety and Health Administration: Integrated Management Information System. 
Table 1-10. Asbestos: Number of MSHA and OSHA inspector samples, percent exceeding the permissible exposure limit (PEL) and average severity level, by industry, 1993-1994

\begin{tabular}{|c|c|c|c|c|}
\hline CIC & $\begin{array}{l}\text { Industries most frequently recorded on } \\
\text { 1991-1992 death certificates with asbestosis }\end{array}$ & $\begin{array}{r}\text { Number of } \\
\text { samples } \\
\end{array}$ & $\%>$ PEL & $\begin{array}{l}\text { Average } \\
\text { severity }\end{array}$ \\
\hline 060 & Construction & 221 & 4.1 & 0.17 \\
\hline 360 & Ship and boat building and repairing & 3 & 0.0 & 0.00 \\
\hline 192 & Industrial and miscellaneous chemicals & 2 & 0.0 & 0.00 \\
\hline 262 & Miscellaneous nonmetallic mineral and stone products & 65 & 10.8 & 0.43 \\
\hline 400 & Railroads & 0 & - & - \\
\hline 901 & General government, n.e.c. & 20 & 0.0 & 0.05 \\
\hline 142 & Yarn, thread, and fabric mills & 1 & 0.0 & 0.00 \\
\hline 211 & Other rubber products, and plastics footwear and belts & 6 & 0.0 & 0.03 \\
\hline 392 & Not specified manufacturing industries & 0 & - & - \\
\hline \multirow[t]{4}{*}{410} & Trucking service & 1 & 0.0 & 0.00 \\
\hline & All other industries & 483 & 0.8 & 0.08 \\
\hline & Industry not reported & 3 & 0.0 & 0.00 \\
\hline & TOTAL & 805 & 2.5 & 0.13 \\
\hline
\end{tabular}

CIC - 1980 Census Industry Code n.e.c. - not elsew here classified - indicates incalculable field

NOTE: See Appendix A for source description, Appendix B for methods, Appendix C for list of 25 states reporting usual industry and years reporting, and Appendix D for agents.

SOURCE: Bureau of Mines: Mine Inspection Data Analysis System. Occupational Safety and Health Administration: Integrated Management Information System. National Center for Health Statistics: multiple cause of death data.

Table 1-11. Asbestos: Number of MSHA and OSHA inspector samples, percent exceeding the permissible exposure limit (PEL) and average severity level, by industry, 1993-1994

\begin{tabular}{|c|c|c|c|c|}
\hline CIC & Industries most frequently sampled in 1993-1994 & $\begin{array}{r}\text { Number of } \\
\text { samples } \\
\end{array}$ & $\%>$ PEL & $\begin{array}{r}\text { Average } \\
\text { severity }\end{array}$ \\
\hline 060 & Construction & 221 & 4.1 & 0.17 \\
\hline 262 & Miscellaneous nonmetallic mineral and stone products & 65 & 10.8 & 0.43 \\
\hline 910 & Justice, public order, and safety & 43 & 0.0 & 0.02 \\
\hline 831 & Ho spitals & 22 & 0.0 & 0.00 \\
\hline 050 & Nonmetallic mining & 21 & 0.0 & 0.04 \\
\hline 901 & General government, n.e.c. & 20 & 0.0 & 0.05 \\
\hline 842 & Elementary and secondary schools & 19 & 0.0 & 0.06 \\
\hline 751 & Automotive repair shops & 18 & 0.0 & 0.03 \\
\hline 591 & Department stores & 16 & 0.0 & 0.00 \\
\hline \multirow[t]{4}{*}{351} & Motor vehicles and motor vehicle equipment & 15 & 0.0 & 0.04 \\
\hline & All other industries & 342 & 1.2 & 0.11 \\
\hline & Industry not reported & 3 & 0.0 & 0.00 \\
\hline & TOTAL & 805 & 2.5 & 0.13 \\
\hline
\end{tabular}

CIC - 1980 Census Industry Code n.e.c. - not elsewhere classified

NOTE: See Appendix A for source description, Appendix B for methods, and Appendix D for agents.

SOURCE: Bureau of Mines: Mine Inspection Data Analysis System. Occupational Safety and Health Administration: Integrated Management Information System. 
Table 1-12 (page 1 of 2). Asbestos: Number of MSHA inspector samples, percent exceeding the permissible exposure limit (PEL) and average severity levels (Avg. Sev.), by state, 1974-1994

\begin{tabular}{|c|c|c|c|c|c|c|c|c|c|c|c|c|}
\hline \multirow[b]{3}{*}{ State } & \multicolumn{4}{|c|}{ 1974-1984 } & \multicolumn{4}{|c|}{ 1985-1994 } & \multicolumn{4}{|c|}{ 1993-1994 } \\
\hline & \multicolumn{2}{|c|}{ Total samples } & \multicolumn{2}{|c|}{ Samples > PEL } & \multicolumn{2}{|c|}{ Total samples } & \multicolumn{2}{|c|}{ Samples > PEL } & \multicolumn{2}{|c|}{ Total samples } & \multicolumn{2}{|c|}{ Samples > PEL } \\
\hline & Number & $\begin{array}{l}\text { Avg. } \\
\text { Sev. }\end{array}$ & $\%$ & $\begin{array}{l}\text { Avg. } \\
\text { Sev. }\end{array}$ & Number & $\begin{array}{l}\text { Avg. } \\
\text { Sev. }\end{array}$ & $\%$ & $\begin{array}{l}\text { Avg. } \\
\text { Sev. }\end{array}$ & Number & $\begin{array}{l}\text { Avg. } \\
\text { Sev. }\end{array}$ & $\%$ & $\begin{array}{l}\text { Avg. } \\
\text { Sev. }\end{array}$ \\
\hline Alabama & 0 & - & - & - & 0 & - & - & - & 0 & - & - & - \\
\hline Alaska & 0 & - & - & - & 0 & - & - & - & 0 & - & - & - \\
\hline Arizona & 86 & 0.94 & 14.0 & 4.78 & 0 & - & - & - & 0 & - & - & - \\
\hline Arkansas & 0 & - & - & - & 0 & - & - & - & 0 & - & - & - \\
\hline California & 156 & 0.73 & 21.8 & 2.33 & 64 & 0.20 & 0.0 & - & 9 & 0.09 & 0.0 & - \\
\hline Colorado & 25 & 0.19 & 0.0 & - & 20 & 0.03 & 0.0 & - & 0 & - & - & - \\
\hline Connecticut & 0 & - & - & - & 0 & - & - & - & 0 & - & - & - \\
\hline Delaware & 0 & - & - & - & 0 & - & - & - & 0 & - & - & - \\
\hline District of Columbia & 0 & - & - & - & 0 & - & - & - & 0 & - & - & - \\
\hline Florida & 4 & 0.03 & 0.0 & - & 0 & - & - & - & 0 & - & - & - \\
\hline Georgia & 16 & 1.79 & 25.0 & 5.45 & 4 & 0.00 & 0.0 & - & 0 & - & - & - \\
\hline Hawaii & 0 & - & - & - & 1 & 0.00 & 0.0 & - & 1 & 0.00 & 0.0 & - \\
\hline Idaho & 0 & - & - & - & 5 & 0.01 & 0.0 & - & 0 & - & - & - \\
\hline Illinois & 55 & 0.05 & 0.0 & - & 2 & 0.00 & 0.0 & - & 1 & 0.00 & 0.0 & - \\
\hline Indiana & 6 & 0.07 & 0.0 & - & 1 & 0.01 & 0.0 & - & 0 & - & - & - \\
\hline Iowa & 0 & - & - & - & 0 & - & - & - & 0 & - & - & - \\
\hline Kansas & 0 & - & - & - & 0 & - & - & - & 0 & - & - & - \\
\hline Kentucky & 0 & - & - & - & 0 & - & - & - & 0 & - & - & - \\
\hline Louisiana & 4 & 0.00 & 0.0 & - & 32 & 0.00 & 0.0 & - & 5 & 0.00 & 0.0 & - \\
\hline Maine & 0 & - & - & - & 0 & - & - & - & 0 & - & - & - \\
\hline Maryland & 91 & 0.27 & 3.3 & 1.58 & 12 & 0.00 & 0.0 & - & 0 & - & - & - \\
\hline Massachusetts & 0 & - & - & - & 1 & 0.00 & 0.0 & - & 0 & - & - & - \\
\hline Michigan & 2 & 0.00 & 0.0 & - & 5 & 0.01 & 0.0 & - & 5 & 0.01 & 0.0 & - \\
\hline Minnesota & 208 & 0.10 & 0.5 & 1.20 & 31 & 0.02 & 0.0 & - & 2 & 0.05 & 0.0 & - \\
\hline Mississippi & 0 & - & - & - & 0 & - & - & - & 0 & - & - & - \\
\hline Missouri & 0 & - & - & - & 4 & 0.00 & 0.0 & - & 0 & - & - & - \\
\hline Montana & 180 & 0.28 & 2.8 & 1.42 & 17 & 0.17 & 0.0 & - & 0 & - & - & - \\
\hline
\end{tabular}


Table 1-12 (page 2 of 2). Asbestos: Number of MSHA inspector samples, percent exceeding the permissible exposure limit (PEL) and average severity levels (Avg. Sev.), by state, 1974-1994

\begin{tabular}{|c|c|c|c|c|c|c|c|c|c|c|c|c|}
\hline \multirow[b]{3}{*}{ State } & \multicolumn{4}{|c|}{ 1974-1984 } & \multicolumn{4}{|c|}{ 1985-1994 } & \multicolumn{4}{|c|}{ 1993-1994 } \\
\hline & \multicolumn{2}{|c|}{ Total samples } & \multicolumn{2}{|c|}{ Samples > PEL } & \multicolumn{2}{|c|}{ Total samples } & \multicolumn{2}{|c|}{ Samples $>$ PEL } & \multicolumn{2}{|c|}{ Total samples } & \multicolumn{2}{|c|}{ Samples > PEL } \\
\hline & Number & $\begin{array}{l}\text { Avg. } \\
\text { Sev. }\end{array}$ & $\%$ & $\begin{array}{l}\text { Avg. } \\
\text { Sev. }\end{array}$ & Number & $\begin{array}{l}\text { Avg. } \\
\text { Sev. }\end{array}$ & $\%$ & $\begin{array}{c}\text { Avg. } \\
\text { Sev. }\end{array}$ & Number & $\begin{array}{l}\text { Avg. } \\
\text { Sev. }\end{array}$ & $\%$ & $\begin{array}{r}\text { Avg. } \\
\text { Sev. }\end{array}$ \\
\hline Nebraska & 0 & - & - & - & 0 & - & - & - & 0 & - & - & - \\
\hline Nevada & 0 & - & - & - & 4 & 0.00 & 0.0 & - & 0 & - & - & - \\
\hline New Hampshire & 0 & - & - & - & 0 & - & - & - & 0 & - & - & - \\
\hline New Jersey & 14 & 0.13 & 0.0 & - & 0 & - & - & - & 0 & - & - & - \\
\hline New Mexico & 96 & 0.18 & 2.1 & 3.30 & 2 & 0.44 & 0.0 & - & 0 & - & - & - \\
\hline New York & 63 & 0.35 & 1.6 & 2.60 & 2 & 0.00 & 0.0 & - & 2 & 0.00 & 0.0 & - \\
\hline North Carolina & 13 & 0.39 & 0.0 & - & 3 & 0.00 & 0.0 & - & 0 & - & - & - \\
\hline North Dakota & 0 & - & - & - & 0 & - & - & - & 0 & - & - & - \\
\hline Ohio & 1 & 0.01 & 0.0 & - & 0 & - & - & - & 0 & - & - & - \\
\hline Oklahoma & 21 & 0.17 & 0.0 & - & 0 & - & - & - & 0 & - & - & - \\
\hline Oregon & 0 & - & - & - & 0 & - & - & - & 0 & - & - & - \\
\hline Pennsylvania & 12 & 0.10 & 0.0 & - & 11 & 0.00 & 0.0 & - & 1 & 0.00 & 0.0 & - \\
\hline Rhode Island & 0 & - & - & - & 0 & - & - & - & 0 & - & - & - \\
\hline South Carolina & 62 & 0.06 & 0.0 & - & 11 & 0.00 & 0.0 & - & 0 & - & - & - \\
\hline South Dakota & 27 & 0.27 & 0.0 & - & 43 & 0.09 & 0.0 & - & 0 & - & - & - \\
\hline Tennessee & 0 & - & - & - & 0 & - & - & - & 0 & - & - & - \\
\hline Texas & 72 & 1.12 & 1.4 & 75.63 & 5 & 0.00 & 0.0 & - & 4 & 0.00 & 0.0 & - \\
\hline Utah & 0 & - & - & - & 2 & 0.00 & 0.0 & - & 0 & - & - & - \\
\hline Vermont & 221 & 0.62 & 14.9 & 2.07 & 34 & 0.55 & 17.6 & 1.96 & 0 & - & - & - \\
\hline Virginia & 12 & 0.16 & 0.0 & - & 8 & 0.00 & 0.0 & - & 2 & 0.00 & 0.0 & - \\
\hline Washington & 1 & 0.02 & 0.0 & - & 10 & 0.00 & 0.0 & - & 0 & - & - & - \\
\hline West Virginia & 0 & - & - & - & 2 & 0.00 & 0.0 & - & 2 & 0.00 & 0.0 & - \\
\hline Wisconsin & 35 & 0.13 & 0.0 & - & 1 & 0.00 & 0.0 & - & 0 & - & - & - \\
\hline Wyoming & 0 & - & - & - & 7 & 0.00 & 0.0 & - & 0 & - & - & - \\
\hline TOTAL & 1,483 & 0.41 & 6.5 & 3.38 & 344 & 0.12 & 1.7 & 1.96 & 34 & 0.03 & 0.0 & - \\
\hline
\end{tabular}

indicates incalculable field.

NOTE: See Appendix A for source description, Appendix B for methods, and Appendix D for agents.

SOURCE: Bureau of Mines: Mine Inspection Data Analysis System. 
Figure 1-8. Asbestos: Number of MSHA inspector samples collected by state, 1993-1994

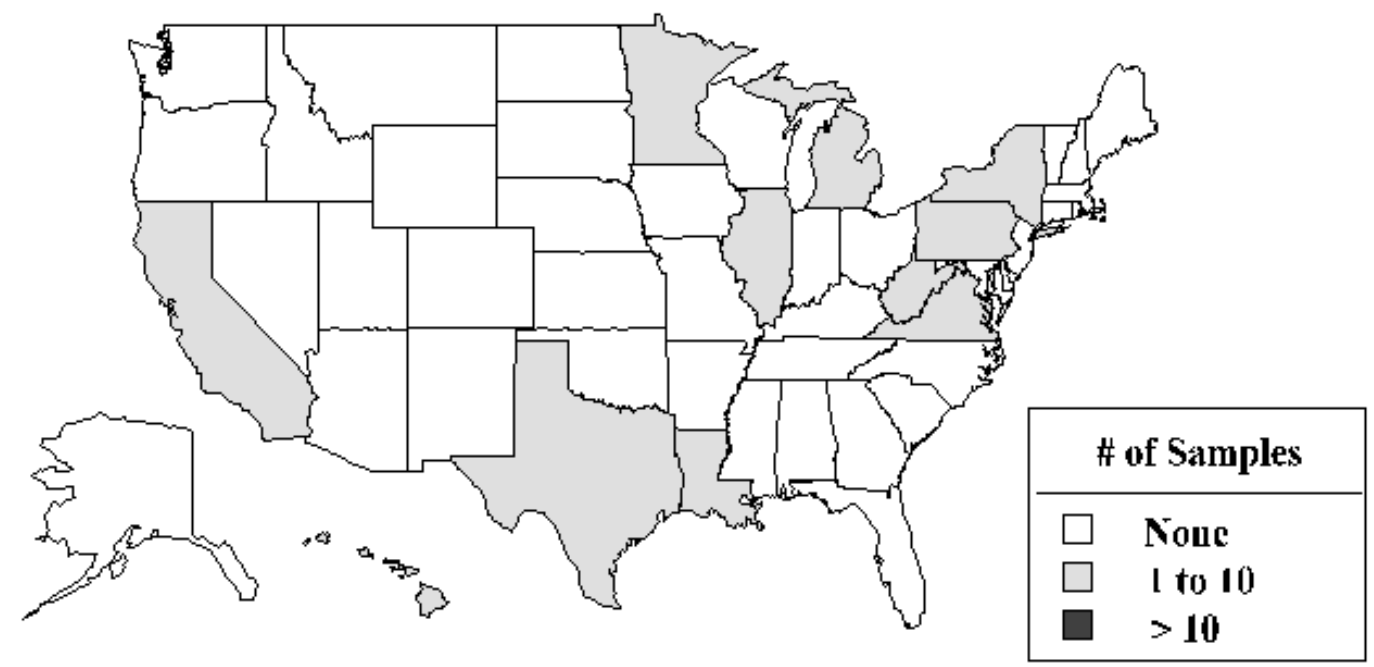

NOTE: See Appendix A for source description, Appendix B for methods, and Appendix D for agents. SOURCE: Bureau of Mines: Mine Inspection Data Analysis System.

Figure 1-9. Asbestos: Percent of MSHA inspector samples that exceeded the permissible exposure limit (PEL), by state, 1993-1994

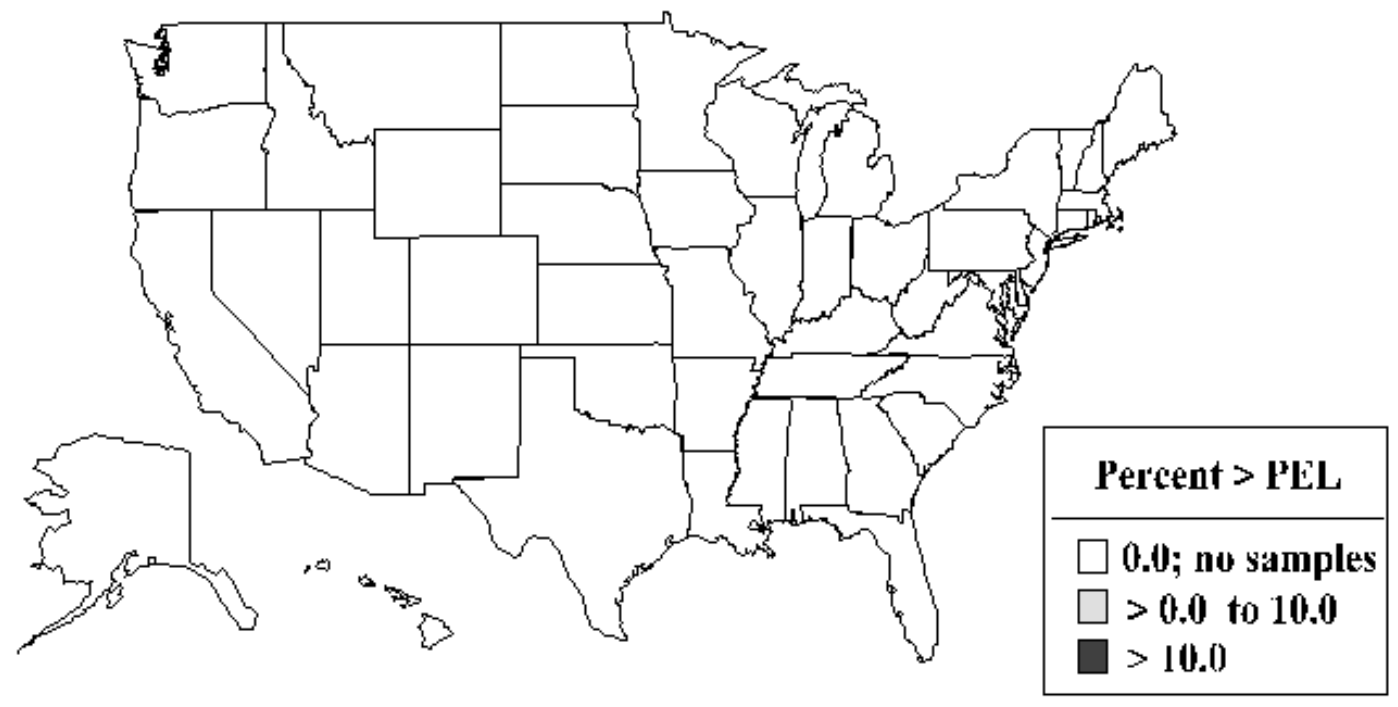

NOTE: No samples collected were above the PEL. See Appendix A for source description, Appendix B for methods, and Appendix D for agents. SOURCE: Bureau of Mines: Mine Inspection Data Analysis System. 
Figure 1-10. Asbestos: Average severity level by state for MSHA inspector samples, 1993-1994

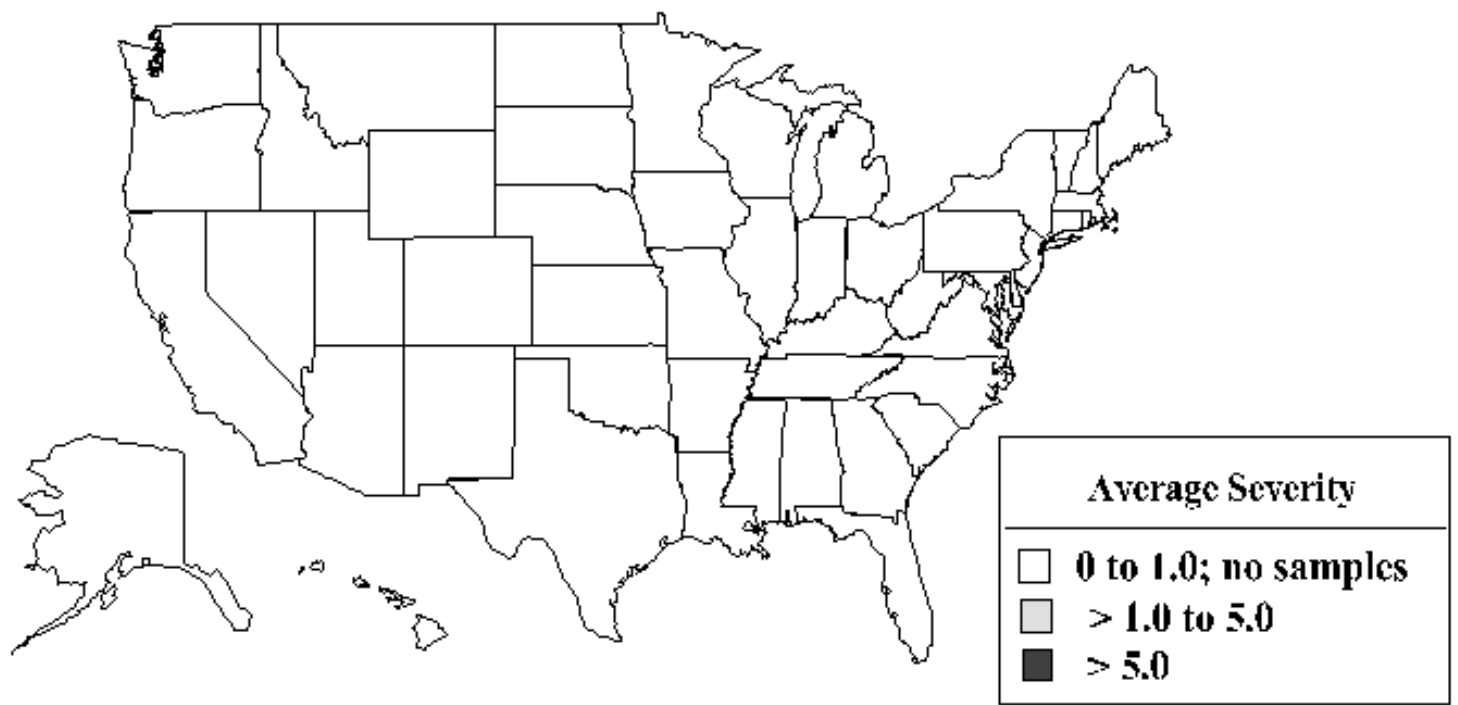

NOTE: See Appendix A for source description, Appendix B for methods, and Appendix D for agents.

SOURCE: Bureau of Mines: Mine Inspection Data Analysis System.

Figure 1-11. Asbestos: Average severity level by state for MSHA inspector samples that exceeded the permissible exposure limit (PEL), 1993-1994

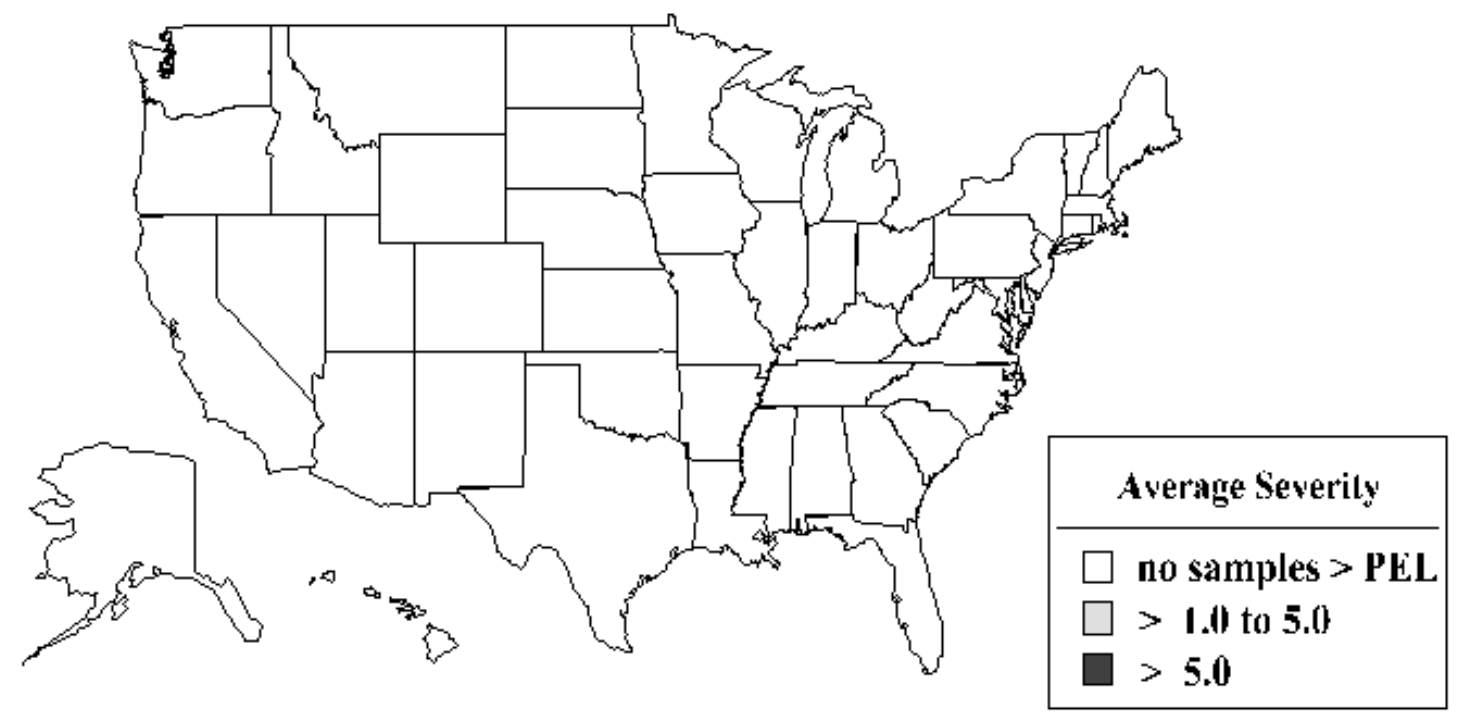

NOTE: No samples collected were above the PEL. See Appendix A for source description, Appendix B for methods, and Appendix D for agents. SOURCE: Bureau of Mines: Mine Inspection Data Analysis System. 
Figure 1-12. Asbestos: Number of MSHA inspector samples

collected by county, 1993-1994

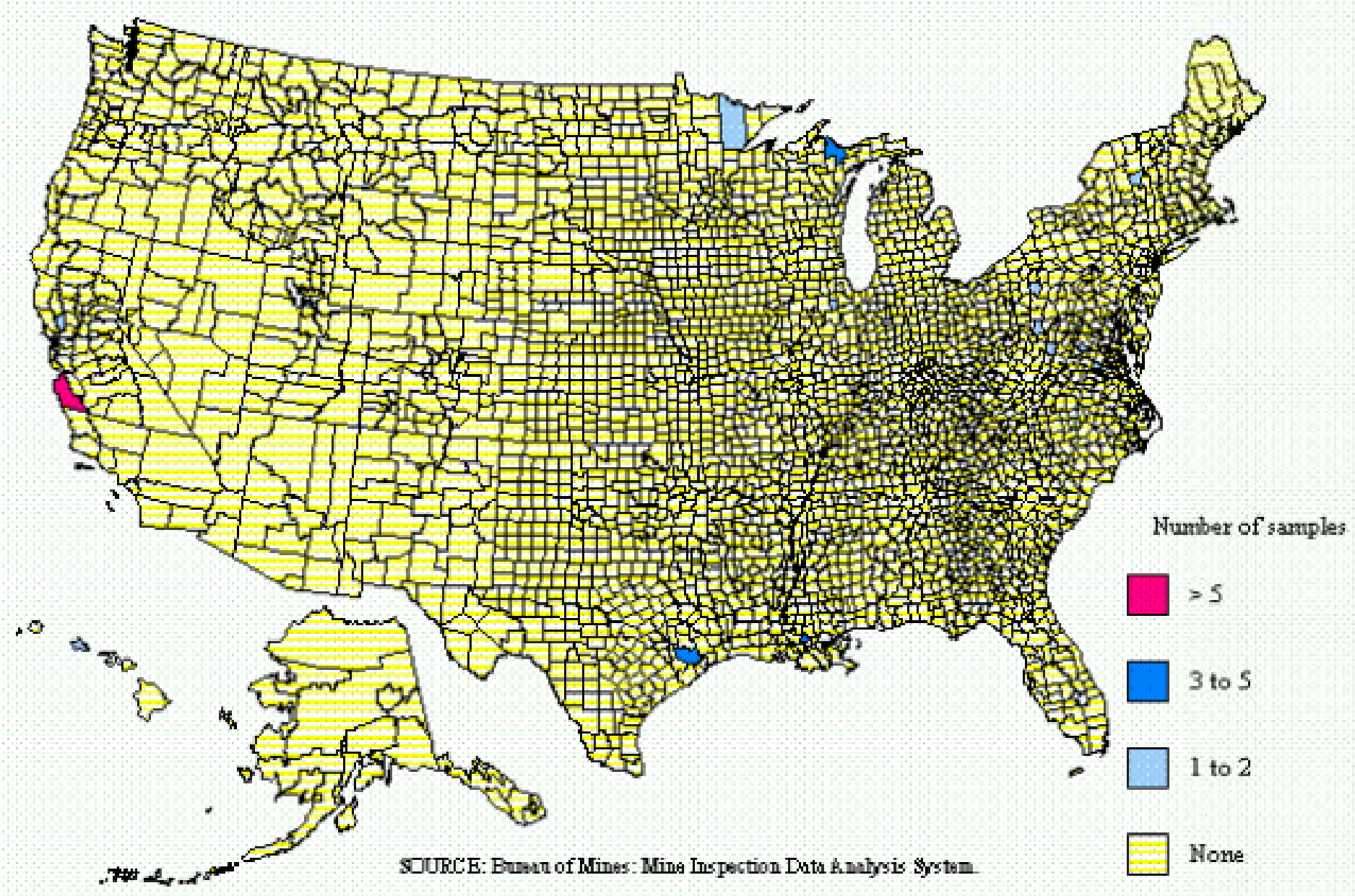


Table 1-13 (page 1 of 2). Asbestos: Number of OSHA inspector samples, percent exceeding the permissible exposure limit (PEL) and average severity levels (Avg. Sev.), by state, 1979-1994

\begin{tabular}{|c|c|c|c|c|c|c|c|c|c|c|c|c|}
\hline \multirow[b]{3}{*}{ State } & \multicolumn{4}{|c|}{ 1979-1984 } & \multicolumn{4}{|c|}{ 1985-1994 } & \multicolumn{4}{|c|}{ 1993-1994 } \\
\hline & \multicolumn{2}{|c|}{ Total samples } & \multicolumn{2}{|c|}{ Samples > PEL } & \multicolumn{2}{|c|}{ Total samples } & \multicolumn{2}{|c|}{ Samples > PEL } & \multicolumn{2}{|c|}{ Total samples } & \multicolumn{2}{|c|}{ Samples > PEL } \\
\hline & Number & $\begin{array}{l}\text { Avg. } \\
\text { Sev. }\end{array}$ & $\%$ & $\begin{array}{c}\text { Avg. } \\
\text { Sev. }\end{array}$ & Number & $\begin{array}{l}\text { Avg. } \\
\text { Sev. }\end{array}$ & $\%$ & $\begin{array}{l}\text { Avg. } \\
\text { Sev. }\end{array}$ & Number & $\begin{array}{l}\text { Avg. } \\
\text { Sev. }\end{array}$ & $\%$ & $\begin{array}{r}\text { Avg. } \\
\text { Sev. }\end{array}$ \\
\hline Alabama & 91 & 0.73 & 9.9 & 6.53 & 82 & 0.06 & 2.4 & 1.71 & 1 & 0.00 & 0.0 & - \\
\hline Alaska & 76 & 0.01 & 0.0 & - & 110 & 0.14 & 2.7 & 4.52 & 4 & 0.00 & 0.0 & - \\
\hline Arizona & 24 & 0.03 & 0.0 & - & 47 & 0.10 & 2.1 & 1.90 & 0 & - & - & - \\
\hline Arkansas & 44 & 0.07 & 0.0 & - & 212 & 0.66 & 7.5 & 7.62 & 67 & 0.40 & 10.4 & 1.90 \\
\hline California & 23 & 0.14 & 4.3 & 1.27 & 153 & 0.55 & 6.5 & 7.13 & 6 & 0.01 & 0.0 & - \\
\hline Colorado & 212 & 0.08 & 0.9 & 3.57 & 123 & 0.14 & 3.3 & 3.23 & 6 & 1.41 & 33.3 & 3.98 \\
\hline Connecticut & 164 & 0.39 & 4.9 & 6.12 & 223 & 0.04 & 0.0 & - & 26 & 0.05 & 0.0 & - \\
\hline Delaware & 20 & 0.13 & 0.0 & - & 4 & 0.01 & 0.0 & - & 0 & - & - & - \\
\hline District of Columbia & 50 & 0.10 & 2.0 & 2.04 & 10 & 0.06 & 0.0 & - & 2 & 0.00 & 0.0 & - \\
\hline Florida & 82 & 1.74 & 7.3 & 23.09 & 177 & 0.13 & 2.3 & 4.73 & 30 & 0.25 & 10.0 & 1.67 \\
\hline Georgia & 197 & 0.45 & 12.2 & 3.01 & 147 & 0.09 & 2.7 & 1.91 & 5 & 0.00 & 0.0 & - \\
\hline Hawaii & 9 & 0.01 & 0.0 & - & 14 & 0.00 & 0.0 & - & 3 & 0.00 & 0.0 & - \\
\hline Idaho & 14 & 0.05 & 0.0 & - & 81 & 0.02 & 0.0 & - & 3 & 0.00 & 0.0 & - \\
\hline Illinois & 222 & 0.06 & 1.8 & 1.73 & 434 & 0.03 & 0.2 & 1.20 & 65 & 0.02 & 0.0 & - \\
\hline Indiana & 170 & 0.28 & 6.5 & 1.44 & 216 & 0.04 & 0.0 & - & 17 & 0.00 & 0.0 & - \\
\hline Iowa & 87 & 0.24 & 6.9 & 2.72 & 209 & 0.03 & 0.5 & 1.15 & 3 & 0.00 & 0.0 & - \\
\hline Kansas & 44 & 0.21 & 9.1 & 1.74 & 33 & 0.04 & 0.0 & - & 3 & 0.00 & 0.0 & - \\
\hline Kentucky & 79 & 0.05 & 1.3 & 1.94 & 132 & 0.08 & 0.0 & - & 13 & 0.03 & 0.0 & - \\
\hline Louisiana & 57 & 0.07 & 1.8 & 1.58 & 82 & 0.07 & 2.4 & 1.63 & 0 & - & - & - \\
\hline Maine & 63 & 0.06 & 0.0 & - & 17 & 0.05 & 0.0 & - & 0 & - & - & - \\
\hline Maryland & 20 & 0.03 & 0.0 & - & 51 & 0.31 & 9.8 & 2.64 & 4 & 0.08 & 0.0 & - \\
\hline Massachusetts & 291 & 0.40 & 15.5 & 1.59 & 241 & 0.39 & 8.7 & 3.71 & 20 & 0.55 & 20.0 & 2.43 \\
\hline Michigan & 0 & - & - & - & 342 & 0.10 & 2.0 & 3.36 & 61 & 0.04 & 0.0 & - \\
\hline Minnesota & 8 & 0.31 & 25.0 & 1.15 & 23 & 0.00 & 0.0 & - & 0 & - & - & - \\
\hline Mississippi & 17 & 0.08 & 0.0 & - & 105 & 0.06 & 1.0 & 1.05 & 16 & 0.09 & 0.0 & - \\
\hline Missouri & 372 & 0.18 & 2.4 & 6.86 & 155 & 0.04 & 1.3 & 1.66 & 11 & 0.00 & 0.0 & - \\
\hline Montana & 120 & 0.09 & 0.8 & 1.95 & 63 & 0.12 & 3.2 & 1.13 & 0 & - & - & - \\
\hline
\end{tabular}


Table 1-13 (page 2 of 2). Asbestos: Number of OSHA inspector samples, percent exceeding the permissible exposure limit (PEL) and average severity levels (Avg. Sev.), by state, 1979-1994

\begin{tabular}{|c|c|c|c|c|c|c|c|c|c|c|c|c|}
\hline \multirow[b]{3}{*}{ State } & \multicolumn{4}{|c|}{ 1979-1984 } & \multicolumn{4}{|c|}{ 1985-1994 } & \multicolumn{4}{|c|}{ 1993-1994 } \\
\hline & \multicolumn{2}{|c|}{ Total samples } & \multicolumn{2}{|c|}{ Samples > PEL } & \multicolumn{2}{|c|}{ Total samples } & \multicolumn{2}{|c|}{ Samples > PEL } & \multicolumn{2}{|c|}{ Total samples } & \multicolumn{2}{|c|}{ Samples > PEL } \\
\hline & Number & $\begin{array}{l}\text { Avg. } \\
\text { Sev. }\end{array}$ & $\%$ & $\begin{array}{l}\text { Avg. } \\
\text { Sev. }\end{array}$ & Number & $\begin{array}{l}\text { Avg. } \\
\text { Sev. }\end{array}$ & $\%$ & $\begin{array}{l}\text { Avg. } \\
\text { Sev. }\end{array}$ & Number & $\begin{array}{l}\text { Avg. } \\
\text { Sev. }\end{array}$ & $\%$ & $\begin{array}{l}\text { Avg. } \\
\text { Sev. }\end{array}$ \\
\hline Nebraska & 36 & 0.04 & 0.0 & - & 109 & 0.01 & 0.0 & - & 3 & 0.14 & 0.0 & - \\
\hline Nevada & 16 & 0.03 & 0.0 & - & 50 & 0.28 & 6.0 & 3.90 & 6 & 0.12 & 0.0 & - \\
\hline New Hampshire & 100 & 0.82 & 12.0 & 5.84 & 70 & 0.05 & 1.4 & 1.50 & 5 & 0.00 & 0.0 & - \\
\hline New Jersey & 268 & 0.23 & 3.4 & 3.39 & 307 & 0.81 & 14.7 & 5.13 & 13 & 0.02 & 0.0 & - \\
\hline New Mexico & 2 & 0.00 & 0.0 & - & 22 & 0.02 & 0.0 & - & 6 & 0.02 & 0.0 & - \\
\hline New York & 613 & 0.08 & 1.8 & 1.25 & 952 & 0.22 & 1.7 & 10.74 & 126 & 0.27 & 2.4 & 10.10 \\
\hline North Carolina & 87 & 0.26 & 4.6 & 2.09 & 231 & 0.20 & 4.3 & 3.34 & 46 & 0.08 & 2.2 & 1.15 \\
\hline North Dakota & 7 & 0.00 & 0.0 & - & 1 & 0.00 & 0.0 & - & 0 & - & - & - \\
\hline Ohio & 282 & 0.46 & 4.3 & 9.27 & 545 & 0.87 & 5.3 & 15.45 & 42 & 0.00 & 0.0 & - \\
\hline Oklahoma & 91 & 0.12 & 2.2 & 4.35 & 78 & 0.14 & 6.4 & 1.83 & 3 & 0.00 & 0.0 & - \\
\hline Oregon & 78 & 0.13 & 5.1 & 1.37 & 76 & 0.09 & 0.0 & - & 15 & 0.08 & 0.0 & - \\
\hline Pennsylvania & 365 & 0.30 & 4.7 & 4.14 & 393 & 0.62 & 6.6 & 8.33 & 11 & 0.00 & 0.0 & - \\
\hline Rhode Island & 46 & 0.38 & 13.0 & 2.43 & 56 & 1.01 & 16.1 & 6.17 & 0 & - & - & - \\
\hline South Carolina & 24 & 0.08 & 0.0 & - & 44 & 0.28 & 9.1 & 2.70 & 8 & 0.00 & 0.0 & - \\
\hline South Dakota & 4 & 0.00 & 0.0 & - & 29 & 0.00 & 0.0 & - & 0 & - & - & - \\
\hline Tennessee & 12 & 0.00 & 0.0 & - & 111 & 4.59 & 0.9 & 500.00 & 29 & 0.07 & 0.0 & - \\
\hline Texas & 317 & 0.05 & 0.6 & 1.30 & 434 & 0.30 & 4.6 & 5.42 & 41 & 0.01 & 0.0 & - \\
\hline Utah & 2 & 0.02 & 0.0 & - & 2 & 0.00 & 0.0 & - & 0 & - & - & - \\
\hline Vermont & 0 & - & - & - & 1 & 0.00 & 0.0 & - & 0 & - & - & - \\
\hline Virginia & 106 & 0.38 & 11.3 & 2.41 & 125 & 0.57 & 16.8 & 2.43 & 0 & - & - & - \\
\hline Washington & 5 & 1.42 & 80.0 & 1.68 & 67 & 0.05 & 0.0 & - & 39 & 0.01 & 0.0 & - \\
\hline W est Virginia & 73 & 0.04 & 0.0 & - & 29 & 1.76 & 27.6 & 5.81 & 0 & - & - & - \\
\hline Wisconsin & 161 & 0.16 & 5.6 & 1.87 & 160 & 0.73 & 6.3 & 10.65 & 12 & 0.03 & 0.0 & - \\
\hline Wyoming & 15 & 0.00 & 0.0 & - & 0 & - & - & - & 0 & - & - & - \\
\hline TOTAL & 5,266 & 0.24 & 4.5 & 3.78 & 7,378 & 0.37 & 4.0 & 8.10 & 771 & 0.14 & 2.6 & 3.37 \\
\hline
\end{tabular}

- indicates incalculable field.

NOTE: $\quad$ See Appendix A for source description, Appendix B for methods, and Appendix D for agents.

SOURCE: Occupational Safety and Health Administration: Integrated Management Information System. 
Figure 1-13. Asbestos: Number of OSHA inspector samples collected by state, 1993-1994

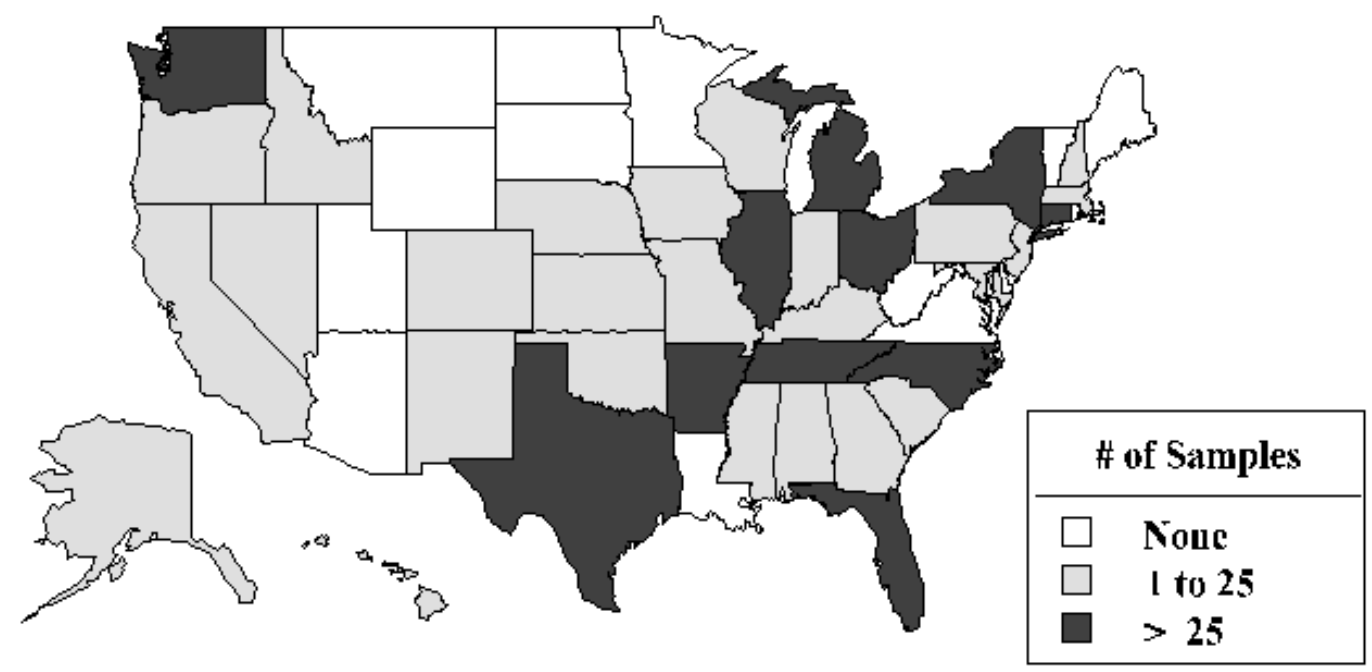

NOTE: See Appendix A for source description, Appendix B for methods, and Appendix D for agents. SOURCE: Occupational Safety and Health Administration: Integrated Management Information System .

Figure 1-14. Asbestos: Percent of OSHA inspector samples that exceeded the permissible exposure limit (PEL), by state, 1993-1994

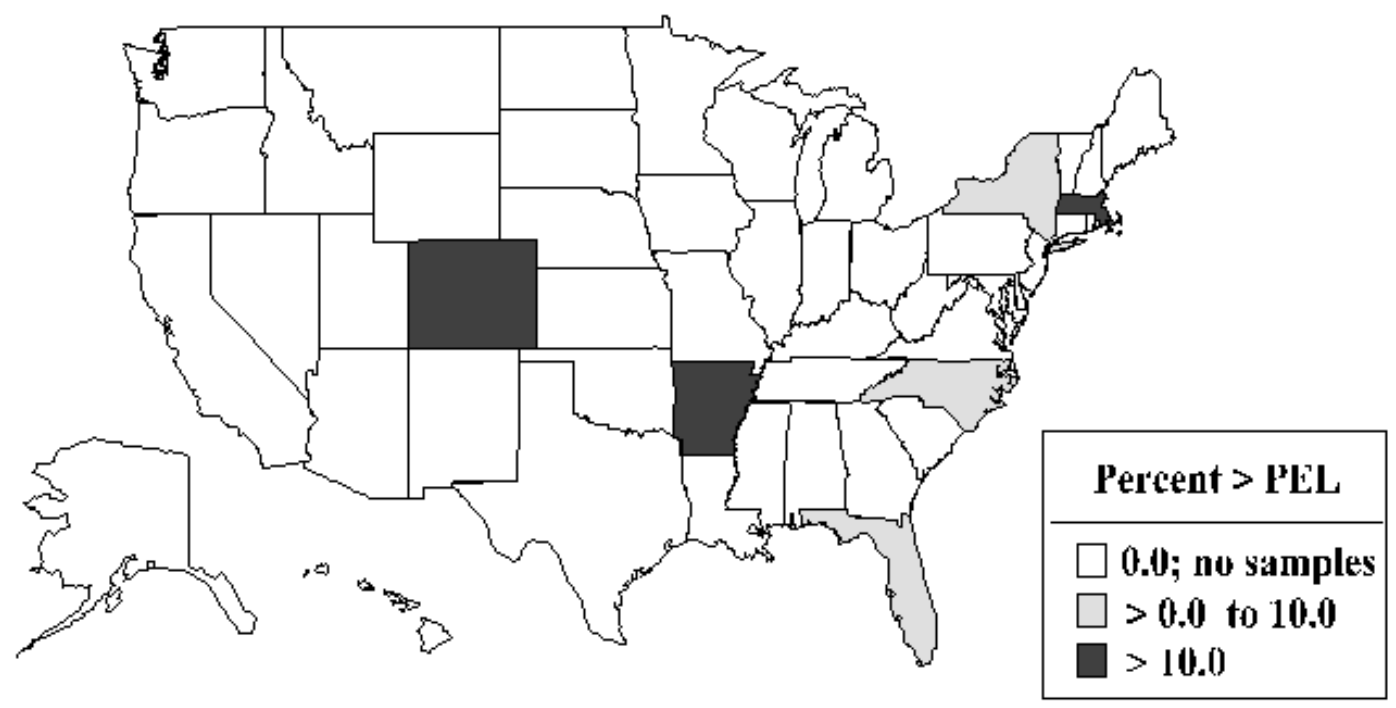

NOTE: See Appendix A for source description, Appendix B for methods, and Appendix D for agents. SOURCE: Occupational Safety and Health Administration: Integrated Management Information System. 
Figure 1-15. Asbestos: Average severity level by state for OSHA inspector samples, 1993-1994

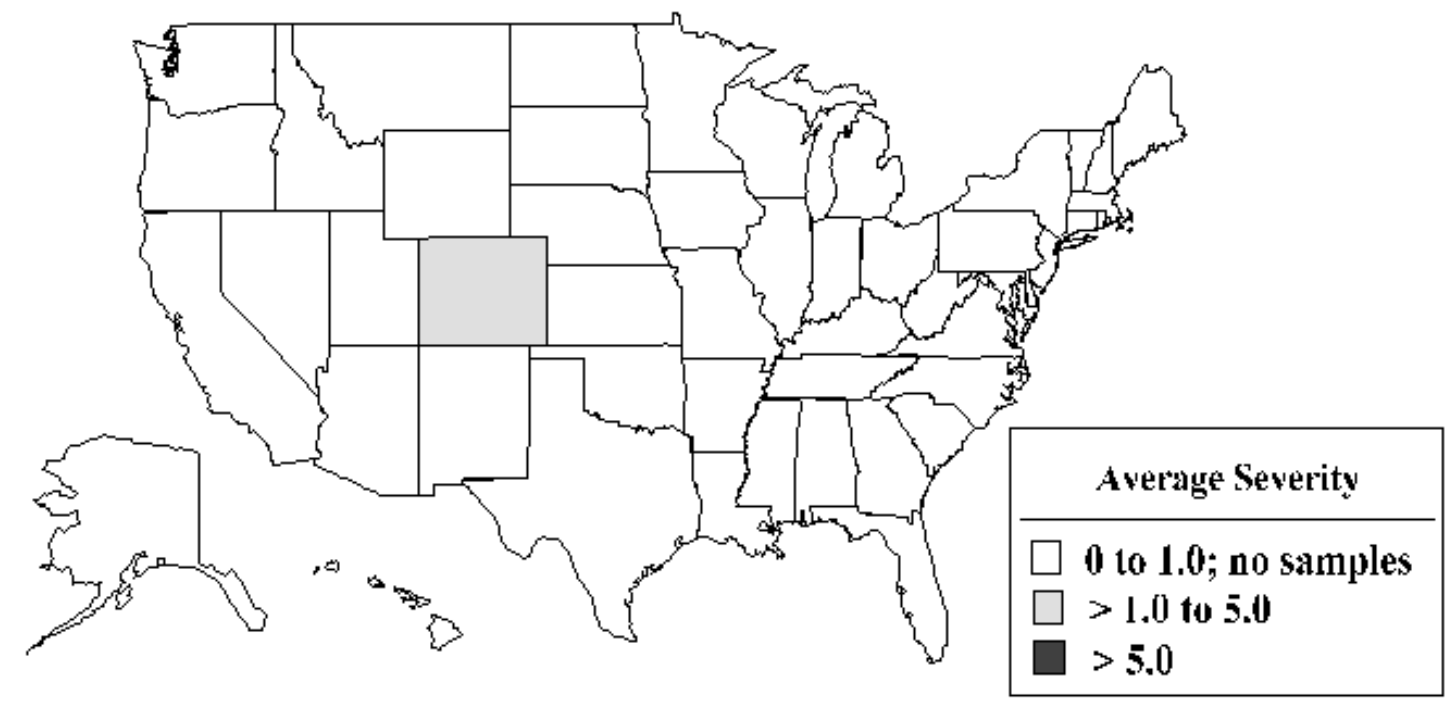

NOTE: See Appendix A for source descriptions, Appendix B for methods, and Appendix D for agents. SOURCE: Occupational Safety and Health Administration: Integrated Management Information System.

Figure 1-16. Asbestos: Average severity level by state for OSHA inspector samples that exceeded the permissible exposure limit (PEL), 1993-1994

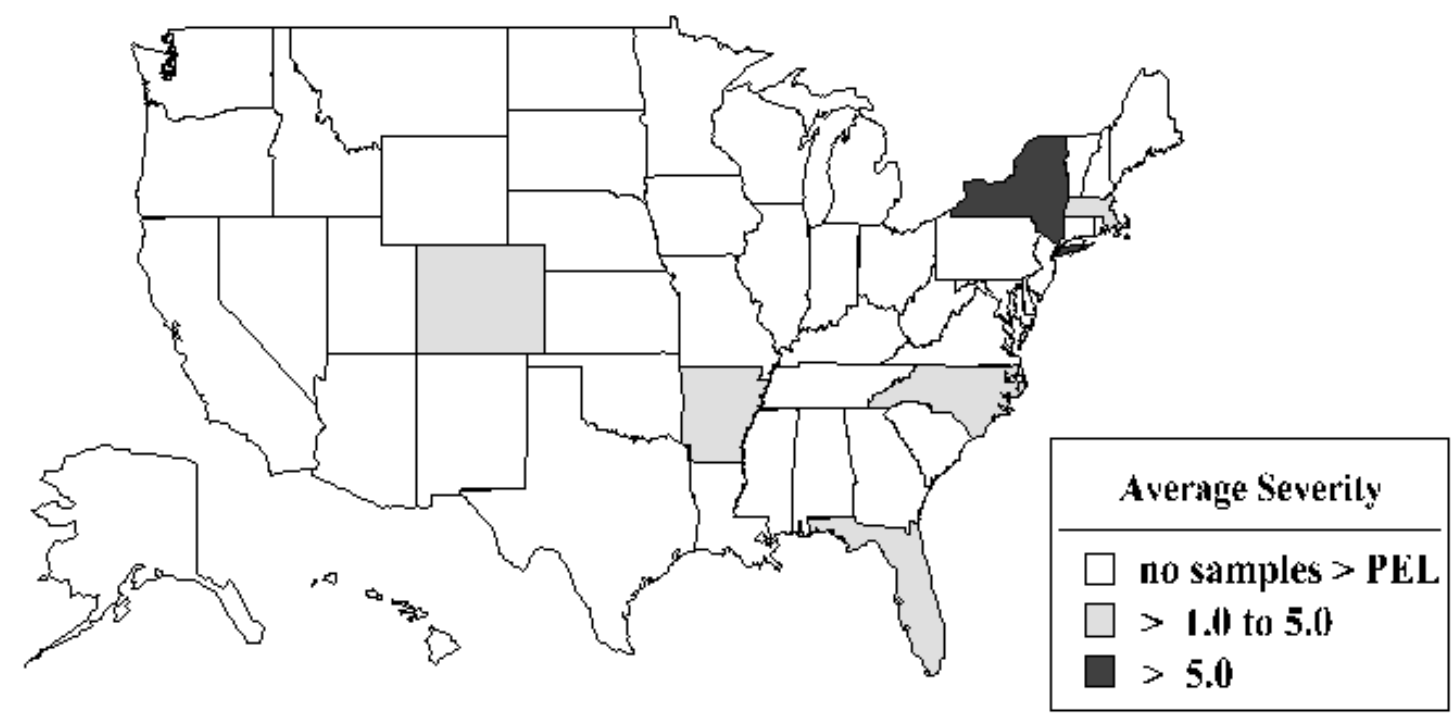

NOTE: See Appendix A for source descriptions, Appendix B for methods, and Appendix D for agents. SOURCE: Occupational Safety and Health Administration: Integrated Management Information System. 
Figure 1-17. Asbestos: Number of OSHA inspector samples

collected by county, 1993-1994

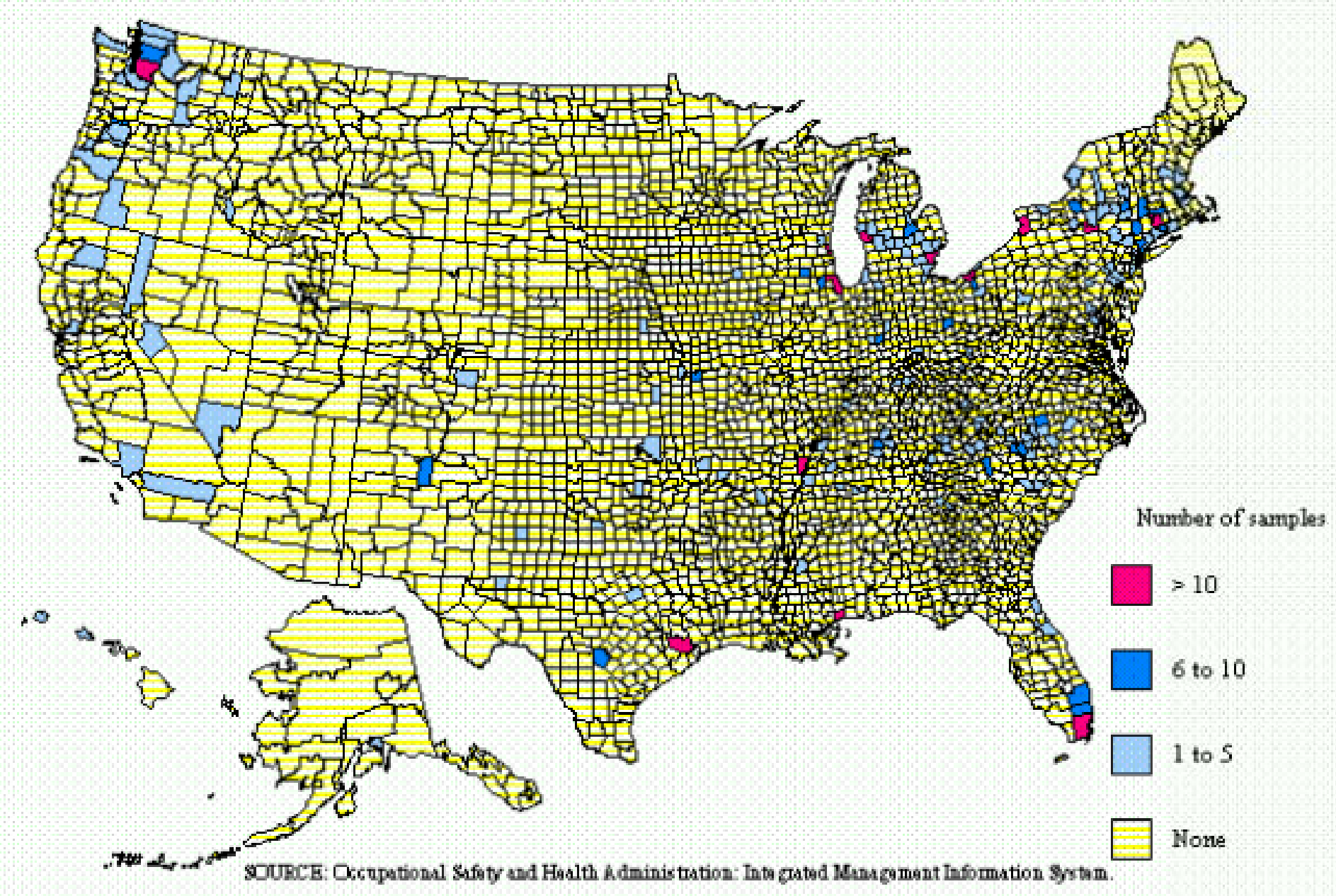


Figure 2-1. Coal workers' pneumoconiosis: Number of deaths, crude and ageadjusted mortality rates, U.S. residents age 15 and over, 1968-1992

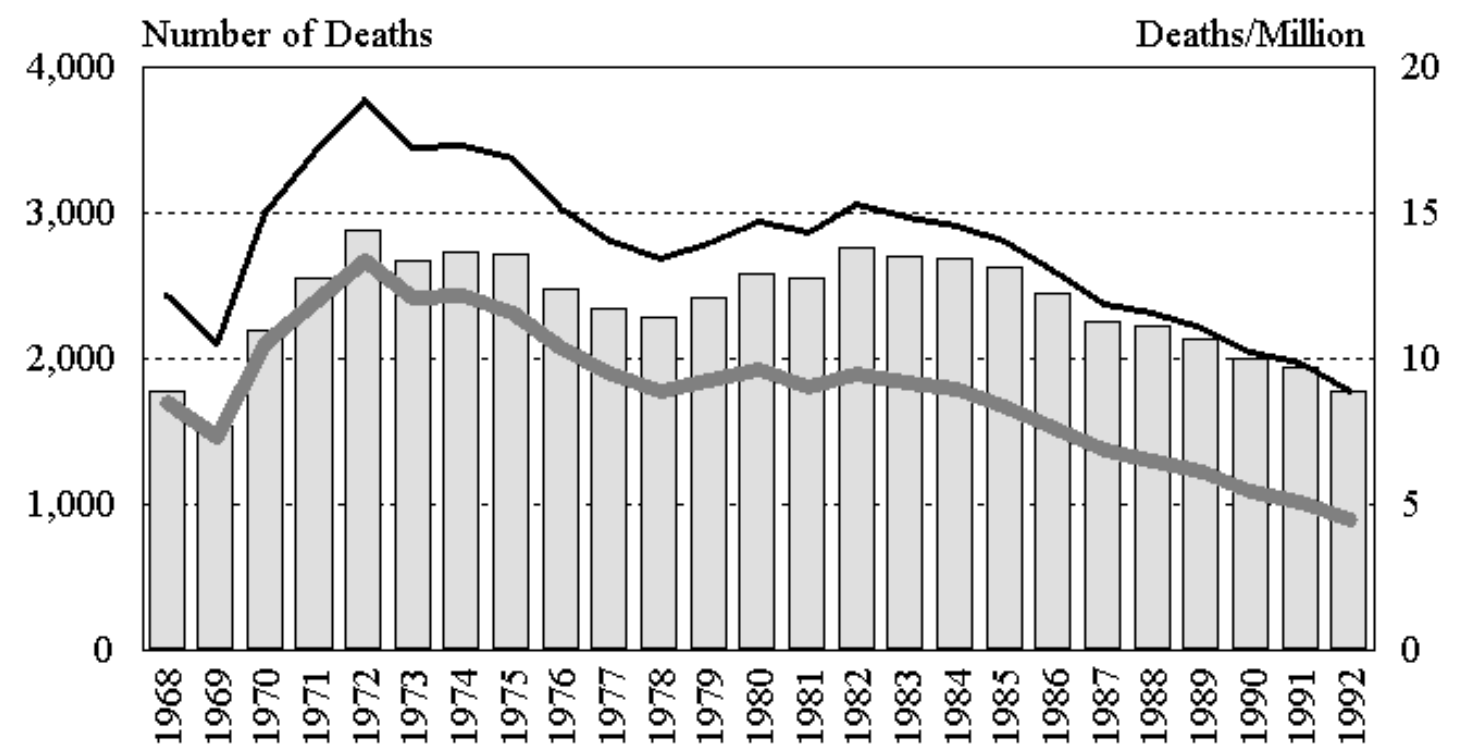

$\square$ Number of Deaths -U.S. Crude Rate - U.S. Age-adjusted Rate

NOTE:

See Appendix A for source description and Appendix B for methods and ICD-8 and ICD-9 codes.

SOURCE: National Center for Health Statistics multiple cause of death data. Population estimates from U.S. Bureau of the Census.

Figure 2-2. Coal workers' pneumoconiosis: Crude mortality rates by state, U.S. residents age 15 and over, 1991-1992

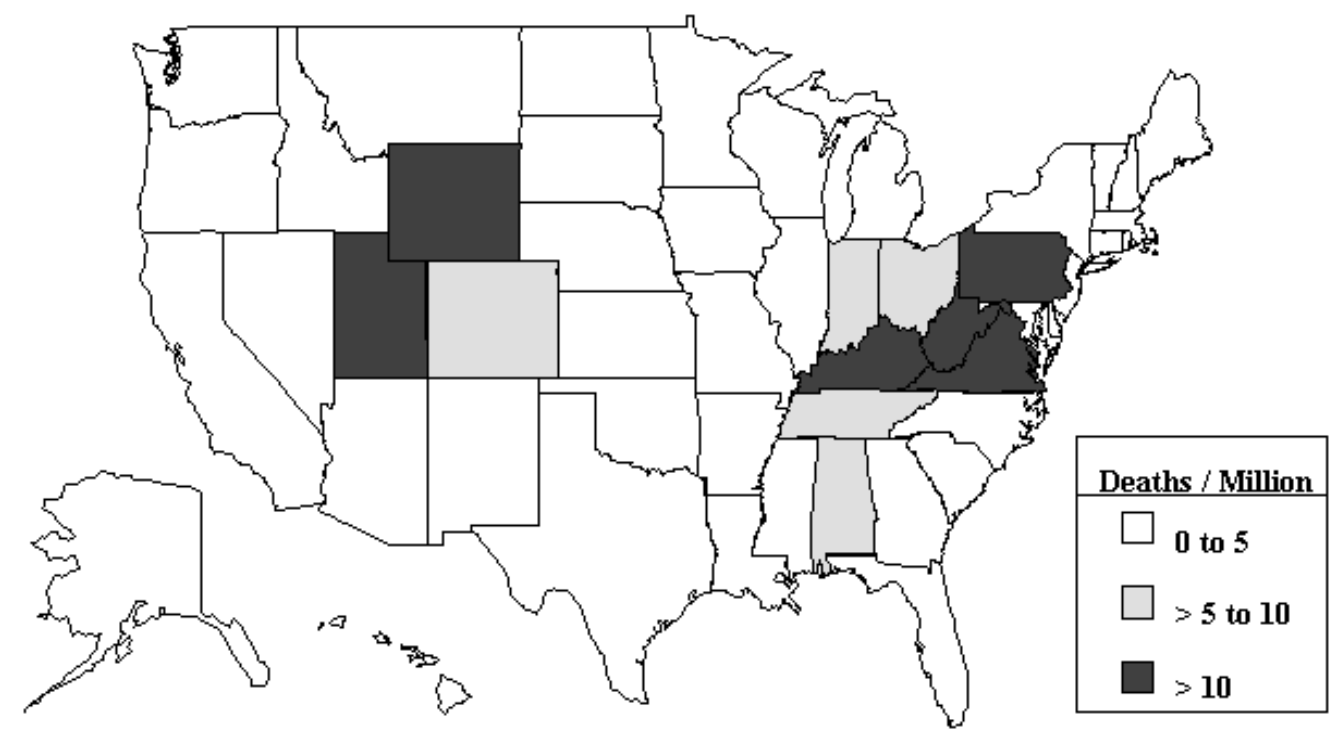

NOTE: $\quad$ See Appendix A for source description and Appendix B for methods and ICD-8 and ICD-9 codes.

SOURCE: National Center for Health Statistics multiple cause of death data. Population estimates from U.S. Bureau of the Census. 
Figure 2-3. Coal workers' pneumoconiosis(CWP): Median age at death, U.S. residents age 15 and over, 1968-1992

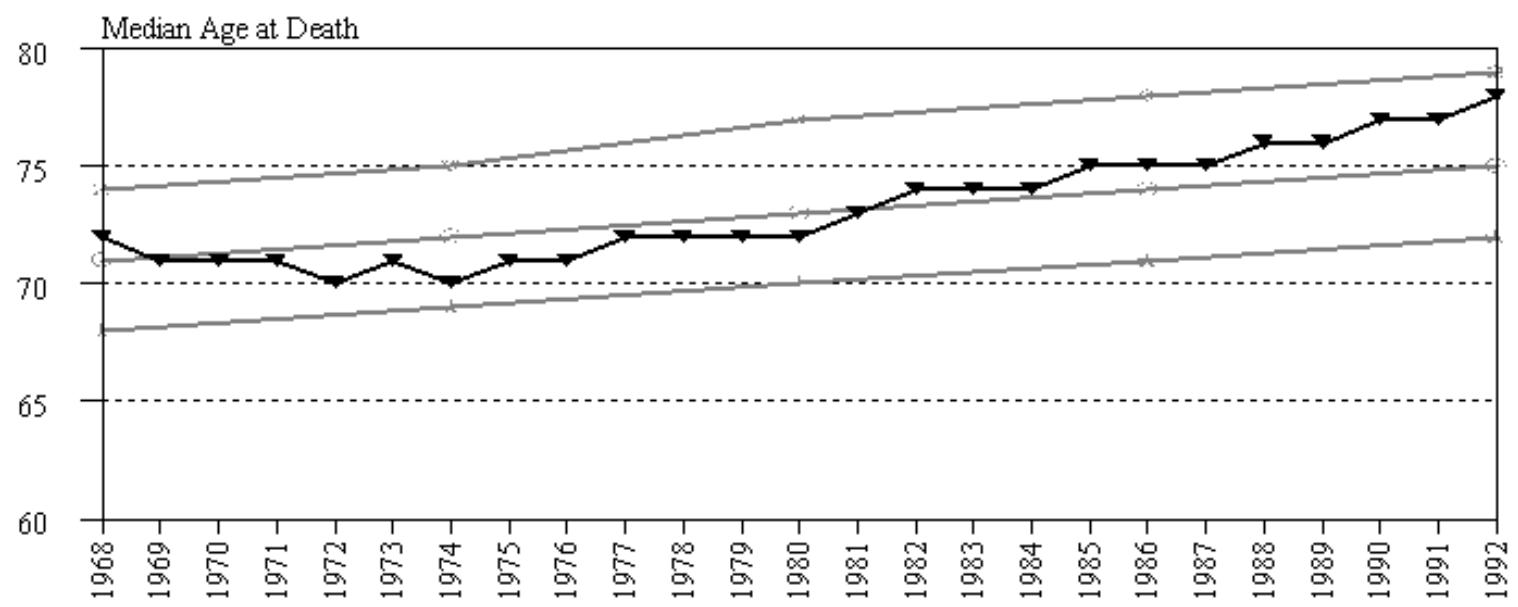

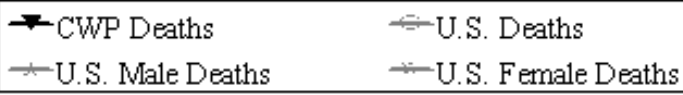

NOTE: See Appendix A for source description and Appendix B for methods and ICD-8 and ICD-9 codes.

SOURCE: National Center for Health Statistics multiple cause of death data.

Table 2-1. Coal workers' pneumoconiosis: Number of deaths by sex, race, and age, U.S. residents age 15 and over, 1991-1992

\begin{tabular}{|c|c|c|c|c|c|c|}
\hline & & & \multicolumn{2}{|c|}{1991} & \multicolumn{2}{|c|}{1992} \\
\hline & & & Number & Percent & Number & Percent \\
\hline \multicolumn{3}{|c|}{ Total deaths } & 1,938 & 100.0 & 1,766 & 100.0 \\
\hline \multirow{2}{*}{\multicolumn{2}{|c|}{ Sex }} & Male & 1,920 & 99.1 & 1,761 & 99.7 \\
\hline & & Female & 18 & 0.9 & 5 & 0.3 \\
\hline \multirow{3}{*}{\multicolumn{2}{|c|}{ Race }} & White & 1,877 & 96.8 & 1,707 & 96.7 \\
\hline & & Black & 60 & 3.1 & 55 & 3.1 \\
\hline & & Other & 1 & 0.1 & 4 & 0.2 \\
\hline \multirow{10}{*}{\multicolumn{2}{|c|}{ Age }} & $15-24$ & 1 & 0.1 & 0 & 0.0 \\
\hline & & $25-34$ & 2 & 0.1 & 2 & 0.1 \\
\hline & & $35-44$ & 14 & 0.7 & 6 & 0.3 \\
\hline & & $45-54$ & 23 & 1.2 & 14 & 0.8 \\
\hline & & $55-64$ & 110 & 5.7 & 90 & 5.1 \\
\hline & & $65-74$ & 536 & 27.7 & 457 & 25.9 \\
\hline & & $75-84$ & 884 & 45.6 & 858 & 48.6 \\
\hline & & 85 and over & 368 & 19.0 & 339 & 19.2 \\
\hline & & Mean age & 76.8 & & 77.5 & \\
\hline & & Range for age & $19-107$ & . & $34-102$ & \\
\hline
\end{tabular}


Table 2-2. Coal workers' pneumoconiosis: Number of deaths by state, U.S. residents age 15 and over, 1968-1992

\begin{tabular}{|c|c|c|c|c|c|}
\hline State & 1968-1978 & 1979-1990 & 1991 & 1992 & TOTAL \\
\hline Alabama & 126 & 328 & 28 & 23 & 505 \\
\hline Alaska & 1 & 2 & - & 1 & 4 \\
\hline Arizona & 32 & 88 & 4 & 2 & 126 \\
\hline Arkansas & 17 & 72 & 7 & 6 & 102 \\
\hline California & 206 & 254 & 41 & 22 & 523 \\
\hline Colorado & 94 & 189 & 15 & 19 & 317 \\
\hline Connecticut & 46 & 55 & 1 & 3 & 105 \\
\hline Delaware & 17 & 26 & 4 & 1 & 48 \\
\hline District of Columbia & 8 & 6 & - & - & 14 \\
\hline Florida & 198 & 399 & 33 & 23 & 653 \\
\hline Georgia & 12 & 43 & 3 & 2 & 60 \\
\hline Hawaii & 1 & 3 & - & - & 4 \\
\hline Idaho & 1 & 3 & - & 1 & 5 \\
\hline Illinois & 305 & 727 & 43 & 46 & 1,121 \\
\hline Indiana & 104 & 263 & 33 & 19 & 419 \\
\hline Iowa & 35 & 85 & 6 & 6 & 132 \\
\hline Kansas & 28 & 36 & 2 & 2 & 68 \\
\hline Kentucky & 737 & 1,971 & 112 & 116 & 2,936 \\
\hline Louisiana & 5 & 14 & 3 & - & 22 \\
\hline Maine & 1 & 2 & - & - & 3 \\
\hline Maryland & 65 & 115 & 5 & 13 & 198 \\
\hline Massachusetts & 26 & 14 & 2 & 2 & 44 \\
\hline Michigan & 135 & 216 & 15 & 10 & 376 \\
\hline Minnesota & 9 & 8 & - & - & 17 \\
\hline Mississippi & 7 & 12 & 3 & 1 & 23 \\
\hline Missouri & 25 & 75 & 6 & 11 & 117 \\
\hline Montana & 12 & 11 & - & - & 23 \\
\hline Nebraska & 4 & 1 & - & - & 5 \\
\hline Nevada & 4 & 12 & 1 & 1 & 18 \\
\hline New Hampshire & 7 & 1 & - & - & 8 \\
\hline New Jersey & 229 & 214 & 8 & 14 & 465 \\
\hline New Mexico & 23 & 44 & 3 & 4 & 74 \\
\hline New York & 167 & 167 & 4 & 2 & 340 \\
\hline North Carolina & 30 & 108 & 13 & 10 & 161 \\
\hline North Dakota & 1 & 4 & - & - & 5 \\
\hline Ohio & 417 & 1,108 & 83 & 74 & 1,682 \\
\hline Oklahoma & 19 & 57 & 4 & 1 & 81 \\
\hline Oregon & 11 & 28 & 1 & 1 & 41 \\
\hline Pennsylvania & 20,965 & 16,994 & 961 & 836 & 39,756 \\
\hline Rhode Island & 5 & 2 & - & - & 7 \\
\hline South Carolina & 11 & 19 & - & 5 & 35 \\
\hline South Dakota & 3 & 10 & - & - & 13 \\
\hline Tennessee & 140 & 420 & 23 & 30 & 613 \\
\hline Texas & 31 & 78 & 7 & 9 & 125 \\
\hline Utah & 24 & 135 & 12 & 13 & 184 \\
\hline Vermont & 1 & 6 & 1 & - & 8 \\
\hline Virginia & 398 & 1,460 & 134 & 147 & 2,139 \\
\hline Washington & 19 & 55 & 1 & 1 & 76 \\
\hline West Virginia & 1,305 & 3,339 & 308 & 283 & 5,235 \\
\hline Wisconsin & 19 & 21 & 1 & - & 41 \\
\hline Wyoming & 37 & 44 & 7 & 6 & 94 \\
\hline TOTAL & 26,123 & 29,344 & 1,938 & 1,766 & 59,171 \\
\hline
\end{tabular}

- indicates no deaths listed.

NOTE: See Appendix A for source description and Appendix B for methods and ICD-8 and ICD-9 codes.

SOURCE: National Center for Health Statistics multiple cause of death data. 
Table 2-3. Coal workers' pneumoconiosis: Mortality rates (per 1,000,000 population) by race and sex, U.S. residents age 15 and over, 1991-1992

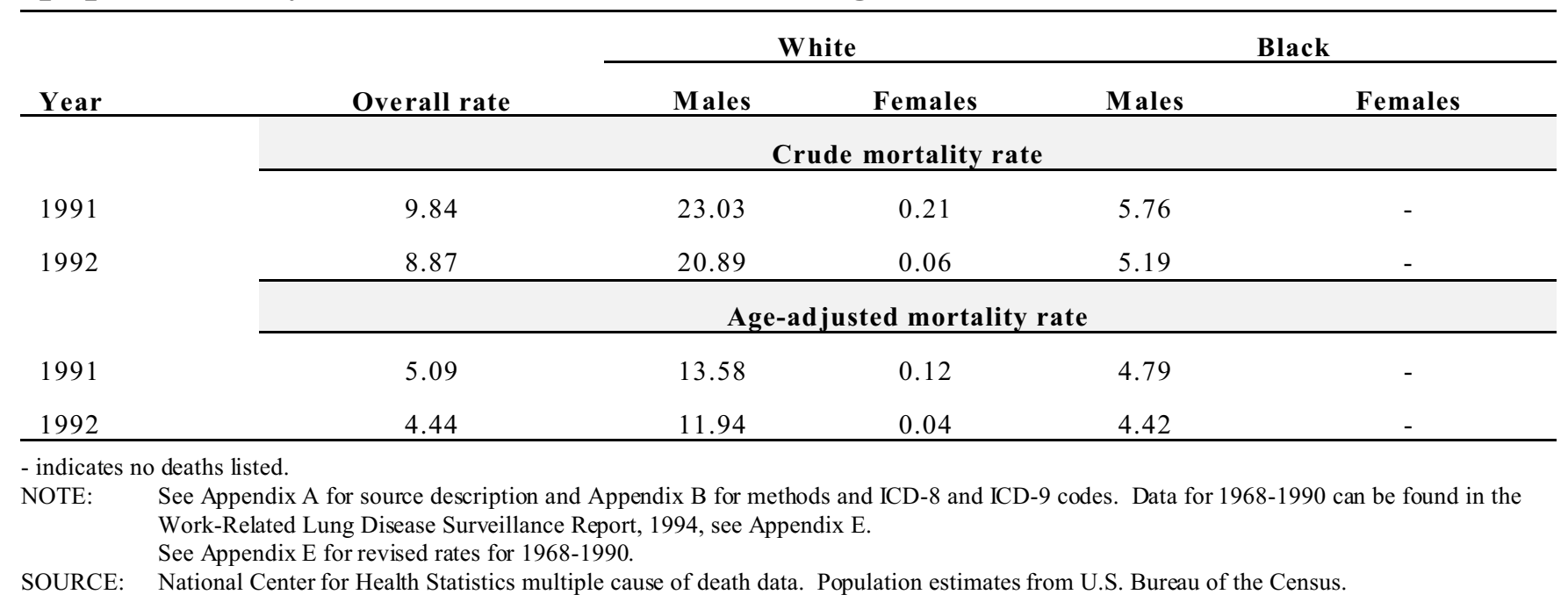

Table 2-4. Coal workers' pneumoconiosis: Years of potential life lost by race and sex, U.S. residents age 15 and over, 1991-1992

\begin{tabular}{|c|c|c|c|c|c|}
\hline \multirow[b]{2}{*}{ Year } & \multirow[b]{2}{*}{ Overall } & \multicolumn{2}{|c|}{ White } & \multicolumn{2}{|c|}{ Black } \\
\hline & & Males & Females & Males & Females \\
\hline & \multicolumn{5}{|c|}{ Years of potential life lost to age 65} \\
\hline 1991 & 1,360 & 1,205 & 115 & 35 & - \\
\hline \multirow[t]{2}{*}{1992} & 880 & 810 & 20 & 50 & - \\
\hline & \multicolumn{5}{|c|}{ Years of potential life lost to life expectancy } \\
\hline 1991 & 20,878 & 17,780 & 336 & 472 & - \\
\hline 1992 & 18,329 & 15,688 & 88 & 458 & - \\
\hline
\end{tabular}

- indicates no deaths listed.

NOTE: $\quad$ See Appendix A for source description and Appendix B for methods and ICD-8 and ICD-9 codes. Data for 1968-1990 can be found in the Work-Related Lung Disease Surveillance Report, 1994, see Appendix E.

SOURCE: National Center for Health Statistics multiple cause of death data. 
Table 2-5. Coal workers' pneumoconiosis: Total number of deaths, crude and age-adjusted mortality rates (per 1,000,000 population), and total years of potential life lost (YPLL) to life expectancy, by state, U.S. residents age 15 and over, 1988-1992

\begin{tabular}{|c|c|c|c|c|c|c|c|c|c|c|}
\hline \multirow[b]{2}{*}{ State } & \multirow{2}{*}{$\begin{array}{r}\text { Total } \\
\text { deaths }\end{array}$} & \multirow[b]{2}{*}{ Rank } & \multicolumn{2}{|c|}{ Crude mortality } & \multicolumn{2}{|c|}{ Age-adjusted mortality } & \multicolumn{4}{|c|}{ YPLL to life expectancy } \\
\hline & & & Rate & Rank & Rate & Rank & Years & Rank & Years/death & Rank \\
\hline Alabama & 146 & 9 & 9.23 & 8 & 3.97 & 9 & 1,362 & 10 & 9.3 & 33 \\
\hline Alaska & 1 & 42 & 0.50 & 39 & 0.82 & 22 & 8 & 46 & 8.0 & 44 \\
\hline Arizona & 20 & 25 & 1.41 & 24 & 0.71 & 26 & 214 & 25 & 10.7 & 22 \\
\hline Arkansas & 29 & 22 & 3.16 & 14 & 1.13 & 19 & 255 & 22 & 8.8 & 41 \\
\hline California & 114 & 11 & 0.98 & 29 & 0.72 & 25 & 1,783 & 8 & 15.6 & 4 \\
\hline Colorado & 87 & 12 & 6.77 & 10 & 3.59 & 10 & 762 & 14 & 8.8 & 42 \\
\hline Connecticut & 13 & 29 & 0.98 & 29 & 0.41 & 32 & 116 & 32 & 8.9 & 40 \\
\hline Delaware & 12 & 30 & 4.54 & 13 & 2.42 & 13 & 114 & 33 & 9.5 & 29 \\
\hline District of Columbia & 1 & 42 & 0.39 & 41 & 0.29 & 37 & 14 & 43 & 14.0 & 5 \\
\hline Florida & 151 & 8 & 2.86 & 16 & 1.17 & 17 & 1,660 & 9 & 11.0 & 17 \\
\hline Georgia & 22 & 24 & 0.87 & 31 & 0.60 & 28 & 245 & 23 & 11.1 & 16 \\
\hline Hawaii & - & - & - & - & - & - & - & - & - & - \\
\hline Idaho & 1 & 42 & 0.27 & 44 & 0.11 & 47 & 8 & 46 & 8.0 & 44 \\
\hline Illinois & 229 & 6 & 5.12 & 12 & 2.67 & 12 & 2,461 & 6 & 10.7 & 20 \\
\hline Indiana & 123 & 10 & 5.68 & 11 & 3.01 & 11 & 1,342 & 11 & 10.9 & 19 \\
\hline Iowa & 33 & 20 & 3.04 & 15 & 1.11 & 21 & 303 & 21 & 9.2 & 37 \\
\hline Kansas & 10 & 32 & 1.05 & 28 & 0.68 & 27 & 178 & 26 & 17.8 & 2 \\
\hline Kentucky & 648 & 4 & 44.80 & 3 & 25.43 & 3 & 7,669 & 4 & 11.8 & 12 \\
\hline Louisiana & 7 & 36 & 0.44 & 40 & 0.27 & 40 & 124 & 31 & 17.7 & 3 \\
\hline Maine & 1 & 42 & 0.21 & 47 & 0.14 & 46 & 14 & 43 & 14.0 & 5 \\
\hline Maryland & 41 & 17 & 2.16 & 20 & 1.37 & 16 & 457 & 17 & 11.1 & 15 \\
\hline Massachusetts & 9 & 34 & 0.37 & 42 & 0.21 & 44 & 113 & 34 & 12.6 & 9 \\
\hline Michigan & 80 & 13 & 2.21 & 19 & 1.17 & 17 & 810 & 13 & 10.1 & 27 \\
\hline Minnesota & - & - & - & - & - & - & - & - & - & - \\
\hline Mississippi & 8 & 35 & 0.82 & 33 & 0.38 & 35 & 75 & 37 & 9.4 & 31 \\
\hline Missouri & 36 & 19 & 1.80 & 23 & 0.80 & 23 & 372 & 19 & 10.3 & 25 \\
\hline Montana & - & - & - & - & - & - & - & - & - & - \\
\hline Nebraska & - & - & - & - & - & - & - & - & - & - \\
\hline Nevada & 4 & 38 & 0.84 & 32 & 0.53 & 31 & 42 & 38 & 10.5 & 24 \\
\hline New Hampshire & 1 & 42 & 0.23 & 46 & 0.24 & 42 & 21 & 41 & 21.0 & 1 \\
\hline New Jersey & 79 & 14 & 2.54 & 18 & 1.38 & 15 & 933 & 12 & 11.8 & 13 \\
\hline New Mexico & 16 & 27 & 2.81 & 17 & 1.50 & 14 & 150 & 28 & 9.4 & 31 \\
\hline New York & 41 & 17 & 0.57 & 37 & 0.25 & 41 & 381 & 18 & 9.3 & 34 \\
\hline North Carolina & 52 & 16 & 1.96 & 21 & 1.12 & 20 & 570 & 16 & 11.0 & 18 \\
\hline North Dakota & 2 & 41 & 0.82 & 33 & 0.28 & 38 & 16 & 42 & 8.0 & 44 \\
\hline Ohio & 428 & 5 & 10.07 & 7 & 5.15 & 7 & 4,540 & 5 & 10.6 & 23 \\
\hline Oklahoma & 17 & 26 & 1.39 & 25 & 0.56 & 29 & 157 & 27 & 9.2 & 35 \\
\hline Oregon & 12 & 30 & 1.07 & 27 & 0.54 & 30 & 148 & 29 & 12.3 & 11 \\
\hline Pennsylvania & 5,062 & 1 & 106.16 & 2 & 45.77 & 2 & 51,640 & 1 & 10.2 & 26 \\
\hline Rhode Island & 1 & 42 & 0.25 & 45 & 0.15 & 45 & 14 & 43 & 14.0 & 5 \\
\hline South Carolina & 10 & 32 & 0.73 & 36 & 0.40 & 33 & 92 & 36 & 9.2 & 36 \\
\hline South Dakota & 3 & 40 & 1.14 & 26 & 0.40 & 33 & 24 & 40 & 8.0 & 44 \\
\hline Tennessee & 168 & 7 & 8.68 & 9 & 4.56 & 8 & 1,804 & 7 & 10.7 & 21 \\
\hline Texas & 33 & 20 & 0.51 & 38 & 0.28 & 38 & 313 & 20 & 9.5 & 30 \\
\hline Utah & 73 & 15 & 12.28 & 6 & 7.59 & 6 & 699 & 15 & 9.6 & 28 \\
\hline Vermont & 4 & 38 & 1.81 & 22 & 0.76 & 24 & 34 & 39 & 8.5 & 43 \\
\hline Virginia & 715 & 3 & 29.01 & 4 & 20.31 & 4 & 8,916 & 3 & 12.5 & 10 \\
\hline Washington & 15 & 28 & 0.79 & 35 & 0.38 & 35 & 134 & 30 & 8.9 & 39 \\
\hline West Virginia & 1,458 & 2 & 203.87 & 1 & 102.05 & 1 & 17,178 & 2 & 11.8 & 14 \\
\hline Wisconsin & 7 & 36 & 0.37 & 42 & 0.23 & 43 & 93 & 35 & 13.3 & 8 \\
\hline Wyoming & 26 & 23 & 15.33 & 5 & 7.81 & 5 & 237 & 24 & 9.1 & 38 \\
\hline
\end{tabular}

- indicates no deaths listed.

NOTE: Ranks are based on unrounded values. See Appendix A for source description and Appendix B for methods and ICD-8 and ICD-9 codes.

SOURCE: National Center for Health Statistics multiple cause of death data. Population es timates from U.S. Bureau of the Census. 
Table 2-6. Coal workers' pneumoconiosis: Most frequently recorded occupations on death certificate, selected states, U.S. residents age 15 and over, 1991-1992

\begin{tabular}{|c|c|c|c|}
\hline $\mathrm{COC}$ & Occupation & Number & Percent \\
\hline 616 & Mining machine operators & 820 & 70.4 \\
\hline 889 & Laborers, except construction & 39 & 3.3 \\
\hline 019 & Managers and administrators, n.e.c. & 15 & 1.3 \\
\hline 804 & Truck drivers, heavy & 15 & 1.3 \\
\hline 453 & Janitors and cleaners & 13 & 1.1 \\
\hline 633 & Supervisors, precision production occupations & 13 & 1.1 \\
\hline 575 & Electricians & 11 & 0.9 \\
\hline 844 & Operating engineers & 10 & 0.9 \\
\hline 243 & Supervisors and proprietors, sales occupations & 9 & 0.8 \\
\hline \multirow[t]{4}{*}{567} & Carpenters & 8 & 0.7 \\
\hline & All other occupations & 168 & 14.4 \\
\hline & Occupation not reported & 44 & 3.8 \\
\hline & TOTAL & 1,165 & 100.0 \\
\hline
\end{tabular}

Table 2-7. Coal workers' pneumoconiosis: Most frequently recorded industries on death certificate, selected states, U.S. residents age 15 and over, 1991-1992

\begin{tabular}{llrr}
\hline CIC & Industry & Number & Percent \\
\hline 041 & Coal mining & 887 & 76.1 \\
060 & Construction & 44 & 3.8 \\
270 & Blast furnaces, steelworks, rolling and finishing mills & 21 & 1.8 \\
400 & Railroads & 13 & 1.1 \\
392 & Not specified manufacturing industries & 12 & 1.0 \\
351 & Motor vehicles and motor vehicle equipment & 9 & 0.8 \\
010 & Agricultural production, crops & 7 & 0.6 \\
192 & Industrial and miscellaneous chemicals & 7 & 0.6 \\
842 & Elementary and secondary schools & 6 & 0.5 \\
961 & Homemaker, student, unemployed, volunteer & 5 & 0.4 \\
& All other industries & 114 & 9.8 \\
& Industry not reported & 40 & 3.4 \\
& TOTAL & 1,165 & 100.0 \\
\hline
\end{tabular}

CIC - 1980 Census Industry Code

NOTE: $\quad$ See Appendix A for source description, Appendix B for methods and ICD-8 and ICD-9 codes, and Appendix C for list of 25 states reporting usual industry and years reporting. Data for 1985-1990 can be found in the Work-Related Lung Disease Surveillance Report, 1994, see Appendix E.

SOURCE: National Center for Health Statistics multiple cause of death data. 
Table 2-8. Coal workers' pneumoconiosis: Proportionate mortality ratio (PMR) by usual occupation, selected states and years, U.S. residents age 15 and over, 1985-1992

\begin{tabular}{|c|c|c|c|c|c|}
\hline \multirow[b]{2}{*}{$\mathrm{COC}$} & \multirow[b]{2}{*}{ Occupation } & \multirow{2}{*}{$\begin{array}{r}\text { Number } \\
\text { of deaths }\end{array}$} & \multirow[b]{2}{*}{ PMR } & \multicolumn{2}{|c|}{$95 \%$ confidence interval } \\
\hline & & & & $\mathrm{LCL}$ & $\mathrm{UCL}$ \\
\hline 616 & Mining machine operators & 2959 & 98.10 & 94.33 & 101.98 \\
\hline 853 & Excavate, loading machine operators & 7 & 7.74 & 3.11 & 15.96 \\
\hline 613 & Supervisors, extractive occupations & 6 & 5.35 & 1.96 & 11.66 \\
\hline 617 & Mining occupations, n.e.c. & 9 & 3.82 & 1.75 & 7.25 \\
\hline 859 & Miscellaneous material moving equipment operators & 9 & 2.79 & 1.28 & 5.29 \\
\hline 824 & Locomotive operating occupations & 14 & 2.62 & 1.43 & 4.40 \\
\hline 719 & Molding and casting machine operators & 9 & 2.25 & 1.03 & 4.27 \\
\hline 844 & Operating engineers & 27 & 1.83 & 1.20 & 2.66 \\
\hline 575 & Electricians & 36 & 1.67 & 1.16 & 2.32 \\
\hline
\end{tabular}


Table 2-9. Coal workers' pneumoconiosis: Proportionate mortality ratio (PMR) by usual industry, selected states and years, U.S. residents age 15 and over, 1985-1992

\begin{tabular}{|c|c|c|c|c|c|}
\hline \multirow[b]{2}{*}{$\mathrm{CIC}$} & \multirow[b]{2}{*}{ Industry } & \multirow{2}{*}{$\begin{array}{l}\text { Number } \\
\text { of deaths }\end{array}$} & \multirow[b]{2}{*}{ PMR } & \multicolumn{2}{|c|}{$95 \%$ confidence interval } \\
\hline & & & & LCL & UCL \\
\hline 041 & Coal mining & 3,158 & 100.22 & 96.64 & 103.85 \\
\hline 040 & Metal mining & 24 & 4.86 & 3.12 & 7.23 \\
\hline
\end{tabular}


Figure 2-4. Coal workers' pneumoconiosis: Total number of deaths by county,

U.S. residents age 15 and over, 1983-1992

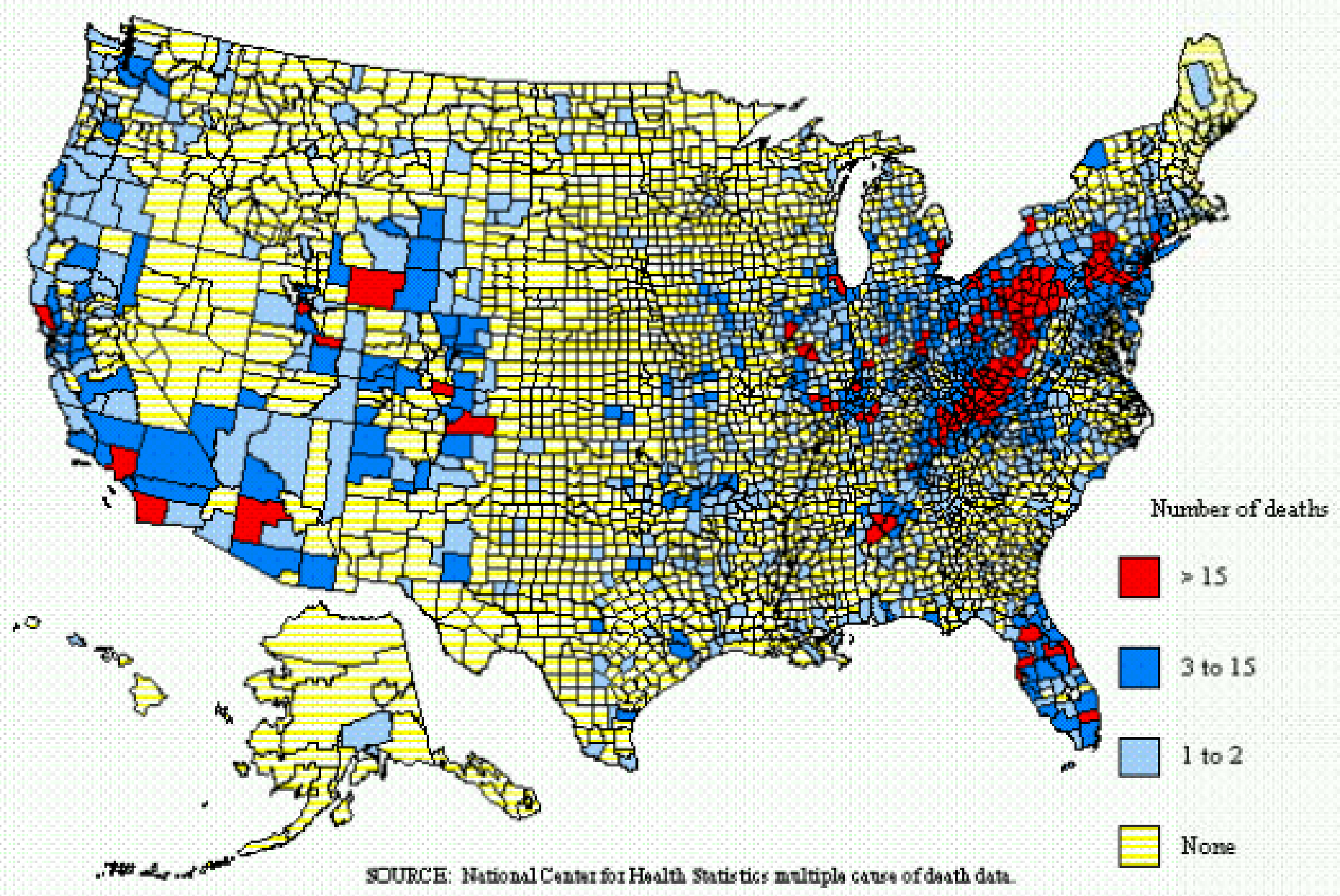


Figure 2-5. Coal workers' pneumoconiosis: Age-adjusted mortality rates

by county, U.S. residents age 15 and over, 1983-1992

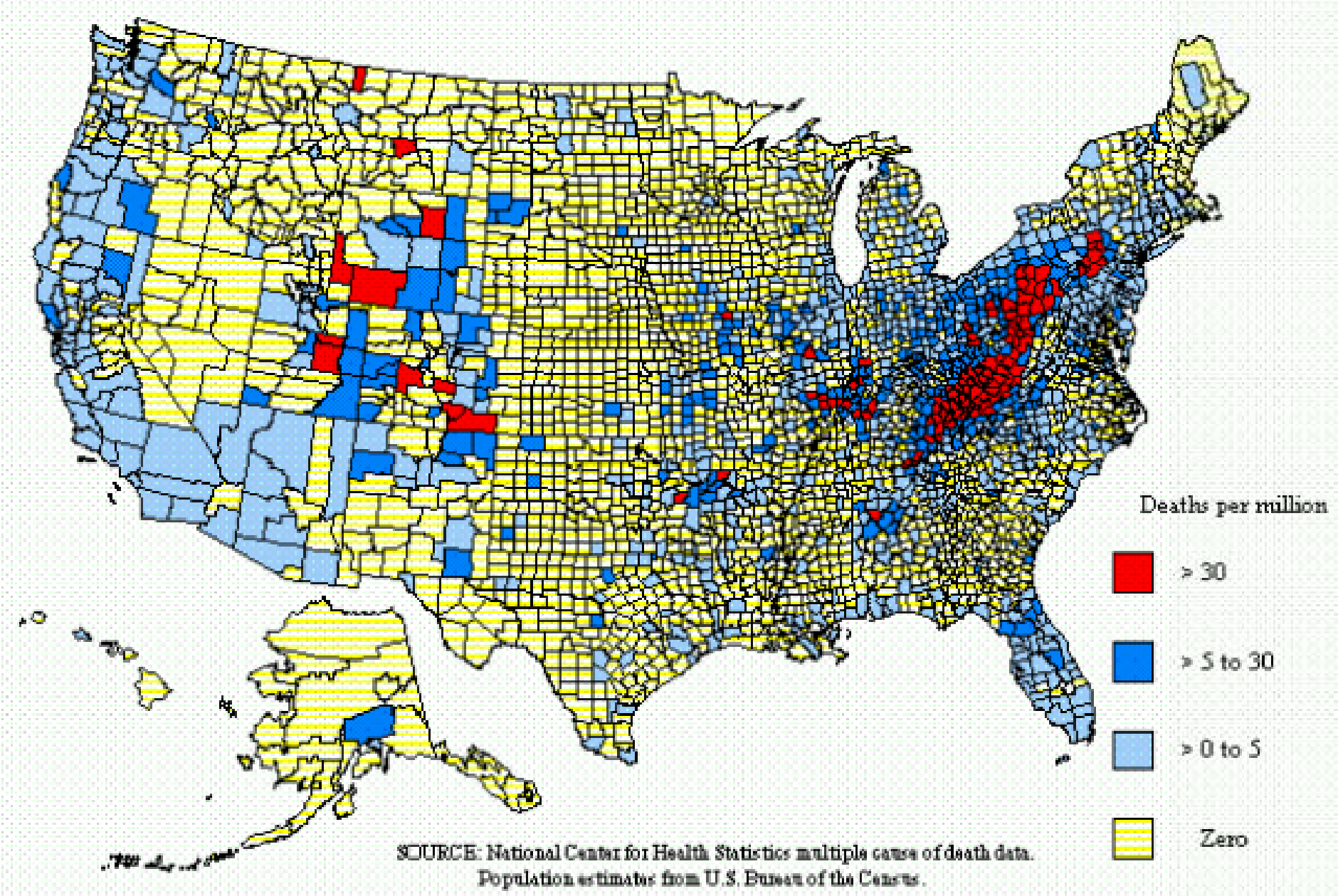


Figure 2-6. Coal and coal mine dust: Number of MSHA and OSHA inspector samples and percent exceeding the permissible exposure limit (PEL), 1970-1994

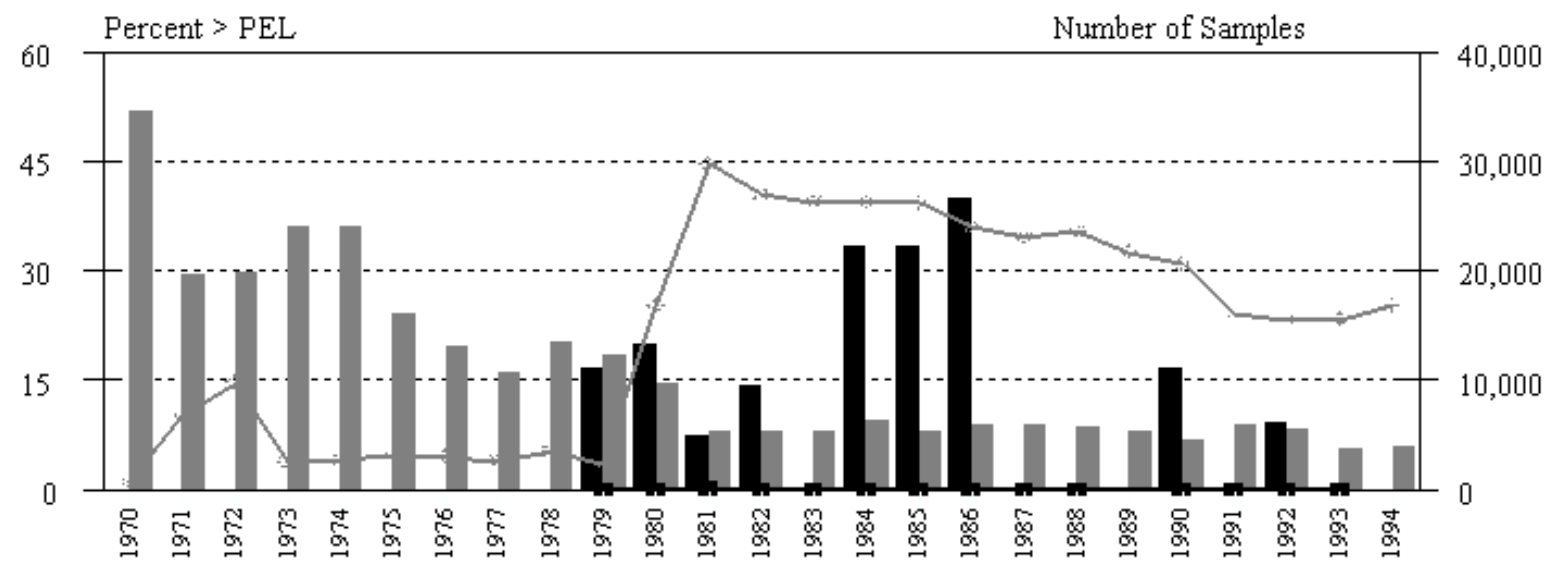

\begin{tabular}{ll|}
- OSHA Percent > PEL & MSHA Percent > PEL \\
- \# OSHA Samples & \#MSHA Samples
\end{tabular}

NOTE: See Appendix A for source description, Appendix B for methods, and Appendix D for agents.

SOURCE: Mine Safety and Health Administration: respirable coal mine dust data. Occupational Safety and Health Administration: Integrated Management Information System.

Figure 2-7. Coal and coal mine dust: Number of MSHA and OSHA inspector samples and average severity levels, 1970-1994

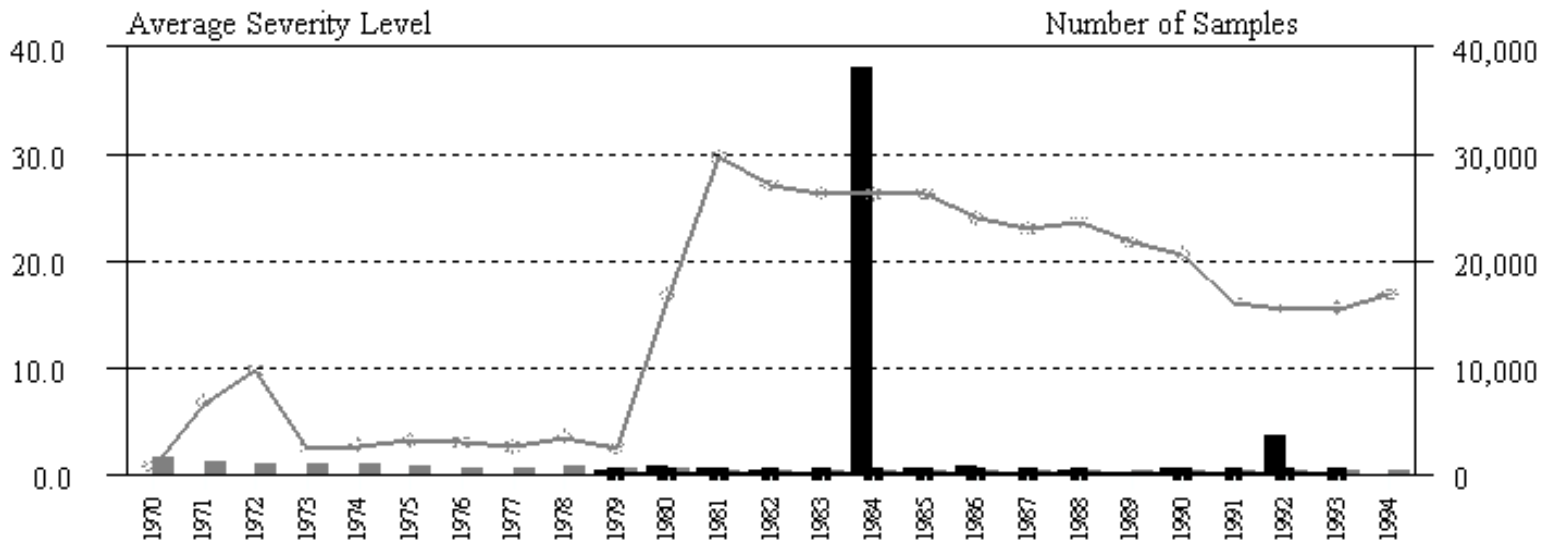

\section{OSHA Severity Levels \# OSHA Samples \\ MSHA Severity Levels \#MSHA Samples}

NOTE: See Appendix A for source description, Appendix B for methods, and Appendix D for agents.

SOURCE: Mine Safety and Health Administration: respirable coal mine dust data. Occupational Safety and Health Administration: Integrated Management Information System. 
Table 2-10. Coal and coal mine dust: Number of MSHA and OSHA inspector samples, percent exceeding the permissible exposure limit (PEL) and average severity level, by industry, 1993-1994

\begin{tabular}{|c|c|c|c|c|}
\hline CIC & $\begin{array}{l}\text { Industries most frequently recorded on } \\
\text { 1991-1992 death certificates with } \\
\text { coal workers' pneumoconiosis }\end{array}$ & $\begin{array}{r}\text { Number of } \\
\text { samples } \\
\end{array}$ & $\%>$ PEL & $\begin{array}{r}\text { Average } \\
\text { severity }\end{array}$ \\
\hline 041 & Coal mining & 32,362 & 5.8 & 0.38 \\
\hline 060 & Construction & 0 & - & - \\
\hline 270 & Blast furnaces, steelworks, rolling and finishing mills & 0 & - & - \\
\hline 400 & Railroads & 0 & - & - \\
\hline 392 & Not specified manufacturing industries & 0 & - & - \\
\hline 351 & Motor vehicles and motor vehicle equipment & 0 & - & - \\
\hline 010 & Agricultural productions, crops & 0 & - & - \\
\hline 192 & Industrial and miscellaneous chemicals & 0 & - & - \\
\hline 842 & Elementary and secondary schools & 0 & - & - \\
\hline \multirow[t]{4}{*}{961} & Homemaker, student, unemployed, volunteer & 0 & - & - \\
\hline & All other industries & 7 & 0.0 & 0.19 \\
\hline & Industry not reported & 0 & - & - \\
\hline & TOTAL & 32,369 & 5.8 & 0.38 \\
\hline \multicolumn{5}{|c|}{ CIC - 1980 Census Industry Code $\quad-$ indicates incalculable field } \\
\hline \multirow{2}{*}{$\begin{array}{l}\text { NOTE: } \\
\text { SOURCE: }\end{array}$} & $\begin{array}{l}\text { See Appendix A for source description, Appendix B for methods, Appendix C for } 1 \\
\text { agents. }\end{array}$ & usual industry & reporting, & endix D for \\
\hline & $\begin{array}{l}\text { Mine Safety and Health Administration: respirable coal mine dust data. Occupationa } \\
\text { National Center for Health Statistics: multiple cause of death data. }\end{array}$ & Administration: Int & ed Management & mation System. \\
\hline
\end{tabular}

Table 2-11. Coal and coal mine dust: Number of MSHA and OSHA inspector samples, percent exceeding the permissible exposure limit (PEL) and average severity level, by industry, 1993-1994

\begin{tabular}{|c|c|c|c|c|}
\hline CIC & Industries most frequently sampled in 1993-1994 & $\begin{array}{r}\text { Number of } \\
\text { samples } \\
\end{array}$ & $\%>$ PEL & $\begin{array}{r}\text { Average } \\
\text { severity }\end{array}$ \\
\hline 041 & Coal mining & 32,362 & 5.8 & 0.38 \\
\hline 160 & Pulp, paper, and paperboard mills & 4 & 0.0 & 0.22 \\
\hline \multirow[t]{4}{*}{891} & Noncommercial educational and scientific research & 3 & 0.0 & 0.14 \\
\hline & All other industries & 0 & - & - \\
\hline & Industry not reported & 0 & - & - \\
\hline & TOTAL & 32,369 & 5.8 & 0.38 \\
\hline
\end{tabular}

CIC - 1980 Census Industry Code

- indicates incalculable field

NOTE: See Appendix A for source description, Appendix B for methods, and Appendix D for agents.

SOURCE: Mine Safety and Health Administration: respirable coal mine dust data. Occupational Safety and Health Administration: Integrated Management Information System. 
Table 2-12 (page 1 of 2). Coal and coal mine dust: Number of MSHA inspector samples, percent exceeding the permissible exposure limit (PEL) and average severity levels (Avg. Sev.), by state, 1970-1994

\begin{tabular}{|c|c|c|c|c|c|c|c|c|c|c|c|c|}
\hline \multirow[b]{3}{*}{ State } & \multicolumn{4}{|c|}{ 1970-1984 } & \multicolumn{4}{|c|}{ 1985-1994 } & \multicolumn{4}{|c|}{ 1993-1994 } \\
\hline & \multicolumn{2}{|c|}{ Total samples } & \multicolumn{2}{|c|}{ Samples > PEL } & \multicolumn{2}{|c|}{ Total samples } & \multicolumn{2}{|c|}{ Samples > PEL } & \multicolumn{2}{|c|}{ Total samples } & \multicolumn{2}{|c|}{ Samples > PEL } \\
\hline & Number & $\begin{array}{l}\text { Avg. } \\
\text { Sev. }\end{array}$ & $\%$ & $\begin{array}{l}\text { Avg. } \\
\text { Sev. }\end{array}$ & Number & $\begin{array}{l}\text { Avg. } \\
\text { Sev. }\end{array}$ & $\%$ & $\begin{array}{l}\text { Avg. } \\
\text { Sev. }\end{array}$ & Number & $\begin{array}{l}\text { Avg. } \\
\text { Sev. }\end{array}$ & $\%$ & $\begin{array}{l}\text { Avg. } \\
\text { Sev. }\end{array}$ \\
\hline Alabama & 3,738 & 0.56 & 13.0 & 1.85 & 6,902 & 0.48 & 9.2 & 1.74 & 1,539 & 0.41 & 4.8 & 2.12 \\
\hline Alaska & 23 & 0.20 & 0.0 & - & 25 & 0.40 & 4.0 & 4.30 & 1 & 0.10 & 0.0 & - \\
\hline Arizona & 46 & 0.37 & 6.5 & 1.47 & 158 & 0.22 & 2.5 & 1.26 & 11 & 0.15 & 0.0 & - \\
\hline Arkansas & 96 & 0.18 & 5.2 & 1.16 & 123 & 0.19 & 4.1 & 1.86 & 17 & 0.09 & 0.0 & - \\
\hline California & 0 & - & - & - & 1 & 0.20 & 0.0 & - & 1 & 0.20 & 0.0 & - \\
\hline Colorado & 2,250 & 1.04 & 30.1 & 2.43 & 2,796 & 0.67 & 15.7 & 1.91 & 381 & 0.50 & 10.0 & 1.40 \\
\hline Connecticut & 0 & - & - & - & 0 & - & - & - & 0 & - & - & - \\
\hline Delaware & 0 & - & - & - & 0 & - & - & - & 0 & - & - & - \\
\hline District of Columbia & 0 & - & - & - & 0 & - & - & - & 0 & - & - & - \\
\hline Florida & 0 & - & - & - & 0 & - & - & - & 0 & - & - & - \\
\hline Georgia & 29 & 0.20 & 0.0 & - & 0 & - & - & - & 0 & - & - & - \\
\hline Hawaii & 0 & - & - & - & 0 & - & - & - & 0 & - & - & - \\
\hline Idaho & 0 & - & - & - & 1 & 0.40 & 0.0 & - & 0 & - & - & - \\
\hline Illinois & 6,279 & 0.83 & 21.5 & 2.09 & 8,249 & 0.54 & 9.0 & 1.54 & 1,354 & 0.46 & 4.8 & 1.42 \\
\hline Indiana & 2,282 & 0.34 & 6.7 & 2.46 & 2,559 & 0.34 & 4.8 & 2.50 & 424 & 0.37 & 7.5 & 1.65 \\
\hline Iowa & 113 & 0.33 & 3.5 & 2.96 & 153 & 0.56 & 16.3 & 1.81 & 13 & 0.28 & 0.0 & - \\
\hline Kansas & 91 & 0.18 & 3.3 & 2.00 & 116 & 0.19 & 1.7 & 2.00 & 5 & 0.12 & 0.0 & - \\
\hline Kentucky & 32,882 & 0.59 & 13.4 & 2.28 & 53,871 & 0.46 & 9.0 & 2.14 & 9,960 & 0.39 & 6.6 & 1.73 \\
\hline Louisiana & 0 & - & - & - & 56 & 0.16 & 0.0 & - & 23 & 0.22 & 0.0 & - \\
\hline Maine & 0 & - & - & - & 0 & - & - & - & 0 & - & - & - \\
\hline Maryland & 602 & 0.42 & 5.0 & 2.17 & 945 & 0.43 & 6.5 & 2.35 & 298 & 0.28 & 3.4 & 1.54 \\
\hline Massachusetts & 0 & - & - & - & 0 & - & - & - & 0 & - & - & - \\
\hline Michigan & 0 & - & - & - & 1 & 0.24 & 0.0 & - & 0 & - & - & - \\
\hline Minnesota & 0 & - & - & - & 3 & 0.15 & 0.0 & - & 0 & - & - & - \\
\hline Mississippi & 0 & - & - & - & 0 & - & - & - & 0 & - & - & - \\
\hline Missouri & 184 & 0.24 & 1.6 & 4.27 & 371 & 0.20 & 1.9 & 1.77 & 74 & 0.21 & 2.7 & 1.53 \\
\hline Montana & 116 & 0.31 & 5.2 & 2.35 & 300 & 0.27 & 3.0 & 3.92 & 52 & 0.15 & 3.8 & 1.70 \\
\hline
\end{tabular}


Table 2-12 (page 2 of 2). Coal and coal mine dust: Number of MSHA inspector samples, percent exceeding the permissible exposure limit (PEL) and average severity levels (Avg. Sev.), by state, 1970-1994

\begin{tabular}{|c|c|c|c|c|c|c|c|c|c|c|c|c|}
\hline \multirow[b]{3}{*}{ State } & \multicolumn{4}{|c|}{ 1970-1984 } & \multicolumn{4}{|c|}{ 1985-1994 } & \multicolumn{4}{|c|}{ 1993-1994 } \\
\hline & \multicolumn{2}{|c|}{ Total samples } & \multicolumn{2}{|c|}{ Samples > PEL } & \multicolumn{2}{|c|}{ Total samples } & \multicolumn{2}{|c|}{ Samples > PEL } & \multicolumn{2}{|c|}{ Total samples } & \multicolumn{2}{|c|}{ Samples > PEL } \\
\hline & Number & $\begin{array}{l}\text { Avg. } \\
\text { Sev. }\end{array}$ & $\%$ & $\begin{array}{l}\text { Avg. } \\
\text { Sev. }\end{array}$ & Number & $\begin{array}{l}\text { Avg. } \\
\text { Sev. }\end{array}$ & $\%$ & $\begin{array}{l}\text { Avg. } \\
\text { Sev. }\end{array}$ & Number & $\begin{array}{l}\text { Avg. } \\
\text { Sev. }\end{array}$ & $\%$ & $\begin{array}{l}\text { Avg. } \\
\text { Sev. }\end{array}$ \\
\hline Nebraska & 0 & - & - & - & 0 & - & - & - & 0 & - & - & - \\
\hline Nevada & 0 & - & - & - & 0 & - & - & - & 0 & - & - & - \\
\hline New Hampshire & 0 & - & - & - & 0 & - & - & - & 0 & - & - & - \\
\hline New Jersey & 0 & - & - & - & 0 & - & - & - & 0 & - & - & - \\
\hline New Mexico & 369 & 1.15 & 28.2 & 2.97 & 558 & 0.40 & 8.4 & 1.68 & 96 & 0.44 & 13.5 & 1.42 \\
\hline New York & 0 & - & - & - & 0 & - & - & - & 0 & - & - & - \\
\hline North Carolina & 0 & - & - & - & 9 & 0.10 & 0.0 & - & 0 & - & - & - \\
\hline North Dakota & 258 & 0.19 & 1.9 & 1.56 & 442 & 0.17 & 1.1 & 3.79 & 34 & 0.11 & 0.0 & - \\
\hline Ohio & 8,574 & 0.69 & 20.6 & 1.76 & 10,524 & 0.33 & 4.6 & 1.83 & 1,566 & 0.23 & 1.6 & 1.54 \\
\hline Oklahoma & 690 & 0.29 & 5.5 & 2.57 & 1,167 & 0.33 & 5.0 & 2.57 & 181 & 0.30 & 3.3 & 2.38 \\
\hline Oregon & 0 & - & - & - & 0 & - & - & - & 0 & - & - & - \\
\hline Pennsylvania & 32,528 & 0.45 & 8.4 & 2.20 & 22,387 & 0.37 & 6.2 & 1.72 & 4,442 & 0.29 & 4.1 & 1.89 \\
\hline Rhode Island & 0 & - & - & - & 0 & - & - & - & 0 & - & - & - \\
\hline South Carolina & 0 & - & - & - & 0 & - & - & - & 0 & - & - & - \\
\hline South Dakota & 0 & - & - & - & 0 & - & - & - & 0 & - & - & - \\
\hline Tennessee & 4,106 & 0.52 & 9.1 & 2.27 & 4,112 & 0.38 & 5.5 & 2.13 & 503 & 0.28 & 3.6 & 1.63 \\
\hline Texas & 540 & 0.18 & 3.0 & 2.04 & 1,210 & 0.14 & 1.5 & 2.07 & 215 & 0.12 & 1.4 & 1.50 \\
\hline Utah & 1,601 & 0.89 & 24.7 & 2.05 & 3,388 & 0.76 & 19.2 & 1.91 & 415 & 0.67 & 14.5 & 1.54 \\
\hline Vermont & 0 & & - & - & 0 & - & - & - & 0 & - & - & - \\
\hline Virginia & 23,490 & 0.52 & 11.7 & 1.98 & 24,565 & 0.44 & 8.8 & 1.80 & 3,296 & 0.40 & 6.2 & 1.80 \\
\hline Washington & 34 & 0.20 & 0.0 & - & 72 & 0.22 & 2.8 & 2.45 & 28 & 0.17 & 0.0 & - \\
\hline West Virginia & 41,525 & 0.63 & 15.1 & 2.08 & 56,576 & 0.43 & 7.1 & 1.82 & 7,105 & 0.42 & 6.9 & 1.68 \\
\hline $\mathrm{W}$ isconsin & 0 & - & - & - & 0 & - & - & - & 0 & - & - & - \\
\hline Wyoming & 548 & 0.55 & 13.7 & 2.00 & 1,209 & 0.34 & 8.1 & 1.54 & 328 & 0.29 & 6.1 & 1.59 \\
\hline TOTAL & 162,994 & 0.58 & 13.3 & 2.12 & 202,849 & 0.44 & 7.9 & 1.91 & 32,362 & 0.38 & 5.8 & 1.73 \\
\hline
\end{tabular}

- indicates incalculable field.

NOTE: $\quad$ See Appendix A for source description, Appendix B for methods, and Appendix D for agents.

SOURCE: Mine Safety and Health Administration: respirable coal mine dust data. 
Figure 2-8. Coal and coal mine dust: Number of MSHA inspector samples collected by state, 1993-1994

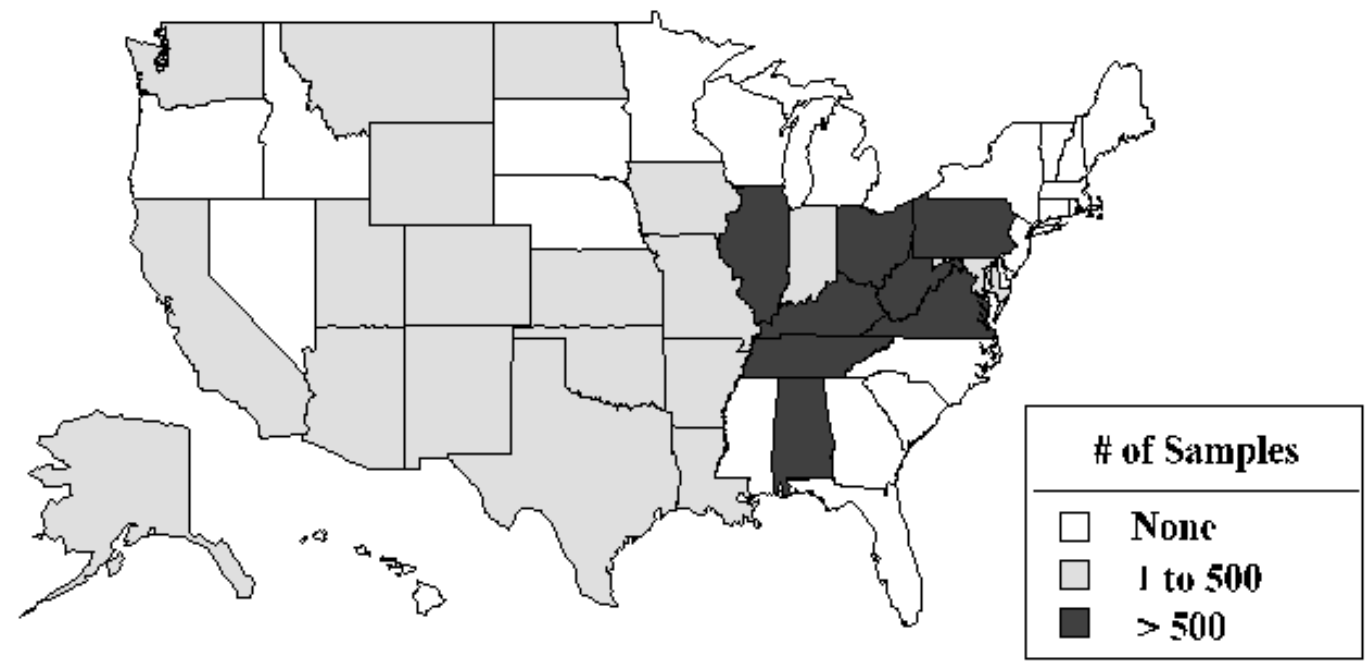

NOTE: See Appendix A for source description, Appendix B for methods, and Appendix D for agents. SOURCE: Mine Safety and Health Administration: respirable coal mine dust data.

Figure 2-9. Coal and coal mine dust: Percent of MSHA inspector samples that exceeded the permissible exposure limit (PEL), by state, 1993-1994

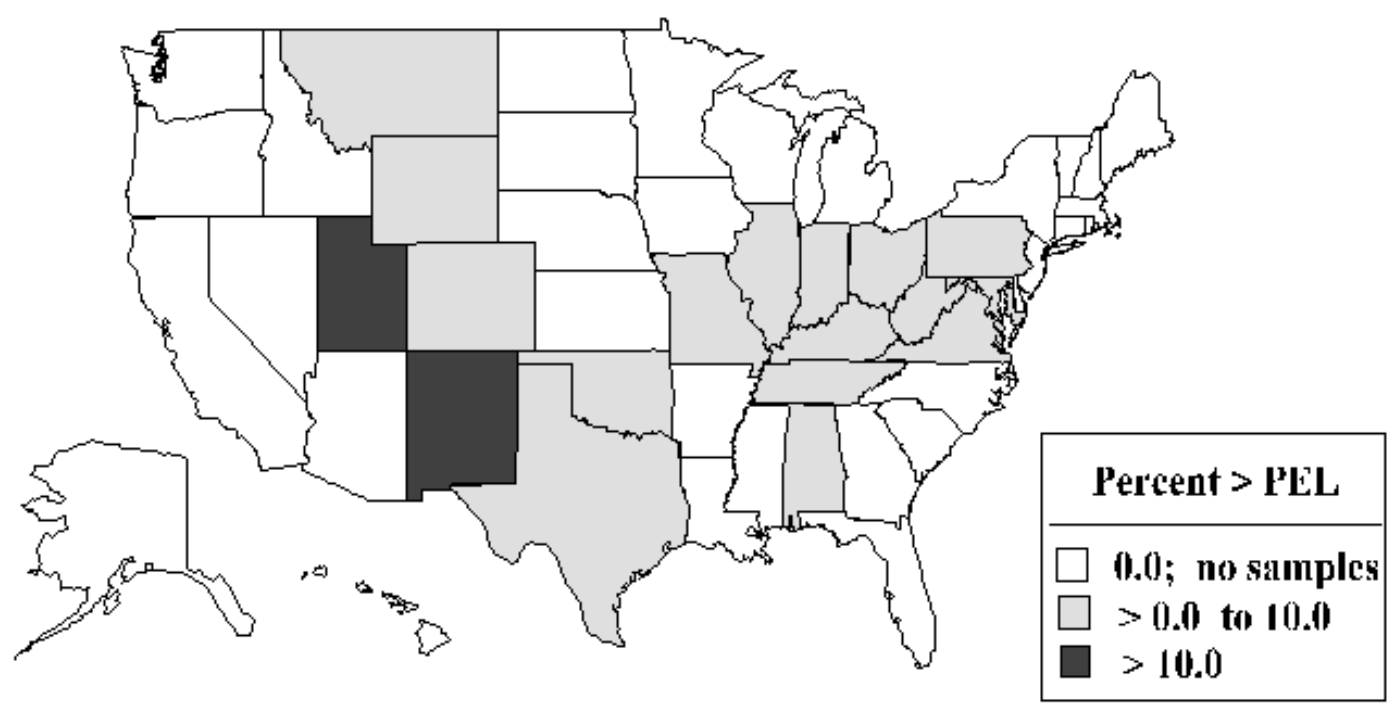

NOTE: See Appendix A for source description, Appendix B for methods, and Appendix D for agents.

SOURCE: Mine Safety and Health Administration: respirable coal mine dust data. 
Figure 2-10. Coal and coal mine dust: Average severity level by state for MSHA inspector samples, 1993-1994

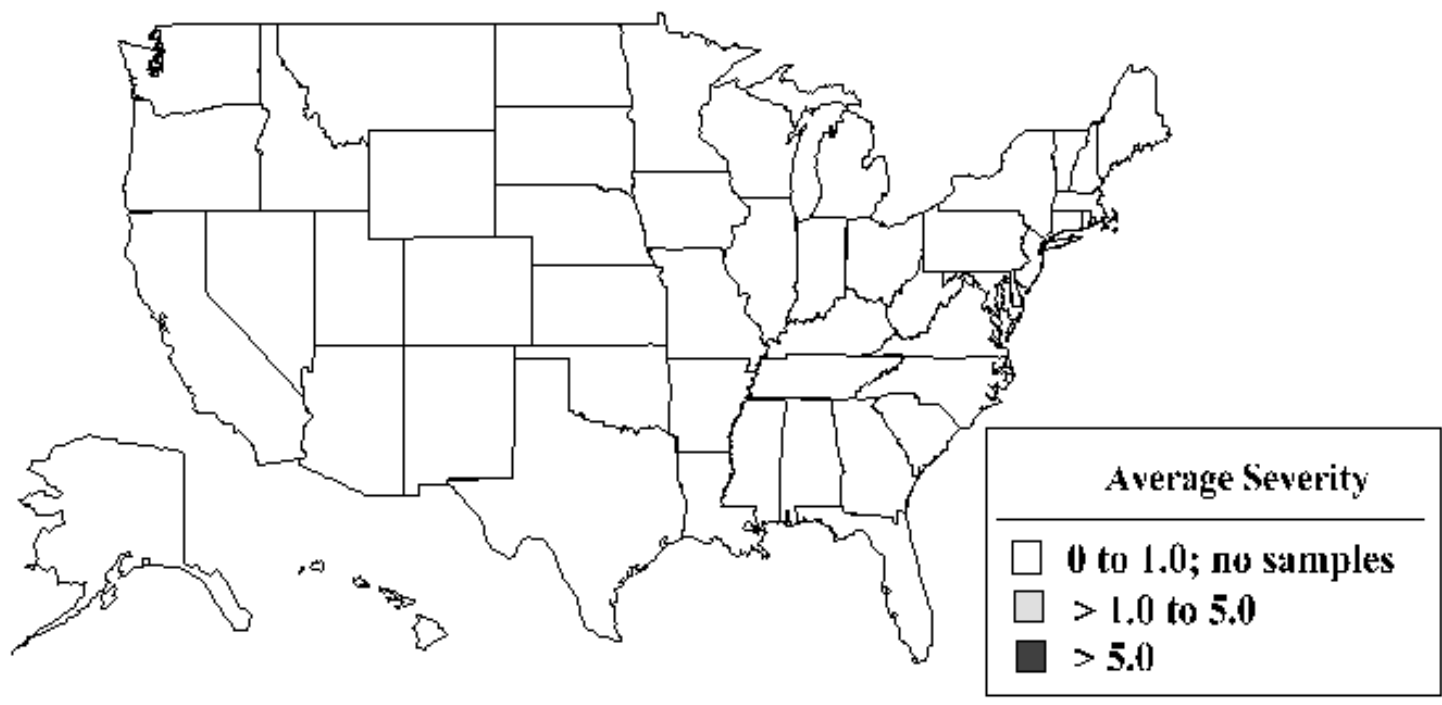

NOTE: See Appendix A for source description, Appendix B for methods, and Appendix D for agents. SOURCE: Mine Safety and Health Administration: respirable coal mine dust data.

Figure 2-11. Coal and coal mine dust: Average severity level by state for MSHA inspector samples that exceeded the permissible exposure limit (PEL), 1993-1994

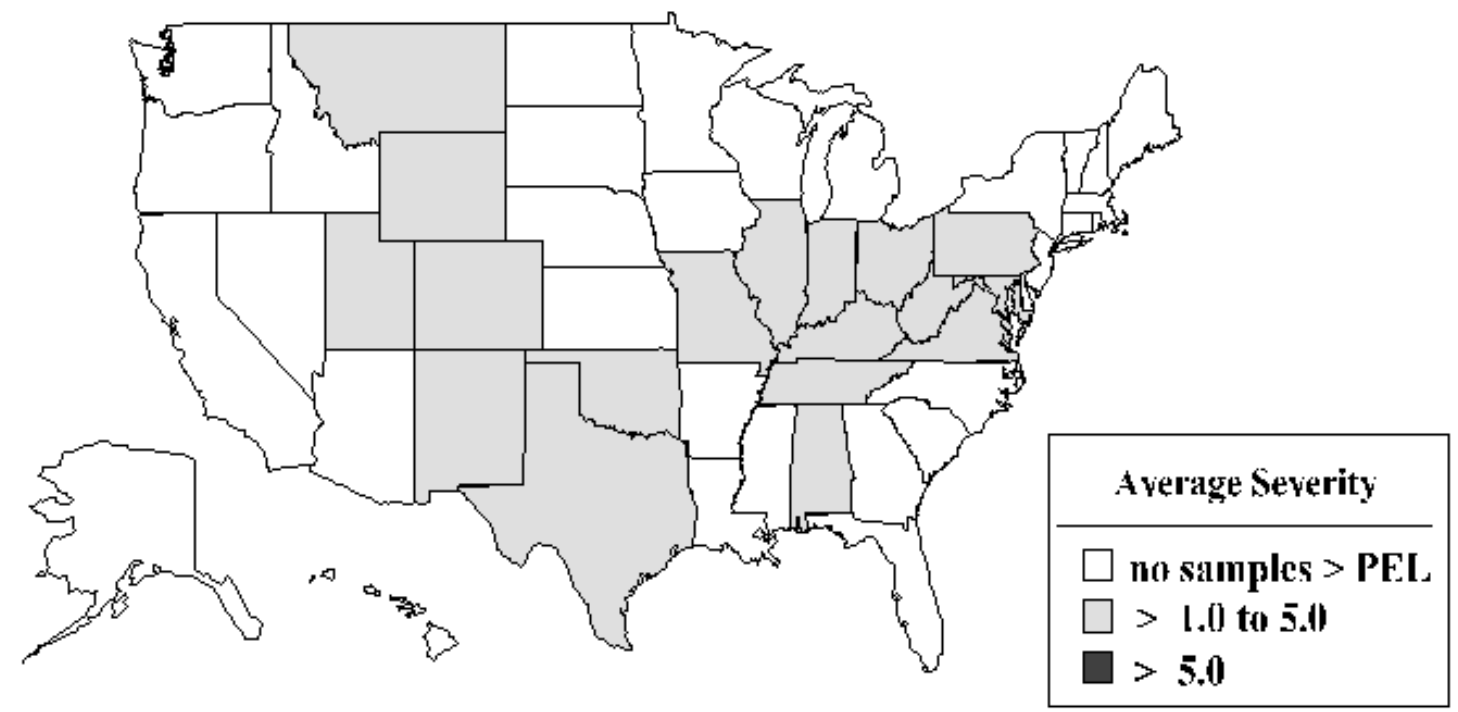

NOTE: See Appendix A for source description, Appendix B for methods, and Appendix D for agents.

SOURCE: Mine Safety and Health Administration: respirable coal mine dust data. 
Figure 2-12. Coal and coal mine dust. Number of MSHA inspector samples collected by county, 1993-1994

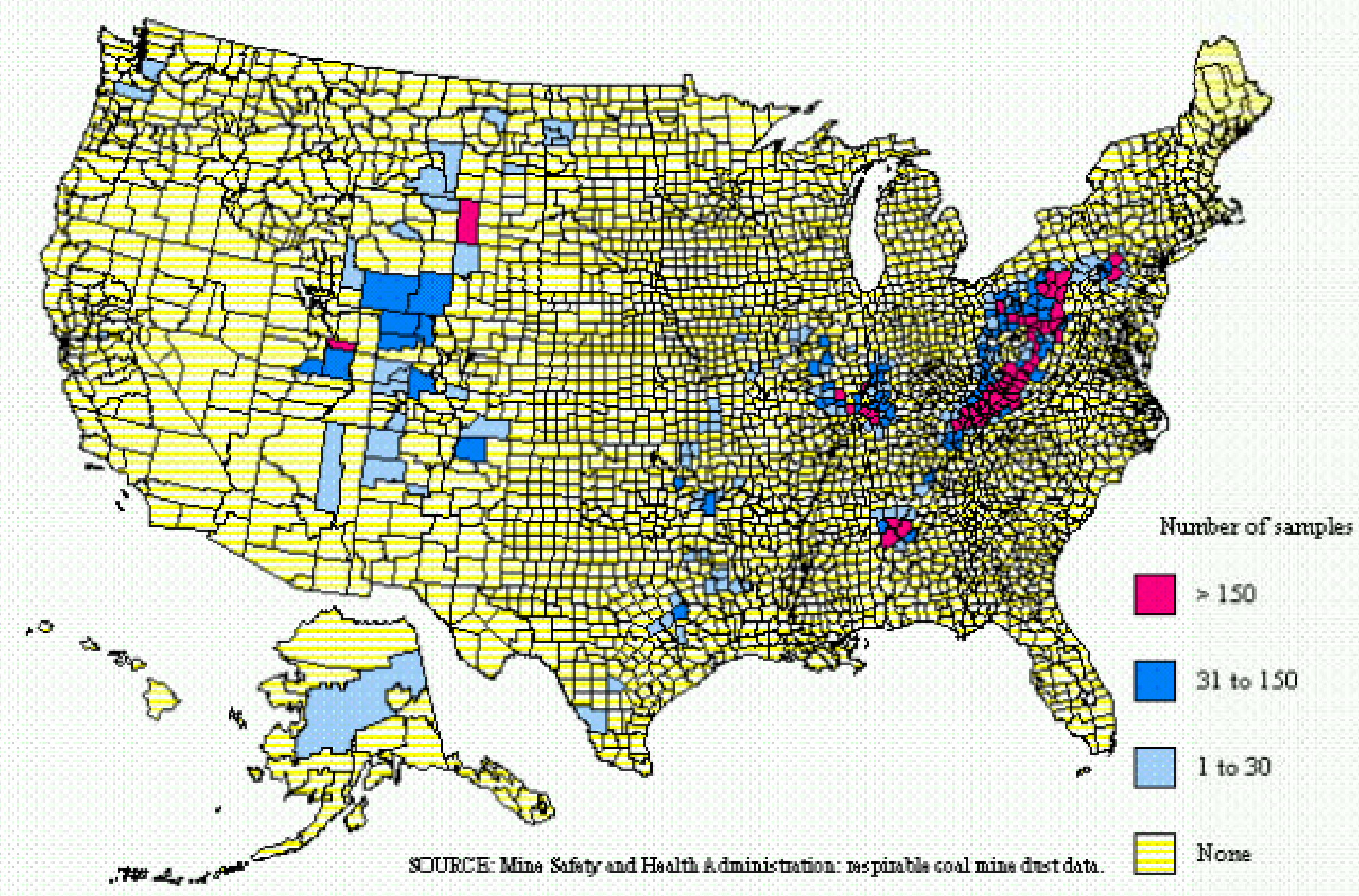


Table 2-13 (page 1 of 2). Coal and coal mine dust: Number of OSHA inspector samples, percent exceeding the permissible exposure limit (PEL) and average severity levels (Avg. Sev.), by state, 1979-1994

\begin{tabular}{|c|c|c|c|c|c|c|c|c|c|c|c|c|}
\hline \multirow[b]{3}{*}{ State } & \multicolumn{4}{|c|}{ 1979-1984 } & \multicolumn{4}{|c|}{ 1985-1994 } & \multicolumn{4}{|c|}{ 1993-1994 } \\
\hline & \multicolumn{2}{|c|}{ Total samples } & \multicolumn{2}{|c|}{ Samples > PEL } & \multicolumn{2}{|c|}{ Total samples } & \multicolumn{2}{|c|}{ Samples > PEL } & \multicolumn{2}{|c|}{ Total samples } & \multicolumn{2}{|c|}{ Samples > PEL } \\
\hline & Number & $\begin{array}{c}\text { Avg. } \\
\text { Sev. }\end{array}$ & $\%$ & $\begin{array}{c}\text { Avg. } \\
\text { Sev. }\end{array}$ & Number & $\begin{array}{l}\text { Avg. } \\
\text { Sev. }\end{array}$ & $\%$ & $\begin{array}{l}\text { Avg. } \\
\text { Sev. }\end{array}$ & Number & $\begin{array}{c}\text { Avg. } \\
\text { Sev. }\end{array}$ & $\%$ & $\begin{array}{l}\text { Avg. } \\
\text { Sev. }\end{array}$ \\
\hline Alabama & 7 & 0.43 & 14.3 & 1.15 & 0 & - & - & - & 0 & - & - & - \\
\hline Alaska & 0 & - & - & - & 0 & - & - & - & 0 & - & - & - \\
\hline Arizona & 0 & - & - & - & 0 & - & - & - & 0 & - & - & - \\
\hline Arkansas & 0 & - & - & - & 0 & - & - & - & 0 & - & - & - \\
\hline California & 0 & - & - & - & 0 & - & - & - & 0 & - & - & - \\
\hline Colorado & 0 & - & - & - & 6 & 0.47 & 0.0 & - & 0 & - & - & - \\
\hline Connecticut & 0 & - & - & - & 0 & - & - & - & 0 & - & - & - \\
\hline Delaware & 0 & - & - & - & 0 & - & - & - & 0 & - & - & - \\
\hline District of Columbia & 0 & - & - & - & 0 & - & - & - & 0 & - & - & - \\
\hline Florida & 2 & 0.11 & 0.0 & - & 0 & - & - & - & 0 & - & - & - \\
\hline Georgia & 0 & - & - & - & 0 & - & - & - & 0 & - & - & - \\
\hline Hawaii & 0 & - & - & - & 0 & - & - & - & 0 & - & - & - \\
\hline Idaho & 0 & - & - & - & 0 & - & - & - & 0 & - & - & - \\
\hline Illinois & 10 & 34.37 & 40.0 & 85.29 & 0 & - & - & - & 0 & - & - & - \\
\hline Indiana & 2 & 0.21 & 0.0 & - & 2 & 0.00 & 0.0 & - & 0 & - & - & - \\
\hline Iowa & 3 & 0.08 & 0.0 & - & 8 & 0.49 & 12.5 & 2.62 & 0 & - & - & - \\
\hline Kansas & 0 & - & - & - & 0 & - & - & - & 0 & - & - & - \\
\hline Kentucky & 0 & - & - & - & 6 & 0.18 & 0.0 & - & 3 & 0.14 & 0.0 & - \\
\hline Louisiana & 2 & 0.21 & 0.0 & - & 0 & - & - & - & 0 & - & - & - \\
\hline Maine & 0 & - & - & - & 0 & - & - & - & 0 & - & - & - \\
\hline Maryland & 0 & - & - & - & 12 & 0.25 & 0.0 & - & 2 & 0.45 & 0.0 & - \\
\hline Massachusetts & 0 & - & - & - & 0 & - & - & - & 0 & - & - & - \\
\hline Michigan & 0 & - & - & - & 3 & 13.09 & 33.3 & 38.50 & 0 & - & - & - \\
\hline Minnesota & 0 & - & - & - & 0 & - & - & - & 0 & - & - & - \\
\hline Mississippi & 0 & - & - & - & 0 & - & - & - & 0 & - & - & - \\
\hline Missouri & 3 & 0.14 & 0.0 & - & 0 & - & - & - & 0 & - & - & - \\
\hline Montana & 0 & - & - & - & 2 & 0.10 & 0.0 & - & 0 & - & - & - \\
\hline
\end{tabular}


Table 2-13 (page 2 of 2). Coal and coal mine dust: Number of OSHA inspector samples, percent exceeding the permissible exposure limit (PEL) and average severity levels (Avg. Sev.), by state, 1979-1994

\begin{tabular}{|c|c|c|c|c|c|c|c|c|c|c|c|c|}
\hline \multirow[b]{3}{*}{ State } & \multicolumn{4}{|c|}{ 1979-1984 } & \multicolumn{4}{|c|}{ 1985-1994 } & \multicolumn{4}{|c|}{ 1993-1994 } \\
\hline & \multicolumn{2}{|c|}{ Total samples } & \multicolumn{2}{|c|}{ Samples > PEL } & \multicolumn{2}{|c|}{ Total samples } & \multicolumn{2}{|c|}{ Samples > PEL } & \multicolumn{2}{|c|}{ Total samples } & \multicolumn{2}{|c|}{ Samples > PEL } \\
\hline & Number & $\begin{array}{l}\text { Avg. } \\
\text { Sev. }\end{array}$ & $\%$ & $\begin{array}{c}\text { Avg. } \\
\text { Sev. }\end{array}$ & Number & $\begin{array}{l}\text { Avg. } \\
\text { Sev. }\end{array}$ & $\%$ & $\begin{array}{l}\text { Avg. } \\
\text { Sev. }\end{array}$ & Number & $\begin{array}{l}\text { Avg. } \\
\text { Sev. }\end{array}$ & $\%$ & $\begin{array}{l}\text { Avg. } \\
\text { Sev. }\end{array}$ \\
\hline Nebraska & 0 & - & - & - & 0 & - & - & - & 0 & - & - & - \\
\hline Nevada & 0 & - & - & - & 0 & - & - & - & 0 & - & - & - \\
\hline New Hampshire & 0 & - & - & - & 0 & - & - & - & 0 & - & - & - \\
\hline New Jersey & 0 & - & - & - & 0 & - & - & - & 0 & - & - & - \\
\hline New Mexico & 0 & - & - & - & 0 & - & - & - & 0 & - & - & - \\
\hline New York & 1 & 0.18 & 0.0 & - & 1 & 1.11 & 100.0 & 1.11 & 0 & - & - & - \\
\hline North Carolina & 0 & - & - & - & 0 & - & - & - & 0 & - & - & - \\
\hline North Dakota & 2 & 4.54 & 100.0 & 4.54 & 0 & - & - & - & 0 & - & - & - \\
\hline Ohio & 16 & 0.34 & 6.3 & 1.75 & 7 & 0.68 & 28.6 & 1.43 & 0 & - & - & - \\
\hline Oklahoma & 0 & - & - & - & 1 & 0.45 & 0.0 & - & 0 & - & - & - \\
\hline Oregon & 0 & - & - & - & 0 & - & - & - & 0 & - & - & - \\
\hline Pennsylvania & 5 & 0.06 & 0.0 & - & 0 & - & - & - & 0 & - & - & - \\
\hline Rhode Island & 0 & - & - & - & 0 & - & - & - & 0 & - & - & - \\
\hline South Carolina & 0 & - & - & - & 0 & - & - & - & 0 & - & - & - \\
\hline South Dakota & 0 & - & - & - & 0 & - & - & - & 0 & - & - & - \\
\hline Tennessee & 0 & - & - & - & 4 & 0.11 & 0.0 & - & 2 & 0.00 & 0.0 & - \\
\hline Texas & 14 & 0.18 & 0.0 & - & 0 & - & - & - & 0 & - & - & - \\
\hline Utah & 0 & - & - & - & 0 & - & - & - & 0 & - & - & - \\
\hline Vermont & 0 & - & - & - & 0 & - & - & - & 0 & - & - & - \\
\hline Virginia & 0 & - & - & - & 4 & 0.00 & 0.0 & - & 0 & - & - & - \\
\hline Washington & 0 & - & - & - & 0 & - & - & - & 0 & - & - & - \\
\hline W est Virginia & 4 & 2.09 & 50.0 & 4.00 & 0 & - & - & - & 0 & - & - & - \\
\hline Wisconsin & 8 & 0.49 & 25.0 & 1.21 & 1 & 0.23 & 0.0 & - & 0 & - & - & - \\
\hline Wyoming & 2 & 0.71 & 0.0 & - & 0 & - & - & - & 0 & - & - & - \\
\hline TOTAL & 81 & 4.69 & 14.8 & 30.30 & 57 & 1.00 & 8.8 & 9.02 & 7 & 0.19 & 0.0 & - \\
\hline
\end{tabular}

- indicates incalculable field.

NOTE: $\quad$ See Appendix A for source description, Appendix B for methods, and Appendix D for agents.

SOURCE: Occupational Safety and Health Administration: Integrated Management Information System. 
Figure 2-13. Coal and coal mine dust: Number of OSHA inspector samples collected by state, 1993-1994

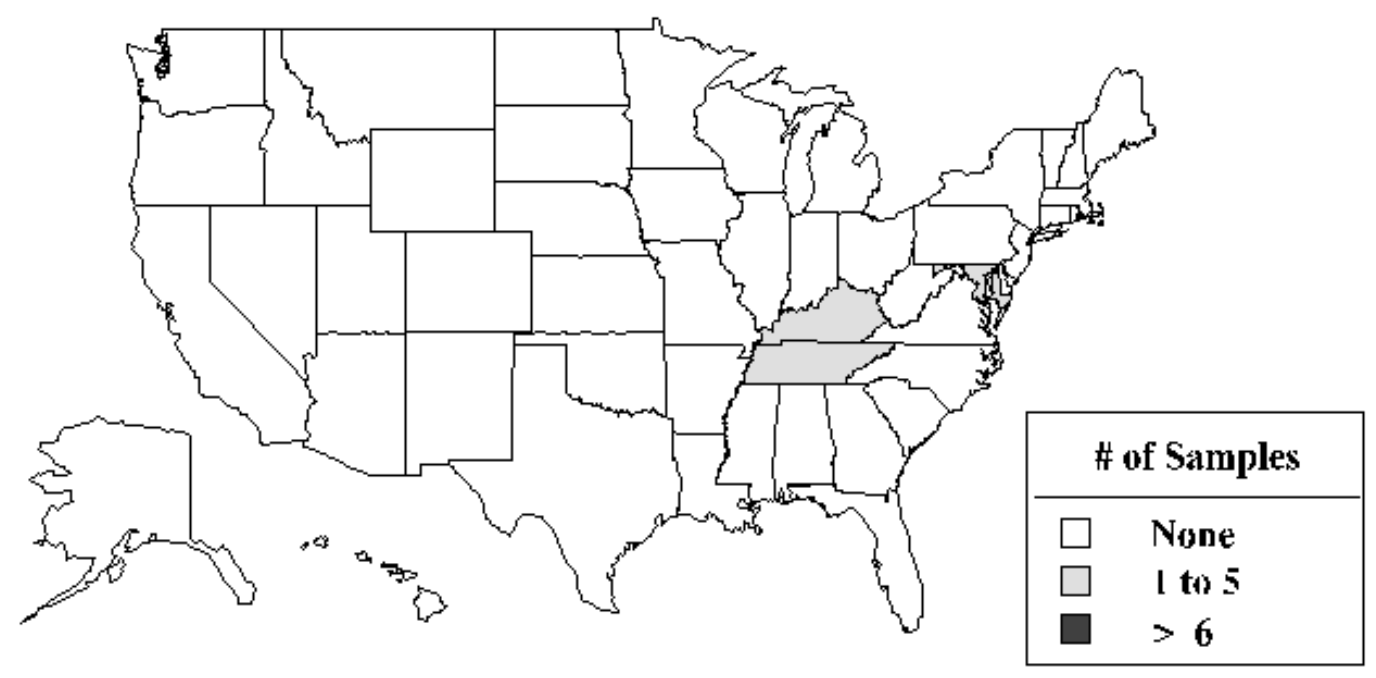

NOTE: See Appendix A for source description, Appendix B for methods, and Appendix D for agents. SOURCE: Occupational Safety and Health Administration: Integrated Management Information System.

Figure 2-14. Coal and coal mine dust: Percent of OSHA inspector samples that exceeded the permissible exposure limit (PEL), by state, 1993-1994

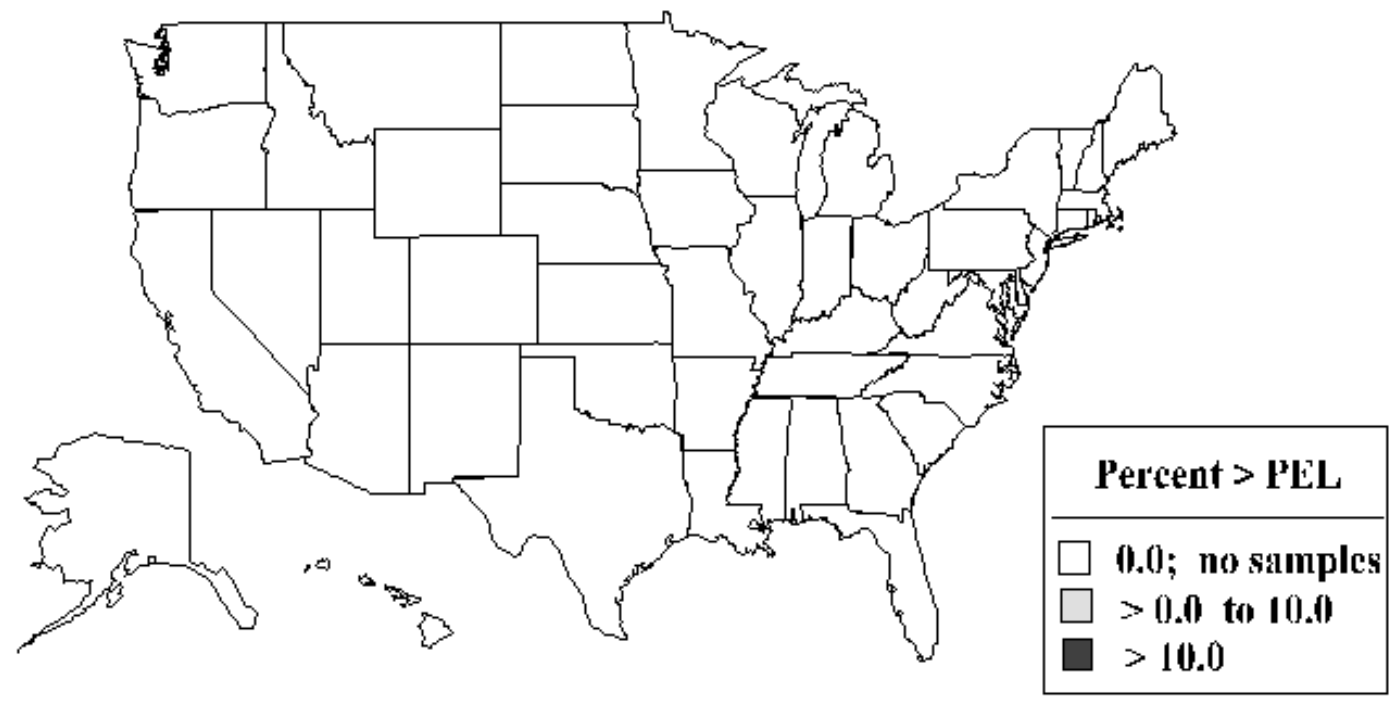

NOTE: No samples collected were above the PEL. See Appendix A for source description, Appendix B for methods, and Appendix D for agents. SOURCE: Occupational Safety and Health Administration: Integrated Management Information System. 
Figure 2-15. Coal and coal mine dust: Average severity level by state for OSHA inspector samples, 1993-1994

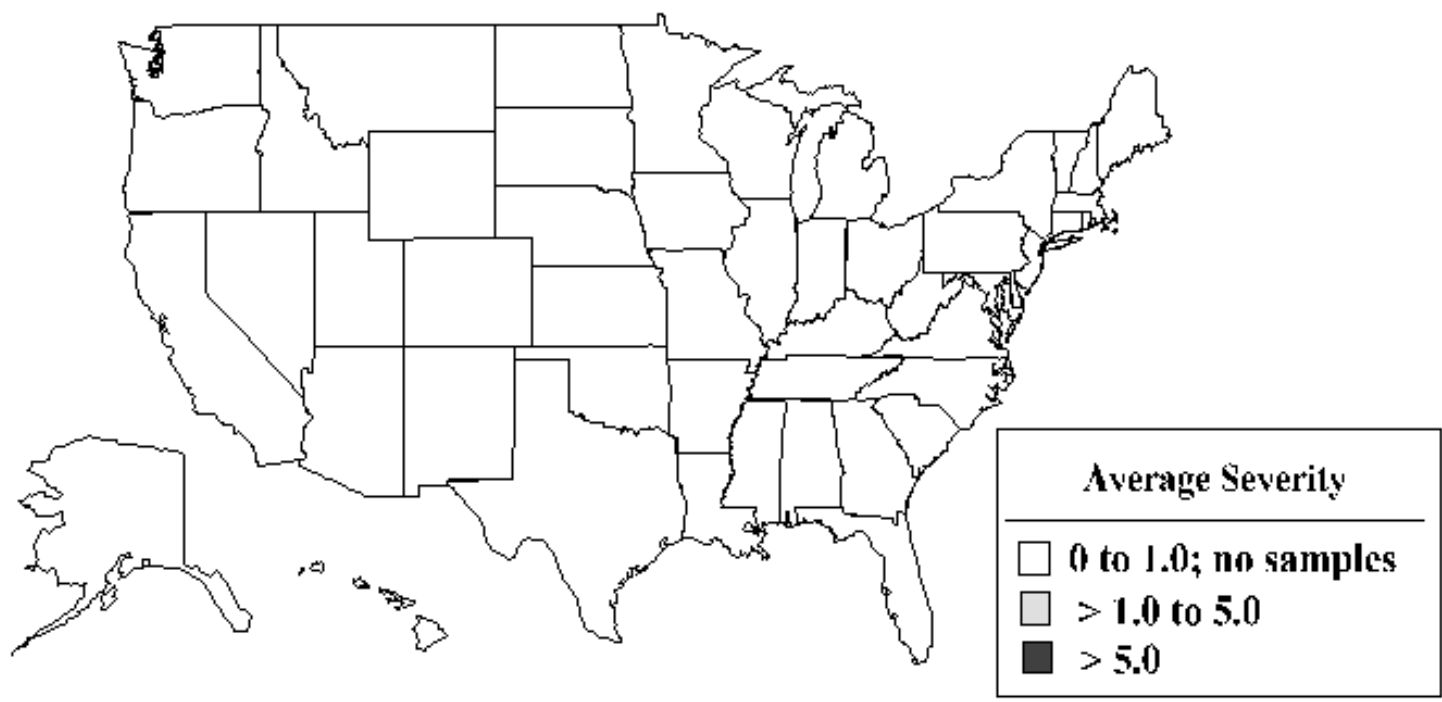

NOTE: $\quad$ No samples collected were above the PEL. See Appendix A for source descriptions, Appendix B for methods, and Appendix D for agents. SOURCE: Occupational Safety and Health Administration: Integrated Management Information System.

Figure 2-16. Coal and coal mine dust: Average severity level by state for OSHA inspector samples that exceeded the permissible exposure limit (PEL), 1993-1994

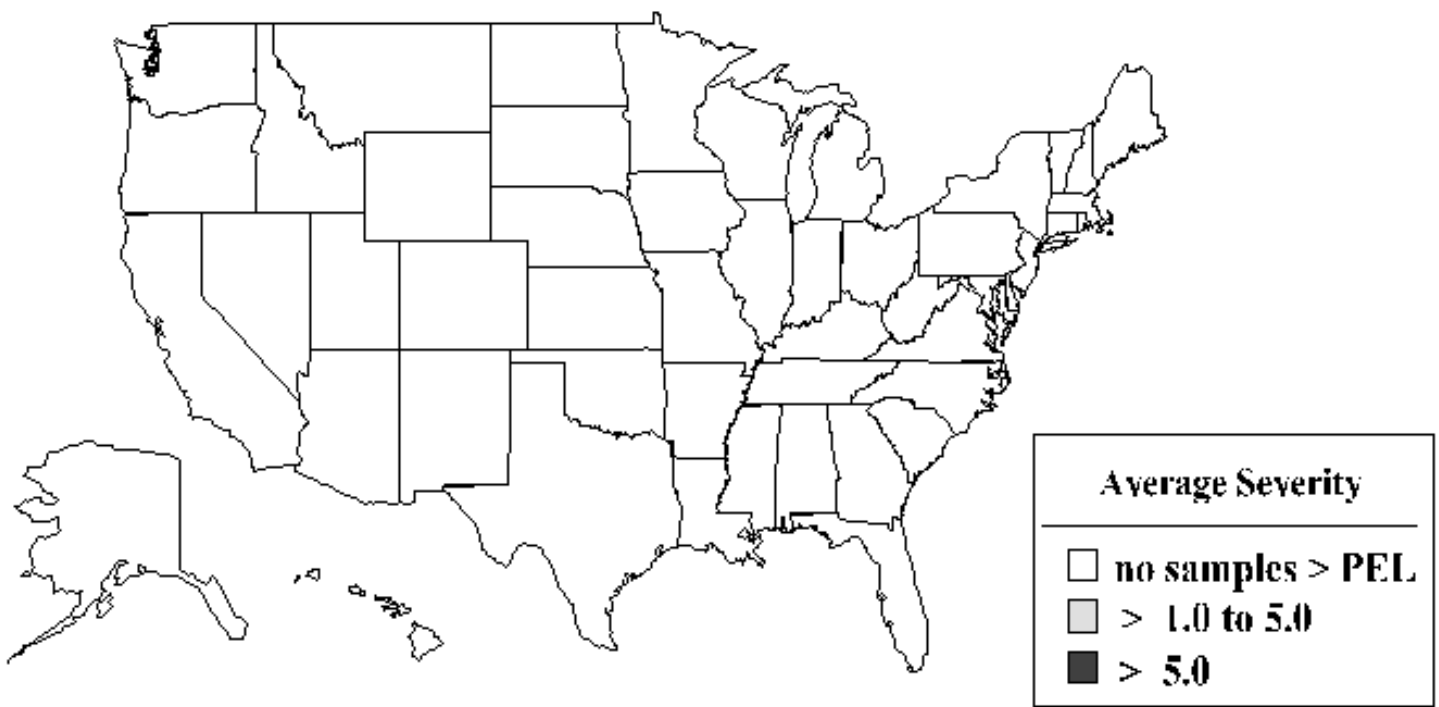

NOTE: No samples collected were above the PEL. See Appendix A for source descriptions, Appendix B for methods, and Appendix D for agents. SOURCE: Occupational Safety and Health Administration: Integrated Management Information System. 
Figure 2-17. Coal and coal mine dust: Number of OSHA inspector samples collected by county, 1993-1994

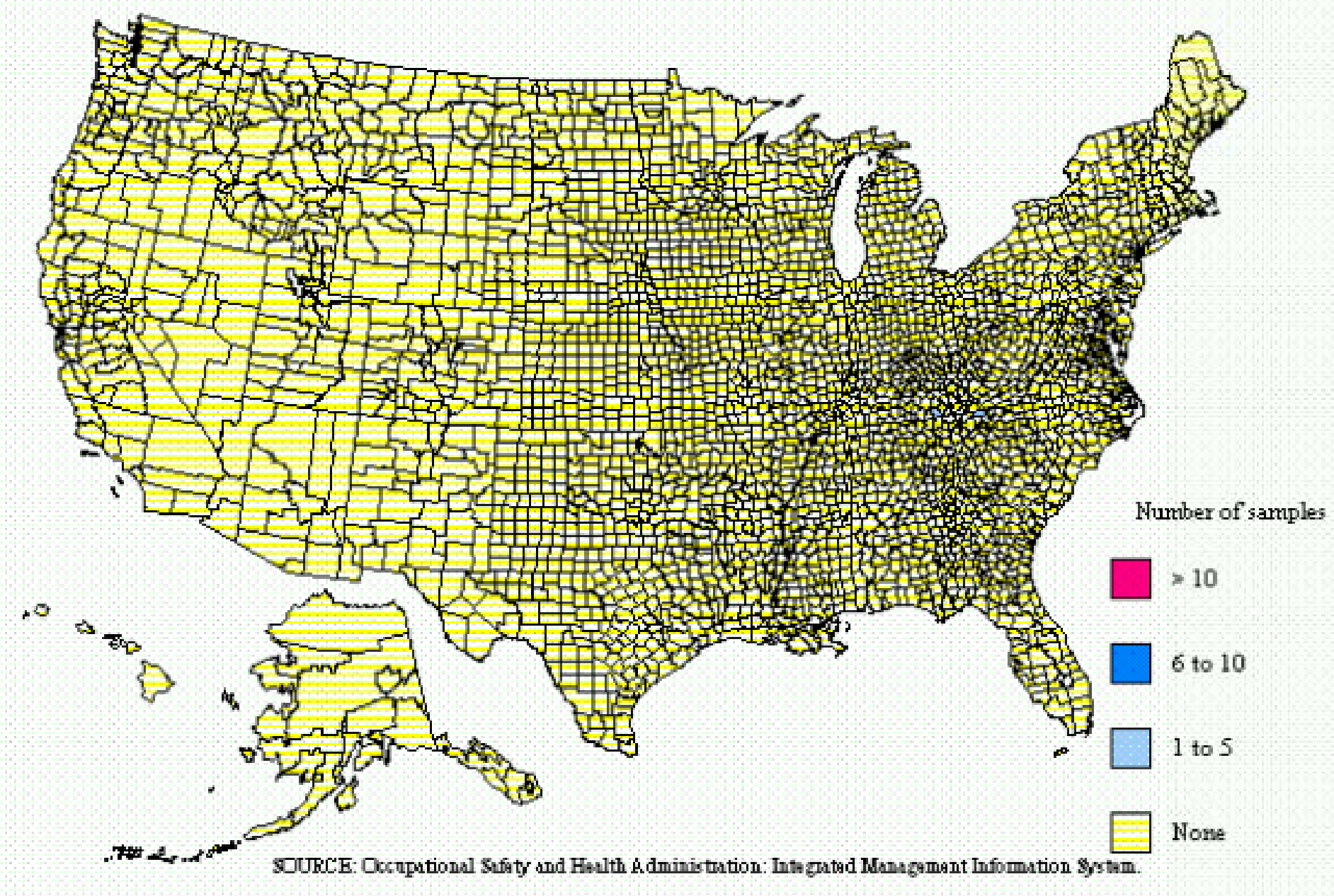


Figure 3-1. Silicosis: Number of deaths, crude and age-adjusted mortality rates, U.S. residents age 15 and over, 1968-1992

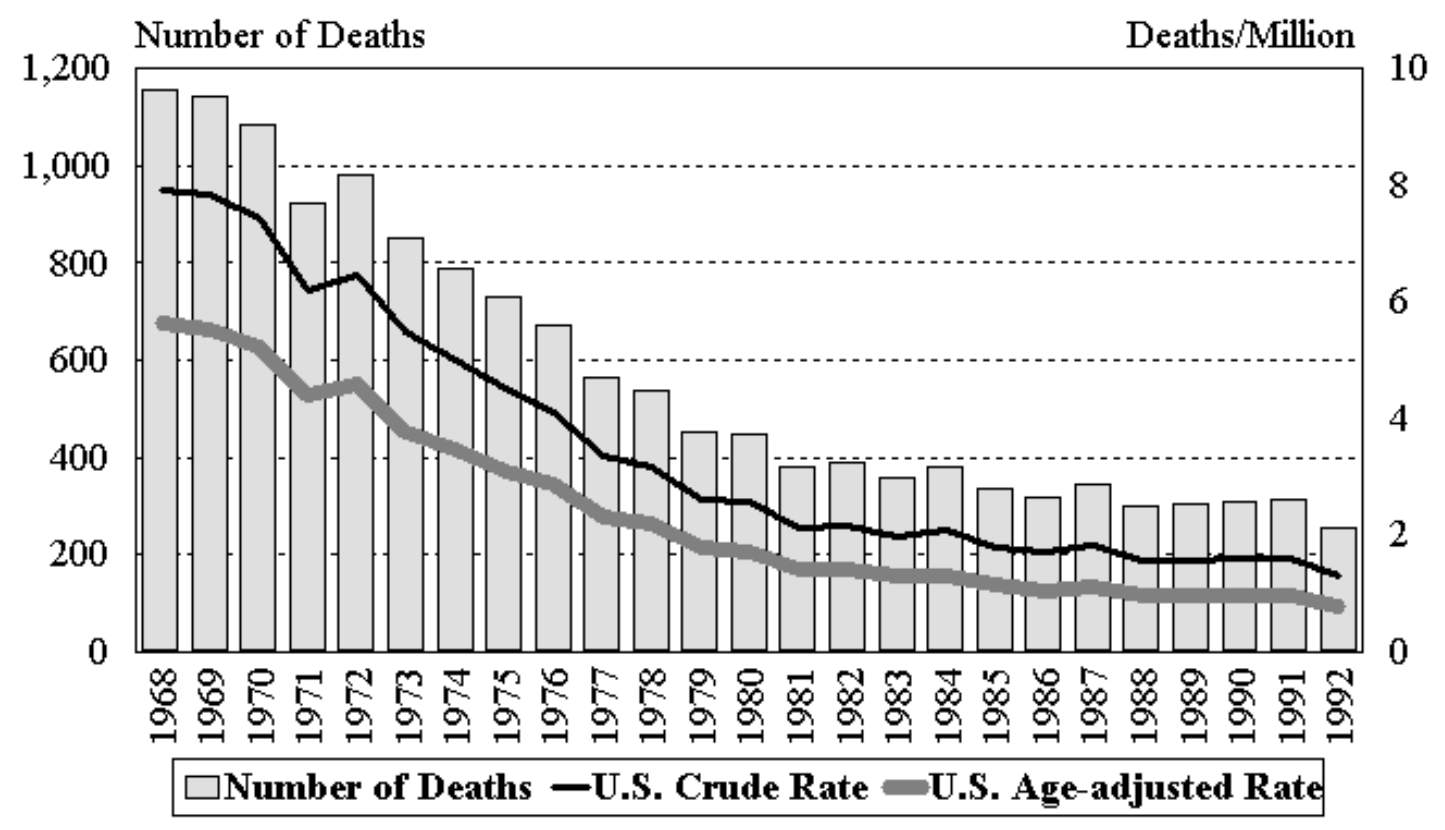

NOTE: $\quad$ See Appendix A for source description and Appendix B for methods and ICD-8 and ICD-9 codes.

SOURCE: National Center for Health Statistics multiple cause of death data. Population estimates from U.S. Bureau of the Census.

Figure 3-2. Silicosis: Crude mortality rates by state, U.S. residents age 15 and over, 1991-1992

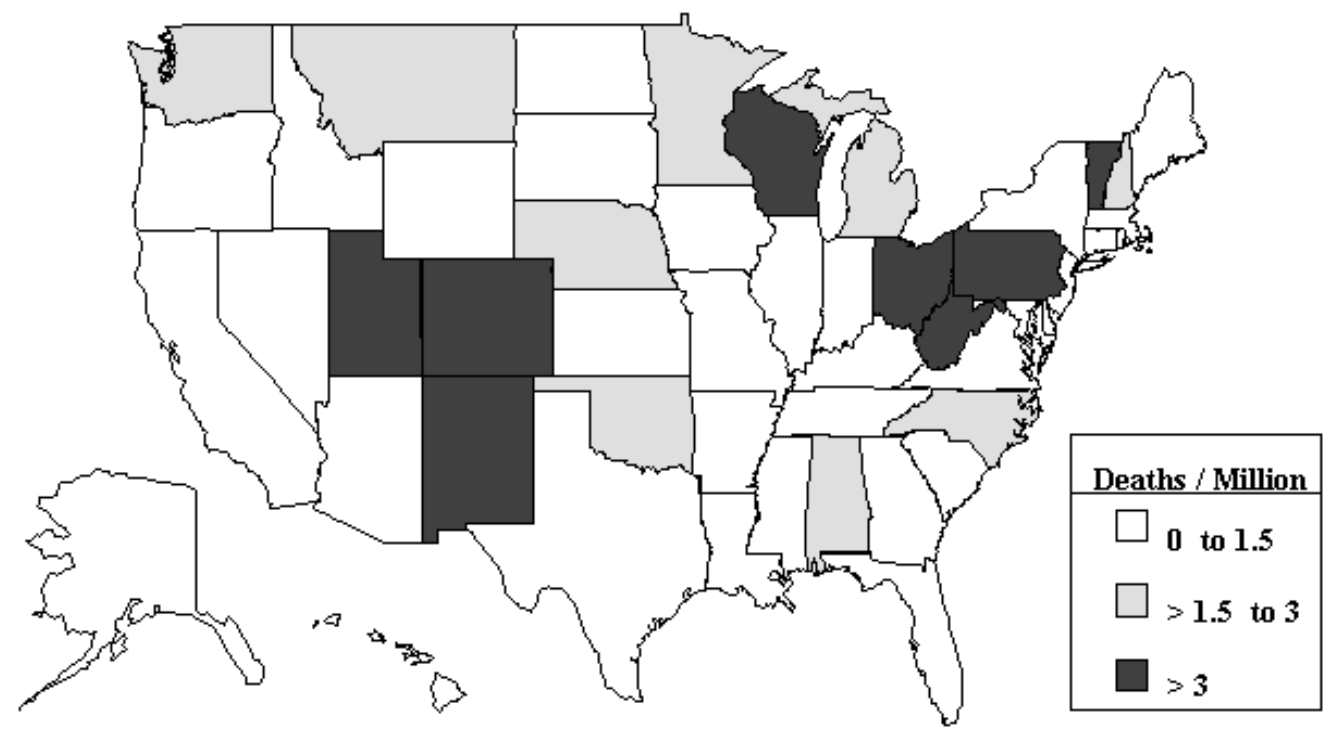

NOTE: $\quad$ See Appendix A for source description and Appendix B for methods and ICD-8 and ICD-9 codes.

SOURCE: National Center for Health Statistics multiple cause of death data. Population estimates from U.S. Bureau of the Census. 
Figure 3-3. Silicosis: Median age at death, U.S. residents age 15 and over, 1968-1992

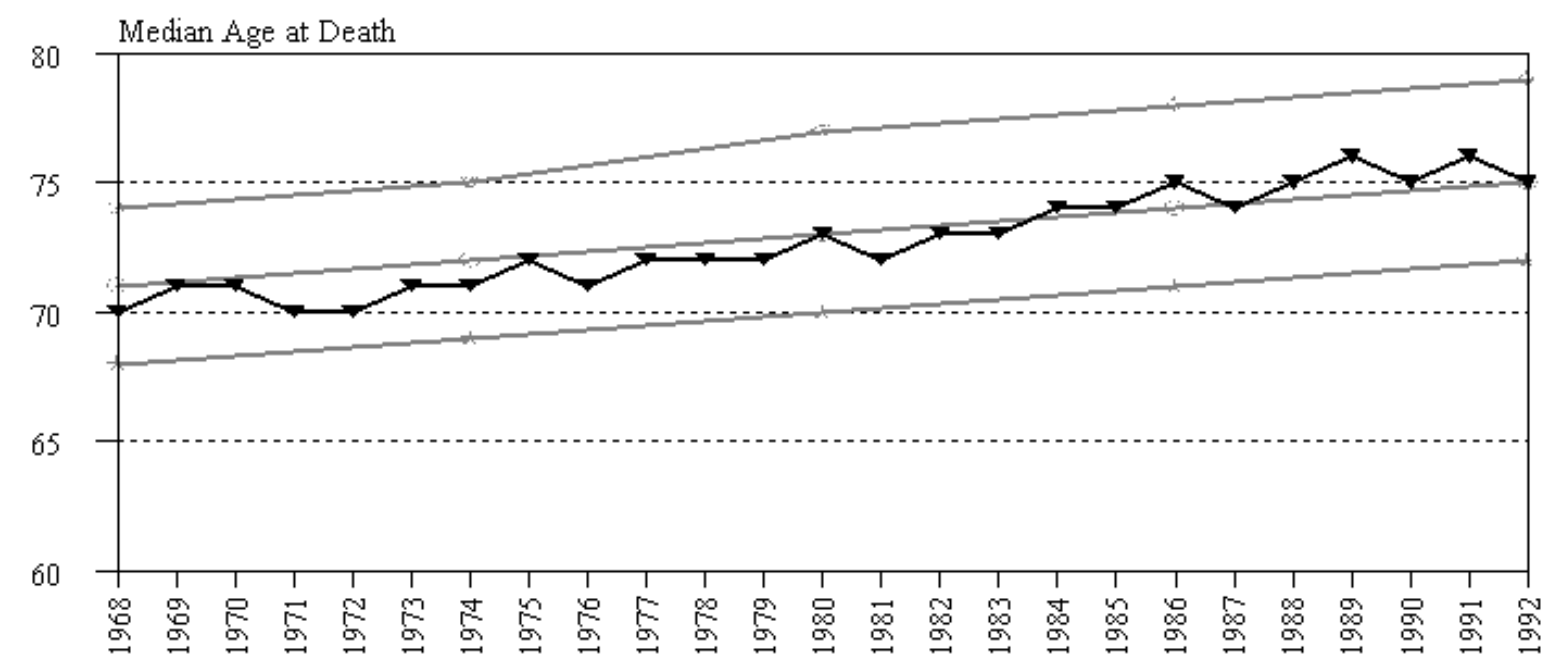

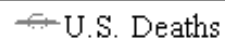

$\rightarrow$ U.S. Male Deaths

$\div$ U.S. Female Deaths

SOURCE: National Center for Health Statistics multiple cause of death data.

Table 3-1. Silicosis: Number of deaths by sex, race, and age, U.S. residents age 15 and over, 1991-1992

\begin{tabular}{|c|c|c|c|c|c|}
\hline & & \multicolumn{2}{|c|}{1991} & \multicolumn{2}{|c|}{1992} \\
\hline & & Number & Percent & Number & Percent \\
\hline \multicolumn{2}{|c|}{ Total deaths } & 314 & 100.0 & 255 & 100.0 \\
\hline \multirow[t]{2}{*}{ Sex } & Male & 305 & 97.1 & 240 & 94.1 \\
\hline & Female & 9 & 2.9 & 15 & 5.9 \\
\hline \multirow[t]{3}{*}{ Race } & White & 257 & 81.9 & 212 & 83.1 \\
\hline & Black & 56 & 17.8 & 39 & 15.3 \\
\hline & Other & 1 & 0.3 & 4 & 1.6 \\
\hline \multirow[t]{10}{*}{ Age } & $15-24$ & 1 & 0.3 & 0 & 0.0 \\
\hline & $25-34$ & 1 & 0.3 & 1 & 0.4 \\
\hline & $35-44$ & 4 & 1.3 & 3 & 1.2 \\
\hline & $45-54$ & 12 & 3.8 & 8 & 3.1 \\
\hline & $55-64$ & 40 & 12.7 & 36 & 14.1 \\
\hline & $65-74$ & 78 & 24.8 & 79 & 31.0 \\
\hline & $75-84$ & 135 & 43.0 & 96 & 37.6 \\
\hline & 85 and over & 43 & 13.7 & 32 & 12.5 \\
\hline & Mean age & 74.2 & & 73.4 & \\
\hline & Range for age & $22-97$ & & $30-96$ & \\
\hline
\end{tabular}


Table 3-2. Silicosis: Number of deaths by state, U.S. residents age 15 and over, 1968-1992

\begin{tabular}{|c|c|c|c|c|c|}
\hline State & 1968-1978 & 1979-1990 & 1991 & 1992 & TOTAL \\
\hline Alabama & 172 & 57 & 9 & 3 & 241 \\
\hline Alaska & 7 & 6 & - & - & 13 \\
\hline Arizona & 177 & 81 & 2 & 1 & 261 \\
\hline Arkansas & 43 & 19 & 2 & 1 & 65 \\
\hline California & 455 & 242 & 16 & 12 & 725 \\
\hline Colorado & 251 & 129 & 15 & 9 & 404 \\
\hline Connecticut & 100 & 54 & 2 & 3 & 159 \\
\hline Delaware & 7 & 5 & - & 1 & 13 \\
\hline District of Columbia & 14 & 10 & - & 1 & 25 \\
\hline Florida & 162 & 142 & 7 & 4 & 315 \\
\hline Georgia & 82 & 69 & 3 & 3 & 157 \\
\hline Hawaii & 6 & 3 & - & - & 9 \\
\hline Idaho & 83 & 27 & - & 2 & 112 \\
\hline Illinois & 191 & 160 & 8 & 11 & 370 \\
\hline Indiana & 143 & 85 & 4 & 4 & 236 \\
\hline Iowa & 30 & 25 & 2 & 2 & 59 \\
\hline Kansas & 70 & 20 & 1 & 1 & 92 \\
\hline Kentucky & 209 & 76 & 2 & 6 & 293 \\
\hline Louisiana & 34 & 45 & 5 & 2 & 86 \\
\hline Maine & 24 & 15 & 2 & - & 41 \\
\hline Maryland & 87 & 41 & 4 & 1 & 133 \\
\hline Massachusetts & 106 & 55 & 3 & 1 & 165 \\
\hline Michigan & 378 & 193 & 9 & 14 & 594 \\
\hline Minnesota & 97 & 90 & 3 & 9 & 199 \\
\hline Mississippi & 14 & 16 & 1 & 1 & 32 \\
\hline Missouri & 112 & 68 & 7 & 4 & 191 \\
\hline Montana & 120 & 49 & - & 3 & 172 \\
\hline Nebraska & 9 & - & 3 & 1 & 13 \\
\hline Nevada & 61 & 29 & - & - & 90 \\
\hline New Hampshire & 17 & 12 & 2 & 1 & 32 \\
\hline New Jersey & 262 & 134 & 11 & 5 & 412 \\
\hline New Mexico & 57 & 40 & 4 & 5 & 106 \\
\hline New York & 447 & 205 & 18 & 6 & 676 \\
\hline North Carolina & 164 & 108 & 14 & 8 & 294 \\
\hline North Dakota & 5 & 3 & - & - & 8 \\
\hline Ohio & 1,036 & 456 & 35 & 24 & 1,551 \\
\hline Oklahoma & 51 & 20 & 4 & 5 & 80 \\
\hline Oregon & 54 & 33 & 3 & 3 & 93 \\
\hline Pennsylvania & 2,483 & 755 & 53 & 53 & 3,344 \\
\hline Rhode Island & 10 & 15 & - & 2 & 27 \\
\hline South Carolina & 30 & 37 & 3 & 1 & 71 \\
\hline South Dakota & 23 & 16 & - & - & 39 \\
\hline Tennessee & 124 & 77 & 3 & 2 & 206 \\
\hline Texas & 105 & 104 & 11 & 11 & 231 \\
\hline Utah & 102 & 54 & 5 & 4 & 165 \\
\hline Vermont & 88 & 38 & 1 & 4 & 131 \\
\hline Virginia & 249 & 79 & 2 & 3 & 333 \\
\hline Washington & 125 & 73 & 7 & 5 & 210 \\
\hline West Virginia & 542 & 77 & 12 & 4 & 635 \\
\hline Wisconsin & 199 & 157 & 16 & 9 & 381 \\
\hline Wyoming & 14 & 9 & - & - & 23 \\
\hline TOTAL & 9,431 & 4,313 & 314 & 255 & 14,313 \\
\hline
\end{tabular}

- indicates no deaths listed.

NOTE: $\quad$ See Appendix A for source description and Appendix B for methods and ICD-8 and ICD-9 codes.

SOURCE: National Center for Health Statistics multiple cause of death data. 
Table 3-3. Silicosis: Mortality rates (per $1,000,000$ population) by race and sex, U.S. residents age 15 and over, 1991-1992

\begin{tabular}{|c|c|c|c|c|c|}
\hline \multirow[b]{2}{*}{ Year } & \multirow[b]{2}{*}{ Overall rate } & \multicolumn{2}{|c|}{ White } & \multicolumn{2}{|c|}{ Black } \\
\hline & & Males & Females & Males & Females \\
\hline & \multicolumn{5}{|c|}{ Crude mortality rate } \\
\hline 1991 & 1.59 & 3.08 & 0.09 & 5.28 & 0.08 \\
\hline \multirow[t]{2}{*}{1992} & 1.28 & 2.46 & 0.14 & 3.49 & 0.16 \\
\hline & \multicolumn{5}{|c|}{ Age-adjusted mortality rate } \\
\hline 1991 & 0.94 & 1.96 & 0.05 & 5.21 & 0.02 \\
\hline 1992 & 0.77 & 1.62 & 0.09 & 3.15 & 0.12 \\
\hline \multirow{2}{*}{ NOTE: } & See Appendix A for source descriptio & $\mathrm{x} \mathrm{B}$ for $\mathrm{m}$ & d ICD-8 and & es. Data & an be found \\
\hline & $\begin{array}{l}\text { Work-Related Lung Disease Surveilla } \\
\text { See Appendix E for revised rates for } \\
\text { National Center for Health Statistics }\end{array}$ & $\begin{array}{l}94 \text {, see } \\
\text { of death }\end{array}$ & tion & B. Bure & \\
\hline
\end{tabular}

Table 3-4. Silicosis: Years of potential life lost by race and sex, U.S. residents age 15 and over, 1991-1992

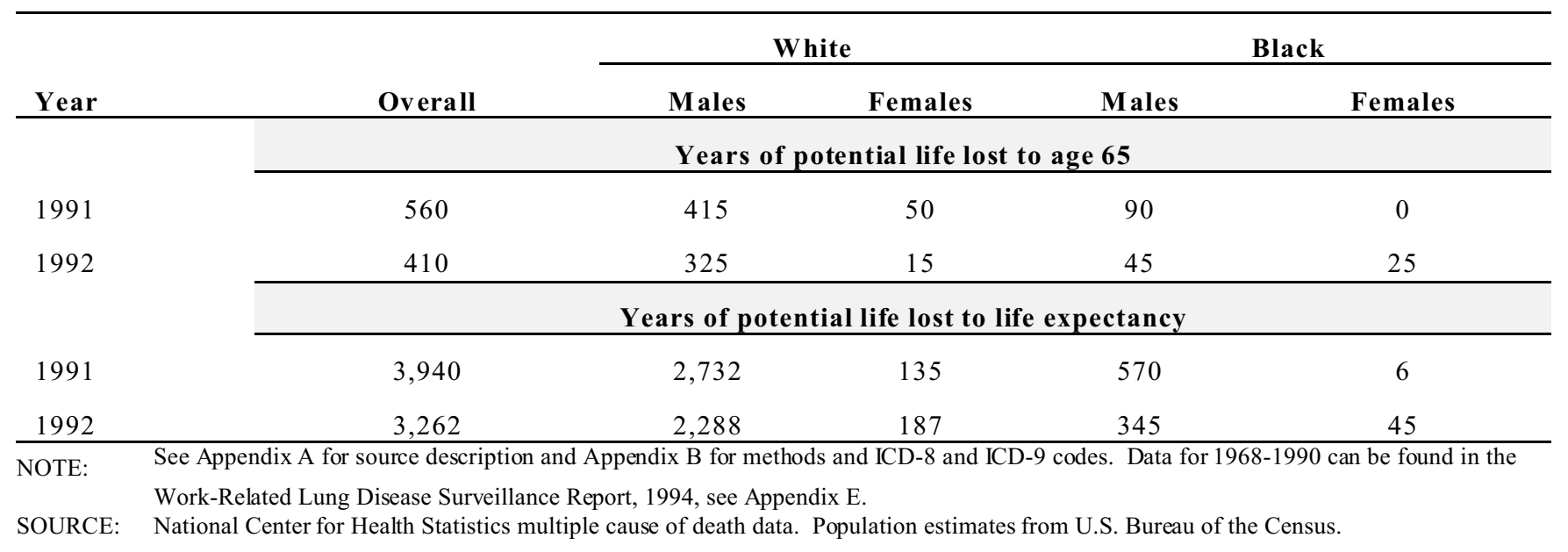


Table 3-5. Silicosis: Total number of deaths, crude and age-adjusted mortality rates (per 1,000,000 population), and total years of potential life lost (YPLL) to life expectancy, by state, U.S. residents age 15 and over, 1988-1992

\begin{tabular}{|c|c|c|c|c|c|c|c|c|c|c|}
\hline \multirow[b]{2}{*}{ State } & \multirow{2}{*}{$\begin{array}{r}\text { Total } \\
\text { deaths }\end{array}$} & \multirow[b]{2}{*}{ Rank } & \multicolumn{2}{|c|}{ Crude mortality } & \multicolumn{2}{|c|}{ Age-adjusted mortality } & \multicolumn{4}{|c|}{ YPLL to life expectancy } \\
\hline & & & Rate & Rank & Rate & Rank & Years & Rank & Years/death & Rank \\
\hline Alabama & 25 & 18 & 1.58 & 18 & 0.96 & 18 & 368 & 16 & 14.7 & 10 \\
\hline Alaska & 1 & 48 & 0.50 & 48 & 0.82 & 22 & 8 & 49 & 8.0 & 49 \\
\hline Arizona & 15 & 28 & 1.06 & 28 & 0.65 & 28 & 190 & 26 & 12.7 & 22 \\
\hline Arkansas & 5 & 40 & 0.55 & 46 & 0.42 & 42 & 102 & 38 & 20.4 & 2 \\
\hline California & 78 & 3 & 0.67 & 41 & 0.40 & 44 & 802 & 4 & 10.3 & 40 \\
\hline Colorado & 58 & 6 & 4.52 & 4 & 3.10 & 2 & 661 & 10 & 11.4 & 33 \\
\hline Connecticut & 13 & 33 & 0.98 & 31 & 0.54 & 36 & 144 & 30 & 11.1 & 34 \\
\hline Delaware & 4 & 42 & 1.51 & 19 & 1.04 & 13 & 53 & 42 & 13.3 & 17 \\
\hline District of Columbia & 2 & 47 & 0.79 & 37 & 0.47 & 38 & 22 & 48 & 11.0 & 35 \\
\hline Florida & 50 & 8 & 0.95 & 33 & 0.53 & 37 & 755 & 7 & 15.1 & 9 \\
\hline Georgia & 23 & 19 & 0.91 & 35 & 0.65 & 28 & 276 & 21 & 12.0 & 27 \\
\hline Hawaii & - & - & - & - & - & - & - & - & - & - \\
\hline Idaho & 13 & 33 & 3.47 & 8 & 1.60 & 10 & 120 & 35 & 9.2 & 46 \\
\hline Illinois & 50 & 8 & 1.12 & 27 & 0.74 & 24 & 689 & 9 & 13.8 & 13 \\
\hline Indiana & 28 & 14 & 1.29 & 22 & 0.85 & 20 & 428 & 14 & 15.3 & 8 \\
\hline Iowa & 9 & 37 & 0.83 & 36 & 0.46 & 39 & 116 & 36 & 12.9 & 20 \\
\hline Kansas & 3 & 44 & 0.31 & 50 & 0.15 & 50 & 30 & 47 & 10.0 & 43 \\
\hline Kentucky & 28 & 14 & 1.94 & 12 & 1.35 & 12 & 465 & 13 & 16.6 & 6 \\
\hline Louisiana & 18 & 23 & 1.13 & 26 & 0.98 & 16 & 322 & 19 & 17.9 & 4 \\
\hline Maine & 5 & 40 & 1.03 & 29 & 0.55 & 34 & 58 & 41 & 11.6 & 31 \\
\hline Maryland & 10 & 35 & 0.53 & 47 & 0.36 & 46 & 123 & 34 & 12.3 & 24 \\
\hline Massachusetts & 17 & 25 & 0.70 & 39 & 0.35 & 47 & 182 & 27 & 10.7 & 38 \\
\hline Michigan & 64 & 5 & 1.77 & 13 & 1.03 & 14 & 758 & 6 & 11.8 & 30 \\
\hline Minnesota & 28 & 14 & 1.66 & 14 & 0.97 & 17 & 352 & 17 & 12.6 & 23 \\
\hline Mississippi & 6 & 39 & 0.62 & 43 & 0.43 & 41 & 86 & 39 & 14.3 & 11 \\
\hline Missouri & 26 & 17 & 1.30 & 21 & 0.74 & 24 & 350 & 18 & 13.5 & 16 \\
\hline Montana & 14 & 29 & 4.58 & 3 & 2.15 & 5 & 140 & 32 & 10.0 & 43 \\
\hline Nebraska & 4 & 42 & 0.66 & 42 & 0.31 & 48 & 43 & 43 & 10.8 & 37 \\
\hline Nevada & 10 & 35 & 2.09 & 11 & 1.43 & 11 & 115 & 37 & 11.5 & 32 \\
\hline New Hampshire & 7 & 38 & 1.61 & 16 & 0.88 & 19 & 71 & 40 & 10.1 & 42 \\
\hline New Jersey & 50 & 8 & 1.61 & 16 & 0.81 & 23 & 513 & 12 & 10.3 & 41 \\
\hline New Mexico & 21 & 21 & 3.69 & 6 & 1.93 & 8 & 202 & 24 & 9.6 & 45 \\
\hline New York & 67 & 4 & 0.93 & 34 & 0.56 & 33 & 863 & 3 & 12.9 & 21 \\
\hline North Carolina & 43 & 12 & 1.62 & 15 & 1.02 & 15 & 562 & 11 & 13.1 & 18 \\
\hline North Dakota & 1 & 48 & 0.41 & 49 & 0.41 & 43 & 38 & 45 & 38.0 & 1 \\
\hline Ohio & 153 & 2 & 3.60 & 7 & 2.07 & 6 & 1,973 & 2 & 12.9 & 19 \\
\hline Oklahoma & 14 & 29 & 1.15 & 24 & 0.55 & 34 & 146 & 29 & 10.4 & 39 \\
\hline Oregon & 16 & 26 & 1.43 & 20 & 0.57 & 32 & 138 & 33 & 8.6 & 48 \\
\hline Pennsylvania & 259 & 1 & 5.43 & 2 & 2.71 & 3 & 3,117 & 1 & 12.0 & 26 \\
\hline Rhode Island & 3 & 44 & 0.74 & 38 & 0.44 & 40 & 41 & 44 & 13.7 & 14 \\
\hline South Carolina & 14 & 29 & 1.03 & 29 & 0.84 & 21 & 257 & 23 & 18.4 & 3 \\
\hline South Dakota & 3 & 44 & 1.14 & 25 & 0.61 & 30 & 36 & 46 & 12.0 & 27 \\
\hline Tennessee & 19 & 22 & 0.98 & 31 & 0.69 & 26 & 306 & 20 & 16.1 & 7 \\
\hline Texas & 45 & 11 & 0.70 & 39 & 0.59 & 31 & 749 & 8 & 16.6 & 5 \\
\hline Utah & 18 & 23 & 3.03 & 10 & 1.97 & 7 & 194 & 25 & 10.8 & 36 \\
\hline Vermont & 16 & 26 & 7.24 & 1 & 3.57 & 1 & 141 & 31 & 8.8 & 47 \\
\hline Virginia & 14 & 29 & 0.57 & 45 & 0.37 & 45 & 168 & 28 & 12.0 & 27 \\
\hline Washington & 22 & 20 & 1.16 & 23 & 0.66 & 27 & 268 & 22 & 12.2 & 25 \\
\hline West Virginia & 29 & 13 & 4.06 & 5 & 2.41 & 4 & 405 & 15 & 14.0 & 12 \\
\hline Wisconsin & 58 & 6 & 3.05 & 9 & 1.88 & 9 & 784 & 5 & 13.5 & 15 \\
\hline Wyoming & 1 & 48 & 0.59 & 44 & 0.31 & 48 & 8 & 49 & 8.0 & 49 \\
\hline
\end{tabular}

- indicates no deaths listed.

NOTE: Ranks are based on unrounded values. See Appendix A for source description and Appendix B for methods and ICD-8 and ICD-9 codes.

SOURCE: National Center for Health Statistics multiple cause of death data. Population estimates from U.S. Burea of the Census. 
Table 3-6. Silicosis: Most frequently recorded occupations on death certificate, U.S. residents age 15 and over, selected states, 1991-1992

\begin{tabular}{|c|c|c|c|}
\hline $\mathrm{COC}$ & Occupation & Number & Percent \\
\hline 616 & Mining machine operators & 39 & $\overline{16.0}$ \\
\hline 889 & Laborers, except construction & 29 & 11.9 \\
\hline 019 & Managers and administrators, n.e.c. & 11 & 4.5 \\
\hline 633 & Supervisors, precision production occupations & 11 & 4.5 \\
\hline 453 & Janitors and cleaners & 8 & 3.3 \\
\hline 719 & Molding, casting machine operators & 8 & 3.3 \\
\hline 243 & Supervisors and proprietors, sales occupations & 6 & 2.5 \\
\hline 844 & Operating engineers & 6 & 2.5 \\
\hline 637 & Machinists & 5 & 2.1 \\
\hline \multirow[t]{4}{*}{787} & Hand molding, casting, and forming occupations & 5 & 2.1 \\
\hline & All other occupations & 109 & 44.9 \\
\hline & Occupation not reported & 6 & 2.5 \\
\hline & TOTAL & 243 & 100.0 \\
\hline
\end{tabular}

Table 3-7. Silicosis: Most frequently recorded industries on death certificate, U.S. residents age 15 and over, selected states, 1991-1992

\begin{tabular}{llrr}
\hline CIC & Industry & Number & Percent \\
\hline 041 & Coal mining & 23 & 9.5 \\
060 & Construction & 23 & 9.5 \\
040 & Metal mining & 21 & 8.6 \\
270 & Blast furnaces, steelworks, rolling and finishing mills & 20 & 8.2 \\
050 & Nonmetallic mining and quarrying, except fuel & 12 & 4.9 \\
262 & Miscellaneous nonmetallic mineral and stone products & 12 & 4.9 \\
392 & Not specified manufacturing industries & 12 & 4.9 \\
271 & Iron and steel foundries & 9 & 3.7 \\
331 & Machinery, except electrical, n.e.c. & 8 & 3.3 \\
252 & Structural clay products & 6 & 2.5 \\
& All other industries & 91 & 37.4 \\
& Industry not reported & 6 & 2.5 \\
& TOTAL & 243 & 100.0 \\
\hline
\end{tabular}

CIC - 1980 Census Industry Code

NOTE: $\quad$ See Appendix A for source description, Appendix B for methods and ICD-8 and ICD-9 codes, and Appendix C for list of 25 states reporting usual industry and years reporting. Data for 1985-1990 can be found in the Work-Related Lung Disease Surveillance Report, 1994, see Appendix E.

SOURCE: National Center for Health Statistics multiple cause of death data. 
Table 3-8. Silicosis: Proportionate mortality ratio (PMR) by usual occupation, selected states and years, U.S. residents age 15 and over, 1985-1992

\begin{tabular}{|c|c|c|c|c|c|}
\hline \multirow[b]{2}{*}{$\mathrm{COC}$} & \multirow[b]{2}{*}{ Occupation } & \multirow{2}{*}{$\begin{array}{r}\text { Number } \\
\text { of deaths }\end{array}$} & \multirow[b]{2}{*}{ PMR } & \multicolumn{2}{|c|}{$95 \%$ confidence interval } \\
\hline & & & & $\mathrm{LCL}$ & $\mathrm{UCL}$ \\
\hline 725 & Miscellaneous metal and plastic machine operators & 11 & 168.44 & 84.22 & 301.32 \\
\hline 675 & Hand molders and shapers, except jewelers & 17 & 64.12 & 37.28 & 102.59 \\
\hline 768 & Crushing and grinding machine operators & 20 & 50.97 & 31.08 & 78.78 \\
\hline 787 & Hand molding, casting, and forming occupations & 10 & 35.70 & 17.16 & 65.62 \\
\hline 719 & Molding and casting machine operators & 27 & 30.60 & 20.13 & 44.54 \\
\hline 616 & Mining machine operators & 128 & 19.61 & 16.26 & 23.46 \\
\hline 617 & Mining occupations, n.e.c. & 8 & 15.33 & 6.61 & 30.18 \\
\hline 599 & Construction trades, n.e.c. & 12 & 14.77 & 7.61 & 25.78 \\
\hline 709 & Grinding, abrading, buffing, and polishing machine operators & 9 & 8.47 & 3.89 & 16.07 \\
\hline 516 & Heavy equipment mechanics & 9 & 7.72 & 3.54 & 14.65 \\
\hline 859 & Miscellaneous material moving equipment operators & 5 & 6.92 & 2.24 & 16.17 \\
\hline 544 & Millwrights & 9 & 6.56 & 3.01 & 12.45 \\
\hline 849 & Crane and tower operators & 8 & 6.02 & 2.59 & 11.85 \\
\hline 563 & Brickmasons and stonemasons & 11 & 4.71 & 2.35 & 8.43 \\
\hline 579 & Painters, construction and maintenance & 19 & 4.50 & 2.71 & 7.03 \\
\hline 766 & Furnace, kiln, oven operators, except food & 6 & 4.10 & 1.50 & 8.93 \\
\hline 889 & Laborers, except construction & 113 & 3.79 & 3.08 & 4.61 \\
\hline 844 & Operating engineers & 12 & 3.56 & 1.84 & 6.21 \\
\hline 783 & Welders and cutters & 13 & 3.01 & 1.60 & 5.15 \\
\hline 779 & Machine operators, not specified & 23 & 2.86 & 1.81 & 4.29 \\
\hline 549 & Not specified mechanics and repairers & 6 & 2.84 & 1.04 & 6.19 \\
\hline 633 & Supervisors, production occupations & 29 & 2.73 & 1.83 & 3.92 \\
\hline 869 & Construction laborers & 21 & 2.14 & 1.32 & 3.27 \\
\hline 637 & Machinists & 17 & 1.79 & 1.04 & 2.86 \\
\hline 453 & Janitors and cleaners & 28 & 1.78 & 1.19 & 2.57 \\
\hline
\end{tabular}


Table 3-9. Silicosis: Proportionate mortality ratio (PMR) by usual industry, selected states and years, U.S. residents age 15 and over, 1985-1992

\begin{tabular}{llrrrr}
\hline & & & \multicolumn{3}{c}{$95 \%$ confidence interval } \\
\cline { 4 - 6 } CIC & Industry & $\begin{array}{r}\text { Number } \\
\text { of deaths }\end{array}$ & PMR & LCL & UCL \\
\hline 040 & Metal mining & 75 & 69.51 & 54.30 & 87.88 \\
262 & Miscellane ous nonmetallic mineral and stone products & 54 & 55.31 & 40.97 & 72.97 \\
050 & Nonmetallic mining and quarrying, except fuel & 47 & 49.77 & 36.33 & 66.63 \\
271 & Iron and steel foundries & 46 & 31.15 & 22.74 & 41.70 \\
261 & Pottery and related products & 21 & 30.73 & 18.97 & 46.99 \\
252 & Structural clay products & 20 & 27.82 & 16.96 & 43.00 \\
041 & Coal mining & 63 & 9.26 & 7.07 & 11.92 \\
270 & Blast furnaces, steelworks, rolling and finishing mills & 67 & 6.49 & 4.95 & 8.35 \\
300 & Miscellaneous fabricated metal products & 15 & 5.87 & 3.28 & 9.69 \\
682 & Miscellaneous retail stores & 10 & 4.63 & 2.23 & 8.51 \\
331 & Machinery, except electrical, n.e.c. & 23 & 3.96 & 2.51 & 5.95 \\
280 & Other primary metal industries & 6 & 3.63 & 1.33 & 7.91 \\
192 & Industrial and miscellaneous chemicals & 11 & 2.72 & 1.36 & 4.87 \\
392 & Not specified manufacturing industries & 37 & 2.67 & 1.85 & 3.71 \\
060 & Construction & 97 & 1.82 & 1.47 & 2.24 \\
\hline
\end{tabular}

CIC - 1980 Census Industry Code n.e.c. - not elsewhere classified LCL - lower confidence limit UCL - upper confidence limit

NOTE: See Appendix A for source description, Appendix B for methods and ICD-8 and ICD-9 codes, and Appendix C for list of 25 states reporting usual industry and years

SOURCE: $\quad$ National Center for Health Statistics multiple cause of death data. 
Figure 3-4. Silicosis: Total number of deaths by county,

U.S. residents age 15 and over, 1983-1992

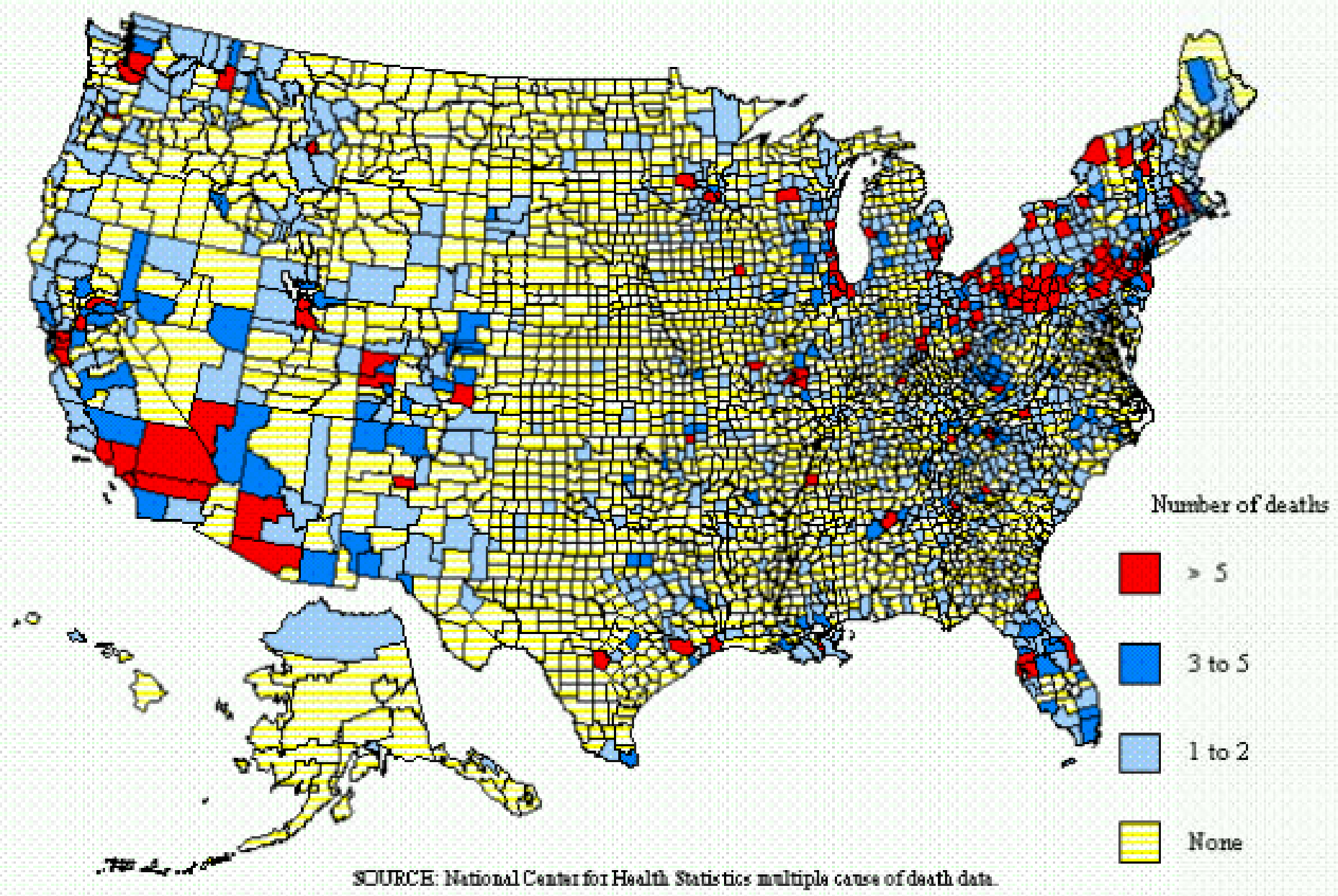


Figure 3-5. Silicosis: Age-adjusted mortality rates by county,

U.S. residents age 15 and over, 1983-1992

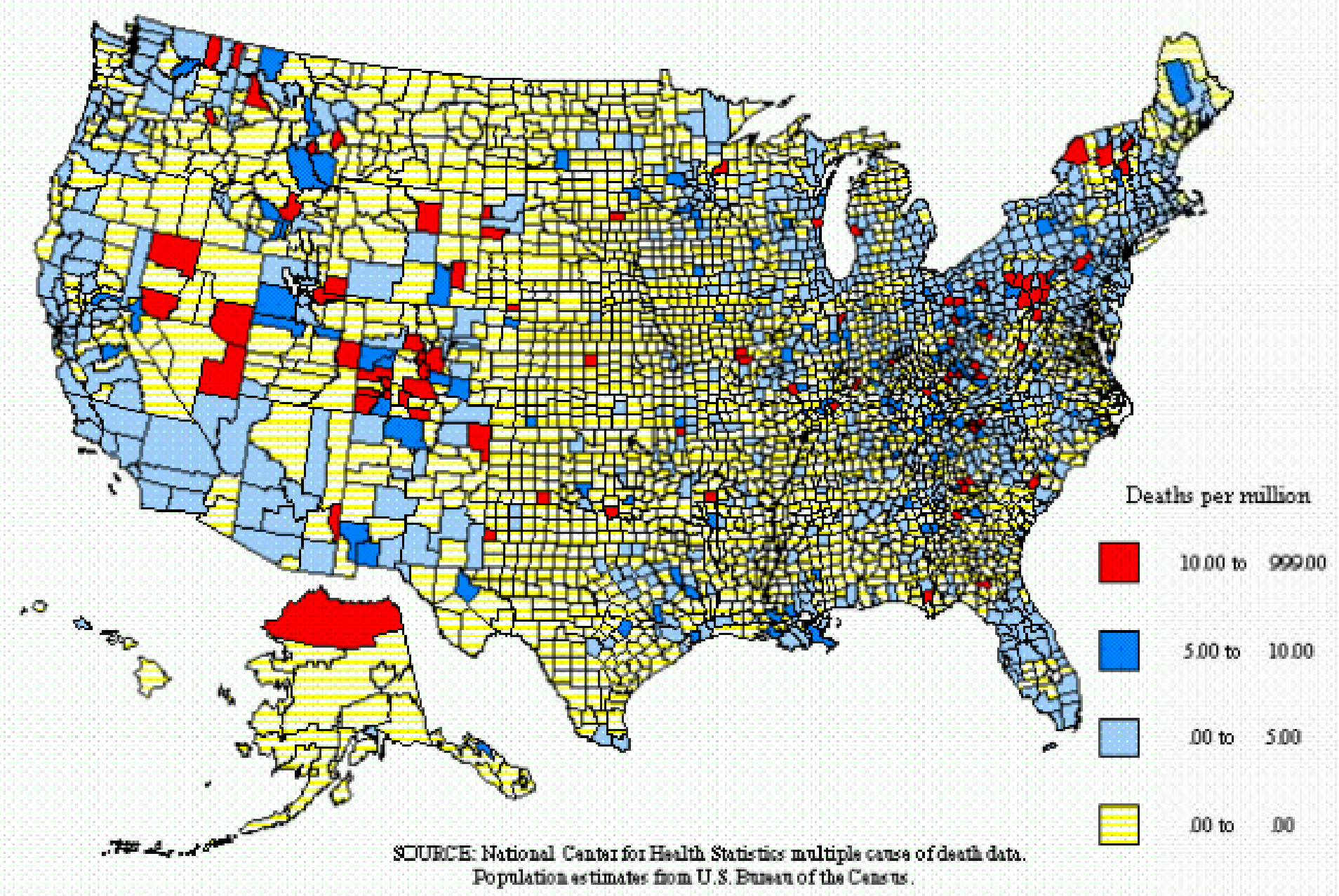


Figure 3-6. Silica: Number of MSHA and OSHA inspector samples and percent exceeding the permissible exposure limit (PEL), 1974-1994

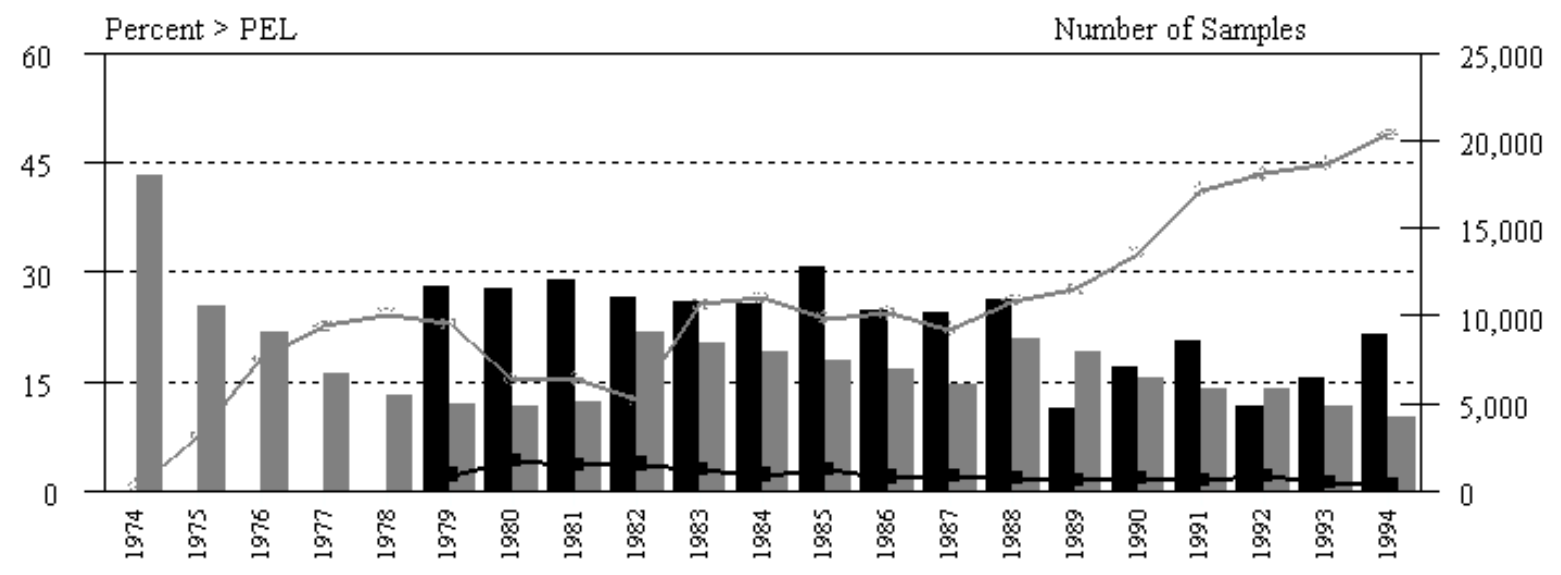

\begin{tabular}{ll|} 
- OSHA Percent > PEL & MSHA Percent > PEL \\
-\# OSHA Samples & \#MSHA Samples \\
\hline
\end{tabular}

NOTE: See Appendix A for source description, Appendix B for methods, and Appendix D for agents.

SOURCE: Mine Safety and Health Administration: respirable coal mine quartz data. Bureau of Mines: Mine Inspection Data Analysis System. Occupational Safety and Health Administration: Integrated Management Information System.

Figure 3-7. Silica: Number of MSHA and OSHA inspector samples and average severity levels, 1974-1994

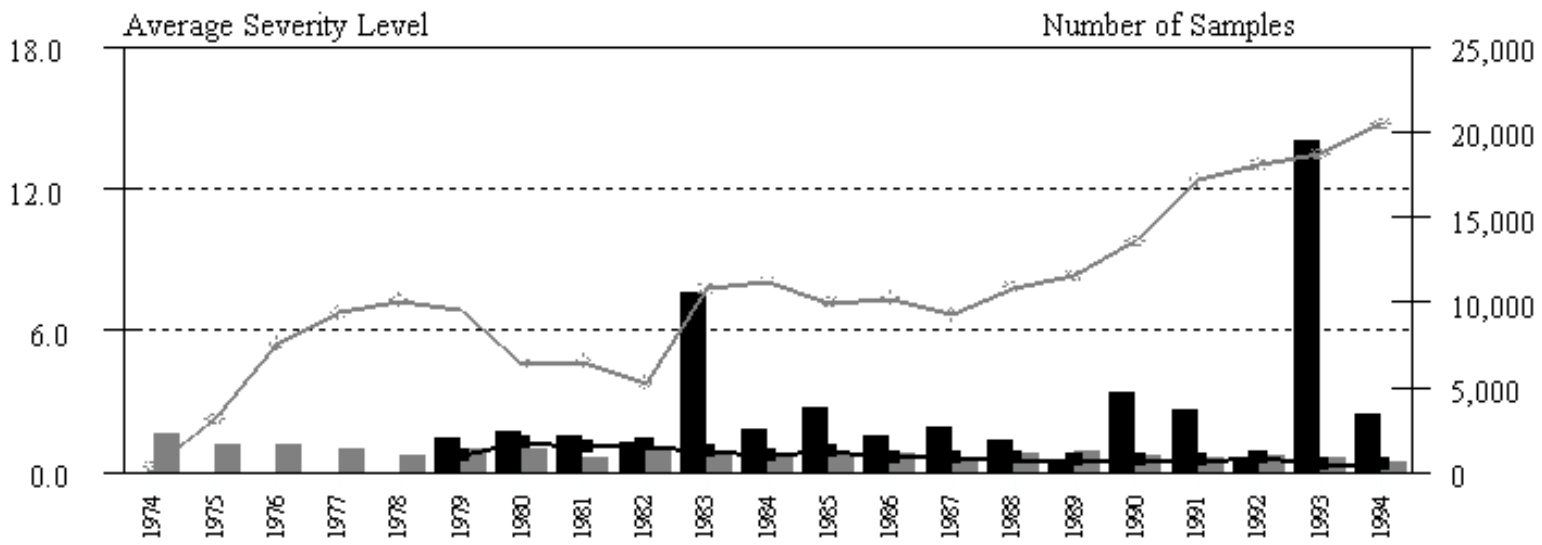

\section{OSHA Severity Levels \\ MSHA Severity Levels}

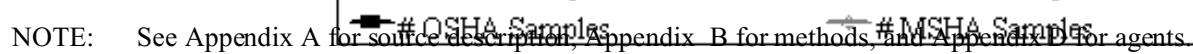

SOURCE: Mine Safety and Health Administration: respirable coal mine quartz data. Bureau of Mines: Mine Inspection Data Analysis System. Occupational Safety and Health Administration: Integrated Management Information System. 
Table 3-10. Silica: Number of MSHA and OSHA inspector samples, percent exceeding the permissible exposure limit (PEL) and average severity level, by industry, 1993-1994

\begin{tabular}{|c|c|c|c|c|}
\hline CIC & $\begin{array}{l}\text { Industries most frequently recorded on } \\
\text { 1991-1992 death certificates with silicosis }\end{array}$ & $\begin{array}{r}\text { Number of } \\
\text { samples } \\
\end{array}$ & $\%>$ PEL & $\begin{array}{r}\text { Average } \\
\text { severity }\end{array}$ \\
\hline 041 & Coal mining & 12,770 & 19.1 & 0.71 \\
\hline 060 & Construction & 58 & 25.9 & 2.85 \\
\hline 040 & Metal mining & 1,857 & 10.4 & 0.43 \\
\hline 270 & Blast furnaces, steelworks, rolling and finishing mills & 7 & 0.0 & 0.00 \\
\hline 050 & Nonmetallic mining & 24,301 & 6.5 & 0.43 \\
\hline 262 & Miscellane ous nonmetallic mineral and stone products & 75 & 10.7 & 0.44 \\
\hline 392 & Not specified manufacturing industries & 0 & - & - \\
\hline 271 & Iron and steel foundries & 254 & 19.7 & 0.85 \\
\hline 331 & Machinery, except electrical, n.e.c. & 16 & 31.3 & 1.05 \\
\hline \multirow[t]{4}{*}{252} & Structural clay products & 28 & 21.4 & 0.87 \\
\hline & All other industries & 416 & 17.3 & 16.79 \\
\hline & Industry not reported & 36 & 5.6 & 0.28 \\
\hline & TOTAL & 39,818 & 10.9 & 0.70 \\
\hline
\end{tabular}

CIC - 1980 Census Industry Code n.e.c. - not elsewhere classified - indicates incalculable field

NOTE: See Appendix A for source description, Appendix B for methods, Appendix C for list of 25 states reporting usual industry and years reporting, and Appendix D for agents.

SOURCE: Mine Safety and Health Administration: respirable coal mine quartz data. Bureau of Mines: Mine Inspection Data Analysis System. Occupational Sa fety and Health Administration: Integrated Management Information System. National Center for Health Statistics: multiple cause of death data.

Table 3-11. Silica: Number of MSHA and OSHA inspector samples, percent exceeding the permissible exposure limit (PEL) and average severity level, by industry, 1993-1994

\begin{tabular}{|c|c|c|c|c|}
\hline CIC & Industries most frequently sampled in 1993-1994 & $\begin{array}{r}\text { Number of } \\
\text { samples } \\
\end{array}$ & $\%>$ PEL & $\begin{array}{r}\text { Average } \\
\text { severity }\end{array}$ \\
\hline 050 & Nonmetallic mining & 24,301 & 6.5 & 0.43 \\
\hline 041 & Coal mining & 12,770 & 19.1 & 0.71 \\
\hline 040 & Metal mining & 1,857 & 10.4 & 0.43 \\
\hline 271 & Iron and steel foundries & 254 & 19.7 & 0.85 \\
\hline 262 & Miscellaneous nonmetallic mineral and stone products & 75 & 10.7 & 0.44 \\
\hline 060 & Construction & 58 & 25.9 & 2.85 \\
\hline 300 & Miscellaneous fabricated metal products & 52 & 46.2 & 17.96 \\
\hline 261 & Pottery and related products & 46 & 17.4 & 0.86 \\
\hline 251 & Cement, concrete, gypsum, and plaster products & 39 & 12.8 & 0.67 \\
\hline \multirow[t]{4}{*}{280} & Other primary metals industries & 30 & 6.7 & 0.28 \\
\hline & All other industries & 300 & 14.7 & 20.06 \\
\hline & Industry not reported & 36 & 5.6 & 0.28 \\
\hline & TOTAL & 39,818 & 10.9 & 0.70 \\
\hline
\end{tabular}

CIC - 1980 Census Industry Code

NOTE: See Appendix A for source description, Appendix B for methods, and Appendix D for agents.

SOURCE: Mine Safety and Health Administration: respirable coal mine quartz data. B ureau of Mines: Mine Inspection Data Analysis System. Occupational Sa fety and Health Administration: Integrated Management Information System. 
Table 3-12 (page 1 of 2). Silica: Number of MSHA inspector samples, percent exceeding the permissible exposure limit (PEL) and average severity levels (Avg. Sev.), by state, 1974-1994

\begin{tabular}{|c|c|c|c|c|c|c|c|c|c|c|c|c|}
\hline \multirow[b]{3}{*}{ State } & \multicolumn{4}{|c|}{ 1974-1984 } & \multicolumn{4}{|c|}{ 1985-1994 } & \multicolumn{4}{|c|}{ 1993-1994 } \\
\hline & \multicolumn{2}{|c|}{ Total samples } & \multicolumn{2}{|c|}{ Samples > PEL } & \multicolumn{2}{|c|}{ Total samples } & \multicolumn{2}{|c|}{ Samples > PEL } & \multicolumn{2}{|c|}{ Total samples } & \multicolumn{2}{|c|}{ Samples > PEL } \\
\hline & Number & $\begin{array}{l}\text { Avg. } \\
\text { Sev. }\end{array}$ & $\%$ & $\begin{array}{r}\text { Avg. } \\
\text { Sev. }\end{array}$ & Number & $\begin{array}{l}\text { Avg. } \\
\text { Sev. }\end{array}$ & $\%$ & $\begin{array}{l}\text { Avg. } \\
\text { Sev. }\end{array}$ & Number & $\begin{array}{l}\text { Avg. } \\
\text { Sev. }\end{array}$ & $\%$ & $\begin{array}{r}\text { Avg. } \\
\text { Sev. }\end{array}$ \\
\hline Alabama & 1,659 & 0.58 & 11.5 & 3.42 & 3,411 & 0.56 & 15.1 & 2.32 & 1,408 & 0.49 & 13.2 & 2.00 \\
\hline Alaska & 79 & 0.67 & 12.7 & 3.40 & 151 & 1.34 & 14.6 & 7.88 & 80 & 0.43 & 5.0 & 5.25 \\
\hline Arizona & 3,548 & 1.14 & 16.4 & 5.43 & 2,169 & 0.83 & 16.4 & 3.72 & 704 & 0.48 & 10.7 & 2.56 \\
\hline Arkansas & 784 & 1.67 & 39.9 & 3.65 & 1,276 & 0.74 & 17.8 & 3.07 & 461 & 0.42 & 11.1 & 2.26 \\
\hline California & 1,834 & 1.73 & 14.5 & 10.16 & 4,239 & 0.63 & 12.2 & 3.34 & 862 & 0.39 & 5.6 & 3.62 \\
\hline Colorado & 3,069 & 0.87 & 19.8 & 3.13 & 4,479 & 0.60 & 14.5 & 2.51 & 877 & 0.54 & 12.4 & 2.32 \\
\hline Connecticut & 330 & 1.25 & 25.2 & 3.96 & 329 & 0.64 & 16.4 & 2.66 & 63 & 0.21 & 3.2 & 2.27 \\
\hline Delaware & 9 & 0.22 & 0.0 & - & 42 & 0.24 & 0.0 & - & 3 & 0.02 & 0.0 & - \\
\hline District of Columbia & 0 & - & - & - & 0 & - & - & - & 0 & - & - & - \\
\hline Florida & 640 & 0.37 & 5.2 & 3.64 & 1,704 & 0.17 & 2.5 & 2.55 & 433 & 0.09 & 1.6 & 1.33 \\
\hline Georgia & 2,254 & 0.59 & 13.4 & 2.59 & 3,342 & 0.46 & 9.5 & 3.08 & 774 & 0.41 & 6.8 & 4.01 \\
\hline Hawaii & 6 & 0.18 & 0.0 & - & 93 & 0.29 & 3.2 & 5.85 & 35 & 0.47 & 2.9 & 13.99 \\
\hline Idaho & 1,653 & 1.29 & 19.2 & 5.11 & 1,235 & 1.01 & 13.9 & 5.67 & 425 & 0.34 & 7.1 & 2.11 \\
\hline Illinois & 4,906 & 0.94 & 19.7 & 3.66 & 5,667 & 0.50 & 12.1 & 2.11 & 1,809 & 0.34 & 5.9 & 2.03 \\
\hline Indiana & 2,479 & 0.40 & 6.9 & 2.92 & 2,261 & 0.36 & 7.6 & 2.34 & 762 & 0.27 & 4.2 & 1.96 \\
\hline Iowa & 1,103 & 0.53 & 11.1 & 2.62 & 1,148 & 0.20 & 3.6 & 1.74 & 351 & 0.16 & 1.1 & 1.95 \\
\hline Kansas & 1,497 & 0.55 & 14.2 & 1.97 & 1,285 & 0.47 & 9.3 & 2.69 & 381 & 0.29 & 5.8 & 1.85 \\
\hline Kentucky & 3,545 & 1.03 & 26.0 & 3.08 & 13,364 & 0.96 & 26.1 & 2.72 & 4,357 & 0.74 & 20.4 & 2.29 \\
\hline Louisiana & 247 & 0.99 & 17.8 & 4.35 & 1,424 & 0.21 & 4.1 & 2.36 & 590 & 0.19 & 3.7 & 1.89 \\
\hline Maine & 424 & 0.61 & 16.0 & 2.03 & 282 & 0.31 & 6.0 & 1.95 & 79 & 0.23 & 3.8 & 1.42 \\
\hline Maryland & 597 & 0.54 & 11.4 & 2.86 & 839 & 0.43 & 8.6 & 2.98 & 173 & 0.67 & 13.3 & 3.53 \\
\hline Massachusetts & 559 & 1.49 & 19.3 & 6.20 & 517 & 0.45 & 10.6 & 2.41 & 153 & 0.32 & 7.2 & 2.01 \\
\hline Michigan & 1,742 & 0.66 & 15.3 & 2.49 & 2,787 & 0.53 & 11.5 & 3.01 & 852 & 0.37 & 9.4 & 2.09 \\
\hline Minnesota & 3,898 & 0.54 & 9.6 & 3.51 & 3,051 & 0.37 & 8.3 & 2.46 & 782 & 0.29 & 5.8 & 2.28 \\
\hline Mississippi & 400 & 0.57 & 8.3 & 4.64 & 916 & 0.32 & 6.6 & 2.88 & 371 & 0.46 & 8.1 & 3.60 \\
\hline Missouri & 2,935 & 1.25 & 15.3 & 6.51 & 4,599 & 1.02 & 10.8 & 7.41 & 1,458 & 0.35 & 6.1 & 2.76 \\
\hline Montana & 1,184 & 0.56 & 12.9 & 2.61 & 1,673 & 0.78 & 15.1 & 3.71 & 363 & 0.51 & 11.0 & 2.56 \\
\hline
\end{tabular}


Table 3-12 (page 2 of 2). Silica: Number of MSHA inspector samples, percent exceeding the permissible exposure limit (PEL) and average severity levels (Avg. Sev.), by state, 1974-1994

\begin{tabular}{|c|c|c|c|c|c|c|c|c|c|c|c|c|}
\hline \multirow[b]{3}{*}{ State } & \multicolumn{4}{|c|}{ 1974-1984 } & \multicolumn{4}{|c|}{ 1985-1994 } & \multicolumn{4}{|c|}{ 1993-1994 } \\
\hline & \multicolumn{2}{|c|}{ Total samples } & \multicolumn{2}{|c|}{ Samples > PEL } & \multicolumn{2}{|c|}{ Total samples } & \multicolumn{2}{|c|}{ Samples > PEL } & \multicolumn{2}{|c|}{ Total samples } & \multicolumn{2}{|c|}{ Samples > PEL } \\
\hline & Number & $\begin{array}{r}\text { Avg. } \\
\text { Sev. }\end{array}$ & $\%$ & $\begin{array}{l}\text { Avg. } \\
\text { Sev. } \\
\end{array}$ & Number & $\begin{array}{l}\text { Avg. } \\
\text { Sev. }\end{array}$ & $\%$ & $\begin{array}{l}\text { Avg. } \\
\text { Sev. }\end{array}$ & Number & $\begin{array}{l}\text { Avg. } \\
\text { Sev. } \\
\end{array}$ & $\%$ & $\begin{array}{l}\text { Avg. } \\
\text { Sev. }\end{array}$ \\
\hline Nebraska & 82 & 0.76 & 15.9 & 2.90 & 443 & 0.18 & 2.5 & 2.28 & 164 & 0.14 & 1.8 & 2.75 \\
\hline Nevada & 984 & 2.53 & 24.7 & 9.23 & 1,984 & 0.96 & 22.3 & 3.19 & 358 & 0.63 & 15.4 & 2.46 \\
\hline New Hampshire & 214 & 1.04 & 23.8 & 3.20 & 226 & 0.37 & 8.8 & 1.70 & 58 & 0.41 & 12.1 & 1.74 \\
\hline New Jersey & 930 & 0.86 & 23.9 & 2.57 & 1,214 & 0.61 & 13.5 & 2.89 & 188 & 0.31 & 7.4 & 1.67 \\
\hline New Mexico & 1,023 & 0.62 & 9.1 & 4.72 & 2,232 & 0.75 & 15.3 & 3.36 & 684 & 0.69 & 13.0 & 3.57 \\
\hline New York & 1,348 & 0.96 & 13.7 & 5.23 & 3,446 & 0.45 & 7.7 & 3.37 & 853 & 0.35 & 6.1 & 3.04 \\
\hline North Carolina & 2,743 & 0.86 & 10.7 & 5.82 & 4,491 & 0.40 & 5.8 & 4.50 & 1,130 & 0.25 & 4.6 & 2.78 \\
\hline North Dakota & 310 & 0.54 & 11.0 & 2.52 & 280 & 0.23 & 3.6 & 1.62 & 94 & 0.12 & 0.0 & - \\
\hline Ohio & 3,549 & 0.77 & 14.8 & 3.75 & 5,193 & 0.55 & 12.6 & 2.69 & 1,431 & 0.37 & 8.7 & 2.12 \\
\hline Oklahoma & 1,410 & 0.72 & 15.4 & 3.44 & 2,711 & 0.68 & 10.7 & 4.68 & 764 & 0.53 & 5.4 & 7.20 \\
\hline Oregon & 340 & 0.48 & 5.9 & 4.86 & 1,575 & 1.37 & 5.1 & 23.90 & 584 & 2.94 & 2.9 & 96.11 \\
\hline Pennsylvania & 5,170 & 0.80 & 18.1 & 3.09 & 8,337 & 0.68 & 15.9 & 2.73 & 2,115 & 0.61 & 13.1 & 2.83 \\
\hline Rhode Island & 71 & 0.82 & 18.3 & 2.69 & 69 & 0.62 & 13.0 & 3.02 & 24 & 0.49 & 16.7 & 2.04 \\
\hline South Carolina & 874 & 1.57 & 23.1 & 5.64 & 2,382 & 0.37 & 8.2 & 2.62 & 784 & 0.35 & 2.9 & 7.39 \\
\hline South Dakota & 1,293 & 0.89 & 17.4 & 3.50 & 1,159 & 0.38 & 9.1 & 2.05 & 234 & 0.26 & 6.0 & 2.05 \\
\hline Tennessee & 2,594 & 0.48 & 10.4 & 2.62 & 4,901 & 0.49 & 11.9 & 2.41 & 1,082 & 0.48 & 10.4 & 2.58 \\
\hline Texas & 1,363 & 1.20 & 17.2 & 5.69 & 6,172 & 0.66 & 6.7 & 7.76 & 1,876 & 0.35 & 5.0 & 4.79 \\
\hline Utah & 978 & 0.65 & 15.0 & 2.44 & 3,779 & 0.78 & 14.3 & 3.91 & 729 & 0.50 & 10.8 & 2.47 \\
\hline Vermont & 494 & 0.61 & 18.6 & 1.78 & 726 & 0.62 & 18.3 & 2.37 & 222 & 0.55 & 14.9 & 2.26 \\
\hline Virginia & 3,121 & 1.08 & 28.2 & 3.08 & 7,533 & 0.94 & 26.9 & 2.61 & 1,752 & 0.72 & 22.3 & 2.06 \\
\hline Washington & 909 & 0.74 & 16.3 & 3.24 & 1,764 & 0.54 & 10.4 & 3.51 & 749 & 0.37 & 8.1 & 2.51 \\
\hline West Virginia & 4,434 & 0.98 & 26.3 & 2.86 & 12,625 & 0.85 & 23.9 & 2.45 & 3,241 & 0.71 & 18.0 & 2.44 \\
\hline Wisconsin & 2,083 & 0.49 & 9.8 & 2.82 & 2,259 & 0.55 & 11.1 & 3.40 & 780 & 0.55 & 6.9 & 5.72 \\
\hline Wyoming & 1,917 & 1.14 & 17.9 & 4.85 & 1,639 & 0.86 & 14.4 & 4.30 & 458 & 0.50 & 12.4 & 2.53 \\
\hline TOTAL & 79,615 & 0.89 & 16.9 & 3.87 & 139,423 & 0.67 & 14.7 & 3.14 & 38,960 & 0.52 & 10.8 & 2.97 \\
\hline
\end{tabular}

- indicates incalculable field.

F for source description, Appendix B for methods, and Appendix D for agen

SOURCE: Mine Safety and Health Administration: respirable coal mine quartz data. Bureau of Mines: Mine Inspection Data Analysis System. 
Figure 3-8. Silica: Number of MSHA inspector samples collected by state, 1993-1994

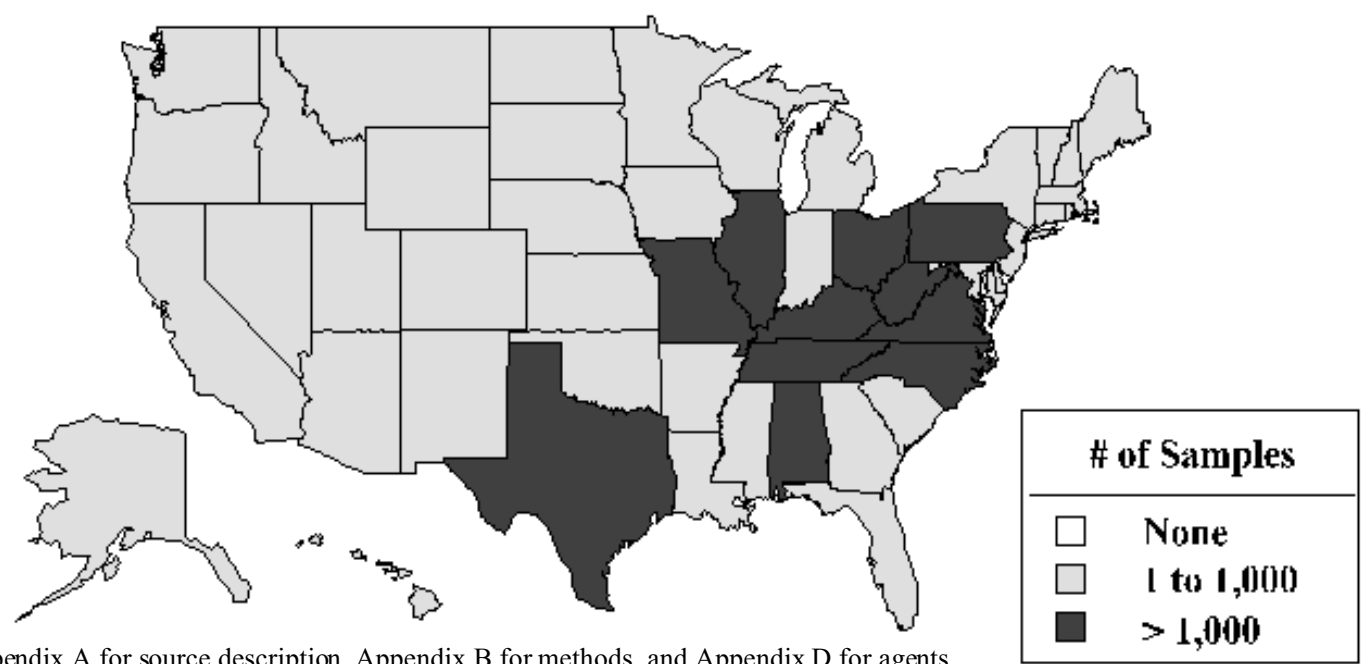

NOTE: See Appendix A for source description, Appendix B for methods, and Appendix D for agents.

SOURCE: Mine Safety and Health Administration: respirable coal mine quartz data. Bureau of Mines: Mine Inspection Data Analysis System.

Figure 3-9. Silica: Percent of MSHA inspector samples that exceeded the permissible exposure limit (PEL), by state, 1993-1994

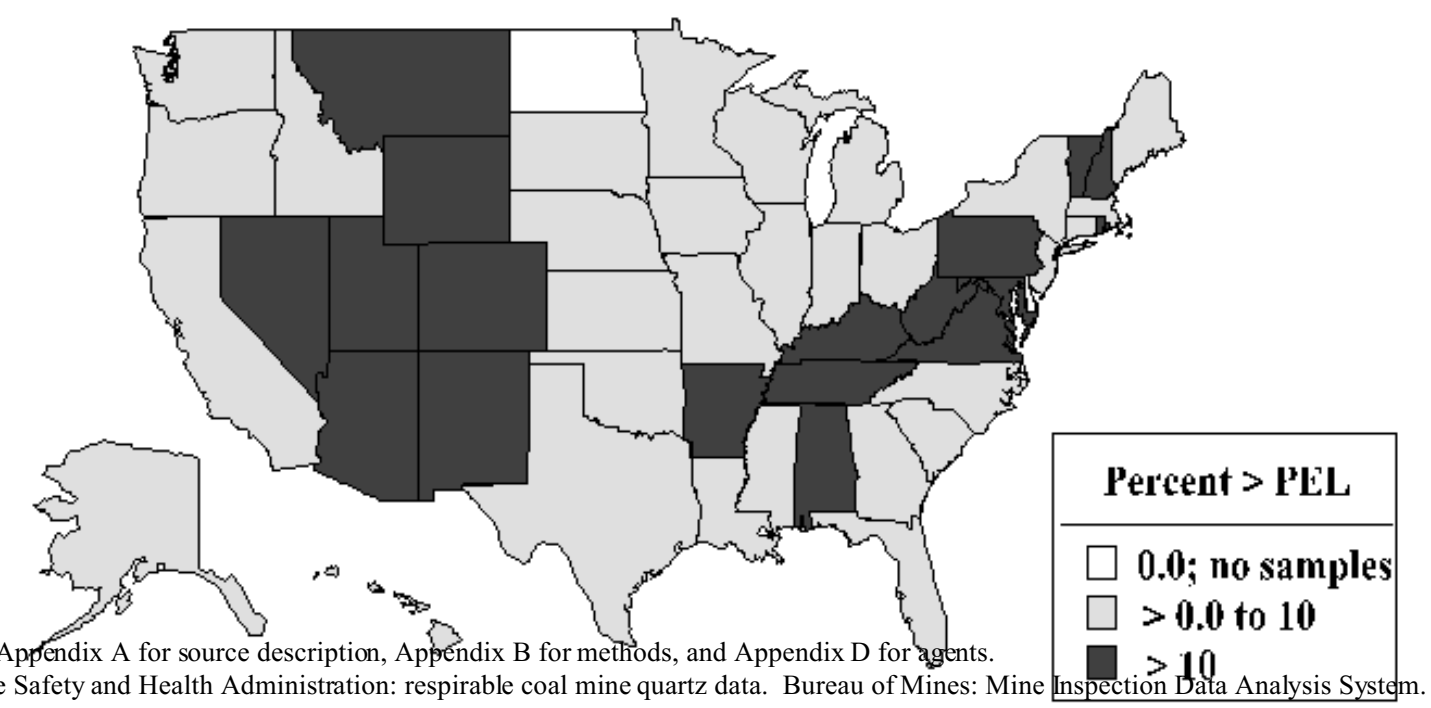


Figure 3-10. Silica: Average severity level by state for MSHA inspector samples, 1993-1994

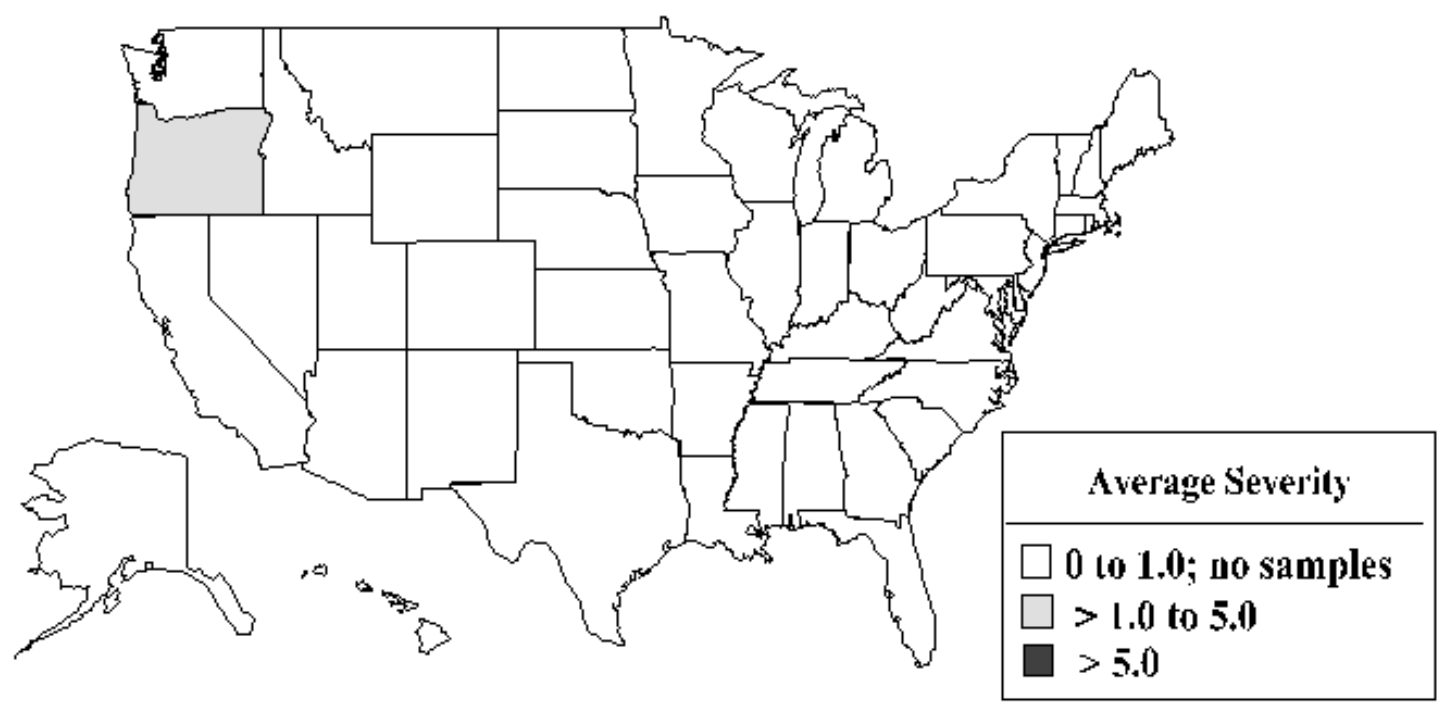

NOTE: See Appendix A for source description, Appendix B for methods, and Appendix D for agents.

SOURCE: Mine Safety and Health Administration: respirable coal mine quartz data. Bureau of Mines: Mine Inspection Data Analysis System.

Figure 3-11. Silica: Average severity level by state for MSHA inspector samples that exceeded the permissible exposure limit (PEL), 1993-1994

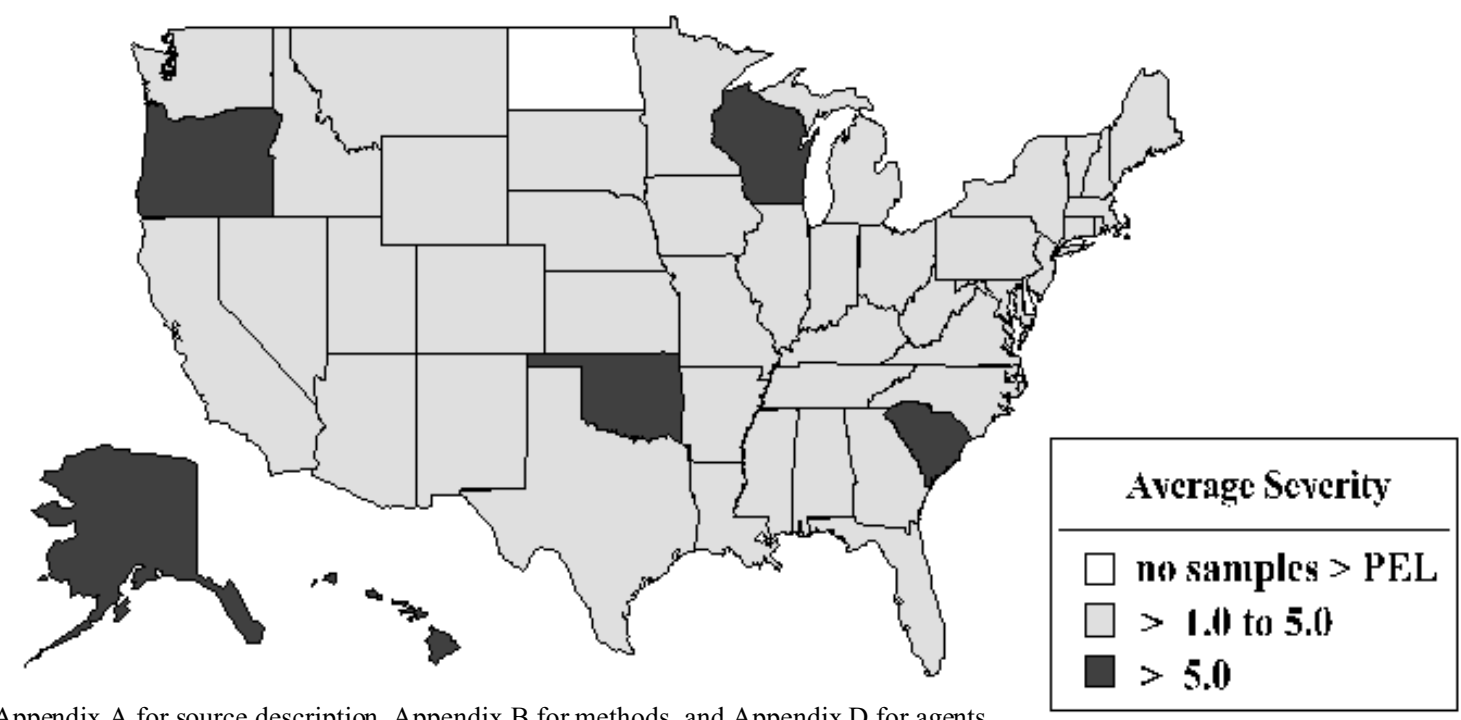

NOTE: See Appendix A for source description, Appendix B for methods, and Appendix D for agents.

SOURCE: Mine Safety and Health Administration: respirable coal mine quartz data. Bureau of Mines: Mine Inspection Data Analysis System. 
Figure 3-12. Silica: Number of MSHA inspector samples collected by county, 1993-1994

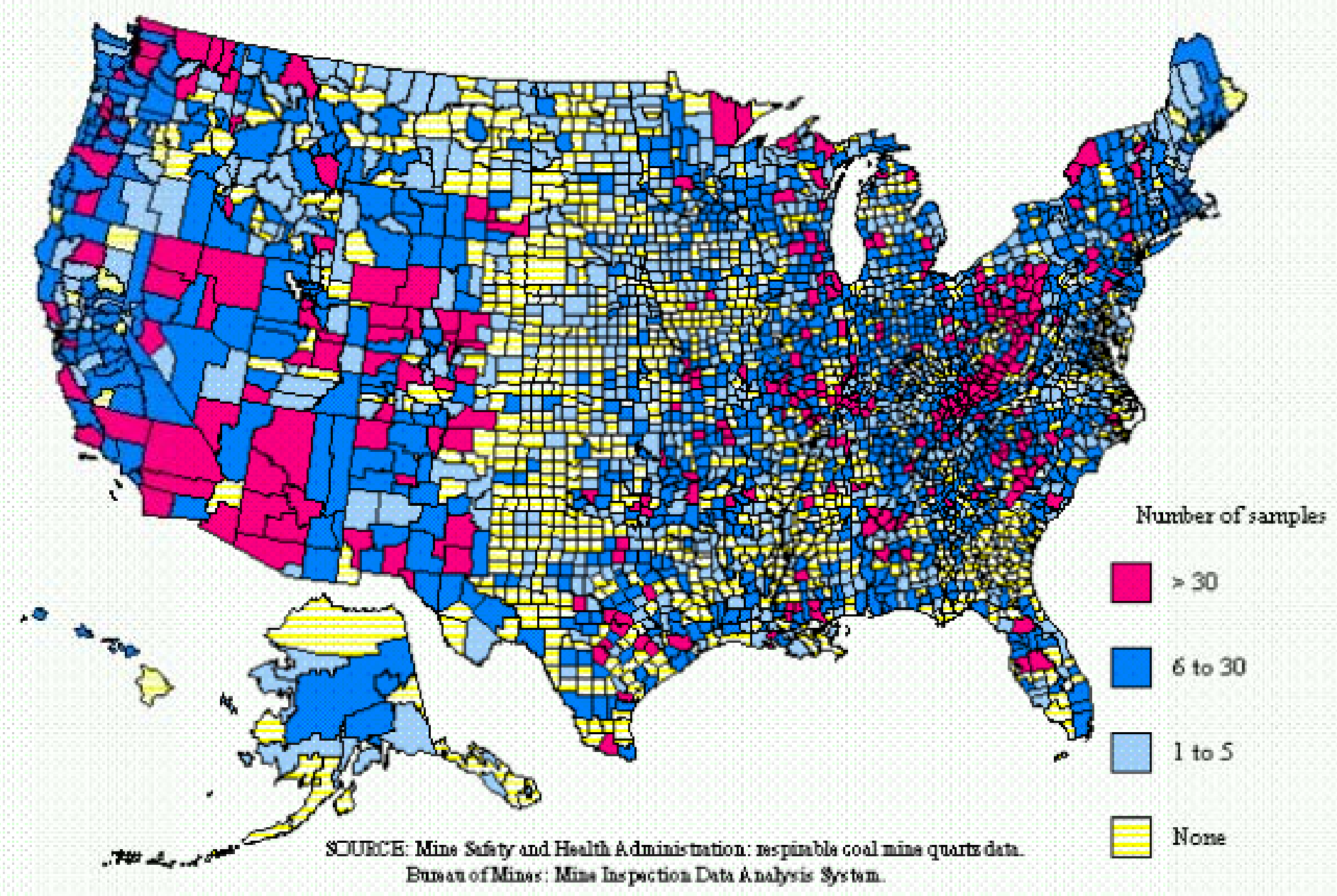


Table 3-13 (page 1 of 2). Silica: Number of OSHA inspector samples, percent exceeding the permissible exposure limit (PEL) and average severity levels (Avg. Sev.), by state, 1979-1994

\begin{tabular}{|c|c|c|c|c|c|c|c|c|c|c|c|c|}
\hline \multirow[b]{3}{*}{ State } & \multicolumn{4}{|c|}{ 1979-1984 } & \multicolumn{4}{|c|}{ 1985-1994 } & \multicolumn{4}{|c|}{ 1993-1994 } \\
\hline & \multicolumn{2}{|c|}{ Total samples } & \multicolumn{2}{|c|}{ Samples > PEL } & \multicolumn{2}{|c|}{ Total samples } & \multicolumn{2}{|c|}{ Samples > PEL } & \multicolumn{2}{|c|}{ Total samples } & \multicolumn{2}{|c|}{ Samples > PEL } \\
\hline & Number & $\begin{array}{c}\text { Avg. } \\
\text { Sev. }\end{array}$ & $\%$ & $\begin{array}{c}\text { Avg. } \\
\text { Sev. }\end{array}$ & Number & $\begin{array}{l}\text { Avg. } \\
\text { Sev. }\end{array}$ & $\%$ & $\begin{array}{c}\text { Avg. } \\
\text { Sev. }\end{array}$ & Number & $\begin{array}{r}\text { Avg. } \\
\text { Sev. }\end{array}$ & $\%$ & $\begin{array}{l}\text { Avg. } \\
\text { Sev. }\end{array}$ \\
\hline Alabama & 258 & 1.10 & 31.0 & 2.84 & 172 & 1.89 & 16.9 & 10.48 & 12 & 8.96 & 41.7 & 21.30 \\
\hline Alaska & 1 & 34.59 & 100.0 & 34.59 & 10 & 0.72 & 30.0 & 2.11 & 0 & - & - & - \\
\hline Arizona & 22 & 1.14 & 36.4 & 2.68 & 25 & 1.55 & 12.0 & 11.75 & 2 & 8.68 & 50.0 & 17.36 \\
\hline Arkansas & 56 & 0.50 & 17.9 & 1.99 & 44 & 2.55 & 25.0 & 9.91 & 3 & 1.70 & 33.3 & 5.09 \\
\hline California & 7 & 6.61 & 42.9 & 15.11 & 143 & 0.97 & 17.5 & 4.98 & 10 & 0.46 & 20.0 & 1.85 \\
\hline Colorado & 233 & 1.58 & 18.0 & 7.54 & 237 & 3.01 & 19.8 & 14.55 & 32 & 0.62 & 18.8 & 2.24 \\
\hline Connecticut & 348 & 0.70 & 16.7 & 2.93 & 138 & 0.61 & 9.4 & 5.21 & 18 & 0.70 & 11.1 & 4.46 \\
\hline Delaware & 17 & 0.32 & 5.9 & 1.90 & 0 & - & - & - & 0 & - & - & - \\
\hline District of Columbia & 0 & - & - & - & 0 & - & - & - & 0 & - & - & - \\
\hline Florida & 60 & 2.05 & 30.0 & 6.30 & 33 & 1.81 & 24.2 & 7.16 & 0 & - & - & - \\
\hline Georgia & 390 & 1.55 & 29.7 & 4.46 & 267 & 0.82 & 18.7 & 3.42 & 32 & 0.47 & 6.3 & 1.74 \\
\hline Hawaii & 3 & 0.07 & 0.0 & - & 1 & 0.00 & 0.0 & - & 0 & - & - & - \\
\hline Idaho & 23 & 3.99 & 43.5 & 8.98 & 23 & 1.80 & 13.0 & 11.67 & 1 & 0.00 & 0.0 & - \\
\hline Illinois & 435 & 1.81 & 28.5 & 5.49 & 341 & 4.48 & 20.8 & 20.56 & 24 & 0.86 & 16.7 & 4.63 \\
\hline Indiana & 106 & 1.15 & 41.5 & 2.25 & 294 & 0.81 & 15.6 & 3.84 & 10 & 0.03 & 0.0 & - \\
\hline Iowa & 259 & 1.09 & 20.1 & 4.14 & 246 & 0.89 & 17.5 & 3.92 & 33 & 1.27 & 51.5 & 2.17 \\
\hline Kansas & 47 & 1.34 & 25.5 & 4.27 & 29 & 0.87 & 17.2 & 3.85 & 0 & - & - & - \\
\hline Kentucky & 78 & 3.38 & 39.7 & 7.93 & 180 & 6.52 & 23.3 & 27.50 & 40 & 1.18 & 17.5 & 6.73 \\
\hline Louisiana & 59 & 1.68 & 39.0 & 3.69 & 38 & 1.12 & 26.3 & 3.58 & 7 & 0.28 & 0.0 & - \\
\hline Maine & 9 & 0.62 & 33.3 & 1.86 & 10 & 5.60 & 40.0 & 13.83 & 0 & - & - & - \\
\hline Maryland & 37 & 2.64 & 21.6 & 11.46 & 164 & 2.35 & 25.6 & 8.80 & 10 & 0.13 & 0.0 & - \\
\hline Massachusetts & 282 & 1.11 & 25.2 & 3.56 & 143 & 0.78 & 21.0 & 2.81 & 12 & 0.70 & 16.7 & 2.63 \\
\hline Michigan & 0 & - & - & - & 570 & 9.06 & 5.1 & 176.08 & 225 & 22.64 & 8.0 & 281.65 \\
\hline Minnesota & 30 & 1.94 & 63.3 & 2.74 & 0 & - & - & - & 0 & - & - & - \\
\hline Mississippi & 36 & 2.49 & 25.0 & 9.06 & 53 & 6.83 & 30.2 & 22.03 & 0 & - & - & - \\
\hline Missouri & 144 & 1.39 & 30.6 & 3.41 & 184 & 0.57 & 16.8 & 2.25 & 0 & - & - & - \\
\hline Montana & 53 & 1.52 & 20.8 & 5.47 & 47 & 3.82 & 31.9 & 11.67 & 0 & - & - & - \\
\hline
\end{tabular}


Table 3-13 (page 2 of 2). Silica: Number of OSHA inspector samples, percent exceeding the permissible exposure limit (PEL) and average severity levels (Avg. Sev.), by state, 1979-1994

\begin{tabular}{|c|c|c|c|c|c|c|c|c|c|c|c|c|}
\hline \multirow[b]{3}{*}{ State } & \multicolumn{4}{|c|}{ 1979-1984 } & \multicolumn{4}{|c|}{ 1985-1994 } & \multicolumn{4}{|c|}{ 1993-1994 } \\
\hline & \multicolumn{2}{|c|}{ Total samples } & \multicolumn{2}{|c|}{ Samples > PEL } & \multicolumn{2}{|c|}{ Total samples } & \multicolumn{2}{|c|}{ Samples > PEL } & \multicolumn{2}{|c|}{ Total samples } & \multicolumn{2}{|c|}{ Samples > PEL } \\
\hline & Number & $\begin{array}{r}\text { Avg. } \\
\text { Sev. }\end{array}$ & $\%$ & $\begin{array}{c}\text { Avg. } \\
\text { Sev. }\end{array}$ & Number & $\begin{array}{c}\text { Avg. } \\
\text { Sev. }\end{array}$ & $\%$ & $\begin{array}{c}\text { Avg. } \\
\text { Sev. }\end{array}$ & Number & $\begin{array}{c}\text { Avg. } \\
\text { Sev. }\end{array}$ & $\%$ & $\begin{array}{r}\text { Avg. } \\
\text { Sev. }\end{array}$ \\
\hline Nebraska & 29 & 1.14 & 24.1 & 3.59 & 49 & 0.44 & 14.3 & 1.98 & 4 & 0.60 & 25.0 & 1.36 \\
\hline Nevada & 10 & 3.03 & 50.0 & 5.84 & 114 & 8.51 & 62.3 & 13.49 & 3 & 0.14 & 0.0 & - \\
\hline New Hampshire & 99 & 0.87 & 21.2 & 2.85 & 114 & 0.93 & 14.9 & 5.05 & 5 & 0.43 & 0.0 & - \\
\hline New Jersey & 194 & 1.32 & 27.8 & 3.91 & 216 & 0.81 & 22.7 & 2.65 & 19 & 0.18 & 5.3 & 1.69 \\
\hline New Mexico & 8 & 1.93 & 75.0 & 2.58 & 4 & 0.00 & 0.0 & - & 0 & - & - & - \\
\hline New York & 639 & 1.61 & 28.8 & 4.79 & 233 & 1.01 & 20.6 & 4.06 & 48 & 0.66 & 16.7 & 2.68 \\
\hline North Carolina & 132 & 61.28 & 12.9 & 473.62 & 122 & 2.70 & 12.3 & 20.69 & 58 & 3.71 & 10.3 & 34.34 \\
\hline North Dakota & 38 & 1.27 & 18.4 & 5.60 & 43 & 0.61 & 18.6 & 1.91 & 0 & - & - & - \\
\hline Ohio & 958 & 1.16 & 28.7 & 3.15 & 693 & 1.93 & 21.6 & 7.96 & 18 & 10.63 & 38.9 & 26.92 \\
\hline Oklahoma & 105 & 1.75 & 17.1 & 8.80 & 70 & 1.03 & 21.4 & 3.26 & 4 & 0.71 & 25.0 & 1.73 \\
\hline Oregon & 86 & 0.61 & 11.6 & 2.53 & 46 & 3.48 & 28.3 & 11.79 & 10 & 0.04 & 0.0 & - \\
\hline Pennsylvania & 1,052 & 1.33 & 26.4 & 3.82 & 754 & 1.76 & 24.4 & 6.24 & 43 & 1.87 & 46.5 & 3.65 \\
\hline Rhode Island & 163 & 1.02 & 24.5 & 3.51 & 86 & 2.81 & 17.4 & 15.21 & 0 & - & - & - \\
\hline South Carolina & 16 & 0.33 & 12.5 & 2.36 & 66 & 1.03 & 15.2 & 6.03 & 3 & 9.20 & 33.3 & 27.59 \\
\hline South Dakota & 21 & 0.27 & 9.5 & 2.26 & 15 & 1.39 & 60.0 & 2.03 & 0 & - & - & - \\
\hline Tennessee & 21 & 0.93 & 19.0 & 2.86 & 200 & 0.77 & 20.0 & 2.89 & 47 & 0.34 & 8.5 & 1.58 \\
\hline Texas & 477 & 3.06 & 23.1 & 12.63 & 363 & 5.29 & 34.2 & 15.13 & 53 & 26.39 & 45.3 & 58.14 \\
\hline Utah & 4 & 1.13 & 25.0 & 4.52 & 43 & 0.31 & 4.7 & 3.60 & 0 & - & - & - \\
\hline Vermont & 0 & - & - & - & 0 & - & - & - & 0 & - & - & - \\
\hline Virginia & 62 & 0.87 & 14.5 & 3.72 & 191 & 1.42 & 29.3 & 4.05 & 0 & - & - & - \\
\hline Washington & 3 & 0.00 & 0.0 & - & 104 & 2.38 & 22.1 & 10.11 & 43 & 1.32 & 30.2 & 3.68 \\
\hline West Virginia & 181 & 2.66 & 35.9 & 6.79 & 77 & 2.86 & 36.4 & 7.50 & 0 & - & - & - \\
\hline Wiscon $\sin$ & 615 & 1.41 & 39.0 & 2.90 & 397 & 1.17 & 27.2 & 3.24 & 29 & 0.44 & 10.3 & 2.15 \\
\hline Wyoming & 5 & 0.87 & 20.0 & 2.57 & 0 & - & - & - & 0 & - & - & - \\
\hline TOTAL & 7,911 & 2.49 & 27.2 & 8.25 & 7,392 & 2.67 & 21.2 & 11.73 & 858 & 8.67 & 18.2 & 46.88 \\
\hline
\end{tabular}

- indicates incalculable field.

NOTE: $\quad$ See Appendix A for source description, Appendix B for methods, and Appendix D for agents.

SOURCE: Occupational Safety and Health Administration: Integrated Management Information System. 
Figure 3-13. Silica: Number of OSHA inspector samples collected by state, 1993-1994

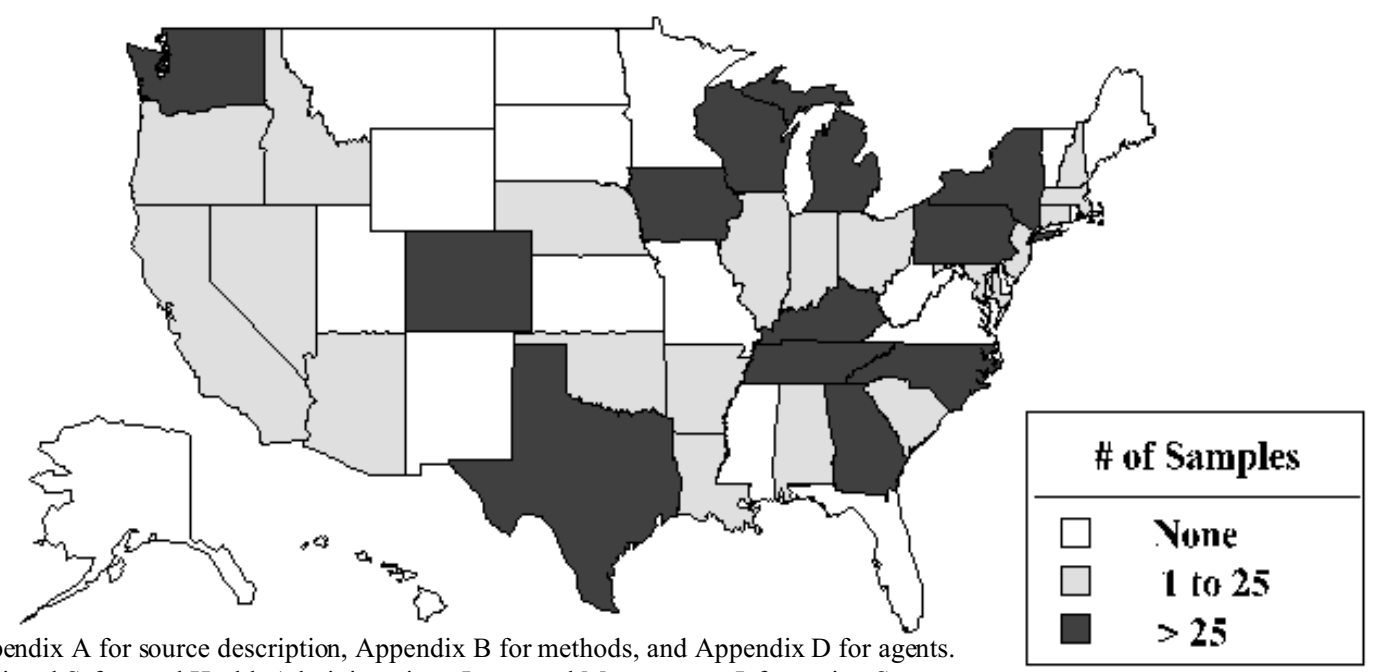

NOTE: See Appendix A for source description, Appendix B for methods, and Appendix D for agents.

SOURCE: Occupational Safety and Health Administration: Integrated Management Information System.

Figure 3-14. Silica: Percent of OSHA inspector samples that exceeded the permissible exposure limit (PEL), by state, 1993-1994

NOTE: See Appendix A for source description, Appendix B for methods, and Appendix D for agents. SOURCE: Occupational Safety and Health Administration: Integrated Management Information System.

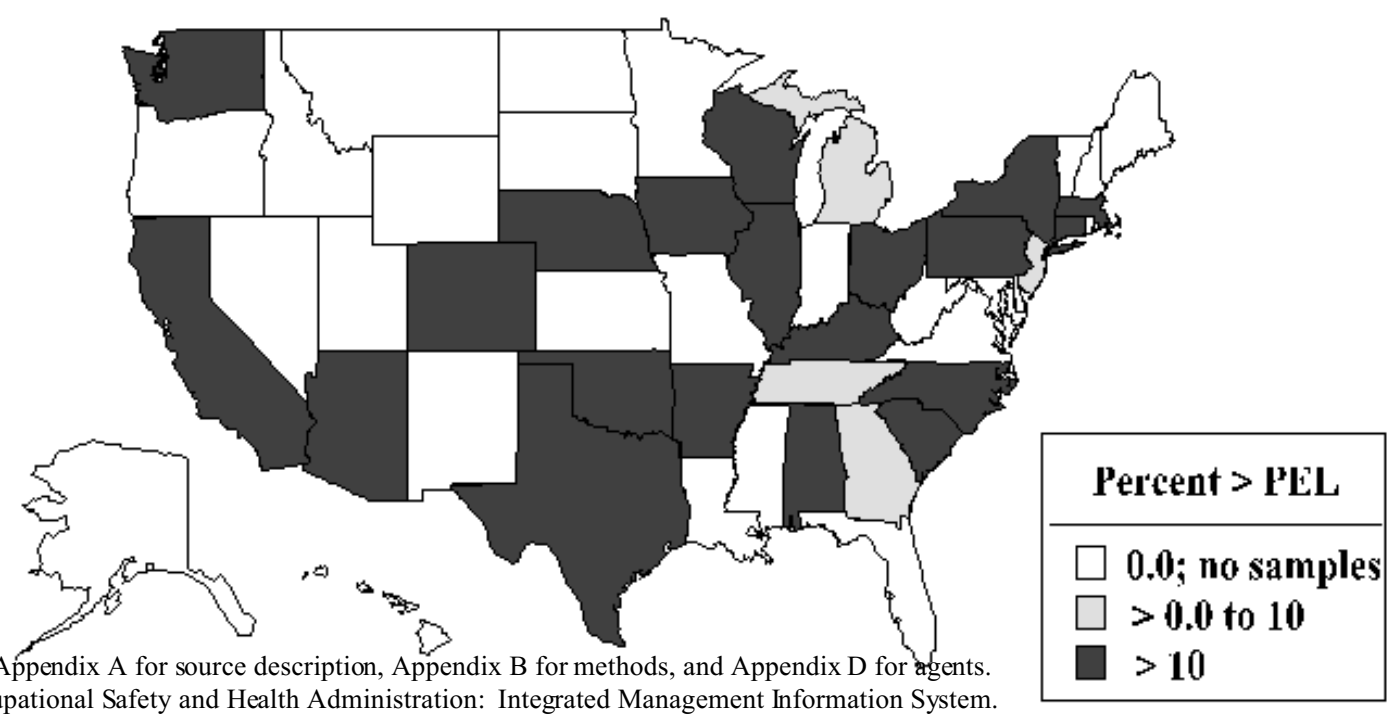


Figure 3-15. Silica: Average severity level by state for OSHA inspector samples, 1993-1994

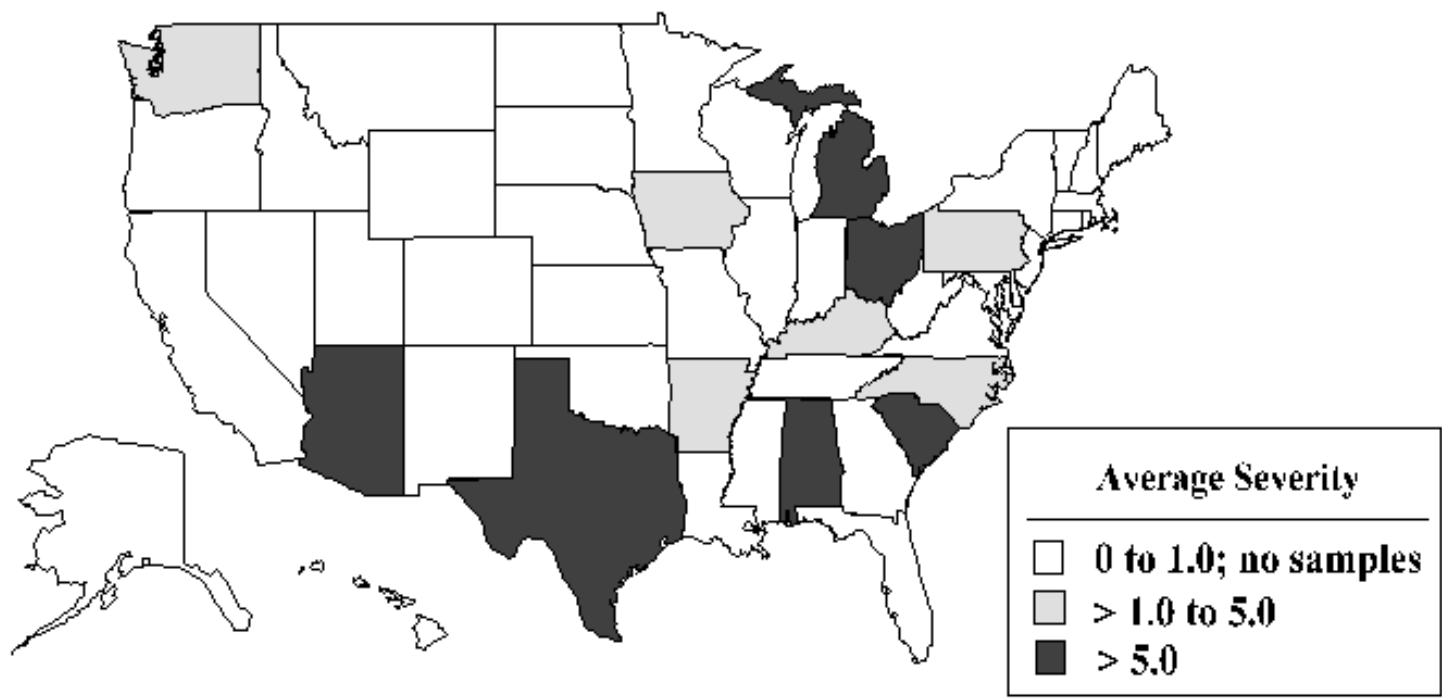

NOTE: See Appendix A for source descriptions, Appendix B for methods, and Appendix D for agents SOURCE: Occupational Safety and Health Administration: Integrated Management Information System.

Figure 3-16. Silica: Average severity level by state for OSHA inspector samples that exceeded the permissible exposure limit (PEL), 1993-1994

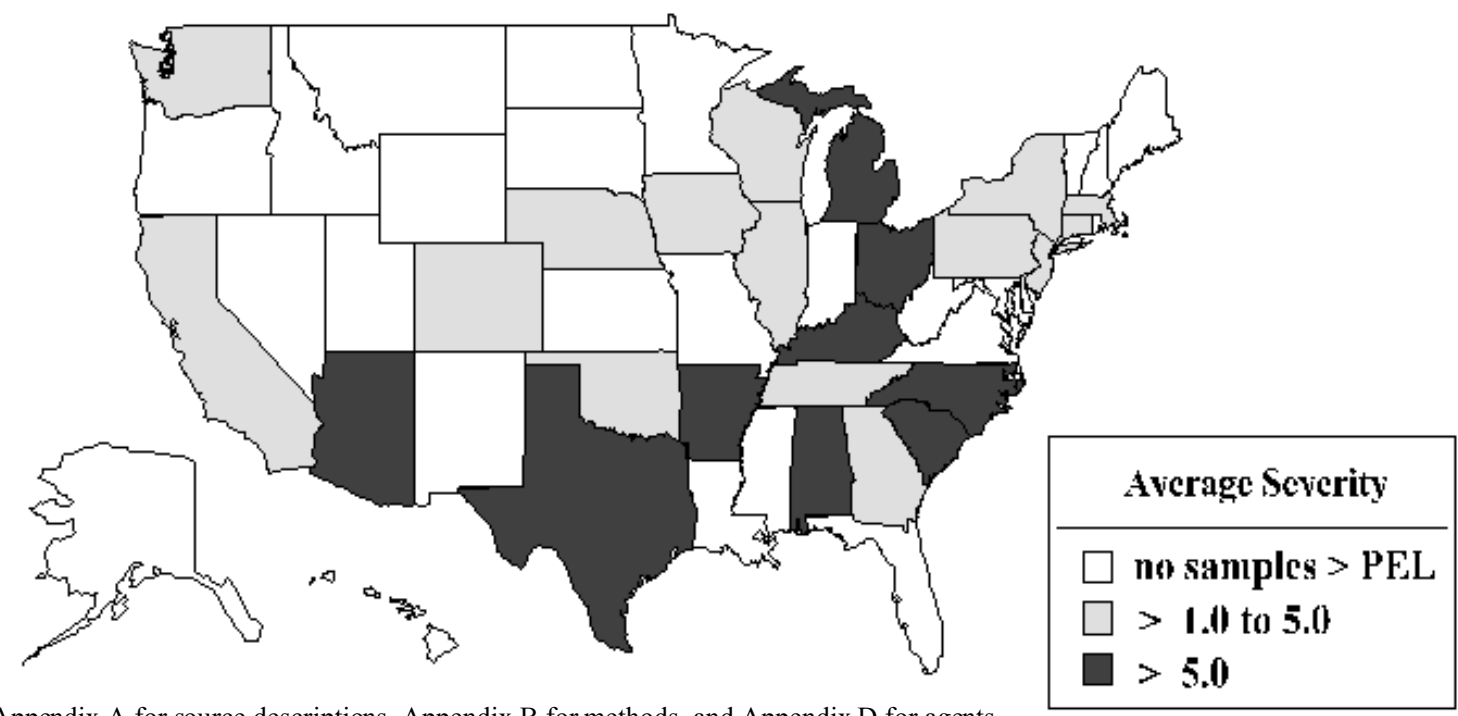

NOTE: See Appendix A for source descriptions, Appendix B for methods, and Appendix D for agents. SOURCE: Occupational Safety and Health Administration: Integrated Management Information System. 
Figure 3-17. Silica: Number of OSHA inspector samples

collected by county, 1993-1994

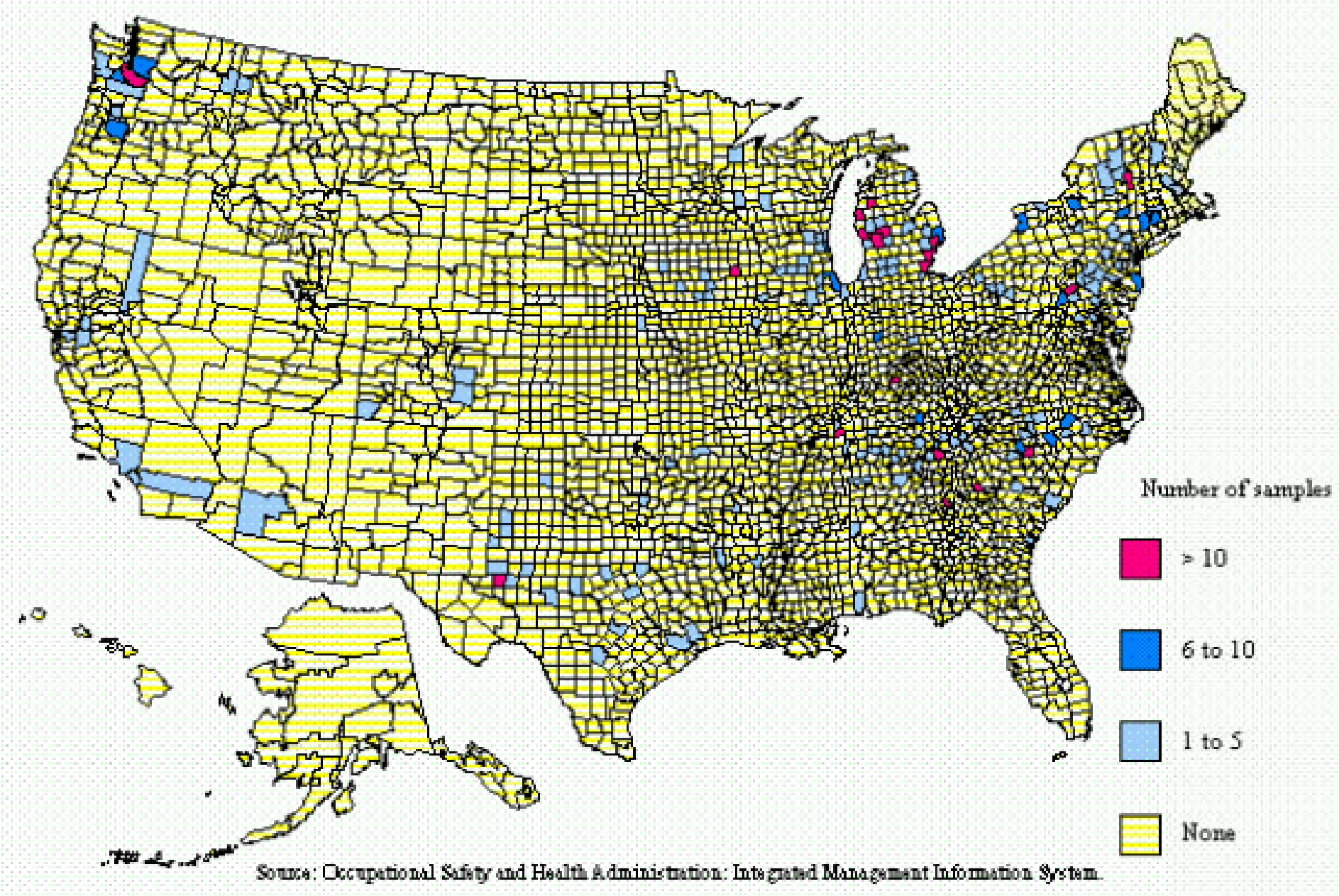


Figure 4-1. Byssinosis: Number of deaths, crude and age-adjusted mortality rates, U.S. residents age 15 and over, 1979-1992

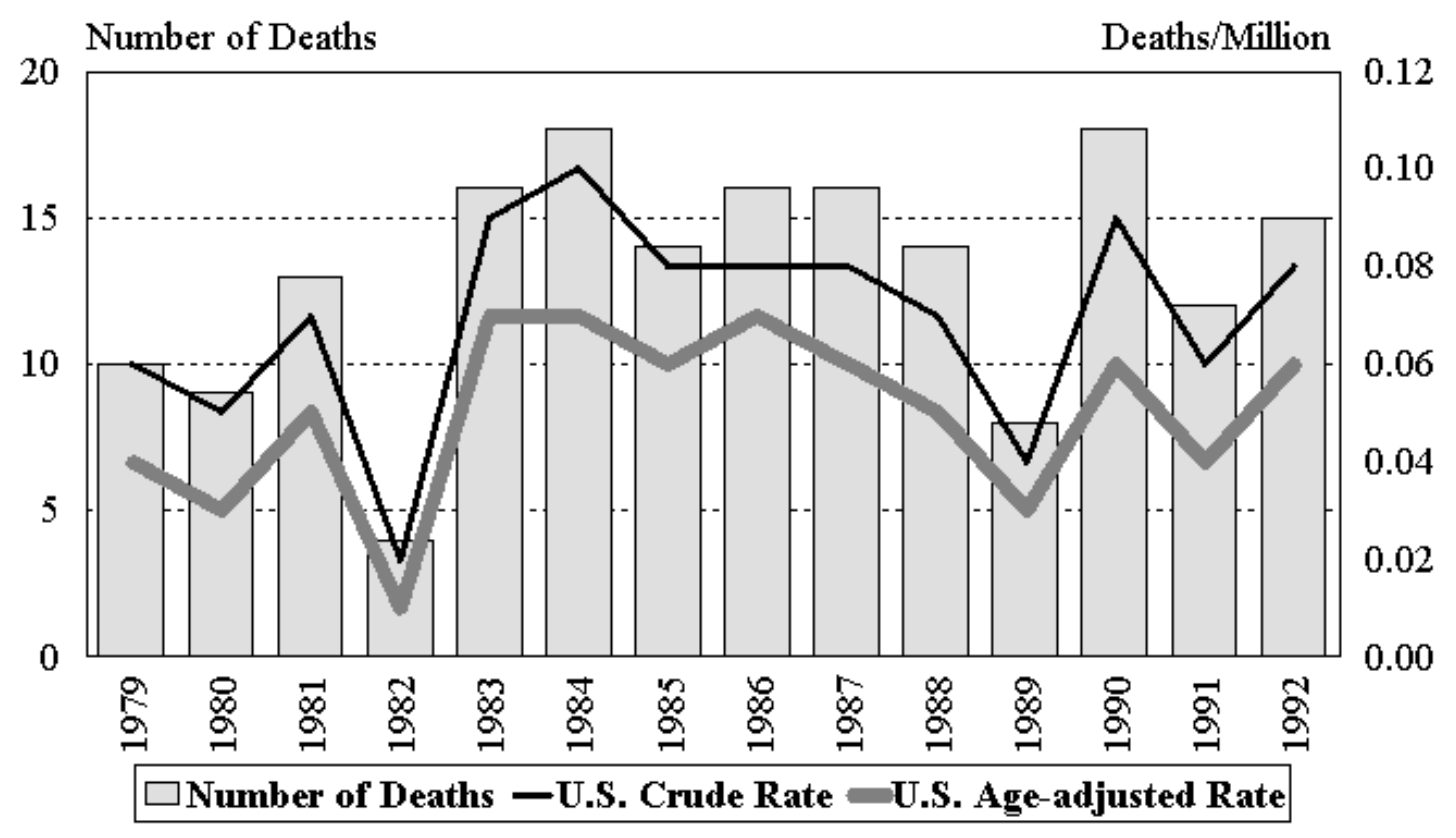

NOTE: $\quad$ See Appendix A for source description and Appendix B for methods and ICD-8 and ICD-9 codes.

SOURCE: National Center for Health Statistics multiple cause of death data. Population estimates from U.S. Bureau of the Census.

Figure 4-2. Byssinosis: Crude mortality rates by state, U.S. residents age 15 and over, 1991-1992

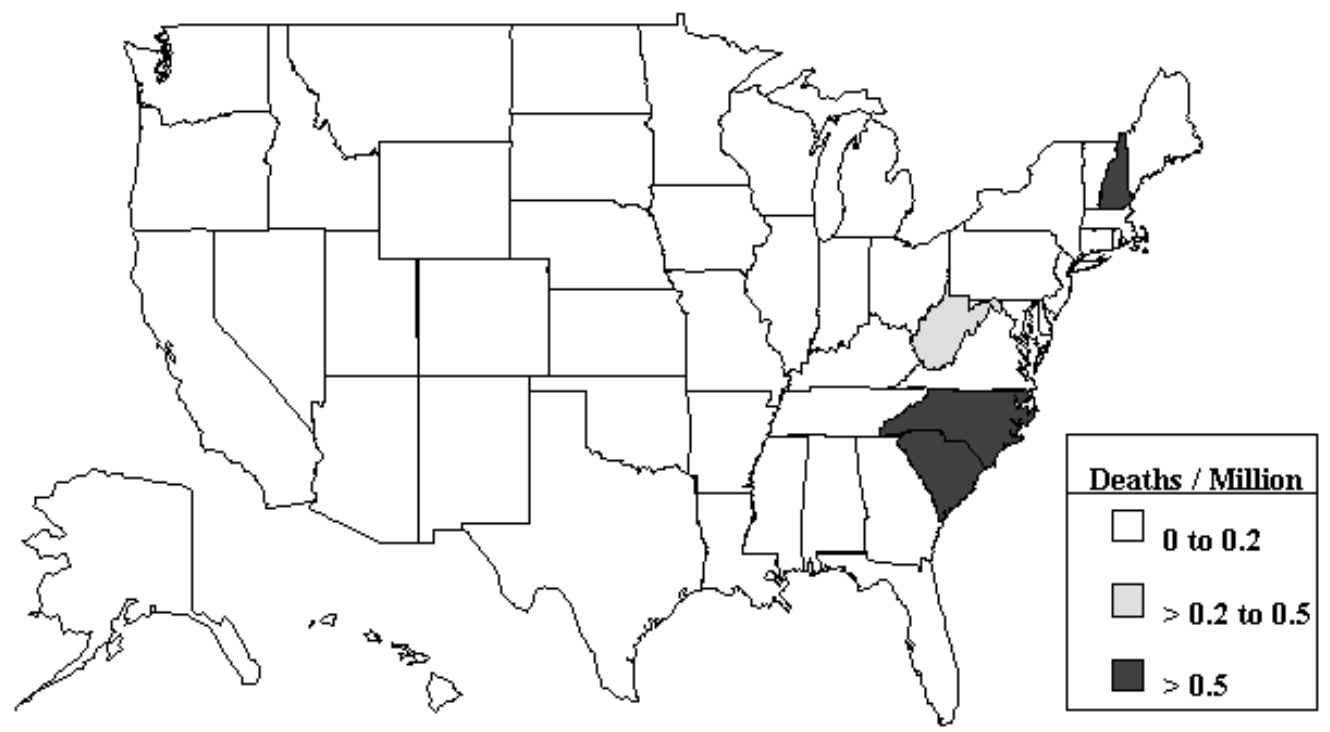

NOTE: $\quad$ See Appendix A for source description and Appendix B for methods and ICD-8 and ICD-9 codes.

SOURCE: National Center for Health Statistics multiple cause of death data. Population estimates from U.S. Bureau of the Census. 
Figure 4-3. Byssinosis: Median age at death, U.S. residents age 15 and over, 1968-1992

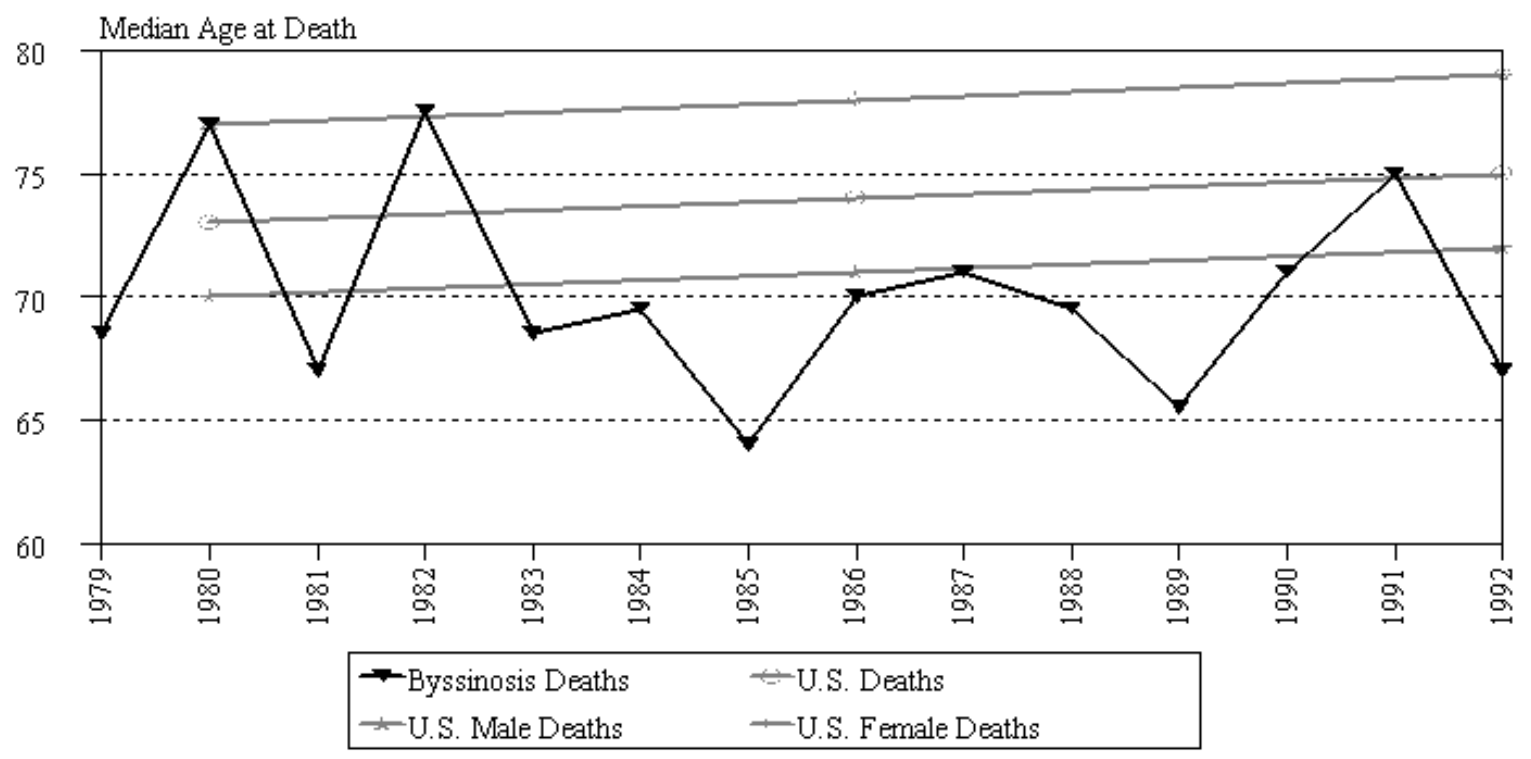

NOTE: See Appendix A for source description and Appendix B for methods and ICD-8 and ICD-9 codes.

SOURCE: National Center for Health Statistics multiple cause of death data.

Table 4-1. Byssinosis: Number of deaths by sex, race, and age, U.S. residents age 15 and over, 1991-1992

\begin{tabular}{|c|c|c|c|c|c|c|}
\hline & & & \multicolumn{2}{|c|}{1991} & \multicolumn{2}{|c|}{1992} \\
\hline & & & Number & Percent & Number & Percent \\
\hline \multicolumn{3}{|c|}{ Total deaths } & 12 & 100.0 & 15 & 100.0 \\
\hline \multirow{2}{*}{\multicolumn{2}{|c|}{ Sex }} & Male & 8 & 66.7 & 9 & 60.0 \\
\hline & & Female & 4 & 33.3 & 6 & 40.0 \\
\hline \multirow{3}{*}{\multicolumn{2}{|c|}{ Race }} & White & 11 & 91.7 & 10 & 66.7 \\
\hline & & Black & 1 & 8.3 & 5 & 33.3 \\
\hline & & Other & 0 & 0.0 & 0 & 0.0 \\
\hline \multirow{10}{*}{\multicolumn{2}{|c|}{ Age }} & $15-24$ & 0 & 0.0 & 3 & 20.0 \\
\hline & & $25-34$ & 0 & 0.0 & 1 & 6.7 \\
\hline & & $35-44$ & 0 & 0.0 & 0 & 0.0 \\
\hline & & $45-54$ & 1 & 8.3 & 0 & 0.0 \\
\hline & & $55-64$ & 1 & 8.3 & 1 & 6.7 \\
\hline & & $65-74$ & 4 & 33.3 & 8 & 53.3 \\
\hline & & $75-84$ & 4 & 33.3 & 2 & 13.3 \\
\hline & & 85 and over & 2 & 16.7 & 0 & 0.0 \\
\hline & & Mean age & 73.7 & & 57.9 & \\
\hline & & Range for age & $53-87$ & & $18-84$ & \\
\hline
\end{tabular}


Table 4-2. Byssinosis: Number of deaths by state, U.S. residents age 15 and over, 1979-1992

\begin{tabular}{|c|c|c|c|c|}
\hline State & 1979-1990 & 1991 & 1992 & TOTAL \\
\hline Alabama & 5 & 1 & - & 6 \\
\hline Alaska & - & - & - & - \\
\hline Arizona & 3 & - & - & 3 \\
\hline Arkansas & - & - & - & - \\
\hline California & 6 & - & 1 & 7 \\
\hline Colorado & - & - & - & - \\
\hline Connecticut & 1 & - & - & 1 \\
\hline Delaware & - & - & - & - \\
\hline District of Columbia & - & - & - & - \\
\hline Florida & 1 & - & 1 & 2 \\
\hline Georgia & 18 & 1 & - & 19 \\
\hline Hawaii & - & - & - & - \\
\hline Idaho & - & - & - & - \\
\hline Illinois & 3 & - & 1 & 4 \\
\hline Indiana & - & 1 & - & 1 \\
\hline Iowa & - & - & - & - \\
\hline Kansas & 1 & - & - & 1 \\
\hline Kentucky & - & - & - & - \\
\hline Louisiana & 1 & - & 1 & 2 \\
\hline Maine & - & - & - & - \\
\hline Maryland & 2 & - & - & 2 \\
\hline Massachusetts & 6 & - & 1 & 7 \\
\hline Michigan & 1 & - & - & 1 \\
\hline Minnesota & 1 & - & - & 1 \\
\hline Mississippi & - & - & - & - \\
\hline Missouri & - & - & - & - \\
\hline Montana & - & - & - & - \\
\hline Nebraska & - & - & - & - \\
\hline Nevada & - & - & - & - \\
\hline New Hampshire & 1 & 1 & - & 2 \\
\hline New Jersey & 2 & - & - & 2 \\
\hline New Mexico & - & - & - & - \\
\hline New York & 1 & - & - & 1 \\
\hline North Carolina & 66 & 2 & 8 & 76 \\
\hline North Dakota & - & - & - & - \\
\hline Ohio & 4 & - & - & 4 \\
\hline Oklahoma & - & 1 & - & 1 \\
\hline Oregon & - & - & - & - \\
\hline Pennsylvania & 8 & 1 & - & 9 \\
\hline Rhode Island & 2 & - & - & 2 \\
\hline South Carolina & 10 & 3 & 2 & 15 \\
\hline South Dakota & - & - & - & - \\
\hline Tennessee & 1 & - & - & 1 \\
\hline Texas & 3 & - & - & 3 \\
\hline Utah & - & - & - & - \\
\hline Vermont & 2 & - & - & 2 \\
\hline Virginia & 4 & - & - & 4 \\
\hline Washington & 1 & - & - & 1 \\
\hline West Virginia & 1 & 1 & - & 2 \\
\hline Wisconsin & 1 & - & - & 1 \\
\hline Wyoming & - & - & - & - \\
\hline TOTAL & 156 & 12 & 15 & 183 \\
\hline
\end{tabular}

- indicates no deaths listed.

NOTE: See Appendix A for source description and Appendix B for methods and ICD-8 and ICD-9 codes.

SOURCE: National Center for Health Statistics multiple cause of death data. 
Table 4-3. Byssinosis: Mortality rates (per 1,000,000 population) by race and sex, U.S. residents age 15 and over, 1991-1992

\begin{tabular}{|c|c|c|c|c|c|}
\hline \multirow[b]{2}{*}{ Year } & \multirow[b]{2}{*}{ Overall rate } & \multicolumn{2}{|c|}{ White } & \multicolumn{2}{|c|}{ Black } \\
\hline & & Males & Females & Males & Females \\
\hline & \multicolumn{5}{|c|}{ Crude mortality rate } \\
\hline 1991 & 0.06 & 0.09 & 0.05 & 0.10 & - \\
\hline \multirow[t]{2}{*}{1992} & 0.08 & 0.06 & 0.06 & 0.38 & 0.08 \\
\hline & \multicolumn{5}{|c|}{ Age-adjusted mortality rate } \\
\hline 1991 & 0.04 & 0.05 & 0.04 & 0.08 & - \\
\hline 1992 & 0.06 & 0.06 & 0.04 & 0.36 & 0.04 \\
\hline
\end{tabular}

- indicates no deaths listed.

NOTE: $\quad$ See Appendix A for source description and Appendix B for methods and ICD-8 and ICD-9 codes. Data for 1979-1990 can be found in the Work-Related Lung Disease Surveillance Report, 1994, see Appendix E. See Appendix E for revised rates for 1979-1990.

SOURCE: National Center for Health Statistics multiple cause of death data. Population estimates from U.S. Bureau of the Census.

Table 4-4. Byssinosis: Years of potential life lost by race and sex, U.S. residents age 15 and over, 1991-1992

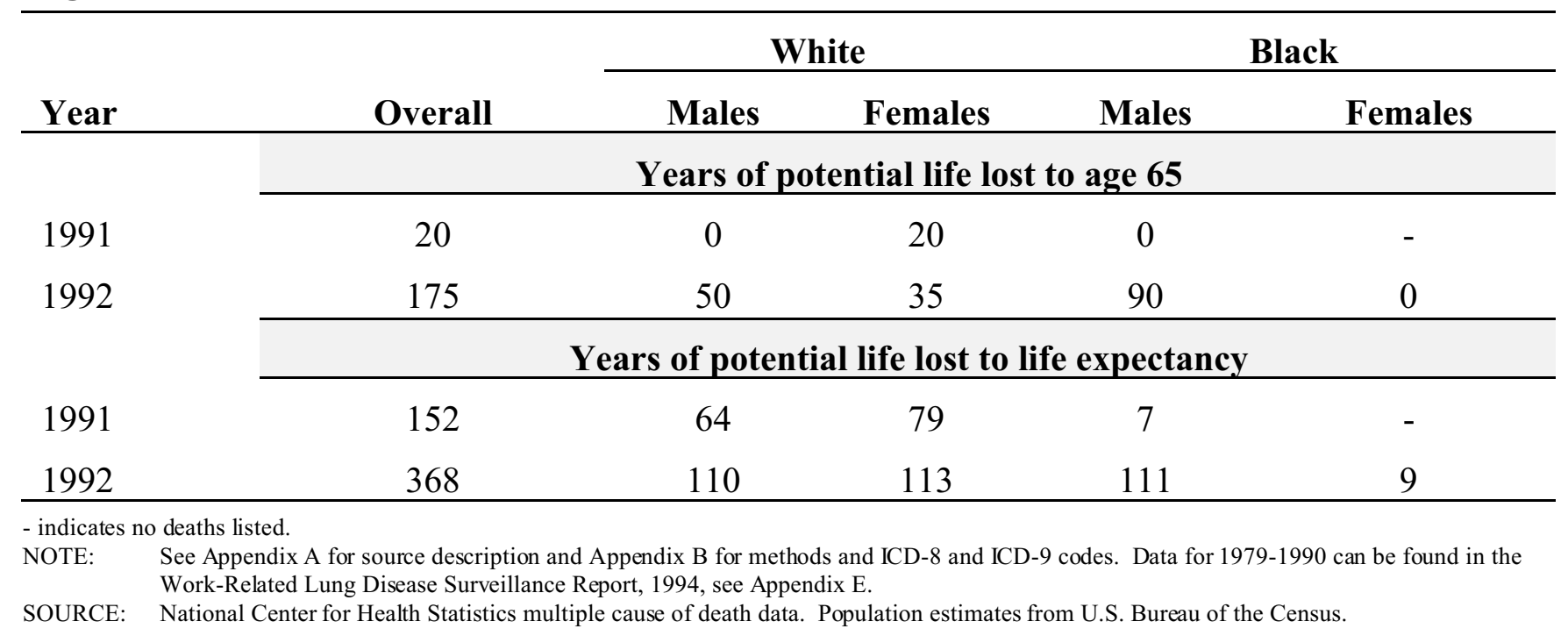


Table 4-5. Byssinosis: Total number of deaths, crude and age-adjusted mortality rates (per 1,000,000 population), and total years of potential life lost (YPLL) to life expectancy, by state, U.S. residents age 15 and over, 1988-1992

\begin{tabular}{|c|c|c|c|c|c|c|c|c|c|c|}
\hline \multirow[b]{2}{*}{ State } & \multirow{2}{*}{$\begin{array}{r}\text { Total } \\
\text { deaths }\end{array}$} & \multirow[b]{2}{*}{ Rank } & \multicolumn{2}{|c|}{ Crude mortality } & \multicolumn{2}{|c|}{ Age-adjusted mortality } & \multicolumn{4}{|c|}{ YPLL to life expectancy } \\
\hline & & & Rate & Rank & Rate & Rank & Years & Rank & Years/death & Rank \\
\hline Alabama & 2 & 5 & 0.13 & 5 & 0.05 & 10 & 16.00 & 16 & 8.0 & 17 \\
\hline Alaska & - & - & - & - & - & - & - & - & - & - \\
\hline Arizona & 1 & 12 & 0.07 & 11 & 0.07 & 5 & 21.00 & 12 & 21.0 & 8 \\
\hline Arkansas & - & - & - & - & - & - & - & - & - & - \\
\hline California & 4 & 3 & 0.03 & 20 & 0.03 & 17 & 105.00 & 3 & 26.3 & 5 \\
\hline Colorado & - & - & - & - & - & - & - & - & - & - \\
\hline Connecticut & - & - & - & - & - & - & - & - & - & - \\
\hline Delaware & - & - & - & - & - & - & - & - & - & - \\
\hline District of Columbia & - & - & - & - & - & - & - & - & - & - \\
\hline Florida & 2 & 5 & 0.04 & 17 & 0.04 & 15 & 71.00 & 5 & 35.5 & 3 \\
\hline Georgia & 2 & 5 & 0.08 & 7 & 0.06 & 6 & 46.00 & 7 & 23.0 & 6 \\
\hline Hawaii & - & - & - & - & - & - & - & - & - & - \\
\hline Idaho & - & - & - & - & - & - & - & - & - & - \\
\hline Illinois & 2 & 5 & 0.04 & 17 & 0.05 & 10 & 104.00 & 4 & 52.0 & 1 \\
\hline Indiana & 1 & 12 & 0.05 & 15 & 0.03 & 17 & 14.00 & 17 & 14.0 & 13 \\
\hline Iowa & - & - & - & - & - & - & - & - & - & - \\
\hline Kansas & 1 & 12 & 0.10 & 6 & 0.04 & 15 & 8.00 & 19 & 8.0 & 17 \\
\hline Kentucky & - & - & - & - & - & - & - & - & - & - \\
\hline Louisiana & 1 & 12 & 0.06 & 13 & 0.06 & 6 & 47.00 & 6 & 47.0 & 2 \\
\hline Maine & - & - & - & - & - & - & - & - & - & - \\
\hline Maryland & - & - & - & - & - & - & - & - & - & - \\
\hline Massachusetts & 2 & 5 & 0.08 & 7 & 0.06 & 6 & 28.00 & 10 & 14.0 & 13 \\
\hline Michigan & 1 & 12 & 0.03 & 20 & 0.01 & 20 & 6.00 & 22 & 6.0 & 22 \\
\hline Minnesota & 1 & 12 & 0.06 & 13 & 0.06 & 6 & 21.00 & 12 & 21.0 & 8 \\
\hline Mississippi & - & - & - & - & - & - & - & - & - & - \\
\hline Missouri & - & - & - & - & - & - & - & - & - & - \\
\hline Montana & - & - & - & - & - & - & - & - & - & - \\
\hline Nebraska & - & - & - & - & - & - & - & - & - & - \\
\hline Nevada & - & - & - & - & - & - & - & - & - & - \\
\hline New Hampshire & 1 & 12 & 0.23 & 3 & 0.28 & 3 & 29.00 & 9 & 29.0 & 4 \\
\hline New Jersey & - & - & - & - & - & - & - & - & - & - \\
\hline New Mexico & - & - & - & - & - & - & - & - & - & - \\
\hline New York & - & - & - & - & - & - & - & - & - & - \\
\hline North Carolina & 29 & 1 & 1.09 & 1 & 0.73 & 1 & 382.00 & 1 & 13.2 & 15 \\
\hline North Dakota & - & - & - & - & - & - & - & - & - & - \\
\hline Ohio & 3 & 4 & 0.07 & 11 & 0.05 & 10 & 43.00 & 8 & 14.3 & 12 \\
\hline Oklahoma & 1 & 12 & 0.08 & 7 & 0.03 & 17 & 8.00 & 19 & 8.0 & 17 \\
\hline Oregon & - & - & - & - & - & - & - & - & - & - \\
\hline Pennsylvania & 2 & 5 & 0.04 & 17 & 0.01 & 20 & 14.00 & 17 & 7.0 & 21 \\
\hline Rhode Island & - & - & - & - & - & - & - & - & - & - \\
\hline South Carolina & 6 & 2 & 0.44 & 2 & 0.36 & 2 & 128.00 & 2 & 21.3 & 7 \\
\hline South Dakota & - & - & - & - & - & - & - & - & - & - \\
\hline Tennessee & - & - & - & - & - & - & - & - & - & - \\
\hline Texas & 1 & 12 & 0.02 & 22 & 0.01 & 20 & 8.00 & 19 & 8.0 & 17 \\
\hline Utah & - & - & - & - & - & - & - & - & - & - \\
\hline Vermont & - & - & - & - & - & - & - & - & - & - \\
\hline Virginia & 2 & 5 & 0.08 & 7 & 0.05 & 10 & 22.00 & 11 & 11.0 & 16 \\
\hline Washington & - & - & - & - & - & - & - & - & - & - \\
\hline West Virginia & 1 & 12 & 0.14 & 4 & 0.12 & 4 & 21.00 & 12 & 21.0 & 8 \\
\hline Wisconsin & 1 & 12 & 0.05 & 15 & 0.05 & 10 & 21.00 & 12 & 21.0 & 8 \\
\hline Wyoming & - & - & - & - & - & - & - & - & - & - \\
\hline
\end{tabular}

indicates no deaths listed.

NOTE: Ranks are based on unrounded values. See Appendix A for source description and Appendix B for methods and ICD-8 and ICD-9 codes.

SOURCE: National Center for Health Statistics multiple cause of death data. Population es timates from U.S. Bureau of the Census. 
Table 4-6. Byssinosis: Most frequently recorded occupations on death certificate, selected states, U.S. residents age 15 and over, 1991-1992

\begin{tabular}{llrr}
\hline COC & Occupation & Number & Percent \\
\hline 749 & Miscellaneous textile machine operators & 3 & 15.0 \\
914 & Homemaker & 3 & 15.0 \\
637 & Machinists & 2 & 10.0 \\
739 & Knitting, looping, taping, and weaving machine operators & 2 & 10.0 \\
889 & Laborers, except construction & 2 & 10.0 \\
203 & Clinical laboratory technologists and technicians & 1 & 5.0 \\
423 & Sheriffs, bailiffs, and other law enforcement officers & 1 & 5.0 \\
479 & Farm workers & 1 & 5.0 \\
518 & Industrial machinery repairers & 1 & 5.0 \\
616 & Mining machine operators & 1 & 5.0 \\
& All other occupations & 3 & 15.0 \\
& Occupation not reported & 0 & 0.0 \\
& TOTAL & 20 & 100.0 \\
\hline
\end{tabular}

COC - 1980 Census Occupation Code

NOTE: $\quad$ See Appendix A for source description, Appendix B for methods and ICD -8 and ICD -9 codes, and Appendix C for list of 25 states reporting usual occupation and years reporting. Data for 1979-1990 can be found in the Work-Related Lung Disease Surveillance Report, 1994, see Appendix E.

SOURCE: National Center for Health Statistics multiple cause of death data.

Table 4-7. Byssinosis: Most frequently recorded industries on death certificate, U.S. residents age 15 and over, selected states, 1991-1992

\begin{tabular}{llrr}
\hline CIC & Industry & Number & Percent \\
\hline 142 & Yarn, thread, and fabric mills & 10 & 50.0 \\
961 & Homemaker, student, unemployed, volunteer & 3 & 15.0 \\
021 & Horticultural services & 1 & 5.0 \\
041 & Coal mining & 1 & 5.0 \\
331 & Machinery, except electrical, n.e.c. & 1 & 5.0 \\
392 & Not specified manufacturing industries & 1 & 5.0 \\
790 & Dressmaking shops & 1 & 5.0 \\
831 & Hospitals & 1 & 5.0 \\
910 & Justice, public order, and safety & 1 & 5.0 \\
& All other industries & 0 & 0.0 \\
& Industry not reported & 0 & 0.0 \\
& TOTAL & 20 & 100.0 \\
\hline
\end{tabular}

CIC - 1980 Census Industry Code

n.e.c. - not elsewhere classified

NOTE: $\quad$ See Appendix A for source description, Appendix B for methods and ICD-8 and ICD-9 codes, and Appendix C for list of 25 states reporting usual industry and years reporting. Data for 1979-1990 can be found in the Work-Related Lung Disease Surveillance Report, 1994, see Appendix E.

SOURCE: National Center for Health Statistics multiple cause of death data. 
Table 4-8. Byssinosis: Proportionate mortality ratio (PMR) by usual occupation, selected states and years, U.S. residents age 15 and over, 1985-1992

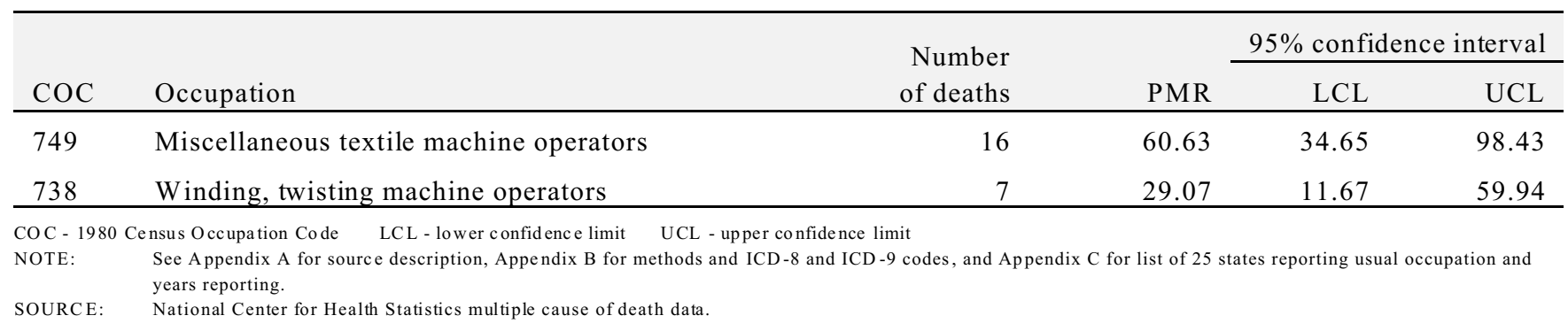


Table 4-9. Byssinosis: Proportionate mortality ratio (PMR) by usual industry, selected states and years, U.S. residents age 15 and over, 1985-1992

\begin{tabular}{|c|c|c|c|c|c|}
\hline \multirow[b]{2}{*}{$\mathrm{CIC}$} & \multirow[b]{2}{*}{ Industry } & \multirow{2}{*}{$\begin{array}{l}\text { Number } \\
\text { of deaths }\end{array}$} & \multirow[b]{2}{*}{ PMR } & \multicolumn{2}{|c|}{$95 \%$ confidence interval } \\
\hline & & & & $\mathrm{LCL}$ & $\mathrm{UCL}$ \\
\hline 142 & Yarn, thread, and fabric mills & 43 & 32.67 & 23.34 & 44.51 \\
\hline
\end{tabular}


Figure 4-4. Byssinosis: Total number of deaths by county,

U.S. residents age 15 and over, 1983-1992

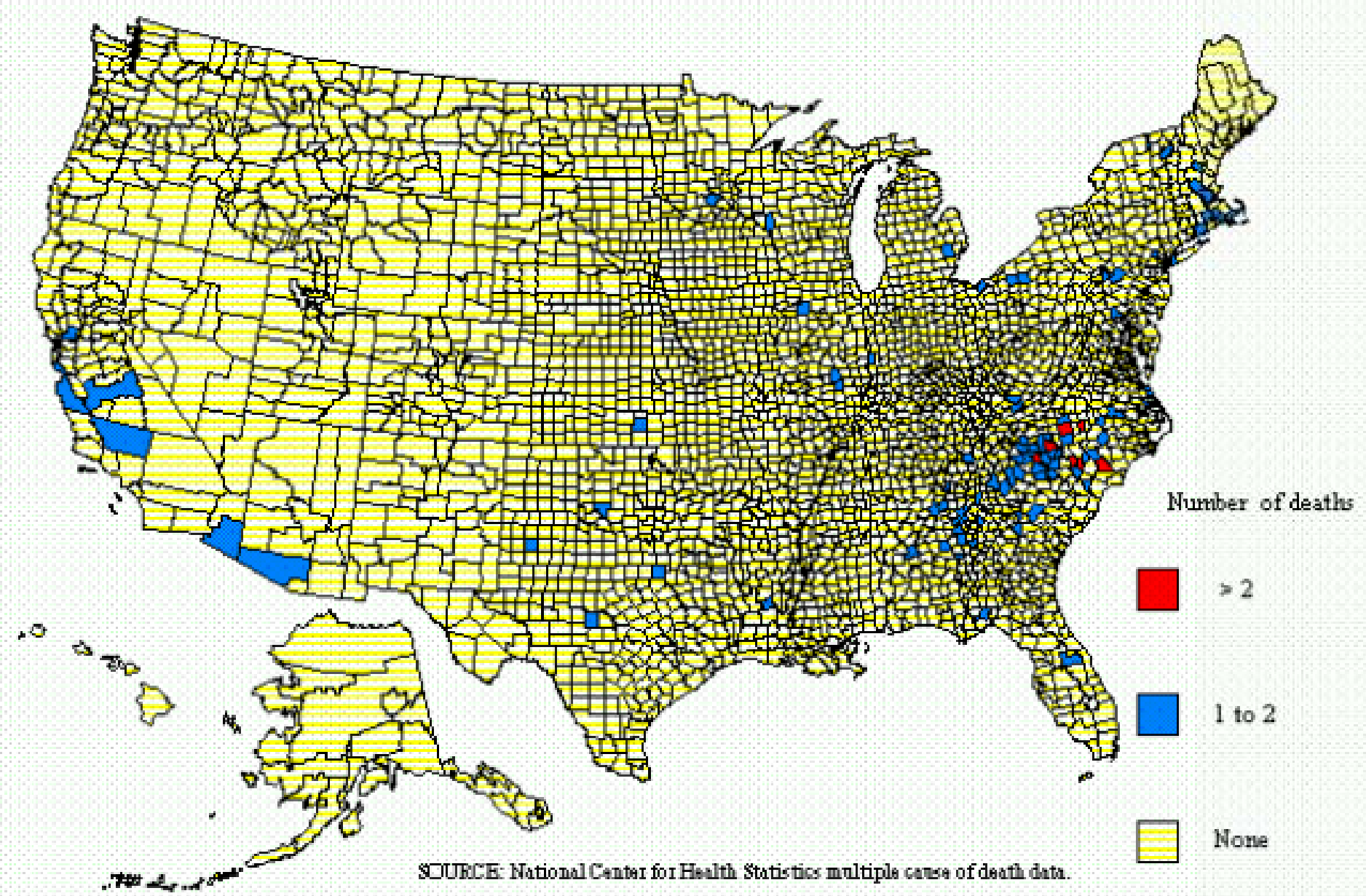


Figure 4-5. Byssinosis: Age-adjusted mortality rates by county,

U.S. residents age 15 and over, 1983-1992

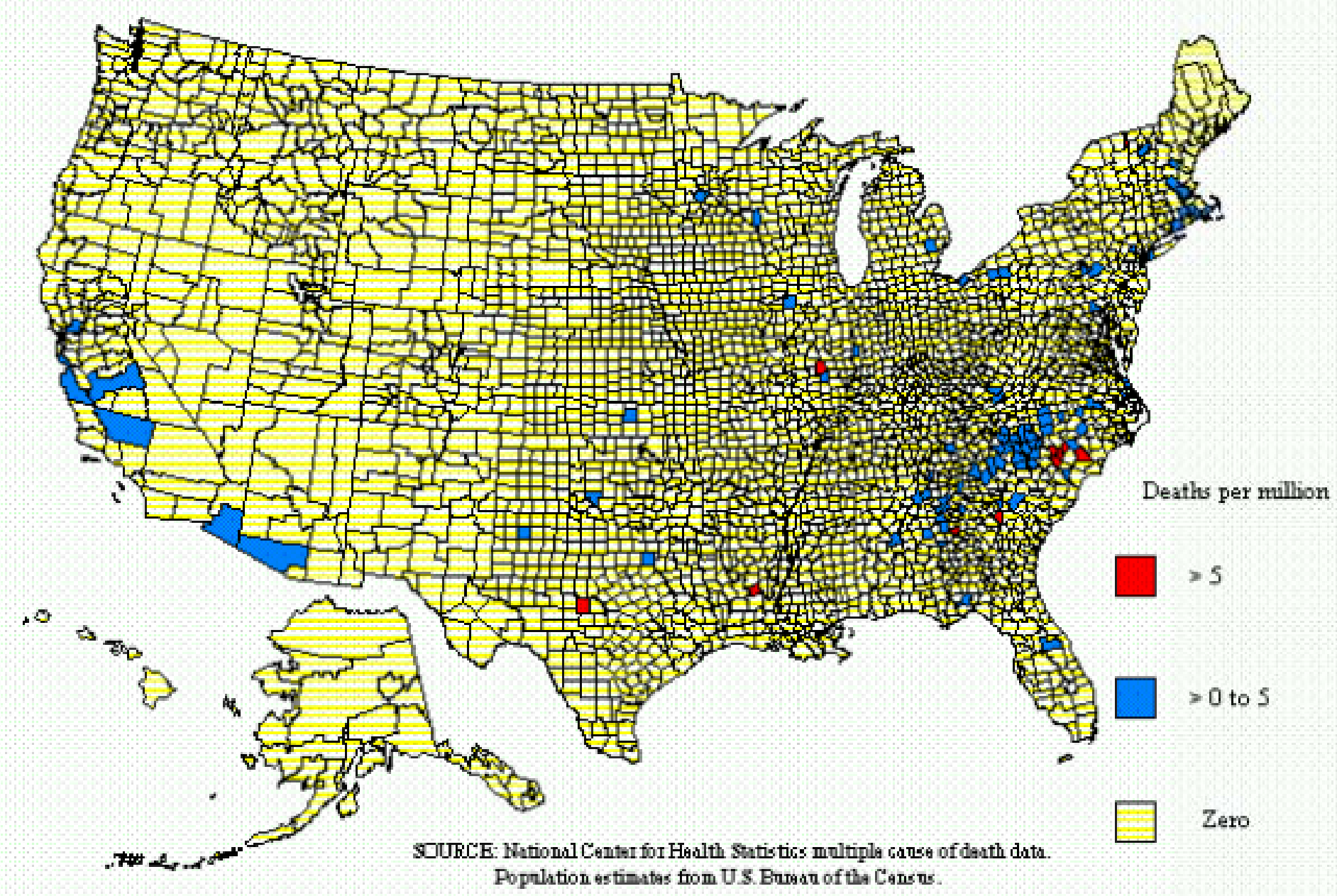


Figure 4-6. Cotton dust: Number of OSHA inspector samples and percent exceeding the permissible exposure limit (PEL), 1979-1994

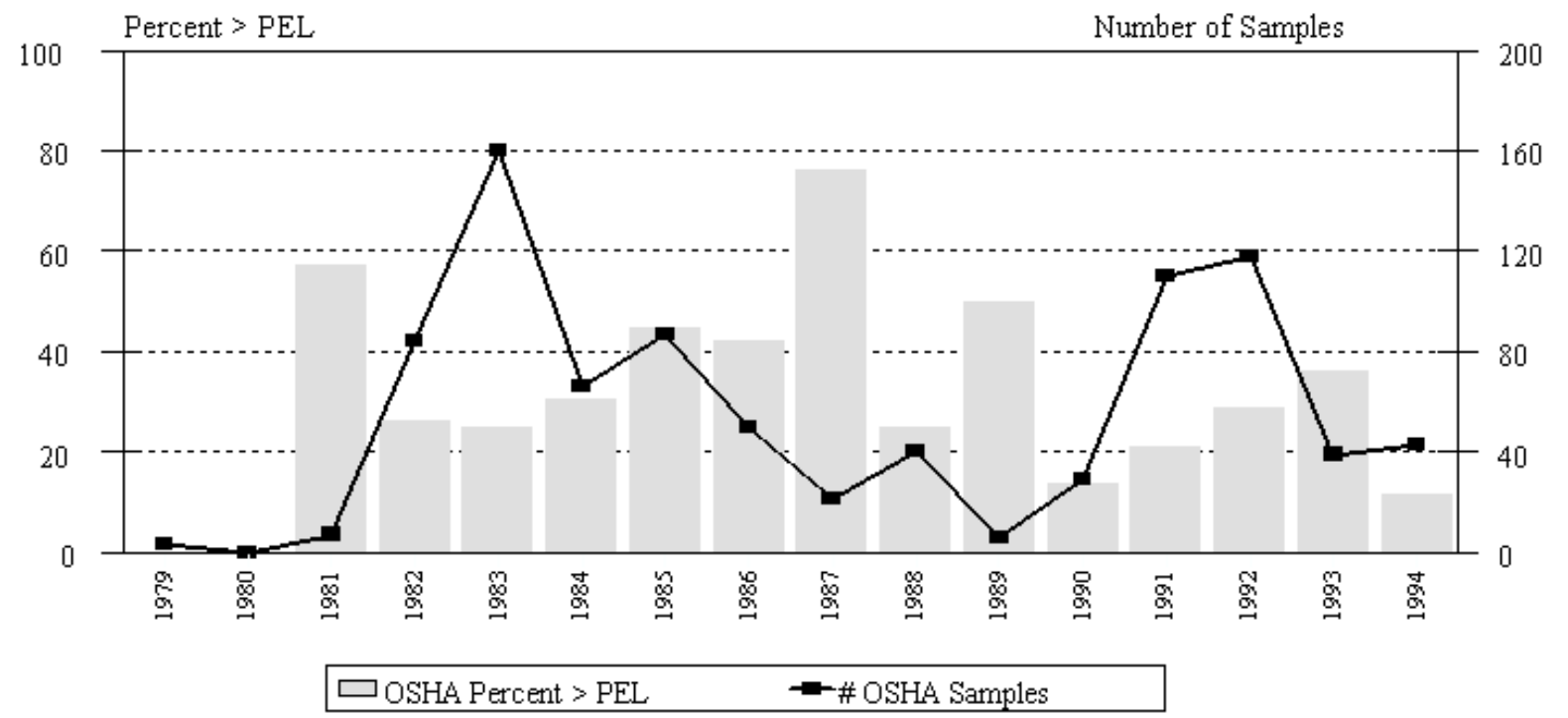

NOTE: See Appendix A for source description, Appendix B for methods, and Appendix D for agents.

SOURCE: Occupational Safety and Health Administration: Integrated Management Information System.

Figure 4-7. Cotton dust: Number of OSHA inspector samples and average severity levels, 1979-1994

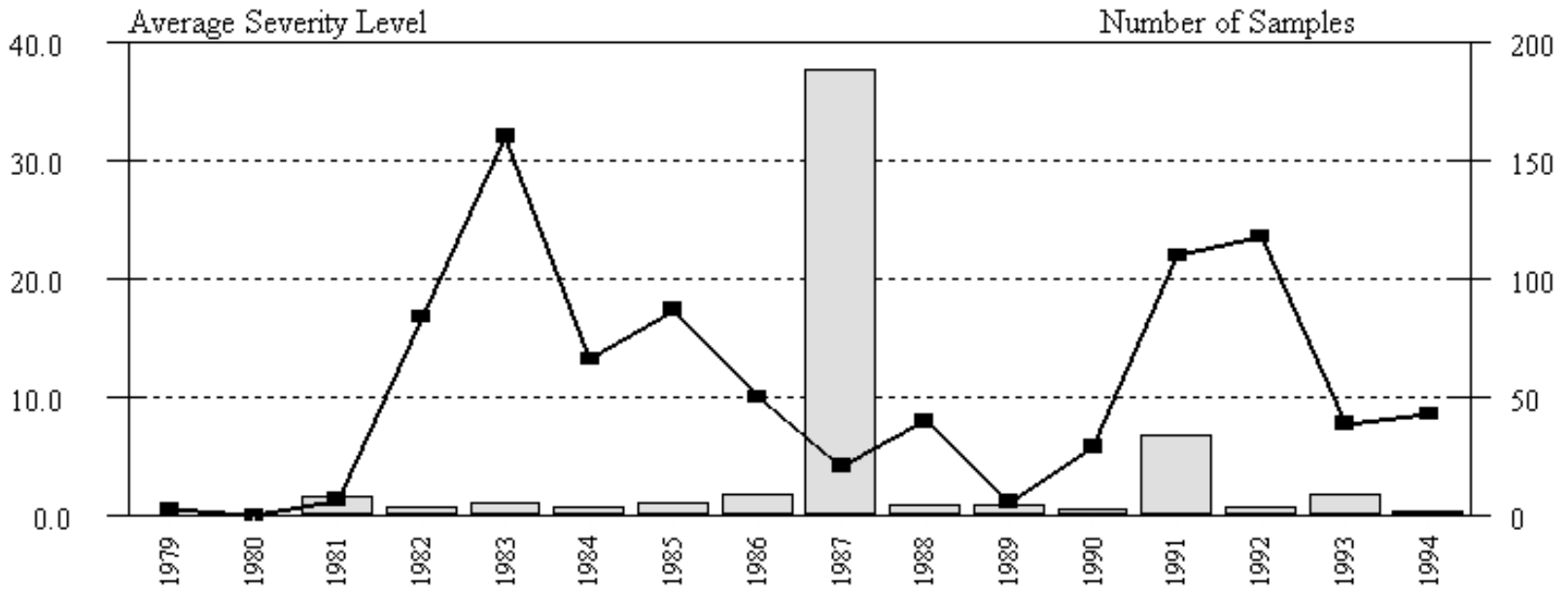

\section{$\square$ OSHA Severity Levels $\quad \rightarrow$ \# OSHA Samples}

NOTE: See Appendix A for source description, Appendix B for methods, and Appendix D for agents.

SOURCE: Occupational Safety and Health Administration: Integrated Management Information System. 
Table 4-10. Cotton dust: Number of OSHA inspector samples, percent exceeding the permissible exposure limit (PEL) and average severity level, by industry, 1993-1994

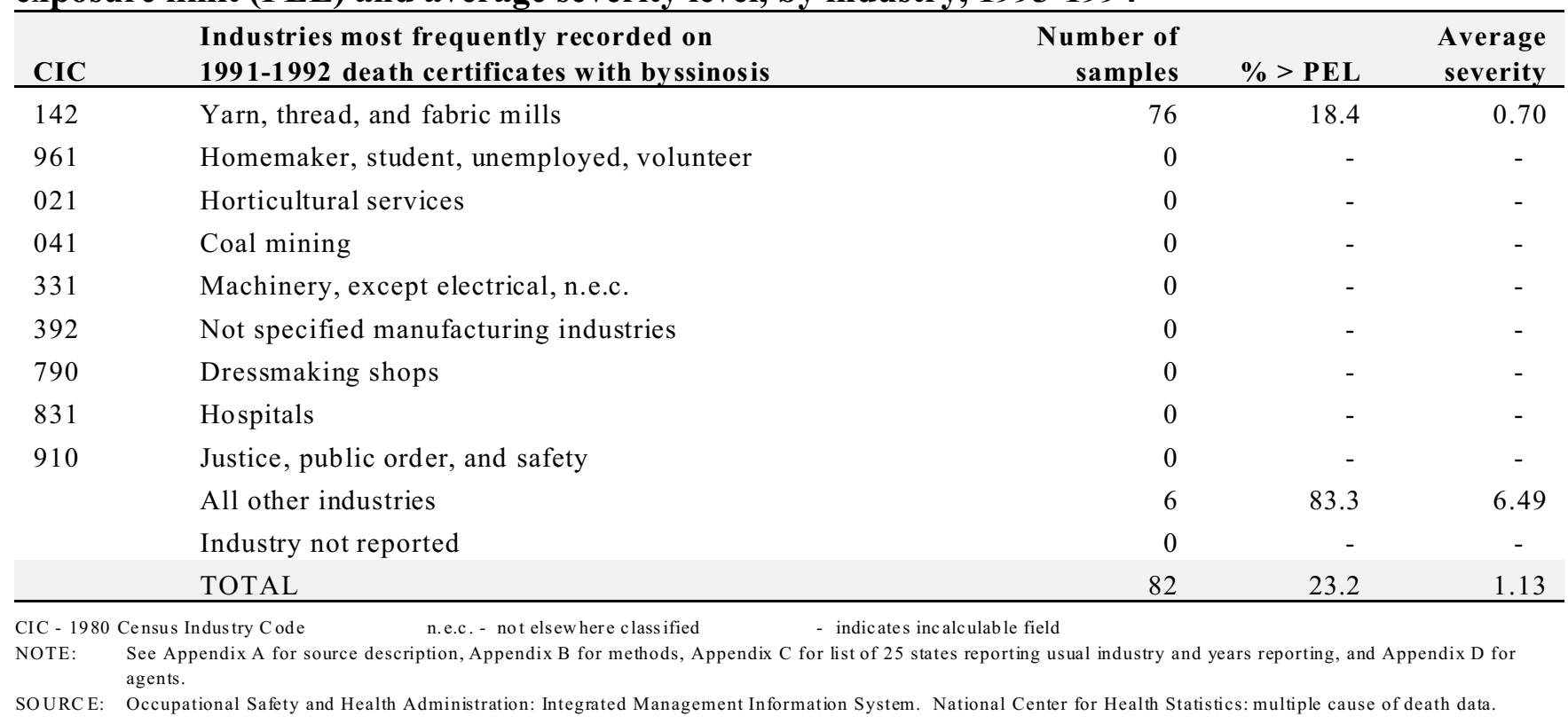

Table 4-11. Cotton dust: Number of OSHA inspector samples, percent exceeding the permissible exposure level (PEL) and average severity level, by industry, 1993-1994

\begin{tabular}{|c|c|c|c|c|}
\hline CIC & Industries most frequently sampled in 1993-1994 & $\begin{array}{r}\text { Number of } \\
\text { samples }\end{array}$ & $\%>$ PEL & $\begin{array}{r}\text { Average } \\
\text { severity } \\
\end{array}$ \\
\hline 142 & Yarn, thread, and fabric mills & 76 & 18.4 & 0.70 \\
\hline 150 & Miscellaneous textile mill products & 5 & 100.0 & 7.79 \\
\hline \multirow[t]{4}{*}{152} & Miscellaneous fabricated textile products & 1 & 0.0 & 0.00 \\
\hline & All other industries & 0 & - & - \\
\hline & Industry not reported & 0 & - & - \\
\hline & TOTAL & 82 & 23.2 & 1.13 \\
\hline
\end{tabular}

CIC - 1980 Census Industry Code

- indicates incalculable field

NOTE: See Appendix A for source description, Appendix B for methods, and Appendix D for agents.

SOURCE: Occupational Safety and Health Administration: Integrated Management Information System. 
Table 4-12 (page 1 of 2). Cotton dust: Number of OSHA inspector samples, percent exceeding the permissible exposure limit (PEL) and average severity levels (Avg. Sev.), by state, 1979-1994

\begin{tabular}{|c|c|c|c|c|c|c|c|c|c|c|c|c|}
\hline \multirow[b]{3}{*}{ State } & \multicolumn{4}{|c|}{ 1979-1984 } & \multicolumn{4}{|c|}{ 1985-1994 } & \multicolumn{4}{|c|}{ 1993-1994 } \\
\hline & \multicolumn{2}{|c|}{ Total samples } & \multicolumn{2}{|c|}{ Samples > PEL } & \multicolumn{2}{|c|}{ Total samples } & \multicolumn{2}{|c|}{ Samples > PEL } & \multicolumn{2}{|c|}{ Total samples } & \multicolumn{2}{|c|}{ Samples > PEL } \\
\hline & Number & $\begin{array}{l}\text { Avg. } \\
\text { Sev. }\end{array}$ & $\%$ & $\begin{array}{l}\text { Avg. } \\
\text { Sev. }\end{array}$ & Number & $\begin{array}{l}\text { Avg. } \\
\text { Sev. }\end{array}$ & $\%$ & $\begin{array}{l}\text { Avg. } \\
\text { Sev. }\end{array}$ & Number & $\begin{array}{l}\text { Avg. } \\
\text { Sev. }\end{array}$ & $\%$ & $\begin{array}{l}\text { Avg. } \\
\text { Sev. }\end{array}$ \\
\hline Alabama & 22 & 0.99 & 36.4 & 1.52 & 41 & 1.15 & 39.0 & 1.94 & 2 & 0.95 & 50.0 & 1.45 \\
\hline Alaska & 0 & - & - & - & 0 & - & - & - & 0 & - & - & - \\
\hline Arizona & 0 & - & - & - & 0 & - & - & - & 0 & - & - & - \\
\hline Arkansas & 0 & - & - & - & 0 & - & - & - & 0 & - & - & - \\
\hline California & 0 & - & - & - & 0 & - & - & - & 0 & - & - & - \\
\hline Colorado & 0 & - & - & - & 0 & - & - & - & 0 & - & - & - \\
\hline Connecticut & 0 & - & - & - & 0 & - & - & - & 0 & - & - & - \\
\hline Delaware & 0 & - & - & - & 0 & - & - & - & 0 & - & - & - \\
\hline District of Columbia & 0 & - & - & - & 0 & - & - & - & 0 & - & - & - \\
\hline Florida & 0 & - & - & - & 0 & - & - & - & 0 & - & - & - \\
\hline Georgia & 27 & 0.79 & 25.9 & 1.81 & 155 & 5.25 & 40.6 & 12.16 & 19 & 0.99 & 31.6 & 1.94 \\
\hline Hawaii & 0 & - & - & - & 0 & - & - & - & 0 & - & - & - \\
\hline Idaho & 0 & - & - & - & 0 & - & - & - & 0 & - & - & - \\
\hline Illinois & 2 & 15.68 & 100.0 & 15.68 & 0 & - & - & - & 0 & - & - & - \\
\hline Indiana & 0 & - & - & - & 0 & - & - & - & 0 & - & - & - \\
\hline Iowa & 0 & - & - & - & 0 & - & - & - & 0 & - & - & - \\
\hline Kansas & 0 & - & - & - & 0 & - & - & - & 0 & - & - & - \\
\hline Kentucky & 0 & - & - & - & 0 & - & - & - & 0 & - & - & - \\
\hline Louisiana & 0 & - & - & - & 0 & - & - & - & 0 & - & - & - \\
\hline Maine & 0 & - & - & - & 0 & - & - & - & 0 & - & - & - \\
\hline Maryland & 0 & - & - & - & 0 & - & - & - & 0 & - & - & - \\
\hline Massachusetts & 0 & - & - & - & 0 & - & - & - & 0 & - & - & - \\
\hline Michigan & 0 & - & - & - & 0 & - & - & - & 0 & - & - & - \\
\hline Minnesota & 0 & - & - & - & 0 & - & - & - & 0 & - & - & - \\
\hline Mississippi & 1 & 0.25 & 0.0 & - & 0 & - & - & - & 0 & - & - & - \\
\hline Missouri & 0 & - & - & - & 0 & - & - & - & 0 & - & - & - \\
\hline Montana & 0 & - & - & - & 0 & - & - & - & 0 & - & - & - \\
\hline
\end{tabular}


Table 4-12 (page 2 of 2). Cotton dust: Number of OSHA inspector samples, percent exceeding the permissible exposure limit (PEL) and average severity levels (Avg. Sev.), by state, 1979-1994

\begin{tabular}{|c|c|c|c|c|c|c|c|c|c|c|c|c|}
\hline \multirow[b]{3}{*}{ State } & \multicolumn{4}{|c|}{ 1979-1984 } & \multicolumn{4}{|c|}{ 1985-1994 } & \multicolumn{4}{|c|}{ 1993-1994 } \\
\hline & \multicolumn{2}{|c|}{ Total samples } & \multicolumn{2}{|c|}{ Samples > PEL } & \multicolumn{2}{|c|}{ Total samples } & \multicolumn{2}{|c|}{ Samples > PEL } & \multicolumn{2}{|c|}{ Total samples } & \multicolumn{2}{|c|}{ Samples > PEL } \\
\hline & Number & $\begin{array}{l}\text { Avg. } \\
\text { Sev. }\end{array}$ & $\%$ & $\begin{array}{l}\text { Avg. } \\
\text { Sev. }\end{array}$ & Number & $\begin{array}{c}\text { Avg. } \\
\text { Sev. }\end{array}$ & $\%$ & $\begin{array}{l}\text { Avg. } \\
\text { Sev. }\end{array}$ & Number & $\begin{array}{l}\text { Avg. } \\
\text { Sev. }\end{array}$ & $\%$ & $\begin{array}{l}\text { Avg. } \\
\text { Sev. }\end{array}$ \\
\hline Nebraska & 0 & - & - & - & 0 & - & - & - & 0 & - & - & - \\
\hline Nevada & 0 & - & - & - & 0 & - & - & - & 0 & - & - & - \\
\hline New Hampshire & 0 & - & - & - & 0 & - & - & - & 0 & - & - & - \\
\hline New Jersey & 0 & - & - & - & 1 & 1.93 & 100.0 & 1.93 & 0 & - & - & - \\
\hline New Mexico & 0 & - & - & - & 0 & - & - & - & 0 & - & - & - \\
\hline New York & 0 & - & - & - & 0 & - & - & - & 0 & - & - & - \\
\hline North Carolina & 240 & 0.84 & 21.3 & 2.09 & 200 & 0.73 & 22.5 & 1.40 & 24 & 0.78 & 20.8 & 1.11 \\
\hline North Dakota & 0 & - & - & - & 0 & - & - & - & 0 & - & - & - \\
\hline Ohio & 0 & - & - & - & 0 & - & - & - & 0 & - & - & - \\
\hline Oklahoma & 1 & 0.00 & 0.0 & - & 0 & - & - & - & 0 & - & - & - \\
\hline Oregon & 0 & - & - & - & 0 & - & - & - & 0 & - & - & - \\
\hline Pennsylvania & 0 & - & - & - & 1 & 0.00 & 0.0 & - & 0 & - & - & - \\
\hline Rhode Island & 0 & - & - & - & 0 & - & - & - & 0 & - & - & - \\
\hline South Carolina & 5 & 0.52 & 20.0 & 1.73 & 88 & 1.18 & 25.0 & 3.33 & 36 & 1.47 & 19.4 & 6.37 \\
\hline South Dakota & 0 & - & - & - & 0 & - & - & - & 0 & - & - & - \\
\hline Tennessee & 0 & - & - & - & 10 & 3.70 & 10.0 & 37.00 & 1 & 0.00 & 0.0 & - \\
\hline Texas & 22 & 1.99 & 77.3 & 2.42 & 47 & 17.56 & 44.7 & 38.57 & 0 & - & - & - \\
\hline Utah & 0 & - & - & - & 0 & - & - & - & 0 & - & - & - \\
\hline Vermont & 0 & - & - & - & 0 & - & - & - & 0 & - & - & - \\
\hline Virginia & 0 & - & - & - & 0 & - & - & - & 0 & - & - & - \\
\hline Washington & 0 & - & - & - & 0 & - & - & - & 0 & - & - & - \\
\hline West Virginia & 0 & - & - & - & 0 & - & - & - & 0 & - & - & - \\
\hline Wisconsin & 0 & - & - & - & 0 & - & - & - & 0 & - & - & - \\
\hline Wyoming & 0 & - & - & - & 0 & - & - & - & 0 & - & - & - \\
\hline TOTAL & 320 & 1.01 & 26.9 & 2.39 & 543 & 3.63 & 31.1 & 10.55 & 82 & 1.13 & 23.2 & 3.33 \\
\hline
\end{tabular}

- indicates incalculable field.

NOTE: $\quad$ See Appendix A for source description, Appendix B for methods, and Appendix D for agents.

SOURCE: Occupational Safety and Health Administration: Integrated Management Information System. 
Figure 4-8. Cotton dust: Number of OSHA inspector samples collected by state, 1993-1994

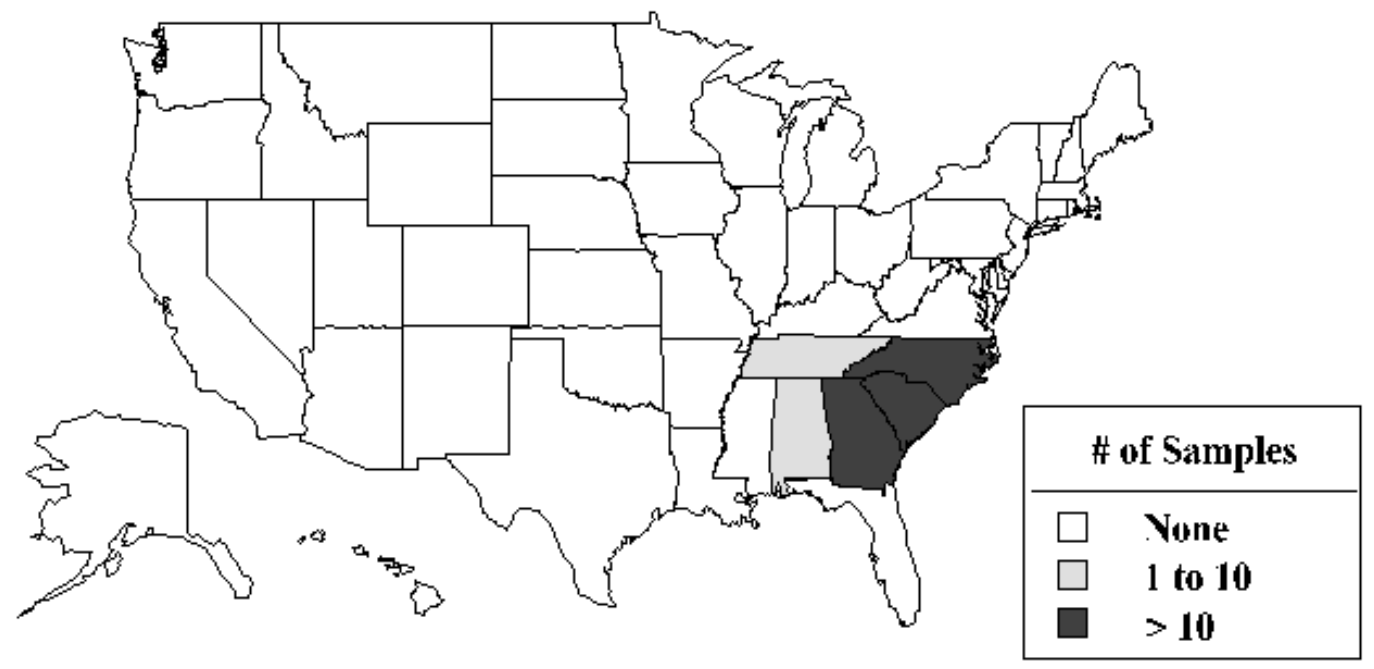

NOTE: See Appendix A for source description, Appendix B for methods, and Appendix D for agents. SOURCE: Occupational Safety and Health Administration: Integrated Management Information System.

Figure 4-9. Cotton dust: Percent of OSHA inspector samples that exceeded the permissible exposure limit (PEL), by state, 1993-1994

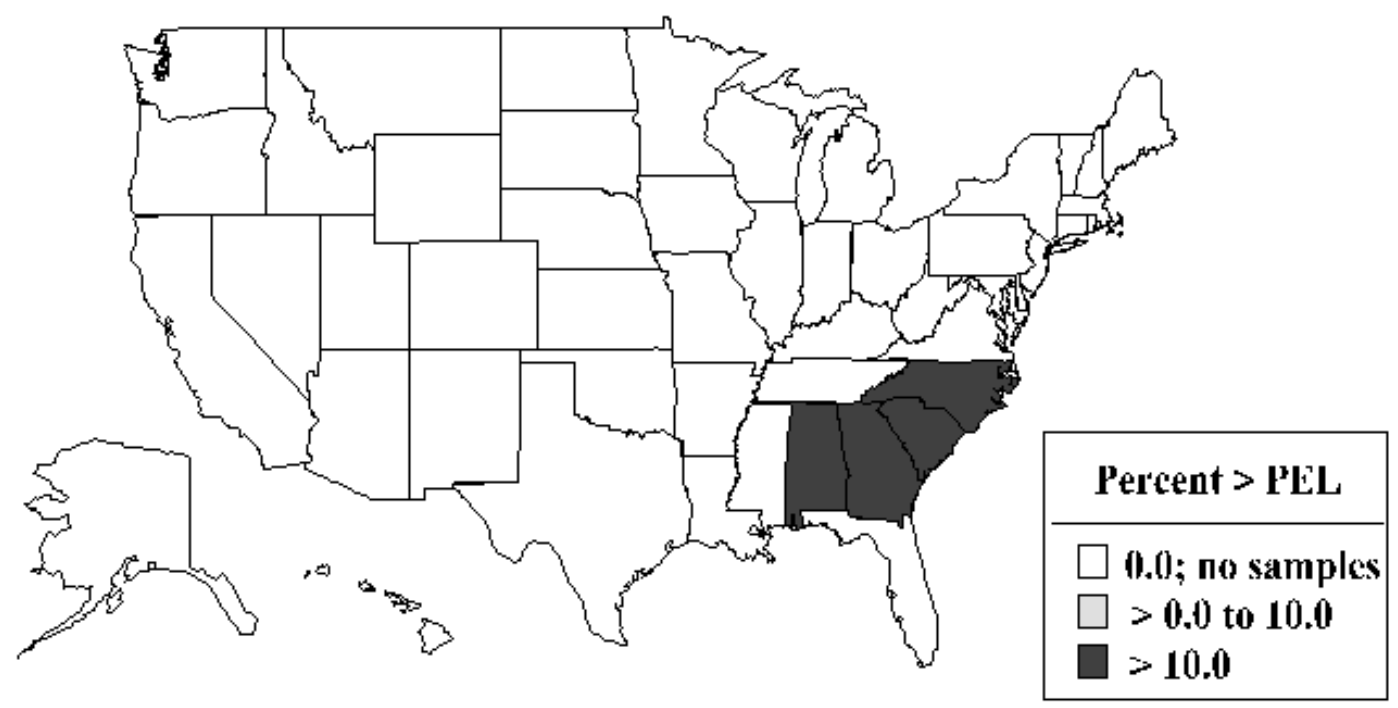

NOTE: See Appendix A for source description, Appendix B for methods, and Appendix D for agents. SOURCE: Occupational Safety and Health Administration: Integrated Management Information System. 
Figure 4-10. Cotton dust: Average severity level by state for OSHA inspector samples, 1993-1994

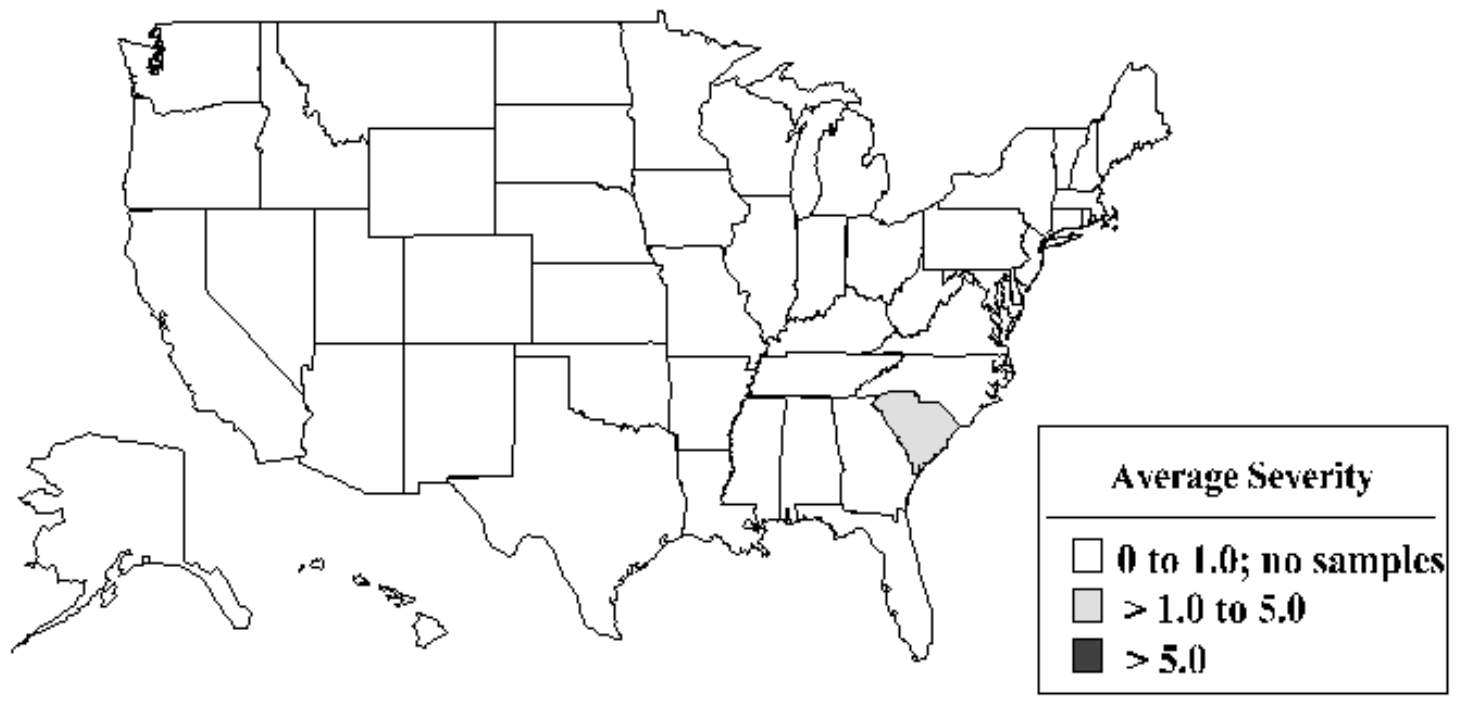

NOTE: See Appendix A for source descriptions, Appendix B for methods, and Appendix D for agents. SOURCE: Occupational Safety and Health Administration: Integrated Management Information System.

Figure 4-11. Cotton dust: Average severity level by state for OSHA inspector samples that exceeded the permissible exposure limit (PEL), 1993-1994

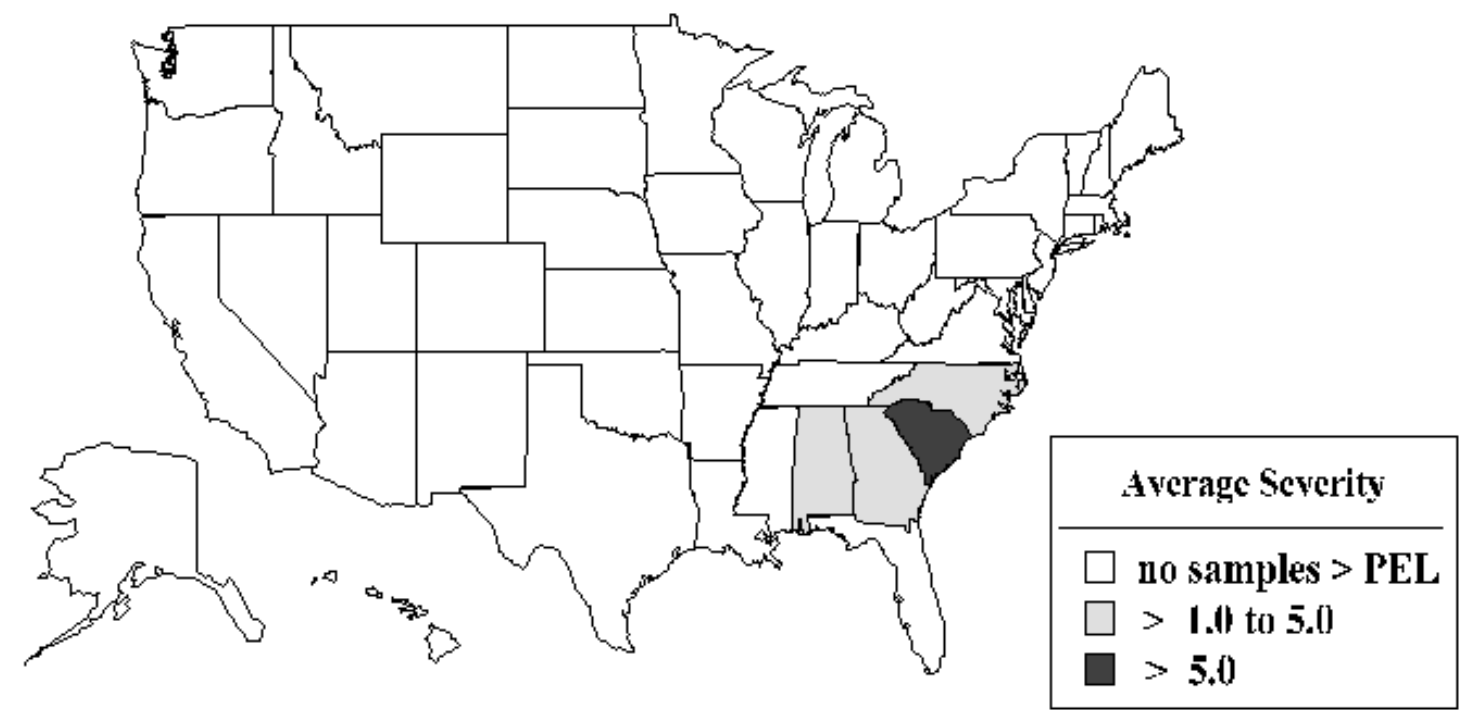

NOTE: See Appendix A for source descriptions, Appendix B for methods, and Appendix D for agents. SOURCE: Occupational Safety and Health Administration: Integrated Management Information System. 
Figure 4-12. Cotton dust: Number of OSHA inspector samples

collected by county, 1993-1994

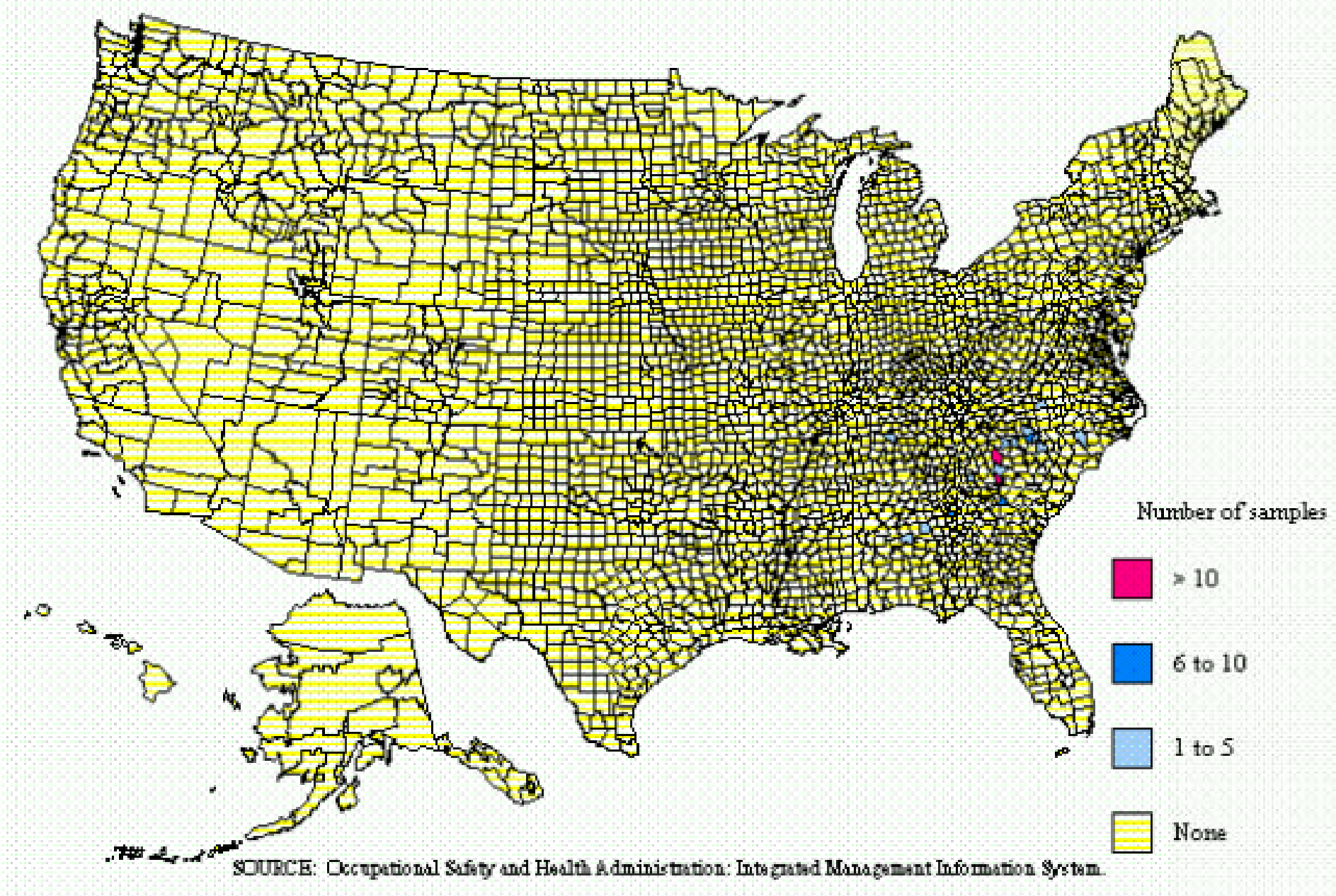



Figure 5-1. Unspecified/Other pneumoconioses: Number of deaths, crude and ageadjusted mortality rates, U.S. residents age 15 and over, 1968-1992

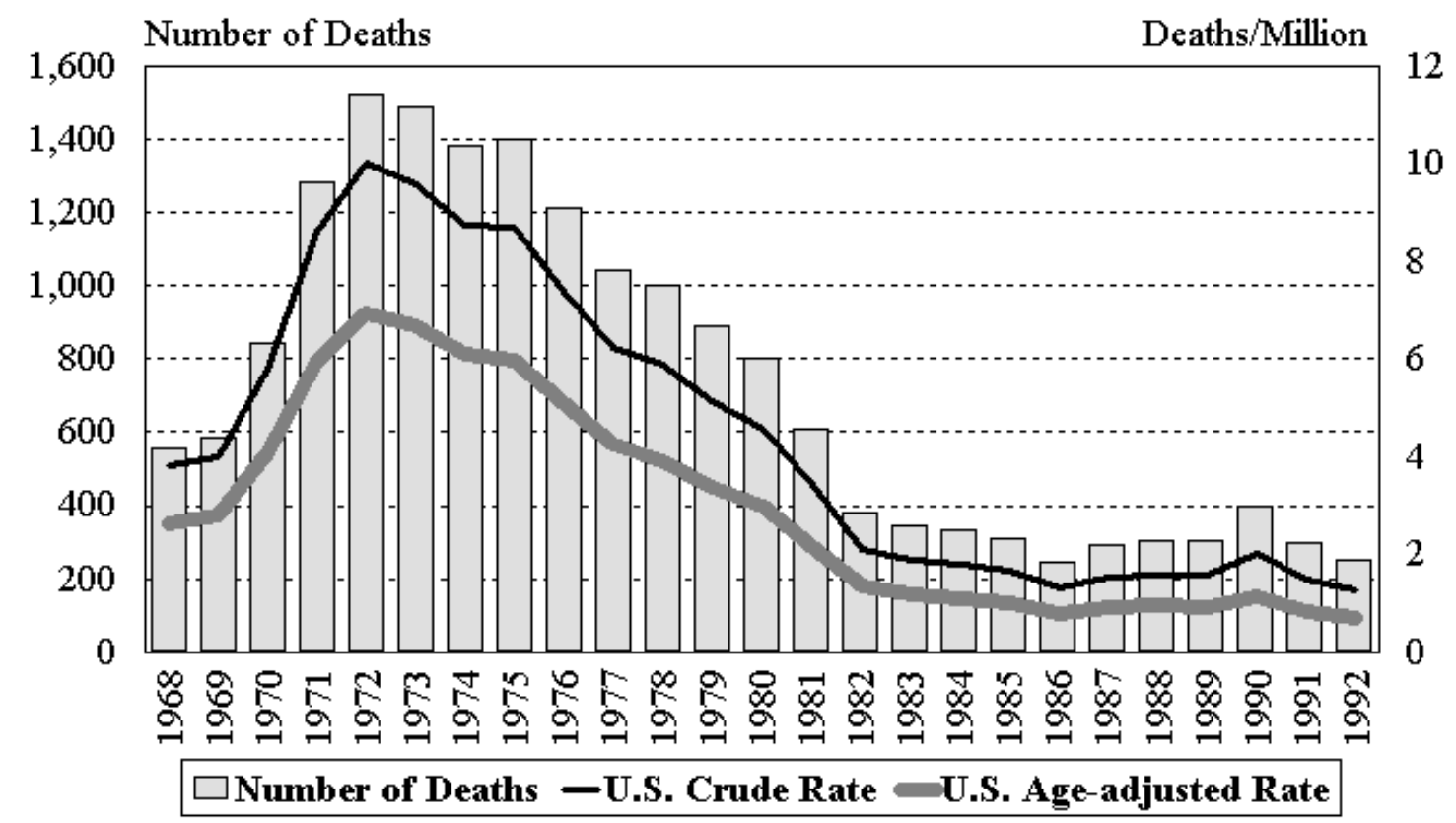

NOTE: $\quad$ See Appendix A for source description and Appendix B for methods and ICD-8 and ICD-9 codes.

SOURCE: National Center for Health Statistics multiple cause of death data. Population estimates from U.S. Bureau of the Census.

Figure 5-2. Unspecified/Other pneumoconioses: Crude mortality rates by state, U.S. residents age 15 and over, 1991-1992

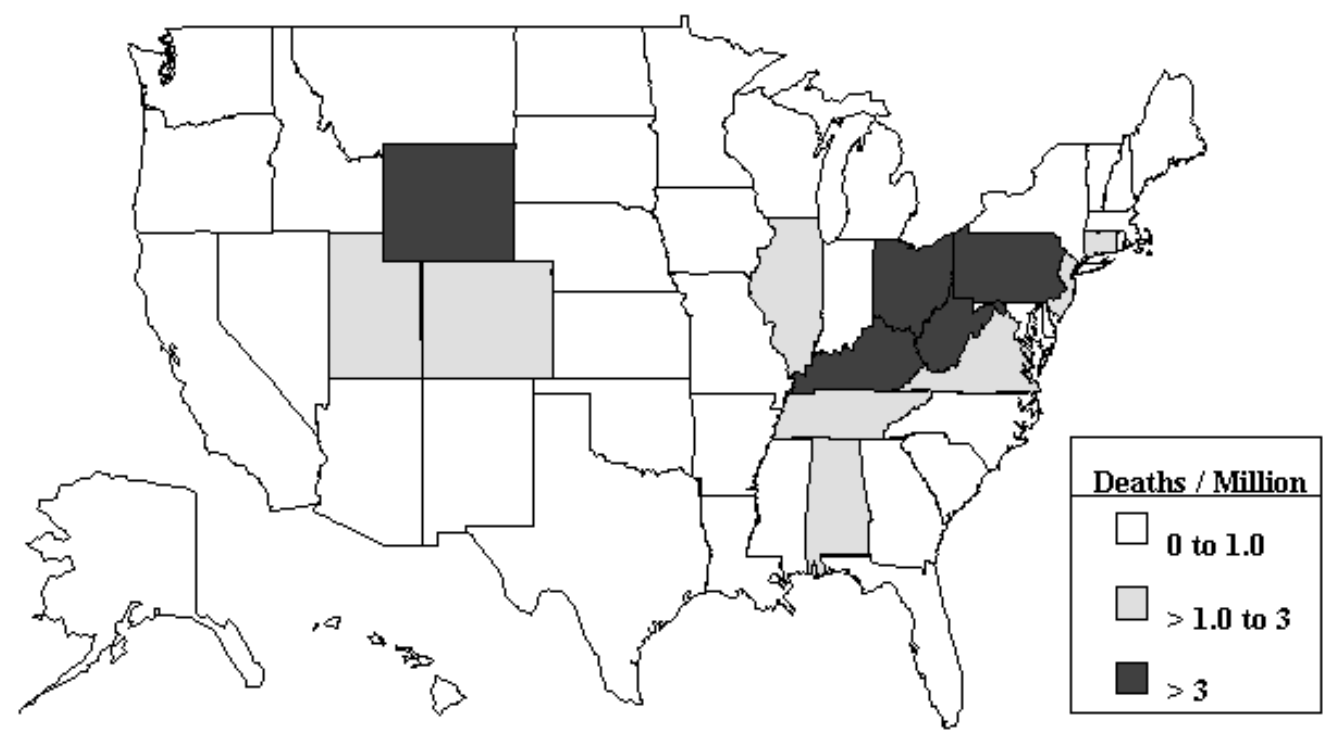

NOTE: $\quad$ See Appendix A for source description and Appendix B for methods and ICD-8 and ICD-9 codes.

OSOURCE: National Center for Health Statistics multiple cause of death data. Population estimates from U.S. Bureau of the Census. 
Figure 5-3. Unspecified/Other pneumoconioses: Median age at death, U.S. residents age 15 and over, 1968-1992

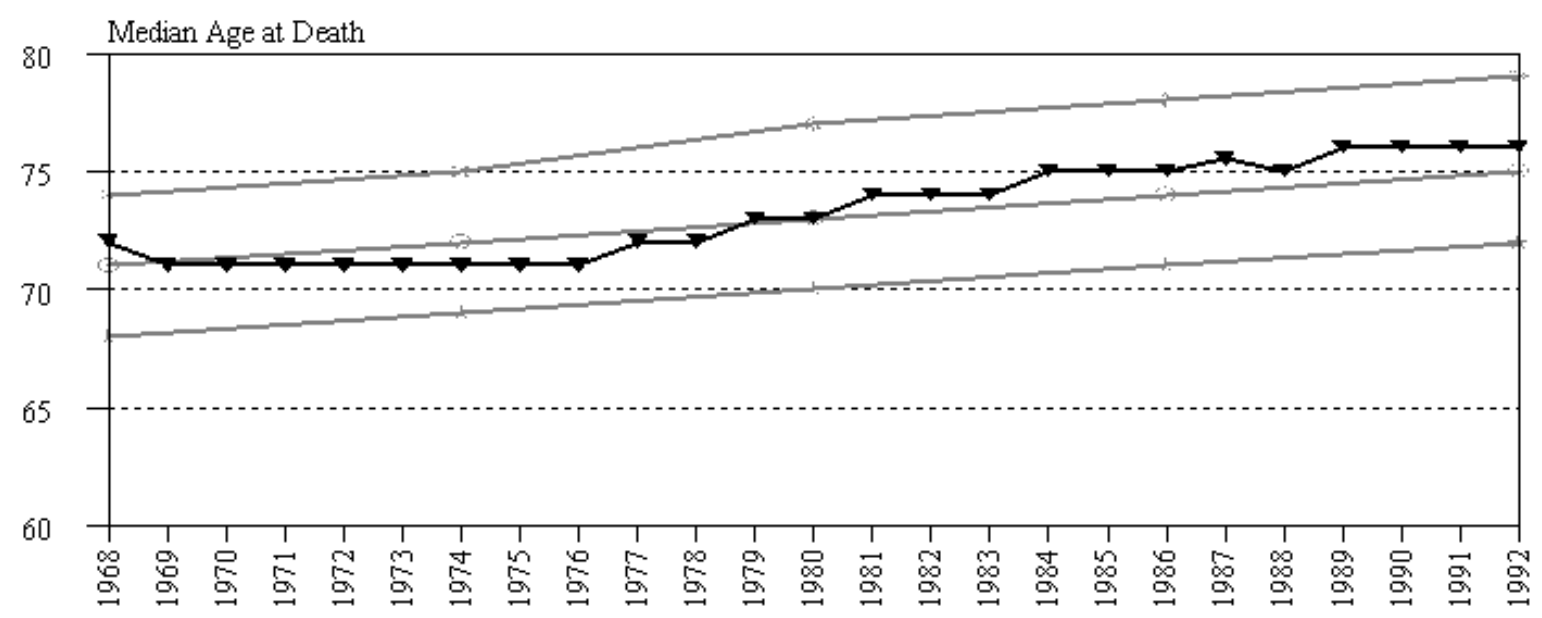

\begin{tabular}{ll|}
$\rightarrow$ Unspecified/Other Deaths & U.S. Deaths \\
$\rightarrow$ U.S. Male Deaths & U.S. Female Deaths \\
\hline
\end{tabular}

NOTE: See Appendix A for source description and Appendix B for methods and ICD-8 and ICD-9 codes.

SOURCE: National Center for Health Statistics multiple cause of death data.

Table 5-1. Unspecified/Other pneumoconioses: Number of deaths by sex, race, and age, U.S. residents age 15 and over, 1991-1992

\begin{tabular}{|c|c|c|c|c|c|}
\hline & & \multicolumn{2}{|c|}{1991} & \multicolumn{2}{|c|}{1992} \\
\hline & & Number & Percent & Number & Percent \\
\hline \multicolumn{2}{|c|}{ Total deaths } & 296 & 100.0 & 249 & 100.0 \\
\hline \multirow[t]{2}{*}{ Sex } & Male & 282 & 95.3 & 234 & 94.0 \\
\hline & Female & 14 & 4.7 & 15 & 6.0 \\
\hline \multirow[t]{3}{*}{ Race } & White & 271 & 91.6 & 231 & 92.8 \\
\hline & Black & 24 & 8.1 & 18 & 7.2 \\
\hline & Other & 1 & 0.3 & 0 & 0.0 \\
\hline \multirow[t]{10}{*}{ Age } & $15-24$ & 0 & 0.0 & 1 & 0.4 \\
\hline & $25-34$ & 1 & 0.3 & 0 & 0.0 \\
\hline & $35-44$ & 6 & 2.0 & 0 & 0.0 \\
\hline & $45-54$ & 3 & 1.0 & 8 & 3.2 \\
\hline & $55-64$ & 22 & 7.4 & 19 & 7.6 \\
\hline & $65-74$ & 103 & 34.8 & 76 & 30.5 \\
\hline & $75-84$ & 110 & 37.2 & 104 & 41.8 \\
\hline & 85 and over & 51 & 17.2 & 41 & 16.5 \\
\hline & Mean age & 75.0 & & 75.3 & \\
\hline & Range for age & $32-94$ & . & $21-95$ & \\
\hline
\end{tabular}


Table 5-2. Unspecified/Other pneumoconioses: Number of deaths by state, U.S. residents age 15 and over, 1968-1992

\begin{tabular}{|c|c|c|c|c|c|}
\hline State & 1968-1978 & 1979-1990 & 1991 & 1992 & TOTAL \\
\hline Alabama & 342 & 133 & 10 & 5 & 490 \\
\hline Alaska & 3 & 1 & - & - & 4 \\
\hline Arizona & 74 & 38 & 1 & 2 & 115 \\
\hline Arkansas & 58 & 17 & 2 & 1 & 78 \\
\hline California & 355 & 154 & 4 & 4 & 517 \\
\hline Colorado & 118 & 48 & 3 & 3 & 172 \\
\hline Connecticut & 81 & 49 & 2 & 4 & 136 \\
\hline Delaware & 10 & 9 & - & - & 19 \\
\hline District of Columbia & 14 & 2 & 1 & - & 17 \\
\hline Florida & 157 & 99 & 10 & 11 & 277 \\
\hline Georgia & 52 & 37 & 1 & 2 & 92 \\
\hline Hawaii & 7 & 1 & - & - & 8 \\
\hline Idaho & 10 & 5 & - & - & 15 \\
\hline Illinois & 732 & 275 & 19 & 13 & 1,039 \\
\hline Indiana & 174 & 96 & 4 & 4 & 278 \\
\hline Iowa & 41 & 8 & - & 4 & 53 \\
\hline Kansas & 43 & 15 & 3 & - & 61 \\
\hline Kentucky & 1,046 & 512 & 32 & 34 & 1,624 \\
\hline Louisiana & 27 & 25 & 4 & - & 56 \\
\hline Maine & 18 & 11 & - & - & 29 \\
\hline Maryland & 71 & 56 & 4 & 1 & 132 \\
\hline Massachusetts & 88 & 45 & 2 & 3 & 138 \\
\hline Michigan & 252 & 126 & 3 & 7 & 388 \\
\hline Minnesota & 54 & 18 & 1 & 1 & 74 \\
\hline Mississippi & 8 & 3 & - & - & 11 \\
\hline Missouri & 64 & 32 & 3 & 1 & 100 \\
\hline Montana & 11 & 9 & - & - & 20 \\
\hline Nebraska & 9 & 4 & 1 & 1 & 15 \\
\hline Nevada & 11 & 4 & - & - & 15 \\
\hline New Hampshire & 11 & 7 & - & 1 & 19 \\
\hline New Jersey & 252 & 159 & 7 & 7 & 425 \\
\hline New Mexico & 49 & 34 & - & 1 & 84 \\
\hline New York & 327 & 139 & 3 & 9 & 478 \\
\hline North Carolina & 71 & 41 & 2 & 4 & 118 \\
\hline North Dakota & 7 & 1 & - & - & 8 \\
\hline Ohio & 672 & 394 & 42 & 27 & 1,135 \\
\hline Oklahoma & 38 & 18 & 1 & 2 & 59 \\
\hline Oregon & 23 & 12 & 1 & - & 36 \\
\hline Pennsylvania & 3,696 & 1,190 & 49 & 33 & 4,968 \\
\hline Rhode Island & 14 & 7 & - & - & 21 \\
\hline South Carolina & 17 & 8 & 3 & - & 28 \\
\hline South Dakota & 10 & 5 & - & - & 15 \\
\hline Tennessee & 267 & 109 & 8 & 3 & 387 \\
\hline Texas & 94 & 42 & 1 & 3 & 140 \\
\hline Utah & 70 & 42 & 1 & 2 & 115 \\
\hline Vermont & 28 & 8 & - & - & 36 \\
\hline Virginia & 582 & 391 & 7 & 4 & 984 \\
\hline Washington & 50 & 19 & 3 & 3 & 75 \\
\hline West Virginia & 1,960 & 699 & 58 & 44 & 2,761 \\
\hline Wisconsin & 91 & 28 & - & 2 & 121 \\
\hline Wyoming & 63 & 17 & - & 3 & 83 \\
\hline TOTAL & 12,322 & 5,202 & 296 & 249 & 18,069 \\
\hline
\end{tabular}

- indicates no deaths listed.

NOTE: See Appendix A for source description and Appendix B for methods and ICD-8 and ICD-9 codes.

SOURCE: National Center for Health Statistics multiple cause of death data. 
Table 5-3. Unspecified/Other pneumoconioses: Mortality rates (per 1,000,000 population) by race and sex, U.S. residents age 15 and over, 1991-1992

\begin{tabular}{|c|c|c|c|c|c|}
\hline \multirow[b]{2}{*}{ Year } & \multirow[b]{2}{*}{ Overall rate } & \multicolumn{2}{|c|}{ White } & \multicolumn{2}{|c|}{ Black } \\
\hline & & Males & Females & Males & Females \\
\hline & \multicolumn{5}{|c|}{ Crude mortality rate } \\
\hline 1991 & 1.50 & 3.20 & 0.15 & 2.21 & 0.08 \\
\hline \multirow[t]{2}{*}{1992} & 1.25 & 2.66 & 0.16 & 1.60 & 0.08 \\
\hline & \multicolumn{5}{|c|}{ Age-adjusted mortality rate } \\
\hline 1991 & 0.83 & 1.96 & 0.07 & 2.05 & 0.07 \\
\hline 1992 & 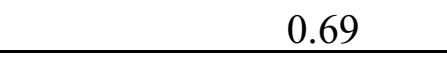 & 1.64 & 0.07 & 1.47 & 0.07 \\
\hline NOTE: & $\begin{array}{l}\text { See Appendix A for source description } \\
\text { Work-Related Lung Disease Surveillan } \\
\text { See Appendix E for revised rates for } 19\end{array}$ & $\begin{array}{l}\text { ix B for } \mathrm{m} \\
994 \text {, see } A\end{array}$ & $\begin{array}{l}\text { nd ICD-8 and I } \\
\text { E. }\end{array}$ & les. Data fo & can be found \\
\hline
\end{tabular}

Table 5-4. Unspecified/Other pneumoconioses: Years of potential life lost by race and sex, U.S. residents age 15 and over, 1991-1992

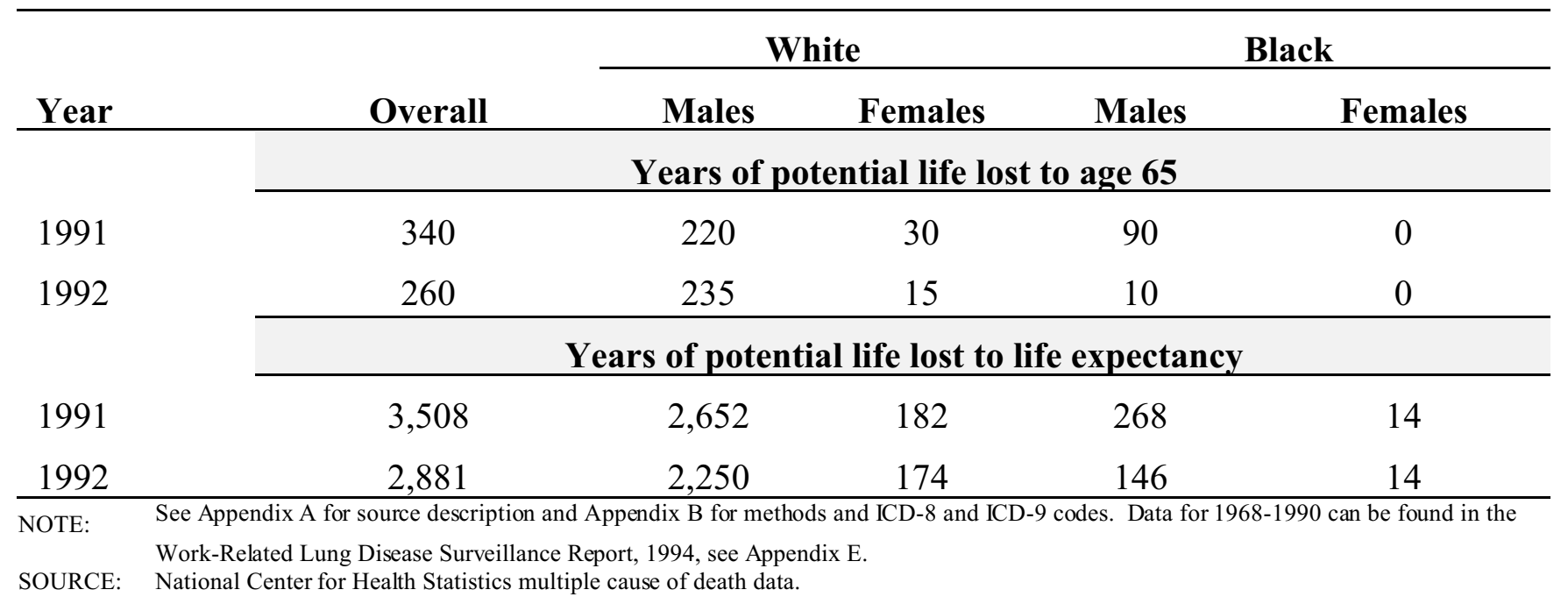


Table 5-5. Unspecified/Other pneumoconioses: Total number of deaths, crude and age-adjusted mortality rates (per $1,000,000$ population), and total years of potential life lost (YPLL) to life expectancy, by state, U.S. residents age 15 and over, 1988-1992

\begin{tabular}{|c|c|c|c|c|c|c|c|c|c|c|}
\hline \multirow[b]{2}{*}{ State } & \multirow{2}{*}{$\begin{array}{r}\text { Total } \\
\text { deaths }\end{array}$} & \multirow[b]{2}{*}{ Rank } & \multicolumn{2}{|c|}{ Crude mortality } & \multicolumn{2}{|c|}{ Age-adjusted mortality } & \multicolumn{4}{|c|}{ YPLL to life expectancy } \\
\hline & & & Rate & Rank & Rate & Rank & Years & Rank & Years/death & Rank \\
\hline Alabama & 36 & 11 & 2.28 & 7 & 1.12 & 12 & 387 & 13 & 10.8 & 33 \\
\hline Alaska & - & - & - & - & - & - & - & - & - & - \\
\hline Arizona & 9 & 27 & 0.63 & 29 & 0.32 & 31 & 94 & 28 & 10.4 & 37 \\
\hline Arkansas & 5 & 33 & 0.55 & 32 & 0.28 & 35 & 58 & 34 & 11.6 & 28 \\
\hline California & 36 & 11 & 0.31 & 43 & 0.22 & 40 & 442 & 12 & 12.3 & 22 \\
\hline Colorado & 8 & 28 & 0.62 & 30 & 0.42 & 24 & 83 & 30 & 10.4 & 38 \\
\hline Connecticut & 15 & 18 & 1.13 & 15 & 0.53 & 20 & 145 & 25 & 9.7 & 41 \\
\hline Delaware & 2 & 40 & 0.76 & 23 & 0.38 & 27 & 16 & 42 & 8.0 & 42 \\
\hline District of Columbia & 2 & 40 & 0.79 & 22 & 0.78 & 14 & 76 & 31 & 38.0 & 1 \\
\hline Florida & 46 & 7 & 0.87 & 19 & 0.34 & 29 & 492 & 10 & 10.7 & 34 \\
\hline Georgia & 10 & 26 & 0.40 & 38 & 0.28 & 35 & 118 & 26 & 11.8 & 26 \\
\hline Hawaii & - & - & - & - & - & - & - & - & - & - \\
\hline Idaho & - & - & - & - & - & - & - & - & - & - \\
\hline Illinois & 102 & 5 & 2.28 & 7 & 1.14 & 11 & 1,045 & 5 & 10.2 & 39 \\
\hline Indiana & 26 & 14 & 1.20 & 14 & 0.61 & 16 & 273 & 16 & 10.5 & 36 \\
\hline Iowa & 4 & 35 & 0.37 & 40 & 0.20 & 42 & 50 & 36 & 12.5 & 18 \\
\hline Kansas & 7 & 30 & 0.73 & 25 & 0.32 & 31 & 70 & 32 & 10.0 & 40 \\
\hline Kentucky & 178 & 3 & 12.31 & 2 & 6.69 & 2 & 2,020 & 3 & 11.3 & 30 \\
\hline Louisiana & 12 & 22 & 0.75 & 24 & 0.55 & 19 & 166 & 20 & 13.8 & 11 \\
\hline Maine & 4 & 35 & 0.83 & 20 & 0.45 & 21 & 44 & 39 & 11.0 & 31 \\
\hline Maryland & 19 & 16 & 1.00 & 16 & 0.62 & 15 & 236 & 17 & 12.4 & 19 \\
\hline Massachusetts & 12 & 22 & 0.49 & 33 & 0.30 & 34 & 151 & 23 & 12.6 & 17 \\
\hline Michigan & 35 & 13 & 0.97 & 17 & 0.61 & 16 & 482 & 11 & 13.8 & 13 \\
\hline Minnesota & 6 & 31 & 0.35 & 41 & 0.24 & 39 & 86 & 29 & 14.3 & 7 \\
\hline Mississippi & 1 & 43 & 0.10 & 48 & 0.07 & 46 & 14 & 43 & 14.0 & 8 \\
\hline Missouri & 13 & 20 & 0.65 & 28 & 0.39 & 26 & 205 & 18 & 15.8 & 5 \\
\hline Montana & 1 & 43 & 0.33 & 42 & 0.07 & 46 & 6 & 47 & 6.0 & 47 \\
\hline Nebraska & 4 & 35 & 0.66 & 27 & 0.44 & 22 & 55 & 35 & 13.8 & 15 \\
\hline Nevada & 1 & 43 & 0.21 & 47 & 0.13 & 43 & 8 & 45 & 8.0 & 42 \\
\hline New Hampshire & 1 & 43 & 0.23 & 45 & 0.06 & 48 & 6 & 47 & 6.0 & 47 \\
\hline New Jersey & 46 & 7 & 1.48 & 12 & 0.89 & 13 & 587 & 7 & 12.8 & 16 \\
\hline New Mexico & 14 & 19 & 2.46 & 6 & 1.53 & 6 & 159 & 21 & 11.4 & 29 \\
\hline New York & 43 & 10 & 0.60 & 31 & 0.35 & 28 & 517 & 9 & 12.0 & 24 \\
\hline North Carolina & 22 & 15 & 0.83 & 20 & 0.59 & 18 & 307 & 14 & 14.0 & 10 \\
\hline North Dakota & 1 & 43 & 0.41 & 37 & 0.27 & 37 & 14 & 43 & 14.0 & 8 \\
\hline Ohio & 145 & 4 & 3.41 & 5 & 1.91 & 3 & 1,700 & 4 & 11.7 & 27 \\
\hline Oklahoma & 11 & 24 & 0.90 & 18 & 0.42 & 24 & 152 & 22 & 13.8 & 12 \\
\hline Oregon & 5 & 33 & 0.45 & 35 & 0.26 & 38 & 62 & 33 & 12.4 & 20 \\
\hline Pennsylvania & 195 & 2 & 4.09 & 3 & 1.88 & 4 & 2,132 & 2 & 10.9 & 32 \\
\hline Rhode Island & 2 & 40 & 0.49 & 33 & 0.34 & 29 & 29 & 40 & 14.5 & 6 \\
\hline South Carolina & 3 & 38 & 0.22 & 46 & 0.10 & 45 & 23 & 41 & 7.7 & 46 \\
\hline South Dakota & 1 & 43 & 0.38 & 39 & 0.13 & 43 & 8 & 45 & 8.0 & 42 \\
\hline Tennessee & 44 & 9 & 2.27 & 9 & 1.28 & 8 & 526 & 8 & 12.0 & 25 \\
\hline Texas & 18 & 17 & 0.28 & 44 & 0.21 & 41 & 285 & 15 & 15.8 & 4 \\
\hline Utah & 11 & 24 & 1.85 & 11 & 1.16 & 10 & 116 & 27 & 10.5 & 35 \\
\hline Vermont & 3 & 38 & 1.36 & 13 & 1.17 & 9 & 48 & 37 & 16.0 & 3 \\
\hline Virginia & 54 & 6 & 2.19 & 10 & 1.49 & 7 & 669 & 6 & 12.4 & 21 \\
\hline Washington & 13 & 20 & 0.68 & 26 & 0.44 & 22 & 179 & 19 & 13.8 & 14 \\
\hline West Virginia & 309 & 1 & 43.21 & 1 & 22.06 & 1 & 3,753 & 1 & 12.1 & 23 \\
\hline Wisconsin & 8 & 28 & 0.42 & 36 & 0.32 & 31 & 147 & 24 & 18.4 & 2 \\
\hline Wyoming & 6 & 31 & 3.54 & 4 & 1.71 & 5 & 48 & 37 & 8.0 & 42 \\
\hline
\end{tabular}

- indicates no deaths listed.

NOTE: Ranks are based on unrounded values. See Appendix A for source description and Appendix B for methods and ICD-8 and ICD-9 codes.

SOURCE: National Center for Health Statistics multiple cause of death data. Population estimates from U.S. Burea u of the Census. 
Table 5-6. Unspecified/Other pneumoconioses: Most frequently recorded occupations on death certificate, selected states, U.S. residents age 15 and over, 1991-1992

\begin{tabular}{llrr}
\hline COC & Occupation & Number & Percent \\
\hline 616 & Mining machine operators & 134 & 45.3 \\
889 & Laborers, except construction & 18 & 6.1 \\
019 & Managers and administrators, n.e.c. & 8 & 2.7 \\
453 & Janitors and cleaners & 8 & 2.7 \\
575 & Electricians & 8 & 2.7 \\
633 & Supervisors, precision production occupations & 7 & 2.4 \\
567 & Carpenters & 6 & 2.0 \\
637 & Machinists & 4 & 1.4 \\
783 & Welders and cutters & 4 & 1.4 \\
156 & Teachers, elementary school & 3 & 1.0 \\
& All other occupations & 89 & 30.1 \\
& Occupation not reported & 7 & 2.4 \\
& TOTAL & 296 & 100.0 \\
\hline COC - 1980 Census Occupation Code & & \\
NOTE: & See Appendix A for source description, Appendix B for methods elsewhere classified ICD-8 and ICD-9 codes, and Appendix C for list of 25 states reporting usual occupation and \\
sourC E: & years reporting. Data for 1985-1990 can be found in the Work-Related Lung Disease Surveillance Report, 1994, see Appendix E. &
\end{tabular}

Table 5-7. Unspecified/Other pneumoconioses: Most frequently recorded industries on death certificate, selected states, U.S. residents age 15 and over, 1991-1992

\begin{tabular}{llrr}
\hline CIC & Industry & Number & Percent \\
\hline 041 & Coal mining & 150 & 50.7 \\
060 & Construction & 19 & 6.4 \\
270 & Blast furnace, steelworks, rolling and finishing mills & 9 & 3.0 \\
351 & Motor vehicles and motor vehicle equipment & 8 & 2.7 \\
392 & Not specified manufacturing industries & 6 & 2.0 \\
842 & Elementary and secondary schools & 5 & 1.7 \\
192 & Industrial and miscellaneous chemicals & 4 & 1.4 \\
271 & Iron and steel foundries & 4 & 1.4 \\
280 & Other primary metal industries & 4 & 1.4 \\
250 & Glass and glass products & 3 & 1.0 \\
& All other industries & 78 & 26.4 \\
& Industry not reported & 6 & 2.0 \\
& TOTAL & 296 & 100.0 \\
\hline
\end{tabular}

CIC - 1980 Census Industry Code

NOTE: $\quad$ See Appendix A for source description, Appendix B for methods and ICD-8 and IC D-9 codes, and Appendix C for list of 25 states reporting usual industry and years reporting. Data for 1985-1990 can be found in the Work-Related Lung Disease Surveillance Report, 1994, see Appendix E.

SOURCE: National Center for Health Statistics multiple cause of death data. 
Table 5-8. Unspecified/Other pneumoconioses: Proportionate mortality ratio (PMR) by usual occupation, selected states and years, U.S. residents age 15 and over, 1985-1992

\begin{tabular}{|c|c|c|c|c|c|}
\hline \multirow[b]{2}{*}{$\mathrm{COC}$} & \multirow[b]{2}{*}{ Occupation } & \multirow{2}{*}{$\begin{array}{l}\text { Number } \\
\text { of deaths }\end{array}$} & \multirow[b]{2}{*}{ PMR } & \multicolumn{2}{|c|}{$95 \%$ confidence interval } \\
\hline & & & & $\mathrm{LCL}$ & UCL \\
\hline 615 & Explosives workers & 5 & 77.07 & 24.94 & 180.07 \\
\hline 616 & Mining machine operators & 423 & 53.96 & 48.79 & 59.49 \\
\hline 719 & Molding and casting machine operators & 14 & 13.22 & 7.22 & 22.18 \\
\hline 768 & Crushing and grinding machine operators & 6 & 12.82 & 4.70 & 27.93 \\
\hline 617 & Mining occupations, n.e.c. & 5 & 8.12 & 2.63 & 18.97 \\
\hline 859 & Miscellaneous material moving equipment operators & 5 & 5.87 & 1.90 & 13.71 \\
\hline 766 & Furnace, kiln, oven operators, except food & 10 & 5.70 & 2.74 & 10.48 \\
\hline 516 & Heavy equipment mechanics & 7 & 5.05 & 2.03 & 10.41 \\
\hline 824 & Locomotive operating occupations & 7 & 5.05 & 2.03 & 10.41 \\
\hline 849 & Crane and tower operators & 7 & 4.37 & 1.76 & 9.01 \\
\hline 575 & Electricians & 22 & 3.83 & 2.39 & 5.80 \\
\hline 563 & Brickmasons and stonemasons & 8 & 2.93 & 1.26 & 5.77 \\
\hline 783 & Welders and cutters & 13 & 2.57 & 1.37 & 4.39 \\
\hline 869 & Construction laborers & 24 & 2.15 & 1.38 & 3.20 \\
\hline 913 & Retired, with no other occupations reported & 19 & 1.88 & 1.13 & 2.94 \\
\hline 889 & Laborers, except construction & 63 & 1.81 & 1.38 & 2.33 \\
\hline 567 & Carpenters & 23 & 1.62 & 1.03 & 2.43 \\
\hline
\end{tabular}


Table 5-9. Unspecified/Other pneumoconioses: Proportionate mortality ratio (PMR) by usual industry, selected states and years, U.S. residents age 15 and over, 1985-1992

\begin{tabular}{|c|c|c|c|c|c|}
\hline \multirow[b]{2}{*}{$\mathrm{CIC}$} & \multirow[b]{2}{*}{ Industry } & \multirow{2}{*}{$\begin{array}{r}\text { Number } \\
\text { of deaths }\end{array}$} & \multirow[b]{2}{*}{ PMR } & \multicolumn{2}{|c|}{$95 \%$ confidence interval } \\
\hline & & & & $\mathrm{LCL}$ & $\mathrm{UCL}$ \\
\hline 041 & Coal mining & 478 & 58.65 & 53.37 & 64.31 \\
\hline 262 & Miscellane ous nonmetallic mineral and stone products & 15 & 12.87 & 7.19 & 21.24 \\
\hline 271 & Iron and steel foundries & 15 & 8.43 & 4.71 & 13.91 \\
\hline 050 & Nonmetallic mining and quarrying, except fuel & 8 & 7.08 & 3.05 & 13.94 \\
\hline 252 & Structural clay products & 6 & 6.98 & 2.56 & 15.21 \\
\hline 040 & Metal mining & 7 & 5.42 & 2.18 & 11.18 \\
\hline 280 & Other primary metal industries & 8 & 4.04 & 1.74 & 7.95 \\
\hline 192 & Industrial and miscellaneous chemicals & 14 & 2.89 & 1.58 & 4.85 \\
\hline 270 & Blast furnaces, steelworks, rolling and finishing mills & 36 & 2.88 & 2.00 & 4.01 \\
\hline 351 & Motor vehicles and motor vehicle equipment & 24 & 1.92 & 1.23 & 2.86 \\
\hline 060 & Construction & 85 & 1.37 & 1.09 & 1.71 \\
\hline
\end{tabular}

CIC - 1980 Census Industry Code $\quad$ LCL - lower confidence limit $\quad$ UCL - upper confidence limit

NOTE: $\quad$ See Appendix A for source description, Appendix B for methods and ICD-8 and ICD-9 codes, and Appendix C for list of 25 states reporting usual industry and years reporting.

SOURCE: National Center for Health Statistics multiple cause of death data 
Figure 5-4. Unspecified/Other pneumoconioses: Total number of deaths

by county, U.S. residents age 15 and over, 1983-1992

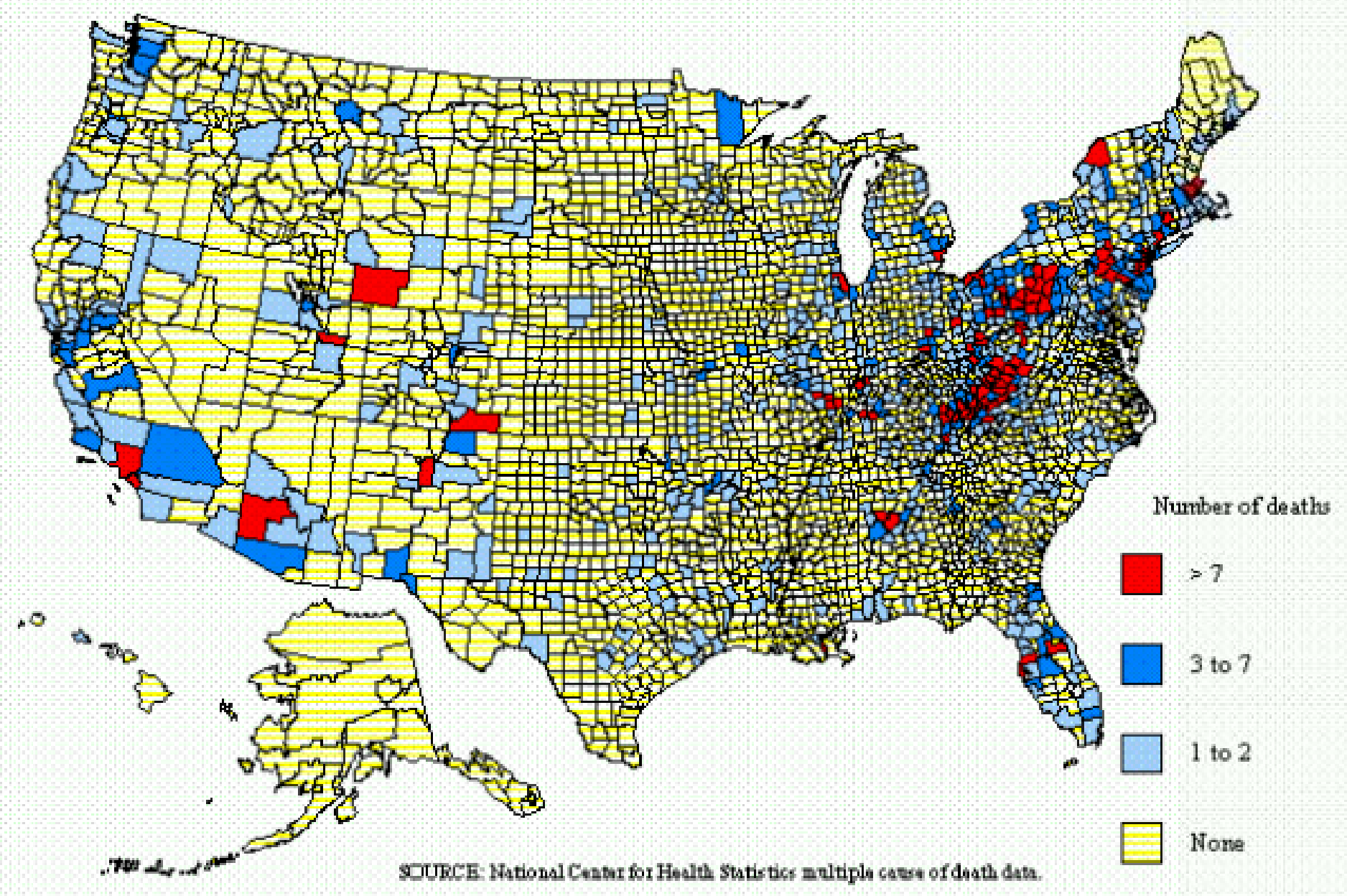


Figure 5-5. Unspecified/Other pneumoconioses: Age-adjusted mortality rates

by county, U.S. residents age 15 and over, 1983-1992

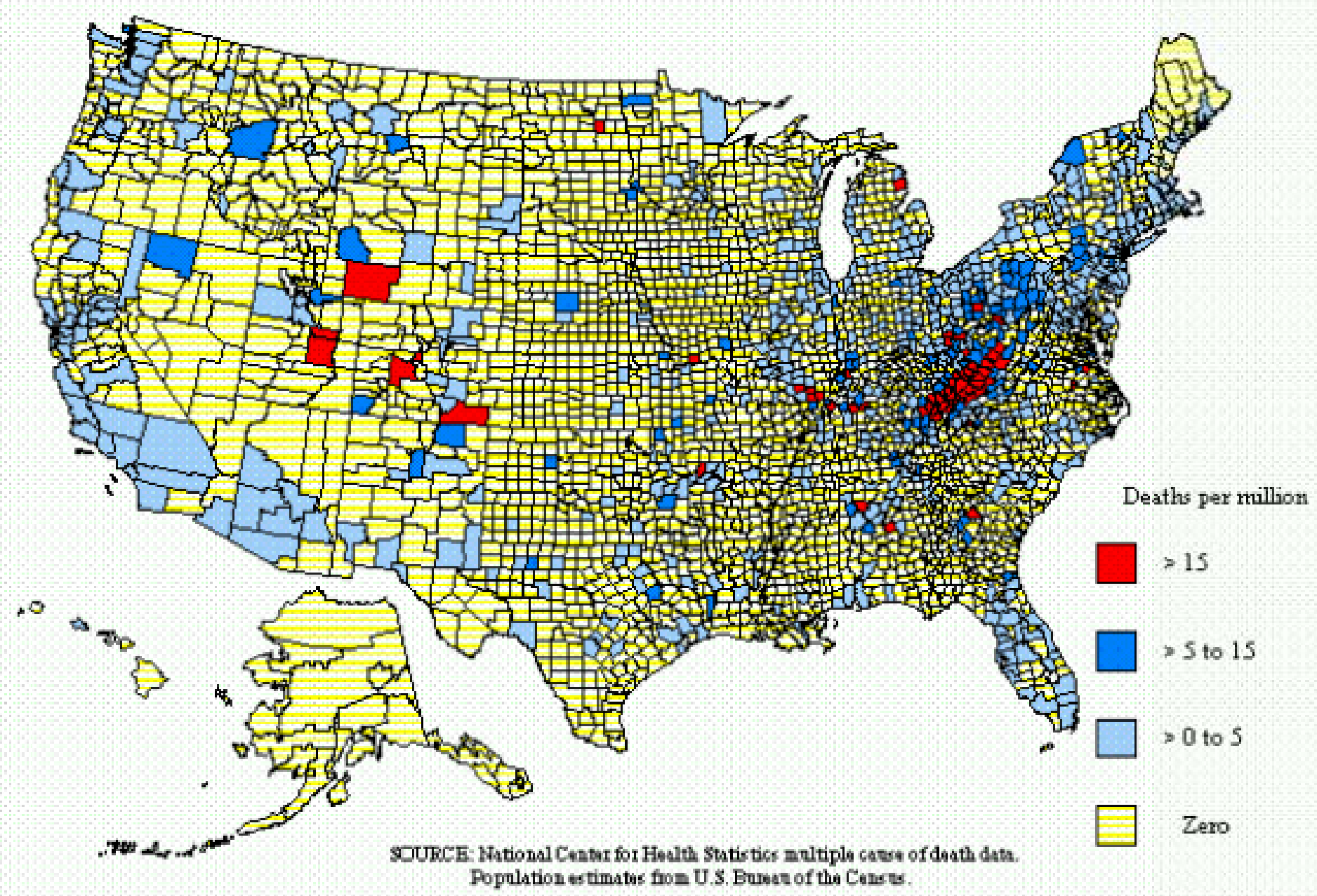


Figure 6-1. All pneumoconioses: Number of deaths, crude and age-adjusted mortality rates, U.S. residents age 15 and over, 1968-1992

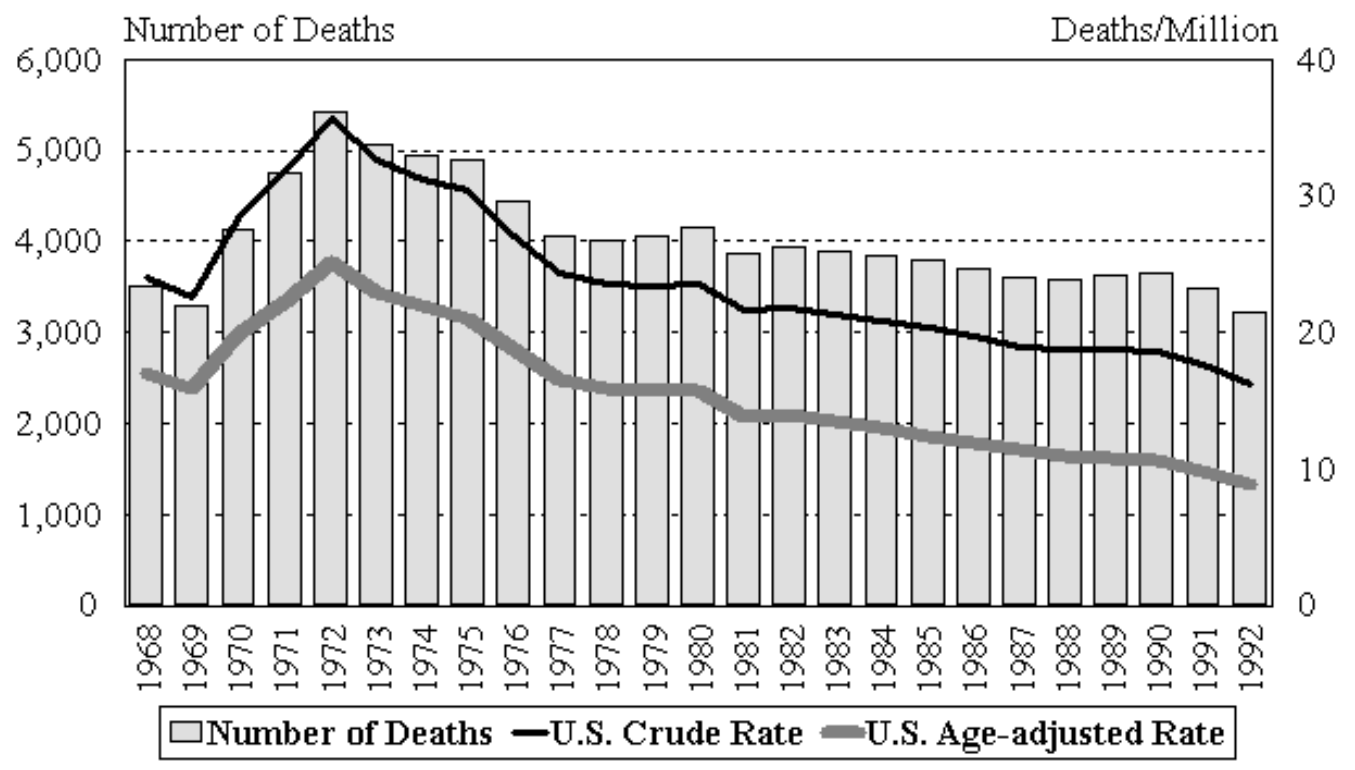

NOTE: $\quad$ See Appendix A for source description and Appendix B for methods and ICD-8 and ICD-9 codes.

SOURCE: National Center for Health Statistics multiple cause of death data. Population estimates from U.S. Bureau of the Census.

Figure 6-2. All pneumoconioses: Crude mortality rates by state, U.S. residents age 15 and over, 1991-1992

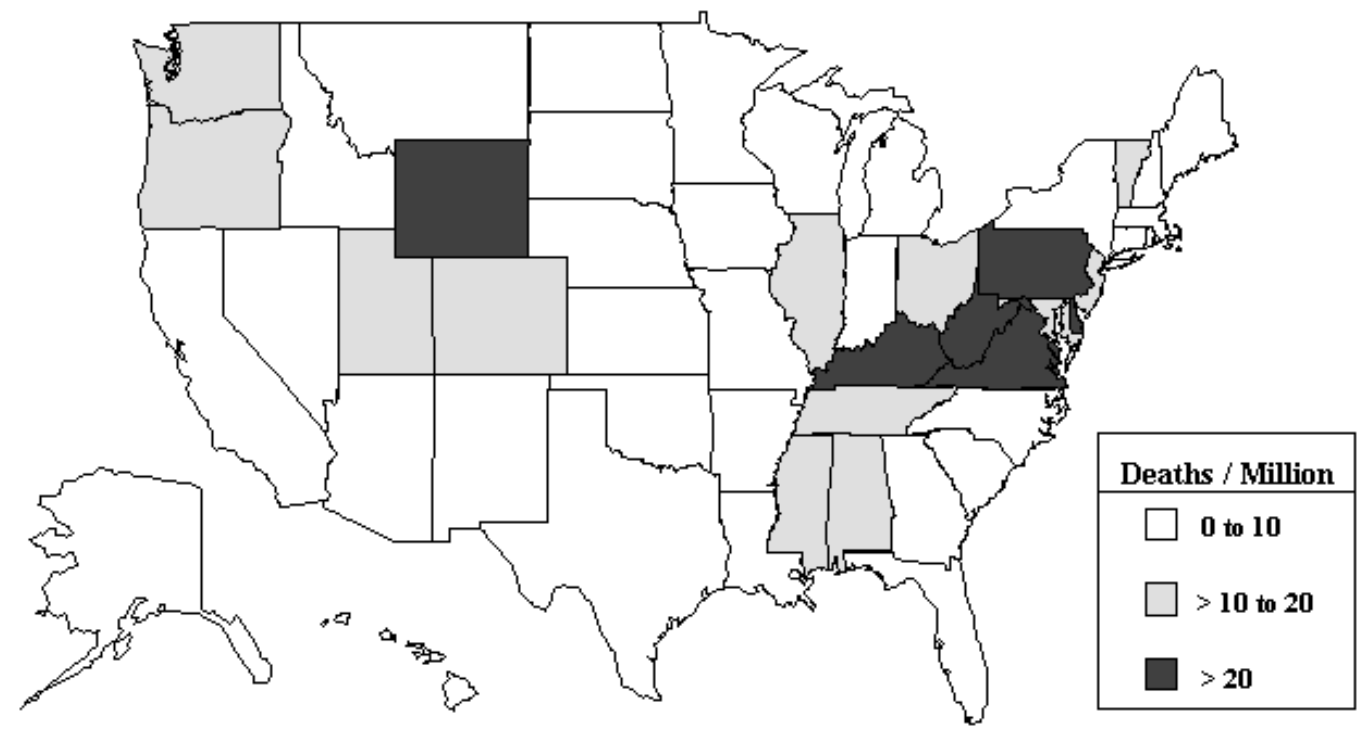

NOTE: $\quad$ See Appendix A for source description and Appendix B for methods and ICD-8 and ICD-9 codes.

SOURCE: National Center for Health Statistics multiple cause of death data. Population estimates from U.S. Bureau of the Census. 
UNITED STATES

All Pneumoconioses

Table 6-1. All pneumoconioses: Number of deaths by state, U.S. residents age 15 and over, 1968-1992

\begin{tabular}{|c|c|c|c|c|c|}
\hline State & 1968-1978 & 1979-1990 & 1991 & 1992 & TOTAL \\
\hline Alabama & 640 & 608 & 71 & 49 & 1,368 \\
\hline Alaska & 14 & 13 & 1 & 2 & 30 \\
\hline Arizona & 293 & 271 & 13 & 13 & 590 \\
\hline Arkansas & 118 & 142 & 17 & 13 & 290 \\
\hline California & 1,138 & 1,587 & 155 & 134 & 3,014 \\
\hline Colorado & 456 & 402 & 38 & 35 & 931 \\
\hline Connecticut & 245 & 251 & 19 & 27 & 542 \\
\hline Delaware & 37 & 75 & 18 & 10 & 140 \\
\hline District of Columbia & 37 & 24 & 2 & 1 & 64 \\
\hline Florida & 540 & 984 & 102 & 91 & 1,717 \\
\hline Georgia & 161 & 244 & 18 & 25 & 448 \\
\hline Hawaii & 14 & 37 & 4 & 4 & 59 \\
\hline Idaho & 96 & 68 & 6 & 6 & 176 \\
\hline Illinois & 1,250 & 1,301 & 90 & 91 & 2,732 \\
\hline Indiana & 422 & 490 & 46 & 31 & 989 \\
\hline Iowa & 110 & 143 & 11 & 19 & 283 \\
\hline Kansas & 141 & 95 & 9 & 10 & 255 \\
\hline Kentucky & 1,956 & 2,586 & 150 & 161 & 4,853 \\
\hline Louisiana & 79 & 193 & 31 & 17 & 320 \\
\hline Maine & 51 & 126 & 10 & 8 & 195 \\
\hline Maryland & 232 & 380 & 40 & 48 & 700 \\
\hline Massachusetts & 317 & 456 & 34 & 55 & 862 \\
\hline Michigan & 769 & 615 & 42 & 47 & 1,473 \\
\hline Minnesota & 167 & 181 & 10 & 26 & 384 \\
\hline Mississippi & 31 & 128 & 29 & 27 & 215 \\
\hline Missouri & 223 & 259 & 27 & 30 & 539 \\
\hline Montana & 145 & 97 & 2 & 7 & 251 \\
\hline Nebraska & 23 & 25 & 7 & 4 & 59 \\
\hline Nevada & 78 & 64 & 4 & 2 & 148 \\
\hline New Hampshire & 40 & 69 & 3 & 6 & 118 \\
\hline New Jersey & 948 & 1,301 & 118 & 105 & 2,472 \\
\hline New Mexico & 132 & 136 & 10 & 11 & 289 \\
\hline New York & 1,007 & 778 & 61 & 46 & 1,892 \\
\hline North Carolina & 285 & 460 & 52 & 54 & 851 \\
\hline North Dakota & 12 & 14 & 3 & - & 29 \\
\hline Ohio & 2,124 & 2,109 & 183 & 156 & 4,572 \\
\hline Oklahoma & 106 & 134 & 16 & 13 & 269 \\
\hline Oregon & 111 & 216 & 27 & 26 & 380 \\
\hline Pennsylvania & 27,017 & 19,524 & 1,143 & 1,018 & 48,702 \\
\hline Rhode Island & 31 & 72 & 1 & 7 & 111 \\
\hline South Carolina & 92 & 173 & 17 & 21 & 303 \\
\hline South Dakota & 37 & 33 & - & - & 70 \\
\hline Tennessee & 533 & 675 & 42 & 39 & 1,289 \\
\hline Texas & 258 & 575 & 112 & 76 & 1,021 \\
\hline Utah & 193 & 246 & 18 & 22 & 479 \\
\hline Vermont & 116 & 65 & 2 & 7 & 190 \\
\hline Virginia & 1,246 & 2,199 & 179 & 196 & 3,820 \\
\hline Washington & 272 & 487 & 60 & 48 & 867 \\
\hline West Virginia & 3,759 & 4,196 & 395 & 359 & 8,709 \\
\hline Wisconsin & 316 & 260 & 27 & 17 & 620 \\
\hline Wyoming & 115 & 74 & 11 & 10 & 210 \\
\hline TOTAL & 48,533 & 45,641 & 3,486 & 3,230 & 100,890 \\
\hline
\end{tabular}

- indicates no deaths listed.

NOTE: See Appendix A for source description and Appendix B for methods and ICD-8 and ICD-9 codes.

SOURCE: National Center for Health Statistics multiple cause of death data. 
Table 6-2. All pneumoconioses: Percent of deaths by condition and state, U.S. residents age 15 and over, $1968-1992$

\begin{tabular}{|c|c|c|c|c|c|}
\hline State & Asbestosis & Silicosis & $\begin{array}{c}\text { Coal workers' } \\
\text { pneumoconiosis }\end{array}$ & Byssinosis & $\begin{array}{r}\text { Unspecified/Other } \\
\text { pneumoconioses }\end{array}$ \\
\hline Alabama & 10.2 & 17.6 & 36.9 & 0.4 & 35.8 \\
\hline Alaska & 30.0 & 43.3 & 13.3 & - & 13.3 \\
\hline Arizona & 16.6 & 44.2 & 21.4 & 0.5 & 19.5 \\
\hline Arkansas & 16.2 & 22.4 & 35.2 & - & 26.9 \\
\hline California & 43.1 & 24.1 & 17.4 & 0.2 & 17.2 \\
\hline Colorado & 5.6 & 43.4 & 34.0 & - & 18.5 \\
\hline Connecticut & 27.1 & 29.3 & 19.4 & 0.2 & 25.1 \\
\hline Delaware & 45.0 & 9.3 & 34.3 & - & 13.6 \\
\hline District of Columbia & 14.1 & 39.1 & 21.9 & - & 26.6 \\
\hline Florida & 28.6 & 18.3 & 38.0 & 0.1 & 16.1 \\
\hline Georgia & 27.2 & 35.0 & 13.4 & 4.2 & 20.5 \\
\hline Hawaii & 67.8 & 15.3 & 6.8 & - & 13.6 \\
\hline Idaho & 25.6 & 63.6 & 2.8 & - & 8.5 \\
\hline Illinois & 8.3 & 13.5 & 41.0 & 0.1 & 38.0 \\
\hline Indiana & 6.7 & 23.9 & 42.4 & 0.1 & 28.1 \\
\hline Iowa & 14.1 & 20.8 & 46.6 & - & 18.7 \\
\hline Kansas & 13.3 & 36.1 & 26.7 & 0.4 & 23.9 \\
\hline Kentucky & 0.9 & 6.0 & 60.5 & - & 33.5 \\
\hline Louisiana & 50.0 & 26.9 & 6.9 & 0.6 & 17.5 \\
\hline Maine & 63.1 & 21.0 & 1.5 & - & 14.9 \\
\hline Maryland & 35.9 & 19.0 & 28.3 & 0.3 & 18.9 \\
\hline Massachusetts & 60.2 & 19.1 & 5.1 & 0.8 & 16.0 \\
\hline Michigan & 9.2 & 40.3 & 25.5 & 0.1 & 26.3 \\
\hline Minnesota & 26.6 & 51.8 & 4.4 & 0.3 & 19.3 \\
\hline Mississippi & 69.3 & 14.9 & 10.7 & - & 5.1 \\
\hline Missouri & 24.5 & 35.4 & 21.7 & - & 18.6 \\
\hline Montana & 15.5 & 68.5 & 9.2 & - & 8.0 \\
\hline Nebraska & 45.8 & 22.0 & 8.5 & - & 25.4 \\
\hline Nevada & 17.6 & 60.8 & 12.2 & - & 10.1 \\
\hline New Hampshire & 49.2 & 27.1 & 6.8 & 1.7 & 16.1 \\
\hline New Jersey & 48.7 & 16.7 & 18.8 & 0.1 & 17.2 \\
\hline New Mexico & 9.3 & 36.7 & 25.6 & - & 29.1 \\
\hline New York & 22.7 & 35.7 & 18.0 & 0.1 & 25.3 \\
\hline North Carolina & 24.7 & 34.5 & 18.9 & 8.9 & 13.9 \\
\hline North Dakota & 31.0 & 27.6 & 17.2 & - & 27.6 \\
\hline Ohio & 5.6 & 33.9 & 36.8 & 0.1 & 24.8 \\
\hline Oklahoma & 19.7 & 29.7 & 30.1 & 0.4 & 21.9 \\
\hline Oregon & 56.1 & 24.5 & 10.8 & - & 9.5 \\
\hline Pennsylvania & 2.0 & 6.9 & 81.6 & - & 10.2 \\
\hline Rhode Island & 53.2 & 24.3 & 6.3 & 1.8 & 18.9 \\
\hline South Carolina & 52.1 & 23.4 & 11.6 & 5.0 & 9.2 \\
\hline South Dakota & 4.3 & 55.7 & 18.6 & - & 21.4 \\
\hline Tennessee & 7.5 & 16.0 & 47.6 & 0.1 & 30.0 \\
\hline Texas & 52.6 & 22.6 & 12.2 & 0.3 & 13.7 \\
\hline Utah & 4.4 & 34.4 & 38.4 & - & 24.0 \\
\hline Vermont & 8.4 & 68.9 & 4.2 & 1.1 & 18.9 \\
\hline Virginia & 10.2 & 8.7 & 56.0 & 0.1 & 25.8 \\
\hline Washington & 59.2 & 24.2 & 8.8 & 0.1 & 8.7 \\
\hline West Virginia & 1.6 & 7.3 & 60.1 & - & 31.7 \\
\hline Wisconsin & 14.4 & 61.5 & 6.6 & 0.2 & 19.5 \\
\hline Wyoming & 5.7 & 11.0 & 44.8 & - & 39.5 \\
\hline TOTAL & 10.0 & 14.2 & 58.6 & 0.2 & 17.9 \\
\hline
\end{tabular}

NOTE: $\quad$ Percentages may total more than $100 \%$ due to deaths with multiple pneumoconioses. See Appendix A for source description and Appendix B for methods and ICD-8 and ICD-9 codes.

SOURCE: National Center for Health Statistics multiple cause of death data. 
Table 6-3. All pneumoconioses: Total number of deaths, crude and age-adjusted mortality rates (per 1,000,000 population), and total years of potential life lost (YPLL) to life expectancy, by state, U.S. residents age 15 and over, 1988-1992

\begin{tabular}{|c|c|c|c|c|c|c|c|c|c|c|}
\hline \multirow[b]{2}{*}{ State } & \multirow{2}{*}{$\begin{array}{r}\text { Total } \\
\text { deaths }\end{array}$} & \multirow[b]{2}{*}{ Rank } & \multicolumn{2}{|c|}{ Crude mortality } & \multicolumn{2}{|c|}{ Age-adjusted mortality } & \multicolumn{4}{|c|}{ YPLL to life expectancy } \\
\hline & & & Rate & Rank & Rate & Rank & Years & Rank & Years/death & Rank \\
\hline Alabama & 303 & 12 & 19.16 & 9 & 9.89 & 10 & 3,388 & 12 & 11.2 & 42 \\
\hline Alaska & 7 & 49 & 3.48 & 47 & 5.61 & 23 & 100 & 50 & 14.3 & 3 \\
\hline Arizona & 75 & 31 & 5.28 & 40 & 3.15 & 40 & 933 & 31 & 12.4 & 17 \\
\hline Arkansas & 59 & 37 & 6.43 & 33 & 3.16 & 39 & 699 & 36 & 11.8 & 28 \\
\hline California & 688 & 6 & 5.93 & 37 & 4.12 & 33 & 8,612 & 6 & 12.5 & 15 \\
\hline Colorado & 175 & 20 & 13.63 & 12 & 8.39 & 11 & 1,818 & 20 & 10.4 & 47 \\
\hline Connecticut & 107 & 28 & 8.07 & 28 & 4.34 & 32 & 1,204 & 28 & 11.3 & 40 \\
\hline Delaware & 65 & 33 & 24.60 & 5 & 16.54 & 5 & 895 & 32 & 13.8 & 6 \\
\hline District of Columbia & 6 & 51 & 2.37 & 51 & 1.84 & 49 & 126 & 49 & 21.0 & 1 \\
\hline Florida & 510 & 8 & 9.66 & 22 & 4.41 & 31 & 6,229 & 8 & 12.2 & 24 \\
\hline Georgia & 112 & 25 & 4.44 & 45 & 3.37 & 37 & 1,492 & 24 & 13.3 & 7 \\
\hline Hawaii & 20 & 46 & 4.58 & 43 & 2.91 & 44 & 227 & 46 & 11.4 & 36 \\
\hline Idaho & 36 & 41 & 9.62 & 23 & 5.43 & 24 & 408 & 41 & 11.3 & 38 \\
\hline Illinois & 476 & 9 & 10.64 & 21 & 5.95 & 20 & 5,544 & 9 & 11.6 & 32 \\
\hline Indiana & 203 & 19 & 9.38 & 24 & 5.20 & 26 & 2,350 & 19 & 11.6 & 33 \\
\hline Iowa & 64 & 34 & 5.90 & 38 & 2.67 & 45 & 701 & 35 & 11.0 & 44 \\
\hline Kansas & 41 & 38 & 4.28 & 46 & 2.25 & 48 & 513 & 38 & 12.5 & 16 \\
\hline Kentucky & 871 & 4 & 60.22 & 3 & 34.22 & 3 & 10,380 & 4 & 11.9 & 26 \\
\hline Louisiana & 123 & 23 & 7.73 & 29 & 5.41 & 25 & 1,751 & 21 & 14.2 & 4 \\
\hline Maine & 63 & 36 & 13.00 & 13 & 7.11 & 15 & 725 & 34 & 11.5 & 34 \\
\hline Maryland & 206 & 18 & 10.84 & 20 & 7.65 & 13 & 2,642 & 17 & 12.8 & 10 \\
\hline Massachusetts & 223 & 17 & 9.16 & 26 & 4.94 & 27 & 2,514 & 18 & 11.3 & 39 \\
\hline Michigan & 245 & 14 & 6.78 & 31 & 4.12 & 33 & 3,029 & 15 & 12.4 & 19 \\
\hline Minnesota & 81 & 30 & 4.79 & 41 & 2.98 & 43 & 1,023 & 30 & 12.6 & 12 \\
\hline Mississippi & 113 & 24 & 11.59 & 17 & 7.10 & 16 & 1,423 & 25 & 12.6 & 13 \\
\hline Missouri & 127 & 21 & 6.34 & 34 & 3.36 & 38 & 1,560 & 22 & 12.3 & 21 \\
\hline Montana & 37 & 40 & 12.10 & 16 & 6.79 & 17 & 454 & 39 & 12.3 & 22 \\
\hline Nebraska & 20 & 46 & 3.29 & 48 & 1.68 & 50 & 225 & 47 & 11.3 & 41 \\
\hline Nevada & 22 & 45 & 4.59 & 42 & 3.15 & 40 & 259 & 45 & 11.8 & 30 \\
\hline New Hampshire & 26 & 44 & 5.96 & 36 & 3.96 & 35 & 321 & 43 & 12.3 & 20 \\
\hline New Jersey & 643 & 7 & 20.68 & 7 & 11.88 & 7 & 7,801 & 7 & 12.1 & 25 \\
\hline New Mexico & 64 & 34 & 11.25 & 19 & 6.52 & 19 & 678 & 37 & 10.6 & 45 \\
\hline New York & 320 & 11 & 4.45 & 44 & 2.56 & 46 & 3,913 & 11 & 12.2 & 23 \\
\hline North Carolina & 244 & 15 & 9.21 & 25 & 5.92 & 21 & 3,113 & 14 & 12.8 & 11 \\
\hline North Dakota & 8 & 48 & 3.27 & 49 & 2.33 & 47 & 155 & 48 & 19.4 & 2 \\
\hline Ohio & 840 & 5 & 19.77 & 8 & 10.96 & 9 & 9,882 & 5 & 11.8 & 31 \\
\hline Oklahoma & 73 & 32 & 5.98 & 35 & 3.00 & 42 & 839 & 33 & 11.5 & 35 \\
\hline Oregon & 126 & 22 & 11.27 & 18 & 5.65 & 22 & 1,400 & 26 & 11.1 & 43 \\
\hline Pennsylvania & 5,926 & 1 & 124.28 & 2 & 54.98 & 2 & 62,129 & 1 & 10.5 & 46 \\
\hline Rhode Island & 35 & 42 & 8.61 & 27 & 4.78 & 30 & 435 & 40 & 12.4 & 18 \\
\hline South Carolina & 97 & 29 & 7.12 & 30 & 4.81 & 28 & 1,285 & 27 & 13.2 & 8 \\
\hline South Dakota & 7 & 49 & 2.66 & 50 & 1.14 & 51 & 69 & 51 & 9.9 & 49 \\
\hline Tennessee & 265 & 13 & 13.69 & 11 & 7.76 & 12 & 3,129 & 13 & 11.8 & 29 \\
\hline Texas & 426 & 10 & 6.59 & 32 & 4.81 & 28 & 5,537 & 10 & 13.0 & 9 \\
\hline Utah & 111 & 26 & 18.67 & 10 & 11.88 & 7 & 1,119 & 29 & 10.1 & 48 \\
\hline Vermont & 28 & 43 & 12.67 & 15 & 6.73 & 18 & 274 & 44 & 9.8 & 50 \\
\hline Virginia & 980 & 3 & 39.76 & 4 & 28.00 & 4 & 12,282 & 3 & 12.5 & 14 \\
\hline Washington & 243 & 16 & 12.77 & 14 & 7.54 & 14 & 2,755 & 16 & 11.3 & 37 \\
\hline West Virginia & 1,878 & 2 & 262.60 & 1 & 132.76 & 1 & 22,378 & 2 & 11.9 & 27 \\
\hline Wisconsin & 109 & 27 & 5.73 & 39 & 3.64 & 36 & 1,513 & 23 & 13.9 & 5 \\
\hline Wyoming & 41 & 38 & 24.17 & 6 & 12.77 & 6 & 382 & 42 & 9.3 & 51 \\
\hline
\end{tabular}

\footnotetext{
NOTE: $\quad$ Ranks are based on unrounded values. See Appendix A for source description and Appendix B for methods and ICD-8 and ICD-9 codes.
} SOURCE: National Center for Health Statistics multiple cause of death data. Population estimates from U.S. Bureau of the Census. 
Table 6-4. All pneumoconioses: Proportionate mortality ratio (PMR) by usual occupation, selected states and years, U.S. residents age 15 and over, 1985-1992

\begin{tabular}{|c|c|c|c|c|c|}
\hline \multirow[b]{2}{*}{$\mathrm{COC}$} & \multirow[b]{2}{*}{ Occupation } & \multirow{2}{*}{$\begin{array}{l}\text { Number } \\
\text { of deaths }\end{array}$} & \multirow[b]{2}{*}{ PMR } & \multicolumn{2}{|c|}{$95 \%$ confidence interval } \\
\hline & & & & LCL & UCL \\
\hline 616 & Mining machine operators & 3,506 & 62.26 & 60.21 & 64.38 \\
\hline 593 & Insulation workers & 111 & 61.57 & 50.06 & 74.90 \\
\hline 725 & Miscellaneous metal and plastic machine operators & 13 & 23.96 & 12.74 & 40.96 \\
\hline 615 & Explosives workers & 8 & 17.15 & 7.39 & 33.76 \\
\hline 643 & Boilermakers & 56 & 12.64 & 9.36 & 16.68 \\
\hline 768 & Crushing and grinding machine operators & 31 & 9.19 & 6.21 & 13.13 \\
\hline 675 & Hand molders and shapers, except jewelers & 20 & 8.75 & 5.34 & 13.52 \\
\hline 046 & Mining engineer & 6 & 7.61 & 2.79 & 16.58 \\
\hline 853 & Excavating and loading machine operators & 13 & 7.40 & 3.94 & 12.65 \\
\hline 719 & Molding and casting machine operators & 52 & 6.83 & 5.06 & 9.01 \\
\hline 646 & Lay-out workers & 9 & 6.65 & 3.05 & 12.62 \\
\hline 613 & Supervisors, extractive occupations & 13 & 6.13 & 3.26 & 10.48 \\
\hline 787 & Hand molding, casting, and forming occupations & 14 & 5.87 & 3.21 & 9.85 \\
\hline 617 & Mining occupations, n.e.c. & 22 & 4.98 & 3.11 & 7.55 \\
\hline 585 & Plumbers, pipefitters, and steamfitters & 174 & 4.76 & 4.05 & 5.55 \\
\hline 653 & Sheet metal workers & 44 & 3.52 & 2.51 & 4.80 \\
\hline 859 & Miscellaneous material moving equipment operators & 21 & 3.42 & 2.11 & 5.23 \\
\hline 575 & Electricians & 137 & 3.31 & 2.74 & 3.96 \\
\hline 516 & Heavy equipment mechanics & 32 & 3.21 & 2.17 & 4.59 \\
\hline 824 & Locomotive operating occupations & 27 & 2.70 & 1.78 & 3.93 \\
\hline 584 & Plasterers & 7 & 2.68 & 1.08 & 5.53 \\
\hline 783 & Welders and cutters & 95 & 2.62 & 2.11 & 3.22 \\
\hline 534 & Heating, air conditioning, and refrigeration mechanics & 11 & 2.52 & 1.26 & 4.51 \\
\hline 599 & Construction trades, n.e.c. & 17 & 2.49 & 1.45 & 3.98 \\
\hline 849 & Crane and tower operators & 28 & 2.43 & 1.62 & 3.51 \\
\hline 544 & Millwrights & 28 & 2.35 & 1.57 & 3.40 \\
\hline 563 & Brickmasons and stonemasons & 45 & 2.29 & 1.67 & 3.07 \\
\hline 759 & Painting, paint spray machine operators & 16 & 2.25 & 1.29 & 3.65 \\
\hline 766 & Furnace, kiln, oven operators, except food & 28 & 2.22 & 1.48 & 3.21 \\
\hline 518 & Industrial machinery repairers & 39 & 2.00 & 1.39 & 2.78 \\
\hline 709 & Grinding, abrading, buffing, and polishing machine operators & 18 & 1.96 & 1.16 & 3.10 \\
\hline 547 & Specified mechanics and repairers, n.e.c. & 21 & 1.86 & 1.15 & 2.84 \\
\hline 844 & Operating engineers & 53 & 1.85 & 1.37 & 2.44 \\
\hline 779 & Machine operators, not specified & 117 & 1.70 & 1.38 & 2.07 \\
\hline 549 & Not specified mechanics and repairers & 29 & 1.62 & 1.09 & 2.33 \\
\hline 889 & Laborers, except construction & 365 & 1.45 & 1.30 & 1.61 \\
\hline 869 & Construction laborers & 110 & 1.37 & 1.11 & 1.67 \\
\hline 633 & Supervisors, production occupations & 122 & 1.33 & 1.10 & 1.59 \\
\hline 637 & Machinists & 106 & 1.29 & 1.05 & 1.57 \\
\hline
\end{tabular}

COC - 1980 Census Occupation Code n.e.c. - not elsewhere classified LCL - lower confidence limit UCL - upper confidence limit

NOTE: $\quad$ See Appendix A for source description, Appendix B for methods and ICD -8 and ICD -9 codes, and Appendix C for list of 25 states reporting usual occupation and years reporting.

SOURCE: National Center for Health Statistics multiple cause of death data. 
Table 6-5. All pneumoconioses: Proportionate mortality ratio (PMR) by usual industry, selected states and years, U.S. residents age 15 and over, 1985-1992

\begin{tabular}{llrrrr}
\hline & & & & & $95 \%$ confidence interval \\
\cline { 4 - 6 } CIC & Industry & $\begin{array}{r}\text { Number } \\
\text { of deaths }\end{array}$ & PMR & LCL & UCL \\
\hline 041 & Coal mining & 3,694 & 62.50 & 60.44 & 64.63 \\
262 & Miscellaneous nonmetallic mineral and stone products & 128 & 15.16 & 12.57 & 18.13 \\
040 & Metal mining & 107 & 11.43 & 9.29 & 13.91 \\
360 & Ship and boat building and repairing & 170 & 9.58 & 8.15 & 11.18 \\
050 & Nonmetallic mining and quarrying, except fuel & 66 & 8.06 & 6.15 & 10.37 \\
271 & Iron and steel foundries & 72 & 5.58 & 4.36 & 7.05 \\
252 & Structural clay products & 33 & 5.29 & 3.57 & 7.56 \\
261 & Pottery and related products & 27 & 4.56 & 3.00 & 6.64 \\
192 & Industrial and miscellaneous chemicals & 95 & 2.70 & 2.18 & 3.32 \\
282 & Fabricated structural metal products & 44 & 2.08 & 1.49 & 2.83 \\
270 & Blast furnaces, steelworks, rolling and finishing mills & 179 & 1.98 & 1.69 & 2.31 \\
300 & Miscellaneous fabricated metal products & 40 & 1.81 & 1.29 & 2.47 \\
060 & Construction & 754 & 1.68 & 1.56 & 1.81 \\
211 & Other rubber products, and plastics footwear and belting & 33 & 1.65 & 1.11 & 2.36 \\
250 & Glass and glass products & 31 & 1.54 & 1.04 & 2.20 \\
392 & Not specified manufacturing industries & 148 & 1.24 & 1.04 & 1.46 \\
\hline
\end{tabular}

CIC - 1980 Census Industry Code $\quad$ LCL - lower confidence limit $\quad$ UCL - upper confidence limit

NOTE: $\quad$ See Appendix A for source description, Appendix B for methods and ICD-8 and ICD-9 codes, and Appendix C for list of 25 states reporting usual industry and years

SOURCE: $\quad$ National Center for Health Statistics multiple cause of death data. 
Figure 6-3. All pneumoconioses: Total number of deaths by county,

U.S. residents age 15 and over, 1983-1992

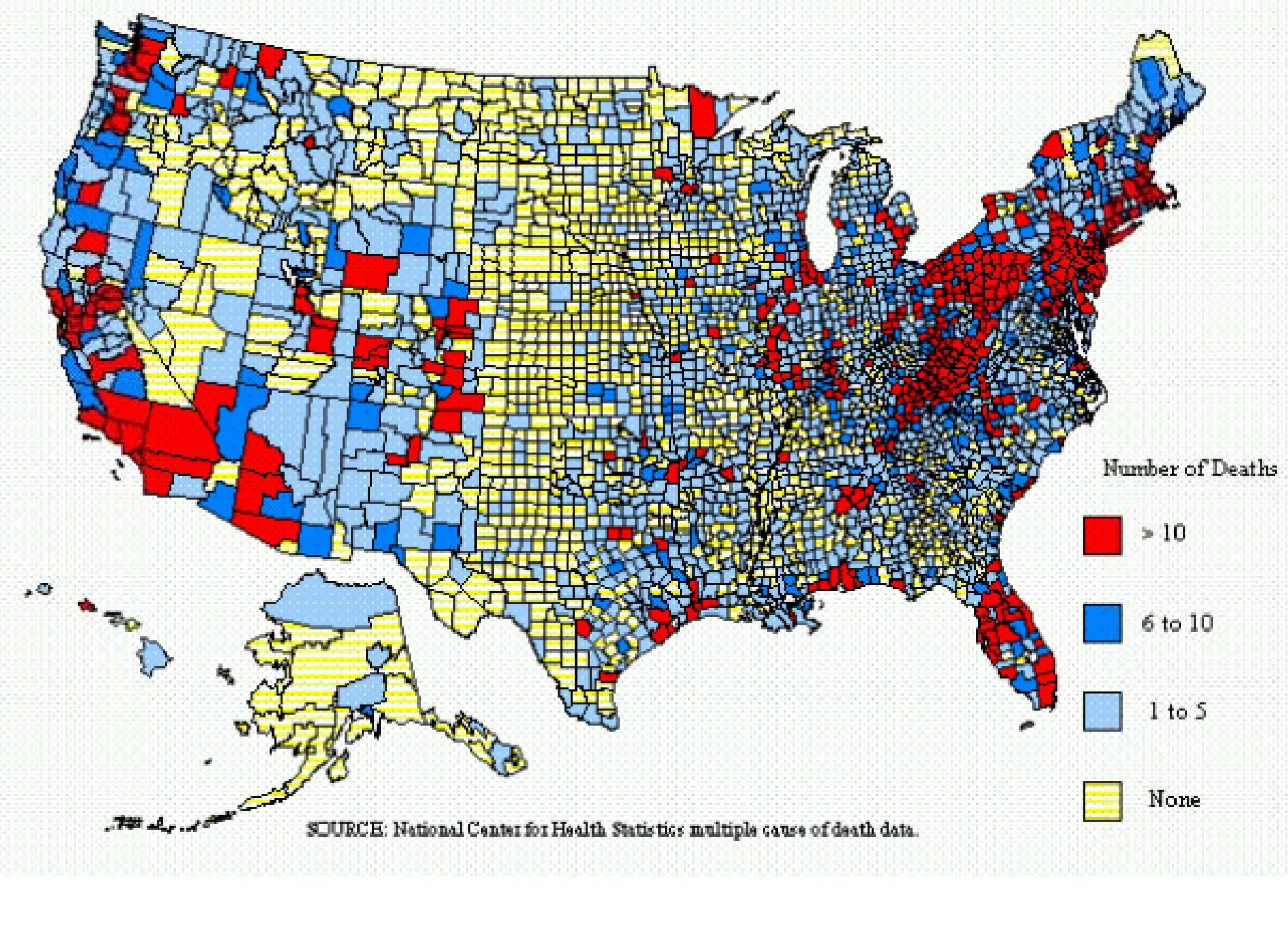


Figure 6-4. All pneumoconioses: Age-adjusted mortality rates by county,

U.S. residents age 15 and over, 1983-1992

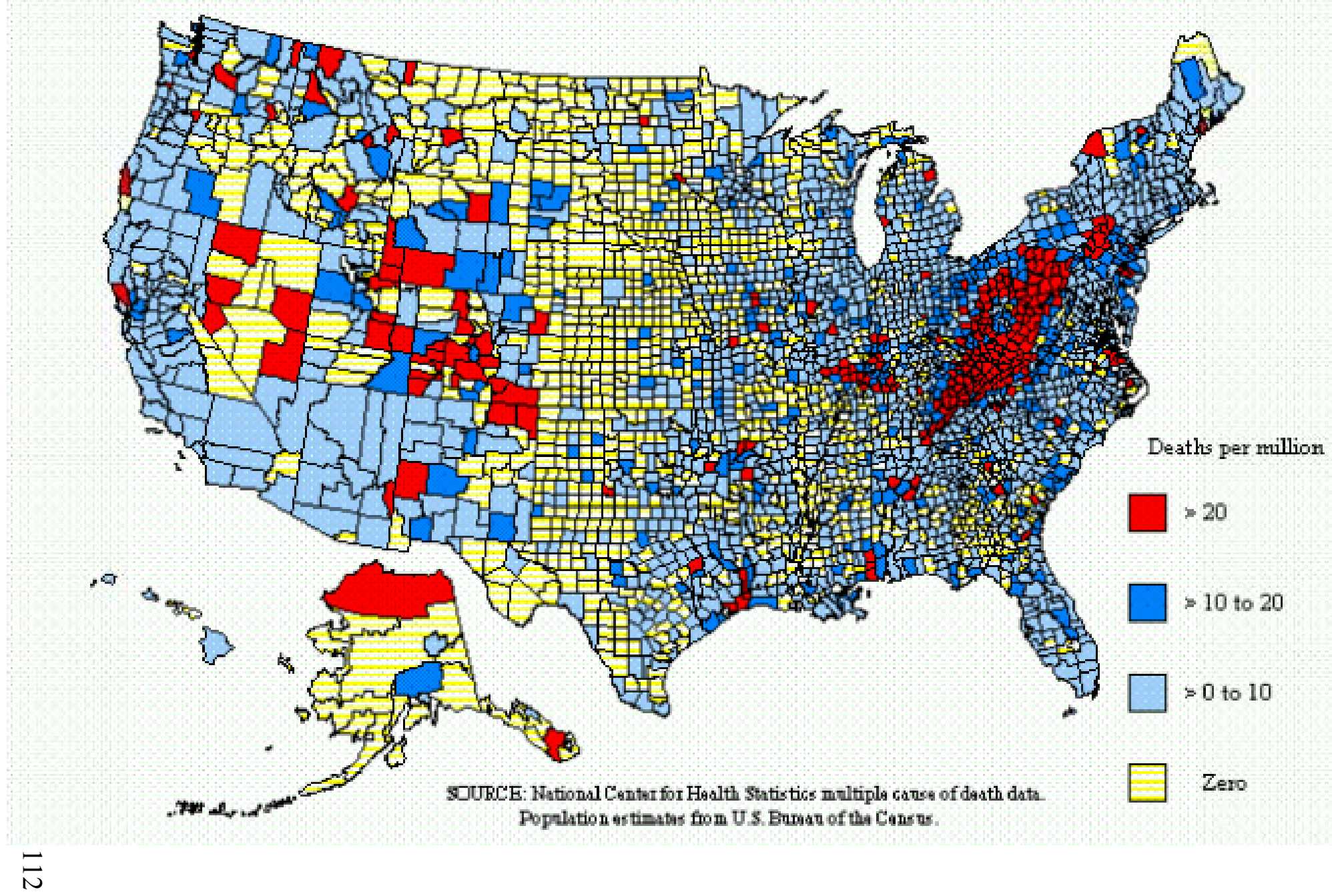


Figure 6-5. Pneumoconiotic agents: Number of MSHA and OSHA inspector samples and percent exceeding the permissible exposure limit (PEL), 1970-1994

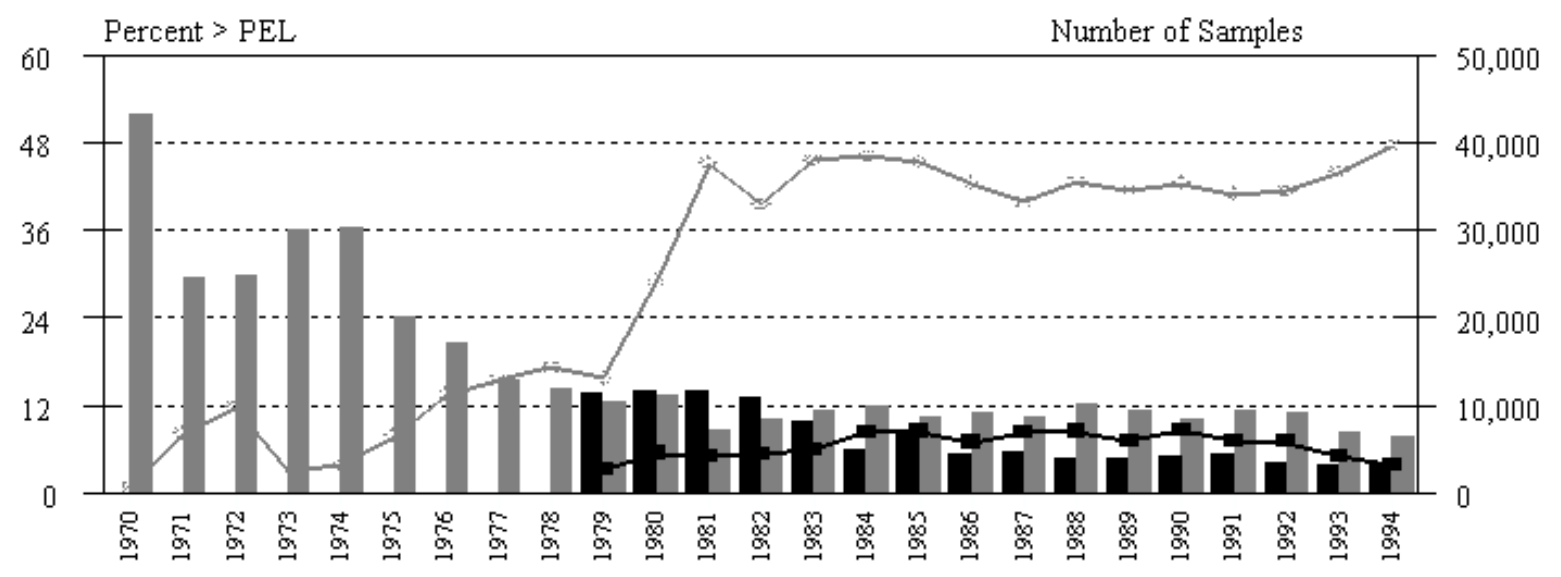

\begin{tabular}{ll} 
OSHA Percent > PEL & MSHA Percent > PEL \\
- \#OSHA Samples & \#MSHA Samples \\
\hline
\end{tabular}

NOTE: See Appendix A for source description, Appendix B for methods, and Appendix D for agents.

SOURCE: Mine Safety and Health Administration: respirable coal mine dust data and respirable coal mine quartz data. Bureau of Mines: Mine Inspection Data Analysis System. Occupational Safety and Health Administration: Integrated Management Information System.

Figure 6-6. Pneumoconiotic agents: Number of MSHA and OSHA inspector samples and average severity levels, 1970-1994

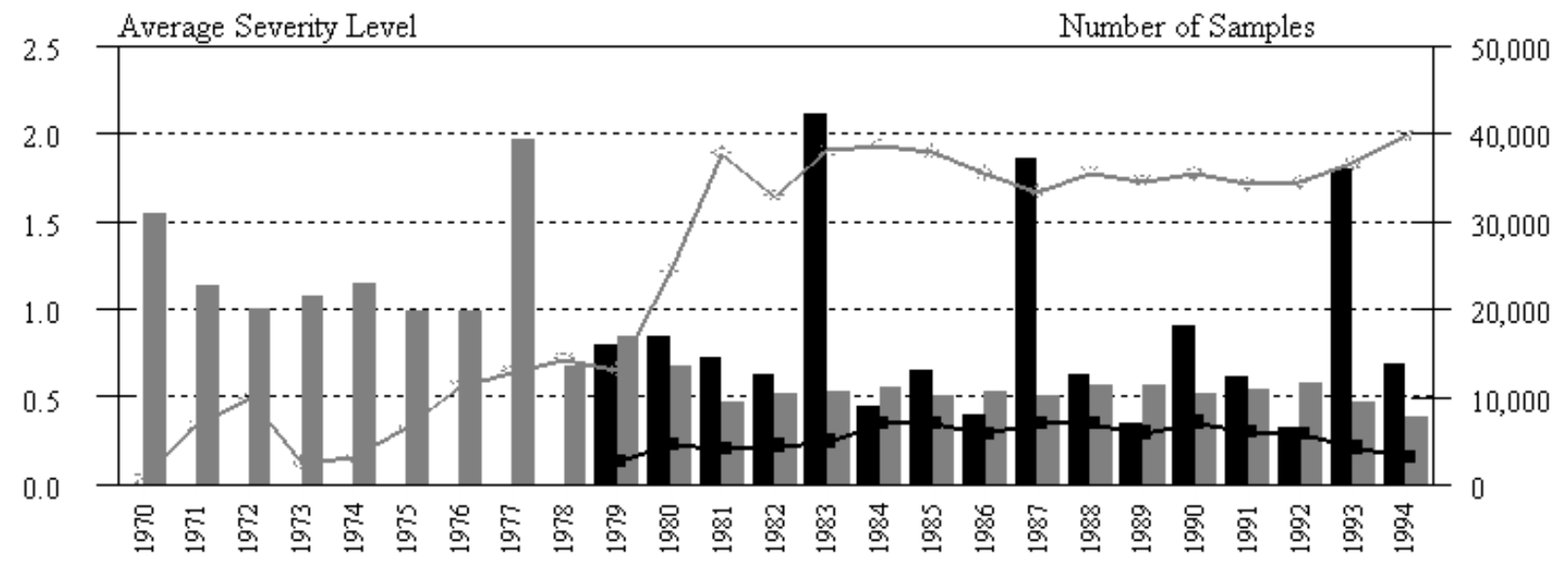

\begin{tabular}{ll|}
\hline OSHA Severity Levels & MSHA Severity Levels \\
-\# OSHA Samples & - \# MSHA Samples \\
\hline
\end{tabular}

NOTE: See Appendix A for source description, Appendix B for methods, and Appendix D for agents.

SOURCE: Mine Safety and Health Administration: respirable coal mine dust data and respirable coal mine quartz data. Bureau of Mines: Mine Inspection Data Analysis System. Occupational Safety and Health Administration: Integrated Management Information System. 
Table 6-6. Pneumoconiotic agents: Number of MSHA and OSHA inspector samples, percent exceeding the permissible exposure limit (PEL) and average severity level, by industry, 1993-1994

\begin{tabular}{|c|c|c|c|c|}
\hline CIC & $\begin{array}{l}\text { Industries most frequently recorded on } \\
\text { 1991-1992 death certificates with any pneumoconiosis }\end{array}$ & $\begin{array}{r}\text { Number of } \\
\text { samples } \\
\end{array}$ & $\%>$ PEL & $\begin{array}{r}\text { Average } \\
\text { severity }\end{array}$ \\
\hline 041 & Coal Mining & 45,132 & 9.6 & 0.47 \\
\hline 060 & Construction & 844 & 5.6 & 1.51 \\
\hline 270 & Blast furnaces, steelworks, rolling and finishing mills & 139 & 0.0 & 0.02 \\
\hline 360 & Ship and boat building and repairing & 101 & 7.9 & 1.00 \\
\hline 392 & Not specified manufacturing industries & 0 & - & - \\
\hline 262 & Miscellane ous nonmetallic mineral and stone products & 181 & 8.3 & 0.34 \\
\hline 192 & Industrial and miscellaneous chemicals & 43 & 4.7 & 4.19 \\
\hline 400 & Railroads & 2 & 0.0 & 0.03 \\
\hline 901 & General government, n.e.c. & 20 & 0.0 & 0.05 \\
\hline \multirow[t]{4}{*}{142} & Yarn, thread, and fabric mills & 77 & 18.2 & 0.69 \\
\hline & All other industries & 36,857 & 5.4 & 0.53 \\
\hline & Industry not reported & 79 & 2.5 & 0.13 \\
\hline & TOTAL & 83,475 & 7.7 & 0.51 \\
\hline
\end{tabular}

CIC - 1980 Census Industry Code

n.e.c. - not elsewhere classified

- indicates incalculable field

NOTE: See Appendix A for source description, Appendix B for methods, Appendix C for list of 25 states reporting usual industry and years reporting, and Appendix D for agents.

SOURCE: Mine Safety and Health Administration: respirable coal mine dust data and respirable coal mine quartz data. Bureau of Mines: Mine Inspection Data Analysis System. Occupational Safety and Health Administration: Integrated Management Information System. National Center for Health Statistics: multiple cause of death data.

Table 6-7. Pneumoconiotic agents: Number of MSHA and OSHA inspector samples, percent exceeding the permissible exposure limit (PEL) and average severity level, by industry, 1993-1994

\begin{tabular}{|c|c|c|c|c|}
\hline CIC & Industries most frequently sampled in 1993-1994 & $\begin{array}{r}\text { Number of } \\
\text { samples } \\
\end{array}$ & $\%>$ PEL & $\begin{array}{r}\text { Average } \\
\text { severity }\end{array}$ \\
\hline 041 & Coal mining & 45,132 & 9.6 & 0.47 \\
\hline 050 & Nonmetallic mining & 27,900 & 5.7 & 0.38 \\
\hline 040 & Metal mining & 3,069 & 6.6 & 0.28 \\
\hline 060 & Construction & 844 & 5.6 & 1.51 \\
\hline 300 & Miscellaneous fabricated metal products & 602 & 4.3 & 1.59 \\
\hline 280 & Other primary metal industries & 587 & 3.6 & 0.18 \\
\hline 271 & Iron and steel foundries & 570 & 9.5 & 1.24 \\
\hline 282 & Fabricated structural metal products & 496 & 2.6 & 10.29 \\
\hline 351 & Motor vehicles and motor vehicle equipment & 458 & 1.3 & 0.26 \\
\hline \multirow[t]{4}{*}{331} & Machinery, except electrical, n.e.c. & 294 & 3.1 & 0.15 \\
\hline & All other industries & 3,444 & 3.3 & 0.41 \\
\hline & Industry not reported & 79 & 2.5 & 0.13 \\
\hline & TOTAL & 83,475 & 7.7 & 0.51 \\
\hline
\end{tabular}

CIC - 1980 Census Industry Code n.e.c. - not elsewhere classified

NOTE: See Appendix A for source description, Appendix B for methods, and Appendix D for agents.

SOURCE: Mine Safety and Health Administration: respirable coal mine dust data and respirable coal mine quartz data. Bureau of Mines: Mine Inspection Data Analysis System. Occupational Safety and Health Administration: Integrated Management Information System. 
Table 6-8 (page 1 of 2). Pneumoconiotic agents: Number of MSHA inspector samples, percent exceeding the permissible exposure limit (PEL) and average severity levels (Avg. Sev.), by state, 1970-1994

\begin{tabular}{|c|c|c|c|c|c|c|c|c|c|c|c|c|}
\hline \multirow[b]{3}{*}{ State } & \multicolumn{4}{|c|}{ 1970-1984 } & \multicolumn{4}{|c|}{ 1985-1994 } & \multicolumn{4}{|c|}{ 1993-1994 } \\
\hline & \multicolumn{2}{|c|}{ Total samples } & \multicolumn{2}{|c|}{ Samples > PEL } & \multicolumn{2}{|c|}{ Total samples } & \multicolumn{2}{|c|}{ Samples > PEL } & \multicolumn{2}{|c|}{ Total samples } & \multicolumn{2}{|c|}{ Samples > PEL } \\
\hline & Number & $\begin{array}{l}\text { Avg. } \\
\text { Sev. }\end{array}$ & $\%$ & $\begin{array}{r}\text { Avg. } \\
\text { Sev. }\end{array}$ & Number & $\begin{array}{l}\text { Avg. } \\
\text { Sev. }\end{array}$ & $\%$ & $\begin{array}{l}\text { Avg. } \\
\text { Sev. }\end{array}$ & Number & $\begin{array}{l}\text { Avg. } \\
\text { Sev. }\end{array}$ & $\%$ & $\begin{array}{r}\text { Avg. } \\
\text { Sev. }\end{array}$ \\
\hline Alabama & 5,472 & 0.56 & 12.4 & 2.29 & 10,394 & 0.50 & 11.1 & 2.00 & 2,990 & 0.44 & 8.7 & 2.03 \\
\hline Alaska & 107 & 0.54 & 9.3 & 3.40 & 303 & 0.71 & 7.6 & 7.72 & 141 & 0.25 & 2.8 & 5.25 \\
\hline Arizona & 3,997 & 1.06 & 15.3 & 5.35 & 2,546 & 0.75 & 14.3 & 3.78 & 800 & 0.44 & 9.6 & 2.62 \\
\hline Arkansas & 954 & 1.40 & 33.4 & 3.60 & 1,545 & 0.64 & 15.5 & 3.02 & 563 & 0.35 & 9.1 & 2.26 \\
\hline California & 2,068 & 1.59 & 14.7 & 9.20 & 4,912 & 0.55 & 10.7 & 3.34 & 1,238 & 0.28 & 4.0 & 3.56 \\
\hline Colorado & 5,711 & 0.89 & 22.7 & 2.76 & 7,635 & 0.60 & 14.3 & 2.27 & 1,292 & 0.51 & 11.4 & 2.08 \\
\hline Connecticut & 410 & 1.11 & 22.2 & 4.01 & 439 & 0.48 & 12.3 & 2.66 & 76 & 0.18 & 2.6 & 2.27 \\
\hline Delaware & 10 & 0.19 & 0.0 & - & 46 & 0.23 & 0.0 & - & 3 & 0.02 & 0.0 & - \\
\hline District of Columbia & 0 & & - & - & 0 & - & - & - & 0 & - & - & - \\
\hline Florida & 1,153 & 4.30 & 5.2 & 80.01 & 1,855 & 0.16 & 2.4 & 2.51 & 511 & 0.09 & 1.8 & 1.42 \\
\hline Georgia & 2,426 & 0.57 & 12.7 & 2.63 & 3,494 & 0.44 & 9.1 & 3.08 & 832 & 0.38 & 6.4 & 4.01 \\
\hline Hawaii & 6 & 0.18 & 0.0 & - & 146 & 0.18 & 2.1 & 5.85 & 88 & 0.19 & 1.1 & 13.99 \\
\hline Idaho & 1,999 & 1.08 & 15.9 & 5.11 & 1,900 & 0.68 & 9.3 & 5.66 & 663 & 0.22 & 4.5 & 2.11 \\
\hline Illinois & 11,544 & 0.85 & 20.1 & 2.75 & 14,338 & 0.51 & 10.0 & 1.82 & 3,320 & 0.38 & 5.3 & 1.83 \\
\hline Indiana & 4,796 & 0.37 & 6.7 & 2.70 & 4,939 & 0.34 & 6.0 & 2.41 & 1,221 & 0.30 & 5.2 & 1.80 \\
\hline Iowa & 1,231 & 0.51 & 10.2 & 2.63 & 1,343 & 0.24 & 5.1 & 1.76 & 370 & 0.16 & 1.1 & 1.95 \\
\hline Kansas & 1,640 & 0.51 & 13.2 & 1.97 & 1,516 & 0.42 & 8.0 & 2.68 & 428 & 0.26 & 5.1 & 1.85 \\
\hline Kentucky & 36,519 & 0.63 & 14.6 & 2.42 & 67,710 & 0.56 & 12.3 & 2.38 & 14,468 & 0.49 & 10.7 & 2.06 \\
\hline Louisiana & 372 & 0.68 & 12.4 & 4.23 & 1,922 & 0.16 & 3.1 & 2.36 & 834 & 0.14 & 2.6 & 1.89 \\
\hline Maine & 441 & 0.59 & 15.4 & 2.03 & 282 & 0.31 & 6.0 & 1.95 & 79 & 0.23 & 3.8 & 1.42 \\
\hline Maryland & 1,290 & 0.46 & 7.8 & 2.62 & 1,917 & 0.40 & 6.9 & 2.69 & 478 & 0.42 & 6.9 & 2.93 \\
\hline Massachusetts & 574 & 1.46 & 19.0 & 6.16 & 621 & 0.38 & 9.0 & 2.39 & 168 & 0.29 & 6.5 & 2.01 \\
\hline Michigan & 2,662 & 3.49 & 11.0 & 29.65 & 3,328 & 0.46 & 9.9 & 3.03 & 1,078 & 0.32 & 7.8 & 2.20 \\
\hline Minnesota & 4,947 & 0.47 & 8.1 & 3.54 & 3,723 & 0.32 & 7.0 & 2.45 & 863 & 0.27 & 5.2 & 2.28 \\
\hline Mississippi & 433 & 0.53 & 7.6 & 4.64 & 932 & 0.32 & 6.4 & 2.88 & 371 & 0.46 & 8.1 & 3.60 \\
\hline Missouri & 3,553 & 1.18 & 13.1 & 7.25 & 5,487 & 0.89 & 9.2 & 7.48 & 1,769 & 0.30 & 5.1 & 2.73 \\
\hline Montana & 1,786 & 0.47 & 10.4 & 2.43 & 2,962 & 0.52 & 9.6 & 3.54 & 538 & 0.38 & 8.2 & 2.52 \\
\hline
\end{tabular}


Table 6-8 (page 2 of 2). Pneumoconiotic agents: Number of MSHA inspector samples, percent exceeding the permissible exposure limit (PEL) and average severity levels (Avg. Sev.), by state, 1970-1994

\begin{tabular}{|c|c|c|c|c|c|c|c|c|c|c|c|c|}
\hline \multirow[b]{3}{*}{ State } & \multicolumn{4}{|c|}{ 1970-1984 } & \multicolumn{4}{|c|}{ 1985-1994 } & \multicolumn{4}{|c|}{ 1993-1994 } \\
\hline & \multicolumn{2}{|c|}{ Total samples } & \multicolumn{2}{|c|}{ Samples > PEL } & \multicolumn{2}{|c|}{ Total samples } & \multicolumn{2}{|c|}{ Samples > PEL } & \multicolumn{2}{|c|}{ Total samples } & \multicolumn{2}{|c|}{ Samples $>$ PEL } \\
\hline & Number & $\begin{array}{l}\text { Avg. } \\
\text { Sev. }\end{array}$ & $\%$ & $\begin{array}{l}\text { Avg. } \\
\text { Sev. }\end{array}$ & Number & $\begin{array}{l}\text { Avg. } \\
\text { Sev. }\end{array}$ & $\%$ & $\begin{array}{l}\text { Avg. } \\
\text { Sev. }\end{array}$ & Number & $\begin{array}{l}\text { Avg. } \\
\text { Sev. }\end{array}$ & $\%$ & $\begin{array}{l}\text { Avg. } \\
\text { Sev. }\end{array}$ \\
\hline Nebraska & 146 & 0.46 & 9.6 & 2.83 & 484 & 0.17 & 2.5 & 2.18 & 174 & 0.13 & 1.7 & 2.75 \\
\hline Nevada & 1,018 & 2.44 & 23.9 & 9.23 & 2,260 & 0.85 & 19.6 & 3.18 & 563 & 0.40 & 9.9 & 2.44 \\
\hline New Hampshire & 214 & 1.04 & 23.8 & 3.20 & 231 & 0.37 & 8.7 & 1.70 & 58 & 0.41 & 12.1 & 1.74 \\
\hline New Jersey & 1,023 & 0.80 & 22.1 & 2.55 & 1,239 & 0.60 & 13.3 & 2.88 & 194 & 0.30 & 7.2 & 1.67 \\
\hline New Mexico & 1,845 & 0.59 & 10.8 & 3.78 & 3,128 & 0.61 & 12.4 & 3.15 & 946 & 0.55 & 10.8 & 3.29 \\
\hline New York & 1,758 & 0.79 & 11.5 & 4.97 & 4,169 & 0.41 & 6.6 & 3.63 & 1,065 & 0.29 & 5.1 & 3.00 \\
\hline North Carolina & 2,877 & 0.82 & 10.4 & 5.75 & 4,757 & 0.38 & 5.5 & 4.48 & 1,260 & 0.23 & 4.3 & 2.75 \\
\hline North Dakota & 568 & 0.38 & 6.9 & 2.39 & 722 & 0.19 & 2.1 & 2.34 & 128 & 0.12 & 0.0 & - \\
\hline Ohio & 12,194 & 0.71 & 18.8 & 2.22 & 15,819 & 0.40 & 7.2 & 2.33 & 3,014 & 0.29 & 4.9 & 2.03 \\
\hline Oklahoma & 2,221 & 0.55 & 11.6 & 3.29 & 4,023 & 0.56 & 8.7 & 4.33 & 1,066 & 0.43 & 4.4 & 6.58 \\
\hline Oregon & 351 & 0.47 & 5.7 & 4.86 & 1,716 & 1.26 & 4.7 & 23.90 & 716 & 2.40 & 2.4 & 96.11 \\
\hline Pennsylvania & 37,716 & 0.50 & 9.7 & 2.43 & 31,159 & 0.45 & 8.7 & 2.21 & 6,588 & 0.39 & 7.0 & 2.46 \\
\hline Rhode Island & 71 & 0.82 & 18.3 & 2.69 & 84 & 0.55 & 11.9 & 2.95 & 24 & 0.49 & 16.7 & 2.04 \\
\hline South Carolina & 1,012 & 2.90 & 21.2 & 12.54 & 2,892 & 0.31 & 6.8 & 2.62 & 995 & 0.28 & 2.3 & 7.39 \\
\hline South Dakota & 1,434 & 0.82 & 16.0 & 3.47 & 1,650 & 0.47 & 7.1 & 4.37 & 361 & 0.19 & 4.2 & 2.02 \\
\hline Tennessee & 6,861 & 0.49 & 9.5 & 2.41 & 9,690 & 0.42 & 8.5 & 2.33 & 1,724 & 0.38 & 7.6 & 2.45 \\
\hline Texas & 2,147 & 0.86 & 11.8 & 5.76 & 8,021 & 0.53 & 5.4 & 7.49 & 2,521 & 0.27 & 3.8 & 4.69 \\
\hline Utah & 2,820 & 0.78 & 20.1 & 2.27 & 7,613 & 0.73 & 15.7 & 2.82 & 1,275 & 0.52 & 11.1 & 2.15 \\
\hline Vermont & 973 & 0.55 & 14.4 & 1.87 & 897 & 0.57 & 15.8 & 2.37 & 249 & 0.51 & 13.3 & 2.26 \\
\hline Virginia & 26,679 & 0.59 & 13.6 & 2.24 & 32,635 & 0.55 & 12.8 & 2.19 & 5,109 & 0.50 & 11.6 & 1.97 \\
\hline Washington & 1,006 & 0.68 & 14.7 & 3.24 & 2,069 & 0.47 & 9.0 & 3.50 & 934 & 0.30 & 6.5 & 2.51 \\
\hline West Virginia & 45,966 & 0.66 & 16.1 & 2.20 & 69,427 & 0.51 & 10.1 & 2.11 & 10,354 & 0.51 & 10.4 & 2.09 \\
\hline $\mathrm{W}$ isconsin & 2,151 & 0.48 & 9.5 & 2.82 & 2,406 & 0.53 & 10.6 & 3.36 & 830 & 0.53 & 6.7 & 5.57 \\
\hline Wyoming & 2,506 & 0.99 & 16.7 & 4.34 & 3,022 & 0.60 & 11.1 & 3.48 & 815 & 0.39 & 9.4 & 2.29 \\
\hline TOTAL & 251,658 & 0.72 & 14.1 & 3.20 & 356,348 & 0.52 & 10.3 & 2.61 & 76,145 & 0.43 & 8.0 & 2.59 \\
\hline
\end{tabular}

NOTE: See Appendix A for source description, Appendix B for methods, and Appendix D for agents.

SOURCE: Mine Safety and Health Administration: respirable coal mine dust data and respirable coal mine quartz data. Bureau of Mines: Mine Inspection Data Analysis System. 
Figure 6-7. Pneumoconiotic agents: Number of MSHA inspector samples collected by state, 1993-1994

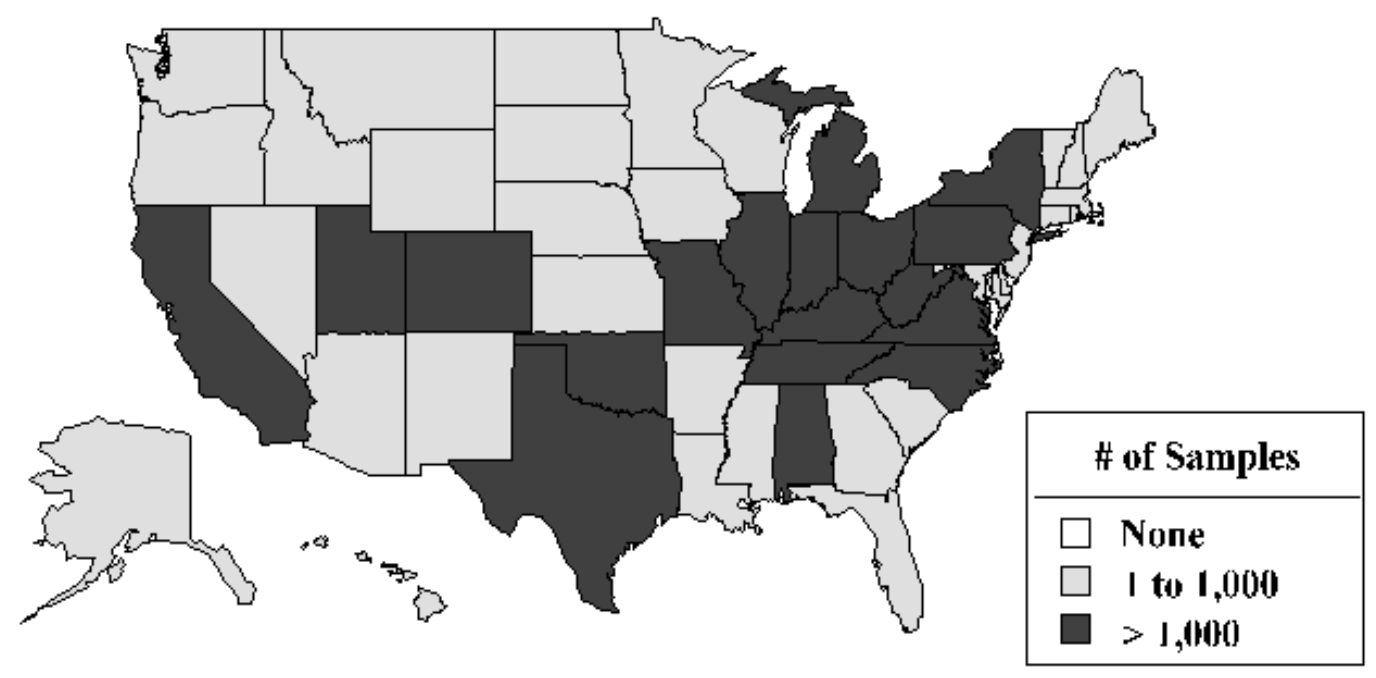

NOTE: See Appendix A for source description, Appendix B for methods, and Appendix D for agents.

SOURCE: Mine Safety and Health Administration: respirable coal mine dust data and respirable coal mine quartz data. Bureau of Mines: Mine Inspection Data Analysis System.

Figure 6-8. Pneumoconiotic agents: Percent of MSHA inspector samples that exceeded the permissible exposure limit (PEL), by state, 1993-1994

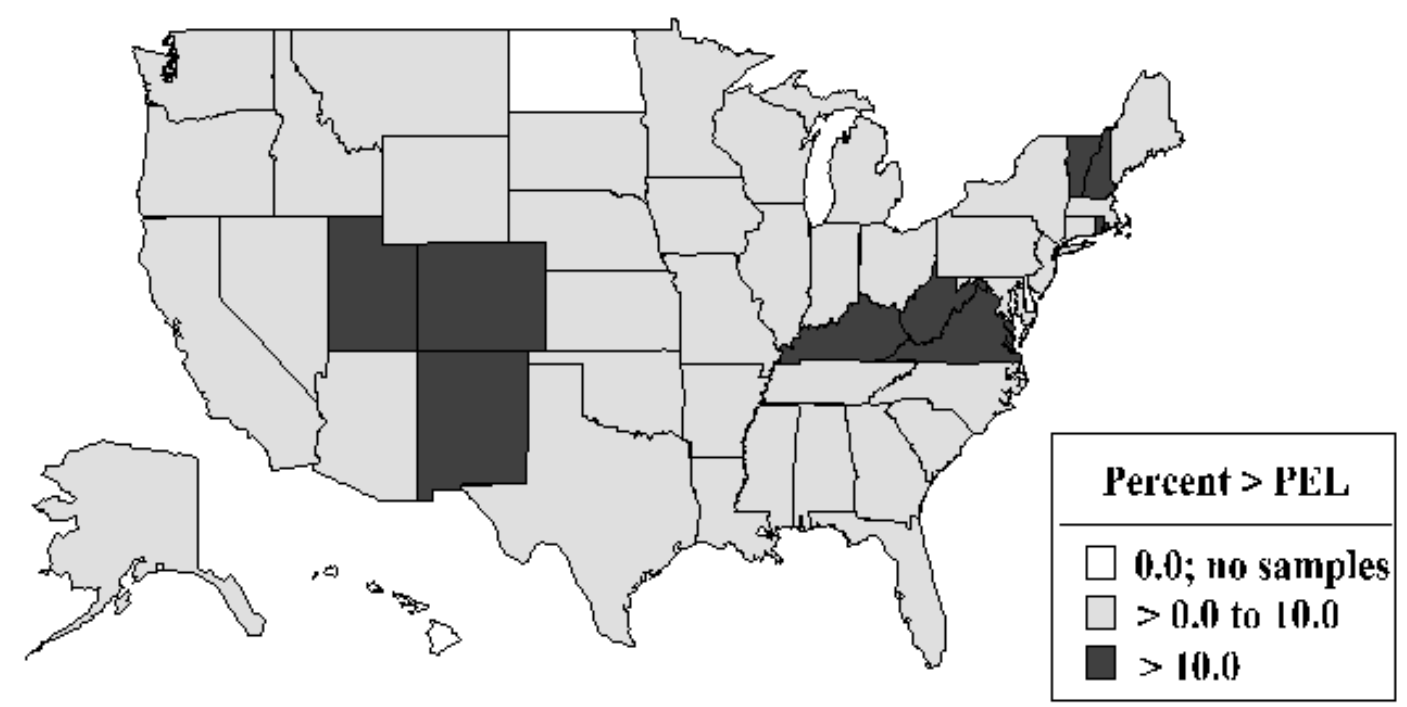

NOTE: See Appendix A for source description, Appendix B for methods, and Appendix D for agents.

SOURCE: Mine Safety and Health Administration: respirable coal mine dust data and respirable coal mine quartz data. Bureau of Mines: Mine Inspection Data Analysis System. 
Figure 6-9. Pneumoconiotic agents: Average severity level by state for MSHA inspector samples, 1993-1994

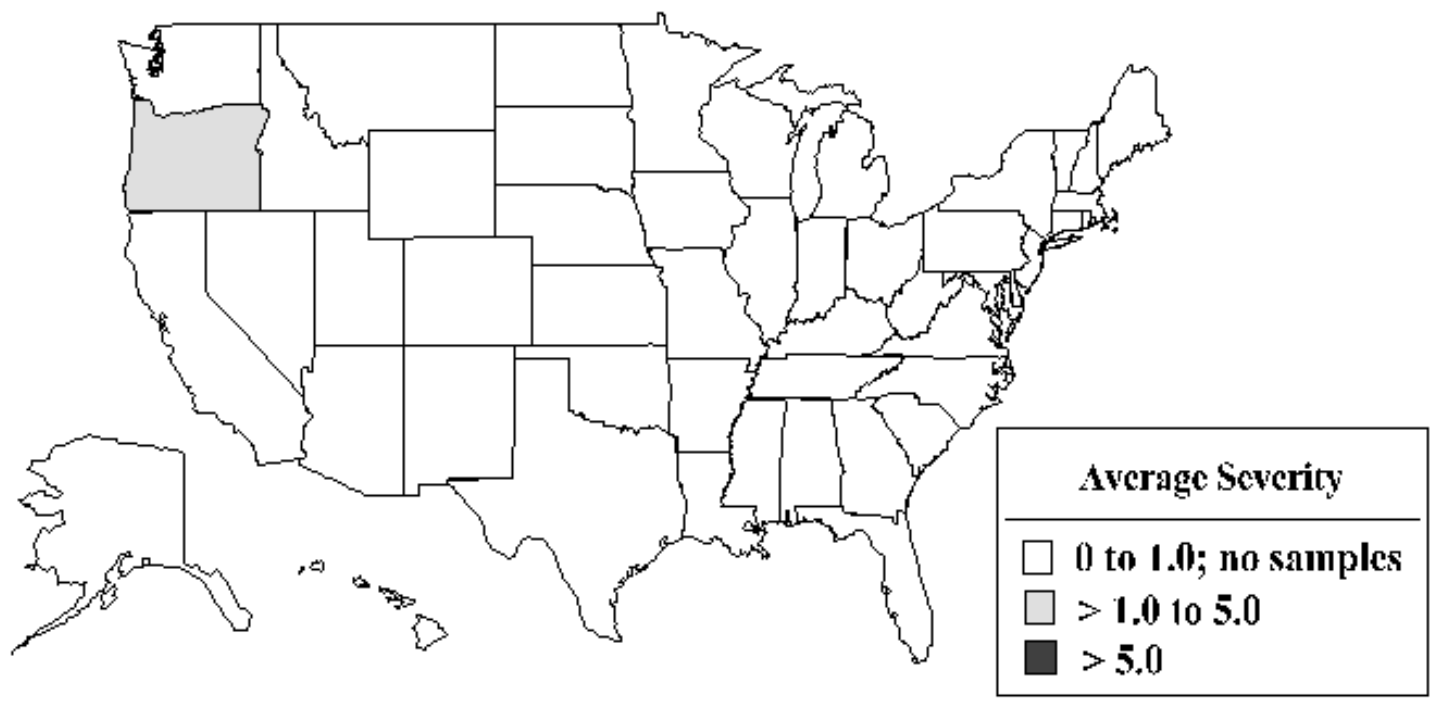

NOTE: See Appendix A for source description, Appendix B for methods, and Appendix D for agents.

SOURCE: Mine Safety and Health Administration: respirable coal mine dust data and respirable coal mine quartz data. Bureau of Mines: Mine Inspection Data Analysis System.

Figure 6-10. Pneumoconiotic agents: Average severity level by state for MSHA inspector samples that exceeded the permissible exposure limit (PEL), 1993-1994

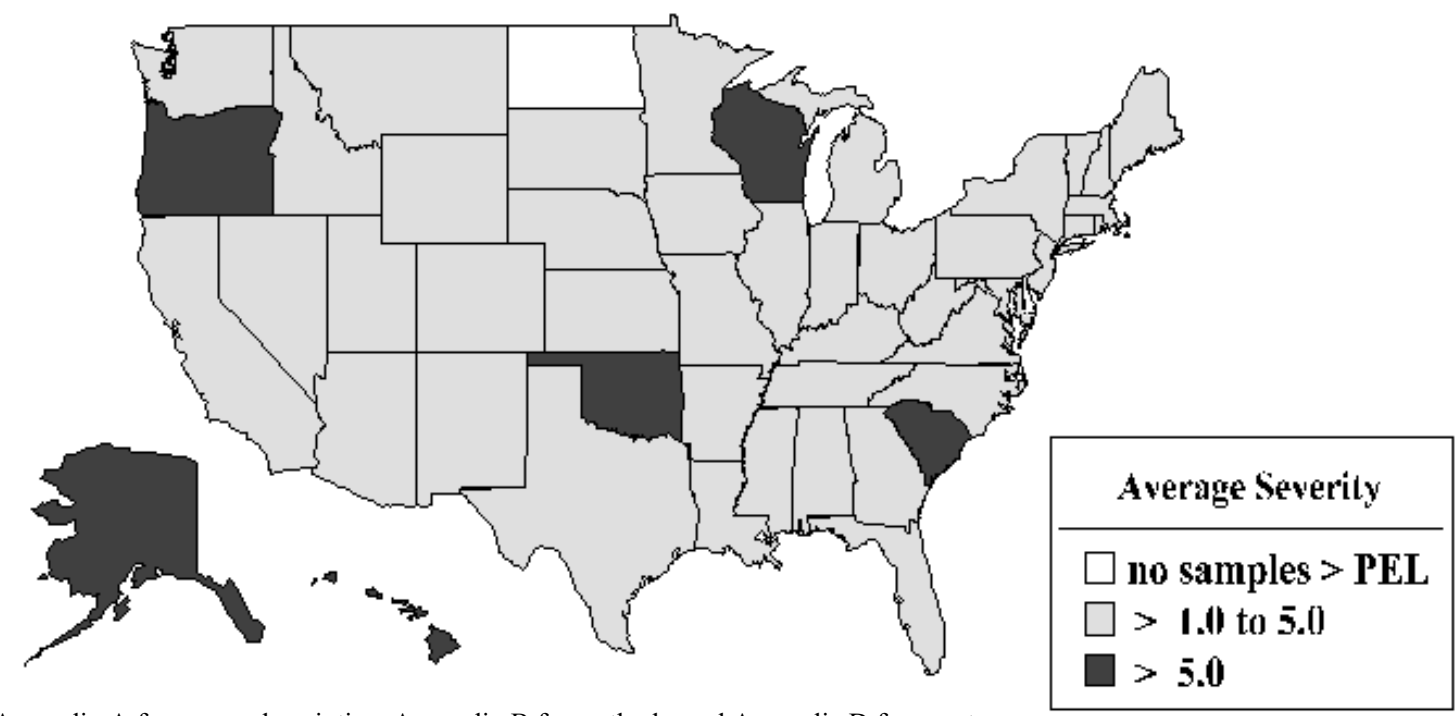

NOTE: See Appendix A for source description, Appendix B for methods, and Appendix D for agents.

SOURCE: Mine Safety and Health Administration: respirable coal mine dust data and respirable coal mine quartz data. Bureau of Mines: Mine Inspection Data Analysis System. 
Figure 6-11. Pneumoconiotic agents: Number of MSHA inspector

samples collected by county, 1993-1994

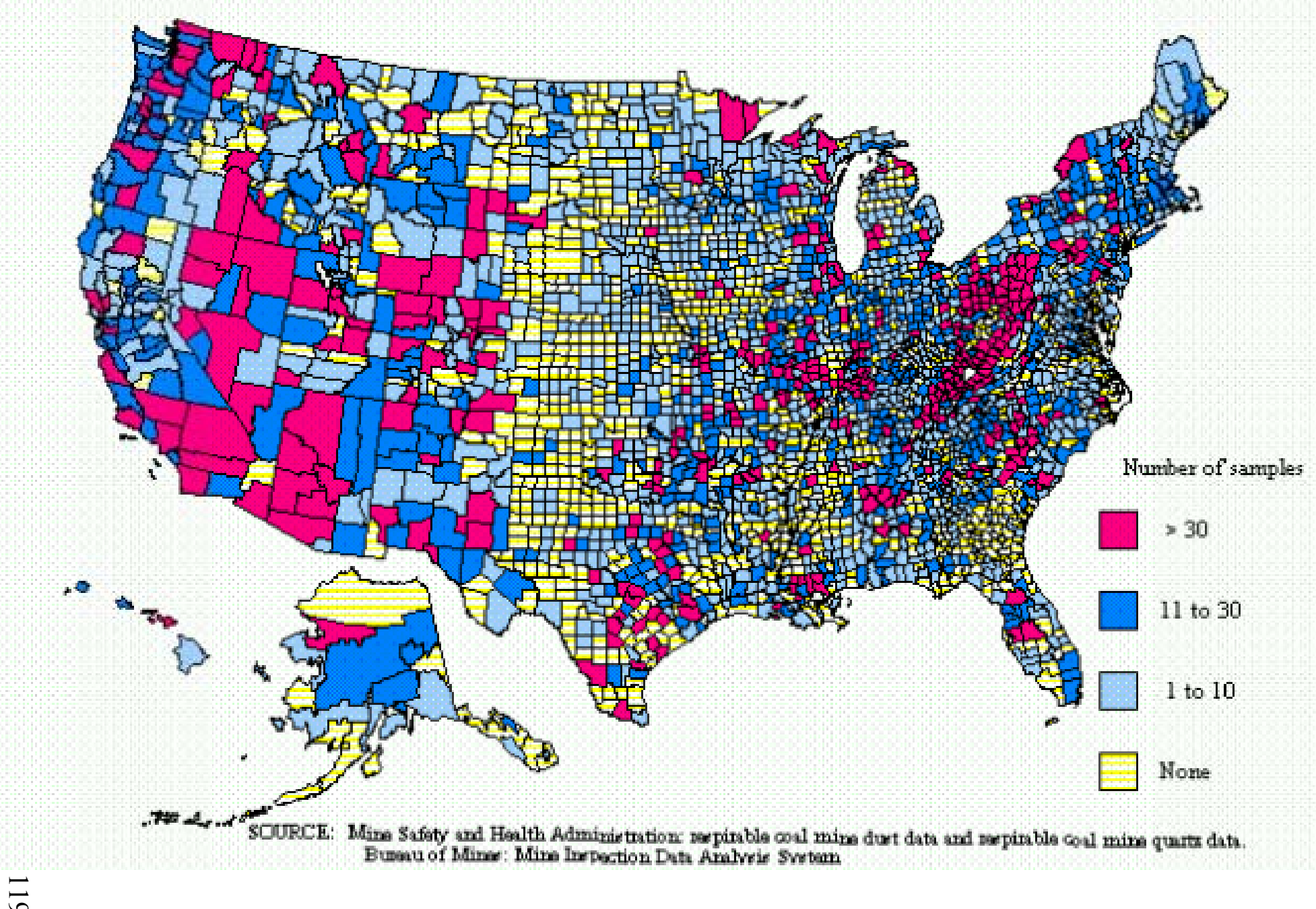


Table 6-9 (page 1 of 2). Pneumoconiotic agents: Number of OSHA inspector samples, percent exceeding the permissible exposure limit (PEL) and average severity levels (Avg. Sev.), by state, 1979-1994

\begin{tabular}{|c|c|c|c|c|c|c|c|c|c|c|c|c|}
\hline \multirow[b]{3}{*}{ State } & \multicolumn{4}{|c|}{ 1979-1984 } & \multicolumn{4}{|c|}{ 1985-1994 } & \multicolumn{4}{|c|}{ 1993-1994 } \\
\hline & \multicolumn{2}{|c|}{ Total samples } & \multicolumn{2}{|c|}{ Samples > PEL } & \multicolumn{2}{|c|}{ Total samples } & \multicolumn{2}{|c|}{ Samples > PEL } & \multicolumn{2}{|c|}{ Total samples } & \multicolumn{2}{|c|}{ Samples > PEL } \\
\hline & Number & $\begin{array}{l}\text { Avg. } \\
\text { Sev. }\end{array}$ & $\%$ & $\begin{array}{r}\text { Avg. } \\
\text { Sev. }\end{array}$ & Number & $\begin{array}{l}\text { Avg. } \\
\text { Sev. }\end{array}$ & $\%$ & $\begin{array}{l}\text { Avg. } \\
\text { Sev. }\end{array}$ & Number & $\begin{array}{l}\text { Avg. } \\
\text { Sev. }\end{array}$ & $\%$ & $\begin{array}{l}\text { Avg. } \\
\text { Sev. }\end{array}$ \\
\hline Alabama & 560 & 0.76 & 19.6 & 2.95 & 839 & 0.55 & 7.2 & 6.40 & 93 & 1.27 & 7.5 & 15.66 \\
\hline Alaska & 93 & 0.38 & 1.1 & 34.59 & 149 & 0.16 & 4.0 & 3.31 & 4 & 0.00 & 0.0 & - \\
\hline Arizona & 303 & 0.15 & 4.6 & 2.35 & 182 & 0.28 & 3.3 & 6.59 & 12 & 1.52 & 8.3 & 17.36 \\
\hline Arkansas & 184 & 0.26 & 7.6 & 1.90 & 690 & 0.41 & 5.1 & 7.06 & 186 & 0.20 & 4.8 & 2.20 \\
\hline California & 52 & 1.05 & 13.5 & 7.30 & 1,462 & 2.11 & 3.6 & 56.84 & 25 & 0.21 & 8.0 & 1.85 \\
\hline Colorado & 1,562 & 0.41 & 5.3 & 5.89 & 2,673 & 4.06 & 2.8 & 141.51 & 126 & 0.24 & 6.3 & 2.67 \\
\hline Connecticut & 698 & 0.49 & 11.0 & 3.06 & 1,285 & 0.26 & 3.1 & 7.12 & 164 & 0.10 & 1.8 & 3.38 \\
\hline Delaware & 90 & 0.22 & 5.6 & 1.49 & 75 & 0.08 & 2.7 & 1.77 & 8 & 0.08 & 0.0 & - \\
\hline District of Columbia & 62 & 0.08 & 1.6 & 2.04 & 47 & 1.96 & 8.5 & 22.75 & 24 & 1.66 & 8.3 & 19.75 \\
\hline Florida & 263 & 1.12 & 9.9 & 10.06 & 1,176 & 0.14 & 2.5 & 4.68 & 109 & 0.08 & 2.8 & 1.67 \\
\hline Georgia & 919 & 0.89 & 18.2 & 3.98 & 2,789 & 0.54 & 5.5 & 8.67 & 340 & 0.39 & 4.1 & 7.27 \\
\hline Hawaii & 14 & 0.08 & 0.0 & - & 20 & 0.00 & 0.0 & - & 3 & 0.00 & 0.0 & - \\
\hline Idaho & 153 & 0.84 & 9.8 & 7.28 & 746 & 0.11 & 1.1 & 5.64 & 171 & 0.04 & 0.0 & - \\
\hline Illinois & 1,451 & 0.98 & 11.4 & 7.35 & 4,549 & 0.41 & 2.9 & 12.51 & 650 & 0.06 & 0.8 & 3.95 \\
\hline Indiana & 516 & 0.53 & 15.1 & 2.09 & 886 & 0.35 & 6.4 & 3.38 & 93 & 0.12 & 3.2 & 1.47 \\
\hline Iowa & 845 & 0.52 & 9.6 & 3.73 & 1,284 & 0.29 & 5.2 & 3.14 & 128 & 0.41 & 14.1 & 2.14 \\
\hline Kansas & 265 & 0.42 & 8.3 & 3.23 & 450 & 0.10 & 1.8 & 2.95 & 72 & 0.02 & 0.0 & - \\
\hline Kentucky & 286 & 1.05 & 12.9 & 7.41 & 767 & 1.73 & 7.8 & 21.08 & 146 & 0.35 & 4.8 & 6.73 \\
\hline Louisiana & 261 & 0.48 & 10.3 & 3.36 & 499 & 0.13 & 2.8 & 3.00 & 25 & 0.12 & 0.0 & - \\
\hline Maine & 111 & 0.28 & 9.0 & 2.19 & 145 & 0.57 & 6.2 & 7.88 & 0 & - & - & - \\
\hline Maryland & 121 & 0.89 & 8.3 & 9.43 & 752 & 0.73 & 9.6 & 6.71 & 122 & 0.25 & 4.1 & 3.49 \\
\hline Massachusetts & 1,015 & 0.60 & 13.0 & 3.57 & 2,695 & 0.13 & 2.9 & 3.34 & 204 & 0.30 & 4.9 & 4.78 \\
\hline Michigan & 0 & - & - & - & 1,781 & 3.05 & 4.6 & 64.42 & 528 & 9.69 & 4.2 & 230.81 \\
\hline Minnesota & 59 & 1.17 & 35.6 & 2.59 & 80 & 0.01 & 0.0 & - & 0 & - & - & - \\
\hline Mississippi & 93 & 1.07 & 9.7 & 9.06 & 535 & 0.78 & 4.3 & 16.78 & 43 & 0.04 & 0.0 & - \\
\hline Missouri & 666 & 0.45 & 8.4 & 3.90 & 1,076 & 2.15 & 4.9 & 42.12 & 18 & 0.00 & 0.0 & - \\
\hline Montana & 692 & 0.18 & 2.0 & 5.33 & 570 & 0.46 & 7.0 & 5.63 & 0 & - & - & - \\
\hline
\end{tabular}


Table 6-9 (page 2 of 2). Pneumoconiotic agents: Number of OSHA inspector samples, percent exceeding the permissible exposure limit (PEL) and average severity levels (Avg. Sev.), by state, 1979-1994

\begin{tabular}{|c|c|c|c|c|c|c|c|c|c|c|c|c|}
\hline \multirow[b]{3}{*}{ State } & \multicolumn{4}{|c|}{ 1979-1984 } & \multicolumn{4}{|c|}{ 1985-1994 } & \multicolumn{4}{|c|}{ 1993-1994 } \\
\hline & \multicolumn{2}{|c|}{ Total samples } & \multicolumn{2}{|c|}{ Samples > PEL } & \multicolumn{2}{|c|}{ Total samples } & \multicolumn{2}{|c|}{ Samples > PEL } & \multicolumn{2}{|c|}{ Total samples } & \multicolumn{2}{|c|}{ Samples > PEL } \\
\hline & Number & $\begin{array}{l}\text { Avg. } \\
\text { Sev. }\end{array}$ & $\%$ & $\begin{array}{l}\text { Avg. } \\
\text { Sev. }\end{array}$ & Number & $\begin{array}{l}\text { Avg. } \\
\text { Sev. }\end{array}$ & $\%$ & $\begin{array}{l}\text { Avg. } \\
\text { Sev. }\end{array}$ & Number & $\begin{array}{l}\text { Avg. } \\
\text { Sev. }\end{array}$ & $\%$ & $\begin{array}{l}\text { Avg. } \\
\text { Sev. }\end{array}$ \\
\hline Nebraska & 123 & 0.31 & 5.7 & 3.59 & 571 & 0.16 & 4.9 & 2.23 & 105 & 0.05 & 1.0 & 1.36 \\
\hline Nevada & 59 & 0.75 & 13.6 & 4.34 & 229 & 4.34 & 33.6 & 12.68 & 15 & 0.08 & 0.0 & - \\
\hline New Hampshire & 325 & 0.58 & 11.4 & 3.69 & 720 & 0.20 & 3.2 & 4.46 & 50 & 0.06 & 0.0 & - \\
\hline New Jersey & 973 & 0.58 & 10.6 & 4.27 & 1,705 & 0.95 & 7.8 & 11.36 & 274 & 3.55 & 4.7 & 74.24 \\
\hline New Mexico & 62 & 0.56 & 16.1 & 2.82 & 31 & 0.06 & 0.0 & - & 8 & 0.21 & 0.0 & - \\
\hline New York & 1,807 & 0.64 & 11.7 & 4.39 & 2,547 & 0.27 & 3.4 & 6.54 & 503 & 0.21 & 3.0 & 5.50 \\
\hline North Carolina & 561 & 14.85 & 13.4 & 108.98 & 1,236 & 0.48 & 6.5 & 5.64 & 514 & 0.49 & 2.5 & 16.58 \\
\hline North Dakota & 204 & 0.42 & 7.8 & 3.68 & 475 & 0.14 & 2.1 & 1.85 & 57 & 0.09 & 0.0 & - \\
\hline Ohio & 2,678 & 0.57 & 12.7 & 3.22 & 6,001 & 0.37 & 4.5 & 6.86 & 390 & 0.66 & 6.2 & 9.52 \\
\hline Oklahoma & 880 & 0.33 & 4.4 & 5.25 & 1,743 & 0.76 & 2.0 & 35.40 & 75 & 0.09 & 1.3 & 1.73 \\
\hline Oregon & 311 & 0.35 & 7.1 & 1.99 & 328 & 2.23 & 9.1 & 23.41 & 90 & 5.41 & 4.4 & 120.36 \\
\hline Pennsylvania & 2,229 & 0.80 & 15.8 & 3.69 & 3,145 & 0.72 & 11.1 & 5.40 & 258 & 0.45 & 10.5 & 3.38 \\
\hline Rhode Island & 401 & 0.90 & 15.0 & 5.22 & 378 & 0.87 & 7.9 & 9.87 & 0 & - & - & - \\
\hline South Carolina & 201 & 0.10 & 2.0 & 2.07 & 859 & 0.44 & 6.3 & 5.43 & 174 & 0.98 & 6.3 & 14.33 \\
\hline South Dakota & 96 & 0.17 & 4.2 & 1.75 & 536 & 0.10 & 2.1 & 1.97 & 82 & 0.10 & 2.4 & 1.74 \\
\hline Tennessee & 48 & 0.53 & 12.5 & 2.32 & 590 & 1.25 & 8.6 & 13.14 & 126 & 0.18 & 4.0 & 2.07 \\
\hline Texas & 2,324 & 0.78 & 7.2 & 9.35 & 3,998 & 0.81 & 5.4 & 14.16 & 523 & 2.71 & 4.8 & 55.86 \\
\hline Utah & 11 & 0.71 & 27.3 & 2.58 & 66 & 0.26 & 4.5 & 2.83 & 7 & 0.35 & 14.3 & 1.30 \\
\hline Vermont & 0 & - & - & - & 3 & 0.10 & 0.0 & - & 0 & - & - & - \\
\hline Virginia & 271 & 0.46 & 11.4 & 2.56 & 462 & 0.87 & 17.7 & 3.92 & 8 & 1.82 & 25.0 & 7.00 \\
\hline Washington & 27 & 0.82 & 37.0 & 1.96 & 285 & 1.16 & 18.9 & 5.56 & 116 & 0.54 & 13.8 & 3.27 \\
\hline West Virginia & 480 & 1.11 & 15.0 & 6.39 & 651 & 0.47 & 6.6 & 6.34 & 8 & 0.00 & 0.0 & - \\
\hline Wisconsin & 2,066 & 0.52 & 13.3 & 2.82 & 3,857 & 0.29 & 5.9 & 3.39 & 650 & 0.09 & 1.5 & 1.61 \\
\hline Wyoming & 23 & 0.27 & 4.3 & 2.57 & 7 & 0.13 & 0.0 & - & 3 & 0.10 & 0.0 & - \\
\hline TOTAL & 27,494 & 0.91 & 11.0 & 6.95 & 58,625 & 0.82 & 5.2 & 14.40 & 7,330 & 1.33 & 3.9 & 32.18 \\
\hline
\end{tabular}

- indicates incalculable field.

NOTE: $\quad$ See Appendix A for source description, Appendix B for methods, and Appendix D for agents.

SOURCE: Occupational Safety and Health Administration: Integrated Management Information System. 
Figure 6-12. Pneumoconiotic agents: Number of OSHA inspector samples collected by state, 1993-1994

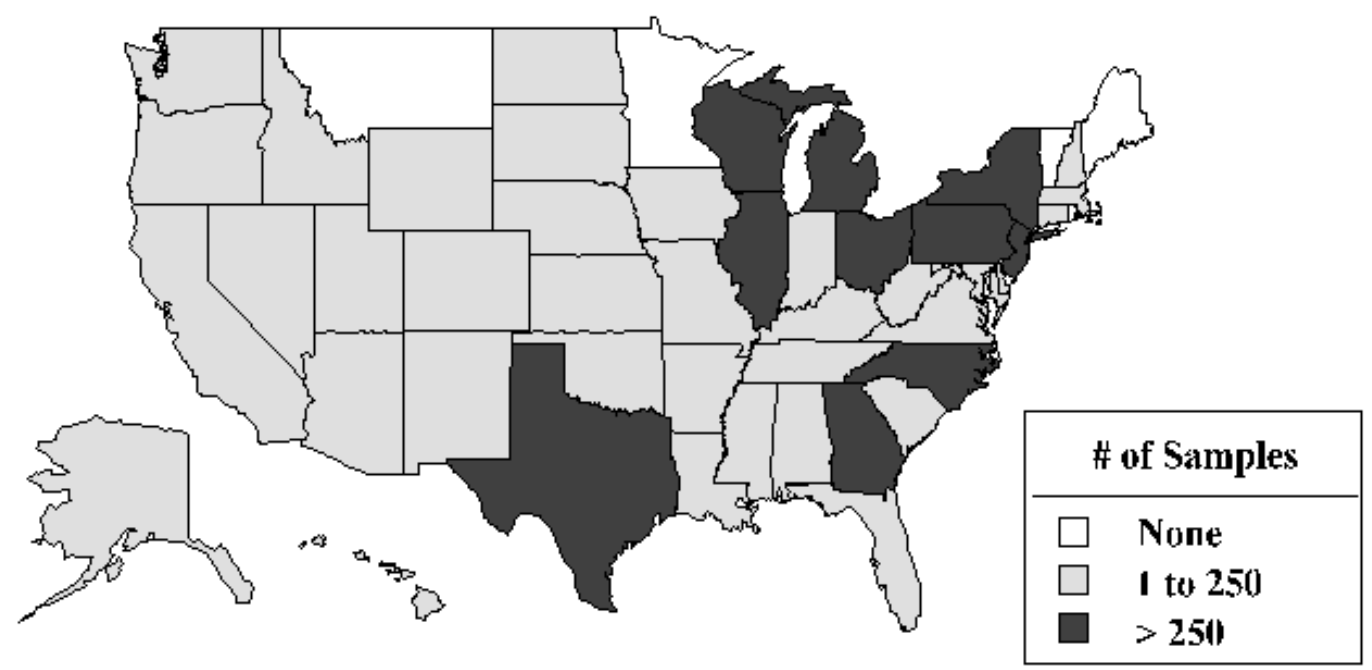

NOTE: See Appendix A for source description, Appendix B for methods, and Appendix D for agents. SOURCE: Occupational Safety and Health Administration: Integrated Management Information System .

Figure 6-13. Pneumoconiotic agents: Percent of OSHA inspector samples that exceeded the permissible exposure limit (PEL), by state, 1993-1994

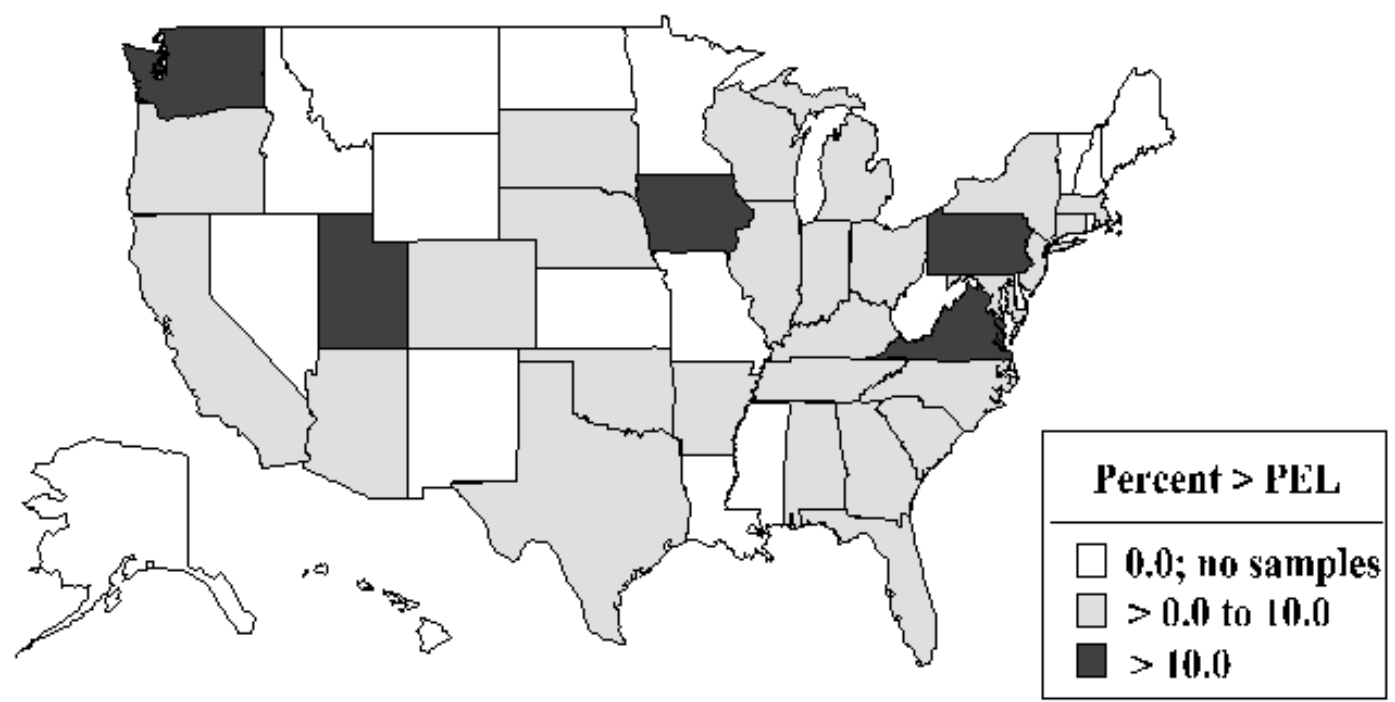

NOTE: See Appendix A for source description, Appendix B for methods, and Appendix D for agents. SOURCE: Occupational Safety and Health Administration: Integrated Management Information System . 
Figure 6-14. Pneumoconiotic agents: Average severity level by state for OSHA inspector samples, 1993-1994

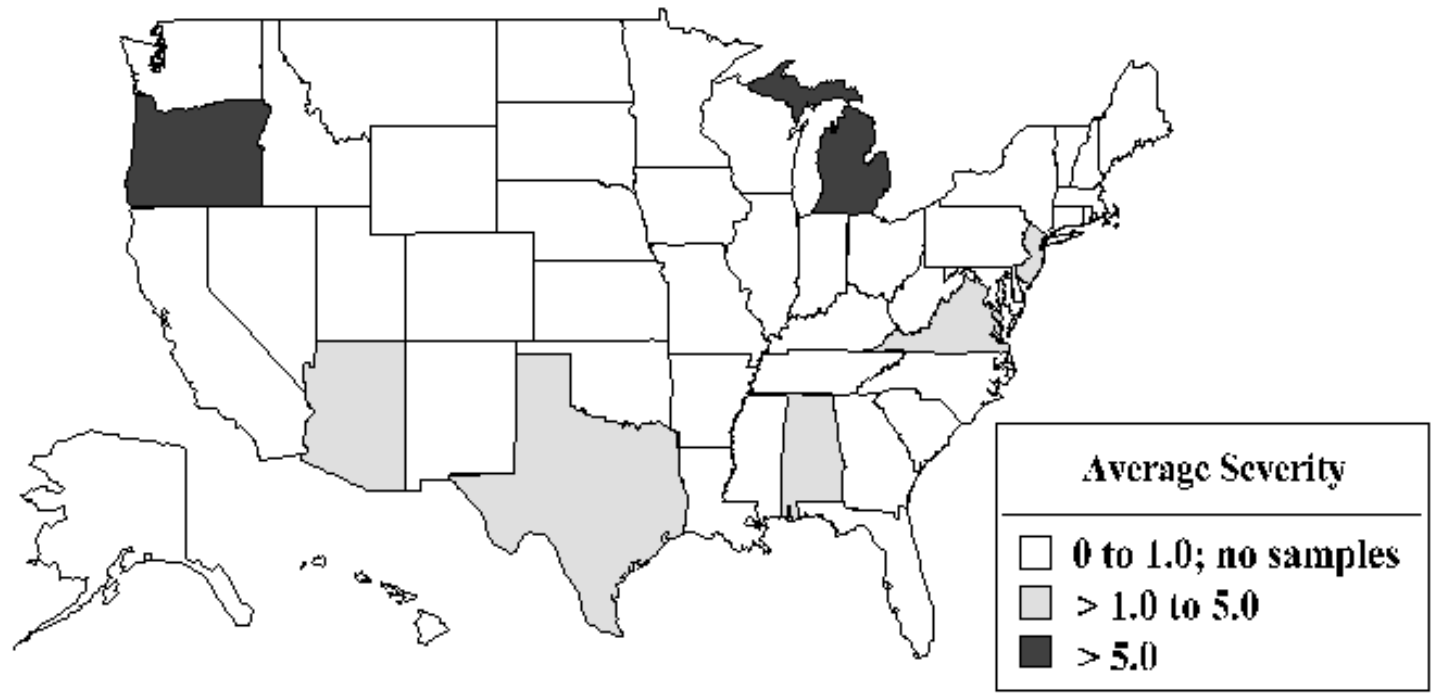

NOTE: See Appendix A for source descriptions, Appendix B for methods, and Appendix D for agents SOURCE: Occupational Safety and Health Administration: Integrated Management Information System.

Figure 6-15. Pneumoconiotic agents: Average severity level by state for OSHA inspector samples that exceeded the permissible exposure limit (PEL), 1993-1994

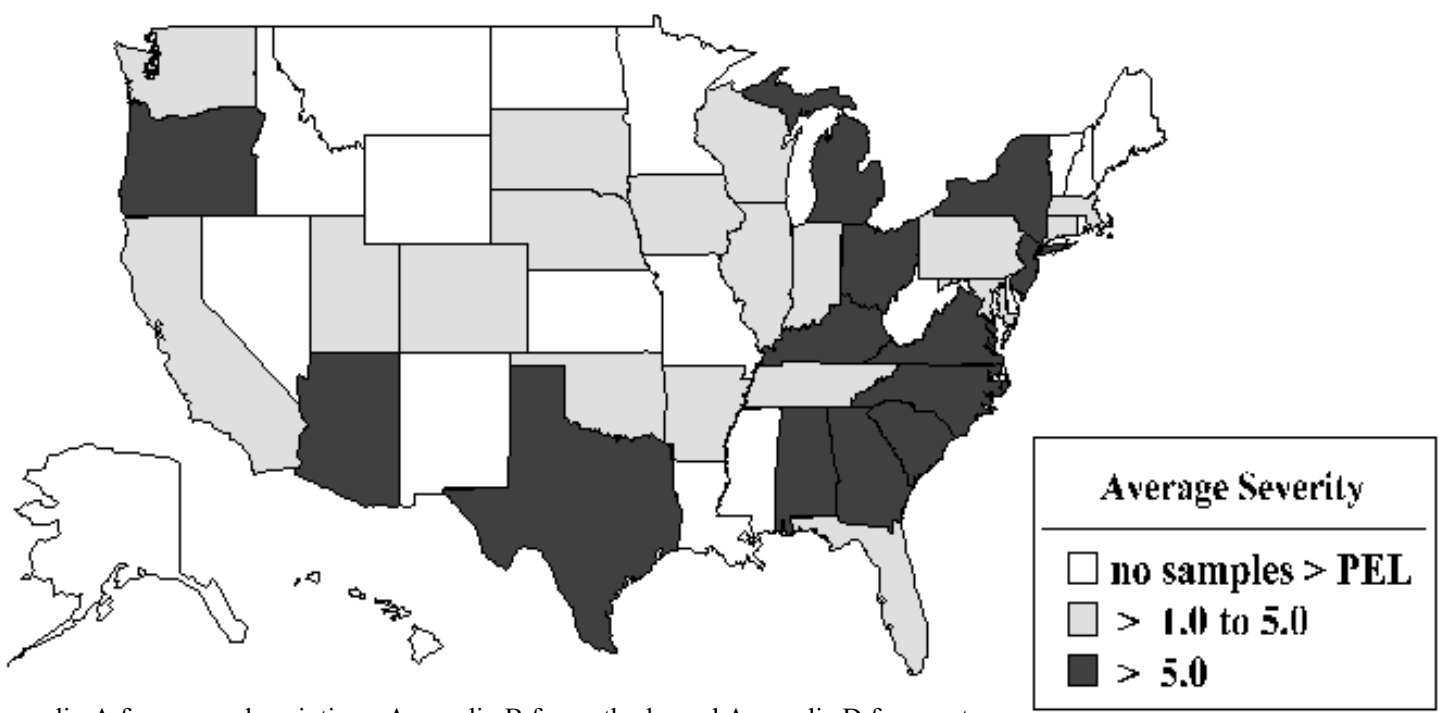

NOTE: See Appendix A for source descriptions, Appendix B for methods, and Appendix D for agents. SOURCE: Occupational Safety and Health Administration: Integrated Management Information System. 
Figure 6-16. Pneumoconiotic agents: Number of OSHA inspector

samples collected by county, 1993-1994

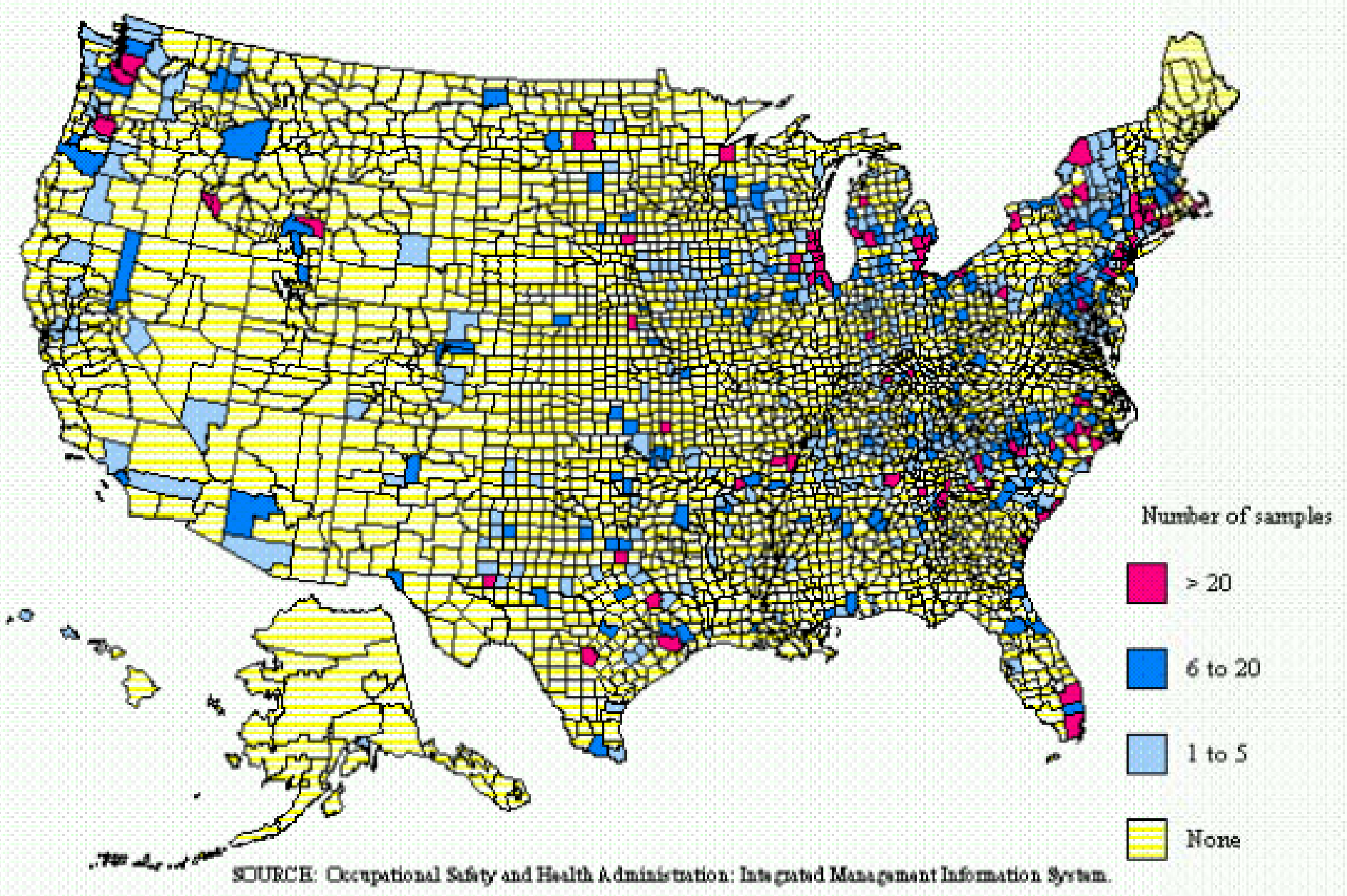




\section{STATE}

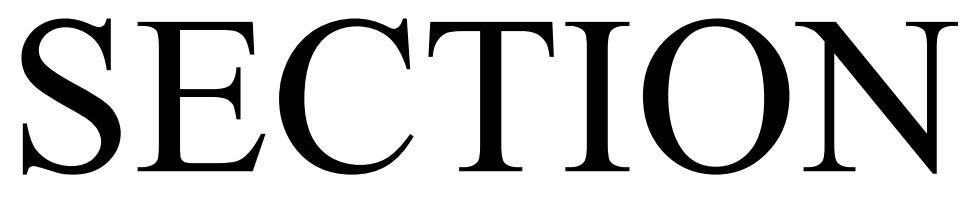




\section{List of Tables and Figures}

\section{State Section}

Each table, figure, and the selected statistics listed below is repeated for each state. All tables and figures for a state are grouped together. A two-letter abbreviation, illustrated below as $[X X]$, is incorporated into the final table/figure identifier. The state name is inserted where designated.

[State Name] Selected statistics from the United States section

Figure $[X X]-1 . \quad$ All pneumoconioses: [State Name] and U.S. age-adjusted mortality rates, residents age 15 and over, 1968-1992

Table $\quad[X X]-1 . \quad$ Number of deaths and age-adjusted mortality rate (per 1,000,000 population) by condition, [State Name] residents age 15 and over, 1968-1992

Figure $[X X]-2 . \quad$ All pneumoconioses: Distribution of deaths by sex and race, [State Name] residents age 15 and over, 1968-1992

Table $\quad[X X]-2 . \quad$ All pneumoconioses: Distribution of deaths by sex, race, and age, [State Name] residents age 15 and over, 1968-1992

Figure $[X X]-3 . \quad$ Pneumoconiosis deaths by condition, [State Name] residents age 15 and over, 1968-1992

Figure $[X X]-4 . \quad$ State Name] and U. S. age-adjusted mortality rates (per 1,000,000 population) by condition, residents age 15 and over, 1983-1992

Table $[X X]-3 . \quad$ Number of deaths and age-adjusted mortality rate (per 1,000,000 population) by condition and county, [State Name] residents age 15 and over, 1983-1992 


\section{Alabama: Selected statistics from the United States section}

All pneumoconioses mortality, 1988-1992

Total deaths

Crude mortality rate (per $1,000,000$ population)

Age-adjusted mortality rate (per $1,000,000$ population)

Total years of potential life lost to life expectancy

Years of potential life lost to life expectancy (per death)
Value

303

19.16

9.89

3,388

11.2

Deaths*

94

146

25

2

36
State rank

12

9

10

12

42

Percent**

distribution

31.0

48.2

Silicosis

Byssinosis

Unspecified/Other pneumoconiosis

* Sum of column may exceed total deaths because of deaths with multiple pneumoconioses.

** Percentages may total more than $100 \%$ due to deaths with multiple pneumoconioses.

NOTE: $\quad$ See Appendix A for source description and Appendix B for methods and ICD-8 and ICD-9 codes.

SOURCE: National Center for Health Statistics multiple cause of death data. Population estimates from U.S. Bureau of the Census.

\section{Figure AL-1. All pneumoconioses: Alabama and U.S. age-adjusted mortality rates, residents age 15 and over, 1968-1992}

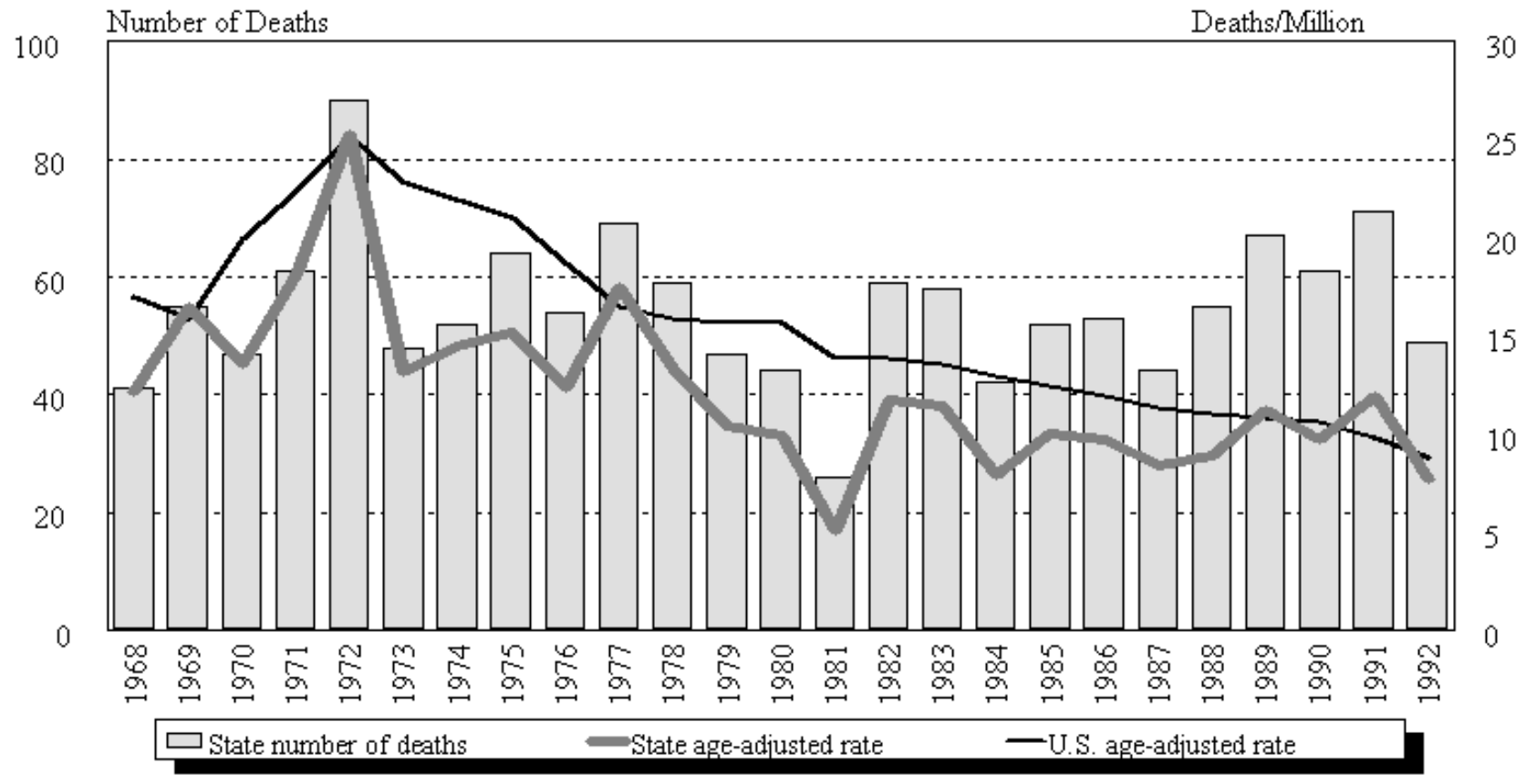

NOTE: $\quad$ See Appendix A for source description and Appendix B for methods and ICD-8 and ICD-9 codes.

SOURCE: $\quad$ National Center for Health Statistics multiple cause of death data. Population estimates from U.S. Bureau of the Census. 
Alabama

Table AL-1. Number of deaths and age-adjusted mortality rate (per 1,000,000 population) by condition, Alabama residents age 15 and over, 1968-1992

\begin{tabular}{|c|c|c|c|c|c|c|c|c|c|c|c|c|}
\hline \multirow[b]{2}{*}{ Year } & \multicolumn{2}{|c|}{ Asbestosis } & \multicolumn{2}{|c|}{$\begin{array}{c}\text { Coal workers' } \\
\text { pneumoconiosis }\end{array}$} & \multicolumn{2}{|c|}{ Silicosis } & \multicolumn{2}{|c|}{ Byssinosis* } & \multicolumn{2}{|c|}{$\begin{array}{c}\text { Unspecified/Other } \\
\text { pneumoconioses }\end{array}$} & \multicolumn{2}{|c|}{ All pneumoconioses } \\
\hline & Number & Rate & Number & Rate & Number & Rate & Number & Rate & Number & Rate & Number & Rate \\
\hline 1968 & - & - & 3 & 0.95 & 21 & 6.47 & & & 17 & 4.66 & 41 & 12.09 \\
\hline 1969 & - & - & 7 & 2.09 & 27 & 8.15 & & & 22 & 6.55 & 55 & 16.48 \\
\hline 1970 & - & - & 2 & 0.55 & 19 & 5.73 & & & 26 & 7.24 & 47 & 13.53 \\
\hline 1971 & - & - & 5 & 1.58 & 15 & 5.11 & & & 42 & 11.75 & 61 & 18.14 \\
\hline 1972 & - & - & 8 & 2.96 & 22 & 6.33 & & & 60 & 15.94 & 90 & 25.23 \\
\hline 1973 & - & - & 6 & 1.65 & 12 & 3.36 & & & 30 & 8.12 & 48 & 13.13 \\
\hline 1974 & 1 & 0.32 & 12 & 3.72 & 9 & 2.45 & & & 31 & 8.21 & 52 & 14.44 \\
\hline 1975 & 1 & 0.21 & 17 & 4.13 & 17 & 4.06 & & & 29 & 6.73 & 64 & 15.14 \\
\hline 1976 & - & - & 22 & 5.13 & 6 & 1.26 & & & 26 & 5.98 & 54 & 12.38 \\
\hline 1977 & 2 & 0.56 & 27 & 7.08 & 14 & 3.65 & & & 29 & 6.93 & 69 & 17.53 \\
\hline 1978 & 2 & 0.54 & 17 & 3.68 & 10 & 2.41 & & & 30 & 6.75 & 59 & 13.38 \\
\hline 1979 & 2 & 0.49 & 19 & 3.91 & 11 & 2.91 & 2 & 0.53 & 17 & 3.56 & 47 & 10.45 \\
\hline 1980 & 2 & 0.53 & 13 & 3.05 & 5 & 1.40 & 1 & 0.23 & 23 & 4.74 & 44 & 9.95 \\
\hline 1981 & - & - & 18 & 3.42 & 2 & 0.35 & - & - & 6 & 1.27 & 26 & 5.03 \\
\hline 1982 & 5 & 1.08 & 40 & 7.20 & 4 & 0.91 & - & - & 10 & 2.54 & 59 & 11.73 \\
\hline 1983 & 4 & 0.91 & 32 & 6.08 & 4 & 0.84 & - & - & 18 & 3.63 & 58 & 11.45 \\
\hline 1984 & 4 & 0.90 & 26 & 4.51 & 4 & 1.09 & - & - & 9 & 1.62 & 42 & 7.89 \\
\hline 1985 & 7 & 1.55 & 30 & 5.78 & 1 & 0.22 & - & - & 15 & 2.72 & 52 & 10.04 \\
\hline 1986 & 9 & 1.90 & 32 & 5.58 & 7 & 1.52 & 1 & 0.22 & 4 & 0.53 & 53 & 9.75 \\
\hline 1987 & 6 & 1.31 & 23 & 4.21 & 6 & 1.09 & - & - & 10 & 2.09 & 44 & 8.42 \\
\hline 1988 & 10 & 2.09 & 33 & 4.86 & 6 & 1.09 & - & - & 6 & 0.92 & 55 & 8.95 \\
\hline 1989 & 22 & 4.29 & 33 & 4.70 & 4 & 0.87 & - & - & 8 & 1.37 & 67 & 11.24 \\
\hline 1990 & 21 & 4.42 & 29 & 3.41 & 3 & 0.74 & 1 & 0.13 & 7 & 1.01 & 61 & 9.71 \\
\hline 1991 & 23 & 4.81 & 28 & 3.71 & 9 & 1.64 & 1 & 0.13 & 10 & 1.61 & 71 & 11.90 \\
\hline 1992 & 18 & 3.28 & 23 & 3.24 & 3 & 0.47 & - & - & 5 & 0.67 & 49 & 7.66 \\
\hline
\end{tabular}

indicates no deaths listed. $\quad *$ no specific code for byssinosis in ICD-8 (1968-1 978).

NOTE: Rates based on small numbers may be unstable. Sum of individual conditions may exceed total because of deaths with multiple pneumoconioses. See Appendix A for source description and Appendix B for methods and ICD-8 and ICD-9 Nodes.

SOURCE: National Center for Health Statistics multiple cause of death data. Population estimates from U.S. Bureau of the Census. 
Figure AL-2. All pneumoconioses: Distribution of deaths by sex and race, Alabama residents age 15 and over, 1968-1992

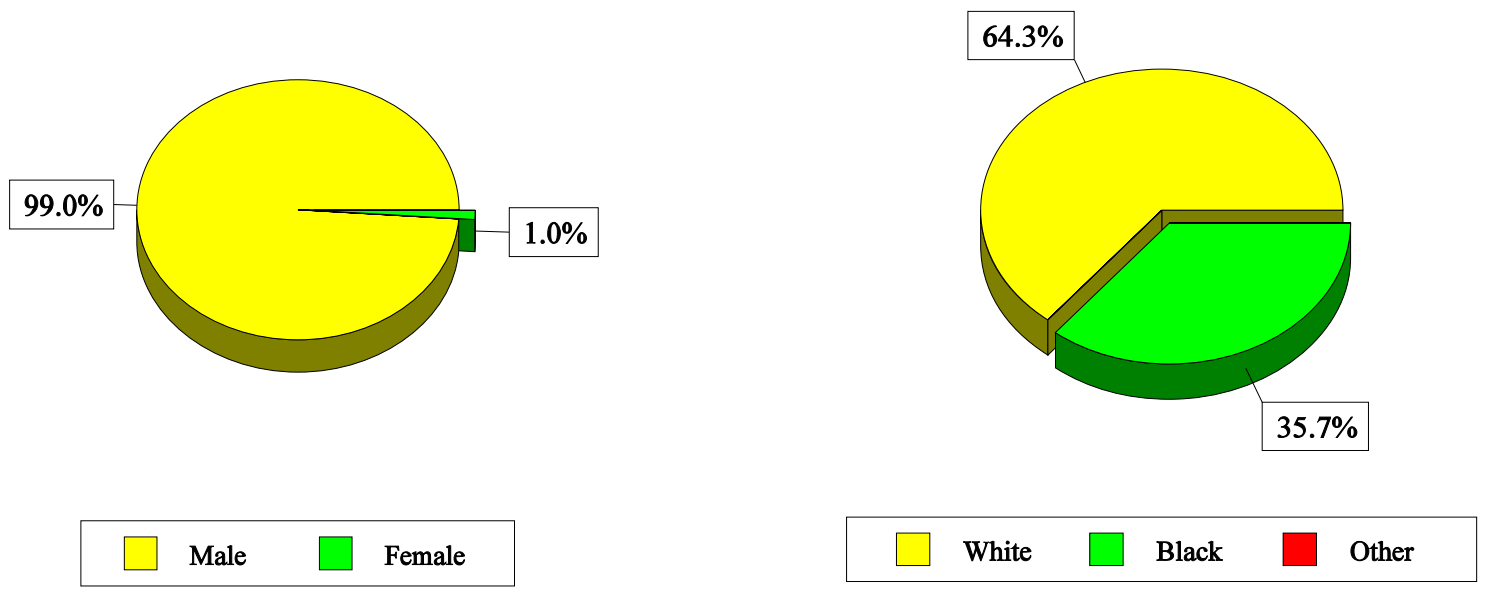

NOTE: $\quad$ See Appendix A for source description and Appendix B for methods and ICD-8 and ICD-9 codes.

SOURCE: National Center for Health Statistics multiple cause of death data.

Table AL-2. All pneumoconioses: Distribution of deaths by sex, race, and age, Alabama residents age 15 and over, 1968-1992

\begin{tabular}{|c|c|c|c|c|c|}
\hline & & \multicolumn{2}{|c|}{ 1968-1992 } & \multicolumn{2}{|c|}{ 1988-1992 } \\
\hline & & Number & Percent & Number & Percent \\
\hline \multicolumn{2}{|l|}{ Total deaths } & 1,368 & 100.0 & 303 & 100.0 \\
\hline \multirow[t]{2}{*}{ Sex } & Male & 1,355 & 99.0 & 299 & 98.7 \\
\hline & Female & 13 & 1.0 & 4 & 1.3 \\
\hline \multirow[t]{3}{*}{ Race } & White & 880 & 64.3 & 227 & 74.9 \\
\hline & Black & 488 & 35.7 & 76 & 25.1 \\
\hline & Other & 0 & 0.0 & 0 & 0.0 \\
\hline \multirow[t]{10}{*}{ Age } & $15-24$ & 1 & 0.1 & 1 & 0.3 \\
\hline & $25-34$ & 2 & 0.1 & 1 & 0.3 \\
\hline & $35-44$ & 14 & 1.0 & 3 & 1.0 \\
\hline & $45-54$ & 42 & 3.1 & 2 & 0.7 \\
\hline & $55-64$ & 191 & 14.0 & 23 & 7.6 \\
\hline & $65-74$ & 489 & 35.7 & 89 & 29.4 \\
\hline & $75-84$ & 464 & 33.9 & 124 & 40.9 \\
\hline & $85+$ & 165 & 12.1 & 60 & 19.8 \\
\hline & Mean age & 73.0 & & 75.9 & \\
\hline & Range for age & $16-101$ & & $16-95$ & \\
\hline
\end{tabular}

NOTE: $\quad$ Percentages may not total to $100 \%$ due to rounding. See Appendix A for source description and Appendix B for methods and ICD-8 and ICD-9 codes.

SOURCE: National Center for Health Statistics multiple cause of death data. 
Figure AL-3. Pneumoconiosis deaths by condition, Alabama residents age 15 and over, 1968-1992

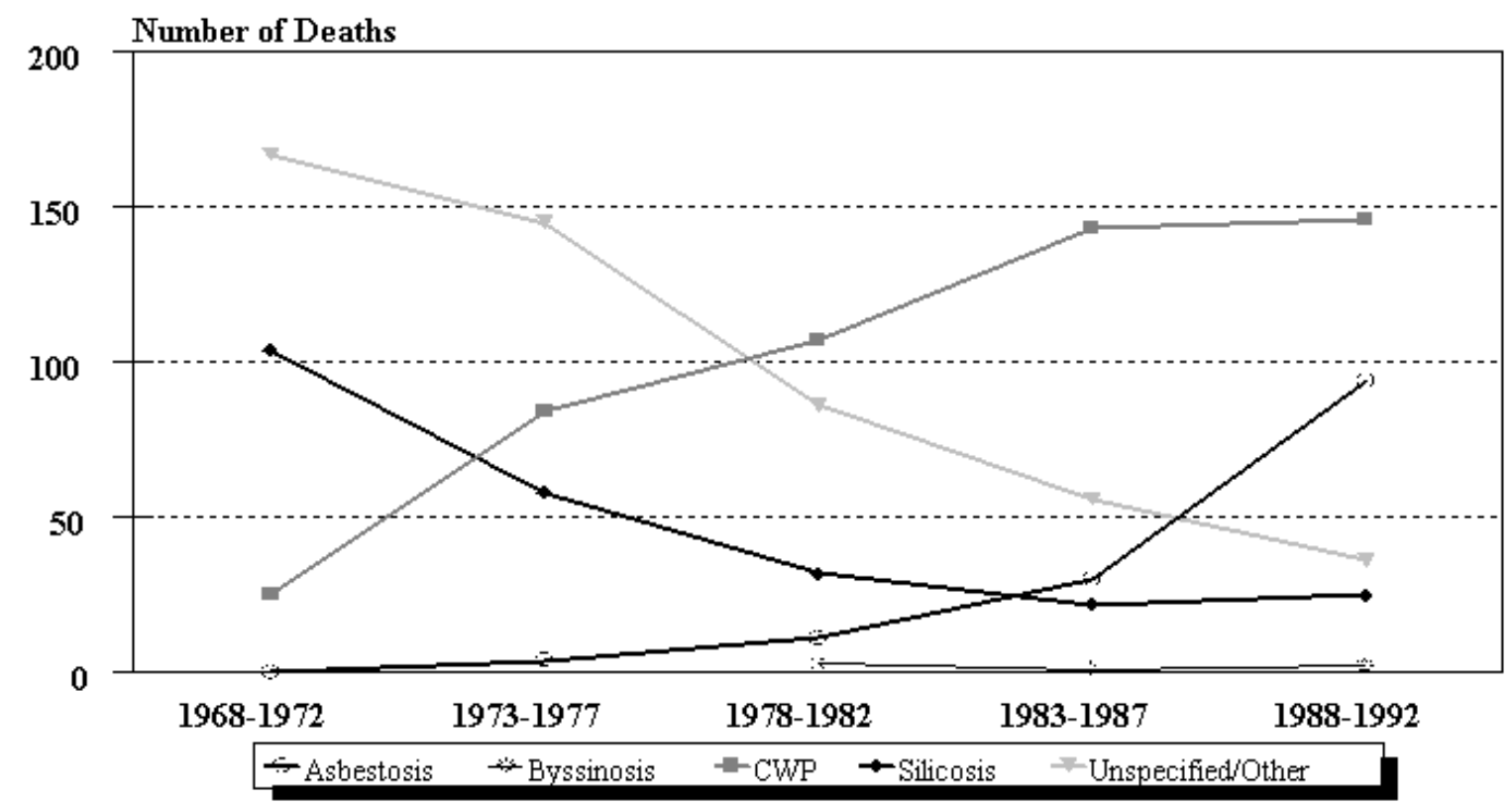

NOTE: See Appendix A for source description and Appendix B for methods and ICD-8 and ICD-9 codes.

SOURCE: National Center for Health Statistics multiple cause of death data.

Figure AL-4. Alabama and U.S. age-adjusted mortality rates (per 1,000,000 population) by condition, residents age 15 and over, 1983-1992

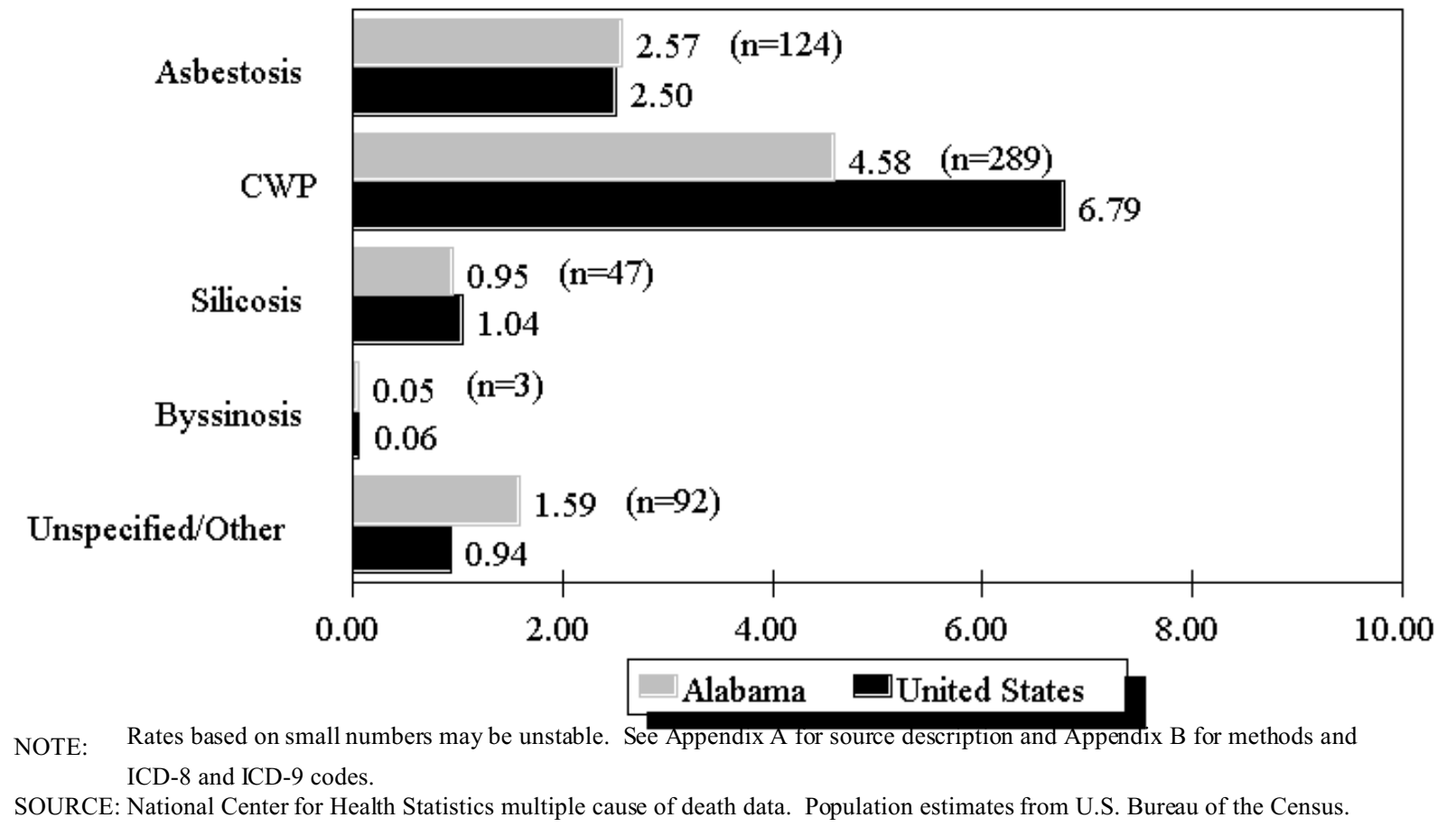


Table AL-3 (page 1 of 2). Number of deaths and age-adjusted mortality rate (per $1,000,000$ population) by condition and county, Alabama residents age 15 and over, 1983-1992

\begin{tabular}{|c|c|c|c|c|c|c|c|c|c|c|c|c|}
\hline \multirow[b]{2}{*}{ County } & \multicolumn{2}{|c|}{ Asbestosis } & \multicolumn{2}{|c|}{$\begin{array}{c}\text { Coal workers' } \\
\text { pneumoconiosis }\end{array}$} & \multicolumn{2}{|c|}{ Silicosis } & \multicolumn{2}{|c|}{ Byssinosis } & \multicolumn{2}{|c|}{$\begin{array}{r}\text { Unspecified/Other } \\
\text { pneumoconioses }\end{array}$} & \multicolumn{2}{|c|}{$\begin{array}{r}\text { All } \\
\text { pneumoconioses }\end{array}$} \\
\hline & $\#$ & Rate & \# & Rate & \# & Rate & $\#$ & Rate & $\#$ & Rate & $\#$ & Rate \\
\hline Autauga & - & - & - & - & 1 & 3.99 & - & - & - & - & 1 & 3.99 \\
\hline Baldwin & 10 & 8.06 & 2 & 1.01 & - & - & - & - & 1 & 0.50 & 13 & 9.58 \\
\hline Barbour & - & - & - & - & 1 & 1.73 & - & - & - & - & 1 & 1.73 \\
\hline Bibb & - & - & - & - & - & - & - & - & 2 & 9.53 & 2 & 9.53 \\
\hline Blount & - & - & 5 & 6.49 & - & - & - & - & - & - & 5 & 6.49 \\
\hline Butler & 1 & 1.59 & - & - & - & - & - & - & - & - & 1 & 1.59 \\
\hline Calhoun & 2 & 1.38 & 6 & 4.15 & 1 & 0.50 & - & - & 1 & 0.50 & 10 & 6.53 \\
\hline Chambers & - & - & - & - & - & - & 1 & 2.37 & - & - & 1 & 2.37 \\
\hline Cherokee & - & - & - & - & - & - & 1 & 2.55 & - & - & 1 & 2.55 \\
\hline Chilton & - & - & 1 & 1.45 & - & - & 1 & 1.45 & - & - & 2 & 2.90 \\
\hline Clarke & 3 & 9.52 & - & - & - & - & - & - & - & - & 3 & 9.52 \\
\hline Cleburne & - & - & - & - & 1 & 7.68 & - & - & - & - & 1 & 7.68 \\
\hline Colbert & 2 & 3.39 & - & - & 2 & 3.39 & - & - & - & - & 4 & 6.79 \\
\hline Coosa & - & - & - & - & - & - & - & - & 2 & 16.25 & 2 & 16.25 \\
\hline Covington & 2 & 3.28 & 2 & 2.00 & - & - & - & - & - & - & 4 & 5.27 \\
\hline Cullman & - & - & 3 & 3.48 & - & - & - & - & - & - & 3 & 3.48 \\
\hline Dale & - & - & - & - & 1 & 2.76 & - & - & - & - & 1 & 2.76 \\
\hline Dallas & - & - & 1 & 0.92 & - & - & - & - & - & - & 1 & 0.92 \\
\hline De Kalb & 1 & 1.73 & 1 & 0.81 & - & - & - & - & 2 & 4.36 & 4 & 6.90 \\
\hline Escambia & 2 & 5.52 & - & - & - & - & - & - & - & - & 2 & 5.52 \\
\hline Etowah & 1 & 0.86 & 3 & 1.75 & - & - & - & - & 2 & 0.89 & 6 & 3.51 \\
\hline Fayette & - & - & 2 & 4.09 & - & - & - & - & 1 & 5.02 & 3 & 9.11 \\
\hline Geneva & - & - & 1 & 3.63 & - & - & - & - & - & - & 1 & 3.63 \\
\hline Henry & 1 & 2.59 & - & - & - & - & - & - & - & - & 1 & 2.59 \\
\hline Houston & 1 & 1.32 & - & - & - & - & - & - & - & - & 1 & 1.32 \\
\hline Jackson & - & - & 2 & 3.27 & 1 & 2.09 & - & - & - & - & 2 & 3.27 \\
\hline Jefferson & 8 & 0.89 & 118 & 11.21 & 25 & 2.95 & - & - & 45 & 4.80 & 196 & 19.85 \\
\hline Lamar & 1 & 5.76 & - & - & - & - & - & - & - & - & 1 & 5.76 \\
\hline Lauderdale & 1 & 1.18 & - & - & - & - & - & - & - & - & 1 & 1.18 \\
\hline Lawrence & 1 & 3.43 & 1 & 1.84 & - & - & - & - & - & - & 2 & 5.27 \\
\hline Limestone & - & - & 1 & 1.12 & 1 & 2.10 & - & - & - & - & 2 & 3.21 \\
\hline Madison & 2 & 1.04 & - & - & - & - & - & - & - & - & 2 & 1.04 \\
\hline
\end{tabular}

See footnotes at end of table. 
Table AL-3 (page 2 of 2). Number of deaths and age-adjusted mortality rate (per $1,000,000$ population) by condition and county, Alabama residents age 15 and over, 1983-1992

\begin{tabular}{|c|c|c|c|c|c|c|c|c|c|c|c|c|}
\hline \multirow[b]{2}{*}{ County } & \multicolumn{2}{|c|}{ Asbestosis } & \multicolumn{2}{|c|}{$\begin{array}{c}\text { Coal workers' } \\
\text { pneumoconiosis }\end{array}$} & \multicolumn{2}{|c|}{ Silicosis } & \multicolumn{2}{|c|}{ Byssinosis } & \multicolumn{2}{|c|}{$\begin{array}{r}\text { Unspecified/Other } \\
\text { pneumoconioses }\end{array}$} & \multicolumn{2}{|c|}{$\begin{array}{r}\text { All } \\
\text { pneumoconioses }\end{array}$} \\
\hline & \# & Rate & \# & Rate & \# & Rate & \# & Rate & \# & Rate & \# & Rate \\
\hline Marion & - & - & 5 & 8.51 & - & - & - & - & 1 & 3.08 & 6 & 11.59 \\
\hline Marshall & 1 & 0.69 & 2 & 2.01 & - & - & - & - & - & - & 3 & 2.70 \\
\hline Mobile & 70 & 17.91 & 1 & 0.16 & 1 & 0.29 & - & - & 1 & 0.16 & 73 & 18.53 \\
\hline Monroe & 2 & 6.54 & - & - & - & - & - & - & 1 & 1.94 & 3 & 8.48 \\
\hline Montgomery & - & - & 2 & 0.82 & - & - & - & - & - & - & 2 & 0.82 \\
\hline Morgan & 2 & 1.69 & 3 & 2.30 & - & - & - & - & 1 & 0.61 & 6 & 4.60 \\
\hline Perry & - & - & 1 & 2.65 & - & - & - & - & - & - & 1 & 2.65 \\
\hline Pickens & 2 & 6.08 & 1 & 4.31 & - & - & - & - & - & - & 3 & 10.39 \\
\hline Pike & - & - & - & - & 1 & 4.46 & - & - & - & - & 1 & 4.46 \\
\hline Russell & 1 & 2.11 & 1 & 2.11 & - & - & - & - & - & - & 2 & 4.22 \\
\hline St. Clair & - & - & 9 & 14.26 & 3 & 4.75 & - & - & 3 & 3.88 & 14 & 20.73 \\
\hline Shelby & 2 & 3.03 & 13 & 18.00 & - & - & - & - & 1 & 1.61 & 16 & 22.64 \\
\hline Sumter & - & - & 1 & 2.38 & - & - & - & - & - & - & 1 & 2.38 \\
\hline Talladega & - & - & - & - & 1 & 1.35 & - & - & - & - & 1 & 1.35 \\
\hline Tallapoosa & 1 & 1.06 & 1 & 2.27 & 1 & 2.27 & - & - & 1 & 2.27 & 4 & 7.88 \\
\hline Tuscaloosa & - & - & 16 & 9.71 & 5 & 3.17 & - & - & 5 & 3.54 & 25 & 15.63 \\
\hline Walker & 2 & 2.82 & 83 & 79.82 & 1 & 0.68 & - & - & 22 & 20.11 & 108 & 103.44 \\
\hline Washington & 2 & 13.46 & - & - & - & - & - & - & - & - & 2 & 13.46 \\
\hline Winston & - & - & 1 & 2.22 & - & - & - & - & - & - & 1 & 2.22 \\
\hline
\end{tabular}




\section{Alaska: Selected statistics from the United States section}

All pneumoconioses mortality, 1988-1992

Total deaths

Crude mortality rate (per $1,000,000$ population)

Age-adjusted mortality rate (per $1,000,000$ population)

Total years of potential life lost to life expectancy

Years of potential life lost to life expectancy (per death)
Value

7

3.48

5.61

100

14.3
Deaths*

5

Coal workers' pneumoconiosis

Silicosis

Byssinosis

\section{Unspecified/Other pneumoconiosis}

, 1988-1992

* Sum of column may exceed total deaths because of deaths with multiple pneumoconioses.

** Percentages may total more than $100 \%$ due to deaths with multiple pneumoconioses.

NOTE: $\quad$ See Appendix A for source description and Appendix B for methods and ICD-8 and ICD-9 codes.

SOURCE: National Center for Health Statistics multiple cause of death data. Population estimates from U.S. Bureau of the Census.
State rank

Percent**

distribution

71.4

14.3

14.3

0.0

0

$\mathbf{0 . 0}$

Figure AK-1. All pneumoconioses: Alaska and U.S. age-adjusted mortality rates, residents age 15 and over, 1968-1992

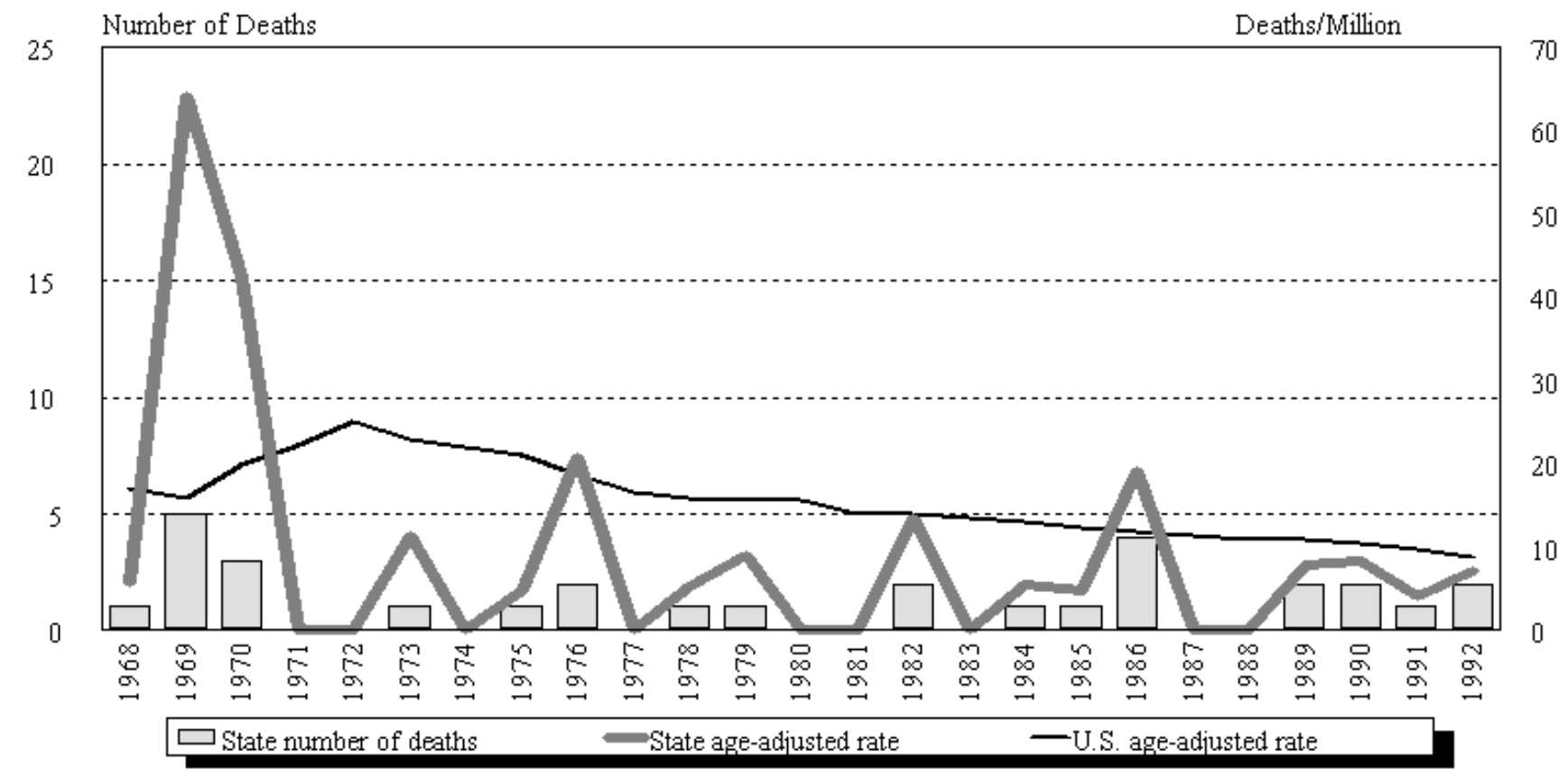

NOTE: $\quad$ See Appendix A for source description and Appendix B for methods and ICD-8 and ICD-9 codes.

SOURCE: $\quad$ National Center for Health Statistics multiple cause of death data. Population estimates from U.S. Bureau of the Census. 


\section{Alaska}

Table AK-1. Number of deaths and age-adjusted mortality rate (per 1,000,000 population) by condition, Alaska residents age 15 and over, 1968-1992

\begin{tabular}{|c|c|c|c|c|c|c|c|c|c|c|c|c|}
\hline \multirow[b]{2}{*}{ Year } & \multicolumn{2}{|c|}{ Asbestosis } & \multicolumn{2}{|c|}{$\begin{array}{l}\text { Coal workers' } \\
\text { pneumoconiosis }\end{array}$} & \multicolumn{2}{|c|}{ Silicosis } & \multicolumn{2}{|c|}{ Byssinosis* } & \multicolumn{2}{|c|}{$\begin{array}{c}\text { Unspecified/Other } \\
\text { pneumoconioses }\end{array}$} & \multicolumn{2}{|c|}{ All pneumoconioses } \\
\hline & Number & Rate & Number & Rate & Number & Rate & Number & Rate & Number & Rate & Number & Rate \\
\hline 1968 & 1 & 5.79 & - & - & - & - & & & - & - & 1 & 5.79 \\
\hline 1969 & - & - & 1 & 13.64 & 4 & 50.48 & & & - & - & 5 & 64.11 \\
\hline 1970 & - & - & - & - & 1 & 15.29 & & & 2 & 27.28 & 3 & 42.56 \\
\hline 1971 & - & - & - & - & - & - & & & - & - & - & - \\
\hline 1972 & - & - & - & - & - & - & & & - & - & - & - \\
\hline 1973 & - & - & - & - & 1 & 11.40 & & & - & - & 1 & 11.40 \\
\hline 1974 & - & - & - & - & - & - & & & - & - & - & - \\
\hline 1975 & 1 & 4.89 & - & - & - & - & & & - & - & 1 & 4.89 \\
\hline 1976 & 1 & 9.73 & - & - & 1 & 11.01 & & & - & - & 2 & 20.74 \\
\hline 1977 & - & - & - & - & - & - & & & - & - & - & - \\
\hline 1978 & - & - & - & - & - & - & & & 1 & 5.29 & 1 & 5.29 \\
\hline 1979 & - & - & - & - & - & - & - & - & 1 & 9.12 & 1 & 9.12 \\
\hline 1980 & - & - & - & - & - & - & - & - & - & - & - & - \\
\hline 1981 & - & - & - & - & - & - & - & - & - & - & - & - \\
\hline 1982 & - & - & - & - & 2 & 13.40 & - & - & - & - & 2 & 13.40 \\
\hline 1983 & - & - & - & - & - & - & - & - & - & - & - & - \\
\hline 1984 & - & - & 1 & 5.58 & - & - & - & - & - & - & 1 & 5.58 \\
\hline 1985 & - & - & - & - & 1 & 4.90 & - & - & - & - & 1 & 4.90 \\
\hline 1986 & 1 & 4.63 & 1 & 4.83 & 2 & 9.67 & - & - & - & - & 4 & 19.13 \\
\hline 1987 & - & - & - & - & - & - & - & - & - & - & - & - \\
\hline 1988 & - & - & - & - & - & - & - & - & - & - & - & - \\
\hline 1989 & 2 & 7.96 & - & - & - & - & - & - & - & - & 2 & 7.96 \\
\hline 1990 & 1 & 4.17 & - & - & 1 & 4.12 & - & - & - & - & 2 & 8.28 \\
\hline 1991 & 1 & 4.03 & - & - & - & - & - & - & - & - & 1 & 4.03 \\
\hline 1992 & 1 & 3.53 & 1 & 3.64 & - & - & - & - & - & - & 2 & 7.17 \\
\hline
\end{tabular}

indicates no deaths listed. ${ }^{*}$ no specific code for byssinosis in ICD-8 (1968-1978)

NOTE: Rates based on small numbers may be unstable. Sum of individual conditions may exceed total because of deaths with multiple pneumoconioses. See Appendix A for source description and Appendix B for methods and ICD-8 and ICD-9 NoTE:

SOURCE: National Center for Health Statistics multiple cause of death data. Population estimates from U.S. Bureau of the Census. 
Figure AK-2. All pneumoconioses: Distribution of deaths by sex and race, Alaska residents age 15 and over, 1968-1992
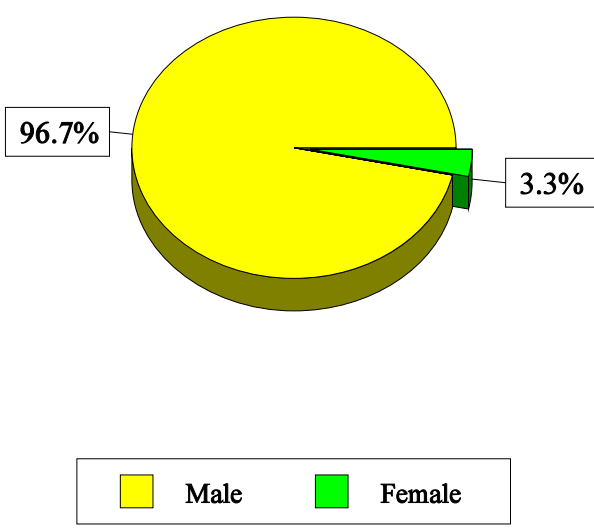

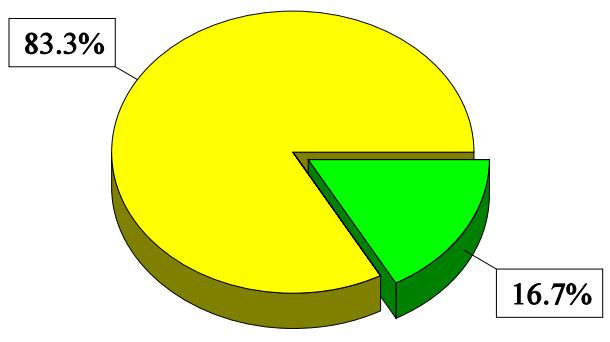

White

NOTE: $\quad$ See Appendix A for source description and Appendix B for methods and ICD-8 and ICD-9 codes.

SOURCE: National Center for Health Statistics multiple cause of death data.

Table AK-2. All pneumoconioses: Distribution of deaths by sex, race, and age, Alaska residents age 15 and over, 1968-1992

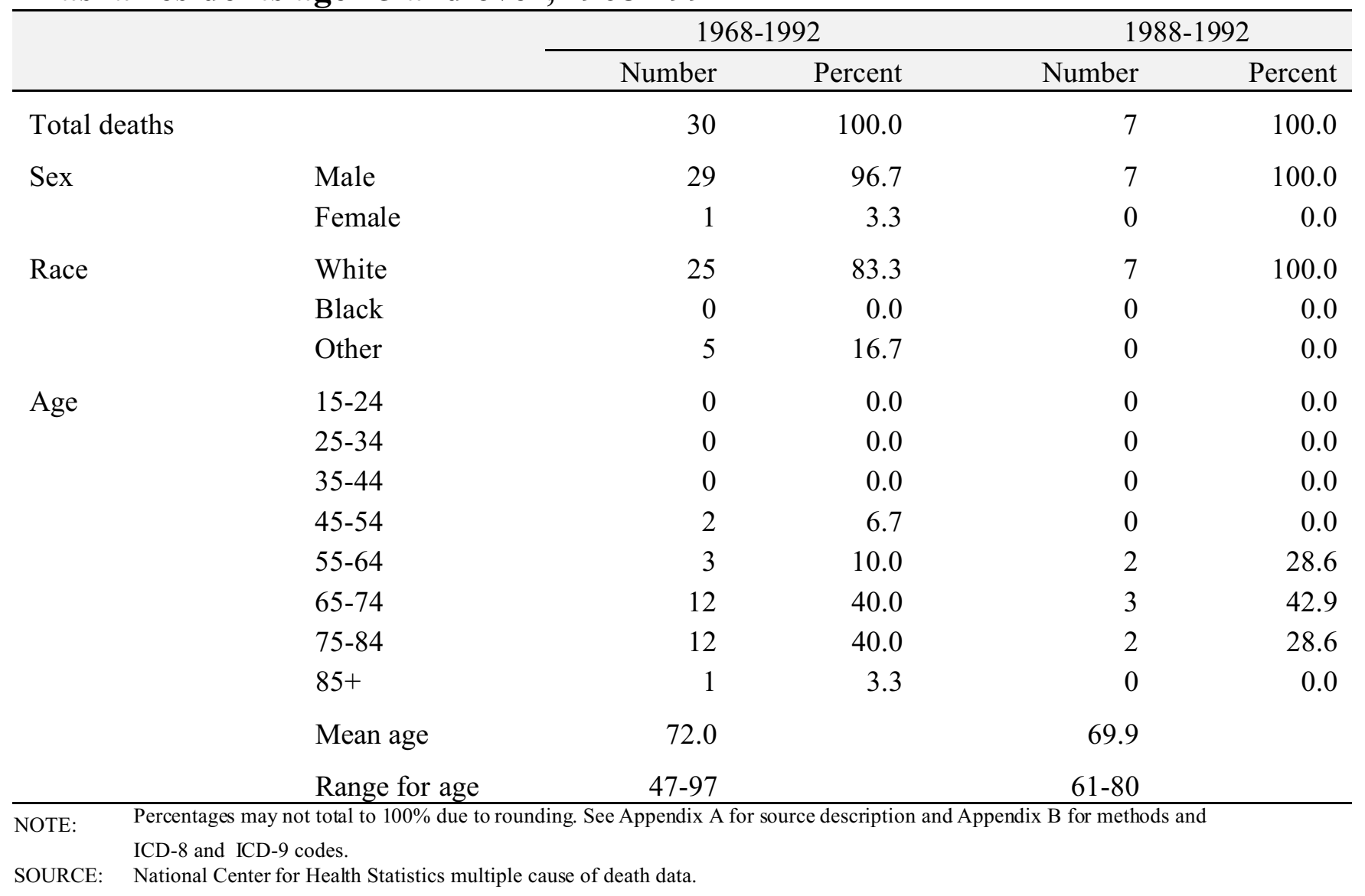


Figure AK-3. Pneumoconiosis deaths by condition, Alaska residents age 15 and over, 1968-1992

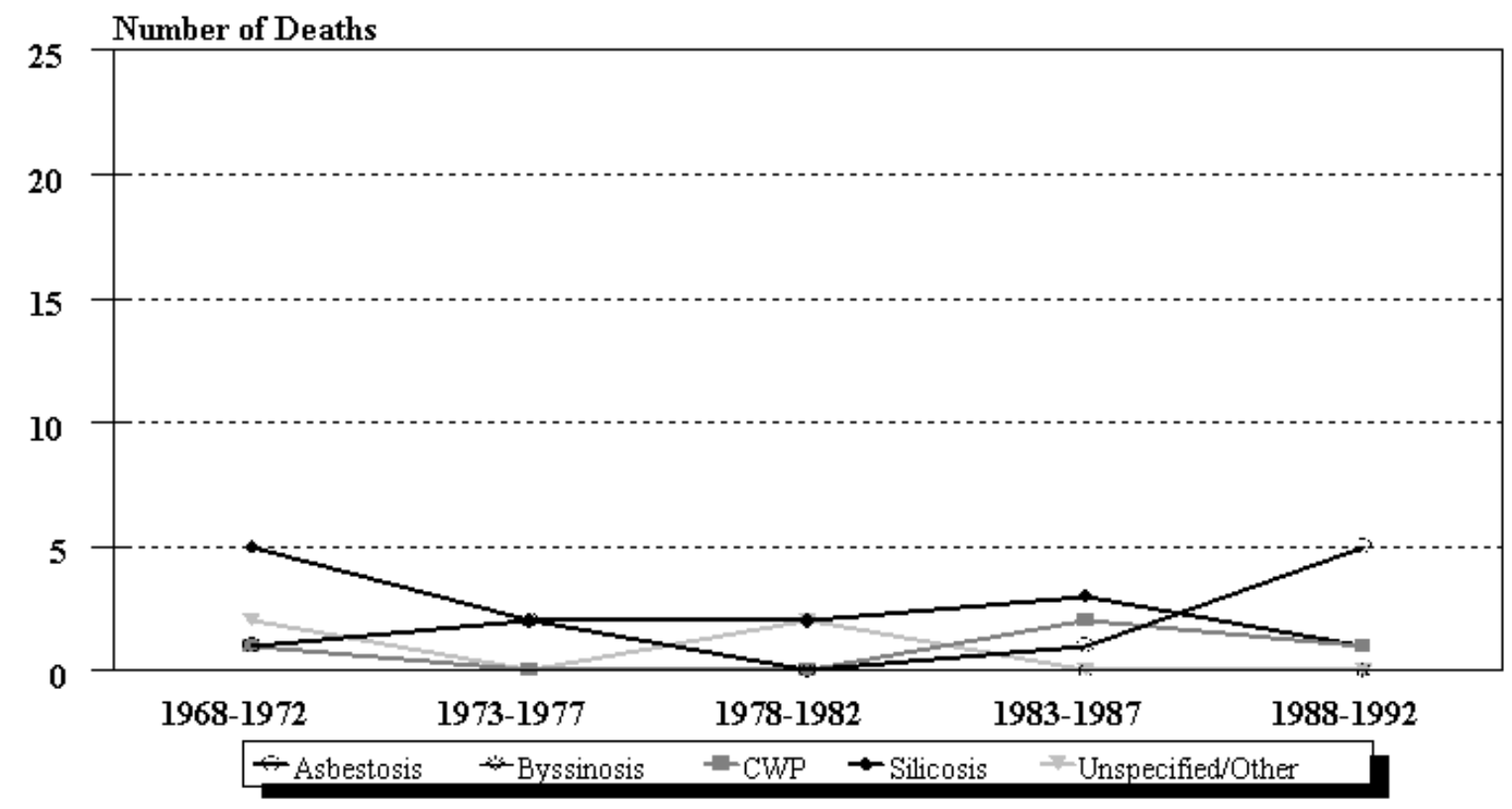

NOTE: See Appendix A for source description and Appendix B for methods and ICD-8 and ICD-9 codes. SOURCE: National Center for Health Statistics multiple cause of death data.

Figure AK-4. Alaska and U.S. age-adjusted mortality rates (per $1,000,000$ population) by condition, residents age 15 and over 1983-1992

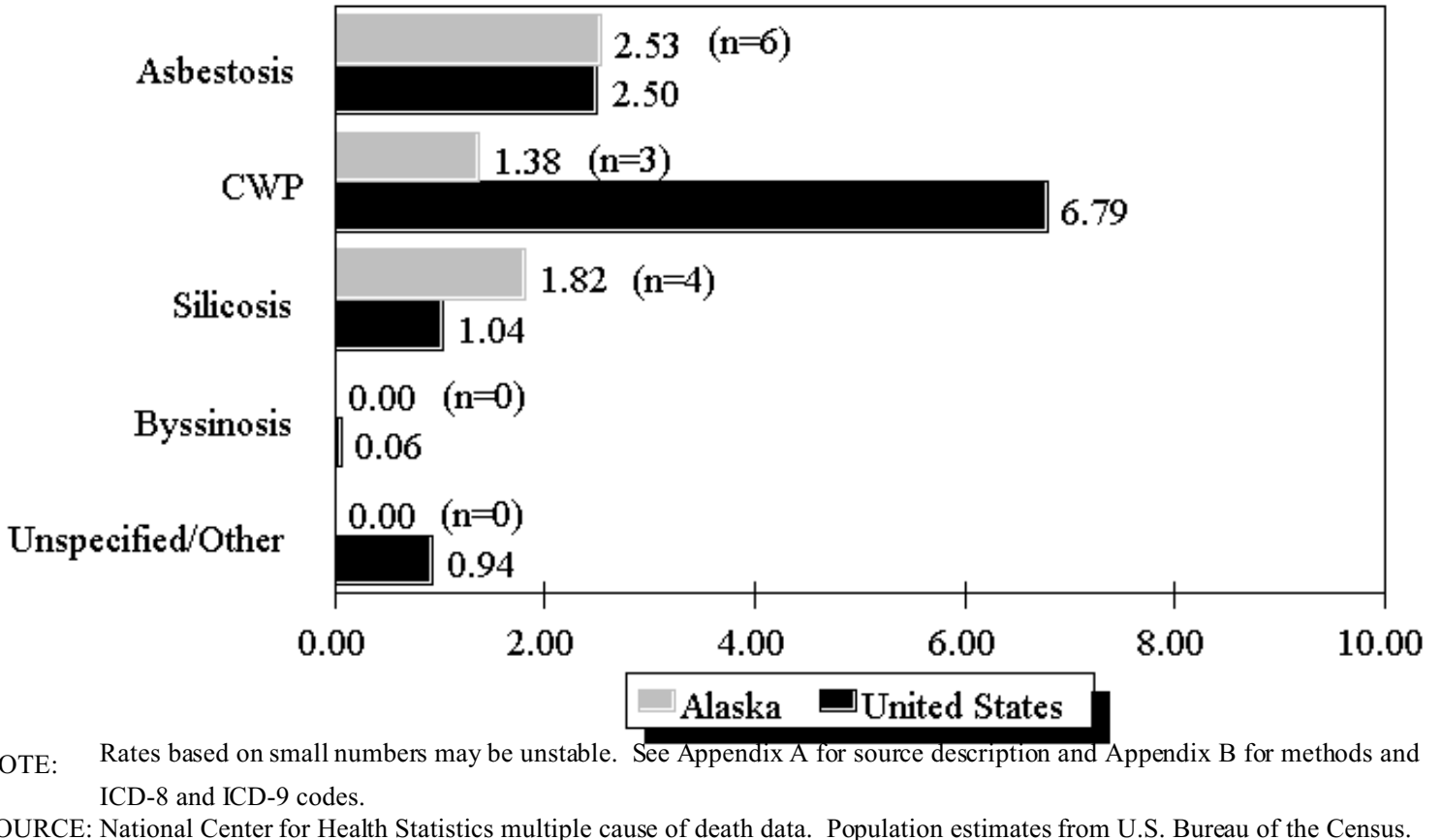


Table AK-3. Number of deaths and age-adjusted mortality rate (per 1,000,000 population) by condition and county, Alaska residents age 15 and over, 1983-1992

\begin{tabular}{|c|c|c|c|c|c|c|c|c|c|c|c|c|}
\hline \multirow[b]{2}{*}{ County } & \multicolumn{2}{|c|}{ Asbestosis } & \multicolumn{2}{|c|}{$\begin{array}{c}\text { Coal workers' } \\
\text { pneumoconiosis }\end{array}$} & \multicolumn{2}{|c|}{ Silicosis } & \multicolumn{2}{|c|}{ Byssinosis } & \multicolumn{2}{|c|}{$\begin{array}{r}\text { Unspecified/Other } \\
\text { pneumoconioses }\end{array}$} & \multicolumn{2}{|c|}{$\begin{array}{r}\text { All } \\
\text { pneumoconioses }\end{array}$} \\
\hline & \# & Rate & \# & Rate & \# & Rate & $\#$ & Rate & \# & Rate & \# & Rate \\
\hline Anchorage & 3 & 2.91 & 1 & 1.30 & 2 & 2.61 & - & - & - & - & 6 & 6.82 \\
\hline Fairbanks & 1 & 3.51 & - & - & - & - & - & - & - & - & 1 & 3.51 \\
\hline Juneau & - & - & - & - & 1 & 7.42 & - & - & - & - & 1 & 7.42 \\
\hline $\begin{array}{l}\text { Matanuska- } \\
\text { Susitna }\end{array}$ & 1 & 4.84 & 2 & 10.34 & - & - & - & - & - & - & 3 & 15.18 \\
\hline North Slope & - & - & - & - & 1 & 46.17 & - & - & - & - & 1 & 46.17 \\
\hline $\begin{array}{l}\text { Wrangell- } \\
\text { Petersburg }\end{array}$ & 1 & 22.65 & - & - & - & - & - & - & - & - & 1 & 22.65 \\
\hline
\end{tabular}

- indicates no deaths listed.

NOTE: Counties with no deaths for all listed conditions are not displayed. Rates based on small numbers may be unstable. See Appendix A for source description and Appendix B for methods and ICD-8 and ICD-9 codes.

SOURCE: National Center for Health Statistics multiple cause of death data. Population estimates from U.S. Bureau of the Census. 


\section{Arizona: Selected statistics from the United States section}

All pneumoconioses mortality, 1988-1992

Total deaths

Crude mortality rate (per $1,000,000$ population)

Age-adjusted mortality rate (per $1,000,000$ population)

Total years of potential life lost to life expectancy

Years of potential life lost to life expectancy (per death)

\begin{tabular}{rr} 
Value & State rank \\
\hline 75 & 31 \\
5.28 & 40 \\
3.15 & 40 \\
933 & 31 \\
12.4 & 17
\end{tabular}

Percent**

Deaths* distribution

30

40.0

Asbestosis

Coal workers' pneumoconiosis

20

26.7

Silicosis

Byssinosis

15

20.0

1.3

1

12.0

Unspecified/Other pneumoconiosis

9

* Sum of column may exceed total deaths because of deaths with multiple pneumoconioses.

** Percentages may total more than $100 \%$ due to deaths with multiple pneumoconioses.

NOTE: $\quad$ See Appendix A for source description and Appendix B for methods and ICD-8 and ICD-9 codes.

SOURCE: $\quad$ National Center for Health Statistics multiple cause of death data. Population estimates from U.S. Bureau of the Census.

Figure AZ-1. All pneumoconioses: Arizona and U.S. age-adjusted mortality rates, residents age 15 and over, 1968-1992

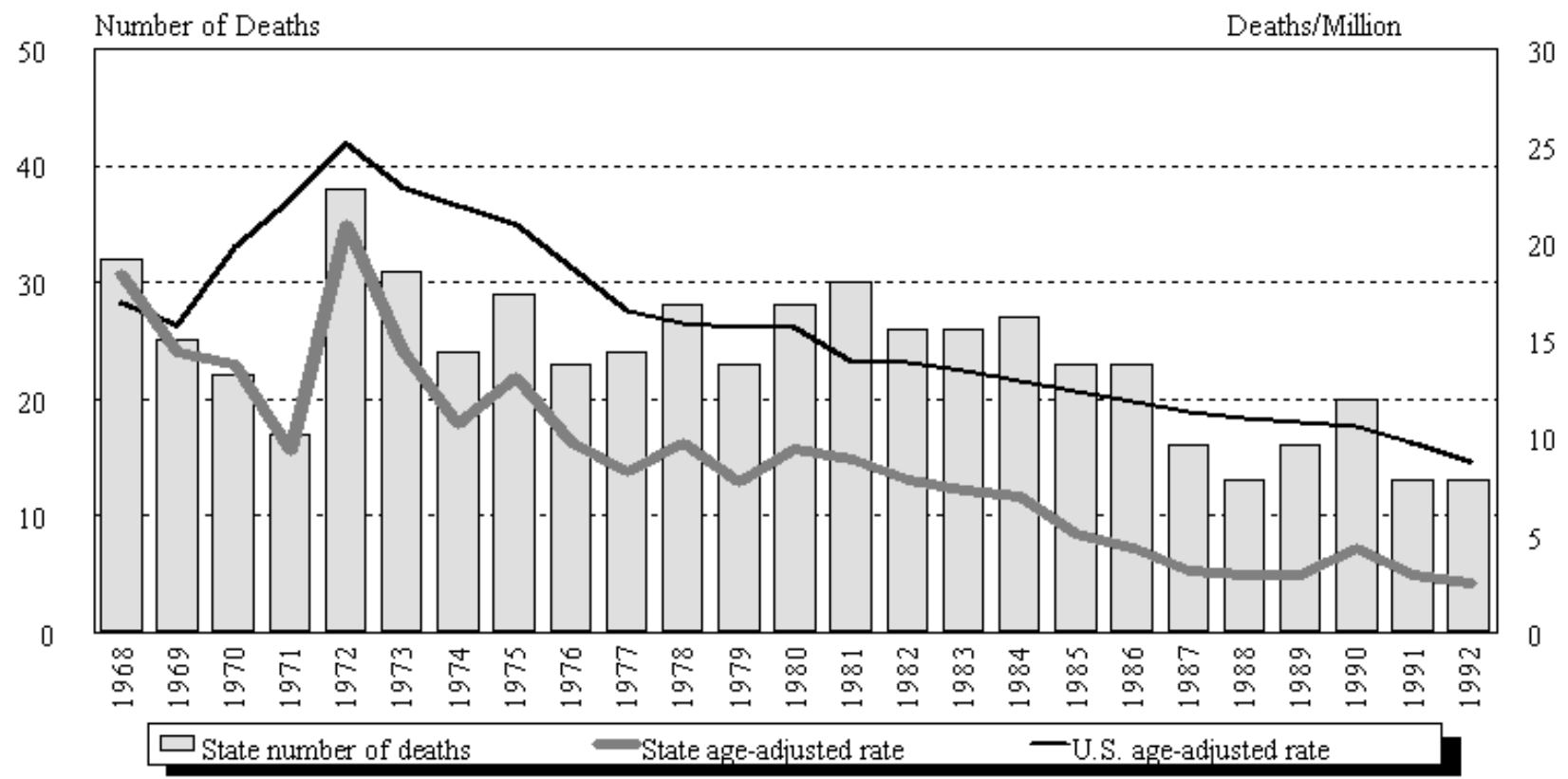

NOTE: $\quad$ See Appendix A for source description and Appendix B for methods and ICD-8 and ICD-9 codes.

SOURCE: $\quad$ National Center for Health Statistics multiple cause of death data. Population estimates from U.S. Bureau of the Census. 


\section{Arizona}

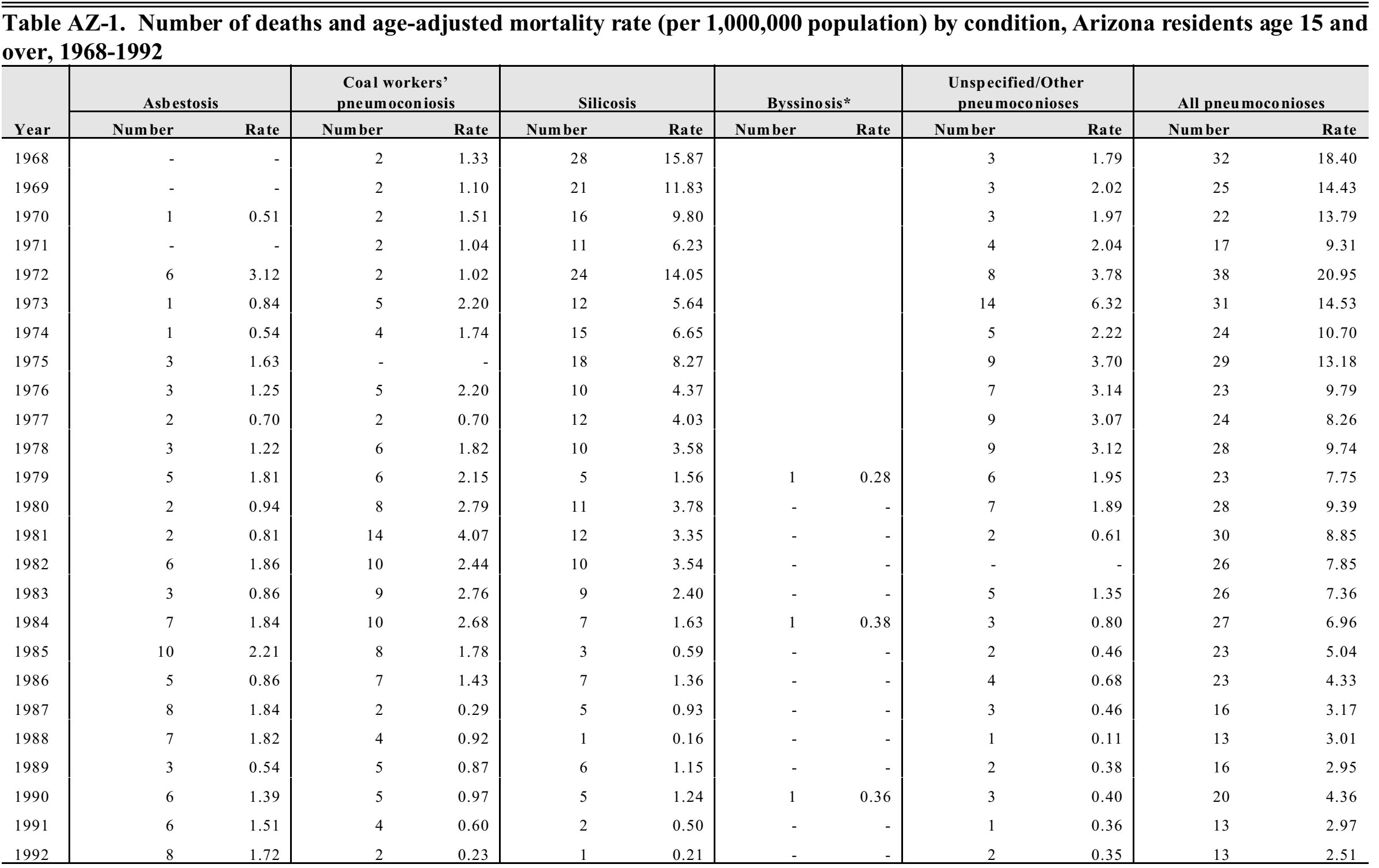

indicates no deaths listed. * no specific code for byssinosis in ICD-8 (1968-1978)

NOTE: $\quad$ Rates based on small numbers may be unstable. Sum of individual conditions may exceed total because of deaths with multiple pneumo conioses. See Appendix A for source description and Appendix B for methods and ICD-8 and ICD-9 codes.

SOURCE: National Center for Health Statistics multiple cause of death data. Population estimates from U.S. Bureau of the Census. 


\section{Figure AZ-2. All pneumoconioses: Distribution of deaths by sex and race,} Arizona residents age 15 and over, 1968-1992
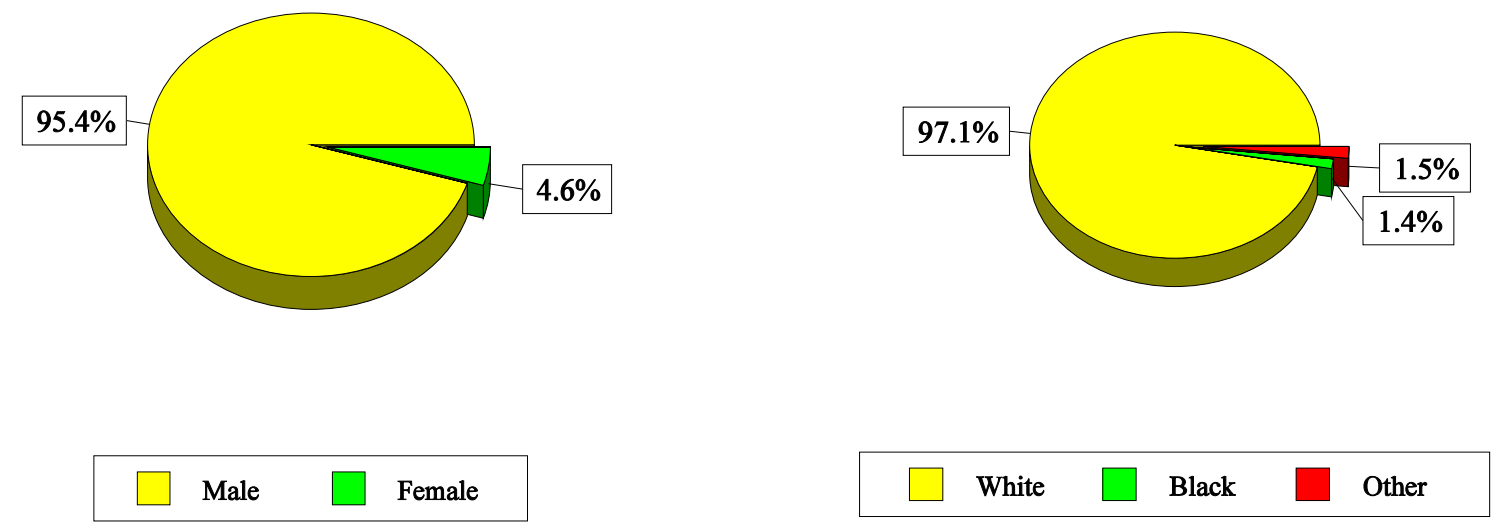

NOTE: See Appendix A for source description and Appendix B for methods and ICD-8 and ICD-9 codes.

SOURCE: National Center for Health Statistics multiple cause of death data.

Table AZ-2. All pneumoconioses: Distribution of deaths by sex, race, and age, Arizona residents age 15 and over, 1968-1992

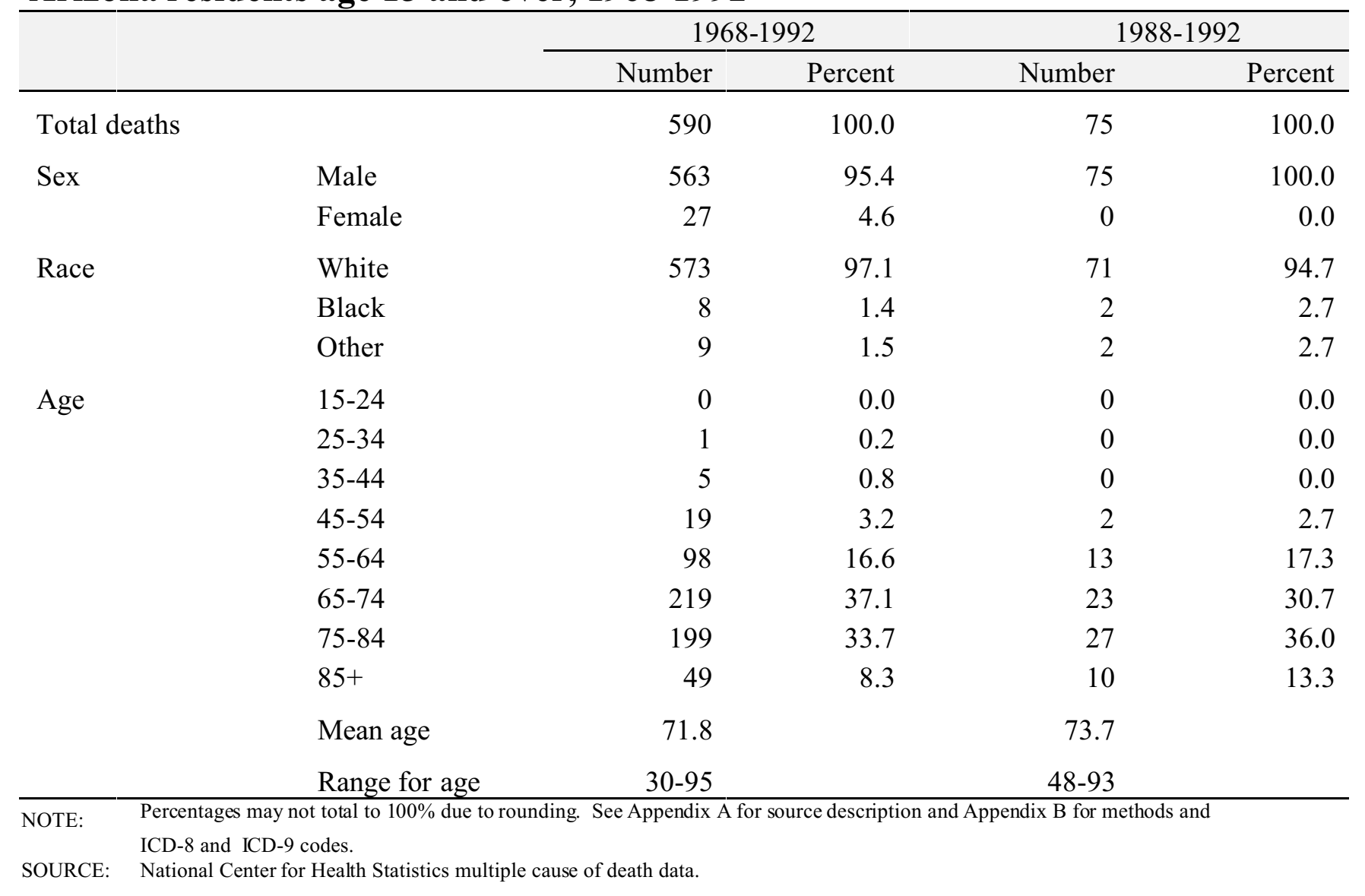


Figure AZ-3. Pneumoconiosis deaths by condition, Arizona residents age 15 and over, 1968-1992

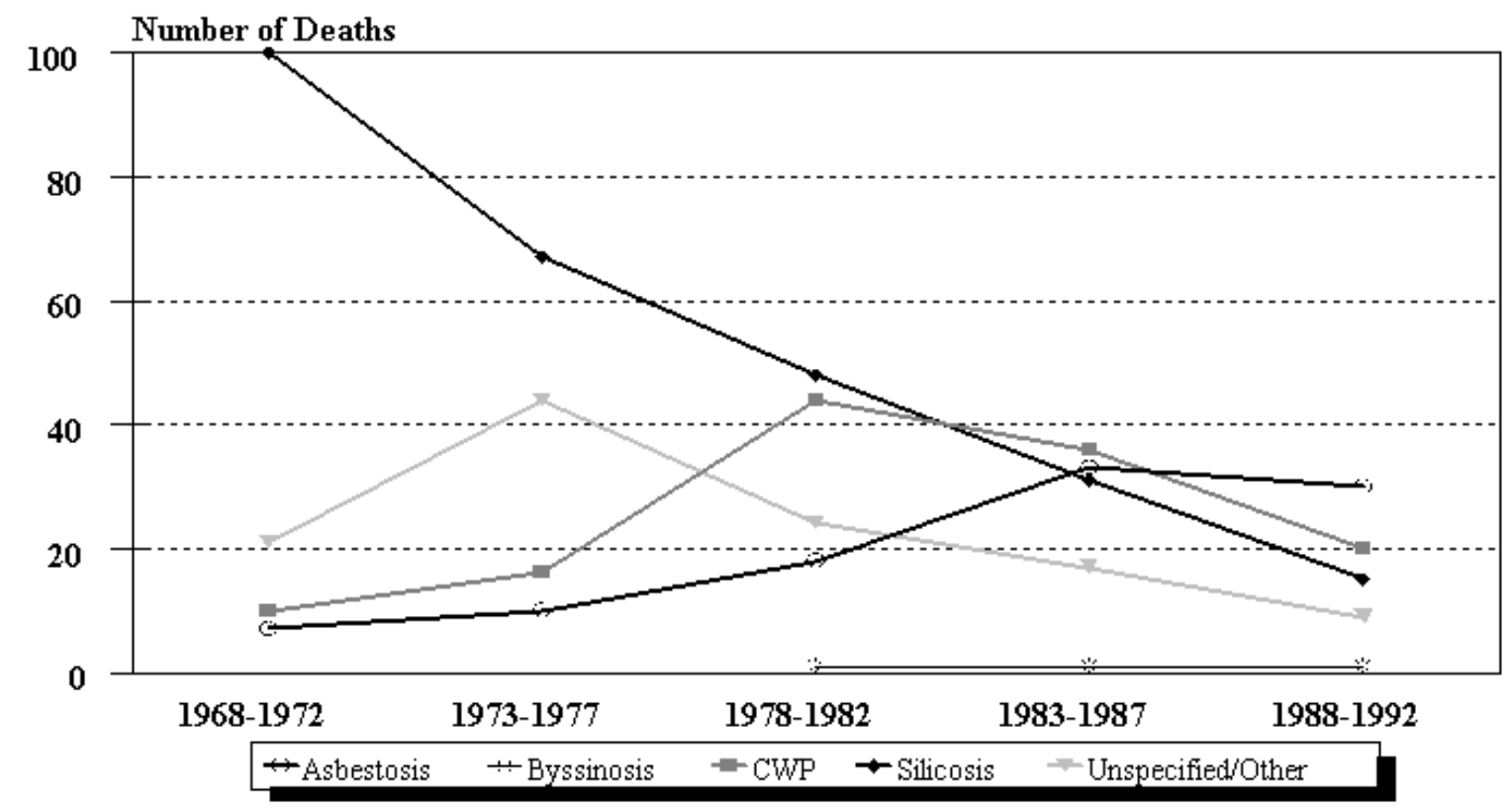

NOTE: See Appendix A for source description and Appendix B for methods and ICD-8 and ICD-9 codes. SOURCE: National Center for Health Statistics multiple cause of death data.

Figure AZ-4. Arizona and U.S. age-adjusted mortality rates (per 1,000,000 population) by condition, residents age 15 and over, 1983-1992

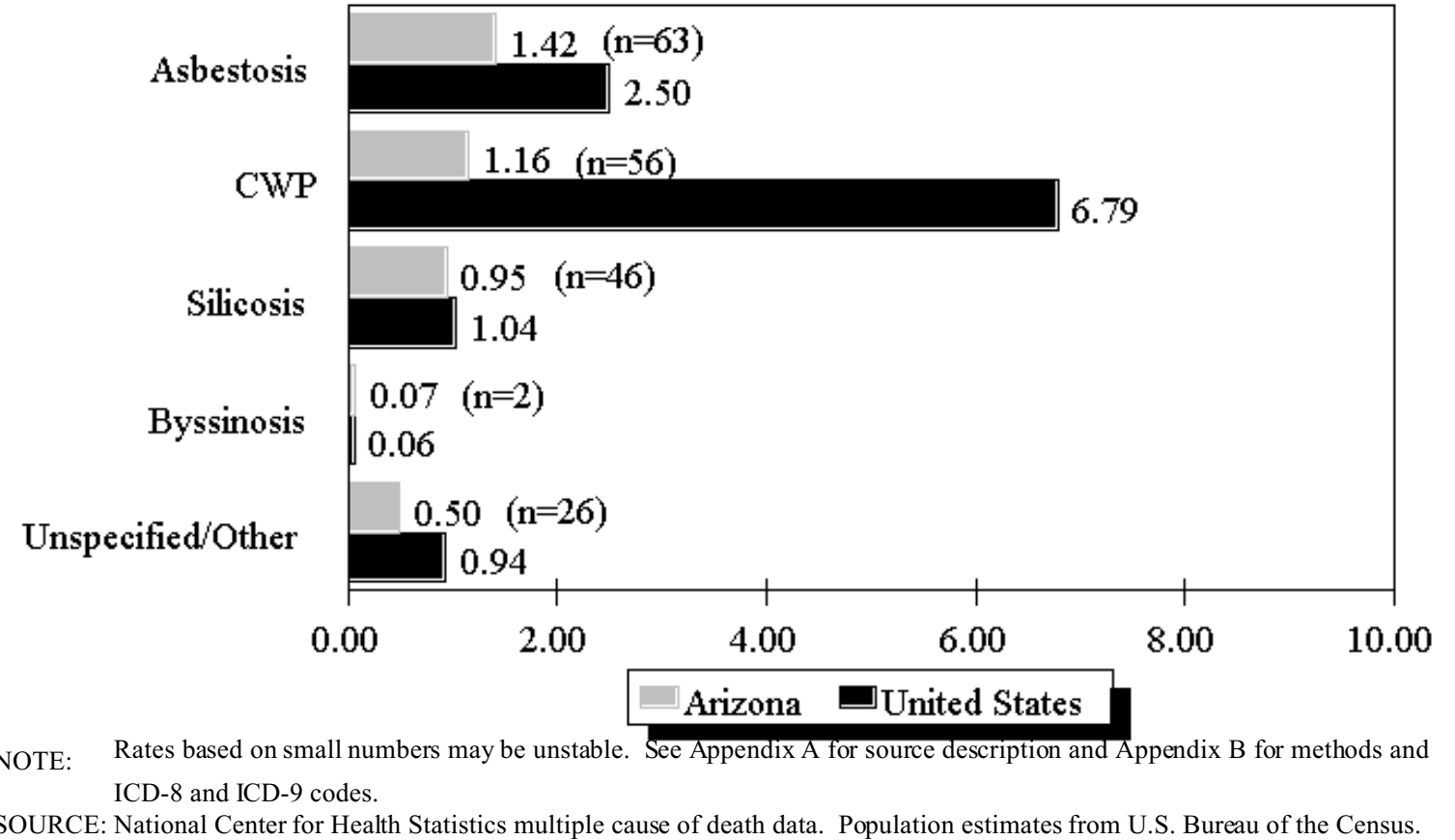


Table AZ-3. Number of deaths and age-adjusted mortality rate (per 1,000,000 population) by condition and county, Arizona residents age 15 and over, 1983-1992

\begin{tabular}{|c|c|c|c|c|c|c|c|c|c|c|c|c|}
\hline \multirow[b]{2}{*}{ County } & \multicolumn{2}{|c|}{ Asbestosis } & \multicolumn{2}{|c|}{$\begin{array}{c}\text { Coal workers' } \\
\text { pneumoconiosis }\end{array}$} & \multicolumn{2}{|c|}{ Silicosis } & \multicolumn{2}{|c|}{ Byssinosis } & \multicolumn{2}{|c|}{$\begin{array}{r}\text { Unspecified/Other } \\
\text { pneumoconioses }\end{array}$} & \multicolumn{2}{|c|}{$\begin{array}{r}\text { All } \\
\text { pneumoconioses }\end{array}$} \\
\hline & \# & Rate & \# & Rate & $\#$ & Rate & \# & Rate & $\#$ & Rate & \# & Rate \\
\hline Apache & - & - & 2 & 4.76 & - & - & - & - & - & - & 2 & 4.76 \\
\hline Cochise & 4 & 3.33 & 3 & 2.30 & 3 & 2.30 & - & - & - & - & 10 & 7.94 \\
\hline Coconino & 1 & 1.55 & 1 & 1.55 & - & - & - & - & - & - & 2 & 3.09 \\
\hline Gila & - & - & - & - & 1 & 1.05 & - & - & 2 & 2.88 & 3 & 3.93 \\
\hline Graham & - & - & - & - & - & - & - & - & 2 & 4.26 & 2 & 4.26 \\
\hline Greenlee & - & - & - & - & 2 & 21.74 & - & - & - & - & 2 & 21.74 \\
\hline Maricopa & 35 & 1.50 & 28 & 1.14 & 21 & 0.74 & - & - & 14 & 0.49 & 96 & 3.81 \\
\hline Mohave & 3 & 1.99 & 2 & 1.26 & 4 & 2.72 & - & - & - & - & 9 & 5.97 \\
\hline Navajo & 1 & 1.83 & - & - & 1 & 1.83 & - & - & - & - & 2 & 3.67 \\
\hline Pima & 9 & 0.96 & 13 & 1.43 & 9 & 1.20 & 1 & 0.16 & 4 & 0.56 & 35 & 4.15 \\
\hline Pinal & 3 & 2.25 & 2 & 1.39 & 2 & 1.06 & - & - & 1 & 0.53 & 8 & 5.24 \\
\hline Yavapai & 3 & 1.58 & 4 & 2.21 & 3 & 1.29 & - & - & 2 & 0.67 & 12 & 5.75 \\
\hline Yuma & 4 & 3.39 & 1 & 0.95 & - & - & 1 & 0.95 & 1 & 0.56 & 7 & 5.72 \\
\hline
\end{tabular}

- indicates no deaths listed.

NOTE: Counties with no deaths for all listed conditions are not displayed. Rates based on small numbers may be unstable. Sum of individual conditions may exceed total because of deaths with multiple pneumoconioses. See Appendix A for source description and Appendix B for methods and ICD-8 and ICD-9 codes.

SOURCE: National Center for Health Statistics multiple cause of death data. Population estimates from U.S. Bureau of the Census. 
Arkansas: Selected statistics from the United States section

All pneumoconioses mortality, 1988-1992

Total deaths

Years of potential life lost to life expectancy (per death)

Crude mortality rate (per $1,000,000$ population)

Age-adjusted mortality rate (per $1,000,000$ population)

Total years of potential life lost to life expectancy rears of potentiallife lost to life expectancy (per death)

Deaths by type of pneumoconiosis, 1988-1992

Asbestosis

Coal workers' pneumoconiosis

Silicosis

Byssinosis
Value

59

6.43

3.16

699

11.8

State rank

33

39

36

28

Percent**

Deaths*

distribution

33.9

49.2

29

8.5

0.0

8.5

* Sum of column may exceed total deaths because of deaths with multiple pneumoconioses.

** Percentages may total more than $100 \%$ due to deaths with multiple pneumoconioses.

NOTE: $\quad$ See Appendix A for source description and Appendix B for methods and ICD-8 and ICD-9 codes.

SOURCE: National Center for Health Statistics multiple cause of death data. Population estimates from U.S. Bureau of the Census.

Figure AR-1. All pneumoconioses: Arkansas and U.S. age-adjusted mortality rates, residents age 15 and over, 1968-1992

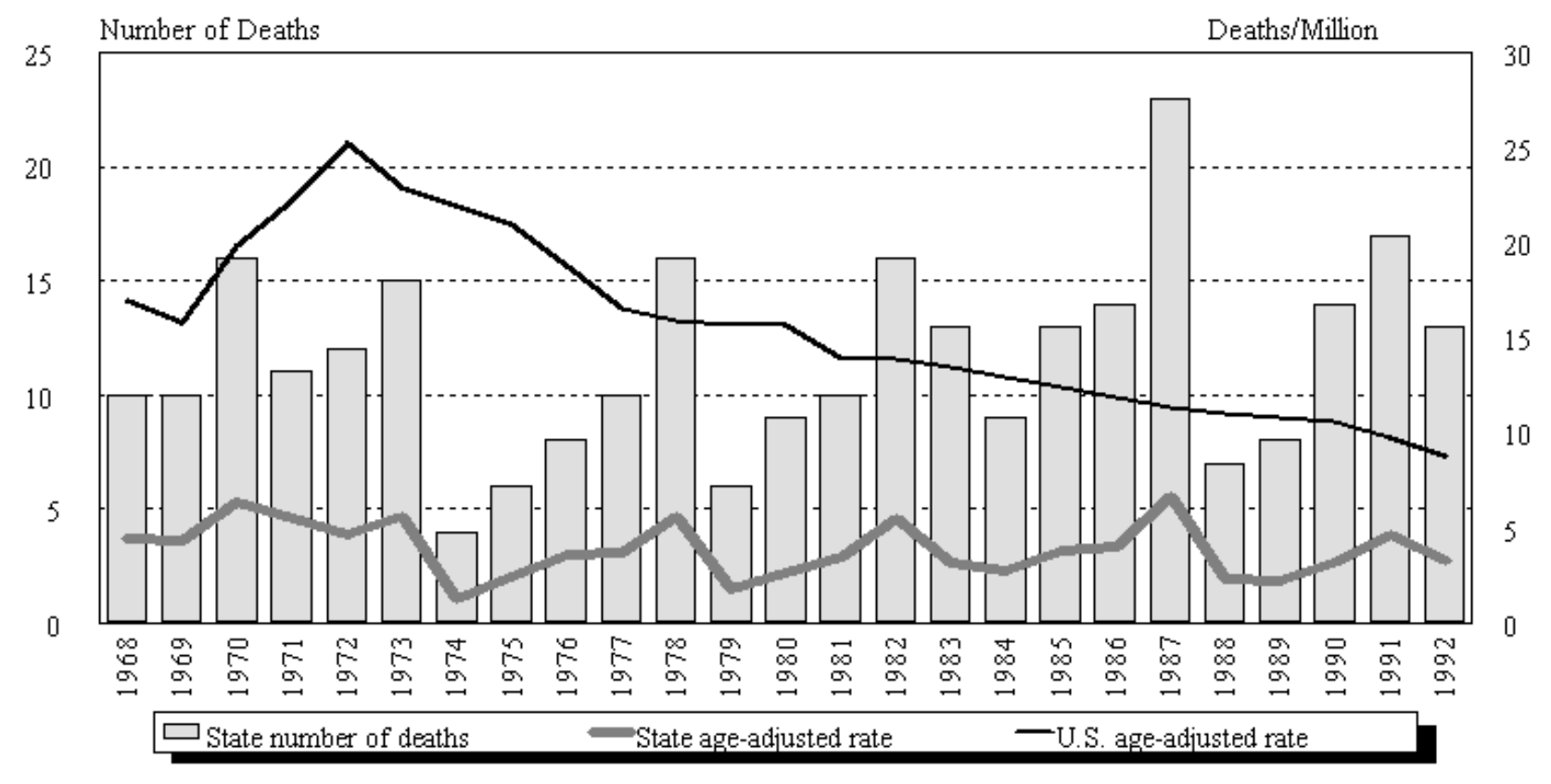

NOTE: $\quad$ See Appendix A for source description and Appendix B for methods and ICD-8 and ICD-9 codes.

SOURCE: National Center for Health Statistics multiple cause of death data. Population estimates from U.S. Bureau of the Census. 
Arkansas

Table AR-1. Number of deaths and age-adjusted mortality rate (per 1,000,000 population) by condition, Arkansas residents age 15 and over, 1968-1992

\begin{tabular}{|c|c|c|c|c|c|c|c|c|c|c|c|c|}
\hline \multirow[b]{2}{*}{ Year } & \multicolumn{2}{|c|}{ Asb estosis } & \multicolumn{2}{|c|}{$\begin{array}{l}\text { Coal workers' } \\
\text { pneumoconiosis }\end{array}$} & \multicolumn{2}{|c|}{ Silicosis } & \multicolumn{2}{|c|}{ Byssinosis* } & \multicolumn{2}{|c|}{$\begin{array}{c}\text { Unspecified/Other } \\
\text { pneumoconioses }\end{array}$} & \multicolumn{2}{|c|}{ All pneumoconioses } \\
\hline & Number & Rate & Number & Rate & Number & Rate & Number & Rate & Number & Rate & Number & Rate \\
\hline 1968 & - & - & - & - & 7 & 3.12 & & & 3 & 1.39 & 10 & 4.51 \\
\hline 1969 & - & - & 2 & 0.75 & 3 & 1.51 & & & 5 & 2.07 & 10 & 4.33 \\
\hline 1970 & - & - & 1 & 0.32 & 11 & 4.40 & & & 4 & 1.61 & 16 & 6.32 \\
\hline 1971 & - & - & 2 & 1.06 & 4 & 2.01 & & & 6 & 2.97 & 11 & 5.52 \\
\hline 1972 & - & - & - & - & 6 & 2.68 & & & 6 & 2.02 & 12 & 4.70 \\
\hline 1973 & - & - & 3 & 1.21 & 4 & 1.62 & & & 8 & 2.77 & 15 & 5.60 \\
\hline 1974 & - & - & - & - & - & - & & & 4 & 1.23 & 4 & 1.23 \\
\hline 1975 & - & - & 1 & 0.37 & 1 & 0.51 & & & 4 & 1.53 & 6 & 2.41 \\
\hline 1976 & - & - & 1 & 0.28 & 3 & 1.45 & & & 4 & 1.87 & 8 & 3.60 \\
\hline 1977 & - & - & 3 & 1.06 & 2 & 0.71 & & & 5 & 1.90 & 10 & 3.67 \\
\hline 1978 & 1 & 0.35 & 4 & 1.21 & 2 & 0.83 & & & 9 & 3.28 & 16 & 5.67 \\
\hline 1979 & - & - & 3 & 1.07 & 1 & 0.34 & - & - & 2 & 0.40 & 6 & 1.81 \\
\hline 1980 & 1 & 0.25 & 7 & 2.11 & 2 & 0.58 & - & - & - & - & 9 & 2.69 \\
\hline 1981 & 2 & 0.67 & 4 & 0.75 & 4 & 2.13 & - & - & - & - & 10 & 3.55 \\
\hline 1982 & 2 & 0.67 & 8 & 2.48 & 2 & 0.95 & - & - & 4 & 1.42 & 16 & 5.52 \\
\hline 1983 & 1 & 0.33 & 8 & 1.83 & 4 & 1.05 & - & - & - & - & 13 & 3.22 \\
\hline 1984 & 3 & 1.13 & 4 & 1.24 & - & - & - & - & 2 & 0.34 & 9 & 2.72 \\
\hline 1985 & - & - & 9 & 1.96 & 2 & 1.20 & - & - & 2 & 0.66 & 13 & 3.82 \\
\hline 1986 & 7 & 2.24 & 6 & 1.50 & - & - & - & - & 1 & 0.33 & 14 & 4.08 \\
\hline 1987 & 10 & 3.65 & 7 & 1.38 & 2 & 0.69 & - & - & 4 & 0.98 & 23 & 6.69 \\
\hline 1988 & 4 & 1.70 & 3 & 0.60 & - & & - & - & - & - & 7 & 2.30 \\
\hline 1989 & 1 & 0.20 & 4 & 0.92 & 2 & 0.83 & - & - & 1 & 0.33 & 8 & 2.28 \\
\hline 1990 & 4 & 1.22 & 9 & 1.66 & - & - & - & - & 1 & 0.33 & 14 & 3.21 \\
\hline 1991 & 6 & 2.05 & 7 & 1.38 & 2 & 0.70 & - & - & 2 & 0.52 & 17 & 4.64 \\
\hline 1992 & 5 & 1.53 & 6 & 1.05 & 1 & 0.54 & - & - & 1 & 0.19 & 13 & 3.31 \\
\hline
\end{tabular}

indicates no deaths listed. ${ }^{*}$ no specific code for by ssinosis in ICD-8 (1968-1 978).

NOTE: Rates based on small numbers may be unstable. Sum of individual conditions may exceed total because of deaths with multiple pneumoconioses. See Appendix A for source description and Appendix B for methods and ICD-8 and ICD-9

SOURCE: National Center for Health Statistics multiple cause of death data. Population estimates from U.S. Bureau of the Census. 
Figure AR-2. All pneumoconioses: Distribution of deaths by sex and race, Arkansas residents age 15 and over, 1968-1992
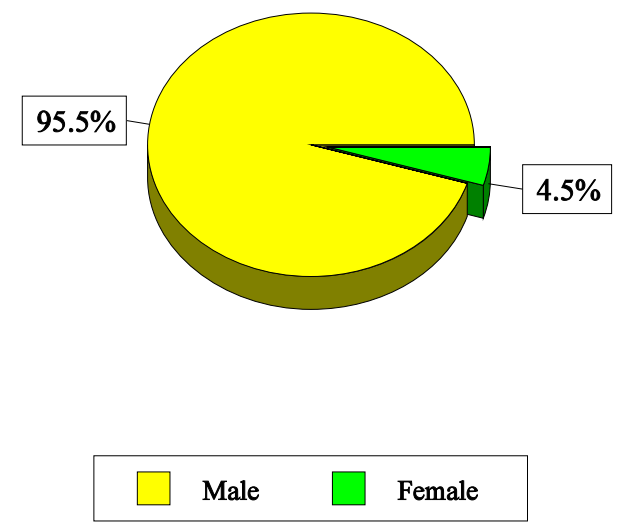
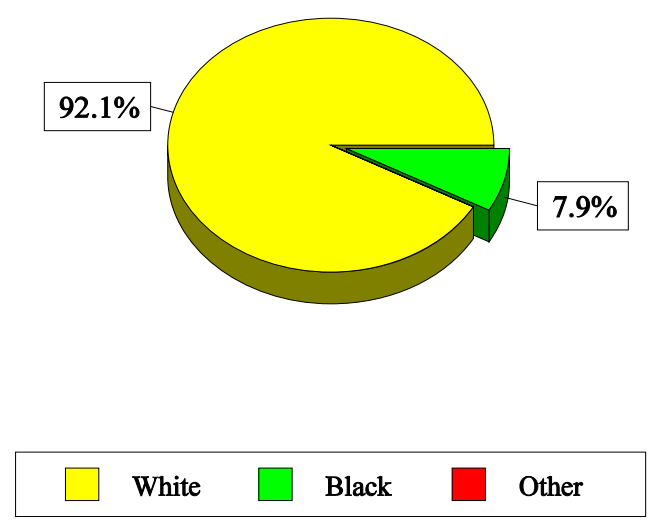

NOTE: $\quad$ See Appendix A for source description and Appendix B for methods and ICD-8 and ICD-9 codes.

SOURCE: National Center for Health Statistics multiple cause of death data.

Table AR-2. All pneumoconioses: Distribution of deaths by sex, race, and age, Arkansas residents age 15 and over, 1968-1992

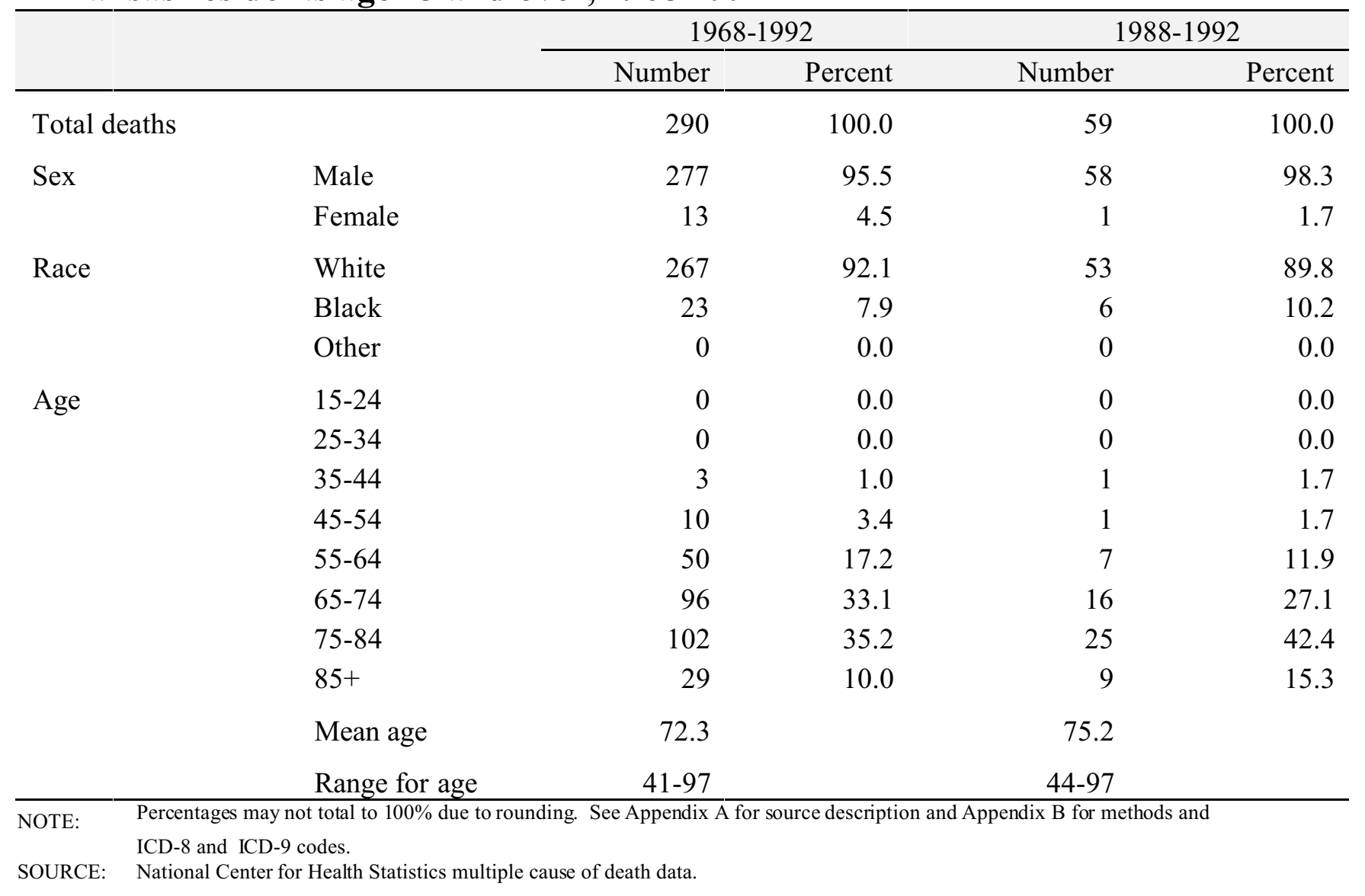




\section{Figure AR-3. Pneumoconiosis deaths by condition, Arkansas residents age 15 and over, 1968-1992}

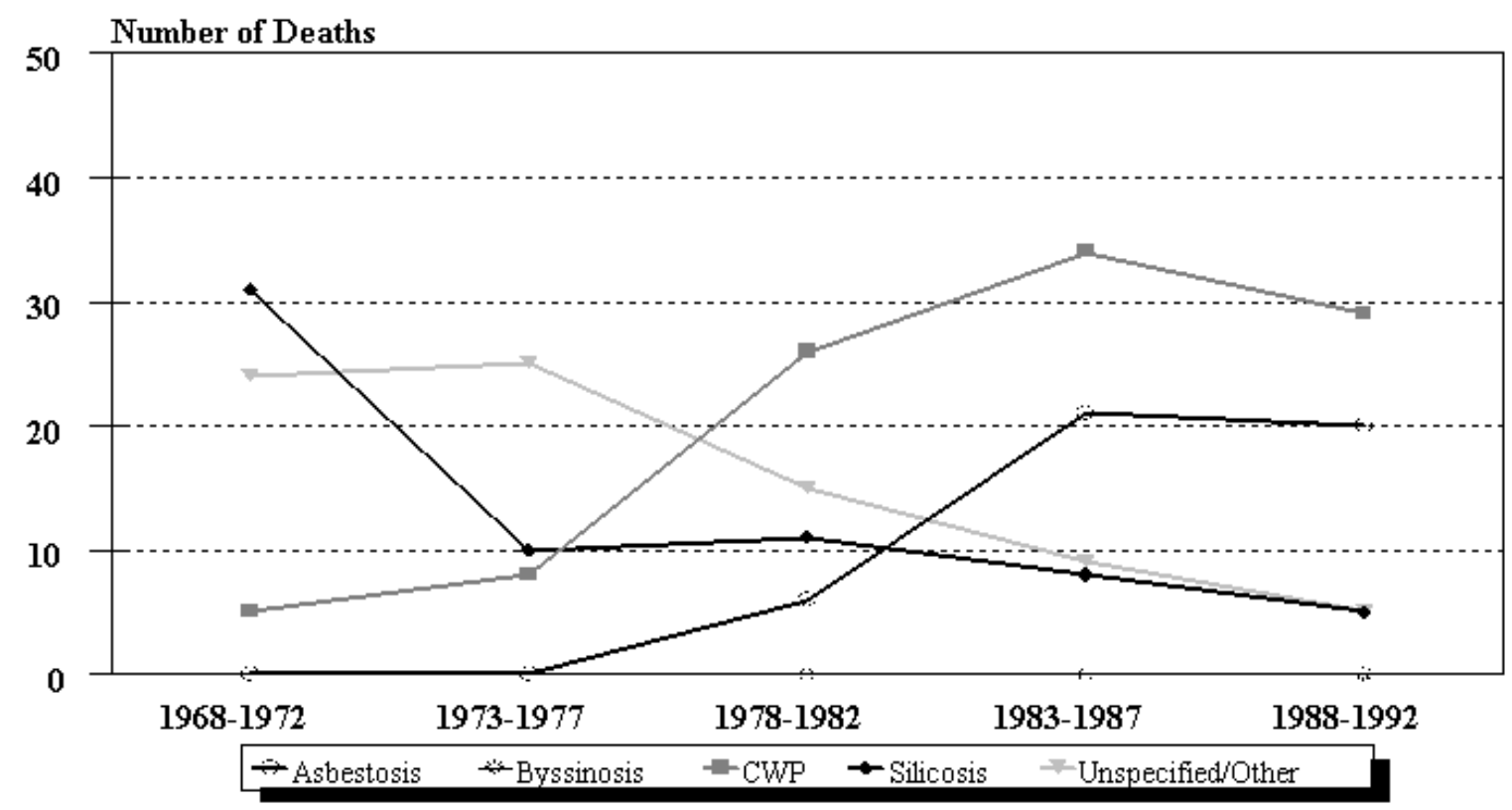

NOTE: See Appendix A for source description and Appendix B for methods and ICD-8 and ICD-9 codes. SOURCE: National Center for Health Statistics multiple cause of death data.

Figure AR-4. Arkansas and U.S. age-adjusted mortality rates (per 1,000,000 population) by condition, residents age 15 and over, 1983-1992

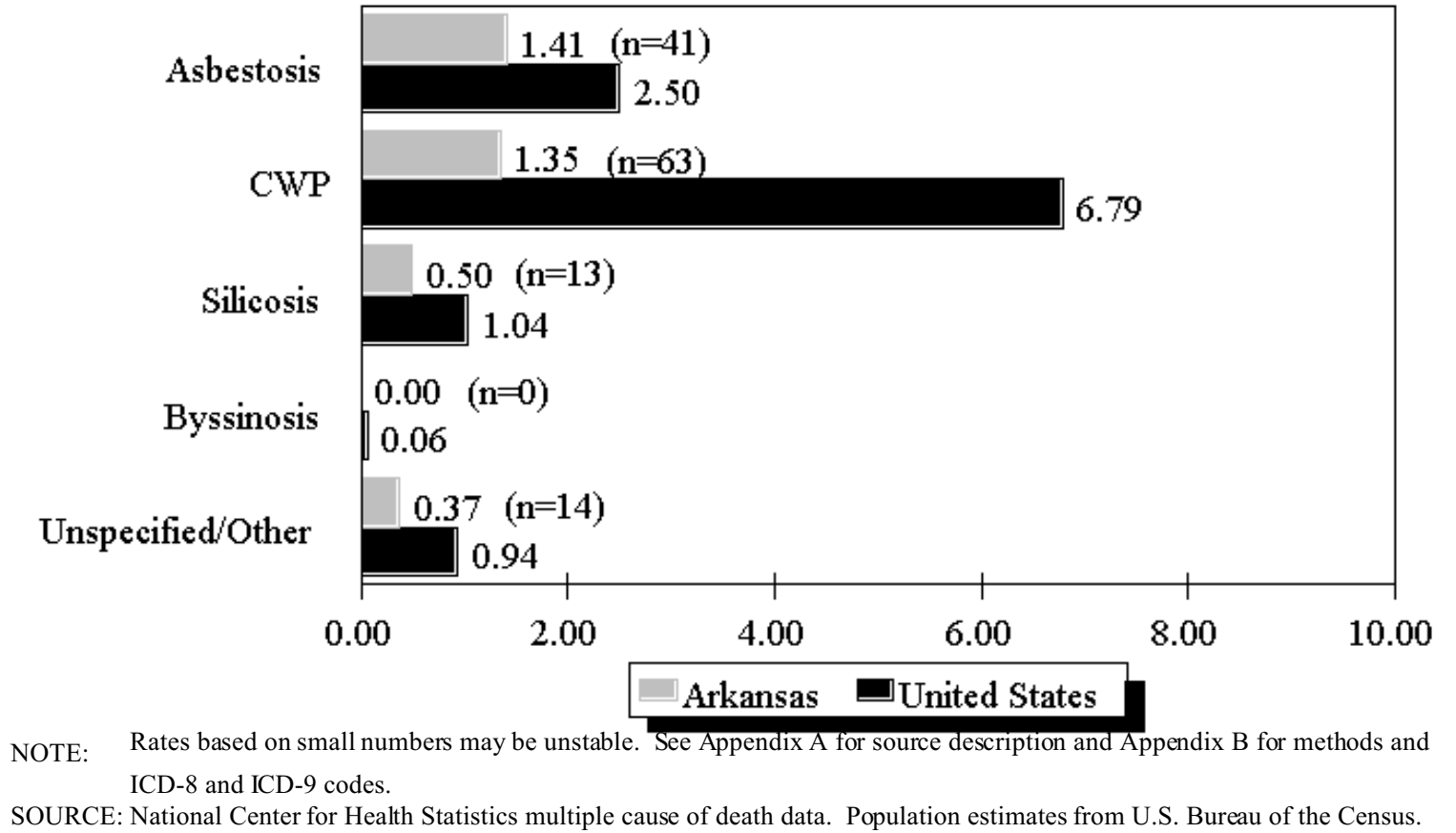


Table AR-3 (page 1 of 2). Number of deaths and age-adjusted mortality rate (per $1,000,000$ population) by condition and county, Arkansas residents age 15 and over, 1983-1992

\begin{tabular}{|c|c|c|c|c|c|c|c|c|c|c|c|c|}
\hline \multirow[b]{2}{*}{ County } & \multicolumn{2}{|c|}{ Asbestosis } & \multicolumn{2}{|c|}{$\begin{array}{c}\text { Coal workers' } \\
\text { pneumoconiosis }\end{array}$} & \multicolumn{2}{|c|}{ Silicosis } & \multicolumn{2}{|c|}{ Byssinosis } & \multicolumn{2}{|c|}{$\begin{array}{r}\text { Unspecified/Other } \\
\text { pneumoconioses }\end{array}$} & \multicolumn{2}{|c|}{$\begin{array}{r}\text { All } \\
\text { pneumoconioses }\end{array}$} \\
\hline & $\#$ & Rate & $\#$ & Rate & $\#$ & Rate & $\#$ & Rate & \# & Rate & $\#$ & Rate \\
\hline Ashley & 1 & 4.02 & - & - & - & - & - & - & - & - & 1 & 4.02 \\
\hline Baxter & 1 & 1.83 & 1 & 0.74 & - & - & - & - & - & - & 2 & 2.57 \\
\hline Benton & 2 & 1.74 & 1 & 0.43 & - & - & - & - & - & - & 3 & 2.17 \\
\hline Clark & 2 & 8.74 & 1 & 4.37 & - & - & - & - & - & - & 3 & 13.12 \\
\hline Clay & - & - & 1 & 1.61 & - & - & - & - & - & - & 1 & 1.61 \\
\hline Cleveland & 1 & 4.97 & - & - & - & - & - & - & - & - & 1 & 4.97 \\
\hline Columbia & 1 & 1.30 & 1 & 1.30 & - & - & - & - & - & - & 2 & 2.59 \\
\hline Crawford & - & - & 1 & 2.69 & - & - & - & - & - & - & 1 & 2.69 \\
\hline Crittenden & - & - & 1 & 2.49 & - & - & - & - & - & - & 1 & 2.49 \\
\hline Dallas & 1 & 3.24 & - & - & - & - & - & - & - & - & 1 & 3.24 \\
\hline Drew & 1 & 6.13 & - & - & - & - & - & - & - & - & 1 & 6.13 \\
\hline Faulkner & 1 & 1.07 & 1 & 1.07 & - & - & - & - & - & - & 2 & 2.14 \\
\hline Franklin & - & - & 2 & 8.42 & - & - & - & - & 4 & 16.84 & 6 & 25.26 \\
\hline Fulton & 1 & 3.13 & 2 & 10.04 & 1 & 3.13 & - & - & - & - & 4 & 16.30 \\
\hline Garland & - & - & 1 & 0.42 & - & - & - & - & 1 & 0.42 & 2 & 0.83 \\
\hline Greene & 1 & 1.28 & - & - & - & - & - & - & - & - & 1 & 1.28 \\
\hline Hempstead & - & - & - & - & 1 & 7.08 & - & - & - & - & 1 & 7.08 \\
\hline Hot Spring & - & - & - & - & 1 & 3.24 & - & - & - & - & 1 & 3.24 \\
\hline Independence & - & - & - & - & 1 & 3.05 & - & - & - & - & 1 & 3.05 \\
\hline Jefferson & 2 & 2.46 & - & - & 1 & 0.54 & - & - & - & - & 3 & 3.00 \\
\hline Johnson & - & - & 14 & 34.80 & - & - & - & - & 1 & 1.88 & 15 & 36.68 \\
\hline Lincoln & - & - & 1 & 3.42 & - & - & - & - & - & - & 1 & 3.42 \\
\hline Logan & 1 & 1.68 & 4 & 9.36 & - & - & - & - & 1 & 4.32 & 6 & 15.36 \\
\hline Lonoke & 2 & 6.06 & 1 & 1.39 & - & - & - & - & - & - & 3 & 7.45 \\
\hline Miller & 1 & 2.60 & - & - & - & - & - & - & - & - & 1 & 2.60 \\
\hline Mississippi & 1 & 2.02 & - & - & - & - & - & - & - & - & 1 & 2.02 \\
\hline Montgomery & - & - & 1 & 4.20 & 2 & 18.22 & - & - & - & - & 3 & 22.42 \\
\hline Nevada & 1 & 8.41 & - & - & 1 & 3.07 & - & - & - & - & 2 & 11.47 \\
\hline Ouachita & 2 & 5.64 & 2 & 4.01 & - & - & - & - & - & - & 4 & 9.65 \\
\hline Perry & - & - & 1 & 5.56 & - & - & - & - & - & - & 1 & 5.56 \\
\hline Phillips & 1 & 5.69 & - & - & - & - & - & - & - & - & 1 & 5.69 \\
\hline
\end{tabular}

See footnotes at end of table. 
Table AR-3 (page 2 of 2). Number of deaths and age-adjusted mortality rate (per $1,000,000$ population) by condition and county, Arkansas residents age 15 and over, 1983-1992

\begin{tabular}{|c|c|c|c|c|c|c|c|c|c|c|c|c|}
\hline \multirow[b]{2}{*}{ County } & \multicolumn{2}{|c|}{ Asbestosis } & \multicolumn{2}{|c|}{$\begin{array}{r}\text { Coal workers' } \\
\text { pneumoconiosis }\end{array}$} & \multicolumn{2}{|c|}{ Silicosis } & \multicolumn{2}{|c|}{ Byssinosis } & \multicolumn{2}{|c|}{$\begin{array}{r}\text { Unspecified/Other } \\
\text { pneumoconioses }\end{array}$} & \multicolumn{2}{|c|}{$\begin{array}{r}\text { All } \\
\text { pneumoconioses }\end{array}$} \\
\hline & $\#$ & Rate & $\#$ & Rate & $\#$ & Rate & $\#$ & Rate & $\#$ & Rate & \# & Rate \\
\hline Poinsett & 1 & 3.73 & - & - & - & - & - & - & - & - & 1 & 3.73 \\
\hline Polk & - & - & 1 & 1.85 & - & - & - & - & - & - & 1 & 1.85 \\
\hline Pope & - & - & 4 & 4.56 & - & - & - & - & 1 & 2.48 & 5 & 7.04 \\
\hline Pulaski & 8 & 2.33 & 1 & 0.17 & 4 & 1.07 & - & - & - & - & 13 & 3.57 \\
\hline St. Francis & 1 & 3.67 & - & - & - & - & - & - & - & - & 1 & 3.67 \\
\hline Saline & 3 & 3.90 & 1 & 1.04 & - & - & - & - & - & - & 4 & 4.94 \\
\hline Scott & - & - & 2 & 11.94 & - & - & - & - & - & - & 2 & 11.94 \\
\hline Sebastian & 1 & 1.04 & 11 & 5.61 & 1 & 1.04 & - & - & 4 & 2.99 & 17 & 10.68 \\
\hline Sharp & - & - & 1 & 4.13 & - & - & - & - & - & - & 1 & 4.13 \\
\hline Union & 1 & 0.78 & - & - & - & - & - & - & - & - & 1 & 0.78 \\
\hline Washington & 1 & 0.51 & 1 & 0.51 & - & - & - & - & - & - & 2 & 1.02 \\
\hline White & 1 & 1.83 & 2 & 2.62 & - & - & - & - & 1 & 1.83 & 4 & 6.28 \\
\hline Yell & - & - & 2 & 4.35 & - & - & - & - & 1 & 4.88 & 3 & 9.23 \\
\hline
\end{tabular}

- indicates no deaths listed.

NOTE: Counties with no deaths for all listed conditions are not displayed. Rates based on small numbers may be unstable. Sum of individual conditions may exceed total because of deaths with multiple pneumoconioses. See Appendix A for source description and Appendix B for methods and ICD-8 and ICD-9 codes.

SOURCE: National Center for Health Statistics multiple cause of death data. Population estimates from U.S. Bureau of the Census. 
California

\section{California: Selected statistics from the United States section}

All pneumoconioses mortality, 1988-1992

Total deaths

Crude mortality rate (per $1,000,000$ population)

Age-adjusted mortality rate (per $1,000,000$ population)

Total years of potential life lost to life expectancy

Years of potential life lost to life expectancy (per death)

$\begin{array}{rr}\frac{\text { Value }}{688} & \text { State rank } \\ 5.93 & 6 \\ 4.12 & 37 \\ 8,612 & 33 \\ 12.5 & 6 \\ & 15\end{array}$

Percent**

Deaths by type of pneumoconiosis, 1988-1992

Asbestosis

$\underline{\text { Deaths* }}$

459

distribution

114

66.7

Coal workers' pneumoconiosis

78

16.6

Silicosis

Byssinosis

Unspecified/Other pneumoconiosis

4

11.3

0.6

36

* Sum of column may exceed total deaths because of deaths with multiple pneumoconioses.

** Percentages may total more than $100 \%$ due to deaths with multiple pneumoconioses.

NOTE: $\quad$ See Appendix A for source description and Appendix B for methods and ICD-8 and ICD-9 codes.

SOURCE: National Center for Health Statistics multiple cause of death data. Population estimates from U.S. Bureau of the Census.

Figure CA-1. All pneumoconioses: California and U.S. age-adjusted mortality rates, residents age 15 and over, 1968-1992

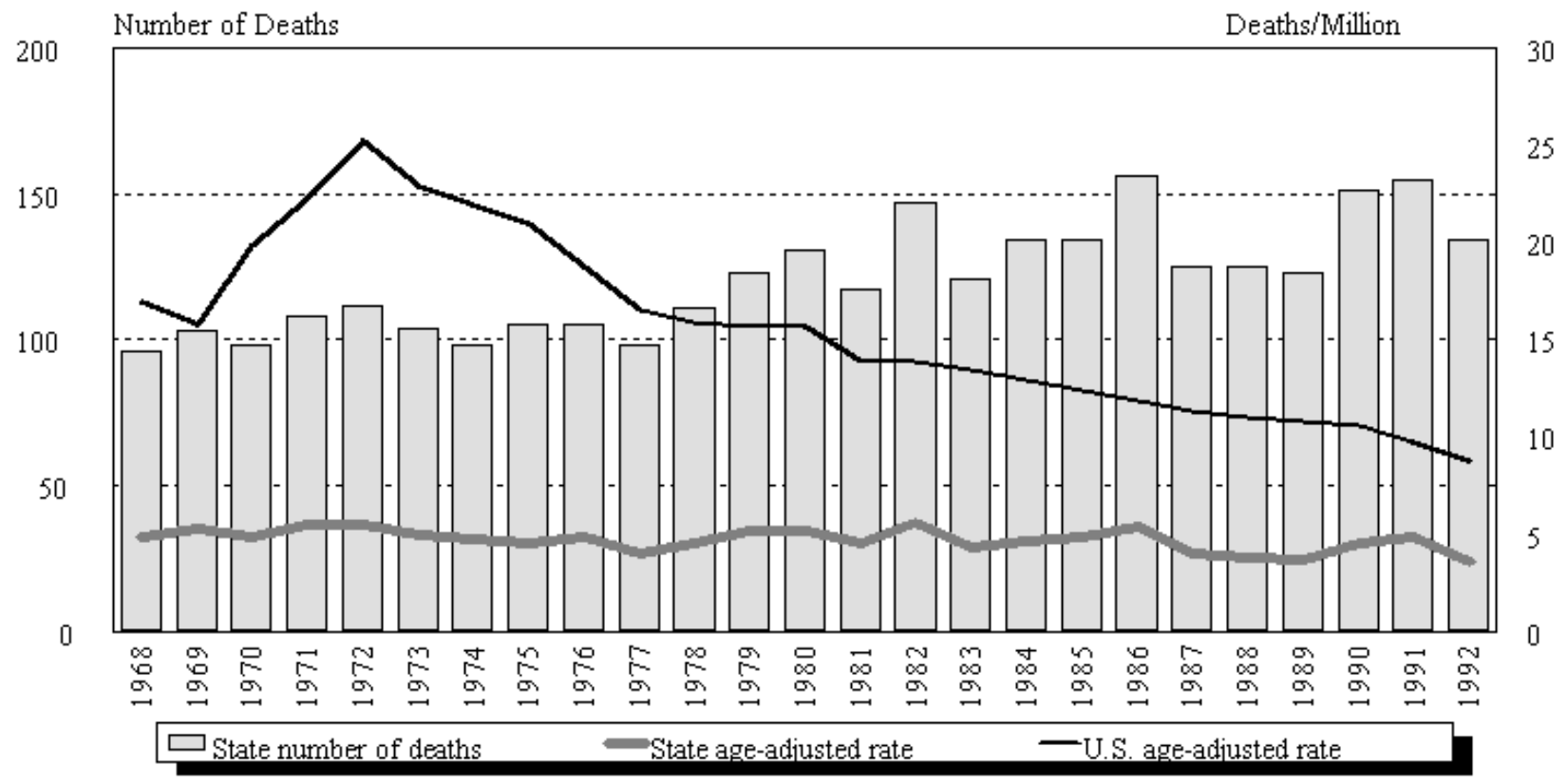


California

\begin{tabular}{|c|c|c|c|c|c|c|c|c|c|c|c|c|}
\hline \multirow[b]{2}{*}{ Year } & \multicolumn{2}{|c|}{ Asb estosis } & \multicolumn{2}{|c|}{$\begin{array}{l}\text { Coal workers' } \\
\text { pneumoconiosis }\end{array}$} & \multicolumn{2}{|c|}{ Silicosis } & \multicolumn{2}{|c|}{ Byssinosis* } & \multicolumn{2}{|c|}{$\begin{array}{l}\text { Unspecified/Other } \\
\text { pneumoconioses }\end{array}$} & \multicolumn{2}{|c|}{ All pneumoconioses } \\
\hline & Number & Rate & Number & Rate & Number & Rate & Number & Rate & Number & Rate & Number & Rate \\
\hline 1968 & 6 & 0.39 & 14 & 0.59 & 46 & 2.34 & & & 32 & 1.70 & 96 & 4.91 \\
\hline 1969 & 10 & 0.60 & 12 & 0.58 & 56 & 2.90 & & & 27 & 1.39 & 103 & 5.35 \\
\hline 1970 & 5 & 0.30 & 18 & 0.93 & 59 & 2.94 & & & 20 & 0.96 & 98 & 4.92 \\
\hline 1971 & 11 & 0.63 & 17 & 0.79 & 51 & 2.56 & & & 33 & 1.72 & 108 & 5.52 \\
\hline 1972 & 8 & 0.46 & 18 & 0.71 & 46 & 2.27 & & & 46 & 2.36 & 112 & 5.53 \\
\hline 1973 & 17 & 0.94 & 20 & 0.87 & 37 & 1.75 & & & 33 & 1.55 & 104 & 4.96 \\
\hline 1974 & 9 & 0.50 & 21 & 0.97 & 41 & 1.90 & & & 30 & 1.52 & 98 & 4.76 \\
\hline 1975 & 11 & 0.54 & 25 & 1.02 & 34 & 1.33 & & & 37 & 1.73 & 105 & 4.54 \\
\hline 1976 & 23 & 1.09 & 24 & 1.02 & 28 & 1.33 & & & 32 & 1.53 & 105 & 4.87 \\
\hline 1977 & 24 & 1.03 & 17 & 0.60 & 27 & 1.08 & & & 30 & 1.29 & 98 & 4.00 \\
\hline 1978 & 33 & 1.47 & 20 & 0.71 & 30 & 1.17 & & & 35 & 1.53 & 111 & 4.59 \\
\hline 1979 & 63 & 2.84 & 24 & 1.04 & 21 & 0.84 & - & - & 17 & 0.62 & 123 & 5.24 \\
\hline 1980 & 59 & 2.50 & 24 & 0.93 & 29 & 1.08 & - & - & 21 & 0.75 & 131 & 5.20 \\
\hline 1981 & 57 & 2.44 & 24 & 0.78 & 22 & 0.80 & - & - & 14 & 0.55 & 117 & 4.56 \\
\hline 1982 & 86 & 3.56 & 21 & 0.71 & 26 & 0.86 & 2 & 0.08 & 18 & 0.62 & 147 & 5.60 \\
\hline 1983 & 67 & 2.59 & 25 & 0.77 & 19 & 0.68 & - & - & 13 & 0.44 & 121 & 4.37 \\
\hline 1984 & 77 & 2.88 & 24 & 0.76 & 22 & 0.70 & - & - & 14 & 0.50 & 134 & 4.72 \\
\hline 1985 & 83 & 3.22 & 20 & 0.61 & 22 & 0.78 & 1 & 0.05 & 11 & 0.33 & 134 & 4.88 \\
\hline 1986 & 112 & 4.08 & 23 & 0.65 & 14 & 0.45 & - & - & 8 & 0.25 & 156 & 5.38 \\
\hline 1987 & 80 & 2.77 & 18 & 0.45 & 17 & 0.58 & - & - & 10 & 0.26 & 125 & 4.06 \\
\hline 1988 & 81 & 2.62 & 16 & 0.40 & 19 & 0.50 & 1 & 0.04 & 10 & 0.26 & 125 & 3.78 \\
\hline 1989 & 87 & 2.64 & 7 & 0.21 & 16 & 0.42 & 2 & 0.10 & 11 & 0.36 & 123 & 3.74 \\
\hline 1990 & 102 & 3.11 & 28 & 0.85 & 15 & 0.36 & - & - & 7 & 0.22 & 151 & 4.52 \\
\hline 1991 & 94 & 2.72 & 41 & 1.56 & 16 & 0.44 & - & - & 4 & 0.14 & 155 & 4.86 \\
\hline 1992 & 95 & 2.66 & 22 & 0.55 & 12 & 0.30 & 1 & 0.03 & 4 & 0.12 & 134 & 3.66 \\
\hline
\end{tabular}

-indicates no deaths listed. $\quad *$ no specific code for byssinosis in ICD-8 (1968-1 978).

NOTE: Rates based on small numbers may be unstable. Sum of individ ual conditions may exceed total because of deaths with multiple pneumoconioses. See Appendix A for source description and Appendix B for methods and ICD-8 and ICD-9

SOURCE: National Center for Health Statistics multiple cause of death data. Population estimates from U.S. Bureau of the Census. 
Figure CA-2. All pneumoconioses: Distribution of deaths by sex and race, California residents age 15 and over, 1968-1992
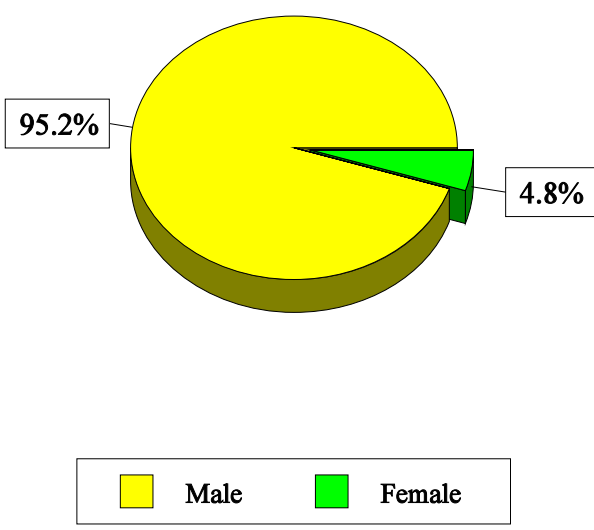
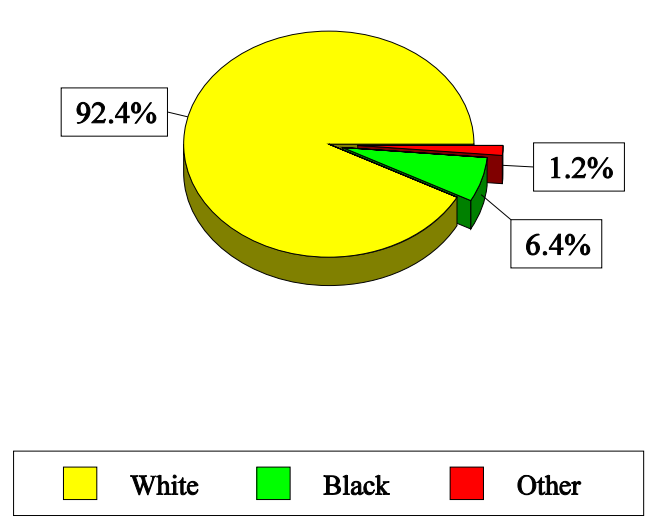

NOTE: See Appendix A for source description and Appendix B for methods and ICD-8 and ICD-9 codes.

SOURCE: National Center for Health Statistics multiple cause of death data.

Table CA-2. All pneumoconioses: Distribution of deaths by sex, race, and age, California residents age 15 and over, 1968-1992

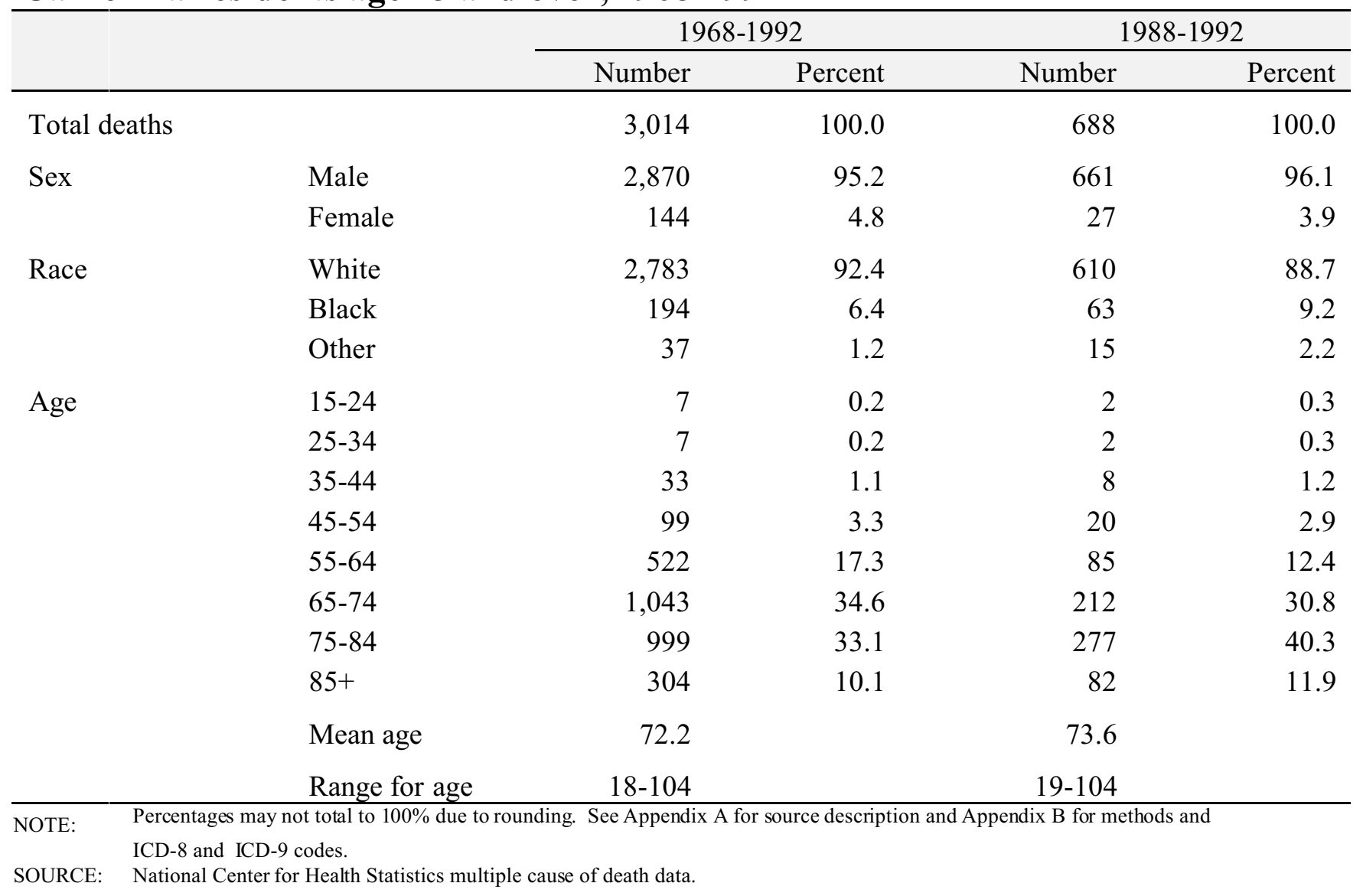




\section{Figure CA-3. Pneumoconiosis deaths by condition, California residents age 15 and over, 1968-1992}

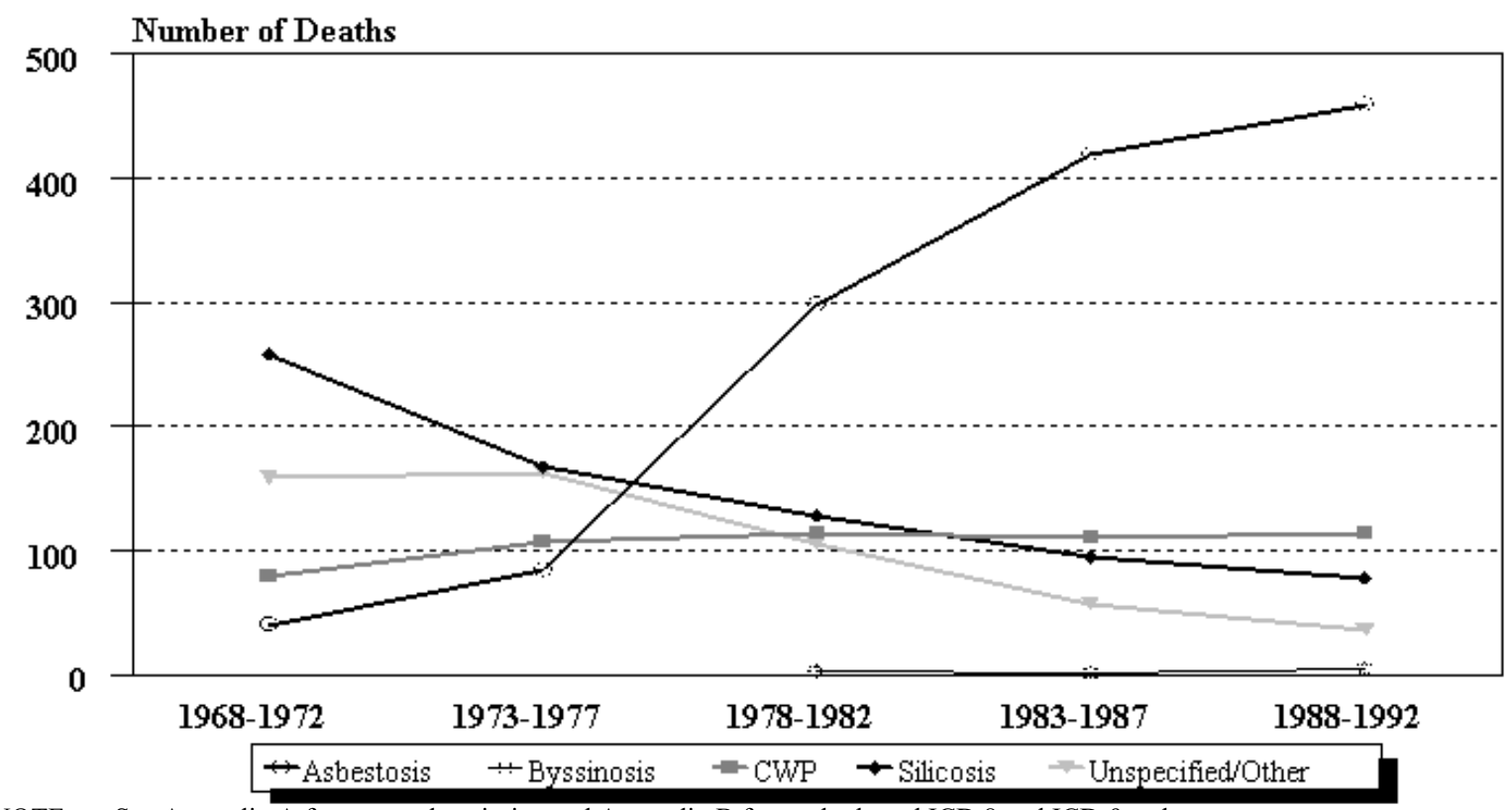

NOTE: See Appendix A for source description and Appendix B for methods and ICD-8 and ICD-9 codes.

SOURCE: National Center for Health Statistics multiple cause of death data.

Figure CA-4. California and U.S. age-adjusted mortality rates (per 1,000,000 population) by condition, residents age 15 and over, 1983-1992

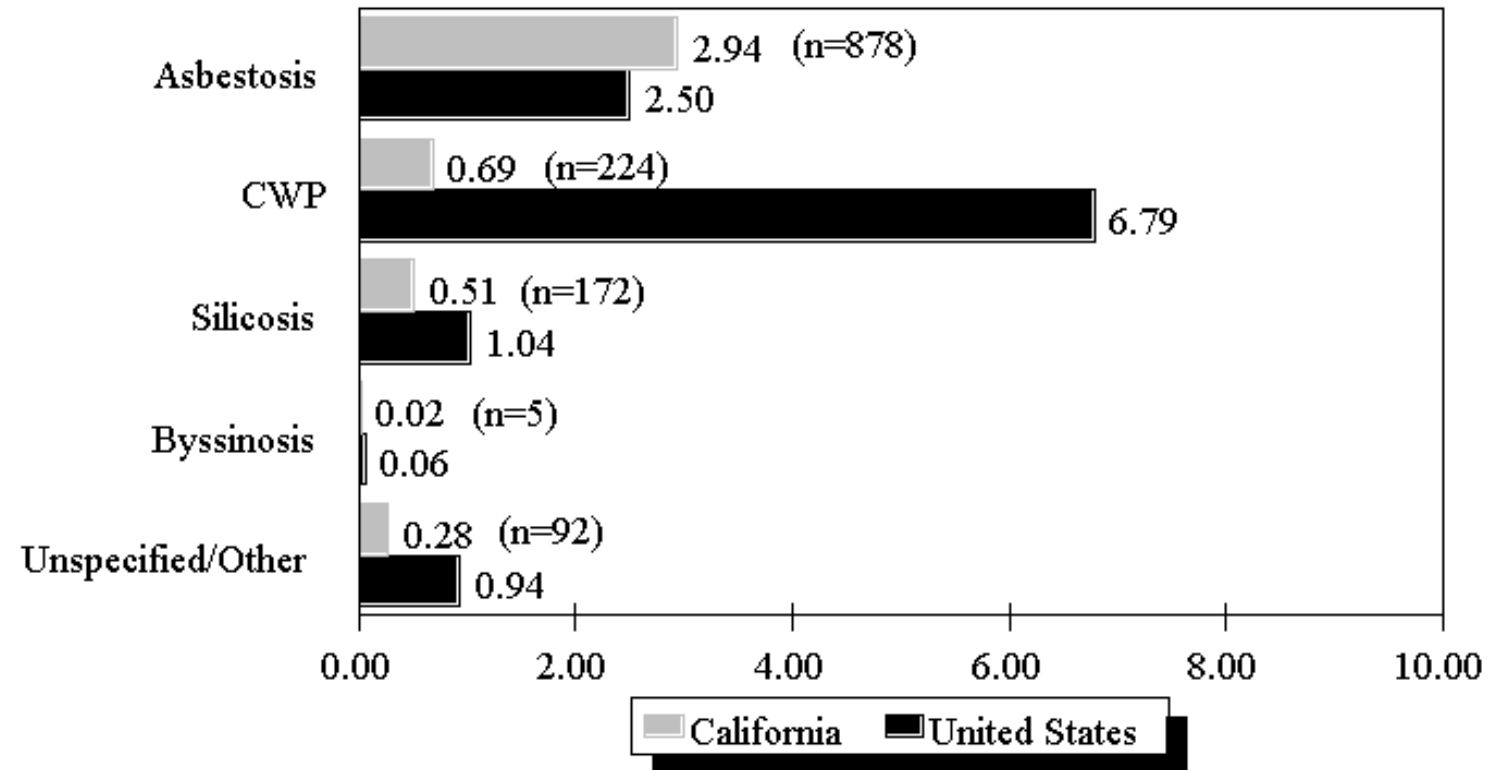

NOTE: $\quad$ Rates based on small numbers may be unstable. See Appendix A for source description and Appendix B for methods and ICD-8 and ICD-9 codes.

SOURCE: National Center for Health Statistics multiple cause of death data. Population estimates from U.S. Bureau of the Census. 
Table CA-3 (page 1 of 2). Number of deaths and age-adjusted mortality rate (per $1,000,000$ population) by condition and county, California residents age 15 and over, 1983-1992

\begin{tabular}{|c|c|c|c|c|c|c|c|c|c|c|c|c|}
\hline \multirow[b]{2}{*}{ County } & \multicolumn{2}{|c|}{ Asbestosis } & \multicolumn{2}{|c|}{$\begin{array}{c}\text { Coal workers' } \\
\text { pneumoconiosis }\end{array}$} & \multicolumn{2}{|c|}{ Silicosis } & \multicolumn{2}{|c|}{ Byssinosis } & \multicolumn{2}{|c|}{$\begin{array}{r}\text { Unspecified/Other } \\
\text { pneumoconioses }\end{array}$} & \multicolumn{2}{|c|}{$\begin{array}{r}\text { All } \\
\text { pneumoconioses }\end{array}$} \\
\hline & \# & Rate & $\#$ & Rate & $\#$ & Rate & \# & Rate & $\#$ & Rate & \# & Rate \\
\hline Alameda & 75 & 5.88 & 3 & 0.20 & 13 & 0.85 & - & - & 3 & 0.30 & 94 & 7.23 \\
\hline Amador & 2 & 5.77 & - & - & - & - & - & - & 2 & 3.14 & 4 & 8.91 \\
\hline Butte & 8 & 3.30 & 3 & 0.68 & 3 & 1.28 & - & - & - & - & 14 & 5.26 \\
\hline Calaveras & 1 & 1.55 & - & - & - & - & - & - & - & - & 1 & 1.55 \\
\hline Colusa & - & - & 1 & 3.46 & - & - & - & - & - & - & 1 & 3.46 \\
\hline Contra Costa & 76 & 9.18 & 2 & 0.24 & 12 & 1.46 & - & - & 2 & 0.17 & 90 & 10.75 \\
\hline El Dorado & 6 & 4.19 & - & - & 4 & 2.41 & - & - & 3 & 2.56 & 12 & 8.56 \\
\hline Fresno & 7 & 1.01 & 10 & 1.27 & 5 & 0.71 & 1 & 0.25 & 3 & 0.51 & 26 & 3.74 \\
\hline Humboldt & 3 & 2.90 & 2 & 1.93 & - & - & - & - & - & - & 5 & 4.83 \\
\hline Imperial & 3 & 2.57 & 1 & 1.56 & 1 & 0.73 & - & - & - & - & 5 & 4.85 \\
\hline Kern & 6 & 1.53 & 6 & 0.86 & 5 & 1.02 & 1 & 0.25 & 1 & 0.25 & 19 & 3.90 \\
\hline Kings & - & - & 1 & 0.93 & - & - & - & - & - & - & 1 & 0.93 \\
\hline Lake & 3 & 2.69 & 3 & 4.46 & 1 & 0.67 & - & - & - & - & 6 & 7.14 \\
\hline Lassen & - & - & 2 & 7.28 & - & - & - & - & - & - & 2 & 7.28 \\
\hline Los Angeles & 174 & 2.22 & 37 & 0.39 & 22 & 0.27 & - & - & 20 & 0.24 & 251 & 3.10 \\
\hline Madera & 3 & 3.32 & - & - & - & - & - & - & - & - & 3 & 3.32 \\
\hline Marin & 11 & 4.04 & 2 & 0.51 & 2 & 0.51 & - & - & 2 & 0.92 & 17 & 5.98 \\
\hline Mariposa & 2 & 8.70 & - & - & 2 & 8.70 & - & - & - & - & 4 & 17.40 \\
\hline Mendocino & 2 & 1.98 & 1 & 1.31 & 2 & 1.98 & - & - & - & - & 4 & 4.60 \\
\hline Merced & 2 & 1.25 & 1 & 0.78 & 2 & 1.25 & - & - & - & - & 5 & 3.28 \\
\hline Modoc & 1 & 8.55 & - & - & - & - & - & - & - & - & 1 & 8.55 \\
\hline Monterey & 4 & 1.17 & 2 & 0.59 & 2 & 0.77 & 1 & 0.38 & 1 & 0.20 & 10 & 3.11 \\
\hline Napa & 24 & 14.91 & 3 & 1.55 & 1 & 0.35 & - & - & - & - & 28 & 16.81 \\
\hline Nevada & 5 & 3.40 & - & - & 8 & 6.10 & - & - & 1 & 1.06 & 13 & 9.50 \\
\hline Orange & 59 & 2.70 & 11 & 0.43 & 13 & 0.52 & - & - & 8 & 0.41 & 90 & 4.02 \\
\hline Placer & 10 & 5.25 & 1 & 0.37 & 4 & 1.79 & - & - & 1 & 0.68 & 16 & 8.09 \\
\hline Plumas & - & - & 1 & 2.59 & - & - & - & - & - & - & 1 & 2.59 \\
\hline Riverside & 31 & 2.30 & 7 & 0.40 & 11 & 0.82 & - & - & 1 & 0.05 & 49 & 3.52 \\
\hline Sacramento & 29 & 2.82 & 3 & 0.21 & 6 & 0.62 & - & - & 4 & 0.33 & 42 & 3.98 \\
\hline San Benito & 1 & 3.72 & - & - & - & - & - & - & - & - & 1 & 3.72 \\
\hline $\begin{array}{l}\text { San } \\
\text { Bernardino }\end{array}$ & 26 & 2.17 & 7 & 0.52 & 7 & 0.62 & - & - & 6 & 0.46 & 46 & 3.78 \\
\hline
\end{tabular}

See footnotes at end of table. 
Table CA-3 (page 2 of 2). Number of deaths and age-adjusted mortality rate (per $1,000,000$ population) by condition and county, California residents age 15 and over, 1983-1992

\begin{tabular}{|c|c|c|c|c|c|c|c|c|c|c|c|c|}
\hline \multirow[b]{2}{*}{ County } & \multicolumn{2}{|c|}{ Asbestosis } & \multicolumn{2}{|c|}{$\begin{array}{r}\text { Coal workers' } \\
\text { pneumoconiosis }\end{array}$} & \multicolumn{2}{|c|}{ Silicosis } & \multicolumn{2}{|c|}{ Byssinosis } & \multicolumn{2}{|c|}{$\begin{array}{r}\text { Unspecified/Other } \\
\text { pneumoconioses }\end{array}$} & \multicolumn{2}{|c|}{$\begin{array}{r}\text { All } \\
\text { pneumoconioses }\end{array}$} \\
\hline & \# & Rate & \# & Rate & $\#$ & Rate & $\#$ & Rate & \# & Rate & \# & Rate \\
\hline San Diego & 59 & 2.62 & 24 & 0.83 & 4 & 0.15 & - & - & 2 & 0.09 & 87 & 3.59 \\
\hline San Francisco & 56 & 5.58 & 3 & 0.25 & 5 & 0.44 & - & - & 1 & 0.06 & 65 & 6.33 \\
\hline San Joaquin & 6 & 1.18 & 3 & 0.39 & 4 & 0.79 & - & - & 7 & 0.92 & 20 & 3.28 \\
\hline $\begin{array}{l}\text { San Luis } \\
\text { Obispo }\end{array}$ & 4 & 1.21 & 1 & 0.50 & 1 & 0.50 & - & - & 1 & 0.24 & 7 & 2.45 \\
\hline San Mateo & 32 & 4.36 & 3 & 0.42 & 3 & 0.34 & - & - & 5 & 0.52 & 43 & 5.65 \\
\hline Santa Barbara & 5 & 1.25 & 6 & 1.40 & 2 & 0.29 & - & - & 5 & 1.25 & 18 & 4.19 \\
\hline Santa Clara & 20 & 1.56 & 2 & 0.11 & 8 & 0.52 & - & - & 3 & 0.21 & 33 & 2.40 \\
\hline Santa Cruz & 8 & 2.90 & 1 & 0.23 & - & - & 1 & 0.58 & - & - & 10 & 3.72 \\
\hline Shasta & 9 & 4.68 & 3 & 1.77 & 2 & 1.07 & - & - & 1 & 0.38 & 15 & 7.90 \\
\hline Siskiyou & 3 & 4.07 & 1 & 1.03 & 1 & 1.03 & - & - & 2 & 3.04 & 7 & 9.18 \\
\hline Solano & 40 & 16.13 & 5 & 2.08 & 1 & 0.29 & 1 & 0.42 & 1 & 0.29 & 48 & 19.21 \\
\hline Sonoma & 28 & 5.93 & 48 & 13.93 & 3 & 0.56 & - & - & 2 & 0.26 & 81 & 20.68 \\
\hline Stanislaus & 4 & 1.07 & 3 & 0.53 & - & - & - & - & - & - & 7 & 1.61 \\
\hline Sutter & 1 & 1.74 & - & - & - & - & - & - & - & - & 1 & 1.74 \\
\hline Tehama & 2 & 2.67 & 1 & 0.89 & 2 & 3.56 & - & - & - & - & 5 & 7.11 \\
\hline Trinity & - & - & - & - & 1 & 4.38 & - & - & - & - & 1 & 4.38 \\
\hline Tulare & 6 & 1.76 & 2 & 0.41 & 2 & 0.41 & - & - & - & - & 10 & 2.58 \\
\hline Toulumne & 1 & 1.78 & - & - & - & - & - & - & - & - & 1 & 1.78 \\
\hline Ventura & 14 & 2.36 & 6 & 0.97 & 7 & 1.08 & - & - & 2 & 0.32 & 28 & 4.53 \\
\hline Yolo & 3 & 2.08 & - & - & - & - & - & - & 2 & 1.55 & 5 & 3.64 \\
\hline Yuba & 3 & 5.47 & 2 & 2.51 & - & - & - & - & - & - & 5 & 7.98 \\
\hline
\end{tabular}

- indicates no deaths listed.

NOTE: Counties with no deaths for all listed conditions are not displayed. Rates based on small numbers may be unstable. Sum of individual conditions may exceed total because of deaths with multiple pneumoconioses. See Appendix A for source description and Appendix B for methods and ICD-8 and ICD-9 codes.

SOURCE: National Center for Health Statistics multiple cause of death data. Population estimates from U.S. Bureau of the Census. 
Colorado

\section{Colorado: Selected statistics from the United States section}

All pneumoconioses mortality, 1988-1992

Total deaths

Crude mortality rate (per $1,000,000$ population)

Age-adjusted mortality rate (per 1,000,000 population)

Total years of potential life lost to life expectancy

Years of potential life lost to life expectancy (per death) $\underline{\text { Value }}$

$\underline{\text { State rank }}$

13.63

8.39

1,818

10.4

20

Percent**

Deaths by type of pneumoconiosis, 1988-1992

Asbestosis

Deaths*

distribution

12.6

Coal workers' pneumoconiosis

87

49.7

Silicosis

58

33.1

Byssinosis

0

0.0

Unspecified/Other pneumoconiosis

* Sum of column may exceed total deaths because of deaths with multiple pneumoconioses.

** Percentages may total more than $100 \%$ due to deaths with multiple pneumoconioses.

NOTE: $\quad$ See Appendix A for source description and Appendix B for methods and ICD-8 and ICD-9 codes.

SOURCE: National Center for Health Statistics multiple cause of death data. Population estimates from U.S. Bureau of the Census.

\section{Figure CO-1. All pneumoconioses: Colorado and U.S. age-adjusted mortality rates, residents age 15 and over, 1968-1992}

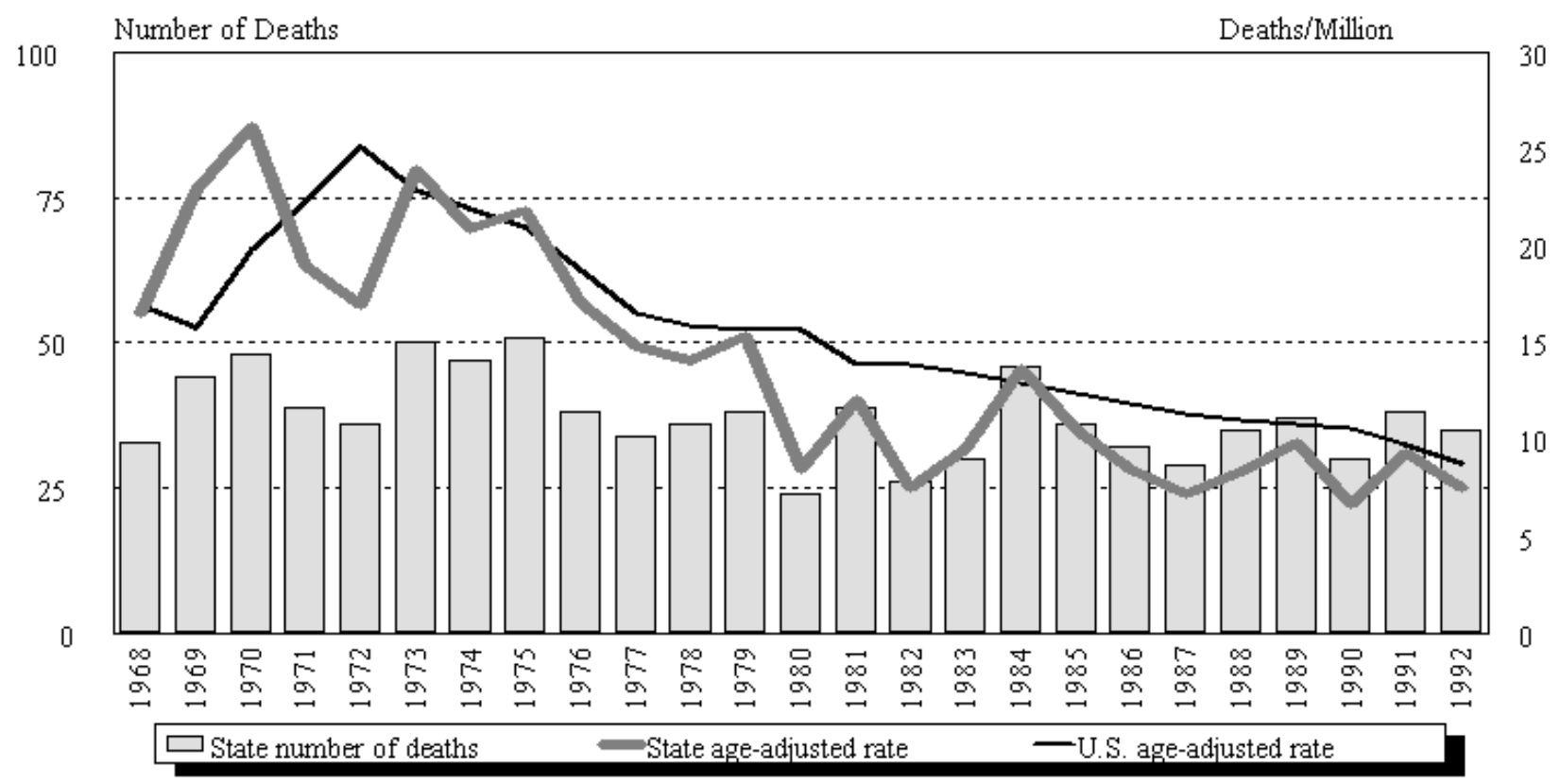

NOTE: $\quad$ See Appendix A for source description and Appendix B for methods and ICD-8 and ICD-9 codes.

SOURCE: $\quad$ National Center for Health Statistics multiple cause of death data. Population estimates from U.S. Bureau of the Census. 
Colorado

\begin{tabular}{|c|c|c|c|c|c|c|c|c|c|c|c|c|}
\hline \multirow[b]{2}{*}{ Year } & \multicolumn{2}{|c|}{ Asbestosis } & \multicolumn{2}{|c|}{$\begin{array}{c}\text { Coal workers' } \\
\text { pneumoconiosis }\end{array}$} & \multicolumn{2}{|c|}{ Silicosis } & \multicolumn{2}{|c|}{ Byssinosis* } & \multicolumn{2}{|c|}{$\begin{array}{c}\text { Unspecified/Other } \\
\text { pneumoconioses }\end{array}$} & \multicolumn{2}{|c|}{ All pneumoconioses } \\
\hline & Number & Rate & Number & Rate & Number & Rate & Number & Rate & Number & Rate & Number & Rate \\
\hline 1968 & 1 & 0.57 & 1 & 0.38 & 26 & 13.34 & & & 5 & 2.29 & 33 & 16.58 \\
\hline 1969 & - & - & 2 & 0.62 & 31 & 16.52 & & & 11 & 5.80 & 44 & 22.94 \\
\hline 1970 & - & - & 6 & 3.09 & 32 & 17.96 & & & 13 & 7.03 & 48 & 26.18 \\
\hline 1971 & - & - & 5 & 2.05 & 28 & 14.07 & & & 8 & 3.85 & 39 & 19.00 \\
\hline 1972 & - & - & 12 & 5.36 & 16 & 8.63 & & & 8 & 3.00 & 36 & 16.99 \\
\hline 1973 & - & - & 10 & 4.35 & 22 & 11.24 & & & 19 & 8.88 & 50 & 23.91 \\
\hline 1974 & - & - & 11 & 4.82 & 26 & 11.69 & & & 12 & 5.51 & 47 & 20.91 \\
\hline 1975 & - & - & 14 & 5.59 & 21 & 9.21 & & & 18 & 7.71 & 51 & 21.78 \\
\hline 1976 & - & - & 11 & 5.43 & 23 & 10.25 & & & 6 & 2.25 & 38 & 17.10 \\
\hline 1977 & 2 & 0.94 & 11 & 4.62 & 13 & 5.75 & & & 8 & 3.49 & 34 & 14.80 \\
\hline 1978 & 2 & 0.95 & 11 & 4.46 & 13 & 5.66 & & & 10 & 3.04 & 36 & 14.11 \\
\hline 1979 & - & - & 8 & 3.66 & 18 & 6.74 & - & - & 12 & 5.02 & 38 & 15.41 \\
\hline 1980 & - & - & 10 & 3.27 & 8 & 2.99 & - & - & 6 & 2.26 & 24 & 8.52 \\
\hline 1981 & - & - & 16 & 5.02 & 16 & 5.60 & - & - & 7 & 1.46 & 39 & 12.07 \\
\hline 1982 & - & - & 15 & 3.66 & 7 & 2.55 & - & - & 4 & 1.28 & 26 & 7.49 \\
\hline 1983 & 6 & 2.50 & 10 & 2.22 & 13 & 4.55 & - & - & 2 & 0.65 & 30 & 9.54 \\
\hline 1984 & 7 & 2.48 & 25 & 6.84 & 10 & 3.27 & - & - & 4 & 1.04 & 46 & 13.63 \\
\hline 1985 & 3 & 0.98 & 22 & 5.97 & 7 & 2.42 & - & - & 4 & 1.10 & 36 & 10.47 \\
\hline 1986 & 6 & 1.92 & 15 & 3.43 & 8 & 2.23 & - & - & 4 & 1.09 & 32 & 8.43 \\
\hline 1987 & 3 & 0.83 & 15 & 3.52 & 8 & 2.19 & - & - & 3 & 0.70 & 29 & 7.24 \\
\hline 1988 & 6 & 1.62 & 21 & 4.71 & 8 & 2.05 & - & - & - & - & 35 & 8.38 \\
\hline 1989 & 3 & 0.91 & 17 & 4.11 & 16 & 4.48 & - & - & 1 & 0.34 & 37 & 9.83 \\
\hline 1990 & 4 & 1.24 & 15 & 3.13 & 10 & 2.12 & - & - & 1 & 0.23 & 30 & 6.72 \\
\hline 1991 & 5 & 1.32 & 15 & 2.82 & 15 & 4.55 & - & - & 3 & 0.65 & 38 & 9.33 \\
\hline 1992 & 4 & 1.28 & 19 & 3.14 & 9 & 2.23 & - & - & 3 & 0.85 & 35 & 7.50 \\
\hline
\end{tabular}

-indicates no deaths listed. ${ }^{*}$ no specific code for by ssinosis in ICD-8 (1968-1978)

NOTE: Rates based on small numbers may be unstable. Sum of individual conditions may exceed total because of deaths with multiple pneumoconioses. See Appendix A for source description and Appendix B for methods and ICD-8 and ICD-9 codes.

SOURCE: National Center for Health Statistics multiple cause of death data. Population estimates from U.S. Bureau of the Census. 
Figure CO-2. All pneumoconioses: Distribution of deaths by sex and race, Colorado residents age 15 and over, 1968-1992
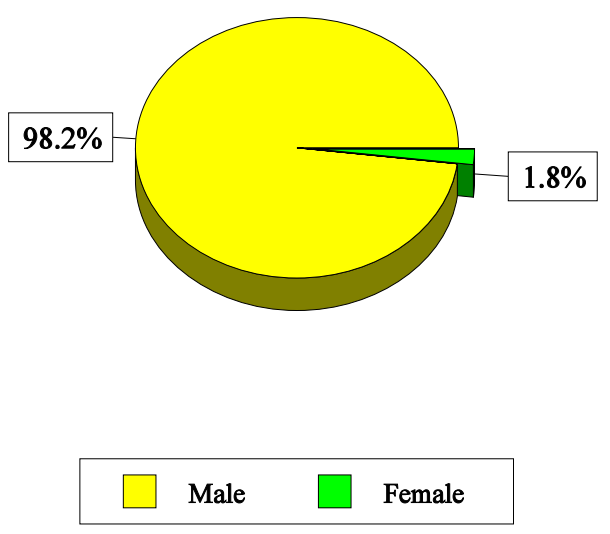
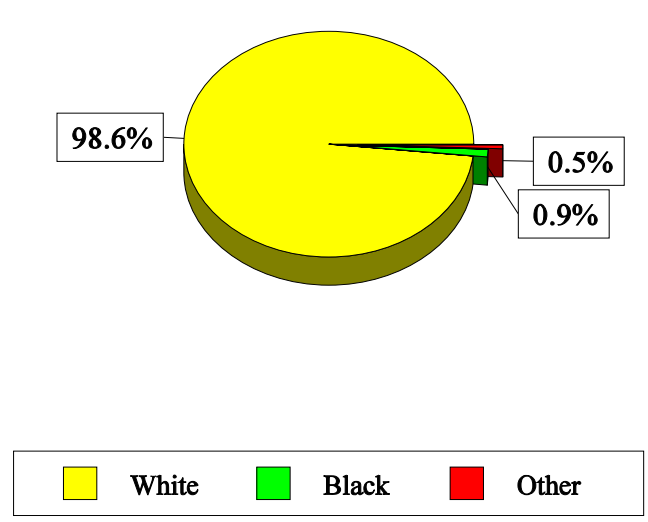

NOTE: $\quad$ See Appendix A for source description and Appendix B for methods and ICD-8 and ICD-9 codes.

SOURCE: National Center for Health Statistics multiple cause of death data.

Table CO-2. All pneumoconioses: Distribution of deaths by sex, race, and age, Colorado residents age 15 and over, 1968-1992

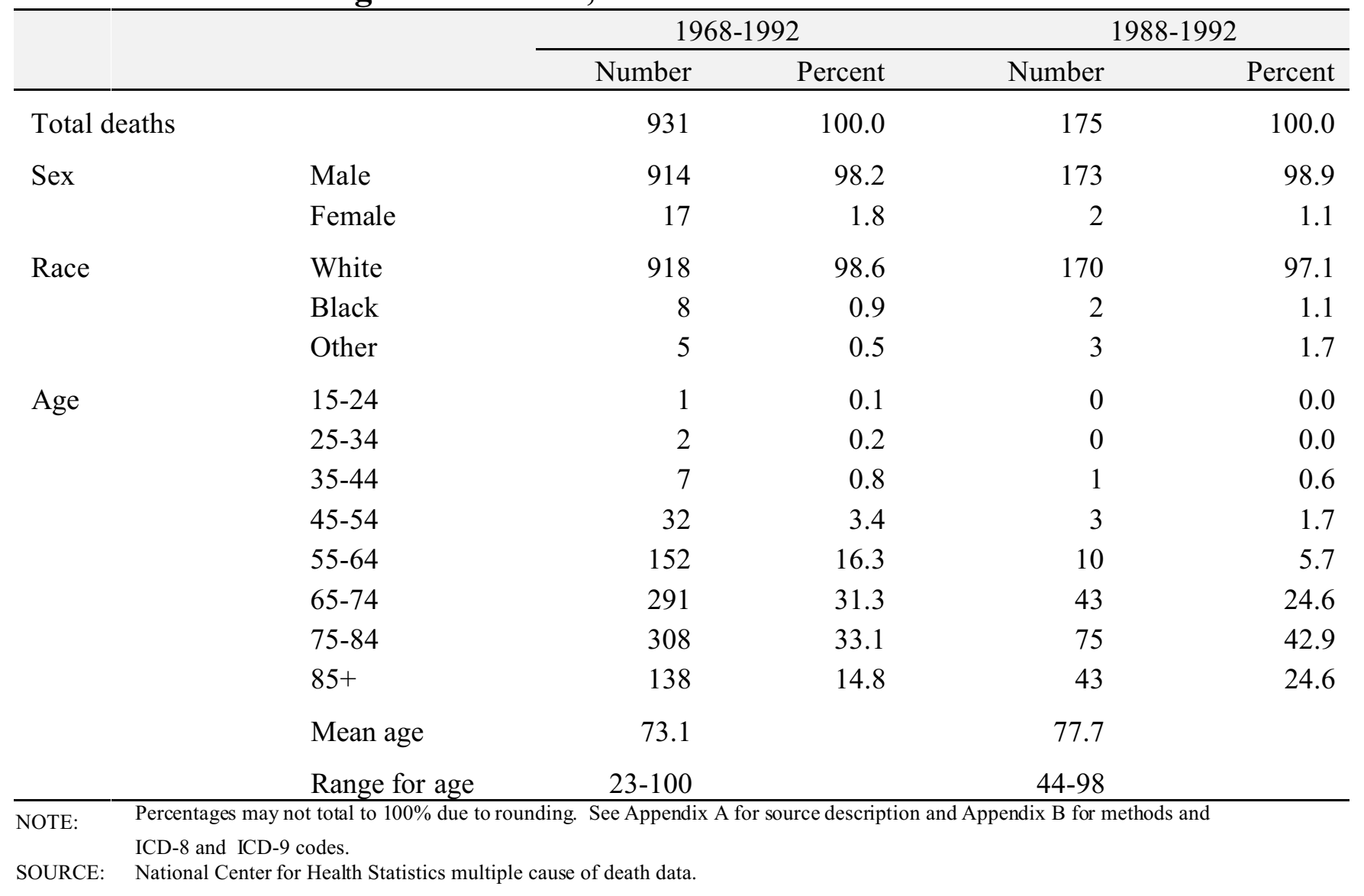




\section{Figure CO-3. Pneumoconiosis deaths by condition, Colorado residents age 15 and over, 1968-1992}

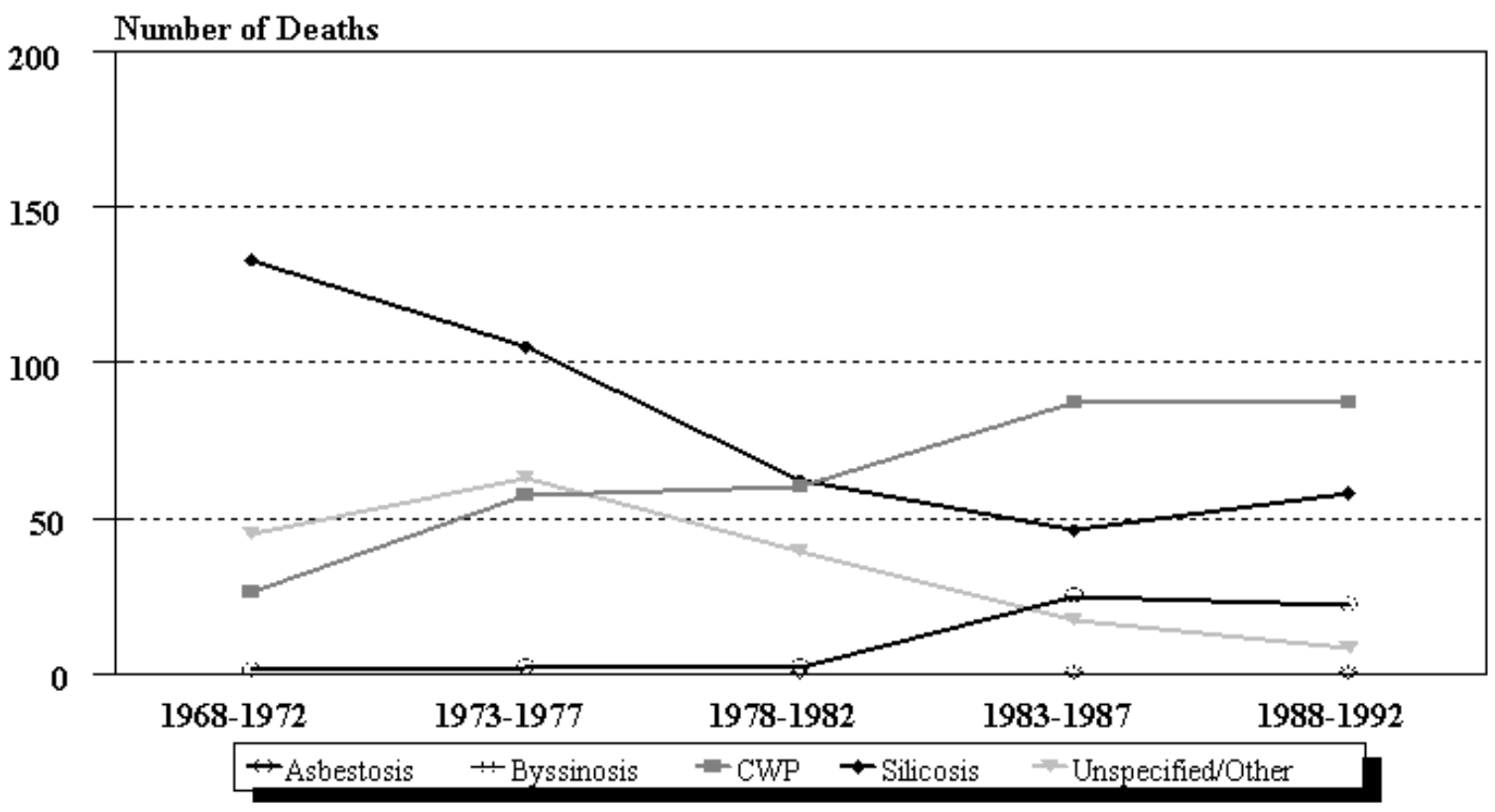

NOTE: See Appendix A for source description and Appendix B for methods and ICD-8 and ICD-9 codes. SOURCE: National Center for Health Statistics multiple cause of death data.

Figure CO-4. Colorado and U.S. age-adjusted mortality rates (per 1,000,000 population) by condition, residents age 15 and over, 1983-1992

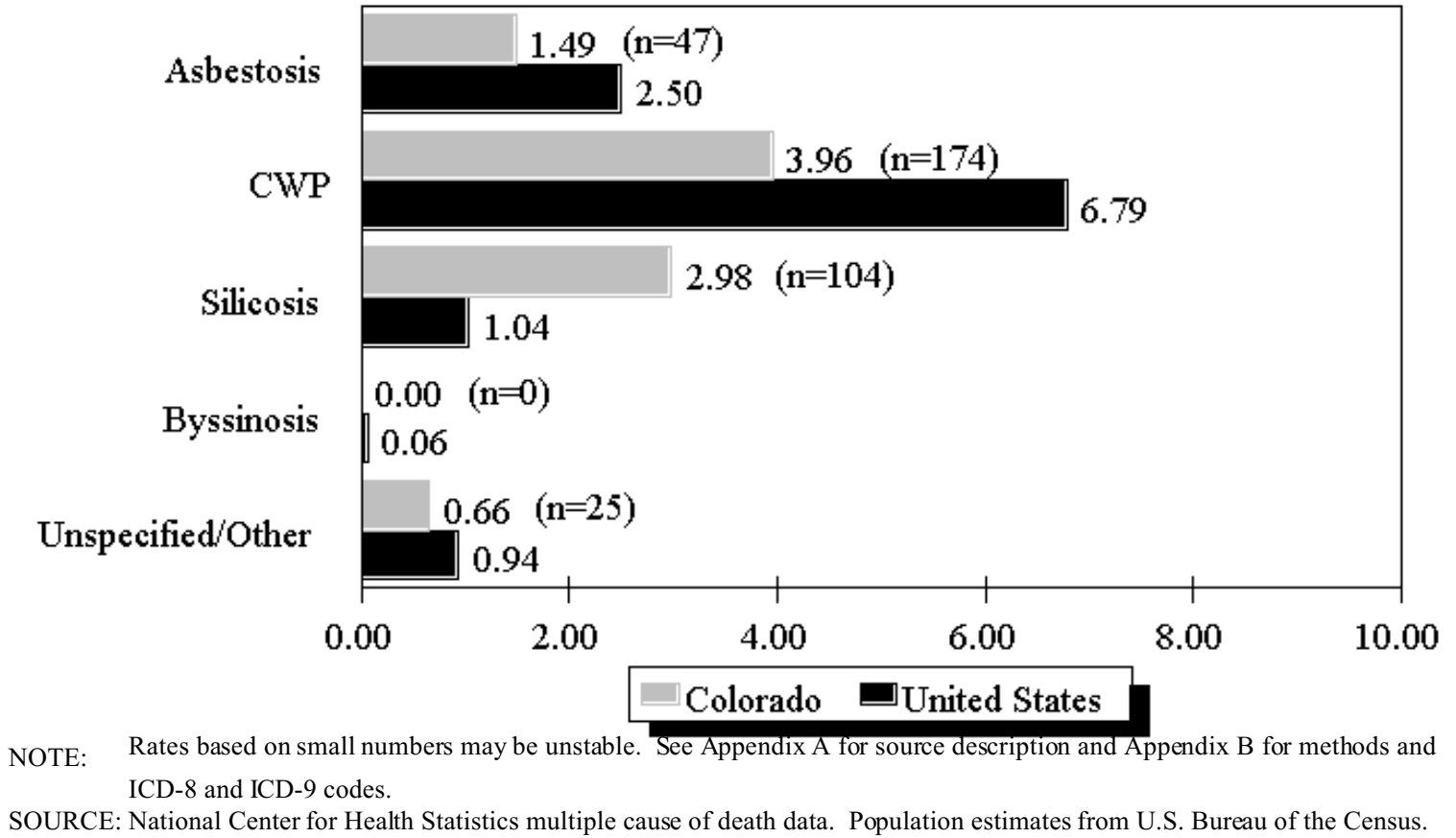


Table CO-3 (page 1 of 2). Number of deaths and age-adjusted mortality rate (per $1,000,000$ population) by condition and county, Colorado residents age 15 and over, 1983-1992

\begin{tabular}{|c|c|c|c|c|c|c|c|c|c|c|c|c|}
\hline \multirow[b]{2}{*}{ County } & \multicolumn{2}{|c|}{ Asbestosis } & \multicolumn{2}{|c|}{$\begin{array}{c}\text { Coal workers' } \\
\text { pneumoconiosis }\end{array}$} & \multicolumn{2}{|r|}{ Silicosis } & \multicolumn{2}{|c|}{ Byssinosis } & \multicolumn{2}{|c|}{$\begin{array}{r}\text { Unspecified/Other } \\
\text { pneumoconioses }\end{array}$} & \multicolumn{2}{|c|}{$\begin{array}{r}\text { All } \\
\text { pneumoconioses }\end{array}$} \\
\hline & \# & Rate & \# & Rate & \# & Rate & \# & Rate & \# & Rate & \# & Rate \\
\hline Adams & 2 & 1.05 & 7 & 3.05 & 5 & 2.37 & - & - & 1 & 0.40 & 15 & 6.87 \\
\hline Alamosa & - & - & 1 & 10.84 & - & - & - & - & - & - & 1 & 10.84 \\
\hline Arapahoe & 4 & 1.54 & 5 & 1.52 & 4 & 1.14 & - & - & - & - & 13 & 4.20 \\
\hline Boulder & 4 & 2.07 & 10 & 4.12 & 3 & 1.49 & - & - & 1 & 0.38 & 17 & 7.69 \\
\hline Chaffee & 1 & 6.71 & - & - & 2 & 10.06 & - & - & - & - & 3 & 16.77 \\
\hline Conejos & - & - & - & - & 1 & 13.30 & - & - & - & - & 1 & 13.30 \\
\hline Crowley & - & - & 1 & 10.22 & - & - & - & - & - & - & 1 & 10.22 \\
\hline Delta & 1 & 3.37 & 10 & 21.36 & 4 & 9.37 & - & - & - & - & 15 & 34.10 \\
\hline Denver & 9 & 1.47 & 16 & 1.99 & 13 & 1.84 & - & - & 2 & 0.19 & 40 & 5.48 \\
\hline Dolores & - & - & - & - & 2 & 85.14 & - & - & - & - & 2 & 85.14 \\
\hline Douglas & - & - & 1 & 3.88 & - & - & - & - & - & - & 1 & 3.88 \\
\hline Eagle & 1 & 14.17 & - & - & 1 & 14.17 & - & - & - & - & 2 & 28.34 \\
\hline El Paso & 1 & 0.37 & 7 & 1.94 & 4 & 1.09 & - & - & - & - & 12 & 3.40 \\
\hline Fremont & 2 & 5.24 & 28 & 40.67 & 4 & 5.58 & - & - & 1 & 2.62 & 35 & 54.11 \\
\hline Garfield & - & - & - & - & 1 & 4.65 & - & - & - & - & 1 & 4.65 \\
\hline Gunnison & - & - & 3 & 40.21 & - & - & - & - & 1 & 17.70 & 4 & 57.91 \\
\hline Huerfano & - & - & 7 & 39.66 & - & - & - & - & 1 & 4.60 & 8 & 44.26 \\
\hline Jefferson & 10 & 2.82 & 8 & 2.03 & 4 & 0.97 & - & - & 3 & 0.75 & 25 & 6.57 \\
\hline Lake & - & - & 1 & 22.27 & 1 & 22.27 & - & - & 1 & 15.85 & 3 & 60.39 \\
\hline Laplata & - & - & - & - & 2 & 7.90 & - & - & - & - & 2 & 7.90 \\
\hline Larimer & 3 & 1.13 & 3 & 1.13 & - & - & - & - & - & - & 6 & 2.27 \\
\hline Las Animas & - & - & 24 & 64.55 & 2 & 4.12 & - & - & 11 & 33.98 & 37 & 102.65 \\
\hline Lincoln & - & - & 1 & 6.64 & - & - & - & - & - & - & 1 & 6.64 \\
\hline Mesa & 5 & 3.76 & 10 & 6.85 & 11 & 9.19 & - & - & - & - & 25 & 18.71 \\
\hline Mineral & - & - & - & - & 3 & 351.61 & - & - & - & - & 3 & 351.61 \\
\hline Montezuma & 1 & 5.43 & 3 & 13.96 & 5 & 24.81 & - & - & 1 & 5.43 & 10 & 49.63 \\
\hline Montrose & - & - & 3 & 7.12 & 10 & 25.01 & - & - & - & - & 13 & 32.13 \\
\hline Morgan & - & - & 1 & 1.76 & - & - & - & - & - & - & 1 & 1.76 \\
\hline Otero & - & - & 1 & 1.66 & - & - & - & - & - & - & 1 & 1.66 \\
\hline Ouray & - & - & - & - & 4 & 134.34 & - & - & - & - & 4 & 134.34 \\
\hline Park & - & - & - & - & 1 & 21.09 & - & - & - & - & 1 & 21.09 \\
\hline
\end{tabular}

See footnotes at end of table. 
Table CO-3 (page 2 of 2). Number of deaths and age-adjusted mortality rate (per $1,000,000$ population) by condition and county, Colorado residents age 15 and over, 1983-1992

\begin{tabular}{|c|c|c|c|c|c|c|c|c|c|c|c|c|}
\hline \multirow[b]{2}{*}{ County } & \multicolumn{2}{|c|}{ Asbestosis } & \multicolumn{2}{|c|}{$\begin{array}{c}\text { Coal workers' } \\
\text { pneumoconiosis }\end{array}$} & \multicolumn{2}{|r|}{ Silicosis } & \multicolumn{2}{|c|}{ Byssinosis } & \multicolumn{2}{|c|}{$\begin{array}{c}\text { Unspecified/Other } \\
\text { pneumoconioses }\end{array}$} & \multicolumn{2}{|c|}{$\begin{array}{r}\text { All } \\
\text { pneumoconioses }\end{array}$} \\
\hline & $\#$ & Rate & \# & Rate & $\#$ & Rate & $\#$ & Rate & $\#$ & Rate & \# & Rate \\
\hline Phillips & - & - & - & - & 1 & 6.03 & - & - & - & - & 1 & 6.03 \\
\hline Pueblo & 2 & 1.11 & 8 & 3.29 & 7 & 5.04 & - & - & 1 & 0.36 & 18 & 9.81 \\
\hline Routt & - & - & 2 & 24.02 & - & - & - & - & - & - & 2 & 24.02 \\
\hline Saguache & - & - & - & - & 2 & 24.46 & - & - & - & - & 2 & 24.46 \\
\hline San Juan & - & - & - & - & 2 & 331.59 & - & - & - & - & 2 & 331.59 \\
\hline Washington & 1 & 30.03 & - & - & - & - & - & - & - & - & 1 & 30.03 \\
\hline Weld & - & - & 13 & 7.80 & 5 & 4.09 & - & - & 1 & 1.01 & 19 & 12.90 \\
\hline
\end{tabular}

- indicates no deaths listed.

NOTE: Counties with no deaths for all listed conditions are not displayed. Rates based on small numbers may be unstable. Sum of individual conditions may exceed total because of deaths with multiple pneumoconioses. See Appendix A for source description and Appendix B for methods and ICD-8 and ICD-9 codes.

SOURCE: National Center for Health Statistics multiple cause of death data. Population estimates from U.S. Bureau of the Census. 
Connecticut

\section{Connecticut: Selected statistics from the United States section}

All pneumoconioses mortality, 1988-1992

Total deaths

Crude mortality rate (per $1,000,000$ population)

Age-adjusted mortality rate (per 1,000,000 population)

Total years of potential life lost to life expectancy

Years of potential life lost to life expectancy (per death) $\underline{\text { Value }}$

107

8.07

4.34

1,204

11.3

$\underline{\text { Deaths* }}$

66

13

13

0

15
State rank

28

28

32

28

40

Percent**

distribution

61.7

12.1

12.1

0.0

14.0

\section{Unspecified/Other pneumoconiosis}

* Sum of column may exceed total deaths because of deaths with multiple pneumoconioses.

** Percentages may total more than $100 \%$ due to deaths with multiple pneumoconioses.

NOTE: $\quad$ See Appendix A for source description and Appendix B for methods and ICD-8 and ICD-9 codes.

SOURCE: National Center for Health Statistics multiple cause of death data. Population estimates from U.S. Bureau of the Census.

Figure CT-1. All pneumoconioses: Connecticut and U.S. age-adjusted mortality rates, residents age 15 and over, 1968-1992

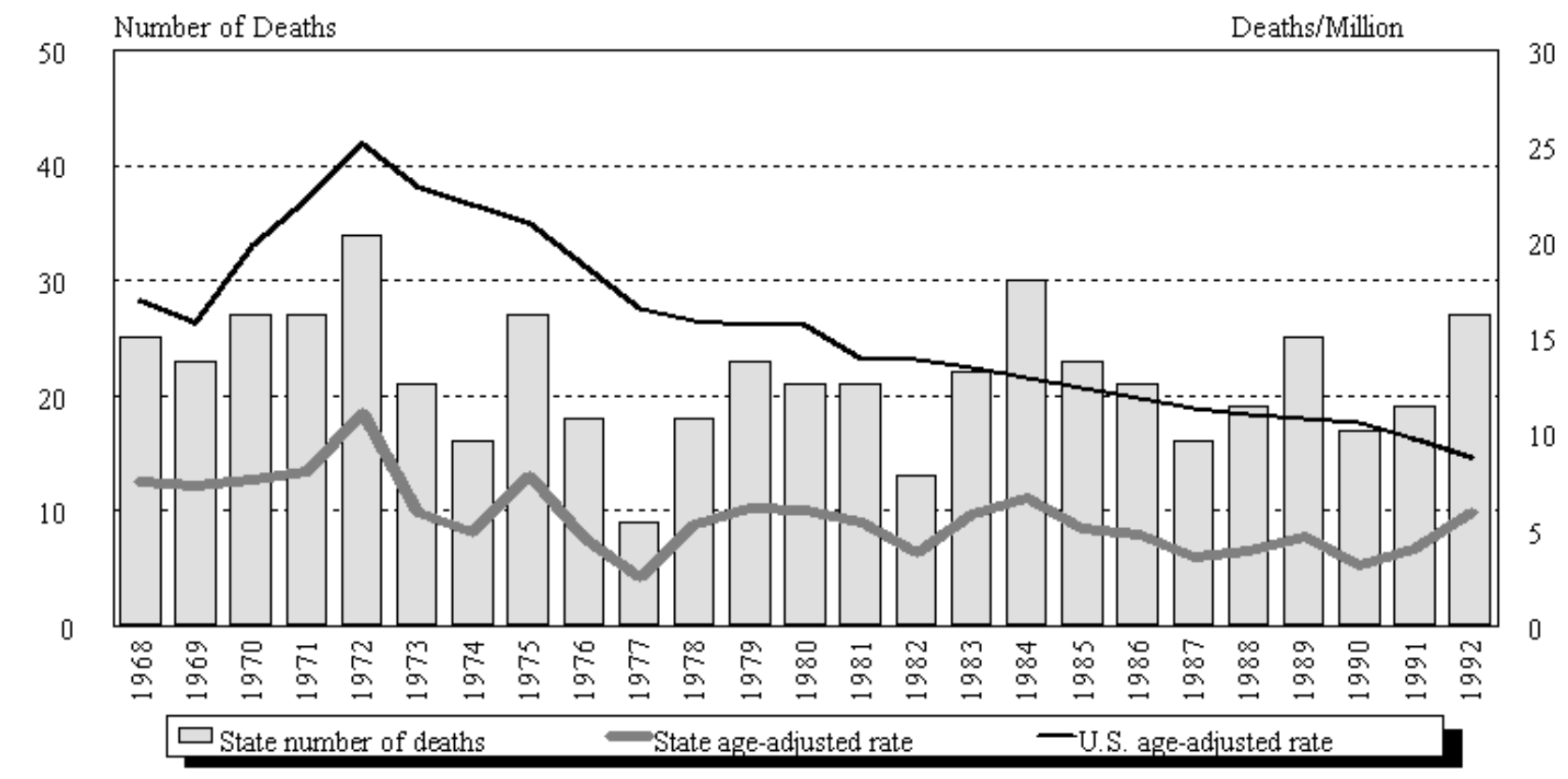

NOTE: $\quad$ See Appendix A for source description and Appendix B for methods and ICD-8 and ICD-9 codes.

SOURCE: National Center for Health Statistics multiple cause of death data. Population estimates from U.S. Bureau of the Census. 


\section{Connecticut}

\begin{tabular}{|c|c|c|c|c|c|c|c|c|c|c|c|c|}
\hline \multirow[b]{2}{*}{ Year } & \multicolumn{2}{|c|}{ Asbestosis } & \multicolumn{2}{|c|}{$\begin{array}{c}\text { Coal workers' } \\
\text { pneumocon iosis }\end{array}$} & \multicolumn{2}{|c|}{ Silicosis } & \multicolumn{2}{|c|}{ Byssinosis* } & \multicolumn{2}{|c|}{$\begin{array}{c}\text { Unsp ecified/Other } \\
\text { pneumoconioses }\end{array}$} & \multicolumn{2}{|c|}{ All pneumoconioses } \\
\hline & Number & Rate & Number & Rate & Number & Rate & Number & Rate & Number & Rate & Number & Rate \\
\hline 1968 & - & - & 6 & 1.67 & 13 & 3.86 & & & 7 & 2.41 & 25 & 7.56 \\
\hline 1969 & 1 & 0.38 & 4 & 1.36 & 12 & 3.55 & & & 6 & 2.07 & 23 & 7.35 \\
\hline 1970 & 1 & 0.38 & 3 & 0.80 & 16 & 4.45 & & & 8 & 2.22 & 27 & 7.67 \\
\hline 1971 & 2 & 0.73 & 7 & 1.91 & 6 & 1.82 & & & 12 & 3.62 & 27 & 8.08 \\
\hline 1972 & 4 & 1.43 & 10 & 3.95 & 10 & 2.54 & & & 10 & 3.21 & 34 & 11.12 \\
\hline 1973 & 2 & 0.38 & 5 & 1.52 & 6 & 1.94 & & & 9 & 2.43 & 21 & 5.92 \\
\hline 1974 & 2 & 0.76 & 2 & 0.69 & 9 & 2.51 & & & 3 & 0.92 & 16 & 4.88 \\
\hline 1975 & 4 & 1.51 & 4 & 1.23 & 10 & 2.63 & & & 9 & 2.49 & 27 & 7.87 \\
\hline 1976 & - & - & 1 & 0.23 & 10 & 2.18 & & & 7 & 2.18 & 18 & 4.58 \\
\hline 1977 & 2 & 0.54 & 2 & 0.44 & 3 & 0.77 & & & 3 & 1.07 & 9 & 2.49 \\
\hline 1978 & 4 & 1.27 & 2 & 0.23 & 5 & 1.40 & & & 7 & 2.35 & 18 & 5.25 \\
\hline 1979 & 3 & 0.99 & 12 & 2.94 & 6 & 1.57 & - & - & 3 & 0.99 & 23 & 6.17 \\
\hline 1980 & 3 & 1.10 & 7 & 1.69 & 6 & 1.63 & - & - & 5 & 1.61 & 21 & 6.03 \\
\hline 1981 & 4 & 1.10 & 3 & 0.87 & 3 & 0.71 & - & - & 11 & 2.72 & 21 & 5.40 \\
\hline 1982 & 2 & 0.64 & 1 & 0.28 & 7 & 2.29 & - & - & 3 & 0.58 & 13 & 3.80 \\
\hline 1983 & 11 & 3.32 & 4 & 0.76 & 4 & 0.99 & - & - & 3 & 0.80 & 22 & 5.87 \\
\hline 1984 & 7 & 1.86 & 7 & 1.22 & 9 & 2.13 & 1 & 0.19 & 7 & 1.49 & 30 & 6.70 \\
\hline 1985 & 8 & 1.93 & 6 & 1.01 & 6 & 1.58 & - & - & 3 & 0.54 & 23 & 5.06 \\
\hline 1986 & 12 & 3.02 & 3 & 0.44 & 3 & 0.71 & - & - & 3 & 0.62 & 21 & 4.80 \\
\hline 1987 & 9 & 2.30 & 3 & 0.43 & 2 & 0.35 & - & - & 2 & 0.51 & 16 & 3.59 \\
\hline 1988 & 10 & 2.23 & 1 & 0.17 & 5 & 1.03 & - & - & 3 & 0.51 & 19 & 3.93 \\
\hline 1989 & 14 & 2.79 & 6 & 0.91 & 1 & 0.16 & - & - & 4 & 0.76 & 25 & 4.62 \\
\hline 1990 & 11 & 1.89 & 2 & 0.51 & 2 & 0.41 & - & - & 2 & 0.41 & 17 & 3.22 \\
\hline 1991 & 14 & 3.42 & 1 & 0.08 & 2 & 0.41 & - & - & 2 & 0.16 & 19 & 4.06 \\
\hline 1992 & 17 & 4.05 & 3 & 0.39 & 3 & 0.66 & . & - & 4 & 0.82 & 27 & 5.91 \\
\hline
\end{tabular}

indicates no deaths listed. * no specific code for byssinosis in ICD-8 (1968-1978).

NOTE: Rates based on small numbers may be unstable. Sum of individual conditions may exceed total because of deaths with multiple pneumoconioses. See Appendix A for source description and Appendix B for methods and ICD-8 and ICD-9 NOTE

SOURCE: National Center for Health Statistics multiple cause of death data. Population estimates from U.S. Bureau of the Census. 


\section{Figure CT-2. All pneumoconioses: Distribution of deaths by sex and race,} Connecticut residents age 15 and over, 1968-1992
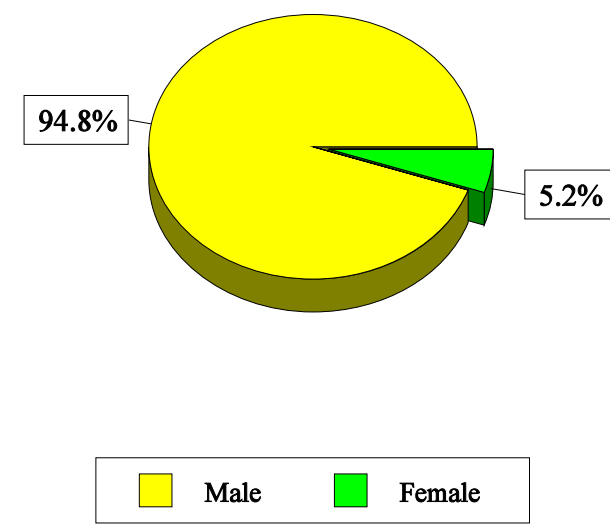
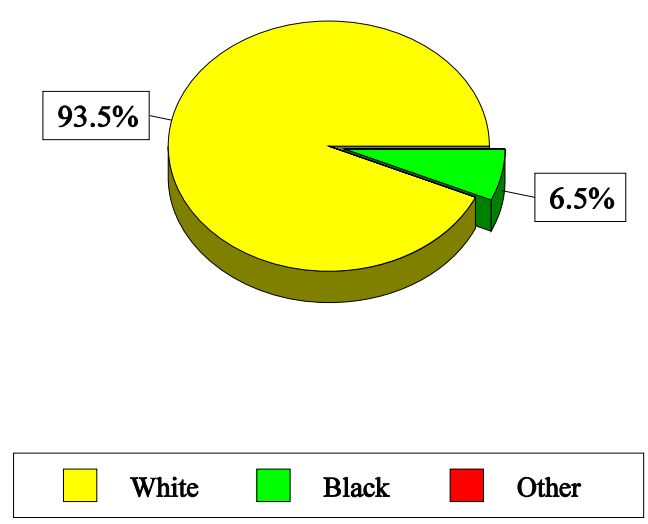

NOTE: $\quad$ See Appendix A for source description and Appendix B for methods and ICD-8 and ICD-9 codes.

SOURCE: National Center for Health Statistics multiple cause of death data.

Table CT-2. All pneumoconioses: Distribution of deaths by sex, race, and age, Connecticut residents age 15 and over, 1968-1992

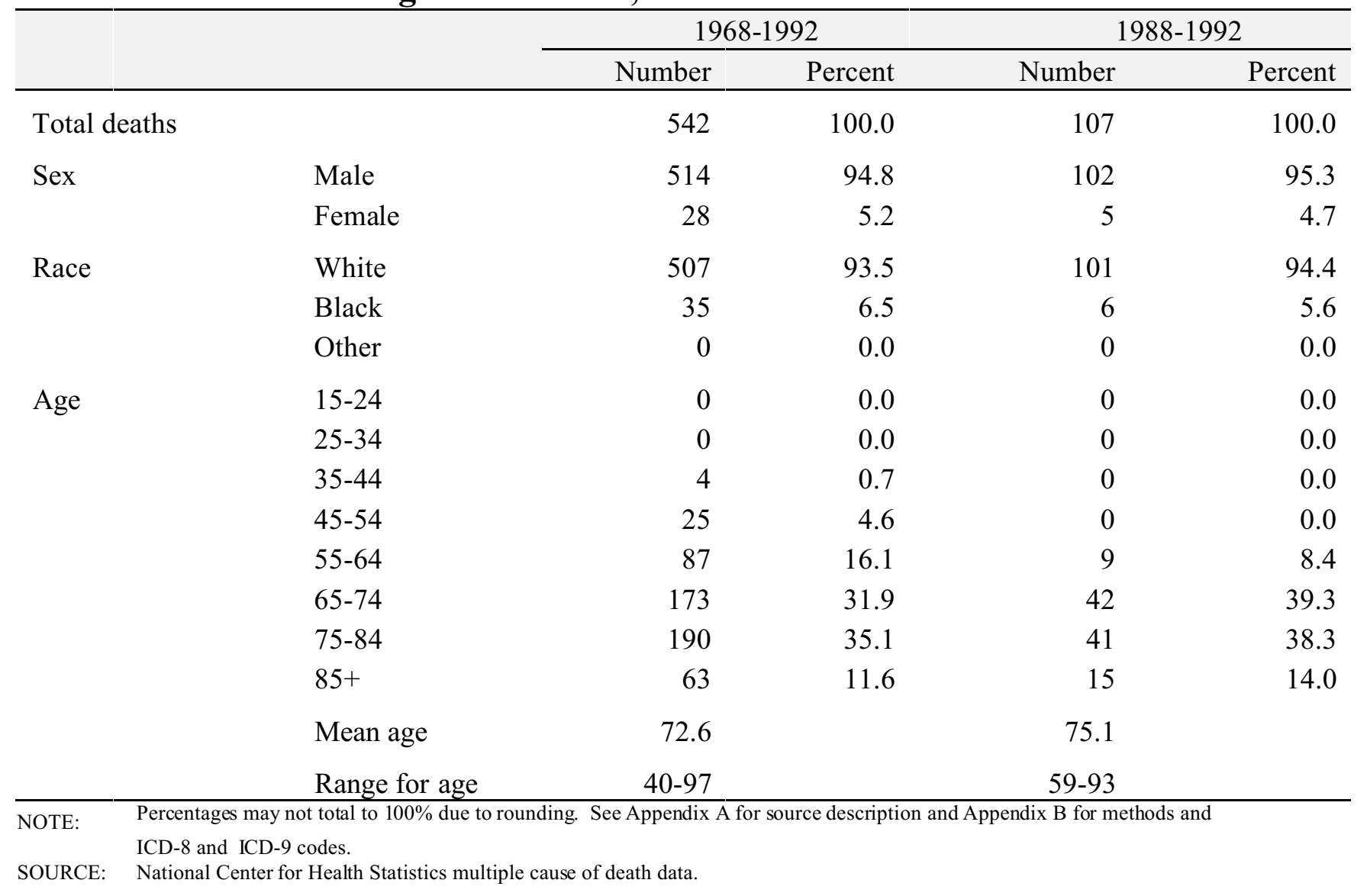




\section{Figure CT-3. Pneumoconiosis deaths by condition, Connecticut residents age 15 and over, 1968-1992}

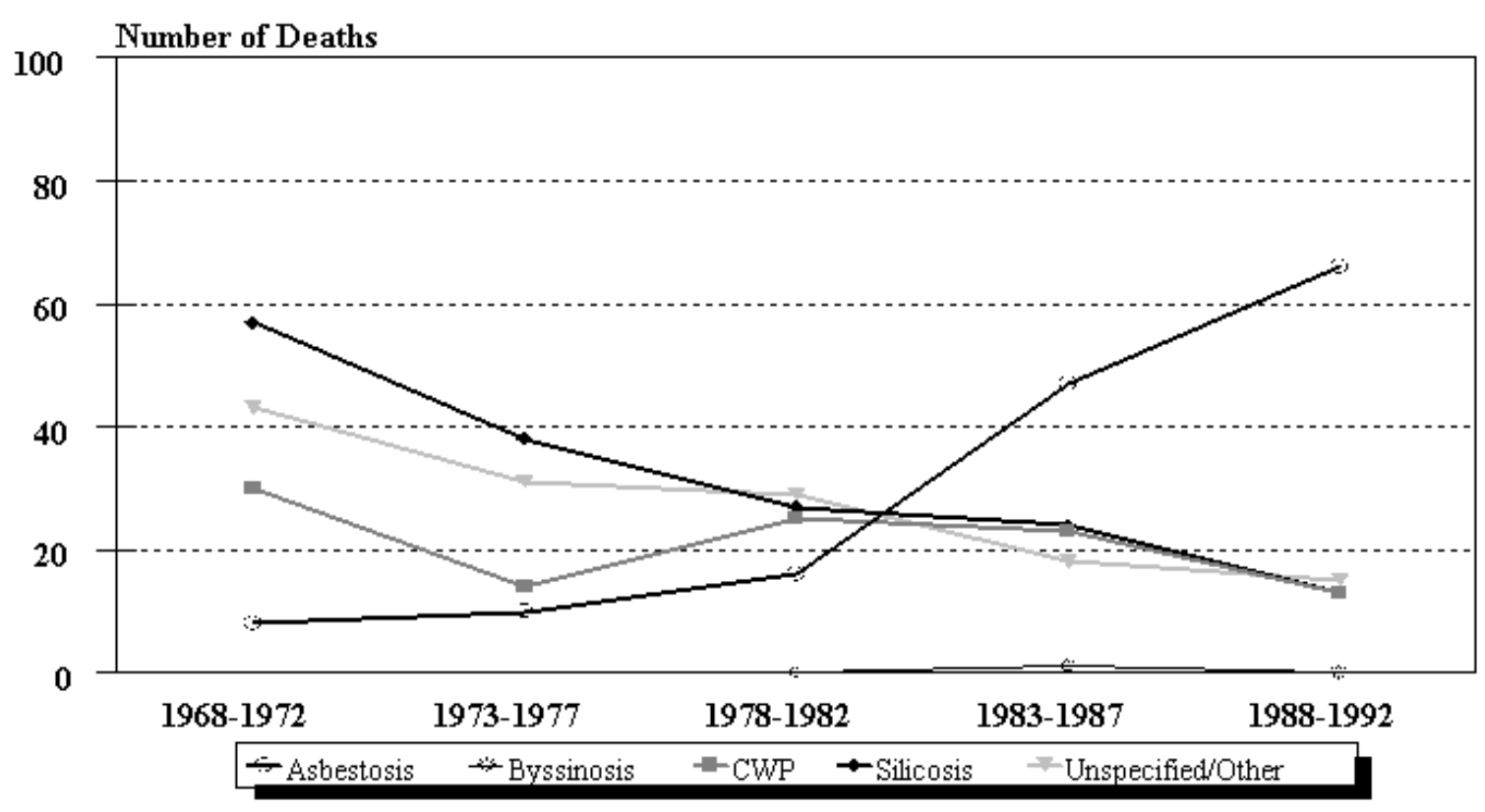

NOTE: See Appendix A for source description and Appendix B for methods and ICD-8 and ICD-9 codes. SOURCE: National Center for Health Statistics multiple cause of death data.

Figure CT-4. Connecticut and U.S. age-adjusted mortality rates (per 1,000,000 population) by condition, residents age 15 and over, 1983-1992

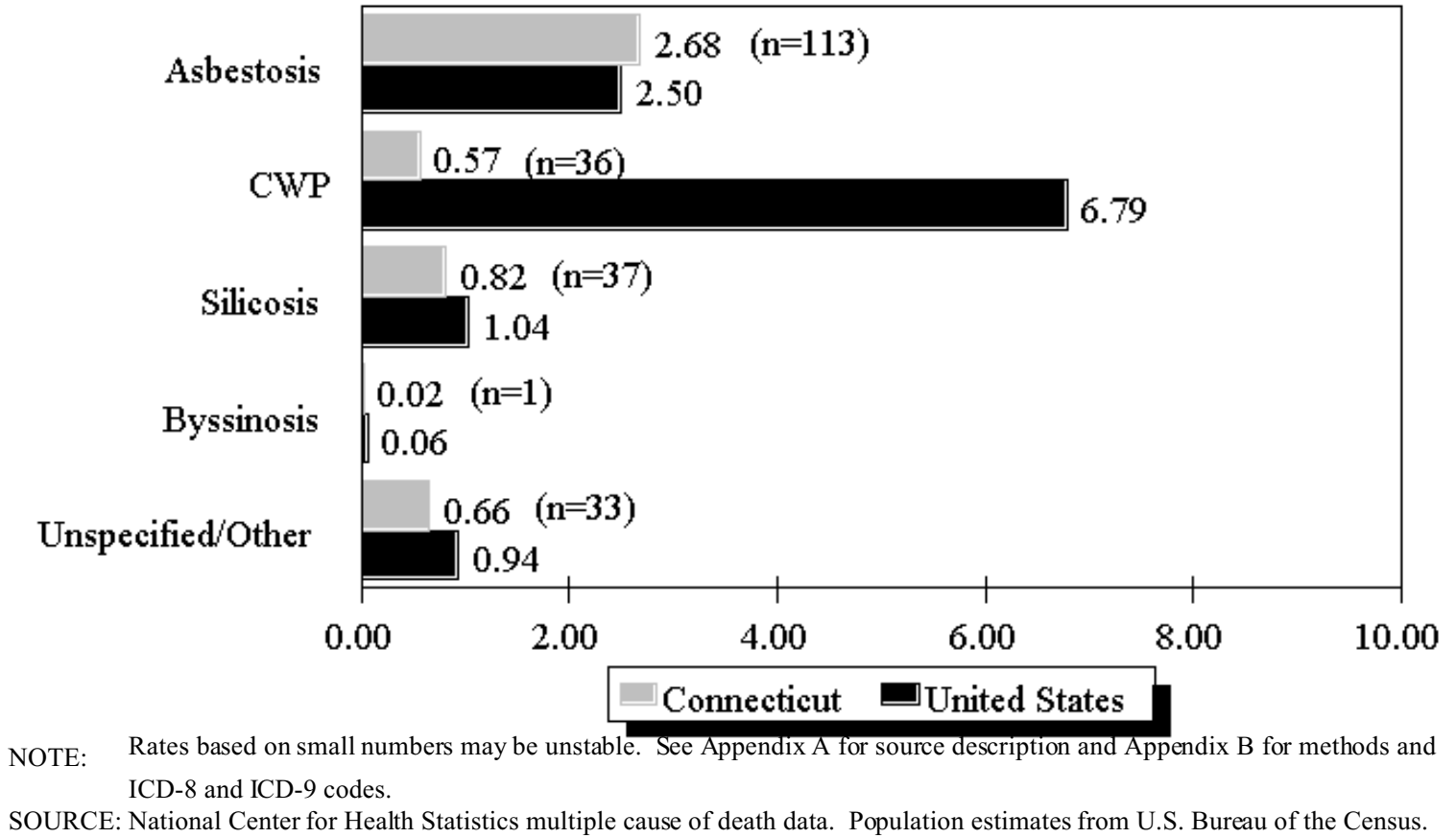


Table CT-3. Number of deaths and age-adjusted mortality rate (per $1,000,000$ population) by condition and county, Connecticut residents age 15 and over, 1983-1992

\begin{tabular}{|c|c|c|c|c|c|c|c|c|c|c|c|c|}
\hline \multirow[b]{2}{*}{ County } & \multicolumn{2}{|c|}{ Asbestosis } & \multicolumn{2}{|c|}{$\begin{array}{r}\text { Coal workers' } \\
\text { pneumoconiosis }\end{array}$} & \multicolumn{2}{|c|}{ Silicosis } & \multicolumn{2}{|c|}{ Byssinosis } & \multicolumn{2}{|c|}{$\begin{array}{r}\text { Unspecified/Other } \\
\text { pneumoconioses }\end{array}$} & \multicolumn{2}{|c|}{$\begin{array}{r}\text { All } \\
\text { pneumoconioses }\end{array}$} \\
\hline & \# & Rate & \# & Rate & \# & Rate & \# & Rate & \# & Rate & \# & Rate \\
\hline Fairfield & 20 & 1.60 & 22 & 1.50 & 11 & 0.78 & - & - & 12 & 1.06 & 64 & 4.88 \\
\hline Hartford & 26 & 2.40 & 5 & 0.28 & 8 & 0.57 & - & - & 10 & 0.80 & 49 & 4.04 \\
\hline Litchfield & 4 & 1.71 & 3 & 0.80 & 1 & 0.59 & - & - & 3 & 0.80 & 11 & 3.89 \\
\hline Middlesex & 4 & 1.79 & - & - & 2 & 0.69 & - & - & - & - & 6 & 2.48 \\
\hline New Haven & 16 & 1.42 & 4 & 0.22 & 9 & 0.97 & - & - & 7 & 0.59 & 36 & 3.21 \\
\hline New London & 38 & 14.97 & - & - & 5 & 2.31 & 1 & 0.23 & 1 & 0.23 & 45 & 17.74 \\
\hline Tolland & 3 & 3.21 & - & - & - & - & - & - & - & - & 3 & 3.21 \\
\hline Windham & 2 & 2.23 & 2 & 1.61 & 1 & 1.12 & - & - & - & - & 5 & 4.96 \\
\hline
\end{tabular}

- indicates no deaths listed.

NOTE: Counties with no deaths for all listed conditions are not displayed. Rates based on small numbers may be unstable. Sum of individual conditions may exceed total because of deaths with multiple pneumoconioses. See Appendix A for source description and Appendix B for methods and ICD-8 and ICD-9 codes.

SOURCE: National Center for Health Statistics multiple cause of death data. Population estimates from U.S. Bureau of the Census. 
All pneumoconioses mortality, 1988-1992

Total deaths

Crude mortality rate (per $1,000,000$ population)

Age-adjusted mortality rate (per $1,000,000$ population)

Total years of potential life lost to life expectancy

Years of potential life lost to life expectancy (per death) $\underline{\text { Value }}$

65

24.60

16.54

895

13.8

Deaths*

49

12

4

0

2 $\underline{\text { State rank }}$

33

5

5

32

6

Percent**

distribution

75.4

18.5

6.2

0.0

3.1

\section{Unspecified/Other pneumoconiosis}

* Sum of column may exceed total deaths because of deaths with multiple pneumoconioses.

** Percentages may total more than $100 \%$ due to deaths with multiple pneumoconioses.

NOTE: $\quad$ See Appendix A for source description and Appendix B for methods and ICD-8 and ICD-9 codes.

SOURCE: National Center for Health Statistics multiple cause of death data. Population estimates from U.S. Bureau of the Census.

\section{Figure DE-1. All pneumoconioses: Delaware and U.S. age-adjusted mortality rates, residents age 15 and over, 1968-1992}

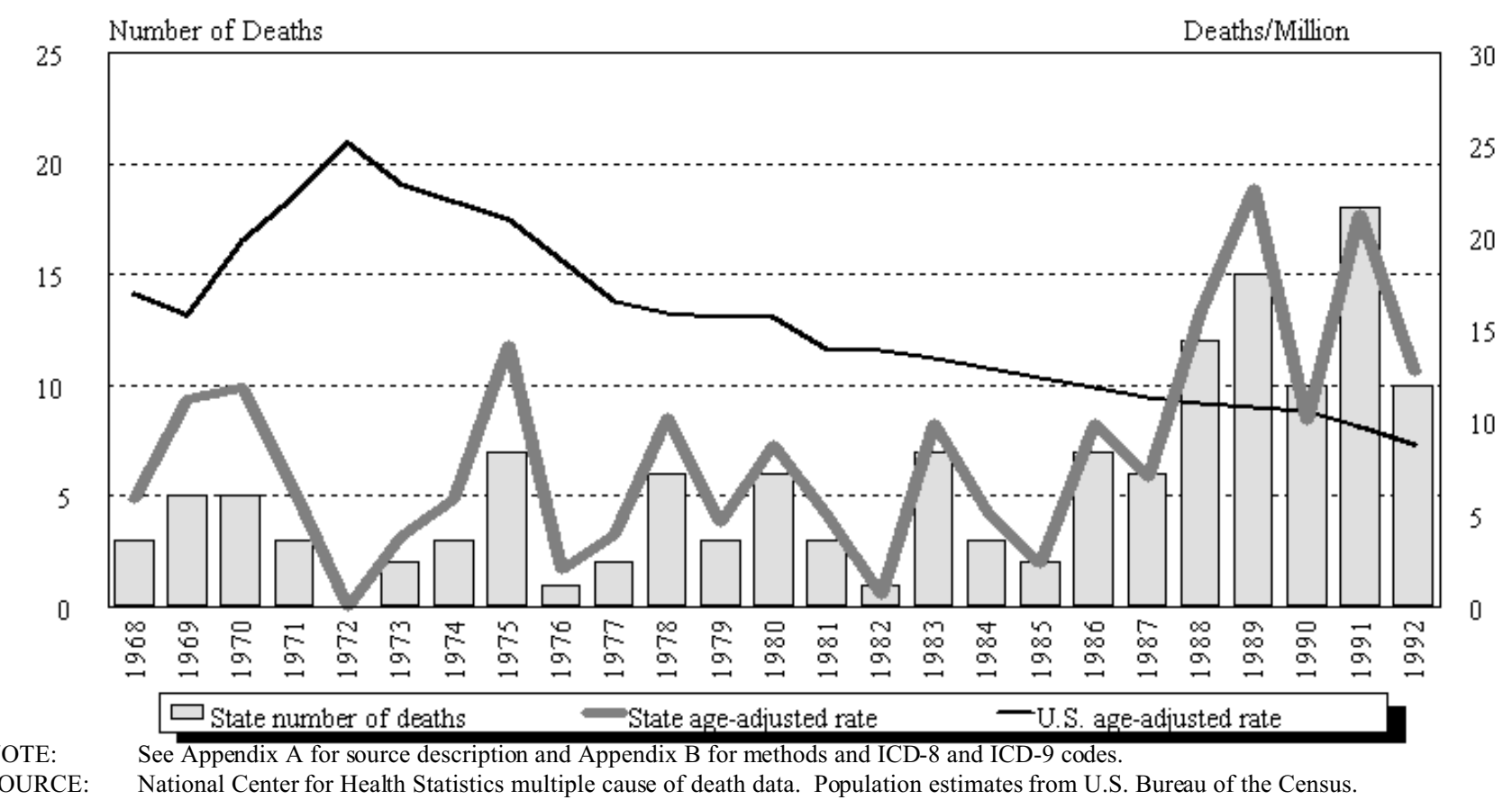


Delaware

\begin{tabular}{|c|c|c|c|c|c|c|c|c|c|c|c|c|}
\hline \multirow[b]{2}{*}{ Year } & \multicolumn{2}{|c|}{ Asbestosis } & \multicolumn{2}{|c|}{$\begin{array}{c}\text { Coal workers' } \\
\text { pneumoconiosis }\end{array}$} & \multicolumn{2}{|c|}{ Silicosis } & \multicolumn{2}{|c|}{ Byssinosis* } & \multicolumn{2}{|c|}{$\begin{array}{c}\text { Unsp ecified/Other } \\
\text { pneumoconioses }\end{array}$} & \multicolumn{2}{|c|}{ All pneumoconioses } \\
\hline & Number & Rate & Number & Rate & Number & Rate & Number & Rate & Number & Rate & Number & Rate \\
\hline 1968 & - & - & 3 & 5.82 & - & - & & & - & - & 3 & 5.82 \\
\hline 1969 & - & - & 1 & 2.36 & 1 & 1.73 & & & 3 & 7.11 & 5 & 11.20 \\
\hline 1970 & - & - & 2 & 4.73 & - & - & & & 3 & 7.19 & 5 & 11.92 \\
\hline 1971 & - & - & 2 & 3.96 & 1 & 2.30 & & & - & - & 3 & 6.26 \\
\hline 1972 & - & - & - & - & - & - & & & - & - & - & - \\
\hline 1973 & - & - & - & - & - & - & & & 2 & 3.77 & 2 & 3.77 \\
\hline 1974 & - & - & 2 & 4.27 & 1 & 1.54 & & & - & - & 3 & 5.80 \\
\hline 1975 & 2 & 4.43 & 3 & 5.63 & 3 & 6.23 & & & - & - & 7 & 14.21 \\
\hline 1976 & 1 & 2.01 & - & - & - & - & & & - & - & 1 & 2.01 \\
\hline 1977 & - & - & 2 & 3.87 & - & - & & & - & - & 2 & 3.87 \\
\hline 1978 & 1 & 1.39 & 2 & 3.27 & 1 & 1.88 & & & 2 & 3.76 & 6 & 10.29 \\
\hline 1979 & - & - & - & - & - & - & - & - & 3 & 4.53 & 3 & 4.53 \\
\hline 1980 & - & - & 3 & 4.26 & 1 & 0.69 & - & - & 2 & 3.81 & 6 & 8.76 \\
\hline 1981 & - & - & 3 & 4.83 & - & - & - & - & - & - & 3 & 4.83 \\
\hline 1982 & - & - & - & - & 1 & 0.66 & - & - & - & - & 1 & 0.66 \\
\hline 1983 & 3 & 4.25 & 4 & 5.68 & - & - & - & - & - & - & 7 & 9.92 \\
\hline 1984 & - & - & 3 & 5.19 & - & - & - & - & - & - & 3 & 5.19 \\
\hline 1985 & 1 & 1.15 & 1 & 1.15 & - & - & - & - & - & - & 2 & 2.29 \\
\hline 1986 & 3 & 4.05 & 4 & 5.90 & - & - & - & - & - & - & 7 & 9.95 \\
\hline 1987 & 3 & 3.91 & 1 & 1.42 & - & - & - & - & 2 & 1.64 & 6 & 6.97 \\
\hline 1988 & 8 & 11.91 & 2 & 2.06 & 1 & 1.03 & - & - & 2 & 2.06 & 12 & 16.02 \\
\hline 1989 & 13 & 18.97 & 1 & 1.34 & 1 & 2.36 & - & - & - & - & 15 & 22.67 \\
\hline 1990 & 6 & 6.68 & 4 & 3.41 & 1 & 0.96 & - & - & - & - & 10 & 10.09 \\
\hline 1991 & 14 & 17.27 & 4 & 4.07 & - & - & - & - & - & - & 18 & 21.35 \\
\hline 1992 & 8 & 10.54 & 1 & 1.26 & 1 & 0.90 & - & - & - & - & 10 & 12.70 \\
\hline
\end{tabular}

-indicates no deaths listed. ${ }^{*}$ no specific code for byssinosis in ICD-8 (1968-1978)

NOTE: Rates based on small numbers may be unstable. Sum of individual conditions may exceed total because of deaths with multiple pneumoconioses. See Appendix A for source description and Appendix B for methods and ICD-8 and ICD-9 codes.

SOURCE: National Center for Health Statistics multiple cause of death data. Population estimates from U.S. Bureau of the Census. 
Figure DE-2. All pneumoconioses: Distribution of deaths by sex and race, Delaware residents age 15 and over, 1968-1992
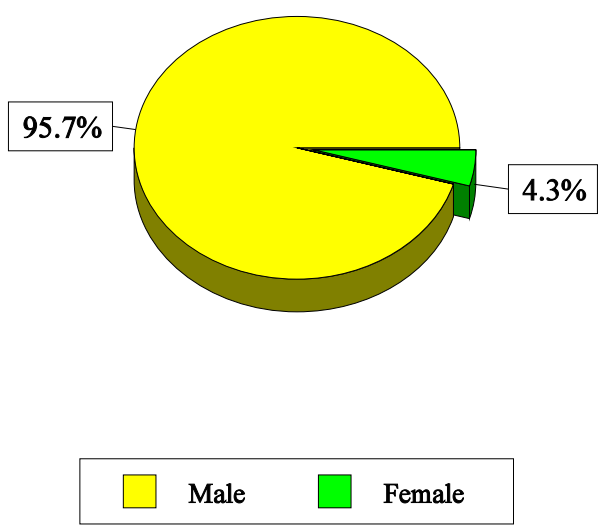
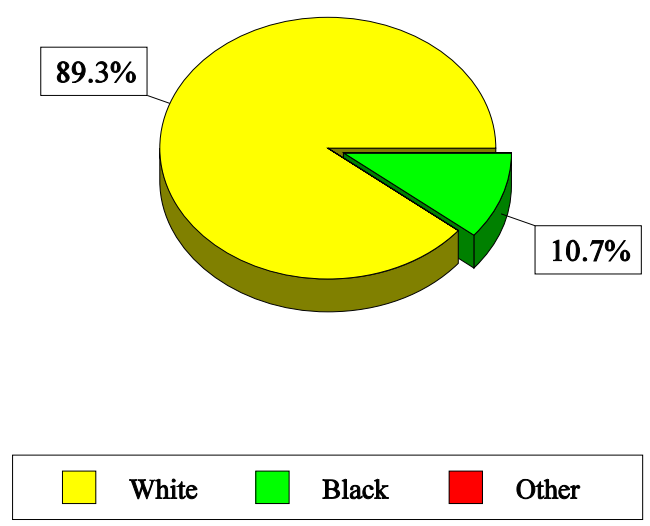

NOTE: $\quad$ See Appendix A for source description and Appendix B for methods and ICD-8 and ICD-9 codes.

SOURCE: National Center for Health Statistics multiple cause of death data.

Table DE-2. All pneumoconioses: Distribution of deaths by sex, race, and age, Delaware residents age 15 and over, 1968-1992

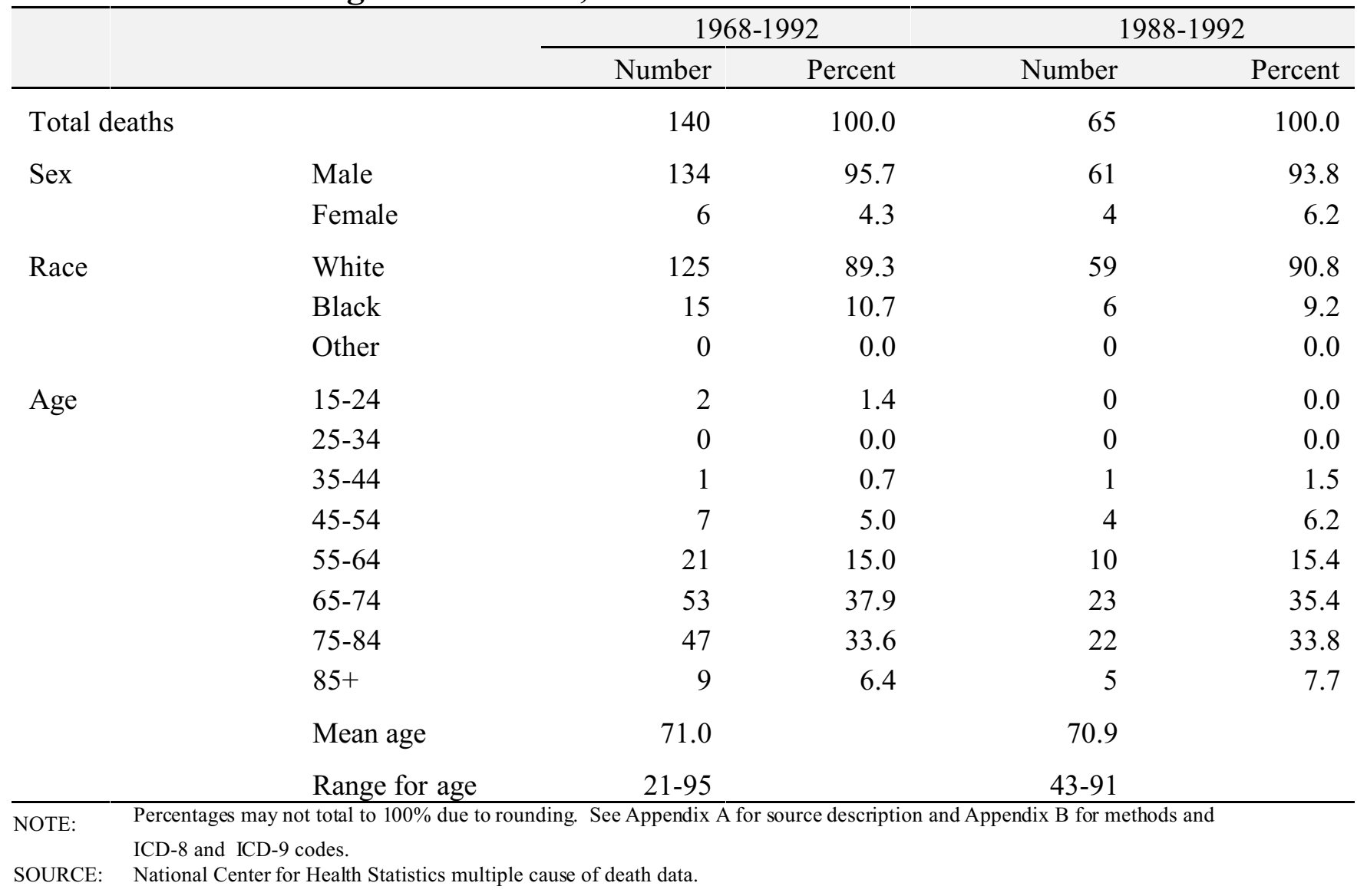




\section{Figure DE-3. Pneumoconiosis deaths by condition, Delaware residents age 15 and over, 1968-1992}

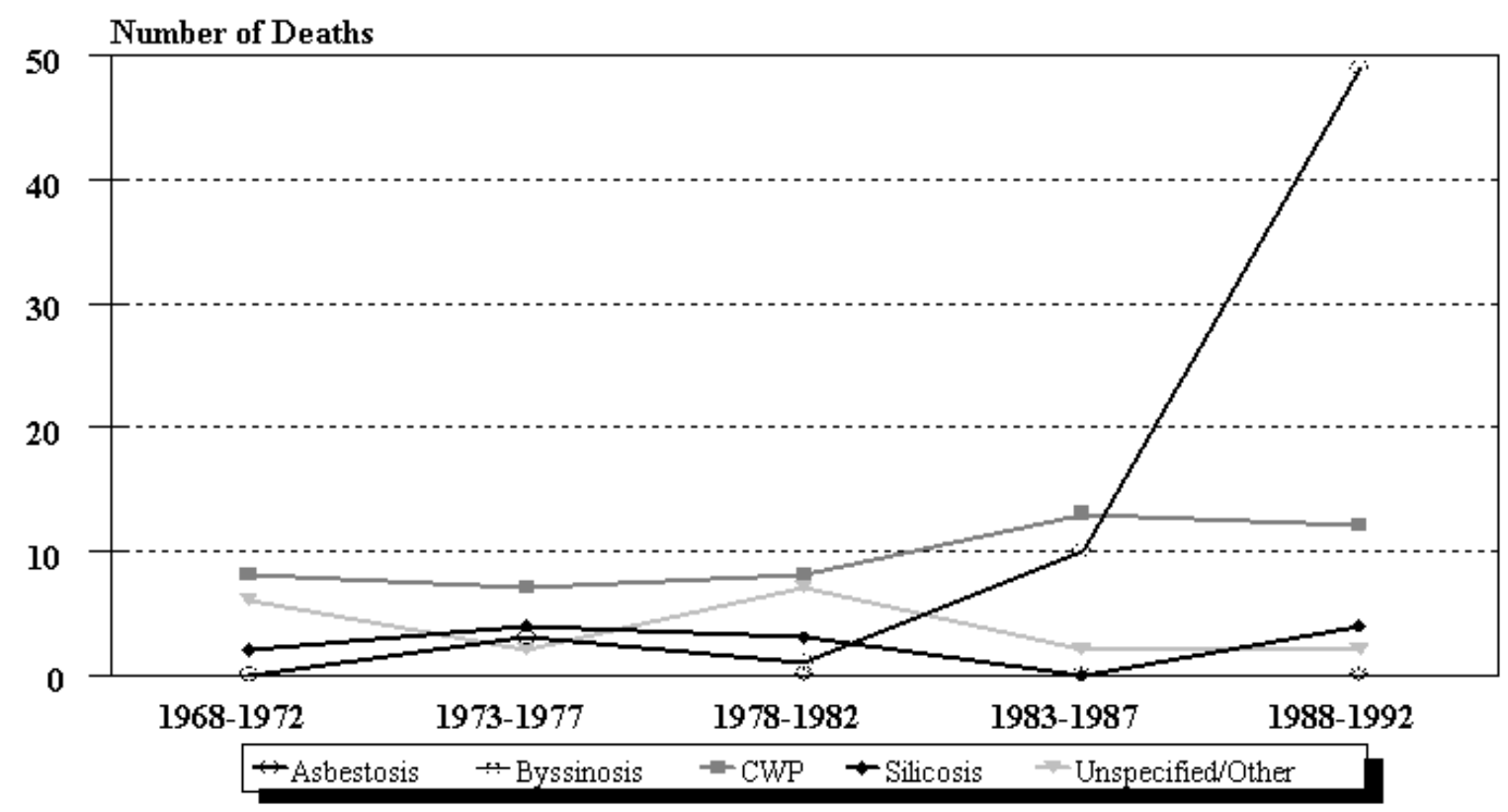

NOTE: See Appendix A for source description and Appendix B for methods and ICD-8 and ICD-9 codes. SOURCE: National Center for Health Statistics multiple cause of death data.

Figure DE-4. Delaware and U.S. age-adjusted mortality rates (per 1,000,000 population) by condition, residents age 15 and over, 1983-1992

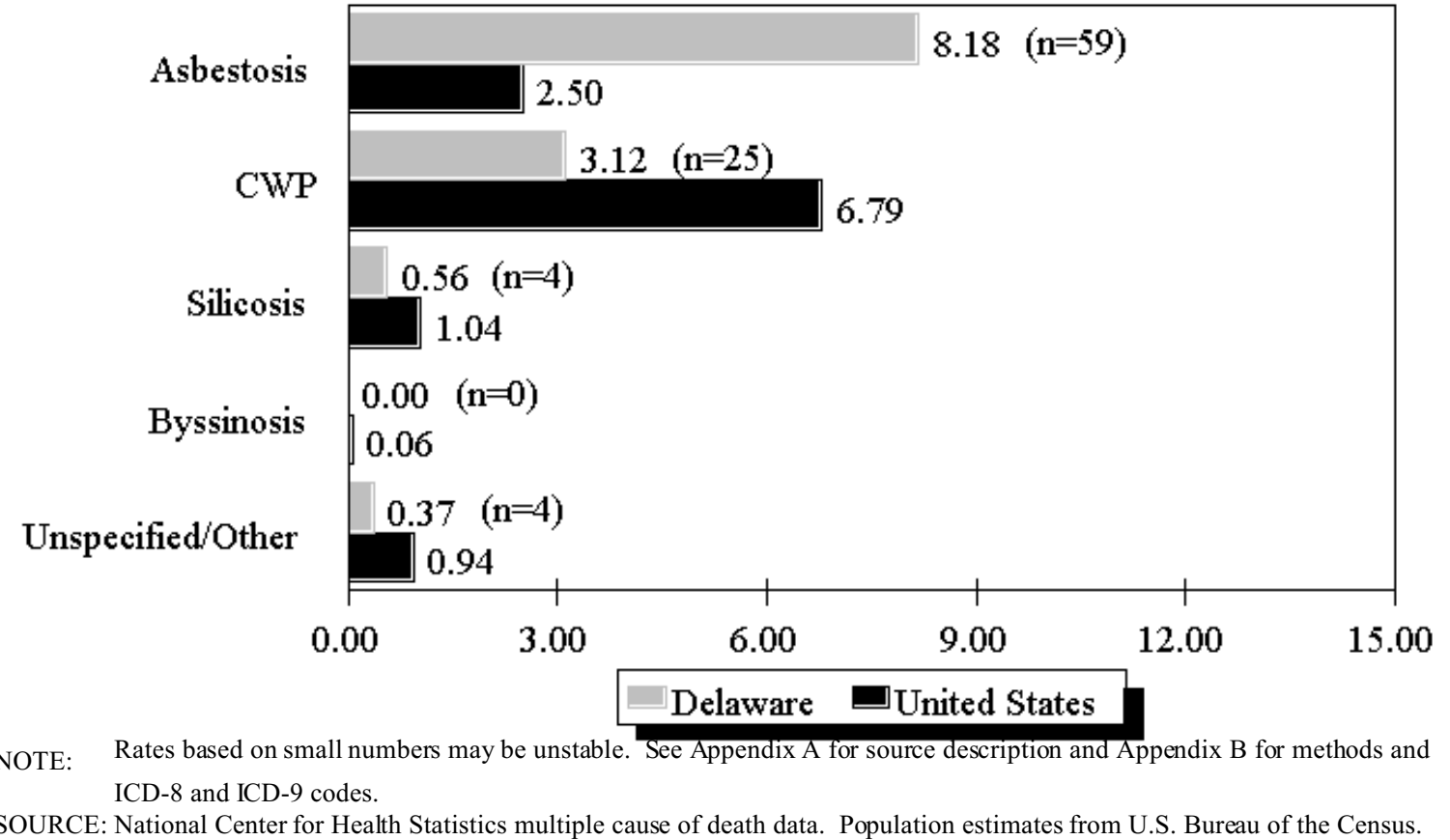


Table DE-3. Number of deaths and age-adjusted mortality rate (per 1,000,000 population) by condition and county, Delaware residents age 15 and over, 1983-1992

\begin{tabular}{|c|c|c|c|c|c|c|c|c|c|c|c|c|}
\hline \multirow[b]{2}{*}{ County } & \multicolumn{2}{|c|}{ Asbestosis } & \multicolumn{2}{|c|}{$\begin{array}{r}\text { Coal workers' } \\
\text { pneumoconiosis }\end{array}$} & \multicolumn{2}{|c|}{ Silicosis } & \multicolumn{2}{|c|}{ Byssinosis } & \multicolumn{2}{|c|}{$\begin{array}{r}\text { Unspecified/Other } \\
\text { pneumoconioses }\end{array}$} & \multicolumn{2}{|c|}{$\begin{array}{r}\text { All } \\
\text { pneumoconioses }\end{array}$} \\
\hline & \# & Rate & \# & Rate & $\#$ & Rate & $\#$ & Rate & \# & Rate & \# & Rate \\
\hline Kent & 6 & 5.71 & 8 & 6.68 & 1 & 0.61 & - & - & - & - & 14 & 12.39 \\
\hline New Castle & 37 & 8.49 & 14 & 2.63 & 2 & 0.30 & - & - & 2 & 0.30 & 54 & 11.56 \\
\hline Sussex & 16 & 9.83 & 3 & 2.29 & 1 & 1.36 & - & - & 2 & 0.84 & 22 & 14.32 \\
\hline
\end{tabular}

- indicates no deaths listed.

NOTE: Counties with no deaths for all listed conditions are not displayed. Rates based on small numbers may be unstable. Sum of individual conditions may exceed total because of deaths with multiple pneumoconioses. See Appendix A for source description and Appendix B for methods and ICD-8 and ICD-9 codes.

SOURCE: National Center for Health Statistics multiple cause of death data. Population estimates from U.S. Bureau of the Census. 


\section{District of Columbia: Selected statistics from the United States section}

All pneumoconioses mortality, 1988-1992

Total deaths

Crude mortality rate (per $1,000,000$ population)

Age-adjusted mortality rate (per 1,000,000 population)

Total years of potential life lost to life expectancy

Years of potential life lost to life expectancy (per death) $\underline{\text { Value }}$

6

2.37

1.84

126

21.0

Deaths by type of pneumoconiosis, 1988-1992

Asbestosis

Coal workers' pneumoconiosis

Silicosis

Byssinosis

Unspecified/Other pneumoconiosis
Deaths*

1

1

2

0

2

State rank
51
51
49
49
1

Percent** distribution

16.7

16.7

33.3

0.0

33.3

* Sum of column may exceed total deaths because of deaths with multiple pneumoconioses.

** Percentages may total more than $100 \%$ due to deaths with multiple pneumoconioses.

NOTE: $\quad$ See Appendix A for source description and Appendix B for methods and ICD-8 and ICD-9 codes.

SOURCE: National Center for Health Statistics multiple cause of death data. Population estimates from U.S. Bureau of the Census.

\section{Figure DC-1. All pneumoconioses: District of Columbia and U.S. age-adjusted mortality rates, residents age 15 and over, 1968-1992}

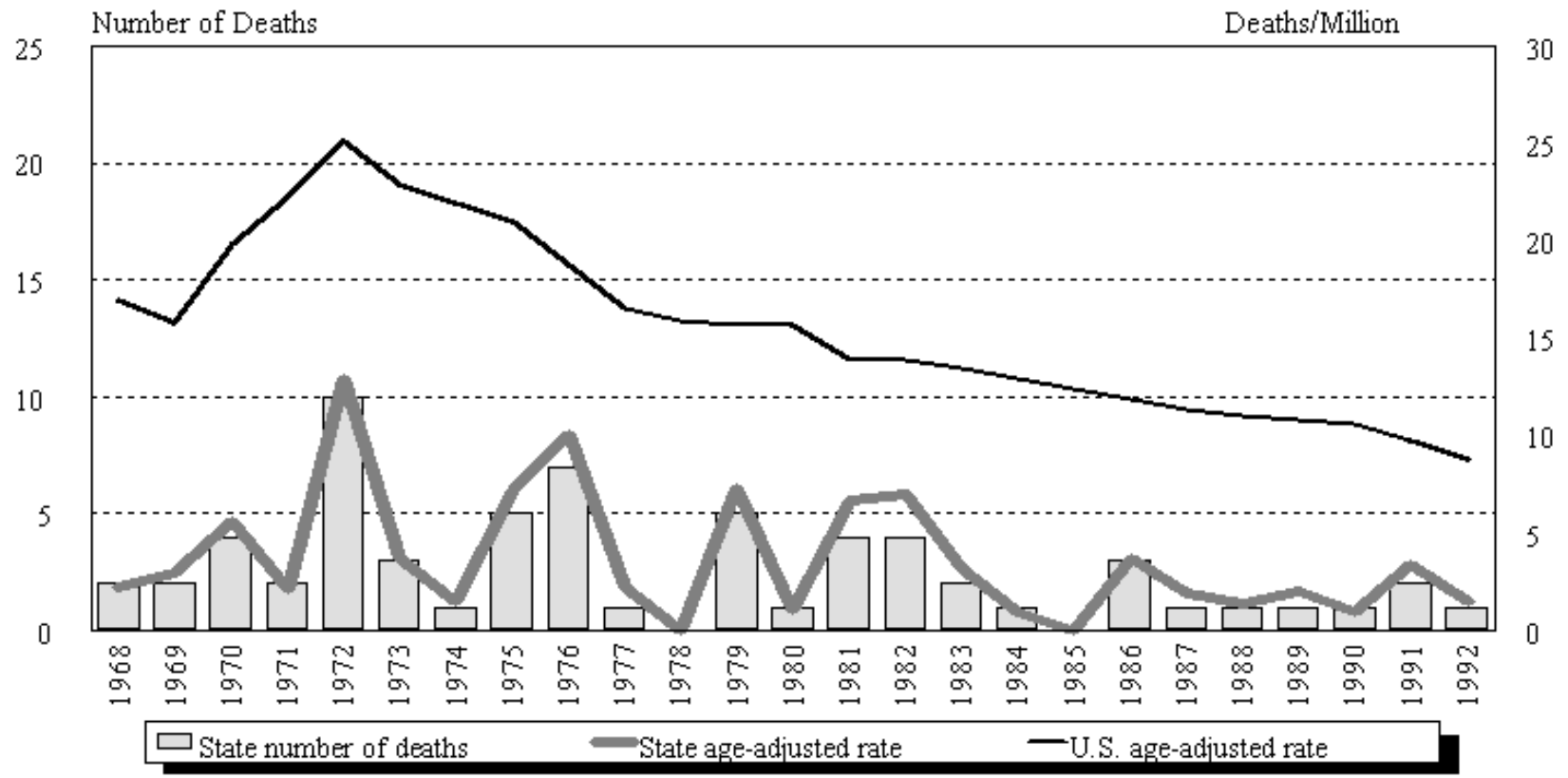

NOTE: $\quad$ See Appendix A for source description and Appendix B for methods and ICD-8 and ICD-9 codes.

SOURCE: National Center for Health Statistics multiple cause of death data. Population estimates from U.S. Bureau of the Census. 
District of Columbia

\begin{tabular}{|c|c|c|c|c|c|c|c|c|c|c|c|c|}
\hline \multirow[b]{2}{*}{ Year } & \multicolumn{2}{|c|}{ Asbestosis } & \multicolumn{2}{|c|}{$\begin{array}{l}\text { Coal workers' } \\
\text { pne umoconiosis }\end{array}$} & \multicolumn{2}{|c|}{ Silicosis } & \multicolumn{2}{|c|}{ Byssinosis* } & \multicolumn{2}{|c|}{$\begin{array}{c}\text { Unspecified/Other } \\
\text { pneumoconioses }\end{array}$} & \multicolumn{2}{|c|}{ All pneumoconioses } \\
\hline & Number & Rate & Number & Rate & Number & Rate & Number & Rate & Number & Rate & Number & Rate \\
\hline 1968 & - & - & 1 & 1.44 & 1 & 0.74 & & & 1 & 1.44 & 2 & 2.18 \\
\hline 1969 & 1 & 1.44 & - & - & 1 & 1.51 & & & - & - & 2 & 2.95 \\
\hline 1970 & - & - & 1 & 1.88 & - & - & & & 3 & 3.75 & 4 & 5.63 \\
\hline 1971 & - & - & 1 & 1.43 & - & - & & & 1 & 0.72 & 2 & 2.15 \\
\hline 1972 & - & - & 2 & 2.87 & 6 & 7.92 & & & 2 & 2.18 & 10 & 12.97 \\
\hline 1973 & 1 & 1.08 & - & & 1 & 1.08 & & & 1 & 1.54 & 3 & 3.69 \\
\hline 1974 & - & - & - & - & - & - & & & 1 & 1.43 & 1 & 1.43 \\
\hline 1975 & - & - & 1 & 1.42 & 1 & 1.58 & & & 3 & 4.22 & 5 & 7.23 \\
\hline 1976 & - & - & 2 & 2.84 & 3 & 4.62 & & & 2 & 2.66 & 7 & 10.11 \\
\hline 1977 & - & - & - & - & 1 & 2.22 & & & - & - & 1 & 2.22 \\
\hline 1978 & - & - & - & - & - & - & & & - & - & - & - \\
\hline 1979 & 1 & 1.39 & 2 & 2.11 & 2 & 3.77 & - & - & - & - & 5 & 7.27 \\
\hline 1980 & - & & - & - & 1 & 1.06 & - & - & - & - & 1 & 1.06 \\
\hline 1981 & 1 & 2.53 & 1 & 0.56 & 1 & 1.04 & - & - & 1 & 2.53 & 4 & 6.66 \\
\hline 1982 & - & & 1 & 1.41 & 3 & 5.54 & - & - & - & - & 4 & 6.95 \\
\hline 1983 & 1 & 1.76 & 1 & 1.41 & - & - & - & - & - & - & 2 & 3.17 \\
\hline 1984 & - & - & - & - & 1 & 0.99 & - & - & - & - & 1 & 0.99 \\
\hline 1985 & - & - & - & - & - & - & - & - & - & - & - & - \\
\hline 1986 & 2 & 1.88 & - & - & 1 & 1.83 & - & - & - & - & 3 & 3.71 \\
\hline 1987 & 1 & 1.87 & - & - & - & - & - & - & - & - & 1 & 1.87 \\
\hline 1988 & - & - & 1 & 1.41 & - & - & - & - & - & - & 1 & 1.41 \\
\hline 1989 & - & - & - & - & - & - & - & - & 1 & 1.97 & 1 & 1.97 \\
\hline 1990 & - & - & - & - & 1 & 0.91 & - & - & - & - & 1 & 0.91 \\
\hline 1991 & 1 & 1.47 & - & - & - & - & - & - & 1 & 1.91 & 2 & 3.38 \\
\hline 1992 & - & & - & & 1 & 1.47 & - & - & - & - & 1 & 1.47 \\
\hline
\end{tabular}

indicates no deaths listed. $\quad *$ no specific code for byssinosis in ICD-8 (1968-1978).

NOTE: Rates based on small numbers may be unstable. Sum of individual conditions may exceed total because of deaths with multiple pneumoconioses. See Appendix A for source description and Appendix B for methods and ICD-8 and ICD-9 codes.

SOURCE: National Center for Health Statistics multiple cause of death data. Population estimates from U.S. Bureau of the Census. 
Figure DC-2. All pneumoconioses: Distribution of deaths by sex and race, District of Columbia residents age 15 and over, 1968-1992

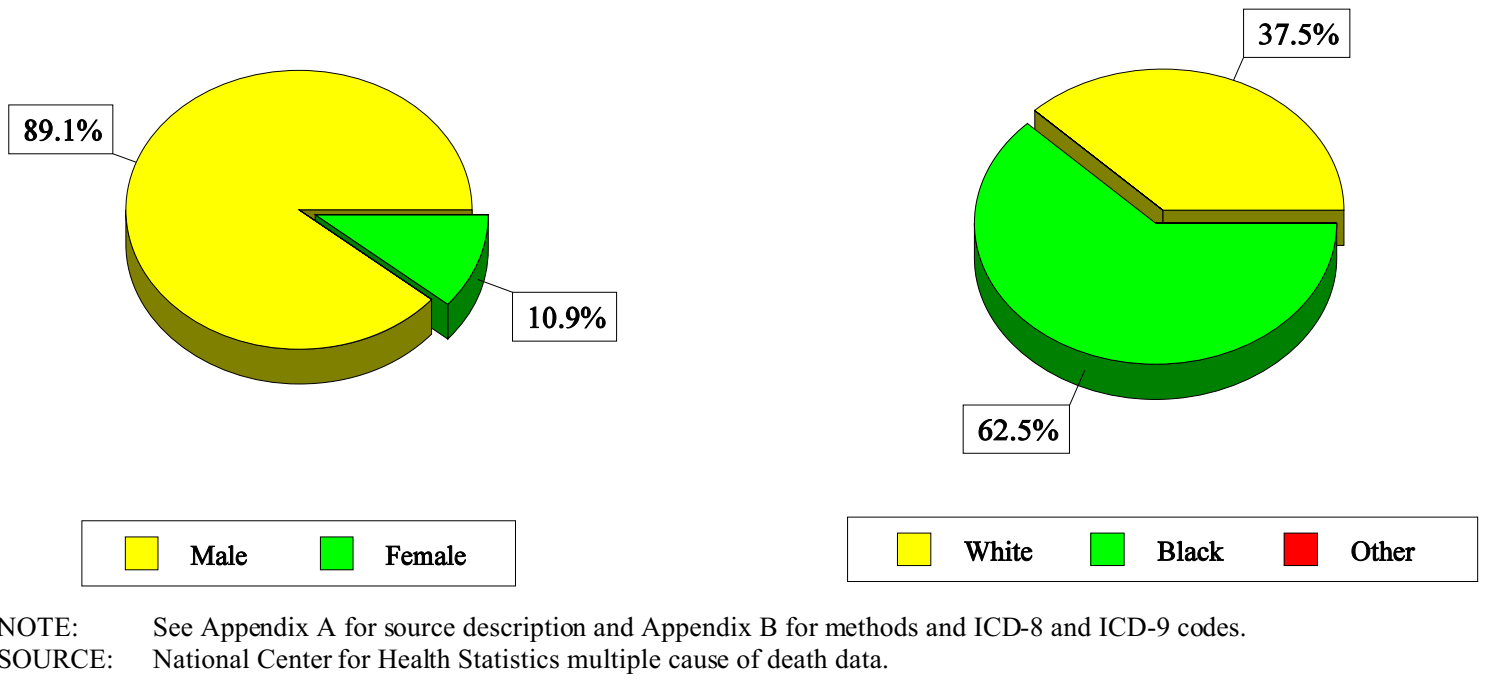

Table DC-2. All pneumoconioses: Distribution of deaths by sex, race, and age, District of Columbia residents age 15 and over, 1968-1992

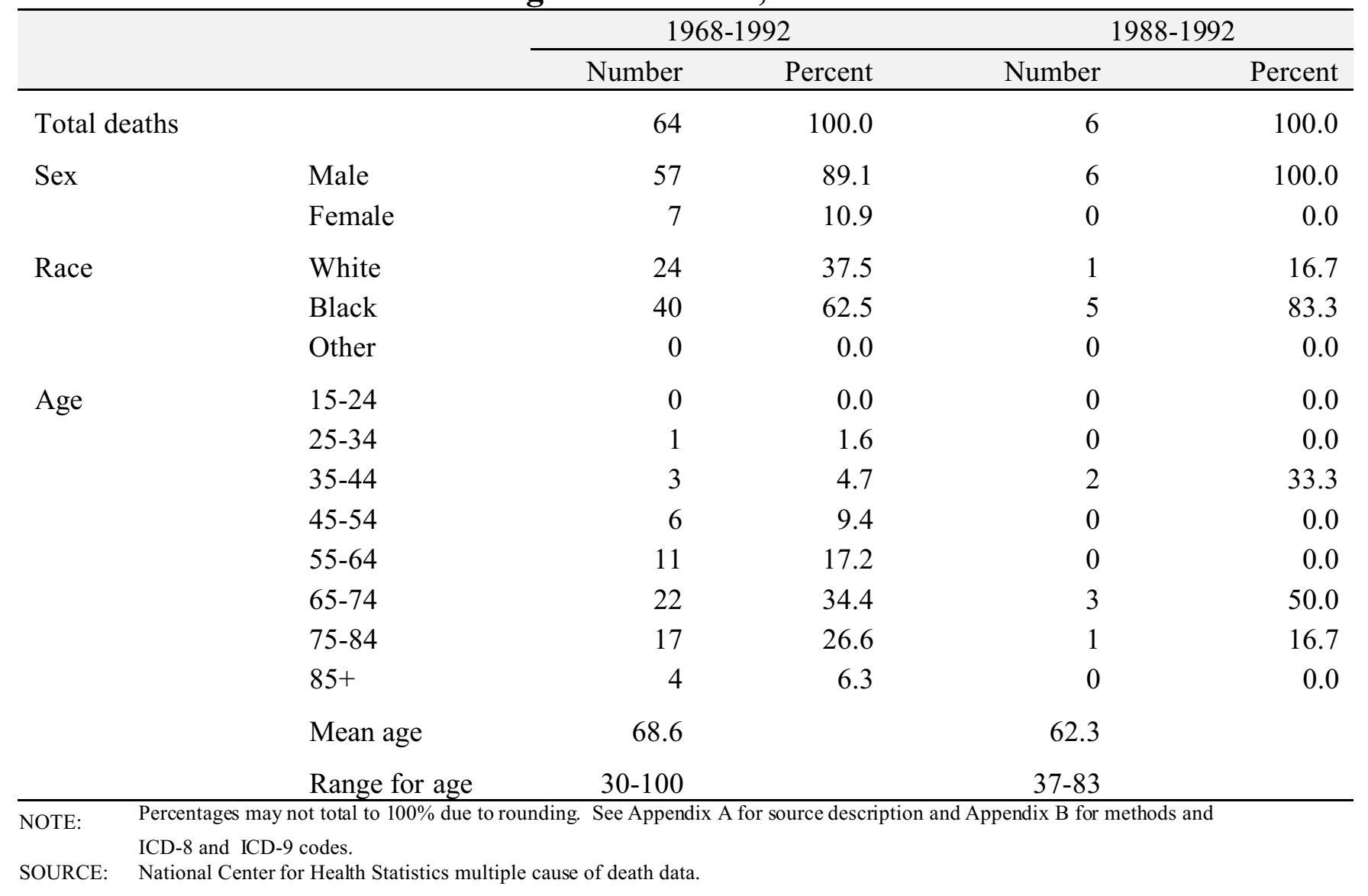


Figure DC-3. Pneumoconiosis deaths by condition, District of Columbia residents age 15 and over, 1968-1992

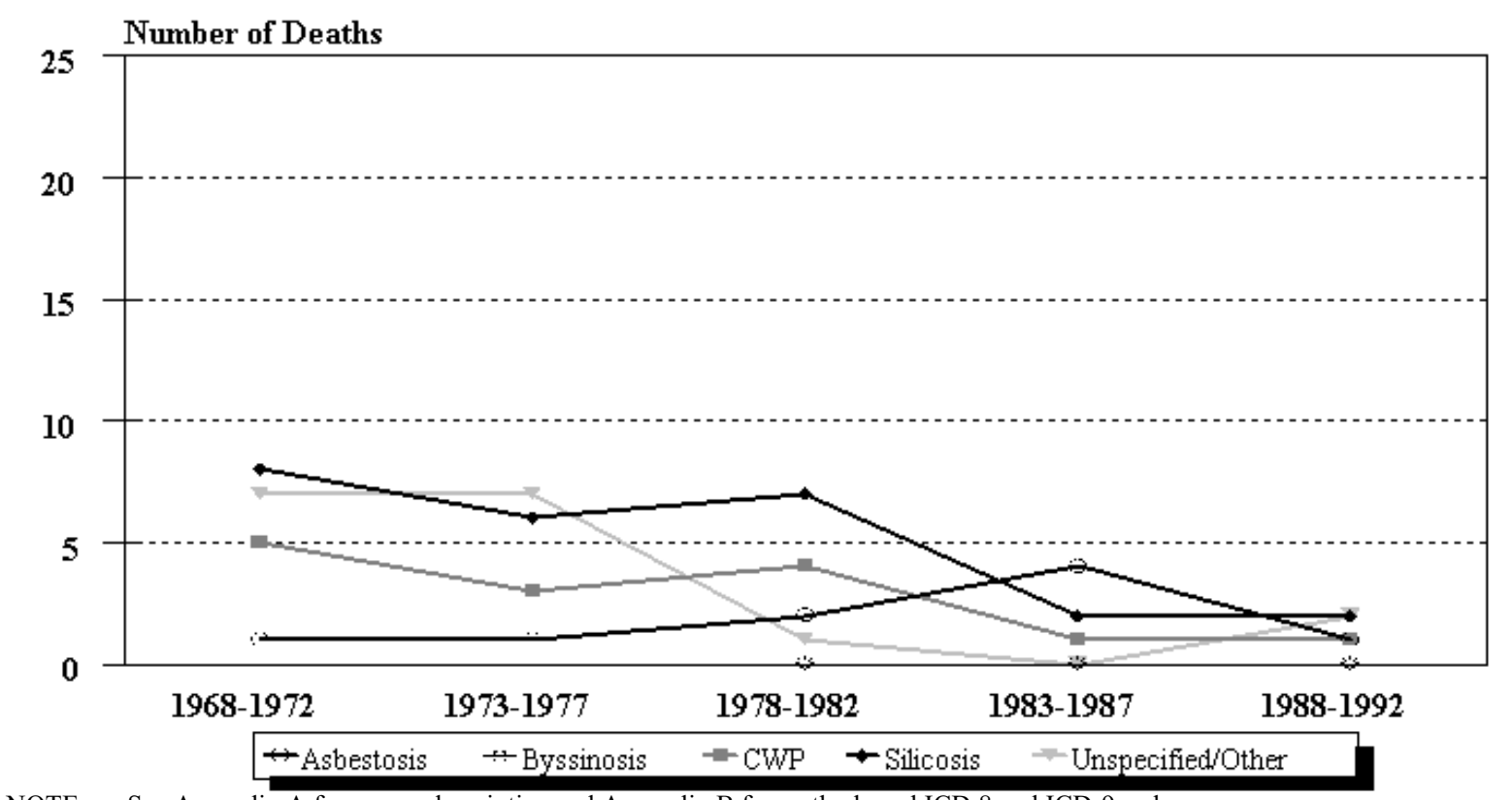

NOTE: See Appendix A for source description and Appendix B for methods and ICD-8 and ICD-9 codes.

SOURCE: National Center for Health Statistics multiple cause of death data.

Figure DC-4. District of Columbia and U.S. age-adjusted mortality rates (per 1,000,000 population) by condition, residents age 15 and over, 1983-1992

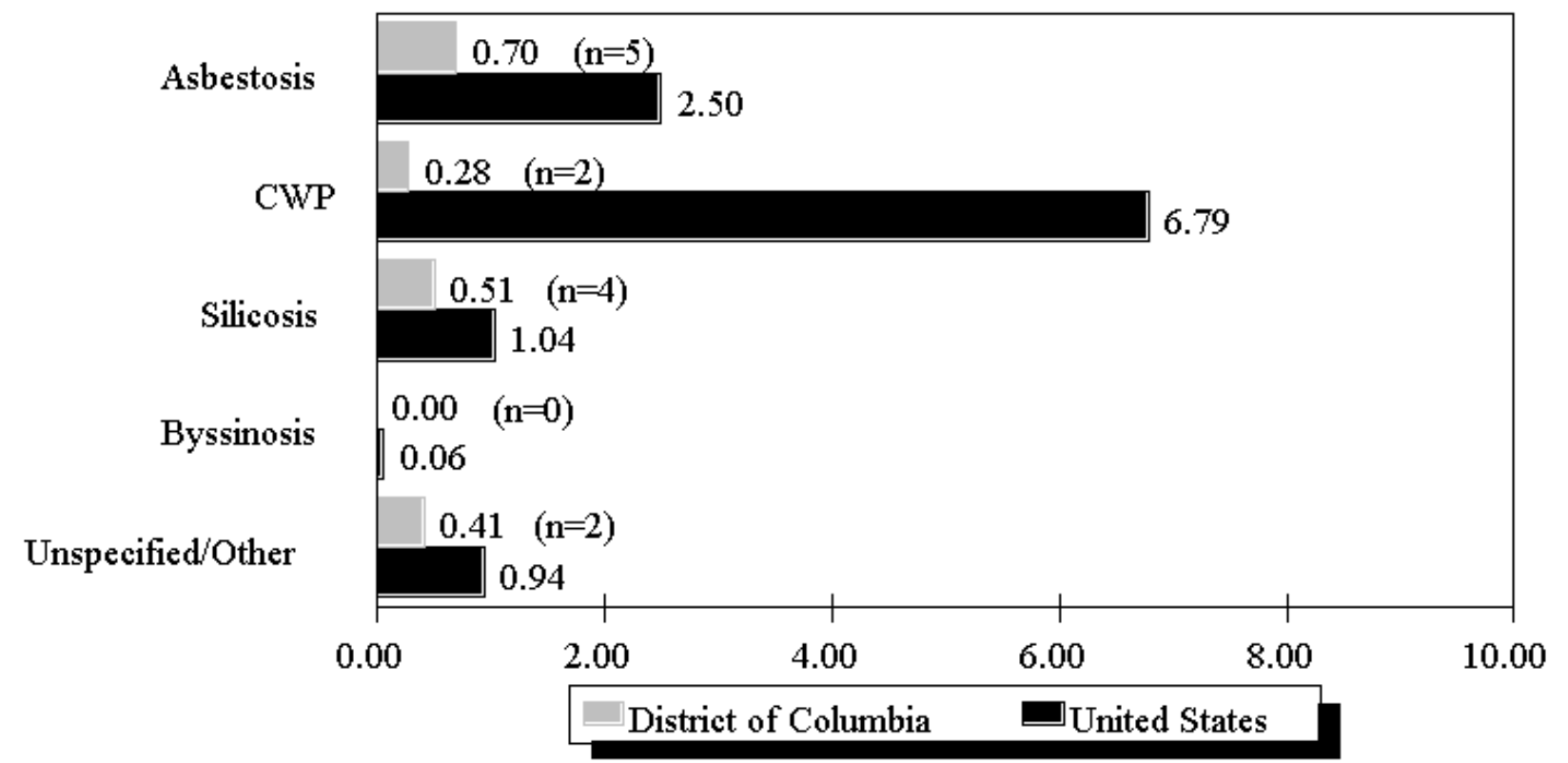

NOTE: Rates based on small numbers may be unstable. See Appendix A for source description and Appendix B for methods and ICD-8 and ICD-9 codes.

SOURCE: National Center for Health Statistics multiple cause of death data. Population estimates from U.S. Bureau of the Census. 
Table DC-3. Number of deaths and age-adjusted mortality rate (per 1,000,000 population) by condition, Washington, D.C. residents age 15 and over, 1983-1992

\begin{tabular}{l} 
Asbestosis \\
\cline { 2 - 8 }
\end{tabular}


Florida

\section{Florida: Selected statistics from the United States section}

All pneumoconioses mortality, 1988-1992

Total deaths

Crude mortality rate (per $1,000,000$ population)

Age-adjusted mortality rate (per $1,000,000$ population)

Total years of potential life lost to life expectancy

Years of potential life lost to life expectancy (per death)

Deaths by type of pneumoconiosis, 1988-1992

Asbestosis

Coal workers' pneumoconiosis

Silicosis

Byssinosis

Unspecified/Other pneumoconiosis

\begin{tabular}{rr} 
Value & State rank \\
\hline 510 & 8 \\
9.66 & 22 \\
4.41 & 31 \\
6,229 & 8 \\
12.2 & 24
\end{tabular}

Percent**

$\underline{\text { Deaths* }}$

264

distribution

151

51.8

29.6

50

9.8

0.4

2

9.0

* Sum of column may exceed total deaths because of deaths with multiple pneumoconioses.

** Percentages may total more than $100 \%$ due to deaths with multiple pneumoconioses.

NOTE: $\quad$ See Appendix A for source description and Appendix B for methods and ICD-8 and ICD-9 codes.

SOURCE: National Center for Health Statistics multiple cause of death data. Population estimates from U.S. Bureau of the Census.

\section{Figure FL-1. All pneumoconioses: Florida and U.S. age-adjusted mortality rates, residents age 15 and over, 1968-1992}

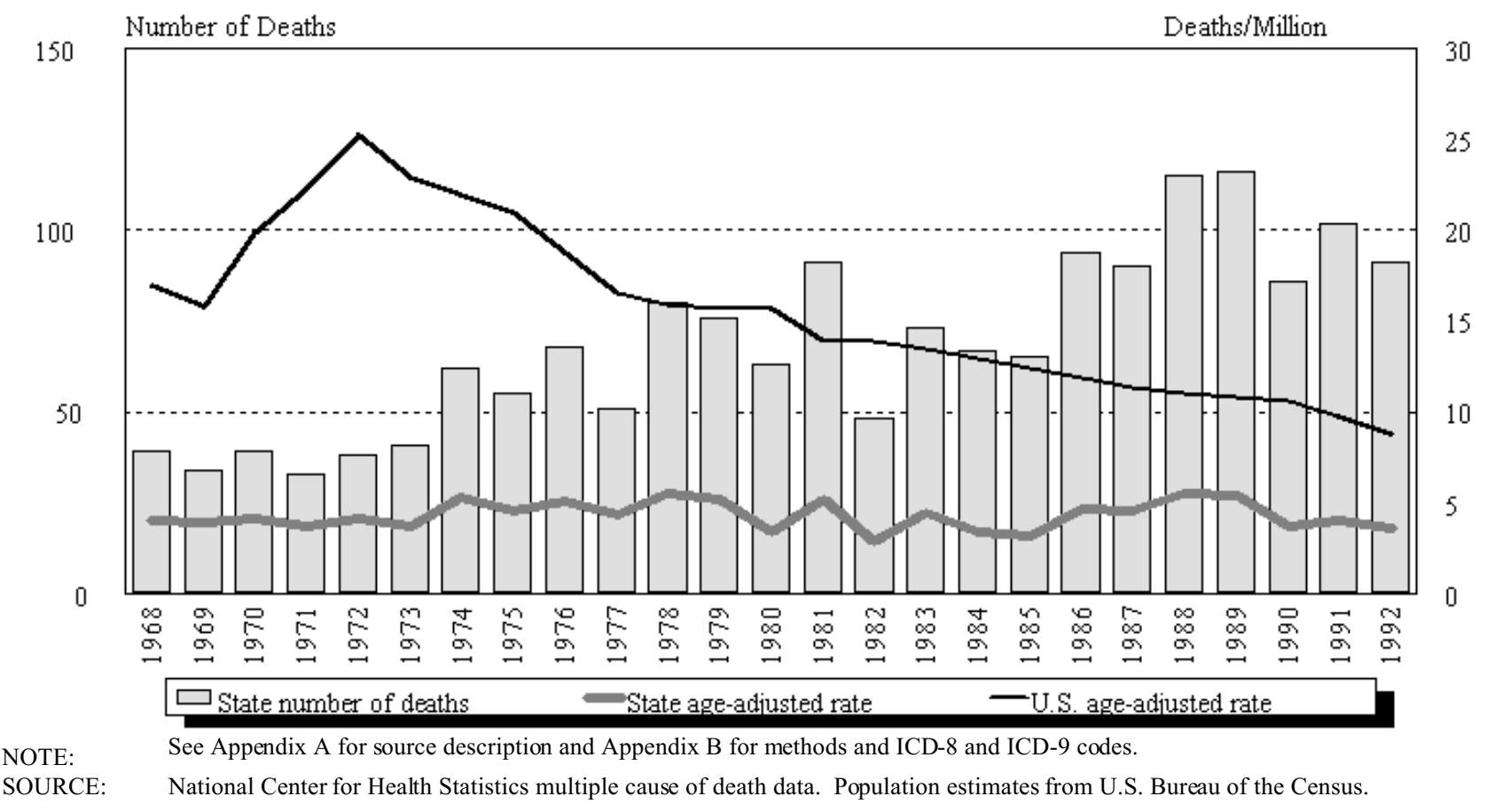




\section{Florida}

\begin{tabular}{|c|c|c|c|c|c|c|c|c|c|c|c|c|}
\hline \multirow[b]{2}{*}{ Year } & \multicolumn{2}{|c|}{ Asbestosis } & \multicolumn{2}{|c|}{$\begin{array}{l}\text { Coal workers' } \\
\text { pneumoconiosis }\end{array}$} & \multicolumn{2}{|c|}{ Silicosis } & \multicolumn{2}{|c|}{ Byssinosis* } & \multicolumn{2}{|c|}{$\begin{array}{c}\text { Unspecified/Other } \\
\text { pneumoconioses }\end{array}$} & \multicolumn{2}{|c|}{ All pneumoconioses } \\
\hline & Number & Rate & Number & Rate & Number & Rate & Number & Rate & Number & Rate & Number & Rate \\
\hline 1968 & 2 & 0.18 & 20 & 2.28 & 13 & 1.25 & & & 7 & 0.64 & 39 & 4.04 \\
\hline 1969 & 2 & 0.30 & 12 & 1.62 & 14 & 1.31 & & & 6 & 0.66 & 34 & 3.89 \\
\hline 1970 & 2 & 0.25 & 10 & 1.11 & 18 & 1.90 & & & 10 & 1.11 & 39 & 4.17 \\
\hline 1971 & - & - & 9 & 1.08 & 13 & 1.38 & & & 11 & 1.26 & 33 & 3.73 \\
\hline 1972 & 2 & 0.27 & 16 & 1.55 & 8 & 1.02 & & & 12 & 1.27 & 38 & 4.11 \\
\hline 1973 & 3 & 0.23 & 9 & 0.75 & 20 & 1.76 & & & 9 & 0.96 & 41 & 3.70 \\
\hline 1974 & 3 & 0.33 & 23 & 1.95 & 16 & 1.30 & & & 22 & 1.83 & 62 & 5.30 \\
\hline 1975 & 2 & 0.17 & 22 & 1.82 & 16 & 1.33 & & & 17 & 1.38 & 55 & 4.53 \\
\hline 1976 & 4 & 0.28 & 24 & 1.78 & 15 & 1.02 & & & 26 & 2.07 & 68 & 5.09 \\
\hline 1977 & 2 & 0.10 & 17 & 1.50 & 16 & 1.33 & & & 18 & 1.47 & 51 & 4.30 \\
\hline 1978 & 15 & 1.23 & 36 & 2.45 & 13 & 0.92 & & & 19 & 1.20 & 80 & 5.52 \\
\hline 1979 & 17 & 1.30 & 35 & 2.27 & 11 & 0.75 & - & - & 13 & 0.90 & 76 & 5.22 \\
\hline 1980 & 10 & 0.61 & 32 & 1.65 & 13 & 0.62 & - & - & 10 & 0.55 & 63 & 3.36 \\
\hline 1981 & 9 & 0.41 & 52 & 3.16 & 17 & 0.91 & - & - & 13 & 0.76 & 91 & 5.25 \\
\hline 1982 & 12 & 0.85 & 26 & 1.42 & 6 & 0.34 & - & - & 4 & 0.23 & 48 & 2.85 \\
\hline 1983 & 20 & 1.39 & 35 & 1.85 & 11 & 0.68 & - & - & 7 & 0.52 & 73 & 4.44 \\
\hline 1984 & 19 & 0.95 & 32 & 1.65 & 10 & 0.48 & - & - & 6 & 0.28 & 67 & 3.36 \\
\hline 1985 & 27 & 1.54 & 20 & 0.95 & 10 & 0.38 & - & - & 9 & 0.35 & 65 & 3.17 \\
\hline 1986 & 35 & 2.03 & 38 & 1.59 & 14 & 0.78 & - & - & 7 & 0.24 & 94 & 4.64 \\
\hline 1987 & 41 & 2.17 & 34 & 1.78 & 11 & 0.49 & - & - & 5 & 0.16 & 90 & 4.52 \\
\hline 1988 & 59 & 3.07 & 35 & 1.49 & 13 & 0.64 & - & - & 9 & 0.32 & 115 & 5.47 \\
\hline 1989 & 56 & 2.55 & 35 & 1.37 & 14 & 1.06 & - & - & 11 & 0.48 & 116 & 5.46 \\
\hline 1990 & 43 & 1.80 & 25 & 1.02 & 12 & 0.64 & 1 & 0.05 & 5 & 0.18 & 86 & 3.69 \\
\hline 1991 & 54 & 2.21 & 33 & 1.27 & 7 & 0.22 & - & - & 10 & 0.39 & 102 & 4.01 \\
\hline 1992 & 52 & 2.20 & 23 & 0.76 & 4 & 0.13 & 1 & 0.14 & 11 & 0.36 & 91 & 3.59 \\
\hline
\end{tabular}

indicates no deaths listed. $\quad *$ no specific code for byssinosis in ICD-8 (1968-1978).

NOTE: Rates based on small numbers may be unstable. Sum of individ ual conditions may exceed total because of deaths with multiple pneumo conioses. See Appendix A for source description and Appendix B for methods and ICD-8 and ICD-9 codes.

SOURCE: National Center for Health Statistics multiple cause of death data. Population estimates from U.S. Bureau of the Census. 
Figure FL-2. All pneumoconioses: Distribution of deaths by sex and race, Florida residents age 15 and over, 1968-1992
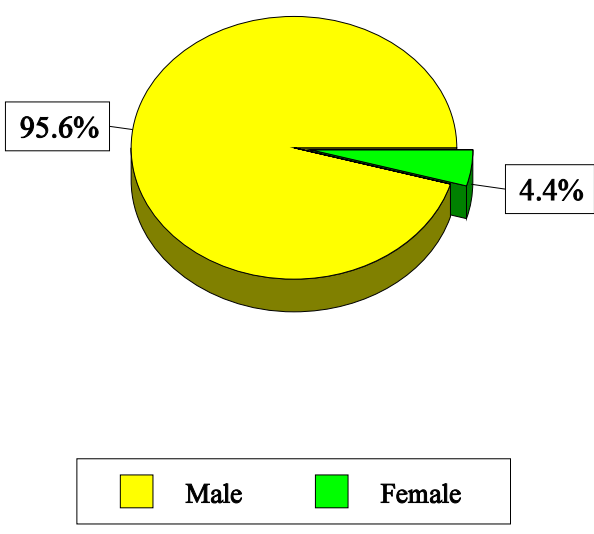

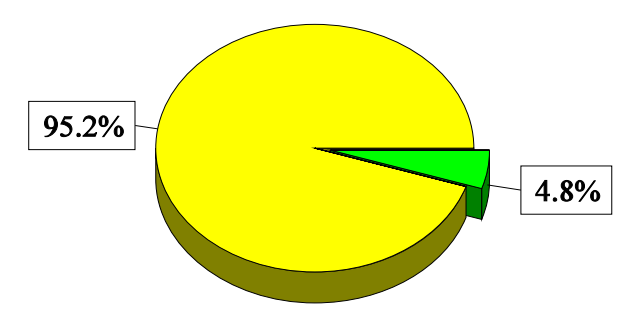

NOTE: $\quad$ See Appendix A for source description and Appendix B for methods and ICD-8 and ICD-9 codes.

SOURCE: National Center for Health Statistics multiple cause of death data.

Table FL-2. All pneumoconioses: Distribution of deaths by sex, race, and age, Florida residents age 15 and over, 1968-1992

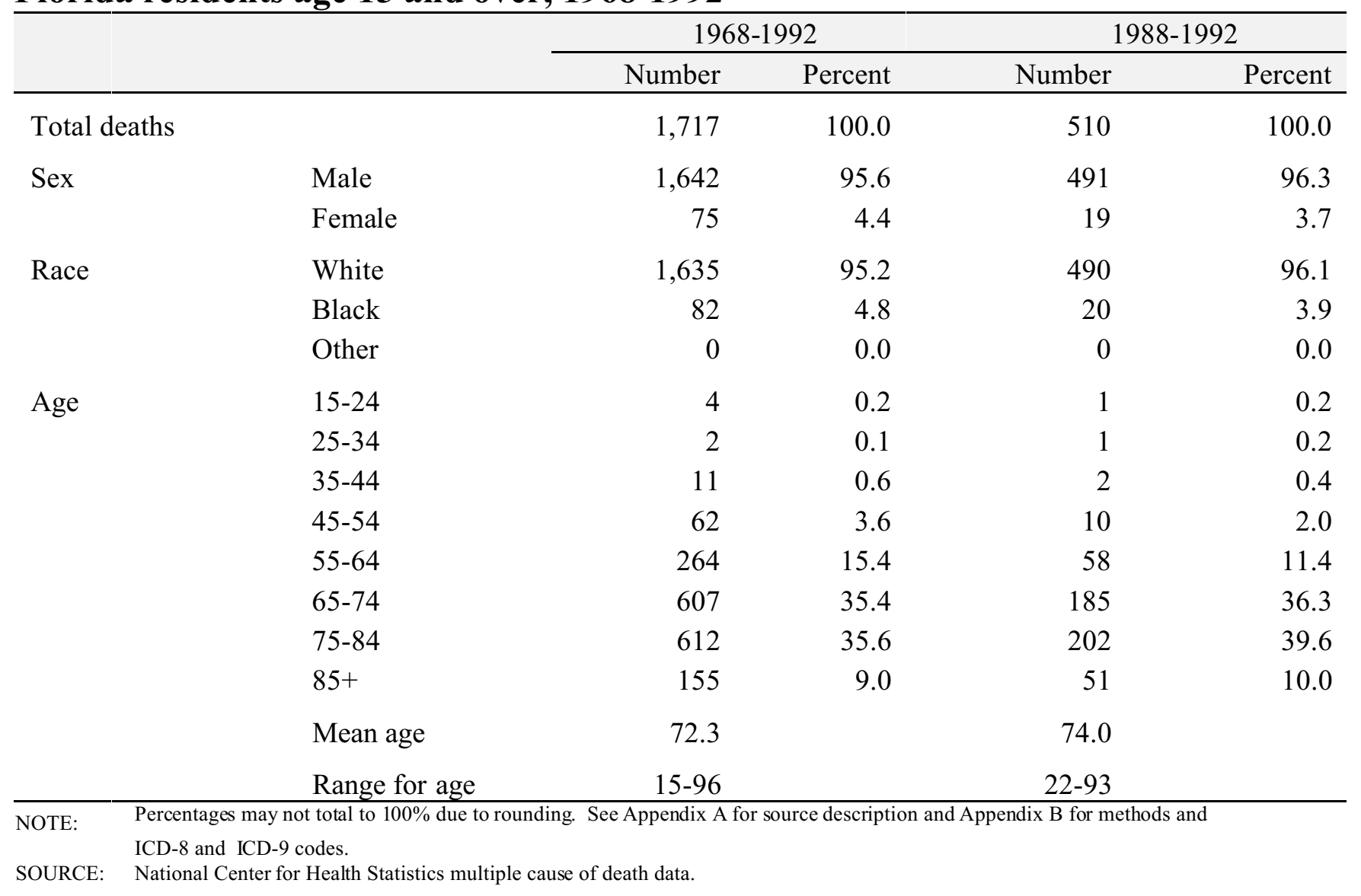


Figure FL-3. Pneumoconiosis deaths by condition, Florida residents age 15 and over, 1968-1992

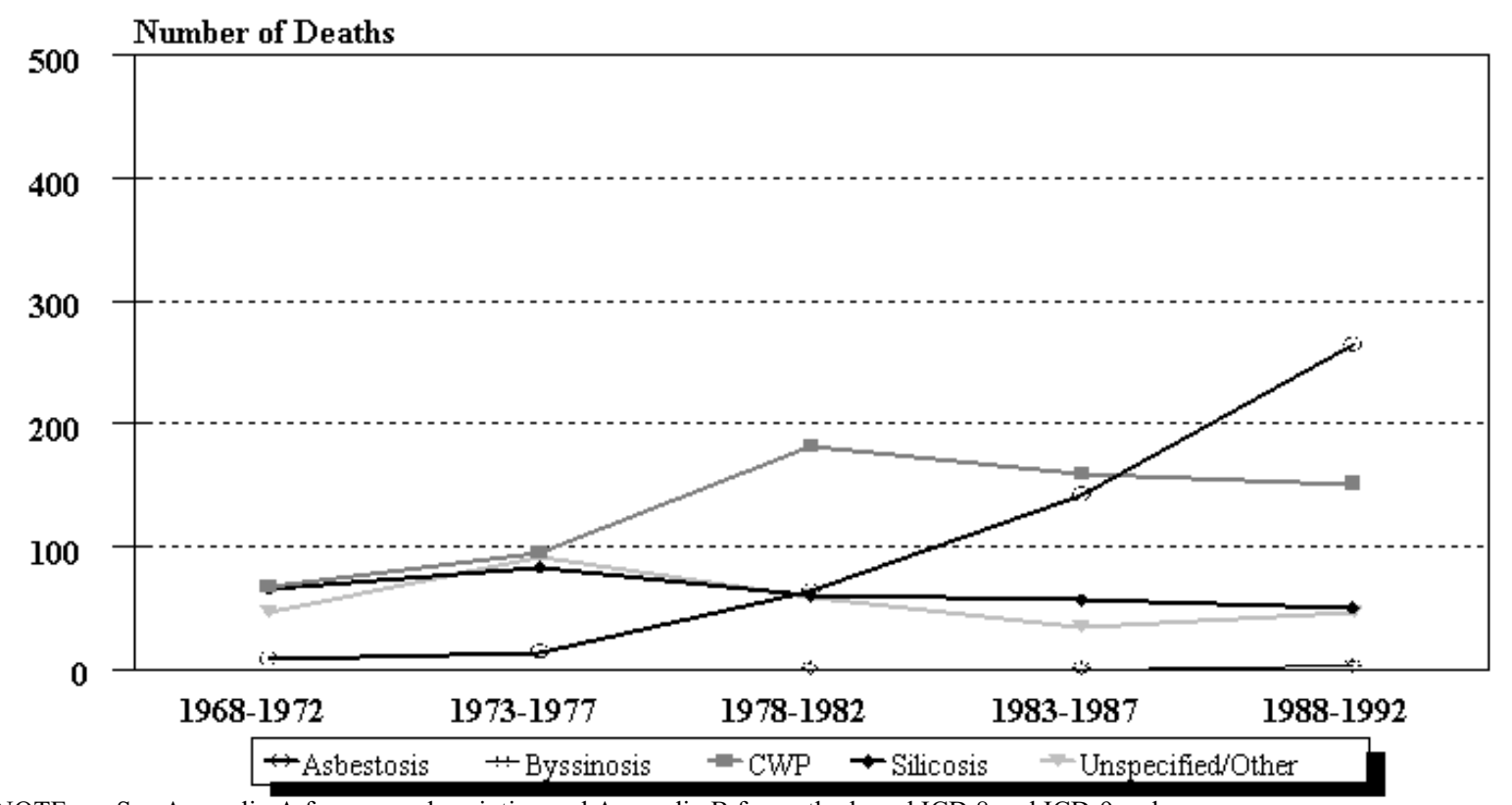

NOTE: See Appendix A for source description and Appendix B for methods and ICD-8 and ICD-9 codes.

SOURCE: National Center for Health Statistics multiple cause of death data.

Figure FL-4. Florida and U.S. age-adjusted mortality rates (per 1,000,000 population) by condition, residents age 15 and over, 1983-1992

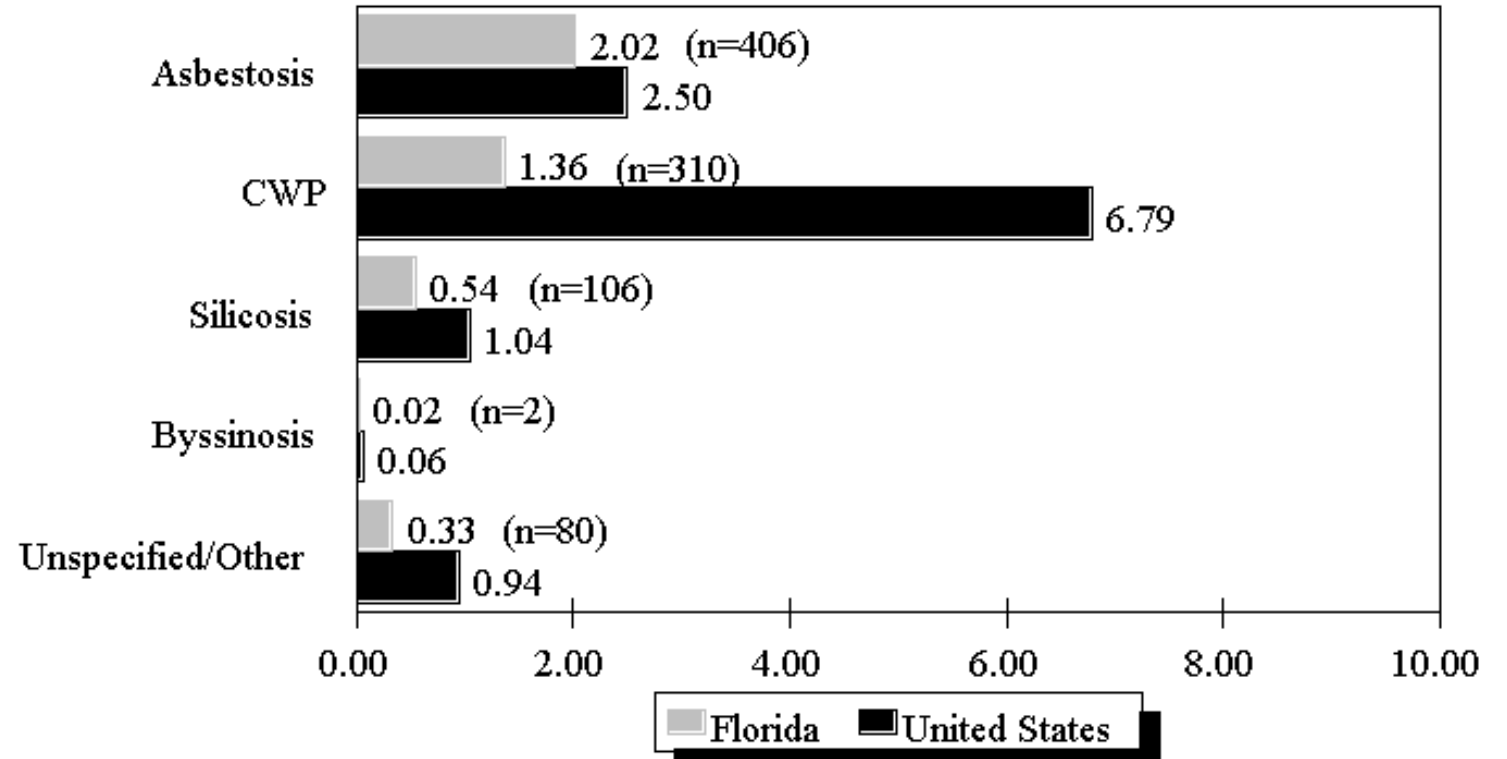

NOTE: Rates based on small numbers may be unstable. See Appendix A for source description and Appendix B for methods and ICD-8 and ICD-9 codes.

SOURCE: National Center for Health Statistics multiple cause of death data. Population estimates from U.S. Bureau of the Census. 
Table FL-3 (page 1 of 2). Number of deaths and age-adjusted mortality rate (per $1,000,000$ population) by condition and county, Florida residents age 15 and over, 1983-1992

\begin{tabular}{|c|c|c|c|c|c|c|c|c|c|c|c|c|}
\hline \multirow[b]{2}{*}{ County } & \multicolumn{2}{|c|}{ Asbestosis } & \multicolumn{2}{|c|}{$\begin{array}{c}\text { Coal workers' } \\
\text { pneumoconiosis }\end{array}$} & \multicolumn{2}{|c|}{ Silicosis } & \multicolumn{2}{|c|}{ Byssinosis } & \multicolumn{2}{|c|}{$\begin{array}{r}\text { Unspecified/Other } \\
\text { pneumoconioses }\end{array}$} & \multicolumn{2}{|c|}{$\begin{array}{r}\text { All } \\
\text { pneumoconioses }\end{array}$} \\
\hline & \# & Rate & \# & Rate & \# & Rate & \# & Rate & \# & Rate & \# & Rate \\
\hline Alachua & 3 & 2.11 & 5 & 2.57 & 1 & 0.84 & - & - & 1 & 0.43 & 9 & 5.12 \\
\hline Bay & 7 & 4.92 & 4 & 3.01 & - & - & - & - & - & - & 11 & 7.93 \\
\hline Bradford & 2 & 7.30 & 2 & 5.27 & - & - & - & - & - & - & 4 & 12.57 \\
\hline Brevard & 8 & 1.87 & 20 & 3.36 & 6 & 1.03 & - & - & 2 & 0.26 & 35 & 6.32 \\
\hline Broward & 41 & 1.78 & 16 & 0.56 & 5 & 0.30 & - & - & 5 & 0.22 & 67 & 2.86 \\
\hline Calhoun & 1 & 4.00 & - & - & 1 & 14.29 & - & - & - & - & 2 & 18.28 \\
\hline Charlotte & 2 & 0.70 & 2 & 0.70 & - & - & - & - & - & - & 4 & 1.40 \\
\hline Citrus & 10 & 4.32 & 8 & 3.74 & 2 & 0.86 & - & - & 2 & 0.86 & 22 & 9.79 \\
\hline Clay & 1 & 1.41 & 2 & 1.72 & 1 & 1.41 & - & - & 1 & 1.41 & 5 & 5.96 \\
\hline Collier & 1 & 0.25 & 5 & 1.71 & 1 & 0.48 & - & - & 1 & 0.48 & 8 & 2.91 \\
\hline Columbia & - & - & 2 & 2.88 & - & - & - & - & 2 & 4.68 & 4 & 7.56 \\
\hline Dade & 28 & 0.98 & 5 & 0.11 & 3 & 0.10 & - & - & 1 & 0.05 & 37 & 1.25 \\
\hline Duval & 34 & 5.48 & 9 & 1.15 & 8 & 1.37 & - & - & 3 & 0.31 & 53 & 8.14 \\
\hline Escambia & 19 & 7.09 & 3 & 0.74 & 1 & 0.25 & - & - & - & - & 23 & 8.08 \\
\hline Flagler & - & - & 1 & 1.86 & 1 & 2.10 & - & - & - & - & 2 & 3.96 \\
\hline Gadsden & 2 & 5.25 & 1 & 1.24 & - & - & - & - & - & - & 3 & 6.48 \\
\hline Gilchrist & 1 & 10.25 & - & - & - & - & - & - & - & - & 1 & 10.25 \\
\hline Gulf & 2 & 14.80 & - & - & - & - & - & - & - & - & 2 & 14.80 \\
\hline Hamilton & - & - & - & - & 1 & 11.27 & - & - & - & - & 1 & 11.27 \\
\hline Hardee & - & - & 1 & 2.53 & - & - & - & - & - & - & 1 & 2.53 \\
\hline Hendry & - & - & 1 & 2.85 & - & - & - & - & - & - & 1 & 2.85 \\
\hline Hernando & 6 & 2.87 & 5 & 3.81 & 4 & 1.98 & - & - & 1 & 0.33 & 16 & 9.00 \\
\hline Highlands & 4 & 1.39 & 2 & 0.69 & - & - & - & - & - & - & 6 & 2.08 \\
\hline Hillsborough & 14 & 1.56 & 15 & 1.44 & 9 & 1.15 & - & - & 2 & 0.14 & 40 & 4.29 \\
\hline Holmes & 1 & 2.74 & - & - & - & - & - & - & - & - & 1 & 2.74 \\
\hline Indian River & 4 & 2.07 & 1 & 0.70 & 2 & 0.67 & - & - & 1 & 0.70 & 8 & 4.14 \\
\hline Jackson & 1 & 2.33 & 1 & 2.33 & 1 & 3.61 & - & - & - & - & 3 & 8.26 \\
\hline Lake & 3 & 1.01 & 10 & 2.97 & 1 & 0.42 & - & - & 3 & 0.77 & 17 & 5.16 \\
\hline Lee & 14 & 2.20 & 4 & 0.70 & 4 & 0.39 & - & - & 1 & 0.10 & 23 & 3.40 \\
\hline Leon & 4 & 3.03 & - & - & 1 & 0.85 & 1 & 0.58 & - & - & 6 & 4.45 \\
\hline Levy & 1 & 2.90 & 2 & 5.79 & 1 & 1.73 & - & - & 1 & 1.73 & 5 & 12.16 \\
\hline Manatee & 7 & 2.60 & 5 & 0.77 & 1 & 0.11 & - & - & 3 & 0.55 & 16 & 4.03 \\
\hline Marion & 3 & 0.94 & 20 & 5.40 & 5 & 1.35 & - & - & 2 & 0.57 & 30 & 8.25 \\
\hline
\end{tabular}

See footnotes at end of table. 
Table FL-3 (page 2 of 2). Number of deaths and age-adjusted mortality rate (per $1,000,000$ population) by condition and county, Florida residents age 15 and over, 1983-1992

\begin{tabular}{|c|c|c|c|c|c|c|c|c|c|c|c|c|}
\hline \multirow[b]{2}{*}{ County } & \multicolumn{2}{|c|}{ Asbestosis } & \multicolumn{2}{|c|}{$\begin{array}{c}\text { Coal workers' } \\
\text { pneumoconiosis }\end{array}$} & \multicolumn{2}{|c|}{ Silicosis } & \multicolumn{2}{|c|}{ Byssinosis } & \multicolumn{2}{|c|}{$\begin{array}{r}\text { Unspecified/Other } \\
\text { pneumoconioses }\end{array}$} & \multicolumn{2}{|c|}{$\begin{array}{r}\text { All } \\
\text { pneumoconioses }\end{array}$} \\
\hline & $\#$ & Rate & \# & Rate & \# & Rate & \# & Rate & $\#$ & Rate & \# & Rate \\
\hline Martin & 7 & 3.75 & - & - & - & - & - & - & - & - & 7 & 3.75 \\
\hline Monroe & 1 & 1.02 & 2 & 1.72 & 1 & 0.71 & - & - & - & - & 4 & 3.45 \\
\hline Nassau & 1 & 2.66 & - & - & - & - & - & - & 3 & 5.63 & 4 & 8.30 \\
\hline Okaloosa & 4 & 3.25 & - & - & 1 & 0.85 & - & - & 1 & 0.70 & 6 & 4.81 \\
\hline Okeechobee & 1 & 5.83 & 2 & 3.78 & - & - & - & - & 1 & 2.95 & 4 & 12.56 \\
\hline Orange & 12 & 1.73 & 26 & 3.89 & 3 & 0.47 & 1 & 0.18 & 10 & 1.37 & 52 & 7.64 \\
\hline Osceola & 2 & 2.05 & 8 & 8.65 & 1 & 1.02 & - & - & - & - & 10 & 10.70 \\
\hline Palm Beach & 30 & 2.11 & 6 & 0.36 & 2 & 0.18 & - & - & 2 & 0.07 & 40 & 2.72 \\
\hline Pasco & 23 & 4.61 & 17 & 2.24 & 12 & 1.59 & - & - & 9 & 1.10 & 60 & 9.45 \\
\hline Pinellas & 41 & 2.35 & 35 & 2.08 & 10 & 0.37 & - & - & 12 & 0.59 & 98 & 5.39 \\
\hline Polk & 6 & 1.15 & 13 & 2.26 & 4 & 0.83 & - & - & 3 & 0.41 & 26 & 4.65 \\
\hline Putnam & 5 & 4.91 & 8 & 7.03 & 2 & 1.87 & - & - & - & - & 15 & 13.81 \\
\hline St. Johns & 4 & 3.34 & 6 & 6.22 & - & - & - & - & - & - & 10 & 9.56 \\
\hline St. Lucie & 7 & 3.15 & 4 & 1.61 & 3 & 2.03 & - & - & - & - & 14 & 6.79 \\
\hline Santa Rosa & 6 & 8.24 & 2 & 3.09 & - & - & - & - & - & - & 8 & 11.32 \\
\hline Sarasota & 9 & 1.76 & 7 & 0.80 & 1 & 0.65 & - & - & 1 & 0.08 & 18 & 3.28 \\
\hline Seminole & 3 & 0.98 & 4 & 1.24 & 3 & 0.79 & - & - & - & - & 10 & 3.01 \\
\hline Sumter & 2 & 3.40 & 1 & 2.16 & 1 & 1.24 & - & - & - & - & 4 & 6.80 \\
\hline Suwannee & 3 & 8.10 & - & - & - & - & - & - & - & - & 3 & 8.10 \\
\hline Taylor & 1 & 3.13 & 1 & 3.13 & - & - & - & - & - & - & 2 & 6.26 \\
\hline Volusia & 11 & 1.64 & 15 & 1.86 & 2 & 0.29 & - & - & 6 & 0.74 & 34 & 4.53 \\
\hline Wakulla & 1 & 7.94 & 1 & 4.37 & - & - & - & - & - & - & 2 & 12.31 \\
\hline Washington & 2 & 9.94 & - & - & - & - & - & - & - & - & 2 & 9.94 \\
\hline
\end{tabular}

- indicates no deaths listed.

NOTE: Counties with no deaths for all listed conditions are not displayed. Rates based on small numbers may be unstable. Sum of individual conditions may exceed total because of deaths with multiple pneumoconioses. See Appendix A for source description and Appendix B for methods and ICD-8 and ICD-9 codes.

SOURCE: National Center for Health Statistics multiple cause of death data. Population estimates from U.S. Bureau of the Census. 
Georgia

\section{Georgia: Selected statistics from the United States section}

All pneumoconioses mortality, 1988-1992

Total deaths

Crude mortality rate (per $1,000,000$ population)

Age-adjusted mortality rate (per 1,000,000 population)

Total years of potential life lost to life expectancy

Years of potential life lost to life expectancy (per death)

\begin{tabular}{rr} 
Value & State rank \\
\hline 112 & 25 \\
4.44 & 45 \\
3.37 & 37 \\
1,492 & 24 \\
13.3 & 7
\end{tabular}

Percent**

$\underline{\text { Deaths* }} \quad \underline{\text { distribution }}$

$55 \quad 49.1$

$22 \quad 19.6$

$23 \quad 20.5$

$2 \quad 1.8$

10

8.9

* Sum of column may exceed total deaths because of deaths with multiple pneumoconioses.

** Percentages may total more than $100 \%$ due to deaths with multiple pneumoconioses.

NOTE: $\quad$ See Appendix A for source description and Appendix B for methods and ICD-8 and ICD-9 codes.

SOURCE: National Center for Health Statistics multiple cause of death data. Population estimates from U.S. Bureau of the Census.

\section{Figure GA-1. All pneumoconioses: Georgia and U.S. age-adjusted mortality rates, residents age 15 and over, 1968-1992}

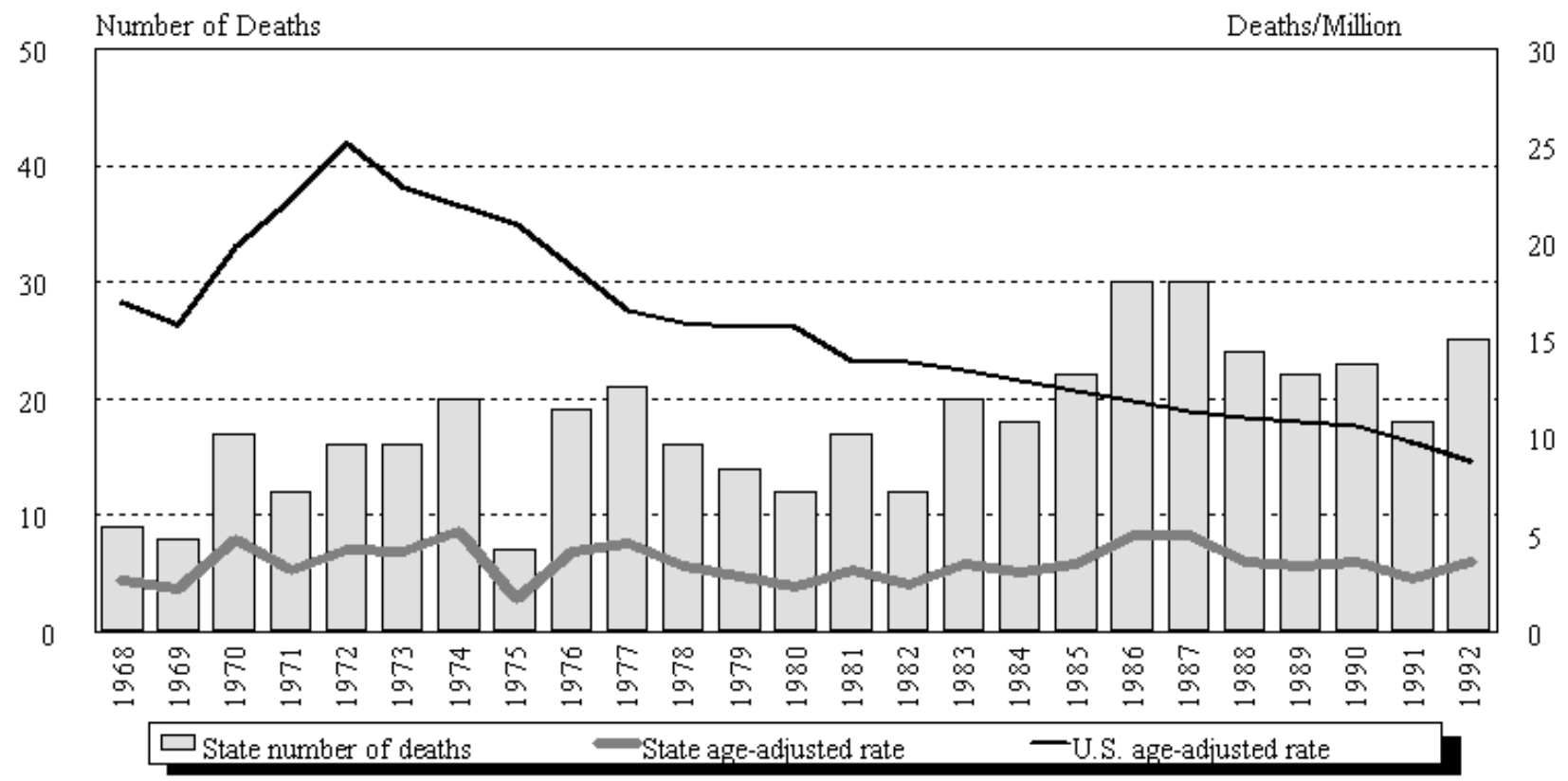

NOTE: $\quad$ See Appendix A for source description and Appendix B for methods and ICD-8 and ICD-9 codes.

SOURCE: National Center for Health Statistics multiple cause of death data. Population estimates from U.S. Bureau of the Census. 


\section{Georgia}

Table GA-1. Number of deaths and age-adjusted mortality rate (per 1,000,000 population) by condition, Georgia residents age 15 and over, 1968-1992

\begin{tabular}{|c|c|c|c|c|c|c|c|c|c|c|c|c|}
\hline \multirow[b]{2}{*}{ Year } & \multicolumn{2}{|c|}{ Asbestosis } & \multicolumn{2}{|c|}{$\begin{array}{l}\text { Coal workers' } \\
\text { pneumoconiosis }\end{array}$} & \multicolumn{2}{|c|}{ Silicosis } & \multicolumn{2}{|c|}{ Byssinosis* } & \multicolumn{2}{|c|}{$\begin{array}{c}\text { Unspecified/Other } \\
\text { pneumoconioses }\end{array}$} & \multicolumn{2}{|c|}{ All pneumoconioses } \\
\hline & Number & Rate & Number & Rate & Number & Rate & Number & Rate & Number & Rate & Number & Rate \\
\hline 1968 & 3 & 0.92 & - & - & 4 & 1.09 & & & 2 & 0.60 & 9 & 2.62 \\
\hline 1969 & - & - & - & - & 5 & 1.31 & & & 3 & 0.87 & 8 & 2.18 \\
\hline 1970 & 1 & 0.27 & - & - & 11 & 3.06 & & & 5 & 1.47 & 17 & 4.80 \\
\hline 1971 & - & - & 2 & 0.42 & 6 & 1.64 & & & 4 & 1.12 & 12 & 3.19 \\
\hline 1972 & 2 & 0.62 & - & - & 8 & 1.95 & & & 6 & 1.66 & 16 & 4.24 \\
\hline 1973 & 3 & 0.80 & 2 & 0.52 & 9 & 2.28 & & & 2 & 0.52 & 16 & 4.12 \\
\hline 1974 & 3 & 0.80 & 1 & 0.26 & 11 & 2.78 & & & 5 & 1.34 & 20 & 5.17 \\
\hline 1975 & 1 & 0.25 & 2 & 0.53 & - & - & & & 4 & 0.94 & 7 & 1.72 \\
\hline 1976 & 1 & 0.22 & 3 & 0.40 & 9 & 1.99 & & & 6 & 1.57 & 19 & 4.18 \\
\hline 1977 & - & - & 1 & 0.24 & 12 & 2.53 & & & 9 & 1.99 & 21 & 4.58 \\
\hline 1978 & 2 & 0.48 & 1 & 0.21 & 7 & 1.50 & & & 6 & 1.20 & 16 & 3.39 \\
\hline 1979 & 3 & 0.73 & 2 & 0.30 & 7 & 1.39 & - & - & 3 & 0.53 & 14 & 2.85 \\
\hline 1980 & 4 & 0.74 & 2 & 0.42 & 1 & 0.16 & - & - & 5 & 1.00 & 12 & 2.32 \\
\hline 1981 & 2 & 0.38 & 4 & 0.77 & 6 & 1.15 & 2 & 0.38 & 3 & 0.49 & 17 & 3.18 \\
\hline 1982 & 8 & 1.79 & 2 & 0.38 & 2 & 0.29 & - & - & - & - & 12 & 2.46 \\
\hline 1983 & 5 & 1.03 & 1 & 0.19 & 7 & 1.08 & 3 & 0.62 & 4 & 0.55 & 20 & 3.47 \\
\hline 1984 & 2 & 0.47 & 4 & 0.53 & 5 & 0.88 & 5 & 0.86 & 2 & 0.37 & 18 & 3.11 \\
\hline 1985 & 4 & 0.67 & 3 & 0.39 & 9 & 1.38 & 1 & 0.28 & 5 & 0.82 & 22 & 3.54 \\
\hline 1986 & 11 & 1.76 & 6 & 1.07 & 9 & 1.41 & 2 & 0.30 & 2 & 0.40 & 30 & 4.94 \\
\hline 1987 & 12 & 1.95 & 2 & 0.29 & 6 & 1.25 & 4 & 0.64 & 6 & 0.88 & 30 & 5.01 \\
\hline 1988 & 8 & 1.32 & 6 & 0.86 & 6 & 0.80 & - & - & 4 & 0.63 & 24 & 3.61 \\
\hline 1989 & 6 & 0.86 & 7 & 1.07 & 7 & 1.09 & 1 & 0.19 & 1 & 0.17 & 22 & 3.37 \\
\hline 1990 & 13 & 2.21 & 4 & 0.56 & 4 & 0.60 & - & - & 2 & 0.18 & 23 & 3.55 \\
\hline 1991 & 10 & 1.74 & 3 & 0.38 & 3 & 0.38 & 1 & 0.11 & 1 & 0.11 & 18 & 2.71 \\
\hline 1992 & 18 & 2.77 & 2 & 0.16 & 3 & 0.38 & - & - & 2 & 0.32 & 25 & 3.64 \\
\hline
\end{tabular}

-indicates no deaths listed. ${ }^{*}$ no specific code for by ssinosis in ICD-8 (1968-1978)

NOTE: Rates based on small numbers may be unstable. Sum of individ ual conditions may exceed total because of deaths with multiple pneumoconioses. See Appendix A for source description and Appendix B for methods and ICD-8 and ICD-9 Nodes.

SOURCE: National Center for Health Statistics multiple cause of death data. Population estimates from U.S. Bureau of the Census. 
Figure GA-2. All pneumoconioses: Distribution of deaths by sex and race, Georgia residents age 15 and over, 1968-1992
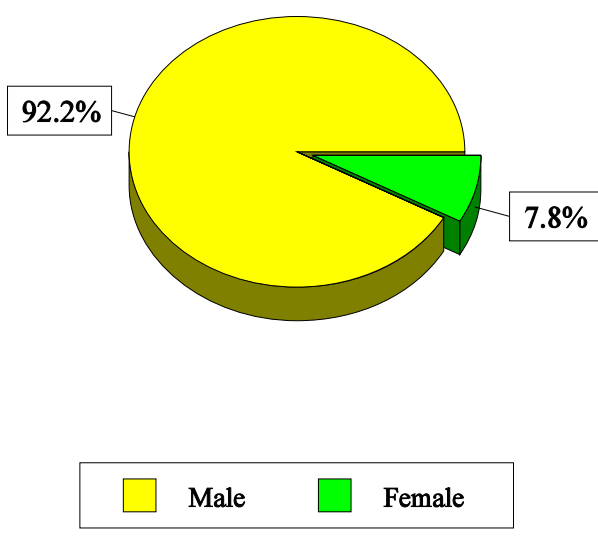

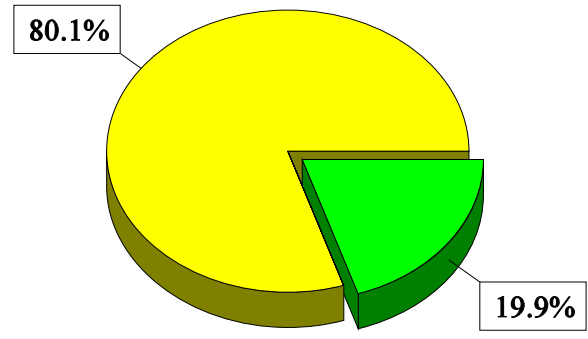

White

Black Other

NOTE: $\quad$ See Appendix A for source description and Appendix B for methods and ICD-8 and ICD-9 codes.

SOURCE: National Center for Health Statistics multiple cause of death data.

Table GA-2. All pneumoconioses: Distribution of deaths by sex, race, and age, Georgia residents age 15 and over, 1968-1992

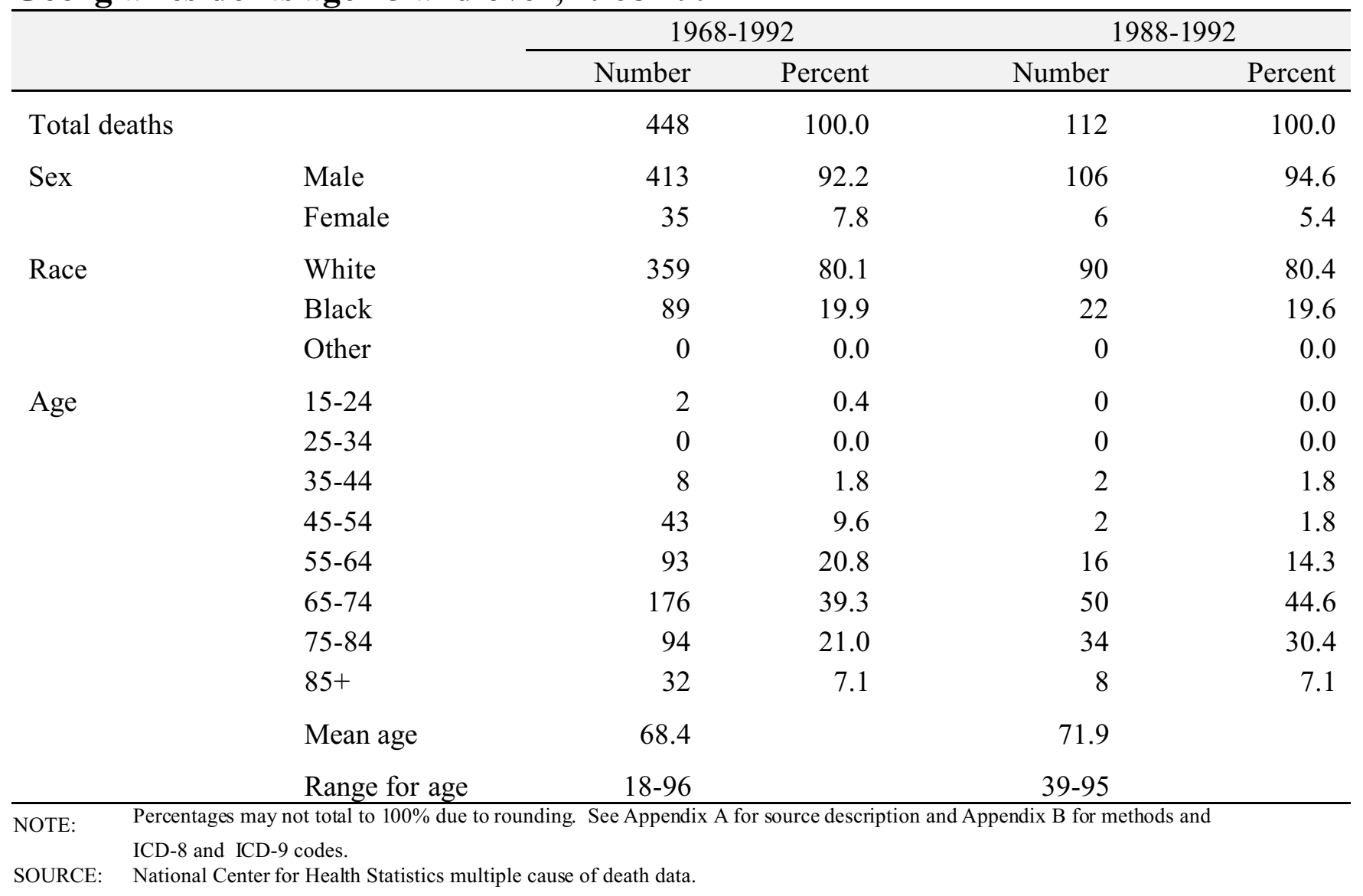




\section{Figure GA-3. Pneumoconiosis deaths by condition, Georgia residents age 15 and over, 1968-1992}

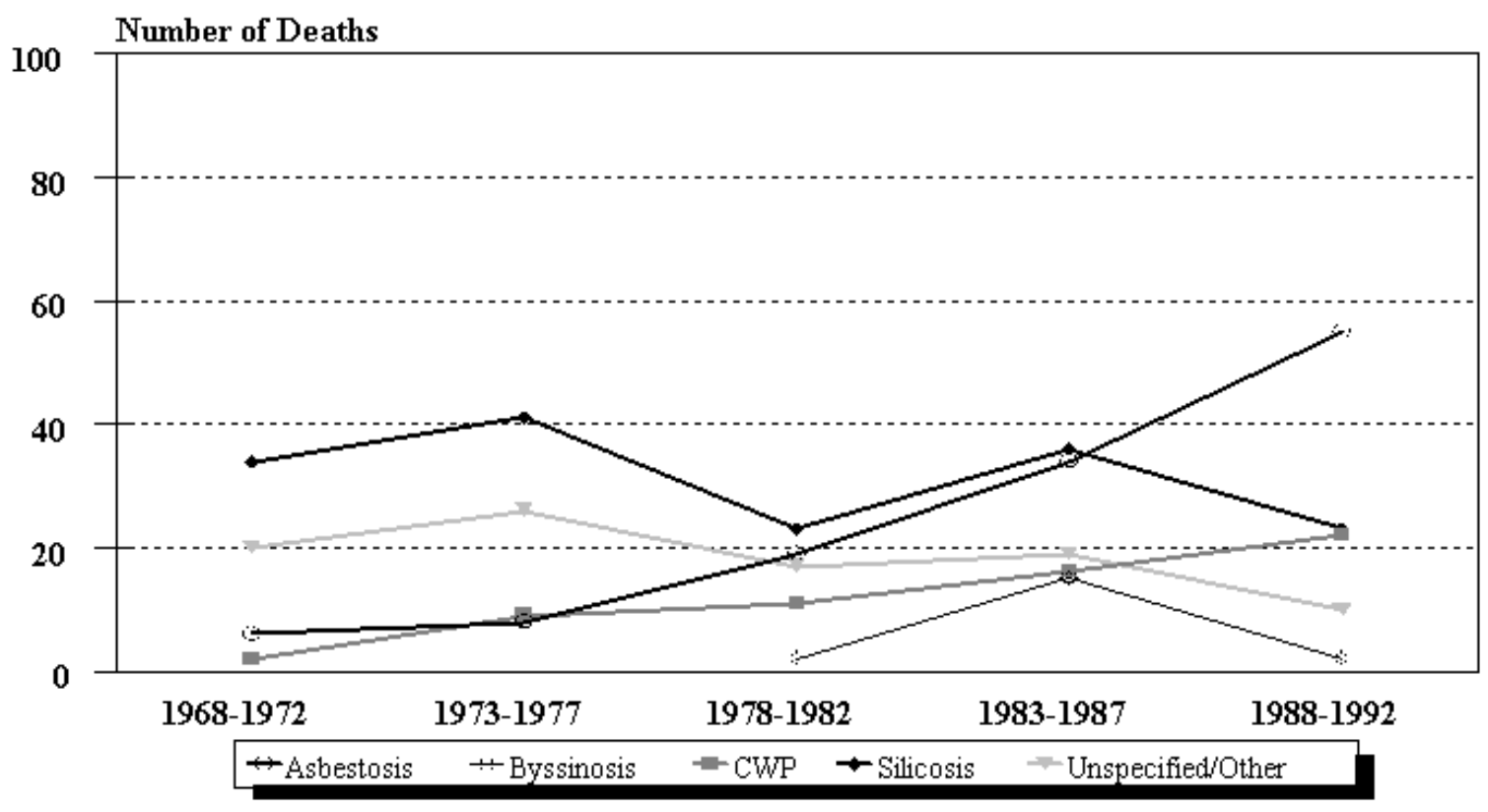

NOTE: See Appendix A for source description and Appendix B for methods and ICD-8 and ICD-9 codes. SOURCE: National Center for Health Statistics multiple cause of death data.

Figure GA-4. Georgia and U.S. age-adjusted mortality rates (per 1,000,000 population) by condition, residents age 15 and over, 1983-1992

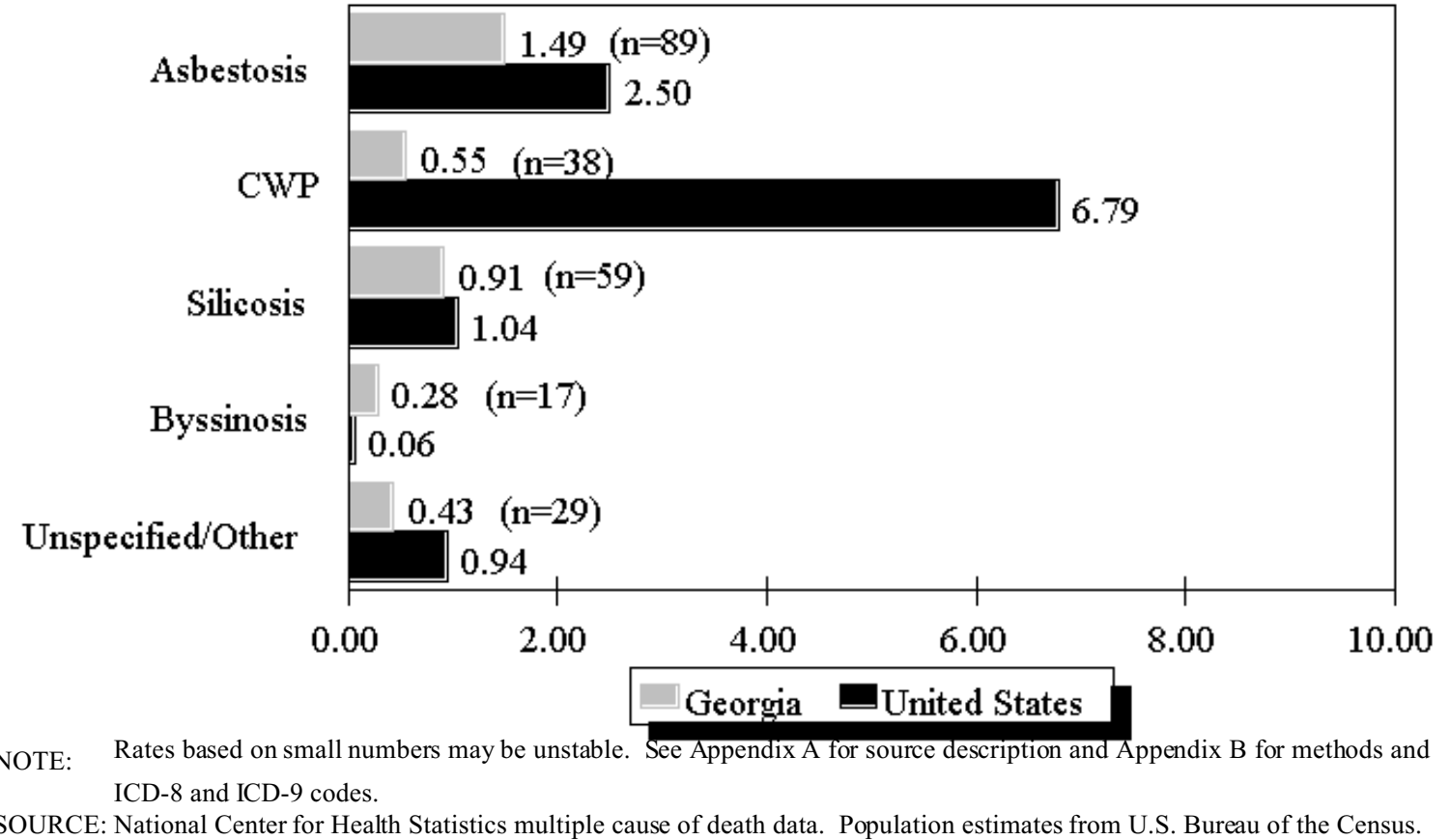


Georgia

Table GA-3 (page 1 of 3). Number of deaths and age-adjusted mortality rate (per $1,000,000$ population) by condition and county, Georgia residents age 15 and over, 1983-1992

\begin{tabular}{|c|c|c|c|c|c|c|c|c|c|c|c|c|}
\hline \multirow[b]{2}{*}{ County } & \multicolumn{2}{|c|}{ Asbestosis } & \multicolumn{2}{|c|}{$\begin{array}{r}\text { Coal workers' } \\
\text { pneumoconiosis }\end{array}$} & \multicolumn{2}{|c|}{ Silicosis } & \multicolumn{2}{|c|}{ Byssinosis } & \multicolumn{2}{|c|}{$\begin{array}{r}\text { Unspecified/Other } \\
\text { pneumoconioses }\end{array}$} & \multicolumn{2}{|c|}{$\begin{array}{r}\text { All } \\
\text { pneumoconioses }\end{array}$} \\
\hline & $\#$ & Rate & $\#$ & Rate & $\#$ & Rate & $\#$ & Rate & $\#$ & Rate & \# & Rate \\
\hline Baldwin & - & - & - & - & 1 & 1.63 & - & - & 1 & 1.63 & 2 & 3.26 \\
\hline Bartow & - & - & 1 & 1.35 & 1 & 2.67 & - & - & - & - & 2 & 4.01 \\
\hline Ben Hill & 1 & 2.78 & 1 & 2.78 & - & - & - & - & - & - & 2 & 5.55 \\
\hline Bibb & 1 & 0.69 & 1 & 0.69 & 1 & 0.36 & - & - & 2 & 0.72 & 5 & 2.46 \\
\hline Brantley & 2 & 22.79 & - & - & - & - & - & - & - & - & 2 & 22.79 \\
\hline Bryan & 1 & 7.83 & - & - & - & - & - & - & - & - & 1 & 7.83 \\
\hline Burke & 1 & 5.63 & - & - & - & - & - & - & - & - & 1 & 5.63 \\
\hline Camden & 1 & 7.66 & - & - & - & - & - & - & - & - & 1 & 7.66 \\
\hline Carroll & 1 & 0.92 & 1 & 1.85 & 1 & 0.92 & - & - & - & - & 3 & 3.70 \\
\hline Catoosa & 2 & 4.32 & - & - & 1 & 2.62 & - & - & - & - & 3 & 6.93 \\
\hline Chatham & 9 & 4.37 & 2 & 0.75 & 1 & 0.49 & - & - & 1 & 0.27 & 13 & 5.88 \\
\hline Cherokee & - & - & 1 & 2.03 & - & - & - & - & 1 & 2.03 & 2 & 4.06 \\
\hline Clarke & - & - & 1 & 1.87 & 3 & 5.91 & - & - & - & - & 4 & 7.78 \\
\hline Clayton & 2 & 1.89 & - & - & 1 & 0.95 & - & - & 3 & 2.65 & 6 & 5.49 \\
\hline Cobb & 4 & 1.36 & 1 & 0.30 & 3 & 0.99 & 2 & 0.69 & 1 & 0.30 & 11 & 3.63 \\
\hline Coffee & - & - & - & - & 1 & 2.15 & - & - & - & - & 1 & 2.15 \\
\hline Columbia & 2 & 5.46 & - & - & - & - & 1 & 2.73 & - & - & 3 & 8.19 \\
\hline Coweta & 1 & 2.38 & - & - & - & - & 2 & 4.77 & - & - & 3 & 7.15 \\
\hline Crisp & - & - & - & - & - & - & - & - & 1 & 2.30 & 1 & 2.30 \\
\hline Dade & - & - & 2 & 14.02 & - & - & - & - & - & - & 2 & 14.02 \\
\hline De Kalb & 3 & 0.76 & 2 & 0.41 & 6 & 1.23 & - & - & 4 & 0.82 & 15 & 3.22 \\
\hline Dodge & - & - & - & - & - & - & - & - & 1 & 5.57 & 1 & 5.57 \\
\hline Dougherty & 1 & 1.24 & - & - & - & - & - & - & - & - & 1 & 1.24 \\
\hline Douglas & 1 & 2.29 & - & - & - & - & 1 & 2.29 & - & - & 2 & 4.59 \\
\hline Effingham & 5 & 28.93 & - & - & - & - & - & - & - & - & 5 & 28.93 \\
\hline Elbert & - & - & - & - & 6 & 18.77 & - & - & - & - & 6 & 18.77 \\
\hline Emanuel & 1 & 4.79 & - & - & - & - & - & - & - & - & 1 & 4.79 \\
\hline Evans & - & - & 1 & 11.91 & - & - & - & - & - & - & 1 & 11.91 \\
\hline Fannin & - & - & 2 & 9.35 & 1 & 2.57 & - & - & - & - & 3 & 11.92 \\
\hline Fayette & - & - & 1 & 2.05 & - & - & - & - & - & - & 1 & 2.05 \\
\hline Floyd & 2 & 2.33 & 2 & 1.75 & - & - & 2 & 2.95 & - & - & 6 & 7.03 \\
\hline Forsyth & - & - & - & - & 1 & 2.04 & - & - & - & - & 1 & 2.04 \\
\hline
\end{tabular}

See footnotes at end of table. 
Table GA-3 (page 2 of 3). Number of deaths and age-adjusted mortality rate (per $1,000,000$ population) by condition and county, Georgia residents age 15 and over, 1983-1992

\begin{tabular}{|c|c|c|c|c|c|c|c|c|c|c|c|c|}
\hline \multirow[b]{2}{*}{ County } & \multicolumn{2}{|c|}{ Asbestosis } & \multicolumn{2}{|c|}{$\begin{array}{r}\text { Coal workers' } \\
\text { pneumoconiosis }\end{array}$} & \multicolumn{2}{|c|}{ Silicosis } & \multicolumn{2}{|c|}{ Byssinosis } & \multicolumn{2}{|c|}{$\begin{array}{r}\text { Unspecified/Other } \\
\text { pneumoconioses }\end{array}$} & \multicolumn{2}{|c|}{$\begin{array}{r}\text { All } \\
\text { pneumoconioses }\end{array}$} \\
\hline & \# & Rate & $\#$ & Rate & $\#$ & Rate & \# & Rate & \# & Rate & $\#$ & Rate \\
\hline Fulton & 1 & 0.10 & 2 & 0.20 & 1 & 0.10 & 2 & 0.30 & 3 & 0.52 & 9 & 1.21 \\
\hline Glynn & 10 & 12.95 & - & - & - & - & - & - & - & - & 10 & 12.95 \\
\hline Gordon & - & - & 1 & 1.91 & - & - & - & - & 1 & 1.91 & 2 & 3.82 \\
\hline Grady & - & - & - & - & 1 & 4.88 & - & - & - & - & 1 & 4.88 \\
\hline Gwinnett & 1 & 0.50 & 1 & 0.50 & 1 & 0.50 & - & - & - & - & 3 & 1.50 \\
\hline Habersham & 1 & 1.94 & - & - & - & - & - & - & - & - & 1 & 1.94 \\
\hline Hall & 2 & 1.96 & 2 & 1.96 & - & - & 1 & 1.25 & - & - & 5 & 5.16 \\
\hline Haralson & 1 & 4.68 & - & - & - & - & - & - & - & - & 1 & 4.68 \\
\hline Harris & - & - & - & - & 1 & 5.30 & - & - & - & - & 1 & 5.30 \\
\hline Henry & 1 & 2.46 & - & - & 1 & 2.46 & - & - & - & - & 2 & 4.93 \\
\hline Houston & - & - & - & - & 1 & 1.31 & - & - & 1 & 1.31 & 2 & 2.62 \\
\hline Jeff Davis & 1 & 9.13 & - & - & - & - & - & - & - & - & 1 & 9.13 \\
\hline Jefferson & 1 & 2.40 & - & - & - & - & 1 & 9.18 & - & - & 2 & 11.57 \\
\hline Jones & - & - & - & - & 2 & 12.83 & - & - & - & - & 2 & 12.83 \\
\hline Mcduffie & 1 & 7.19 & - & - & - & - & - & - & - & - & 1 & 7.19 \\
\hline Mcintosh & - & - & 2 & 18.41 & - & - & - & - & - & - & 2 & 18.41 \\
\hline Madison & 1 & 5.75 & - & - & 2 & 11.50 & - & - & - & - & 3 & 17.25 \\
\hline Meriwether & - & - & - & - & - & - & 1 & 2.26 & - & - & 1 & 2.26 \\
\hline Murray & - & - & 1 & 5.66 & 1 & 3.55 & - & - & 1 & 5.66 & 3 & 14.88 \\
\hline Muscogee & - & - & 2 & 1.26 & 3 & 1.63 & 1 & 0.63 & - & - & 6 & 3.51 \\
\hline Newton & - & - & - & - & - & - & 1 & 1.57 & - & - & 1 & 1.57 \\
\hline Oglethorpe & - & - & - & - & 3 & 30.63 & - & - & - & - & 3 & 30.63 \\
\hline Paulding & - & - & 1 & 2.46 & - & - & - & - & - & - & 1 & 2.46 \\
\hline Pickens & 1 & 6.94 & - & - & - & - & - & - & - & - & 1 & 6.94 \\
\hline Polk & - & - & - & - & 1 & 2.83 & - & - & - & - & 1 & 2.83 \\
\hline Putnam & 1 & 6.93 & - & - & - & - & - & - & - & - & 1 & 6.93 \\
\hline Rabun & 1 & 12.06 & - & - & - & - & - & - & - & - & 1 & 12.06 \\
\hline Richmond & 3 & 1.14 & 1 & 0.64 & 2 & 1.02 & - & - & 1 & 0.38 & 7 & 3.18 \\
\hline Rockdale & 4 & 9.98 & - & - & 8 & 17.03 & - & - & - & - & 12 & 27.01 \\
\hline Stephens & - & - & 1 & 2.10 & - & - & - & - & - & - & 1 & 2.10 \\
\hline Talbot & - & - & - & - & 1 & 6.88 & - & - & - & - & 1 & 6.88 \\
\hline Tattnall & 2 & 11.73 & - & - & - & - & - & - & - & - & 2 & 11.73 \\
\hline Telfair & 1 & 8.08 & - & - & - & - & - & - & - & - & 1 & 8.08 \\
\hline
\end{tabular}

See footnotes at end of table. 
Table GA-3 (page 3 of 3). Number of deaths and age-adjusted mortality rate (per $1,000,000$ population) by condition and county, Georgia residents age 15 and over, 1983-1992

\begin{tabular}{|c|c|c|c|c|c|c|c|c|c|c|c|c|}
\hline \multirow[b]{2}{*}{ County } & \multicolumn{2}{|c|}{ Asbestosis } & \multicolumn{2}{|c|}{$\begin{array}{c}\text { Coal workers' } \\
\text { pneumoconiosis }\end{array}$} & \multicolumn{2}{|c|}{ Silicosis } & \multicolumn{2}{|c|}{ Byssinosis } & \multicolumn{2}{|c|}{$\begin{array}{r}\text { Unspecified/Other } \\
\text { pneumoconioses }\end{array}$} & \multicolumn{2}{|c|}{$\begin{array}{r}\text { All } \\
\text { pneumoconioses }\end{array}$} \\
\hline & \# & Rate & \# & Rate & $\#$ & Rate & $\#$ & Rate & \# & Rate & \# & Rate \\
\hline Tift & 1 & 1.74 & 2 & 5.15 & - & - & - & - & - & - & 3 & 6.89 \\
\hline Troup & 4 & 5.41 & - & - & - & - & - & - & - & - & 4 & 5.41 \\
\hline Twiggs & - & - & - & - & - & - & - & - & 1 & 11.39 & 1 & 11.39 \\
\hline Upson & - & - & - & - & - & - & 1 & 5.65 & - & - & 1 & 5.65 \\
\hline Walker & 1 & 1.69 & 3 & 4.30 & - & - & - & - & 2 & 2.62 & 6 & 8.61 \\
\hline Walton & 1 & 3.18 & - & - & - & - & - & - & - & - & 1 & 3.18 \\
\hline Ware & 5 & 10.27 & - & - & - & - & - & - & - & - & 5 & 10.27 \\
\hline Washington & 2 & 7.93 & - & - & 1 & 5.61 & - & - & 3 & 16.83 & 6 & 30.37 \\
\hline Wayne & - & - & - & - & 1 & 2.60 & - & - & - & - & 1 & 2.60 \\
\hline Whitfield & - & - & - & - & - & - & 1 & 1.01 & - & - & 1 & 1.01 \\
\hline Wilkinson & - & - & - & - & - & - & - & - & 1 & 10.71 & 1 & 10.71 \\
\hline
\end{tabular}

- indicates no deaths listed.

NOTE: Counties with no deaths for all listed conditions are not displayed. Rates based on small numbers may be unstable. Sum of individual conditions may exceed total because of deaths with multiple pneumoconioses. See Appendix A for source description and Appendix B for methods and ICD-8 and ICD-9 codes.

SOURCE: National Center for Health Statistics multiple cause of death data. Population estimates from U.S. Bureau of the Census. 
Hawaii

\section{Hawaii: Selected statistics from the United States section}

All pneumoconioses mortality, 1988-1992

Total deaths

Crude mortality rate (per $1,000,000$ population)

Age-adjusted mortality rate (per 1,000,000 population)

Total years of potential life lost to life expectancy

Years of potential life lost to life expectancy (per death)

\begin{tabular}{rr} 
Value & State rank \\
\hline 20 & 46 \\
4.58 & 43 \\
2.91 & 44 \\
227 & 46 \\
11.4 & 36
\end{tabular}

Percent**

Deaths*

distribution

20

100.0

Asbestosis

0

0.0

0

0.0

Silicosis

Byssinosis

Unspecified/Other pneumoconiosis

* Sum of column may exceed total deaths because of deaths with multiple pneumoconioses.

** Percentages may total more than $100 \%$ due to deaths with multiple pneumoconioses.

NOTE: $\quad$ See Appendix A for source description and Appendix B for methods and ICD-8 and ICD-9 codes.

SOURCE: National Center for Health Statistics multiple cause of death data. Population estimates from U.S. Bureau of the Census.

Figure HI-1. All pneumoconioses: Hawaii and U.S. age-adjusted mortality rates, residents age 15 and over, 1968-1992

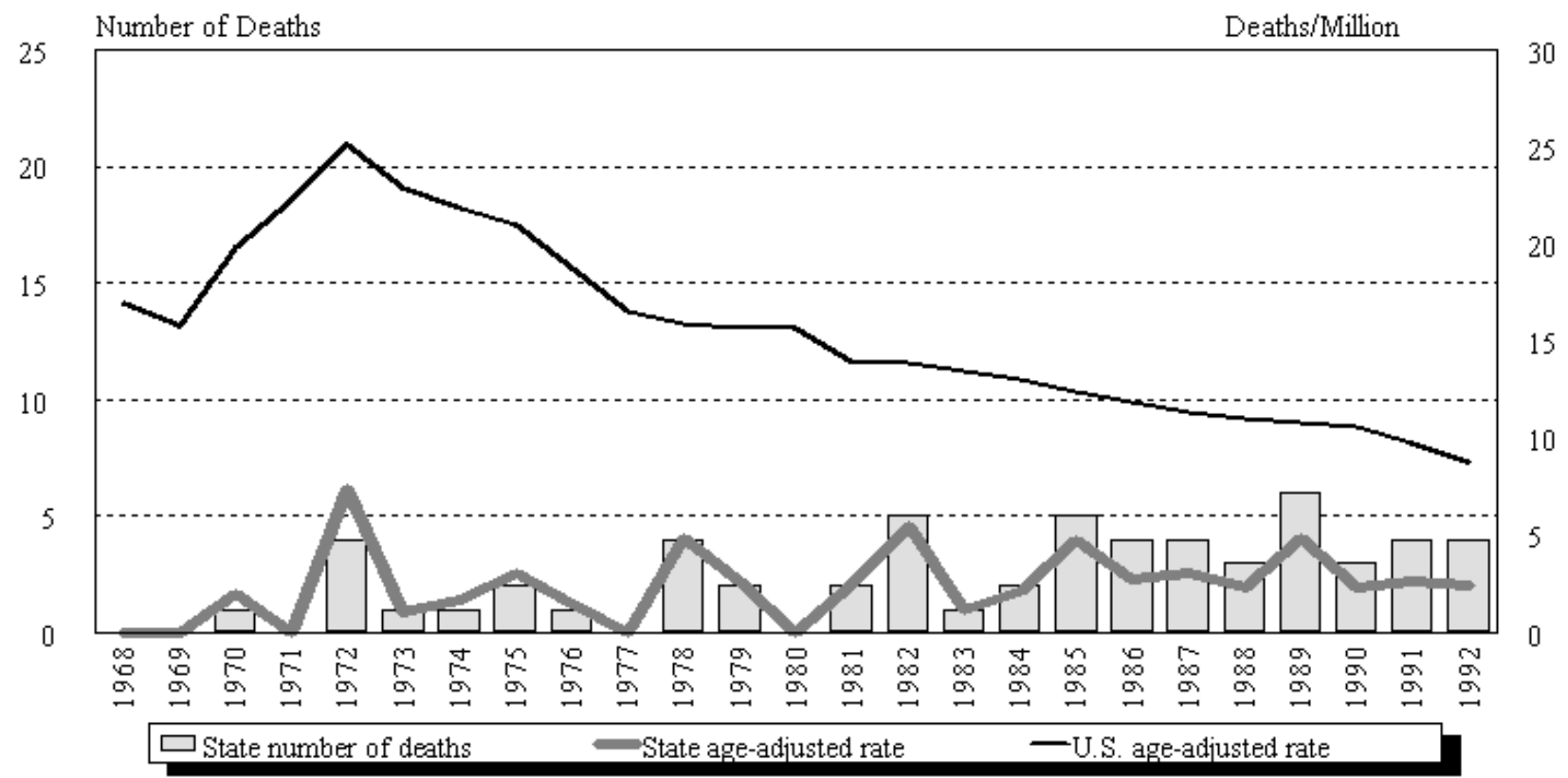

NOTE: $\quad$ See Appendix A for source description and Appendix B for methods and ICD-8 and ICD-9 codes.

SOURCE: $\quad$ National Center for Health Statistics multiple cause of death data. Population estimates from U.S. Bureau of the Census. 


\section{Hawaii}

Table HI-1. Number of deaths and age-adjusted mortality rate (per 1,000,000 population) by condition, Hawaii residents age 15 and over, 1968-1992

\begin{tabular}{|c|c|c|c|c|c|c|c|c|c|c|c|c|}
\hline \multirow[b]{2}{*}{ Year } & \multicolumn{2}{|c|}{ Asbestosis } & \multicolumn{2}{|c|}{$\begin{array}{c}\text { Coal workers' } \\
\text { pneumoconiosis }\end{array}$} & \multicolumn{2}{|c|}{ Silicosis } & \multicolumn{2}{|c|}{ Byssinosis* } & \multicolumn{2}{|c|}{$\begin{array}{c}\text { Unspecified/Other } \\
\text { pneumoconioses }\end{array}$} & \multicolumn{2}{|c|}{ All pneumoconioses } \\
\hline & Number & Rate & Number & Rate & Number & Rate & Number & Rate & Number & Rate & Number & Rate \\
\hline 1968 & - & - & - & - & - & - & & & - & - & - & - \\
\hline 1969 & - & - & - & - & - & - & & & - & - & - & - \\
\hline 1970 & - & - & - & - & - & - & & & 1 & 1.95 & 1 & 1.95 \\
\hline 1971 & - & - & - & - & - & - & & & - & - & - & - \\
\hline 1972 & - & - & - & - & 2 & 3.88 & & & 2 & 3.49 & 4 & 7.37 \\
\hline 1973 & - & - & - & - & 1 & 1.04 & & & - & - & 1 & 1.04 \\
\hline 1974 & - & - & - & - & 1 & 1.66 & & & 1 & 1.66 & 1 & 1.66 \\
\hline 1975 & - & - & 1 & 1.45 & 1 & 1.64 & & & - & - & 2 & 3.09 \\
\hline 1976 & - & - & - & - & - & - & & & 1 & 1.45 & 1 & 1.45 \\
\hline 1977 & - & - & - & - & - & - & & & - & - & - & - \\
\hline 1978 & 1 & 1.42 & - & - & 1 & 0.73 & & & 2 & 2.69 & 4 & 4.84 \\
\hline 1979 & 2 & 2.64 & - & - & - & - & - & - & - & - & 2 & 2.64 \\
\hline 1980 & - & - & - & - & - & - & - & - & - & - & - & - \\
\hline 1981 & 1 & 1.24 & 1 & 1.24 & - & - & - & - & - & - & 2 & 2.48 \\
\hline 1982 & 3 & 3.33 & 1 & 0.97 & 1 & 1.22 & - & - & - & - & 5 & 5.52 \\
\hline 1983 & 1 & 1.20 & - & - & - & - & - & - & - & - & 1 & 1.20 \\
\hline 1984 & 2 & 2.15 & - & - & 1 & 1.08 & - & - & - & - & 2 & 2.15 \\
\hline 1985 & 5 & 4.76 & - & - & - & - & - & - & - & - & 5 & 4.76 \\
\hline 1986 & 2 & 1.77 & 1 & 0.47 & 1 & 0.47 & - & - & - & - & 4 & 2.70 \\
\hline 1987 & 3 & 2.30 & - & - & - & - & - & - & 1 & 0.74 & 4 & 3.04 \\
\hline 1988 & 3 & 2.30 & - & - & - & - & - & - & - & - & 3 & 2.30 \\
\hline 1989 & 6 & 4.87 & - & - & - & - & - & - & - & - & 6 & 4.87 \\
\hline 1990 & 3 & 2.32 & - & - & - & - & - & - & - & - & 3 & 2.32 \\
\hline 1991 & 4 & 2.64 & - & - & - & - & - & - & - & - & 4 & 2.64 \\
\hline 1992 & 4 & 2.46 & - & - & - & - & - & - & - & - & 4 & 2.46 \\
\hline
\end{tabular}

indicates no deaths listed. ${ }^{*}$ no specific code for byssinosis in ICD-8 (1968-1978).

NOTE: Rates based on small numbers may be unstable. Sum of individual conditions may exceed total because of deaths with multiple pneumoconioses. See Appendix A for source description and Appendix B for methods and ICD-8 and ICD-9 codes.

SOURCE: National Center for Health Statistics multiple cause of death data. Population estimates from U.S. Bureau of the Census. 


\section{Figure HI-2. All pneumoconioses: Distribution of deaths by sex and race,} Hawaii residents age 15 and over, 1968-1992

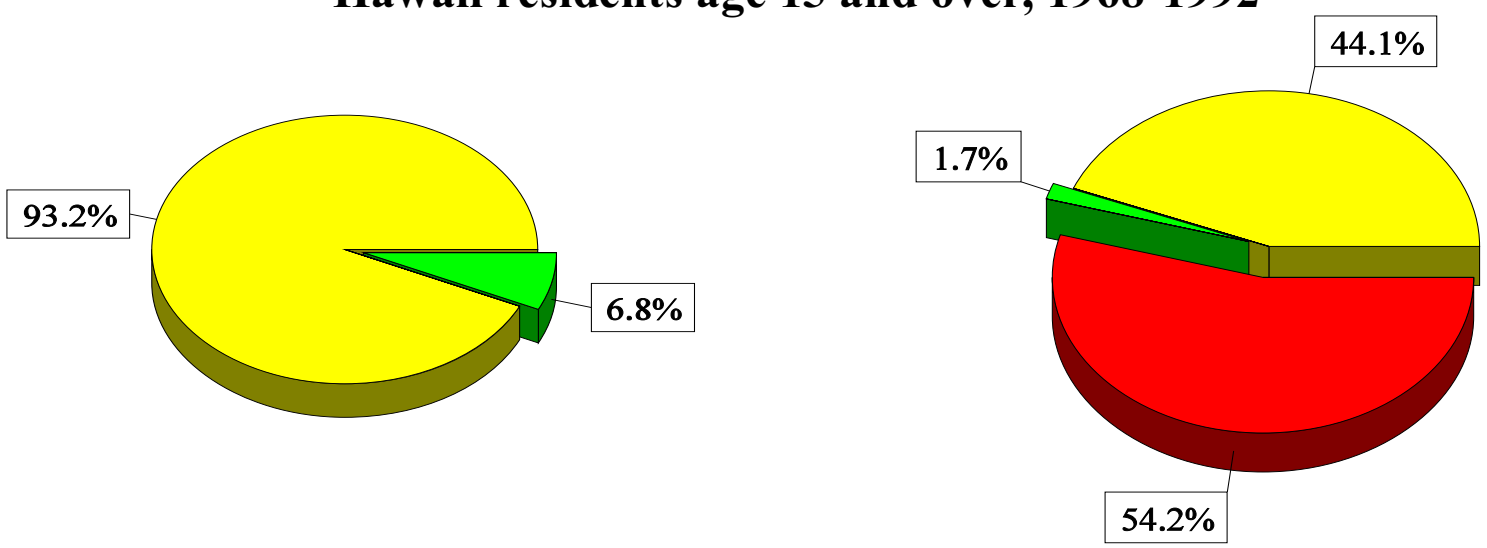

NOTE: $\quad$ See Appendix A for source description and Appendix B for methods and ICD-8 and ICD-9 codes.

SOURCE: National Center for Health Statistics multiple cause of death data.

Table HI-2. All pneumoconioses: Distribution of deaths by sex, race, and age, Hawaii residents age 15 and over, 1968-1992

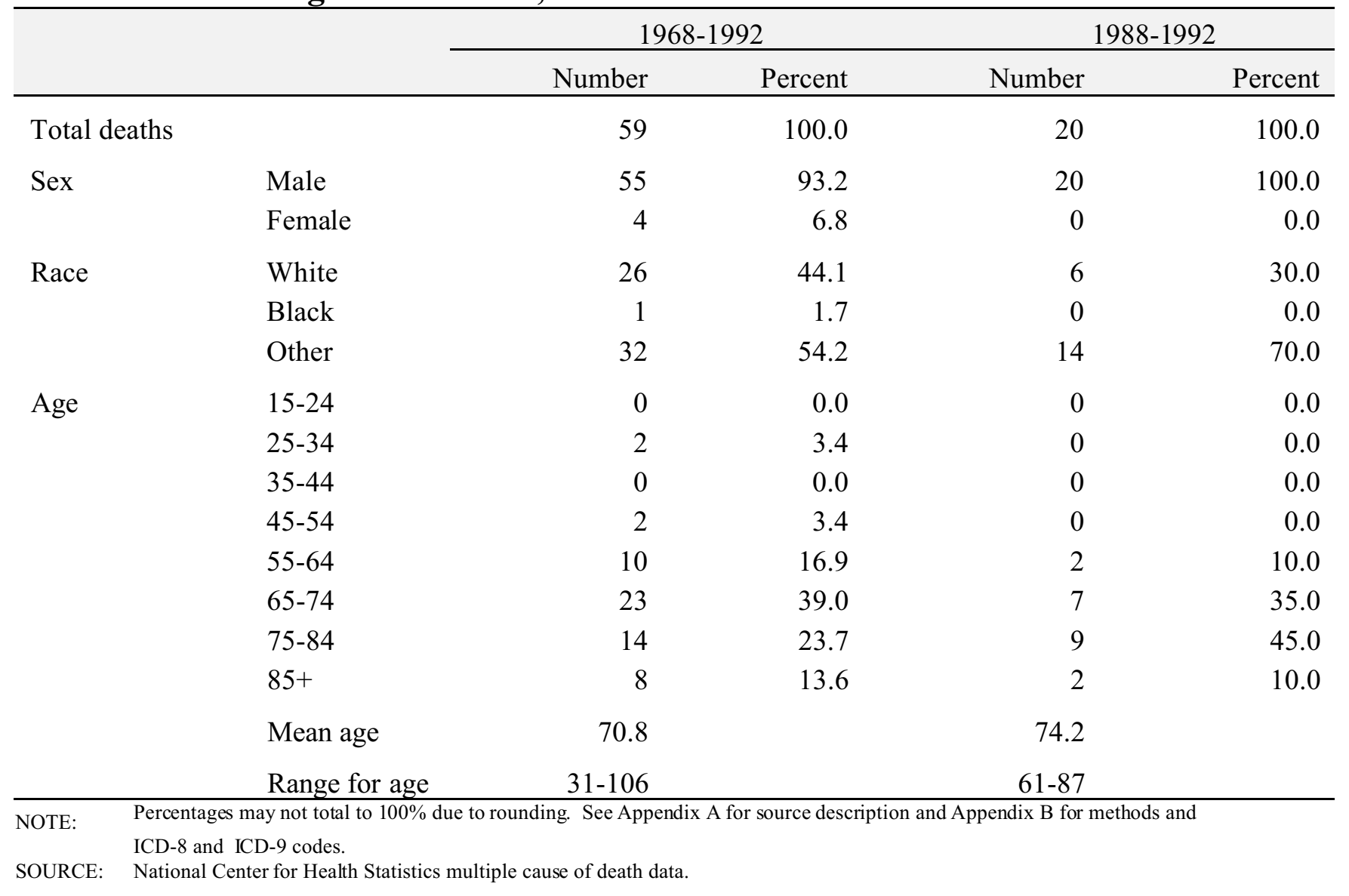


Figure HI-3. Pneumoconiosis deaths by condition, Hawaii residents age 15 and over, 1968-1992

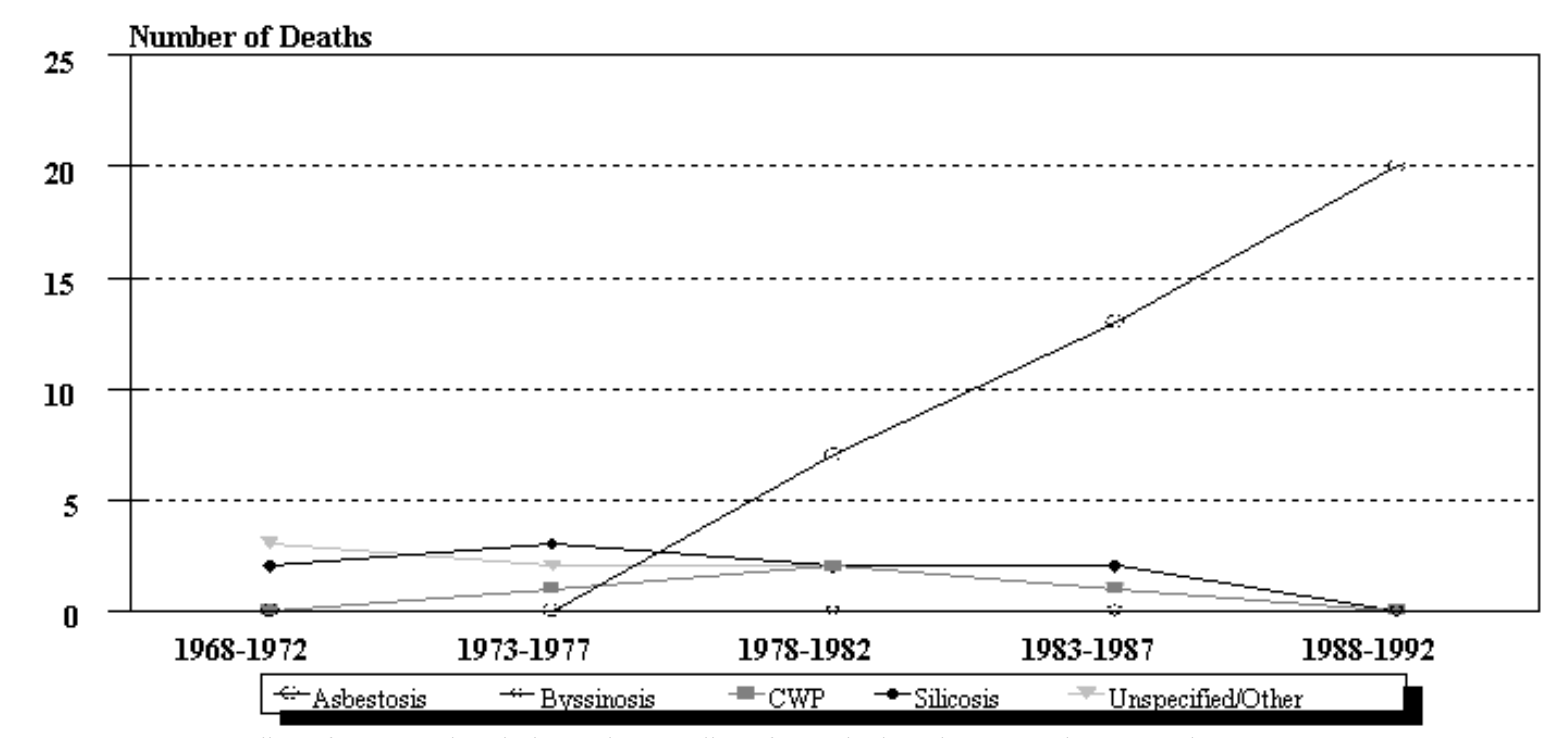

NOTE: See Appendix A for source description and Appendix B for methods and ICD-8 and ICD-9 codes. SOURCE: National Center for Health Statistics multiple cause of death data.

Figure HI-4. Hawaii and. U.S. age-adjusted mortality rates (per 1,000,000 population) by condition, residents age 15 and over, 1983-1992

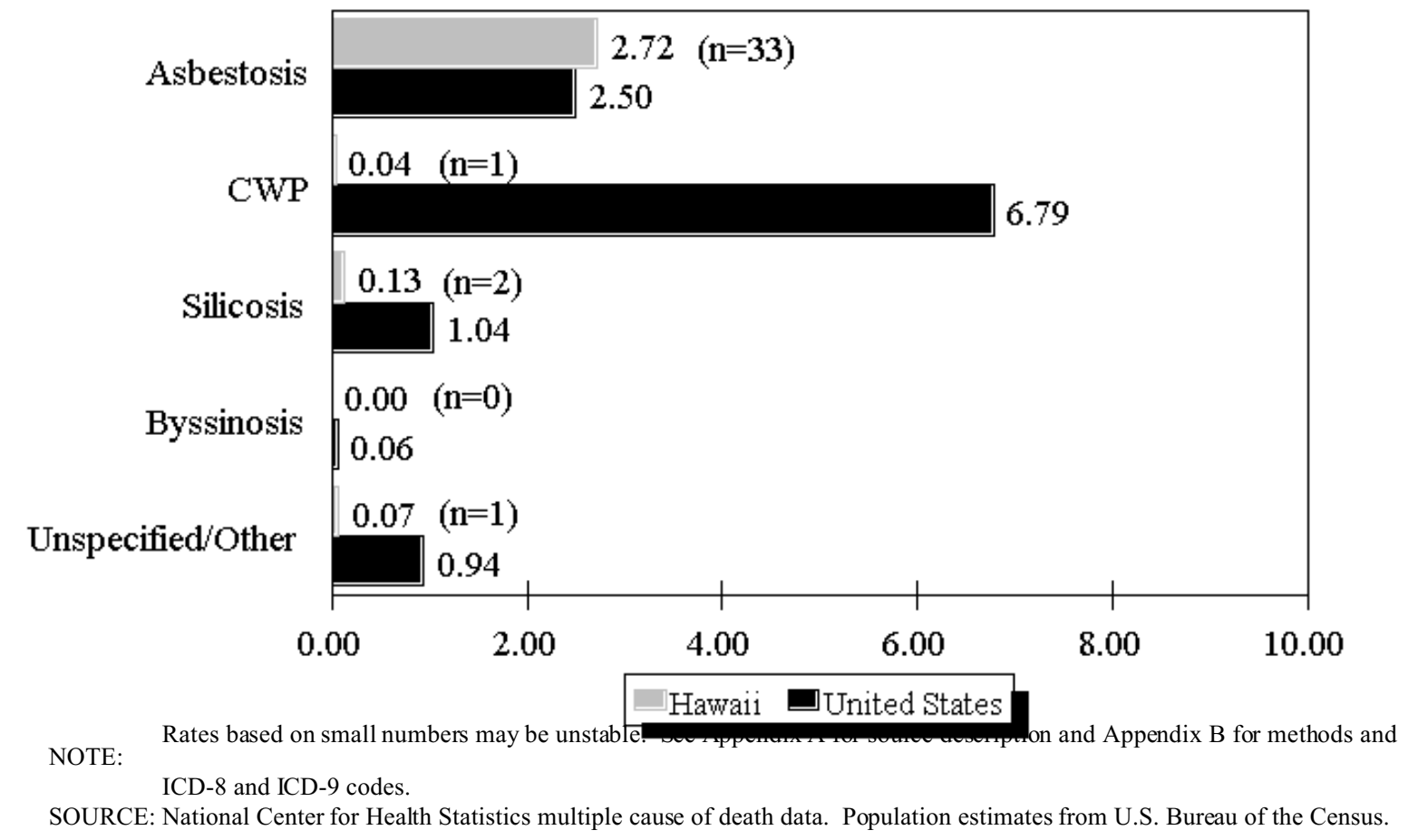


Table HI-3. Number of deaths and age-adjusted mortality rate (per 1,000,000 population) by condition and county, Hawaii residents age 15 and over, 19831992

\begin{tabular}{|c|c|c|c|c|c|c|c|c|c|c|c|c|}
\hline \multirow[b]{2}{*}{ County } & \multicolumn{2}{|c|}{ Asbestosis } & \multicolumn{2}{|c|}{$\begin{array}{r}\text { Coal workers' } \\
\text { pneumoconiosis }\end{array}$} & \multicolumn{2}{|c|}{ Silicosis } & \multicolumn{2}{|c|}{ Byssinosis } & \multicolumn{2}{|c|}{$\begin{array}{r}\text { Unspecified/Other } \\
\text { pneumoconioses }\end{array}$} & \multicolumn{2}{|c|}{$\begin{array}{r}\text { All } \\
\text { pneumoconioses }\end{array}$} \\
\hline & \# & Rate & $\#$ & Rate & $\#$ & Rate & \# & Rate & \# & Rate & \# & Rate \\
\hline Hawaii & 1 & 0.53 & - & - & - & - & - & - & - & - & 1 & 0.53 \\
\hline Honolulu & 30 & 3.66 & 1 & 0.09 & 2 & 0.23 & - & - & 1 & 0.09 & 33 & 3.93 \\
\hline Kauai & 2 & 2.24 & - & - & - & - & - & - & - & - & 2 & 2.24 \\
\hline
\end{tabular}

- indicates no deaths listed.

NOTE: Counties with no deaths for all listed conditions are not displayed. Rates based on small numbers may be unstable. Sum of individual conditions may exceed total because of deaths with multiple pneumoconioses. See Appendix A for source description and Appendix B for methods and ICD-8 and ICD-9 codes.

SOURCE: National Center for Health Statistics multiple cause of death data. Population estimates from U.S. Bureau of the Census. 
Idaho

\section{Idaho: Selected statistics from the United States section}

All pneumoconioses mortality, 1988-1992

Total deaths

Crude mortality rate (per $1,000,000$ population)

Age-adjusted mortality rate (per 1,000,000 population)

Total years of potential life lost to life expectancy

Years of potential life lost to life expectancy (per death)

\begin{tabular}{rr} 
Value & State rank \\
\hline 36 & 41 \\
9.62 & 23 \\
5.43 & 24 \\
408 & 41 \\
11.3 & 38
\end{tabular}

Percent**

$\underline{\text { Deaths* }} \underline{\text { distribution }}$

2261.1

12.8

$13 \quad 36.1$

$0 \quad 0.0$

$0 \quad 0.0$

Unspecified/Other pneumoconiosis

* Sum of column may exceed total deaths because of deaths with multiple pneumoconioses.

** Percentages may total more than $100 \%$ due to deaths with multiple pneumoconioses.

NOTE: $\quad$ See Appendix A for source description and Appendix B for methods and ICD-8 and ICD-9 codes.

SOURCE: National Center for Health Statistics multiple cause of death data. Population estimates from U.S. Bureau of the Census.

\section{Figure ID-1. All pneumoconioses: Idaho and U.S. age-adjusted mortality rates, residents age 15 and over, 1968-1992}

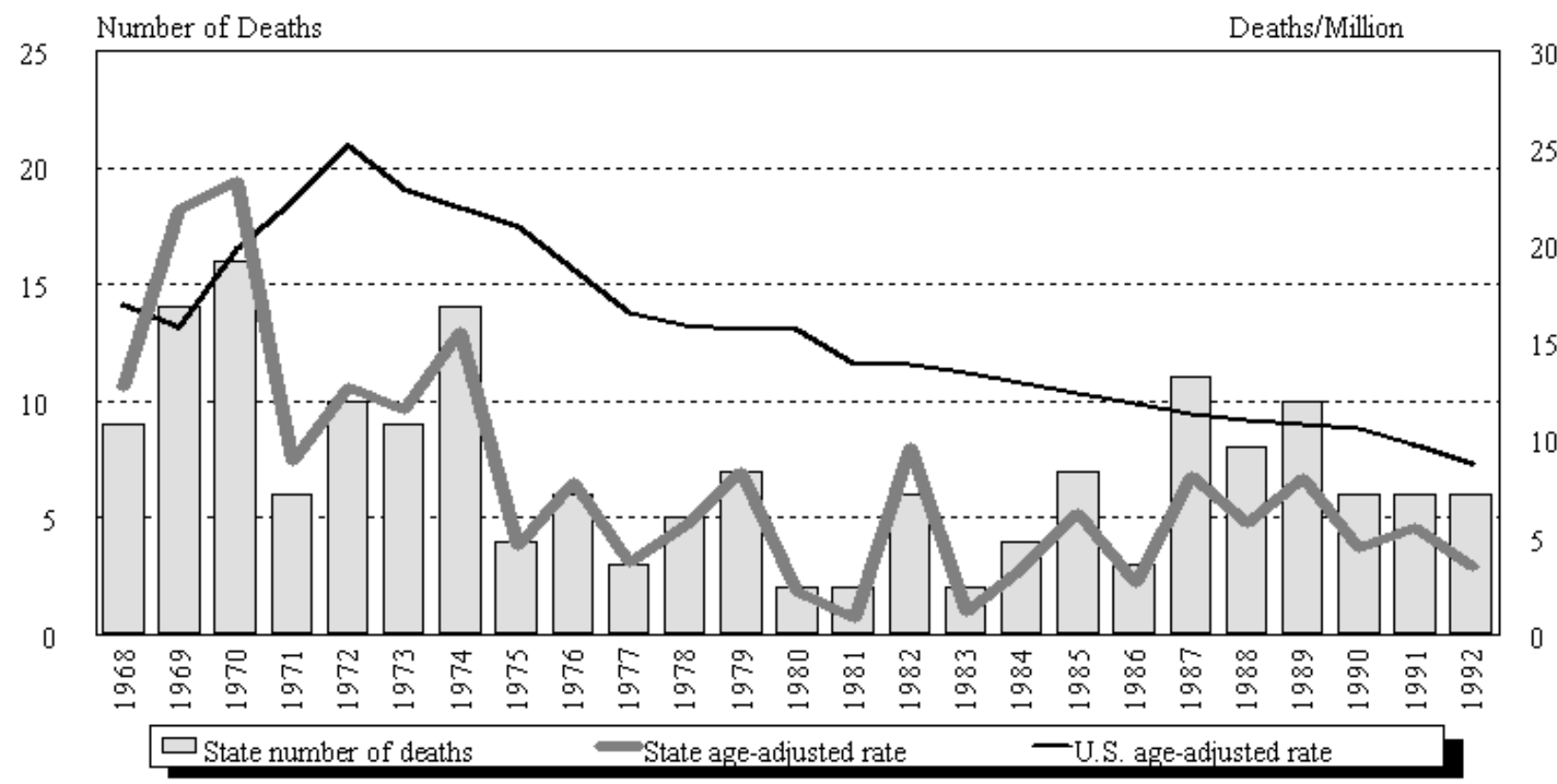

NOTE: See Appendix A for source description and Appendix B for methods and ICD-8 and ICD-9 codes.

SOURCE: National Center for Health Statistics multiple cause of death data. Population estimates from U.S. Bureau of the Census. 


\section{Idaho}

Table ID-1. Number of deaths and age-adjusted mortality rate (per $1,000,000$ population) by condition, Idaho residents age 15 and over, 1968-1992

\begin{tabular}{|c|c|c|c|c|c|c|c|c|c|c|c|c|}
\hline \multirow[b]{2}{*}{ Year } & \multicolumn{2}{|c|}{ Asbestosis } & \multicolumn{2}{|c|}{$\begin{array}{l}\text { Coal workers' } \\
\text { pneumoconiosis }\end{array}$} & \multicolumn{2}{|c|}{ Silicosis } & \multicolumn{2}{|c|}{ Byssinosis* } & \multicolumn{2}{|c|}{$\begin{array}{c}\text { Unspecified/Other } \\
\text { pneu moconioses }\end{array}$} & \multicolumn{2}{|c|}{ All pneumoconioses } \\
\hline & Number & Rate & Number & Rate & Number & Rate & Number & Rate & Number & Rate & Number & Rate \\
\hline 1968 & - & - & - & - & 9 & 12.75 & & & - & - & 9 & 12.75 \\
\hline 1969 & - & - & - & - & 14 & 21.87 & & & - & - & 14 & 21.87 \\
\hline 1970 & - & - & - & - & 15 & 21.71 & & & 1 & 1.63 & 16 & 23.34 \\
\hline 1971 & - & - & - & - & 5 & 7.25 & & & 1 & 1.60 & 6 & 8.85 \\
\hline 1972 & - & - & - & - & 8 & 10.64 & & & 2 & 2.04 & 10 & 12.68 \\
\hline 1973 & 1 & 1.55 & 1 & 1.43 & 7 & 8.56 & & & - & - & 9 & 11.54 \\
\hline 1974 & 1 & 1.51 & - & - & 11 & 12.93 & & & 2 & 1.10 & 14 & 15.54 \\
\hline 1975 & - & - & - & - & 4 & 4.60 & & & - & - & 4 & 4.60 \\
\hline 1976 & 1 & 1.28 & - & - & 5 & 6.56 & & & 1 & 1.28 & 6 & 7.84 \\
\hline 1977 & - & - & - & - & 2 & 2.46 & & & 1 & 1.23 & 3 & 3.69 \\
\hline 1978 & - & - & - & - & 3 & 3.74 & & & 2 & 1.85 & 5 & 5.59 \\
\hline 1979 & 2 & 2.50 & - & - & 3 & 3.16 & - & - & 2 & 2.72 & 7 & 8.38 \\
\hline 1980 & - & - & - & - & 1 & 1.10 & - & - & 1 & 1.10 & 2 & 2.20 \\
\hline 1981 & - & - & - & - & 2 & 0.83 & - & - & - & - & 2 & 0.83 \\
\hline 1982 & 4 & 7.56 & - & - & 2 & 2.09 & - & - & - & - & 6 & 9.65 \\
\hline 1983 & 1 & 0.73 & - & - & 1 & 0.38 & - & - & - & - & 2 & 1.12 \\
\hline 1984 & 2 & 1.70 & - & - & 2 & 1.70 & - & - & - & - & 4 & 3.40 \\
\hline 1985 & 2 & 2.27 & 2 & 1.35 & 1 & 0.99 & - & - & 2 & 1.66 & 7 & 6.26 \\
\hline 1986 & 3 & 2.61 & - & - & - & - & - & - & - & - & 3 & 2.61 \\
\hline 1987 & 6 & 5.23 & 1 & 0.35 & 4 & 2.59 & - & - & - & - & 11 & 8.18 \\
\hline 1988 & 4 & 3.56 & - & - & 4 & 2.20 & - & - & - & - & 8 & 5.75 \\
\hline 1989 & 5 & 4.37 & - & - & 5 & 3.67 & - & - & - & - & 10 & 8.04 \\
\hline 1990 & 4 & 3.19 & - & - & 2 & 1.25 & - & - & - & - & 6 & 4.44 \\
\hline 1991 & 6 & 5.52 & - & - & - & - & - & - & - & - & 6 & 5.52 \\
\hline 1992 & 3 & 1.95 & 1 & 0.53 & 2 & 1.06 & - & - & - & - & 6 & 3.54 \\
\hline
\end{tabular}

indicates no deaths listed. $\quad *$ no specific code for byssinosis in ICD-8 (1968-1978)

NOTE: Rates based on small numbers may be unstable. Sum of individ ual conditions may exceed total because of deaths with multiple pneumoconioses. See Appendix A for source description and Appendix B for methods and ICD-8 and ICD-9 NoTe:

SOURCE: National Center for Health Statistics multiple cause of death data. Population estimates from U.S. Bureau of the Census. 
Figure ID-2. All pneumoconioses: Distribution of deaths by sex and race, Idaho residents age 15 and over, 1968-1992
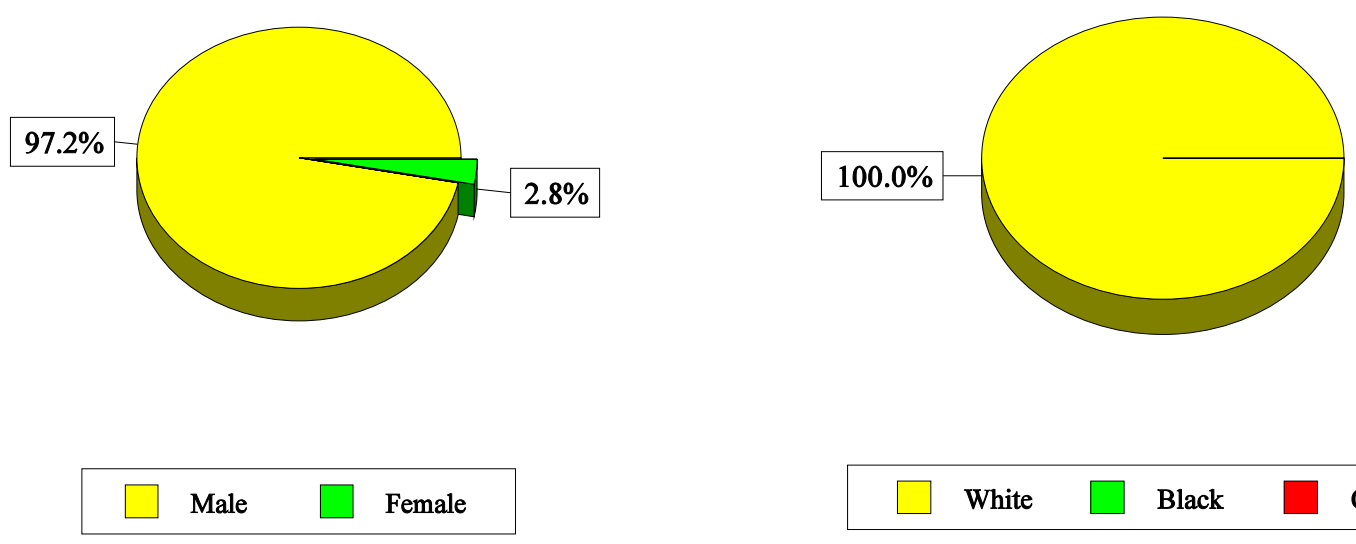

NOTE:

See Appendix A for source description and Appendix B for methods and ICD-8 and ICD-9 codes.

SOURCE: National Center for Health Statistics multiple cause of death data.

Table ID-2. All pneumoconioses: Distribution of deaths by sex, race, and age, Idaho residents age 15 and over, 1968-1992

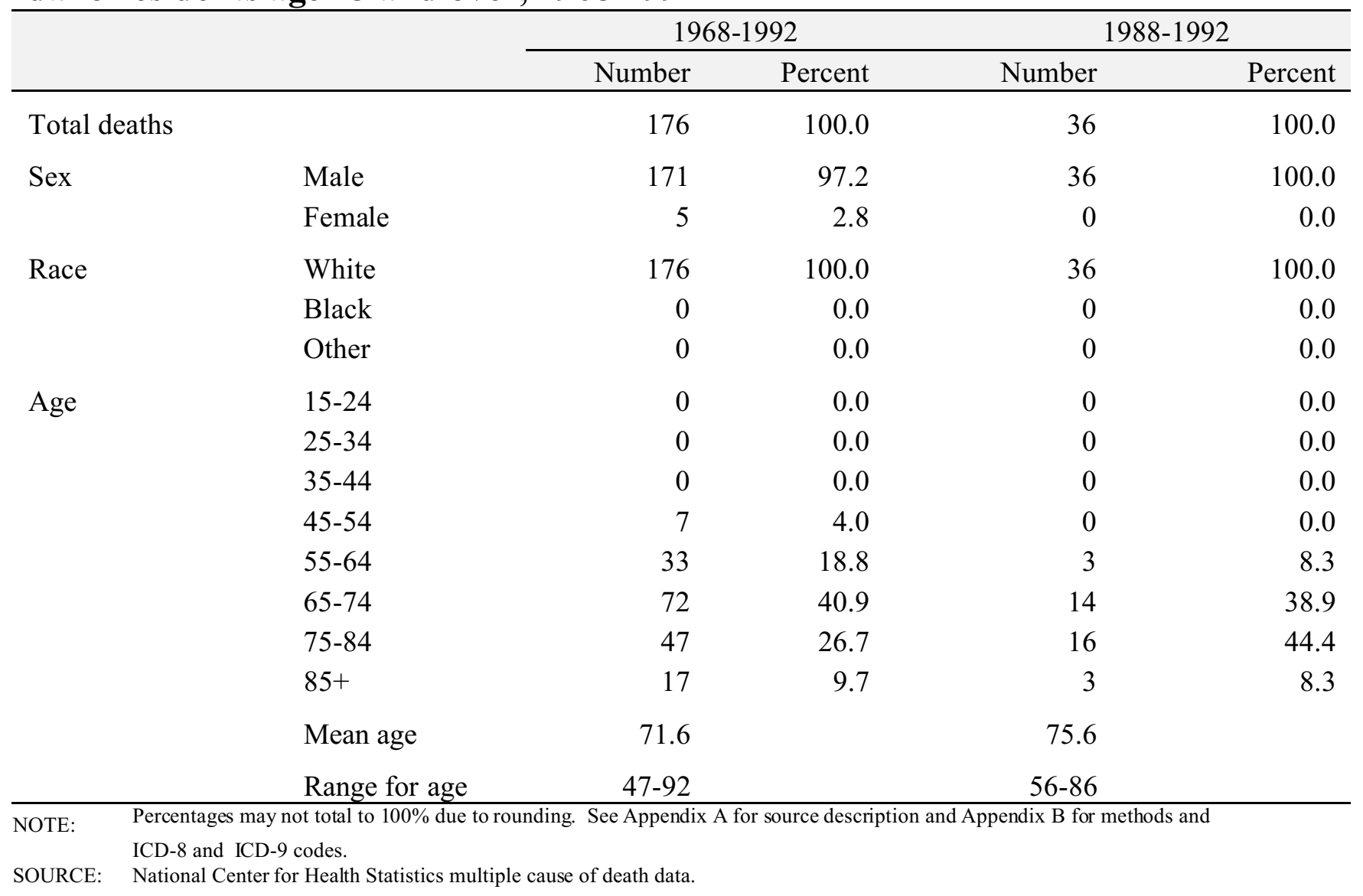


Figure ID-3. Pneumoconiosis deaths by condition, Idaho residents age 15 and over, 1968-1992

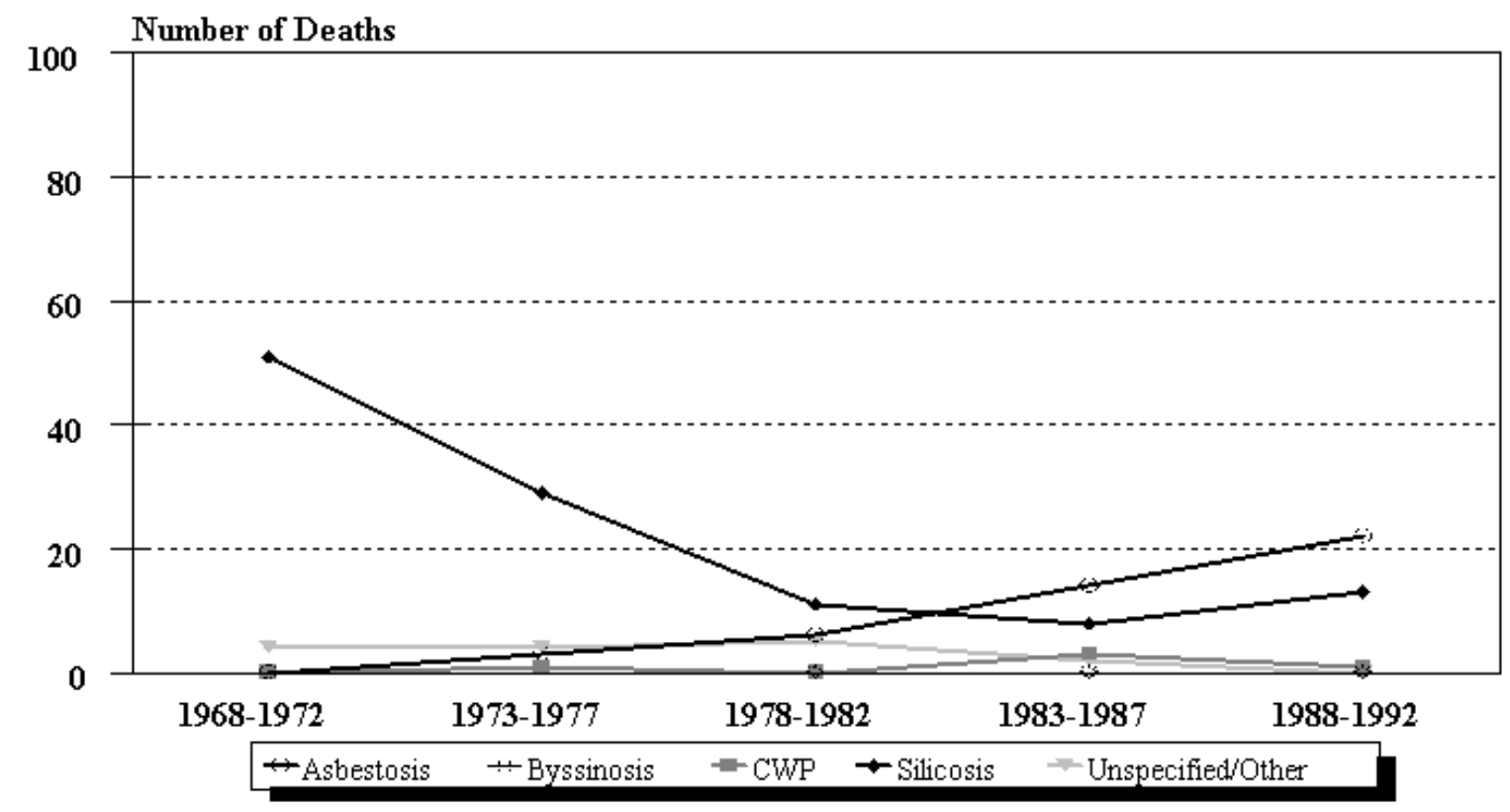

NOTE: See Appendix A for source description and Appendix B for methods and ICD-8 and ICD-9 codes. SOURCE: National Center for Health Statistics multiple cause of death data.

Figure ID-4. Idaho and U.S. age-adjusted mortality rates (per 1,000,000 population) by condition, residents age 15 and over, 1983-1992

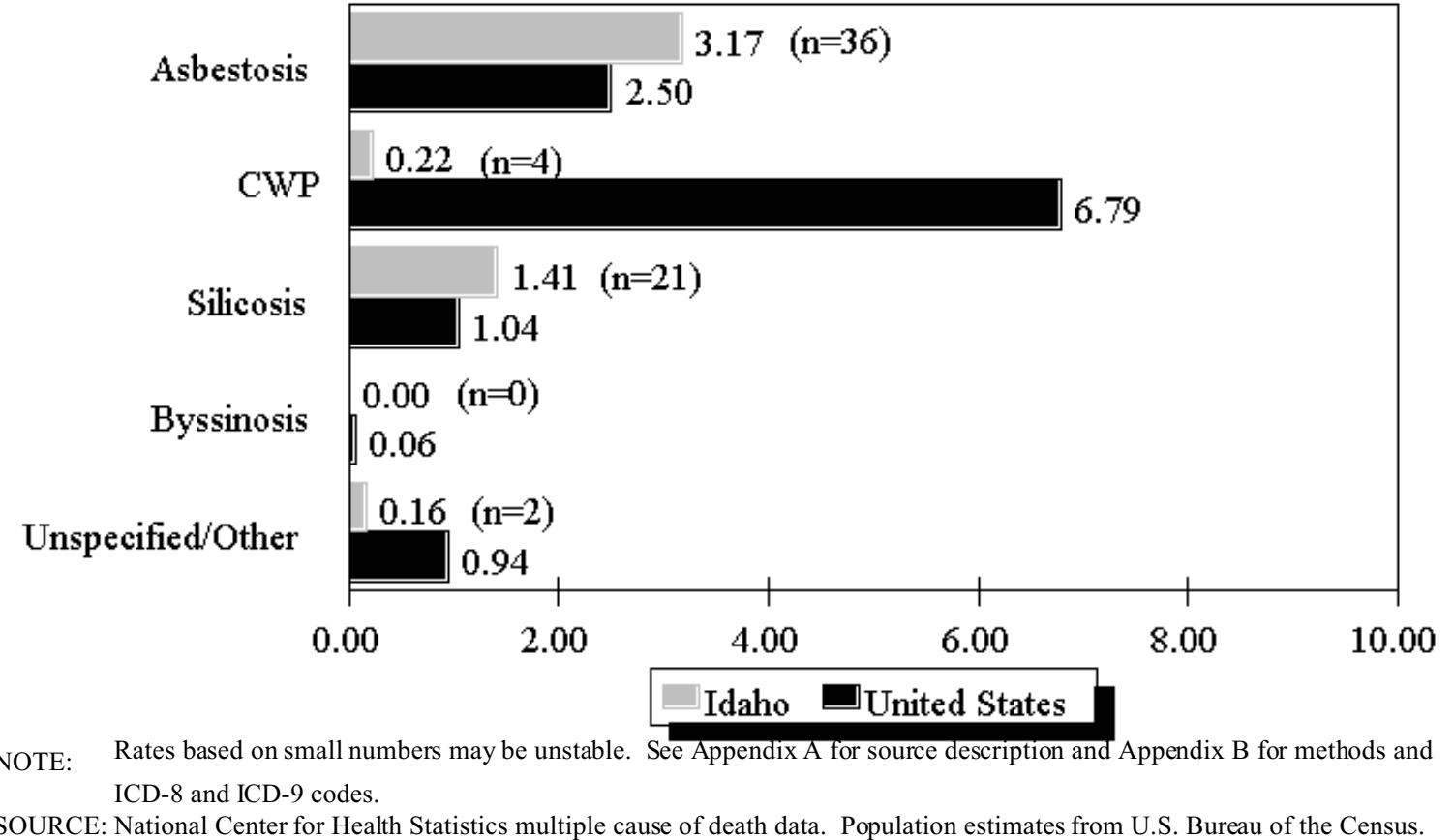


Table ID-3. Number of deaths and age-adjusted mortality rate (per 1,000,000 population) by condition and county, Idaho residents age 15 and over, 1983-1992

\begin{tabular}{|c|c|c|c|c|c|c|c|c|c|c|c|c|}
\hline \multirow[b]{2}{*}{ County } & \multicolumn{2}{|c|}{ Asbestosis } & \multicolumn{2}{|c|}{$\begin{array}{c}\text { Coal workers' } \\
\text { pneumoconiosis }\end{array}$} & \multicolumn{2}{|c|}{ Silicosis } & \multicolumn{2}{|c|}{ Byssinosis } & \multicolumn{2}{|c|}{$\begin{array}{r}\text { Unspecified/Other } \\
\text { pneumoconioses }\end{array}$} & \multicolumn{2}{|c|}{$\begin{array}{r}\text { All } \\
\text { pneumoconioses }\end{array}$} \\
\hline & $\#$ & Rate & $\#$ & Rate & $\#$ & Rate & \# & Rate & \# & Rate & \# & Rate \\
\hline Ada & 2 & 0.66 & 1 & 0.33 & 3 & 1.33 & - & - & 1 & 0.33 & 7 & 2.65 \\
\hline Bannock & 5 & 7.20 & 1 & 1.03 & - & - & - & - & - & - & 6 & 8.22 \\
\hline Bingham & - & - & - & - & 1 & 3.48 & - & - & - & - & 1 & 3.48 \\
\hline Blaine & 1 & 9.14 & - & - & 1 & 9.14 & - & - & - & - & 2 & 18.28 \\
\hline Bonner & 1 & 3.59 & - & - & 1 & 1.97 & - & - & - & - & 2 & 5.56 \\
\hline Bonneville & 2 & 3.92 & - & - & - & - & - & - & - & - & 2 & 3.92 \\
\hline Boundary & 1 & 13.61 & - & - & - & - & - & - & - & - & 1 & 13.61 \\
\hline Butte & - & - & - & - & 1 & 33.41 & - & - & - & - & 1 & 33.41 \\
\hline Canyon & 4 & 4.26 & - & - & 4 & 2.09 & - & - & - & - & 8 & 6.35 \\
\hline Cassia & 2 & 8.60 & - & - & - & - & - & - & - & - & 2 & 8.60 \\
\hline Clearwater & 1 & 10.24 & - & - & - & - & - & - & - & - & 1 & 10.24 \\
\hline Idaho & - & - & - & - & - & - & - & - & 1 & 6.45 & 1 & 6.45 \\
\hline Kootenai & 6 & 7.57 & - & - & 2 & 1.56 & - & - & - & - & 8 & 9.13 \\
\hline Latah & 1 & 1.95 & - & - & - & - & - & - & - & - & 1 & 1.95 \\
\hline Minidoka & 2 & 10.95 & - & - & 1 & 5.47 & - & - & - & - & 3 & 16.42 \\
\hline Nez Perce & 2 & 5.65 & - & - & 1 & 1.21 & - & - & - & - & 3 & 6.86 \\
\hline Owyhee & 1 & 6.07 & - & - & - & - & - & - & - & - & 1 & 6.07 \\
\hline Payette & 2 & 8.21 & - & - & - & - & - & - & - & - & 2 & 8.21 \\
\hline Shoshone & 2 & 11.88 & - & - & 5 & 20.78 & - & - & - & - & 7 & 32.66 \\
\hline Twin Falls & 1 & 1.89 & 2 & 1.56 & 1 & 0.78 & - & - & - & - & 4 & 4.23 \\
\hline
\end{tabular}




\section{Illinois: Selected statistics from the United States section}

\section{All pneumoconioses mortality, 1988-1992}

Total deaths

Crude mortality rate (per $1,000,000$ population)

Age-adjusted mortality rate (per 1,000,000 population)

Total years of potential life lost to life expectancy

Years of potential life lost to life expectancy (per death)

\begin{tabular}{rr} 
Value & State rank \\
\hline 476 & 9 \\
10.64 & 21 \\
5.95 & 20 \\
5,544 & 9 \\
11.6 & 32
\end{tabular}

Percent**

Deaths*

distribution

96

20.2

229

48.1

50

10.5

2

0.4

102

21.4

\section{Unspecified/Other pneumoconiosis}

* Sum of column may exceed total deaths because of deaths with multiple pneumoconioses.

** Percentages may total more than $100 \%$ due to deaths with multiple pneumoconioses.

NOTE: $\quad$ See Appendix A for source description and Appendix B for methods and ICD-8 and ICD-9 codes.

SOURCE: $\quad$ National Center for Health Statistics multiple cause of death data. Population estimates from U.S. Bureau of the Census.

\section{Figure IL-1. All pneumoconioses: Illinois and U.S. age-adjusted mortality rates, residents age 15 and over, 1968-1992}

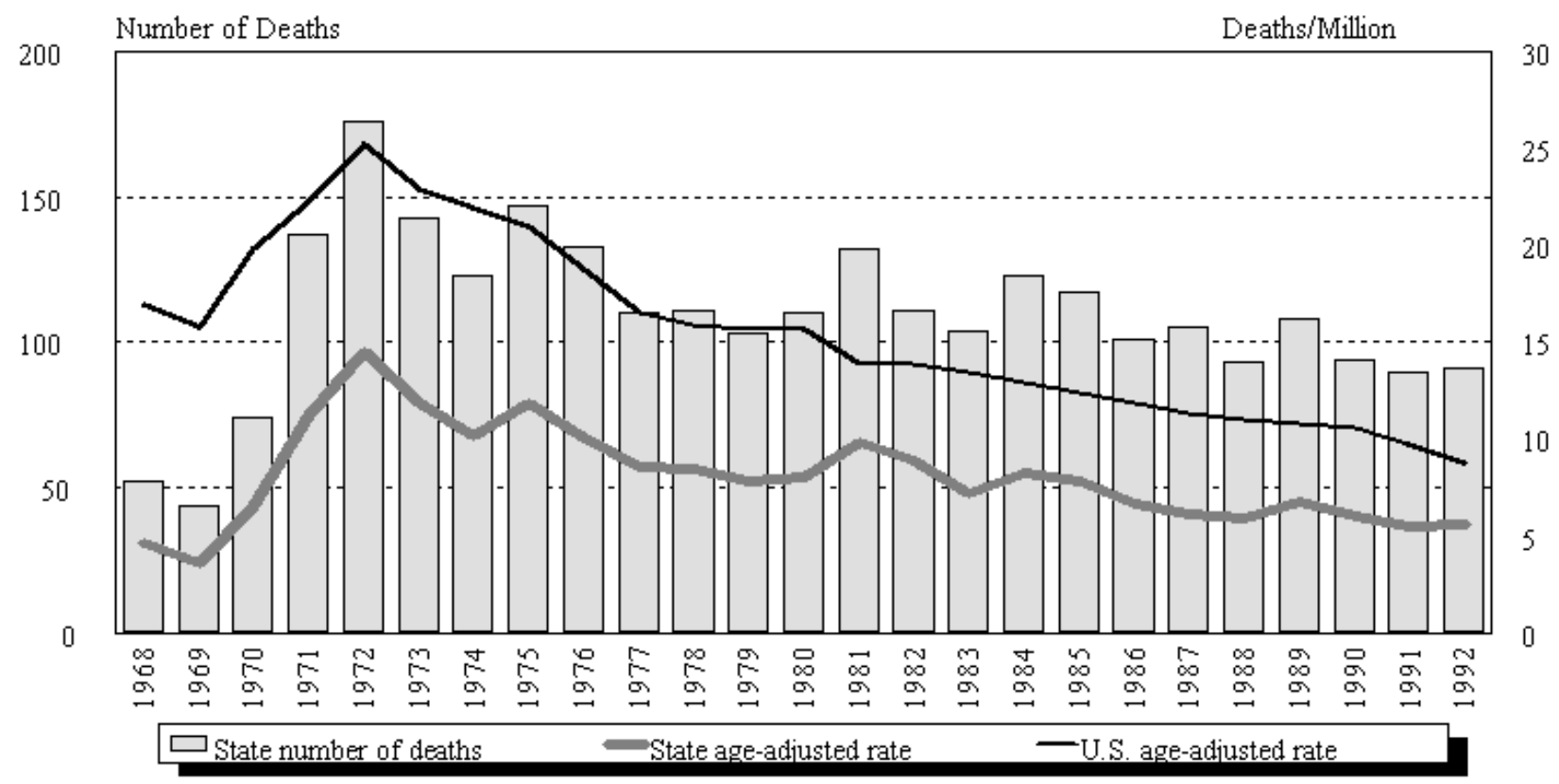

NOTE: $\quad$ See Appendix A for source description and Appendix B for methods and ICD-8 and ICD-9 codes.

SOURCE: $\quad$ National Center for Health Statistics multiple cause of death data. Population estimates from U.S. Bureau of the Census. 
Illinois

Table IL-1. Number of deaths and age-adjusted mortality rate (per 1,000,000 population) by condition, Illinois residents age 15 and over, 1968-1992

\begin{tabular}{|c|c|c|c|c|c|c|c|c|c|c|c|c|}
\hline \multirow[b]{2}{*}{ Year } & \multicolumn{2}{|c|}{ Asbestosis } & \multicolumn{2}{|c|}{$\begin{array}{l}\text { Coal workers' } \\
\text { pne umocon iosis }\end{array}$} & \multicolumn{2}{|c|}{ Silicosis } & \multicolumn{2}{|c|}{ Byssinosis* } & \multicolumn{2}{|c|}{$\begin{array}{l}\text { Unsp ecified/Other } \\
\text { pneumoconioses }\end{array}$} & \multicolumn{2}{|c|}{ All pneumoconioses } \\
\hline & Number & Rate & Number & Rate & Number & Rate & Number & Rate & Number & Rate & Number & Rate \\
\hline 1968 & 3 & 0.33 & 6 & 0.39 & 27 & 2.51 & & & 17 & 1.50 & 52 & 4.65 \\
\hline 1969 & 1 & 0.10 & 8 & 0.58 & 20 & 1.72 & & & 15 & 1.19 & 44 & 3.60 \\
\hline 1970 & 5 & 0.57 & 24 & 1.99 & 17 & 1.55 & & & 29 & 2.54 & 74 & 6.55 \\
\hline 1971 & 3 & 0.29 & 40 & 3.39 & 19 & 1.69 & & & 76 & 5.92 & 137 & 11.19 \\
\hline 1972 & 6 & 0.59 & 36 & 2.97 & 24 & 2.09 & & & 112 & 8.96 & 176 & 14.48 \\
\hline 1973 & 1 & 0.09 & 33 & 2.96 & 20 & 1.67 & & & 89 & 7.11 & 143 & 11.83 \\
\hline 1974 & 5 & 0.48 & 20 & 1.86 & 15 & 1.27 & & & 86 & 6.85 & 123 & 10.15 \\
\hline 1975 & 2 & 0.22 & 40 & 3.09 & 21 & 1.77 & & & 86 & 6.96 & 147 & 11.92 \\
\hline 1976 & 3 & 0.30 & 33 & 2.53 & 13 & 1.08 & & & 88 & 6.53 & 133 & 10.07 \\
\hline 1977 & 6 & 0.57 & 34 & 2.53 & 6 & 0.43 & & & 65 & 5.12 & 110 & 8.55 \\
\hline 1978 & 5 & 0.42 & 31 & 2.45 & 9 & 0.64 & & & 69 & 5.23 & 111 & 8.51 \\
\hline 1979 & 6 & 0.55 & 36 & 2.63 & 13 & 1.11 & - & - & 48 & 3.60 & 103 & 7.90 \\
\hline 1980 & 8 & 0.70 & 41 & 2.81 & 15 & 1.37 & 1 & 0.06 & 45 & 3.17 & 110 & 8.11 \\
\hline 1981 & 13 & 1.29 & 86 & 6.01 & 11 & 0.90 & - & - & 22 & 1.63 & 132 & 9.83 \\
\hline 1982 & 4 & 0.39 & 68 & 4.85 & 21 & 2.27 & - & - & 20 & 1.58 & 111 & 8.92 \\
\hline 1983 & 11 & 0.89 & 68 & 4.50 & 11 & 0.84 & - & - & 14 & 0.96 & 104 & 7.19 \\
\hline 1984 & 11 & 0.85 & 83 & 5.41 & 17 & 1.22 & - & - & 14 & 0.92 & 123 & 8.26 \\
\hline 1985 & 14 & 1.08 & 78 & 5.19 & 15 & 1.09 & - & - & 12 & 0.60 & 117 & 7.80 \\
\hline 1986 & 15 & 1.33 & 64 & 4.10 & 11 & 0.71 & 1 & 0.13 & 12 & 0.64 & 101 & 6.71 \\
\hline 1987 & 10 & 0.79 & 63 & 3.46 & 15 & 1.10 & - & - & 18 & 0.85 & 105 & 6.11 \\
\hline 1988 & 11 & 0.97 & 53 & 3.20 & 12 & 0.74 & - & - & 18 & 1.08 & 93 & 5.90 \\
\hline 1989 & 26 & 1.78 & 38 & 2.52 & 9 & 0.54 & - & - & 36 & 1.95 & 108 & 6.74 \\
\hline 1990 & 18 & 1.22 & 49 & 2.83 & 10 & 0.88 & 1 & 0.11 & 16 & 0.99 & 94 & 6.03 \\
\hline 1991 & 20 & 1.51 & 43 & 2.29 & 8 & 0.69 & - & - & 19 & 1.04 & 90 & 5.53 \\
\hline 1992 & 21 & 1.51 & 46 & 2.52 & 11 & 0.86 & 1 & 0.15 & 13 & 0.67 & 91 & 5.59 \\
\hline
\end{tabular}

-indicates no deaths listed. * * no sp ecific code for byssinosis in ICD-8 (1968-1978)

NOTE: Rates based on small numbers may be unstable. Sum of individual conditions may exceed total because of deaths with multiple pneumoconioses. See Appendix A for source description and Appendix B for methods and ICD-8 and ICD-9 codes.

SOURCE: National Center for Health Statistics multiple cause of death data. Population estimates from U.S. Bureau of the Census. 


\section{Figure IL-2. All pneumoconioses: Distribution of deaths by sex and race, Illinois residents age 15 and over, 1968-1992}
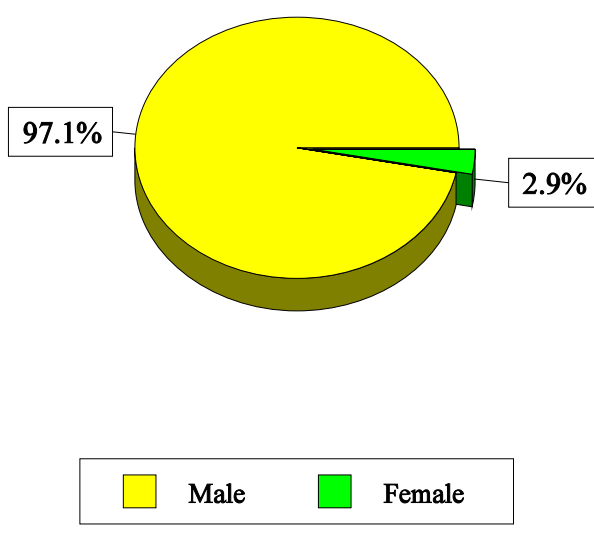

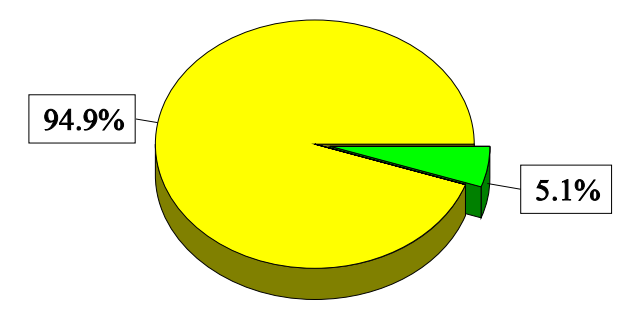

NOTE: $\quad$ See Appendix A for source description and Appendix B for methods and ICD-8 and ICD-9 codes.

SOURCE: National Center for Health Statistics multiple cause of death data.

Table IL-2. All pneumoconioses: Distribution of deaths by sex, race, and age, Illinois residents age 15 and over, 1968-1992

\begin{tabular}{|c|c|c|c|c|c|}
\hline & & \multicolumn{2}{|c|}{ 1968-1992 } & \multicolumn{2}{|c|}{ 1988-1992 } \\
\hline & & Number & Percent & Number & Percent \\
\hline Total deaths & & 2,732 & 100.0 & 476 & 100.0 \\
\hline \multirow[t]{2}{*}{ Sex } & Male & 2,652 & 97.1 & 459 & 96.4 \\
\hline & Female & 80 & 2.9 & 17 & 3.6 \\
\hline \multirow[t]{3}{*}{ Race } & White & 2,592 & 94.9 & 444 & 93.3 \\
\hline & Black & 140 & 5.1 & 32 & 6.7 \\
\hline & Other & 0 & 0.0 & 0 & 0.0 \\
\hline \multirow[t]{10}{*}{ Age } & $15-24$ & 2 & 0.1 & 1 & 0.2 \\
\hline & $25-34$ & 6 & 0.2 & 1 & 0.2 \\
\hline & $35-44$ & 24 & 0.9 & 4 & 0.8 \\
\hline & $45-54$ & 107 & 3.9 & 11 & 2.3 \\
\hline & $55-64$ & 382 & 14.0 & 43 & 9.0 \\
\hline & $65-74$ & 887 & 32.5 & 141 & 29.6 \\
\hline & $75-84$ & 975 & 35.7 & 199 & 41.8 \\
\hline & $85+$ & 349 & 12.8 & 76 & 16.0 \\
\hline & Mean age & 73.2 & & 75.2 & \\
\hline & Range for age & $24-118$ & & $24-95$ & \\
\hline
\end{tabular}

NOTE: $\quad$ Percentages may not total to $100 \%$ due to rounding. See Appendix A for source description and Appendix B for methods and ICD-8 and ICD-9 codes.

SOURCE: National Center for Health Statistics multiple cause of death data. 


\section{Illinois}

Figure IL-3. Pneumoconiosis deaths by condition, Illinois residents age 15 and over, 1968-1992

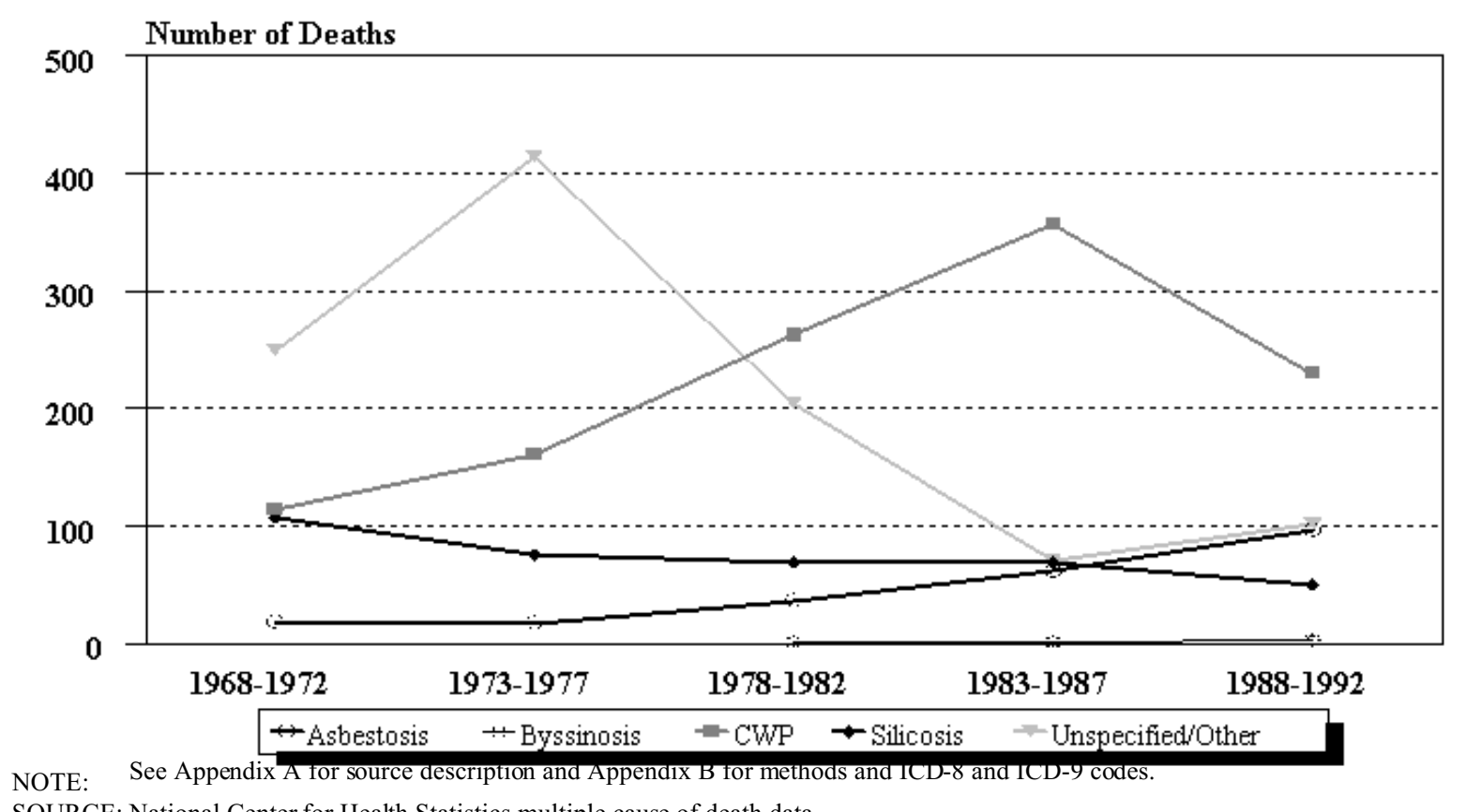

SOURCE: National Center for Health Statistics multiple cause of death data.

Figure IL-4. Illinois and U.S. age-adjusted mortality rates (per 1,000,000 population) by condition, residents age 15 and over, 1983-1992

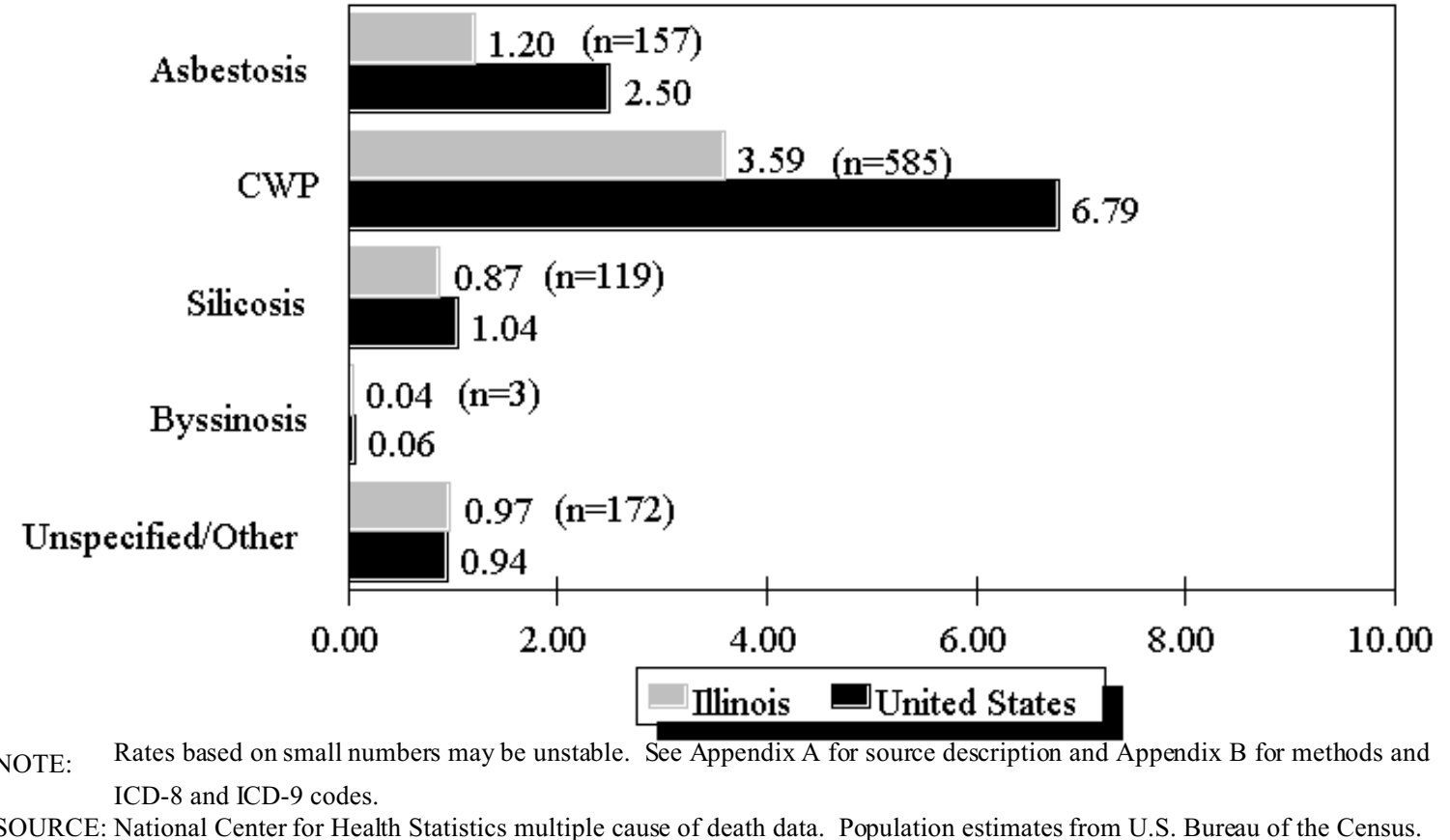


Table IL-3 (page 1 of 3). Number of deaths and age-adjusted mortality rate (per $1,000,000$ population) by condition and county, Illinois residents age 15 and over, 1983-1992

\begin{tabular}{|c|c|c|c|c|c|c|c|c|c|c|c|c|}
\hline \multirow[b]{2}{*}{ County } & \multicolumn{2}{|c|}{ Asbestosis } & \multicolumn{2}{|c|}{$\begin{array}{c}\text { Coal workers' } \\
\text { pneumoconiosis }\end{array}$} & \multicolumn{2}{|c|}{ Silicosis } & \multicolumn{2}{|c|}{ Byssinosis } & \multicolumn{2}{|c|}{$\begin{array}{r}\text { Unspecified/Other } \\
\text { pneumoconioses }\end{array}$} & \multicolumn{2}{|c|}{$\begin{array}{r}\text { All } \\
\text { pneumoconioses }\end{array}$} \\
\hline & \# & Rate & \# & Rate & \# & Rate & $\#$ & Rate & \# & Rate & \# & Rate \\
\hline Adams & - & - & - & - & 2 & 1.02 & - & - & - & - & 2 & 1.02 \\
\hline Alexander & - & - & 1 & 7.79 & 1 & 7.79 & - & - & - & - & 2 & 15.58 \\
\hline Bond & 1 & 5.87 & - & - & - & - & - & - & - & - & 1 & 5.87 \\
\hline Boone & - & - & - & - & 1 & 1.83 & - & - & - & - & 1 & 1.83 \\
\hline Bureau & 1 & 2.43 & - & - & - & - & - & - & - & - & 1 & 2.43 \\
\hline Champaign & 1 & 0.89 & 2 & 0.87 & - & - & - & - & 1 & 0.89 & 4 & 2.65 \\
\hline Christian & 2 & 5.28 & 32 & 63.83 & 3 & 2.87 & - & - & 3 & 4.44 & 39 & 75.46 \\
\hline Clinton & 1 & 3.07 & 1 & 1.34 & 1 & 1.34 & - & - & 1 & 1.34 & 4 & 7.09 \\
\hline Coles & 1 & 0.85 & - & - & 1 & 2.14 & - & - & - & - & 2 & 3.00 \\
\hline Cook & 34 & 0.62 & 45 & 0.70 & 30 & 0.55 & - & - & 22 & 0.35 & 131 & 2.22 \\
\hline Crawford & 1 & 4.18 & - & - & - & - & - & - & - & - & 1 & 4.18 \\
\hline De Kalb & 2 & 2.61 & - & - & - & - & - & - & - & - & 2 & 2.61 \\
\hline De Witt & - & - & 1 & 2.27 & 1 & 8.76 & - & - & - & - & 2 & 11.03 \\
\hline Douglas & - & - & 4 & 13.61 & - & - & - & - & - & - & 4 & 13.61 \\
\hline du Page & 5 & 0.82 & 2 & 0.29 & 3 & 0.40 & - & - & 2 & 0.29 & 12 & 1.79 \\
\hline Edgar & - & - & 1 & 4.19 & 1 & 7.43 & - & - & 1 & 4.19 & 3 & 15.80 \\
\hline Effingham & 1 & 1.38 & - & - & - & - & - & - & - & - & 1 & 1.38 \\
\hline Fayette & - & - & - & - & - & - & 1 & 7.27 & - & - & 1 & 7.27 \\
\hline Ford & 1 & 5.86 & - & - & - & - & - & - & - & - & 1 & 5.86 \\
\hline Franklin & 2 & 3.90 & 152 & 162.55 & 2 & 3.90 & - & - & 56 & 56.31 & 212 & 226.65 \\
\hline Fulton & - & - & 17 & 23.97 & - & - & - & - & 1 & 0.84 & 18 & 24.81 \\
\hline Gallatin & - & - & 3 & 35.72 & - & - & - & - & - & - & 3 & 35.72 \\
\hline Greene & - & - & 1 & 2.02 & - & - & - & - & 1 & 2.02 & 2 & 4.05 \\
\hline Grundy & - & - & 3 & 6.61 & 1 & 1.59 & - & - & - & - & 4 & 8.20 \\
\hline Hamilton & - & - & 2 & 26.65 & - & - & - & - & - & - & 2 & 26.65 \\
\hline Hardin & - & - & 1 & 14.87 & 1 & 7.03 & - & - & 1 & 14.87 & 3 & 36.76 \\
\hline Henderson & - & - & 1 & 10.36 & - & - & - & - & - & - & 1 & 10.36 \\
\hline Henry & - & - & - & - & 1 & 0.72 & 1 & 3.12 & - & - & 2 & 3.84 \\
\hline Iroquois & - & - & 1 & 1.09 & - & - & - & - & 1 & 1.09 & 2 & 2.17 \\
\hline Jackson & 1 & 2.26 & 8 & 10.07 & - & - & - & - & 1 & 0.93 & 10 & 13.26 \\
\hline Jefferson & 2 & 2.00 & 12 & 19.35 & - & - & - & - & 2 & 4.94 & 16 & 26.29 \\
\hline Johnson & - & - & 2 & 7.29 & - & - & - & - & 1 & 8.15 & 3 & 15.43 \\
\hline Kane & 1 & 0.22 & 5 & 1.78 & 3 & 0.88 & - & - & 1 & 0.22 & 10 & 3.11 \\
\hline
\end{tabular}

See footnotes at end of table. 
Table IL-3 (page 2 of 3). Number of deaths and age-adjusted mortality rate (per $1,000,000$ population) by condition and county, Illinois residents age 15 and over, 1983-1992

\begin{tabular}{|c|c|c|c|c|c|c|c|c|c|c|c|c|}
\hline \multirow[b]{2}{*}{ County } & \multicolumn{2}{|c|}{ Asbestosis } & \multicolumn{2}{|c|}{$\begin{array}{r}\text { Coal workers' } \\
\text { pneumoconiosis }\end{array}$} & \multicolumn{2}{|c|}{ Silicosis } & \multicolumn{2}{|c|}{ Byssinosis } & \multicolumn{2}{|c|}{$\begin{array}{r}\text { Unspecified/Other } \\
\text { pneumoconioses }\end{array}$} & \multicolumn{2}{|c|}{$\begin{array}{r}\text { All } \\
\text { pneumoconioses }\end{array}$} \\
\hline & \# & Rate & \# & Rate & \# & Rate & \# & Rate & $\#$ & Rate & \# & Rate \\
\hline Kankakee & - & - & 1 & 0.52 & 1 & 0.52 & - & - & - & - & 2 & 1.03 \\
\hline Kendall & - & - & - & - & 1 & 3.63 & - & - & - & - & 1 & 3.63 \\
\hline Knox & - & - & 8 & 8.73 & - & - & - & - & 2 & 1.24 & 10 & 9.97 \\
\hline Lake & 38 & 8.93 & 8 & 1.77 & 6 & 1.64 & - & - & 2 & 0.55 & 50 & 11.90 \\
\hline La Salle & 2 & 0.68 & 3 & 1.49 & 5 & 3.61 & - & - & 2 & 0.68 & 12 & 6.45 \\
\hline Lawrence & 1 & 1.59 & - & - & 1 & 9.47 & - & - & - & - & 2 & 11.06 \\
\hline Livingston & 1 & 2.52 & 1 & 2.52 & - & - & - & - & 1 & 2.52 & 3 & 7.55 \\
\hline Logan & 1 & 1.17 & - & - & - & - & - & - & - & - & 1 & 1.17 \\
\hline McDonough & - & - & 1 & 1.21 & - & - & - & - & - & - & 1 & 1.21 \\
\hline McHenry & 2 & 1.18 & 3 & 1.95 & - & - & - & - & - & - & 5 & 3.13 \\
\hline McLean & 9 & 9.80 & 2 & 0.94 & - & - & - & - & - & - & 11 & 10.75 \\
\hline Macon & 2 & 1.17 & - & - & - & - & - & - & - & - & 2 & 1.17 \\
\hline Macoupin & 2 & 5.02 & 5 & 6.77 & 3 & 5.70 & - & - & 2 & 2.48 & 11 & 16.76 \\
\hline Madison & 16 & 5.08 & 8 & 2.53 & 8 & 2.34 & - & - & 1 & 0.19 & 32 & 9.75 \\
\hline Marion & - & - & 1 & 0.83 & - & - & 1 & 3.78 & - & - & 2 & 4.61 \\
\hline Marshall & - & - & 3 & 7.42 & - & - & - & - & - & - & 3 & 7.42 \\
\hline Massac & 1 & 2.15 & - & - & 1 & 5.48 & - & - & - & - & 2 & 7.63 \\
\hline Menard & - & - & 2 & 6.61 & - & - & - & - & - & - & 2 & 6.61 \\
\hline Mercer & - & - & 1 & 5.40 & - & - & - & - & - & - & 1 & 5.40 \\
\hline Monroe & - & - & 1 & 4.66 & - & - & - & - & - & - & 1 & 4.66 \\
\hline Montgomery & - & - & 2 & 3.87 & - & - & - & - & - & - & 2 & 3.87 \\
\hline Morgan & 2 & 3.65 & - & - & - & - & - & - & 1 & 1.00 & 3 & 4.65 \\
\hline Moultrie & - & - & 1 & 2.01 & - & - & - & - & - & - & 1 & 2.01 \\
\hline Ogle & - & - & - & - & - & - & - & - & 1 & 2.26 & 1 & 2.26 \\
\hline Peoria & 2 & 1.11 & 9 & 2.83 & 2 & 0.80 & - & - & 1 & 0.25 & 14 & 4.98 \\
\hline Perry & - & - & 16 & 38.43 & 2 & 5.76 & - & - & 8 & 15.40 & 25 & 55.44 \\
\hline Piatt & - & - & 1 & 2.63 & 1 & 2.63 & - & - & - & - & 2 & 5.26 \\
\hline Pike & 1 & 4.52 & - & - & - & - & - & - & 1 & 1.59 & 2 & 6.11 \\
\hline Pope & - & - & - & - & 1 & 7.46 & - & - & - & - & 1 & 7.46 \\
\hline Putnam & - & - & 1 & 7.81 & - & - & - & - & - & - & 1 & 7.81 \\
\hline Randolph & - & - & 10 & 23.80 & 3 & 10.21 & - & - & 1 & 1.10 & 14 & 35.11 \\
\hline Richland & - & - & 1 & 2.00 & - & - & - & - & - & - & 1 & 2.00 \\
\hline
\end{tabular}

See footnotes at end of table. 
Table IL-3 (page 3 of 3). Number of deaths and age-adjusted mortality rate (per $1,000,000$ population) by condition and county, Illinois residents age 15 and over, 1983-1992

\begin{tabular}{|c|c|c|c|c|c|c|c|c|c|c|c|c|}
\hline \multirow[b]{2}{*}{ County } & \multicolumn{2}{|c|}{ Asbestosis } & \multicolumn{2}{|c|}{$\begin{array}{r}\text { Coal workers' } \\
\text { pneumoconiosis }\end{array}$} & \multicolumn{2}{|c|}{ Silicosis } & \multicolumn{2}{|c|}{ Byssinosis } & \multicolumn{2}{|c|}{$\begin{array}{r}\text { Unspecified/Other } \\
\text { pneumoconioses }\end{array}$} & \multicolumn{2}{|c|}{$\begin{array}{r}\text { All } \\
\text { pneumoconioses }\end{array}$} \\
\hline & $\#$ & Rate & \# & Rate & $\#$ & Rate & $\#$ & Rate & \# & Rate & \# & Rate \\
\hline Rock Island & 2 & 0.92 & 5 & 2.46 & 4 & 1.50 & - & - & - & - & 11 & 4.88 \\
\hline St Clair & 7 & 1.99 & 11 & 2.36 & 10 & 3.38 & - & - & 3 & 0.79 & 30 & 8.11 \\
\hline Saline & 1 & 2.95 & 48 & 104.15 & - & - & - & - & 19 & 31.26 & 68 & 138.36 \\
\hline Sangamon & 2 & 1.17 & 18 & 7.76 & 1 & 0.59 & - & - & 1 & 0.25 & 21 & 9.19 \\
\hline Schuyler & - & - & 1 & 10.95 & - & - & - & - & - & - & 1 & 10.95 \\
\hline Shelby & - & - & 2 & 5.32 & 1 & 3.78 & - & - & - & - & 3 & 9.10 \\
\hline Stark & - & - & - & - & - & - & - & - & 1 & 4.62 & 1 & 4.62 \\
\hline Stephenson & - & - & - & - & 1 & 0.81 & - & - & - & - & 1 & 0.81 \\
\hline Tazewell & 1 & 0.82 & 1 & 0.41 & - & - & - & - & 2 & 0.82 & 4 & 2.04 \\
\hline Vermilion & - & - & 4 & 1.86 & 1 & 0.47 & - & - & - & - & 5 & 2.33 \\
\hline Warren & 1 & 4.72 & 2 & 3.51 & 1 & 4.72 & - & - & - & - & 4 & 12.94 \\
\hline Washington & - & - & - & - & - & - & - & - & 1 & 2.12 & 1 & 2.12 \\
\hline White & - & - & 4 & 12.44 & 1 & 4.57 & - & - & - & - & 5 & 17.01 \\
\hline Whiteside & 1 & 1.57 & - & - & 1 & 0.73 & - & - & - & - & 2 & 2.30 \\
\hline Will & 2 & 0.82 & 3 & 0.85 & 8 & 2.38 & - & - & 4 & 1.46 & 17 & 5.51 \\
\hline Williamson & - & - & 93 & 89.26 & - & - & - & - & 22 & 20.60 & 115 & 109.86 \\
\hline Winnebago & 2 & 0.84 & 5 & 1.27 & 3 & 0.64 & - & - & 1 & 0.21 & 11 & 2.97 \\
\hline Woodford & 1 & 3.33 & 2 & 2.64 & - & - & - & - & - & - & 3 & 5.98 \\
\hline
\end{tabular}




\section{Indiana: Selected statistics from the United States section}

All pneumoconioses mortality, 1988-1992

Total deaths

Crude mortality rate (per $1,000,000$ population)

Age-adjusted mortality rate (per 1,000,000 population)

Total years of potential life lost to life expectancy

Years of potential life lost to life expectancy (per death)

Deaths by type of pneumoconiosis, 1988-1992

Asbestosis

Coal workers' pneumoconiosis

Silicosis

Byssinosis

Unspecified/Other pneumoconiosis

\begin{tabular}{rr} 
Value & State rank \\
\hline 203 & 19 \\
9.38 & 24 \\
5.20 & 26 \\
2,350 & 19 \\
11.6 & 33
\end{tabular}

Percent**

Deaths*

distribution

26

12.8

123

60.6

28

13.8

0.5

12.8

* Sum of column may exceed total deaths because of deaths with multiple pneumoconioses.

** Percentages may total more than $100 \%$ due to deaths with multiple pneumoconioses.

NOTE: $\quad$ See Appendix A for source description and Appendix B for methods and ICD-8 and ICD-9 codes.

SOURCE: National Center for Health Statistics multiple cause of death data. Population estimates from U.S. Bureau of the Census.

\section{Figure IN-1. All pneumoconioses: Indiana and U.S. age-adjusted mortality rates, residents age 15 and over, 1968-1992}

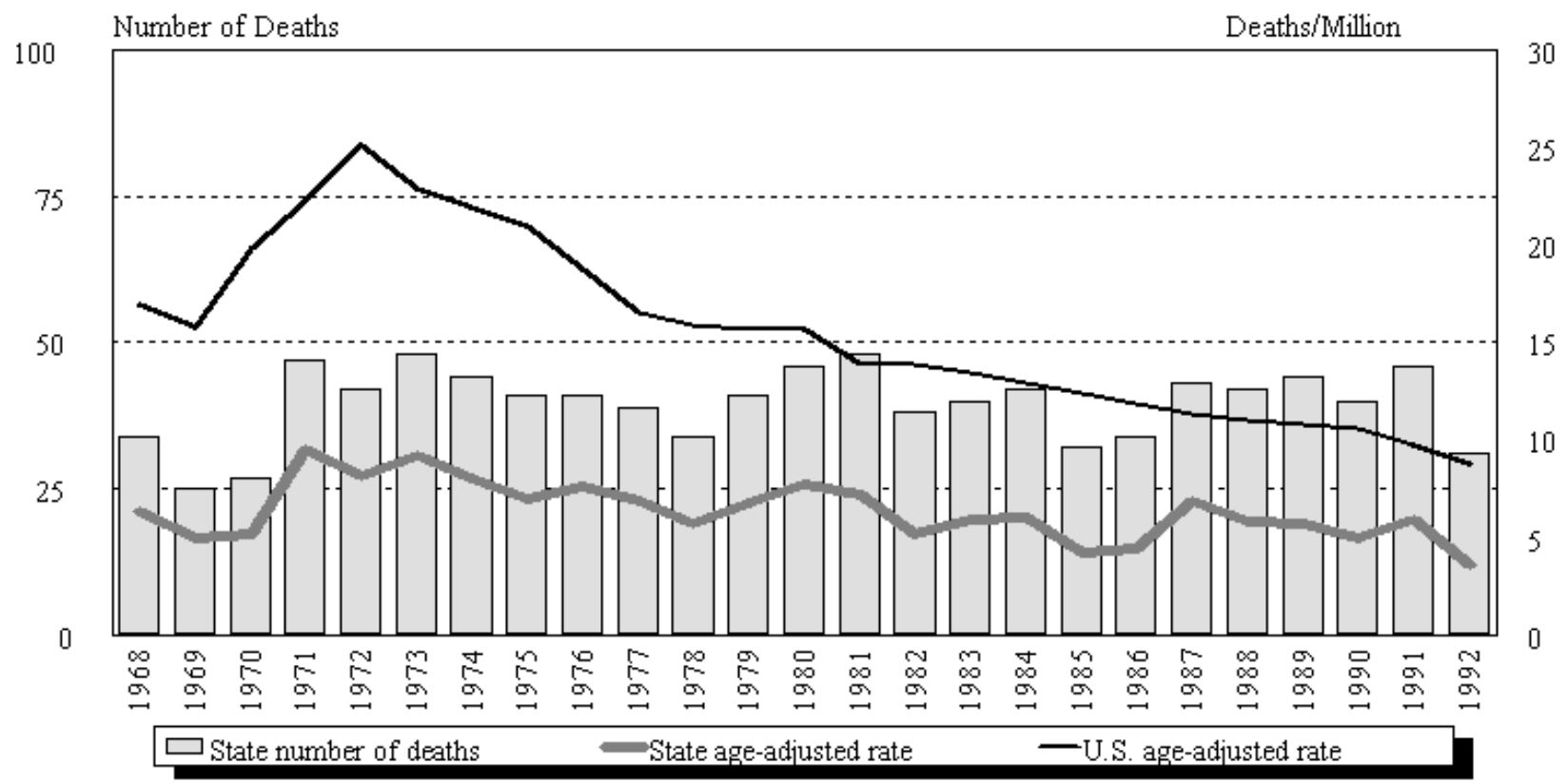

NOTE: $\quad$ See Appendix A for source description and Appendix B for methods and ICD-8 and ICD-9 codes.

SOURCE: $\quad$ National Center for Health Statistics multiple cause of death data. Population estimates from U.S. Bureau of the Census. 


\section{Indiana}

Table IN-1. Number of deaths and age-adjusted mortality rate (per 1,000,000 population) by condition, Indiana residents age 15 and over, 1968-1992

\begin{tabular}{|c|c|c|c|c|c|c|c|c|c|c|c|c|}
\hline \multirow[b]{2}{*}{ Year } & \multicolumn{2}{|c|}{ Asb estosis } & \multicolumn{2}{|c|}{$\begin{array}{l}\text { Coal workers' } \\
\text { pne umoconiosis }\end{array}$} & \multicolumn{2}{|c|}{ Silicosis } & \multicolumn{2}{|c|}{ Byssinosis* } & \multicolumn{2}{|c|}{$\begin{array}{c}\text { Unspecified/Other } \\
\text { pneumoconioses }\end{array}$} & \multicolumn{2}{|c|}{ All pneumoconioses } \\
\hline & Number & Rate & Number & Rate & Number & Rate & Number & Rate & Number & Rate & Number & Rate \\
\hline 1968 & 1 & 0.22 & 4 & 0.66 & 20 & 3.76 & & & 9 & 1.71 & 34 & 6.35 \\
\hline 1969 & - & - & 3 & 0.67 & 17 & 3.31 & & & 5 & 0.96 & 25 & 4.94 \\
\hline 1970 & 1 & 0.22 & 2 & 0.36 & 15 & 3.00 & & & 9 & 1.65 & 27 & 5.22 \\
\hline 1971 & 1 & 0.21 & 9 & 1.95 & 17 & 3.27 & & & 21 & 4.30 & 47 & 9.50 \\
\hline 1972 & - & - & 6 & 1.26 & 14 & 3.00 & & & 22 & 3.91 & 42 & 8.17 \\
\hline 1973 & - & - & 14 & 2.81 & 13 & 2.49 & & & 21 & 3.91 & 48 & 9.21 \\
\hline 1974 & 1 & 0.23 & 12 & 2.37 & 10 & 2.02 & & & 23 & 3.82 & 44 & 8.01 \\
\hline 1975 & 1 & 0.20 & 14 & 2.29 & 7 & 1.27 & & & 21 & 3.60 & 41 & 6.95 \\
\hline 1976 & - & - & 14 & 2.68 & 10 & 1.83 & & & 19 & 3.42 & 41 & 7.63 \\
\hline 1977 & - & - & 18 & 3.48 & 11 & 1.84 & & & 11 & 1.81 & 39 & 6.91 \\
\hline 1978 & 4 & 0.86 & 8 & 1.45 & 9 & 1.47 & & & 13 & 1.96 & 34 & 5.75 \\
\hline 1979 & 3 & 0.71 & 15 & 2.27 & 12 & 2.10 & - & - & 11 & 1.66 & 41 & 6.74 \\
\hline 1980 & 4 & 0.76 & 22 & 3.69 & 9 & 1.63 & - & - & 11 & 1.66 & 46 & 7.74 \\
\hline 1981 & 2 & 0.36 & 24 & 3.89 & 6 & 0.68 & - & - & 18 & 2.64 & 48 & 7.16 \\
\hline 1982 & 6 & 0.96 & 16 & 2.30 & 6 & 0.74 & - & - & 10 & 1.22 & 38 & 5.22 \\
\hline 1983 & 3 & 0.45 & 22 & 3.21 & 8 & 1.32 & - & - & 7 & 0.99 & 40 & 5.97 \\
\hline 1984 & 4 & 0.53 & 25 & 3.66 & 7 & 1.22 & - & - & 6 & 0.59 & 42 & 6.00 \\
\hline 1985 & 3 & 0.52 & 21 & 2.70 & 3 & 0.35 & - & - & 5 & 0.64 & 32 & 4.20 \\
\hline 1986 & 3 & 0.55 & 22 & 2.66 & 6 & 0.82 & - & - & 3 & 0.40 & 34 & 4.44 \\
\hline 1987 & 3 & 0.60 & 25 & 4.08 & 8 & 1.45 & - & - & 7 & 0.72 & 43 & 6.84 \\
\hline 1988 & 5 & 0.71 & 25 & 3.40 & 6 & 0.95 & - & - & 6 & 0.77 & 42 & 5.83 \\
\hline 1989 & 5 & 0.59 & 27 & 3.47 & 6 & 0.86 & - & - & 6 & 0.75 & 44 & 5.68 \\
\hline 1990 & 8 & 1.19 & 19 & 2.17 & 8 & 1.19 & - & - & 6 & 0.68 & 40 & 5.01 \\
\hline 1991 & 4 & 0.64 & 33 & 3.96 & 4 & 0.64 & 1 & 0.16 & 4 & 0.52 & 46 & 5.93 \\
\hline 1992 & 4 & 0.51 & 19 & 2.09 & 4 & 0.63 & - & - & 4 & 0.35 & 31 & 3.59 \\
\hline
\end{tabular}

indicates no deaths listed. $\quad *$ no specific code for by ssinosis in ICD-8 (1968-1978).

NOTE: Rates based on small numbers may be unstable. Sum of individual conditions may exceed total because of deaths with multiple pneumoconioses. See Appendix A for source description and Appendix B for methods and ICD-8 and ICD-9 codes.

SOURCE: National Center for Health Statistics multiple cause of death data. Population estimates from U.S. Bureau of the Census. 


\section{Figure IN-2. All pneumoconioses: Distribution of deaths by sex and race,} Indiana residents age 15 and over, 1968-1992
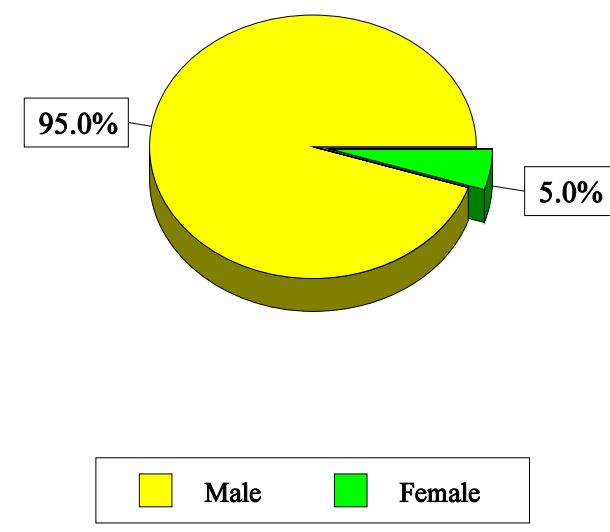
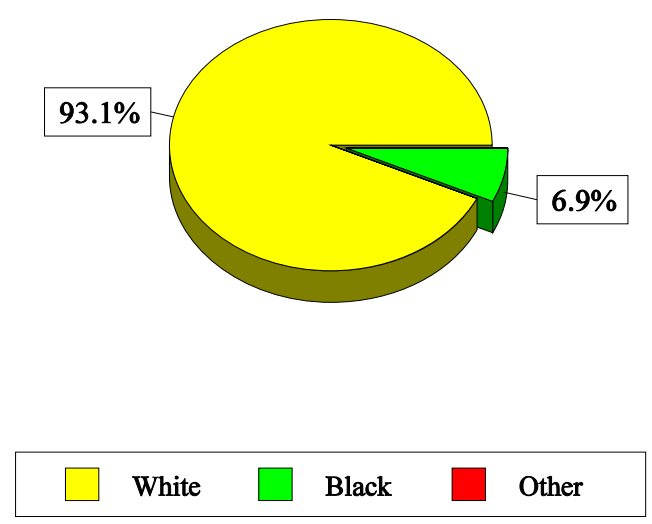

NOTE: See Appendix A for source description and Appendix B for methods and ICD-8 and ICD-9 codes.

SOURCE: National Center for Health Statistics multiple cause of death data.

Table IN-2. All pneumoconioses: Distribution of deaths by sex, race, and age, Indiana residents age 15 and over, 1968-1992

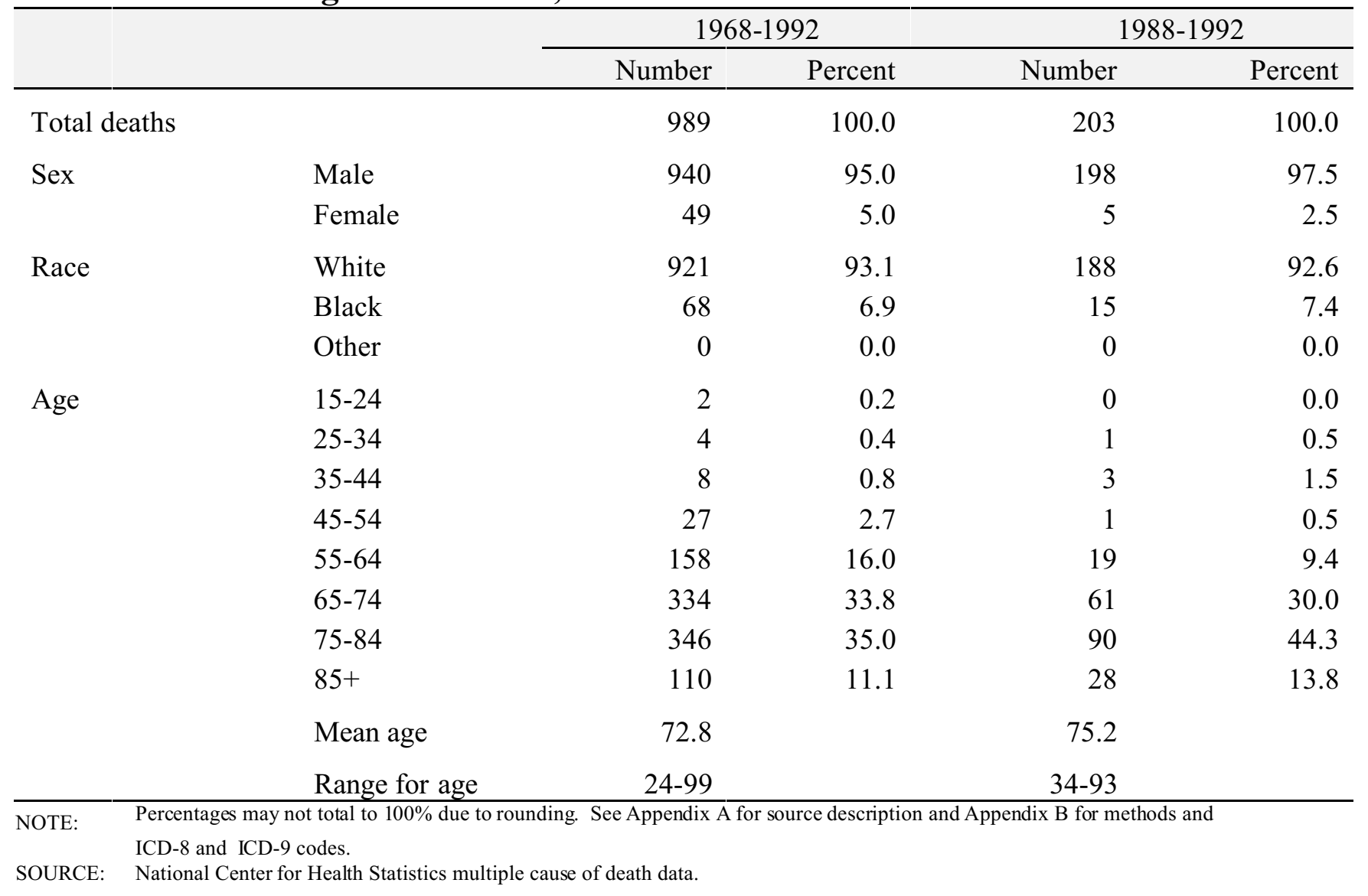


Figure IN-3. Pneumoconiosis deaths by condition, Indiana residents age 15 and over, 1968-1992

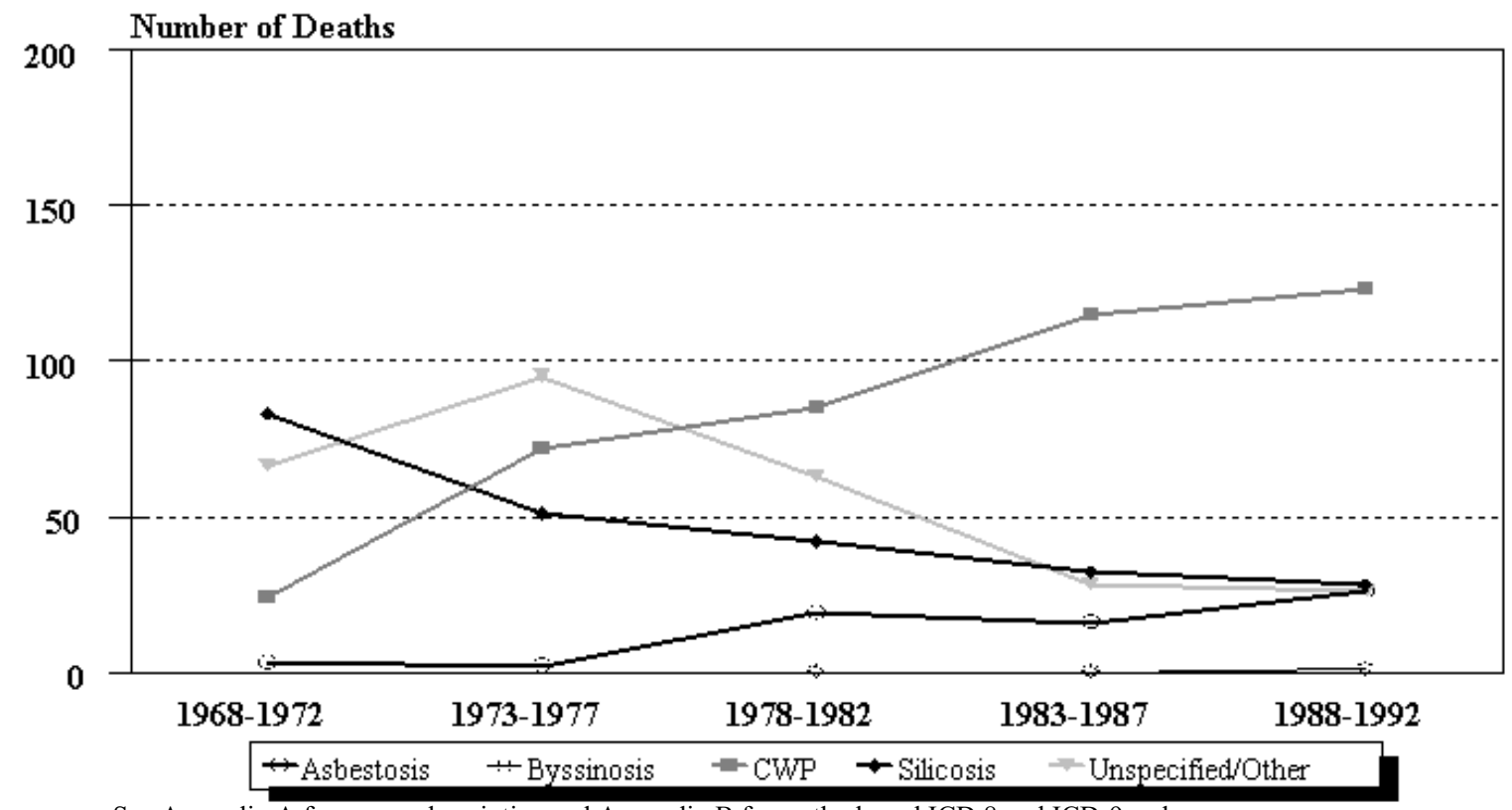

NOTE: See Appendix A for source description and Appendix B for methods and ICD-8 and ICD-9 codes.

SOURCE: National Center for Health Statistics multiple cause of death data.

Figure IN-4. Indiana and U.S. age-adjusted mortality rates (per 1,000,000 population) by condition, residents age 15 and over, 1983-1992

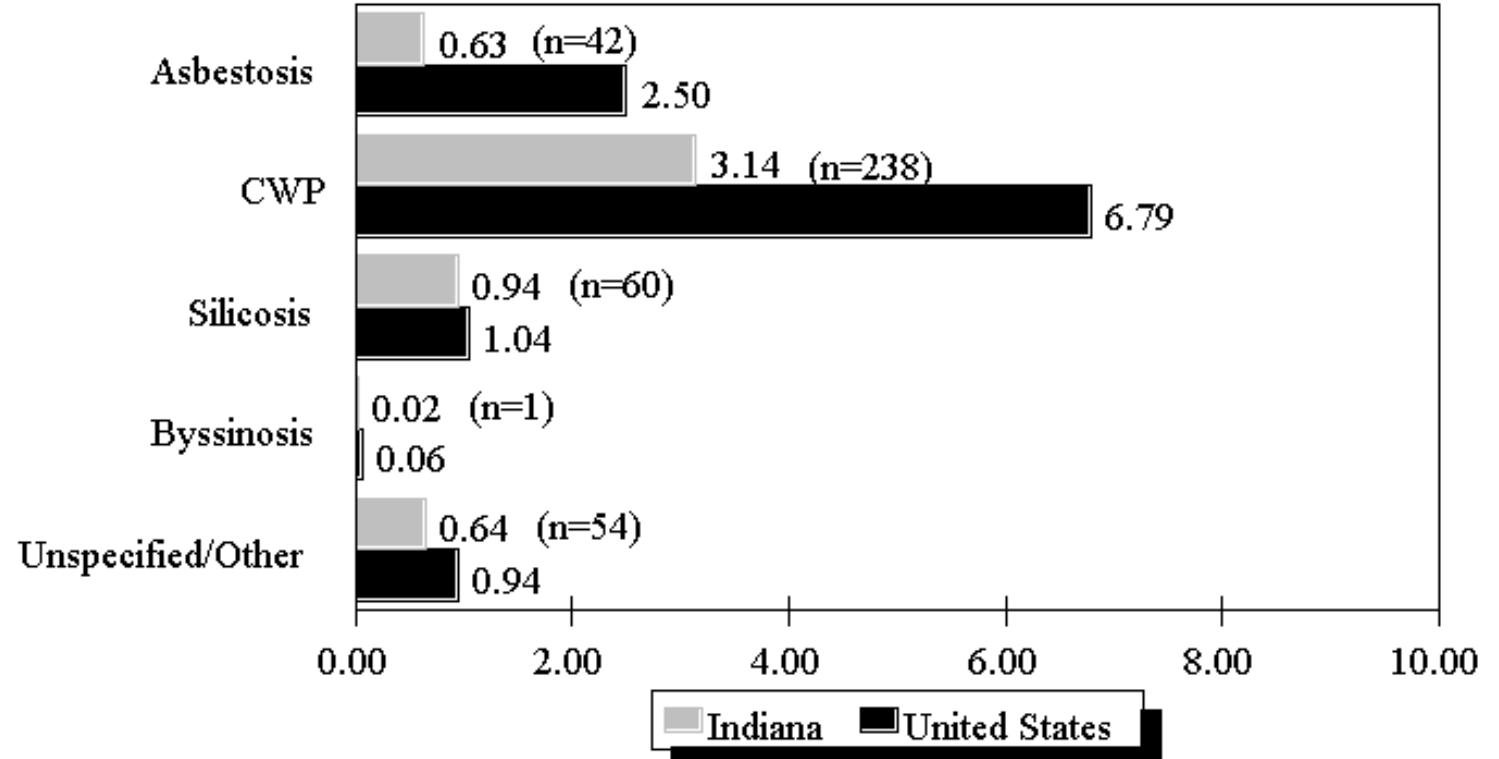

NOTE: Rates based on small numbers may be unstable. See Appendix A for source description and Appendix B for methods and ICD-8 and ICD-9 codes.

SOURCE: National Center for Health Statistics multiple cause of death data. Population estimates from U.S. Bureau of the Census. 
Table IN-3 (page 1 of 3). Number of deaths and age-adjusted mortality rate (per $1,000,000$ population) by condition and county, Indiana residents age 15 and over, 1983-1992

\begin{tabular}{|c|c|c|c|c|c|c|c|c|c|c|c|c|}
\hline \multirow[b]{2}{*}{ County } & \multicolumn{2}{|c|}{ Asbestosis } & \multicolumn{2}{|c|}{$\begin{array}{c}\text { Coal workers' } \\
\text { pneumoconiosis }\end{array}$} & \multicolumn{2}{|c|}{ Silicosis } & \multicolumn{2}{|c|}{ Byssinosis } & \multicolumn{2}{|c|}{$\begin{array}{r}\text { Unspecified/Other } \\
\text { pneumoconioses }\end{array}$} & \multicolumn{2}{|c|}{$\begin{array}{r}\text { All } \\
\text { pneumoconioses }\end{array}$} \\
\hline & $\#$ & Rate & \# & Rate & $\#$ & Rate & $\#$ & Rate & \# & Rate & \# & Rate \\
\hline Allen & 1 & 0.39 & 10 & 3.15 & - & - & - & - & - & - & 11 & 3.55 \\
\hline Bartholomew & - & - & - & - & 1 & 0.94 & - & - & 1 & 1.75 & 2 & 2.69 \\
\hline Boone & - & - & 1 & 1.22 & - & - & - & - & - & - & 1 & 1.22 \\
\hline Cass & - & - & 1 & 1.17 & 2 & 3.55 & - & - & - & - & 3 & 4.72 \\
\hline Clark & 1 & 0.66 & 1 & 1.23 & 2 & 1.90 & - & - & 1 & 1.23 & 5 & 5.03 \\
\hline Clay & - & - & 5 & 9.54 & 2 & 5.14 & - & - & - & - & 7 & 14.69 \\
\hline Daviess & - & - & 2 & 6.80 & - & - & - & - & 2 & 6.80 & 4 & 13.60 \\
\hline Dearborn & - & - & 3 & 5.87 & 1 & 1.50 & - & - & - & - & 4 & 7.37 \\
\hline De Kalb & - & - & 1 & 1.53 & - & - & - & - & - & - & 1 & 1.53 \\
\hline Delaware & - & - & 4 & 2.67 & 1 & 0.44 & - & - & - & - & 5 & 3.11 \\
\hline Dubois & - & - & 3 & 8.72 & - & - & - & - & - & - & 3 & 8.72 \\
\hline Elkhart & - & - & 6 & 3.50 & 1 & 0.78 & - & - & - & - & 7 & 4.28 \\
\hline Fayette & - & - & 3 & 7.15 & - & - & - & - & - & - & 3 & 7.15 \\
\hline Floyd & - & - & 1 & 1.68 & 1 & 2.15 & - & - & - & - & 2 & 3.83 \\
\hline Fountain & - & - & - & - & 2 & 4.34 & - & - & - & - & 2 & 4.34 \\
\hline Fulton & - & - & 1 & 4.67 & - & - & - & - & - & - & 1 & 4.67 \\
\hline Gibson & 1 & 2.88 & 26 & 44.62 & - & - & - & - & 9 & 14.15 & 36 & 61.66 \\
\hline Grant & 1 & 1.27 & 4 & 4.47 & - & - & - & - & - & - & 5 & 5.74 \\
\hline Greene & - & - & 20 & 40.01 & 1 & 2.95 & - & - & 5 & 7.84 & 26 & 50.80 \\
\hline Hamilton & - & - & 2 & 2.21 & - & - & - & - & - & - & 2 & 2.21 \\
\hline Hancock & - & - & 2 & 5.27 & - & - & - & - & - & - & 2 & 5.27 \\
\hline Harrison & - & - & 3 & 7.75 & - & - & - & - & - & - & 3 & 7.75 \\
\hline Hendricks & 1 & 1.66 & 3 & 3.67 & - & - & - & - & - & - & 4 & 5.33 \\
\hline Henry & 1 & 1.91 & 2 & 1.86 & - & - & - & - & 2 & 1.86 & 5 & 5.62 \\
\hline Howard & 1 & 0.73 & 1 & 0.73 & 4 & 3.47 & - & - & 1 & 0.73 & 7 & 5.65 \\
\hline Huntington & 1 & 1.20 & 1 & 2.99 & - & - & - & - & - & - & 2 & 4.18 \\
\hline Jackson & - & - & 1 & 1.20 & - & - & - & - & - & - & 1 & 1.20 \\
\hline Jay & 1 & 4.31 & - & - & - & - & - & - & - & - & 1 & 4.31 \\
\hline Jefferson & - & - & 1 & 3.47 & 1 & 1.69 & - & - & - & - & 2 & 5.16 \\
\hline Johnson & - & - & 1 & 0.68 & 2 & 3.03 & - & - & - & - & 3 & 3.71 \\
\hline Knox & 2 & 4.63 & 7 & 7.85 & - & - & - & - & - & - & 8 & 10.17 \\
\hline Kosciusko & - & - & 6 & 8.73 & - & - & - & - & - & - & 6 & 8.73 \\
\hline Lagrange & - & - & 1 & 4.36 & - & - & - & - & - & - & 1 & 4.36 \\
\hline
\end{tabular}

See footnotes at end of table. 
Table IN-3 (page 2 of 3). Number of deaths and age-adjusted mortality rate (per $1,000,000$ population) by condition and county, Indiana residents age 15 and over, 1983-1992

\begin{tabular}{|c|c|c|c|c|c|c|c|c|c|c|c|c|}
\hline \multirow[b]{2}{*}{ County } & \multicolumn{2}{|c|}{ Asbestosis } & \multicolumn{2}{|c|}{$\begin{array}{r}\text { Coal workers' } \\
\text { pneumoconiosis }\end{array}$} & \multicolumn{2}{|c|}{ Silicosis } & \multicolumn{2}{|c|}{ Byssinosis } & \multicolumn{2}{|c|}{$\begin{array}{r}\text { Unspecified/Other } \\
\text { pneumoconioses }\end{array}$} & \multicolumn{2}{|c|}{$\begin{array}{r}\text { All } \\
\text { pneumoconioses }\end{array}$} \\
\hline & $\#$ & Rate & $\#$ & Rate & $\#$ & Rate & $\#$ & Rate & $\#$ & Rate & $\#$ & Rate \\
\hline Lake & 5 & 0.98 & 7 & 1.33 & 9 & 1.43 & - & - & 6 & 0.96 & 27 & 4.69 \\
\hline La Porte & 1 & 0.51 & - & - & 1 & 0.94 & - & - & 1 & 0.51 & 3 & 1.96 \\
\hline Lawrence & 1 & 1.04 & - & - & - & - & - & - & 1 & 2.27 & 2 & 3.30 \\
\hline Madison & - & - & 2 & 0.75 & 2 & 0.75 & - & - & 1 & 0.38 & 5 & 1.88 \\
\hline Marion & 7 & 0.86 & 16 & 1.57 & 13 & 1.84 & - & - & 2 & 0.22 & 38 & 4.48 \\
\hline Marshall & - & - & 1 & 2.48 & - & - & - & - & - & - & 1 & 2.48 \\
\hline Martin & - & - & 1 & 9.20 & - & - & - & - & 1 & 5.06 & 2 & 14.26 \\
\hline Montgomery & - & - & 2 & 4.13 & - & - & - & - & - & - & 2 & 4.13 \\
\hline Morgan & 1 & 2.14 & - & - & - & - & - & - & - & - & 1 & 2.14 \\
\hline Newton & - & - & - & - & - & - & - & - & 1 & 7.76 & 1 & 7.76 \\
\hline Noble & - & - & 2 & 4.44 & - & - & - & - & - & - & 2 & 4.44 \\
\hline Ohio & - & - & 1 & 8.32 & - & - & - & - & - & - & 1 & 8.32 \\
\hline Parke & - & - & - & - & 1 & 5.63 & - & - & - & - & 1 & 5.63 \\
\hline Pike & - & - & 8 & 36.24 & - & - & - & - & 8 & 24.40 & 16 & 60.64 \\
\hline Porter & - & - & 5 & 4.19 & 1 & 0.61 & - & - & - & - & 6 & 4.80 \\
\hline Posey & - & - & 4 & 12.75 & - & - & - & - & - & - & 4 & 12.75 \\
\hline Putnam & - & - & - & - & - & - & - & - & 1 & 1.61 & 1 & 1.61 \\
\hline Randolph & 1 & 3.33 & 2 & 4.86 & 1 & 1.53 & - & - & - & - & 4 & 9.72 \\
\hline Ripley & - & - & 2 & 5.93 & 1 & 1.72 & - & - & - & - & 3 & 7.65 \\
\hline St Joseph & 1 & 0.19 & 1 & 0.19 & 1 & 0.41 & - & - & - & - & 3 & 0.79 \\
\hline Scott & - & - & 5 & 19.90 & - & - & - & - & - & - & 5 & 19.90 \\
\hline Shelby & - & - & 2 & 4.02 & - & - & - & - & - & - & 2 & 4.02 \\
\hline Spencer & - & - & - & - & 2 & 10.63 & - & - & - & - & 2 & 10.63 \\
\hline Starke & - & - & 1 & 4.35 & - & - & - & - & 1 & 2.05 & 2 & 6.41 \\
\hline Sullivan & 1 & 4.43 & 8 & 19.66 & - & - & - & - & 1 & 4.43 & 10 & 28.52 \\
\hline Tippecanoe & 1 & 1.13 & 1 & 0.54 & 2 & 2.25 & - & - & - & - & 4 & 3.92 \\
\hline Union & 1 & 15.13 & - & - & 1 & 6.38 & - & - & - & - & 2 & 21.51 \\
\hline Vanderburgh & 6 & 1.79 & 13 & 4.76 & 2 & 0.49 & - & - & 3 & 1.36 & 24 & 8.41 \\
\hline Vermillion & - & - & 4 & 8.38 & - & - & - & - & 2 & 10.56 & 6 & 18.93 \\
\hline Vigo & 2 & 1.31 & 8 & 3.18 & - & - & 1 & 0.92 & 2 & 1.31 & 13 & 6.72 \\
\hline Wabash & - & - & 4 & 6.40 & - & - & - & - & - & - & 4 & 6.40 \\
\hline
\end{tabular}

See footnotes at end of table. 


\section{Table IN-3 (page 3 of 3). Number of deaths and age-adjusted mortality rate (per $1,000,000$ population) by condition and county, Indiana residents age 15 and over, 1983-1992}

\begin{tabular}{|c|c|c|c|c|c|c|c|c|c|c|c|c|}
\hline \multirow[b]{2}{*}{ County } & \multicolumn{2}{|c|}{ Asbestosis } & \multicolumn{2}{|c|}{$\begin{array}{c}\text { Coal workers' } \\
\text { pneumoconiosis }\end{array}$} & \multicolumn{2}{|c|}{ Silicosis } & \multicolumn{2}{|c|}{ Byssinosis } & \multicolumn{2}{|c|}{$\begin{array}{r}\text { Unspecified/Other } \\
\text { pneumoconioses }\end{array}$} & \multicolumn{2}{|c|}{$\begin{array}{r}\text { All } \\
\text { pneumoconioses }\end{array}$} \\
\hline & $\#$ & Rate & $\#$ & Rate & \# & Rate & \# & Rate & \# & Rate & $\#$ & Rate \\
\hline Warren & - & - & - & - & 1 & 19.59 & - & - & - & - & 1 & 19.59 \\
\hline Warrick & 2 & 5.68 & 8 & 18.58 & - & - & - & - & 2 & 2.93 & 12 & 27.19 \\
\hline Washington & - & - & 1 & 4.51 & 1 & 6.29 & - & - & - & - & 2 & 10.80 \\
\hline Wayne & 1 & 1.29 & 2 & 1.89 & - & - & - & - & - & - & 3 & 3.18 \\
\hline Wells & - & - & 4 & 12.22 & - & - & - & - & - & - & 4 & 12.22 \\
\hline
\end{tabular}

- indicates no deaths listed.

NOTE: Counties with no deaths for all listed conditions are not displayed. Rates based on small numbers may be unstable. Sum of individual conditions may exceed total because of deaths with multiple pneumoconioses. See Appendix A for source description and Appendix B for methods and ICD-8 and ICD-9 codes.

SOURCE: National Center for Health Statistics multiple cause of death data. Population estimates from U.S. Bureau of the Census. 


\section{Iowa: Selected statistics from the United States section}

All pneumoconioses mortality, 1988-1992

Total deaths

Crude mortality rate (per $1,000,000$ population)

Age-adjusted mortality rate (per 1,000,000 population)

Total years of potential life lost to life expectancy

Years of potential life lost to life expectancy (per death) $\underline{\text { Value }}$

64

5.90

2.67

701

11.0

Deaths*

18

33

Coal workers' pneumoconiosis

Silicosis

Byssinosis

Unspecified/Other pneumoconiosis $\underline{\text { State rank }}$

34

38

45

35

44

Percent**

distribution

28.1

51.6

14.1

0.0

6.3

* Sum of column may exceed total deaths because of deaths with multiple pneumoconioses.

** Percentages may total more than $100 \%$ due to deaths with multiple pneumoconioses.

NOTE: $\quad$ See Appendix A for source description and Appendix B for methods and ICD-8 and ICD-9 codes.

SOURCE: National Center for Health Statistics multiple cause of death data. Population estimates from U.S. Bureau of the Census.

\section{Figure IA-1. All pneumoconioses: Iowa and U.S. age-adjusted mortality rates, residents age 15 and over, 1968-1992}

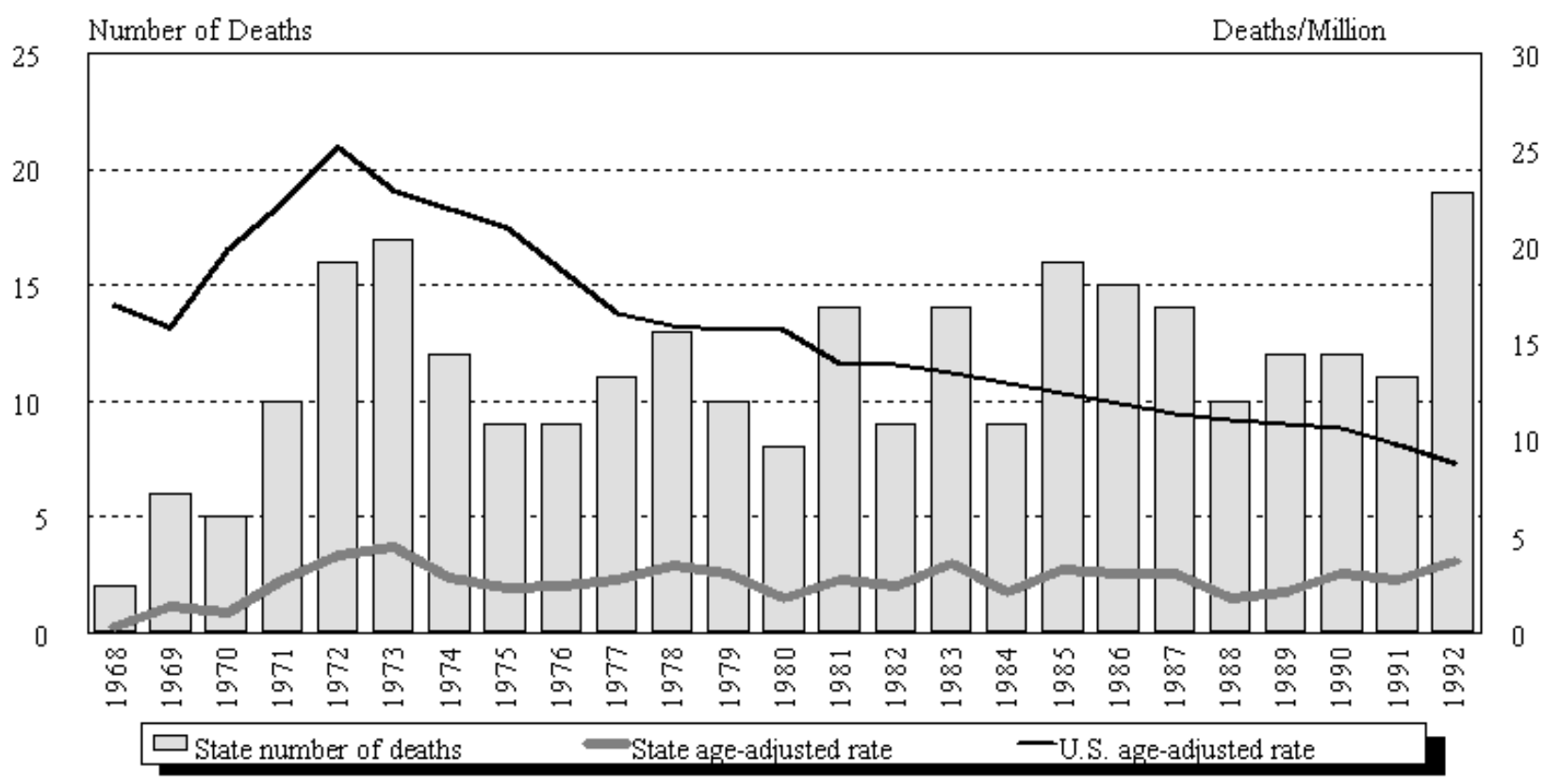

NOTE: $\quad$ See Appendix A for source description and Appendix B for methods and ICD-8 and ICD-9 codes.

SOURCE: $\quad$ National Center for Health Statistics multiple cause of death data. Population estimates from U.S. Bureau of the Census. 


\section{Iowa}

\begin{tabular}{|c|c|c|c|c|c|c|c|c|c|c|c|c|}
\hline \multirow[b]{2}{*}{ Year } & \multicolumn{2}{|c|}{ Asb estosis } & \multicolumn{2}{|c|}{$\begin{array}{l}\text { Coal workers' } \\
\text { pneumoconiosis }\end{array}$} & \multicolumn{2}{|c|}{ Silicosis } & \multicolumn{2}{|c|}{ Byssinosis* } & \multicolumn{2}{|c|}{$\begin{array}{l}\text { Unspecified/Other } \\
\text { pneumoconioses }\end{array}$} & \multicolumn{2}{|c|}{ All pneu moconioses } \\
\hline & Number & Rate & Number & Rate & Number & Rate & Number & Rate & Number & Rate & Number & Rate \\
\hline 1968 & 1 & 0.12 & - & - & - & - & & & 1 & 0.20 & 2 & 0.32 \\
\hline 1969 & - & - & - & - & 6 & 1.42 & & & - & - & 6 & 1.42 \\
\hline 1970 & - & - & 1 & 0.20 & 3 & 0.51 & & & 1 & 0.32 & 5 & 1.03 \\
\hline 1971 & - & - & 1 & 0.19 & 2 & 0.72 & & & 7 & 1.89 & 10 & 2.80 \\
\hline 1972 & - & - & 4 & 0.61 & 6 & 1.81 & & & 6 & 1.62 & 16 & 4.04 \\
\hline 1973 & - & - & 7 & 1.92 & 4 & 1.22 & & & 7 & 1.66 & 17 & 4.41 \\
\hline 1974 & 1 & 0.40 & 3 & 0.56 & 4 & 1.06 & & & 4 & 0.88 & 12 & 2.90 \\
\hline 1975 & 1 & 0.32 & 2 & 0.63 & 2 & 0.37 & & & 4 & 1.01 & 9 & 2.33 \\
\hline 1976 & 1 & 0.52 & 6 & 1.19 & - & & & & 2 & 0.70 & 9 & 2.41 \\
\hline 1977 & 1 & 0.53 & 7 & 1.57 & 1 & 0.18 & & & 2 & 0.48 & 11 & 2.76 \\
\hline 1978 & - & - & 4 & 0.98 & 2 & 0.92 & & & 7 & 1.54 & 13 & 3.44 \\
\hline 1979 & 1 & 0.08 & 7 & 2.33 & - & - & - & - & 2 & 0.61 & 10 & 3.02 \\
\hline 1980 & - & - & 5 & 1.13 & 3 & 0.68 & - & - & - & - & 8 & 1.81 \\
\hline 1981 & 1 & 0.38 & 11 & 1.91 & 1 & 0.30 & - & - & 1 & 0.18 & 14 & 2.77 \\
\hline 1982 & - & - & 6 & 1.66 & 1 & 0.38 & - & - & 2 & 0.35 & 9 & 2.39 \\
\hline 1983 & 1 & 0.17 & 8 & 1.98 & 3 & 0.85 & - & - & 2 & 0.56 & 14 & 3.57 \\
\hline 1984 & 1 & 0.39 & 6 & 1.18 & 2 & 0.56 & - & - & - & - & 9 & 2.12 \\
\hline 1985 & 3 & 1.20 & 10 & 1.54 & 2 & 0.34 & - & - & 1 & 0.17 & 16 & 3.25 \\
\hline 1986 & 4 & 1.05 & 8 & 1.63 & 3 & 0.41 & - & - & - & - & 15 & 3.09 \\
\hline 1987 & 6 & 1.19 & 3 & 0.75 & 5 & 1.11 & - & - & - & - & 14 & 3.05 \\
\hline 1988 & 2 & 0.46 & 6 & 1.05 & 2 & 0.33 & - & - & - & - & 10 & 1.84 \\
\hline 1989 & 2 & 0.45 & 9 & 1.62 & 1 & 0.07 & - & - & - & - & 12 & 2.14 \\
\hline 1990 & 4 & 1.14 & 6 & 1.02 & 2 & 0.86 & - & - & - & - & 12 & 3.02 \\
\hline 1991 & 3 & 1.15 & 6 & 1.13 & 2 & 0.44 & - & - & - & - & 11 & 2.72 \\
\hline 1992 & 7 & 1.36 & 6 & 0.75 & 2 & 0.60 & - & - & 4 & 1.01 & 19 & 3.71 \\
\hline
\end{tabular}

- indicates no deaths listed. $\quad *$ no specific code for byssinosis in ICD-8 (1968-1978)

NOTE: Rates based on small numbers may be unstable. Sum of individual conditions may exceed total because of deaths with multiple pneumoconioses. See Appendix A for source description and Appendix B for methods and ICD-8 and ICD-9 codes.

SOURCE: National Center for Health Statistics multiple cause of death data. Population estimates from U.S. Bureau of the Census. 


\section{Figure IA-2. All pneumoconioses: Distribution of deaths by sex and race,} Iowa residents age 15 and over, 1968-1992
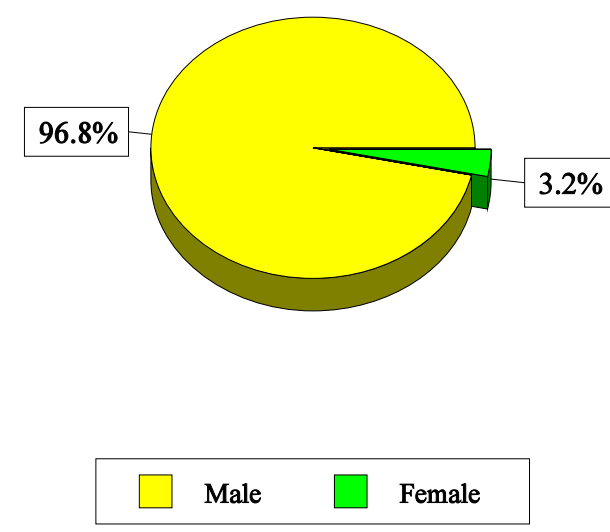

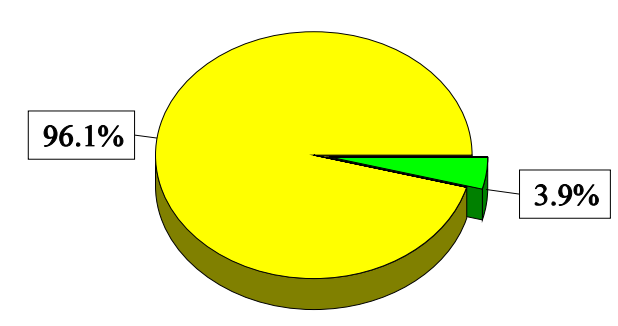

NOTE: $\quad$ See Appendix A for source description and Appendix B for methods and ICD-8 and ICD-9 codes.

SOURCE: National Center for Health Statistics multiple cause of death data.

Table IA-2. All pneumoconioses: Distribution of deaths by sex, race, and age, Iowa residents age 15 and over, 1968-1992

\begin{tabular}{|c|c|c|c|c|c|c|}
\hline & & & \multicolumn{2}{|c|}{ 1968-1992 } & \multicolumn{2}{|c|}{ 1988-1992 } \\
\hline & & & Number & Percent & Number & Percent \\
\hline \multicolumn{3}{|c|}{ Total deaths } & 283 & 100.0 & 64 & 100.0 \\
\hline \multirow{2}{*}{\multicolumn{2}{|c|}{ Sex }} & Male & 274 & 96.8 & 63 & 98.4 \\
\hline & & Female & 9 & 3.2 & 1 & 1.6 \\
\hline \multirow{3}{*}{\multicolumn{2}{|c|}{ Race }} & White & 272 & 96.1 & 61 & 95.3 \\
\hline & & Black & 11 & 3.9 & 3 & 4.7 \\
\hline & & Other & 0 & 0.0 & 0 & 0.0 \\
\hline \multirow{10}{*}{\multicolumn{2}{|c|}{ Age }} & $15-24$ & 0 & 0.0 & 0 & 0.0 \\
\hline & & $25-34$ & 0 & 0.0 & 0 & 0.0 \\
\hline & & $35-44$ & 0 & 0.0 & 0 & 0.0 \\
\hline & & $45-54$ & 9 & 3.2 & 1 & 1.6 \\
\hline & & $55-64$ & 28 & 9.9 & 5 & 7.8 \\
\hline & & $65-74$ & 89 & 31.4 & 20 & 31.3 \\
\hline & & $75-84$ & 112 & 39.6 & 26 & 40.6 \\
\hline & & $85+$ & 45 & 15.9 & 12 & 18.8 \\
\hline & & Mean age & 75.1 & & 76.1 & \\
\hline & & Range for age & $45-100$ & & $47-100$ & \\
\hline & $\begin{array}{l}\text { Percen } \\
\text { ICD-8 }\end{array}$ & $\begin{array}{l}\text { total to } 100 \% \text { due } t \\
\text { odes. }\end{array}$ & e Appendix & descr & ndix $B$ for $n$ & \\
\hline
\end{tabular}


Figure IA-3. Pneumoconiosis deaths by condition, Iowa residents age 15 and over, 1968-1992

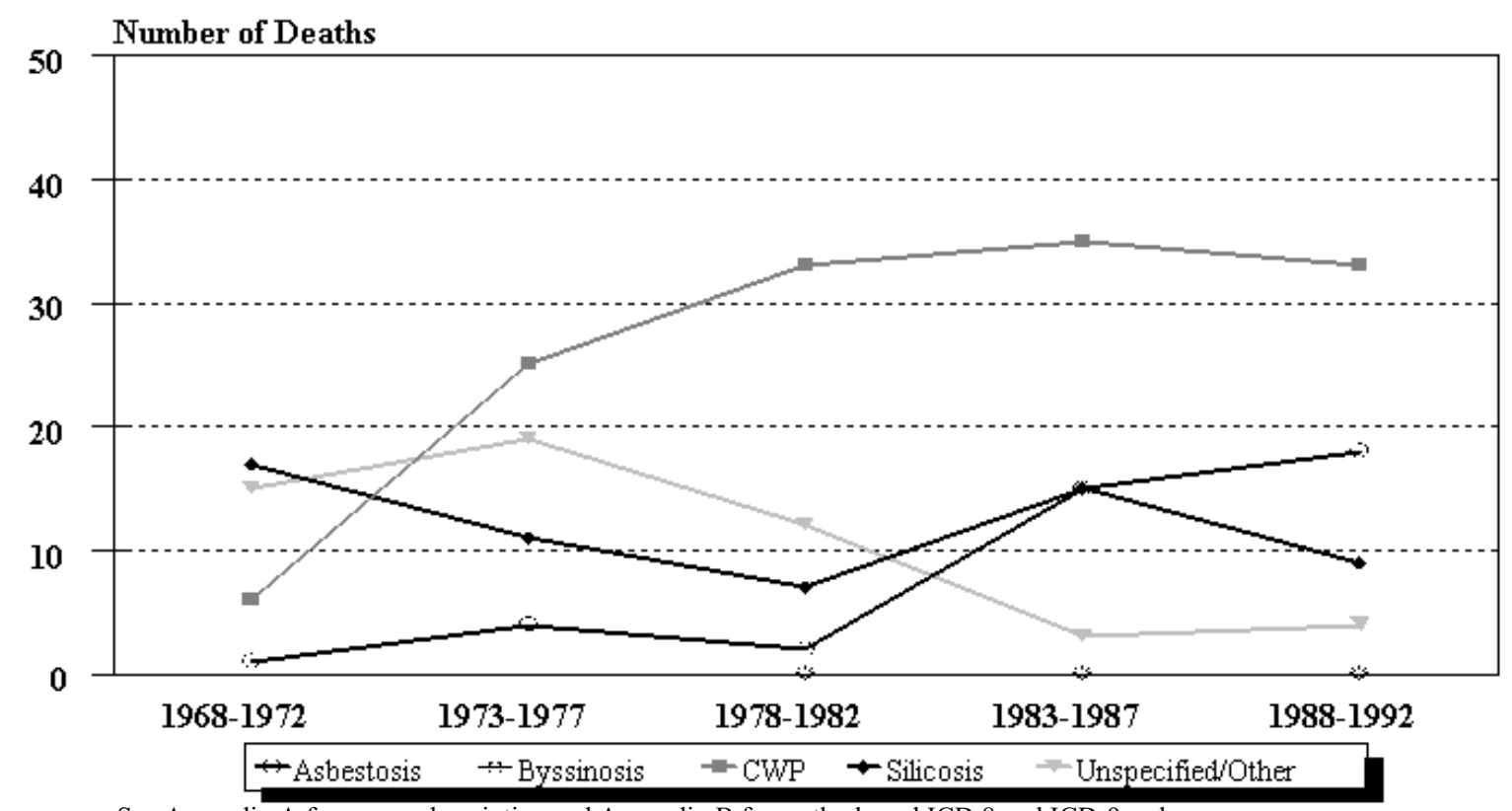

NOTE: See Appendix A for source description and Appendix B for methods and ICD-8 and ICD-9 codes.

SOURCE: National Center for Health Statistics multiple cause of death data.

Figure IA-4. Iowa and U.S. age-adjusted mortality rates (per 1,000,000 population) by condition, residents age 15 and over, 1983-1992

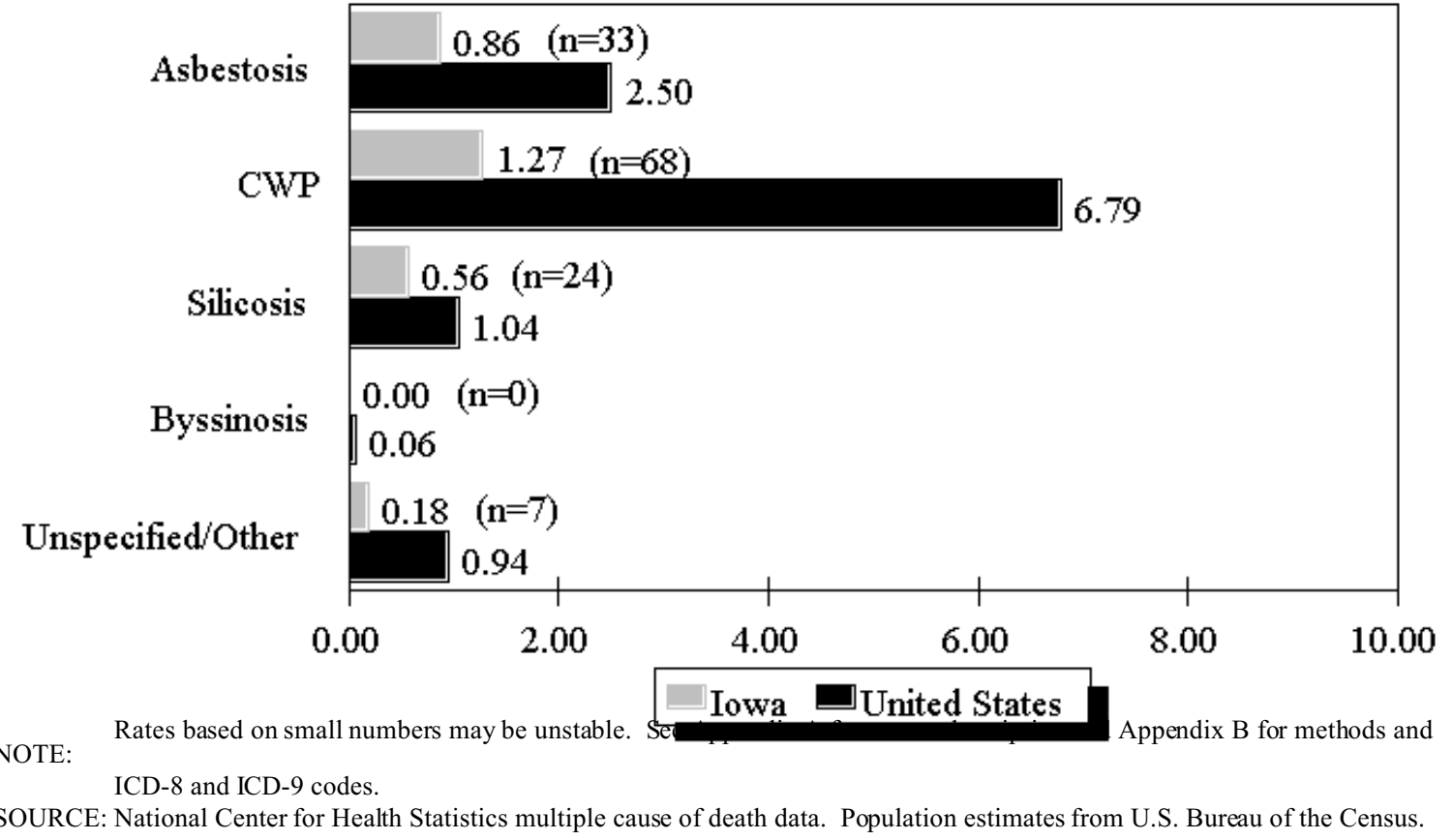


Table IA-3 (page 1 of 2). Number of deaths and age-adjusted mortality rate (per $1,000,000$ population) by condition and county, Iowa residents age 15 and over, 1983-1992

\begin{tabular}{|c|c|c|c|c|c|c|c|c|c|c|c|c|}
\hline \multirow[b]{2}{*}{ County } & \multicolumn{2}{|c|}{ Asbestosis } & \multicolumn{2}{|c|}{$\begin{array}{c}\text { Coal workers' } \\
\text { pneumoconiosis }\end{array}$} & \multicolumn{2}{|c|}{ Silicosis } & \multicolumn{2}{|c|}{ Byssinosis } & \multicolumn{2}{|c|}{$\begin{array}{r}\text { Unspecified/Other } \\
\text { pneumoconioses }\end{array}$} & \multicolumn{2}{|c|}{$\begin{array}{r}\text { All } \\
\text { pneumoconioses }\end{array}$} \\
\hline & $\#$ & Rate & $\#$ & Rate & $\#$ & Rate & $\#$ & Rate & \# & Rate & $\#$ & Rate \\
\hline Adams & - & - & 1 & 5.30 & - & - & - & - & - & - & 1 & 5.30 \\
\hline Appanoose & - & - & 7 & 17.55 & 1 & 5.79 & - & - & - & - & 8 & 23.34 \\
\hline Black Hawk & 2 & 1.24 & 2 & 0.78 & 6 & 3.71 & - & - & 1 & 0.85 & 11 & 6.58 \\
\hline Boone & - & - & 6 & 12.16 & - & - & - & - & - & - & 6 & 12.16 \\
\hline Carroll & 1 & 4.24 & - & - & - & - & - & - & - & - & 1 & 4.24 \\
\hline Cedar & 2 & 10.48 & - & - & - & - & - & - & - & - & 2 & 10.48 \\
\hline Clinton & - & - & - & - & - & - & - & - & 1 & 1.83 & 1 & 1.83 \\
\hline Dallas & - & - & 2 & 2.76 & - & - & - & - & - & - & 2 & 2.76 \\
\hline Davis & - & - & 2 & 14.04 & - & - & - & - & - & - & 2 & 14.04 \\
\hline Delaware & 1 & 2.36 & - & - & 1 & 2.36 & - & - & - & - & 2 & 4.72 \\
\hline Des Moines & 3 & 6.59 & - & - & 1 & 0.87 & - & - & - & - & 4 & 7.46 \\
\hline Emmet & - & - & 1 & 7.48 & - & - & - & - & - & - & 1 & 7.48 \\
\hline Guthrie & - & - & 1 & 2.46 & - & - & - & - & - & - & 1 & 2.46 \\
\hline Hamilton & - & - & - & - & 1 & 5.13 & - & - & - & - & 1 & 5.13 \\
\hline Henry & 1 & 5.28 & - & - & - & - & - & - & - & - & 1 & 5.28 \\
\hline Iowa & - & - & 1 & 2.10 & - & - & - & - & - & - & 1 & 2.10 \\
\hline Jackson & 1 & 7.62 & 1 & 1.78 & - & - & - & - & - & - & 2 & 9.40 \\
\hline Jasker & 1 & 2.61 & 3 & 4.84 & 1 & 1.12 & - & - & - & - & 5 & 8.57 \\
\hline Jefferson & - & - & 2 & 4.65 & - & - & - & - & - & - & 2 & 4.65 \\
\hline Johnson & - & - & - & - & 1 & 0.89 & - & - & - & - & 1 & 0.89 \\
\hline Lee & 2 & 4.64 & - & - & 1 & 0.95 & - & - & 2 & 3.27 & 5 & 8.87 \\
\hline Linn & 2 & 1.37 & 1 & 0.68 & 2 & 1.53 & - & - & - & - & 5 & 3.58 \\
\hline Lucas & - & - & 2 & 19.19 & - & - & - & - & - & - & 2 & 19.19 \\
\hline Madison & - & - & 1 & 2.47 & - & - & - & - & - & - & 1 & 2.47 \\
\hline Marion & - & - & 5 & 12.40 & - & - & - & - & - & - & 5 & 12.40 \\
\hline Mitchell & - & - & - & - & 1 & 2.27 & - & - & - & - & 1 & 2.27 \\
\hline Monroe & - & - & 5 & 35.91 & - & - & - & - & - & - & 5 & 35.91 \\
\hline Montgomery & - & - & 1 & 2.01 & - & - & - & - & - & - & 1 & 2.01 \\
\hline Muscatine & 1 & 1.13 & - & - & 2 & 2.26 & - & - & - & - & 3 & 3.39 \\
\hline Page & 1 & 4.71 & - & - & - & - & - & - & - & - & 1 & 4.71 \\
\hline Polk & 5 & 1.45 & 13 & 2.46 & 1 & 0.17 & - & - & 1 & 0.37 & 20 & 4.45 \\
\hline Pottawattamie & 2 & 1.79 & - & - & - & - & - & - & - & - & 2 & 1.79 \\
\hline
\end{tabular}

See footnotes at end of table. 
Table IA-3 (page 2 of 2). Number of deaths and age-adjusted mortality rate (per $1,000,000$ population) by condition and county, Iowa residents age 15 and over, 1983-1992

\begin{tabular}{|c|c|c|c|c|c|c|c|c|c|c|c|c|}
\hline \multirow[b]{2}{*}{ County } & \multicolumn{2}{|c|}{ Asbestosis } & \multicolumn{2}{|c|}{$\begin{array}{c}\text { Coal workers' } \\
\text { pneumoconiosis }\end{array}$} & \multicolumn{2}{|c|}{ Silicosis } & \multicolumn{2}{|c|}{ Byssinosis } & \multicolumn{2}{|c|}{$\begin{array}{r}\text { Unspecified/Other } \\
\text { pneumoconioses }\end{array}$} & \multicolumn{2}{|c|}{$\begin{array}{r}\text { All } \\
\text { pneumoconioses }\end{array}$} \\
\hline & $\#$ & Rate & \# & Rate & $\#$ & Rate & $\#$ & Rate & \# & Rate & $\#$ & Rate \\
\hline Poweshiek & 1 & 5.07 & - & - & - & - & - & - & - & - & 1 & 5.07 \\
\hline Scott & 2 & 0.78 & 2 & 1.17 & 3 & 1.56 & - & - & - & - & 7 & 3.50 \\
\hline Sioux & 1 & 3.53 & - & - & - & - & - & - & - & - & 1 & 3.53 \\
\hline Story & - & - & 2 & 4.26 & - & - & - & - & - & - & 2 & 4.26 \\
\hline Tama & - & - & - & - & 1 & 4.77 & - & - & - & - & 1 & 4.77 \\
\hline Union & - & - & 1 & 2.23 & - & - & - & - & - & - & 1 & 2.23 \\
\hline Van Buren & - & - & 1 & 3.57 & - & - & - & - & - & - & 1 & 3.57 \\
\hline Wapello & 1 & 0.87 & 2 & 1.74 & - & - & - & - & - & - & 3 & 2.61 \\
\hline Wayne & - & - & 1 & 2.90 & - & - & - & - & - & - & 1 & 2.90 \\
\hline Webster & - & - & 2 & 3.00 & - & - & - & - & 2 & 1.64 & 4 & 4.64 \\
\hline Woodbury & 3 & 1.88 & - & - & 1 & 0.43 & - & - & - & - & 4 & 2.31 \\
\hline
\end{tabular}

- indicates no deaths listed.

NOTE: Counties with no deaths for all listed conditions are not displayed. Rates based on small numbers may be unstable. Sum of individual conditions may exceed total

because of deaths with multiple pneumoconioses. See Appendix A for source description and Appendix B for methods and ICD-8 and ICD-9 codes

SOURCE: National Center for Health Statistics multiple cause of death data. Population estimates from U.S. Bureau of the Census. 
Kansas: Selected statistics from the United States section

All pneumoconioses mortality, 1988-1992

Total deaths

Crude mortality rate (per $1,000,000$ population)

Age-adjusted mortality rate (per $1,000,000$ population)

Total years of potential life lost to life expectancy

Years of potential life lost to life expectancy (per death)

Deaths by type of pneumoconiosis, 1988-1992

$\underline{\text { Value }}$

41

4.28

2.25

513

12.5

Deaths*

20

10

3

1

7 $\underline{\text { State rank }}$

38

46

48

38

16

Percent** distribution

48.8

24.4

7.3

2.4

17.1

Unspecified/Other pneumoconiosis

* Sum of column may exceed total deaths because of deaths with multiple pneumoconioses.

** Percentages may total more than $100 \%$ due to deaths with multiple pneumoconioses.

NOTE: $\quad$ See Appendix A for source description and Appendix B for methods and ICD-8 and ICD-9 codes.

SOURCE: National Center for Health Statistics multiple cause of death data. Population estimates from U.S. Bureau of the Census.

\section{Figure KS-1. All pneumoconioses: Kansas and U.S. age-adjusted mortality rates, residents age 15 and over, 1968-1992}

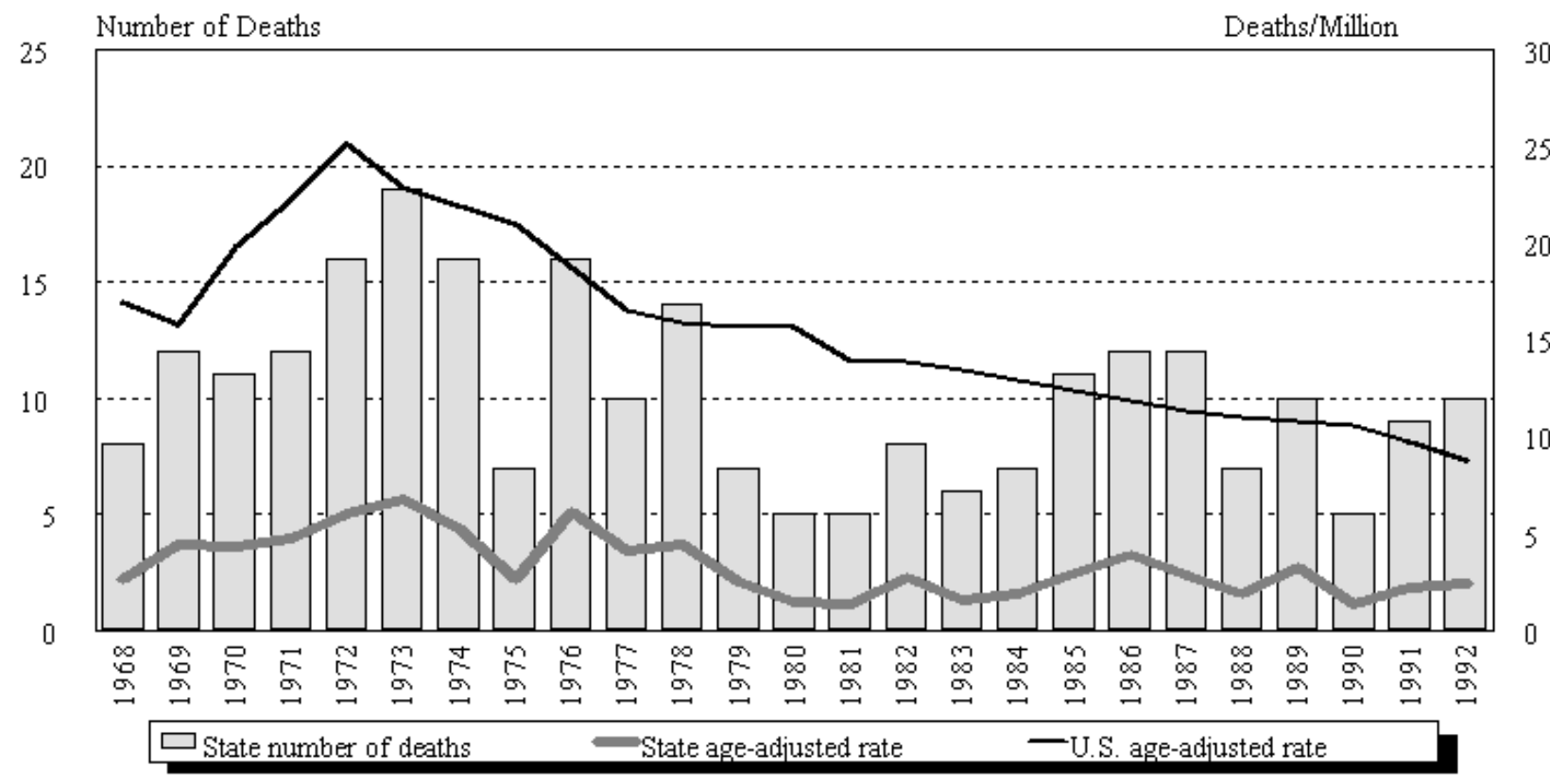

NOTE: $\quad$ See Appendix A for source description and Appendix B for methods and ICD-8 and ICD-9 codes.

SOURCE: $\quad$ National Center for Health Statistics multiple cause of death data. Population estimates from U.S. Bureau of the Census. 


\section{Kansas}

Table KS-1. Number of deaths and age-adjusted mortality rate (per 1,000,000 population) by condition, Kansas residents age 15 and over, 1968-1992

\begin{tabular}{|c|c|c|c|c|c|c|c|c|c|c|c|c|}
\hline \multirow[b]{2}{*}{ Year } & \multicolumn{2}{|c|}{ Asb estosis } & \multicolumn{2}{|c|}{$\begin{array}{l}\text { Coal workers' } \\
\text { pneumoconiosis }\end{array}$} & \multicolumn{2}{|c|}{ Silicosis } & \multicolumn{2}{|c|}{ Byssinosis* } & \multicolumn{2}{|c|}{$\begin{array}{c}\text { Unspecified/Other } \\
\text { pneumoconioses }\end{array}$} & \multicolumn{2}{|c|}{ All pneumoconioses } \\
\hline & Number & Rate & Number & Rate & Number & Rate & Number & Rate & Number & Rate & Number & Rate \\
\hline 1968 & - & - & 1 & 0.16 & 6 & 2.28 & & & 1 & 0.16 & 8 & 2.61 \\
\hline 1969 & - & - & - & - & 9 & 3.06 & & & 3 & 1.34 & 12 & 4.40 \\
\hline 1970 & - & - & - & - & 11 & 4.32 & & & - & - & 11 & 4.32 \\
\hline 1971 & - & - & 1 & 0.26 & 8 & 3.31 & & & 3 & 1.17 & 12 & 4.74 \\
\hline 1972 & - & - & 4 & 1.02 & 6 & 2.90 & & & 6 & 2.12 & 16 & 6.04 \\
\hline 1973 & - & - & 5 & 1.68 & 9 & 3.70 & & & 5 & 1.44 & 19 & 6.82 \\
\hline 1974 & - & - & 7 & 2.06 & 4 & 1.70 & & & 5 & 1.54 & 16 & 5.30 \\
\hline 1975 & 1 & 0.24 & 1 & 0.50 & - & - & & & 5 & 1.89 & 7 & 2.63 \\
\hline 1976 & - & - & 2 & 0.88 & 9 & 3.58 & & & 5 & 1.64 & 16 & 6.10 \\
\hline 1977 & - & - & 2 & 1.18 & 4 & 1.39 & & & 5 & 1.77 & 10 & 4.10 \\
\hline 1978 & - & - & 5 & 1.35 & 4 & 1.58 & & & 5 & 1.49 & 14 & 4.43 \\
\hline 1979 & 1 & 0.48 & 2 & 0.75 & 2 & 0.85 & - & - & 2 & 0.47 & 7 & 2.55 \\
\hline 1980 & - & - & 1 & 0.23 & 1 & 0.23 & - & - & 3 & 0.98 & 5 & 1.44 \\
\hline 1981 & - & - & 1 & 0.11 & 3 & 0.95 & - & - & 1 & 0.37 & 5 & 1.42 \\
\hline 1982 & 1 & 0.47 & 3 & 0.69 & 4 & 1.64 & - & - & - & - & 8 & 2.80 \\
\hline 1983 & 1 & 0.36 & 2 & 0.46 & 2 & 0.46 & - & - & 1 & 0.36 & 6 & 1.64 \\
\hline 1984 & - & - & 6 & 1.72 & - & - & - & - & 1 & 0.22 & 7 & 1.94 \\
\hline 1985 & 2 & 0.69 & 6 & 1.32 & 2 & 0.57 & - & - & 1 & 0.36 & 11 & 2.94 \\
\hline 1986 & 3 & 0.93 & 7 & 2.16 & - & - & - & - & 2 & 0.84 & 12 & 3.94 \\
\hline 1987 & 5 & 1.33 & 2 & 0.30 & 5 & 1.21 & - & - & - & - & 12 & 2.84 \\
\hline 1988 & 3 & 0.76 & - & - & 1 & 0.35 & - & - & 3 & 0.76 & 7 & 1.87 \\
\hline 1989 & 5 & 1.87 & 4 & 1.28 & - & - & - & - & 1 & 0.09 & 10 & 3.24 \\
\hline 1990 & 2 & 0.40 & 2 & 0.76 & - & - & 1 & 0.20 & - & - & 5 & 1.35 \\
\hline 1991 & 3 & 0.59 & 2 & 0.69 & 1 & 0.20 & - & - & 3 & 0.78 & 9 & 2.25 \\
\hline 1992 & 7 & 1.59 & 2 & 0.69 & 1 & 0.19 & - & - & - & - & 10 & 2.48 \\
\hline
\end{tabular}

indicates no deaths listed. ${ }^{*}$ no specific code for byssinosis in ICD-8 (1968-1978).

NOTE: Rates based on small numbers may be unstable. Sum of individual conditions may exceed total because of deaths with multiple pneumoconioses. See Appendix A for source description and Appendix B for methods and ICD-8 and ICD-9 codes.

SOURCE: National Center for Health Statistics multiple cause of death data. Population estimates from U.S. Bureau of the Census. 
Figure KS-2. All pneumoconioses: Distribution of deaths by sex and race, Kansas residents age 15 and over, 1968-1992
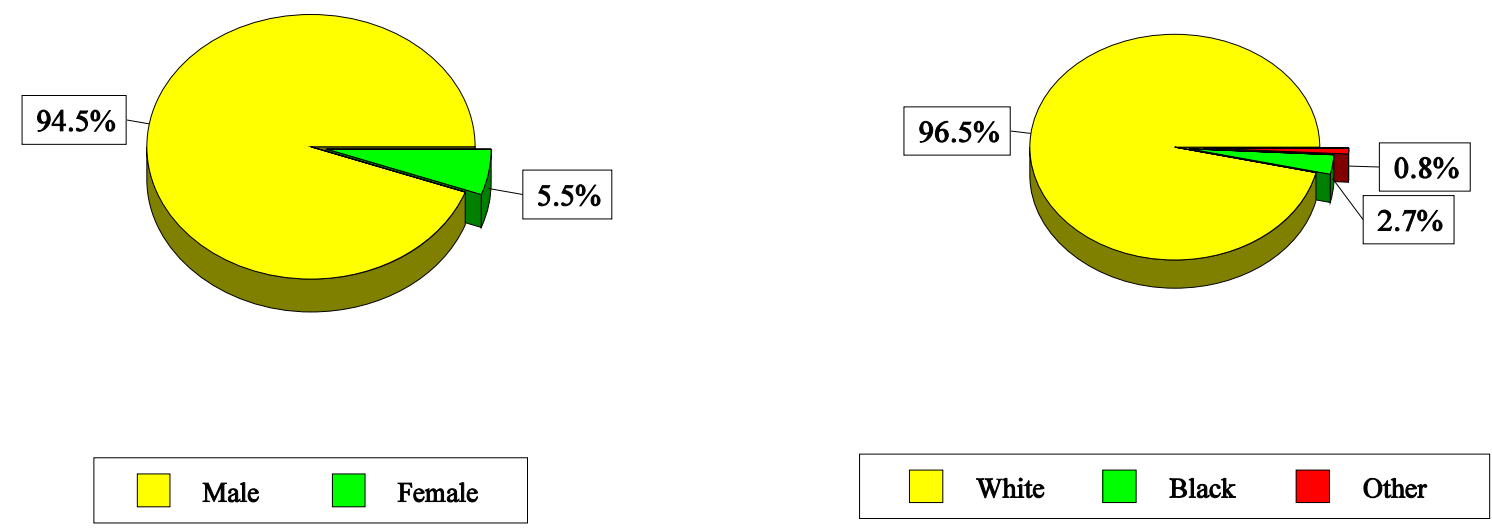

NOTE: $\quad$ See Appendix A for source description and Appendix B for methods and ICD-8 and ICD-9 codes.

SOURCE: National Center for Health Statistics multiple cause of death data.

Table KS-2. All pneumoconioses: Distribution of deaths by sex, race, and age, Kansas residents age 15 and over, 1968-1992

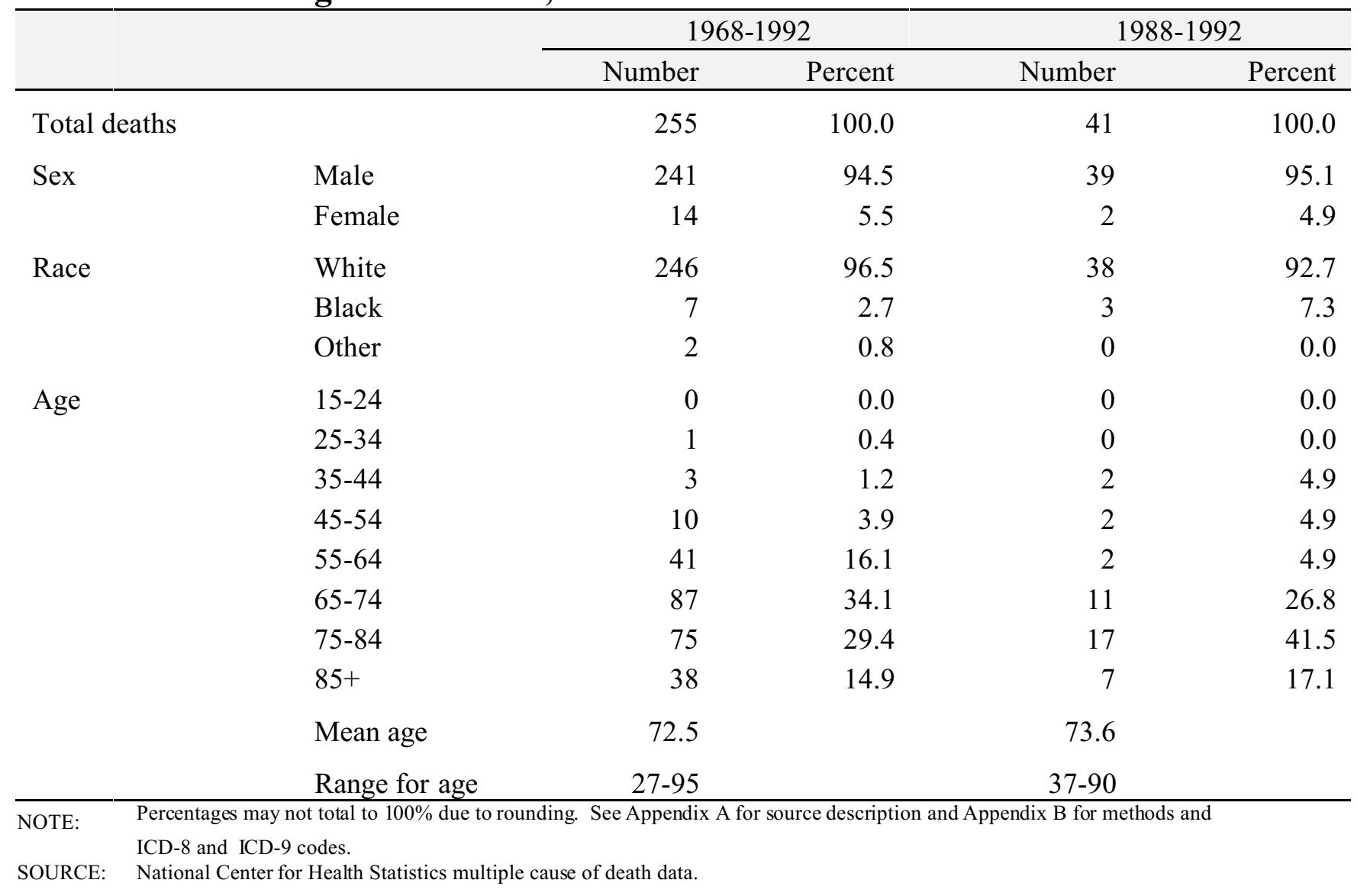




\section{Figure KS-3. Pneumoconiosis deaths by condition, Kansas residents age 15 and over, 1968-1992}

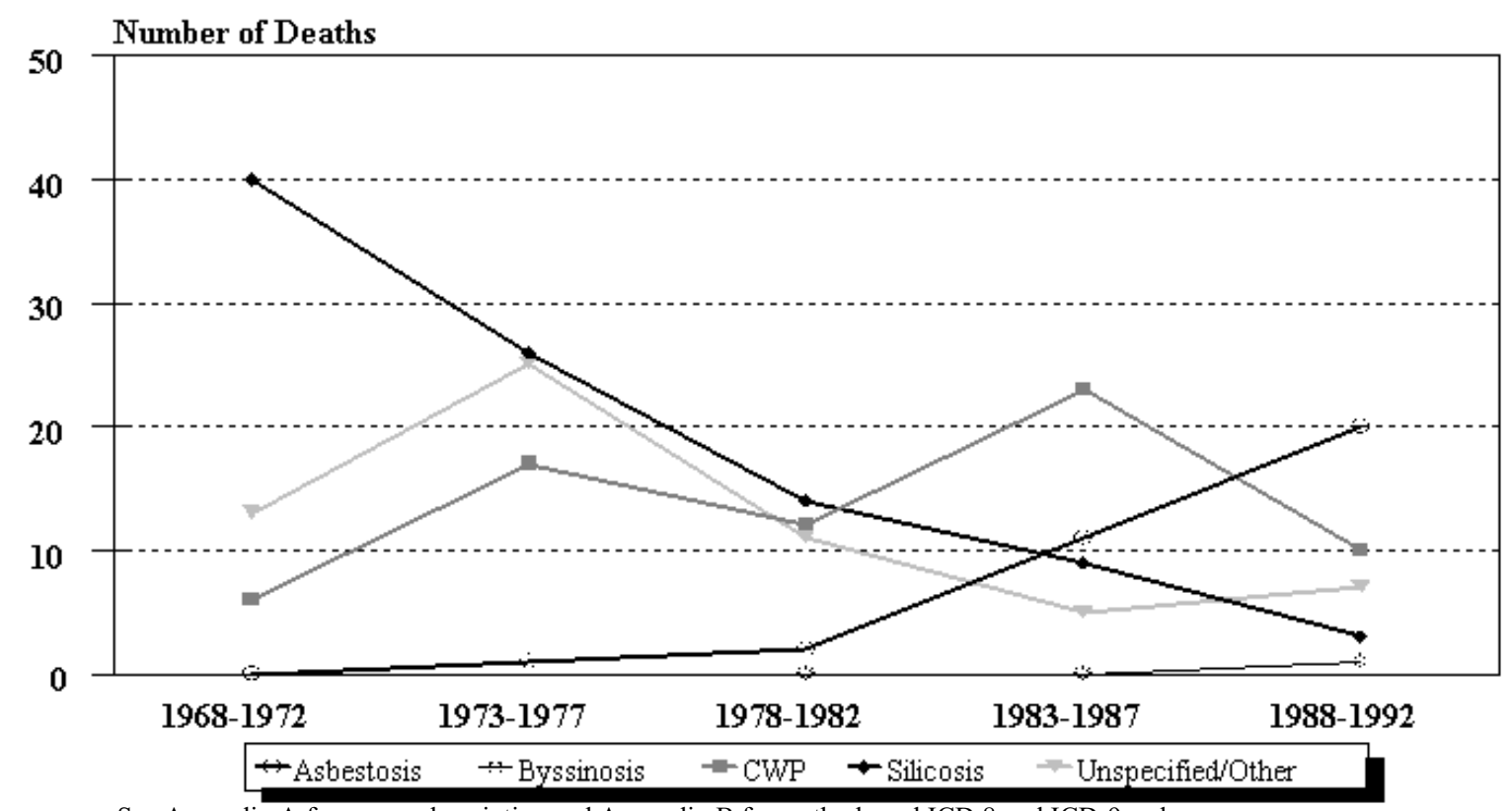

NOTE: See Appendix A for source description and Appendix B for methods and ICD-8 and ICD-9 codes.

SOURCE: National Center for Health Statistics multiple cause of death data.

Figure KS-4. Kansas and U.S. age-adjusted mortality rates (per 1,000,000 population) by condition, residents age 15 and over, 1983-1992

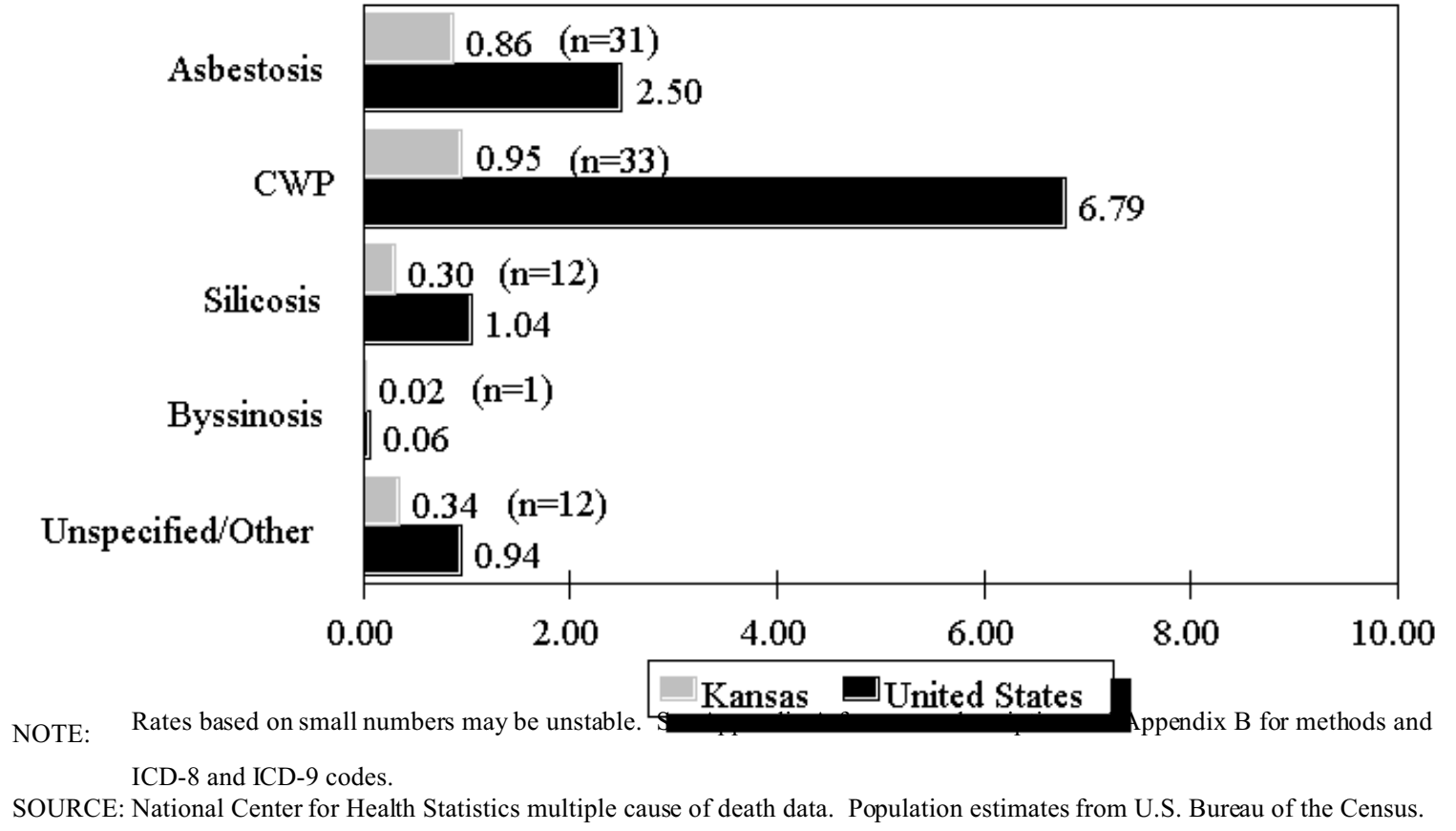


Kansas

\begin{tabular}{|c|c|c|c|c|c|c|c|c|c|c|c|c|}
\hline \multirow[b]{2}{*}{ County } & \multicolumn{2}{|c|}{ Asbestosis } & \multicolumn{2}{|c|}{$\begin{array}{r}\text { Coal workers' } \\
\text { pneumoconiosis }\end{array}$} & \multicolumn{2}{|c|}{ Silicosis } & \multicolumn{2}{|c|}{ Byssinosis } & \multicolumn{2}{|c|}{$\begin{array}{l}\text { Unspecified/Other } \\
\text { pneumoconioses }\end{array}$} & \multicolumn{2}{|c|}{$\begin{array}{r}\text { All } \\
\text { pneumoconiose }\end{array}$} \\
\hline & $\#$ & Rate & $\#$ & Rate & $\#$ & Rate & $\#$ & Rate & $\#$ & Rate & $\#$ & Rate \\
\hline Atchison & - & - & - & - & 2 & 3.92 & - & - & 2 & 7.47 & 4 & 11.39 \\
\hline Bourbon & - & - & 1 & 5.46 & - & - & - & - & - & - & 1 & 5.46 \\
\hline Bulter & 5 & 6.98 & - & - & - & - & - & - & - & - & 5 & 6.98 \\
\hline Cherokee & - & - & 1 & 4.03 & 3 & 7.13 & - & - & 2 & 8.06 & 6 & 19.23 \\
\hline Cowley & 1 & 0.92 & - & - & - & - & - & - & - & - & 1 & 0.92 \\
\hline Crawford & - & - & 6 & 6.32 & 1 & 2.48 & - & - & 2 & 3.25 & 9 & 12.05 \\
\hline Dickson & - & - & - & - & - & - & - & - & 1 & 4.43 & 1 & 4.43 \\
\hline Douglas & 2 & 3.27 & 1 & 0.96 & - & - & - & - & - & - & 3 & 4.23 \\
\hline Franklin & - & - & 1 & 1.55 & - & - & - & - & - & - & 1 & 1.55 \\
\hline Geary & 1 & 5.14 & - & - & - & - & - & - & - & - & 1 & 5.14 \\
\hline Harvey & 1 & 3.23 & - & - & - & - & - & - & - & - & 1 & 3.23 \\
\hline Jefferson & - & - & 1 & 2.56 & - & - & - & - & - & - & 1 & 2.56 \\
\hline Jewell & 1 & 14.93 & - & - & - & - & - & - & - & - & 1 & 14.93 \\
\hline Johnson & 3 & 0.85 & 2 & 0.61 & - & - & - & - & - & - & 5 & 1.46 \\
\hline Kingman & 1 & 3.51 & - & - & - & - & - & - & - & - & 1 & 3.51 \\
\hline Labette & 1 & 1.27 & 1 & 3.72 & - & - & - & - & - & - & 2 & 4.99 \\
\hline Leavenworth & - & - & - & - & 2 & 3.24 & - & - & - & - & 2 & 3.24 \\
\hline Linn & 1 & 3.21 & 2 & 6.43 & - & - & - & - & - & - & 3 & 9.64 \\
\hline Marshall & 2 & 8.21 & - & - & - & - & - & - & - & - & 2 & 8.21 \\
\hline Miami & - & - & - & - & - & - & - & - & 1 & 4.50 & 1 & 4.50 \\
\hline Montgomery & 1 & 0.75 & - & - & - & - & - & - & - & - & 1 & 0.75 \\
\hline Morris & 1 & 12.04 & - & - & - & - & - & - & - & - & 1 & 12.04 \\
\hline Osage & 1 & 2.06 & 3 & 6.18 & - & - & - & - & 1 & 2.06 & 5 & 10.31 \\
\hline Osborne & - & - & - & - & 1 & 13.56 & - & - & - & - & 1 & 13.56 \\
\hline Pratt & 1 & 2.88 & - & - & - & - & - & - & - & - & 1 & 2.88 \\
\hline Reno & - & - & 6 & 10.58 & - & - & - & - & - & - & 6 & 10.58 \\
\hline Riley & 1 & 3.41 & - & - & - & - & - & - & - & - & 1 & 3.41 \\
\hline Sedgwick & 2 & 0.44 & 3 & 0.82 & 1 & 0.29 & 1 & 0.15 & 2 & 0.44 & 9 & 2.14 \\
\hline Shawnee & 4 & 1.54 & 3 & 1.25 & - & - & - & - & 1 & 0.30 & 8 & 3.09 \\
\hline Stevens & - & - & - & - & 1 & 9.11 & - & - & - & - & 1 & 9.11 \\
\hline Wilson & - & - & - & - & 1 & 2.40 & - & - & - & - & 1 & 2.40 \\
\hline Woodson & - & - & 1 & 15.67 & - & - & - & - & - & - & 1 & 15.67 \\
\hline Wyandotte & 1 & 0.64 & 1 & 0.30 & - & - & - & - & - & - & 2 & 0.94 \\
\hline
\end{tabular}

NOTE: Counties with no deaths for all listed conditions are not displayed. Rates based on small numbers may be unstable. Sum of individual conditions may exceed total because of deaths with multiple pneumoconioses. See Appendix A for source description and Appendix B for methods and ICD-8 and ICD-9 codes.

SOURCE: National Center for Health Statistics multiple cause of death data. Population estimates from U.S. Bureau of the Census. 
Kentucky

\section{Kentucky: Selected statistics from the United States section}

All pneumoconioses mortality, 1988-1992

Total deaths

Crude mortality rate (per $1,000,000$ population)

Age-adjusted mortality rate (per $1,000,000$ population)

Total years of potential life lost to life expectancy

Years of potential life lost to life expectancy (per death) $\underline{\text { Value }}$

871

60.22

34.22

10,380

11.9

Deaths*

19

648

28

0

178
State rank

4

3

Percent**

distribution

2.2

74.4

3.2

0.0

20.4

\section{Unspecified/Other pneumoconiosis}

* Sum of column may exceed total deaths because of deaths with multiple pneumoconioses.

** Percentages may total more than $100 \%$ due to deaths with multiple pneumoconioses.

NOTE: $\quad$ See Appendix A for source description and Appendix B for methods and ICD-8 and ICD-9 codes.

SOURCE: National Center for Health Statistics multiple cause of death data. Population estimates from U.S. Bureau of the Census.

Figure KY-1. All pneumoconioses: Kentucky and U.S. age-adjusted mortality rates, residents age 15 and over, 1968-1992

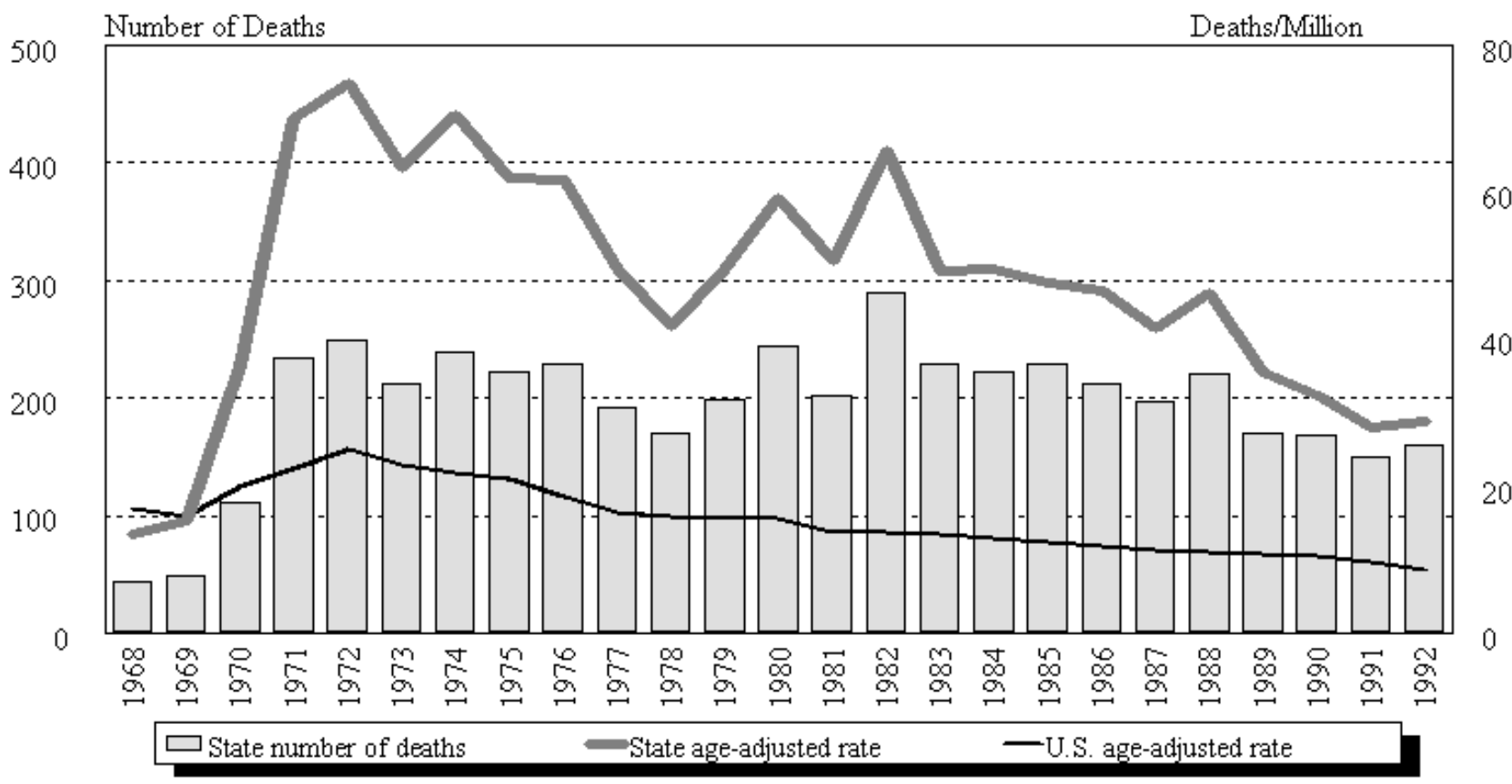

NOTE: $\quad$ See Appendix A for source description and Appendix B for methods and ICD-8 and ICD-9 codes.

SOURCE: National Center for Health Statistics multiple cause of death data. Population estimates from U.S. Bureau of the Census. 
Kentucky

\begin{tabular}{|c|c|c|c|c|c|c|c|c|c|c|c|c|}
\hline \multirow[b]{2}{*}{ Year } & \multicolumn{2}{|c|}{ Asb estosis } & \multicolumn{2}{|c|}{$\begin{array}{l}\text { Coal workers' } \\
\text { pneumoconiosis }\end{array}$} & \multicolumn{2}{|c|}{ Silicosis } & \multicolumn{2}{|c|}{ Byssinosis* } & \multicolumn{2}{|c|}{$\begin{array}{c}\text { Unspecified/Other } \\
\text { pneumoconioses }\end{array}$} & \multicolumn{2}{|c|}{ All pneumoconioses } \\
\hline & Number & Rate & Number & Rate & Number & Rate & Number & Rate & Number & Rate & Number & Rate \\
\hline 1968 & - & - & 3 & 0.66 & 23 & 7.91 & & & 18 & 5.09 & 44 & 13.67 \\
\hline 1969 & - & - & 7 & 2.41 & 22 & 6.75 & & & 22 & 6.88 & 49 & 15.37 \\
\hline 1970 & 1 & 0.45 & 34 & 11.34 & 31 & 10.06 & & & 51 & 16.06 & 112 & 36.36 \\
\hline 1971 & - & - & 80 & 24.29 & 25 & 8.14 & & & 138 & 40.56 & 234 & 69.92 \\
\hline 1972 & - & - & 88 & 27.54 & 30 & 8.46 & & & 140 & 41.71 & 250 & 74.98 \\
\hline 1973 & - & - & 72 & 22.00 & 24 & 6.57 & & & 119 & 35.42 & 213 & 63.28 \\
\hline 1974 & - & - & 89 & 27.26 & 10 & 2.67 & & & 143 & 41.79 & 239 & 70.62 \\
\hline 1975 & - & - & 89 & 24.56 & 15 & 4.16 & & & 123 & 34.39 & 222 & 61.87 \\
\hline 1976 & - & - & 97 & 26.29 & 12 & 3.49 & & & 121 & 32.22 & 229 & 61.72 \\
\hline 1977 & - & - & 94 & 24.16 & 13 & 3.25 & & & 89 & 22.97 & 193 & 49.66 \\
\hline 1978 & 1 & 0.33 & 84 & 20.64 & 4 & 0.76 & & & 82 & 20.01 & 171 & 41.73 \\
\hline 1979 & 1 & 0.26 & 109 & 26.91 & 10 & 3.23 & - & - & 80 & 19.76 & 199 & 49.73 \\
\hline 1980 & - & - & 130 & 31.32 & 4 & 1.10 & - & - & 111 & 26.97 & 245 & 59.39 \\
\hline 1981 & 6 & 1.63 & 159 & 39.89 & 4 & 1.00 & - & - & 34 & 8.14 & 203 & 50.66 \\
\hline 1982 & 2 & 0.63 & 242 & 55.19 & 12 & 2.73 & - & - & 34 & 7.29 & 290 & 65.84 \\
\hline 1983 & - & - & 196 & 42.34 & 6 & 1.19 & - & - & 27 & 5.85 & 229 & 49.38 \\
\hline 1984 & 2 & 0.62 & 177 & 39.66 & 3 & 0.66 & - & - & 40 & 8.54 & 222 & 49.48 \\
\hline 1985 & 4 & 1.18 & 195 & 39.71 & 10 & 2.63 & - & - & 21 & 4.28 & 229 & 47.63 \\
\hline 1986 & 4 & 0.74 & 182 & 39.54 & 5 & 1.48 & - & - & 22 & 5.07 & 212 & 46.66 \\
\hline 1987 & 3 & 0.65 & 161 & 32.93 & 2 & 0.57 & - & - & 31 & 7.36 & 197 & 41.50 \\
\hline 1988 & 4 & 0.55 & 168 & 35.11 & 11 & 2.65 & - & - & 39 & 8.23 & 221 & 46.30 \\
\hline 1989 & 4 & 1.24 & 137 & 28.59 & 3 & 0.81 & - & - & 26 & 5.03 & 170 & 35.67 \\
\hline 1990 & 1 & 0.15 & 115 & 23.03 & 6 & 1.20 & - & - & 47 & 7.93 & 169 & 32.32 \\
\hline 1991 & 5 & 1.40 & 112 & 19.95 & 2 & 0.55 & - & - & 32 & 6.56 & 150 & 28.12 \\
\hline 1992 & 5 & 1.01 & 116 & 20.58 & 6 & 1.54 & - & - & 34 & 5.68 & 161 & 28.80 \\
\hline
\end{tabular}

- indicates no deaths listed. $\quad *$ no sp ecific code for by ssinosis in ICD-8 (1968-1978).

NOTE: Rates based on small numbers may be unstable. Sum of individual conditions may exceed total because of deaths with multiple pneumoconioses. See Appendix A for source description and Appendix B for methods and ICD-8 and ICD-9 codes.

SOURCE: National Center for Health Statistics multiple cause of death data. Population estimates from U.S. Bureau of the Census. 
Figure KY-2. All pneumoconioses: Distribution of deaths by sex and race, Kentucky residents age 15 and over, 1968-1992
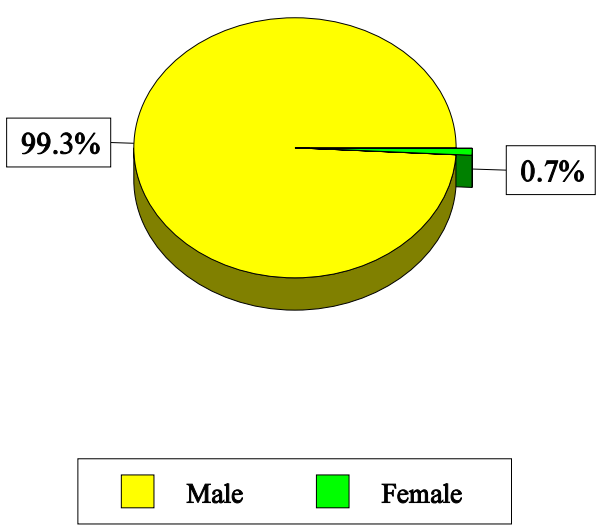

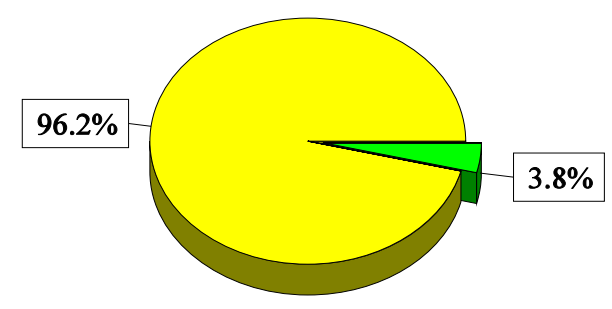

NOTE: $\quad$ See Appendix A for source description and Appendix B for methods and ICD-8 and ICD-9 codes.

SOURCE: National Center for Health Statistics multiple cause of death data.

Table KY-2. All pneumoconioses: Distribution of deaths by sex, race, and age, Kentucky residents age 15 and over, 1968-1992

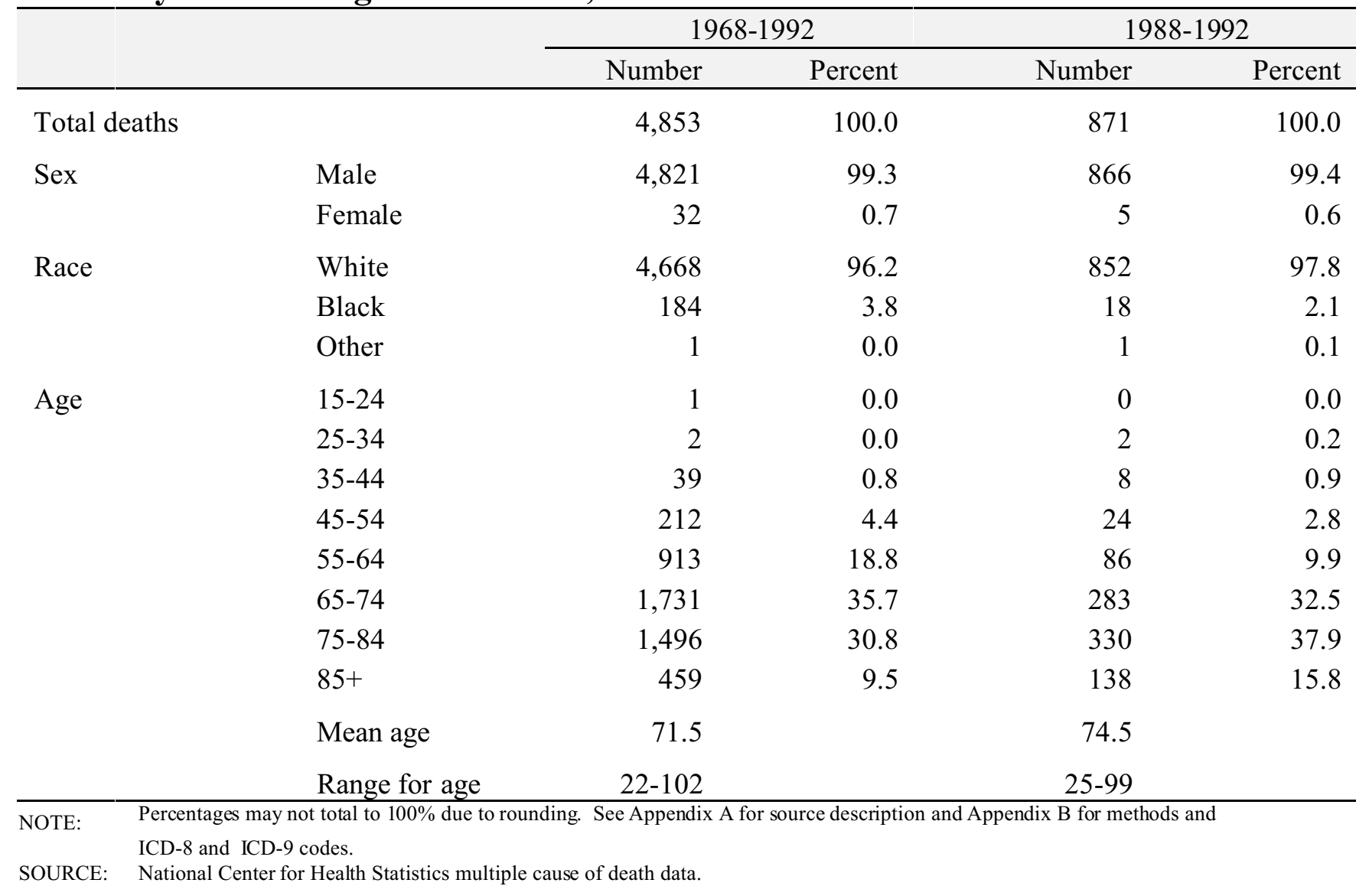




\section{Figure KY-3. Pneumoconiosis deaths by condition, Kentucky residents age 15 and over, 1968-1992}

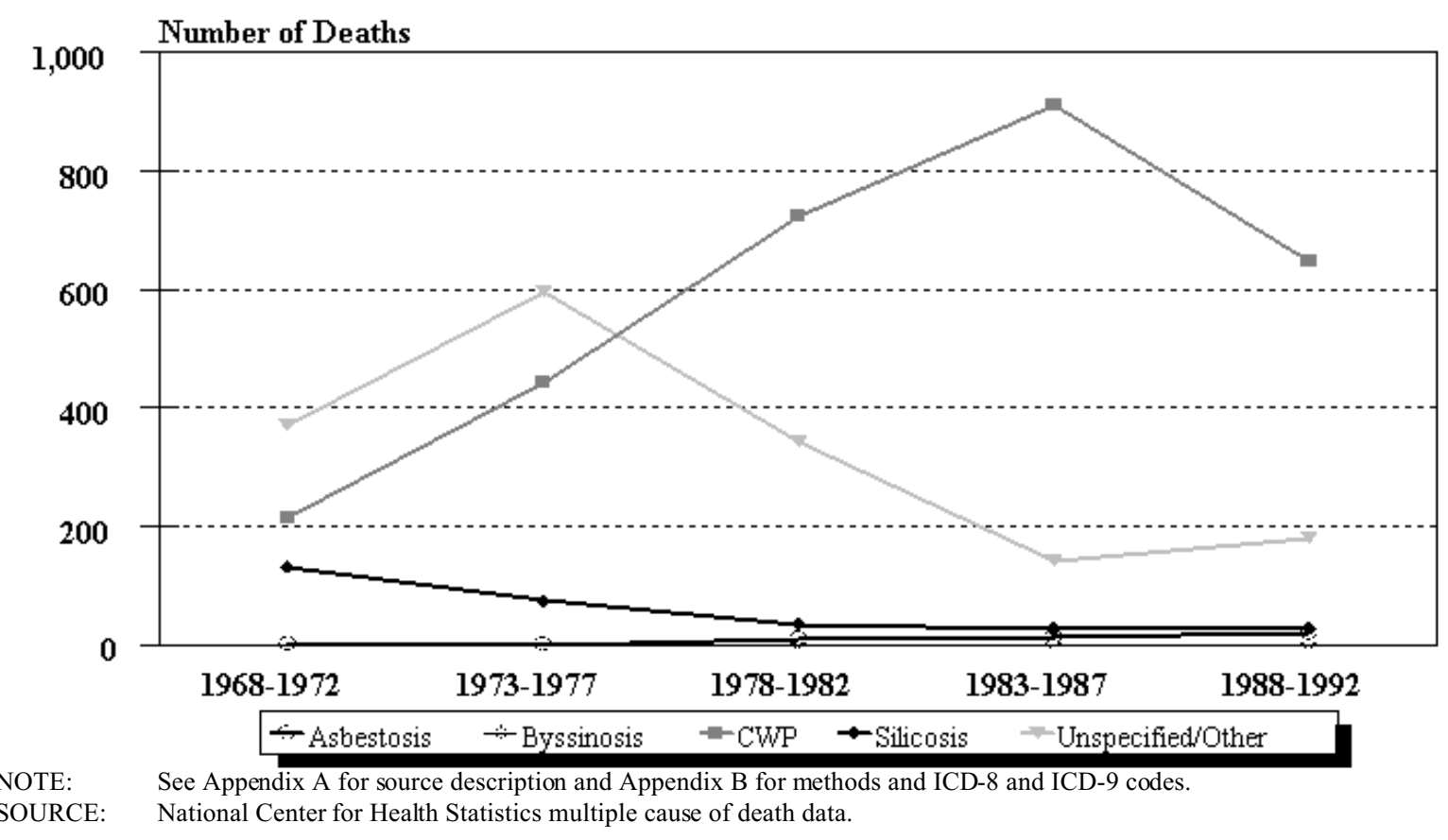

Figure KY-4. Kentucky and U.S. age-adjusted mortality rates (per 1,000,000 population) by condition, residents age 15 and over, 1983-1992

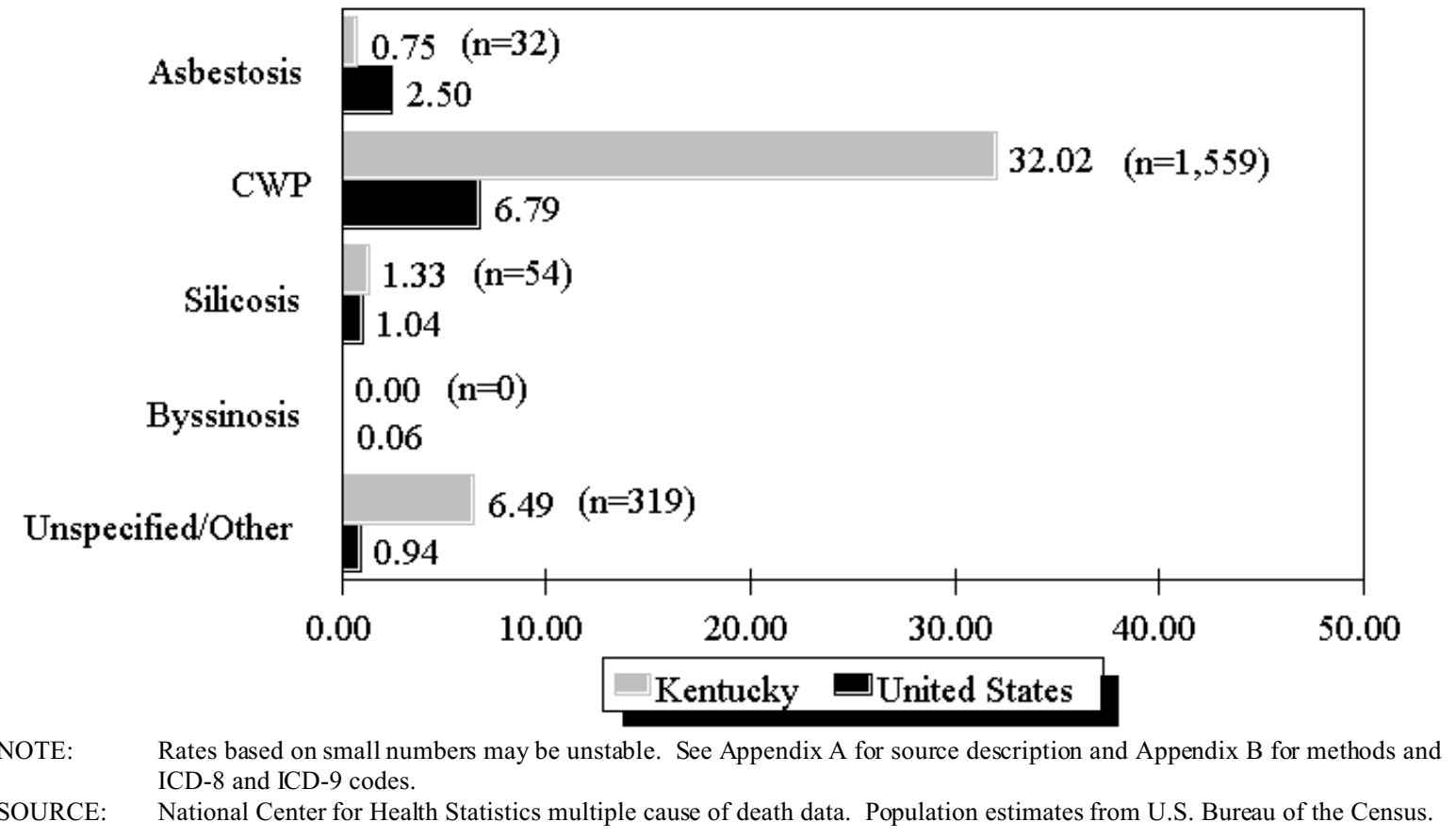


Kentucky

Table KY-3 (page 1 of 3). Number of deaths and age-adjusted mortality rate (per $1,000,000$ population) by condition and county, Kentucky residents age 15 and over, 1983-1992

\begin{tabular}{|c|c|c|c|c|c|c|c|c|c|c|c|c|}
\hline \multirow[b]{2}{*}{ County } & \multicolumn{2}{|c|}{ Asbestosis } & \multicolumn{2}{|c|}{$\begin{array}{c}\text { Coal workers' } \\
\text { pneumoconiosis }\end{array}$} & \multicolumn{2}{|c|}{ Silicosis } & \multicolumn{2}{|c|}{ Byssinosis } & \multicolumn{2}{|c|}{$\begin{array}{r}\text { Unspecified/Other } \\
\text { pneumoconioses }\end{array}$} & \multicolumn{2}{|c|}{$\begin{array}{r}\text { All } \\
\text { pneumoconioses }\end{array}$} \\
\hline & $\#$ & Rate & $\#$ & Rate & $\#$ & Rate & $\#$ & Rate & $\#$ & Rate & $\#$ & Rate \\
\hline Adair & - & - & - & - & 2 & 8.45 & - & - & - & - & 2 & 8.45 \\
\hline Barren & - & - & - & - & 1 & 2.70 & - & - & - & - & 1 & 2.70 \\
\hline Bell & 1 & 1.57 & 105 & 244.67 & - & - & - & - & 36 & 85.61 & 142 & 331.85 \\
\hline Boone & 2 & 4.12 & 3 & 7.60 & 1 & 2.53 & - & - & 1 & 2.53 & 7 & 16.79 \\
\hline Bourbon & - & - & 1 & 5.19 & - & - & - & - & - & - & 1 & 5.19 \\
\hline Boyd & 1 & 0.91 & 6 & 7.07 & - & - & - & - & 3 & 3.53 & 10 & 11.51 \\
\hline Boyle & - & - & 2 & 5.54 & - & - & - & - & - & - & 2 & 5.54 \\
\hline Breathitt & - & - & 8 & 40.41 & - & - & - & - & 3 & 10.93 & 11 & 51.34 \\
\hline Breckinridge & - & - & 1 & 5.52 & - & - & - & - & 1 & 2.68 & 2 & 8.20 \\
\hline Butler & - & - & 2 & 12.51 & - & - & - & - & 7 & 51.43 & 9 & 63.94 \\
\hline Caldwell & - & - & 2 & 4.78 & - & - & - & - & - & - & 2 & 4.78 \\
\hline Campbell & 1 & 1.23 & 7 & 7.98 & - & - & - & - & - & - & 8 & 9.21 \\
\hline Carter & - & - & 4 & 8.56 & 2 & 4.28 & - & - & 1 & 4.33 & 6 & 15.04 \\
\hline Casey & - & - & 2 & 5.90 & - & - & - & - & - & - & 2 & 5.90 \\
\hline Christian & - & - & 5 & 5.69 & 1 & 0.90 & - & - & 2 & 2.98 & 8 & 9.57 \\
\hline Clay & - & - & 12 & 55.56 & 1 & 5.54 & - & - & 4 & 19.43 & 17 & 80.52 \\
\hline Clinton & - & - & 1 & 9.78 & - & - & - & - & - & - & 1 & 9.78 \\
\hline Crittenden & - & - & 4 & 26.43 & 2 & 19.02 & - & - & 3 & 22.73 & 8 & 58.67 \\
\hline Daviess & 3 & 3.55 & 14 & 12.50 & - & - & - & - & 1 & 0.60 & 18 & 16.65 \\
\hline Elliott & - & - & 2 & 24.83 & - & - & - & - & - & - & 2 & 24.83 \\
\hline Estill & - & - & 2 & 10.04 & - & - & - & - & - & - & 2 & 10.04 \\
\hline Fayette & 2 & 0.90 & 6 & 2.43 & 1 & 0.59 & - & - & 2 & 0.90 & 11 & 4.83 \\
\hline Fleming & - & - & 1 & 7.63 & - & - & - & - & 1 & 7.63 & 2 & 15.25 \\
\hline Floyd & - & - & 344 & 673.59 & 2 & 4.95 & - & - & 25 & 53.43 & 371 & 731.97 \\
\hline Franklin & - & - & 2 & 2.65 & - & - & - & - & 1 & 1.33 & 3 & 3.98 \\
\hline Fulton & - & - & 1 & 9.71 & - & - & - & - & - & - & 1 & 9.71 \\
\hline Garrard & - & - & 1 & 3.51 & - & - & - & - & - & - & 1 & 3.51 \\
\hline Grant & - & - & 3 & 14.95 & - & - & - & - & - & - & 3 & 14.95 \\
\hline Graves & 1 & 0.99 & - & - & - & - & - & - & 1 & 0.99 & 2 & 1.97 \\
\hline Green & - & - & - & - & 1 & 7.86 & - & - & - & - & 1 & 7.86 \\
\hline Greenup & 1 & 1.68 & 6 & 12.10 & 2 & 3.36 & - & - & 1 & 1.68 & 10 & 18.83 \\
\hline Hardin & - & - & 1 & 1.70 & 1 & 1.76 & - & - & 1 & 1.76 & 3 & 5.22 \\
\hline Harlan & 1 & 3.89 & 177 & 365.53 & 1 & 1.40 & - & - & 53 & 115.64 & 232 & 486.45 \\
\hline
\end{tabular}

See footnotes at end of table. 
Table KY-3 (page 2 of 3). Number of deaths and age-adjusted mortality rate (per $1,000,000$ population) by condition and county, Kentucky residents age 15 and over, 1983-1992

\begin{tabular}{|c|c|c|c|c|c|c|c|c|c|c|c|c|}
\hline \multirow[b]{2}{*}{ County } & \multicolumn{2}{|c|}{ Asbestosis } & \multicolumn{2}{|c|}{$\begin{array}{c}\text { Coal workers' } \\
\text { pneumoconiosis }\end{array}$} & \multicolumn{2}{|c|}{ Silicosis } & \multicolumn{2}{|c|}{ Byssinosis } & \multicolumn{2}{|c|}{$\begin{array}{r}\text { Unspecified/Other } \\
\text { pneumoconioses }\end{array}$} & \multicolumn{2}{|c|}{$\begin{array}{r}\text { All } \\
\text { pneumoconioses }\end{array}$} \\
\hline & \# & Rate & \# & Rate & \# & Rate & \# & Rate & \# & Rate & \# & Rate \\
\hline Hart & - & - & - & $\begin{array}{l}- \\
-\end{array}$ & 1 & 5.86 & - & - & - & - & 1 & 5.86 \\
\hline Henderson & - & - & 9 & 11.99 & - & - & - & - & - & - & 9 & 11.99 \\
\hline Henry & - & - & 1 & 3.55 & - & - & - & - & - & - & 1 & 3.55 \\
\hline Hopkins & - & - & 32 & 52.14 & - & - & - & - & 10 & 14.92 & 42 & 67.06 \\
\hline Jackson & - & - & 10 & 68.90 & 1 & 12.67 & - & - & - & - & 11 & 81.57 \\
\hline Jefferson & 7 & 0.82 & 7 & 0.75 & 11 & 1.39 & - & - & 6 & 0.53 & 31 & 3.49 \\
\hline Jessamine & 1 & 2.68 & - & - & - & - & - & - & 1 & 2.68 & 2 & 5.35 \\
\hline Johnson & - & - & 46 & 150.02 & 5 & 22.79 & - & - & 3 & 9.33 & 54 & 182.14 \\
\hline Kenton & 1 & 0.81 & 12 & 6.92 & 2 & 1.38 & - & - & - & - & 14 & 8.71 \\
\hline Knott & - & - & 51 & 237.97 & - & - & - & - & 4 & 17.72 & 55 & 255.69 \\
\hline Knox & - & - & 73 & 200.30 & - & - & - & - & 17 & 42.59 & 90 & 242.89 \\
\hline Laurel & 1 & 1.36 & 33 & 58.34 & - & - & - & - & 9 & 14.79 & 43 & 74.50 \\
\hline Lawrence & - & - & 11 & 57.94 & 1 & 7.39 & - & - & - & - & 12 & 65.33 \\
\hline Lee & - & - & 4 & 20.68 & 1 & 5.17 & - & - & - & - & 5 & 25.85 \\
\hline Leslie & - & - & 18 & 146.56 & - & - & - & - & 9 & 64.13 & 27 & 210.69 \\
\hline Letcher & - & - & 131 & 418.13 & - & - & - & - & 15 & 43.18 & 146 & 461.31 \\
\hline Lewis & - & - & 3 & 19.44 & 1 & 7.79 & - & - & 1 & 7.79 & 5 & 35.03 \\
\hline Lincoln & - & - & 4 & 11.86 & 1 & 4.86 & - & - & 1 & 2.33 & 6 & 19.05 \\
\hline Logan & - & - & 1 & 3.82 & - & - & - & - & - & - & 1 & 3.82 \\
\hline McCracken & 1 & 0.63 & 4 & 2.51 & - & - & - & - & 1 & 1.44 & 6 & 4.57 \\
\hline McCreary & - & - & 13 & 67.99 & - & - & - & - & 1 & 3.94 & 14 & 71.93 \\
\hline McLean & - & - & 2 & 13.67 & - & - & - & - & - & - & 2 & 13.67 \\
\hline Madison & - & - & 8 & 15.02 & 1 & 2.33 & - & - & 1 & 1.12 & 10 & 18.47 \\
\hline Magoffin & 3 & 30.85 & 6 & 48.69 & 1 & 11.49 & - & - & 1 & 11.49 & 11 & 102.52 \\
\hline Martin & - & - & 9 & 91.15 & 2 & 17.99 & - & - & 2 & 16.84 & 13 & 125.98 \\
\hline Meade & - & - & 1 & 7.15 & 1 & 7.15 & - & - & - & - & 2 & 14.29 \\
\hline Mercer & - & - & 1 & 4.83 & - & - & - & - & - & - & 1 & 4.83 \\
\hline Metcalfe & 1 & 9.83 & 2 & 9.03 & 1 & 9.83 & - & - & - & - & 4 & 28.69 \\
\hline Montgomery & - & - & 1 & 2.57 & - & - & - & - & - & - & 1 & 2.57 \\
\hline Morgan & - & - & 3 & 17.41 & - & - & - & - & 1 & 4.19 & 4 & 21.60 \\
\hline Muhlenberg & - & - & 85 & 185.73 & 1 & 3.00 & - & - & 27 & 52.37 & 113 & 241.10 \\
\hline Ohio & - & - & 16 & 45.55 & - & - & - & - & 4 & 10.08 & 20 & 55.63 \\
\hline
\end{tabular}

See footnotes at end of table. 
Kentucky

Table KY-3 (page 3 of 3). Number of deaths and age-adjusted mortality rate (per $1,000,000$ population) by condition and county, Kentucky residents age 15 and over, 1983-1992

\begin{tabular}{|c|c|c|c|c|c|c|c|c|c|c|c|c|}
\hline \multirow[b]{2}{*}{ County } & \multicolumn{2}{|c|}{ Asbestosis } & \multicolumn{2}{|c|}{$\begin{array}{r}\text { Coal workers' } \\
\text { pneumoconiosis }\end{array}$} & \multicolumn{2}{|c|}{ Silicosis } & \multicolumn{2}{|c|}{ Byssinosis } & \multicolumn{2}{|c|}{$\begin{array}{r}\text { Unspecified/Other } \\
\text { pneumoconioses }\end{array}$} & \multicolumn{2}{|c|}{$\begin{array}{r}\text { All } \\
\text { pneumoconioses }\end{array}$} \\
\hline & $\#$ & Rate & \# & Rate & $\#$ & Rate & $\#$ & Rate & \# & Rate & \# & Rate \\
\hline Owen & - & - & 1 & 10.00 & - & - & - & - & - & - & 1 & 10.00 \\
\hline Owsley & - & - & 1 & 18.71 & - & - & - & - & - & - & 1 & 18.71 \\
\hline Pendleton & 1 & 13.13 & 1 & 9.13 & - & - & - & - & - & - & 2 & 22.26 \\
\hline Perry & 1 & 3.86 & 29 & 96.27 & - & - & - & - & 7 & 22.27 & 36 & 118.54 \\
\hline Pike & - & - & 112 & 141.46 & 1 & 0.93 & - & - & 29 & 37.36 & 142 & 179.75 \\
\hline Powell & - & - & 2 & 16.01 & - & - & - & - & 1 & 5.28 & 3 & 21.29 \\
\hline Pulaski & 1 & 0.96 & 6 & 9.51 & 1 & 1.90 & - & - & 3 & 3.81 & 11 & 16.17 \\
\hline Rockcastle & - & - & 3 & 17.08 & - & - & - & - & - & - & 3 & 17.08 \\
\hline Rowan & - & - & 1 & 3.25 & - & - & - & - & - & - & 1 & 3.25 \\
\hline Russell & - & - & - & - & - & - & - & - & 2 & 12.92 & 2 & 12.92 \\
\hline Scott & - & - & 3 & 12.88 & - & - & - & - & - & - & 3 & 12.88 \\
\hline Shelby & - & - & 1 & 4.32 & 1 & 1.93 & - & - & - & - & 2 & 6.25 \\
\hline Trimble & - & - & - & - & 1 & 16.64 & - & - & - & - & 1 & 16.64 \\
\hline Union & - & - & 8 & 43.47 & - & - & - & - & - & - & 8 & 43.47 \\
\hline Warren & - & - & 2 & 2.65 & - & - & - & - & 2 & 3.12 & 4 & 5.77 \\
\hline Wayne & - & - & 3 & 10.99 & - & - & - & - & - & - & 3 & 10.99 \\
\hline Webster & - & - & 8 & 19.20 & - & - & - & - & - & - & 8 & 19.20 \\
\hline Whitley & 1 & 3.17 & 51 & 104.00 & 1 & 3.17 & - & - & 14 & 33.76 & 67 & 144.10 \\
\hline Wolfe & - & - & 4 & 62.31 & - & - & - & - & - & - & 4 & 62.31 \\
\hline Woodford & - & - & 1 & 3.25 & - & - & - & - & - & - & 1 & 3.25 \\
\hline
\end{tabular}




\section{Louisiana: Selected statistics from the United States section}

All pneumoconioses mortality, 1988-1992

Total deaths

Crude mortality rate (per $1,000,000$ population)

Age-adjusted mortality rate (per $1,000,000$ population)

Total years of potential life lost to life expectancy

Years of potential life lost to life expectancy (per death) $\underline{\text { Value }}$

123

7.73

1,751

14.2

Deaths*

86

7

18

1
5.41

State rank

23

29

25

21

4

Percent**

distribution

69.9

5.7

Silicosis

Byssinosis

Unspecified/Other pneumoconiosis

* Sum of column may exceed total deaths because of deaths with multiple pneumoconioses.

** Percentages may total more than $100 \%$ due to deaths with multiple pneumoconioses.

NOTE: $\quad$ See Appendix A for source description and Appendix B for methods and ICD-8 and ICD-9 codes.

SOURCE: National Center for Health Statistics multiple cause of death data. Population estimates from U.S. Bureau of the Census.

Figure LA-1. All pneumoconioses: Louisiana and U.S. age-adjusted mortality rates, residents age 15 and over, 1968-1992

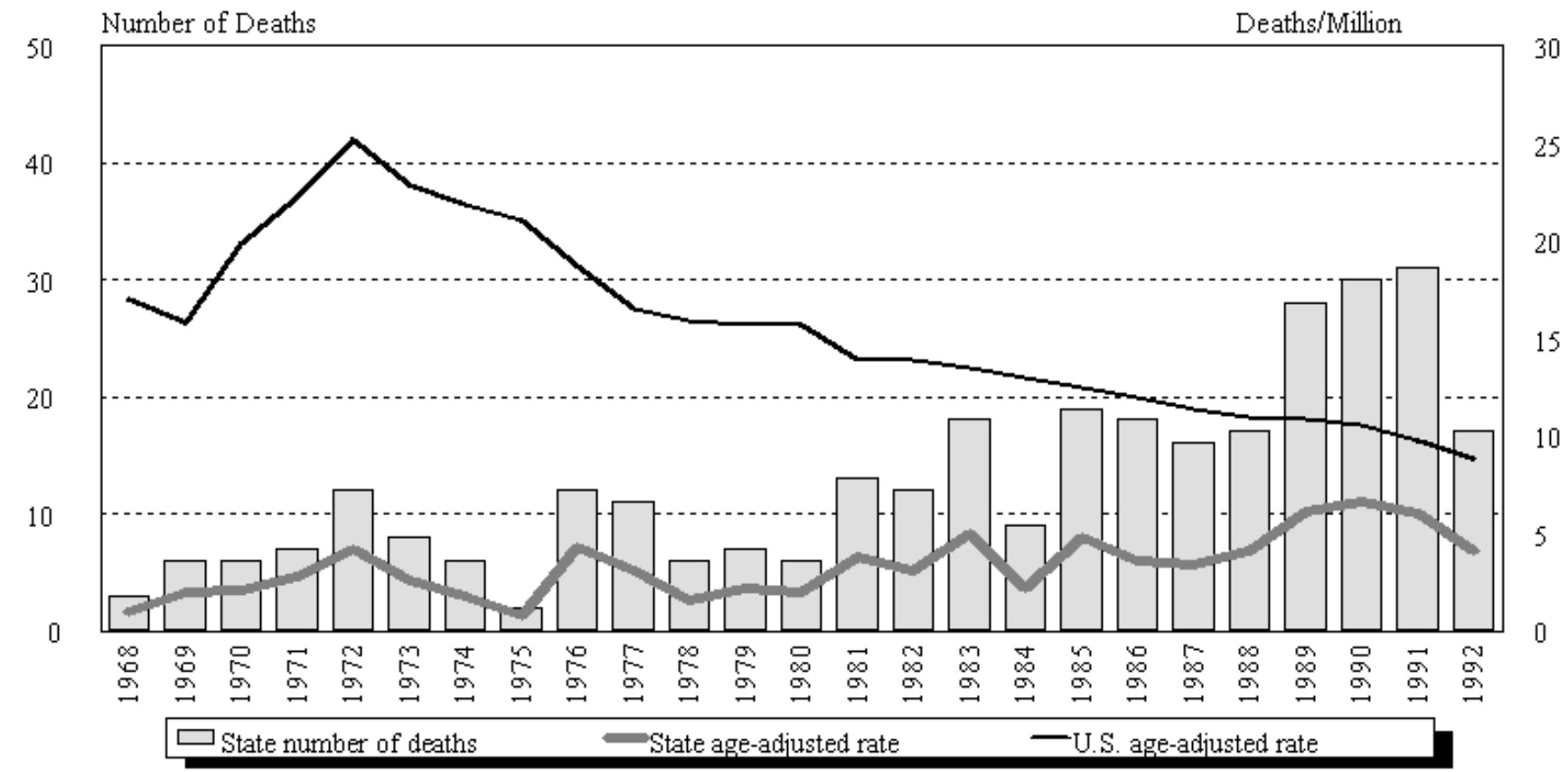

NOTE: See Appendix A for source description and Appendix B for methods and ICD-8 and ICD-9 codes.

SOURCE: National Center for Health Statistics multiple cause of death data. Population estimates from U.S. Bureau of the Census. 


\section{Louisiana}

Table LA-1. Number of deaths and age-adjusted mortality rate (per 1,000,000 population) by condition, Louisiana residents age 15 and over, 1968-1992

\begin{tabular}{|c|c|c|c|c|c|c|c|c|c|c|c|c|}
\hline \multirow[b]{2}{*}{ Year } & \multicolumn{2}{|c|}{ Asbestosis } & \multicolumn{2}{|c|}{$\begin{array}{l}\text { Coal workers' } \\
\text { pneumoconiosis }\end{array}$} & \multicolumn{2}{|c|}{ Silicosis } & \multicolumn{2}{|c|}{ Byssinosis* } & \multicolumn{2}{|c|}{$\begin{array}{c}\text { Unsp ecified/Other } \\
\text { pneumoconioses }\end{array}$} & \multicolumn{2}{|c|}{ All pneumoconioses } \\
\hline & Number & Rate & Number & Rate & Number & Rate & Number & Rate & Number & Rate & Number & Rate \\
\hline 1968 & 1 & 0.27 & - & - & 1 & 0.50 & & & 1 & 0.18 & 3 & 0.95 \\
\hline 1969 & 1 & 0.35 & 1 & 0.27 & 1 & 0.32 & & & 3 & 1.02 & 6 & 1.97 \\
\hline 1970 & - & - & 1 & 0.35 & 3 & 0.99 & & & 2 & 0.70 & 6 & 2.05 \\
\hline 1971 & 1 & 0.35 & - & - & 6 & 2.47 & & & - & - & 7 & 2.82 \\
\hline 1972 & 2 & 0.82 & - & - & 8 & 2.71 & & & 2 & 0.68 & 12 & 4.21 \\
\hline 1973 & 2 & 0.59 & - & - & 3 & 1.12 & & & 3 & 0.83 & 8 & 2.54 \\
\hline 1974 & - & - & - & - & 3 & 1.00 & & & 3 & 0.80 & 6 & 1.80 \\
\hline 1975 & - & - & 1 & 0.33 & - & - & & & 1 & 0.40 & 2 & 0.73 \\
\hline 1976 & 3 & 1.12 & 1 & 0.33 & 3 & 1.15 & & & 5 & 1.66 & 12 & 4.25 \\
\hline 1977 & 1 & 0.32 & 1 & 0.21 & 4 & 1.27 & & & 5 & 1.25 & 11 & 3.06 \\
\hline 1978 & 2 & 0.58 & - & - & 2 & 0.44 & & & 2 & 0.52 & 6 & 1.54 \\
\hline 1979 & 2 & 0.51 & - & - & 4 & 1.39 & - & - & 1 & 0.26 & 7 & 2.16 \\
\hline 1980 & 3 & 0.91 & - & - & 2 & 0.70 & - & - & 1 & 0.40 & 6 & 2.02 \\
\hline 1981 & 5 & 1.28 & - & - & 6 & 1.83 & 1 & 0.30 & 2 & 0.58 & 13 & 3.74 \\
\hline 1982 & 9 & 2.35 & - & - & 2 & 0.58 & - & - & 1 & 0.18 & 12 & 3.11 \\
\hline 1983 & 9 & 2.15 & 3 & 0.74 & 5 & 1.66 & - & - & 1 & 0.40 & 18 & 4.95 \\
\hline 1984 & 4 & 0.86 & 2 & 0.63 & 2 & 0.45 & - & - & 1 & 0.24 & 9 & 2.18 \\
\hline 1985 & 11 & 2.86 & 2 & 0.33 & 5 & 1.38 & - & - & 4 & 1.09 & 19 & 4.80 \\
\hline 1986 & 11 & 2.46 & 1 & 0.16 & 3 & 0.61 & - & - & 4 & 0.57 & 18 & 3.57 \\
\hline 1987 & 7 & 1.68 & 2 & 0.39 & 5 & 1.09 & - & - & 2 & 0.25 & 16 & 3.40 \\
\hline 1988 & 12 & 2.73 & - & - & 3 & 1.00 & - & - & 2 & 0.39 & 17 & 4.12 \\
\hline 1989 & 20 & 3.92 & 3 & 0.71 & 2 & 0.62 & - & - & 3 & 0.85 & 28 & 6.10 \\
\hline 1990 & 20 & 4.10 & 1 & 0.31 & 6 & 1.48 & - & - & 3 & 0.70 & 30 & 6.59 \\
\hline 1991 & 20 & 3.94 & 3 & 0.32 & 5 & 1.15 & - & - & 4 & 0.77 & 31 & 6.03 \\
\hline 1992 & 14 & 3.16 & - & - & 2 & 0.59 & 1 & 0.32 & - & - & 17 & 4.07 \\
\hline
\end{tabular}

indicates no deaths listed. ${ }^{*}$ no sp ecific code for byssinosis in ICD-8 (1968-1978)

NOTE: Rates based on small numbers may be unstable. Sum of individual conditions may exceed total because of deaths with multiple pneumoconioses. See Appendix A for source description and Appendix B for methods and ICD-8 and ICD-9 codes.

SOURCE: National Center for Health Statistics multiple cause of death data. Population estimates from U.S. Bureau of the Census. 
Figure LA-2. All pneumoconioses: Distribution of deaths by sex and race, Louisiana residents age 15 and over, 1968-1992
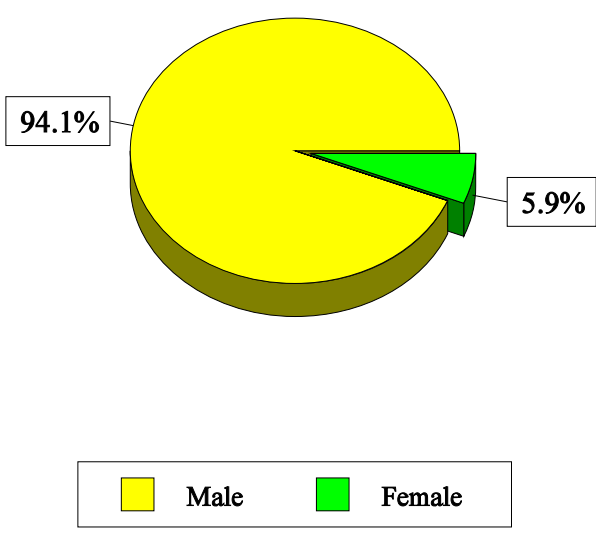

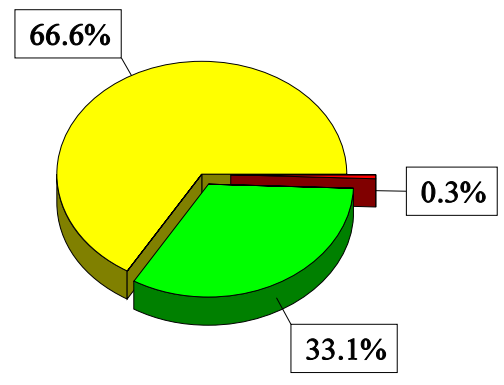

White
Black Other

NOTE: See Appendix A for source description and Appendix B for methods and ICD-8 and ICD-9 codes.

SOURCE: National Center for Health Statistics multiple cause of death data.

Table LA-2. All pneumoconioses: Distribution of deaths by sex, race, and age, Louisiana residents age 15 and over, 1968-1992

\begin{tabular}{llrrrr}
\hline & & \multicolumn{2}{c}{$1968-1992$} & \multicolumn{2}{c}{$1988-1992$} \\
\cline { 3 - 5 } Total deaths & & Number & Percent & Number & Percent \\
Sex & Male & 320 & 100.0 & 123 & 100.0 \\
& Female & 301 & 94.1 & 115 & 93.5 \\
Race & White & 19 & 5.9 & 8 & 6.5 \\
& Black & 213 & 66.6 & 89 & 72.4 \\
& Other & 106 & 33.1 & 33 & 26.8 \\
Age & $15-24$ & 1 & 0.3 & 1 & 0.8 \\
& $25-34$ & 0 & 0.0 & 0 & 0.0 \\
& $35-44$ & 4 & 1.3 & 1 & 0.8 \\
& $45-54$ & 13 & 4.1 & 2 & 1.6 \\
& $55-64$ & 30 & 9.4 & 7 & 17.9 \\
& $65-74$ & 80 & 25.0 & 22 & 35.0 \\
& $75-84$ & 103 & 32.2 & 43 & 27.6 \\
& $85+$ & 67 & 20.9 & 34 & 11.4 \\
& Mean age & 23 & 7.2 & & 70.6 \\
\end{tabular}

NOTE: ICD-8 and ICD-9 codes.

SOURCE: National Center for Health Statistics multiple cause of death data. 
Figure LA-3. Pneumoconiosis deaths by condition, Louisiana residents age 15 and over, 1968-1992

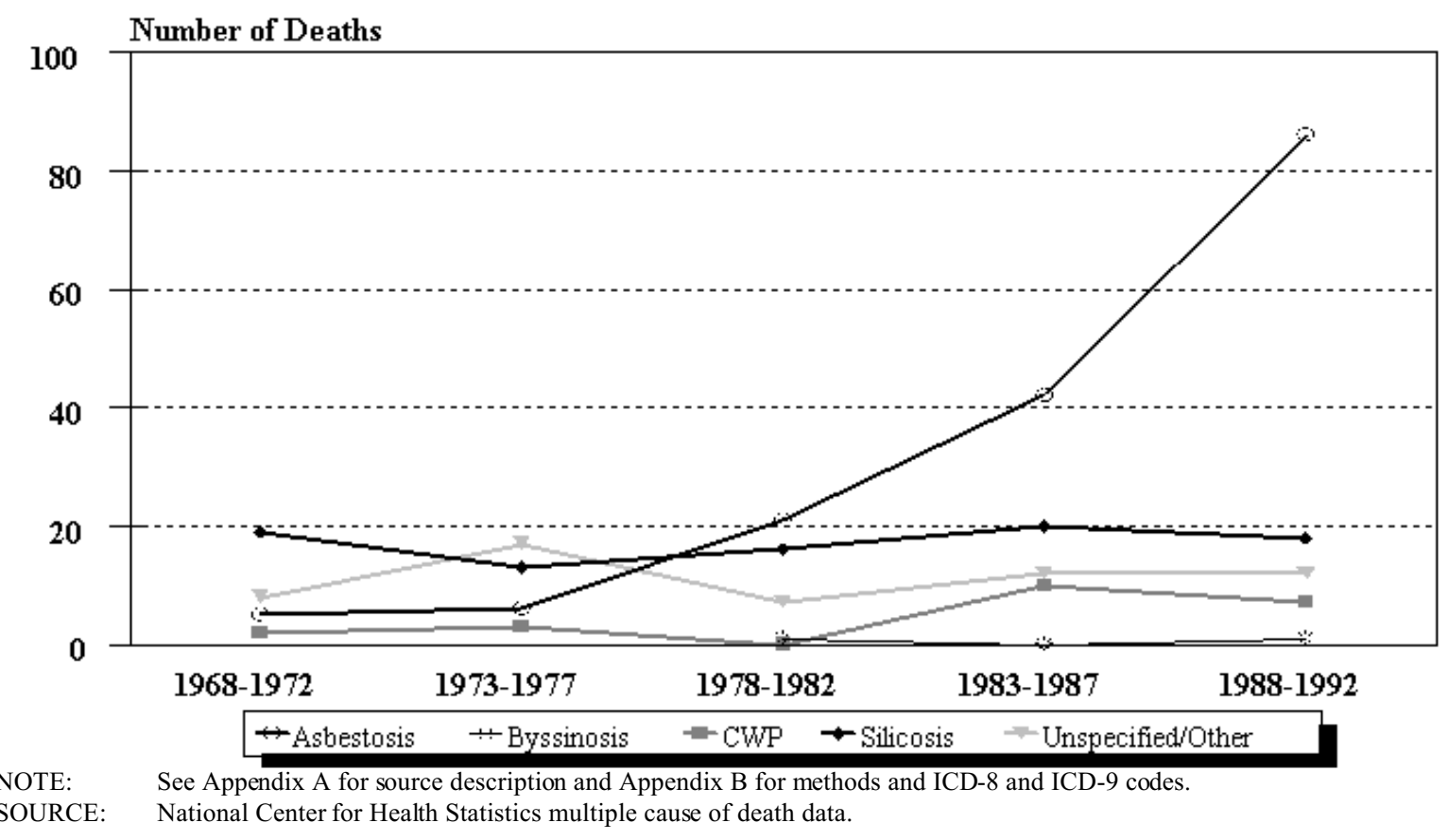

Figure LA-4. Louisiana and U.S. age-adjusted mortality rates (per 1,000,000 population) by condition, residents age 15 and over, 1983-1992

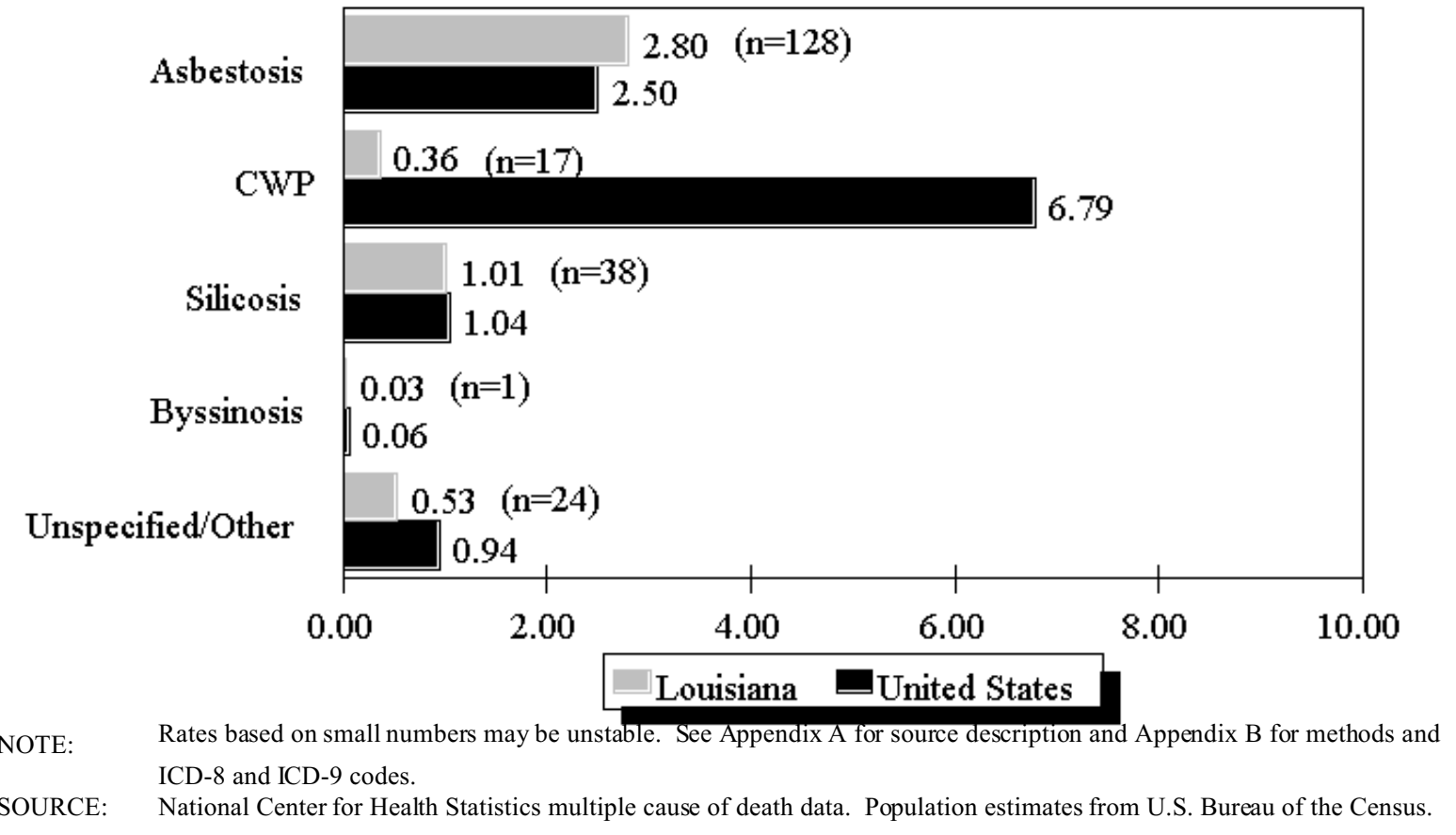


Table LA-3 (page 1 of 2). Number of deaths and age-adjusted mortality rate (per $1,000,000$ population) by condition and county, Louisiana residents age 15 and over, 1983-1992

\begin{tabular}{|c|c|c|c|c|c|c|c|c|c|c|c|c|}
\hline \multirow[b]{2}{*}{ County } & \multicolumn{2}{|c|}{ Asbestosis } & \multicolumn{2}{|c|}{$\begin{array}{c}\text { Coal workers' } \\
\text { pneumoconiosis }\end{array}$} & \multicolumn{2}{|c|}{ Silicosis } & \multicolumn{2}{|c|}{ Byssinosis } & \multicolumn{2}{|c|}{$\begin{array}{r}\text { Unspecified/Other } \\
\text { pneumoconioses }\end{array}$} & \multicolumn{2}{|c|}{$\begin{array}{r}\text { All } \\
\text { pneumoconioses }\end{array}$} \\
\hline & \# & Rate & $\#$ & Rate & $\#$ & Rate & \# & Rate & \# & Rate & \# & Rate \\
\hline Acadia & 2 & 3.86 & - & - & - & - & - & - & - & - & 2 & 3.86 \\
\hline Ascension & 1 & 2.55 & 1 & 2.52 & - & - & - & - & - & - & 2 & 5.07 \\
\hline Beauregard & 1 & 1.99 & - & - & - & - & - & - & - & - & 1 & 1.99 \\
\hline Bossier & - & - & 2 & 1.85 & - & - & - & - & - & - & 2 & 1.85 \\
\hline Caddo & 6 & 1.76 & - & - & - & - & - & - & - & - & 6 & 1.76 \\
\hline Calcasieu & 10 & 6.07 & 1 & 0.66 & 1 & 0.66 & - & - & - & - & 12 & 7.39 \\
\hline Cameron & 1 & 11.67 & - & - & - & - & - & - & - & - & 1 & 11.67 \\
\hline Catahoula & - & - & - & - & - & - & - & - & 1 & 4.03 & 1 & 4.03 \\
\hline $\begin{array}{l}\text { East Baton } \\
\text { Rouge }\end{array}$ & 7 & 2.32 & 1 & 0.21 & - & - & - & - & 1 & 0.21 & 9 & 2.74 \\
\hline East Feliciana & 1 & 6.29 & - & - & - & - & - & - & - & - & 1 & 6.29 \\
\hline Grant & - & - & - & - & - & - & 1 & 8.62 & - & - & 1 & 8.62 \\
\hline Iberia & 3 & 5.22 & - & - & - & - & - & - & - & - & 3 & 5.22 \\
\hline Iberville & 1 & 3.70 & - & - & 2 & 7.41 & - & - & - & - & 3 & 11.11 \\
\hline Jefferson & 34 & 7.95 & - & - & 8 & 2.06 & - & - & 13 & 2.96 & 53 & 12.54 \\
\hline Jefferson Davis & 2 & 6.52 & - & - & - & - & - & - & - & - & 2 & 6.52 \\
\hline Lafayette & 3 & 1.85 & - & - & 1 & 0.84 & - & - & - & - & 4 & 2.68 \\
\hline Lafourche & 1 & 0.93 & 1 & 1.77 & 1 & 1.47 & - & - & - & - & 3 & 4.17 \\
\hline Lincoln & 1 & 1.34 & - & - & - & - & - & - & - & - & 1 & 1.34 \\
\hline Livingston & 1 & 1.97 & 1 & 1.33 & 3 & 5.28 & - & - & - & - & 5 & 8.58 \\
\hline Morehouse & 1 & 1.32 & 2 & 6.31 & - & - & - & - & - & - & 3 & 7.63 \\
\hline Orleans & 21 & 3.69 & 1 & 0.30 & 6 & 1.24 & - & - & 3 & 0.51 & 29 & 5.33 \\
\hline Ouachita & 2 & 0.81 & 1 & 0.41 & 1 & 0.41 & - & - & 1 & 0.82 & 5 & 2.44 \\
\hline Plaquemines & - & - & - & - & 1 & 5.85 & - & - & - & - & 1 & 5.85 \\
\hline Rapides & 2 & 0.83 & - & - & - & - & - & - & 1 & 0.42 & 3 & 1.25 \\
\hline Red River & 1 & 4.13 & - & - & - & - & - & - & - & - & 1 & 4.13 \\
\hline Richland & 2 & 3.56 & - & - & - & - & - & - & 1 & 4.45 & 3 & 8.01 \\
\hline Sabine & - & - & - & - & - & - & - & - & 1 & 3.54 & 1 & 3.54 \\
\hline St Bernard & 6 & 7.98 & 2 & 2.66 & 1 & 1.52 & - & - & - & - & 9 & 12.16 \\
\hline St Charles & 2 & 6.66 & - & - & - & - & - & - & 1 & 3.33 & 2 & 6.66 \\
\hline $\begin{array}{l}\text { St John the } \\
\text { Baptist }\end{array}$ & - & - & - & - & 1 & 4.18 & - & - & - & - & 1 & 4.18 \\
\hline
\end{tabular}

See footnotes at end of table. 
Table LA-3 (page 2 of 2). Number of deaths and age-adjusted mortality rate (per $1,000,000$ population) by condition and county, Louisiana residents age 15 and over, 1983-1992

\begin{tabular}{|c|c|c|c|c|c|c|c|c|c|c|c|c|}
\hline \multirow[b]{2}{*}{ County } & \multicolumn{2}{|c|}{ Asbestosis } & \multicolumn{2}{|c|}{$\begin{array}{r}\text { Coal workers' } \\
\text { pneumoconiosis }\end{array}$} & \multicolumn{2}{|c|}{ Silicosis } & \multicolumn{2}{|c|}{ Byssinosis } & \multicolumn{2}{|c|}{$\begin{array}{r}\text { Unspecified/Other } \\
\text { pneumoconioses }\end{array}$} & \multicolumn{2}{|c|}{$\begin{array}{r}\text { All } \\
\text { pneumoconioses }\end{array}$} \\
\hline & \# & Rate & $\#$ & Rate & $\#$ & Rate & \# & Rate & $\#$ & Rate & $\#$ & Rate \\
\hline St Landry & 2 & 3.85 & - & - & - & - & - & - & - & - & 2 & 3.85 \\
\hline St Mary & 1 & 1.22 & - & - & 3 & 5.51 & - & - & - & - & 4 & 6.73 \\
\hline St Tammany & 4 & 3.31 & 1 & 0.61 & 4 & 3.02 & - & - & - & - & 9 & 6.94 \\
\hline Tangipahoa & - & - & - & - & 1 & 1.56 & - & - & - & - & 1 & 1.56 \\
\hline Terrebonne & 2 & 2.82 & - & - & 1 & 1.41 & - & - & - & - & 3 & 4.23 \\
\hline Vernon & 2 & 6.44 & 1 & 3.22 & - & - & - & - & - & - & 3 & 9.66 \\
\hline Washington & 2 & 4.27 & 1 & 1.09 & 2 & 4.27 & - & - & 1 & 1.09 & 6 & 10.73 \\
\hline $\begin{array}{l}\text { West Baton } \\
\text { Rouge }\end{array}$ & 1 & 6.46 & - & - & 1 & 7.48 & - & - & - & - & 2 & 13.94 \\
\hline West Carroll & 1 & 3.27 & 1 & 3.27 & - & - & - & - & - & - & 2 & 6.53 \\
\hline West Feliciana & 1 & 7.44 & - & - & - & - & - & - & - & - & 1 & 7.44 \\
\hline
\end{tabular}

- indicates no deaths listed.

NOTE: Counties with no deaths for all listed conditions are not displayed. Rates based on small numbers may be unstable. Sum of individual conditions may exceed total because of deaths with multiple pneumoconioses. See Appendix A for source description and Appendix B for methods and ICD-8 and ICD-9 codes.

SOURCE: National Center for Health Statistics multiple cause of death data. Population estimates from U.S. Bureau of the Census. 


\section{Maine: Selected statistics from the United States section}

All pneumoconioses mortality, 1988-1992

Total deaths

Crude mortality rate (per $1,000,000$ population)

Age-adjusted mortality rate (per $1,000,000$ population)

Total years of potential life lost to life expectancy

Years of potential life lost to life expectancy (per death) $\underline{\text { Value }}$

63

13.00

7.11

725

11.5

$\underline{\text { Deaths* }}$

53

1

1

5

0

4 $\underline{\text { State rank }}$

36

13

15

34

34

Percent**

distribution

84.1

1.6

7.9

Byssinosis

Unspecified/Other pneumoconiosis

* Sum of column may exceed total deaths because of deaths with multiple pneumoconioses.

** Percentages may total more than $100 \%$ due to deaths with multiple pneumoconioses.

NOTE: $\quad$ See Appendix A for source description and Appendix B for methods and ICD-8 and ICD-9 codes.

SOURCE: National Center for Health Statistics multiple cause of death data. Population estimates from U.S. Bureau of the Census.

Figure ME-1. All pneumoconioses: Maine and U.S. age-adjusted mortality rates, residents age 15 and over, 1968-1992

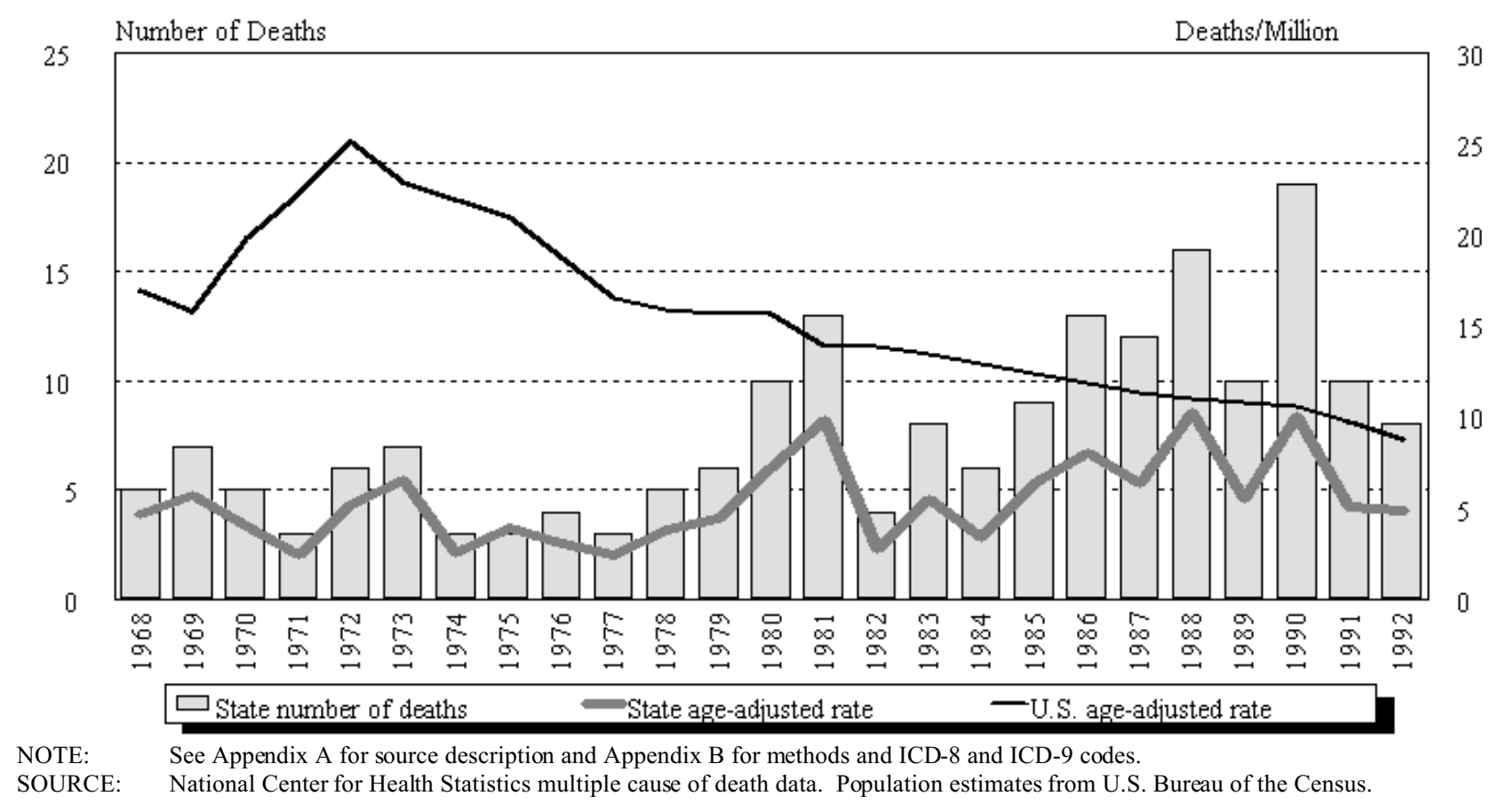


Maine

\begin{tabular}{|c|c|c|c|c|c|c|c|c|c|c|c|c|}
\hline \multirow[b]{2}{*}{ Year } & \multicolumn{2}{|c|}{ Asbestosis } & \multicolumn{2}{|c|}{$\begin{array}{l}\text { Coal workers' } \\
\text { pneumoconiosis }\end{array}$} & \multicolumn{2}{|c|}{ Silicosis } & \multicolumn{2}{|c|}{ Byssinosis* } & \multicolumn{2}{|c|}{$\begin{array}{c}\text { Unspecified/Other } \\
\text { pneumoconioses }\end{array}$} & \multicolumn{2}{|c|}{ All pneumoconioses } \\
\hline & Number & Rate & Number & Rate & Number & Rate & Number & Rate & Number & Rate & Number & Rate \\
\hline 1968 & - & - & - & - & 2 & 2.36 & & & 3 & 2.27 & 5 & 4.63 \\
\hline 1969 & 1 & 0.94 & - & - & 4 & 3.04 & & & 2 & 1.76 & 7 & 5.74 \\
\hline 1970 & 1 & 1.13 & - & - & 3 & 2.27 & & & 2 & 1.57 & 5 & 4.03 \\
\hline 1971 & 1 & 0.62 & - & - & 2 & 1.84 & & & - & - & 3 & 2.46 \\
\hline 1972 & - & - & - & - & 2 & 1.22 & & & 4 & 4.00 & 6 & 5.22 \\
\hline 1973 & 1 & 1.10 & 1 & 0.60 & 3 & 2.38 & & & 2 & 2.48 & 7 & 6.55 \\
\hline 1974 & 1 & 1.09 & - & - & 2 & 1.46 & & & - & - & 3 & 2.54 \\
\hline 1975 & 1 & 1.37 & - & - & 1 & 0.85 & & & 1 & 1.70 & 3 & 3.93 \\
\hline 1976 & 2 & 1.68 & - & - & 1 & 0.56 & & & 1 & 0.84 & 4 & 3.08 \\
\hline 1977 & - & - & - & - & 2 & 1.37 & & & 1 & 1.03 & 3 & 2.40 \\
\hline 1978 & 1 & 1.37 & - & - & 2 & 1.35 & & & 2 & 1.10 & 5 & 3.82 \\
\hline 1979 & 3 & 2.32 & - & - & 1 & 0.80 & - & - & 2 & 1.32 & 6 & 4.44 \\
\hline 1980 & 6 & 4.64 & - & - & 3 & 1.81 & - & - & 1 & 0.78 & 10 & 7.24 \\
\hline 1981 & 11 & 8.59 & - & - & 1 & 0.78 & - & - & 1 & 0.51 & 13 & 9.88 \\
\hline 1982 & 3 & 1.79 & - & - & 1 & 0.98 & - & - & - & - & 4 & 2.78 \\
\hline 1983 & 8 & 5.53 & - & - & - & - & - & - & - & - & 8 & 5.53 \\
\hline 1984 & 3 & 2.21 & - & - & 1 & 0.49 & - & - & 2 & 0.72 & 6 & 3.42 \\
\hline 1985 & 7 & 5.42 & 1 & 0.48 & 1 & 0.48 & - & - & - & - & 9 & 6.38 \\
\hline 1986 & 11 & 7.32 & - & - & 1 & 0.47 & - & - & 1 & 0.23 & 13 & 8.02 \\
\hline 1987 & 9 & 4.69 & - & - & 3 & 1.66 & - & - & - & - & 12 & 6.35 \\
\hline 1988 & 12 & 7.68 & - & - & 1 & 0.98 & - & - & 3 & 1.62 & 16 & 10.28 \\
\hline 1989 & 8 & 4.05 & 1 & 0.71 & - & - & - & - & 1 & 0.71 & 10 & 5.47 \\
\hline 1990 & 17 & 8.90 & - & - & 2 & 1.13 & - & - & - & - & 19 & 10.04 \\
\hline 1991 & 8 & 4.51 & - & - & 2 & 0.62 & - & - & - & - & 10 & 5.13 \\
\hline 1992 & 8 & 4.85 & - & - & - & - & - & - & - & - & 8 & 4.85 \\
\hline
\end{tabular}

indicates no deaths listed. ${ }^{*}$ no specific code for by ssinosis in ICD-8 (1968-1978)

NOTE: Rates based on small numbers may be unstable. Sum of individual conditions may exceed total because of deaths with multiple pneumoconioses. See Appendix A for source description and Appendix B for methods and ICD-8 and ICD-9 codes.

SOURCE: National Center for Health Statistics multiple cause of death data. Population estimates from U.S. Bureau of the Census. 
Figure ME-2. All pneumoconioses: Distribution of deaths by sex and race, Maine residents age 15 and over, 1968-1992
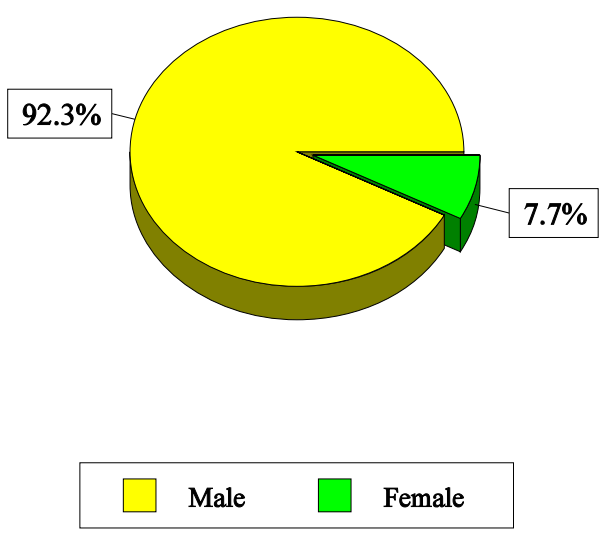
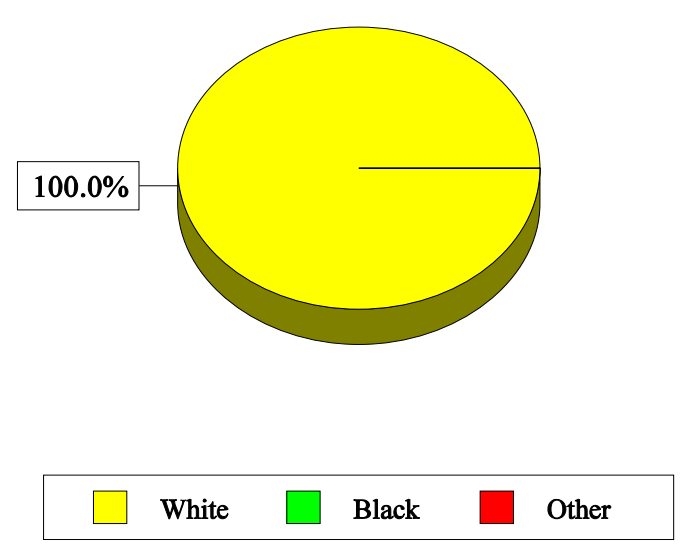

NOTE: $\quad$ See Appendix A for source description and Appendix B for methods and ICD-8 and ICD-9 codes.

SOURCE: National Center for Health Statistics multiple cause of death data.

Table ME-2. All pneumoconioses: Distribution of deaths by sex, race, and age, Maine residents age 15 and over, 1968-1992

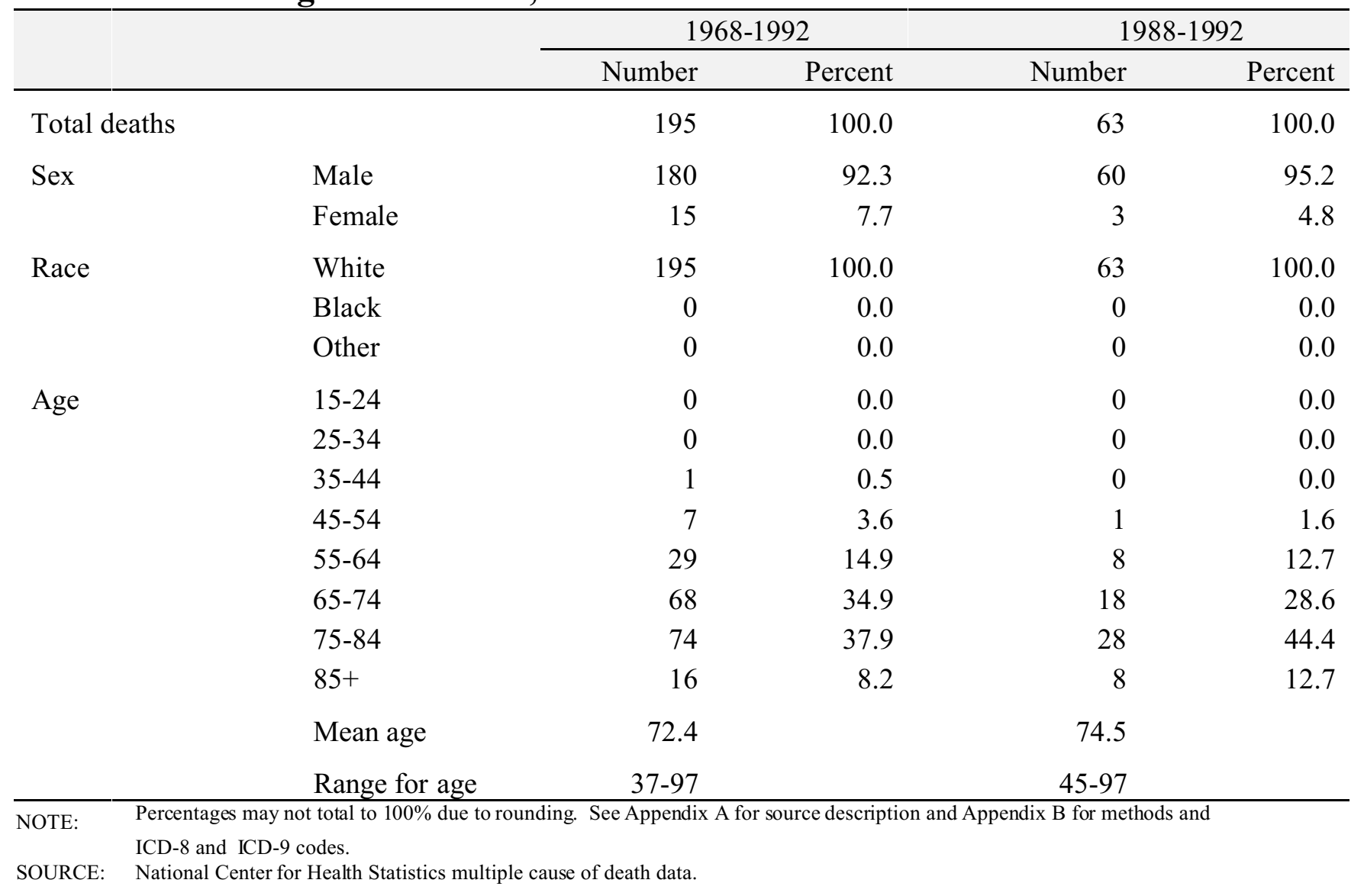


Figure ME-3. Pneumoconiosis deaths by condition, Maine residents age 15 and over, 1968-1992

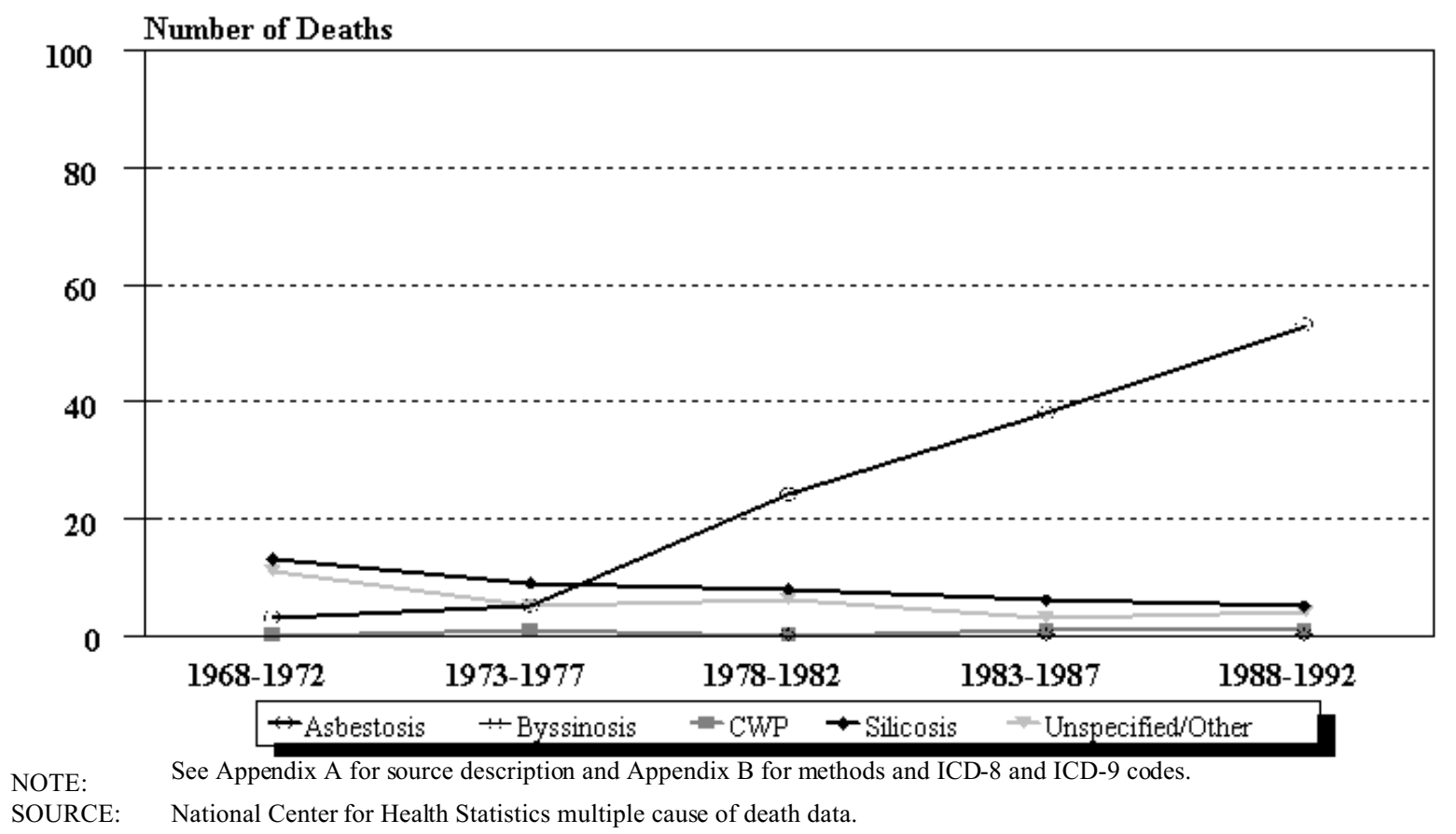

Figure ME-4. Maine and U.S. age-adjusted mortality rates (per 1,000,000 population) by condition, residents age 15 and over, 1983-1992

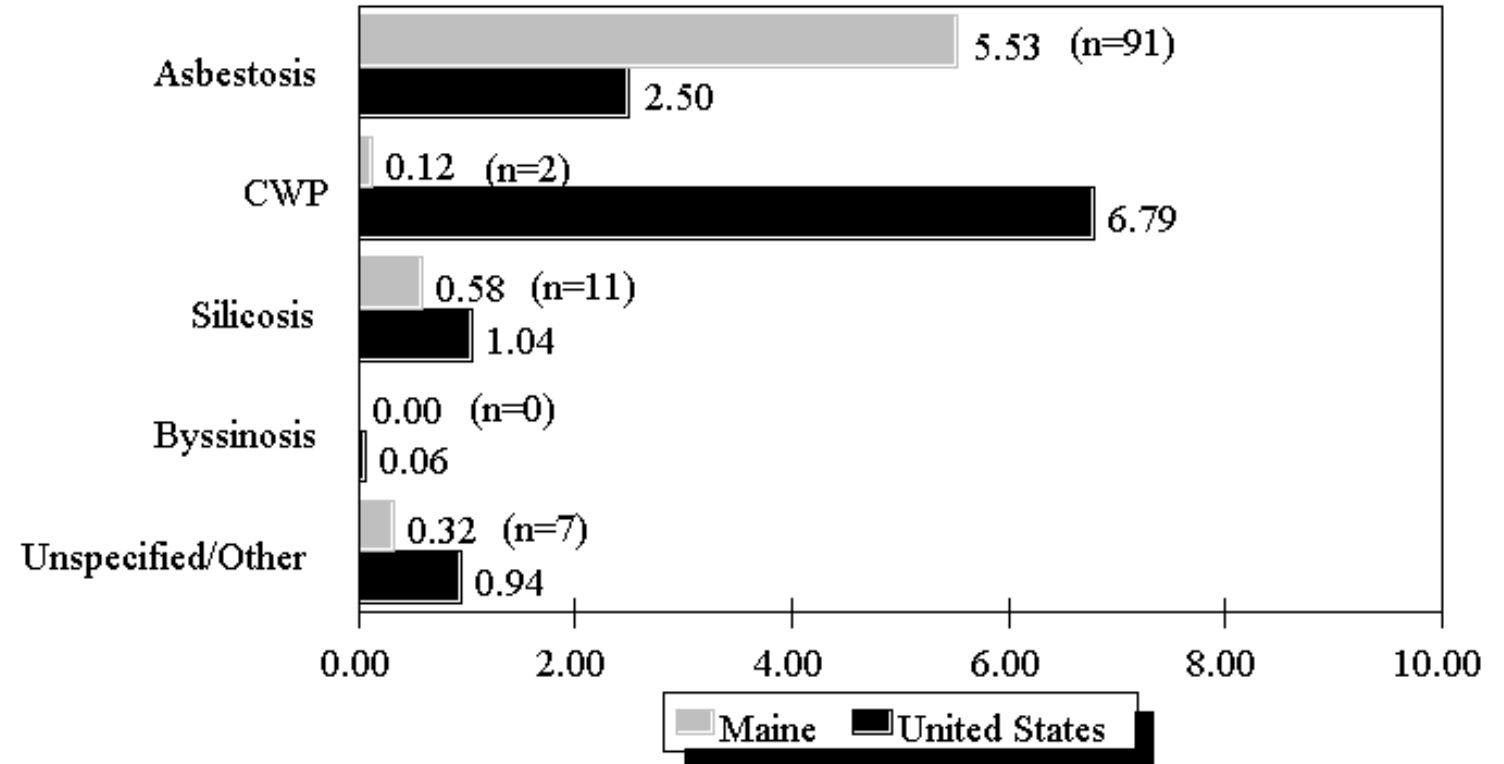

NOTE: $\quad$ Rates based on small numbers may be unstable. See Appendix A for source description and Appendix B for methods and ICD-8 and ICD-9 codes.

SOURCE: National Center for Health Statistics multiple cause of death data. Population estimates from U.S. Bureau of the Census. 
Table ME-3. Number of deaths and age-adjusted mortality rate (per 1,000,000 population) by condition and county, Maine residents age 15 and over, 1983-1992

\begin{tabular}{|c|c|c|c|c|c|c|c|c|c|c|c|c|}
\hline \multirow[b]{2}{*}{ County } & \multicolumn{2}{|c|}{ Asbestosis } & \multicolumn{2}{|c|}{$\begin{array}{c}\text { Coal workers' } \\
\text { pneumoconiosis }\end{array}$} & \multicolumn{2}{|c|}{ Silicosis } & \multicolumn{2}{|c|}{ Byssinosis } & \multicolumn{2}{|c|}{$\begin{array}{r}\text { Unspecified/Other } \\
\text { pneumoconioses }\end{array}$} & \multicolumn{2}{|c|}{$\begin{array}{r}\text { All } \\
\text { pneumoconioses }\end{array}$} \\
\hline & $\#$ & Rate & $\#$ & Rate & $\#$ & Rate & $\#$ & Rate & $\#$ & Rate & \# & Rate \\
\hline Androscoggin & 5 & 2.80 & - & - & 2 & 2.04 & - & - & 1 & 0.44 & 8 & 5.28 \\
\hline Cumberland & 27 & 7.71 & - & - & - & - & - & - & 1 & 0.20 & 28 & 7.91 \\
\hline Franklin & 1 & 1.74 & - & - & 1 & 1.74 & - & - & - & - & 2 & 3.47 \\
\hline Hancock & 3 & 3.80 & - & - & 2 & 2.94 & - & - & 2 & 1.71 & 7 & 8.46 \\
\hline Kennebec & 10 & 8.13 & - & - & - & - & - & - & - & - & 10 & 8.13 \\
\hline Knox & 5 & 8.04 & - & - & - & - & - & - & - & - & 5 & 8.04 \\
\hline Lincoln & 5 & 13.19 & 1 & 2.99 & - & - & - & - & 2 & 4.29 & 8 & 20.48 \\
\hline Oxford & 1 & 0.82 & - & - & - & - & - & - & - & - & 1 & 0.82 \\
\hline Penobscot & 3 & 1.95 & - & - & 1 & 0.39 & - & - & - & - & 4 & 2.34 \\
\hline Piscataquis & 1 & 2.14 & 1 & 2.14 & 4 & 8.58 & - & - & - & - & 6 & 12.86 \\
\hline Sagadahoc & 22 & 62.55 & - & - & - & - & - & - & 1 & 3.78 & 23 & 66.33 \\
\hline Somerset & 1 & 2.12 & - & - & - & - & - & - & - & - & 1 & 2.12 \\
\hline Washington & 1 & 1.11 & - & - & - & - & - & - & - & - & 1 & 1.11 \\
\hline York & 6 & 3.67 & - & - & 1 & 0.32 & - & - & - & - & 7 & 3.99 \\
\hline
\end{tabular}


Maryland

\section{Maryland: Selected statistics from the United States section}

All pneumoconioses mortality, 1988-1992

Total deaths

Crude mortality rate (per $1,000,000$ population)

Age-adjusted mortality rate (per $1,000,000$ population)

Total years of potential life lost to life expectancy

Years of potential life lost to life expectancy (per death)
Value

206

10.84

7.65

2,642

12.8

Deaths*

136

41

10

0

19 $\underline{\text { State rank }}$

18

20

13

17

10

Percent**

distribution

66.0

19.9

Silicosis

4.9

Byssinosis

Unspecified/Other pneumoconiosis

* Sum of column may exceed total deaths because of deaths with multiple pneumoconioses.

** Percentages may total more than $100 \%$ due to deaths with multiple pneumoconioses.

NOTE: $\quad$ See Appendix A for source description and Appendix B for methods and ICD-8 and ICD-9 codes.

SOURCE: National Center for Health Statistics multiple cause of death data. Population estimates from U.S. Bureau of the Census.

\section{Figure MD-1. All pneumoconioses: Maryland and U.S. age-adjusted mortality rates, residents age 15 and over, 1968-1992}

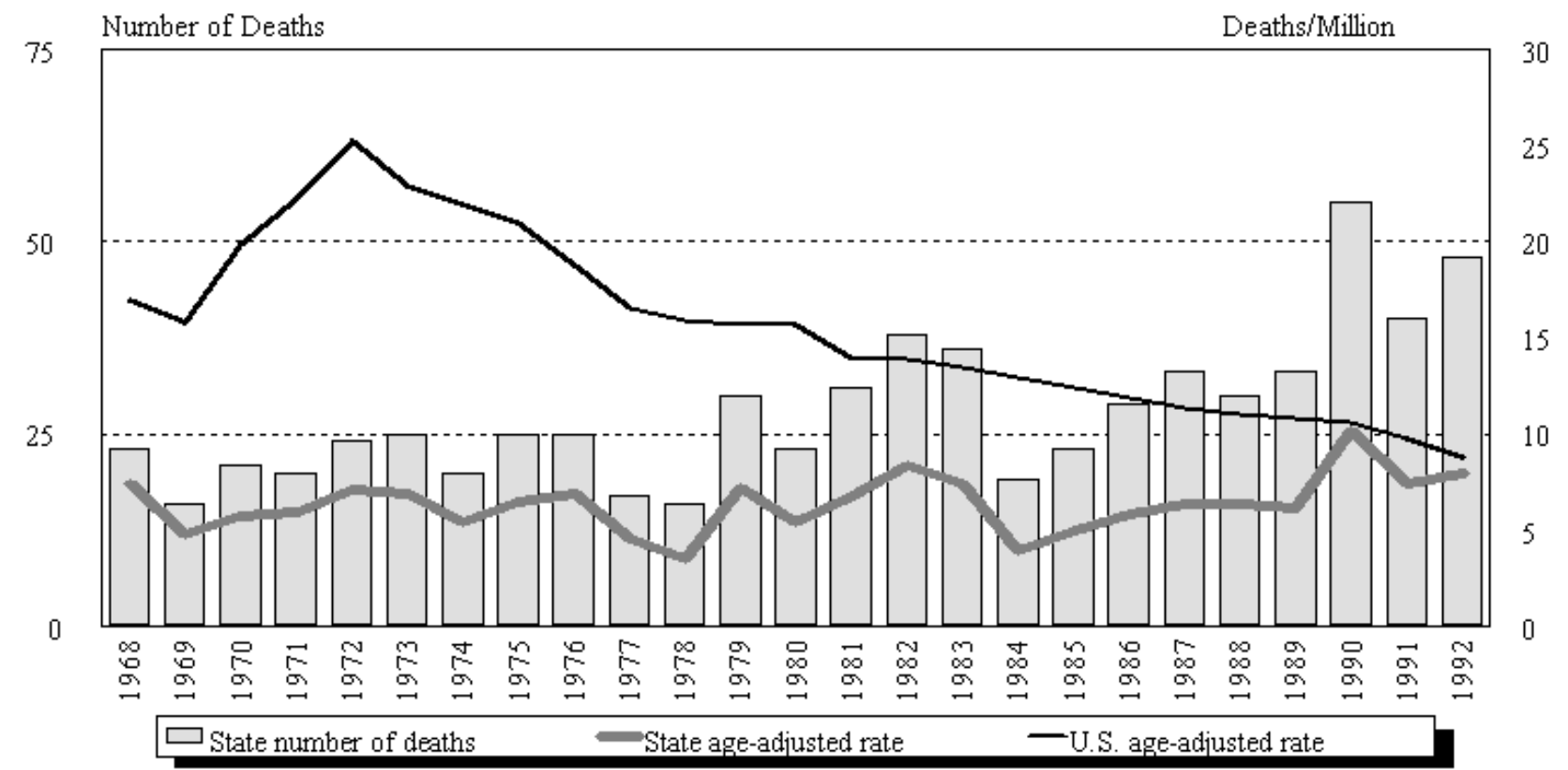

NOTE: $\quad$ See Appendix A for source description and Appendix B for methods and ICD-8 and ICD-9 codes.

SOURCE: National Center for Health Statistics multiple cause of death data. Population estimates from U.S. Bureau of the Census. 
Maryland

\begin{tabular}{|c|c|c|c|c|c|c|c|c|c|c|c|c|}
\hline \multirow[b]{2}{*}{ Year } & \multicolumn{2}{|c|}{ Asb estosis } & \multicolumn{2}{|c|}{$\begin{array}{l}\text { Coal workers' } \\
\text { pneumoconiosis }\end{array}$} & \multicolumn{2}{|c|}{ Silicosis } & \multicolumn{2}{|c|}{ Byssinosis* } & \multicolumn{2}{|c|}{$\begin{array}{c}\text { Unspecified/Other } \\
\text { pneumoconioses }\end{array}$} & \multicolumn{2}{|c|}{ All pneumoconioses } \\
\hline & Number & Rate & Number & Rate & Number & Rate & Number & Rate & Number & Rate & Number & Rate \\
\hline 1968 & - & - & 4 & 1.27 & 14 & 4.45 & & & 5 & 1.68 & 23 & 7.40 \\
\hline 1969 & 1 & 0.34 & 5 & 1.46 & 9 & 2.66 & & & 3 & 0.92 & 16 & 4.79 \\
\hline 1970 & - & - & 6 & 1.51 & 13 & 3.69 & & & 4 & 0.90 & 21 & 5.72 \\
\hline 1971 & 2 & 0.64 & 5 & 1.63 & 7 & 2.03 & & & 7 & 1.97 & 20 & 5.95 \\
\hline 1972 & - & - & 12 & 3.48 & 6 & 1.89 & & & 6 & 1.75 & 24 & 7.12 \\
\hline 1973 & 4 & 1.18 & 6 & 1.64 & 8 & 2.24 & & & 7 & 1.81 & 25 & 6.87 \\
\hline 1974 & 1 & 0.30 & 6 & 1.59 & 7 & 1.85 & & & 7 & 1.88 & 20 & 5.39 \\
\hline 1975 & - & - & 8 & 2.08 & 7 & 2.02 & & & 11 & 2.58 & 25 & 6.45 \\
\hline 1976 & 5 & 1.28 & 3 & 0.79 & 6 & 1.73 & & & 13 & 3.59 & 25 & 6.89 \\
\hline 1977 & 4 & 1.23 & 4 & 0.97 & 6 & 1.58 & & & 4 & 0.96 & 17 & 4.61 \\
\hline 1978 & 2 & 0.48 & 6 & 1.21 & 4 & 0.79 & & & 4 & 1.03 & 16 & 3.52 \\
\hline 1979 & 8 & 1.94 & 9 & 2.06 & 8 & 2.03 & 1 & 0.12 & 7 & 1.80 & 30 & 7.22 \\
\hline 1980 & 2 & 0.52 & 8 & 1.87 & 6 & 1.50 & - & - & 7 & 1.49 & 23 & 5.39 \\
\hline 1981 & 7 & 1.61 & 13 & 2.74 & 6 & 1.48 & - & - & 6 & 1.27 & 31 & 6.84 \\
\hline 1982 & 11 & 2.50 & 19 & 3.97 & 3 & 0.86 & - & - & 5 & 1.01 & 38 & 8.34 \\
\hline 1983 & 12 & 2.57 & 19 & 3.63 & 4 & 0.80 & - & - & 3 & 0.79 & 36 & 7.42 \\
\hline 1984 & 9 & 1.96 & 3 & 0.60 & 2 & 0.36 & - & - & 5 & 1.00 & 19 & 3.92 \\
\hline 1985 & 13 & 2.80 & 5 & 1.08 & 3 & 0.67 & - & - & 2 & 0.41 & 23 & 4.96 \\
\hline 1986 & 16 & 3.40 & 8 & 1.43 & 2 & 0.39 & 1 & 0.23 & 2 & 0.43 & 29 & 5.88 \\
\hline 1987 & 18 & 3.80 & 8 & 1.28 & 2 & 0.36 & - & - & 5 & 0.97 & 33 & 6.41 \\
\hline 1988 & 16 & 3.59 & 6 & 1.22 & 2 & 0.54 & - & - & 6 & 1.04 & 30 & 6.38 \\
\hline 1989 & 24 & 4.54 & 5 & 1.05 & - & - & - & - & 4 & 0.59 & 33 & 6.17 \\
\hline 1990 & 36 & 7.14 & 12 & 2.05 & 3 & 0.43 & - & - & 4 & 0.59 & 55 & 10.22 \\
\hline 1991 & 27 & 5.19 & 5 & 0.71 & 4 & 0.70 & - & - & 4 & 0.86 & 40 & 7.45 \\
\hline 1992 & 33 & 5.97 & 13 & 1.82 & 1 & 0.14 & - & - & 1 & 0.07 & 48 & 8.00 \\
\hline
\end{tabular}

- indicates no deaths listed. ${ }^{*}$ no sp ecific code for byssinosis in ICD-8 (1968-1978)

NOTE: Rates based on small numbers may be unstable. Sum of individual conditions may exceed total because of deaths with multiple pneumoconioses. See Appendix A for source description and Appendix B for methods and ICD-8 and ICD-9 codes.

SOURCE: National Center for Health Statistics multiple cause of death data. Population estimates from U.S. Bureau of the Census. 
Figure MD-2. All pneumoconioses: Distribution of deaths by sex and race, Maryland residents age 15 and over, 1968-1992
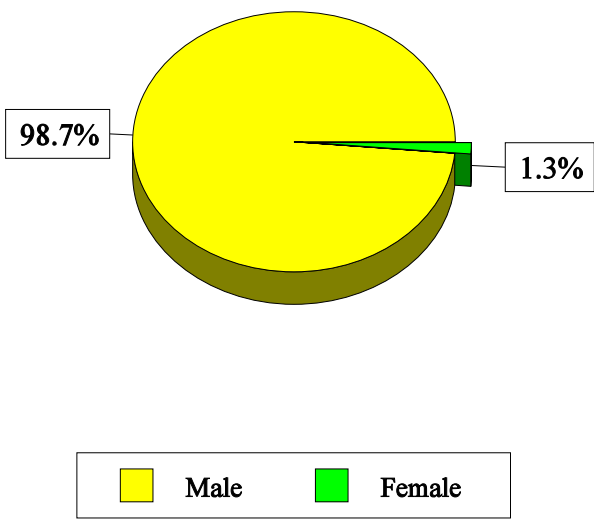

\section{$87.5 \%$}

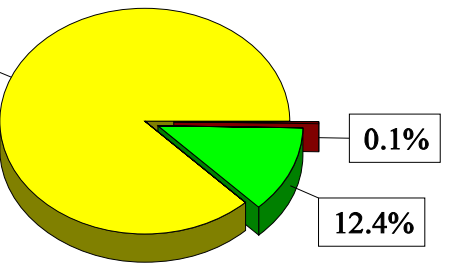

White

Black

Other

$\square$ White $\square$ Black $\square$ Other

NOTE: $\quad$ See Appendix A for source description and Appendix B for methods and ICD-8 and ICD-9 codes.

SOURCE: National Center for Health Statistics multiple cause of death data.

Table MD-2. All pneumoconioses: Distribution of deaths by sex, race, and age, Maryland residents age 15 and over, 1968-1992

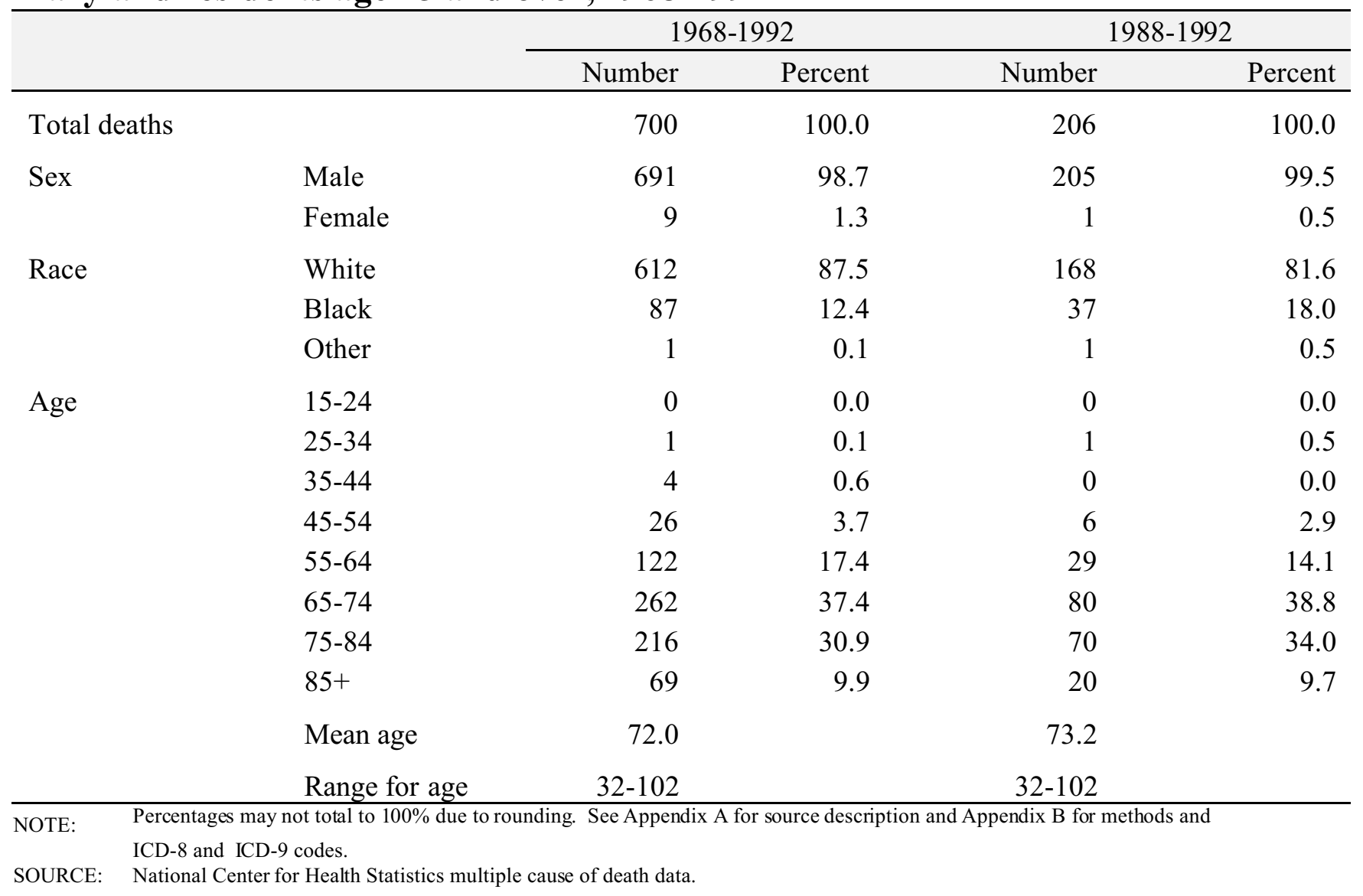


Figure MD-3. Pneumoconiosis deaths by condition, Maryland residents age 15 and over, 1968-1992

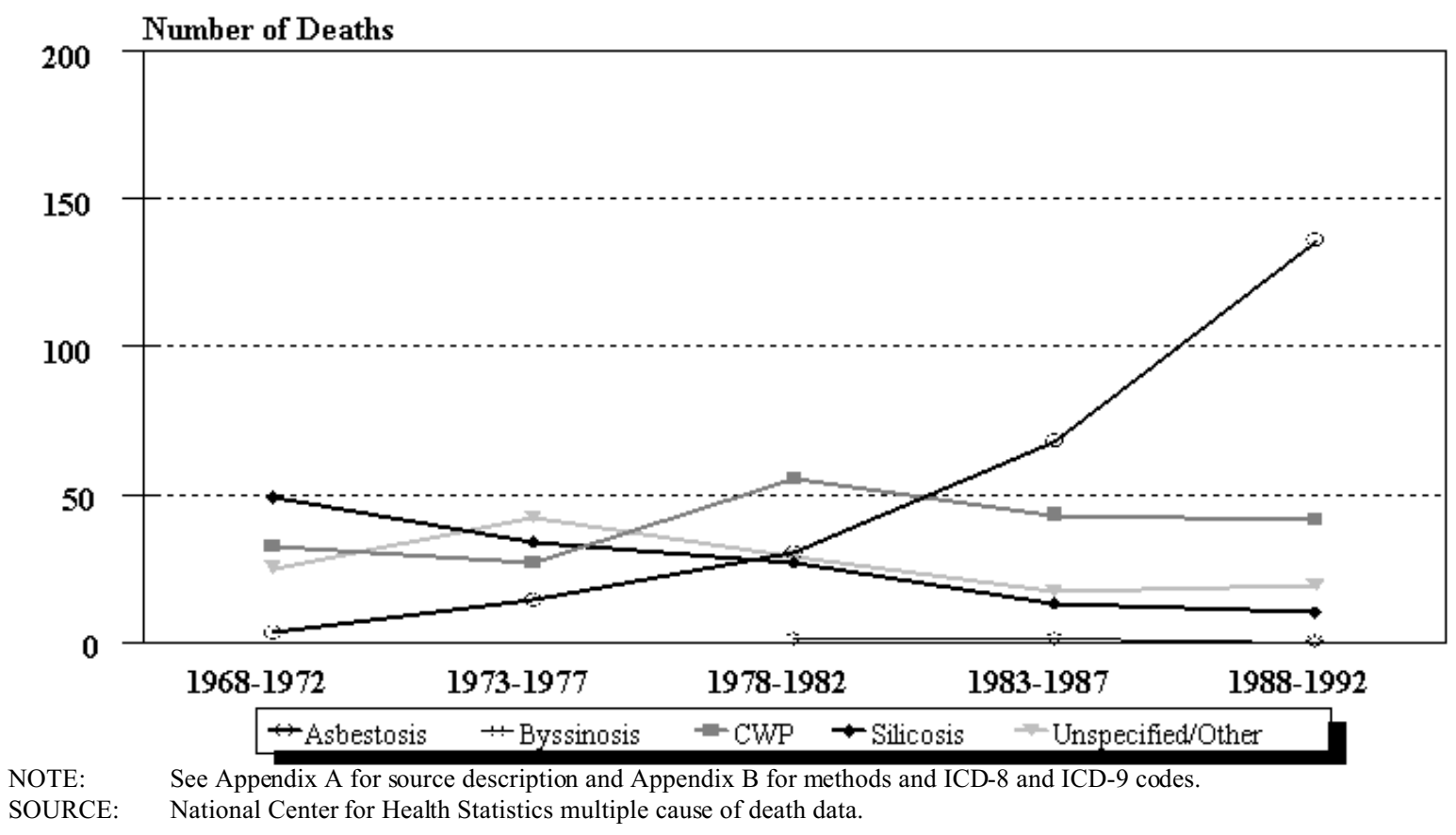

Figure MD-4. Maryland and U.S. age-adjusted mortality rates (per 1,000,000 population) by condition, residents age 15 and over, 1983-1992

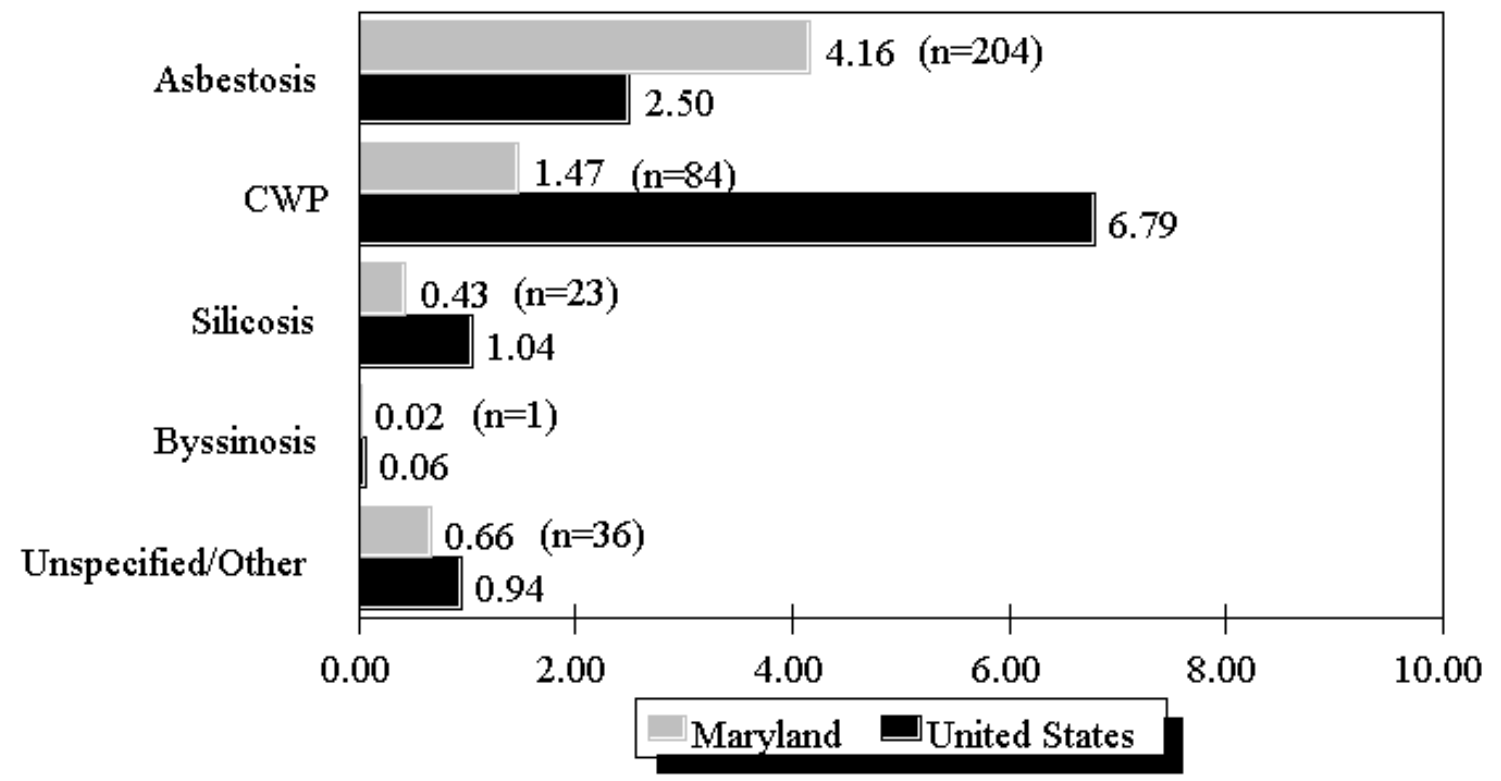

NOTE: $\quad$ Rates based on small numbers may be unstable. See Appendix A for source description and Appendix B for methods and ICD-8 and ICD-9 codes.

SOURCE: National Center for Health Statistics multiple cause of death data. Population estimates from U.S. Bureau of the Census. 
Table MD-3. Number of deaths and age-adjusted mortality rate (per 1,000,000 population) by condition and county, Maryland residents age 15 and over, 1983-1992

\begin{tabular}{|c|c|c|c|c|c|c|c|c|c|c|c|c|}
\hline \multirow[b]{2}{*}{ County } & \multicolumn{2}{|c|}{ Asbestosis } & \multicolumn{2}{|c|}{$\begin{array}{c}\text { Coal workers' } \\
\text { pneumoconiosis }\end{array}$} & \multicolumn{2}{|c|}{ Silicosis } & \multicolumn{2}{|c|}{ Byssinosis } & \multicolumn{2}{|c|}{$\begin{array}{r}\text { Unspecified/Other } \\
\text { pneumoconioses }\end{array}$} & \multicolumn{2}{|c|}{$\begin{array}{r}\text { All } \\
\text { pneumoconioses }\end{array}$} \\
\hline & \# & Rate & \# & Rate & \# & Rate & $\#$ & Rate & \# & Rate & \# & Rate \\
\hline Allegany & 14 & 10.10 & 10 & 5.92 & 3 & 3.14 & - & - & 5 & 2.96 & 32 & 22.11 \\
\hline Anne Arundel & 20 & 5.44 & 4 & 1.04 & 4 & 1.12 & - & - & 2 & 0.52 & 30 & 8.13 \\
\hline Baltimore & 63 & 7.67 & 8 & 0.80 & 2 & 0.21 & 1 & 0.14 & 4 & 0.37 & 78 & 9.20 \\
\hline Calvert & 1 & 2.65 & - & - & - & - & - & - & 2 & 4.47 & 3 & 7.12 \\
\hline Carroll & 3 & 2.70 & 5 & 3.80 & 1 & 0.55 & - & - & - & - & 9 & 7.04 \\
\hline Cecil & 2 & 2.72 & 4 & 4.86 & - & - & - & - & - & - & 6 & 7.58 \\
\hline Charles & 1 & 1.74 & 1 & 1.27 & - & - & - & - & - & - & 2 & 3.00 \\
\hline Dorchester & 1 & 1.28 & - & - & - & - & - & - & - & - & 1 & 1.28 \\
\hline Frederick & 2 & 1.44 & 4 & 2.87 & 2 & 1.44 & - & - & 2 & 1.44 & 10 & 7.18 \\
\hline Garrett & 1 & 1.76 & 10 & 26.86 & - & - & - & - & 4 & 8.88 & 15 & 37.50 \\
\hline Harford & 6 & 4.04 & 4 & 2.49 & 1 & 0.57 & - & - & 1 & 0.77 & 12 & 7.88 \\
\hline Howard & 4 & 3.17 & 2 & 1.71 & 1 & 0.73 & - & - & 1 & 0.73 & 7 & 5.61 \\
\hline Kent & 1 & 2.34 & 1 & 4.60 & - & - & - & - & - & - & 2 & 6.94 \\
\hline Montgomery & 8 & 1.18 & 11 & 1.33 & 1 & 0.10 & - & - & 2 & 0.19 & 21 & 2.70 \\
\hline Prince Georges & 4 & 0.84 & 11 & 2.06 & 1 & 0.16 & - & - & 3 & 0.56 & 19 & 3.62 \\
\hline Queen Annes & 1 & 1.83 & - & - & - & - & - & - & - & - & 1 & 1.83 \\
\hline St. Marys & 1 & 1.25 & - & - & - & - & - & - & - & - & 1 & 1.25 \\
\hline Somerset & 1 & 1.93 & - & - & - & - & - & - & - & - & 1 & 1.93 \\
\hline Talbot & 2 & 3.66 & - & - & - & - & - & - & - & - & 2 & 3.66 \\
\hline Washington & 1 & 0.41 & 2 & 1.23 & - & - & - & - & 1 & 0.41 & 4 & 2.05 \\
\hline Wicomico & 3 & 3.60 & 1 & 0.72 & - & - & - & - & 1 & 1.91 & 5 & 6.23 \\
\hline Worcester & 2 & 4.46 & - & - & - & - & - & - & - & - & 2 & 4.46 \\
\hline \multicolumn{13}{|c|}{ Independent city: } \\
\hline Baltimore & 62 & 6.94 & 6 & 0.46 & 7 & 0.67 & - & - & 8 & 0.87 & 83 & 8.94 \\
\hline
\end{tabular}

- indicates no deaths listed.

NOTE: Counties with no deaths for all listed conditions are not displayed. Rates based on small numbers may be unstable. Sum of individual conditions may exceed total because of deaths with multiple pneumoconioses. See Appendix A for source description and Appendix B for methods and ICD-8 and ICD-9 codes

SOURCE: National Center for Health Statistics multiple cause of death data. Population estimates from U.S. Bureau of the Census. 


\section{Massachusetts: Selected statistics from the United States section}

All pneumoconioses mortality, 1988-1992

Total deaths

Crude mortality rate (per $1,000,000$ population)

Age-adjusted mortality rate (per $1,000,000$ population)

Total years of potential life lost to life expectancy

Years of potential life lost to life expectancy (per death)
Value

223

9.16

4.94

2,514

11.3

Deaths*

184

9

17

2

12 $\underline{\text { State rank }}$

17

26

27

18

39

Percent**

distribution

82.5

4.0

7.6

0.9

5.4

* Sum of column may exceed total deaths because of deaths with multiple pneumoconioses.

** Percentages may total more than $100 \%$ due to deaths with multiple pneumoconioses.

NOTE: $\quad$ See Appendix A for source description and Appendix B for methods and ICD-8 and ICD-9 codes.

SOURCE: National Center for Health Statistics multiple cause of death data. Population estimates from U.S. Bureau of the Census.

Figure MA-1. All pneumoconioses: Massachusetts and U.S. age-adjusted mortality rates, residents age 15 and over, 1968-1992

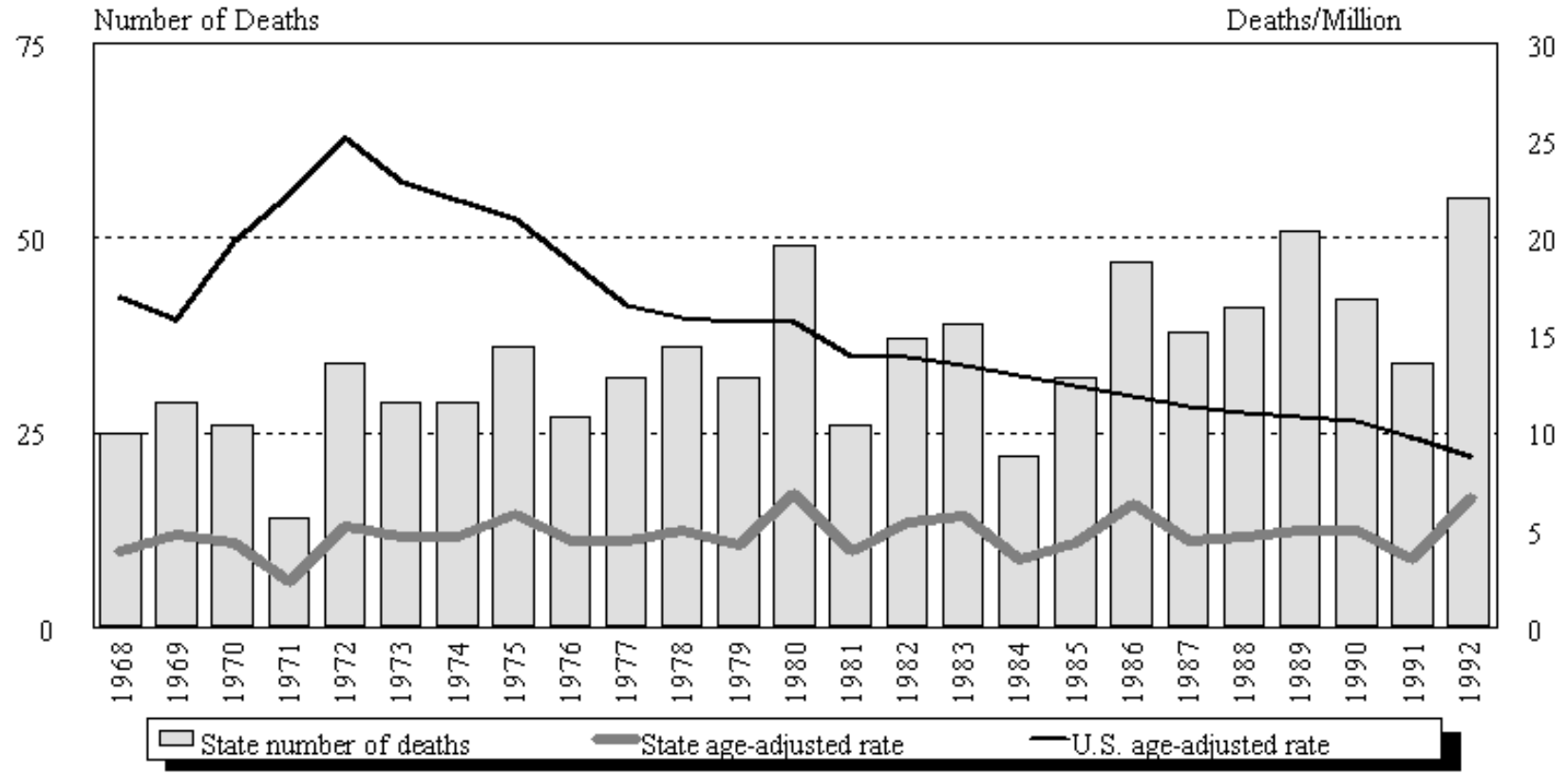

NOTE: $\quad$ See Appendix A for source description and Appendix B for methods and ICD-8 and ICD-9 codes.

SOURCE: National Center for Health Statistics multiple cause of death data. Population estimates from U.S. Bureau of the Census. 
Massachusetts

Table MA-1. Number of deaths and age-adjusted mortality rate (per 1,000,000 population) by condition, Massachusetts residents age 15 and over, 1968-1992

\begin{tabular}{|c|c|c|c|c|c|c|c|c|c|c|c|c|}
\hline \multirow[b]{2}{*}{ Year } & \multicolumn{2}{|c|}{ Asbestosis } & \multicolumn{2}{|c|}{$\begin{array}{l}\text { Coal workers' } \\
\text { pneumoconiosis }\end{array}$} & \multicolumn{2}{|c|}{ Silicosis } & \multicolumn{2}{|c|}{ Byssinosis* } & \multicolumn{2}{|c|}{$\begin{array}{c}\text { Unspecified/Other } \\
\text { pneumoconioses }\end{array}$} & \multicolumn{2}{|c|}{ All pneumoconioses } \\
\hline & Number & Rate & Number & Rate & Number & Rate & Number & Rate & Number & Rate & Number & Rate \\
\hline 1968 & 4 & 0.61 & 1 & 0.11 & 14 & 2.17 & & & 7 & 1.15 & 25 & 3.87 \\
\hline 1969 & 6 & 1.19 & 1 & 0.11 & 14 & 1.86 & & & 11 & 2.15 & 29 & 4.81 \\
\hline 1970 & 4 & 0.80 & 4 & 0.74 & 10 & 1.37 & & & 8 & 1.39 & 26 & 4.31 \\
\hline 1971 & 3 & 0.50 & - & - & 2 & 0.36 & & & 9 & 1.44 & 14 & 2.30 \\
\hline 1972 & 16 & 2.72 & 8 & 1.16 & 4 & 0.44 & & & 6 & 0.89 & 34 & 5.20 \\
\hline 1973 & 11 & 1.94 & 4 & 0.68 & 11 & 1.48 & & & 4 & 0.62 & 29 & 4.61 \\
\hline 1974 & 8 & 1.52 & 1 & 0.06 & 9 & 1.35 & & & 11 & 1.75 & 29 & 4.68 \\
\hline 1975 & 14 & 2.43 & 2 & 0.35 & 13 & 1.85 & & & 8 & 1.25 & 36 & 5.78 \\
\hline 1976 & 10 & 1.65 & - & - & 9 & 1.44 & & & 8 & 1.37 & 27 & 4.47 \\
\hline 1977 & 13 & 1.92 & 1 & 0.10 & 11 & 1.43 & & & 7 & 1.01 & 32 & 4.46 \\
\hline 1978 & 15 & 2.29 & 4 & 0.42 & 9 & 1.06 & & & 9 & 1.36 & 36 & 5.02 \\
\hline 1979 & 21 & 2.96 & 2 & 0.26 & 8 & 0.92 & - & - & 2 & 0.28 & 32 & 4.27 \\
\hline 1980 & 33 & 4.56 & 3 & 0.52 & 8 & 1.17 & 1 & 0.05 & 6 & 0.87 & 49 & 6.84 \\
\hline 1981 & 14 & 2.07 & 1 & 0.15 & 5 & 0.96 & - & - & 6 & 0.79 & 26 & 3.97 \\
\hline 1982 & 26 & 4.08 & 2 & 0.25 & 4 & 0.53 & 1 & 0.05 & 4 & 0.48 & 37 & 5.39 \\
\hline 1983 & 29 & 4.63 & - & - & 2 & 0.25 & 2 & 0.09 & 6 & 0.76 & 39 & 5.73 \\
\hline 1984 & 16 & 2.54 & - & - & 2 & 0.24 & - & - & 4 & 0.67 & 22 & 3.45 \\
\hline 1985 & 24 & 3.20 & - & - & 3 & 0.57 & 1 & 0.09 & 4 & 0.47 & 32 & 4.34 \\
\hline 1986 & 37 & 5.13 & 1 & 0.15 & 5 & 0.46 & - & - & 4 & 0.62 & 47 & 6.36 \\
\hline 1987 & 31 & 3.47 & - & - & 5 & 0.66 & - & - & 2 & 0.34 & 38 & 4.47 \\
\hline 1988 & 30 & 3.25 & 2 & 0.24 & 5 & 0.72 & - & - & 4 & 0.41 & 41 & 4.62 \\
\hline 1989 & 43 & 4.27 & 1 & 0.04 & 4 & 0.37 & - & - & 3 & 0.27 & 51 & 4.95 \\
\hline 1990 & 36 & 4.41 & 2 & 0.18 & 4 & 0.41 & 1 & 0.14 & - & - & 42 & 5.00 \\
\hline 1991 & 27 & 2.78 & 2 & 0.17 & 3 & 0.21 & - & - & 2 & 0.28 & 34 & 3.44 \\
\hline 1992 & 48 & 5.57 & 2 & 0.39 & 1 & 0.04 & 1 & 0.14 & 3 & 0.53 & 55 & 6.66 \\
\hline
\end{tabular}

indicates no deaths listed. $\quad *$ no specific code for byssinosis in ICD-8 (1968-1 978).

NOTE: Rates based on small numbers may be unstable. Sum of individ ual conditions may exceed total because of deaths with multiple pneumo conioses. See Appendix A for source description and Appendix B for methods and ICD-8 and ICD-9 codes.

SOURCE: National Center for Health Statistics multiple cause of death data. Population estimates from U.S. Bureau of the Census. 
Figure MA-2. All pneumoconioses: Distribution of deaths by sex and race, Massachusetts residents age 15 and over, 1968-1992
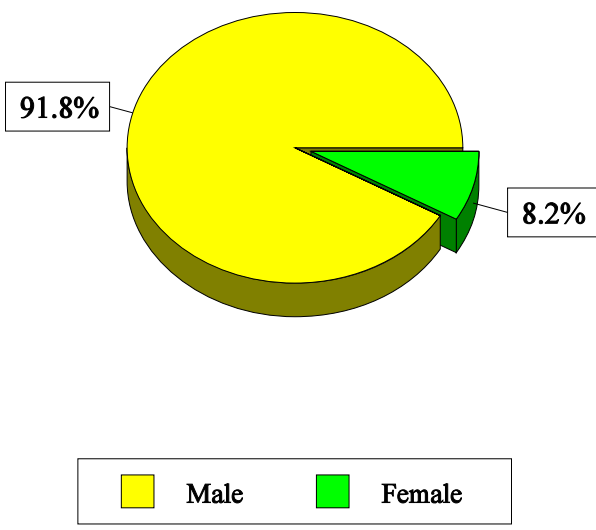
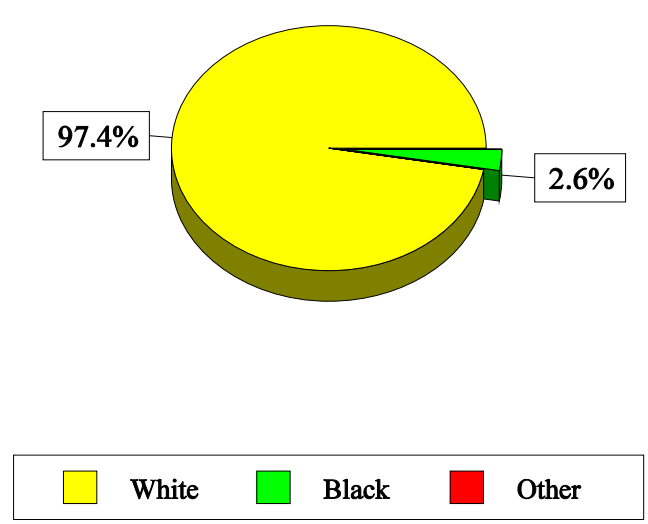

NOTE: $\quad$ See Appendix A for source description and Appendix B for methods and ICD-8 and ICD-9 codes.

SOURCE: National Center for Health Statistics multiple cause of death data.

Table MA-2. All pneumoconioses: Distribution of deaths by sex, race, and age, Massachusetts residents age 15 and over, 1968-1992

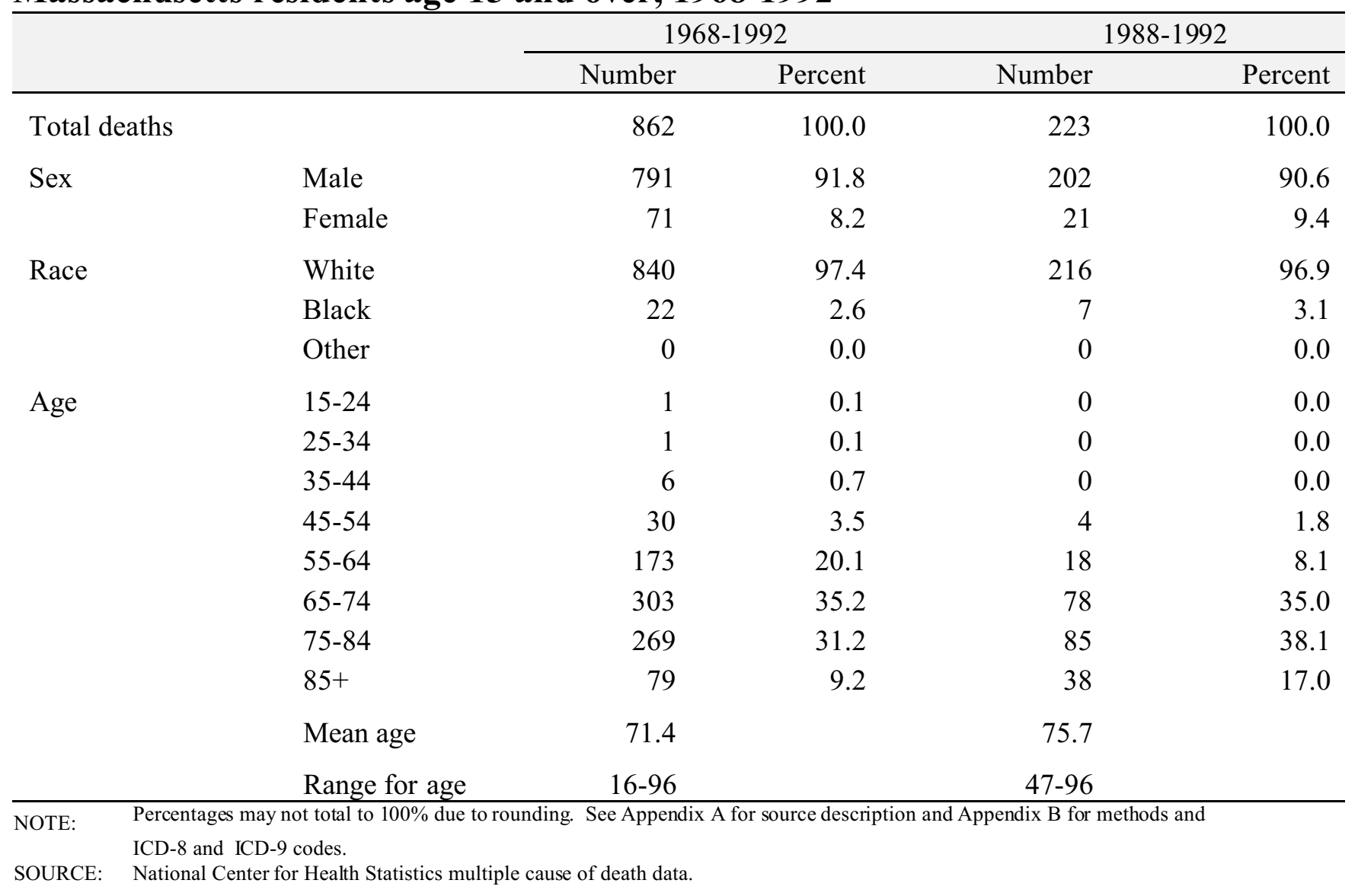


Figure MA-3. Pneumoconiosis deaths by condition, Massachusetts residents age 15 and over, 1968-1992

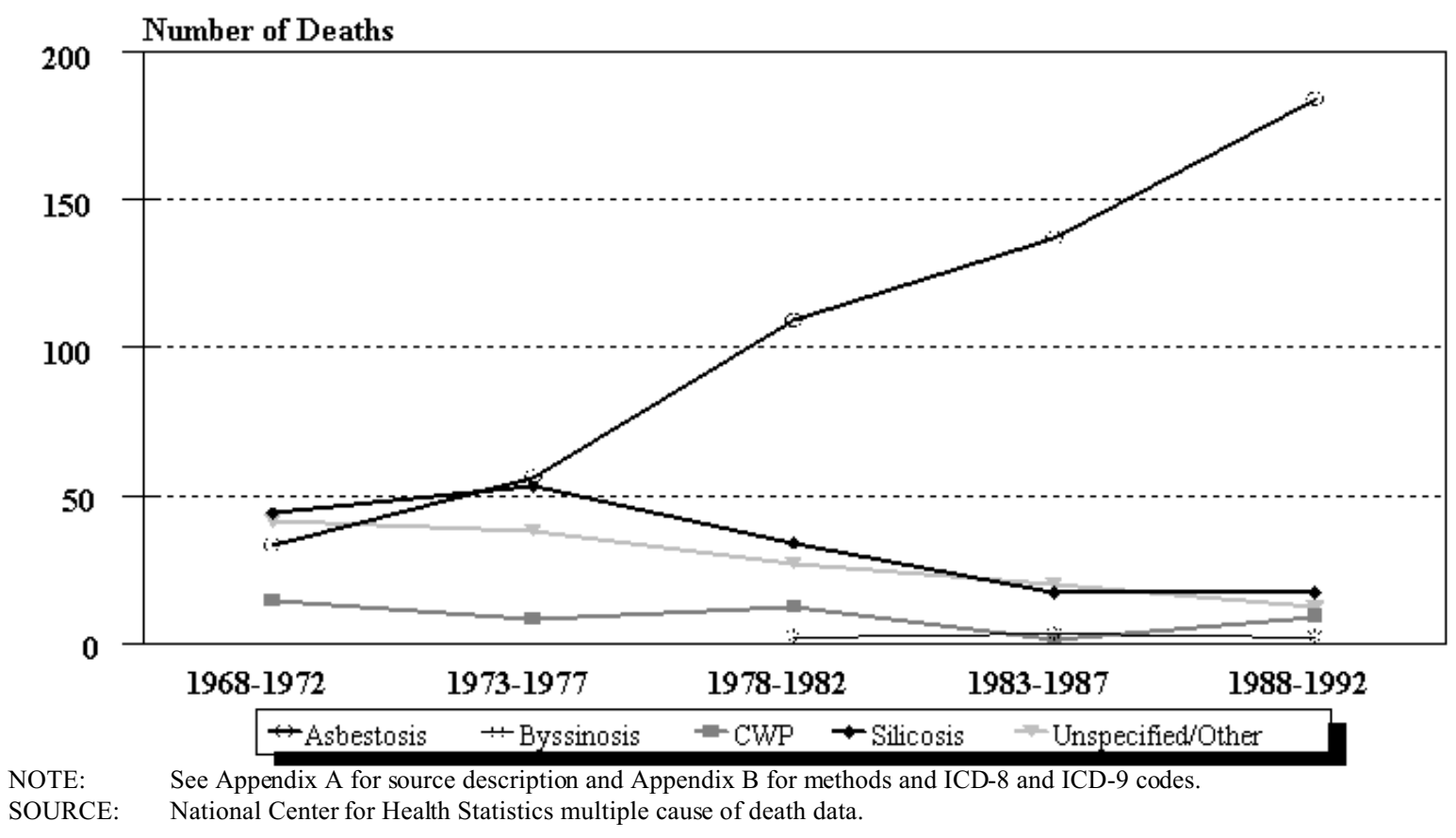

Figure MA-4. Massachusetts and U.S. age-adjusted mortality rates (per 1,000,000 population) by condition, residents age 15 and over, 1983-1992

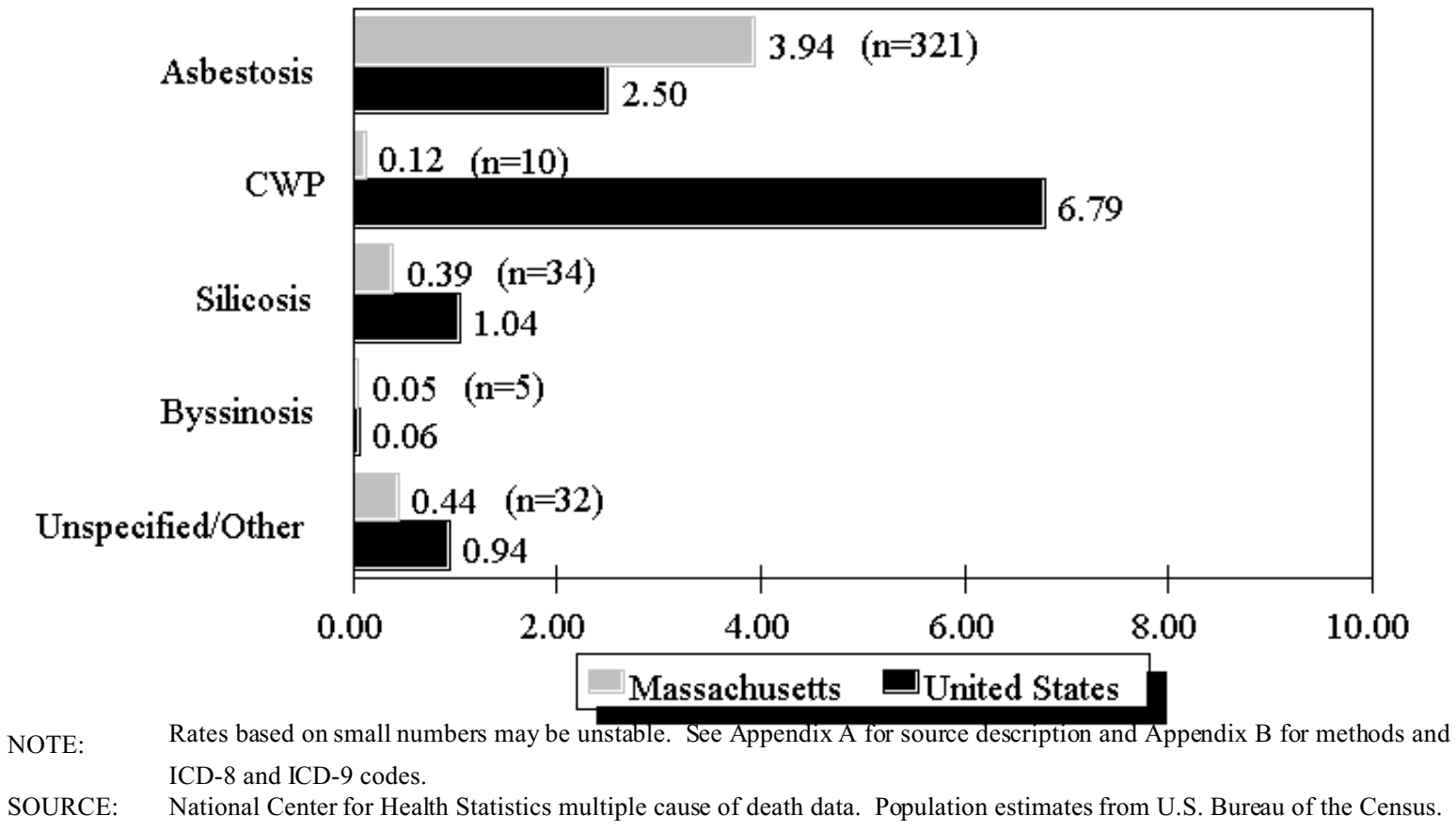


Table MA-3. Number of deaths and age-adjusted mortality rate (per 1,000,000 population) by condition and county, Massachusetts residents age 15 and over, 1983-1992

\begin{tabular}{|c|c|c|c|c|c|c|c|c|c|c|c|c|}
\hline \multirow[b]{2}{*}{ County } & \multicolumn{2}{|c|}{ Asbestosis } & \multicolumn{2}{|c|}{$\begin{array}{r}\text { Coal workers' } \\
\text { pneumoconiosis }\end{array}$} & \multicolumn{2}{|c|}{ Silicosis } & \multicolumn{2}{|c|}{ Byssinosis } & \multicolumn{2}{|c|}{$\begin{array}{r}\text { Unspecified/Other } \\
\text { pneumoconioses }\end{array}$} & \multicolumn{2}{|c|}{$\begin{array}{r}\text { All } \\
\text { pneumoconioses }\end{array}$} \\
\hline & \# & Rate & \# & Rate & $\#$ & Rate & \# & Rate & $\#$ & Rate & \# & Rate \\
\hline Barnstable & 17 & 5.22 & - & - & 1 & 0.41 & 1 & 0.16 & 1 & 0.41 & 20 & 6.20 \\
\hline Berkshire & 3 & 0.79 & 1 & 0.26 & 2 & 0.53 & - & - & - & - & 6 & 1.58 \\
\hline Bristol & 17 & 2.48 & 1 & 0.20 & 3 & 0.27 & 1 & 0.09 & 1 & 0.20 & 23 & 3.24 \\
\hline Essex & 30 & 3.24 & - & - & 2 & 0.13 & - & - & 11 & 1.56 & 43 & 4.93 \\
\hline Franklin & - & - & - & - & 1 & 0.64 & - & - & - & - & 1 & 0.64 \\
\hline Hampden & 15 & 2.50 & 2 & 0.43 & 3 & 0.52 & - & - & 1 & 0.10 & 21 & 3.56 \\
\hline Hampshire & 1 & 0.40 & 2 & 1.24 & 1 & 0.40 & - & - & - & - & 4 & 2.04 \\
\hline Middlesex & 75 & 4.31 & 2 & 0.11 & 1 & 0.08 & 2 & 0.15 & 9 & 0.47 & 89 & 5.12 \\
\hline Norfolk & 68 & 7.95 & - & - & 4 & 0.46 & - & - & 1 & 0.16 & 73 & 8.58 \\
\hline Plymouth & 33 & 6.91 & - & - & - & - & - & - & 1 & 0.27 & 34 & 7.18 \\
\hline Suffolk & 34 & 4.22 & 1 & 0.07 & 2 & 0.35 & 1 & 0.07 & 2 & 0.15 & 40 & 4.87 \\
\hline Worcester & 28 & 3.63 & 1 & 0.15 & 14 & 1.41 & - & - & 5 & 0.73 & 47 & 5.78 \\
\hline
\end{tabular}

- indicates no deaths listed.

NOTE: $\quad$ Counties with no deaths for all listed conditions are not displayed. Rates based on small numbers may be unstable. Sum of individual conditions may exceed total because of deaths with multiple pneumoconioses. See Appendix A for source description and Appendix B for methods and ICD-8 and ICD-9 codes.

SOURCE: National Center for Health Statistics multiple cause of death data. Population estimates from U.S. Bureau of the Census. 


\section{Michigan: Selected statistics from the United States section}

All pneumoconioses mortality, 1988-1992

Total deaths

Crude mortality rate (per $1,000,000$ population)

Age-adjusted mortality rate (per $1,000,000$ population)

Total years of potential life lost to life expectancy

Years of potential life lost to life expectancy (per death)

Deaths by type of pneumoconiosis, 1988-1992

Asbestosis

Coal workers' pneumoconiosis

Silicosis

Byssinosis

Unspecified/Other pneumoconiosis

\begin{tabular}{rr} 
Value & State rank \\
\hline 245 & 14 \\
6.78 & 31 \\
4.12 & 33 \\
3,029 & 15 \\
12.4 & 19
\end{tabular}

Percent**

Deaths* $\quad \underline{\text { distribution }}$

26.9

32.7

26.1

0.4

14.3

* Sum of column may exceed total deaths because of deaths with multiple pneumoconioses.

** Percentages may total more than $100 \%$ due to deaths with multiple pneumoconioses.

NOTE: $\quad$ See Appendix A for source description and Appendix B for methods and ICD-8 and ICD-9 codes.

SOURCE: National Center for Health Statistics multiple cause of death data. Population estimates from U.S. Bureau of the Census.

\section{Figure MI-1. All pneumoconioses: Michigan and U.S. age-adjusted mortality rates, residents age 15 and over, 1968-1992}

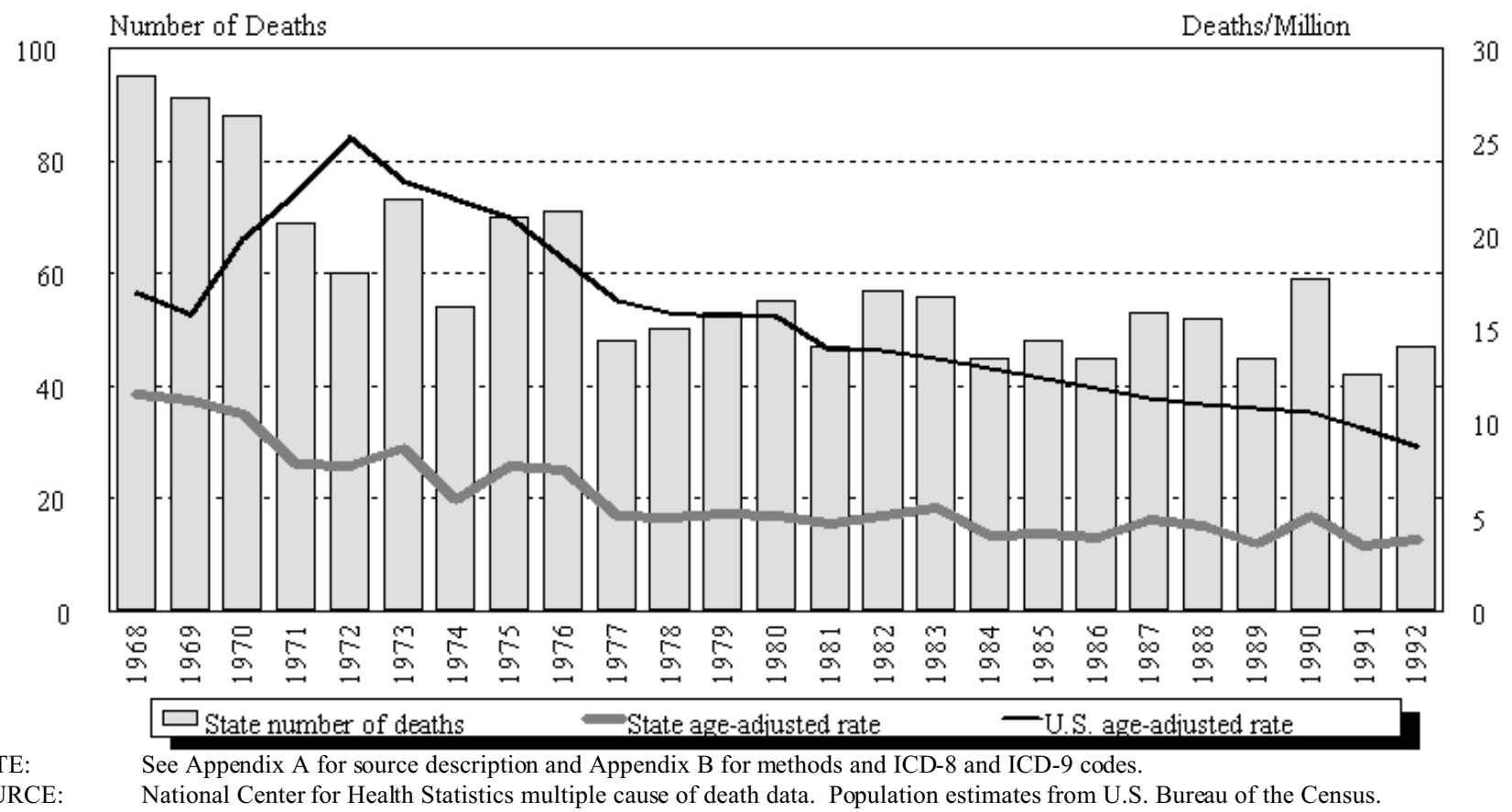




\section{Michigan}

Table MI-1. Number of deaths and age-adjusted mortality rate (per 1,000,000 population) by condition, Michigan residents age 15 and over, 1968-1992

\begin{tabular}{|c|c|c|c|c|c|c|c|c|c|c|c|c|}
\hline \multirow[b]{2}{*}{ Year } & \multicolumn{2}{|c|}{ Asbestosis } & \multicolumn{2}{|c|}{$\begin{array}{c}\text { Coal workers' } \\
\text { pneumoconiosis }\end{array}$} & \multicolumn{2}{|c|}{ Silicosis } & \multicolumn{2}{|c|}{ Byssinosis* } & \multicolumn{2}{|c|}{$\begin{array}{c}\text { Unspecified/Other } \\
\text { pneumoconioses }\end{array}$} & \multicolumn{2}{|c|}{ All pneumoconioses } \\
\hline & Number & Rate & Number & Rate & Number & Rate & Number & Rate & Number & Rate & Number & Rate \\
\hline 1968 & 1 & 0.14 & 7 & 0.87 & 55 & 6.65 & & & 32 & 3.87 & 95 & 11.53 \\
\hline 1969 & - & - & 9 & 1.13 & 52 & 6.42 & & & 32 & 3.96 & 91 & 11.27 \\
\hline 1970 & 2 & 0.28 & 17 & 2.11 & 43 & 5.30 & & & 26 & 2.85 & 88 & 10.54 \\
\hline 1971 & 1 & 0.15 & 16 & 1.64 & 34 & 4.23 & & & 23 & 2.48 & 69 & 7.89 \\
\hline 1972 & - & - & 12 & 1.67 & 32 & 4.18 & & & 18 & 2.15 & 60 & 7.73 \\
\hline 1973 & 2 & 0.27 & 10 & 1.14 & 38 & 4.47 & & & 23 & 2.84 & 73 & 8.72 \\
\hline 1974 & - & - & 9 & 0.99 & 25 & 2.64 & & & 23 & 2.71 & 54 & 5.94 \\
\hline 1975 & 2 & 0.27 & 12 & 1.36 & 35 & 3.69 & & & 22 & 2.47 & 70 & 7.72 \\
\hline 1976 & 3 & 0.42 & 22 & 2.28 & 24 & 2.62 & & & 24 & 2.44 & 71 & 7.54 \\
\hline 1977 & 4 & 0.50 & 11 & 1.28 & 20 & 2.05 & & & 13 & 1.26 & 48 & 5.09 \\
\hline 1978 & 4 & 0.54 & 10 & 1.14 & 20 & 1.95 & & & 16 & 1.38 & 50 & 5.01 \\
\hline 1979 & 3 & 0.36 & 12 & 1.15 & 19 & 1.86 & - & - & 19 & 1.78 & 53 & 5.16 \\
\hline 1980 & 4 & 0.47 & 15 & 1.34 & 23 & 2.06 & - & - & 14 & 1.25 & 55 & 5.07 \\
\hline 1981 & 4 & 0.49 & 19 & 1.99 & 15 & 1.41 & - & - & 11 & 1.00 & 47 & 4.66 \\
\hline 1982 & 5 & 0.61 & 23 & 1.86 & 21 & 1.77 & - & - & 9 & 0.92 & 57 & 5.11 \\
\hline 1983 & 8 & 0.93 & 24 & 2.12 & 17 & 1.60 & - & - & 8 & 0.86 & 56 & 5.47 \\
\hline 1984 & 3 & 0.32 & 14 & 1.09 & 12 & 1.15 & - & - & 16 & 1.43 & 45 & 3.99 \\
\hline 1985 & 6 & 0.57 & 17 & 1.46 & 15 & 1.20 & - & - & 10 & 0.94 & 48 & 4.17 \\
\hline 1986 & 6 & 0.62 & 16 & 1.35 & 14 & 1.17 & - & - & 9 & 0.76 & 45 & 3.90 \\
\hline 1987 & 11 & 1.29 & 21 & 1.83 & 16 & 1.23 & - & - & 5 & 0.51 & 53 & 4.86 \\
\hline 1988 & 7 & 0.74 & 20 & 1.62 & 15 & 1.21 & - & - & 10 & 0.96 & 52 & 4.52 \\
\hline 1989 & 11 & 1.21 & 14 & 1.02 & 14 & 1.09 & - & - & 7 & 0.44 & 45 & 3.66 \\
\hline 1990 & 17 & 1.80 & 21 & 1.37 & 12 & 1.16 & 1 & 0.03 & 8 & 0.77 & 59 & 5.14 \\
\hline 1991 & 15 & 1.44 & 15 & 1.13 & 9 & 0.68 & - & - & 3 & 0.30 & 42 & 3.55 \\
\hline 1992 & 16 & 1.40 & 10 & 0.74 & 14 & 1.04 & - & - & 7 & 0.62 & 47 & 3.80 \\
\hline
\end{tabular}

NOTE: Rates based on small numbers may be unstable. Sum of individual conditions may exceed total because of deaths with multiple pneumoconioses. See Appendix A for source description and Appendix B for methods and ICD-8 and ICD-9 codes.

SOURCE: National Center for Health Statistics multiple cause of death data. Population estimates from U.S. Bureau of the Census. 
Figure MI-2. All pneumoconioses: Distribution of deaths by sex and race, Michigan residents age 15 and over, 1968-1992
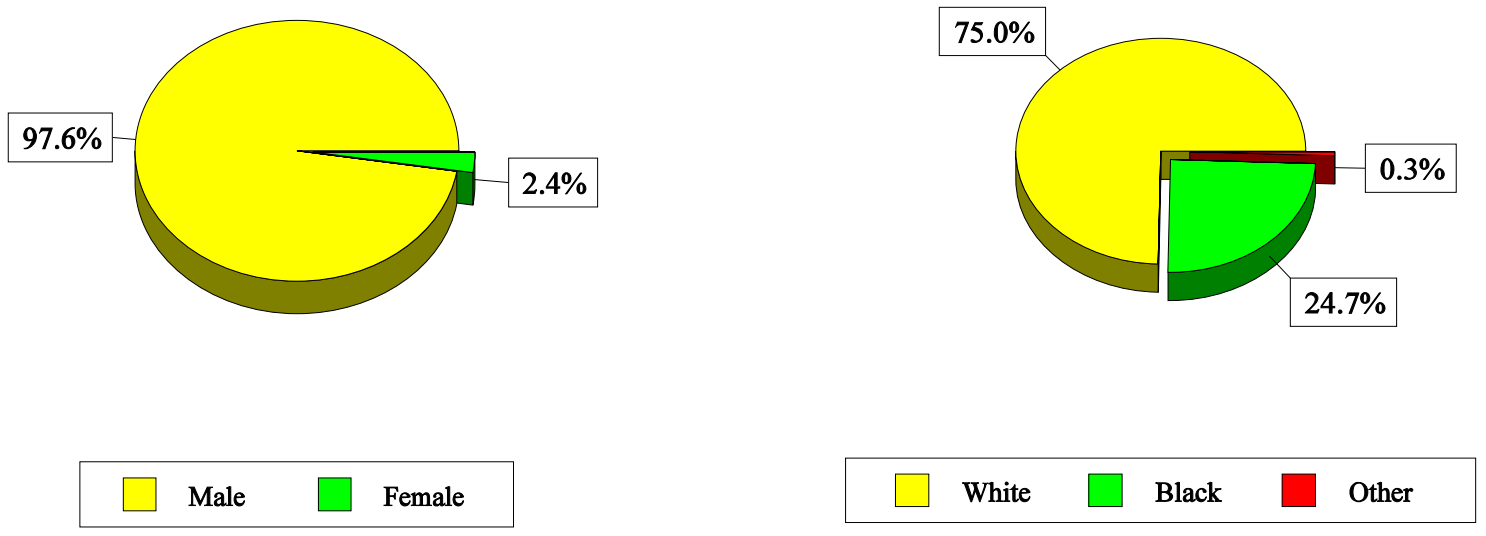

NOTE: $\quad$ See Appendix A for source description and Appendix B for methods and ICD-8 and ICD-9 codes.

SOURCE: National Center for Health Statistics multiple cause of death data.

Table MI-2. All pneumoconioses: Distribution of deaths by sex, race, and age, Michigan residents age 15 and over, 1968-1992

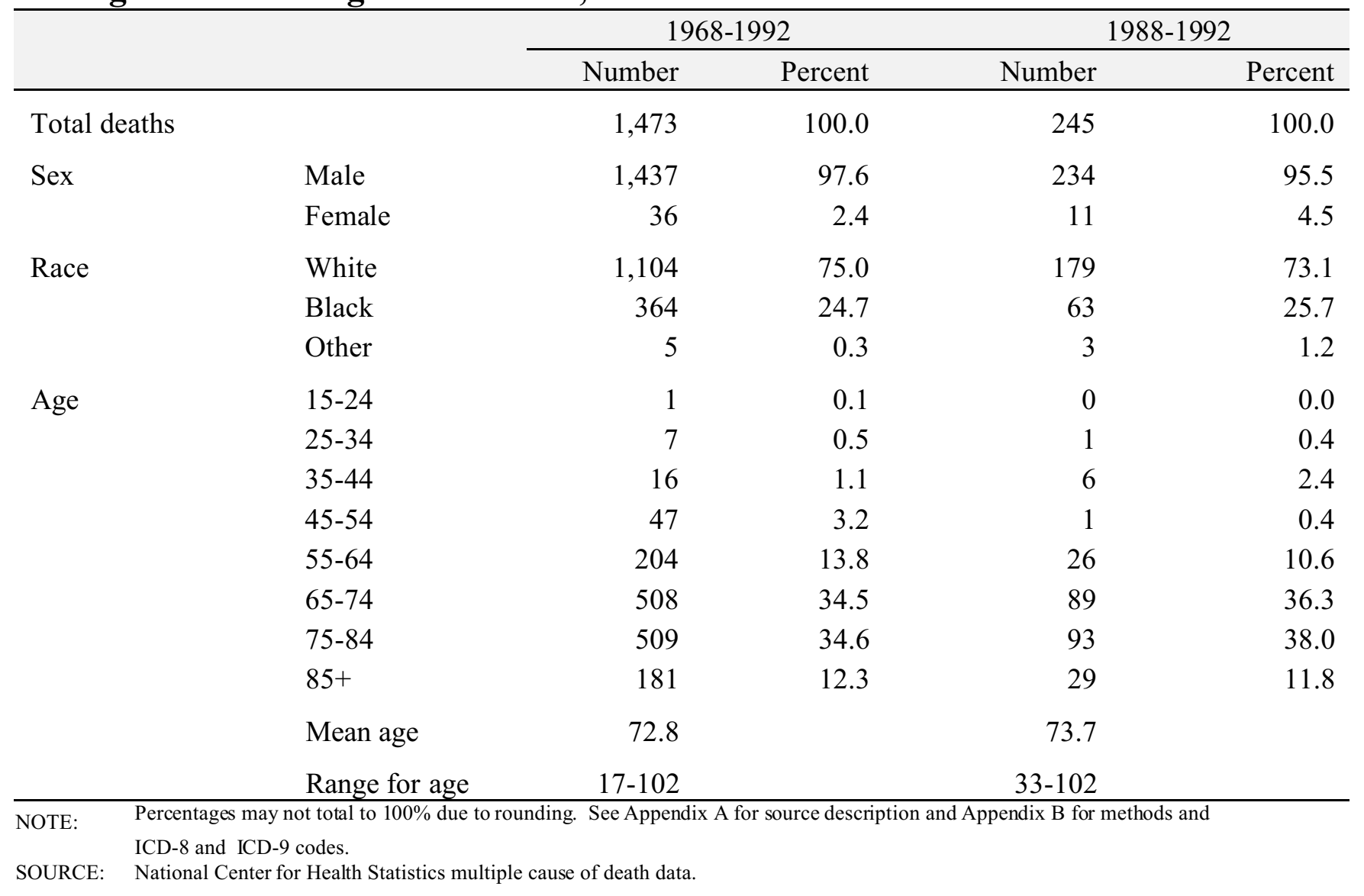


Figure MI-3. Pneumoconiosis deaths by condition, Michigan residents age 15 and over, 1968-1992

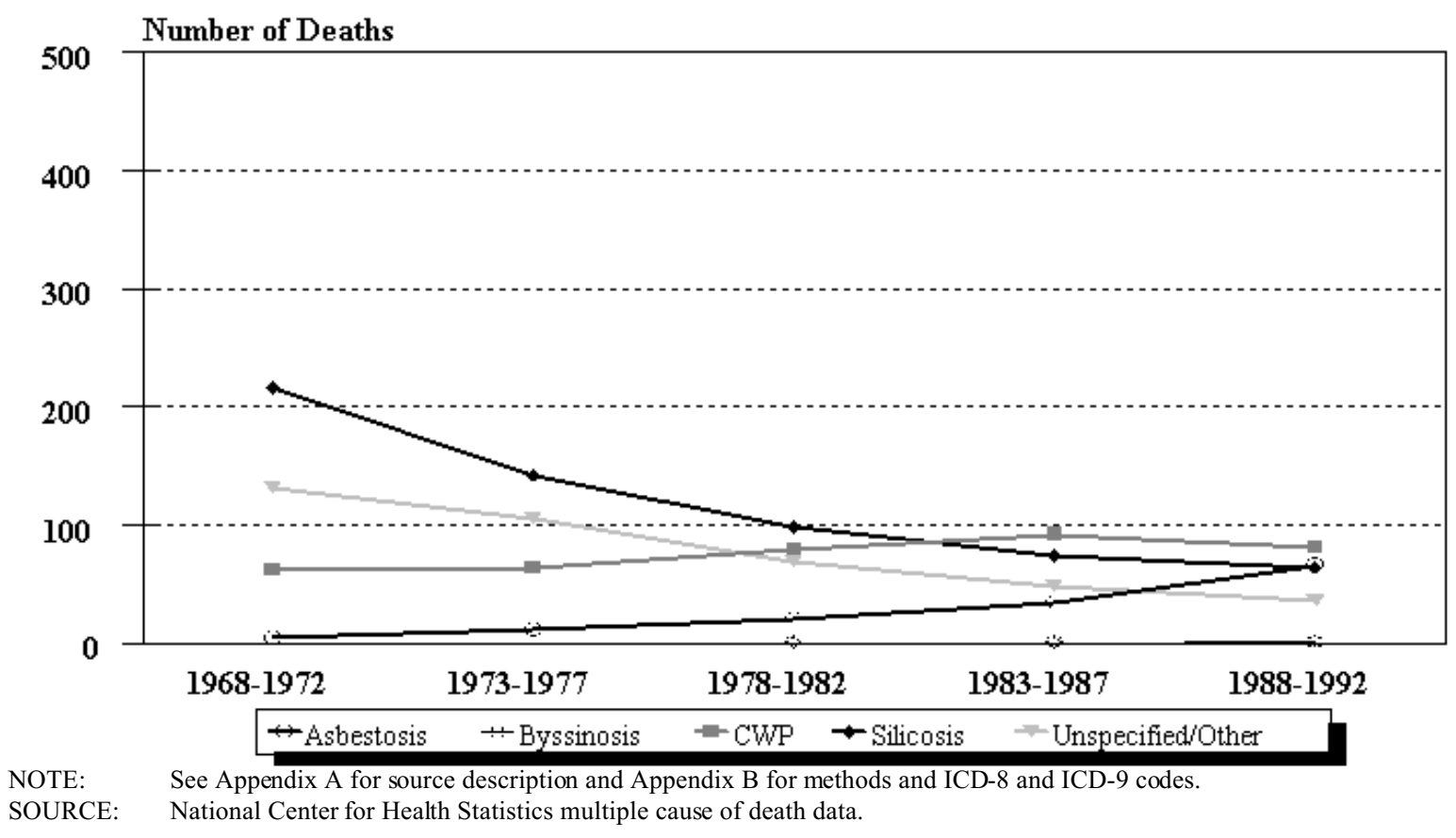

Figure MI-4. Michigan and U.S. age-adjusted mortality rates (per 1,000,000 population) by condition, residents age 15 and over, 1983-1992

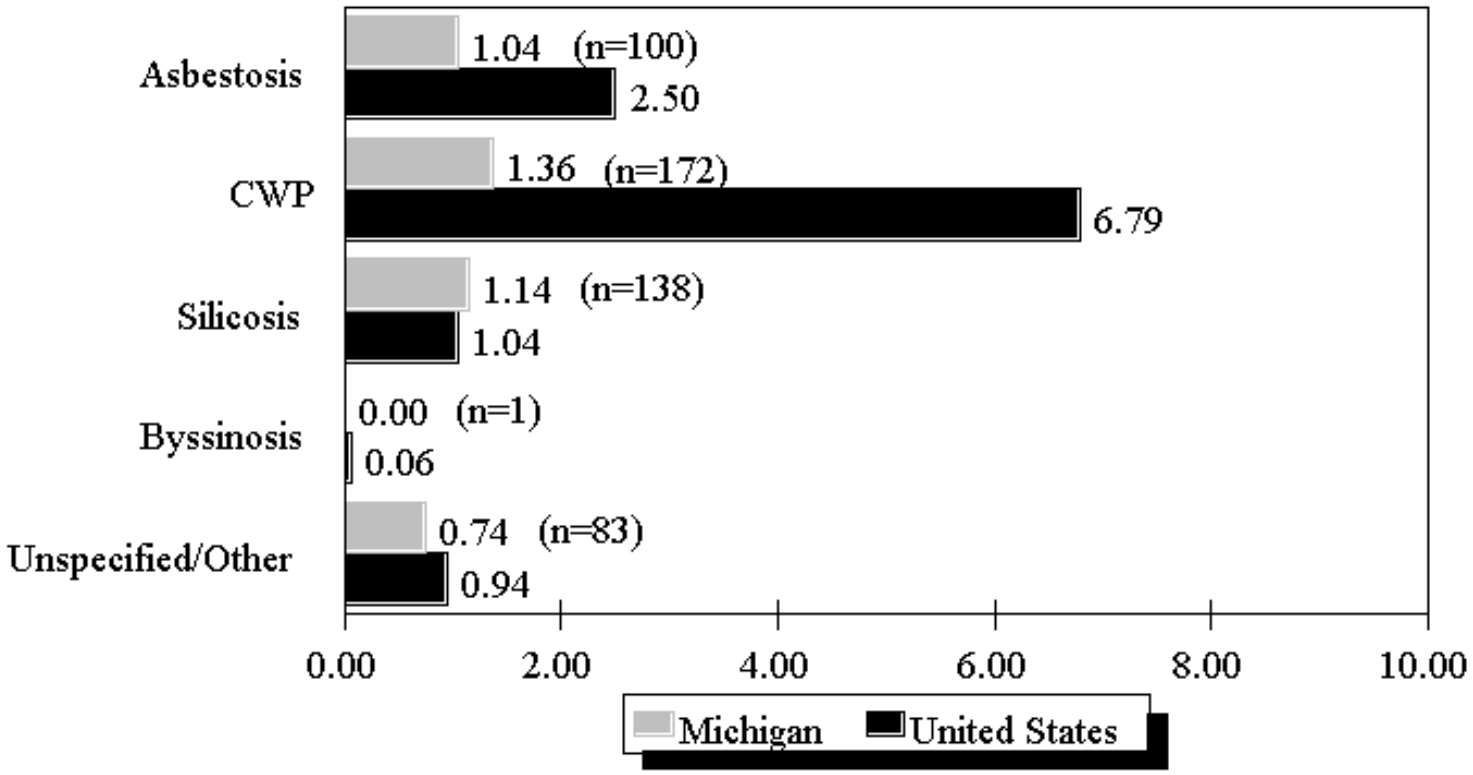

NOTE: $\quad$ Rates based on small numbers may be unstable. See Appendix A for source description and Appendix B for methods and ICD-8 and ICD-9 codes.

SOURCE: National Center for Health Statistics multiple cause of death data. Population estimates from U.S. Bureau of the Census. 
Table MI-3 (page 1 of 2). Number of deaths and age-adjusted mortality rate (per 1,000,000 population) by condition and county, Michigan residents age 15 and over, 1983-1992

\begin{tabular}{|c|c|c|c|c|c|c|c|c|c|c|c|c|}
\hline \multirow[b]{2}{*}{ County } & \multicolumn{2}{|c|}{ Asbestosis } & \multicolumn{2}{|c|}{$\begin{array}{c}\text { Coal workers' } \\
\text { pneumoconiosis }\end{array}$} & \multicolumn{2}{|c|}{ Silicosis } & \multicolumn{2}{|c|}{ Byssinosis } & \multicolumn{2}{|c|}{$\begin{array}{r}\text { Unspecified/Other } \\
\text { pneumoconioses }\end{array}$} & \multicolumn{2}{|c|}{$\begin{array}{r}\text { All } \\
\text { pneumoconioses }\end{array}$} \\
\hline & $\#$ & Rate & \# & Rate & $\#$ & Rate & $\#$ & Rate & $\#$ & Rate & $\#$ & Rate \\
\hline Alcona & - & - & - & - & - & - & - & - & 1 & 20.66 & 1 & 20.66 \\
\hline Allegan & - & - & - & - & 1 & 0.65 & - & - & - & - & 1 & 0.65 \\
\hline Alpena & - & - & - & - & - & - & - & - & 1 & 3.01 & 1 & 3.01 \\
\hline Arenac & 1 & 5.53 & - & - & - & - & - & - & - & - & 1 & 5.53 \\
\hline Baraga & 1 & 11.00 & - & - & - & - & - & - & - & - & 1 & 11.00 \\
\hline Bay & 6 & 4.58 & - & - & - & - & - & - & - & - & 6 & 4.58 \\
\hline Berrien & - & - & 2 & 0.62 & 4 & 1.55 & - & - & 4 & 1.86 & 10 & 4.03 \\
\hline Branch & 1 & 2.48 & - & - & - & - & - & - & 1 & 1.23 & 2 & 3.71 \\
\hline Calhoun & 2 & 1.47 & 2 & 0.74 & 1 & 0.73 & - & - & - & - & 5 & 2.94 \\
\hline Charlevoix & - & - & 1 & 2.29 & - & - & - & - & - & - & 1 & 2.29 \\
\hline Clare & 2 & 6.17 & 1 & 1.75 & - & - & - & - & - & - & 3 & 7.92 \\
\hline Clinton & 1 & 1.30 & 3 & 4.89 & - & - & - & - & - & - & 4 & 6.19 \\
\hline Crawford & - & - & 1 & 4.18 & - & - & - & - & - & - & 1 & 4.18 \\
\hline Delta & 1 & 2.46 & - & - & - & - & - & - & - & - & 1 & 2.46 \\
\hline Dickinson & - & - & - & - & 1 & 1.24 & - & - & - & - & 1 & 1.24 \\
\hline Eaton & 2 & 2.81 & 2 & 1.48 & - & - & - & - & - & - & 4 & 4.29 \\
\hline Emmet & 1 & 4.23 & 2 & 6.05 & 1 & 4.23 & - & - & - & - & 3 & 10.28 \\
\hline Genesee & 2 & 0.60 & 6 & 1.41 & 1 & 0.16 & - & - & 3 & 0.82 & 12 & 2.98 \\
\hline Gladwin & - & - & - & - & 1 & 2.09 & - & - & - & - & 1 & 2.09 \\
\hline Grand Traver & - & - & - & - & 1 & 0.85 & - & - & - & - & 1 & 0.85 \\
\hline Gratiot & 1 & 2.78 & - & - & - & - & - & - & - & - & 1 & 2.78 \\
\hline Hillsdale & 1 & 1.12 & - & - & 1 & 2.43 & - & - & - & - & 2 & 3.55 \\
\hline Houghton & - & - & 1 & 0.93 & - & - & - & - & - & - & 1 & 0.93 \\
\hline Huron & 2 & 4.66 & - & - & 1 & 1.04 & - & - & 1 & 1.04 & 4 & 6.74 \\
\hline Ingram & 5 & 2.44 & 2 & 0.81 & 2 & 0.54 & - & - & - & - & 9 & 3.79 \\
\hline Iosco & 1 & 2.96 & - & - & - & - & - & - & - & - & 1 & 2.96 \\
\hline Isabella & 1 & 1.55 & - & - & - & - & - & - & - & - & 1 & 1.55 \\
\hline Jackson & 2 & 1.68 & - & - & 2 & 1.10 & - & - & 1 & 0.37 & 5 & 3.15 \\
\hline Kalamazoo & 3 & 1.42 & - & - & 3 & 1.13 & - & - & - & - & 6 & 2.55 \\
\hline Kent & 2 & 0.24 & - & - & 4 & 0.75 & - & - & 1 & 0.26 & 7 & 1.25 \\
\hline Lapeer & - & - & - & - & 1 & 1.13 & - & - & - & - & 1 & 1.13 \\
\hline Lenawee & 2 & 1.84 & 2 & 2.44 & 1 & 0.62 & - & - & - & - & 5 & 4.91 \\
\hline
\end{tabular}

See footnotes at end of table. 
Table MI-3 (page 2 of 2). Number of deaths and age-adjusted mortality rate (per 1,000,000 population) by condition and county, Michigan residents age 15 and over, 1983-1992

\begin{tabular}{|c|c|c|c|c|c|c|c|c|c|c|c|c|}
\hline \multirow[b]{2}{*}{ County } & \multicolumn{2}{|c|}{ Asbestosis } & \multicolumn{2}{|c|}{$\begin{array}{c}\text { Coal workers' } \\
\text { pneumoconiosis }\end{array}$} & \multicolumn{2}{|c|}{ Silicosis } & \multicolumn{2}{|c|}{ Byssinosis } & \multicolumn{2}{|c|}{$\begin{array}{l}\text { Unspecified/Other } \\
\text { pneumoconioses }\end{array}$} & \multicolumn{2}{|c|}{$\begin{array}{r}\text { All } \\
\text { pneumoconioses }\end{array}$} \\
\hline & $\#$ & Rate & $\#$ & Rate & \# & Rate & $\#$ & Rate & \# & Rate & \# & Rate \\
\hline Livingston & 2 & 2.55 & - & - & - & - & - & - & 1 & 1.28 & 3 & 3.83 \\
\hline Mackinac & 1 & 14.27 & - & - & - & - & - & - & - & - & 1 & 14.27 \\
\hline Macomb & 4 & 0.58 & 21 & 2.32 & 6 & 0.65 & - & - & 5 & 0.56 & 36 & 4.11 \\
\hline Manistee & - & - & - & - & - & - & - & - & 1 & 1.66 & 1 & 1.66 \\
\hline Marquette & 2 & 3.60 & - & - & 1 & 1.80 & - & - & - & - & 3 & 5.40 \\
\hline Mason & 3 & 12.51 & - & - & - & - & - & - & 1 & 3.37 & 4 & 15.88 \\
\hline Mecosta & - & - & - & - & 1 & 1.72 & - & - & - & - & 1 & 1.72 \\
\hline Menominee & 1 & 3.54 & 1 & 1.48 & 1 & 3.54 & - & - & 1 & 3.54 & 4 & 12.09 \\
\hline Midland & 7 & 10.86 & 1 & 1.65 & - & - & - & - & - & - & 8 & 12.51 \\
\hline Missaukee & - & - & 1 & 4.18 & - & - & - & - & - & - & 1 & 4.18 \\
\hline Monroe & 5 & 3.72 & 9 & 5.89 & 2 & 1.01 & - & - & - & - & 16 & 10.62 \\
\hline Muskigon & 1 & 0.66 & 7 & 3.01 & 35 & 16.28 & - & - & 6 & 3.31 & 48 & 22.92 \\
\hline Newaygo & - & - & 1 & 1.35 & 1 & 1.35 & - & - & - & - & 2 & 2.70 \\
\hline Oakland & 4 & 0.33 & 14 & 0.99 & 7 & 0.52 & 1 & 0.06 & 6 & 0.55 & 32 & 2.45 \\
\hline Oceana & - & - & - & - & 1 & 4.39 & - & - & 1 & 2.31 & 2 & 6.69 \\
\hline Ogemaw & - & - & 1 & 2.32 & - & - & - & - & - & - & 1 & 2.32 \\
\hline Osceola & - & - & 1 & 2.54 & - & - & - & - & - & - & 1 & 2.54 \\
\hline Oscoda & - & - & 1 & 8.74 & - & - & - & - & - & - & 1 & 8.74 \\
\hline Ottawa & 1 & 0.75 & 3 & 1.49 & 1 & 0.37 & - & - & - & - & 5 & 2.61 \\
\hline Roscommon & 1 & 2.92 & - & - & - & - & - & - & - & - & 1 & 2.92 \\
\hline Saginaw & 4 & 2.09 & 4 & 1.98 & 3 & 1.05 & - & - & 6 & 2.10 & 17 & 7.22 \\
\hline St Clair & 1 & 0.39 & 1 & 0.39 & 2 & 0.78 & - & - & - & - & 4 & 1.56 \\
\hline St Joseph & - & - & - & - & - & - & - & - & 1 & 1.79 & 1 & 1.79 \\
\hline Shiawassee & 1 & 1.71 & 1 & 1.71 & 1 & 1.71 & - & - & - & - & 3 & 5.13 \\
\hline Tuscola & - & - & - & - & 1 & 2.05 & - & - & - & - & 1 & 2.05 \\
\hline Van Buren & - & - & - & - & 2 & 1.45 & - & - & 1 & 1.55 & 3 & 3.00 \\
\hline Washtenaw & 1 & 0.32 & 2 & 1.18 & 1 & 0.59 & - & - & 1 & 0.59 & 5 & 2.69 \\
\hline Wayne & 21 & 0.88 & 78 & 2.80 & 46 & 1.76 & - & - & 38 & 1.46 & 183 & 6.89 \\
\hline Wexford & - & - & - & - & - & - & - & - & 1 & 1.84 & 1 & 1.84 \\
\hline
\end{tabular}




\section{Minnesota: Selected statistics from the United States section}

All pneumoconioses mortality, 1988-1992

Total deaths
Years of potential life lost to life expectancy (per death)

Crude mortality rate (per $1,000,000$ population)

Age-adjusted mortality rate (per $1,000,000$ population)

Total years of potential life lost to life expectancy
Deaths by type of pneumoconiosis, 1988-1992

Asbestosis

Coal workers' pneumoconiosis

Silicosis

Byssinosis

Unspecified/Other pneumoconiosis $\underline{\text { Value }}$

81

4.79

2.98

1,023

12.6

$\underline{\text { State rank }}$

30

41

43

30

12

Percent**

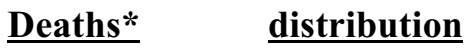

58.0

0.0

34.6

1.2

7.4

* Sum of column may exceed total deaths because of deaths with multiple pneumoconioses.

** Percentages may total more than $100 \%$ due to deaths with multiple pneumoconioses.

NOTE: $\quad$ See Appendix A for source description and Appendix B for methods and ICD-8 and ICD-9 codes.

SOURCE: $\quad$ National Center for Health Statistics multiple cause of death data. Population estimates from U.S. Bureau of the Census.

Figure MN-1. All pneumoconioses: Minnesota and U.S. age-adjusted mortality rates, residents age 15 and over, 1968-1992

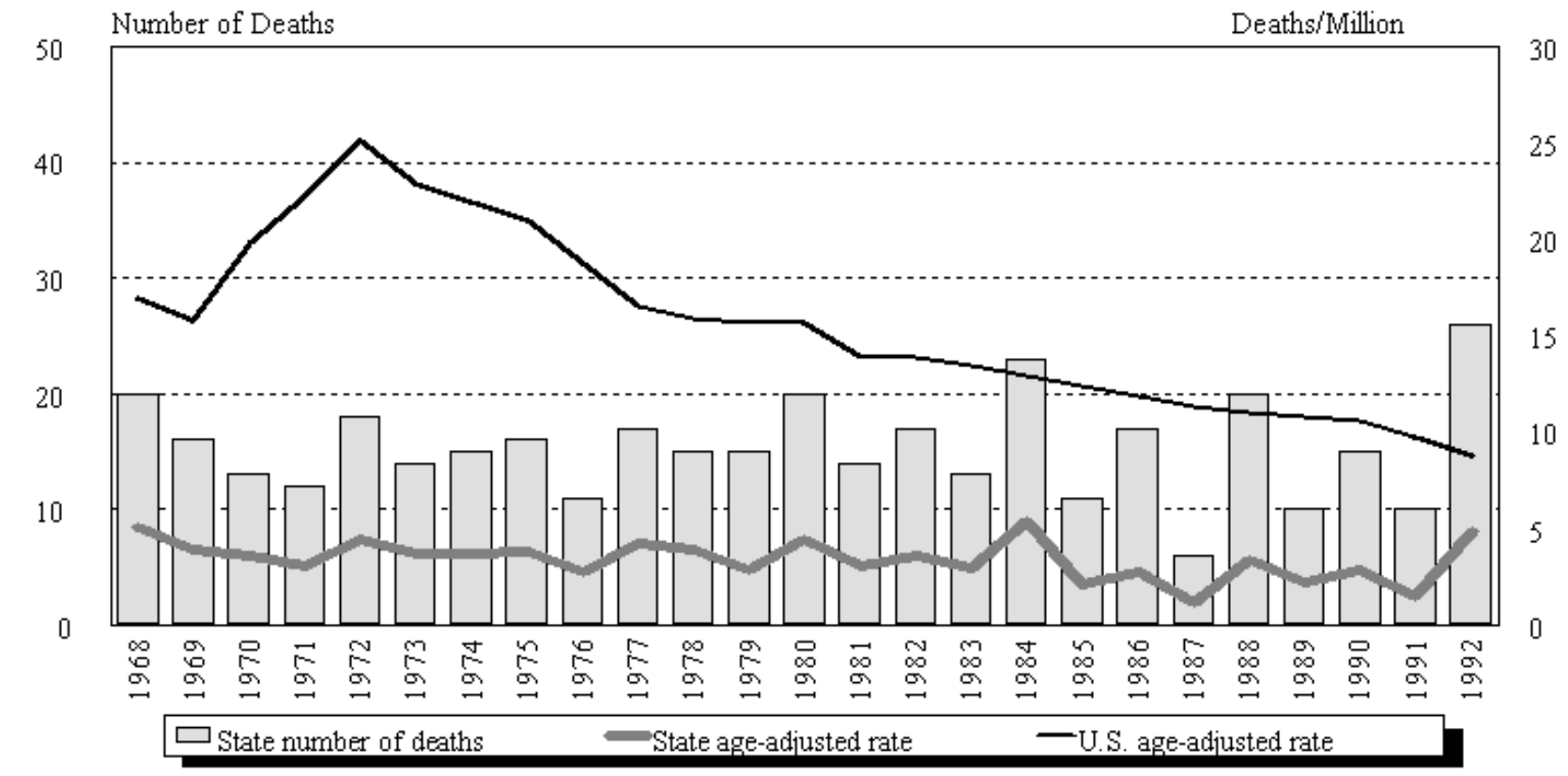

NOTE: $\quad$ See Appendix A for source description and Appendix B for methods and ICD-8 and ICD-9 codes.

SOURCE: $\quad$ National Center for Health Statistics multiple cause of death data. Population estimates from U.S. Bureau of the Census. 
Minnesota

Table MN-1. Number of deaths and age-adjusted mortality rate (per 1,000,000 population) by condition, Minnesota residents age 15 and over, 1968-1992

\begin{tabular}{|c|c|c|c|c|c|c|c|c|c|c|c|c|}
\hline \multirow[b]{2}{*}{ Year } & \multicolumn{2}{|c|}{ Asb estosis } & \multicolumn{2}{|c|}{$\begin{array}{l}\text { Coal workers' } \\
\text { pneumoconiosis }\end{array}$} & \multicolumn{2}{|c|}{ Silicosis } & \multicolumn{2}{|c|}{ Byssinosis* } & \multicolumn{2}{|c|}{$\begin{array}{c}\text { Unspecified/Other } \\
\text { pneumoconioses }\end{array}$} & \multicolumn{2}{|c|}{ All pneumoconioses } \\
\hline & Number & Rate & Number & Rate & Number & Rate & Number & Rate & Number & Rate & Number & Rate \\
\hline 1968 & 1 & 0.32 & 2 & 0.59 & 18 & 4.61 & & & 1 & 0.17 & 20 & 5.10 \\
\hline 1969 & - & - & - & - & 13 & 3.07 & & & 3 & 0.83 & 16 & 3.90 \\
\hline 1970 & 2 & 0.54 & - & - & 6 & 1.41 & & & 5 & 1.64 & 13 & 3.59 \\
\hline 1971 & 1 & 0.32 & 1 & 0.27 & 5 & 1.34 & & & 5 & 1.16 & 12 & 3.08 \\
\hline 1972 & - & - & 2 & 0.33 & 12 & 2.98 & & & 4 & 1.12 & 18 & 4.43 \\
\hline 1973 & 1 & 0.31 & 1 & 0.26 & 3 & 0.48 & & & 9 & 2.65 & 14 & 3.71 \\
\hline 1974 & 2 & 0.57 & 1 & 0.40 & 9 & 2.28 & & & 4 & 0.74 & 15 & 3.73 \\
\hline 1975 & - & - & - & - & 11 & 2.68 & & & 5 & 1.12 & 16 & 3.80 \\
\hline 1976 & 1 & 0.31 & - & - & 6 & 1.52 & & & 4 & 0.97 & 11 & 2.80 \\
\hline 1977 & 1 & 0.25 & 1 & 0.45 & 6 & 1.16 & & & 9 & 2.41 & 17 & 4.27 \\
\hline 1978 & 2 & 0.65 & 1 & 0.15 & 8 & 1.83 & & & 5 & 1.41 & 15 & 3.90 \\
\hline 1979 & 5 & 1.01 & 1 & 0.07 & 6 & 0.99 & - & - & 3 & 0.79 & 15 & 2.86 \\
\hline 1980 & 6 & 1.84 & 1 & 0.07 & 10 & 1.93 & - & - & 3 & 0.60 & 20 & 4.45 \\
\hline 1981 & 2 & 0.56 & - & - & 11 & 2.22 & - & - & 1 & 0.24 & 14 & 3.02 \\
\hline 1982 & 4 & 0.77 & 4 & 0.74 & 9 & 2.13 & - & - & 1 & 0.29 & 17 & 3.64 \\
\hline 1983 & 9 & 2.07 & 1 & 0.42 & 3 & 0.42 & - & - & - & - & 13 & 2.92 \\
\hline 1984 & 8 & 2.21 & 1 & 0.30 & 13 & 2.78 & - & - & 2 & 0.37 & 23 & 5.36 \\
\hline 1985 & 1 & 0.42 & - & - & 9 & 1.44 & - & - & 1 & 0.23 & 11 & 2.09 \\
\hline 1986 & 5 & 0.95 & - & - & 11 & 1.93 & - & - & 3 & 0.42 & 17 & 2.77 \\
\hline 1987 & 4 & 0.87 & - & - & 2 & 0.27 & - & - & - & - & 6 & 1.14 \\
\hline 1988 & 11 & 2.08 & - & - & 8 & 1.09 & - & - & 1 & 0.22 & 20 & 3.39 \\
\hline 1989 & 5 & 1.28 & - & - & 3 & 0.48 & - & - & 2 & 0.44 & 10 & 2.19 \\
\hline 1990 & 8 & 1.23 & - & - & 5 & 1.15 & 1 & 0.31 & 1 & 0.13 & 15 & 2.82 \\
\hline 1991 & 6 & 1.02 & - & - & 3 & 0.46 & - & - & 1 & 0.05 & 10 & 1.53 \\
\hline 1992 & 17 & 3.26 & - & - & 9 & 1.64 & - & - & 1 & 0.34 & 26 & 4.92 \\
\hline
\end{tabular}

indicates no deaths listed. * no specific code for byssinosis in ICD-8 (1968-1978)

NOTE: Rates based on small numbers may be unstable. Sum of individ ual conditions may exceed total because of deaths with multiple pneumoconioses. See Appendix A for source description and Appendix B for methods and ICD-8 and ICD-9 codes.

SOURCE: National Center for Health Statistics multiple cause of death data. Population estimates from U.S. Bureau of the Census. 
Figure MN-2. All pneumoconioses: Distribution of deaths by sex and race, Minnesota residents age 15 and over, 1968-1992
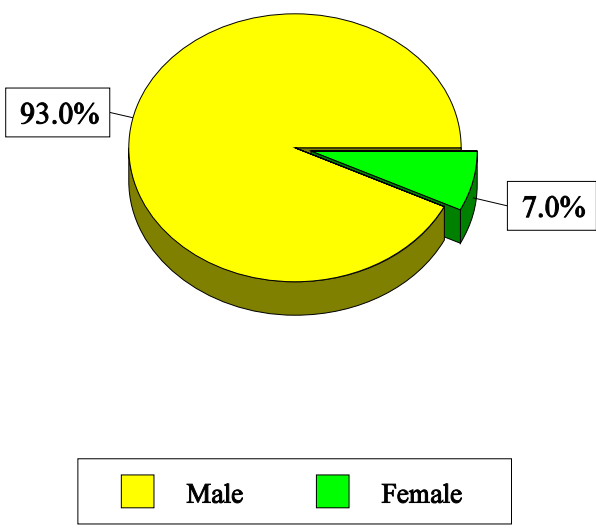
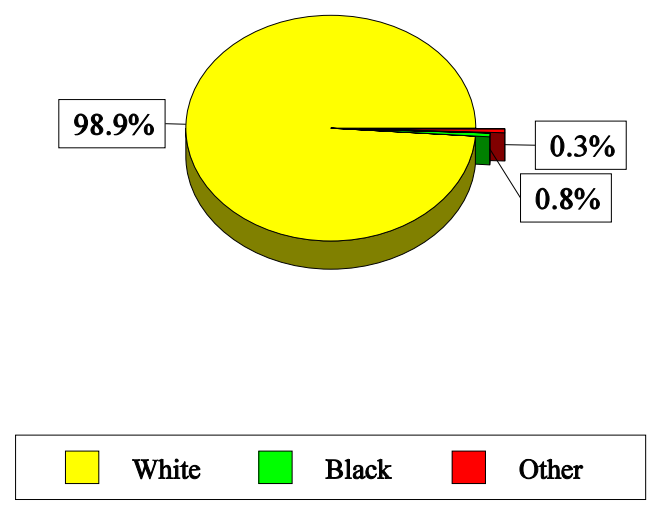

NOTE: $\quad$ See Appendix A for source description and Appendix B for methods and ICD-8 and ICD-9 codes.

SOURCE: National Center for Health Statistics multiple cause of death data.

Table MN-2. All pneumoconioses: Distribution of deaths by sex, race, and age, Minnesota residents age 15 and over, 1968-1992

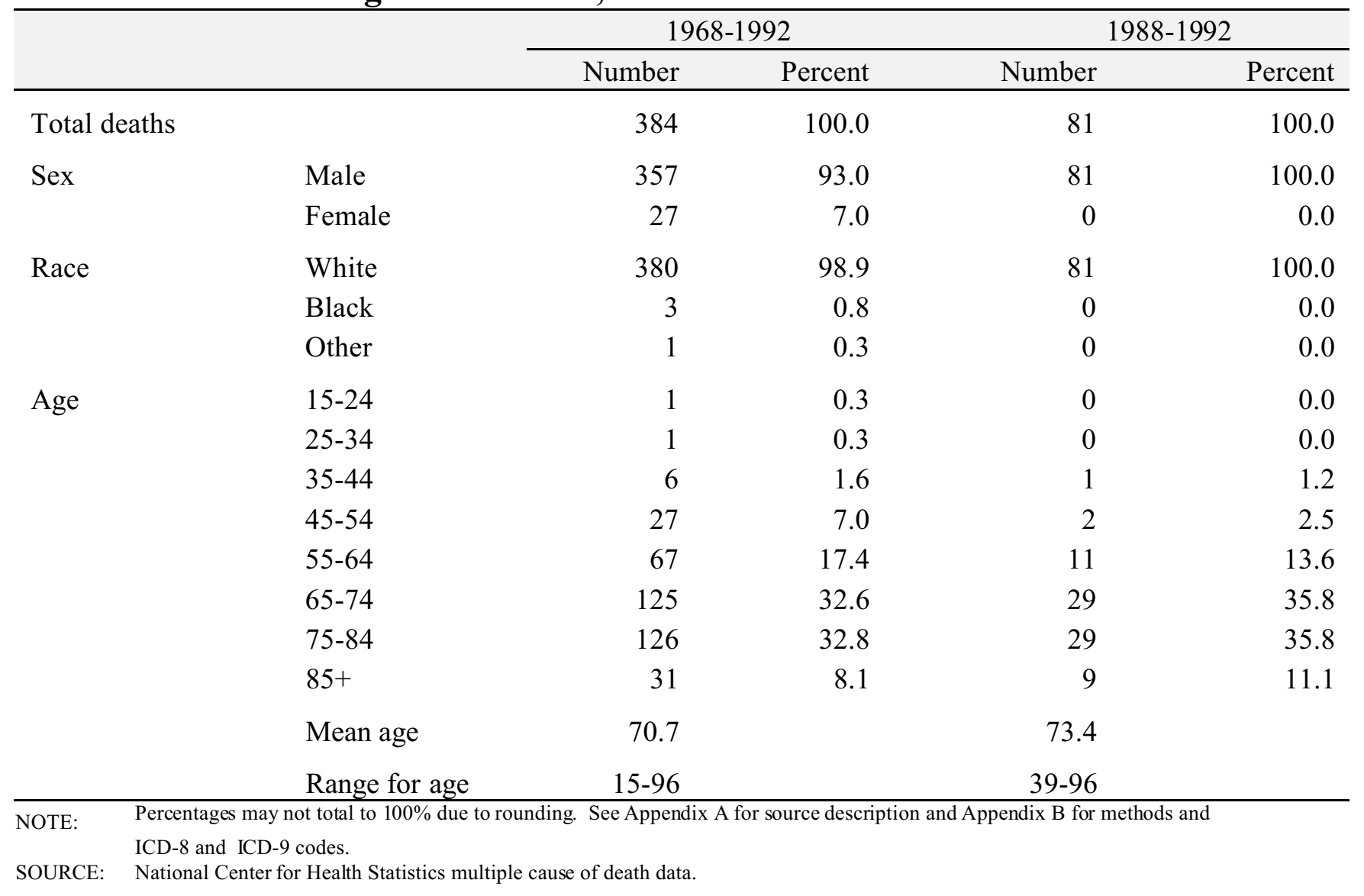


Figure MN-3. Pneumoconiosis deaths by condition, Minnesota residents age 15 and over, 1968-1992

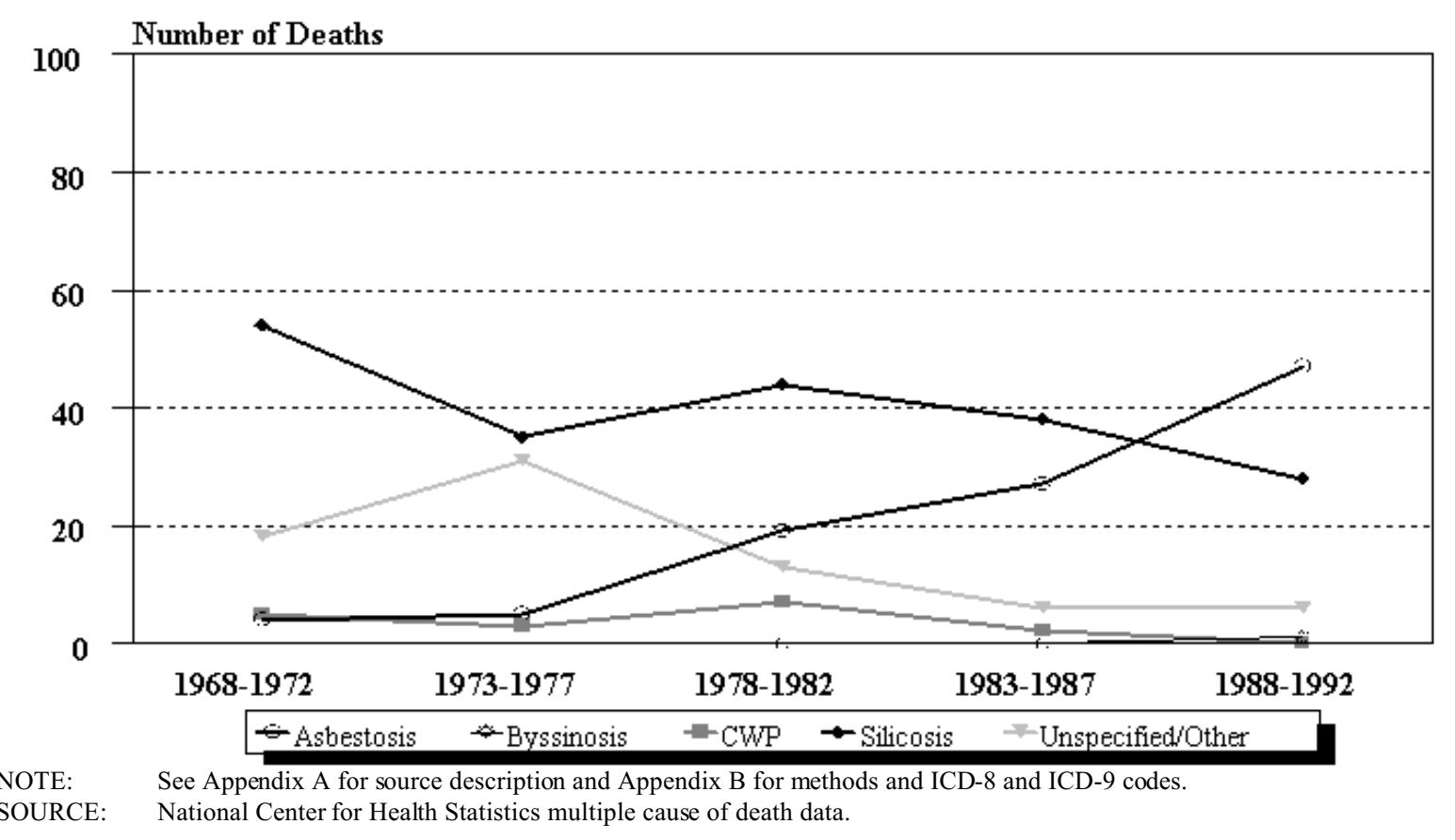

Figure MN-4. Minnesota and U.S. age-adjusted mortality rates (per 1,000,000 population) by condition, residents age 15 and over, 1983-1992

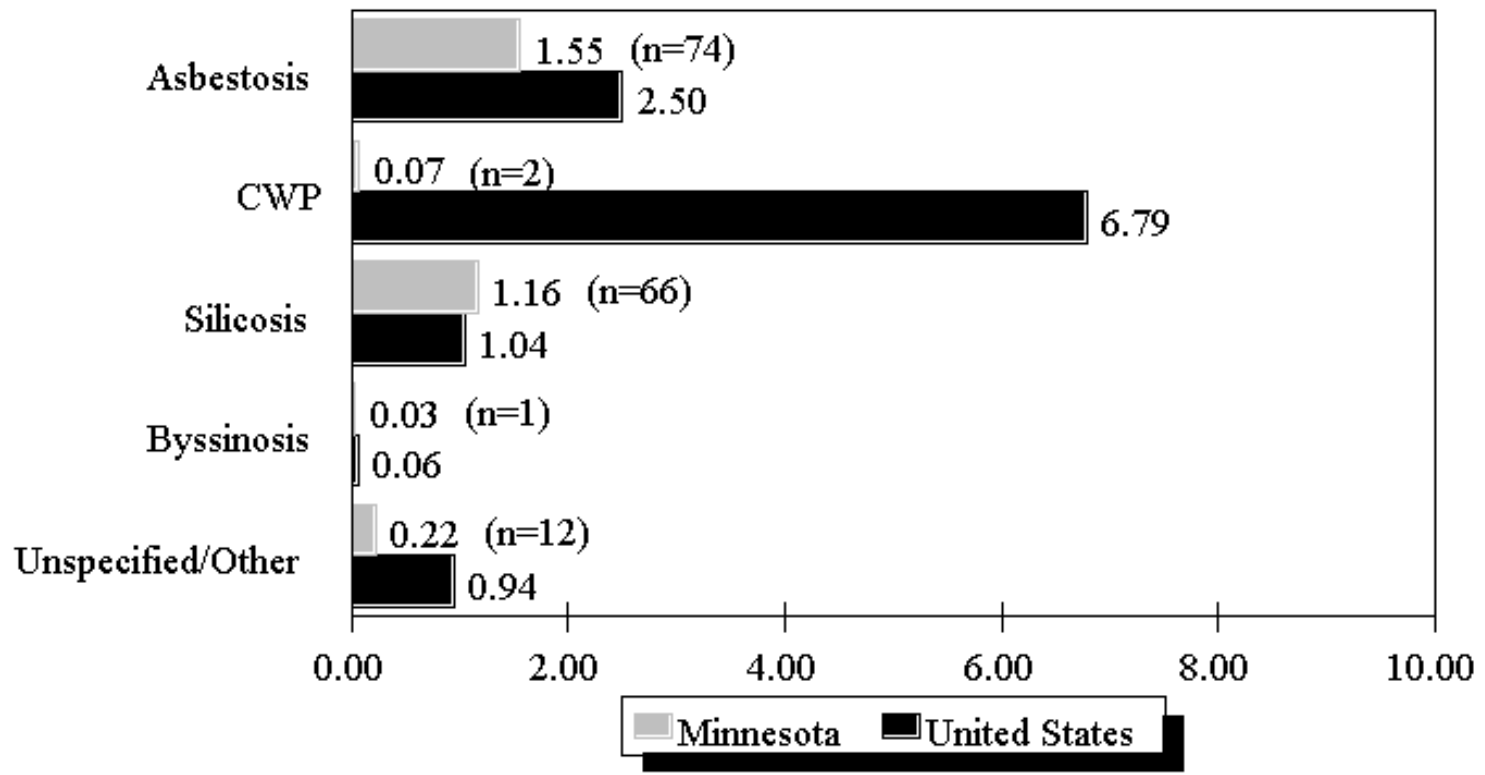

NOTE: $\quad$ Rates based on small numbers may be unstable. See Appendix A for source description and Appendix B for methods and ICD-8 and ICD-9 codes.

SOURCE: National Center for Health Statistics multiple cause of death data. Population estimates from U.S. Bureau of the Census. 
Table MN-3 (page 1 of 2). Number of deaths and age-adjusted mortality rate (per 1,000,000 population) by condition and county, Minnesota residents age 15 and over, 1983-1992

\begin{tabular}{|c|c|c|c|c|c|c|c|c|c|c|c|c|}
\hline \multirow[b]{2}{*}{ County } & \multicolumn{2}{|c|}{ Asbestosis } & \multicolumn{2}{|c|}{$\begin{array}{c}\text { Coal workers' } \\
\text { pneumoconiosis }\end{array}$} & \multicolumn{2}{|c|}{ Silicosis } & \multicolumn{2}{|c|}{ Byssinosis } & \multicolumn{2}{|c|}{$\begin{array}{r}\text { Unspecified/Other } \\
\text { pneumoconioses }\end{array}$} & \multicolumn{2}{|c|}{$\begin{array}{r}\text { All } \\
\text { pneumoconioses }\end{array}$} \\
\hline & $\#$ & Rate & \# & Rate & $\#$ & Rate & $\#$ & Rate & $\#$ & Rate & $\#$ & Rate \\
\hline Anoka & 1 & 0.61 & - & - & 3 & 2.16 & - & - & 1 & 0.77 & 5 & 3.54 \\
\hline Benton & 1 & 1.61 & - & - & 2 & 3.22 & - & - & - & - & 3 & 4.84 \\
\hline Big Stone & 1 & 11.52 & - & - & 1 & 3.41 & - & - & 1 & 11.52 & 3 & 26.46 \\
\hline Carlton & 1 & 3.23 & - & - & 1 & 1.44 & - & - & - & - & 2 & 4.68 \\
\hline Carver & - & - & - & - & 2 & 3.16 & - & - & - & - & 2 & 3.16 \\
\hline Chippewa & - & - & - & - & 1 & 1.95 & - & - & - & - & 1 & 1.95 \\
\hline Chisago & 1 & 4.18 & - & - & 1 & 1.65 & - & - & - & - & 2 & 5.83 \\
\hline Clay & 1 & 2.48 & - & - & 2 & 4.96 & - & - & - & - & 2 & 4.96 \\
\hline Crow Wing & 1 & 1.97 & - & - & - & - & - & - & - & - & 1 & 1.97 \\
\hline Dakota & 1 & 0.70 & - & - & - & - & - & - & - & - & 1 & 0.70 \\
\hline Douglas & 1 & 1.12 & - & - & 1 & 1.12 & - & - & - & - & 2 & 2.24 \\
\hline Fillmore & 1 & 1.30 & - & - & - & - & - & - & - & - & 1 & 1.30 \\
\hline Freeborn & - & - & - & - & 1 & 4.42 & - & - & - & - & 1 & 4.42 \\
\hline Goodhue & 1 & 0.84 & - & - & - & - & - & - & 1 & 0.84 & 2 & 1.68 \\
\hline Hennepin & 18 & 1.56 & 2 & 0.26 & 9 & 0.85 & - & - & 1 & 0.12 & 29 & 2.66 \\
\hline Hubbard & 1 & 9.83 & - & - & - & - & - & - & - & - & 1 & 9.83 \\
\hline Itasca & 4 & 7.65 & - & - & 1 & 2.20 & - & - & - & - & 4 & 7.65 \\
\hline Lake & 1 & 7.15 & - & - & - & - & - & - & - & - & 1 & 7.15 \\
\hline Le Sueur & 1 & 4.38 & - & - & 2 & 6.04 & - & - & - & - & 3 & 10.41 \\
\hline McLeod & - & - & - & - & 1 & 1.23 & - & - & - & - & 1 & 1.23 \\
\hline Marshall & - & - & - & - & - & - & - & - & 1 & 13.43 & 1 & 13.43 \\
\hline Martin & 1 & 1.22 & - & - & 1 & 1.22 & - & - & - & - & 2 & 2.43 \\
\hline Mille Lacs & - & - & - & - & 1 & 1.76 & - & - & 1 & 1.76 & 2 & 3.52 \\
\hline Morrison & - & - & - & - & 2 & 2.66 & - & - & - & - & 2 & 2.66 \\
\hline Mower & - & - & - & - & - & - & - & - & 1 & 0.81 & 1 & 0.81 \\
\hline Nicollet & - & - & - & - & 1 & 4.70 & - & - & - & - & 1 & 4.70 \\
\hline Nobles & 1 & 4.33 & - & - & 1 & 4.33 & - & - & - & - & 1 & 4.33 \\
\hline Olmsted & 1 & 1.35 & - & - & - & - & - & - & - & - & 1 & 1.35 \\
\hline Pine & 1 & 1.87 & - & - & - & - & - & - & - & - & 1 & 1.87 \\
\hline Polk & 1 & 2.87 & - & - & - & - & - & - & - & - & 1 & 2.87 \\
\hline Ramsey & 14 & 2.71 & - & - & 8 & 1.10 & - & - & 1 & 0.24 & 23 & 4.05 \\
\hline St Louis & 12 & 4.98 & - & - & 2 & 0.37 & - & - & 3 & 0.83 & 17 & 6.19 \\
\hline
\end{tabular}

See footnotes at end of table. 
Table MN-3 (page 2 of 2). Number of deaths and age-adjusted mortality rate (per 1,000,000 population) by condition and county, Minnesota residents age 15 and over, 1983-1992

\begin{tabular}{|c|c|c|c|c|c|c|c|c|c|c|c|c|}
\hline \multirow[b]{2}{*}{ County } & \multicolumn{2}{|c|}{ Asbestosis } & \multicolumn{2}{|c|}{$\begin{array}{c}\text { Coal workers' } \\
\text { pneumoconiosis }\end{array}$} & \multicolumn{2}{|c|}{ Silicosis } & \multicolumn{2}{|c|}{ Byssinosis } & \multicolumn{2}{|c|}{$\begin{array}{r}\text { Unspecified/Other } \\
\text { pneumoconioses }\end{array}$} & \multicolumn{2}{|c|}{$\begin{array}{r}\text { All } \\
\text { pneumoconioses }\end{array}$} \\
\hline & \# & Rate & \# & Rate & $\#$ & Rate & \# & Rate & $\#$ & Rate & \# & Rate \\
\hline Scott & - & - & - & - & 1 & 3.17 & - & - & - & - & 1 & 3.17 \\
\hline Sherburne & 1 & 4.55 & - & - & 1 & 2.12 & - & - & - & - & 2 & 6.68 \\
\hline Sibley & - & - & - & - & 1 & 6.08 & - & - & - & - & 1 & 6.08 \\
\hline Stearns & 2 & 1.65 & - & - & 12 & 8.05 & - & - & 1 & 0.52 & 15 & 10.22 \\
\hline Steele & - & - & - & - & 2 & 4.95 & - & - & - & - & 2 & 4.95 \\
\hline Waseca & - & - & - & - & 1 & 5.74 & - & - & - & - & 1 & 5.74 \\
\hline Washington & 3 & 3.64 & - & - & 2 & 1.53 & - & - & - & - & 5 & 5.17 \\
\hline Wright & 1 & 2.19 & - & - & 2 & 4.37 & 1 & 2.19 & - & - & 4 & 8.74 \\
\hline $\begin{array}{l}\text { ndicates no death } \\
\text { OTE: } \quad \text { Count } \\
\\
\quad \text { becau }\end{array}$ & & n1St & 101101 & Ispra & Ade & on & 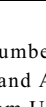 & Iay & C. $\mathrm{S}$ & $\begin{array}{l}1 \text { Idualc } \\
8 \text { and }\end{array}$ & 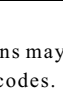 & total \\
\hline
\end{tabular}


Mississippi

\section{Mississippi: Selected statistics from the United States section}

All pneumoconioses mortality, 1988-1992

Total deaths

Crude mortality rate (per $1,000,000$ population)

Age-adjusted mortality rate (per $1,000,000$ population)

Total years of potential life lost to life expectancy

Years of potential life lost to life expectancy (per death)

\begin{tabular}{rr} 
Value & State rank \\
\hline 113 & 24 \\
11.59 & 17 \\
7.10 & 16 \\
1,423 & 25 \\
12.6 & 13
\end{tabular}

Percent**

$\underline{\text { Deaths* }} \quad \underline{\text { distribution }}$

$98 \quad 86.7$

Asbestosis

8

7.1

Silicosis

Byssinosis

Unspecified/Other pneumoconiosis

$6 \quad 5.3$

$0 \quad 0.0$

1

0.9

* Sum of column may exceed total deaths because of deaths with multiple pneumoconioses.

** Percentages may total more than $100 \%$ due to deaths with multiple pneumoconioses.

NOTE: $\quad$ See Appendix A for source description and Appendix B for methods and ICD-8 and ICD-9 codes.

SOURCE: National Center for Health Statistics multiple cause of death data. Population estimates from U.S. Bureau of the Census.

\section{Figure MS-1. All pneumoconioses: Mississippi and U.S. age-adjusted mortality rates, residents age 15 and over, 1968-1992}

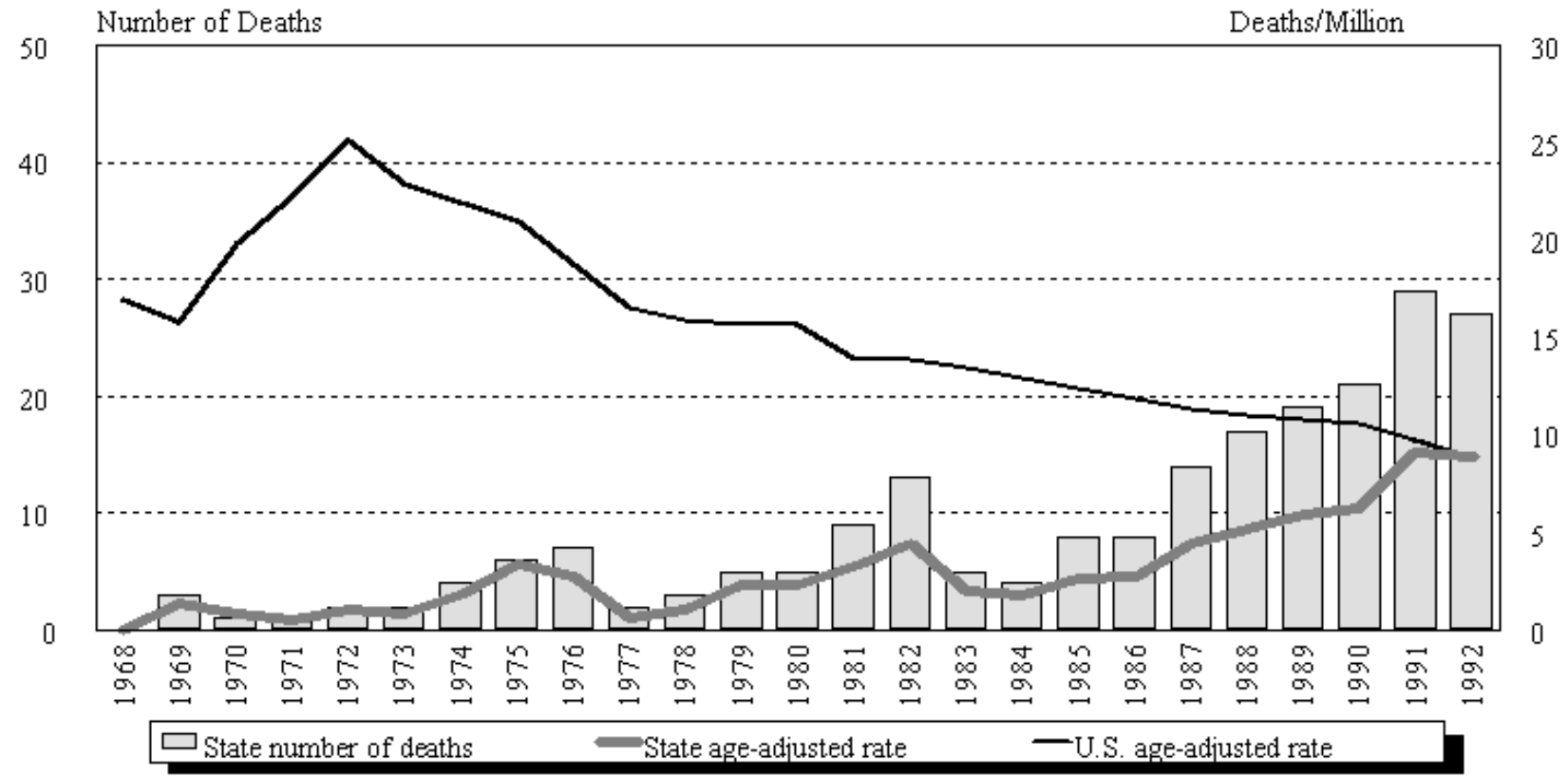

NOTE: $\quad$ See Appendix A for source description and Appendix B for methods and ICD-8 and ICD-9 codes.

SOURCE: $\quad$ National Center for Health Statistics multiple cause of death data. Population estimates from U.S. Bureau of the Census. 
Mississippi

Table MS-1. Number of deaths and age-adjusted mortality rate (per 1,000,000 population) by condition, Mississippi residents age 15 and over, 1968-1992

\begin{tabular}{|c|c|c|c|c|c|c|c|c|c|c|c|c|}
\hline \multirow[b]{2}{*}{ Year } & \multicolumn{2}{|c|}{ Asb estosis } & \multicolumn{2}{|c|}{$\begin{array}{l}\text { Coal workers' } \\
\text { pneumoconiosis }\end{array}$} & \multicolumn{2}{|c|}{ Silicosis } & \multicolumn{2}{|c|}{ Byssinosis* } & \multicolumn{2}{|c|}{$\begin{array}{c}\text { Unspecified/Other } \\
\text { pneumoconioses }\end{array}$} & \multicolumn{2}{|c|}{ All pneumoconioses } \\
\hline & Number & Rate & Number & Rate & Number & Rate & Number & Rate & Number & Rate & Number & Rate \\
\hline 1968 & - & - & - & - & - & - & & & - & - & - & - \\
\hline 1969 & - & - & 2 & 0.98 & 1 & 0.36 & & & - & - & 3 & 1.34 \\
\hline 1970 & - & - & - & - & 1 & 0.83 & & & - & - & 1 & 0.83 \\
\hline 1971 & - & - & - & - & 1 & 0.52 & & & - & - & 1 & 0.52 \\
\hline 1972 & - & - & - & - & - & - & & & 2 & 1.04 & 2 & 1.04 \\
\hline 1973 & - & - & - & - & 2 & 0.82 & & & - & - & 2 & 0.82 \\
\hline 1974 & - & - & - & - & 3 & 1.23 & & & 1 & 0.52 & 4 & 1.75 \\
\hline 1975 & - & - & 2 & 1.03 & 4 & 2.40 & & & - & - & 6 & 3.43 \\
\hline 1976 & 1 & 0.30 & 2 & 0.76 & 1 & 0.38 & & & 3 & 1.27 & 7 & 2.71 \\
\hline 1977 & - & - & 1 & 0.29 & 1 & 0.37 & & & - & - & 2 & 0.67 \\
\hline 1978 & 1 & 0.37 & - & & - & - & & & 2 & 0.73 & 3 & 1.10 \\
\hline 1979 & 2 & 0.77 & 1 & 0.36 & 1 & 0.50 & - & - & 1 & 0.68 & 5 & 2.30 \\
\hline 1980 & 2 & 0.71 & - & - & 3 & 1.67 & - & - & - & - & 5 & 2.38 \\
\hline 1981 & 5 & 1.75 & 2 & 0.72 & 2 & 0.84 & - & - & - & - & 9 & 3.31 \\
\hline 1982 & 10 & 3.62 & 2 & 0.61 & 1 & 0.25 & - & - & - & - & 13 & 4.48 \\
\hline 1983 & 4 & 1.49 & - & - & 1 & 0.48 & - & - & - & - & 5 & 1.97 \\
\hline 1984 & 1 & 0.35 & 1 & 0.24 & 1 & 0.69 & - & - & 1 & 0.54 & 4 & 1.82 \\
\hline 1985 & 5 & 1.52 & 1 & 0.13 & 2 & 1.04 & - & - & - & - & 8 & 2.69 \\
\hline 1986 & 8 & 2.79 & - & & - & - & - & - & - & - & 8 & 2.79 \\
\hline 1987 & 12 & 3.98 & 1 & 0.22 & 1 & 0.22 & - & - & - & - & 14 & 4.42 \\
\hline 1988 & 15 & 4.73 & 2 & 0.44 & - & - & - & - & - & - & 17 & 5.16 \\
\hline 1989 & 17 & 5.36 & - & - & 1 & 0.22 & - & - & 1 & 0.36 & 19 & 5.94 \\
\hline 1990 & 16 & 4.47 & 2 & 0.57 & 3 & 1.22 & - & - & - & - & 21 & 6.26 \\
\hline 1991 & 25 & 8.09 & 3 & 0.77 & 1 & 0.21 & - & - & - & - & 29 & 9.07 \\
\hline 1992 & 25 & 8.30 & 1 & 0.11 & 1 & 0.51 & - & - & - & - & 27 & 8.92 \\
\hline
\end{tabular}

- indicates no deaths listed. ${ }^{*}$ no specific code for byssinosis in ICD-8 (1968-1978)

NOTE: Rates based on small numbers may be unstable. Sum of individual conditions may exceed total because of deaths with multiple pneumoconioses. See Appendix A for source description and Appendix B for methods and ICD-8 and ICD-9 codes.

SOURCE: National Center for Health Statistics multiple cause of death data. Population estimates from U.S. Bureau of the Census. 


\section{Figure MS-2. All pneumoconioses: Distribution of deaths by sex and race, Mississippi residents age 15 and over, 1968-1992}
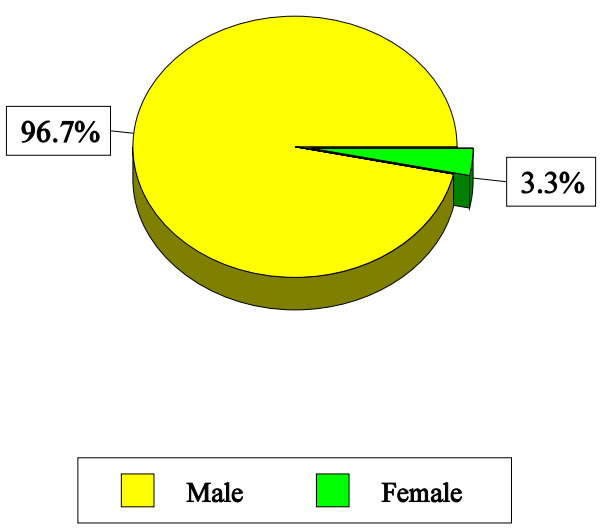
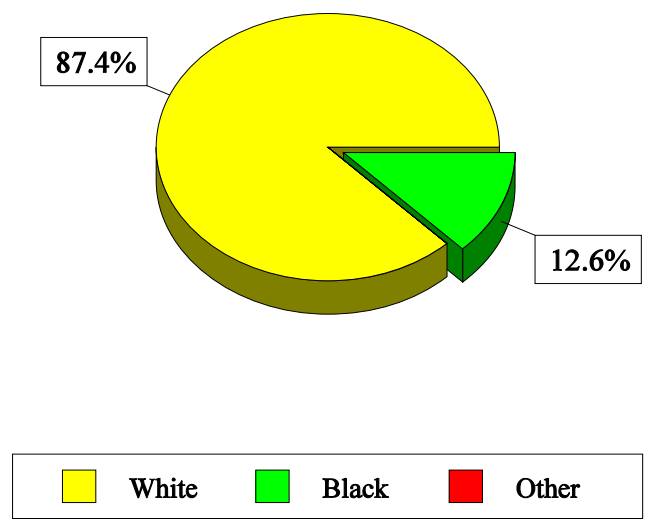

NOTE: $\quad$ See Appendix A for source description and Appendix B for methods and ICD-8 and ICD-9 codes.

SOURCE: National Center for Health Statistics multiple cause of death data.

Table MS-2. All pneumoconioses: Distribution of deaths by sex, race, and age, Mississippi residents age 15 and over, 1968-1992

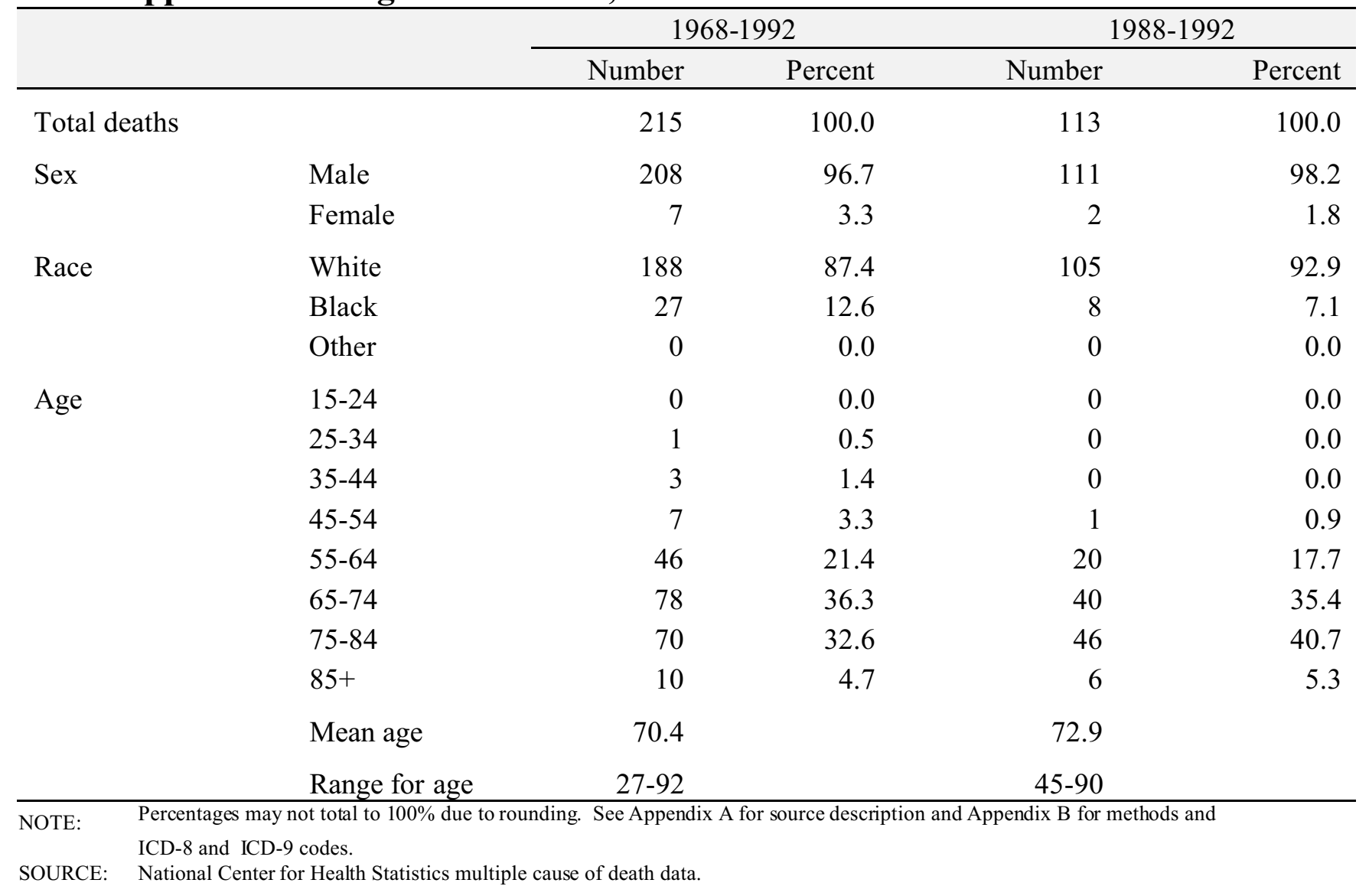


Figure MS-3. Pneumoconiosis deaths by condition, Mississippi residents age 15 and over, 1968-1992

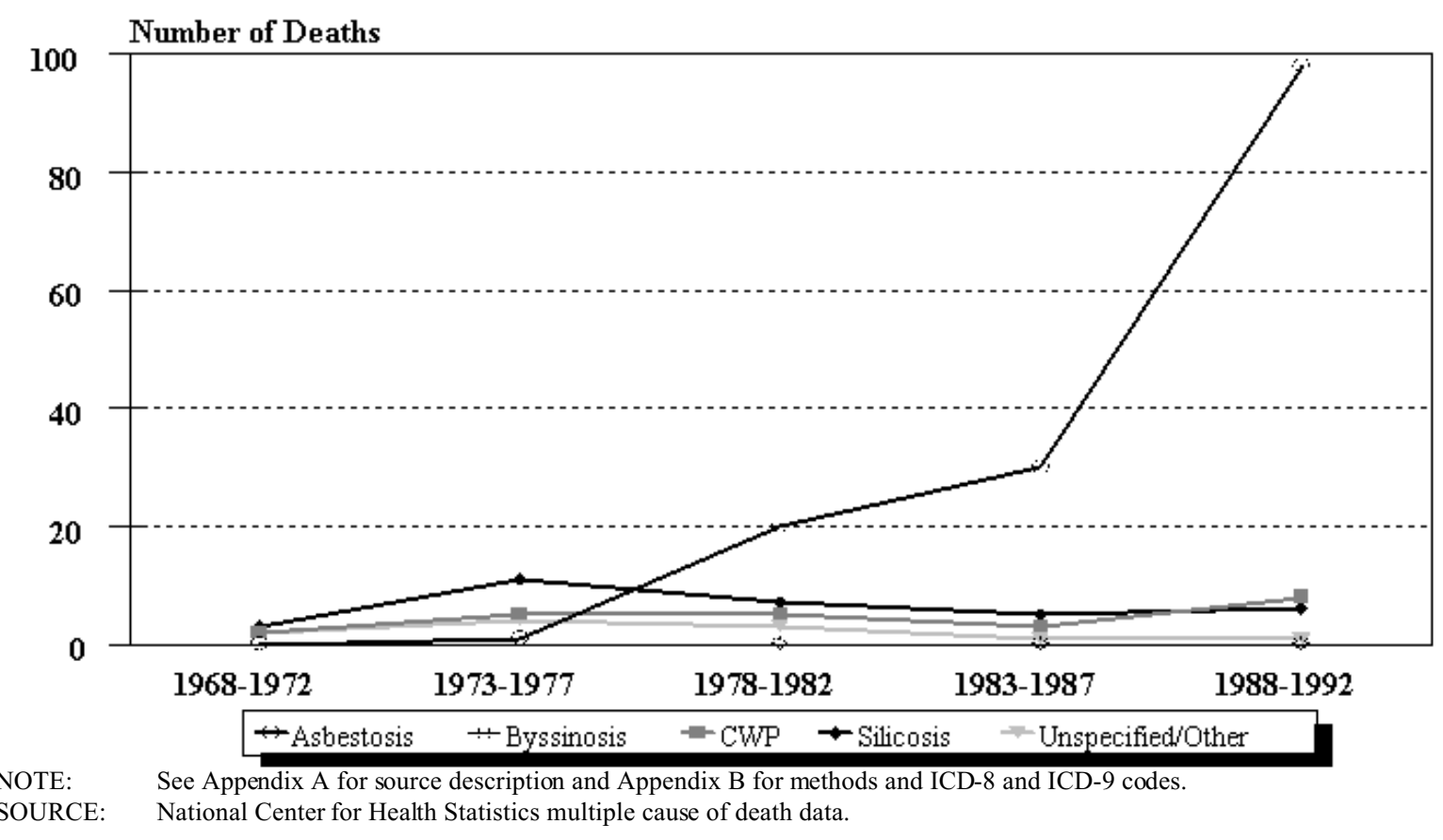

Figure MS-4. Mississippi and U.S. age-adjusted mortality rates (per 1,000,000 population) by condition, residents age 15 and over, 1983-1992

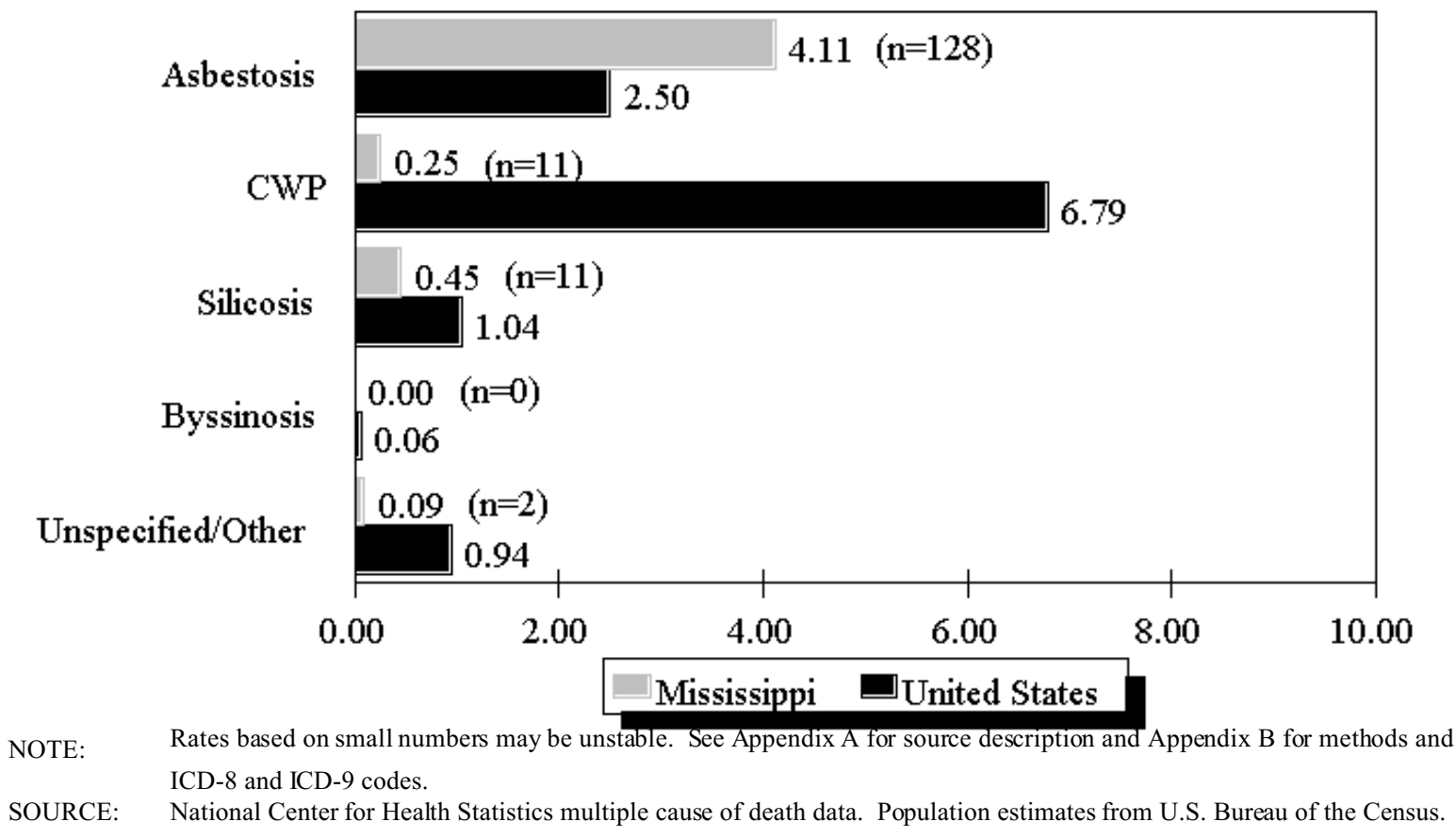


Table MS-3 (page 1 of 2). Number of deaths and age-adjusted mortality rate (per $1,000,000$ population) by condition and county, Mississippi residents age 15 and over, 1983-1992

\begin{tabular}{|c|c|c|c|c|c|c|c|c|c|c|c|c|}
\hline \multirow[b]{2}{*}{ County } & \multicolumn{2}{|c|}{ Asbestosis } & \multicolumn{2}{|c|}{$\begin{array}{r}\text { Coal workers' } \\
\text { pneumoconiosis }\end{array}$} & \multicolumn{2}{|c|}{ Silicosis } & \multicolumn{2}{|c|}{ Byssinosis } & \multicolumn{2}{|c|}{$\begin{array}{r}\text { Unspecified/Other } \\
\text { pneumoconioses }\end{array}$} & \multicolumn{2}{|c|}{$\begin{array}{r}\text { All } \\
\text { pneumoconioses }\end{array}$} \\
\hline & \# & Rate & \# & Rate & $\#$ & Rate & \# & Rate & \# & Rate & \# & Rate \\
\hline Adams & - & - & 1 & 1.30 & - & - & - & - & - & - & 1 & 1.30 \\
\hline Attala & 2 & 9.09 & 1 & 4.55 & 1 & 4.55 & - & - & - & - & 4 & 18.19 \\
\hline Choctaw & 1 & 11.04 & - & - & - & - & - & - & - & - & 1 & 11.04 \\
\hline Coahoma & - & - & 1 & 1.27 & - & - & - & - & - & - & 1 & 1.27 \\
\hline Copiah & 1 & 3.93 & - & - & - & - & - & - & - & - & 1 & 3.93 \\
\hline Covington & 1 & 6.48 & - & - & 1 & 9.54 & - & - & - & - & 2 & 16.02 \\
\hline De Soto & 1 & 2.07 & - & - & - & - & - & - & - & - & 1 & 2.07 \\
\hline Forrest & 1 & 0.78 & 1 & 0.78 & - & - & - & - & - & - & 2 & 1.56 \\
\hline George & 8 & 41.86 & - & - & - & - & - & - & - & - & 8 & 41.86 \\
\hline Greene & 3 & 28.02 & - & - & - & - & - & - & - & - & 3 & 28.02 \\
\hline Hancock & 3 & 5.67 & - & - & - & - & - & - & - & - & 3 & 5.67 \\
\hline Harrison & 17 & 10.24 & 1 & 0.69 & - & - & - & - & - & - & 18 & 10.93 \\
\hline Hinds & 1 & 0.47 & 1 & 0.23 & 1 & 0.23 & - & - & - & - & 3 & 0.92 \\
\hline Humphreys & 1 & 3.48 & - & - & - & - & - & - & - & - & 1 & 3.48 \\
\hline Itawamba & 2 & 9.18 & - & - & - & - & - & - & - & - & 2 & 9.18 \\
\hline Jackson & 54 & 46.36 & 1 & 0.74 & 1 & 1.04 & - & - & 1 & 1.04 & 57 & 49.17 \\
\hline Jasper & 1 & 5.94 & - & - & - & - & - & - & - & - & 1 & 5.94 \\
\hline Jones & 5 & 7.73 & - & - & - & - & - & - & - & - & 5 & 7.73 \\
\hline Lauderdale & 3 & 4.11 & - & - & - & - & - & - & - & - & 3 & 4.11 \\
\hline Lawrence & - & - & - & - & 1 & 3.76 & - & - & - & - & 1 & 3.76 \\
\hline Lee & 1 & 0.86 & 1 & 0.86 & - & - & - & - & - & - & 2 & 1.72 \\
\hline Lowndes & - & - & 3 & 3.21 & 2 & 4.79 & - & - & - & - & 5 & 8.00 \\
\hline Marion & - & - & - & - & 1 & 3.74 & - & - & - & - & 1 & 3.74 \\
\hline Marshall & - & - & - & - & - & - & - & - & 1 & 4.44 & 1 & 4.44 \\
\hline Montgomery & 1 & 7.12 & - & - & - & - & - & - & - & - & 1 & 7.12 \\
\hline Neshoba & 1 & 6.32 & - & - & 1 & 1.72 & - & - & - & - & 2 & 8.05 \\
\hline Newton & 1 & 4.58 & - & - & - & - & - & - & - & - & 1 & 4.58 \\
\hline Panola & 2 & 5.13 & - & - & - & - & - & - & - & - & 2 & 5.13 \\
\hline Perry & 1 & 5.30 & - & - & - & - & - & - & - & - & 1 & 5.30 \\
\hline Pike & 3 & 6.44 & - & - & 1 & 2.64 & - & - & - & - & 4 & 9.07 \\
\hline Prentiss & 1 & 4.15 & - & - & - & - & - & - & - & - & 1 & 4.15 \\
\hline
\end{tabular}

See footnotes at end of table. 
Table MS-3 (page 2 of 2). Number of deaths and age-adjusted mortality rate (per $1,000,000$ population) by condition and county, Mississippi residents age 15 and over, 1983-1992

\begin{tabular}{|c|c|c|c|c|c|c|c|c|c|c|c|c|}
\hline \multirow[b]{2}{*}{ County } & \multicolumn{2}{|c|}{ Asbestosis } & \multicolumn{2}{|c|}{$\begin{array}{r}\text { Coal workers' } \\
\text { pneumoconiosis }\end{array}$} & \multicolumn{2}{|c|}{ Silicosis } & \multicolumn{2}{|c|}{ Byssinosis } & \multicolumn{2}{|c|}{$\begin{array}{r}\text { Unspecified/Other } \\
\text { pneumoconioses }\end{array}$} & \multicolumn{2}{|c|}{$\begin{array}{r}\text { All } \\
\text { pneumoconioses }\end{array}$} \\
\hline & \# & Rate & \# & Rate & \# & Rate & \# & Rate & \# & Rate & $\#$ & Rate \\
\hline Rankin & 3 & 3.93 & - & - & - & - & - & - & - & - & 3 & 3.93 \\
\hline Tishomingo & 3 & 14.42 & - & - & - & - & - & - & - & - & 3 & 14.42 \\
\hline Union & - & - & - & - & 1 & 4.31 & - & - & - & - & 1 & 4.31 \\
\hline Wayne & 4 & 17.27 & - & - & - & - & - & - & - & - & 4 & 17.27 \\
\hline Webster & 1 & 8.52 & - & - & - & - & - & - & - & - & 1 & 8.52 \\
\hline Yazoo & 1 & 3.85 & - & - & - & - & - & - & - & - & 1 & 3.85 \\
\hline
\end{tabular}

- indicates no deaths listed.

NOTE: Counties with no deaths for all listed conditions are not displayed. Rates based on small numbers may be unstable. Sum of individual conditions may exceed total because of deaths with multiple pneumoconioses. See Appendix A for source description and Appendix B for methods and ICD-8 and ICD-9 codes.

SOURCE: National Center for Health Statistics multiple cause of death data. Population estimates from U.S. Bureau of the Census. 


\section{Missouri: Selected statistics from the United States section}

All pneumoconioses mortality, 1988-1992

Total deaths

Crude mortality rate (per $1,000,000$ population)

Age-adjusted mortality rate (per 1,000,000 population)

Total years of potential life lost to life expectancy

Years of potential life lost to life expectancy (per death)

\begin{tabular}{rr} 
Value & State rank \\
\hline 127 & 21 \\
6.34 & 34 \\
3.36 & 38 \\
1,560 & 22 \\
12.3 & 21
\end{tabular}

Percent**

Deaths by type of pneumoconiosis, 1988-1992

Asbestosis

$\underline{\text { Deaths* }}$

distribution

Coal workers' pneumoconiosis

52

40.9

36

28.3

Silicosis

26

20.5

0

$\mathbf{0 . 0}$

13

\section{Unspecified/Other pneumoconiosis}

* Sum of column may exceed total deaths because of deaths with multiple pneumoconioses.

** Percentages may total more than $100 \%$ due to deaths with multiple pneumoconioses.

NOTE: $\quad$ See Appendix A for source description and Appendix B for methods and ICD-8 and ICD-9 codes.

SOURCE: National Center for Health Statistics multiple cause of death data. Population estimates from U.S. Bureau of the Census.

\section{Figure MO-1. All pneumoconioses: Missouri and U.S. age-adjusted mortality rates, residents age 15 and over, 1968-1992}

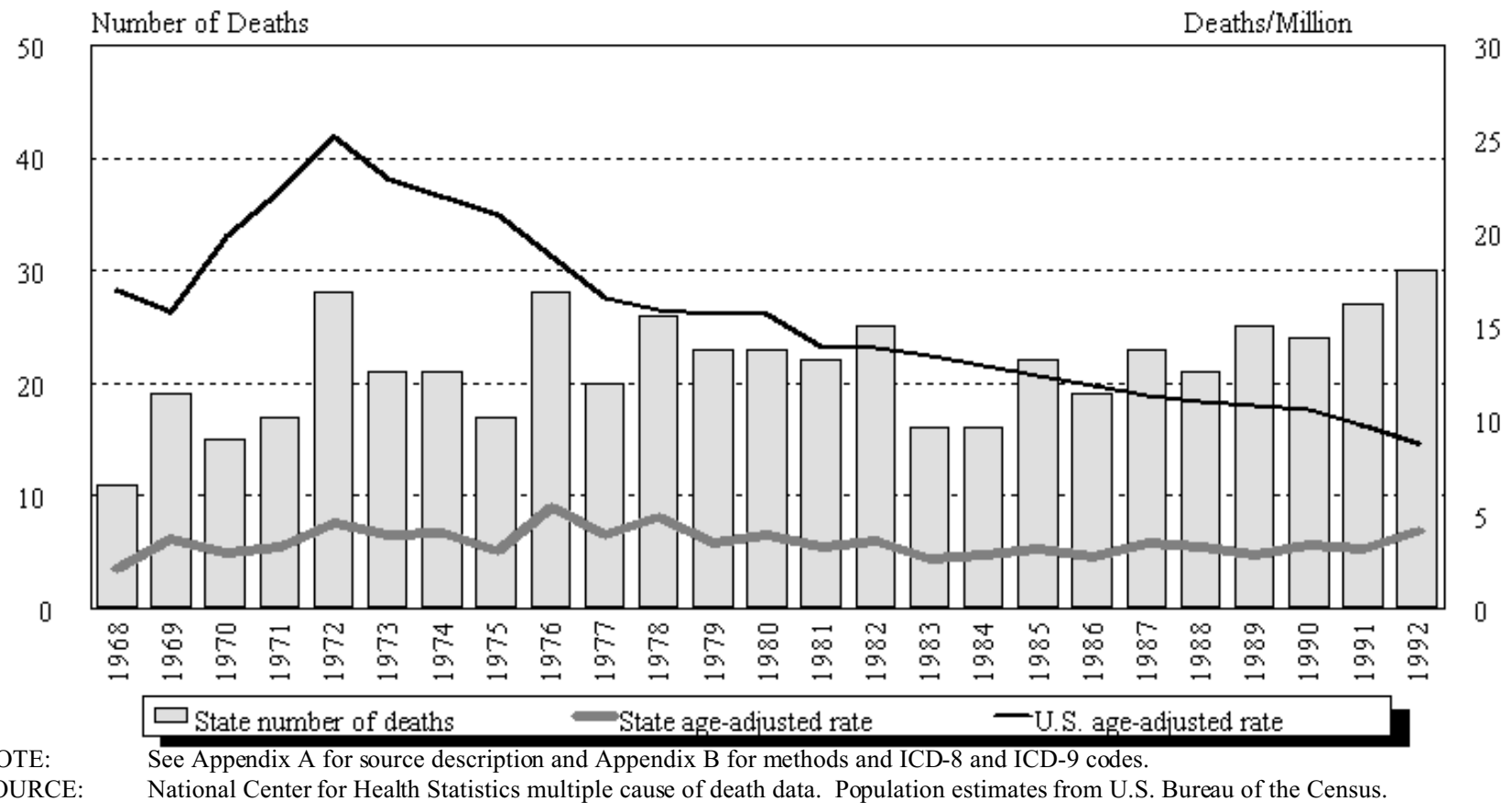


Missouri

\begin{tabular}{|c|c|c|c|c|c|c|c|c|c|c|c|c|}
\hline \multirow[b]{2}{*}{ Year } & \multicolumn{2}{|c|}{ Asbestosis } & \multicolumn{2}{|c|}{$\begin{array}{l}\text { Coal workers' } \\
\text { pneumoconiosis }\end{array}$} & \multicolumn{2}{|c|}{ Silicosis } & \multicolumn{2}{|c|}{ Byssinosis* } & \multicolumn{2}{|c|}{$\begin{array}{c}\text { Unspecified/Other } \\
\text { pneumoconioses }\end{array}$} & \multicolumn{2}{|c|}{ All pneumoconioses } \\
\hline & Number & Rate & Number & Rate & Number & Rate & Number & Rate & Number & Rate & Number & Rate \\
\hline 1968 & - & - & 2 & 0.38 & 7 & 1.40 & & & 2 & 0.32 & 11 & 2.09 \\
\hline 1969 & 1 & 0.19 & 1 & 0.30 & 14 & 2.63 & & & 3 & 0.61 & 19 & 3.74 \\
\hline 1970 & - & - & 3 & 0.41 & 5 & 1.07 & & & 7 & 1.46 & 15 & 2.94 \\
\hline 1971 & 1 & 0.23 & 2 & 0.21 & 8 & 1.69 & & & 6 & 1.20 & 17 & 3.33 \\
\hline 1972 & 2 & 0.46 & 2 & 0.25 & 16 & 2.49 & & & 8 & 1.33 & 28 & 4.53 \\
\hline 1973 & - & - & 3 & 0.54 & 12 & 2.30 & & & 6 & 1.04 & 21 & 3.88 \\
\hline 1974 & 3 & 0.71 & 5 & 0.79 & 10 & 2.01 & & & 3 & 0.55 & 21 & 4.06 \\
\hline 1975 & 4 & 0.89 & 2 & 0.30 & 7 & 1.26 & & & 4 & 0.65 & 17 & 3.10 \\
\hline 1976 & 5 & 0.93 & 1 & 0.17 & 13 & 2.28 & & & 9 & 1.98 & 28 & 5.36 \\
\hline 1977 & 2 & 0.46 & 3 & 0.52 & 9 & 1.90 & & & 6 & 1.01 & 20 & 3.89 \\
\hline 1978 & 5 & 0.91 & 1 & 0.17 & 11 & 2.11 & & & 10 & 1.80 & 26 & 4.82 \\
\hline 1979 & 5 & 1.11 & 9 & 1.08 & 6 & 0.80 & - & - & 3 & 0.54 & 23 & 3.53 \\
\hline 1980 & 2 & 0.39 & 5 & 0.79 & 9 & 1.57 & - & - & 7 & 1.16 & 23 & 3.91 \\
\hline 1981 & 5 & 0.78 & 9 & 1.28 & 6 & 0.96 & - & - & 2 & 0.28 & 22 & 3.30 \\
\hline 1982 & 8 & 1.34 & 10 & 1.34 & 5 & 0.72 & - & - & 2 & 0.23 & 25 & 3.62 \\
\hline 1983 & 6 & 1.11 & 5 & 0.77 & 2 & 0.34 & - & - & 3 & 0.44 & 16 & 2.66 \\
\hline 1984 & 3 & 0.57 & 2 & 0.27 & 9 & 1.73 & - & - & 2 & 0.27 & 16 & 2.85 \\
\hline 1985 & 9 & 1.41 & 5 & 0.66 & 7 & 0.93 & - & - & 1 & 0.17 & 22 & 3.16 \\
\hline 1986 & 12 & 1.97 & 4 & 0.42 & 2 & 0.33 & - & - & 1 & 0.05 & 19 & 2.77 \\
\hline 1987 & 7 & 1.24 & 7 & 0.85 & 7 & 1.24 & - & - & 2 & 0.15 & 23 & 3.48 \\
\hline 1988 & 11 & 1.87 & 8 & 1.03 & 2 & 0.33 & - & - & - & - & 21 & 3.23 \\
\hline 1989 & 7 & 0.71 & 7 & 0.60 & 8 & 0.99 & - & - & 3 & 0.56 & 25 & 2.86 \\
\hline 1990 & 9 & 1.27 & 4 & 0.59 & 5 & 0.82 & - & - & 6 & 0.75 & 24 & 3.43 \\
\hline 1991 & 11 & 1.42 & 6 & 0.53 & 7 & 0.89 & - & - & 3 & 0.30 & 27 & 3.14 \\
\hline 1992 & 14 & 1.88 & 11 & 1.21 & 4 & 0.68 & - & - & 1 & 0.35 & 30 & 4.13 \\
\hline
\end{tabular}

- indicates no deaths listed. $\quad *$ no sp ecific code for byssinosis in ICD-8 (1968-1978).

NOTE: Rates based on small numbers may be unstable. Sum of individual conditions may exceed total because of deaths with multiple pneumoconioses. See Appendix A for source description and Appendix B for methods and ICD-8 and ICD-9 codes.

SOURCE: National Center for Health Statistics multiple cause of death data. Population estimates from U.S. Bureau of the Census. 


\section{Figure MO-2. All pneumoconioses: Distribution of deaths by sex and race, Missouri residents age 15 and over, 1968-1992}

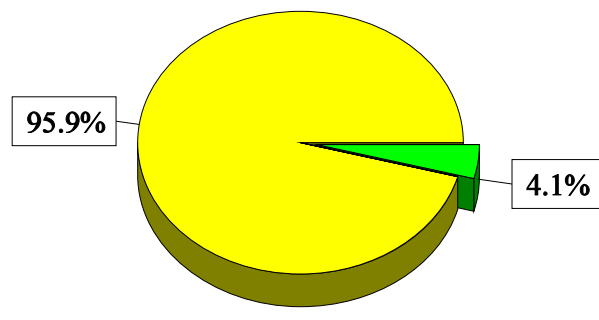

$\square$ Male $\square$ Female

\section{$87.4 \%$}

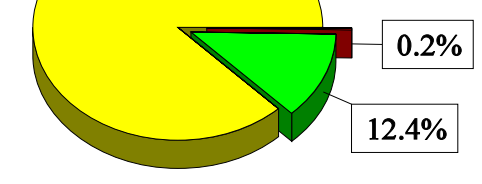

NOTE:

See Appendix A for source description and Appendix B for methods and ICD-8 and ICD-9 codes.

SOURCE: National Center for Health Statistics multiple cause of death data.

Table MO-2. All pneumoconioses: Distribution of deaths by sex, race, and age, Missouri residents age 15 and over, 1968-1992

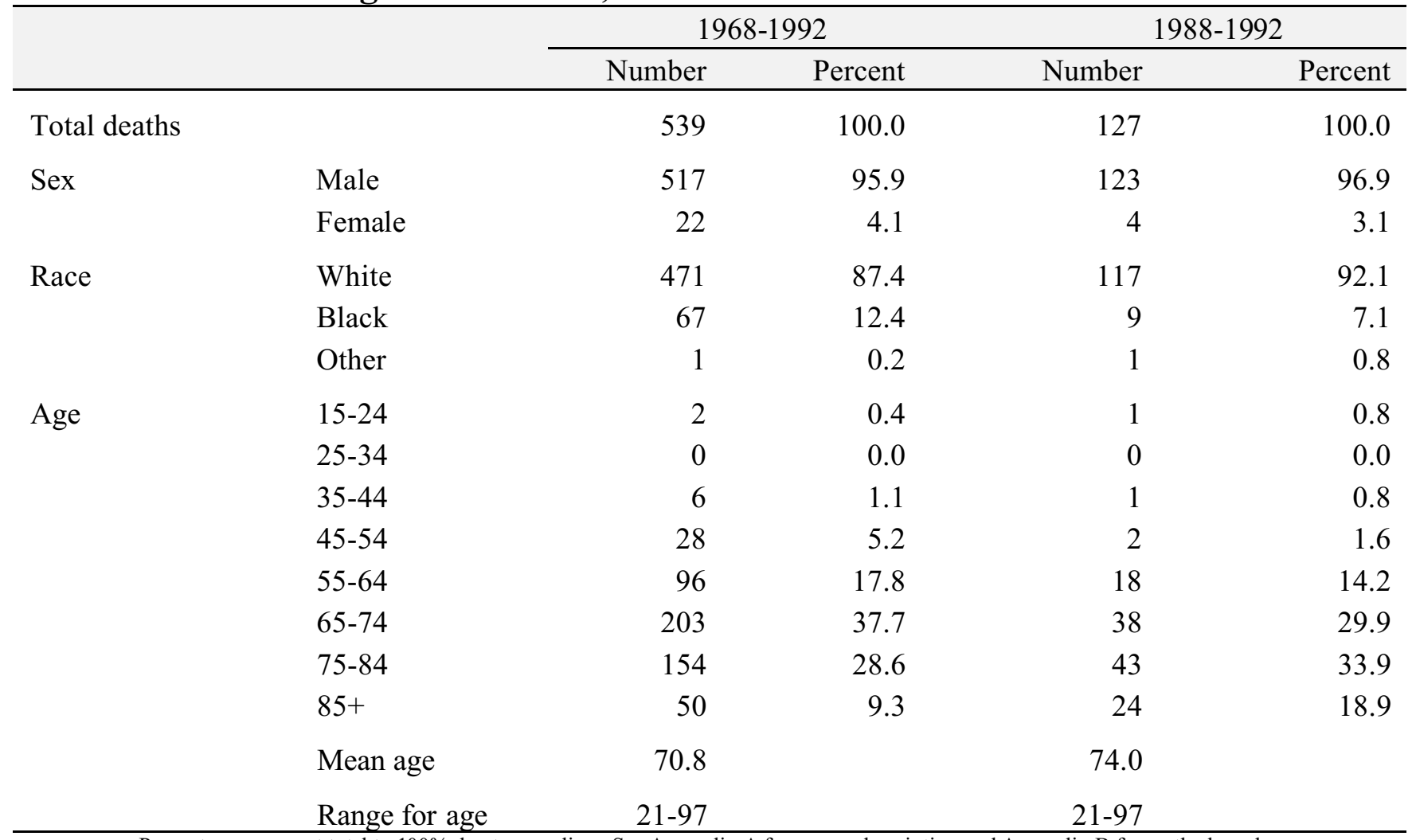

NOTE: $\quad$ Percentages may not total to $100 \%$ due to rounding. See Appendix A for source description and Appendix B for methods and ICD-8 and ICD-9 codes.

SOURCE: National Center for Health Statistics multiple cause of death data. 
Figure MO-3. Pneumoconiosis deaths by condition, Missouri residents age 15 and over, 1968-1992

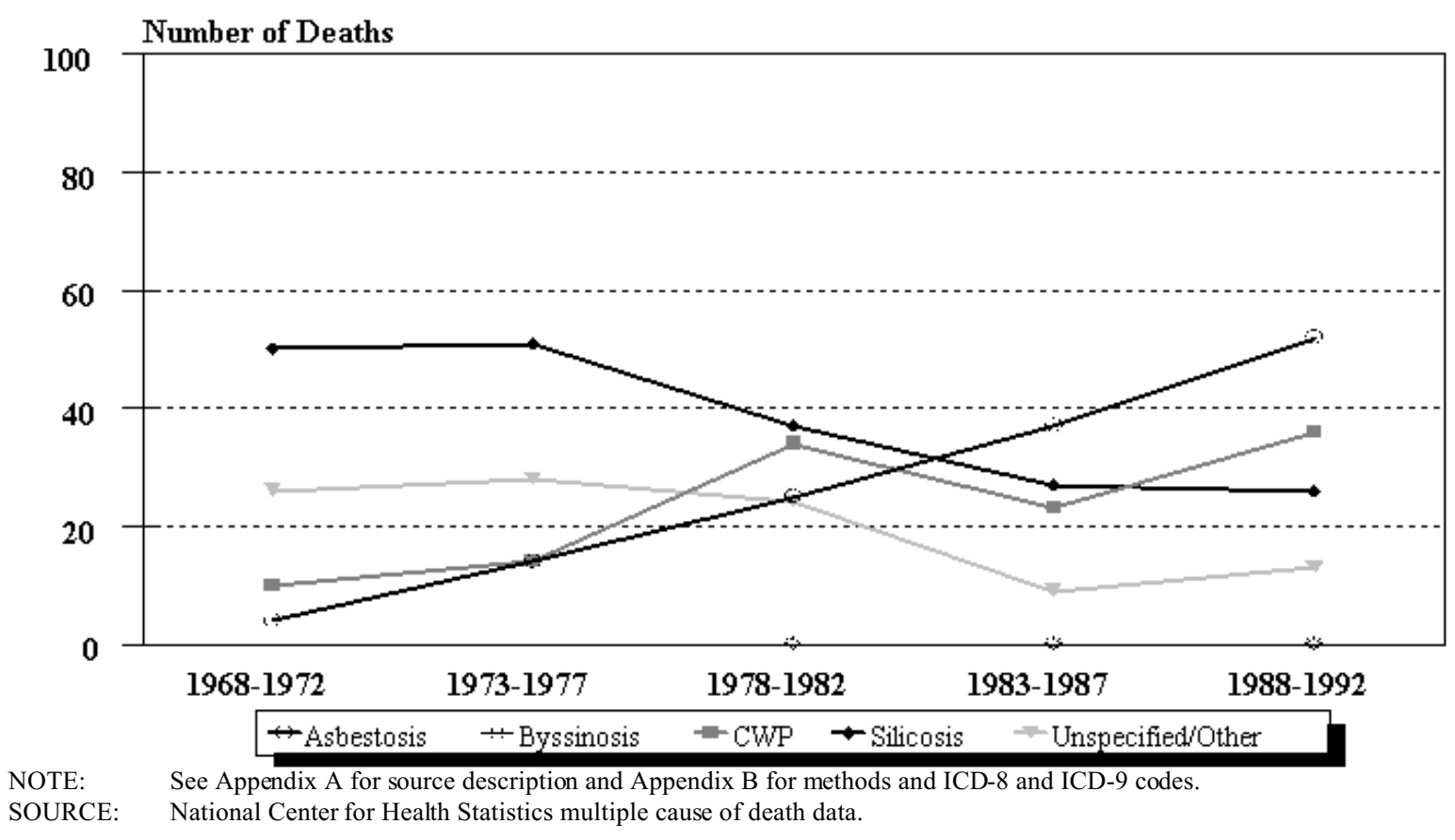

Figure MO-4. Missouri and U.S. age-adjusted mortality rates (per 1,000,000 population) by condition, residents age 15 and over, 1983-1992

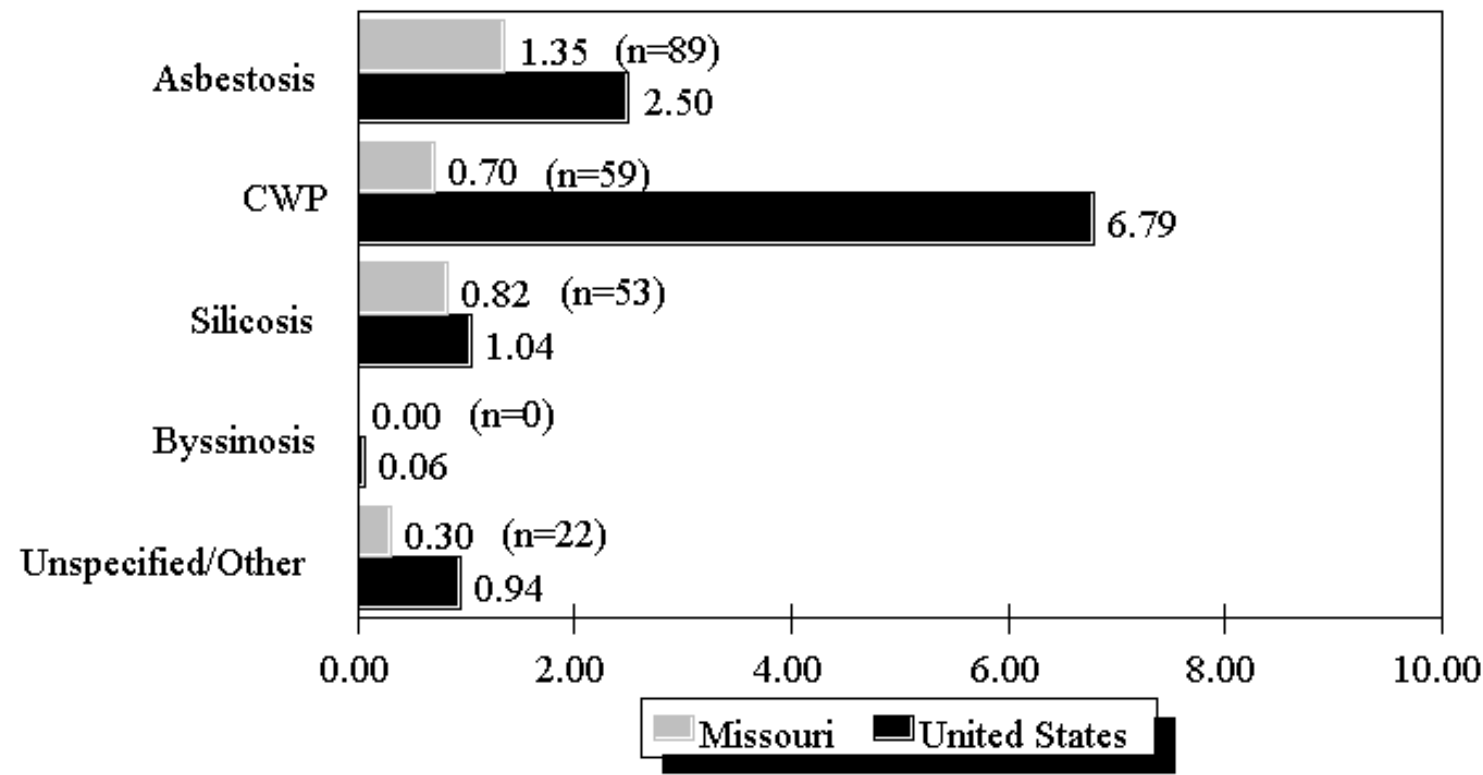

NOTE: $\quad$ Rates based on small numbers may be unstable. See Appendix A for source description and Appendix B for methods and ICD-8 and ICD-9 codes.

SOURCE: National Center for Health Statistics multiple cause of death data. Population estimates from U.S. Bureau of the Census. 
Table MO-3 (page 1 of 2). Number of deaths and age-adjusted mortality rate (per 1,000,000 population) by condition and county, Missouri residents age 15 and over, 1983-1992

\begin{tabular}{|c|c|c|c|c|c|c|c|c|c|c|c|c|}
\hline \multirow[b]{2}{*}{ County } & \multicolumn{2}{|c|}{ Asbestosis } & \multicolumn{2}{|c|}{$\begin{array}{r}\text { Coal workers' } \\
\text { pneumoconiosis }\end{array}$} & \multicolumn{2}{|c|}{ Silicosis } & \multicolumn{2}{|c|}{ Byssinosis } & \multicolumn{2}{|c|}{$\begin{array}{r}\text { Unspecified/Other } \\
\text { pneumoconioses }\end{array}$} & \multicolumn{2}{|c|}{$\begin{array}{r}\text { All } \\
\text { pneumoconioses }\end{array}$} \\
\hline & \# & Rate & \# & Rate & \# & Rate & \# & Rate & \# & Rate & \# & Rate \\
\hline Adair & - & - & 2 & 3.46 & - & - & - & - & - & - & 2 & 3.46 \\
\hline Andrew & 1 & 6.73 & - & - & - & - & - & - & - & - & 1 & 6.73 \\
\hline Audrain & 1 & 3.45 & - & - & 6 & 16.48 & - & - & - & - & 7 & 19.93 \\
\hline Barton & 1 & 7.38 & - & - & - & - & - & - & - & - & 1 & 7.38 \\
\hline Bates & - & - & 3 & 5.56 & - & - & - & - & - & - & 3 & 5.56 \\
\hline Benton & 1 & 2.24 & - & - & - & - & - & - & - & - & 1 & 2.24 \\
\hline Boone & 1 & 1.49 & - & - & - & - & - & - & - & - & 1 & 1.49 \\
\hline Callaway & - & - & - & - & 1 & 3.46 & - & - & - & - & 1 & 3.46 \\
\hline $\begin{array}{l}\text { Cape } \\
\text { Girardeau }\end{array}$ & 1 & 1.78 & - & - & - & - & - & - & - & - & 1 & 1.78 \\
\hline Cass & 2 & 2.98 & - & - & - & - & - & - & - & - & 2 & 2.98 \\
\hline Christian & - & - & 1 & 3.95 & 1 & 1.85 & - & - & - & - & 2 & 5.80 \\
\hline Clark & - & - & 1 & 11.83 & - & - & - & - & - & - & 1 & 11.83 \\
\hline Clay & 5 & 3.00 & - & - & - & - & - & - & - & - & 5 & 3.00 \\
\hline Crawford & 2 & 6.42 & - & - & 1 & 4.54 & - & - & - & - & 3 & 10.97 \\
\hline Douglas & 1 & 7.19 & - & - & 1 & 2.91 & - & - & - & - & 2 & 10.10 \\
\hline Dunklin & 1 & 4.53 & - & - & - & - & - & - & - & - & 1 & 4.53 \\
\hline Franklin & 2 & 2.05 & - & - & 1 & 1.39 & - & - & - & - & 3 & 3.44 \\
\hline Gasconade & 1 & 5.56 & - & - & - & - & - & - & - & - & 1 & 5.56 \\
\hline Greene & 2 & 1.07 & 1 & 0.54 & 1 & 0.23 & - & - & 1 & 0.63 & 5 & 2.46 \\
\hline Harrison & - & - & 1 & 2.51 & - & - & - & - & - & - & 1 & 2.51 \\
\hline Henry & 1 & 4.03 & 4 & 13.44 & - & - & - & - & - & - & 5 & 17.47 \\
\hline Hickory & - & - & - & - & - & - & - & - & 1 & 7.03 & 1 & 7.03 \\
\hline Jackson & 10 & 1.22 & 3 & 0.47 & 5 & 0.90 & - & - & 4 & 0.49 & 22 & 3.08 \\
\hline Jasper & 2 & 1.50 & 1 & 0.43 & 4 & 2.37 & - & - & - & - & 7 & 4.30 \\
\hline Jefferson & 4 & 2.71 & 1 & 0.51 & 1 & 0.84 & - & - & - & - & 6 & 4.06 \\
\hline Johnson & 1 & 3.62 & - & - & - & - & - & - & - & - & 1 & 3.62 \\
\hline Lafayette & - & - & 5 & 5.30 & - & - & - & - & - & - & 5 & 5.30 \\
\hline Lincoln & 1 & 4.14 & - & - & - & - & - & - & 1 & 1.84 & 2 & 5.98 \\
\hline Linn & 1 & 5.25 & 1 & 5.25 & - & - & - & - & - & - & 2 & 10.49 \\
\hline Livingston & 1 & 1.91 & - & - & - & - & - & - & - & - & 1 & 1.91 \\
\hline
\end{tabular}

See footnotes at end of table. 
Table MO-3 (page 2 of 2). Number of deaths and age-adjusted mortality rate (per 1,000,000 population) by condition and county, Missouri residents age 15 and over, 1983-1992

\begin{tabular}{|c|c|c|c|c|c|c|c|c|c|c|c|c|}
\hline \multirow[b]{2}{*}{ County } & \multicolumn{2}{|c|}{ Asbestosis } & \multicolumn{2}{|c|}{$\begin{array}{r}\text { Coal workers' } \\
\text { pneumoconiosis }\end{array}$} & \multicolumn{2}{|c|}{ Silicosis } & \multicolumn{2}{|c|}{ Byssinosis } & \multicolumn{2}{|c|}{$\begin{array}{r}\text { Unspecified/Other } \\
\text { pneumoconioses }\end{array}$} & \multicolumn{2}{|c|}{$\begin{array}{r}\text { All } \\
\text { pneumoconioses }\end{array}$} \\
\hline & $\#$ & Rate & \# & Rate & \# & Rate & \# & Rate & \# & Rate & \# & Rate \\
\hline McDonald & - & - & 1 & 2.63 & - & - & - & - & - & - & 1 & 2.63 \\
\hline Macon & - & - & 8 & 16.80 & - & - & - & - & 3 & 8.41 & 11 & 25.21 \\
\hline Marion & 1 & 1.10 & 1 & 1.10 & - & - & - & - & 1 & 3.33 & 3 & 5.52 \\
\hline Miller & - & - & - & - & - & - & - & - & 1 & 1.81 & 1 & 1.81 \\
\hline Monroe & - & - & - & - & 2 & 12.80 & - & - & - & - & 2 & 12.80 \\
\hline Morgan & 1 & 4.25 & - & - & - & - & - & - & - & - & 1 & 4.25 \\
\hline Newton & - & - & - & - & 1 & 1.00 & - & - & 1 & 1.00 & 2 & 1.99 \\
\hline Nodaway & 1 & 1.65 & - & - & - & - & - & - & - & - & 1 & 1.65 \\
\hline Oregon & - & - & 2 & 18.30 & - & - & - & - & - & - & 2 & 18.30 \\
\hline Pettis & - & - & - & - & 1 & 2.56 & - & - & - & - & 1 & 2.56 \\
\hline Phelps & 1 & 3.00 & - & - & - & - & - & - & 1 & 1.33 & 2 & 4.33 \\
\hline Putnam & - & - & 2 & 17.75 & - & - & - & - & - & - & 2 & 17.75 \\
\hline Randolph & - & - & 3 & 6.65 & - & - & - & - & - & - & 3 & 6.65 \\
\hline Ray & - & - & 3 & 8.81 & - & - & - & - & 5 & 18.25 & 8 & 27.06 \\
\hline St Charles & 3 & 2.43 & - & - & 1 & 0.81 & - & - & 1 & 0.52 & 5 & 3.77 \\
\hline St Clair & - & - & 1 & 7.97 & - & - & - & - & - & - & 1 & 7.97 \\
\hline Ste Genevieve & 1 & 2.82 & - & - & - & - & - & - & - & - & 1 & 2.82 \\
\hline St Francois & - & - & - & - & - & - & - & - & 1 & 1.90 & 1 & 1.90 \\
\hline St Louis & 26 & 2.28 & 4 & 0.31 & 8 & 0.72 & - & - & 1 & 0.10 & 39 & 3.41 \\
\hline Scott & - & - & 2 & 5.24 & - & - & - & - & - & - & 2 & 5.24 \\
\hline Sullivan & - & - & 1 & 11.02 & - & - & - & - & - & - & 1 & 11.02 \\
\hline Taney & - & - & 2 & 3.94 & - & - & - & - & - & - & 2 & 3.94 \\
\hline Vernon & - & - & - & - & 1 & 1.77 & - & - & - & - & 1 & 1.77 \\
\hline Warren & 1 & 5.29 & - & - & - & - & - & - & - & - & 1 & 5.29 \\
\hline Washington & - & - & 1 & 2.89 & - & - & - & - & - & - & 1 & 2.89 \\
\hline Wayne & 1 & 2.80 & 1 & 2.80 & - & - & - & - & - & - & 2 & 5.60 \\
\hline \multicolumn{13}{|c|}{ Independent city: } \\
\hline St Louis & 10 & 1.10 & 3 & 0.54 & 17 & 3.44 & - & - & - & - & 30 & 5.08 \\
\hline
\end{tabular}




\section{Montana: Selected statistics from the United States section}

All pneumoconioses mortality, 1988-1992

Total deaths

Crude mortality rate (per $1,000,000$ population)

Age-adjusted mortality rate (per $1,000,000$ population)

Total years of potential life lost to life expectancy

Years of potential life lost to life expectancy (per death)
Value

37

12.10

6.79

454

12.3

Deaths*

22

0

14

0

1 $\underline{\text { State rank }}$

40

16

17

39

22

Percent**

distribution

59.5

0.0

37.8

0.0

2.7

Unspecified/Other pneumoconiosis

* Sum of column may exceed total deaths because of deaths with multiple pneumoconioses.

** Percentages may total more than $100 \%$ due to deaths with multiple pneumoconioses.

NOTE: $\quad$ See Appendix A for source description and Appendix B for methods and ICD-8 and ICD-9 codes.

SOURCE: $\quad$ National Center for Health Statistics multiple cause of death data. Population estimates from U.S. Bureau of the Census.

\section{Figure MT-1. All pneumoconioses: Montana and U.S. age-adjusted mortality rates, residents age 15 and over, 1968-1992}

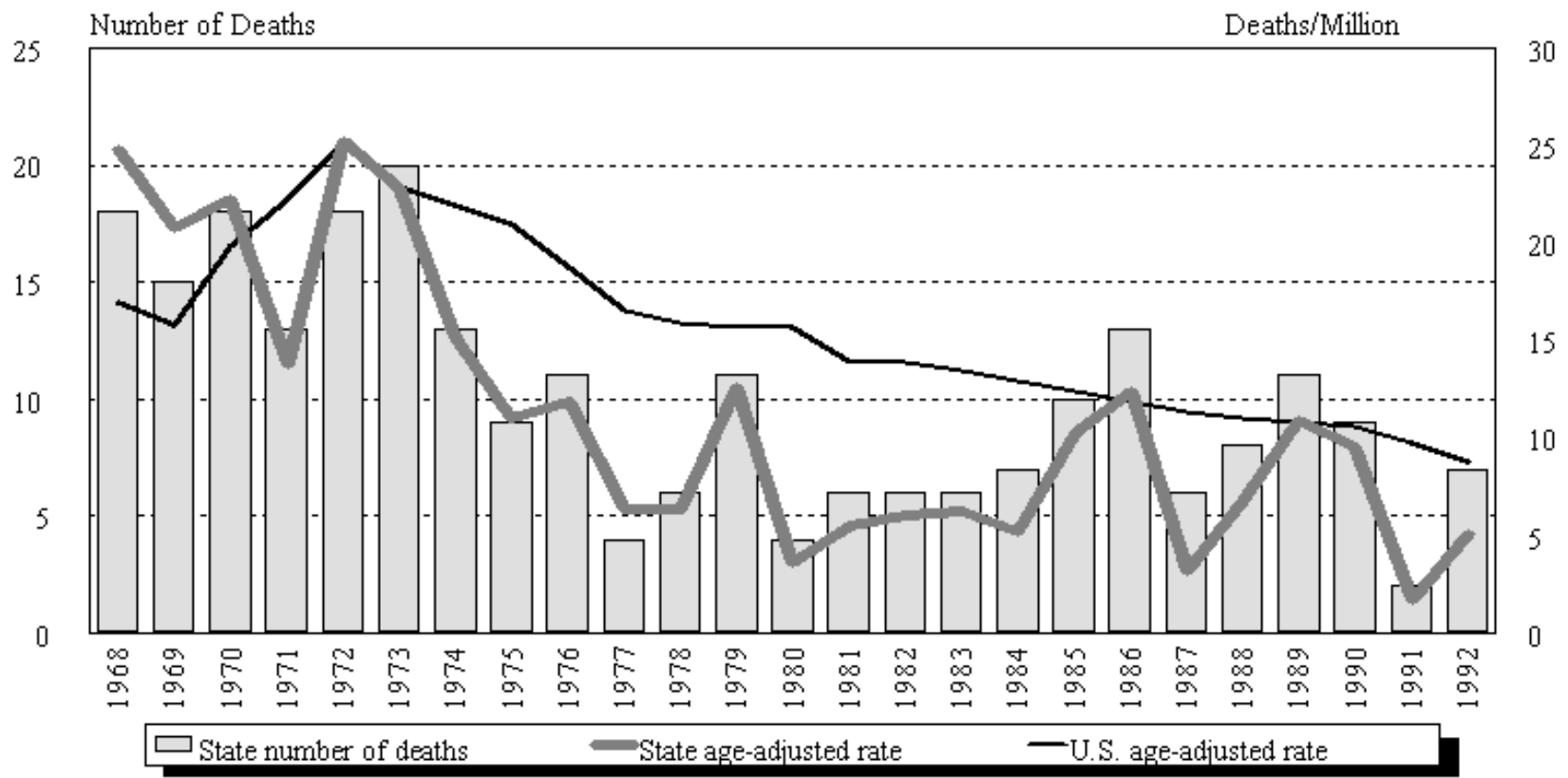

NOTE: $\quad$ See Appendix A for source description and Appendix B for methods and ICD-8 and ICD-9 codes.

SOURCE: National Center for Health Statistics multiple cause of death data. Population estimates from U.S. Bureau of the Census. 
Montana

Table MT-1. Number of deaths and age-adjusted mortality rate (per 1,000,000 population) by condition, Montana residents age 15 and over, 1968-1992

\begin{tabular}{|c|c|c|c|c|c|c|c|c|c|c|c|c|}
\hline \multirow[b]{2}{*}{ Year } & \multicolumn{2}{|c|}{ Asb estosis } & \multicolumn{2}{|c|}{$\begin{array}{l}\text { Coal workers' } \\
\text { pneumoconiosis }\end{array}$} & \multicolumn{2}{|c|}{ Silicosis } & \multicolumn{2}{|c|}{ Byssinosis* } & \multicolumn{2}{|c|}{$\begin{array}{c}\text { Unspecified/Other } \\
\text { pneumoconioses }\end{array}$} & \multicolumn{2}{|c|}{ All pneumoconioses } \\
\hline & Number & Rate & Number & Rate & Number & Rate & Number & Rate & Number & Rate & Number & Rate \\
\hline 1968 & - & - & - & - & 17 & 23.13 & & & 1 & 1.69 & 18 & 24.82 \\
\hline 1969 & - & - & 2 & 3.34 & 13 & 17.42 & & & - & - & 15 & 20.76 \\
\hline 1970 & - & - & - & - & 18 & 22.26 & & & - & - & 18 & 22.26 \\
\hline 1971 & - & - & - & - & 13 & 13.73 & & & - & - & 13 & 13.73 \\
\hline 1972 & - & - & 4 & 6.42 & 10 & 12.46 & & & 4 & 6.32 & 18 & 25.20 \\
\hline 1973 & - & - & 1 & 1.00 & 17 & 18.68 & & & 3 & 4.70 & 20 & 22.77 \\
\hline 1974 & 2 & 3.09 & 1 & 1.50 & 10 & 10.57 & & & - & - & 13 & 15.15 \\
\hline 1975 & 1 & 2.04 & 2 & 2.92 & 6 & 6.02 & & & - & - & 9 & 10.98 \\
\hline 1976 & - & - & 2 & 2.02 & 9 & 9.85 & & & - & - & 11 & 11.87 \\
\hline 1977 & - & - & - & - & 3 & 4.29 & & & 1 & 2.05 & 4 & 6.34 \\
\hline 1978 & - & - & - & - & 4 & 3.90 & & & 2 & 2.48 & 6 & 6.38 \\
\hline 1979 & - & - & 2 & 2.25 & 10 & 11.65 & - & - & - & - & 11 & 12.62 \\
\hline 1980 & - & - & - & - & 3 & 3.15 & - & - & 1 & 0.41 & 4 & 3.57 \\
\hline 1981 & 2 & 2.96 & 2 & 0.81 & 2 & 1.80 & - & - & - & - & 6 & 5.57 \\
\hline 1982 & 2 & 2.92 & - & - & 4 & 3.15 & - & - & - & - & 6 & 6.07 \\
\hline 1983 & 2 & 2.30 & - & - & 4 & 3.93 & - & - & - & - & 6 & 6.23 \\
\hline 1984 & 2 & 2.55 & 1 & 0.37 & 2 & 1.56 & - & - & 2 & 0.74 & 7 & 5.22 \\
\hline 1985 & 3 & 2.96 & 2 & 2.53 & 5 & 5.49 & - & - & 1 & 0.75 & 10 & 10.31 \\
\hline 1986 & 2 & 2.56 & 3 & 3.29 & 5 & 4.39 & - & - & 3 & 2.20 & 13 & 12.43 \\
\hline 1987 & 1 & 0.36 & 1 & 0.72 & 3 & 1.79 & - & - & 1 & 0.36 & 6 & 3.23 \\
\hline 1988 & 2 & 1.06 & - & - & 6 & 5.78 & - & - & - & - & 8 & 6.84 \\
\hline 1989 & 8 & 9.54 & - & - & 3 & 1.39 & - & - & - & - & 11 & 10.92 \\
\hline 1990 & 6 & 7.44 & - & - & 2 & 1.72 & - & - & 1 & 0.35 & 9 & 9.51 \\
\hline 1991 & 2 & 1.70 & - & - & - & - & - & - & - & - & 2 & 1.70 \\
\hline 1992 & 4 & 3.20 & - & - & 3 & 1.90 & - & - & - & - & 7 & 5.10 \\
\hline
\end{tabular}

indicates no deaths listed. ${ }^{*}$ no specific code for byssinosis in ICD-8 (1968-1978)

NOTE: Rates based on small numbers may be unstable. Sum of individual conditions may exceed total because of deaths with multiple pneumo conioses. See Appendix A for source description and Appendix B for methods and ICD-8 and ICD-9 NOTE:
codes.

SOURCE: National Center for Health Statistics multiple cause of death data. Population estimates from U.S. Bureau of the Census. 


\section{Figure MT-2. All pneumoconioses: Distribution of deaths by sex and race, Montana residents age 15 and over, 1968-1992}
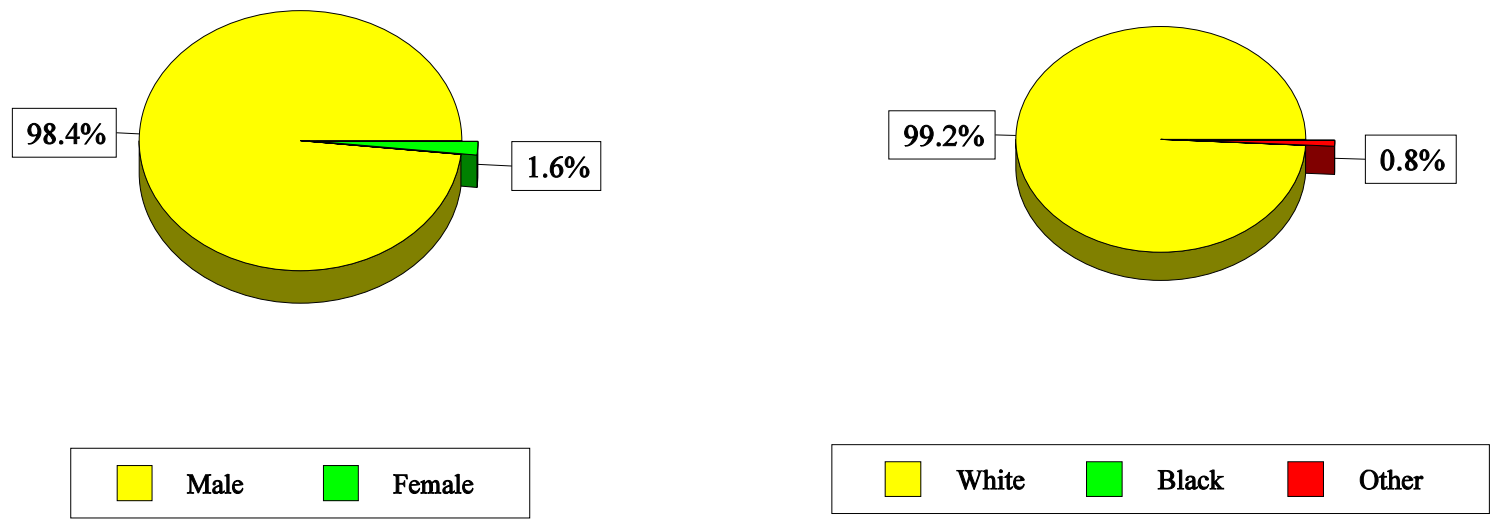

NOTE:

See Appendix A for source description and Appendix B for methods and ICD-8 and ICD-9 codes.

SOURCE: National Center for Health Statistics multiple cause of death data.

Table MT-2. All pneumoconioses: Distribution of deaths by sex, race, and age, Montana residents age 15 and over, 1968-1992

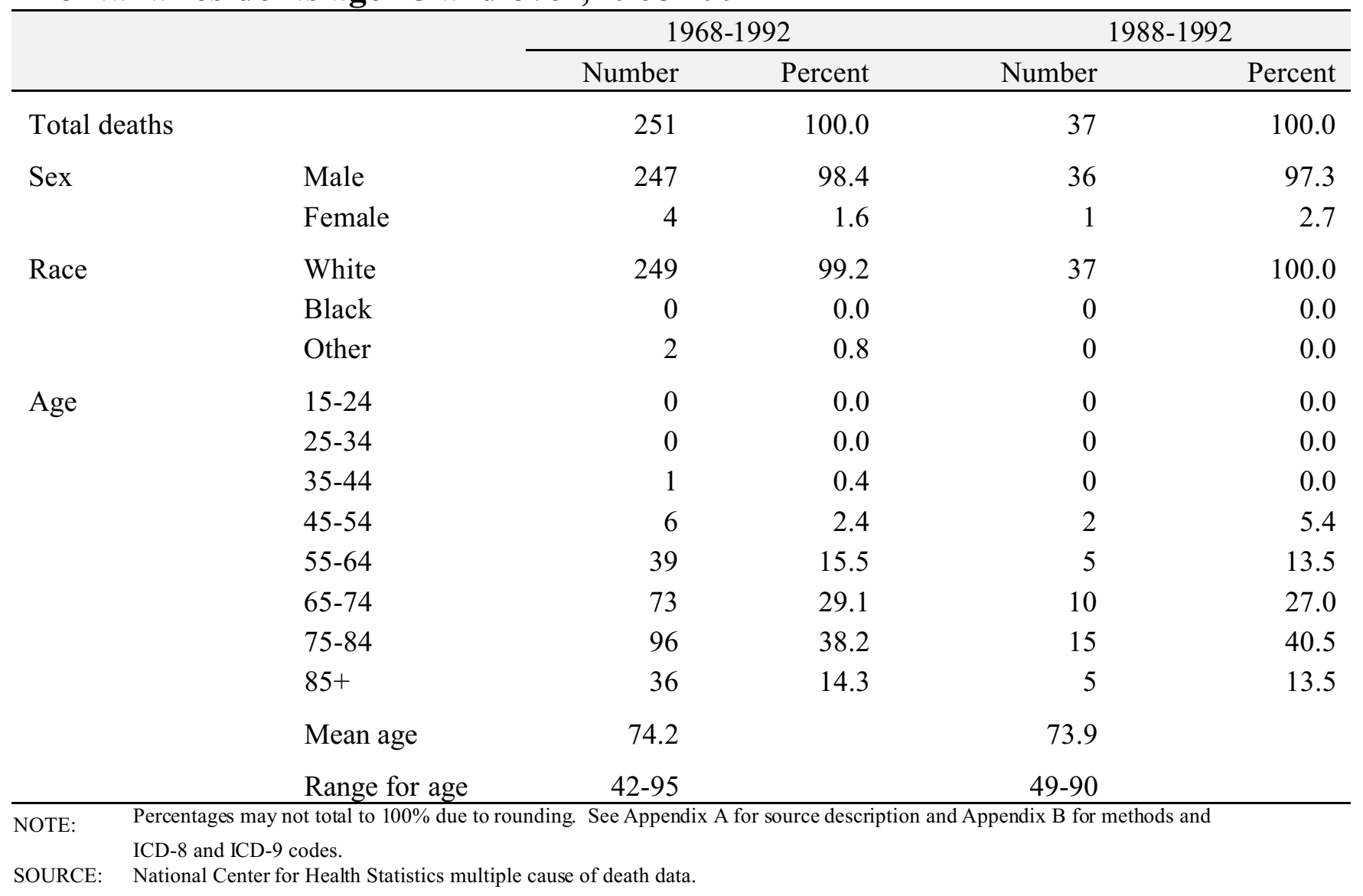


Figure MT-3. Pneumoconiosis deaths by condition, Montana residents age 15 and over, 1968-1992

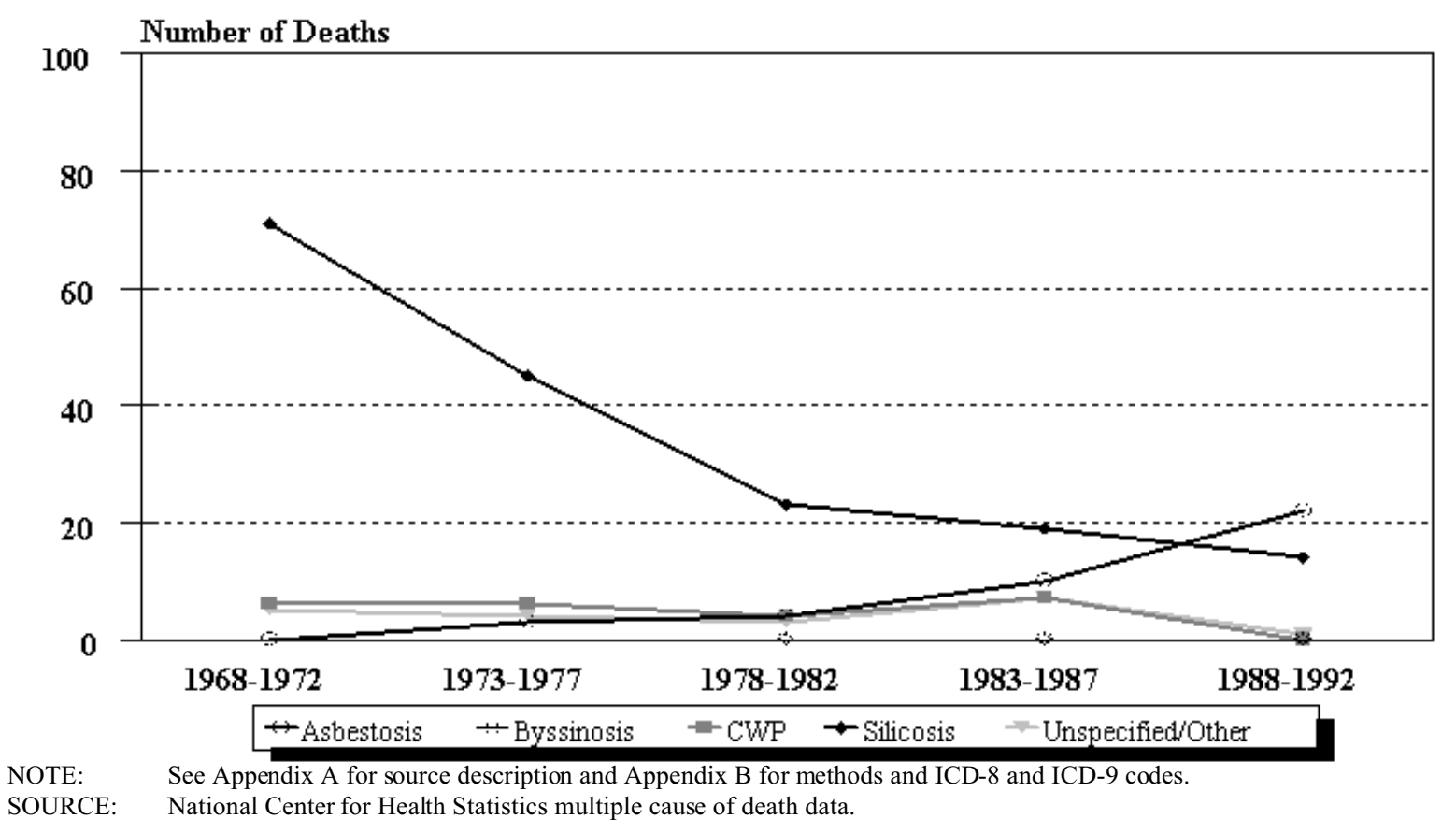

Figure MT-4. Montana and U.S. age-adjusted mortality rates (per 1,000,000 population) by condition, residents age 15 and over, 1983-1992

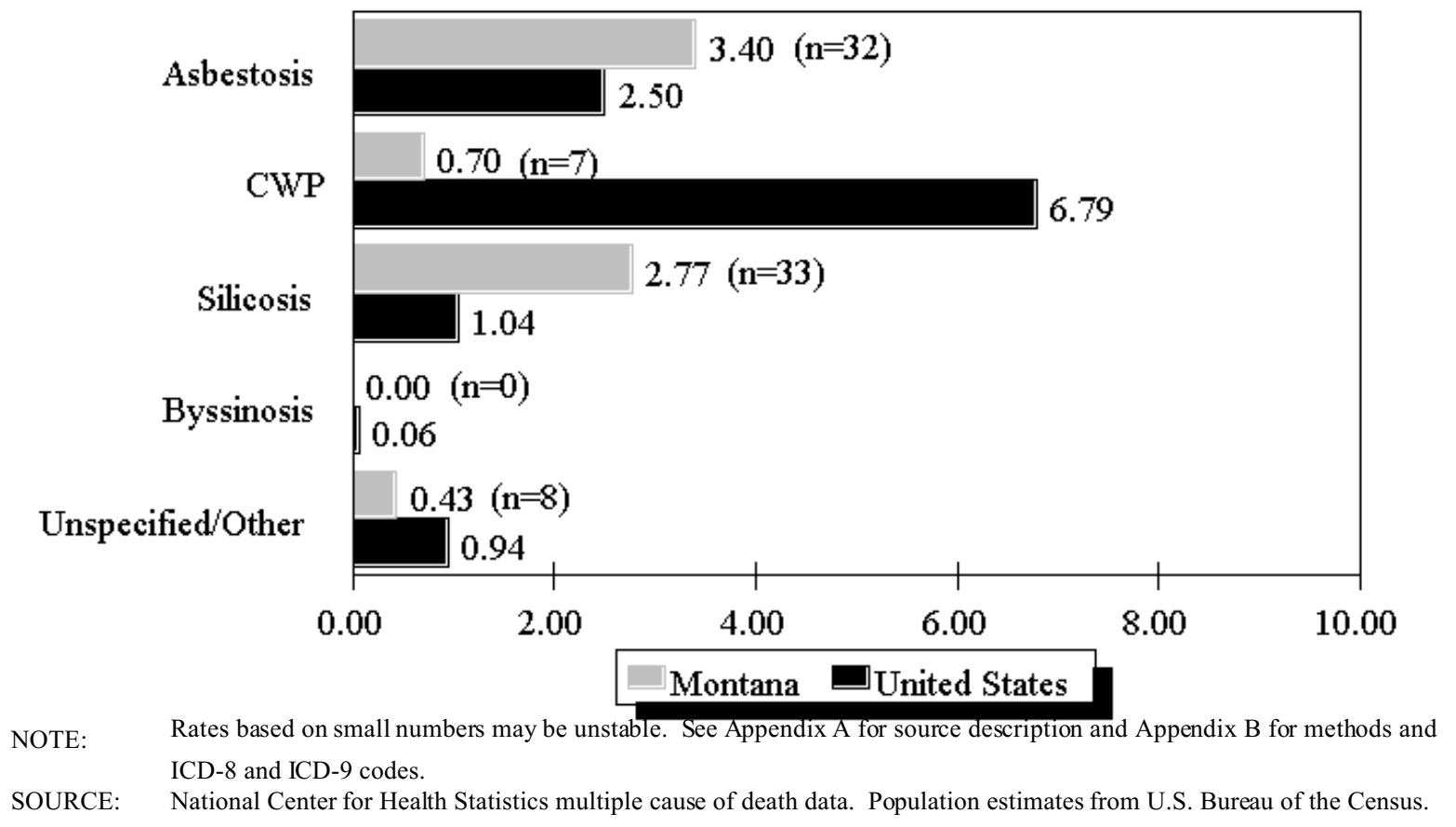


Table MT-3. Number of deaths and age-adjusted mortality rate (per 1,000,000 population) by condition and county, Montana residents age 15 and over, 1983-1992

\begin{tabular}{|c|c|c|c|c|c|c|c|c|c|c|c|c|}
\hline \multirow[b]{2}{*}{ County } & \multicolumn{2}{|c|}{ Asbestosis } & \multicolumn{2}{|c|}{$\begin{array}{c}\text { Coal workers' } \\
\text { pneumoconiosis }\end{array}$} & \multicolumn{2}{|c|}{ Silicosis } & \multicolumn{2}{|c|}{ Byssinosis } & \multicolumn{2}{|c|}{$\begin{array}{r}\text { Unspecified/Other } \\
\text { pneumoconioses }\end{array}$} & \multicolumn{2}{|c|}{$\begin{array}{r}\text { All } \\
\text { pneumoconioses }\end{array}$} \\
\hline & $\#$ & Rate & \# & Rate & $\#$ & Rate & $\#$ & Rate & \# & Rate & $\#$ & Rate \\
\hline Beaverhead & - & - & - & - & 1 & 5.67 & - & - & - & - & 1 & 5.67 \\
\hline Broadwater & - & - & - & - & 2 & 39.15 & - & - & - & - & 2 & 39.15 \\
\hline Cascade & 3 & 2.75 & 2 & 1.35 & 2 & 2.07 & - & - & 3 & 2.03 & 10 & 8.20 \\
\hline Custer & - & - & 1 & 3.05 & - & - & - & - & - & - & 1 & 3.05 \\
\hline Dawson & 1 & 9.44 & - & - & - & - & - & - & - & - & 1 & 9.44 \\
\hline Deer Lodge & 1 & 7.17 & - & - & 1 & 3.41 & - & - & - & - & 2 & 10.58 \\
\hline Fergus & - & - & - & - & - & - & - & - & 1 & 2.45 & 1 & 2.45 \\
\hline Flathead & 1 & 1.82 & - & - & - & - & - & - & - & - & 1 & 1.82 \\
\hline Gallatin & - & - & - & - & - & - & - & - & 1 & 1.57 & 1 & 1.57 \\
\hline Jefferson & 1 & 8.67 & - & - & - & - & - & - & - & - & 1 & 8.67 \\
\hline Lake & - & - & - & - & 1 & 4.49 & - & - & - & - & 1 & 4.49 \\
\hline $\begin{array}{l}\text { Lewis and } \\
\text { Clark }\end{array}$ & 2 & 4.96 & - & - & 2 & 2.48 & - & - & - & - & 4 & 7.43 \\
\hline Liberty & - & - & 1 & 40.98 & - & - & - & - & - & - & 1 & 40.98 \\
\hline Lincoln & 11 & 60.23 & - & - & 1 & 5.96 & - & - & - & - & 12 & 66.19 \\
\hline Madison & 1 & 5.99 & - & - & 1 & 5.99 & - & - & - & - & 2 & 11.98 \\
\hline Missoula & 2 & 3.49 & - & - & - & - & - & - & - & - & 2 & 3.49 \\
\hline Musselshell & - & - & 2 & 35.33 & - & - & - & - & 1 & 7.67 & 3 & 43.01 \\
\hline Park & 2 & 9.28 & - & - & - & - & - & - & - & - & 2 & 9.28 \\
\hline Powell & - & - & - & - & 1 & 6.78 & - & - & - & - & 1 & 6.78 \\
\hline Sanders & - & - & - & - & 1 & 4.62 & - & - & - & - & 1 & 4.62 \\
\hline Silver Bow & 1 & 1.08 & 1 & 2.56 & 20 & 34.92 & - & - & 2 & 3.64 & 23 & 39.64 \\
\hline Toole & 1 & 8.58 & - & - & - & - & - & - & - & - & 1 & 8.58 \\
\hline Yellowstone & 5 & 4.57 & - & - & - & - & - & - & - & - & 5 & 4.57 \\
\hline
\end{tabular}

- indicates no deaths listed.

NOTE: Counties with no deaths for all listed conditions are not displayed. Rates based on small numbers may be unstable. Sum of individual conditions may exceed total because of deaths with multiple pneumoconioses. See Appendix A for source description and Appendix B for methods and ICD-8 and ICD-9 codes.

SOURCE: National Center for Health Statistics multiple cause of death data. Population estimates from U.S. Bureau of the Census. 


\section{Nebraska: Selected statistics from the United States section}

All pneumoconioses mortality, 1988-1992

Total deaths

$\underline{\text { Value }}$

20

3.29

1.68

225

11.3
Years of potential life lost to life expectancy (per death)

Crude mortality rate (per $1,000,000$ population)

Age-adjusted mortality rate (per $1,000,000$ population)

Total years of potential life lost to life expectancy
Deaths by type of pneumoconiosis, 1988-1992

Asbestosis

Coal workers' pneumoconiosis

Silicosis

Byssinosis $\underline{\text { State rank }}$

46

48

50

47

41

Percent**

distribution

60.0

0.0

20.0

0.0

20.0

* Sum of column may exceed total deaths because of deaths with multiple pneumoconioses.

** Percentages may total more than $100 \%$ due to deaths with multiple pneumoconioses.

NOTE: $\quad$ See Appendix A for source description and Appendix B for methods and ICD-8 and ICD-9 codes.

SOURCE: $\quad$ National Center for Health Statistics multiple cause of death data. Population estimates from U.S. Bureau of the Census.

\section{Figure NE-1. All pneumoconioses: Nebraska and U.S. age-adjusted mortality rates, residents age 15 and over, 1968-1992}

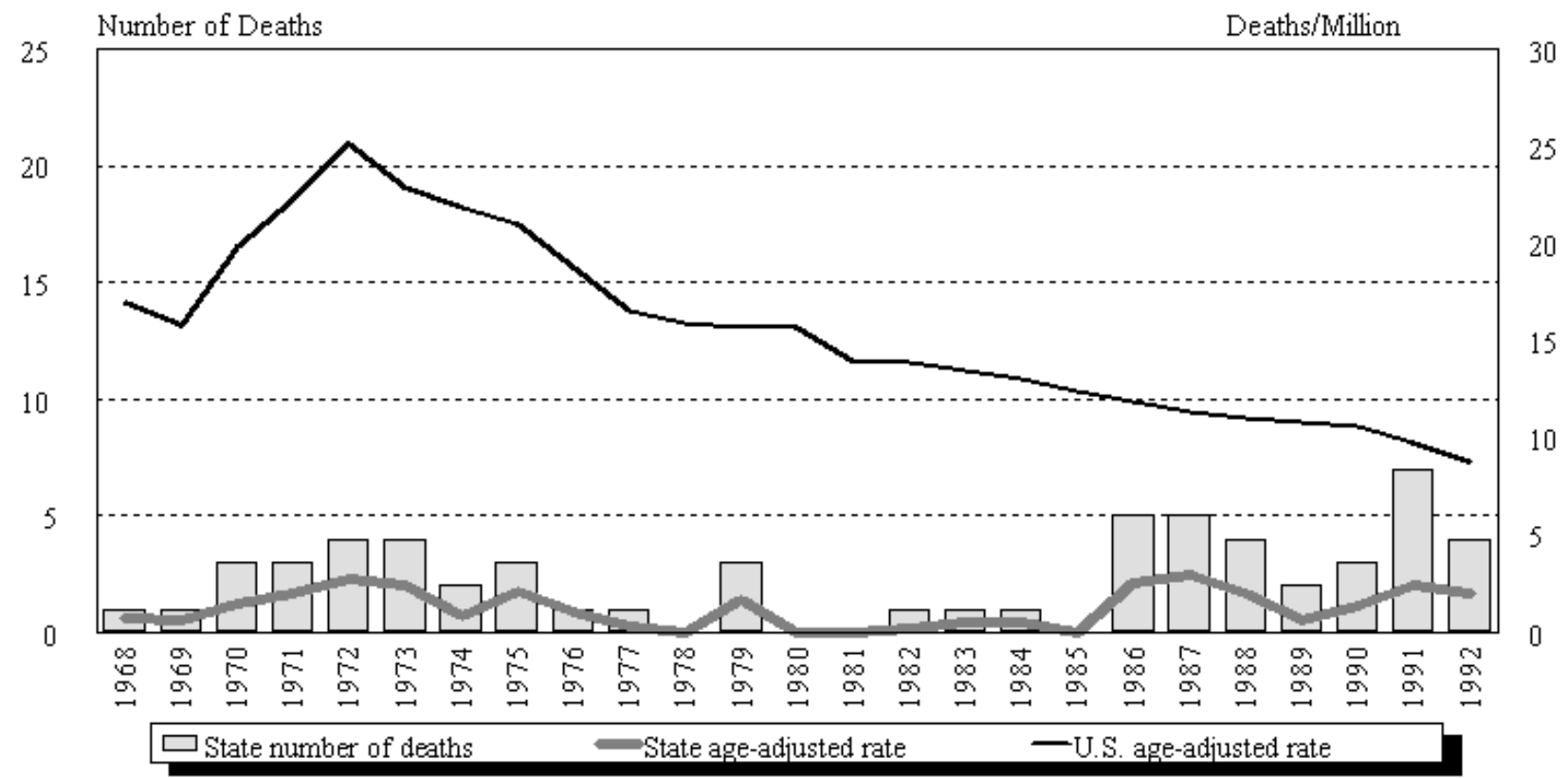

NOTE: $\quad$ See Appendix A for source description and Appendix B for methods and ICD-8 and ICD-9 codes.

SOURCE: National Center for Health Statistics multiple cause of death data. Population estimates from U.S. Bureau of the Census. 


\section{Nebraska}

Table NE-1. Number of deaths and age-adjusted mortality rate (per 1,000,000 population) by condition, Nebraska residents age 15 and over, 1968-1992

\begin{tabular}{|c|c|c|c|c|c|c|c|c|c|c|c|c|}
\hline \multirow[b]{2}{*}{ Year } & \multicolumn{2}{|c|}{ Asbestosis } & \multicolumn{2}{|c|}{$\begin{array}{l}\text { Coal workers' } \\
\text { pneumoconiosis }\end{array}$} & \multicolumn{2}{|c|}{ Silicosis } & \multicolumn{2}{|c|}{ Byssinosis* } & \multicolumn{2}{|c|}{$\begin{array}{c}\text { Unspecified/Other } \\
\text { pneumoconioses }\end{array}$} & \multicolumn{2}{|c|}{ All pneumoconioses } \\
\hline & Number & Rate & Number & Rate & Number & Rate & Number & Rate & Number & Rate & Number & Rate \\
\hline 1968 & - & - & - & - & 1 & 0.77 & & & - & - & 1 & 0.77 \\
\hline 1969 & - & - & - & - & 1 & 0.61 & & & - & - & 1 & 0.61 \\
\hline 1970 & - & - & 2 & 0.85 & 1 & 0.61 & & & - & - & 3 & 1.46 \\
\hline 1971 & - & - & 1 & 0.77 & 1 & 0.77 & & & 2 & 1.22 & 3 & 1.99 \\
\hline 1972 & 2 & 1.54 & - & - & 2 & 1.21 & & & - & - & 4 & 2.75 \\
\hline 1973 & - & - & - & - & - & - & & & 4 & 2.40 & 4 & 2.40 \\
\hline 1974 & - & - & - & - & 2 & 0.80 & & & - & - & 2 & 0.80 \\
\hline 1975 & - & - & 1 & 0.77 & 1 & 0.77 & & & 1 & 0.60 & 3 & 2.14 \\
\hline 1976 & - & - & - & - & - & - & & & 1 & 1.00 & 1 & 1.00 \\
\hline 1977 & - & - & - & - & - & - & & & 1 & 0.35 & 1 & 0.35 \\
\hline 1978 & - & - & - & - & - & - & & & - & - & - & - \\
\hline 1979 & 3 & 1.67 & - & - & - & - & - & - & - & - & 3 & 1.67 \\
\hline 1980 & - & - & - & - & - & - & - & - & - & - & - & - \\
\hline 1981 & - & - & - & - & - & - & - & - & - & - & - & - \\
\hline 1982 & - & - & 1 & 0.15 & - & - & - & - & - & - & 1 & 0.15 \\
\hline 1983 & - & - & - & - & - & - & - & - & 1 & 0.56 & 1 & 0.56 \\
\hline 1984 & 1 & 0.55 & - & - & - & - & - & - & - & - & 1 & 0.55 \\
\hline 1985 & - & - & - & - & - & - & - & - & - & - & - & - \\
\hline 1986 & 4 & 1.94 & - & - & - & - & - & - & 1 & 0.55 & 5 & 2.49 \\
\hline 1987 & 5 & 2.98 & - & - & - & - & - & - & - & - & 5 & 2.98 \\
\hline 1988 & 2 & 0.86 & - & - & - & - & - & - & 2 & 1.11 & 4 & 1.97 \\
\hline 1989 & 2 & 0.61 & - & - & - & - & - & - & - & - & 2 & 0.61 \\
\hline 1990 & 3 & 1.40 & - & - & - & - & - & - & - & - & 3 & 1.40 \\
\hline 1991 & 3 & 0.89 & - & - & 3 & 0.97 & - & - & 1 & 0.55 & 7 & 2.40 \\
\hline 1992 & 2 & 0.93 & - & - & 1 & 0.54 & - & - & 1 & 0.54 & 4 & 2.02 \\
\hline
\end{tabular}

-indicates no deaths listed. ${ }^{*}$ no specific code for byssinosis in ICD-8 (1968-1978)

NOTE: Rates based on small numbers may be unstable. Sum of individual conditions may exceed total because of deaths with multiple pneumoconioses. See Appendix A for source description and Appendix B for methods and ICD-8 and ICD-9 NoTe:

SOURCE: National Center for Health Statistics multiple cause of death data. Population estimates from U.S. Bureau of the Census. 


\section{Figure NE-2. All pneumoconioses: Distribution of deaths by sex and race,} Nebraska residents age 15 and over, 1968-1992
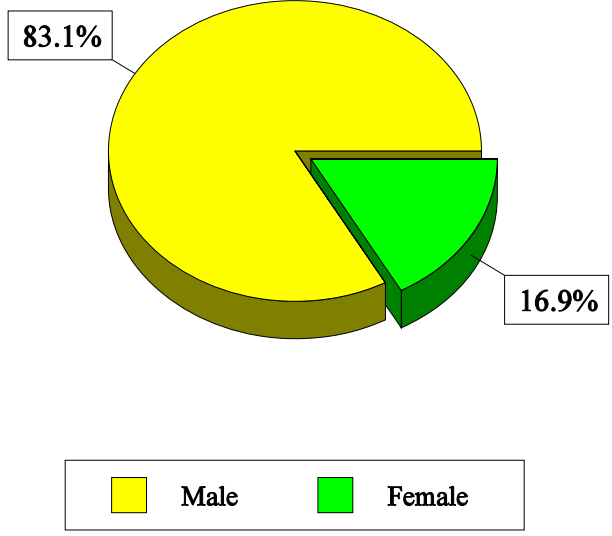
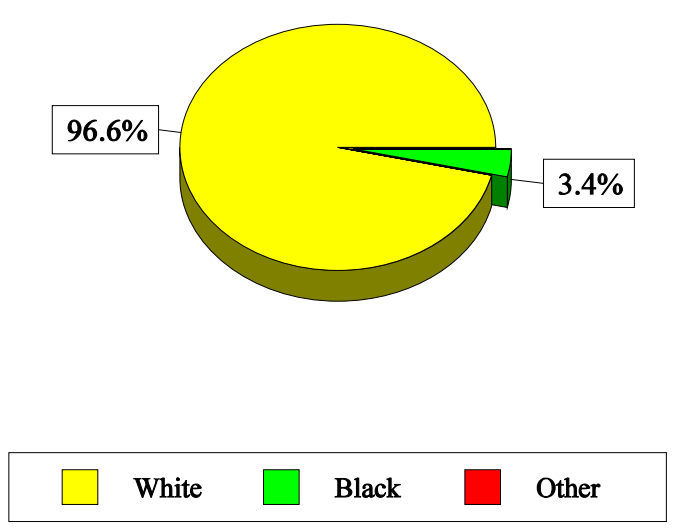

NOTE:

See Appendix A for source description and Appendix B for methods and ICD-8 and ICD-9 codes.

SOURCE: National Center for Health Statistics multiple cause of death data.

Table NE-2. All pneumoconioses: Distribution of deaths by sex, race, and age, Nebraska residents age 15 and over, 1968-1992

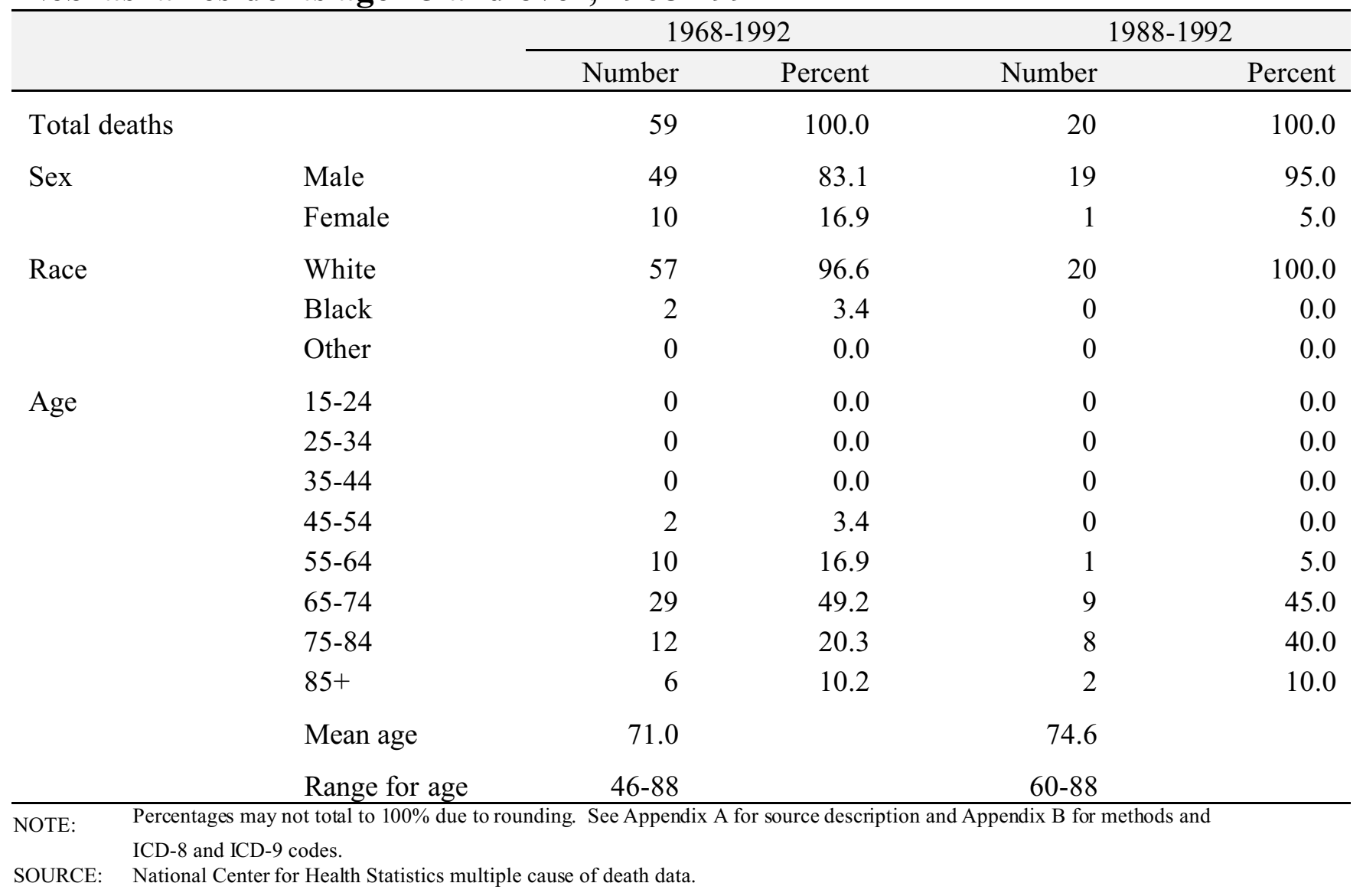




\section{Figure NE-3. Pneumoconiosis deaths by condition, Nebraska residents age 15 and over, 1968-1992}

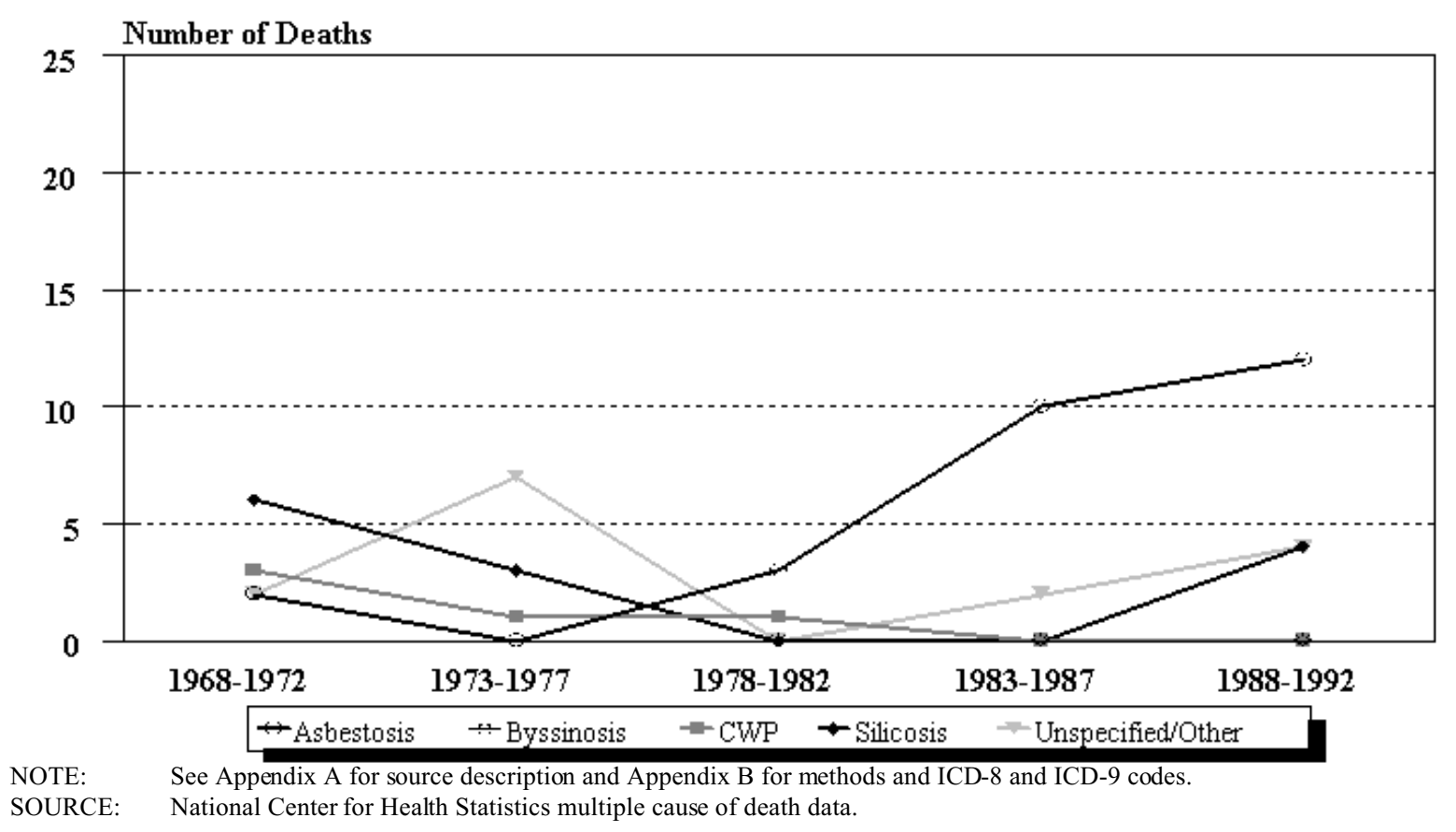

Figure NE-4. Nebraska and U.S. age-adjusted mortality rates (per 1,000,000 population) by condition, residents age 15 and over, 1983-1992

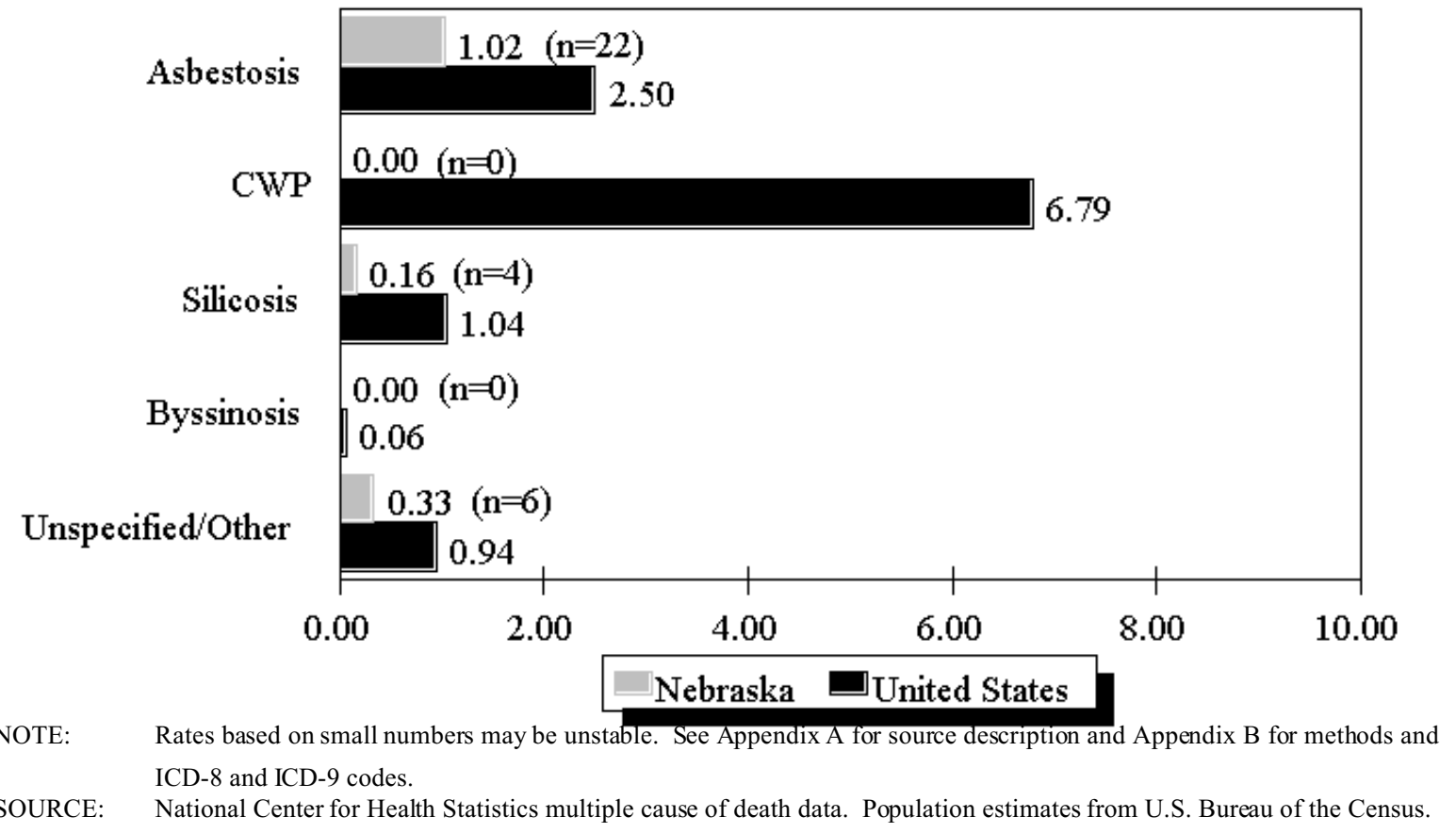


Table NE-3. Number of deaths and age-adjusted mortality rate (per 1,000,000 population) by condition and county, Nebraska residents age 15 and over, 1983-1992

\begin{tabular}{|c|c|c|c|c|c|c|c|c|c|c|c|c|}
\hline \multirow[b]{2}{*}{ County } & \multicolumn{2}{|c|}{ Asbestosis } & \multicolumn{2}{|c|}{$\begin{array}{c}\text { Coal workers' } \\
\text { pneumoconiosis }\end{array}$} & \multicolumn{2}{|c|}{ Silicosis } & \multicolumn{2}{|c|}{ Byssinosis } & \multicolumn{2}{|c|}{$\begin{array}{r}\text { Unspecified/Other } \\
\text { pneumoconioses }\end{array}$} & \multicolumn{2}{|c|}{$\begin{array}{r}\text { All } \\
\text { pneumoconioses }\end{array}$} \\
\hline & $\#$ & Rate & \# & Rate & $\#$ & Rate & $\#$ & Rate & $\#$ & Rate & \# & Rate \\
\hline Boone & 1 & 12.08 & - & - & - & - & - & - & - & - & 1 & 12.08 \\
\hline Cass & - & - & - & - & - & - & - & - & 1 & 4.96 & 1 & 4.96 \\
\hline Custer & - & - & - & - & - & - & - & - & 1 & 6.24 & 1 & 6.24 \\
\hline Dawson & - & - & - & - & - & - & - & - & 1 & 4.57 & 1 & 4.57 \\
\hline Deuel & - & - & - & - & 1 & 10.71 & - & - & - & - & 1 & 10.71 \\
\hline Douglas & 18 & 3.80 & - & - & 2 & 0.57 & - & - & 2 & 0.57 & 22 & 4.93 \\
\hline Hall & - & - & - & - & 1 & 0.87 & - & - & - & - & 1 & 0.87 \\
\hline Lancaster & 1 & 0.27 & - & - & - & - & - & - & 1 & 0.62 & 2 & 0.89 \\
\hline Sarpy & 2 & 4.14 & - & - & - & - & - & - & - & - & 2 & 4.14 \\
\hline
\end{tabular}

- indicates no deaths listed.

NOTE: Counties with no deaths for all listed conditions are not displayed. Rates based on small numbers may be unstable. Sum of individual conditions may exceed total because of deaths with multiple pneumoconioses. See Appendix A for source description and Appendix B for methods and ICD-8 and ICD-9 codes.

SOURCE: National Center for Health Statistics multiple cause of death data. Population estimates from U.S. Bureau of the Census. 


\section{Nevada: Selected statistics from the United States section}

All pneumoconioses mortality, 1988-1992

Total deaths

Crude mortality rate (per $1,000,000$ population)

Age-adjusted mortality rate (per $1,000,000$ population)

Total years of potential life lost to life expectancy

Years of potential life lost to life expectancy (per death)

Deaths by type of pneumoconiosis, 1988-1992

Asbestosis

Coal workers' pneumoconiosis

Silicosis

Byssinosis

Unspecified/Other pneumoconiosis

\begin{tabular}{rr} 
Value & State rank \\
\hline 22 & 45 \\
4.59 & 42 \\
3.15 & 40 \\
259 & 45 \\
11.8 & 30
\end{tabular}

Percent**

distribution

31.8

18.2

45.5

0.0

4.5

* Sum of column may exceed total deaths because of deaths with multiple pneumoconioses.

** Percentages may total more than $100 \%$ due to deaths with multiple pneumoconioses.

NOTE: $\quad$ See Appendix A for source description and Appendix B for methods and ICD-8 and ICD-9 codes.

SOURCE: $\quad$ National Center for Health Statistics multiple cause of death data. Population estimates from U.S. Bureau of the Census.

\section{Figure NV-1. All pneumoconioses: Nevada and U.S. age-adjusted mortality rates, residents age 15 and over, 1968-1992}

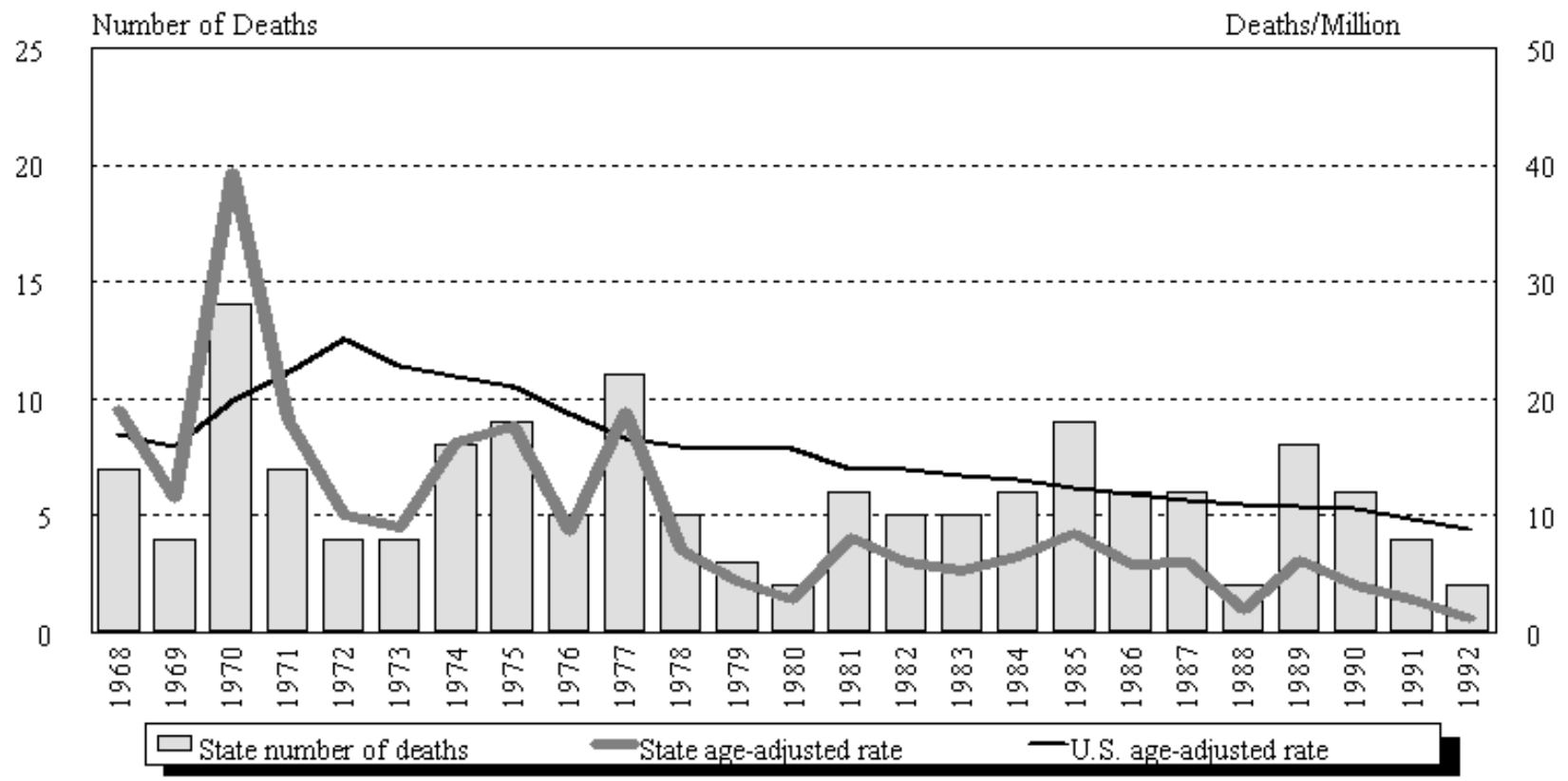

NOTE: $\quad$ See Appendix A for source description and Appendix B for methods and ICD-8 and ICD-9 codes.

SOURCE: National Center for Health Statistics multiple cause of death data. Population estimates from U.S. Bureau of the Census. 


\section{Nevada}

\begin{tabular}{|c|c|c|c|c|c|c|c|c|c|c|c|c|}
\hline \multirow[b]{2}{*}{ Year } & \multicolumn{2}{|c|}{ Asb estosis } & \multicolumn{2}{|c|}{$\begin{array}{l}\text { Coal workers' } \\
\text { pneumoconiosis }\end{array}$} & \multicolumn{2}{|c|}{ Silicosis } & \multicolumn{2}{|c|}{ Byssinosis* } & \multicolumn{2}{|c|}{$\begin{array}{c}\text { Unspecified/Other } \\
\text { pneumoconioses }\end{array}$} & \multicolumn{2}{|c|}{ All pneumoconioses } \\
\hline & Number & Rate & Number & Rate & Number & Rate & Number & Rate & Number & Rate & Number & Rate \\
\hline 1968 & - & - & - & - & 7 & 19.02 & & & - & - & 7 & 19.02 \\
\hline 1969 & 1 & 3.02 & - & - & 3 & 8.41 & & & - & - & 4 & 11.43 \\
\hline 1970 & - & - & 1 & 2.95 & 11 & 30.58 & & & 2 & 5.83 & 14 & 39.35 \\
\hline 1971 & 1 & 2.78 & - & - & 6 & 15.45 & & & - & - & 7 & 18.22 \\
\hline 1972 & - & - & - & - & 4 & 10.06 & & & - & - & 4 & 10.06 \\
\hline 1973 & - & - & - & - & 4 & 9.08 & & & - & - & 4 & 9.08 \\
\hline 1974 & - & - & - & - & 4 & 7.96 & & & 4 & 8.21 & 8 & 16.17 \\
\hline 1975 & - & - & 1 & 1.46 & 8 & 16.26 & & & 1 & 2.07 & 9 & 17.72 \\
\hline 1976 & - & - & - & - & 5 & 8.72 & & & - & - & 5 & 8.72 \\
\hline 1977 & 1 & 1.67 & - & - & 7 & 12.14 & & & 3 & 5.09 & 11 & 18.90 \\
\hline 1978 & - & - & 2 & 3.17 & 2 & 2.77 & & & 1 & 1.16 & 5 & 7.09 \\
\hline 1979 & - & - & 1 & 1.49 & 2 & 2.94 & - & - & - & - & 3 & 4.43 \\
\hline 1980 & 1 & 1.39 & - & - & 1 & 1.42 & - & - & - & - & 2 & 2.81 \\
\hline 1981 & 2 & 3.04 & 1 & 1.27 & 1 & 1.30 & - & - & 2 & 2.54 & 6 & 8.16 \\
\hline 1982 & 1 & 1.16 & 2 & 2.45 & 2 & 2.45 & - & - & - & - & 5 & 6.06 \\
\hline 1983 & 1 & 1.23 & 1 & 1.09 & 2 & 1.92 & - & - & 1 & 1.13 & 5 & 5.37 \\
\hline 1984 & 1 & 1.20 & 3 & 3.14 & 2 & 2.29 & - & - & - & - & 6 & 6.62 \\
\hline 1985 & 3 & 3.03 & 2 & 1.73 & 4 & 3.73 & - & - & - & - & 9 & 8.49 \\
\hline 1986 & 4 & 4.02 & - & - & 2 & 1.81 & - & - & - & - & 6 & 5.83 \\
\hline 1987 & 3 & 3.31 & - & - & 3 & 2.68 & - & - & - & - & 6 & 5.99 \\
\hline 1988 & 1 & 1.08 & - & - & 1 & 0.78 & - & - & - & - & 2 & 1.87 \\
\hline 1989 & - & - & 1 & 0.54 & 6 & 4.92 & - & - & 1 & 0.73 & 8 & 6.18 \\
\hline 1990 & 2 & 1.50 & 1 & 0.67 & 3 & 1.91 & - & - & - & - & 6 & 4.07 \\
\hline 1991 & 3 & 2.04 & 1 & 0.71 & - & - & - & - & - & - & 4 & 2.76 \\
\hline 1992 & 1 & 0.58 & 1 & 0.68 & - & - & - & - & - & - & 2 & 1.27 \\
\hline
\end{tabular}

- indicates no deaths listed. $\quad *$ no specific code for byssinosis in ICD-8 (1968-1978)

NOTE: Rates based on small numbers may be unstable. Sum of individual conditions may exceed total because of deaths with multiple pneumoconioses. See Appendix A for source description and Appendix B for methods and ICD-8 and ICD-9 codes.

SOURCE: National Center for Health Statistics multiple cause of death data. Population estimates from U.S. Bureau of the Census. 
Figure NV-2. All pneumoconioses: Distribution of deaths by sex and race, Nevada residents age 15 and over, 1968-1992
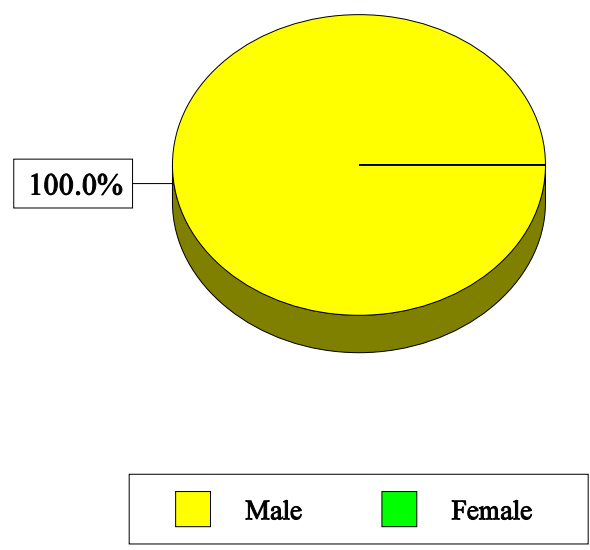
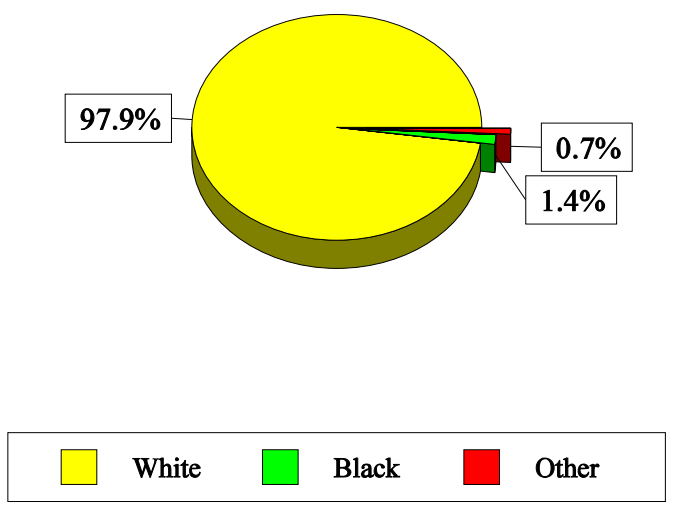

NOTE: See Appendix A for source description and Appendix B for methods and ICD-8 and ICD-9 codes.

SOURCE: National Center for Health Statistics multiple cause of death data.

Table NV-2. All pneumoconioses: Distribution of deaths by sex, race, and age, Nevada residents age 15 and over, 1968-1992

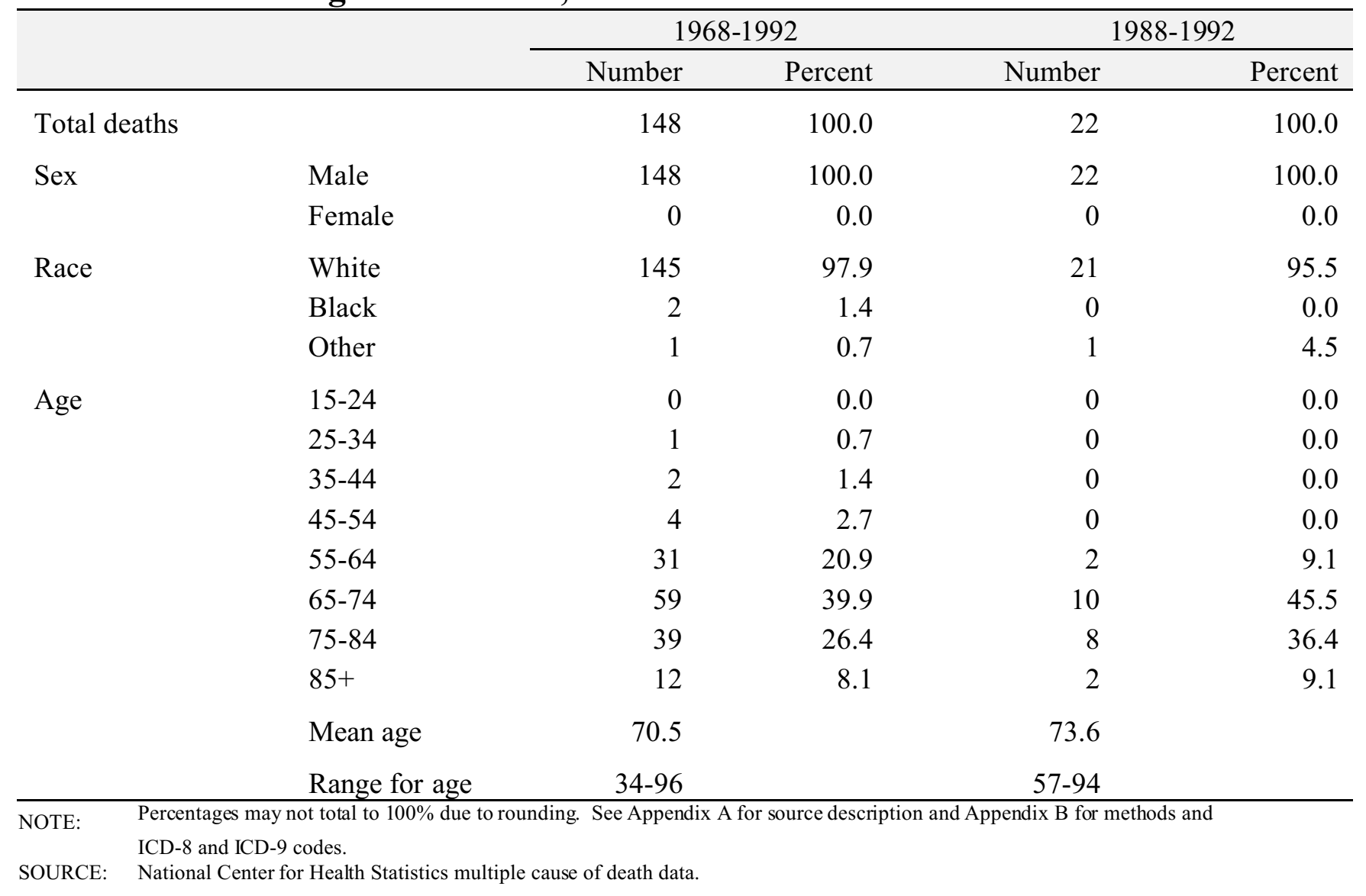


Figure NV-3. Pneumoconiosis deaths by condition, Nevada residents age 15 and over, 1968-1992

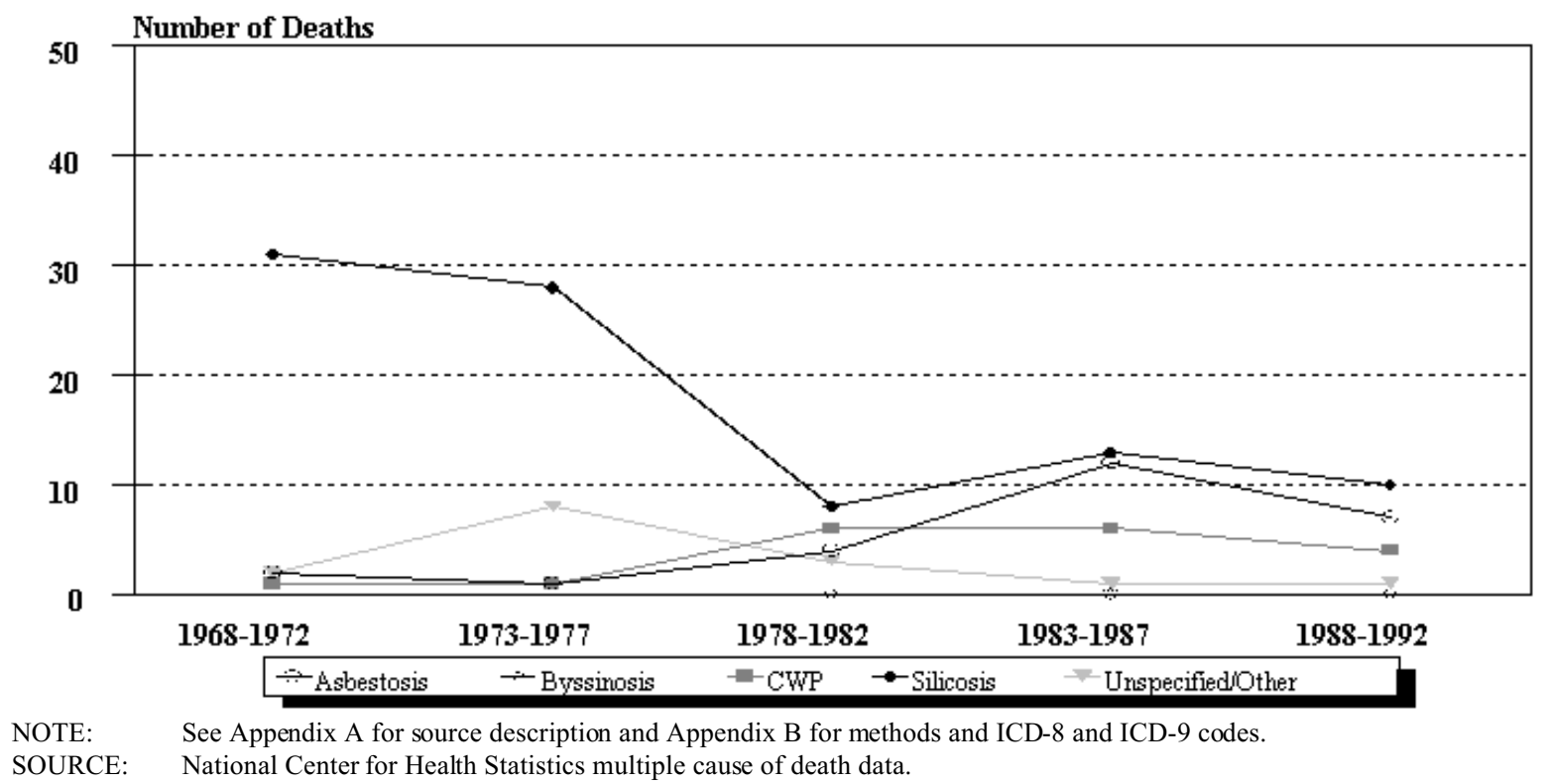

Figure NV-4. Nevada and U.S. age-adjusted mortality rates (per 1,000,000 population) by condition, residents age 15 and over, 1983-1992

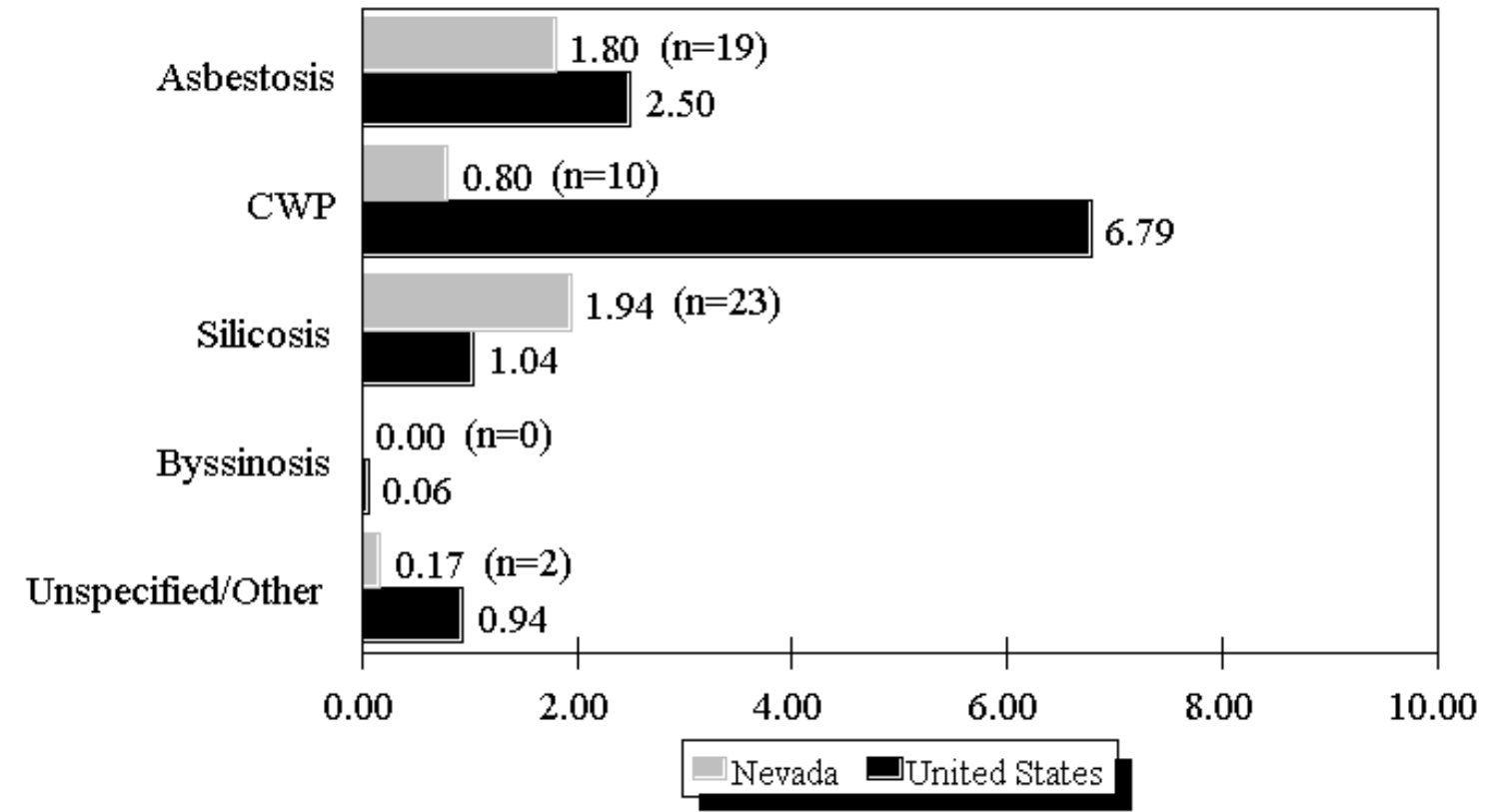

NOTE: $\quad$ Rates based on small numbers may be unstable. See Appendix A for source description and Appendix B for methods and ICD-8 and ICD-9 codes.

SOURCE: National Center for Health Statistics multiple cause of death data. Population estimates from U.S. Bureau of the Census. 
Table NV-3. Number of deaths and age-adjusted mortality rate (per 1,000,000 population) by condition and county, Nevada residents age 15 and over, 1983-1992

\begin{tabular}{|c|c|c|c|c|c|c|c|c|c|c|c|c|}
\hline \multirow[b]{2}{*}{ County } & \multicolumn{2}{|c|}{ Asbestosis } & \multicolumn{2}{|c|}{$\begin{array}{r}\text { Coal workers' } \\
\text { pneumoconiosis }\end{array}$} & \multicolumn{2}{|c|}{ Silicosis } & \multicolumn{2}{|c|}{ Byssinosis } & \multicolumn{2}{|c|}{$\begin{array}{r}\text { Unspecified/Other } \\
\text { pneumoconioses }\end{array}$} & \multicolumn{2}{|c|}{$\begin{array}{r}\text { All } \\
\text { pneumoconioses }\end{array}$} \\
\hline & $\#$ & Rate & \# & Rate & \# & Rate & \# & Rate & $\#$ & Rate & \# & Rate \\
\hline Churchill & 1 & 3.34 & - & - & 4 & 22.11 & - & - & - & - & 5 & 25.45 \\
\hline Clark & 14 & 2.27 & 7 & 1.04 & 6 & 0.94 & - & - & - & - & 27 & 4.25 \\
\hline Douglas & 1 & 3.33 & - & - & - & - & - & - & - & - & 1 & 3.33 \\
\hline Humboldt & - & - & - & - & 1 & 10.18 & - & - & 1 & 12.09 & 2 & 22.28 \\
\hline Lincoln & - & - & - & - & 1 & 24.64 & - & - & - & - & 1 & 24.64 \\
\hline Lyon & - & - & - & - & 2 & 7.92 & - & - & - & - & 2 & 7.92 \\
\hline Mineral & 1 & 23.70 & - & - & - & - & - & - & - & - & 1 & 23.70 \\
\hline Washoe & 2 & 0.97 & 3 & 1.16 & 4 & 1.50 & - & - & 1 & 0.34 & 10 & 3.97 \\
\hline White Pine & - & - & - & - & 3 & 25.99 & - & - & - & - & 3 & 25.99 \\
\hline \multicolumn{13}{|c|}{ Independent city: } \\
\hline Carson City & - & - & - & - & 2 & 3.84 & - & - & - & - & 2 & 3.84 \\
\hline
\end{tabular}

- indicates no deaths listed.

NOTE: Counties with no deaths for all listed conditions are not displayed. Rates based on small numbers may be unstable. Sum of individual conditions may exceed total because of deaths with multiple pneumoconioses. See Appendix A for source description and Appendix B for methods and ICD-8 and ICD-9 codes.

SOURCE: National Center for Health Statistics multiple cause of death data. Population estimates from U.S. Bureau of the Census. 


\section{New Hampshire: Selected statistics from the United States section}

All pneumoconioses mortality, 1988-1992

Total deaths

Years of potential life lost to life expectancy (per death)

Deaths by type of pneumoconiosis, 1988-1992

Asbestosis

Coal workers' pneumoconiosis

Silicosis

Byssinosis

Unspecified/Other pneumoconiosis $\underline{\text { Value }}$

26

5.96

3.96

321

12.3

$\underline{\text { State rank }}$

44

36

35

43

20

Percent**

$\underline{\text { Deaths* }} \quad \underline{\text { distribution }}$

65.4

3.8

26.9

3.8

3.8

* Sum of column may exceed total deaths because of deaths with multiple pneumoconioses.

** Percentages may total more than $100 \%$ due to deaths with multiple pneumoconioses.

NOTE: $\quad$ See Appendix A for source description and Appendix B for methods and ICD-8 and ICD-9 codes.

SOURCE: National Center for Health Statistics multiple cause of death data. Population estimates from U.S. Bureau of the Census.

\section{Figure NH-1. All pneumoconioses: New Hampshire and U.S. age-adjusted mortality rates, residents age 15 and over, 1968-1992}

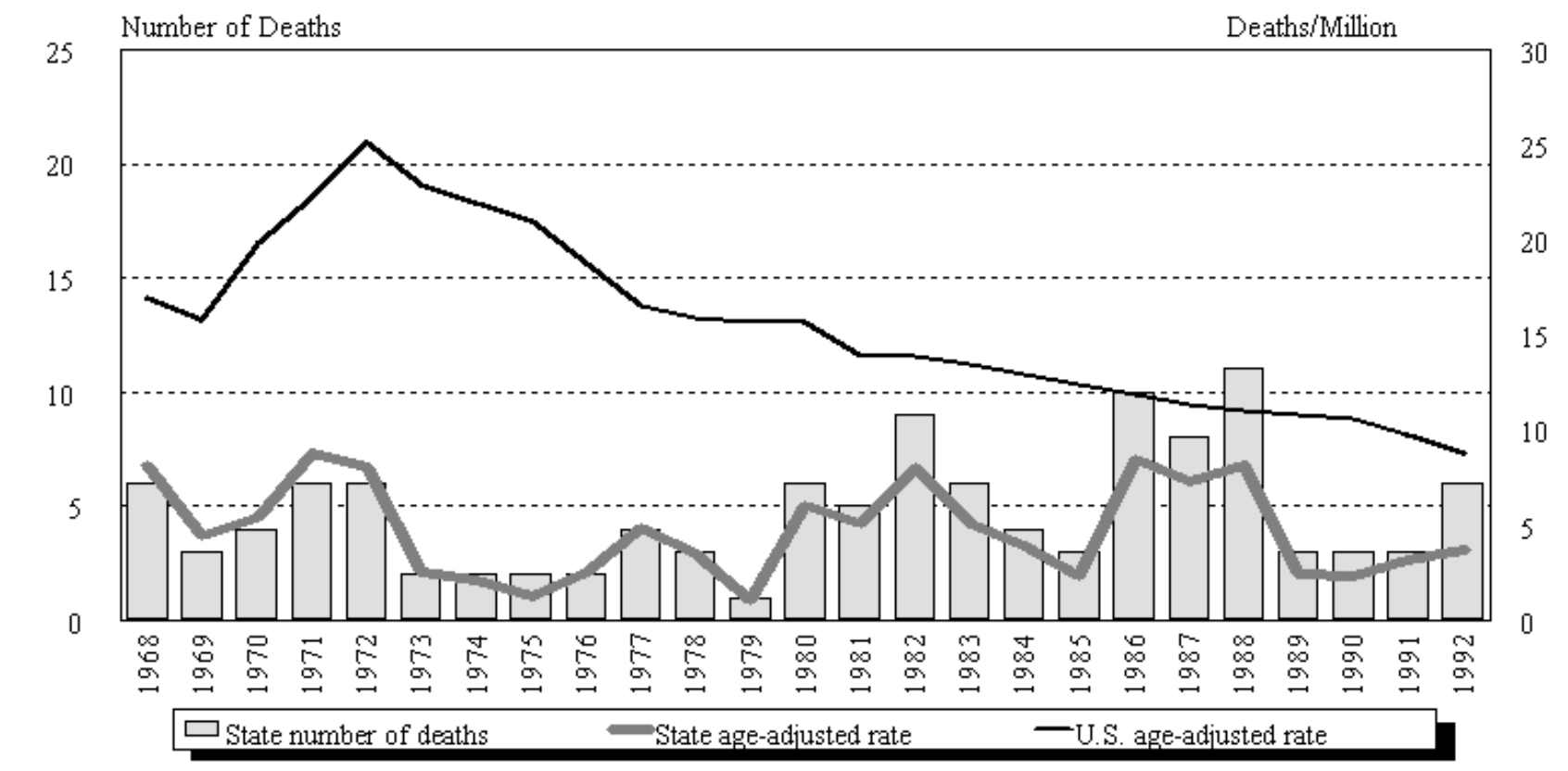

NOTE: $\quad$ See Appendix A for source description and Appendix B for methods and ICD-8 and ICD-9 codes.

SOURCE: National Center for Health Statistics multiple cause of death data. Population estimates from U.S. Bureau of the Census. 


\section{New Hampshire}

\begin{tabular}{|c|c|c|c|c|c|c|c|c|c|c|c|c|}
\hline \multirow[b]{2}{*}{ Year } & \multicolumn{2}{|c|}{ Asbestosis } & \multicolumn{2}{|c|}{$\begin{array}{l}\text { Coal workers' } \\
\text { pneumoconiosis }\end{array}$} & \multicolumn{2}{|c|}{ Silicosis } & \multicolumn{2}{|c|}{ Byssinosis* } & \multicolumn{2}{|c|}{$\begin{array}{c}\text { Unspecified/Other } \\
\text { pneumoconioses }\end{array}$} & \multicolumn{2}{|c|}{ All pneumoconioses } \\
\hline & Number & Rate & Number & Rate & Number & Rate & Number & Rate & Number & Rate & Number & Rate \\
\hline 1968 & - & - & - & - & 3 & 4.28 & & & 3 & 3.87 & 6 & 8.15 \\
\hline 1969 & - & - & - & - & 1 & 1.58 & & & 2 & 2.86 & 3 & 4.45 \\
\hline 1970 & - & - & - & - & 2 & 2.86 & & & 2 & 2.52 & 4 & 5.38 \\
\hline 1971 & 1 & 1.32 & 1 & 1.55 & 4 & 5.97 & & & - & - & 6 & 8.84 \\
\hline 1972 & - & - & 2 & 3.71 & 2 & 2.57 & & & 2 & 1.78 & 6 & 8.06 \\
\hline 1973 & - & - & 1 & 1.25 & - & - & & & 1 & 1.25 & 2 & 2.51 \\
\hline 1974 & - & - & - & - & 1 & 0.85 & & & 1 & 1.22 & 2 & 2.07 \\
\hline 1975 & - & - & 1 & 0.83 & 1 & 0.47 & & & - & - & 2 & 1.30 \\
\hline 1976 & 1 & 1.41 & - & - & 1 & 1.15 & & & - & - & 2 & 2.56 \\
\hline 1977 & 2 & 2.72 & 2 & 2.14 & - & - & & & - & - & 4 & 4.86 \\
\hline 1978 & 1 & 1.32 & - & - & 2 & 2.19 & & & - & - & 3 & 3.51 \\
\hline 1979 & 1 & 1.07 & - & - & - & - & - & - & - & - & 1 & 1.07 \\
\hline 1980 & 5 & 5.02 & - & - & - & - & - & - & 1 & 1.04 & 6 & 6.06 \\
\hline 1981 & 5 & 5.13 & - & - & - & - & - & - & - & - & 5 & 5.13 \\
\hline 1982 & 3 & 3.08 & - & - & 3 & 1.77 & - & - & 3 & 3.20 & 9 & 8.05 \\
\hline 1983 & 5 & 4.35 & - & - & 1 & 0.69 & - & - & - & - & 6 & 5.04 \\
\hline 1984 & 1 & 1.01 & - & - & 1 & 1.01 & 1 & 0.68 & 1 & 1.23 & 4 & 3.92 \\
\hline 1985 & 1 & 0.99 & - & - & 1 & 0.99 & - & - & 1 & 0.33 & 3 & 2.31 \\
\hline 1986 & 9 & 7.22 & - & - & 1 & 1.21 & - & - & - & - & 10 & 8.43 \\
\hline 1987 & 6 & 5.20 & - & - & 1 & 0.94 & - & - & 1 & 1.21 & 8 & 7.34 \\
\hline 1988 & 8 & 6.37 & 1 & 1.20 & 2 & 0.59 & - & - & - & - & 11 & 8.16 \\
\hline 1989 & 2 & 1.49 & - & - & 1 & 0.91 & - & - & - & - & 3 & 2.39 \\
\hline 1990 & 2 & 1.79 & - & - & 1 & 0.57 & - & - & - & - & 3 & 2.36 \\
\hline 1991 & 1 & 1.24 & - & - & 2 & 1.80 & 1 & 1.39 & - & - & 3 & 3.19 \\
\hline 1992 & 4 & 2.90 & - & - & 1 & 0.55 & - & - & 1 & 0.26 & 6 & 3.71 \\
\hline
\end{tabular}

- indicates no deaths listed. $\quad *$ no sp ecific code for byssinosis in ICD-8 (1968-1978)

NOTE: Rates based on small numbers may be unstable. Sum of individual conditions may exceed total because of deaths with multiple pneumoconioses. See Appendix A for source description and Appendix B for methods and ICD-8 and ICD-9 codes.

SOURCE: National Center for Health Statistics multiple cause of death data. Population estimates from U.S. Bureau of the Census. 
Figure NH-2. All pneumoconioses: Distribution of deaths by sex and race, New Hampshire residents age 15 and over, 1968-1992
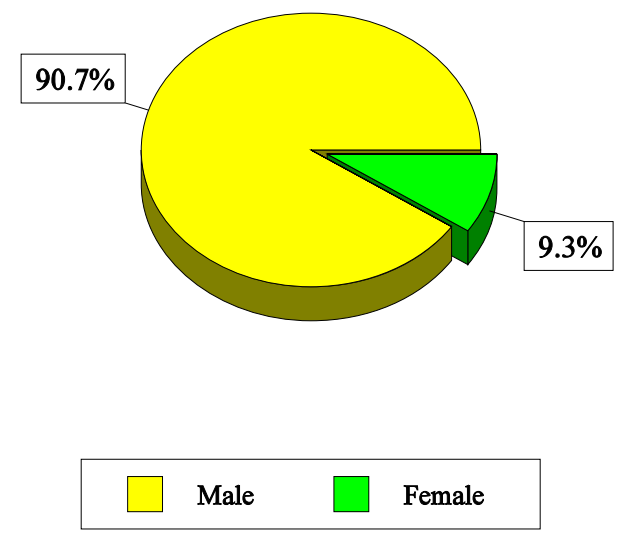
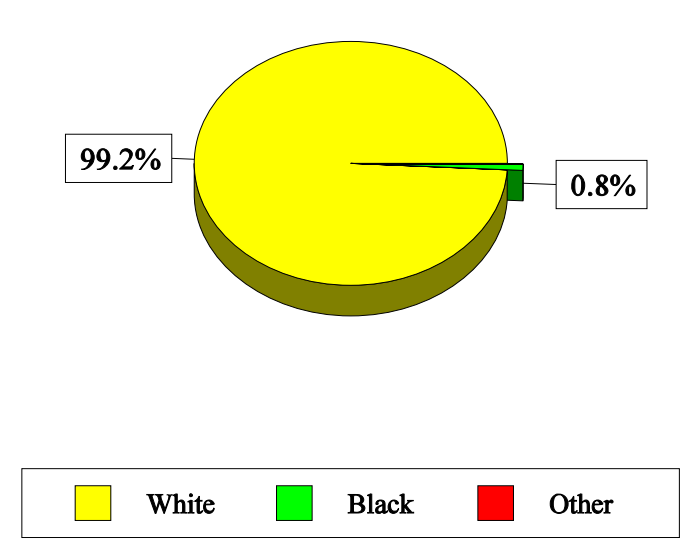

NOTE: $\quad$ See Appendix A for source description and Appendix B for methods and ICD-8 and ICD-9 codes.

SOURCE: National Center for Health Statistics multiple cause of death data.

Table NH-2. All pneumoconioses: Distribution of deaths by sex, race, and age, New Hampshire residents age 15 and over, 1968-1992

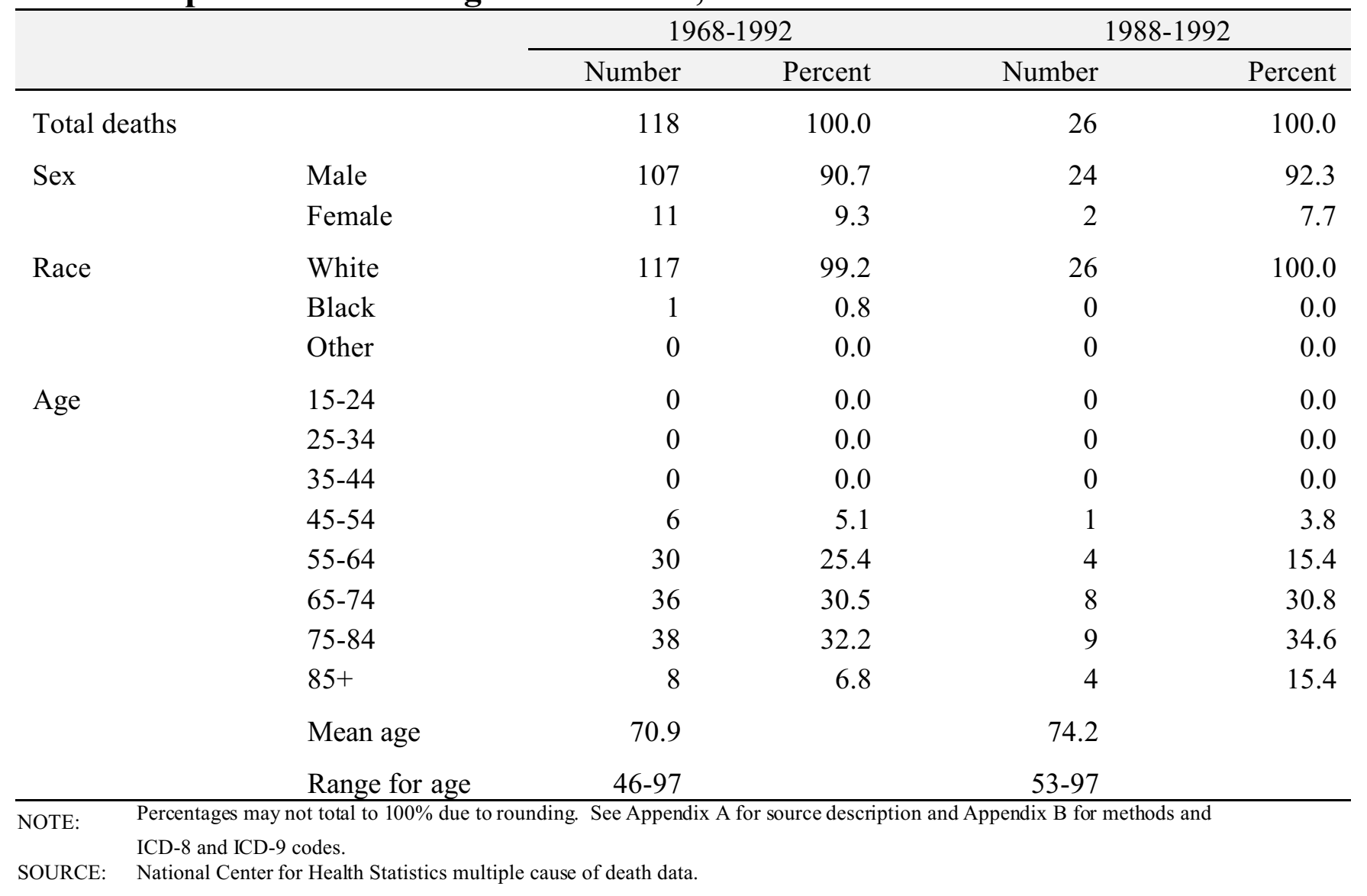


Figure NH-3. Pneumoconiosis deaths by condition, New Hampshire residents age 15 and over, 1968-1992

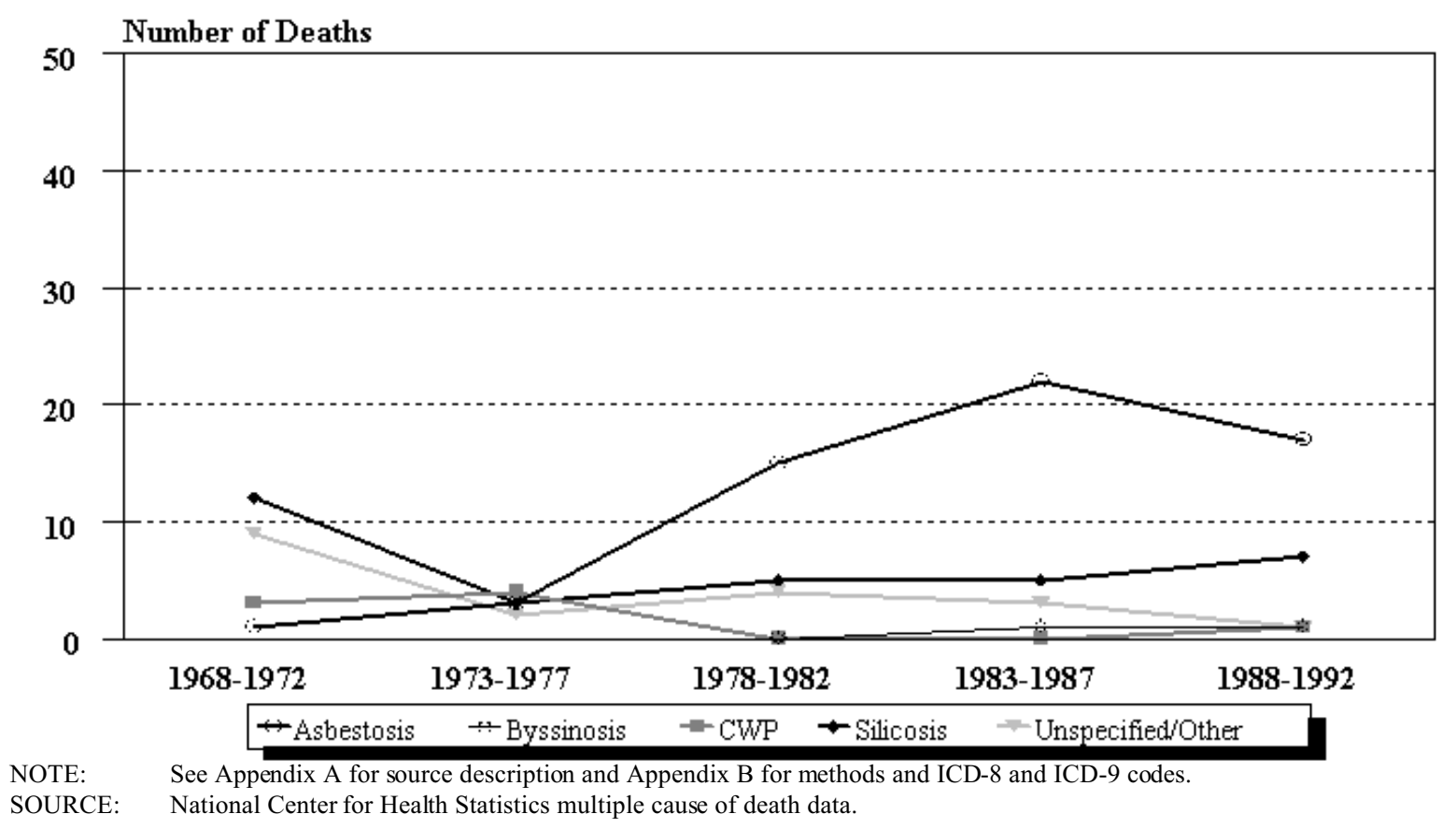

Figure NH-4. New Hampshire and U.S. age-adjusted mortality rates (per 1,000,000 population) by condition, residents age 15 and over, 1983-1992

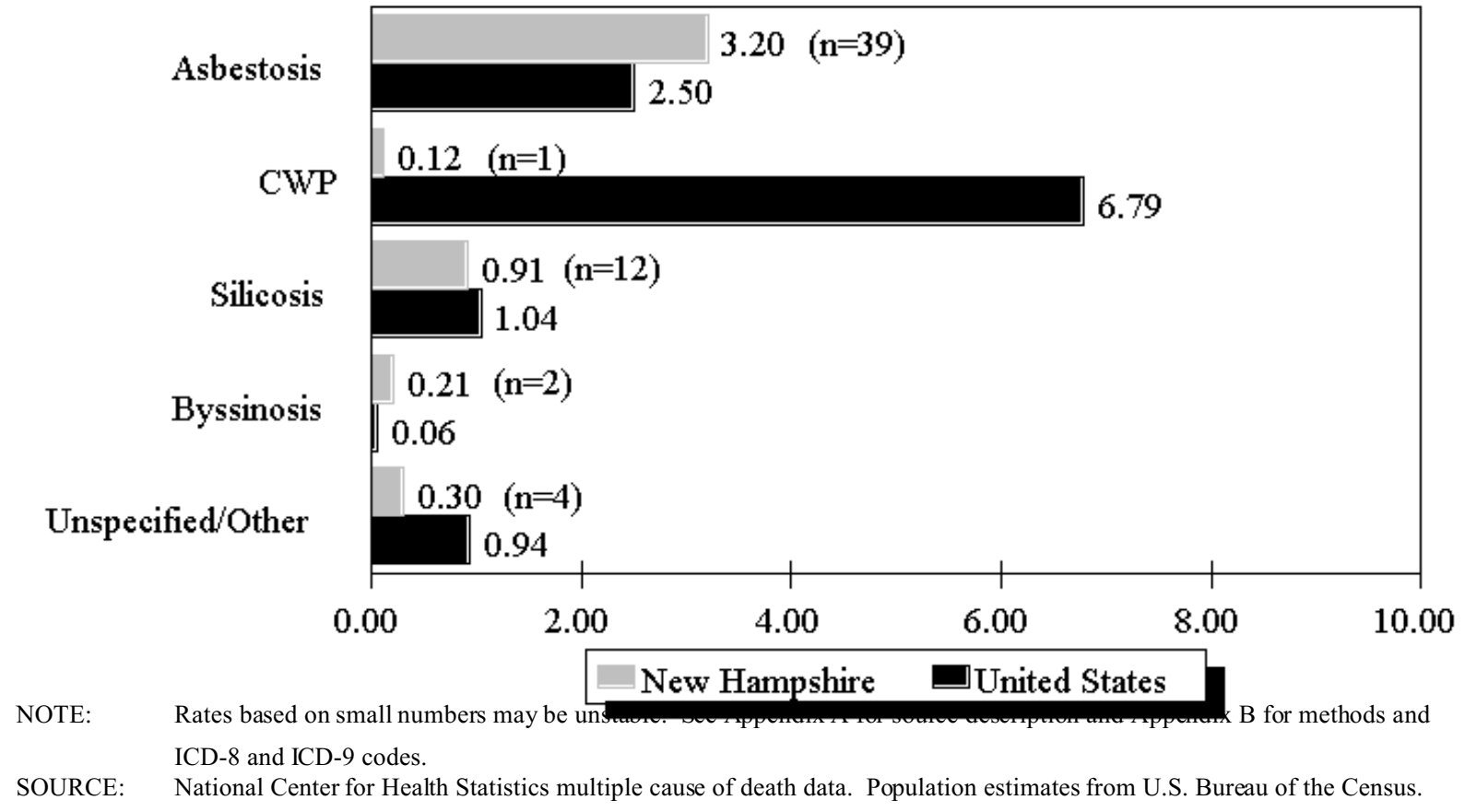


Table NH-3. Number of deaths and age-adjusted mortality rate (per 1,000,000 population) by condition and county, New Hampshire residents age 15 and over, 1983-1992

\begin{tabular}{|c|c|c|c|c|c|c|c|c|c|c|c|c|}
\hline \multirow[b]{2}{*}{ County } & \multicolumn{2}{|c|}{ Asbestosis } & \multicolumn{2}{|c|}{$\begin{array}{r}\text { Coal workers' } \\
\text { pneumoconiosis }\end{array}$} & \multicolumn{2}{|c|}{ Silicosis } & \multicolumn{2}{|c|}{ Byssinosis } & \multicolumn{2}{|c|}{$\begin{array}{r}\text { Unspecified/Other } \\
\text { pneumoconioses }\end{array}$} & \multicolumn{2}{|c|}{$\begin{array}{r}\text { All } \\
\text { pneumoconioses }\end{array}$} \\
\hline & $\#$ & Rate & \# & Rate & \# & Rate & $\#$ & Rate & \# & Rate & \# & Rate \\
\hline Belknap & 5 & 9.10 & - & - & 2 & 3.04 & 1 & 1.02 & - & - & 8 & 13.17 \\
\hline Cheshire & 3 & 3.78 & 1 & 1.53 & 1 & 0.71 & - & - & - & - & 5 & 6.02 \\
\hline Coos & 2 & 3.59 & - & - & - & - & - & - & 1 & 2.45 & 3 & 6.04 \\
\hline Grafton & 3 & 2.90 & - & - & 2 & 2.21 & - & - & 1 & 0.70 & 6 & 5.81 \\
\hline Hillsborough & 7 & 1.73 & - & - & - & - & 1 & 0.42 & 1 & 0.19 & 9 & 2.34 \\
\hline Merrimack & 2 & 1.42 & - & - & 2 & 1.42 & - & - & - & - & 4 & 2.83 \\
\hline Rockingham & 11 & 4.65 & - & - & 2 & 1.13 & - & - & - & - & 12 & 5.21 \\
\hline Strafford & 6 & 6.07 & - & - & 1 & 0.61 & - & - & 1 & 1.21 & 8 & 7.89 \\
\hline Sullivan & - & - & - & - & 2 & 3.67 & - & - & - & - & 2 & 3.67 \\
\hline
\end{tabular}

- indicates no deaths listed.

NOTE: Counties with no deaths for all listed conditions are not displayed. Rates based on small numbers may be unstable. Sum of individual conditions may exceed total because of deaths with multiple pneumoconioses. See Appendix A for source description and Appendix B for methods and ICD-8 and ICD-9 codes.

SOURCE: National Center for Health Statistics multiple cause of death data. Population estimates from U.S. Bureau of the Census. 
New Jersey

\section{New Jersey: Selected statistics from the United States section}

All pneumoconioses mortality, 1988-1992

Total deaths

Crude mortality rate (per $1,000,000$ population)

Age-adjusted mortality rate (per $1,000,000$ population)

Total years of potential life lost to life expectancy

Years of potential life lost to life expectancy (per death)

\begin{tabular}{rr} 
Value & State rank \\
\hline 643 & 7 \\
20.68 & 7 \\
11.88 & 7 \\
7,801 & 7 \\
12.1 & 25
\end{tabular}

Percent**

Deaths* distribution

473

73.6

12.3

Coal workers' pneumoconiosis

79

7.8

Silicosis

50

0.0

Byssinosis

Unspecified/Other pneumoconiosis

0

7.2

* Sum of column may exceed total deaths because of deaths with multiple pneumoconioses.

** Percentages may total more than $100 \%$ due to deaths with multiple pneumoconioses.

NOTE: $\quad$ See Appendix A for source description and Appendix B for methods and ICD-8 and ICD-9 codes.

SOURCE: National Center for Health Statistics multiple cause of death data. Population estimates from U.S. Bureau of the Census.

\section{Figure NJ-1. All pneumoconioses: New Jersey and U.S. age-adjusted mortality rates, residents age 15 and over, 1968-1992}

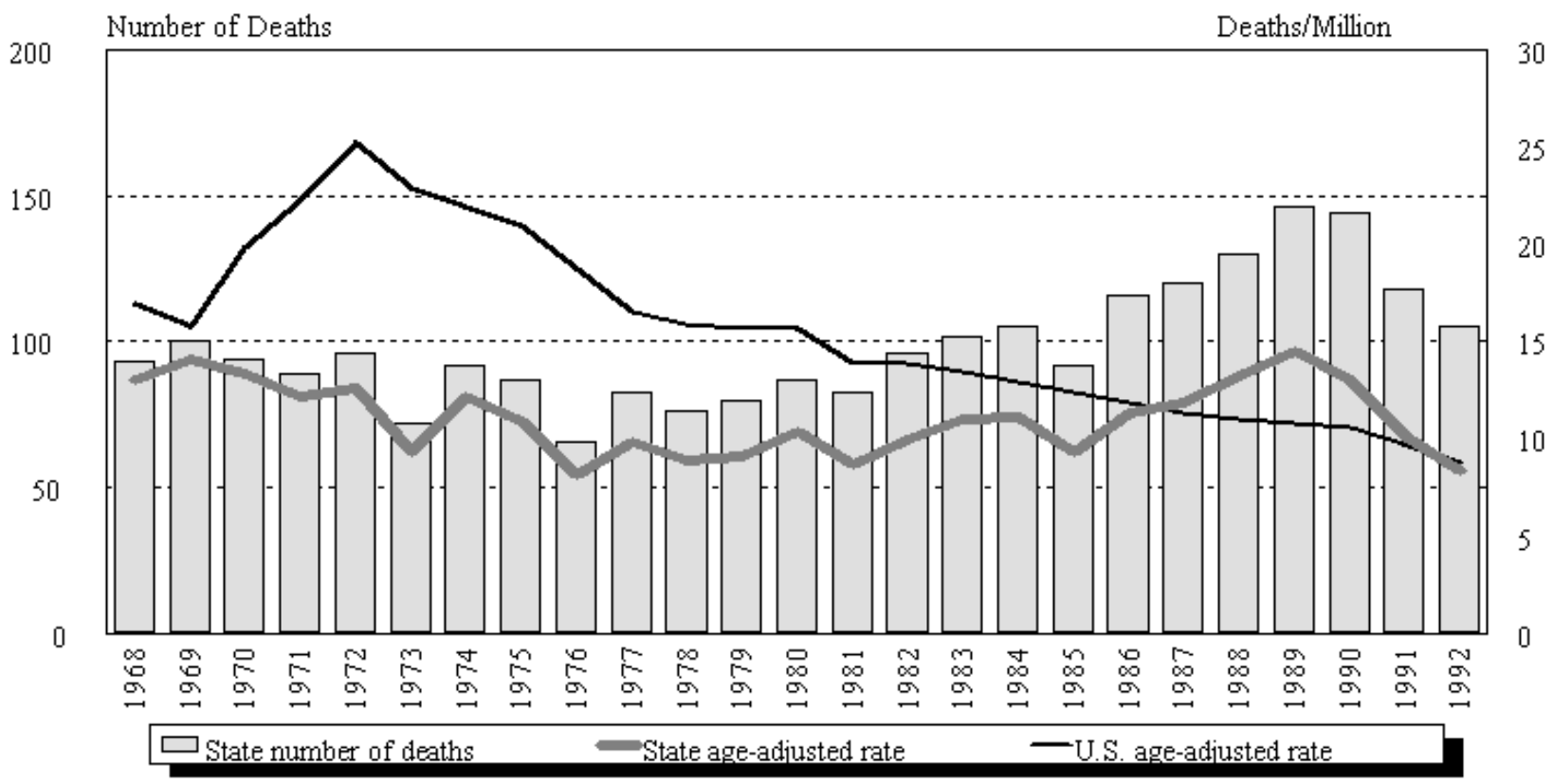

NOTE: $\quad$ See Appendix A for source description and Appendix B for methods and ICD-8 and ICD-9 codes.

SOURCE: National Center for Health Statistics multiple cause of death data. Population estimates from U.S. Bureau of the Census. 
New Jersey

\begin{tabular}{|c|c|c|c|c|c|c|c|c|c|c|c|c|}
\hline \multirow[b]{2}{*}{ Year } & \multicolumn{2}{|c|}{ Asb estosis } & \multicolumn{2}{|c|}{$\begin{array}{l}\text { Coal workers' } \\
\text { pneumocon iosis }\end{array}$} & \multicolumn{2}{|c|}{ Silicosis } & \multicolumn{2}{|c|}{ Byssinosis* } & \multicolumn{2}{|c|}{$\begin{array}{l}\text { Unspecified/Other } \\
\text { pneumoconioses }\end{array}$} & \multicolumn{2}{|c|}{ All pneu moconioses } \\
\hline & Number & Rate & Number & Rate & Number & Rate & Number & Rate & Number & Rate & Number & Rate \\
\hline 1968 & 18 & 2.81 & 24 & 3.34 & 32 & 4.41 & & & 24 & 3.20 & 93 & 13.00 \\
\hline 1969 & 18 & 2.67 & 19 & 2.63 & 32 & 4.50 & & & 36 & 4.86 & 100 & 14.05 \\
\hline 1970 & 17 & 2.60 & 23 & 2.98 & 31 & 4.30 & & & 26 & 3.90 & 94 & 13.35 \\
\hline 1971 & 15 & 2.18 & 25 & 3.13 & 29 & 3.99 & & & 21 & 3.03 & 89 & 12.20 \\
\hline 1972 & 28 & 4.00 & 22 & 2.92 & 28 & 3.50 & & & 20 & 2.48 & 96 & 12.61 \\
\hline 1973 & 14 & 1.98 & 15 & 2.00 & 26 & 3.22 & & & 19 & 2.43 & 72 & 9.38 \\
\hline 1974 & 24 & 3.32 & 29 & 3.98 & 23 & 2.96 & & & 19 & 2.20 & 92 & 12.16 \\
\hline 1975 & 18 & 2.21 & 20 & 2.67 & 22 & 2.64 & & & 29 & 3.67 & 87 & 10.91 \\
\hline 1976 & 21 & 2.75 & 18 & 1.94 & 14 & 1.84 & & & 15 & 1.93 & 66 & 8.19 \\
\hline 1977 & 26 & 3.21 & 15 & 1.92 & 18 & 2.09 & & & 24 & 2.65 & 83 & 9.87 \\
\hline 1978 & 33 & 4.10 & 19 & 2.02 & 7 & 0.91 & & & 19 & 2.13 & 76 & 8.90 \\
\hline 1979 & 34 & 4.11 & 23 & 2.62 & 8 & 0.79 & - & - & 16 & 1.72 & 80 & 9.14 \\
\hline 1980 & 46 & 5.60 & 17 & 1.98 & 13 & 1.47 & - & - & 11 & 1.28 & 87 & 10.34 \\
\hline 1981 & 43 & 4.82 & 15 & 1.38 & 13 & 1.25 & - & - & 13 & 1.37 & 83 & 8.71 \\
\hline 1982 & 52 & 5.82 & 18 & 1.59 & 10 & 1.06 & 1 & 0.05 & 15 & 1.42 & 96 & 9.94 \\
\hline 1983 & 51 & 5.67 & 21 & 2.14 & 10 & 1.17 & - & - & 20 & 2.09 & 102 & 11.07 \\
\hline 1984 & 49 & 5.53 & 22 & 2.16 & 17 & 1.72 & - & - & 18 & 1.85 & 105 & 11.14 \\
\hline 1985 & 63 & 6.69 & 11 & 0.98 & 8 & 0.70 & 1 & 0.11 & 9 & 0.80 & 92 & 9.29 \\
\hline 1986 & 82 & 8.36 & 12 & 0.82 & 9 & 0.86 & - & - & 14 & 1.34 & 116 & 11.31 \\
\hline 1987 & 80 & 8.63 & 18 & 1.36 & 12 & 1.00 & - & - & 11 & 0.96 & 120 & 11.91 \\
\hline 1988 & 83 & 8.47 & 24 & 2.55 & 12 & 0.95 & - & - & 12 & 1.37 & 130 & 13.27 \\
\hline 1989 & 102 & 10.56 & 22 & 1.97 & 12 & 0.95 & - & - & 11 & 1.09 & 146 & 14.50 \\
\hline 1990 & 115 & 10.25 & 11 & 1.05 & 10 & 0.83 & - & - & 9 & 0.97 & 144 & 13.03 \\
\hline 1991 & 93 & 8.28 & 8 & 0.50 & 11 & 0.89 & - & - & 7 & 0.50 & 118 & 10.13 \\
\hline 1992 & 80 & 6.75 & 14 & 0.84 & 5 & 0.43 & - & - & 7 & 0.50 & 105 & 8.37 \\
\hline
\end{tabular}

- indicates no deaths listed. $\quad *$ no specific code for byssinosis in ICD-8 (1968-1978).

NOTE: Rates based on small numbers may be unstable. Sum of individual conditions may exceed total because of deaths with multiple pneumoconioses. See Appendix A for source description and Appendix B for methods and ICD-8 and ICD-9 codes.

SOURCE: National Center for Health Statistics multiple cause of death data. Population estimates from U.S. Bureau of the Census. 


\section{Figure NJ-2. All pneumoconioses: Distribution of deaths by sex and race, New Jersey residents age 15 and over, 1968-1992}
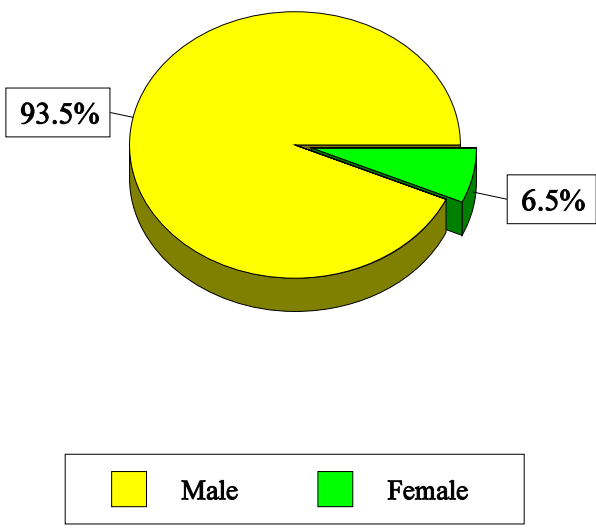
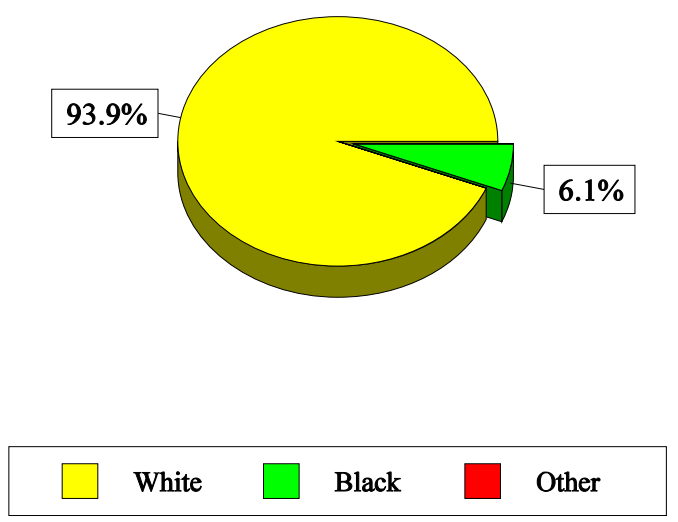

NOTE:

See Appendix A for source description and Appendix B for methods and ICD-8 and ICD-9 codes.

SOURCE: National Center for Health Statistics multiple cause of death data.

Table NJ-2. All pneumoconioses: Distribution of deaths by sex, race, and age, New Jersey residents age 15 and over, 1968-1992

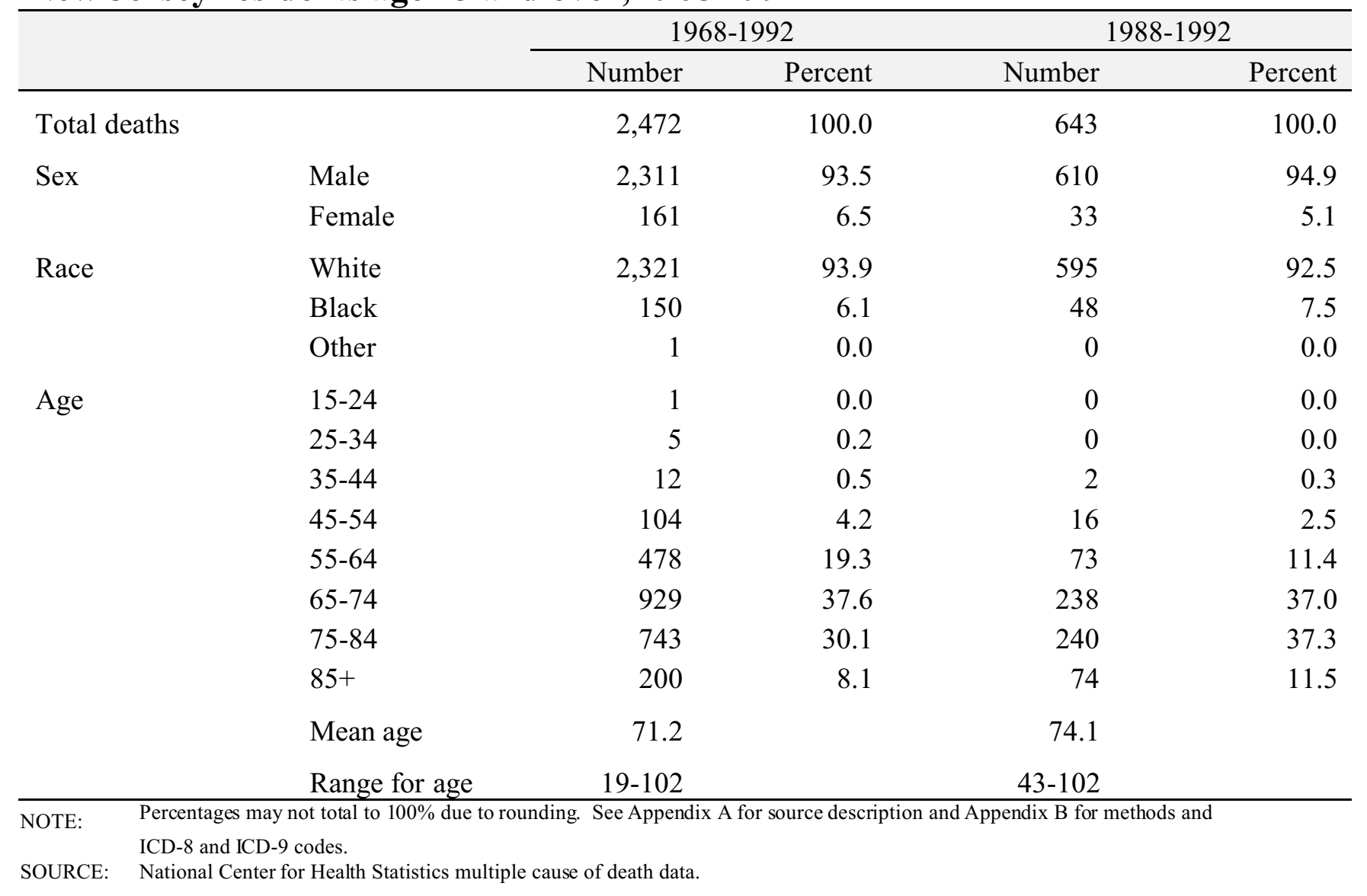


Figure NJ-3. Pneumoconiosis deaths by condition, New Jersey residents age 15 and over, 1968-1992

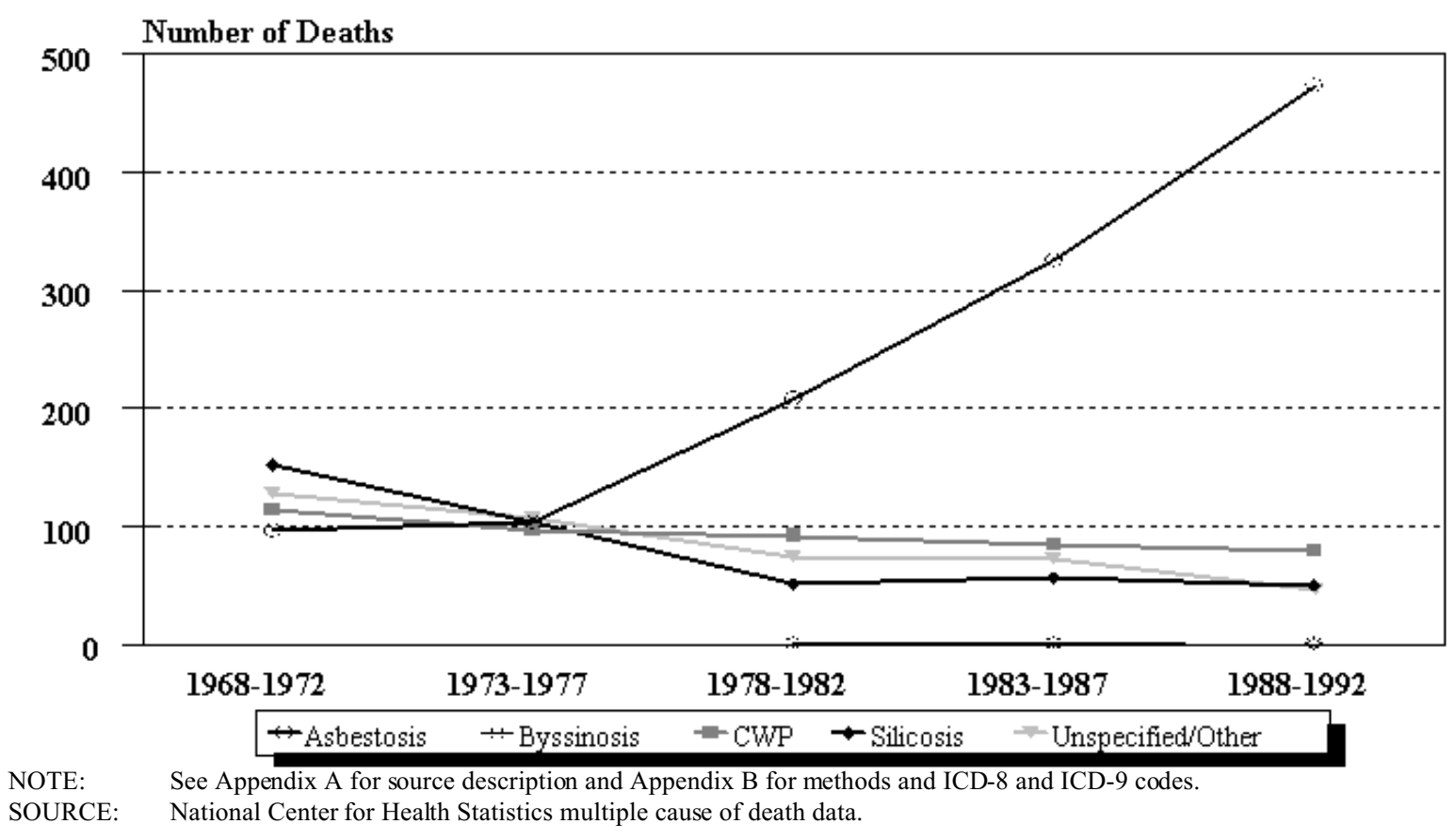

Figure NJ-4. New Jersey and U.S. age-adjusted mortality rates (per 1,000,000 population) by condition, residents age 15 and over, 1983-1992

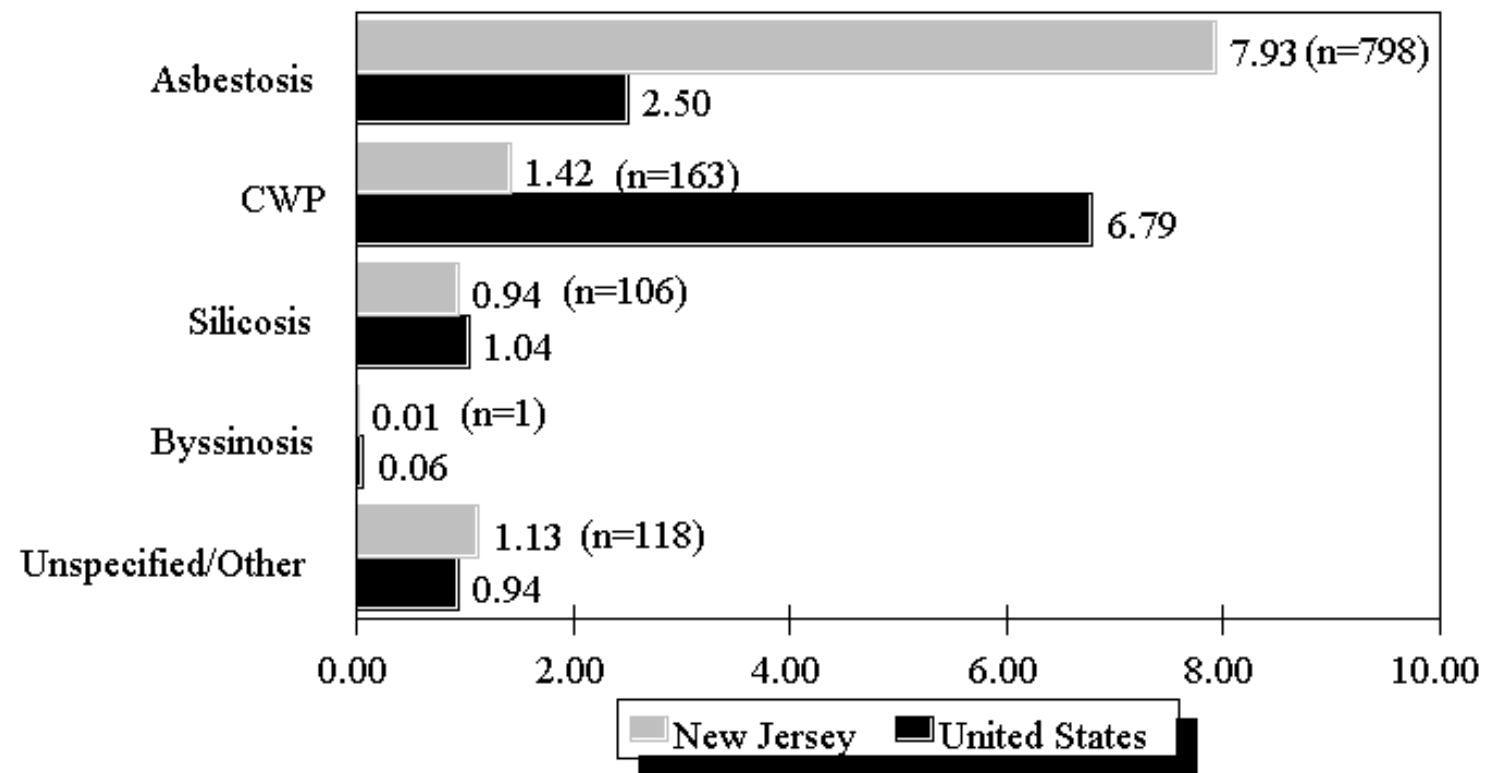

NOTE: $\quad$ Rates based on small numbers may be unstable. See Appendix A for source description and Appendix B for methods and ICD-8 and ICD-9 codes.

SOURCE: National Center for Health Statistics multiple cause of death data. Population estimates from U.S. Bureau of the Census. 
Table NJ-3. Number of deaths and age-adjusted mortality rate (per 1,000,000 population) by condition and county, New Jersey residents age 15 and over, 1983-1992

\begin{tabular}{|c|c|c|c|c|c|c|c|c|c|c|c|c|}
\hline \multirow[b]{2}{*}{ County } & \multicolumn{2}{|c|}{ Asbestosis } & \multicolumn{2}{|c|}{$\begin{array}{c}\text { Coal workers' } \\
\text { pneumoconiosis }\end{array}$} & \multicolumn{2}{|c|}{ Silicosis } & \multicolumn{2}{|c|}{ Byssinosis } & \multicolumn{2}{|c|}{$\begin{array}{r}\text { Unspecified/Other } \\
\text { pneumoconioses }\end{array}$} & \multicolumn{2}{|c|}{$\begin{array}{r}\text { All } \\
\text { pneumoconioses }\end{array}$} \\
\hline & $\#$ & Rate & \# & Rate & $\#$ & Rate & \# & Rate & $\#$ & Rate & $\#$ & Rate \\
\hline Atlantic & 21 & 5.58 & 3 & 0.83 & 2 & 0.63 & - & - & 4 & 1.74 & 29 & 8.58 \\
\hline Bergen & 25 & 2.01 & 11 & 0.69 & 4 & 0.26 & - & - & 8 & 0.53 & 48 & 3.49 \\
\hline Burlington & 41 & 10.55 & 6 & 1.31 & 3 & 0.55 & - & - & 5 & 1.03 & 55 & 13.45 \\
\hline Camden & 188 & 33.75 & 6 & 0.98 & 5 & 0.77 & - & - & 4 & 0.66 & 202 & 35.96 \\
\hline Cape May & 13 & 7.67 & 3 & 1.50 & 1 & 0.35 & - & - & - & - & 17 & 9.51 \\
\hline Cumberland & 11 & 7.64 & 3 & 1.47 & 4 & 2.56 & - & - & - & - & 18 & 11.66 \\
\hline Essex & 17 & 1.87 & 17 & 1.96 & 8 & 0.71 & - & - & 8 & 0.96 & 50 & 5.51 \\
\hline Gloucester & 65 & 28.45 & 1 & 0.32 & - & - & - & - & 1 & 0.32 & 67 & 29.08 \\
\hline Hudson & 16 & 2.42 & 13 & 2.14 & 6 & 0.82 & - & - & 4 & 0.38 & 39 & 5.76 \\
\hline Hunterdon & 10 & 9.67 & 1 & 0.67 & 1 & 1.16 & - & - & 2 & 1.35 & 14 & 12.86 \\
\hline Mercer & 10 & 2.39 & 14 & 2.91 & 15 & 2.80 & - & - & 10 & 2.39 & 48 & 10.32 \\
\hline Middlesex & 66 & 8.96 & 23 & 2.99 & 12 & 1.61 & - & - & 24 & 3.21 & 123 & 16.51 \\
\hline Monmouth & 16 & 2.59 & 10 & 1.03 & 1 & 0.19 & - & - & 3 & 0.38 & 30 & 4.18 \\
\hline Morris & 19 & 4.21 & 11 & 2.11 & 4 & 0.61 & - & - & 4 & 0.96 & 37 & 7.73 \\
\hline Ocean & 29 & 3.41 & 8 & 0.65 & 8 & 1.11 & - & - & 5 & 0.57 & 50 & 5.74 \\
\hline Passaic & 21 & 3.77 & 7 & 1.23 & 8 & 1.56 & 1 & 0.22 & 5 & 0.78 & 42 & 7.56 \\
\hline Salem & 6 & 7.24 & 1 & 0.74 & - & - & - & - & - & - & 7 & 7.98 \\
\hline Somerset & 180 & 71.40 & 11 & 4.16 & 10 & 3.54 & - & - & 14 & 5.84 & 213 & 84.36 \\
\hline Sussex & 2 & 1.66 & - & - & 1 & 0.55 & - & - & - & - & 3 & 2.22 \\
\hline Union & 40 & 4.93 & 9 & 0.92 & 7 & 0.89 & - & - & 12 & 1.44 & 68 & 8.17 \\
\hline Warren & 2 & 2.62 & 5 & 4.50 & 6 & 5.06 & - & - & 5 & 5.07 & 18 & 17.25 \\
\hline
\end{tabular}




\section{New Mexico: Selected statistics from the United States section}

All pneumoconioses mortality, 1988-1992

Total deaths

Crude mortality rate (per $1,000,000$ population)

Age-adjusted mortality rate (per $1,000,000$ population)

Total years of potential life lost to life expectancy

Years of potential life lost to life expectancy (per death)

Deaths by type of pneumoconiosis, 1988-1992

Asbestosis

Coal workers' pneumoconiosis

Silicosis

Byssinosis

Unspecified/Other pneumoconiosis

\begin{tabular}{rr} 
Value & State rank \\
\hline 64 & 34 \\
11.25 & 19 \\
6.52 & 19 \\
678 & 37 \\
10.6 & 45
\end{tabular}

Percent**

Deaths* distribution

20.3

25.0

32.8

0.0

21.9

* Sum of column may exceed total deaths because of deaths with multiple pneumoconioses.

** Percentages may total more than $100 \%$ due to deaths with multiple pneumoconioses.

NOTE: $\quad$ See Appendix A for source description and Appendix B for methods and ICD-8 and ICD-9 codes.

SOURCE: $\quad$ National Center for Health Statistics multiple cause of death data. Population estimates from U.S. Bureau of the Census.

Figure NM-1. All pneumoconioses: New Mexico and U.S. age-adjusted mortality rates, residents age 15 and over, 1968-1992

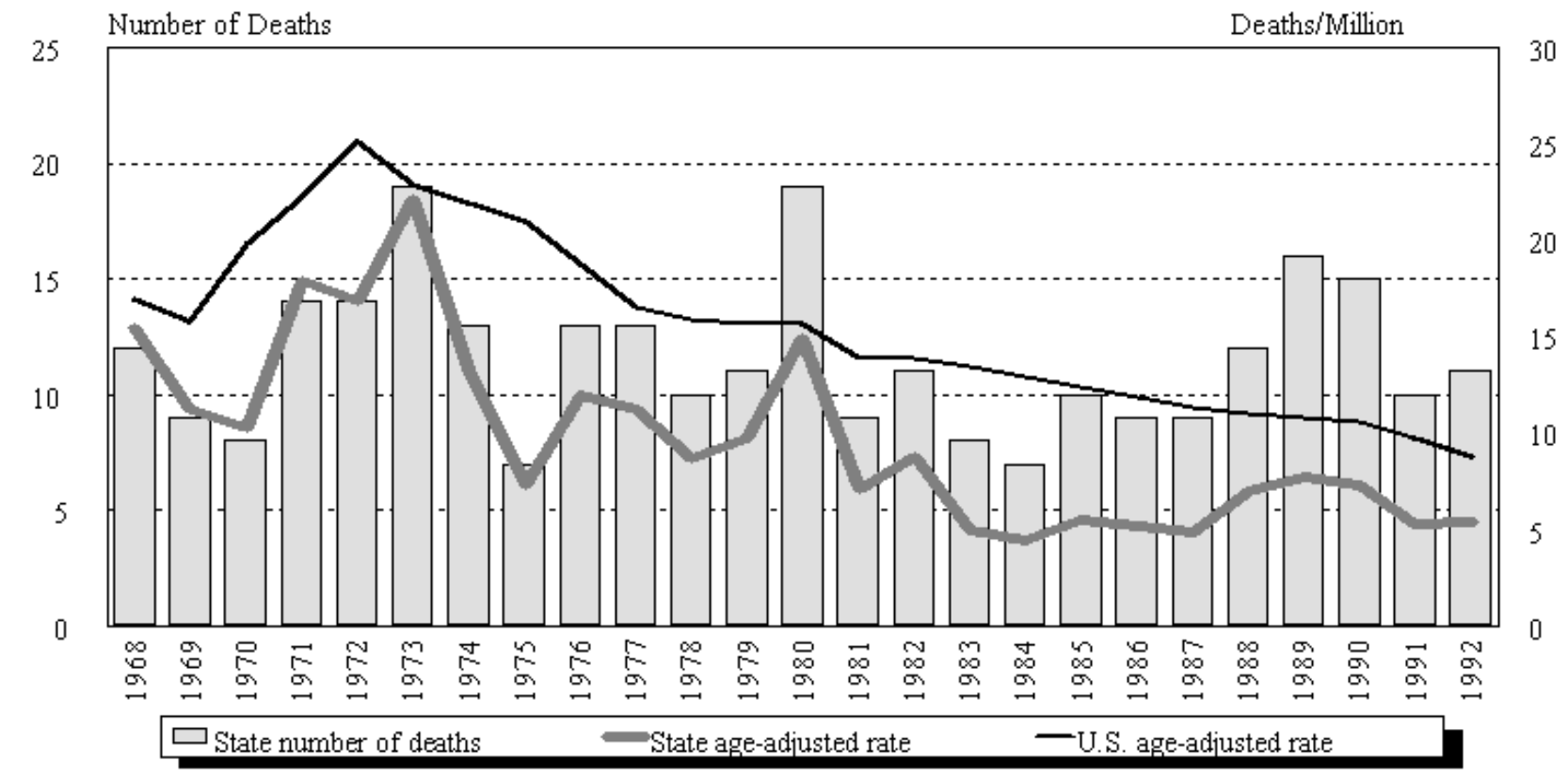

NOTE: $\quad$ See Appendix A for source description and Appendix B for methods and ICD-8 and ICD-9 codes.

SOURCE: National Center for Health Statistics multiple cause of death data. Population estimates from U.S. Bureau of the Census. 
New Mexico

Table NM-1. Number of deaths and age-adjusted mortality rate (per 1,000,000 population) by condition, New Mexico residents age 15 and over, 1968-1992

\begin{tabular}{|c|c|c|c|c|c|c|c|c|c|c|c|c|}
\hline \multirow[b]{2}{*}{ Year } & \multicolumn{2}{|c|}{ Asbestosis } & \multicolumn{2}{|c|}{$\begin{array}{l}\text { Coal workers' } \\
\text { pneumoconiosis }\end{array}$} & \multicolumn{2}{|c|}{ Silicosis } & \multicolumn{2}{|c|}{ Byssinosis* } & \multicolumn{2}{|c|}{$\begin{array}{c}\text { Unspecified/Other } \\
\text { pneumoconioses }\end{array}$} & \multicolumn{2}{|c|}{ All pneumoconioses } \\
\hline & Number & Rate & Number & Rate & Number & Rate & Number & Rate & Number & Rate & Number & Rate \\
\hline 1968 & - & - & - & & 6 & 7.29 & & & 6 & 8.15 & 12 & 15.44 \\
\hline 1969 & - & - & - & - & 7 & 8.67 & & & 2 & 2.53 & 9 & 11.20 \\
\hline 1970 & - & - & 1 & 1.11 & 4 & 5.76 & & & 3 & 3.44 & 8 & 10.31 \\
\hline 1971 & - & - & 3 & 3.73 & 7 & 9.34 & & & 4 & 4.79 & 14 & 17.86 \\
\hline 1972 & - & - & 2 & 2.52 & 8 & 9.76 & & & 4 & 4.54 & 14 & 16.82 \\
\hline 1973 & 1 & 1.43 & 3 & 3.20 & 6 & 6.41 & & & 9 & 11.12 & 19 & 22.16 \\
\hline 1974 & - & - & 4 & 4.21 & 5 & 5.00 & & & 4 & 4.18 & 13 & 13.39 \\
\hline 1975 & - & - & 1 & 1.08 & 3 & 3.04 & & & 4 & 4.22 & 7 & 7.26 \\
\hline 1976 & - & - & 4 & 3.76 & 5 & 5.07 & & & 4 & 3.11 & 13 & 11.95 \\
\hline 1977 & - & - & 3 & 2.41 & 3 & 2.77 & & & 7 & 6.03 & 13 & 11.22 \\
\hline 1978 & 3 & 2.64 & 2 & 1.71 & 3 & 2.77 & & & 2 & 1.56 & 10 & 8.67 \\
\hline 1979 & - & - & 6 & 5.44 & 3 & 2.81 & - & - & 2 & 1.46 & 11 & 9.70 \\
\hline 1980 & 1 & 0.71 & 2 & 1.57 & 10 & 8.25 & - & - & 7 & 5.25 & 19 & 14.92 \\
\hline 1981 & 1 & 0.67 & 2 & 1.64 & 4 & 3.76 & - & - & 2 & 1.06 & 9 & 7.12 \\
\hline 1982 & 1 & 0.94 & 4 & 3.00 & 3 & 3.02 & - & - & 3 & 1.80 & 11 & 8.76 \\
\hline 1983 & 1 & 0.59 & 6 & 3.75 & 1 & 0.59 & - & - & - & - & 8 & 4.94 \\
\hline 1984 & - & - & 4 & 2.45 & 2 & 1.46 & - & - & 1 & 0.57 & 7 & 4.48 \\
\hline 1985 & 1 & 0.89 & 5 & 2.90 & 2 & 1.08 & - & - & 2 & 0.63 & 10 & 5.49 \\
\hline 1986 & 1 & 0.52 & 4 & 2.26 & 2 & 1.39 & - & - & 2 & 1.03 & 9 & 5.20 \\
\hline 1987 & 4 & 2.57 & 2 & 0.79 & 1 & 0.70 & - & - & 2 & 0.79 & 9 & 4.84 \\
\hline 1988 & 2 & 1.57 & 2 & 0.96 & 2 & 0.97 & - & - & 6 & 3.49 & 12 & 6.99 \\
\hline 1989 & 5 & 2.57 & 4 & 2.28 & 4 & 1.49 & - & - & 3 & 1.39 & 16 & 7.73 \\
\hline 1990 & 2 & 1.11 & 3 & 1.16 & 6 & 2.57 & - & - & 4 & 2.44 & 15 & 7.28 \\
\hline 1991 & 3 & 1.96 & 3 & 1.12 & 4 & 2.17 & - & - & - & - & 10 & 5.25 \\
\hline 1992 & 1 & 0.64 & 4 & 1.96 & 5 & 2.33 & - & - & 1 & 0.42 & 11 & 5.35 \\
\hline
\end{tabular}

indicates no deaths listed. * no specific code for byssinosis in ICD-8 (1968-1978).
NOTE: Rates based on small numbers may be unstable. Sum of individual conditions may exceed total because of deaths with multiple pneumoconioses. See Appendix A for source description and Appendix B for methods and ICD-8 and ICD-9 NOTE:

SOURCE: National Center for Health Statistics multiple cause of death data. Population estimates from U.S. Bureau of the Census. 
Figure NM-2. All pneumoconioses: Distribution of deaths by sex and race, New Mexico residents age 15 and over, 1968-1992
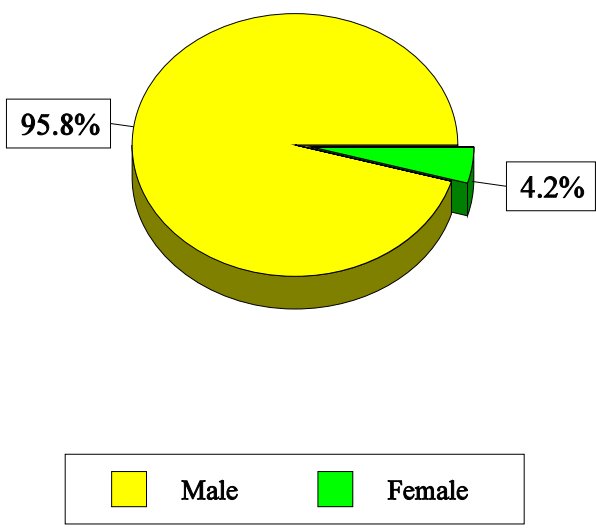
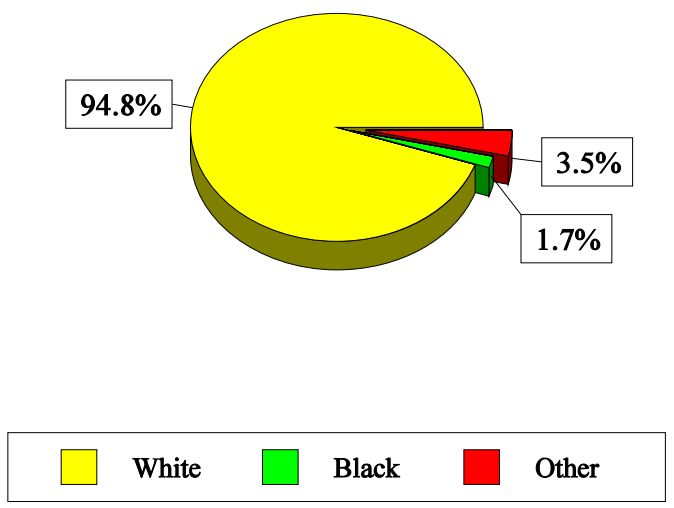

NOTE:

See Appendix A for source description and Appendix B for methods and ICD-8 and ICD-9 codes.

SOURCE: National Center for Health Statistics multiple cause of death data.

Table NM-2. All pneumoconioses: Distribution of deaths by sex, race, and age, New Mexico residents age 15 and over, 1968-1992

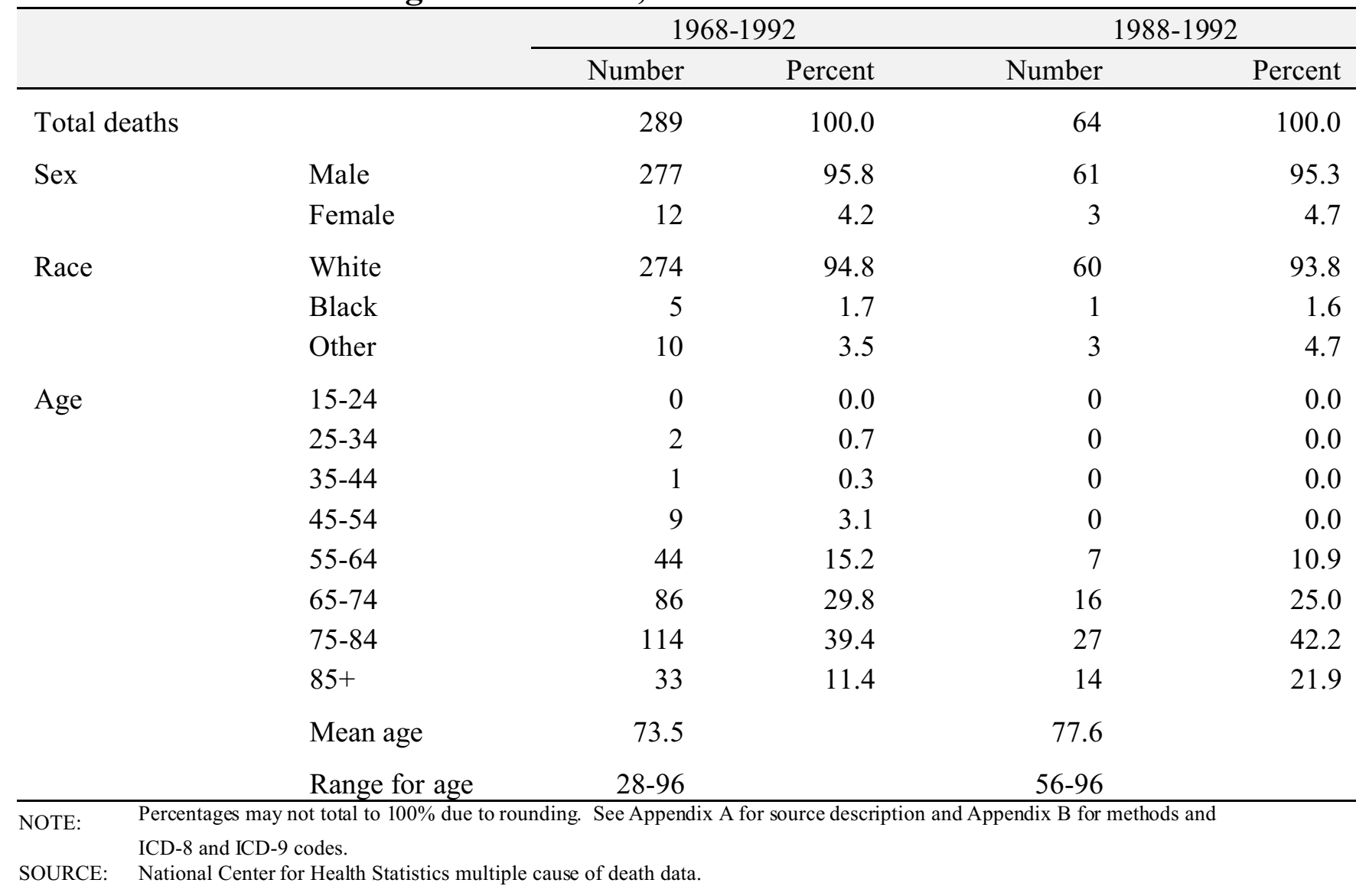


Figure NM-3. Pneumoconiosis deaths by condition, New Mexico residents age 15 and over, 1968-1992

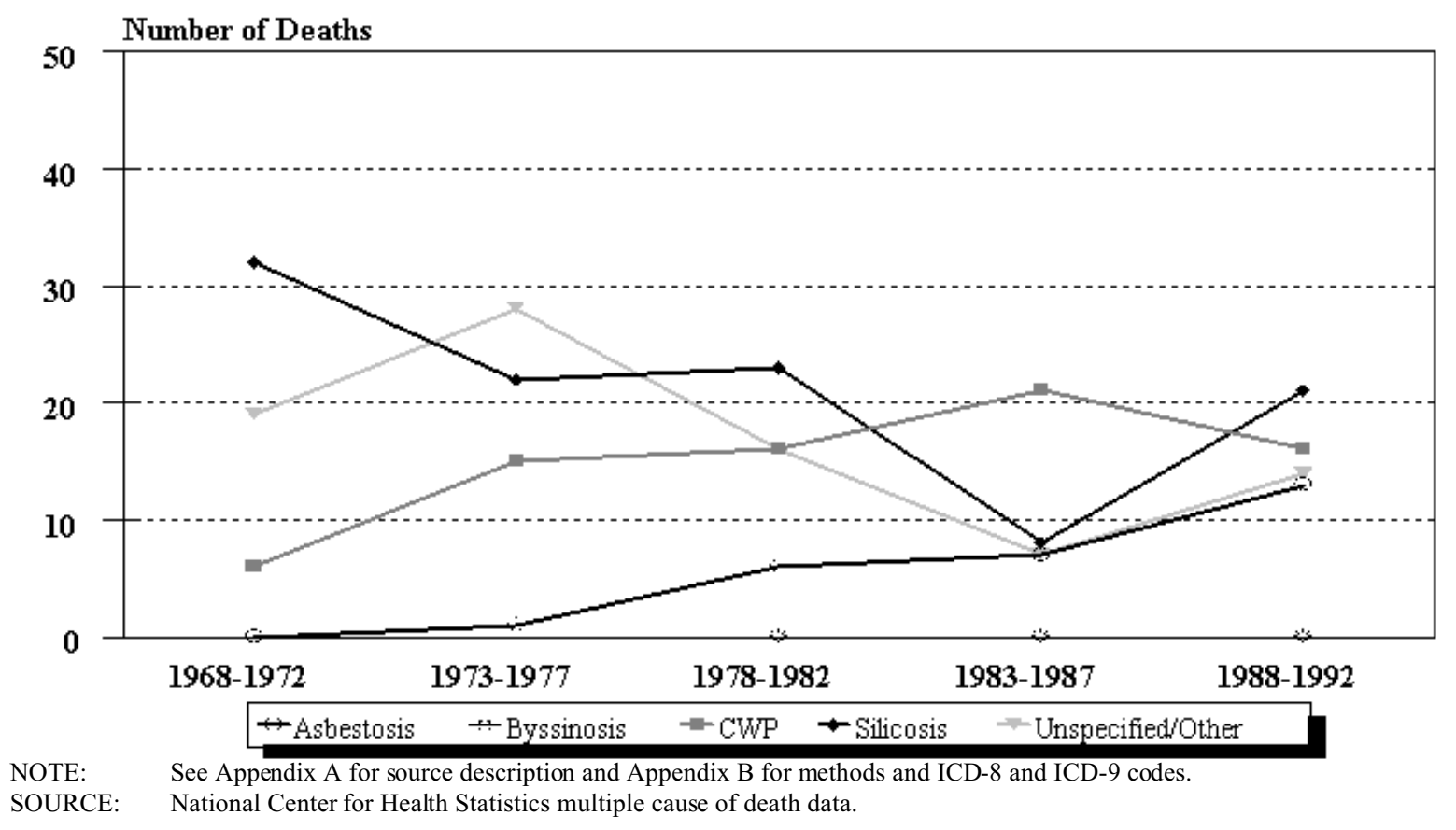

Figure NM-4. New Mexico and U.S. age-adjusted mortality rates (per 1,000,000 population) by condition, residents age 15 and over, 1983-1992

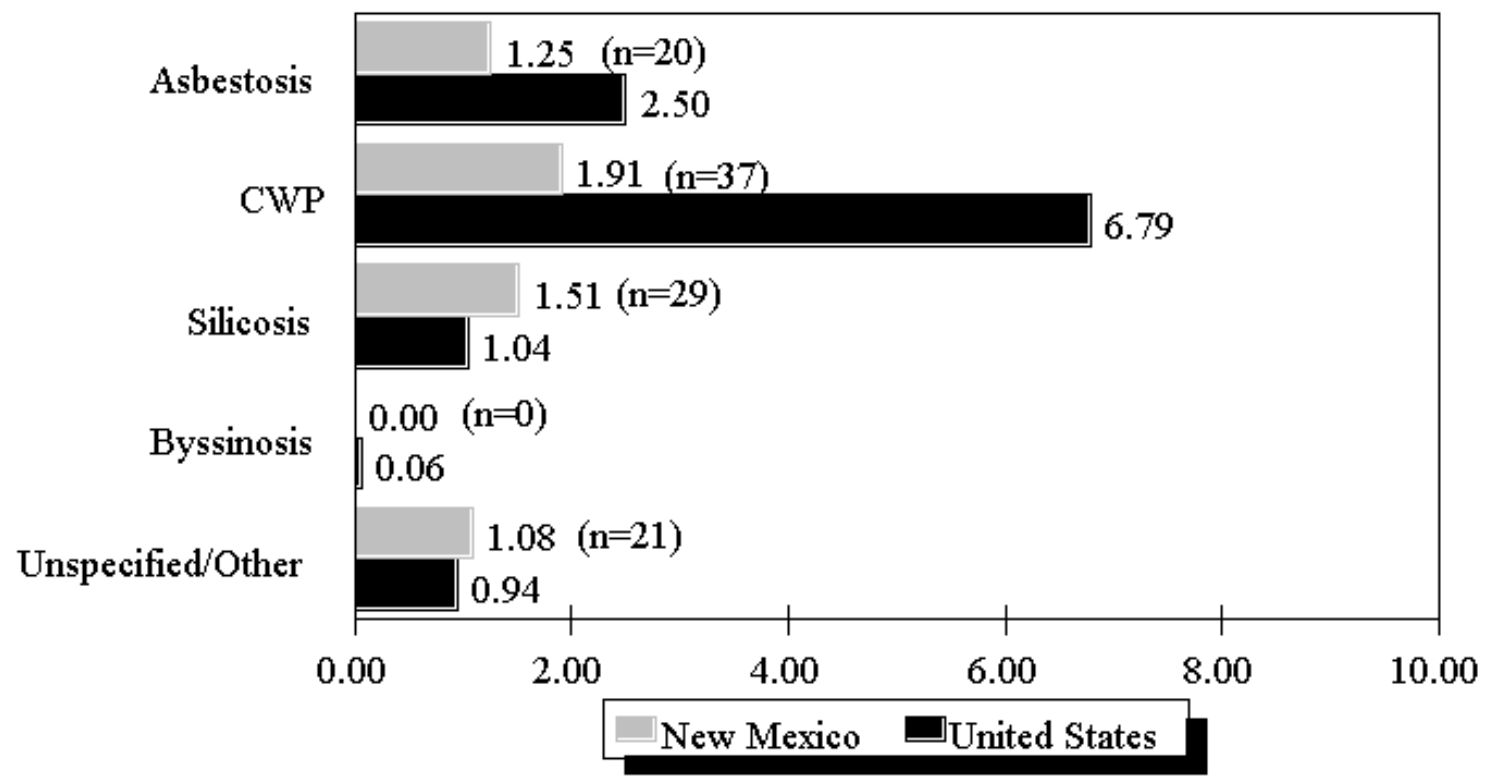

NOTE: $\quad$ Rates based on small numbers may be unstable. See Appendix A for source description and Appendix B for methods and SOURCE: National Center for Health Statistics multiple cause of death data. Population estimates from U.S. Bureau of the Census. 
Table NM-3. Number of deaths and age-adjusted mortality rate (per 1,000,000 population) by condition and county, New Mexico residents age 15 and over, 1983-1992

\begin{tabular}{|c|c|c|c|c|c|c|c|c|c|c|c|c|}
\hline \multirow[b]{2}{*}{ County } & \multicolumn{2}{|c|}{ Asbestosis } & \multicolumn{2}{|c|}{$\begin{array}{c}\text { Coal workers' } \\
\text { pneumoconiosis }\end{array}$} & \multicolumn{2}{|c|}{ Silicosis } & \multicolumn{2}{|c|}{ Byssinosis } & \multicolumn{2}{|c|}{$\begin{array}{r}\text { Unspecified/Other } \\
\text { pneumoconioses }\end{array}$} & \multicolumn{2}{|c|}{$\begin{array}{r}\text { All } \\
\text { pneumoconioses }\end{array}$} \\
\hline & $\#$ & Rate & $\#$ & Rate & $\#$ & Rate & $\#$ & Rate & \# & Rate & $\#$ & Rate \\
\hline Bernalillo & 5 & 1.18 & 9 & 1.56 & 6 & 1.11 & - & - & 1 & 0.15 & 21 & 4.00 \\
\hline Catron & 1 & 32.58 & - & - & - & - & - & - & - & - & 1 & 32.58 \\
\hline Chaves & - & - & 1 & 0.78 & 1 & 1.70 & - & - & - & - & 2 & 2.49 \\
\hline Cibola & 1 & 3.60 & - & - & - & - & - & - & - & - & 1 & 3.60 \\
\hline Colfax & - & - & 7 & 28.32 & 1 & 2.96 & - & - & 3 & 8.89 & 11 & 40.17 \\
\hline Curry & - & - & - & - & - & - & - & - & 1 & 2.99 & 1 & 2.99 \\
\hline Dona Ana & 1 & 0.64 & - & - & 2 & 1.28 & - & - & 3 & 2.68 & 6 & 4.60 \\
\hline Eddy & 4 & 4.45 & 5 & 5.29 & - & - & - & - & 1 & 0.84 & 10 & 10.58 \\
\hline Grant & - & - & - & - & 3 & 5.65 & - & - & 1 & 1.88 & 4 & 7.53 \\
\hline Lea & - & - & - & - & 1 & 1.18 & - & - & 1 & 1.18 & 2 & 2.37 \\
\hline Luna & 1 & 4.03 & - & - & 3 & 8.12 & - & - & - & - & 4 & 12.16 \\
\hline McKinley & - & - & 3 & 6.76 & - & - & - & - & - & - & 3 & 6.76 \\
\hline Mora & - & - & 1 & 9.36 & - & - & - & - & - & - & 1 & 9.36 \\
\hline Otero & - & - & - & - & 1 & 2.50 & - & - & - & - & 1 & 2.50 \\
\hline Rio Arriba & - & - & - & - & 3 & 7.97 & - & - & - & - & 3 & 7.97 \\
\hline Sandoval & 1 & 2.25 & 1 & 1.36 & - & - & - & - & - & - & 2 & 3.61 \\
\hline San Juan & 1 & 1.60 & 3 & 3.77 & 3 & 3.77 & - & - & - & - & 7 & 9.14 \\
\hline San Miguel & 1 & 2.07 & 1 & 4.75 & - & - & - & - & 1 & 2.07 & 3 & 8.90 \\
\hline Santa Fe & 1 & 1.30 & 5 & 4.26 & 2 & 1.48 & - & - & 8 & 7.04 & 16 & 14.08 \\
\hline Sierra & 1 & 5.44 & - & - & - & - & - & - & - & - & 1 & 5.44 \\
\hline Socorro & 1 & 8.48 & - & - & 1 & 4.63 & - & - & - & - & 2 & 13.11 \\
\hline Union & - & - & 1 & 7.20 & 1 & 19.06 & - & - & - & - & 2 & 26.26 \\
\hline Valencia & 1 & 1.83 & - & - & - & - & - & - & - & - & 1 & 1.83 \\
\hline
\end{tabular}




\section{New York: Selected statistics from the United States section}

All pneumoconioses mortality, 1988-1992

Total deaths

Crude mortality rate (per $1,000,000$ population)

Age-adjusted mortality rate (per $1,000,000$ population)

Total years of potential life lost to life expectancy

Years of potential life lost to life expectancy (per death) $\frac{\text { Value }}{320}$

4.45

2.56

3,913

12.2

$\underline{\text { Deaths* }}$

172

41

67

0

43 $\underline{\text { State rank }}$

11

44

46

11

23

Percent**

distribution

53.8

12.8

20.9

0.0

13.4

\section{Unspecified/Other pneumoconiosis}

* Sum of column may exceed total deaths because of deaths with multiple pneumoconioses.

** Percentages may total more than $100 \%$ due to deaths with multiple pneumoconioses.

NOTE: $\quad$ See Appendix A for source description and Appendix B for methods and ICD-8 and ICD-9 codes.

SOURCE: National Center for Health Statistics multiple cause of death data. Population estimates from U.S. Bureau of the Census.

Figure NY-1. All pneumoconioses: New York and U.S. age-adjusted mortality rates, residents age 15 and over, 1968-1992

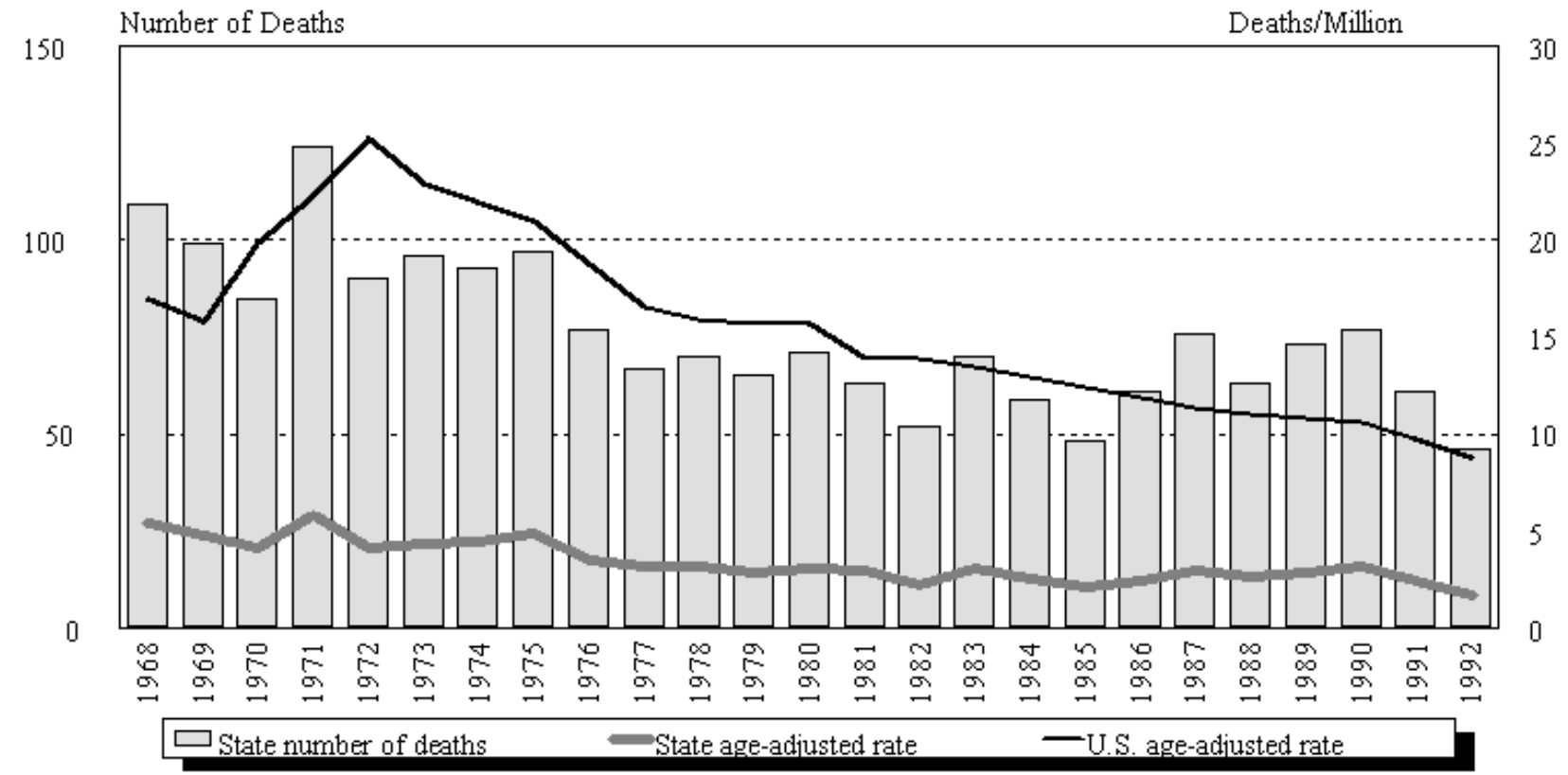

NOTE: $\quad$ See Appendix A for source description and Appendix B for methods and ICD-8 and ICD-9 codes.

SOURCE: National Center for Health Statistics multiple cause of death data. Population estimates from U.S. Bureau of the Census. 
New York

\begin{tabular}{|c|c|c|c|c|c|c|c|c|c|c|c|c|}
\hline \multirow[b]{2}{*}{ Year } & \multicolumn{2}{|c|}{ Asbestosis } & \multicolumn{2}{|c|}{$\begin{array}{l}\text { Coal workers' } \\
\text { pne umocon iosis }\end{array}$} & \multicolumn{2}{|c|}{ Silicosis } & \multicolumn{2}{|c|}{ Byssinosis* } & \multicolumn{2}{|c|}{$\begin{array}{c}\text { Unspecified/Other } \\
\text { pneumoconioses }\end{array}$} & \multicolumn{2}{|c|}{ All pneumoconioses } \\
\hline & Number & Rate & Number & Rate & Number & Rate & Number & Rate & Number & Rate & Number & Rate \\
\hline 1968 & 8 & 0.44 & 18 & 0.85 & 59 & 2.91 & & & 27 & 1.31 & 109 & 5.39 \\
\hline 1969 & 4 & 0.22 & 12 & 0.58 & 56 & 2.63 & & & 31 & 1.50 & 99 & 4.73 \\
\hline 1970 & 7 & 0.35 & 17 & 0.82 & 44 & 2.13 & & & 20 & 0.95 & 85 & 4.13 \\
\hline 1971 & 14 & 0.78 & 17 & 0.78 & 57 & 2.63 & & & 37 & 1.75 & 124 & 5.88 \\
\hline 1972 & 4 & 0.18 & 22 & 1.02 & 42 & 1.94 & & & 24 & 1.06 & 90 & 4.13 \\
\hline 1973 & 8 & 0.40 & 9 & 0.42 & 46 & 2.11 & & & 37 & 1.68 & 96 & 4.39 \\
\hline 1974 & 6 & 0.28 & 18 & 0.80 & 38 & 1.83 & & & 35 & 1.68 & 93 & 4.44 \\
\hline 1975 & 9 & 0.46 & 18 & 0.93 & 27 & 1.25 & & & 43 & 2.24 & 97 & 4.89 \\
\hline 1976 & 11 & 0.53 & 17 & 0.83 & 21 & 0.87 & & & 30 & 1.37 & 77 & 3.51 \\
\hline 1977 & 6 & 0.37 & 10 & 0.45 & 31 & 1.41 & & & 22 & 1.05 & 67 & 3.19 \\
\hline 1978 & 14 & 0.71 & 9 & 0.39 & 26 & 1.18 & & & 21 & 0.94 & 70 & 3.23 \\
\hline 1979 & 12 & 0.61 & 16 & 0.75 & 23 & 0.94 & - & - & 15 & 0.56 & 65 & 2.83 \\
\hline 1980 & 13 & 0.64 & 24 & 0.93 & 17 & 0.84 & - & - & 18 & 0.77 & 71 & 3.12 \\
\hline 1981 & 12 & 0.69 & 16 & 0.67 & 20 & 0.99 & - & - & 15 & 0.61 & 63 & 2.96 \\
\hline 1982 & 16 & 0.71 & 15 & 0.63 & 11 & 0.43 & - & - & 10 & 0.42 & 52 & 2.19 \\
\hline 1983 & 25 & 1.18 & 15 & 0.64 & 22 & 0.91 & - & - & 8 & 0.35 & 70 & 3.09 \\
\hline 1984 & 24 & 1.13 & 6 & 0.23 & 17 & 0.74 & - & - & 12 & 0.50 & 59 & 2.60 \\
\hline 1985 & 10 & 0.49 & 11 & 0.46 & 16 & 0.72 & - & - & 11 & 0.45 & 48 & 2.11 \\
\hline 1986 & 25 & 1.02 & 16 & 0.59 & 9 & 0.38 & 1 & 0.03 & 10 & 0.37 & 61 & 2.39 \\
\hline 1987 & 30 & 1.24 & 13 & 0.48 & 27 & 0.99 & - & - & 9 & 0.39 & 76 & 3.00 \\
\hline 1988 & 29 & 1.24 & 13 & 0.46 & 12 & 0.59 & - & - & 9 & 0.37 & 63 & 2.65 \\
\hline 1989 & 32 & 1.32 & 13 & 0.36 & 16 & 0.69 & - & - & 12 & 0.49 & 73 & 2.86 \\
\hline 1990 & 44 & 1.98 & 9 & 0.30 & 15 & 0.60 & - & - & 10 & 0.36 & 77 & 3.21 \\
\hline 1991 & 37 & 1.50 & 4 & 0.12 & 18 & 0.69 & - & - & 3 & 0.16 & 61 & 2.40 \\
\hline 1992 & 30 & 1.07 & 2 & 0.04 & 6 & 0.26 & - & - & 9 & 0.35 & 46 & 1.67 \\
\hline
\end{tabular}

- indicates no deaths listed. $\quad *$ no sp ecific code for byssinosis in ICD-8 (1968-1978).

NOTE: Rates based on small numbers may be unstable. Sum of individual conditions may exceed total because of deaths with multiple pneumoconioses. See Appendix A for source description and Appendix B for methods and ICD-8 and ICD-9 codes.

SOURCE: National Center for Health Statistics multiple cause of death data. Population estimates from U.S. Bureau of the Census. 
Figure NY-2. All pneumoconioses: Distribution of deaths by sex and race, New York residents age 15 and over, 1968-1992
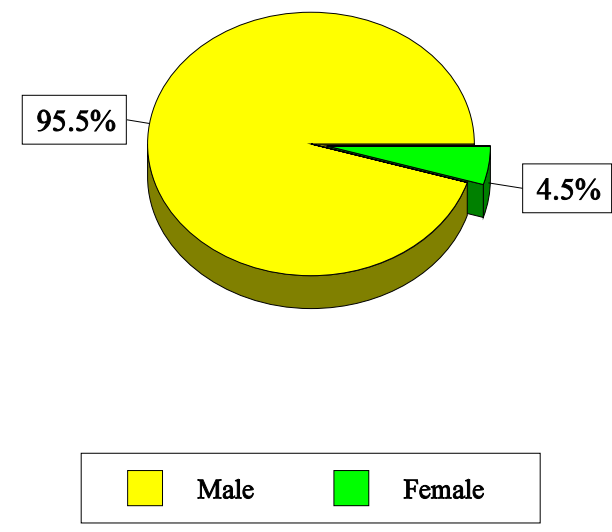

\section{$88.4 \%$}
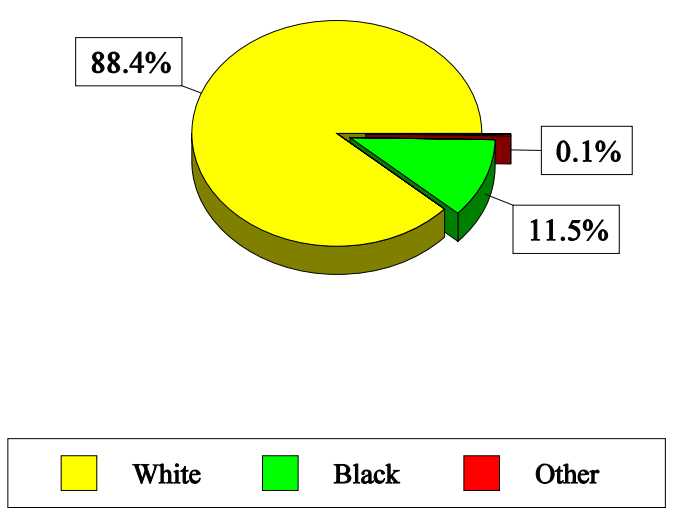

NOTE:

See Appendix A for source description and Appendix B for methods and ICD-8 and ICD-9 codes.

SOURCE: National Center for Health Statistics multiple cause of death data.

Table NY-2. All pneumoconioses: Distribution of deaths by sex, race, and age, New York residents age 15 and over, 1968-1992

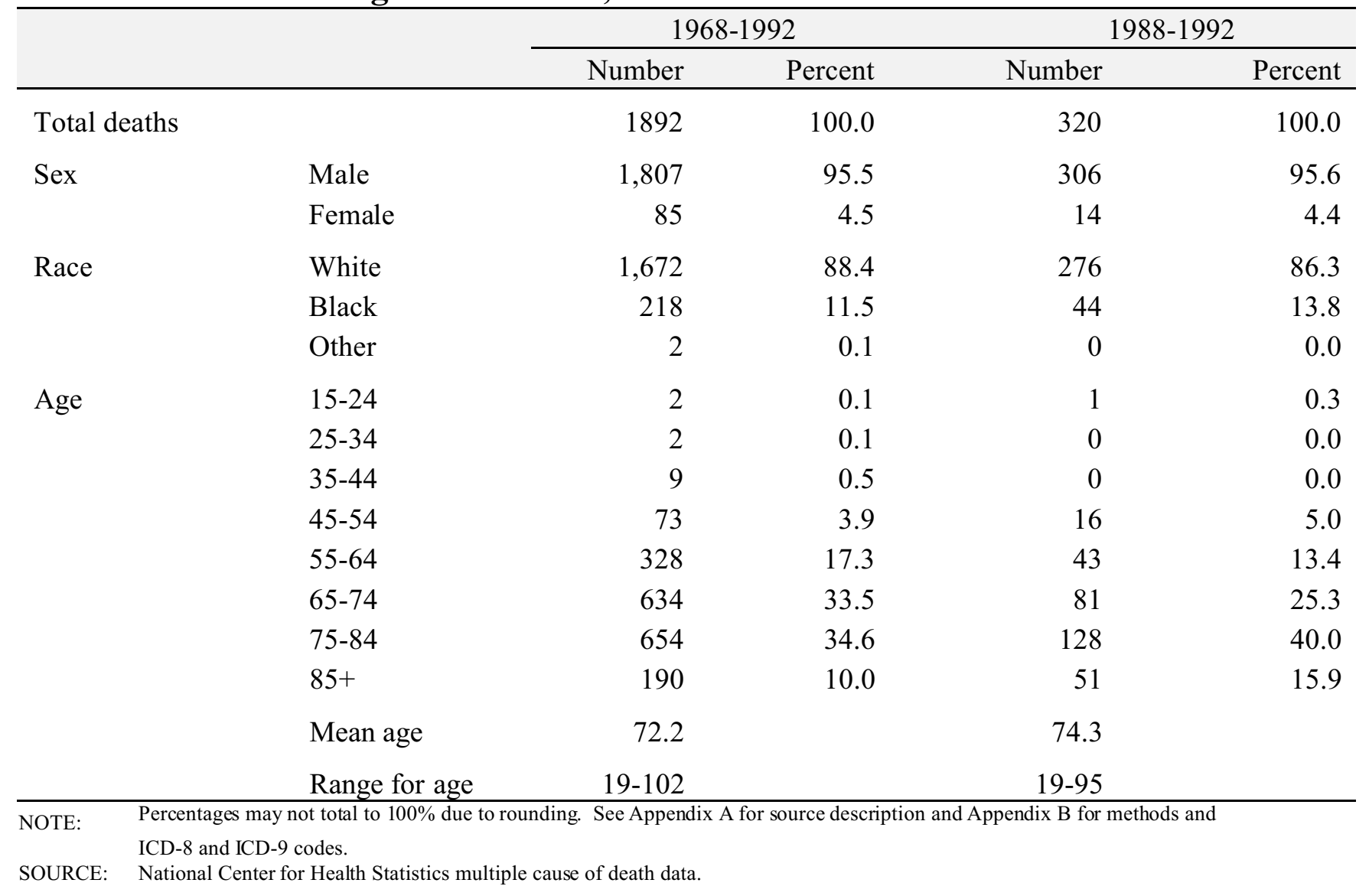




\section{Figure NY-3. Pneumoconiosis deaths by condition, New York residents age 15 and over, 1968-1992}

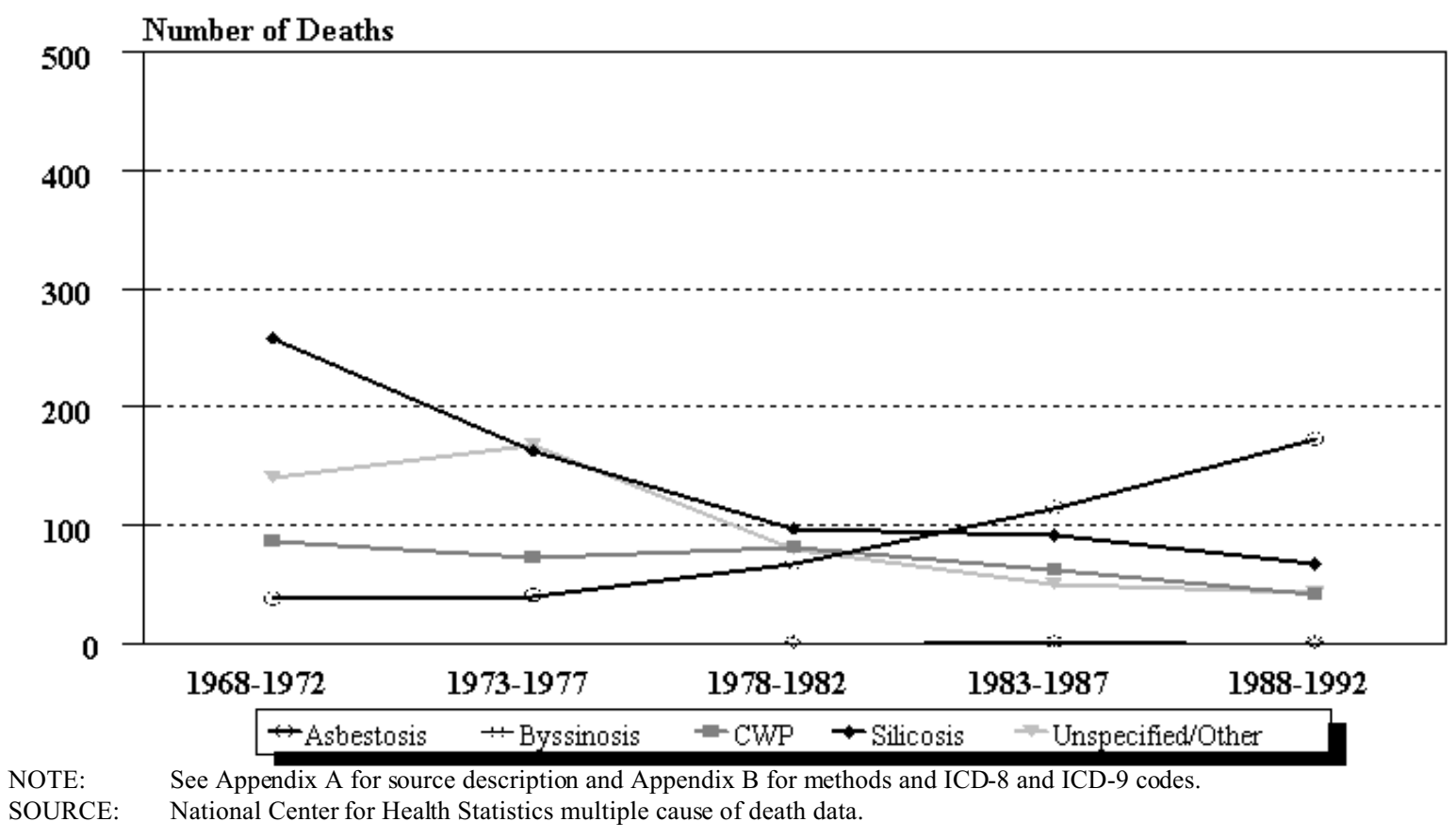

Figure NY-4. New York and U.S. age-adjusted mortality rates (per 1,000,000 population) by condition, residents age 15 and over, 1983-1992

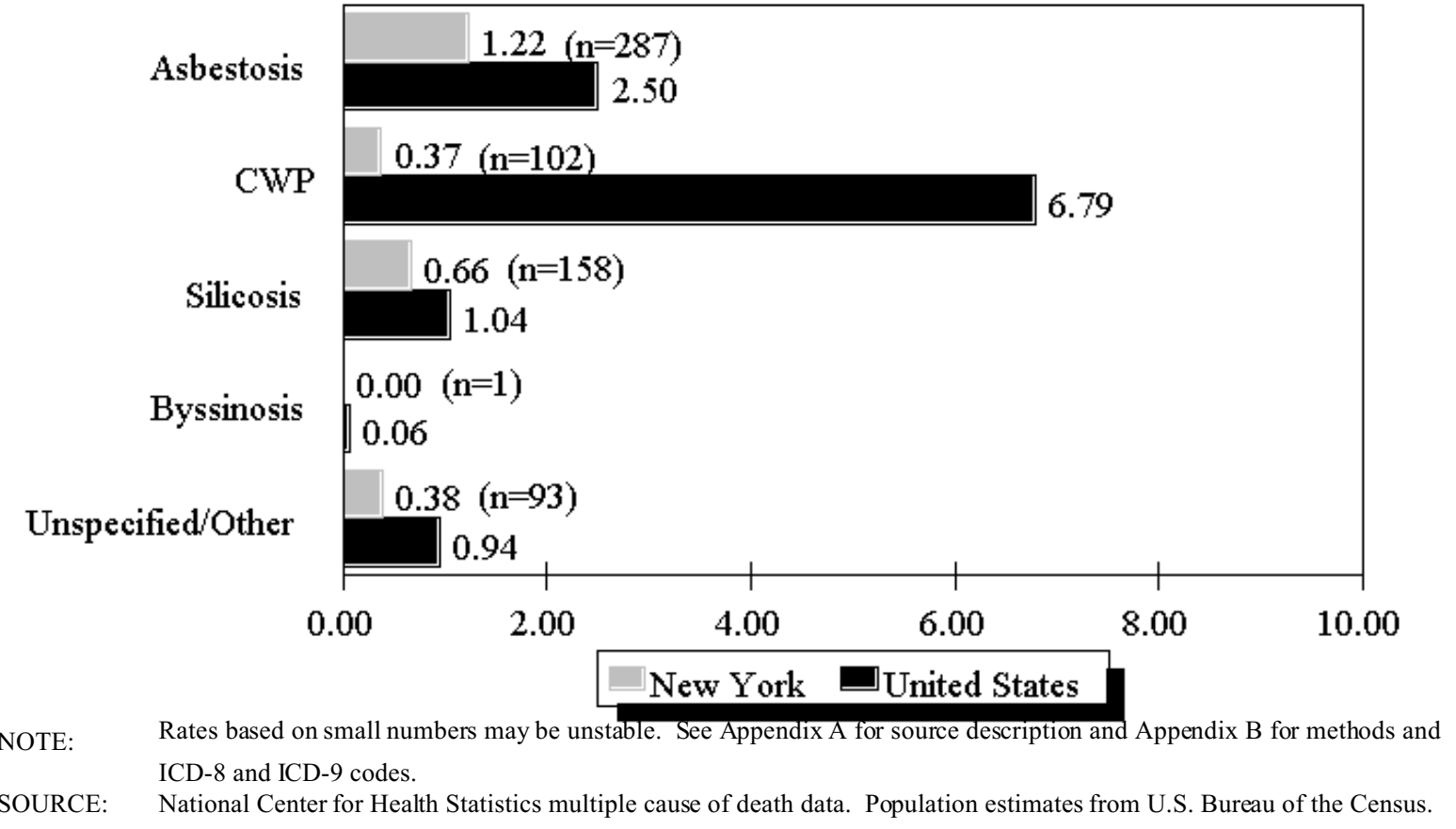


Table NY-3 (page 1 of 2). Number of deaths and age-adjusted mortality rate (per $1,000,000$ population) by condition and county, New York residents age 15 and over, 1983-1992

\begin{tabular}{|c|c|c|c|c|c|c|c|c|c|c|c|c|}
\hline \multirow[b]{2}{*}{ County } & \multicolumn{2}{|c|}{ Asbestosis } & \multicolumn{2}{|c|}{$\begin{array}{r}\text { Coal workers' } \\
\text { pneumoconiosis }\end{array}$} & \multicolumn{2}{|c|}{ Silicosis } & \multicolumn{2}{|c|}{ Byssinosis } & \multicolumn{2}{|c|}{$\begin{array}{r}\text { Unspecified/Other } \\
\text { pneumoconioses }\end{array}$} & \multicolumn{2}{|c|}{$\begin{array}{r}\text { All } \\
\text { pneumoconioses }\end{array}$} \\
\hline & $\#$ & Rate & \# & Rate & $\#$ & Rate & $\#$ & Rate & \# & Rate & $\#$ & Rate \\
\hline Albany & 10 & 3.00 & 1 & 0.34 & 2 & 0.29 & - & - & 3 & 0.63 & 16 & 4.26 \\
\hline Allegany & 1 & 0.92 & 1 & 0.92 & 1 & 0.92 & - & - & - & - & 3 & 2.76 \\
\hline Bronx & 13 & 0.87 & 5 & 0.37 & 7 & 0.60 & - & - & 5 & 0.32 & 30 & 2.17 \\
\hline Broome & 3 & 1.35 & 12 & 3.63 & - & - & - & - & 7 & 2.14 & 22 & 7.12 \\
\hline Cattaraugus & - & - & 1 & 0.55 & 2 & 1.70 & - & - & 1 & 1.15 & 4 & 3.39 \\
\hline Cayuga & - & - & - & - & 2 & 1.79 & - & - & 2 & 2.47 & 3 & 3.03 \\
\hline Chautauqua & 1 & 0.65 & 3 & 0.82 & 1 & 0.65 & - & - & 1 & 0.27 & 6 & 2.39 \\
\hline Chemung & 2 & 1.46 & 2 & 0.94 & 1 & 0.47 & - & - & 2 & 1.99 & 7 & 4.85 \\
\hline Chenango & 1 & 1.99 & 2 & 1.78 & - & - & - & - & - & - & 3 & 3.77 \\
\hline Clinton & - & - & - & - & 1 & 0.79 & - & - & - & - & 1 & 0.79 \\
\hline Columbia & 4 & 3.26 & 1 & 1.45 & - & - & - & - & - & - & 5 & 4.71 \\
\hline Cortland & 1 & 1.06 & - & - & 1 & 3.24 & - & - & - & - & 2 & 4.30 \\
\hline Delaware & - & - & 1 & 0.83 & - & - & - & - & - & - & 1 & 0.83 \\
\hline Dutchess & 1 & 0.44 & 1 & 0.44 & 1 & 0.44 & - & - & 1 & 0.22 & 4 & 1.54 \\
\hline Erie & 18 & 1.62 & 17 & 1.17 & 22 & 1.53 & - & - & 5 & 0.28 & 60 & 4.47 \\
\hline Essex & 1 & 2.52 & - & - & 7 & 13.56 & - & - & - & - & 8 & 16.08 \\
\hline Fulton & 1 & 0.71 & - & - & - & - & - & - & - & - & 1 & 0.71 \\
\hline Genesee & 2 & 2.49 & 1 & 1.70 & 2 & 1.58 & - & - & - & - & 5 & 5.77 \\
\hline Greene & - & - & - & - & - & - & - & - & 1 & 2.06 & 1 & 2.06 \\
\hline Herkimer & - & - & - & - & - & - & - & - & 3 & 3.29 & 3 & 3.29 \\
\hline Jefferson & 3 & 3.57 & - & - & 2 & 2.38 & - & - & 1 & 1.19 & 6 & 7.14 \\
\hline Kings & 26 & 0.90 & 2 & 0.09 & 7 & 0.23 & - & - & 2 & 0.05 & 37 & 1.27 \\
\hline Lewis & - & - & - & - & 1 & 2.00 & - & - & 1 & 2.00 & 2 & 4.00 \\
\hline Madison & - & - & - & - & 1 & 0.86 & - & - & - & - & 1 & 0.86 \\
\hline Monroe & 9 & 1.11 & 8 & 0.66 & 9 & 0.81 & - & - & 5 & 0.44 & 31 & 3.02 \\
\hline Montgomery & 1 & 1.55 & 1 & 0.63 & - & - & - & - & - & - & 2 & 2.18 \\
\hline Nassau & 31 & 1.69 & 2 & 0.08 & 4 & 0.21 & 1 & 0.04 & 4 & 0.17 & 41 & 2.16 \\
\hline New York & 9 & 0.37 & 6 & 0.26 & 4 & 0.16 & - & - & 3 & 0.09 & 22 & 0.88 \\
\hline Niagara & 9 & 2.70 & 2 & 0.42 & 2 & 0.62 & - & - & 5 & 2.06 & 18 & 5.81 \\
\hline Oneida & 1 & 0.37 & - & - & 5 & 1.04 & - & - & 1 & 0.17 & 7 & 1.58 \\
\hline Onondaga & 5 & 0.98 & 1 & 0.11 & 7 & 1.11 & - & - & 2 & 0.33 & 15 & 2.53 \\
\hline
\end{tabular}

See footnotes at end of table. 
Table NY-3 (page 2 of 2). Number of deaths and age-adjusted mortality rate (per $1,000,000$ population) by condition and county, New York residents age 15 and over, 1983-1992

\begin{tabular}{|c|c|c|c|c|c|c|c|c|c|c|c|c|}
\hline \multirow[b]{2}{*}{ County } & \multicolumn{2}{|c|}{ Asbestosis } & \multicolumn{2}{|c|}{$\begin{array}{c}\text { Coal workers' } \\
\text { pneumoconiosis }\end{array}$} & \multicolumn{2}{|c|}{ Silicosis } & \multicolumn{2}{|c|}{ Byssinosis } & \multicolumn{2}{|c|}{$\begin{array}{r}\text { Unspecified/Other } \\
\text { pneumoconioses }\end{array}$} & \multicolumn{2}{|c|}{$\begin{array}{r}\text { All } \\
\text { pneumoconioses }\end{array}$} \\
\hline & $\#$ & Rate & \# & Rate & $\#$ & Rate & $\#$ & Rate & \# & Rate & \# & Rate \\
\hline Ontario & 4 & 3.86 & - & - & 3 & 3.09 & - & - & 1 & 1.11 & 8 & 8.05 \\
\hline Orange & 8 & 1.85 & 2 & 0.62 & 1 & 0.21 & - & - & 3 & 1.25 & 14 & 3.94 \\
\hline Oswego & 2 & 1.52 & - & - & - & - & - & - & - & - & 2 & 1.52 \\
\hline Otsego & - & - & - & - & 1 & 1.65 & - & - & - & - & 1 & 1.65 \\
\hline Putnam & - & - & - & - & - & - & - & - & 1 & 0.84 & 1 & 0.84 \\
\hline Queens & 30 & 1.16 & 11 & 0.34 & 14 & 0.56 & - & - & 11 & 0.39 & 65 & 2.40 \\
\hline Rensselaer & 3 & 1.66 & - & - & 3 & 1.66 & - & - & 2 & 1.64 & 8 & 4.96 \\
\hline Richmond & 5 & 0.95 & - & - & 1 & 0.16 & - & - & 2 & 0.53 & 8 & 1.65 \\
\hline Rockland & 4 & 1.33 & 1 & 0.42 & 1 & 0.42 & - & - & - & - & 6 & 2.17 \\
\hline St. Lawrence & 3 & 2.43 & 3 & 2.43 & 14 & 11.65 & - & - & 9 & 7.28 & 28 & 23.31 \\
\hline Saratoga & 2 & 1.39 & - & - & - & - & - & - & - & - & 2 & 1.39 \\
\hline Schenectady & 1 & 0.26 & 1 & 0.26 & 2 & 0.51 & - & - & - & - & 4 & 1.03 \\
\hline Schoharie & 1 & 1.44 & 1 & 1.44 & - & - & - & - & - & - & 2 & 2.88 \\
\hline Schuyler & - & - & - & - & 1 & 7.46 & - & - & - & - & 1 & 7.46 \\
\hline Seneca & 1 & 2.81 & - & - & 1 & 2.81 & - & - & - & - & 2 & 5.63 \\
\hline Steuben & 3 & 2.86 & 1 & 0.95 & 6 & 5.77 & - & - & 2 & 1.91 & 12 & 11.50 \\
\hline Suffolk & 40 & 2.74 & 3 & 0.18 & - & - & - & - & 2 & 0.13 & 45 & 3.06 \\
\hline Sullivan & 2 & 2.02 & - & - & 1 & 1.35 & - & - & 1 & 1.35 & 4 & 4.73 \\
\hline Tioga & - & - & 1 & 1.20 & 2 & 4.31 & - & - & - & - & 3 & 5.51 \\
\hline Tompkins & - & - & - & - & 1 & 1.64 & - & - & 1 & 0.75 & 2 & 2.40 \\
\hline Ulster & 5 & 1.81 & 1 & 0.63 & 4 & 1.84 & - & - & - & - & 10 & 4.28 \\
\hline Warren & 1 & 0.76 & - & - & - & - & - & - & - & - & 1 & 0.76 \\
\hline Washington & 1 & 1.77 & - & - & 5 & 7.02 & - & - & 2 & 1.70 & 8 & 10.49 \\
\hline Wayne & 3 & 3.47 & 1 & 1.29 & 1 & 0.63 & - & - & - & - & 5 & 5.39 \\
\hline Westchester & 14 & 1.09 & 6 & 0.47 & 4 & 0.31 & - & - & 1 & 0.11 & 25 & 1.97 \\
\hline
\end{tabular}




\section{North Carolina: Selected statistics from the United States section}

All pneumoconioses mortality, 1988-1992

Total deaths

Crude mortality rate (per $1,000,000$ population)

Age-adjusted mortality rate (per $1,000,000$ population)

Total years of potential life lost to life expectancy

Years of potential life lost to life expectancy (per death)

\begin{tabular}{rr} 
Value & State rank \\
\hline 244 & 15 \\
9.21 & 25 \\
5.92 & 21 \\
3,113 & 14 \\
12.8 & 11
\end{tabular}

Percent**

Deaths by type of pneumoconiosis, 1988-1992

Asbestosis

Deaths*

100

distribution

Coal workers' pneumoconiosis

41.0

21.3

Silicosis

52

17.6

Byssinosis

29

11.9

22

* Sum of column may exceed total deaths because of deaths with multiple pneumoconioses.

** Percentages may total more than $100 \%$ due to deaths with multiple pneumoconioses.

NOTE: $\quad$ See Appendix A for source description and Appendix B for methods and ICD-8 and ICD-9 codes.

SOURCE: $\quad$ National Center for Health Statistics multiple cause of death data. Population estimates from U.S. Bureau of the Census.

Figure NC-1. All pneumoconioses: North Carolina and U.S. age-adjusted mortality rates, residents age 15 and over, 1968-1992

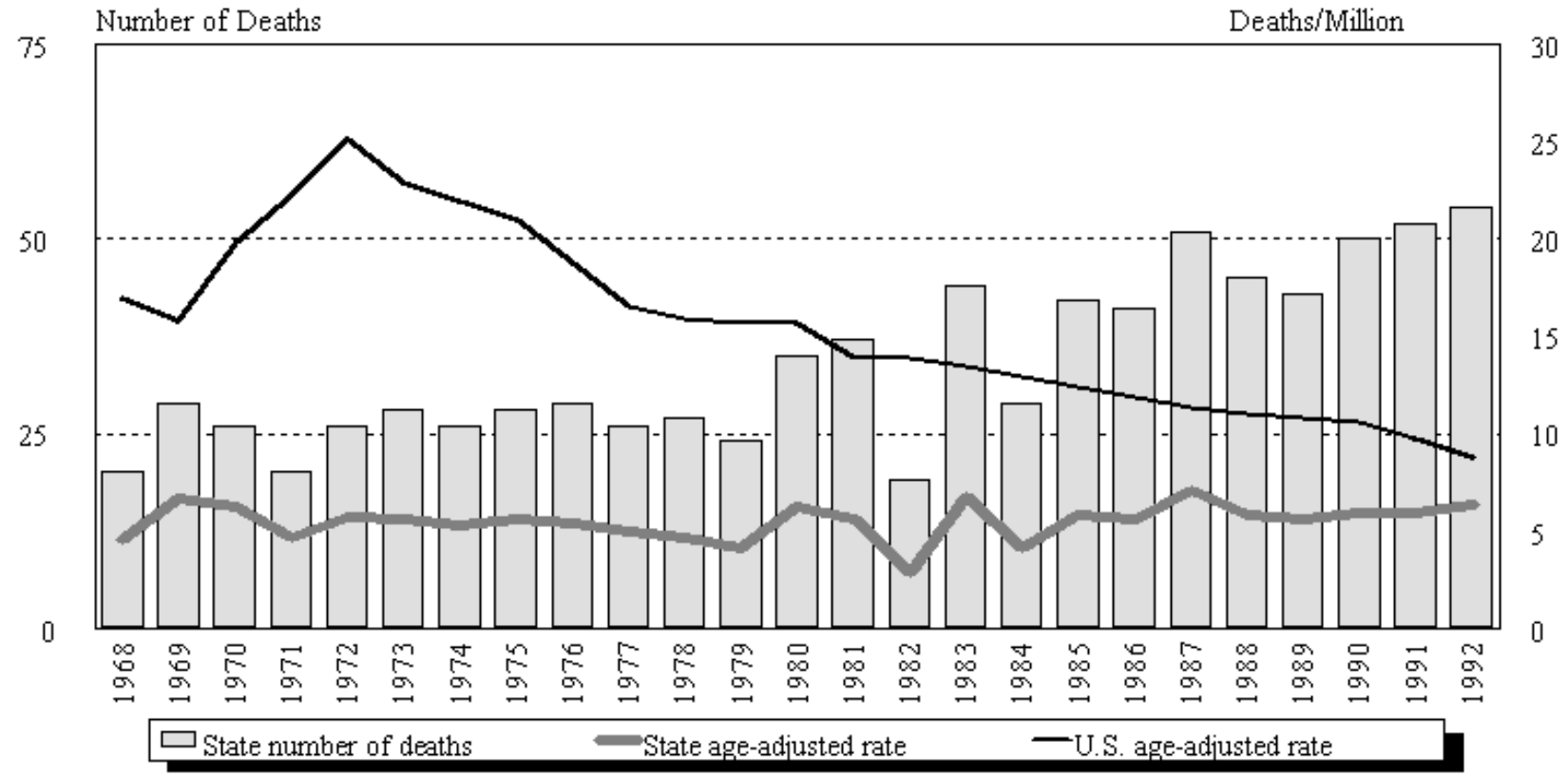

NOTE: $\quad$ See Appendix A for source description and Appendix B for methods and ICD-8 and ICD-9 codes.

SOURCE: $\quad$ National Center for Health Statistics multiple cause of death data. Population estimates from U.S. Bureau of the Census. 
North Carolina

\begin{tabular}{|c|c|c|c|c|c|c|c|c|c|c|c|c|}
\hline \multirow[b]{2}{*}{ Year } & \multicolumn{2}{|c|}{ Asbestosis } & \multicolumn{2}{|c|}{$\begin{array}{l}\text { Coal workers' } \\
\text { pneumoconiosis }\end{array}$} & \multicolumn{2}{|c|}{ Silicosis } & \multicolumn{2}{|c|}{ Byssinosis* } & \multicolumn{2}{|c|}{$\begin{array}{c}\text { Unspecified/Other } \\
\text { pneumoconioses }\end{array}$} & \multicolumn{2}{|c|}{ All pneumoconioses } \\
\hline & Number & Rate & Number & Rate & Number & Rate & Number & Rate & Number & Rate & Number & Rate \\
\hline 1968 & 2 & 0.48 & 2 & 0.38 & 13 & 3.02 & & & 3 & 0.72 & 20 & 4.60 \\
\hline 1969 & 4 & 1.00 & 3 & 0.63 & 19 & 4.37 & & & 4 & 0.96 & 29 & 6.72 \\
\hline 1970 & 5 & 1.31 & 3 & 0.76 & 18 & 4.26 & & & 2 & 0.44 & 26 & 6.26 \\
\hline 1971 & 1 & 0.24 & 1 & 0.23 & 15 & 3.44 & & & 3 & 0.73 & 20 & 4.64 \\
\hline 1972 & - & - & - & & 24 & 5.32 & & & 2 & 0.36 & 26 & 5.68 \\
\hline 1973 & 3 & 0.67 & 4 & 0.83 & 11 & 2.07 & & & 10 & 2.10 & 28 & 5.66 \\
\hline 1974 & 3 & 0.75 & 2 & 0.44 & 13 & 2.65 & & & 8 & 1.49 & 26 & 5.32 \\
\hline 1975 & 1 & 0.22 & 4 & 0.87 & 13 & 2.57 & & & 11 & 2.19 & 28 & 5.64 \\
\hline 1976 & 2 & 0.35 & 4 & 0.66 & 16 & 3.04 & & & 7 & 1.32 & 29 & 5.36 \\
\hline 1977 & 1 & 0.26 & 3 & 0.45 & 11 & 2.00 & & & 11 & 2.32 & 26 & 5.03 \\
\hline 1978 & 2 & 0.35 & 4 & 0.65 & 11 & 1.93 & & & 10 & 1.72 & 27 & 4.65 \\
\hline 1979 & 4 & 0.71 & 2 & 0.34 & 11 & 1.80 & 3 & 0.57 & 4 & 0.74 & 24 & 4.17 \\
\hline 1980 & 8 & 1.47 & 12 & 1.97 & 8 & 1.48 & 3 & 0.47 & 5 & 0.97 & 35 & 6.20 \\
\hline 1981 & 4 & 0.53 & 9 & 1.45 & 9 & 1.45 & 7 & 1.10 & 9 & 1.31 & 37 & 5.65 \\
\hline 1982 & 6 & 0.90 & 8 & 1.14 & 4 & 0.70 & - & - & 1 & 0.07 & 19 & 2.81 \\
\hline 1983 & 9 & 1.36 & 15 & 2.22 & 11 & 1.67 & 7 & 1.29 & 2 & 0.19 & 44 & 6.73 \\
\hline 1984 & 10 & 1.43 & 4 & 0.60 & 11 & 1.58 & 4 & 0.53 & - & - & 29 & 4.15 \\
\hline 1985 & 15 & 2.23 & 7 & 0.86 & 12 & 1.57 & 7 & 1.10 & 1 & 0.11 & 42 & 5.87 \\
\hline 1986 & 13 & 1.92 & 10 & 1.13 & 9 & 0.98 & 7 & 1.31 & 2 & 0.24 & 41 & 5.57 \\
\hline 1987 & 17 & 2.57 & 12 & 1.37 & 12 & 1.71 & 9 & 1.28 & 1 & 0.14 & 51 & 7.07 \\
\hline 1988 & 12 & 1.59 & 6 & 0.81 & 9 & 1.01 & 10 & 1.31 & 8 & 1.13 & 45 & 5.86 \\
\hline 1989 & 17 & 2.29 & 11 & 1.29 & 7 & 0.97 & 4 & 0.59 & 4 & 0.51 & 43 & 5.65 \\
\hline 1990 & 25 & 3.04 & 12 & 1.28 & 5 & 0.60 & 5 & 0.59 & 4 & 0.54 & 50 & 5.92 \\
\hline 1991 & 21 & 2.54 & 13 & 1.31 & 14 & 1.64 & 2 & 0.14 & 2 & 0.26 & 52 & 5.90 \\
\hline 1992 & 25 & 3.06 & 10 & 0.93 & 8 & 0.88 & 8 & 1.05 & 4 & 0.53 & 54 & 6.32 \\
\hline
\end{tabular}

- indicates no deaths listed. $\quad$ * no specific code for byssinosis in ICD-8 (1968-1 978).

NOTE: Rates based on small numbers may be unstable. Sum of individual conditions may exceed total because of deaths with multiple pneumoconioses. See Appendix A for source description and Appendix B for methods and ICD-8 and ICD-9 codes.

SOURCE: National Center for Health Statistics multiple cause of death data. Population estimates from U.S. Bureau of the Census. 
Figure NC-2. All pneumoconioses: Distribution of deaths by sex and race, North Carolina residents age 15 and over, 1968-1992
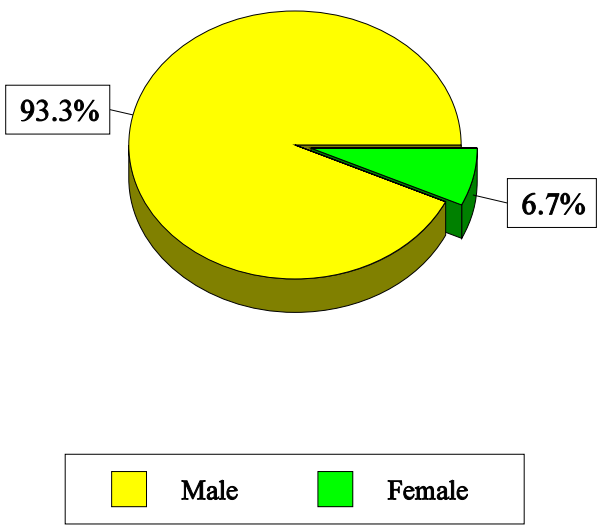

$86.2 \%$
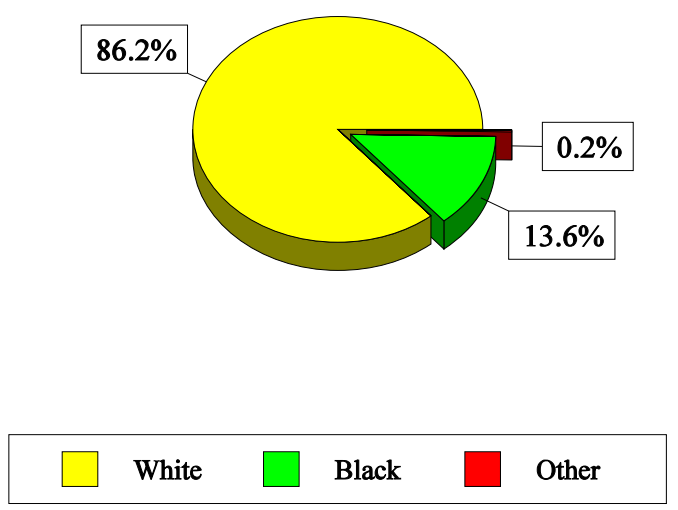

NOTE: $\quad$ See Appendix A for source description and Appendix B for methods and ICD-8 and ICD-9 codes.

SOURCE: National Center for Health Statistics multiple cause of death data.

Table NC-2. All pneumoconioses: Distribution of deaths by sex, race, and age, North Carolina residents age 15 and over, 1968-1992

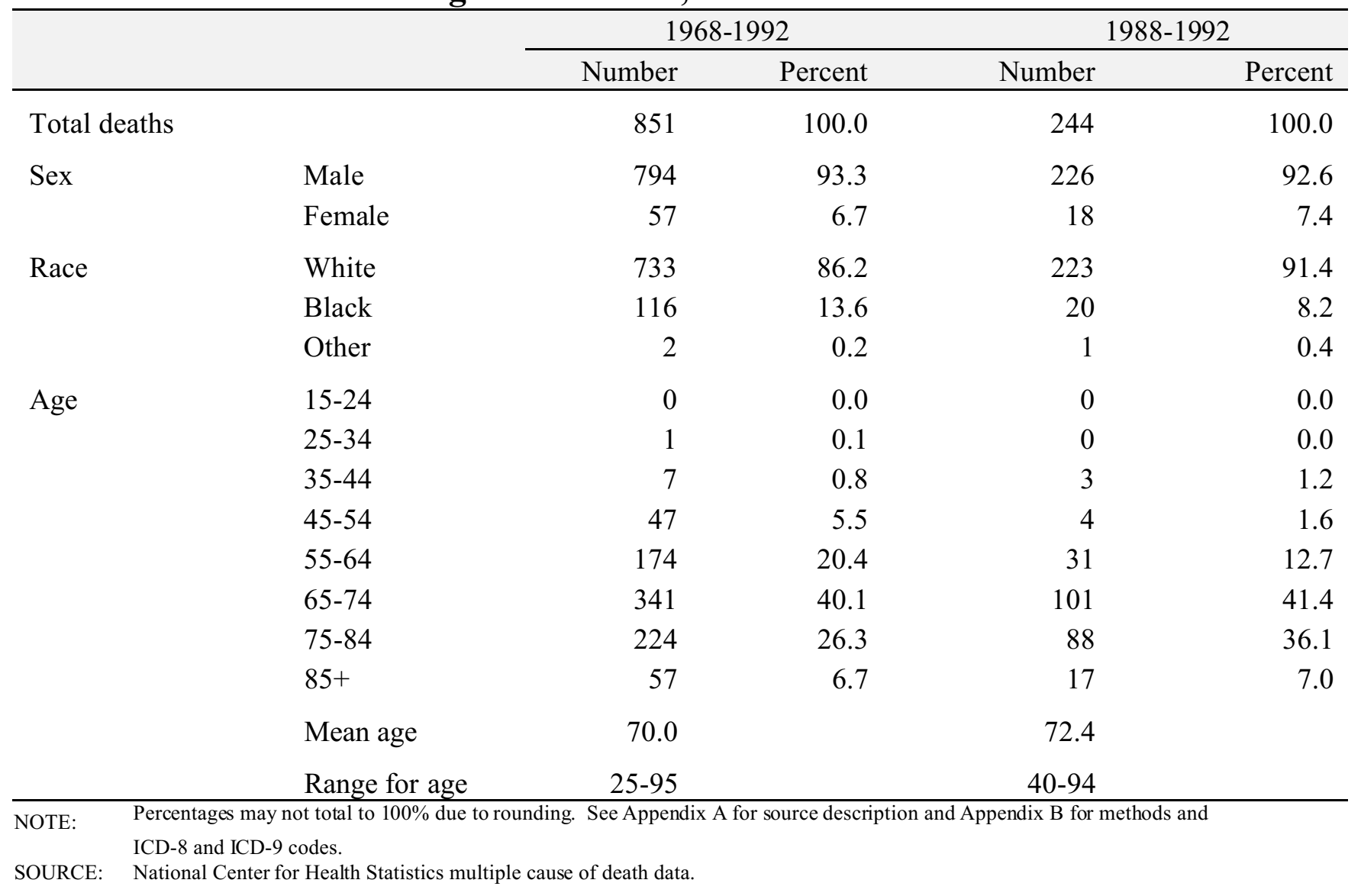


Figure NC-3. Pneumoconiosis deaths by condition, North Carolina residents age 15 and over, 1968-1992

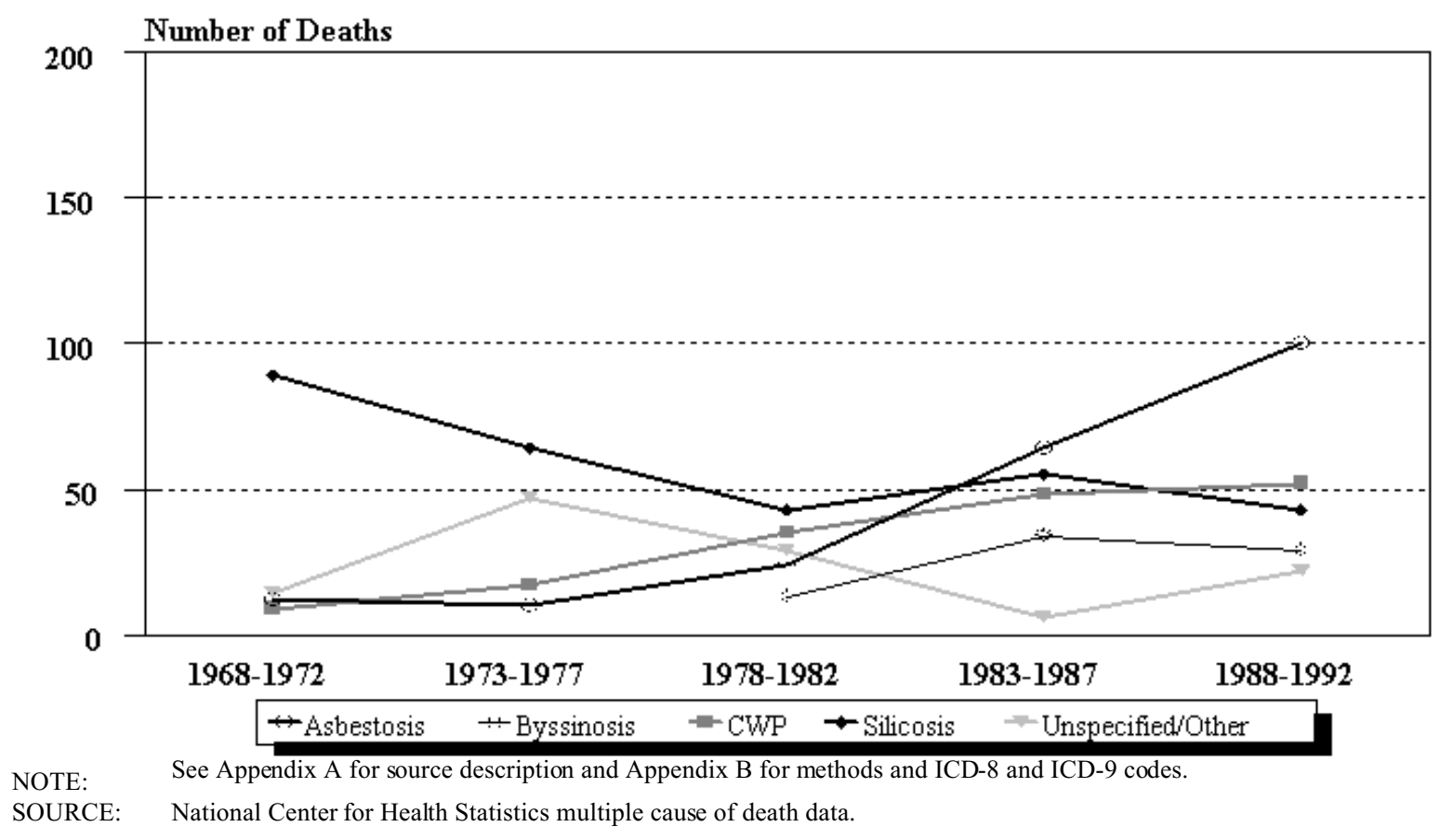

Figure NC-4. North Carolina and U.S. age-adjusted mortality rates (per 1,000,000 population) by condition, residents age 15 and over, 1983-1992

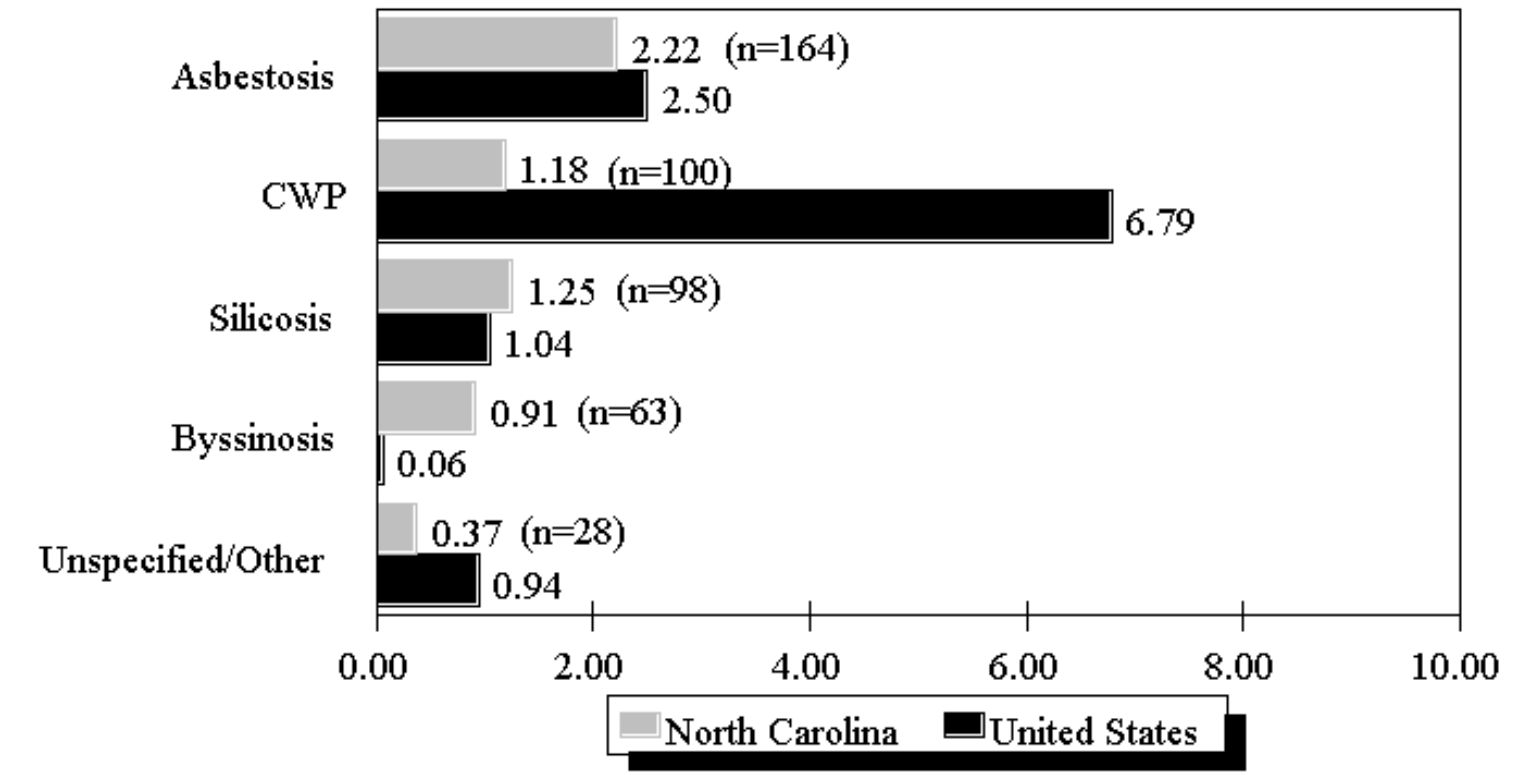

NOTE: $\quad$ Rates based on small numbers may be unstable. See Appendix A for source description and Appendix B for methods and ICD-8 and ICD-9 codes.

SOURCE: National Center for Health Statistics multiple cause of death data. Population estimates from U.S. Bureau of the Census. 
Table NC-3 (page 1 of 3). Number of deaths and age-adjusted mortality rate (per $1,000,000$ population) by condition and county, North Carolina residents age 15 and over, 1983-1992

\begin{tabular}{|c|c|c|c|c|c|c|c|c|c|c|c|c|}
\hline \multirow[b]{2}{*}{ County } & \multicolumn{2}{|c|}{ Asbestosis } & \multicolumn{2}{|c|}{$\begin{array}{r}\text { Coal workers' } \\
\text { pneumoconiosis }\end{array}$} & \multicolumn{2}{|c|}{ Silicosis } & \multicolumn{2}{|c|}{ Byssinosis } & \multicolumn{2}{|c|}{$\begin{array}{r}\text { Unspecified/Other } \\
\text { pneumoconioses }\end{array}$} & \multicolumn{2}{|c|}{$\begin{array}{r}\text { All } \\
\text { pneumoconioses }\end{array}$} \\
\hline & $\#$ & Rate & $\#$ & Rate & $\#$ & Rate & $\#$ & Rate & $\#$ & Rate & $\#$ & Rate \\
\hline Alamance & 3 & 2.16 & - & - & - & - & - & - & - & - & 3 & 2.16 \\
\hline Alexander & 1 & 4.09 & 1 & 4.09 & 1 & 2.36 & - & - & - & - & 3 & 10.54 \\
\hline Anson & 3 & 9.59 & - & - & - & - & - & - & - & - & 3 & 9.59 \\
\hline Ashe & 1 & 5.98 & 6 & 14.21 & - & - & - & - & 1 & 1.66 & 8 & 21.85 \\
\hline Avery & - & - & 3 & 16.14 & 5 & 19.56 & - & - & 2 & 9.71 & 10 & 45.41 \\
\hline Beaufort & 4 & 7.41 & - & - & - & - & - & - & - & - & 4 & 7.41 \\
\hline Bertie & 1 & 2.33 & - & - & - & - & - & - & - & - & 1 & 2.33 \\
\hline Bladen & - & - & - & - & - & - & 4 & 13.02 & - & - & 4 & 13.02 \\
\hline Brunswick & 2 & 2.94 & 3 & 4.81 & - & - & - & - & 1 & 1.60 & 6 & 9.36 \\
\hline Buncombe & 2 & 0.76 & 4 & 1.52 & 2 & 1.04 & - & - & - & - & 8 & 3.31 \\
\hline Burke & 2 & 1.50 & 1 & 0.75 & 3 & 2.25 & 2 & 2.06 & - & - & 8 & 6.56 \\
\hline Cabarrus & 4 & 3.15 & - & - & 1 & 0.55 & 1 & 1.02 & - & - & 6 & 4.73 \\
\hline Caldwell & 2 & 2.93 & 2 & 2.93 & 1 & 0.87 & 2 & 2.33 & - & - & 7 & 9.06 \\
\hline Carteret & 3 & 4.44 & 1 & 1.09 & - & - & - & - & 1 & 1.67 & 5 & 7.20 \\
\hline Caswell & - & - & - & - & 1 & 2.42 & - & - & - & - & 1 & 2.42 \\
\hline Catawba & 2 & 1.78 & 2 & 1.07 & 1 & 0.54 & 1 & 0.54 & - & - & 6 & 3.92 \\
\hline Cherokee & 1 & 1.89 & - & - & 2 & 5.78 & - & - & - & - & 3 & 7.67 \\
\hline Chowan & 1 & 6.17 & 1 & 6.17 & - & - & - & - & - & - & 2 & 12.34 \\
\hline Cleveland & 1 & 1.16 & - & - & 2 & 1.78 & 1 & 1.16 & - & - & 4 & 4.09 \\
\hline Columbus & 1 & 1.91 & - & - & - & - & - & - & - & - & 1 & 1.91 \\
\hline Craven & 1 & 1.41 & - & - & 4 & 5.62 & - & - & - & - & 5 & 7.03 \\
\hline Cumberland & 1 & 0.62 & 2 & 1.24 & 3 & 1.86 & 2 & 1.03 & 1 & 0.62 & 8 & 4.75 \\
\hline Currituck & 1 & 7.16 & - & - & - & - & - & - & - & - & 1 & 7.16 \\
\hline Dare & 1 & 4.39 & - & - & - & - & - & - & - & - & 1 & 4.39 \\
\hline Davidson & 1 & 0.83 & 3 & 1.82 & 2 & 1.33 & - & - & - & - & 6 & 3.98 \\
\hline Davie & 2 & 6.87 & 1 & 3.44 & - & - & 1 & 3.44 & 1 & 1.88 & 5 & 15.63 \\
\hline Duplin & - & - & 1 & 2.33 & - & - & - & - & 1 & 1.26 & 2 & 3.60 \\
\hline Durham & 6 & 2.82 & - & - & - & - & - & - & - & - & 6 & 2.82 \\
\hline Edgecombe & 1 & 1.87 & - & - & - & - & - & - & - & - & 1 & 1.87 \\
\hline Forsyth & 4 & 1.23 & 5 & 1.25 & 1 & 0.21 & - & - & 2 & 0.61 & 12 & 3.29 \\
\hline Franklin & 2 & 6.90 & 2 & 2.81 & - & - & 1 & 1.40 & - & - & 5 & 11.12 \\
\hline
\end{tabular}

See footnotes at end of table. 
Table NC-3 (page 2 of 3). Number of deaths and age-adjusted mortality rate (per $1,000,000$ population) by condition and county, North Carolina residents age 15 and over, 1983-1992

\begin{tabular}{|c|c|c|c|c|c|c|c|c|c|c|c|c|}
\hline \multirow[b]{2}{*}{ County } & \multicolumn{2}{|c|}{ Asbestosis } & \multicolumn{2}{|c|}{$\begin{array}{c}\text { Coal workers' } \\
\text { pneumoconiosis }\end{array}$} & \multicolumn{2}{|c|}{ Silicosis } & \multicolumn{2}{|c|}{ Byssinosis } & \multicolumn{2}{|c|}{$\begin{array}{r}\text { Unspecified/Other } \\
\text { pneumoconioses }\end{array}$} & \multicolumn{2}{|c|}{$\begin{array}{r}\text { All } \\
\text { pneumoconioses }\end{array}$} \\
\hline & \# & Rate & \# & Rate & \# & Rate & $\#$ & Rate & \# & Rate & \# & Rate \\
\hline Gaston & 4 & 2.14 & - & - & 1 & 0.60 & 4 & 2.60 & - & - & 9 & 5.34 \\
\hline Graham & 1 & 11.84 & - & - & - & - & - & - & - & - & 1 & 11.84 \\
\hline Granville & - & - & - & - & 1 & 3.63 & - & - & - & - & 1 & 3.63 \\
\hline Greene & 1 & 6.44 & 1 & 6.44 & 1 & 3.94 & - & - & - & - & 3 & 16.82 \\
\hline Guilford & - & - & 6 & 1.17 & 2 & 0.49 & 8 & 2.33 & 1 & 0.32 & 17 & 4.30 \\
\hline Halifax & 2 & 3.40 & - & - & - & - & 1 & 1.70 & - & - & 3 & 5.11 \\
\hline Harnett & 1 & 1.65 & 3 & 4.23 & - & - & - & - & 1 & 1.65 & 5 & 7.54 \\
\hline Haywood & 4 & 4.94 & - & - & - & - & - & - & - & - & 4 & 4.94 \\
\hline Henderson & 2 & 1.54 & 2 & 2.62 & - & - & - & - & - & - & 4 & 4.16 \\
\hline Hertford & 2 & 10.82 & - & - & - & - & - & - & - & - & 2 & 10.82 \\
\hline Hoke & - & - & 1 & 3.62 & - & - & 2 & 9.25 & 1 & 3.62 & 4 & 16.50 \\
\hline Iredell & 2 & 1.65 & 7 & 5.05 & 1 & 1.07 & 1 & 1.07 & 2 & 2.14 & 13 & 10.99 \\
\hline Jackson & - & - & - & - & 1 & 3.86 & - & - & - & - & 1 & 3.86 \\
\hline Johnston & 1 & 1.27 & 1 & 0.74 & 1 & 1.27 & 1 & 1.27 & 1 & 0.74 & 5 & 5.29 \\
\hline Lee & - & - & 2 & 3.84 & - & - & - & - & 1 & 2.38 & 3 & 6.22 \\
\hline Lenoir & 3 & 4.23 & - & - & 1 & 0.95 & - & - & - & - & 4 & 5.18 \\
\hline Lincoln & 1 & 2.18 & - & - & - & - & 1 & 1.33 & - & - & 2 & 3.52 \\
\hline McDonwell & - & - & - & - & 3 & 5.31 & - & - & - & - & 3 & 5.31 \\
\hline Macon & 1 & 1.31 & - & - & 3 & 5.54 & - & - & 1 & 2.91 & 5 & 9.77 \\
\hline Madison & 1 & 5.17 & - & - & - & - & - & - & - & - & 1 & 5.17 \\
\hline Martin & 1 & 3.61 & - & - & - & - & - & - & - & - & 1 & 3.61 \\
\hline Mecklenberg & 22 & 4.71 & 1 & 0.16 & 2 & 0.42 & 2 & 0.43 & 2 & 0.42 & 29 & 6.14 \\
\hline Mitchell & - & - & 1 & 2.54 & 17 & 76.64 & - & - & 2 & 15.08 & 20 & 94.25 \\
\hline Montgomery & - & - & 1 & 2.21 & - & - & - & - & - & - & 1 & 2.21 \\
\hline Moore & 1 & 1.25 & 1 & 0.69 & - & - & - & - & - & - & 2 & 1.94 \\
\hline Nash & - & - & - & - & 3 & 3.99 & - & - & - & - & 3 & 3.99 \\
\hline New Hanover & 15 & 11.63 & 2 & 1.38 & 1 & 0.51 & - & - & - & - & 18 & 13.52 \\
\hline Onslow & - & - & 1 & 1.63 & - & - & - & - & 1 & 1.29 & 2 & 2.92 \\
\hline Orange & 1 & 0.88 & 1 & 0.88 & - & - & 3 & 4.26 & 1 & 0.88 & 6 & 6.91 \\
\hline Pasquotank & 1 & 3.30 & - & - & - & - & 1 & 3.30 & - & - & 2 & 6.60 \\
\hline Perquimans & 1 & 7.41 & - & - & - & - & - & - & - & - & 1 & 7.41 \\
\hline Person & - & - & 1 & 1.64 & - & - & - & - & - & - & 1 & 1.64 \\
\hline
\end{tabular}

See footnotes at end of table. 
Table NC-3 (page 3 of 3). Number of deaths and age-adjusted mortality rate (per 1,000,000 population) by condition and county, North Carolina residents age 15 and over, 1983-1992

\begin{tabular}{|c|c|c|c|c|c|c|c|c|c|c|c|c|}
\hline \multirow[b]{2}{*}{ County } & \multicolumn{2}{|c|}{ Asbestosis } & \multicolumn{2}{|c|}{$\begin{array}{r}\text { Coal workers' } \\
\text { pneumoconiosis }\end{array}$} & \multicolumn{2}{|c|}{ Silicosis } & \multicolumn{2}{|c|}{ Byssinosis } & \multicolumn{2}{|c|}{$\begin{array}{r}\text { Unspecified/Other } \\
\text { pneumoconioses }\end{array}$} & \multicolumn{2}{|c|}{$\begin{array}{r}\text { All } \\
\text { pneumoconioses }\end{array}$} \\
\hline & $\#$ & Rate & \# & Rate & \# & Rate & $\#$ & Rate & \# & Rate & \# & Rate \\
\hline Pitt & 1 & 1.52 & - & - & 1 & 1.23 & - & - & - & - & 2 & 2.75 \\
\hline Randolph & 1 & 1.00 & 5 & 4.59 & - & - & 1 & 1.00 & - & - & 7 & 6.59 \\
\hline Richmond & 3 & 6.20 & 3 & 5.31 & - & - & 8 & 16.86 & - & - & 13 & 26.31 \\
\hline Rockingham & 2 & 1.16 & 2 & 1.67 & - & - & 2 & 2.18 & 1 & 1.09 & 7 & 6.10 \\
\hline Rowan & 8 & 5.40 & 3 & 2.08 & 2 & 0.83 & 5 & 4.15 & - & - & 18 & 12.45 \\
\hline Rutherford & 2 & 3.17 & - & - & - & - & 1 & 1.59 & - & - & 3 & 4.76 \\
\hline Sampson & 2 & 3.01 & - & - & 1 & 2.99 & - & - & - & - & 3 & 6.00 \\
\hline Scotland & 1 & 3.46 & - & - & - & - & 3 & 7.52 & - & - & 4 & 10.98 \\
\hline Stanly & 2 & 2.75 & 1 & 1.79 & - & - & 2 & 3.57 & - & - & 5 & 8.10 \\
\hline Stokes & 1 & 3.06 & 2 & 4.66 & 1 & 1.60 & - & - & - & - & 4 & 9.32 \\
\hline Surry & 1 & 0.74 & 4 & 5.18 & 7 & 7.41 & - & - & - & - & 12 & 13.33 \\
\hline Swain & - & - & - & - & 1 & 3.67 & - & - & - & - & 1 & 3.67 \\
\hline Tyrrell & 1 & 21.33 & - & - & - & - & - & - & - & - & 1 & 21.33 \\
\hline Union & 8 & 10.89 & 2 & 2.43 & - & - & 2 & 2.43 & - & - & 12 & 15.76 \\
\hline Vance & - & - & - & - & 2 & 5.21 & - & - & - & - & 2 & 5.21 \\
\hline Wake & 7 & 2.03 & 3 & 0.97 & 3 & 0.83 & - & - & - & - & 13 & 3.83 \\
\hline Watauga & - & - & 1 & 1.76 & - & - & - & - & - & - & 1 & 1.76 \\
\hline Wayne & 2 & 2.23 & 2 & 1.84 & 1 & 1.12 & - & - & 1 & 1.12 & 6 & 6.30 \\
\hline Wilkes & - & - & - & - & - & - & - & - & 2 & 2.58 & 2 & 2.58 \\
\hline Wilson & - & - & - & - & 4 & 6.73 & - & - & - & - & 4 & 6.73 \\
\hline Yadkin & - & - & 2 & 3.02 & - & - & - & - & - & - & 2 & 3.02 \\
\hline Yancey & - & - & - & - & 8 & 37.71 & - & - & - & - & 8 & 37.71 \\
\hline
\end{tabular}




\section{North Dakota: Selected statistics from the United States section}

All pneumoconioses mortality, 1988-1992

Total deaths

Crude mortality rate (per $1,000,000$ population)

Age-adjusted mortality rate (per $1,000,000$ population)

Total years of potential life lost to life expectancy

Years of potential life lost to life expectancy (per death) $\underline{\text { Value }}$

8

3.27

2.33

155

19.4

Deaths*

\section{4}

2

1

0

1

\begin{tabular}{r} 
State rank \\
\hline 48 \\
49 \\
47 \\
48 \\
2
\end{tabular}

Percent** distribution

50.0

25.0

12.5

0.0

12.5

\section{Unspecified/Other pneumoconiosis}

* Sum of column may exceed total deaths because of deaths with multiple pneumoconioses.

** Percentages may total more than $100 \%$ due to deaths with multiple pneumoconioses.

NOTE: $\quad$ See Appendix A for source description and Appendix B for methods and ICD-8 and ICD-9 codes.

SOURCE: National Center for Health Statistics multiple cause of death data. Population estimates from U.S. Bureau of the Census.

Figure ND-1. All pneumoconioses: North Dakota and U.S. age-adjusted mortality rates, residents age 15 and over, 1968-1992

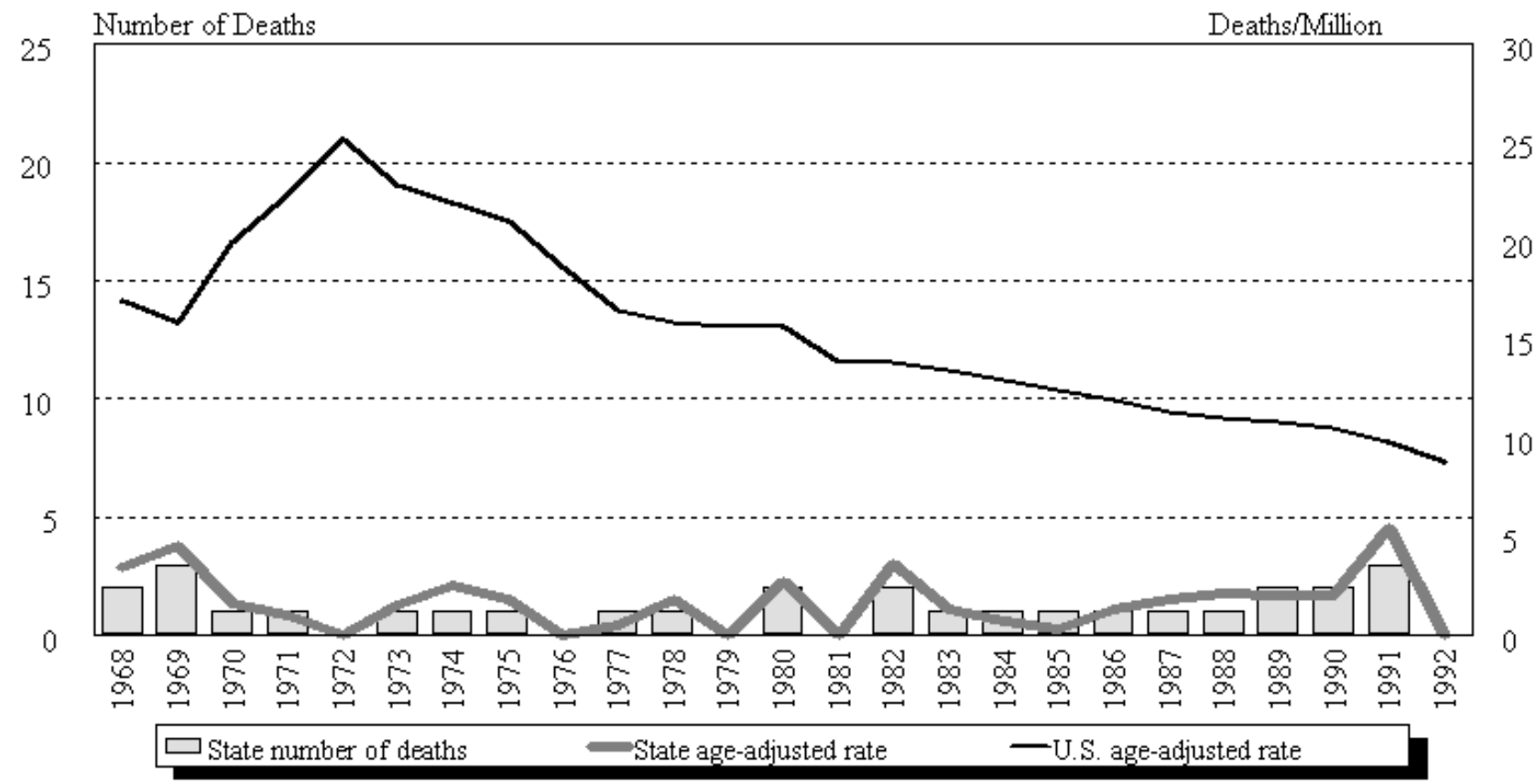

NOTE: $\quad$ See Appendix A for source description and Appendix B for methods and ICD-8 and ICD-9 codes.

SOURCE: National Center for Health Statistics multiple cause of death data. Population estimates from U.S. Bureau of the Census. 
North Dakota

Table ND-1. Number of deaths and age-adjusted mortality rate (per 1,000,000 population) by condition, North Dakota residents age 15 and over, 1968-1992

\begin{tabular}{|c|c|c|c|c|c|c|c|c|c|c|c|c|}
\hline \multirow[b]{2}{*}{ Year } & \multicolumn{2}{|c|}{ Asbestosis } & \multicolumn{2}{|c|}{$\begin{array}{l}\text { Coal workers' } \\
\text { pneumoconiosis }\end{array}$} & \multicolumn{2}{|c|}{ Silicosis } & \multicolumn{2}{|c|}{ Byssinosis* } & \multicolumn{2}{|c|}{$\begin{array}{c}\text { Unspecified/Other } \\
\text { pneumoconioses }\end{array}$} & \multicolumn{2}{|c|}{ All pneumoconioses } \\
\hline & Number & Rate & Number & Rate & Number & Rate & Number & Rate & Number & Rate & Number & Rate \\
\hline 1968 & - & - & - & - & 1 & 1.83 & & & 2 & 3.48 & 2 & 3.48 \\
\hline 1969 & - & - & 1 & 1.07 & 2 & 3.48 & & & - & - & 3 & 4.55 \\
\hline 1970 & - & - & - & - & - & - & & & 1 & 1.64 & 1 & 1.64 \\
\hline 1971 & - & - & - & - & - & - & & & 1 & 1.06 & 1 & 1.06 \\
\hline 1972 & - & - & - & - & - & - & & & - & - & - & - \\
\hline 1973 & - & - & - & - & - & - & & & 1 & 1.54 & 1 & 1.54 \\
\hline 1974 & - & - & - & - & - & - & & & 1 & 2.51 & 1 & 2.51 \\
\hline 1975 & - & - & - & - & 1 & 1.84 & & & - & - & 1 & 1.84 \\
\hline 1976 & - & - & - & - & - & - & & & - & - & - & - \\
\hline 1977 & - & - & - & - & 1 & 0.50 & & & - & - & 1 & 0.50 \\
\hline 1978 & - & - & - & - & - & - & & & 1 & 1.85 & 1 & 1.85 \\
\hline 1979 & - & - & - & - & - & - & - & - & - & - & - & - \\
\hline 1980 & 2 & 2.79 & - & - & - & - & - & - & - & - & 2 & 2.79 \\
\hline 1981 & - & - & - & - & - & - & - & - & - & - & - & - \\
\hline 1982 & 2 & 3.62 & - & - & - & - & - & - & - & - & 2 & 3.62 \\
\hline 1983 & 1 & 1.31 & - & - & - & - & - & - & - & - & 1 & 1.31 \\
\hline 1984 & - & - & - & - & 1 & 0.78 & - & - & - & - & 1 & 0.78 \\
\hline 1985 & - & - & - & - & 1 & 0.36 & - & - & - & - & 1 & 0.36 \\
\hline 1986 & - & - & 1 & 1.31 & - & - & - & - & - & - & 1 & 1.31 \\
\hline 1987 & - & - & 1 & 1.87 & - & - & - & - & - & - & 1 & 1.87 \\
\hline 1988 & - & - & - & - & 1 & 2.13 & - & - & - & - & 1 & 2.13 \\
\hline 1989 & 1 & 1.34 & 1 & 0.72 & - & - & - & - & - & - & 2 & 2.06 \\
\hline 1990 & - & - & 1 & 0.71 & - & - & - & - & 1 & 1.36 & 2 & 2.07 \\
\hline 1991 & 3 & 5.43 & - & - & - & - & - & - & - & - & 3 & 5.43 \\
\hline 1992 & - & - & - & - & - & - & - & - & - & - & - & - \\
\hline
\end{tabular}

- indicates no deaths listed. $\quad *$ no specific code for byssinosis in ICD-8 (1968-1978).

NOTE: Rates based on small numbers may be unstable. Sum of individual conditions may exceed total because of deaths with multiple pneumoconioses. See Appendix A for source description and Appendix B for methods and ICD-8 and ICD-9 codes.

SOURCE: National Center for Health Statistics multiple cause of death data. Population estimates from U.S. Bureau of the Census. 
Figure ND-2. All pneumoconioses: Distribution of deaths by sex and race, North Dakota residents age 15 and over, 1968-1992
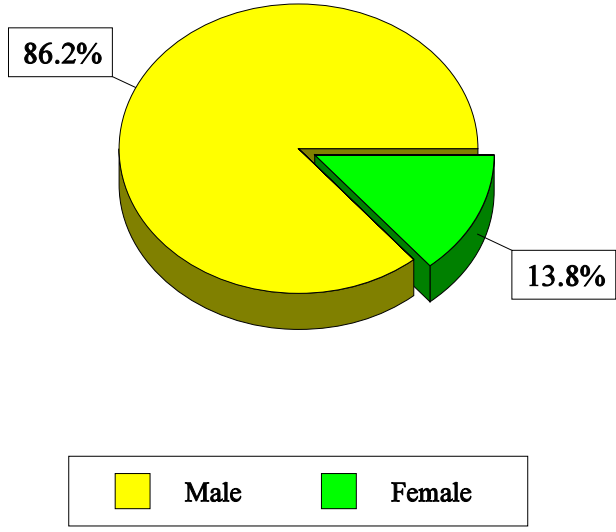

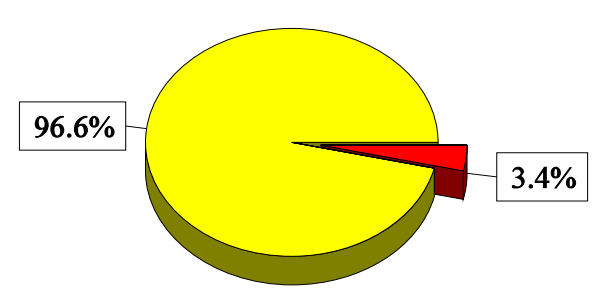

NOTE:

See Appendix A for source description and Appendix B for methods and ICD-8 and ICD-9 codes.

SOURCE: National Center for Health Statistics multiple cause of death data.

Table ND-2. All pneumoconioses: Distribution of deaths by sex, race, and age, North Dakota residents age 15 and over, 1968-1992

\begin{tabular}{|c|c|c|c|c|c|}
\hline & & \multicolumn{2}{|c|}{ 1968-1992 } & \multicolumn{2}{|c|}{ 1988-1992 } \\
\hline & & Number & Percent & Number & Percent \\
\hline \multicolumn{2}{|l|}{ Total deaths } & 29 & 100.0 & 8 & 100.0 \\
\hline \multirow[t]{2}{*}{ Sex } & Male & 25 & 86.2 & 8 & 100.0 \\
\hline & Female & 4 & 13.8 & 0 & 0.0 \\
\hline \multirow[t]{3}{*}{ Race } & White & 28 & 96.6 & 8 & 100.0 \\
\hline & Black & 0 & 0.0 & 0 & 0.0 \\
\hline & Other & 1 & 3.4 & 0 & 0.0 \\
\hline \multirow[t]{10}{*}{ Age } & $15-24$ & 0 & 0.0 & 0 & 0.0 \\
\hline & $25-34$ & 0 & 0.0 & 0 & 0.0 \\
\hline & $35-44$ & 2 & 6.9 & 2 & 25.0 \\
\hline & $45-54$ & 1 & 3.4 & 0 & 0.0 \\
\hline & $55-64$ & 9 & 31.0 & 1 & 12.5 \\
\hline & $65-74$ & 9 & 31.0 & 3 & 37.5 \\
\hline & $75-84$ & 6 & 20.7 & 2 & 25.0 \\
\hline & $85+$ & 2 & 6.9 & 0 & 0.0 \\
\hline & Mean age & 66.9 & & 64.3 & \\
\hline & Range for age & $38-85$ & & $38-82$ & \\
\hline $\begin{array}{l}\text { Perc } \\
\text { ICD }\end{array}$ & $\begin{array}{l}\text { ot total to } 100 \% \text { due to } \\
\text { todes. }\end{array}$ & See Append & ce descri] & $\operatorname{dix} B$ for $n$ & \\
\hline $\begin{array}{ll} & \text { ICD } \\
\text { CE: } & \text { Nati }\end{array}$ & $\begin{array}{l}\text { des. } \\
\text { Health Statistics mul }\end{array}$ & death data. & & & \\
\hline
\end{tabular}


Figure ND-3. Pneumoconiosis deaths by condition, North Dakota residents age 15 and over, 1968-1992

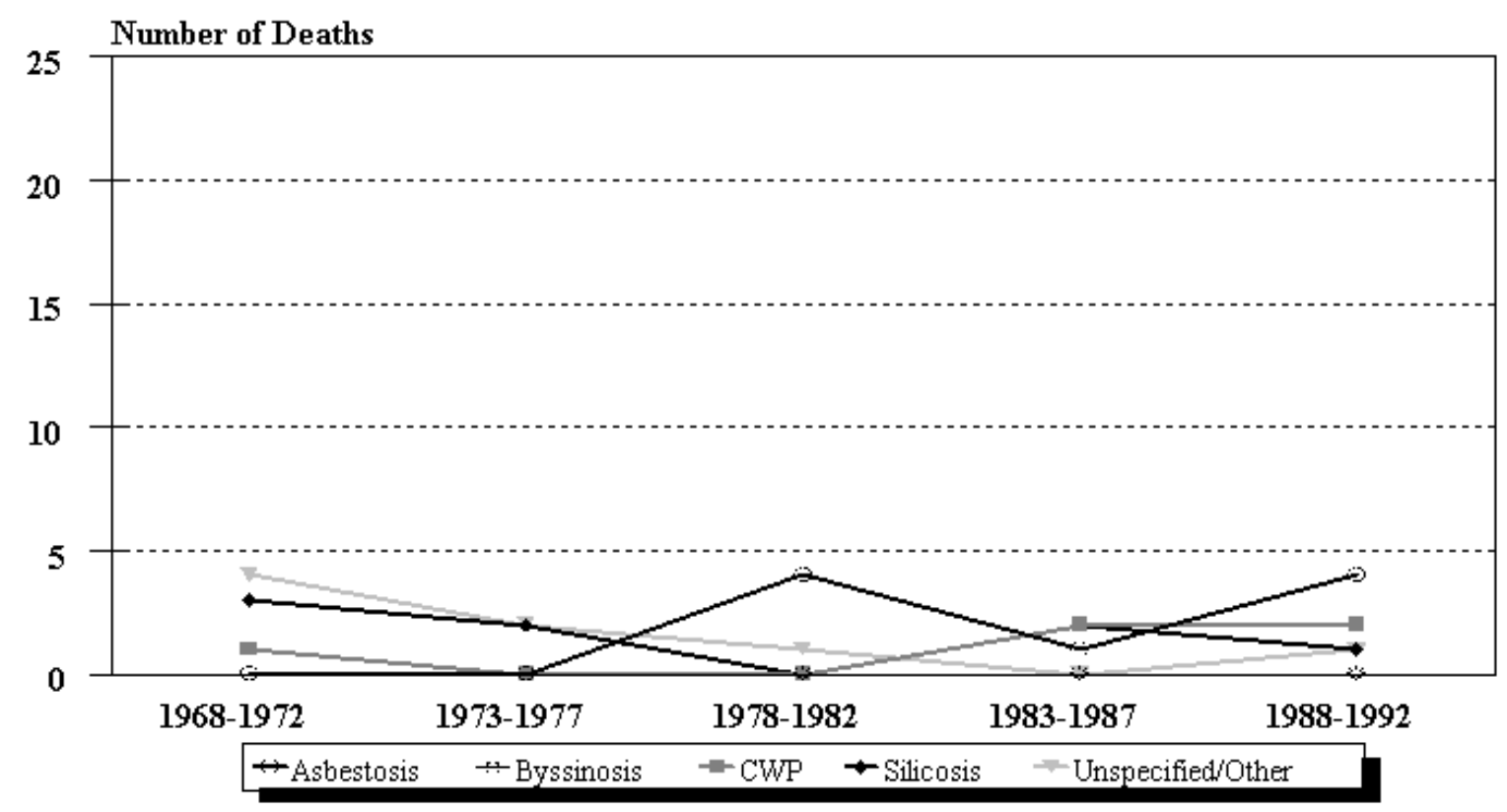

NOTE: See Appendix A for source description and Appendix B for methods and ICD-8 and ICD-9 codes.

SOURCE: National Center for Health Statistics multiple cause of death data.

Figure ND-4. North Dakota and U.S. age-adjusted mortality rates (per 1,000,000 population) by condition, residents age 15 and over, 1983-1992

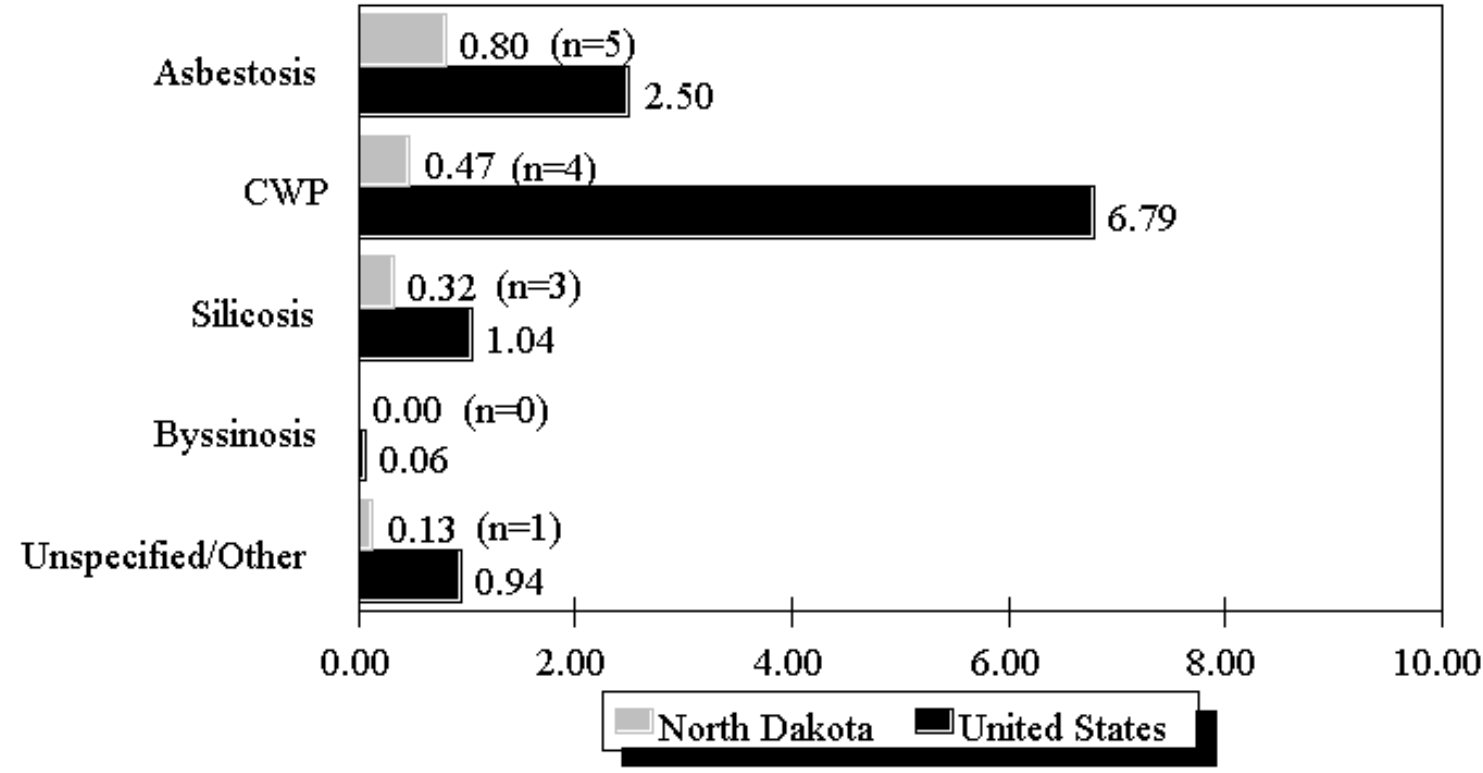

NOTE: Rates based on small numbers may be unstable. See Appendix A for source description and Appendix B for methods and ICD-8 and ICD-9 codes.

SOURCE: National Center for Health Statistics multiple cause of death data. Population estimates from U.S. Bureau of the Census. 
Table ND-3. Number of deaths and age-adjusted mortality rate (per 1,000,000 population) by condition and county, North Dakota residents age 15 and over, 1983-1992

\begin{tabular}{|c|c|c|c|c|c|c|c|c|c|c|c|c|}
\hline \multirow[b]{2}{*}{ County } & \multicolumn{2}{|c|}{ Asbestosis } & \multicolumn{2}{|c|}{$\begin{array}{c}\text { Coal workers' } \\
\text { pneumoconiosis }\end{array}$} & \multicolumn{2}{|c|}{ Silicosis } & \multicolumn{2}{|c|}{ Byssinosis } & \multicolumn{2}{|c|}{$\begin{array}{r}\text { Unspecified/Other } \\
\text { pneumoconioses }\end{array}$} & \multicolumn{2}{|c|}{$\begin{array}{r}\text { All } \\
\text { pneumoconioses }\end{array}$} \\
\hline & $\#$ & Rate & $\#$ & Rate & $\#$ & Rate & $\#$ & Rate & \# & Rate & \# & Rate \\
\hline Bottineau & 1 & 9.98 & - & - & - & - & - & - & - & - & 1 & 9.98 \\
\hline Burleigh & - & - & 1 & 2.08 & - & - & - & - & - & - & 1 & 2.08 \\
\hline Cass & 2 & 2.89 & - & - & 1 & 1.51 & - & - & - & - & 3 & 4.41 \\
\hline Emmons & - & - & - & - & 1 & 5.78 & - & - & - & - & 1 & 5.78 \\
\hline Grand Forks & - & - & 1 & 2.33 & - & - & - & - & - & - & 1 & 2.33 \\
\hline Griggs & - & - & - & - & - & - & - & - & 1 & 20.59 & 1 & 20.59 \\
\hline La Moure & 1 & 12.96 & - & - & - & - & - & - & - & - & 1 & 12.96 \\
\hline McHenry & - & - & 1 & 4.09 & - & - & - & - & - & - & 1 & 4.09 \\
\hline Morton & 1 & 4.30 & - & - & - & - & - & - & - & - & 1 & 4.30 \\
\hline Stutsman & - & - & - & - & 1 & 1.63 & - & - & - & - & 1 & 1.63 \\
\hline Traill & - & - & 1 & 2.85 & - & - & - & - & - & - & 1 & 2.85 \\
\hline
\end{tabular}

- indicates no deaths listed.

NOTE: Counties with no deaths for all listed conditions are not displayed. Rates based on small numbers may be unstable. Sum of individual conditions may exceed total because of deaths with multiple pneumoconioses. See Appendix A for source description and Appendix B for methods and ICD-8 and ICD-9 codes.

SOURCE: National Center for Health Statistics multiple cause of death data. Population estimates from U.S. Bureau of the Census. 


\section{Ohio: Selected statistics from the United States section}

All pneumoconioses mortality, 1988-1992

Total deaths

Crude mortality rate (per $1,000,000$ population)

Age-adjusted mortality rate (per 1,000,000 population)

Total years of potential life lost to life expectancy

Years of potential life lost to life expectancy (per death)

Deaths by type of pneumoconiosis, 1988-1992

Asbestosis

Coal workers' pneumoconiosis

Silicosis

Byssinosis

Unspecified/Other pneumoconiosis

\begin{tabular}{rr} 
Value & State rank \\
\hline 840 & 5 \\
19.77 & 8 \\
10.96 & 9 \\
9,882 & 5 \\
11.8 & 31
\end{tabular}

Percent**

$\underline{\text { Deaths* } \quad \text { distribution }}$

$118 \quad 14.0$

$428 \quad 51.0$

$153 \quad 18.2$

$3 \quad 0.4$

145

* Sum of column may exceed total deaths because of deaths with multiple pneumoconioses.

** Percentages may total more than $100 \%$ due to deaths with multiple pneumoconioses.

NOTE: $\quad$ See Appendix A for source description and Appendix B for methods and ICD-8 and ICD-9 codes.

SOURCE: $\quad$ National Center for Health Statistics multiple cause of death data. Population estimates from U.S. Bureau of the Census.

\section{Figure OH-1. All pneumoconioses: Ohio and U.S. age-adjusted mortality rates, residents age 15 and over, 1968-1992}

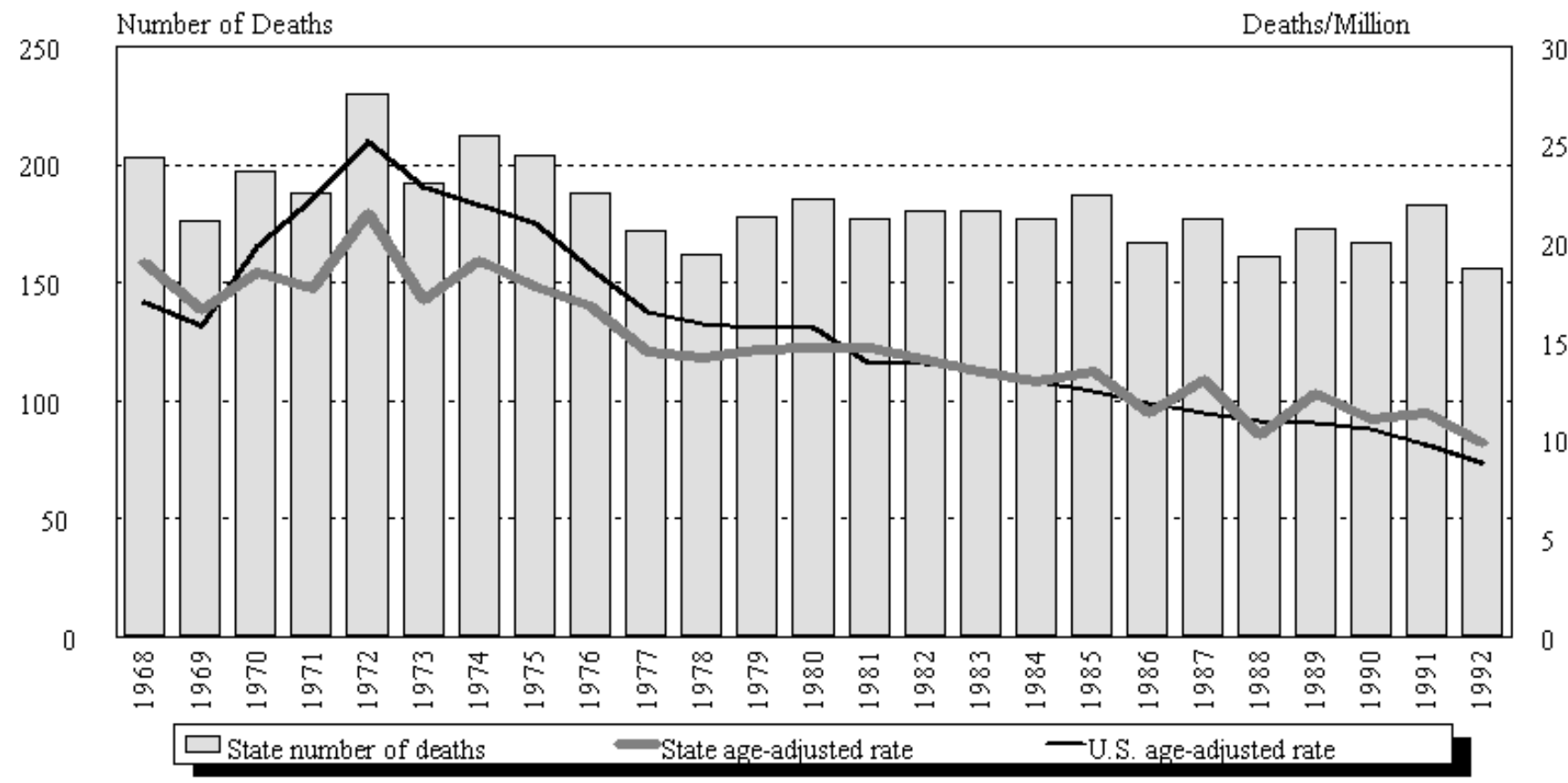

NOTE: $\quad$ See Appendix A for source description and Appendix B for methods and ICD-8 and ICD-9 codes.

SOURCE: $\quad$ National Center for Health Statistics multiple cause of death data. Population estimates from U.S. Bureau of the Census. 
Ohio

\begin{tabular}{|c|c|c|c|c|c|c|c|c|c|c|c|c|}
\hline \multirow[b]{2}{*}{ Year } & \multicolumn{2}{|c|}{ Asbestosis } & \multicolumn{2}{|c|}{$\begin{array}{l}\text { Coal workers' } \\
\text { pneumoconiosis }\end{array}$} & \multicolumn{2}{|c|}{ Silicosis } & \multicolumn{2}{|c|}{ Byssinosis* } & \multicolumn{2}{|c|}{$\begin{array}{c}\text { Unspecified/Other } \\
\text { pneumoconioses }\end{array}$} & \multicolumn{2}{|c|}{ All pneu moconioses } \\
\hline & Number & Rate & Number & Rate & Number & Rate & Number & Rate & Number & Rate & Number & Rate \\
\hline 1968 & 2 & 0.23 & 24 & 2.26 & 143 & 13.44 & & & 39 & 3.63 & 203 & 19.06 \\
\hline 1969 & - & - & 16 & 1.52 & 124 & 11.69 & & & 39 & 3.65 & 176 & 16.60 \\
\hline 1970 & 5 & 0.62 & 28 & 2.52 & 132 & 12.36 & & & 33 & 3.12 & 197 & 18.54 \\
\hline 1971 & 3 & 0.32 & 30 & 2.91 & 103 & 9.79 & & & 54 & 4.93 & 188 & 17.74 \\
\hline 1972 & 6 & 0.64 & 28 & 2.46 & 126 & 12.25 & & & 70 & 6.17 & 230 & 21.53 \\
\hline 1973 & 1 & 0.11 & 35 & 3.43 & 83 & 7.11 & & & 78 & 6.91 & 192 & 17.15 \\
\hline 1974 & 1 & 0.10 & 48 & 4.52 & 86 & 7.81 & & & 82 & 7.15 & 212 & 19.08 \\
\hline 1975 & 3 & 0.33 & 56 & 5.08 & 66 & 5.72 & & & 80 & 6.74 & 204 & 17.78 \\
\hline 1976 & 1 & 0.11 & 50 & 4.82 & 62 & 5.48 & & & 77 & 6.66 & 188 & 16.83 \\
\hline 1977 & 4 & 0.36 & 61 & 5.26 & 54 & 4.63 & & & 56 & 4.49 & 172 & 14.53 \\
\hline 1978 & 6 & 0.61 & 41 & 3.55 & 57 & 5.13 & & & 64 & 5.43 & 162 & 14.19 \\
\hline 1979 & 7 & 0.68 & 68 & 5.66 & 52 & 4.22 & 1 & 0.09 & 50 & 3.93 & 178 & 14.59 \\
\hline 1980 & 9 & 0.82 & 92 & 7.34 & 42 & 3.35 & - & - & 46 & 3.58 & 185 & 14.74 \\
\hline 1981 & 8 & 0.74 & 105 & 8.87 & 38 & 3.15 & - & - & 30 & 2.34 & 177 & 14.74 \\
\hline 1982 & 10 & 1.10 & 86 & 6.26 & 50 & 3.98 & - & - & 34 & 2.71 & 180 & 14.04 \\
\hline 1983 & 9 & 0.87 & 105 & 7.53 & 32 & 2.64 & - & - & 36 & 2.60 & 180 & 13.52 \\
\hline 1984 & 10 & 0.82 & 93 & 6.83 & 47 & 3.65 & - & - & 28 & 1.81 & 177 & 13.01 \\
\hline 1985 & 17 & 1.50 & 105 & 7.29 & 31 & 2.35 & - & - & 36 & 2.54 & 187 & 13.51 \\
\hline 1986 & 14 & 1.24 & 92 & 6.11 & 41 & 2.72 & - & - & 22 & 1.48 & 167 & 11.40 \\
\hline 1987 & 21 & 1.97 & 91 & 6.08 & 29 & 2.59 & - & - & 36 & 2.41 & 177 & 13.06 \\
\hline 1988 & 12 & 0.98 & 102 & 5.91 & 26 & 1.89 & 2 & 0.13 & 20 & 1.37 & 161 & 10.23 \\
\hline 1989 & 23 & 1.93 & 90 & 6.30 & 35 & 2.37 & - & - & 27 & 1.90 & 173 & 12.37 \\
\hline 1990 & 27 & 2.12 & 79 & 4.82 & 33 & 2.20 & 1 & 0.11 & 29 & 1.93 & 167 & 11.04 \\
\hline 1991 & 24 & 1.91 & 83 & 4.91 & 35 & 2.11 & - & & 42 & 2.55 & 183 & 11.40 \\
\hline 1992 & 32 & 2.48 & 74 & 3.89 & 24 & 1.80 & - & & 27 & 1.79 & 156 & 9.86 \\
\hline
\end{tabular}

indicates no deaths listed. $\quad{ }^{*}$ no specific code for by ssinosis in ICD-8 (1968-1 978).

NOTE: Rates based on small numbers may be unstable. Sum of individ ual conditions may exceed total because of deaths with multiple pneumo conioses. See Appendix A for source description and Appendix B for methods and ICD-8 and ICD-9

SOURCE: National Center for Health Statistics multiple cause of death data. Population estimates from U.S. Bureau of the Census. 
Figure OH-2. All pneumoconioses: Distribution of deaths by sex and race, Ohio residents age 15 and over, 1968-1992
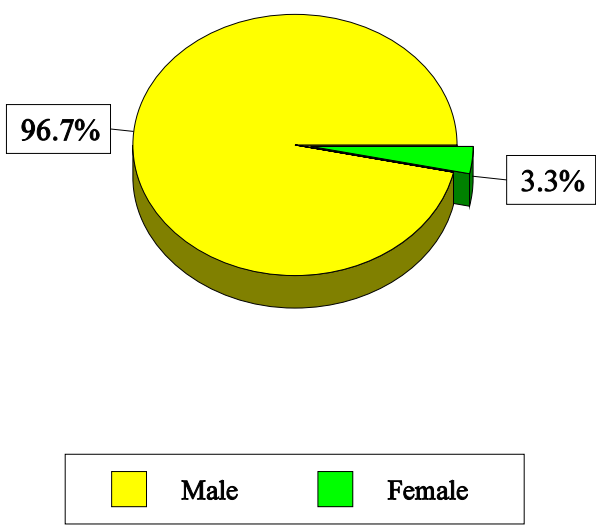

$86.0 \%$
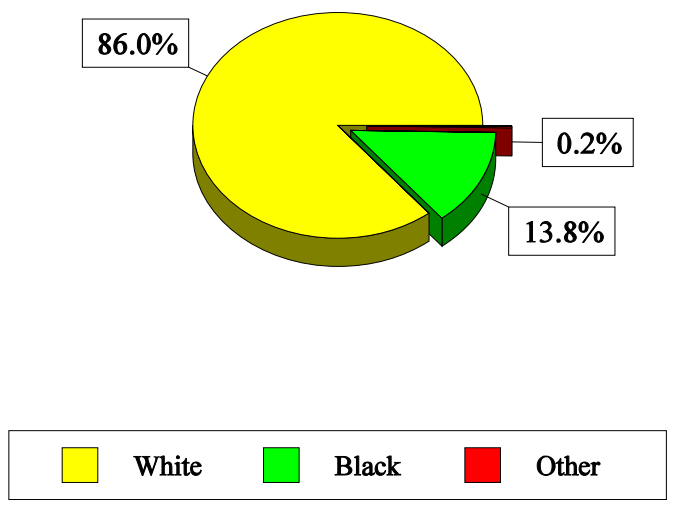

NOTE: See Appendix A for source description and Appendix B for methods and ICD-8 and ICD-9 codes.

SOURCE: National Center for Health Statistics multiple cause of death data.

Table OH-2. All pneumoconioses: Distribution of deaths by sex, race, and age, Ohio residents age 15 and over, 1968-1992

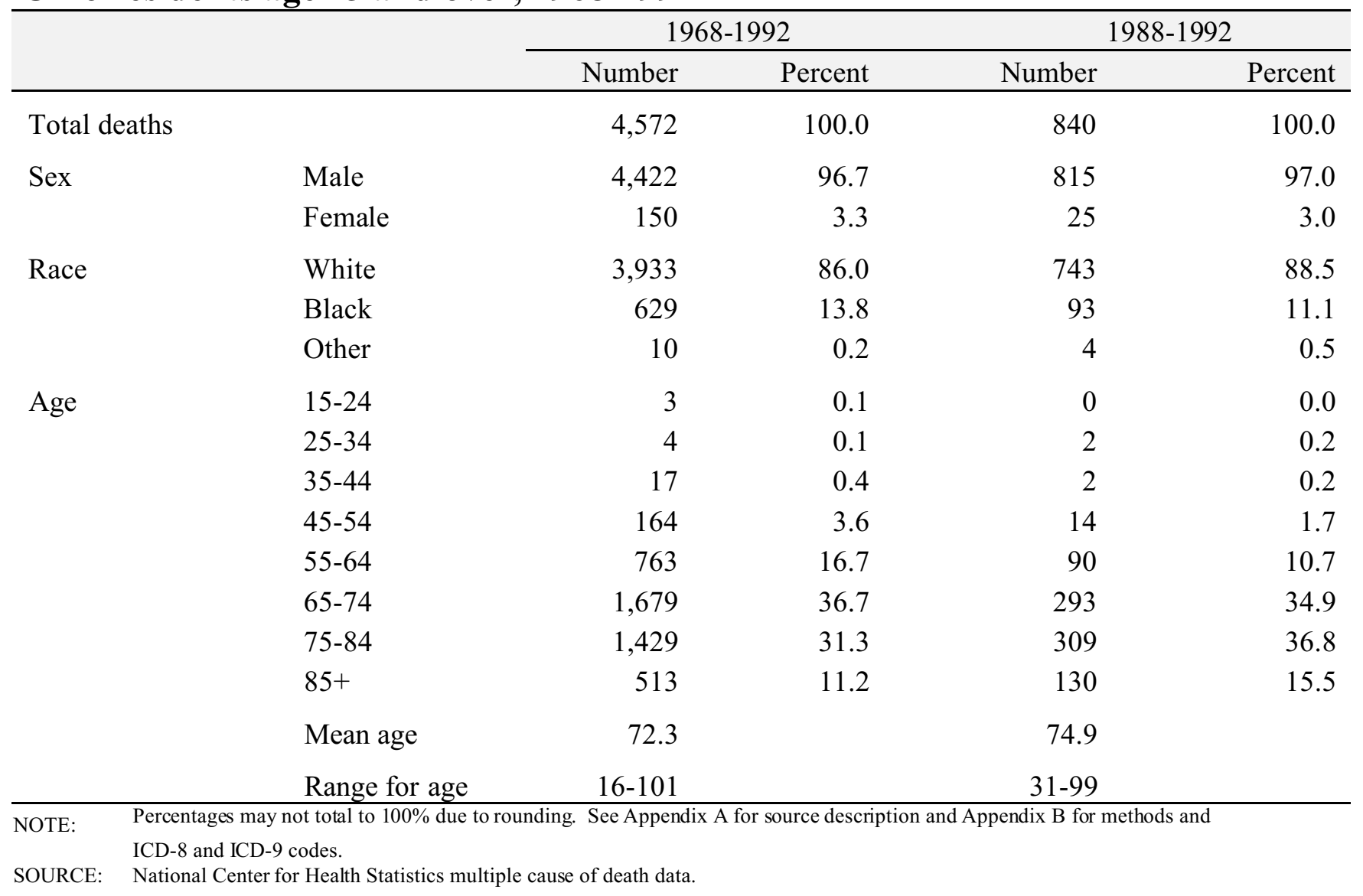




\section{Figure OH-3. Pneumoconiosis deaths by condition, Ohio residents age 15 and over, 1968-1992}

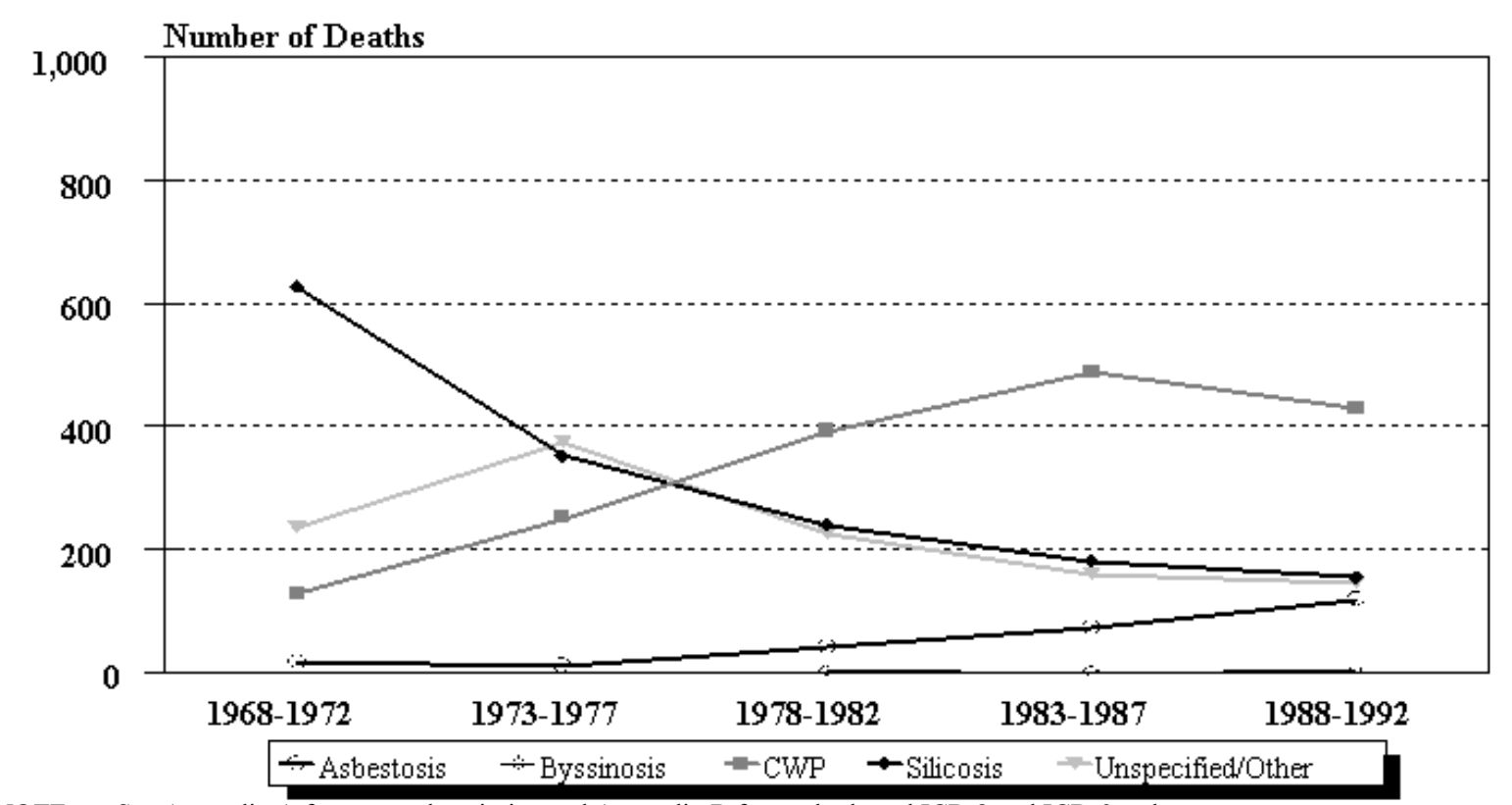

NOTE: See Appendix A for source description and Appendix B for methods and ICD-8 and ICD-9 codes.

SOURCE: National Center for Health Statistics multiple cause of death data.

Figure OH-4. Ohio and U.S. age-adjusted mortality rates (per 1,000,000 population) by condition, residents age 15 and over, 1983-1992

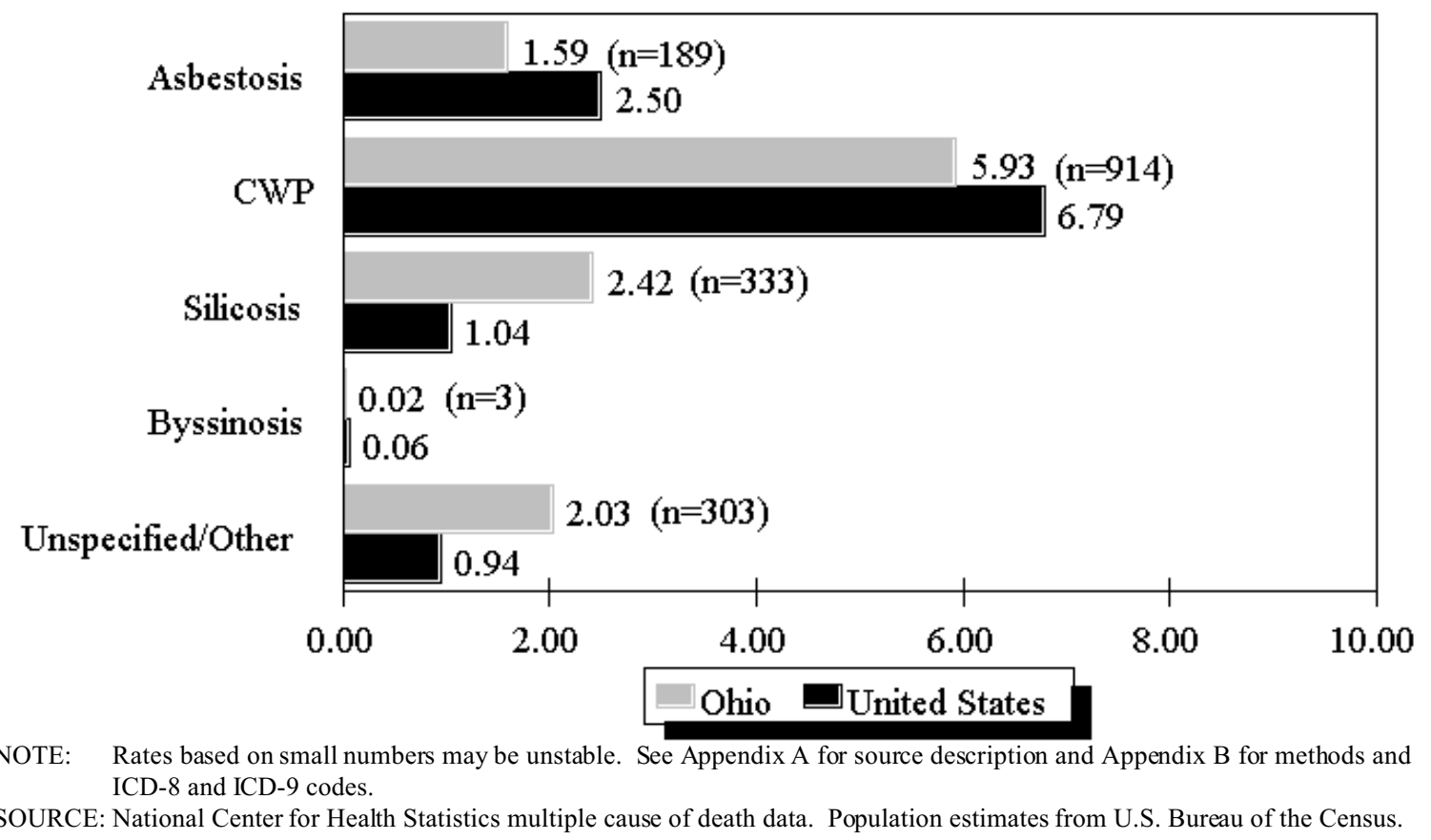


Table OH-3 (page 1 of 3). Number of deaths and age-adjusted mortality rate (per 1,000,000 population) by condition and county, Ohio residents age 15 and over, 1983-1992

\begin{tabular}{|c|c|c|c|c|c|c|c|c|c|c|c|c|}
\hline \multirow[b]{2}{*}{ County } & \multicolumn{2}{|c|}{ Asbestosis } & \multicolumn{2}{|c|}{$\begin{array}{r}\text { Coal workers' } \\
\text { pneumoconiosis }\end{array}$} & \multicolumn{2}{|c|}{ Silicosis } & \multicolumn{2}{|c|}{ Byssinosis } & \multicolumn{2}{|c|}{$\begin{array}{r}\text { Unspecified/Other } \\
\text { pneumoconioses }\end{array}$} & \multicolumn{2}{|c|}{$\begin{array}{r}\text { All } \\
\text { pneumoconioses }\end{array}$} \\
\hline & \# & Rate & $\#$ & Rate & $\#$ & Rate & $\#$ & Rate & $\#$ & Rate & $\#$ & Rate \\
\hline Adams & - & - & 3 & 7.89 & - & - & - & - & 2 & 3.85 & 5 & 11.73 \\
\hline Allen & 2 & 1.87 & 4 & 2.29 & 1 & 0.45 & - & - & 1 & 0.94 & 8 & 5.55 \\
\hline Ashland & 1 & 2.17 & 6 & 8.32 & 3 & 5.34 & - & - & 1 & 1.00 & 11 & 16.83 \\
\hline Ashtabula & 6 & 4.70 & 6 & 2.78 & 1 & 0.46 & - & - & 1 & 0.94 & 14 & 8.89 \\
\hline Athens & - & - & 27 & 45.81 & - & - & - & - & 8 & 11.27 & 35 & 57.08 \\
\hline Avglaize & - & - & 6 & 7.76 & 3 & 7.09 & - & - & - & - & 9 & 14.84 \\
\hline Belmont & 2 & 1.00 & 64 & 44.17 & 1 & 0.50 & - & - & 25 & 20.94 & 91 & 66.11 \\
\hline Brown & 1 & 3.08 & 1 & 1.43 & - & - & - & - & - & - & 2 & 4.51 \\
\hline Butler & 4 & 1.49 & 22 & 7.03 & 13 & 4.34 & - & - & 3 & 0.89 & 40 & 12.92 \\
\hline Carroll & 1 & 3.69 & 6 & 15.31 & 2 & 7.38 & - & - & - & - & 9 & 26.39 \\
\hline Champaign & - & - & 4 & 10.52 & 1 & 1.44 & - & - & - & - & 5 & 11.95 \\
\hline Clark & - & - & 7 & 2.65 & 4 & 2.68 & - & - & 2 & 1.35 & 13 & 6.67 \\
\hline Clermont & 2 & 1.51 & 2 & 1.51 & - & - & - & - & 4 & 2.66 & 8 & 5.67 \\
\hline Clinton & - & - & 4 & 10.52 & - & - & - & - & - & - & 4 & 10.52 \\
\hline Columbiana & 3 & 2.98 & 17 & 10.27 & 24 & 14.63 & - & - & 10 & 5.60 & 52 & 32.21 \\
\hline Coshocton & - & - & 12 & 25.09 & 4 & 10.62 & - & - & 1 & 1.31 & 17 & 37.01 \\
\hline Crawford & - & - & 4 & 5.88 & 1 & 0.93 & - & - & 4 & 6.96 & 9 & 13.78 \\
\hline Cuyahoga & 17 & 0.98 & 75 & 3.19 & 57 & 2.59 & - & - & 39 & 1.76 & 187 & 8.45 \\
\hline Darke & - & - & 4 & 3.18 & - & - & - & - & 1 & 0.80 & 5 & 3.98 \\
\hline Defiance & 1 & 1.47 & 1 & 1.47 & - & - & - & - & 2 & 5.83 & 4 & 8.76 \\
\hline Delaware & 2 & 4.01 & 3 & 4.26 & 2 & 4.01 & - & - & 1 & 1.13 & 8 & 13.40 \\
\hline Erie & 2 & 1.94 & 5 & 4.59 & 4 & 4.42 & - & - & - & - & 10 & 9.71 \\
\hline Fairfield & 1 & 1.13 & 4 & 2.86 & 2 & 1.71 & - & - & - & - & 7 & 5.70 \\
\hline Franklin & 10 & 1.20 & 61 & 6.68 & 18 & 1.85 & - & - & 19 & 1.80 & 108 & 11.53 \\
\hline Fulton & - & - & - & - & - & - & - & - & 1 & 1.31 & 1 & 1.31 \\
\hline Gallia & 3 & 10.11 & 4 & 10.04 & 2 & 3.30 & - & - & 6 & 13.34 & 15 & 36.79 \\
\hline Geauga & 1 & 1.43 & 4 & 4.69 & 2 & 2.86 & - & - & 2 & 2.35 & 9 & 11.33 \\
\hline Greene & 4 & 3.33 & 13 & 9.71 & - & - & - & - & 3 & 2.35 & 20 & 15.40 \\
\hline Guernsey & - & - & 14 & 24.32 & 2 & 3.47 & - & - & 8 & 11.29 & 24 & 39.08 \\
\hline Hamilton & 22 & 2.22 & 51 & 4.40 & 20 & 1.68 & - & - & 12 & 0.85 & 104 & 9.10 \\
\hline Hancock & - & - & - & - & 2 & 2.41 & - & - & 1 & 0.76 & 3 & 3.17 \\
\hline
\end{tabular}

See footnotes at end of table. 
Table OH-3 (page 2 of 3). Number of deaths and age-adjusted mortality rate (per 1,000,000 population) by condition and county, Ohio residents age 15 and over, 1983-1992

\begin{tabular}{|c|c|c|c|c|c|c|c|c|c|c|c|c|}
\hline \multirow[b]{2}{*}{ County } & \multicolumn{2}{|c|}{ Asbestosis } & \multicolumn{2}{|c|}{$\begin{array}{r}\text { Coal workers' } \\
\text { pneumoconiosis }\end{array}$} & \multicolumn{2}{|c|}{ Silicosis } & \multicolumn{2}{|c|}{ Byssinosis } & \multicolumn{2}{|c|}{$\begin{array}{r}\text { Unspecified/Other } \\
\text { pneumoconioses }\end{array}$} & \multicolumn{2}{|c|}{$\begin{array}{r}\text { All } \\
\text { pneumoconioses }\end{array}$} \\
\hline & \# & Rate & $\#$ & Rate & $\#$ & Rate & $\#$ & Rate & $\#$ & Rate & $\#$ & Rate \\
\hline Hardin & - & - & 2 & 4.89 & 1 & 3.41 & - & - & - & - & 3 & 8.30 \\
\hline Harrison & - & - & 10 & 49.86 & - & - & - & - & 3 & 12.69 & 13 & 62.55 \\
\hline Henry & 1 & 1.55 & 1 & 3.57 & - & - & - & - & 1 & 3.57 & 3 & 8.70 \\
\hline Highland & - & - & 2 & 3.88 & - & - & - & - & 1 & 2.66 & 3 & 6.54 \\
\hline Hocking & 1 & 5.55 & 3 & 9.82 & 1 & 1.97 & - & - & 1 & 1.97 & 6 & 19.32 \\
\hline Holmes & - & - & 2 & 7.08 & 1 & 1.76 & - & - & - & - & 3 & 8.84 \\
\hline Huron & - & - & 2 & 2.02 & 2 & 2.02 & - & - & 1 & 1.01 & 5 & 5.06 \\
\hline Jackson & 1 & 3.17 & 10 & 21.82 & 8 & 23.73 & - & - & 10 & 25.12 & 28 & 70.68 \\
\hline Jefferson & 3 & 3.19 & 29 & 21.66 & 3 & 3.63 & - & - & 9 & 6.87 & 44 & 35.35 \\
\hline Knox & 1 & 2.04 & 1 & 0.94 & 7 & 13.16 & - & - & - & - & 9 & 16.14 \\
\hline Lake & 1 & 0.31 & 11 & 4.07 & 4 & 1.88 & - & - & 3 & 1.44 & 19 & 7.70 \\
\hline Lawrence & 3 & 4.67 & 10 & 12.08 & 1 & 0.86 & - & - & 10 & 14.17 & 24 & 31.78 \\
\hline Licking & - & - & 2 & 0.90 & 2 & 1.30 & - & - & 1 & 0.45 & 5 & 2.65 \\
\hline Logan & - & - & - & - & 1 & 2.28 & - & - & - & - & 1 & 2.28 \\
\hline Lorain & 6 & 2.38 & 14 & 4.50 & 8 & 3.36 & 1 & 0.41 & 11 & 3.90 & 40 & 14.56 \\
\hline Lucas & 14 & 2.94 & 14 & 2.59 & 9 & 2.11 & - & - & 5 & 1.02 & 42 & 8.65 \\
\hline Madison & - & - & 3 & 7.19 & - & - & - & - & - & - & 3 & 7.19 \\
\hline Mahoning & 6 & 1.68 & 15 & 3.00 & 6 & 1.11 & - & - & 6 & 1.26 & 33 & 7.05 \\
\hline Marion & - & - & 4 & 4.14 & 8 & 9.60 & - & - & 2 & 3.00 & 14 & 16.74 \\
\hline Medina & - & - & 17 & 13.65 & 2 & 1.23 & - & - & 4 & 3.83 & 22 & 18.09 \\
\hline Meigs & - & - & 10 & 31.72 & 1 & 4.01 & - & - & 12 & 31.37 & 23 & 67.10 \\
\hline Mercer & - & - & 1 & 2.63 & - & - & - & - & - & - & 1 & 2.63 \\
\hline Miami & 1 & 0.59 & 2 & 1.72 & 1 & 0.59 & - & - & 1 & 1.13 & 4 & 3.44 \\
\hline Monroe & - & - & 1 & 2.50 & - & - & - & - & 1 & 5.80 & 2 & 8.30 \\
\hline Montgomery & 8 & 1.35 & 51 & 6.52 & 14 & 2.09 & - & - & 8 & 1.20 & 81 & 11.16 \\
\hline Morgan & - & - & 5 & 14.67 & 2 & 5.87 & - & - & - & - & 7 & 20.53 \\
\hline Morrow & - & - & 6 & 19.11 & 1 & 4.17 & - & - & 1 & 4.17 & 8 & 27.46 \\
\hline Muskingum & 1 & 1.21 & 29 & 27.87 & 14 & 9.83 & - & - & 3 & 2.34 & 47 & 41.24 \\
\hline Noble & - & - & 1 & 3.42 & 1 & 8.22 & - & - & 2 & 6.85 & 4 & 18.49 \\
\hline Ottawa & - & - & 1 & 1.20 & - & - & - & - & 1 & 2.16 & 2 & 3.35 \\
\hline Paulding & - & - & 4 & 20.80 & - & - & - & - & - & - & 4 & 20.80 \\
\hline Perry & - & - & 38 & 97.51 & 8 & 17.80 & - & - & 1 & 3.34 & 47 & 118.65 \\
\hline
\end{tabular}

See footnotes at end of table. 
Table OH-3 (page 3 of 3). Number of deaths and age-adjusted mortality rate (per 1,000,000 population) by condition and county, Ohio residents age 15 and over, 1983-1992

\begin{tabular}{|c|c|c|c|c|c|c|c|c|c|c|c|c|}
\hline \multirow[b]{2}{*}{ County } & \multicolumn{2}{|c|}{ Asbestosis } & \multicolumn{2}{|c|}{$\begin{array}{r}\text { Coal workers' } \\
\text { pneumoconiosis }\end{array}$} & \multicolumn{2}{|c|}{ Silicosis } & \multicolumn{2}{|c|}{ Byssinosis } & \multicolumn{2}{|c|}{$\begin{array}{r}\text { Unspecified/Other } \\
\text { pneumoconioses }\end{array}$} & \multicolumn{2}{|c|}{$\begin{array}{r}\text { All } \\
\text { pneumoconioses }\end{array}$} \\
\hline & $\#$ & Rate & \# & Rate & $\#$ & Rate & $\#$ & Rate & $\#$ & Rate & $\#$ & Rate \\
\hline Pickaway & 5 & 12.47 & 7 & 13.56 & 3 & 7.75 & - & - & 1 & 2.49 & 16 & 36.27 \\
\hline Pike & 1 & 6.25 & 4 & 14.63 & - & - & - & - & 1 & 4.25 & 6 & 25.12 \\
\hline Portage & 4 & 3.76 & 13 & 9.05 & - & - & - & - & 3 & 2.04 & 20 & 14.84 \\
\hline Preble & - & - & 4 & 9.22 & - & - & - & - & - & - & 4 & 9.22 \\
\hline Putnam & - & - & 1 & 3.42 & 1 & 3.42 & - & - & - & - & 2 & 6.84 \\
\hline Richland & - & - & 11 & 7.18 & 3 & 2.30 & - & - & 2 & 0.86 & 16 & 10.34 \\
\hline Ross & 1 & 1.51 & 1 & 0.80 & - & - & - & - & - & - & 2 & 2.32 \\
\hline Sandusky & 1 & 1.66 & 3 & 3.27 & - & - & - & - & - & - & 4 & 4.92 \\
\hline Scioto & 1 & 1.16 & 4 & 4.02 & 7 & 5.60 & - & - & 2 & 2.37 & 14 & 13.16 \\
\hline Seneca & - & - & 1 & 1.73 & 2 & 3.45 & - & - & - & - & 3 & 5.18 \\
\hline Shelby & - & - & 6 & 10.22 & 1 & 2.57 & - & - & 1 & 1.27 & 8 & 14.05 \\
\hline Stark & 4 & 1.03 & 14 & 2.73 & 20 & 4.14 & - & - & 6 & 1.17 & 44 & 9.06 \\
\hline Summit & 26 & 4.08 & 32 & 4.16 & 8 & 1.50 & - & - & 8 & 1.15 & 73 & 10.70 \\
\hline Trumbull & 2 & 0.79 & 20 & 6.25 & 6 & 2.04 & 2 & 0.63 & 6 & 1.55 & 34 & 10.63 \\
\hline Tuscarawas & - & - & 33 & 26.63 & 1 & 1.11 & - & - & 7 & 6.00 & 41 & 33.74 \\
\hline Union & 1 & 4.37 & 3 & 7.41 & - & - & - & - & - & - & 4 & 11.78 \\
\hline Van Wert & - & - & 1 & 3.21 & - & - & - & - & - & - & 1 & 3.21 \\
\hline Vinton & 2 & 17.78 & 1 & 4.22 & - & - & - & - & 2 & 17.78 & 5 & 39.78 \\
\hline Warren & 2 & 1.87 & 4 & 3.29 & 2 & 2.33 & - & - & 5 & 5.83 & 13 & 13.32 \\
\hline Washington & 1 & 1.59 & 4 & 3.87 & 1 & 0.76 & - & - & - & - & 6 & 6.22 \\
\hline Wayne & 1 & 1.15 & 7 & 6.02 & 1 & 1.15 & - & - & 3 & 2.87 & 12 & 11.19 \\
\hline Williams & 2 & 4.12 & - & - & - & - & - & - & - & - & 2 & 4.12 \\
\hline Wood & 3 & 3.14 & 4 & 4.62 & 1 & 0.59 & - & - & 1 & 0.59 & 9 & 8.94 \\
\hline Wyandot & - & - & 1 & 4.35 & 1 & 6.62 & - & - & - & - & 2 & 10.97 \\
\hline
\end{tabular}

- indicates no deaths listed.

NOTE: Counties with no deaths for all listed conditions are not displayed. Rates based on small numbers may be unstable. Sum of individual conditions may exceed total because of deaths with multiple pneumoconioses. See Appendix A for source description and Appendix B for methods and ICD-8 and ICD-9 codes.

SOURCE: National Center for Health Statistics multiple cause of death data. Population estimates from U.S. Bureau of the Census. 


\section{Oklahoma: Selected statistics from the United States section}

All pneumoconioses mortality, 1988-1992

Total deaths

$\underline{\text { Value }}$

$\underline{\text { State rank }}$

Crude mortality rate (per $1,000,000$ population)

73

Age-adjusted mortality rate (per $1,000,000$ population)

5.98

35

Total years of potential life lost to life expectancy

3.00

Years of potential life lost to life expectancy (per death)

Deaths by type of pneumoconiosis, 1988-1992

Percent**

Asbestosis

$\underline{\text { Deaths* }}$

distribution

Coal workers' pneumoconiosis

Silicosis

Byssinosis

\section{Unspecified/Other pneumoconiosis}

* Sum of column may exceed total deaths because of deaths with multiple pneumoconioses.

** Percentages may total more than $100 \%$ due to deaths with multiple pneumoconioses.

NOTE: $\quad$ See Appendix A for source description and Appendix B for methods and ICD-8 and ICD-9 codes.

SOURCE: National Center for Health Statistics multiple cause of death data. Population estimates from U.S. Bureau of the Census.

Figure OK-1. All pneumoconioses: Oklahoma and U.S. age-adjusted mortality rates, residents age 15 and over, 1968-1992

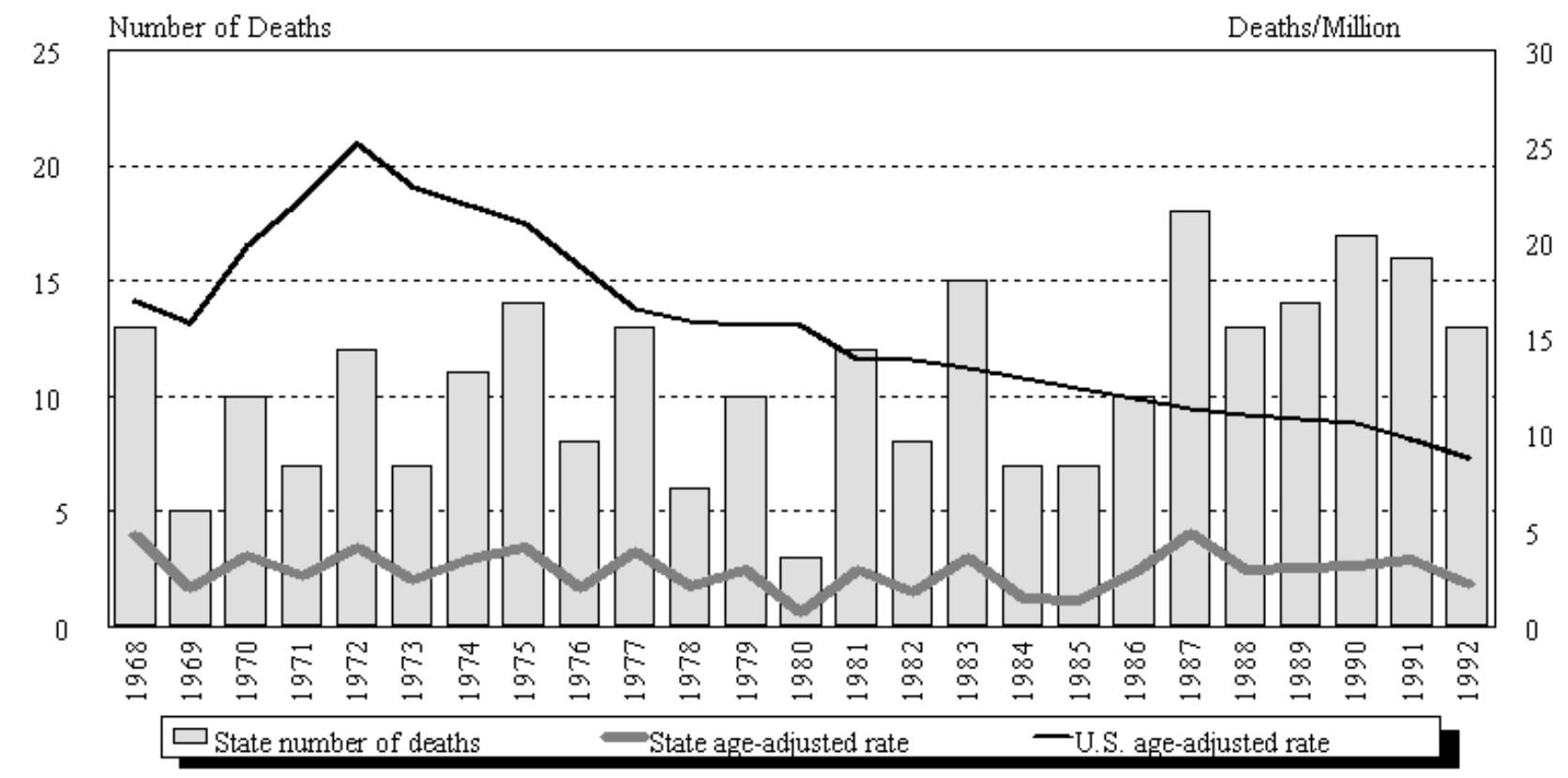

NOTE: 
Oklahoma

\begin{tabular}{|c|c|c|c|c|c|c|c|c|c|c|c|c|}
\hline \multirow[b]{2}{*}{ Year } & \multicolumn{2}{|c|}{ Asb estosis } & \multicolumn{2}{|c|}{$\begin{array}{l}\text { Coal workers' } \\
\text { pne umoconiosis }\end{array}$} & \multicolumn{2}{|c|}{ Silicosis } & \multicolumn{2}{|c|}{ Byssinosis* } & \multicolumn{2}{|c|}{$\begin{array}{c}\text { Unspecified/Other } \\
\text { pneumoconioses }\end{array}$} & \multicolumn{2}{|c|}{ All pneumoconioses } \\
\hline & Number & Rate & Number & Rate & Number & Rate & Number & Rate & Number & Rate & Number & Rate \\
\hline 1968 & - & - & - & - & 10 & 3.59 & & & 3 & 1.19 & 13 & 4.78 \\
\hline 1969 & - & - & - & - & 4 & 1.61 & & & 1 & 0.42 & 5 & 2.03 \\
\hline 1970 & - & - & - & - & 7 & 2.77 & & & 3 & 0.95 & 10 & 3.72 \\
\hline 1971 & - & - & 1 & 0.24 & 5 & 1.97 & & & 2 & 0.84 & 7 & 2.63 \\
\hline 1972 & - & - & 4 & 1.31 & 6 & 2.14 & & & 4 & 1.50 & 12 & 4.11 \\
\hline 1973 & - & - & 1 & 0.14 & 2 & 0.91 & & & 4 & 1.42 & 7 & 2.46 \\
\hline 1974 & - & - & 1 & 0.13 & 8 & 2.77 & & & 3 & 0.77 & 11 & 3.45 \\
\hline 1975 & 1 & 0.31 & 3 & 0.97 & 5 & 1.20 & & & 5 & 1.60 & 14 & 4.09 \\
\hline 1976 & - & - & 2 & 0.34 & 1 & 0.30 & & & 5 & 1.39 & 8 & 2.04 \\
\hline 1977 & - & - & 4 & 1.21 & 3 & 1.04 & & & 7 & 2.01 & 13 & 3.96 \\
\hline 1978 & 2 & 0.82 & 3 & 0.79 & - & - & & & 1 & 0.53 & 6 & 2.15 \\
\hline 1979 & 1 & 0.39 & 3 & 0.97 & 3 & 0.97 & - & - & 3 & 0.60 & 10 & 2.92 \\
\hline 1980 & - & - & 2 & 0.48 & 1 & 0.29 & - & - & - & - & 3 & 0.77 \\
\hline 1981 & - & - & 10 & 2.27 & - & - & - & - & 2 & 0.74 & 12 & 3.01 \\
\hline 1982 & 4 & 1.38 & 4 & 0.38 & - & - & - & - & - & - & 8 & 1.77 \\
\hline 1983 & 6 & 1.73 & 6 & 0.96 & 2 & 0.69 & - & - & 1 & 0.26 & 15 & 3.65 \\
\hline 1984 & - & - & 3 & 0.70 & 2 & 0.44 & - & - & 2 & 0.34 & 7 & 1.47 \\
\hline 1985 & 2 & 0.61 & 4 & 0.61 & - & - & - & - & 1 & 0.17 & 7 & 1.38 \\
\hline 1986 & 2 & 0.43 & 5 & 1.31 & 3 & 1.13 & - & - & - & - & 10 & 2.87 \\
\hline 1987 & 5 & 1.63 & 8 & 1.55 & 4 & 1.46 & - & - & 1 & 0.27 & 18 & 4.90 \\
\hline 1988 & 5 & 1.67 & 4 & 0.66 & - & - & - & - & 4 & 0.63 & 13 & 2.95 \\
\hline 1989 & 8 & 1.77 & 3 & 0.52 & 1 & 0.16 & - & - & 2 & 0.58 & 14 & 3.04 \\
\hline 1990 & 6 & 1.30 & 5 & 0.68 & 4 & 0.91 & - & - & 2 & 0.24 & 17 & 3.13 \\
\hline 1991 & 6 & 1.21 & 4 & 0.90 & 4 & 0.86 & 1 & 0.16 & 1 & 0.39 & 16 & 3.53 \\
\hline 1992 & 5 & 1.13 & 1 & 0.08 & 5 & 0.81 & - & - & 2 & 0.23 & 13 & 2.24 \\
\hline
\end{tabular}

- indicates no deaths listed. $\quad{ }^{*}$ no specific code for byssinosis in ICD-8 (1968-1978)

NOTE: Rates based on small numbers may be unstable. Sum of individ ual conditions may exceed total because of deaths with multiple pneumoconioses. See Appendix A for source description and Appendix B for methods and ICD-8 and ICD-9

SOURCE: National Center for Health Statistics multiple cause of death data. Population estimates from U.S. Bureau of the Census. 


\section{Figure OK-2. All pneumoconioses: Distribution of deaths by sex and race,} Oklahoma residents age 15 and over, 1968-1992
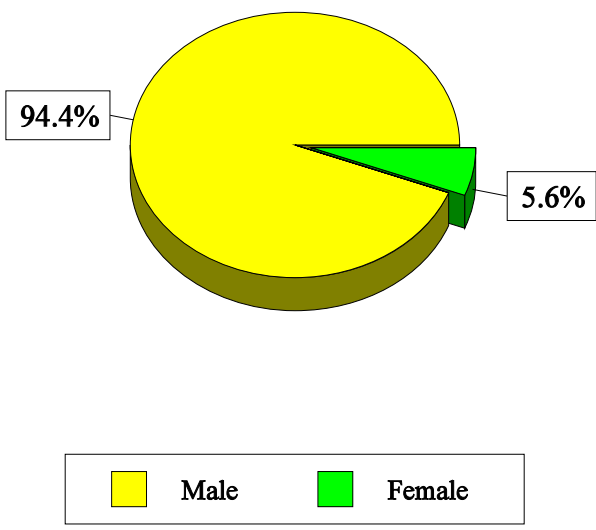
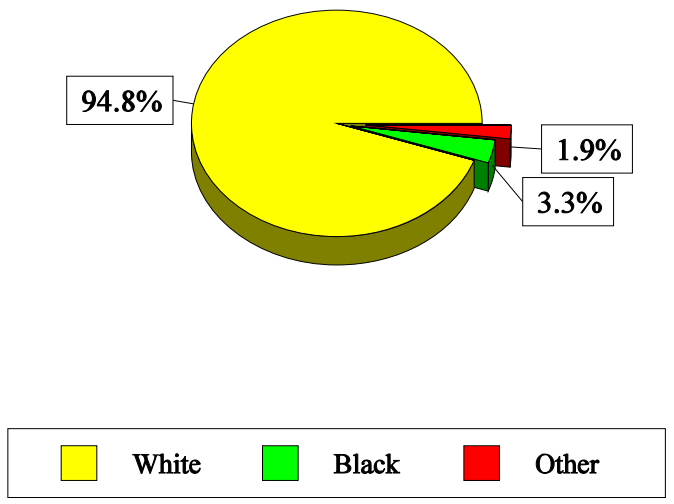

NOTE: See Appendix A for source description and Appendix B for methods and ICD-8 and ICD-9 codes.

SOURCE: National Center for Health Statistics multiple cause of death data.

Table OK-2. All pneumoconioses: Distribution of deaths by sex, race, and age, Oklahoma residents age 15 and over, 1968-1992

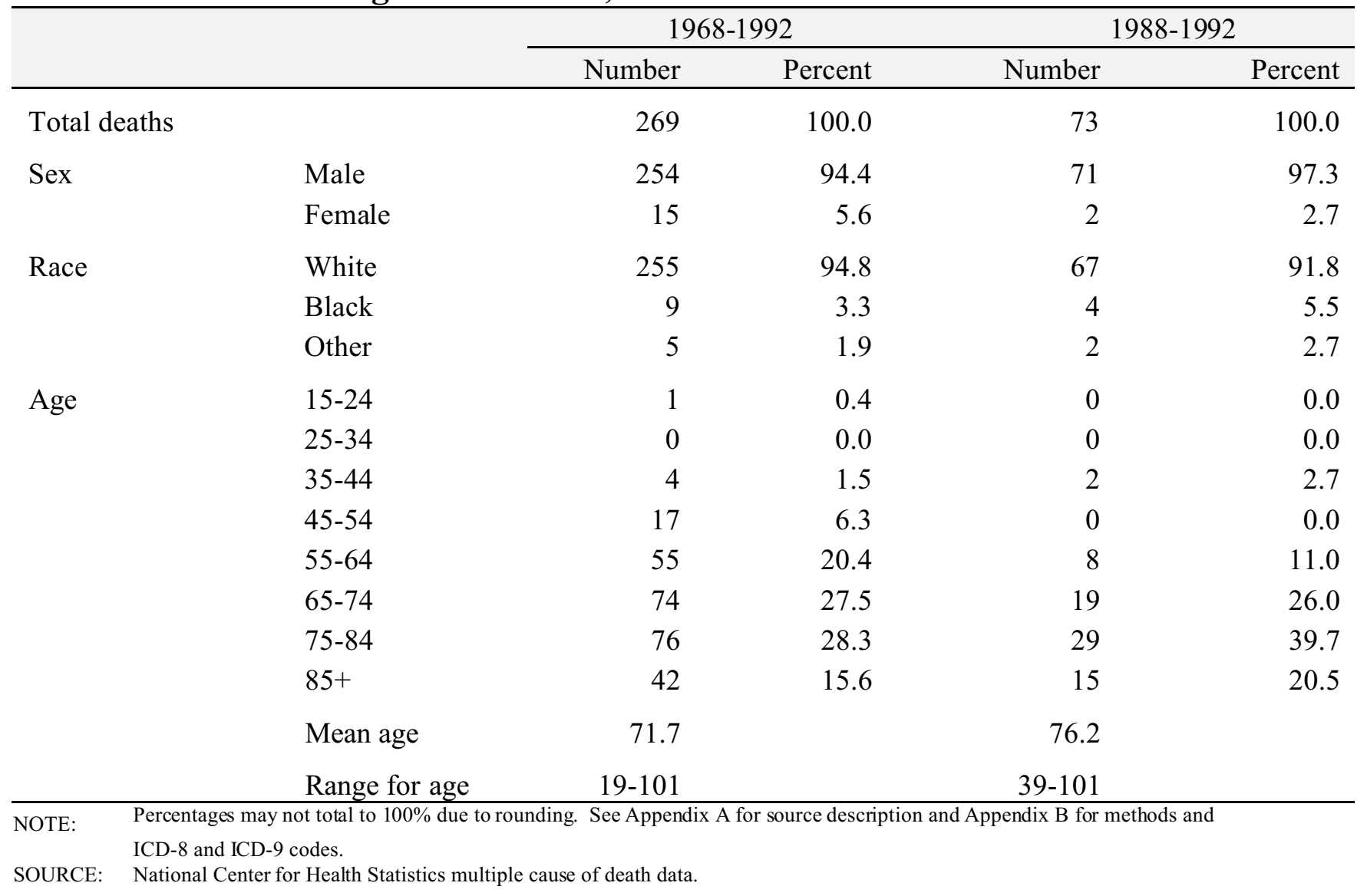




\section{Figure OK-3. Pneumoconiosis deaths by condition, Oklahoma residents age 15 and over, 1968-1992}

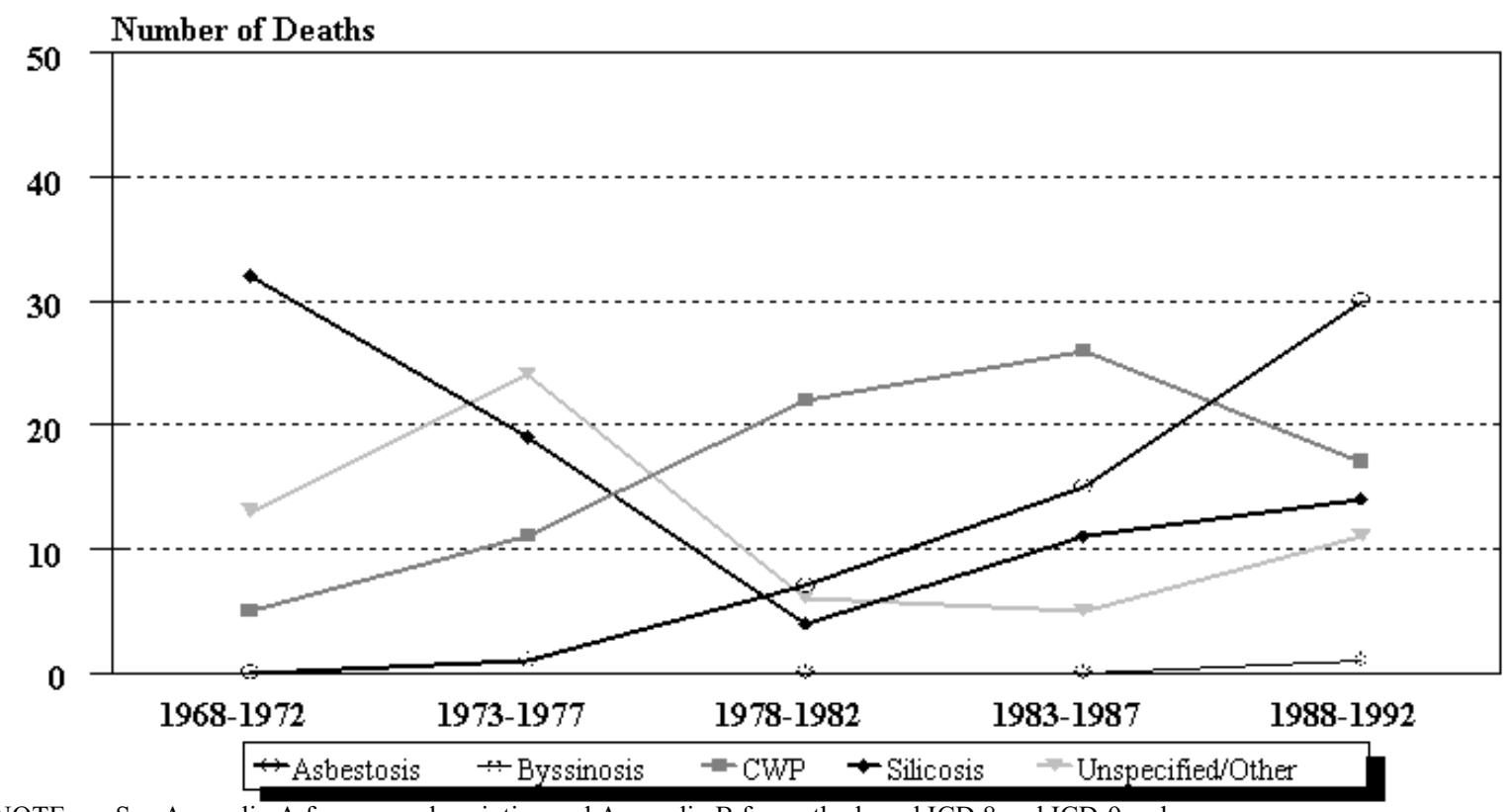

NOTE: See Appendix A for source description and Appendix B for methods and ICD-8 and ICD-9 codes.

SOURCE: National Center for Health Statistics multiple cause of death data.

Figure OK-4. Oklahoma and U.S. age-adjusted mortality rates (per 1,000,000 population) by condition, residents age 15 and over, 1983-1992

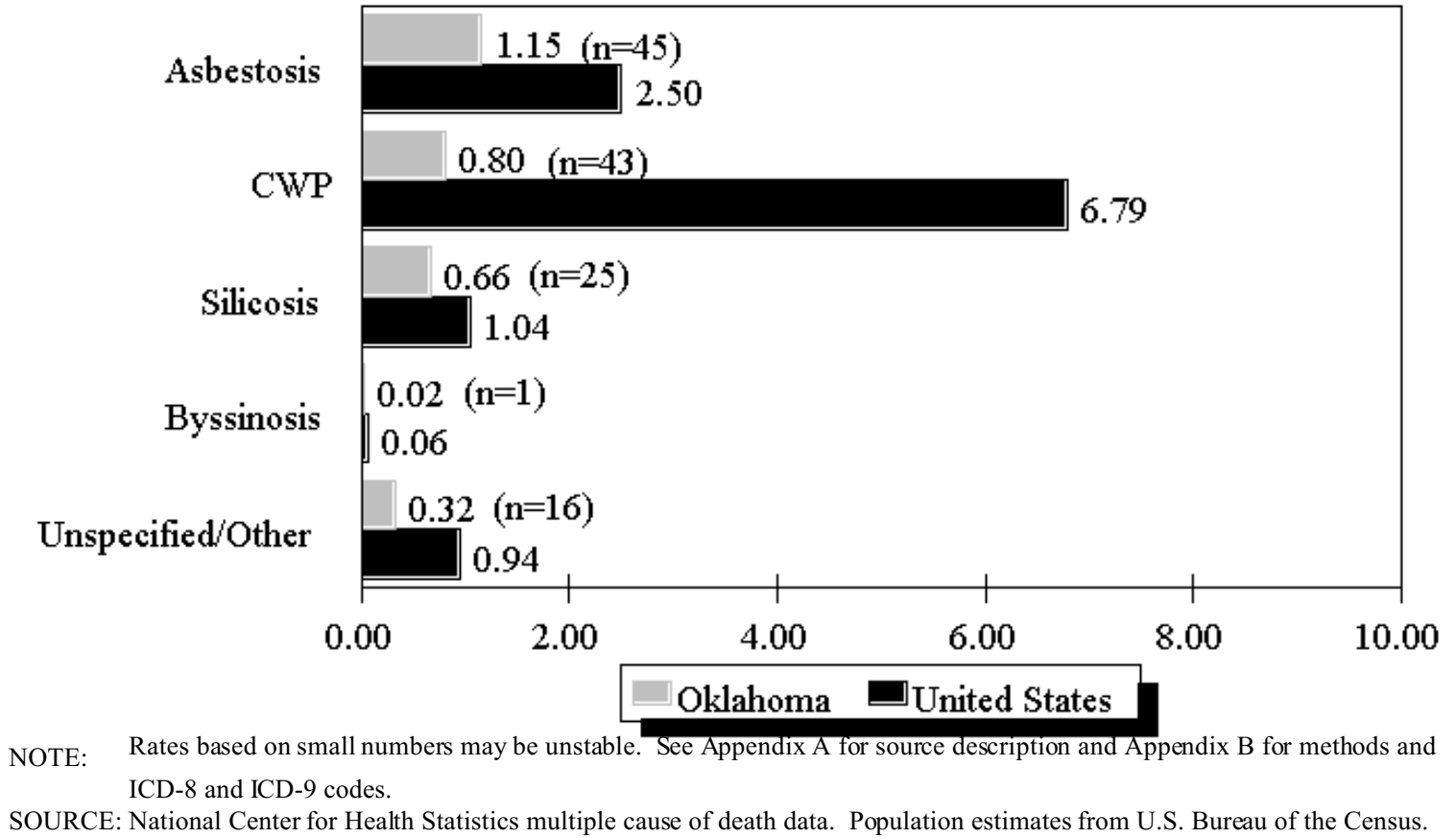


Table OK-3 (page 1 of 2). Number of deaths and age-adjusted mortality rate (per 1,000,000 population) by condition and county, Oklahoma residents age 15 and over, 1983-1992

\begin{tabular}{|c|c|c|c|c|c|c|c|c|c|c|c|c|}
\hline \multirow[b]{2}{*}{ County } & \multicolumn{2}{|c|}{ Asbestosis } & \multicolumn{2}{|c|}{$\begin{array}{c}\text { Coal workers' } \\
\text { pneumoconiosis }\end{array}$} & \multicolumn{2}{|c|}{ Silicosis } & \multicolumn{2}{|c|}{ Byssinosis } & \multicolumn{2}{|c|}{$\begin{array}{r}\text { Unspecified/Other } \\
\text { pneumoconioses }\end{array}$} & \multicolumn{2}{|c|}{$\begin{array}{r}\text { All } \\
\text { pneumoconioses }\end{array}$} \\
\hline & $\#$ & Rate & $\#$ & Rate & $\#$ & Rate & $\#$ & Rate & \# & Rate & $\#$ & Rate \\
\hline Adair & 1 & 5.53 & - & - & - & - & - & - & - & - & 1 & 5.53 \\
\hline Bryan & 3 & 6.64 & - & - & - & - & - & - & - & - & 3 & 6.64 \\
\hline Canadian & - & - & 1 & 2.01 & - & - & - & - & - & - & 1 & 2.01 \\
\hline Carter & 1 & 0.80 & - & - & - & - & - & - & - & - & 1 & 0.80 \\
\hline Choctaw & 1 & 2.09 & - & - & - & - & - & - & - & - & 1 & 2.09 \\
\hline Cleveland & - & - & 2 & 1.60 & 1 & 0.97 & - & - & - & - & 3 & 2.57 \\
\hline Creek & 3 & 4.93 & - & - & - & - & - & - & - & - & 3 & 4.93 \\
\hline Delaware & - & - & 1 & 1.29 & 1 & 1.29 & - & - & - & - & 2 & 2.59 \\
\hline Garfield & 1 & 0.65 & - & - & - & - & - & - & - & - & 1 & 0.65 \\
\hline Grady & 2 & 4.98 & - & - & - & - & - & - & - & - & 2 & 4.98 \\
\hline Grant & - & - & 1 & 3.93 & - & - & - & - & - & - & 1 & 3.93 \\
\hline Hughes & 1 & 5.47 & - & - & - & - & - & - & - & - & 1 & 5.47 \\
\hline Jackson & 1 & 4.17 & - & - & - & - & - & - & - & - & 1 & 4.17 \\
\hline Jefferson & - & - & - & - & 2 & 13.92 & - & - & - & - & 2 & 13.92 \\
\hline Kiowa & - & - & - & - & 2 & 8.72 & 1 & 2.01 & - & - & 3 & 10.73 \\
\hline Latimer & - & - & 7 & 30.10 & - & - & - & - & 1 & 3.60 & 8 & 33.70 \\
\hline Le Flore & - & - & 9 & 9.61 & 1 & 2.26 & - & - & 1 & 0.92 & 11 & 12.79 \\
\hline Lincoln & 1 & 1.37 & - & - & - & - & - & - & - & - & 1 & 1.37 \\
\hline McCurtain & 1 & 2.95 & 2 & 4.24 & - & - & - & - & - & - & 3 & 7.20 \\
\hline Major & 1 & 3.49 & - & - & - & - & - & - & - & - & 1 & 3.49 \\
\hline Marshall & 1 & 14.68 & - & - & - & - & - & - & - & - & 1 & 14.68 \\
\hline Mayes & - & - & - & - & 1 & 4.31 & - & - & 1 & 4.31 & 2 & 8.61 \\
\hline Murray & 1 & 2.46 & - & - & 1 & 6.44 & - & - & - & - & 2 & 8.91 \\
\hline Muskogee & - & - & - & - & 1 & 1.35 & - & - & 2 & 1.92 & 3 & 3.27 \\
\hline Noble & 1 & 2.85 & - & - & - & - & - & - & - & - & 1 & 2.85 \\
\hline Nowata & - & - & - & - & - & - & - & - & 2 & 5.96 & 2 & 5.96 \\
\hline Oklahoma & 8 & 1.25 & 3 & 0.60 & 4 & 0.69 & - & - & 1 & 0.09 & 16 & 2.63 \\
\hline Okmulgee & 2 & 4.70 & 6 & 6.91 & - & - & - & - & - & - & 8 & 11.61 \\
\hline Osage & 1 & 2.32 & - & - & - & - & - & - & - & - & 1 & 2.32 \\
\hline Ottawa & 1 & 2.54 & - & - & 6 & 11.21 & - & - & - & - & 7 & 13.74 \\
\hline Payne & 1 & 0.87 & - & - & - & - & - & - & - & - & 1 & 0.87 \\
\hline Pittsburg & 1 & 1.95 & 3 & 3.59 & - & - & - & - & 2 & 2.77 & 6 & 8.31 \\
\hline
\end{tabular}

See footnotes at end of table. 
Table OK-3 (page 2 of 2). Number of deaths and age-adjusted mortality rate (per 1,000,000 population) by condition and county, Oklahoma residents age 15 and over, 1983-1992

\begin{tabular}{|c|c|c|c|c|c|c|c|c|c|c|c|c|}
\hline \multirow[b]{2}{*}{ County } & \multicolumn{2}{|c|}{ Asbestosis } & \multicolumn{2}{|c|}{$\begin{array}{c}\text { Coal workers' } \\
\text { pneumoconiosis }\end{array}$} & \multicolumn{2}{|c|}{ Silicosis } & \multicolumn{2}{|c|}{ Byssinosis } & \multicolumn{2}{|c|}{$\begin{array}{r}\text { Unspecified/Other } \\
\text { pneumoconioses }\end{array}$} & \multicolumn{2}{|c|}{$\begin{array}{r}\text { All } \\
\text { pneumoconioses }\end{array}$} \\
\hline & $\#$ & Rate & \# & Rate & $\#$ & Rate & \# & Rate & \# & Rate & \# & Rate \\
\hline Pontotoc & 1 & 2.70 & 1 & 1.00 & - & - & - & - & - & - & 2 & 3.69 \\
\hline Pushmataha & - & - & - & - & - & - & - & - & 1 & 7.03 & 1 & 7.03 \\
\hline Rogers & 2 & 3.50 & - & - & - & - & - & - & - & - & 2 & 3.50 \\
\hline Seminole & - & - & 1 & 1.19 & - & - & - & - & - & - & 1 & 1.19 \\
\hline Sequoyah & - & - & 2 & 4.45 & - & - & - & - & - & - & 2 & 4.45 \\
\hline Stephens & - & - & - & - & - & - & - & - & 1 & 0.79 & 1 & 0.79 \\
\hline Texas & - & - & 1 & 5.99 & - & - & - & - & - & - & 1 & 5.99 \\
\hline Tillman & - & - & - & - & 1 & 2.59 & - & - & - & - & 1 & 2.59 \\
\hline Tulsa & 6 & 0.91 & 2 & 0.34 & 3 & 0.62 & - & - & 4 & 0.62 & 15 & 2.49 \\
\hline Wagoner & - & - & 1 & 1.50 & - & - & - & - & - & - & 1 & 1.50 \\
\hline Washington & 1 & 0.88 & - & - & - & - & - & - & - & - & 1 & 0.88 \\
\hline Washita & 1 & 6.67 & - & - & - & - & - & - & - & - & 1 & 6.67 \\
\hline Woodward & - & - & - & - & 1 & 2.35 & - & - & - & - & 1 & 2.35 \\
\hline \multicolumn{13}{|c|}{ indicates no deaths listed. } \\
\hline $\begin{array}{ll}\text { OTE: } & \text { Counti } \\
& \text { becaus } \\
\text { DURCE: } & \text { Nation }\end{array}$ & & 1 liste & & $\begin{array}{l}\text { display } \\
\text { Append }\end{array}$ & & test & & be & $\begin{array}{l}\text { e. Sur } \\
\text { thods } \\
\text { Censu }\end{array}$ & idualc & & \\
\hline
\end{tabular}




\section{Oregon: Selected statistics from the United States section}

All pneumoconioses mortality, 1988-1992

Total deaths

$\underline{\text { Value }}$

126

State rank

22

Crude mortality rate (per $1,000,000$ population)

Age-adjusted mortality rate (per $1,000,000$ population)

Total years of potential life lost to life expectancy

Years of potential life lost to life expectancy (per death)

Deaths by type of pneumoconiosis, 1988-1992

\section{Asbestosis}

Coal workers' pneumoconiosis

Silicosis

Byssinosis

Unspecified/Other pneumoconiosis
11.27

5.65

1,400

11.1

\section{8}

22

26

43

Percent**

$\underline{\text { Deaths* }} \quad \underline{\text { distribution }}$

$94 \quad 74.6$

$16 \quad 12.7$

$\begin{array}{ll}0 & 0.0\end{array}$

5

4.0
$12 \quad 9.5$

* Sum of column may exceed total deaths because of deaths with multiple pneumoconioses.

** Percentages may total more than $100 \%$ due to deaths with multiple pneumoconioses.

NOTE: $\quad$ See Appendix A for source description and Appendix B for methods and ICD-8 and ICD-9 codes.

SOURCE: National Center for Health Statistics multiple cause of death data. Population estimates from U.S. Bureau of the Census.

\section{Figure OR-1. All pneumoconioses: Oregon and U.S. age-adjusted mortality rates, residents age 15 and over, 1968-1992}

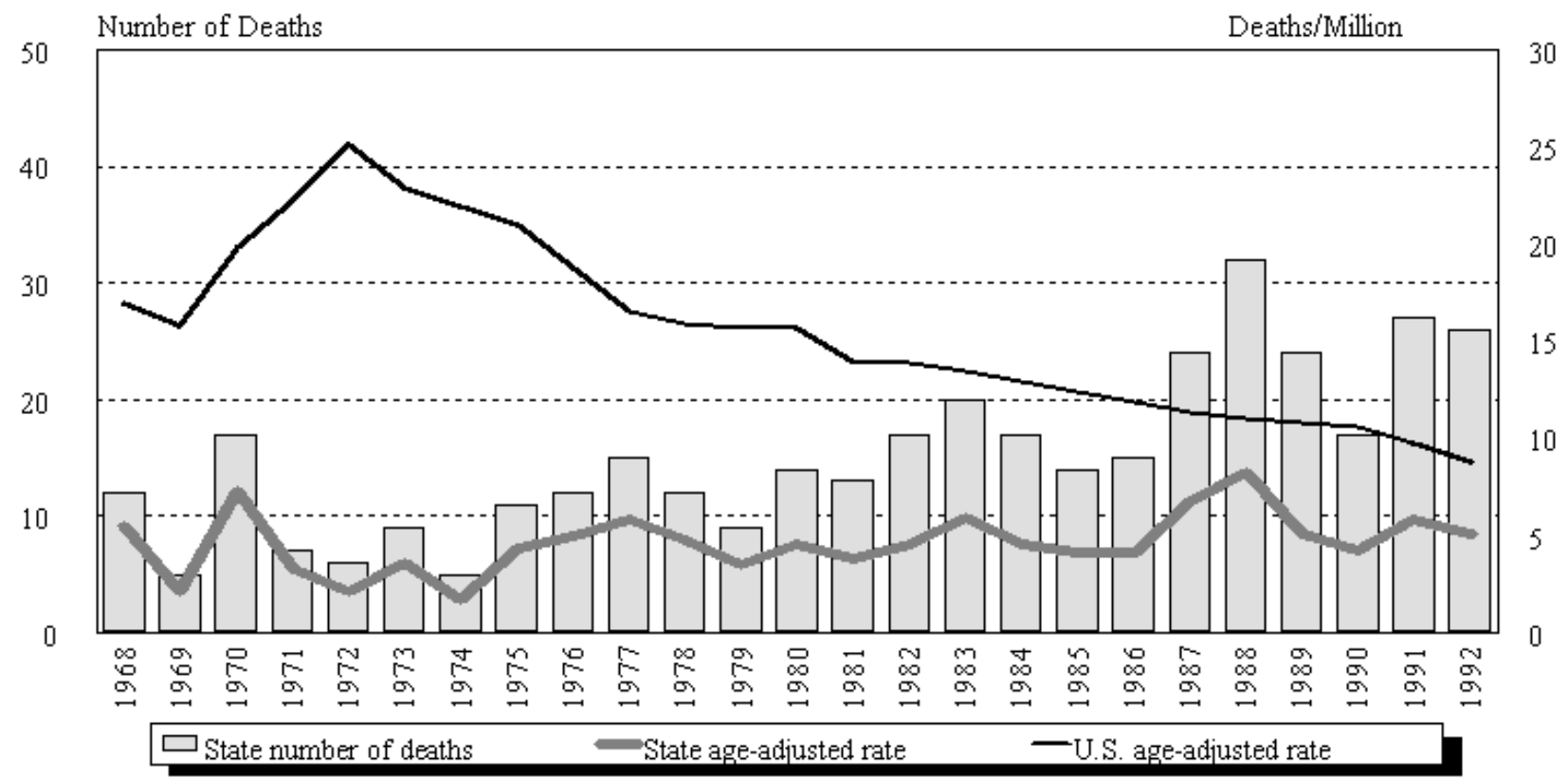

NOTE: $\quad$ See Appendix A for source description and Appendix B for methods and ICD-8 and ICD-9 codes.

SOURCE: National Center for Health Statistics multiple cause of death data. Population estimates from U.S. Bureau of the Census. 


\section{Oregon}

Table OR-1. Number of deaths and age-adjusted mortality rate (per 1,000,000 population) by condition, Oregon residents age 15 and over, 1968-1992

\begin{tabular}{|c|c|c|c|c|c|c|c|c|c|c|c|c|}
\hline \multirow[b]{2}{*}{ Year } & \multicolumn{2}{|c|}{ Asbestosis } & \multicolumn{2}{|c|}{$\begin{array}{l}\text { Coal workers' } \\
\text { pneumoconiosis }\end{array}$} & \multicolumn{2}{|c|}{ Silicosis } & \multicolumn{2}{|c|}{ Byssinosis* } & \multicolumn{2}{|c|}{$\begin{array}{c}\text { Unspecified/Other } \\
\text { pneumoconioses }\end{array}$} & \multicolumn{2}{|c|}{ All pneumoconioses } \\
\hline & Number & Rate & Number & Rate & Number & Rate & Number & Rate & Number & Rate & Number & Rate \\
\hline 1968 & 1 & 0.64 & - & - & 8 & 3.47 & & & 3 & 1.42 & 12 & 5.54 \\
\hline 1969 & - & - & - & - & 5 & 2.10 & & & - & - & 5 & 2.10 \\
\hline 1970 & 2 & 0.99 & - & - & 13 & 5.56 & & & 2 & 0.79 & 17 & 7.34 \\
\hline 1971 & 1 & 0.51 & 1 & 0.64 & 3 & 1.38 & & & 2 & 0.77 & 7 & 3.30 \\
\hline 1972 & 2 & 0.38 & 2 & 0.90 & 2 & 0.90 & & & - & - & 6 & 2.17 \\
\hline 1973 & 2 & 0.73 & - & - & 5 & 2.04 & & & 2 & 0.87 & 9 & 3.65 \\
\hline 1974 & 1 & 0.29 & 1 & 0.29 & 3 & 1.14 & & & - & - & 5 & 1.72 \\
\hline 1975 & 2 & 0.82 & 2 & 0.89 & 6 & 2.39 & & & 2 & 0.77 & 11 & 4.39 \\
\hline 1976 & 3 & 1.27 & 2 & 0.68 & 5 & 2.23 & & & 3 & 1.19 & 12 & 4.98 \\
\hline 1977 & 4 & 1.84 & 3 & 1.12 & 3 & 1.04 & & & 5 & 1.81 & 15 & 5.81 \\
\hline 1978 & 7 & 2.58 & - & - & 1 & 0.37 & & & 4 & 1.86 & 12 & 4.81 \\
\hline 1979 & 5 & 1.86 & - & - & 3 & 1.24 & - & - & 1 & 0.36 & 9 & 3.46 \\
\hline 1980 & 6 & 1.71 & 3 & 1.43 & 5 & 1.42 & - & - & - & - & 14 & 4.56 \\
\hline 1981 & 9 & 2.95 & 1 & 0.24 & 3 & 0.59 & - & - & - & - & 13 & 3.78 \\
\hline 1982 & 8 & 2.60 & 4 & 0.81 & 4 & 0.93 & - & - & 1 & 0.24 & 17 & 4.58 \\
\hline 1983 & 13 & 3.59 & 3 & 1.21 & 3 & 0.90 & - & - & 1 & 0.23 & 20 & 5.93 \\
\hline 1984 & 13 & 3.63 & 2 & 0.55 & 2 & 0.34 & - & - & - & - & 17 & 4.52 \\
\hline 1985 & 10 & 3.13 & - & - & 1 & 0.22 & - & - & 3 & 0.81 & 14 & 4.16 \\
\hline 1986 & 12 & 3.65 & 2 & 0.32 & - & - & - & - & 1 & 0.11 & 15 & 4.08 \\
\hline 1987 & 18 & 4.61 & 3 & 0.93 & 2 & 1.06 & - & - & 1 & 0.20 & 24 & 6.81 \\
\hline 1988 & 21 & 5.76 & 7 & 1.49 & 2 & 0.39 & - & - & 2 & 0.61 & 32 & 8.25 \\
\hline 1989 & 17 & 3.33 & 2 & 0.48 & 5 & 0.96 & - & - & 1 & 0.46 & 24 & 5.04 \\
\hline 1990 & 12 & 3.16 & 1 & 0.39 & 3 & 0.54 & - & - & 1 & 0.18 & 17 & 4.26 \\
\hline 1991 & 22 & 5.09 & 1 & 0.17 & 3 & 0.44 & - & - & 1 & 0.09 & 27 & 5.79 \\
\hline 1992 & 22 & 4.32 & 1 & 0.28 & 3 & 0.53 & - & - & - & - & 26 & 5.13 \\
\hline
\end{tabular}

indicates no deaths listed. ${ }^{*}$ no sp ecific code for byssinosis in ICD-8 (1968-1978)

NOTE: Rates based on small numbers may be unstable. Sum of individual conditions may exceed total because of deaths with multiple pneumoconioses. See Appendix A for source description and Appendix B for methods and ICD-8 and ICD-9 NOTE

SOURCE: National Center for Health Statistics multiple cause of death data. Population estimates from U.S. Bureau of the Census. 
Figure OR-2. All pneumoconioses: Distribution of deaths by sex and race, Oregon residents age 15 and over, 1968-1992
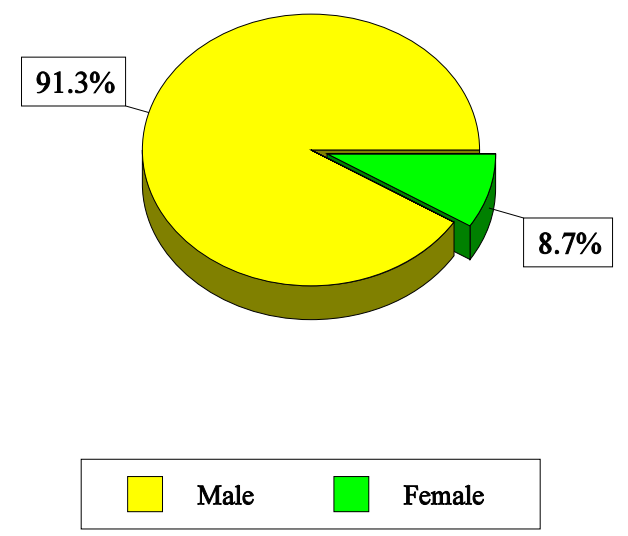
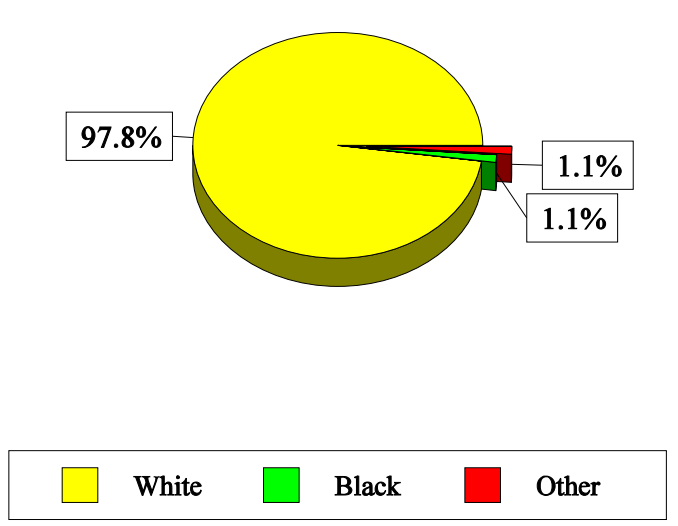

NOTE: See Appendix A for source description and Appendix B for methods and ICD-8 and ICD-9 codes.

SOURCE: National Center for Health Statistics multiple cause of death data.

Table OR-2. All pneumoconioses: Distribution of deaths by sex, race, and age, Oregon residents age 15 and over, 1968-1992

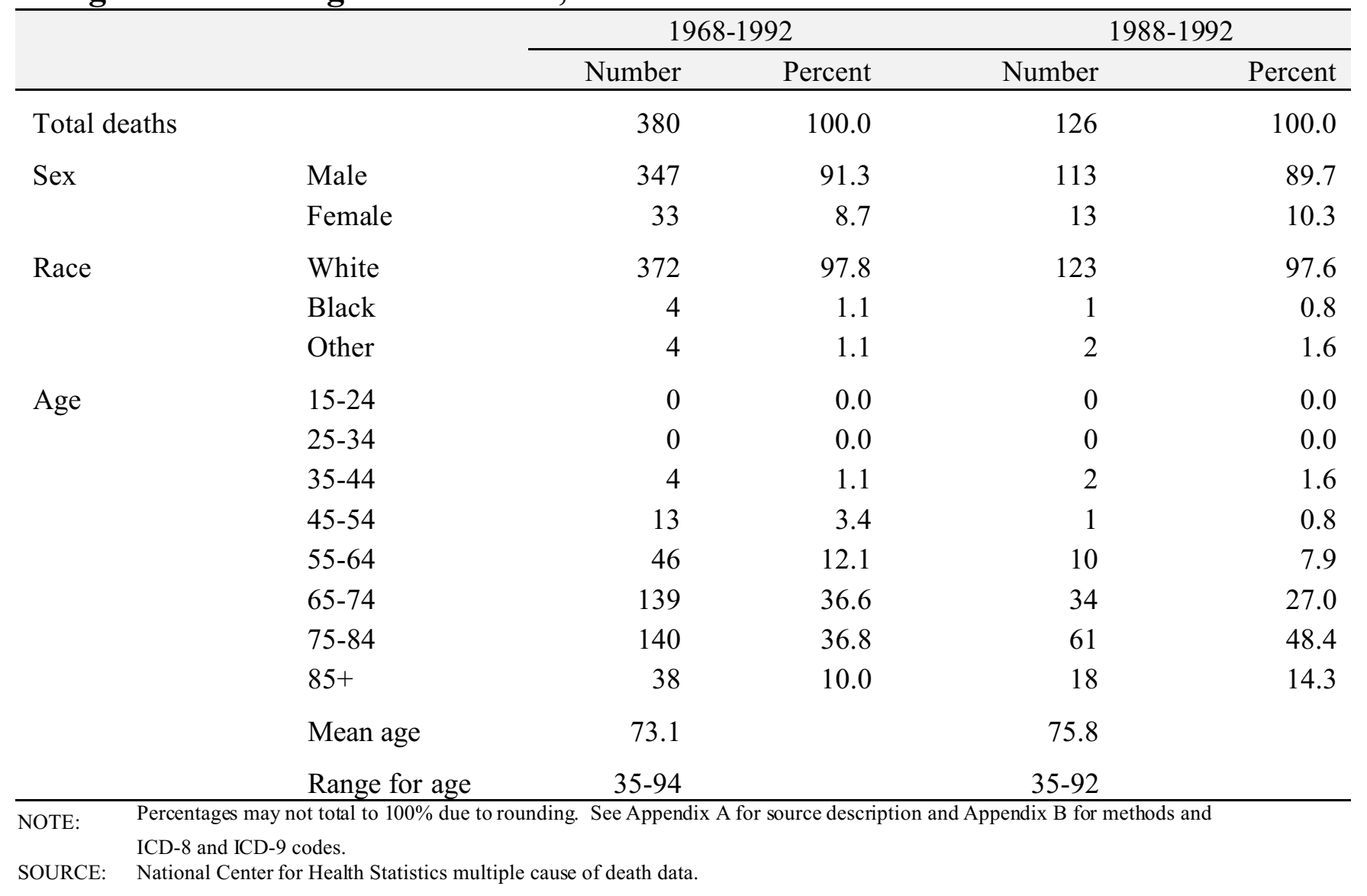




\section{Figure OR-3. Pneumoconiosis deaths by condition, Oregon residents age 15 and over, 1968-1992}

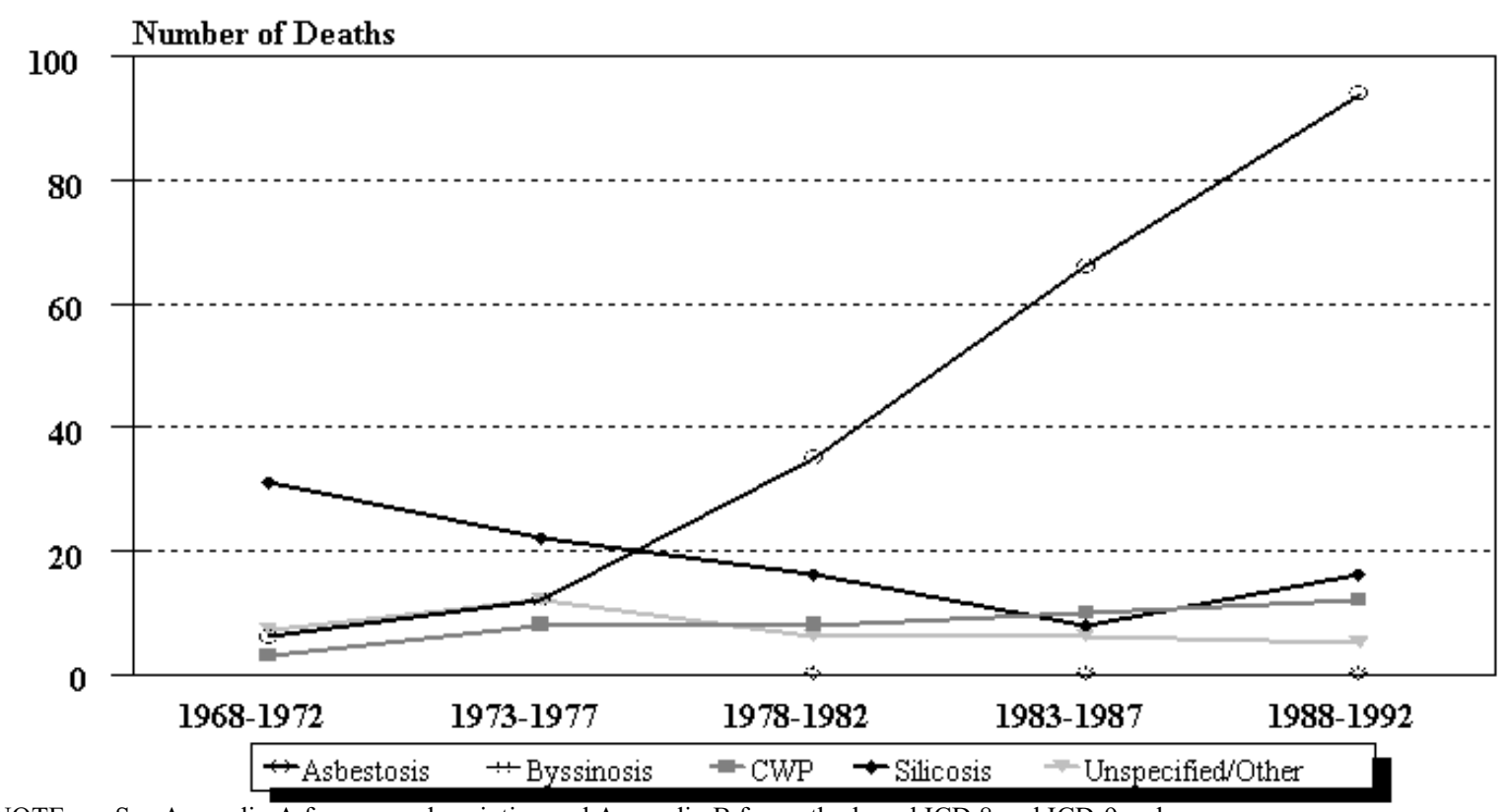

NOTE: See Appendix A for source description and Appendix B for methods and ICD-8 and ICD-9 codes.

SOURCE: National Center for Health Statistics multiple cause of death data.

Figure OR-4. Oregon and U.S. age-adjusted mortality rates (per 1,000,000 population) by condition, residents age 15 and over, 1983-1992

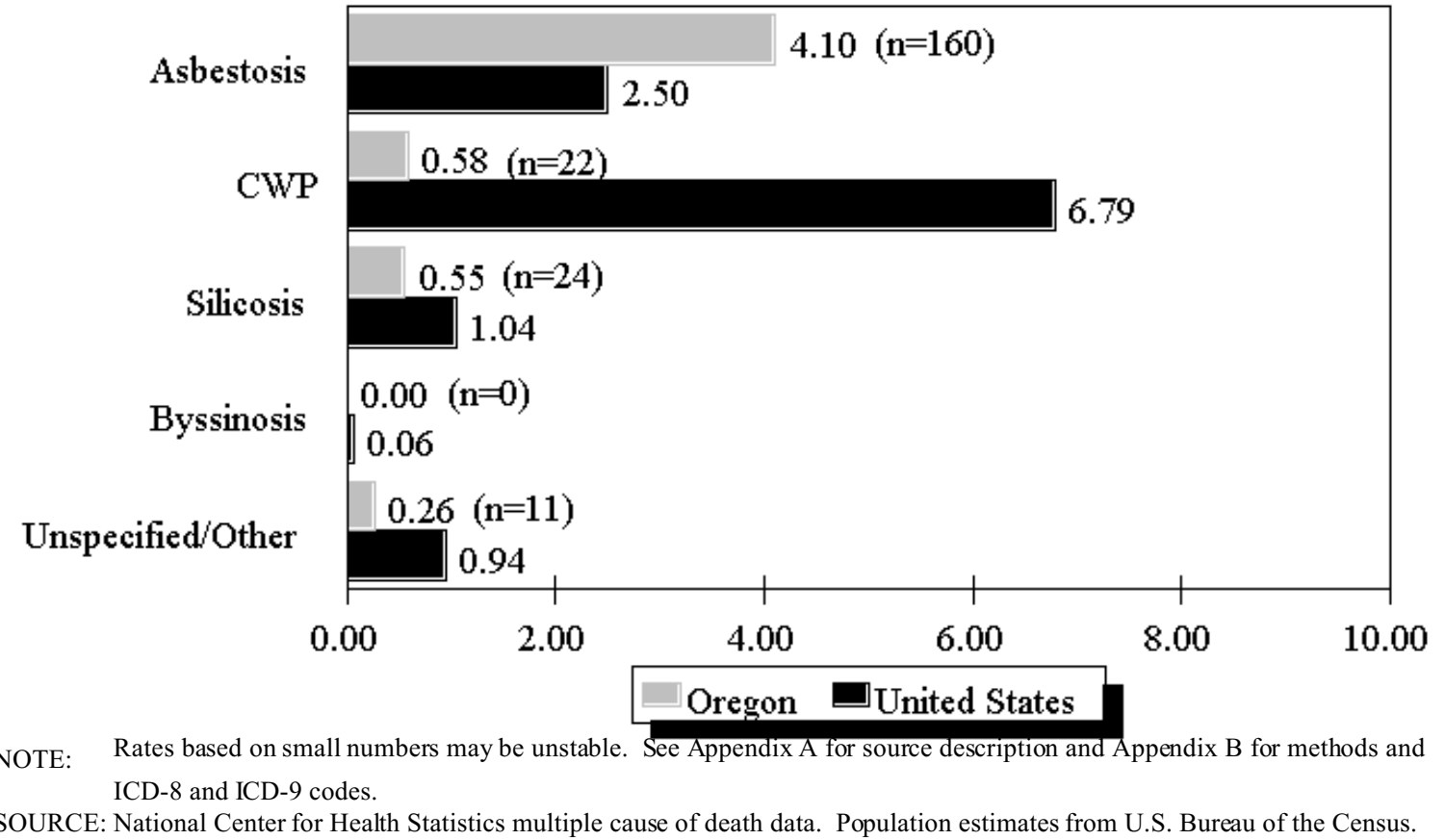


Table OR-3. Number of deaths and age-adjusted mortality rate (per 1,000,000 population) by condition and county, Oregon residents age 15 and over, 1983-1992

\begin{tabular}{|c|c|c|c|c|c|c|c|c|c|c|c|c|}
\hline \multirow[b]{2}{*}{ County } & \multicolumn{2}{|c|}{ Asbestosis } & \multicolumn{2}{|c|}{$\begin{array}{c}\text { Coal workers' } \\
\text { pneumoconiosis }\end{array}$} & \multicolumn{2}{|c|}{ Silicosis } & \multicolumn{2}{|c|}{ Byssinosis } & \multicolumn{2}{|c|}{$\begin{array}{r}\text { Unspecified/Other } \\
\text { pneumoconioses }\end{array}$} & \multicolumn{2}{|c|}{$\begin{array}{r}\text { All } \\
\text { pneumoconioses }\end{array}$} \\
\hline & $\#$ & Rate & \# & Rate & $\#$ & Rate & $\#$ & Rate & $\#$ & Rate & $\#$ & Rate \\
\hline Clackamas & 10 & 2.90 & 3 & 1.10 & - & - & - & - & 2 & 0.45 & 15 & 4.45 \\
\hline Clatsop & 1 & 2.81 & 2 & 2.49 & 1 & 1.25 & - & - & - & - & 4 & 6.55 \\
\hline Columbia & - & - & - & - & - & - & - & - & 1 & 1.52 & 1 & 1.52 \\
\hline Coos & 2 & 2.14 & 3 & 5.18 & 1 & 0.72 & - & - & 1 & 1.42 & 7 & 9.45 \\
\hline Curry & 8 & 24.03 & - & - & - & - & - & - & - & - & 8 & 24.03 \\
\hline Deschutes & 5 & 5.11 & 1 & 1.37 & 1 & 0.79 & - & - & - & - & 7 & 7.27 \\
\hline Douglas & 1 & 0.98 & 2 & 1.50 & - & - & - & - & 1 & 0.52 & 4 & 3.00 \\
\hline Hood River & 5 & 24.45 & - & - & - & - & - & - & - & - & 5 & 24.45 \\
\hline Jackson & 10 & 4.10 & 1 & 0.65 & 2 & 0.96 & - & - & - & - & 13 & 5.71 \\
\hline Josephine & 6 & 4.79 & 1 & 0.57 & - & - & - & - & 1 & 0.57 & 8 & 5.94 \\
\hline Klamath & 2 & 3.36 & 1 & 0.91 & - & - & - & - & - & - & 3 & 4.28 \\
\hline Lake & - & - & 1 & 12.58 & - & - & - & - & - & - & 1 & 12.58 \\
\hline Lane & 8 & 2.52 & 1 & 0.20 & 1 & 0.41 & - & - & - & - & 10 & 3.12 \\
\hline Lincoln & 2 & 2.95 & 1 & 1.90 & 1 & 1.05 & - & - & - & - & 4 & 5.89 \\
\hline Linn & 8 & 6.01 & 1 & 0.53 & - & - & - & - & - & - & 9 & 6.54 \\
\hline Malheur & 1 & 5.92 & - & - & - & - & - & - & - & - & 1 & 5.92 \\
\hline Marion & 19 & 6.11 & - & - & 1 & 0.20 & - & - & 1 & 0.20 & 20 & 6.31 \\
\hline Multnomah & 55 & 7.27 & 2 & 0.32 & 12 & 1.44 & - & - & 3 & 0.46 & 72 & 9.50 \\
\hline Polk & 3 & 4.83 & - & - & - & - & - & - & - & - & 3 & 4.83 \\
\hline Tillamook & 2 & 3.51 & - & - & 2 & 3.51 & - & - & - & - & 4 & 7.01 \\
\hline Umatilla & 1 & 0.83 & 1 & 0.83 & - & - & - & - & 1 & 2.48 & 3 & 4.15 \\
\hline Wasco & 1 & 4.16 & - & - & 1 & 1.72 & - & - & - & - & 2 & 5.87 \\
\hline Washington & 8 & 2.29 & 1 & 0.22 & 1 & 0.22 & - & - & - & - & 10 & 2.74 \\
\hline Yamhill & 2 & 3.63 & - & - & - & - & - & - & - & - & 2 & 3.63 \\
\hline
\end{tabular}

- indicates no deaths listed.

NOTE: Counties with no deaths for all listed conditions are not displayed. Rates based on small numbers may be unstable. Sum of individual conditions may exceed total because of deaths with multiple pneumoconioses. See Appendix A for source description and Appendix B for methods and ICD-8 and ICD-9 codes.

SOURCE: National Center for Health Statistics multiple cause of death data. Population estimates from U.S. Bureau of the Census. 


\section{Pennsylvania: Selected statistics from the United States section}

All pneumoconioses mortality, 1988-1992

Total deaths

Years of potential life lost to life expectancy (per death)

Deaths by type of pneumoconiosis, 1988-1992

Asbestosis

Coal workers' pneumoconiosis

Silicosis

Byssinosis

Unspecified/Other pneumoconiosis $\underline{\text { Value }}$

5,926

124.28

54.98

62,129

10.5

$\underline{\text { State rank }}$

\section{1}

2

2

Percent**

$\underline{\text { Deaths* }} \quad \underline{\text { distribution }}$

430

7.3

5,062

85.4

259

4.4

2

0.0

195

* Sum of column may exceed total deaths because of deaths with multiple pneumoconioses.

** Percentages may total more than $100 \%$ due to deaths with multiple pneumoconioses.

NOTE: $\quad$ See Appendix A for source description and Appendix B for methods and ICD-8 and ICD-9 codes.

SOURCE: National Center for Health Statistics multiple cause of death data. Population estimates from U.S. Bureau of the Census.

Figure PA-1. All pneumoconioses: Pennsylvania and U.S. age-adjusted mortality rates, residents age 15 and over, 1968-1992

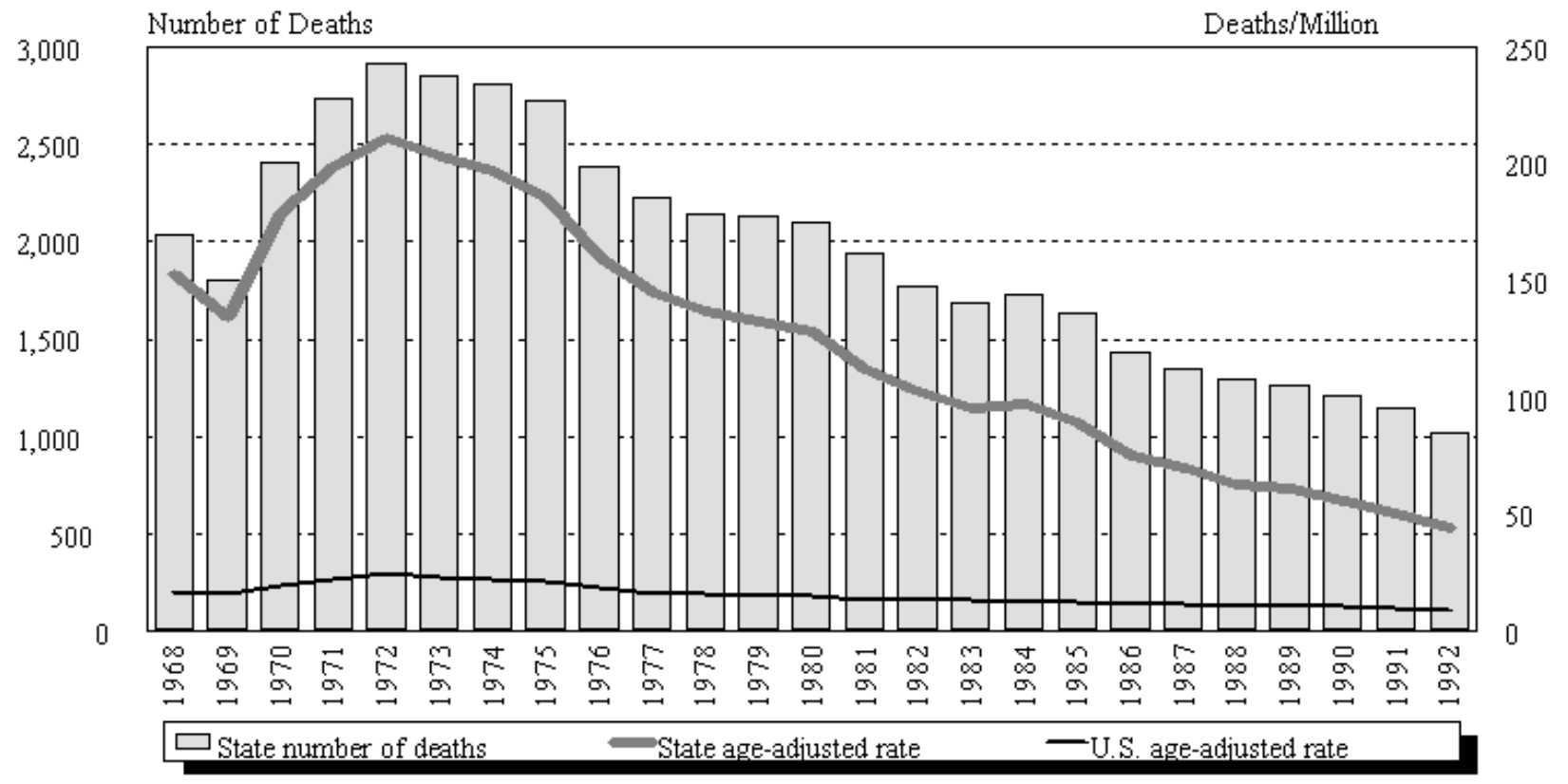

NOTE: $\quad$ See Appendix A for source description and Appendix B for methods and ICD-8 and ICD-9 codes.

SOURCE: National Center for Health Statistics multiple cause of death data. Population estimates from U.S. Bureau of the Census. 


\section{Pennsylvania}

\begin{tabular}{|c|c|c|c|c|c|c|c|c|c|c|c|c|}
\hline \multirow[b]{2}{*}{ Year } & \multicolumn{2}{|c|}{ Asbestosis } & \multicolumn{2}{|c|}{$\begin{array}{l}\text { Coal workers' } \\
\text { pneumoconiosis }\end{array}$} & \multicolumn{2}{|c|}{ Silicosis } & \multicolumn{2}{|c|}{ Byssinosis* } & \multicolumn{2}{|c|}{$\begin{array}{c}\text { Unspecified/Other } \\
\text { pneumoconioses }\end{array}$} & \multicolumn{2}{|c|}{ All pneumoconioses } \\
\hline & Number & Rate & Number & Rate & Number & Rate & Number & Rate & Number & Rate & Number & Rate \\
\hline 1968 & 6 & 0.53 & 1,589 & 119.86 & 284 & 21.73 & & & 176 & 12.51 & 2,030 & 152.72 \\
\hline 1969 & 9 & 0.75 & 1,347 & 100.42 & 278 & 20.96 & & & 179 & 13.02 & 1,800 & 134.21 \\
\hline 1970 & 11 & 0.88 & 1,878 & 140.13 & 291 & 21.71 & & & 266 & 19.35 & 2,410 & 179.30 \\
\hline 1971 & 11 & 0.91 & 2,087 & 153.44 & 270 & 19.53 & & & 402 & 28.12 & 2,729 & 198.85 \\
\hline 1972 & 22 & 1.87 & 2,274 & 166.34 & 240 & 17.05 & & & 418 & 28.90 & 2,910 & 210.78 \\
\hline 1973 & 20 & 1.52 & 2,136 & 153.34 & 227 & 16.10 & & & 494 & 34.35 & 2,852 & 203.32 \\
\hline 1974 & 17 & 1.33 & 2,158 & 152.45 & 212 & 14.59 & & & 451 & 30.70 & 2,809 & 197.03 \\
\hline 1975 & 18 & 1.35 & 2,088 & 142.74 & 200 & 13.39 & & & 439 & 29.24 & 2,723 & 185.17 \\
\hline 1976 & 11 & 0.77 & 1,879 & 126.69 & 186 & 12.47 & & & 326 & 21.20 & 2,381 & 159.70 \\
\hline 1977 & 15 & 1.00 & 1,785 & 116.65 & 143 & 9.33 & & & 305 & 19.00 & 2,230 & 144.92 \\
\hline 1978 & 25 & 2.02 & 1,744 & 111.50 & 152 & 10.10 & & & 240 & 14.80 & 2,143 & 137.32 \\
\hline 1979 & 24 & 1.72 & 1,753 & 109.62 & 85 & 5.45 & 1 & 0.08 & 266 & 16.05 & 2,126 & 132.77 \\
\hline 1980 & 35 & 2.45 & 1,719 & 105.98 & 94 & 5.52 & 1 & 0.05 & 252 & 14.55 & 2,094 & 128.12 \\
\hline 1981 & 32 & 2.09 & 1,602 & 93.30 & 75 & 4.30 & 3 & 0.21 & 228 & 12.58 & 1,937 & 112.27 \\
\hline 1982 & 33 & 2.29 & 1,594 & 91.70 & 64 & 3.65 & - & - & 84 & 5.23 & 1,770 & 102.60 \\
\hline 1983 & 36 & 2.35 & 1,515 & 86.08 & 68 & 3.95 & 1 & 0.07 & 65 & 3.63 & 1,681 & 95.82 \\
\hline 1984 & 60 & 3.85 & 1,552 & 86.80 & 60 & 3.37 & - & - & 64 & 3.67 & 1,731 & 97.39 \\
\hline 1985 & 54 & 3.47 & 1,480 & 79.70 & 55 & 3.18 & 1 & 0.08 & 49 & 2.82 & 1,634 & 88.99 \\
\hline 1986 & 54 & 3.46 & 1,303 & 67.77 & 49 & 2.87 & - & - & 36 & 1.88 & 1,435 & 75.44 \\
\hline 1987 & 65 & 4.01 & 1,211 & 61.71 & 52 & 2.83 & - & - & 33 & 1.70 & 1,351 & 69.69 \\
\hline 1988 & 79 & 4.67 & 1,153 & 55.34 & 45 & 2.25 & - & - & 26 & 1.31 & 1,295 & 63.15 \\
\hline 1989 & 101 & 6.04 & 1,082 & 50.89 & 47 & 2.52 & - & - & 36 & 1.75 & 1,264 & 61.05 \\
\hline 1990 & 67 & 3.85 & 1,030 & 46.70 & 61 & 3.08 & 1 & 0.04 & 51 & 2.53 & 1,206 & 56.00 \\
\hline 1991 & 83 & 4.48 & 961 & 41.42 & 53 & 2.90 & 1 & 0.02 & 49 & 2.28 & 1,143 & 50.87 \\
\hline 1992 & 100 & 5.16 & 836 & 35.26 & 53 & 2.82 & - & - & 33 & 1.53 & 1,018 & 44.56 \\
\hline
\end{tabular}

- indicates no deaths listed. * no sp ecific code for by ssinosis in ICD -8 (1968-1978)

NOTE: $\quad$ Rates based on small numbers may be unstable. Sum of individual conditions may exceed total because of deaths with multiple pneumoconioses. See Appendix A for source description and Appendix B for methods and ICD-8 and ICD-9 NOTE:

SOURCE: National Center for Health Statistics multiple cause of death data. Population estimates from U.S. Bureau of the Census. 
Pennsylvania

\section{Figure PA-2. All pneumoconioses: Distribution of deaths by sex and race, Pennsylvania residents age 15 and over, 1968-1992}
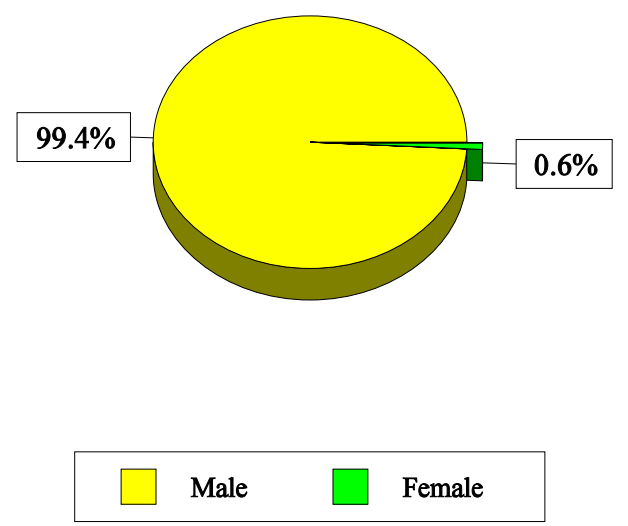

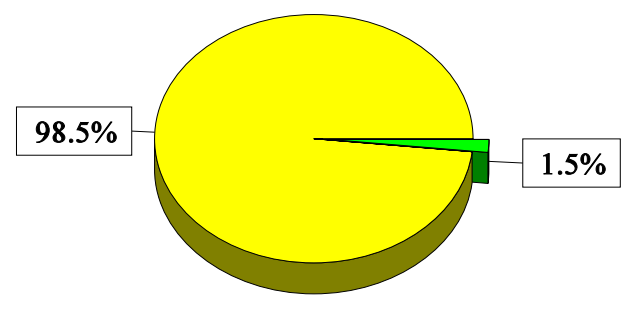

NOTE: $\quad$ See Appendix A for source description and Appendix B for methods and ICD-8 and ICD-9 codes. SOURCE: National Center for Health Statistics multiple cause of death data.

Table PA-2. All pneumoconioses: Distribution of deaths by sex, race, and age, Pennsylvania residents age 15 and over, 1968-1992

\begin{tabular}{|c|c|c|c|c|c|}
\hline & & \multicolumn{2}{|c|}{ 1968-1992 } & \multicolumn{2}{|c|}{ 1988-1992 } \\
\hline & & Number & Percent & Number & Percent \\
\hline Total deaths & & 48,702 & 100.0 & 5,926 & 100.0 \\
\hline \multirow[t]{2}{*}{ Sex } & Male & 48,402 & 99.4 & 5,878 & 99.2 \\
\hline & Female & 300 & 0.6 & 48 & 0.8 \\
\hline \multirow[t]{3}{*}{ Race } & White & 47,993 & 98.5 & 5,831 & 98.4 \\
\hline & Black & 708 & 1.5 & 95 & 1.6 \\
\hline & Other & 1 & 0.0 & 0 & 0.0 \\
\hline \multirow[t]{10}{*}{ Age } & $15-24$ & 6 & 0.0 & 0 & 0.0 \\
\hline & $25-34$ & 20 & 0.0 & 2 & 0.0 \\
\hline & $35-44$ & 90 & 0.2 & 7 & 0.1 \\
\hline & $45-54$ & 1,204 & 2.5 & 37 & 0.6 \\
\hline & $55-64$ & 7,801 & 16.0 & 333 & 5.6 \\
\hline & $65-74$ & 17,934 & 36.8 & 1,771 & 29.9 \\
\hline & $75-84$ & 16,684 & 34.3 & 2,803 & 47.3 \\
\hline & $85+$ & 4,963 & 10.2 & 973 & 16.4 \\
\hline & Mean age & 72.8 & & 76.8 & \\
\hline & Range for age & $15-105$ & & $30-104$ & \\
\hline
\end{tabular}

NOTE: Percentages may not total to $100 \%$ due to rounding. See Appendix A for source description and Appendix B for methods and ICD-8 and ICD-9 codes.

SOURCE: National Center for Health Statistics multiple cause of death data. 
Figure PA-3. Pneumoconiosis deaths by condition, Pennsylvania residents age 15 and over, 1968-1992

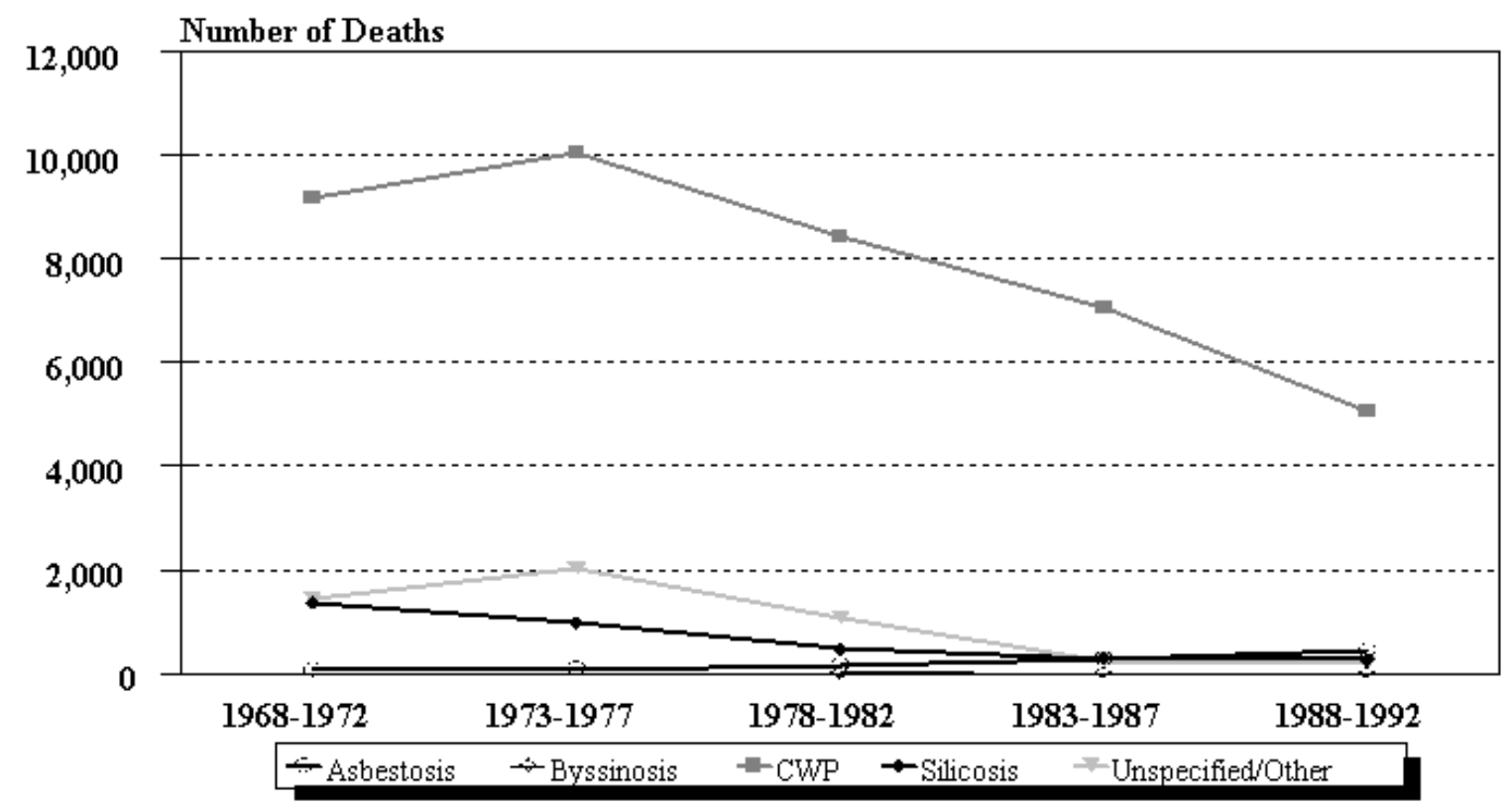

NOTE: See Appendix A for source description and Appendix B for methods and ICD-8 and ICD-9 codes. SOURCE: National Center for Health Statistics multiple cause of death data.

Figure PA-4. Pennsylvania and U.S. age-adjusted mortality rates (per 1,000,000 population) by condition, residents age 15 and over, 1983-1992

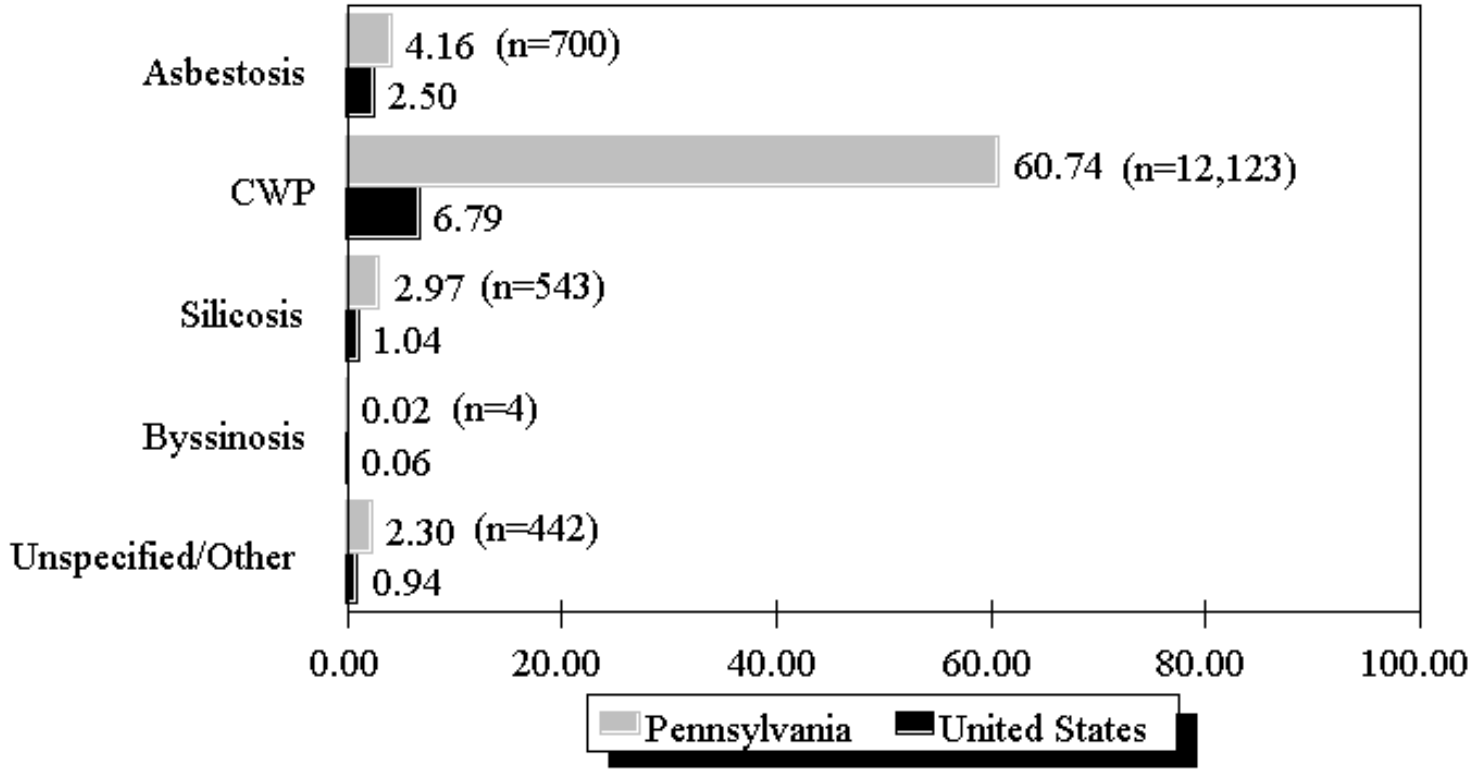

NOTE: Rates based on small numbers may be unstable. See Appendix A for source description and Appendix B for methods and ICD-8 and ICD-9 codes.

SOURCE: National Center for Health Statistics multiple cause of death data. Population estimates from U.S. Bureau of the Census. 
Pennsylvania

Table PA-3 (page 1 of 2). Number of deaths and age-adjusted mortality rate (per $1,000,000$ population) by condition and county, Pennsylvania residents age 15 and over, 1983-1992

\begin{tabular}{|c|c|c|c|c|c|c|c|c|c|c|c|c|}
\hline \multirow[b]{2}{*}{ County } & \multicolumn{2}{|c|}{ Asbestosis } & \multicolumn{2}{|c|}{$\begin{array}{c}\text { Coal workers' } \\
\text { pneumoconiosis }\end{array}$} & \multicolumn{2}{|c|}{ Silicosis } & \multicolumn{2}{|c|}{ Byssinosis } & \multicolumn{2}{|c|}{$\begin{array}{r}\text { Unspecified/Other } \\
\text { pneumoconioses }\end{array}$} & \multicolumn{2}{|c|}{$\begin{array}{r}\text { All } \\
\text { pneumoconioses }\end{array}$} \\
\hline & \# & Rate & \# & Rate & \# & Rate & \# & Rate & \# & Rate & \# & Rate \\
\hline Adams & 1 & 1.37 & 1 & 1.37 & 2 & 2.01 & - & - & - & - & 4 & 4.74 \\
\hline Allegheny & 31 & 1.69 & 288 & 11.27 & 47 & 2.36 & - & - & 23 & 0.93 & 387 & 16.12 \\
\hline Armstrong & 1 & 1.11 & 69 & 51.70 & 12 & 10.34 & - & - & 11 & 8.65 & 92 & 70.70 \\
\hline Beaver & 4 & 1.49 & 17 & 6.56 & 8 & 3.33 & - & - & 3 & 1.08 & 30 & 11.63 \\
\hline Bedford & 3 & 4.63 & 33 & 44.93 & 6 & 10.13 & - & - & 6 & 9.57 & 48 & 69.26 \\
\hline Berks & 17 & 3.88 & 18 & 2.85 & 32 & 6.37 & - & - & 20 & 4.13 & 86 & 17.10 \\
\hline Blair & 18 & 7.82 & 17 & 10.07 & 9 & 4.78 & - & - & 7 & 3.48 & 51 & 26.15 \\
\hline Bradford & 1 & 0.73 & 1 & 0.73 & 2 & 1.47 & - & - & - & - & 4 & 2.93 \\
\hline Bucks & 25 & 4.60 & 31 & 5.14 & 6 & 1.10 & - & - & 7 & 1.39 & 69 & 12.23 \\
\hline Butler & 1 & 0.68 & 22 & 9.41 & 2 & 1.01 & - & - & - & - & 25 & 11.10 \\
\hline Cambria & 9 & 3.49 & 924 & 323.29 & 42 & 13.97 & - & - & 44 & 14.67 & 1,008 & 351.46 \\
\hline Cameron & - & - & 1 & 6.53 & - & - & - & - & - & - & 1 & 6.53 \\
\hline Carbon & 5 & 9.47 & 310 & 291.00 & 11 & 11.67 & - & - & 11 & 12.53 & 336 & 323.31 \\
\hline Centre & 2 & 1.80 & 14 & 11.29 & 3 & 2.97 & - & - & 6 & 3.80 & 25 & 19.87 \\
\hline Chester & 5 & 1.43 & 9 & 2.17 & 2 & 0.63 & - & - & - & - & 16 & 4.23 \\
\hline Clarion & - & - & 45 & 74.68 & - & - & - & - & 5 & 6.87 & 50 & 81.56 \\
\hline Clearfield & 1 & 1.09 & 115 & 94.95 & 6 & 4.30 & - & - & 13 & 10.26 & 135 & 110.60 \\
\hline Clinton & 1 & 1.20 & 4 & 7.04 & - & - & - & - & - & - & 5 & 8.25 \\
\hline Columbia & 3 & 4.57 & 88 & 93.83 & 2 & 1.39 & - & - & 6 & 4.91 & 99 & 104.71 \\
\hline Crawford & 2 & 1.57 & 1 & 0.49 & 3 & 2.06 & - & - & - & - & 6 & 4.12 \\
\hline Cumberland & - & - & 7 & 2.36 & - & - & - & - & 1 & 0.52 & 8 & 2.88 \\
\hline Dauphin & 3 & 1.00 & 55 & 14.61 & 3 & 0.60 & - & - & 2 & 0.60 & 63 & 16.81 \\
\hline Delaware & 130 & 17.77 & 28 & 3.00 & 17 & 2.13 & - & - & 2 & 0.24 & 175 & 22.89 \\
\hline Elk & - & - & 9 & 13.57 & - & - & - & - & 1 & 1.26 & 10 & 14.83 \\
\hline Erie & 3 & 0.73 & 12 & 2.93 & 11 & 3.07 & - & - & 3 & 0.57 & 29 & 7.30 \\
\hline Fayette & 6 & 2.14 & 642 & 251.31 & 7 & 3.22 & - & - & 13 & 6.17 & 667 & 262.57 \\
\hline Forest & - & - & 1 & 7.61 & - & - & - & - & - & - & 1 & 7.61 \\
\hline Franklin & 2 & 1.58 & 2 & 1.18 & 2 & 1.18 & - & - & 1 & 0.79 & 7 & 4.74 \\
\hline Fulton & - & - & 2 & 11.03 & 1 & 10.36 & - & - & 2 & 11.03 & 5 & 32.42 \\
\hline Greene & 1 & 1.00 & 117 & 204.61 & 2 & 4.53 & - & - & 4 & 7.80 & 123 & 215.68 \\
\hline Huntingdon & 2 & 2.22 & 26 & 44.28 & 16 & 25.51 & - & - & 3 & 4.44 & 47 & 76.44 \\
\hline Indiana & 1 & 0.55 & 192 & 153.95 & 20 & 16.30 & - & - & 14 & 14.81 & 226 & 185.06 \\
\hline Jefferson & 2 & 3.67 & 40 & 47.56 & 1 & 1.83 & - & - & 8 & 9.87 & 51 & 62.94 \\
\hline Juniata & - & - & 1 & 4.70 & 2 & 6.88 & - & - & 2 & 4.36 & 5 & 15.94 \\
\hline
\end{tabular}

See footnotes at end of table. 
Table PA-3 (page 2 of 2). Number of deaths and age-adjusted mortality rate (per $1,000,000$ population) by condition and county, Pennsylvania residents age 15 and over, 1983-1992

\begin{tabular}{|c|c|c|c|c|c|c|c|c|c|c|c|c|}
\hline \multirow[b]{2}{*}{ County } & \multicolumn{2}{|c|}{ Asbestosis } & \multicolumn{2}{|c|}{$\begin{array}{r}\text { Coal workers' } \\
\text { pneumoconiosis }\end{array}$} & \multicolumn{2}{|c|}{ Silicosis } & \multicolumn{2}{|c|}{ Byssinosis } & \multicolumn{2}{|c|}{$\begin{array}{r}\text { Unspecified/Other } \\
\text { pneumoconioses }\end{array}$} & \multicolumn{2}{|c|}{$\begin{array}{r}\text { All } \\
\text { pneumoconioses }\end{array}$} \\
\hline & $\#$ & Rate & $\#$ & Rate & $\#$ & Rate & $\#$ & Rate & $\#$ & Rate & \# & Rate \\
\hline Lackawanna & 9 & 2.90 & 1172 & 258.11 & 25 & 5.60 & - & - & 31 & 6.72 & 1,237 & 273.33 \\
\hline Lancaster & 33 & 5.88 & 13 & 2.26 & 8 & 1.66 & - & - & 6 & 1.00 & 60 & 10.81 \\
\hline Lawrence & 1 & 0.80 & 3 & 1.99 & 2 & 1.59 & - & - & 2 & 0.80 & 8 & 5.18 \\
\hline Lebanon & 4 & 2.87 & 4 & 2.43 & 2 & 1.22 & - & - & - & - & 10 & 6.51 \\
\hline Lehigh & 5 & 1.25 & 21 & 4.17 & 13 & 2.96 & - & - & 6 & 0.91 & 43 & 8.83 \\
\hline Luzerne & 25 & 4.48 & 3,506 & 540.04 & 24 & 3.62 & - & - & 53 & 9.37 & 3,602 & 556.63 \\
\hline Lycoming & 1 & 0.79 & 2 & 0.74 & 1 & 0.37 & - & - & - & - & 4 & 1.90 \\
\hline McKean & 8 & 13.76 & 1 & 0.76 & 2 & 2.59 & - & - & - & - & 11 & 17.11 \\
\hline Mercer & 1 & 0.68 & 12 & 6.39 & 4 & 2.20 & 1 & 0.33 & 7 & 2.64 & 25 & 12.23 \\
\hline Mifflin & - & - & 6 & 9.46 & 11 & 16.03 & - & - & 1 & 0.92 & 18 & 26.40 \\
\hline Monroe & 3 & 3.33 & 12 & 11.40 & 3 & 2.85 & - & - & 1 & 1.11 & 18 & 17.58 \\
\hline Montgomery & 110 & 11.62 & 31 & 3.10 & 14 & 1.56 & - & - & 8 & 0.74 & 162 & 16.88 \\
\hline Montour & - & - & 5 & 13.41 & - & - & - & - & - & - & 5 & 13.41 \\
\hline Northampton & 2 & 0.75 & 10 & 2.87 & 6 & 2.08 & - & - & 5 & 0.99 & 23 & 6.69 \\
\hline $\begin{array}{l}\text { North- } \\
\text { umberland }\end{array}$ & 6 & 3.93 & 663 & 386.78 & 6 & 3.04 & 2 & 1.16 & 3 & 1.52 & 680 & 396.43 \\
\hline Perry & - & - & 4 & 8.74 & - & - & - & - & - & - & 4 & 8.74 \\
\hline Philadelphia & 146 & 7.18 & 36 & 1.47 & 27 & 1.11 & - & - & 10 & 0.49 & 217 & 10.14 \\
\hline Pike & 1 & 3.15 & 4 & 7.86 & - & - & - & - & - & - & 5 & 11.01 \\
\hline Potter & 2 & 7.56 & 2 & 7.56 & 1 & 2.42 & - & - & - & - & 5 & 17.54 \\
\hline Schuylkill & 19 & 8.72 & 2,387 & 834.68 & 39 & 13.45 & 1 & 0.47 & 27 & 9.29 & 2,463 & 862.64 \\
\hline Snyder & 1 & 2.91 & 6 & 11.99 & 2 & 3.08 & - & - & - & - & 9 & 17.98 \\
\hline Somerset & 6 & 7.16 & 358 & 281.03 & 17 & 17.12 & - & - & 14 & 9.51 & 393 & 312.69 \\
\hline Sullivan & - & - & 4 & 19.44 & 1 & 4.86 & - & - & - & - & 5 & 24.30 \\
\hline Susquehanna & 2 & 3.45 & 40 & 55.28 & 2 & 5.95 & - & - & 7 & 12.70 & 51 & 77.38 \\
\hline Tioga & 1 & 1.10 & 4 & 6.85 & 1 & 3.63 & - & - & - & - & 6 & 11.59 \\
\hline Union & - & - & 1 & 1.38 & - & - & - & - & - & - & 1 & 1.38 \\
\hline Venango & 3 & 4.39 & 10 & 11.08 & 1 & 1.46 & - & - & 3 & 3.68 & 17 & 20.61 \\
\hline Warren & 1 & 1.93 & 4 & 5.70 & 2 & 2.85 & - & - & - & - & 6 & 9.55 \\
\hline Washington & 7 & 2.63 & 332 & 92.41 & 10 & 2.57 & - & - & 15 & 4.98 & 361 & 101.80 \\
\hline Wayne & 5 & 8.93 & 18 & 23.90 & 1 & 2.19 & - & - & - & - & 24 & 35.03 \\
\hline Westmoreland & 10 & 1.66 & 283 & 43.39 & 35 & 6.26 & - & - & 19 & 3.10 & 346 & 54.20 \\
\hline Wyoming & 3 & 9.75 & 21 & 54.58 & - & - & - & - & 1 & 1.95 & 25 & 66.28 \\
\hline York & 4 & 1.07 & 6 & 1.38 & 6 & 1.78 & - & - & 5 & 1.07 & 20 & 5.00 \\
\hline
\end{tabular}




\section{Rhode Island: Selected statistics from the United States section}

All pneumoconioses mortality, 1988-1992

Total deaths

Crude mortality rate (per $1,000,000$ population)

Age-adjusted mortality rate (per $1,000,000$ population)

Total years of potential life lost to life expectancy

Years of potential life lost to life expectancy (per death)

\begin{tabular}{rr} 
Value & State rank \\
\hline 35 & 42 \\
8.61 & 27 \\
4.78 & 30 \\
435 & 40 \\
12.4 & 18
\end{tabular}

Percent**

Deaths by type of pneumoconiosis, 1988-1992

Deaths*

distribution

29

82.9

Coal workers' pneumoconiosis

1

2.9

Silicosis

3

8.6

Byssinosis

Unspecified/Other pneumoconiosis

$0 \quad 0.0$

* Sum of column may exceed total deaths because of deaths with multiple pneumoconioses.

** Percentages may total more than $100 \%$ due to deaths with multiple pneumoconioses.

NOTE: $\quad$ See Appendix A for source description and Appendix B for methods and ICD-8 and ICD-9 codes.

SOURCE: National Center for Health Statistics multiple cause of death data. Population estimates from U.S. Bureau of the Census.

Figure RI-1. All pneumoconioses: Rhode Island and U.S. age-adjusted mortality rates, residents age 15 and over, 1968-1992

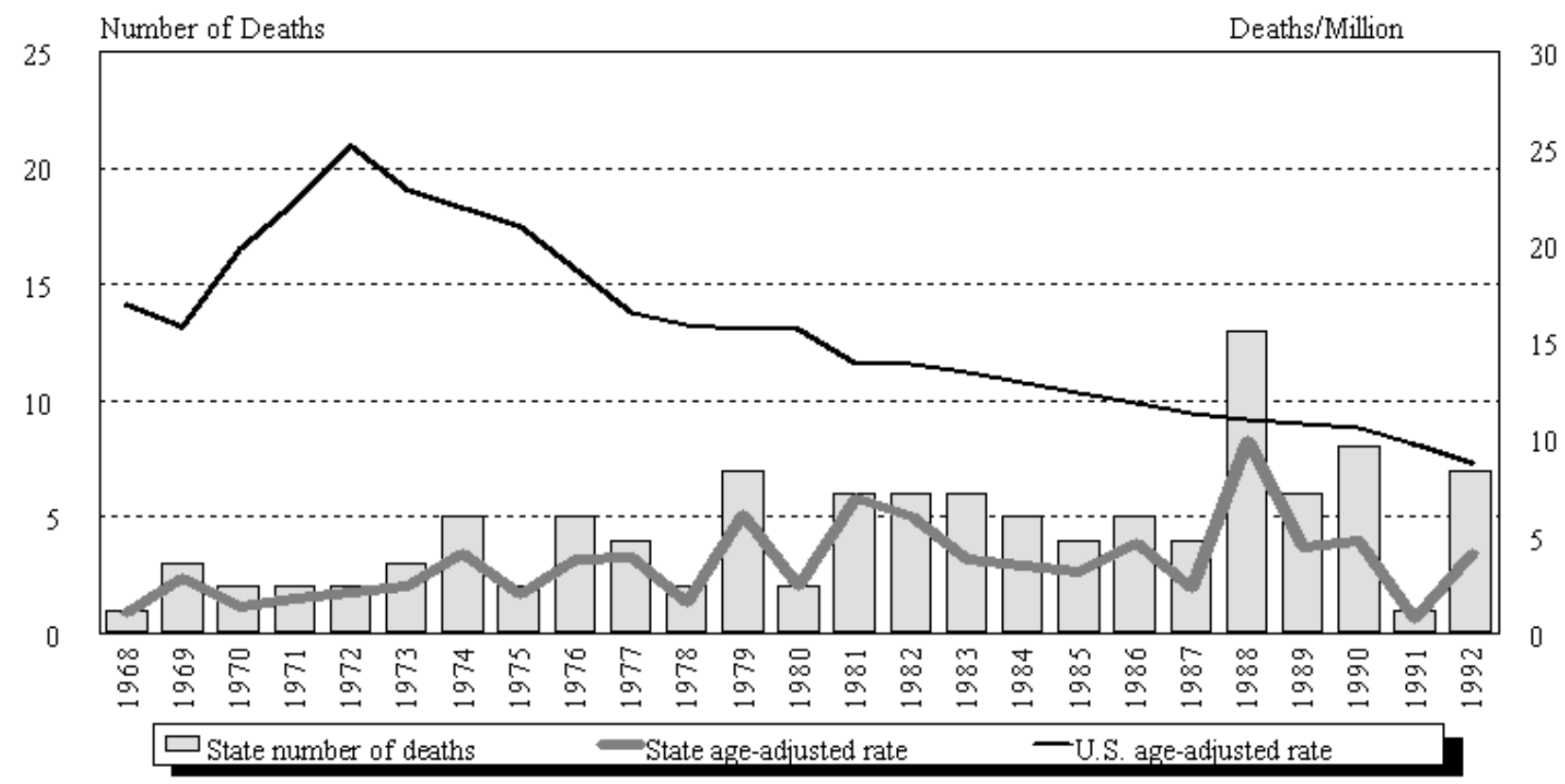

NOTE: $\quad$ See Appendix A for source description and Appendix B for methods and ICD-8 and ICD-9 codes.

SOURCE: National Center for Health Statistics multiple cause of death data. Population estimates from U.S. Bureau of the Census. 
Rhode Island

Table RI-1. Number of deaths and age-adjusted mortality rate (per 1,000,000 population) by condition, Rhode Island residents age 15 and over, 1968-1992

\begin{tabular}{|c|c|c|c|c|c|c|c|c|c|c|c|c|}
\hline \multirow[b]{2}{*}{ Year } & \multicolumn{2}{|c|}{ Asbestosis } & \multicolumn{2}{|c|}{$\begin{array}{c}\text { Coal workers' } \\
\text { pneumoconiosis }\end{array}$} & \multicolumn{2}{|c|}{ Silicosis } & \multicolumn{2}{|c|}{ Byssinosis* } & \multicolumn{2}{|c|}{$\begin{array}{c}\text { Unspecified/Other } \\
\text { pneumoconioses }\end{array}$} & \multicolumn{2}{|c|}{ All pneumoconioses } \\
\hline & Number & Rate & Number & Rate & Number & Rate & Number & Rate & Number & Rate & Number & Rate \\
\hline 1968 & - & - & - & & 1 & 1.01 & & & - & - & 1 & 1.01 \\
\hline 1969 & - & - & - & - & 1 & 0.71 & & & 2 & 2.14 & 3 & 2.85 \\
\hline 1970 & - & - & 1 & 0.71 & 1 & 0.71 & & & 1 & 0.71 & 2 & 1.42 \\
\hline 1971 & 1 & 1.11 & - & - & - & - & & & 1 & 0.69 & 2 & 1.80 \\
\hline 1972 & - & - & - & - & - & - & & & 2 & 2.17 & 2 & 2.17 \\
\hline 1973 & - & - & 2 & 1.73 & - & - & & & 1 & 0.66 & 3 & 2.39 \\
\hline 1974 & 1 & 0.95 & 2 & 1.60 & 1 & 0.95 & & & 1 & 0.65 & 5 & 4.14 \\
\hline 1975 & - & - & - & - & 2 & 2.04 & & & - & - & 2 & 2.04 \\
\hline 1976 & - & - & - & - & 3 & 2.32 & & & 3 & 2.07 & 5 & 3.76 \\
\hline 1977 & 2 & 1.52 & - & - & 1 & 1.47 & & & 2 & 2.36 & 4 & 3.88 \\
\hline 1978 & 1 & 0.61 & - & - & - & - & & & 1 & 1.03 & 2 & 1.63 \\
\hline 1979 & 2 & 1.46 & 1 & 1.02 & 4 & 3.65 & - & - & - & - & 7 & 6.13 \\
\hline 1980 & 1 & 1.62 & - & - & 1 & 0.85 & - & - & - & - & 2 & 2.47 \\
\hline 1981 & 6 & 7.04 & - & - & - & - & - & - & - & - & 6 & 7.04 \\
\hline 1982 & 4 & 5.46 & - & - & 4 & 2.66 & - & - & - & - & 6 & 6.04 \\
\hline 1983 & 4 & 2.44 & - & - & 1 & 0.55 & 1 & 0.83 & - & - & 6 & 3.82 \\
\hline 1984 & 3 & 2.15 & - & - & 1 & 0.27 & 1 & 1.06 & - & - & 5 & 3.48 \\
\hline 1985 & 2 & 1.61 & - & - & 1 & 1.08 & - & - & 1 & 0.53 & 4 & 3.21 \\
\hline 1986 & 3 & 3.07 & - & - & 1 & 0.79 & - & - & 1 & 0.79 & 5 & 4.65 \\
\hline 1987 & - & - & - & - & 1 & 0.25 & - & - & 3 & 2.12 & 4 & 2.37 \\
\hline 1988 & 11 & 8.72 & 1 & 0.77 & - & - & - & - & 1 & 0.49 & 13 & 9.98 \\
\hline 1989 & 5 & 3.24 & - & - & - & - & - & - & 1 & 1.18 & 6 & 4.42 \\
\hline 1990 & 7 & 4.49 & - & - & 1 & 0.23 & - & - & - & - & 8 & 4.72 \\
\hline 1991 & 1 & 0.76 & - & - & - & - & - & - & - & - & 1 & 0.76 \\
\hline 1992 & 5 & 2.11 & - & - & 2 & 1.99 & - & - & - & - & 7 & 4.10 \\
\hline
\end{tabular}

indicates no deaths listed. ${ }^{*}$ no specific code for by ssinosis in ICD-8 (1968-1 978).

NOTE: Rates based on small numbers may be unstable. Sum of individual conditions may exceed total because of deaths with multiple pneumoconioses. See Appendix A for source description and Appendix B for methods and ICD-8 and ICD-9 codes.

SOURCE: National Center for Health Statistics multiple cause of death data. Population estimates from U.S. Bureau of the Census. 
Figure RI-2. All pneumoconioses: Distribution of deaths by sex and race, Rhode Island residents age 15 and over, 1968-1992
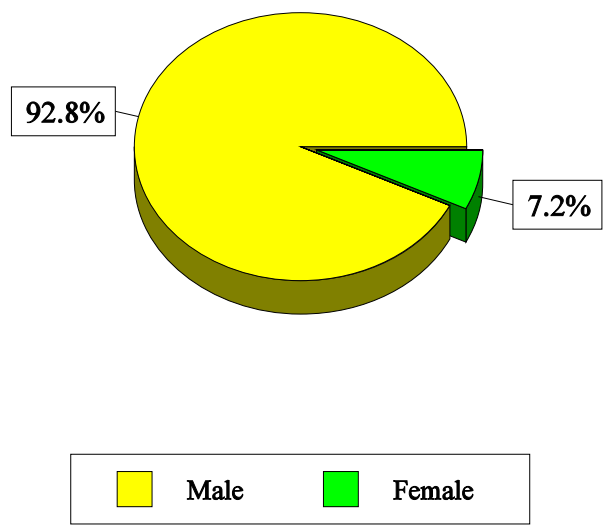

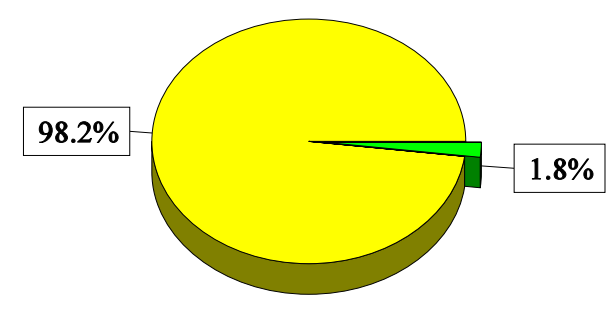

NOTE:

See Appendix A for source description and Appendix B for methods and ICD-8 and ICD-9 codes.

SOURCE: National Center for Health Statistics multiple cause of death data.

Table RI-2. All pneumoconioses: Distribution of deaths by sex, race, and age, Rhode Island residents age 15 and over, 1968-1992

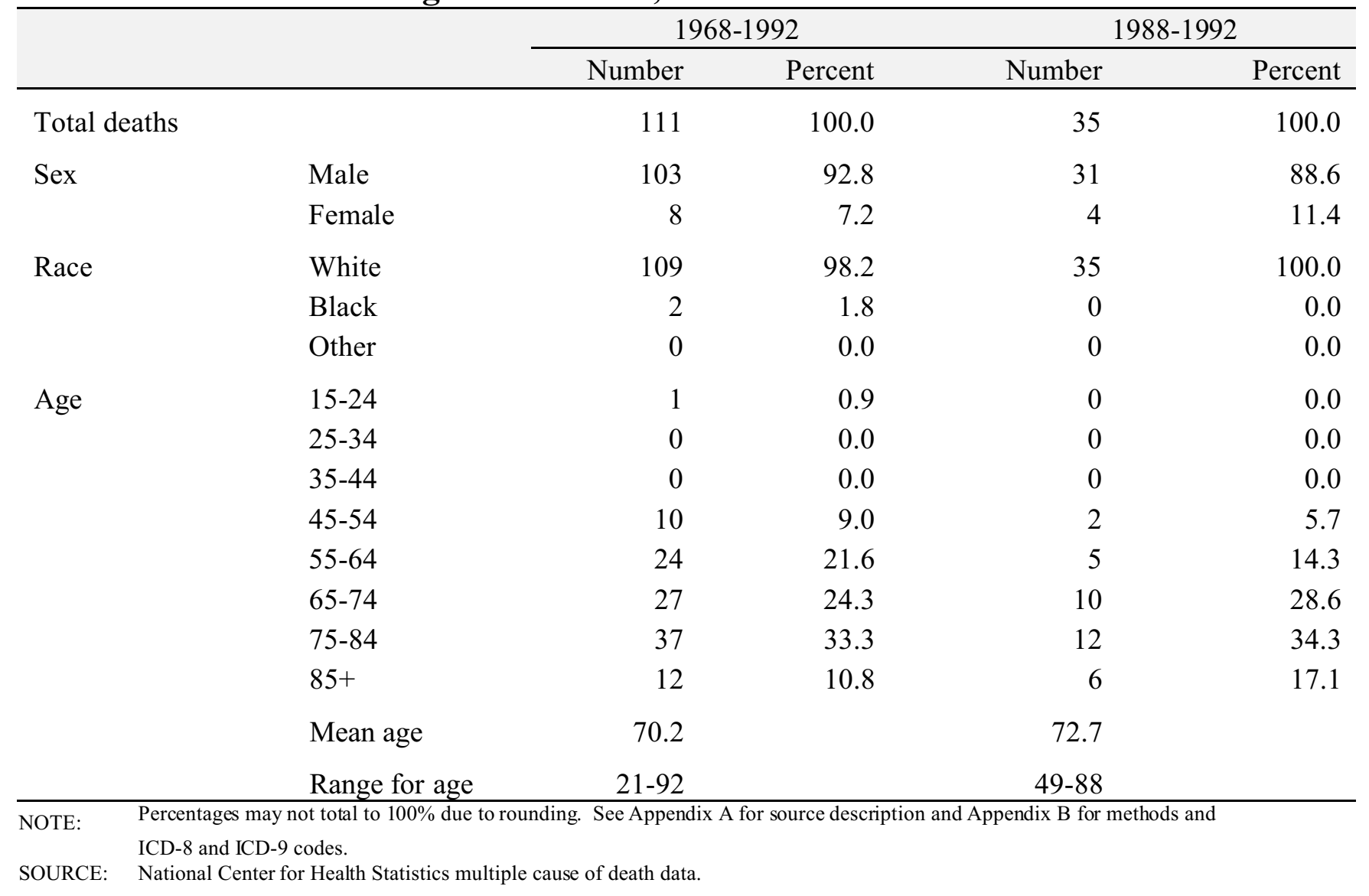


Figure RI-3. Pneumoconiosis deaths by condition, Rhode Island residents age 15 and over, 1968-1992

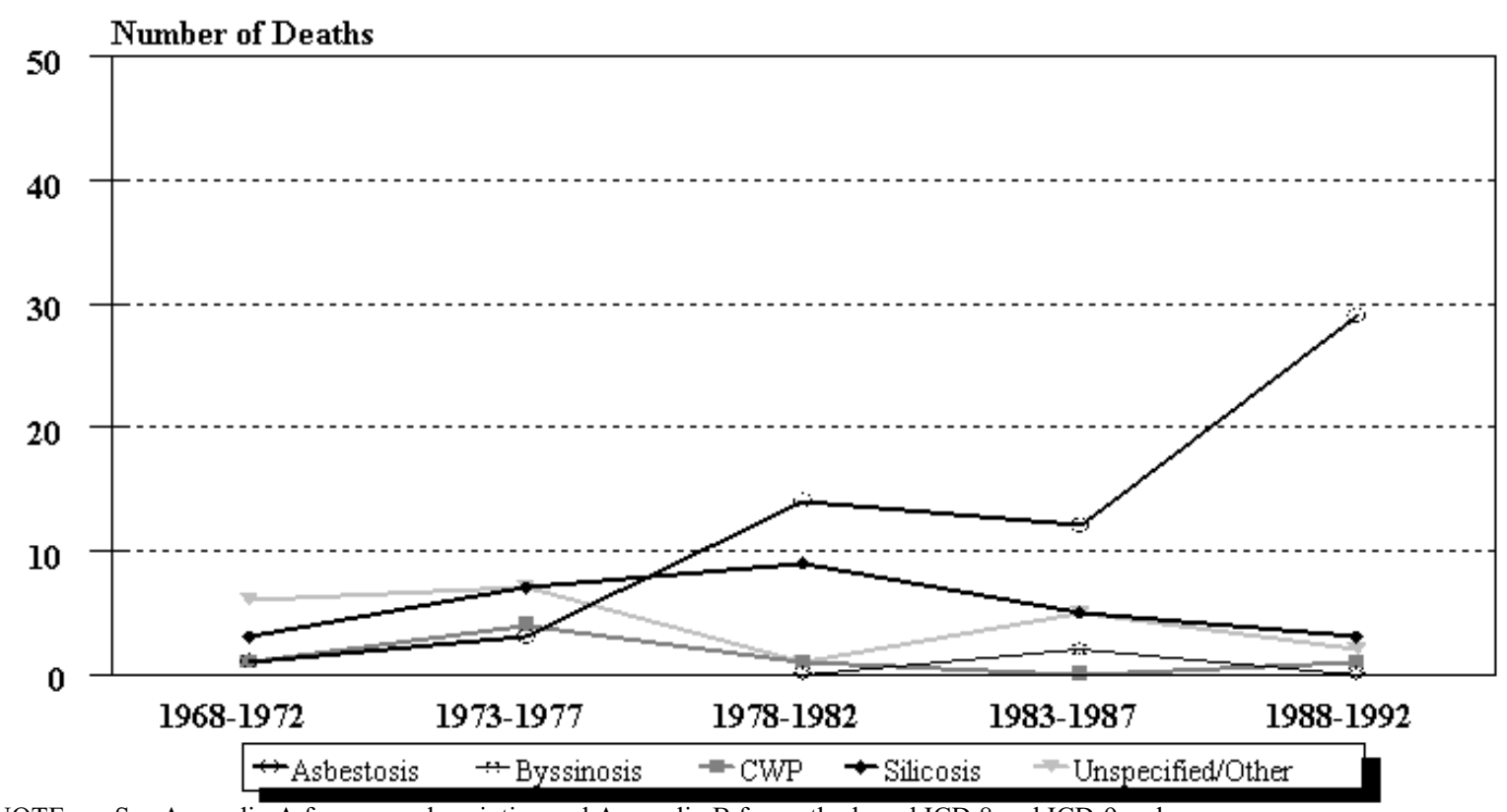

NOTE: See Appendix A for source description and Appendix B for methods and ICD-8 and ICD-9 codes.

SOURCE: National Center for Health Statistics multiple cause of death data.

Figure RI-4. Rhode Island and U.S. age-adjusted mortality rates (per 1,000,000 population) by condition, residents age 15 and over, 1983-1992

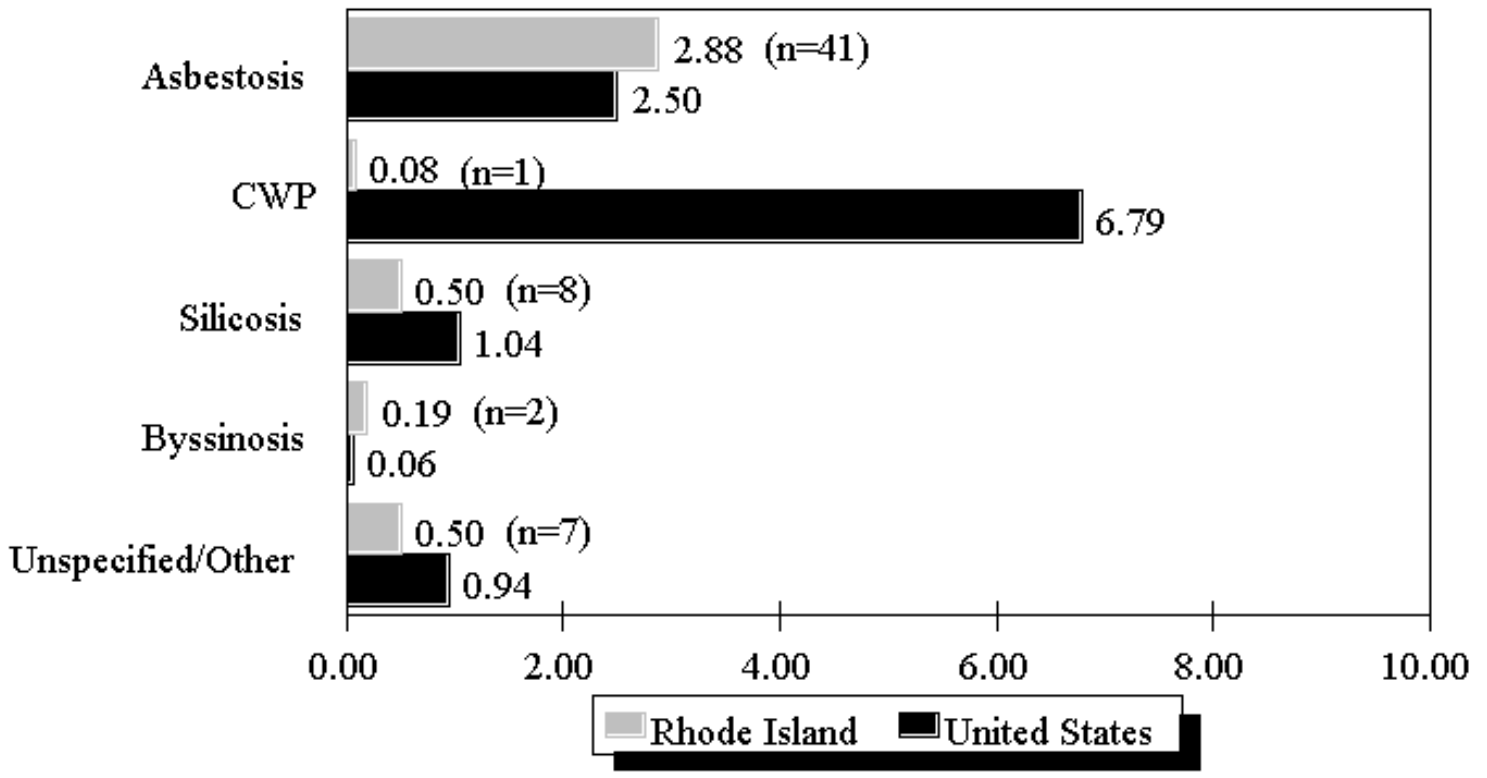

NOTE: Rates based on small numbers may be unstable. See Appendix A for source description and Appendix B for methods and ICD-8 and ICD-9 codes.

SOURCE: National Center for Health Statistics multiple cause of death data. Population estimates from U.S. Bureau of the Census. 
Table RI-3. Number of deaths and age-adjusted mortality rate (per 1,000,000 population) by condition and county, Rhode Island residents age 15 and over, 1983-1992

\begin{tabular}{|c|c|c|c|c|c|c|c|c|c|c|c|c|}
\hline \multirow[b]{2}{*}{ County } & \multicolumn{2}{|c|}{ Asbestosis } & \multicolumn{2}{|c|}{$\begin{array}{r}\text { Coal workers' } \\
\text { pneumoconiosis }\end{array}$} & \multicolumn{2}{|c|}{ Silicosis } & \multicolumn{2}{|c|}{ Byssinosis } & \multicolumn{2}{|c|}{$\begin{array}{r}\text { Unspecified/Other } \\
\text { pneumoconioses }\end{array}$} & \multicolumn{2}{|c|}{$\begin{array}{r}\text { All } \\
\text { pneumoconioses }\end{array}$} \\
\hline & \# & Rate & \# & Rate & \# & Rate & $\#$ & Rate & \# & Rate & \# & Rate \\
\hline Bristol & - & - & - & - & 1 & 1.79 & - & - & 3 & 4.51 & 4 & 6.30 \\
\hline Kent & 3 & 0.88 & - & - & - & - & - & - & 1 & 0.29 & 4 & 1.17 \\
\hline Newport & 2 & 1.82 & - & - & - & - & - & - & - & - & 2 & 1.82 \\
\hline Providence & 30 & 3.23 & 1 & 0.16 & 6 & 0.58 & 2 & 0.32 & 2 & 0.13 & 41 & 4.42 \\
\hline Washington & 6 & 6.64 & - & - & 1 & 1.06 & - & - & 1 & 1.06 & 8 & 8.76 \\
\hline
\end{tabular}

- indicates no deaths listed.

NOTE: Counties with no deaths for all listed conditions are not displayed. Rates based on small numbers may be unstable. Sum of individual conditions may exceed total because of deaths with multiple pneumoconioses. See Appendix A for source description and Appendix B for methods and ICD-8 and ICD-9 codes.

SOURCE: National Center for Health Statistics multiple cause of death data. Population estimates from U.S. Bureau of the Census. 


\section{South Carolina: Selected statistics from the United States section}

All pneumoconioses mortality, 1988-1992

Total deaths

Crude mortality rate (per $1,000,000$ population)

Age-adjusted mortality rate (per $1,000,000$ population)

Total years of potential life lost to life expectancy

Years of potential life lost to life expectancy (per death)

\begin{tabular}{rr} 
Value & State rank \\
\hline 97 & 29 \\
7.12 & 30 \\
4.81 & 28 \\
1,285 & 27 \\
13.2 & 8
\end{tabular}

Percent**

Deaths by type of pneumoconiosis, 1988-1992

Deaths*

distribution

Asbestosis

64

66.0

10

10.3

Coal workers' pneumoconiosis

14

14.4

Byssinosis

Unspecified/Other pneumoconiosis

* Sum of column may exceed total deaths because of deaths with multiple pneumoconioses.

** Percentages may total more than $100 \%$ due to deaths with multiple pneumoconioses.

NOTE: $\quad$ See Appendix A for source description and Appendix B for methods and ICD-8 and ICD-9 codes.

SOURCE: National Center for Health Statistics multiple cause of death data. Population estimates from U.S. Bureau of the Census.

Figure SC-1. All pneumoconioses: South Carolina and U.S. age-adjusted mortality rates, residents age 15 and over, 1968-1992

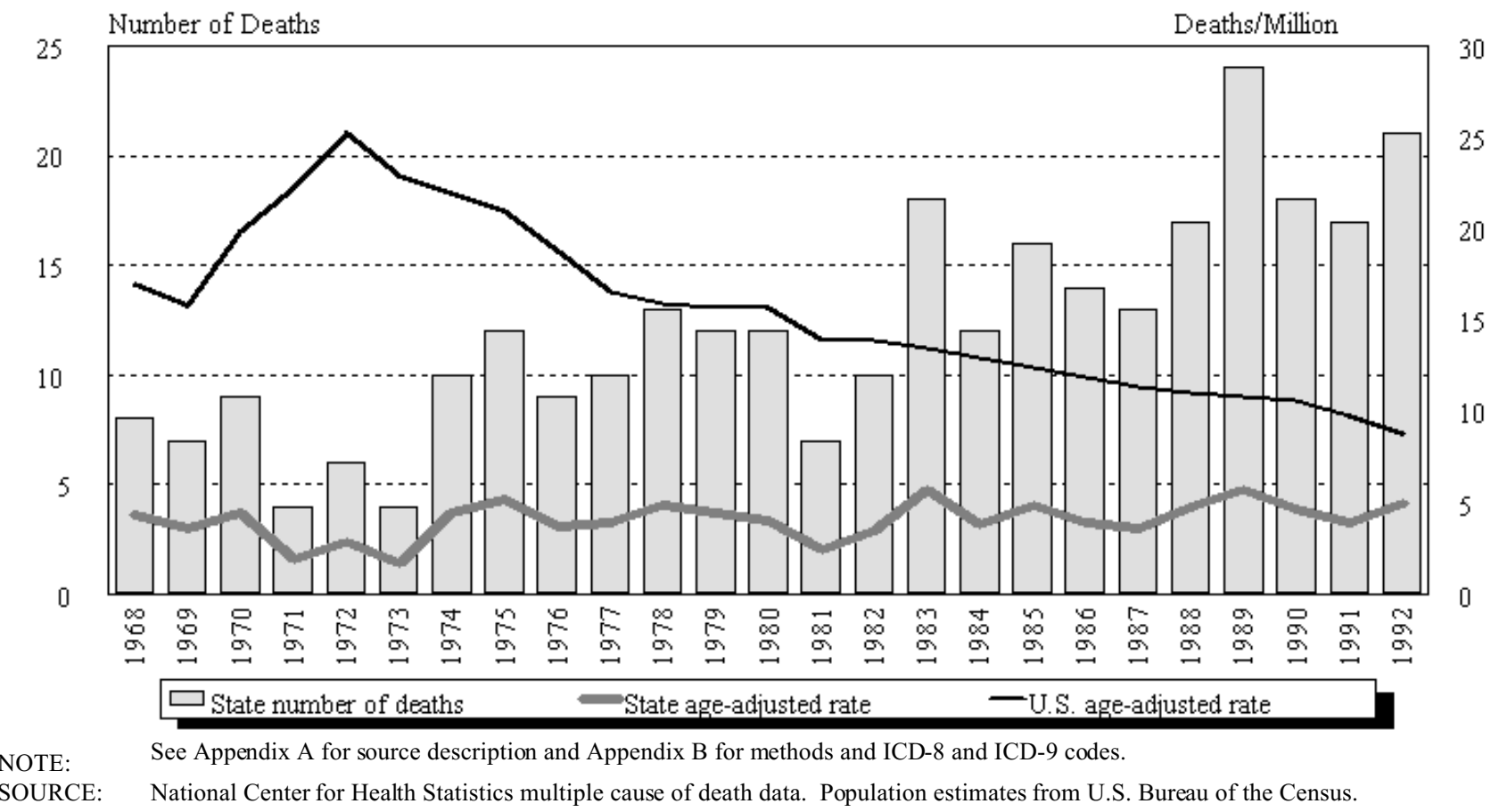


South Carolina

Table SC-1. Number of deaths and age-adjusted mortality rate (per 1,000,000 population) by condition, South Carolina residents age 15 and over, 1968-1992

\begin{tabular}{|c|c|c|c|c|c|c|c|c|c|c|c|c|}
\hline \multirow[b]{2}{*}{ Year } & \multicolumn{2}{|c|}{ Asbestosis } & \multicolumn{2}{|c|}{$\begin{array}{l}\text { Coal workers' } \\
\text { pneumoconiosis }\end{array}$} & \multicolumn{2}{|c|}{ Silicosis } & \multicolumn{2}{|c|}{ Byssinosis* } & \multicolumn{2}{|c|}{$\begin{array}{c}\text { Unspecified/Other } \\
\text { pneumoconioses }\end{array}$} & \multicolumn{2}{|c|}{ All pneumoconioses } \\
\hline & Number & Rate & Number & Rate & Number & Rate & Number & Rate & Number & Rate & Number & Rate \\
\hline 1968 & 3 & 1.58 & - & - & 5 & 2.72 & & & - & - & 8 & 4.31 \\
\hline 1969 & 5 & 2.55 & - & - & 2 & 1.02 & & & - & - & 7 & 3.57 \\
\hline 1970 & 3 & 1.53 & 2 & 0.87 & 4 & 2.08 & & & 1 & 0.43 & 9 & 4.47 \\
\hline 1971 & 1 & 0.49 & 1 & 0.49 & - & - & & & 2 & 0.98 & 4 & 1.96 \\
\hline 1972 & 2 & 0.95 & - & - & - & - & & & 4 & 1.91 & 6 & 2.86 \\
\hline 1973 & 2 & 0.91 & - & - & 2 & 0.84 & & & - & - & 4 & 1.74 \\
\hline 1974 & 3 & 1.43 & 1 & 0.43 & 6 & 2.76 & & & 1 & 0.37 & 10 & 4.46 \\
\hline 1975 & 3 & 1.24 & 2 & 0.79 & 4 & 1.84 & & & 3 & 1.35 & 12 & 5.22 \\
\hline 1976 & 2 & 0.85 & 2 & 0.85 & 2 & 0.82 & & & 3 & 1.20 & 9 & 3.72 \\
\hline 1977 & 5 & 2.22 & 1 & 0.38 & 3 & 0.93 & & & 1 & 0.38 & 10 & 3.90 \\
\hline 1978 & 8 & 3.09 & 2 & 0.72 & 2 & 0.72 & & & 2 & 0.68 & 13 & 4.90 \\
\hline 1979 & 7 & 2.61 & - & - & 5 & 1.85 & - & - & 1 & 0.39 & 12 & 4.42 \\
\hline 1980 & 4 & 1.44 & 4 & 1.15 & 2 & 0.72 & 1 & 0.34 & 1 & 0.34 & 12 & 3.99 \\
\hline 1981 & 2 & 0.61 & 1 & 0.28 & 4 & 1.59 & - & - & - & - & 7 & 2.48 \\
\hline 1982 & 4 & 1.74 & 1 & 0.48 & 4 & 0.96 & - & - & 1 & 0.27 & 10 & 3.45 \\
\hline 1983 & 8 & 2.93 & 2 & 0.57 & 3 & 0.67 & 2 & 0.68 & 3 & 0.94 & 18 & 5.78 \\
\hline 1984 & 6 & 1.92 & 1 & 0.30 & 1 & 0.37 & 4 & 1.22 & - & - & 12 & 3.80 \\
\hline 1985 & 9 & 2.61 & 2 & 0.53 & 3 & 1.09 & - & - & 2 & 0.66 & 16 & 4.89 \\
\hline 1986 & 9 & 2.48 & 1 & 0.22 & 3 & 0.67 & 1 & 0.50 & - & - & 14 & 3.88 \\
\hline 1987 & 8 & 2.28 & 2 & 0.58 & 2 & 0.53 & 1 & 0.28 & - & - & 13 & 3.66 \\
\hline 1988 & 14 & 3.80 & 1 & 0.13 & 2 & 0.84 & - & - & - & - & 17 & 4.77 \\
\hline 1989 & 18 & 4.47 & 3 & 0.60 & 3 & 0.69 & - & - & - & - & 24 & 5.75 \\
\hline 1990 & 11 & 2.40 & 1 & 0.19 & 5 & 1.71 & 1 & 0.37 & - & - & 18 & 4.66 \\
\hline 1991 & 8 & 1.78 & - & - & 3 & 0.88 & 3 & 0.78 & 3 & 0.49 & 17 & 3.93 \\
\hline 1992 & 13 & 3.20 & 5 & 1.05 & 1 & 0.11 & 2 & 0.62 & - & - & 21 & 4.99 \\
\hline
\end{tabular}

indicates no deaths listed. * no specific code for byssinosis in ICD-8 (1968-1978).

NOTE: Rates based on small numbers may be unstable. Sum of individual conditions may exceed total because of deaths with multiple pneumoconioses. See Appendix A for source description and Appendix B for methods and ICD-8 and ICD-9 codes.

SOURCE: National Center for Health Statistics multiple cause of death data. Population estimates from U.S. Bureau of the Census. 


\section{Figure SC-2. All pneumoconioses: Distribution of deaths by sex and race, South Carolina residents age 15 and over, 1968-1992}
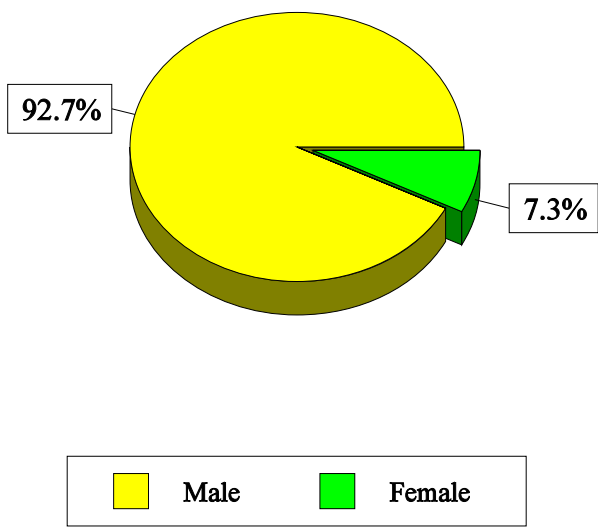
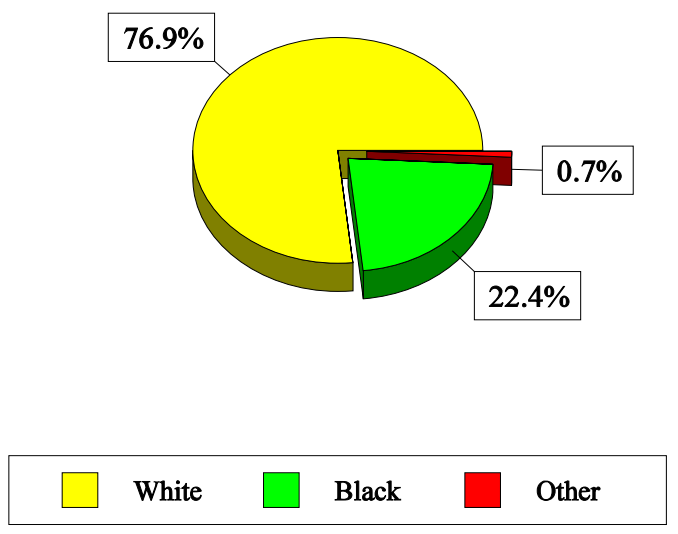

NOTE: See Appendix A for source description and Appendix B for methods and ICD-8 and ICD-9 codes.

SOURCE: National Center for Health Statistics multiple cause of death data.

Table SC-2. All pneumoconioses: Distribution of deaths by sex, race, and age, South Carolina residents age 15 and over, 1968-1992

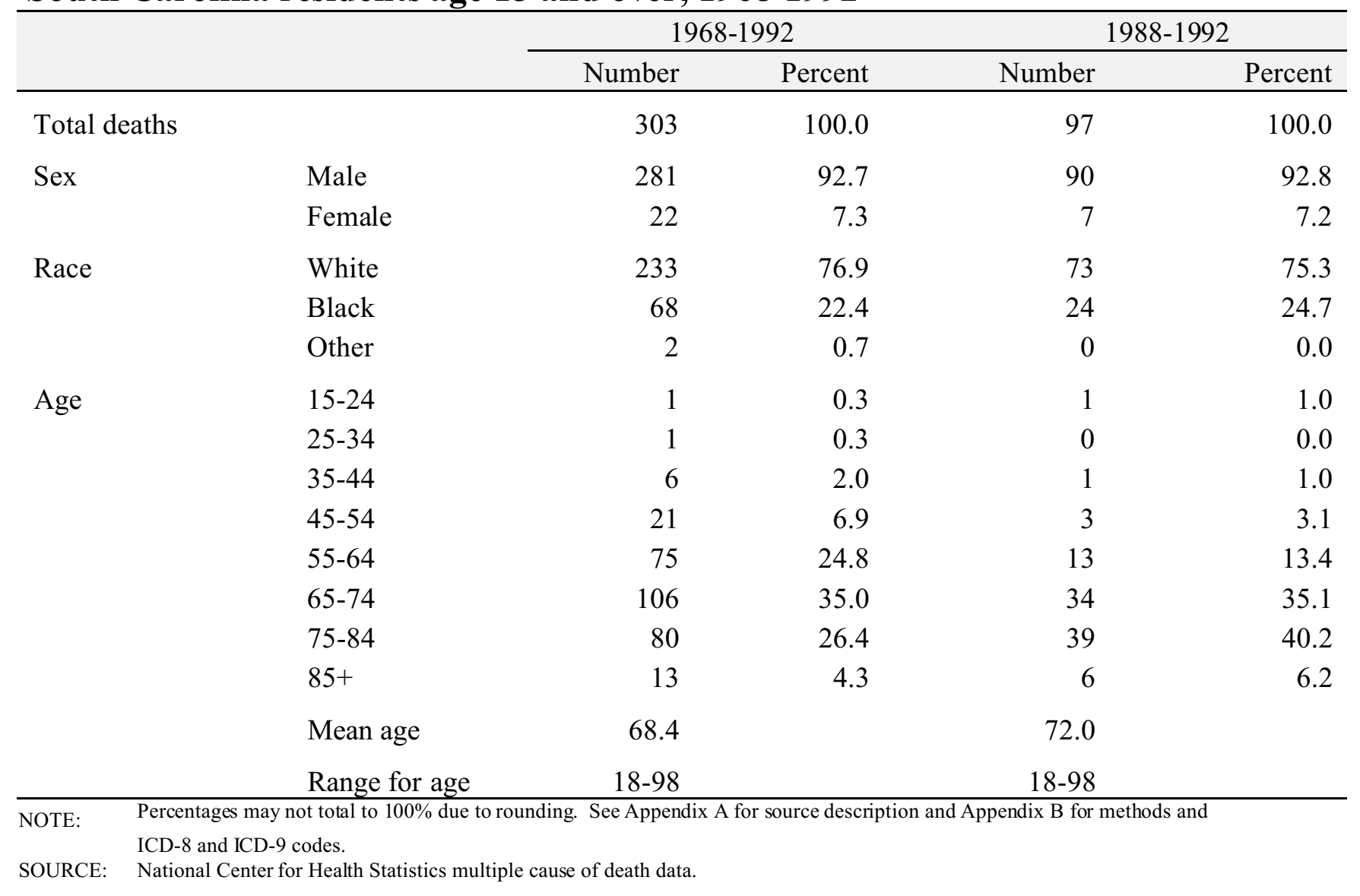


Figure SC-3. Pneumoconiosis deaths by condition, South Carolina residents age 15 and over, 1968-1992

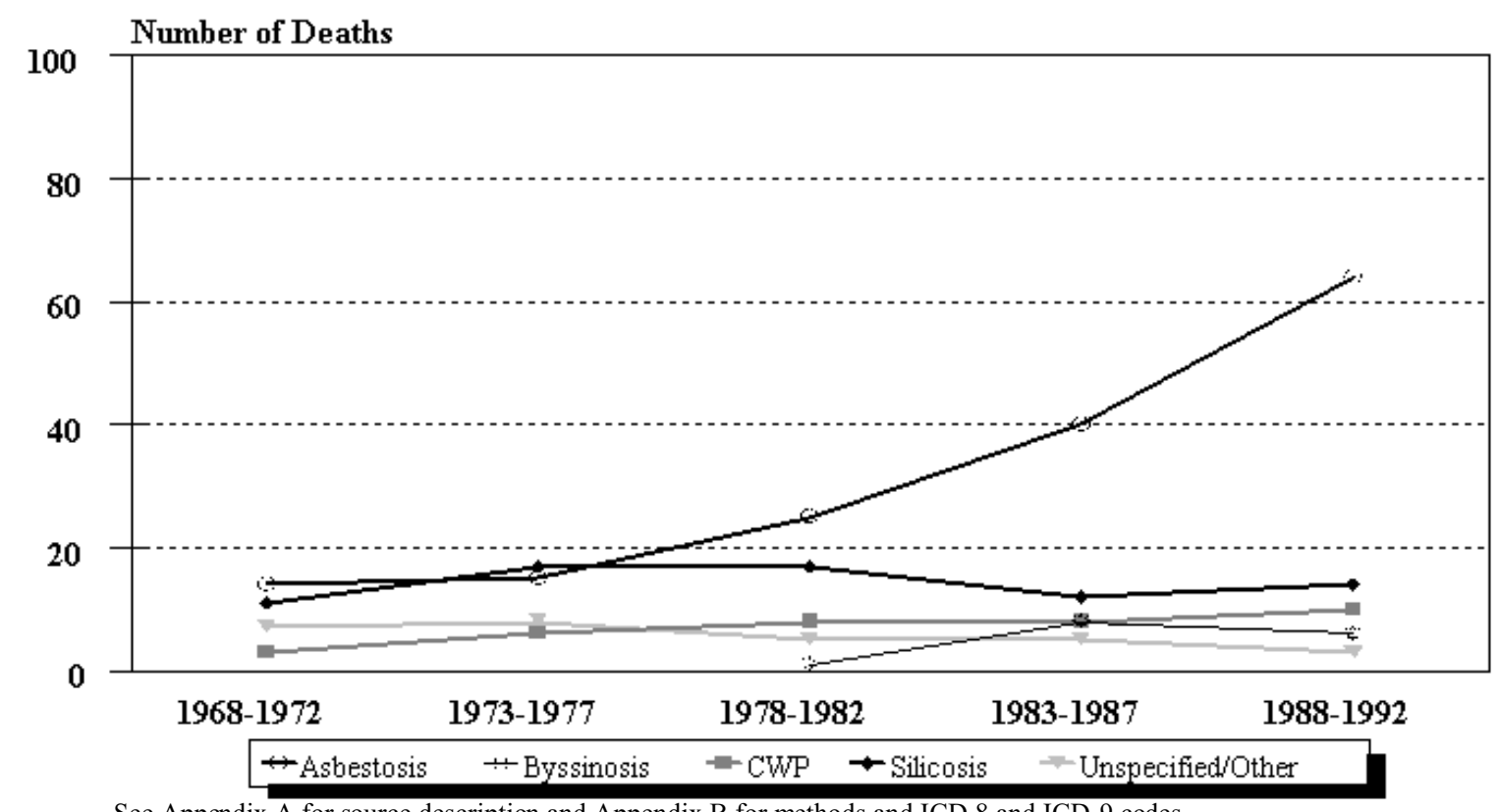

NOTE: See Appendix A for source description and Appendix B for methods and ICD-8 and ICD-9 codes.

SOURCE: National Center for Health Statistics multiple cause of death data.

Figure SC-4. South Carolina and U.S. age-adjusted mortality rates (per 1,000,000 population) by condition, residents age 15 and over, 1983-1992

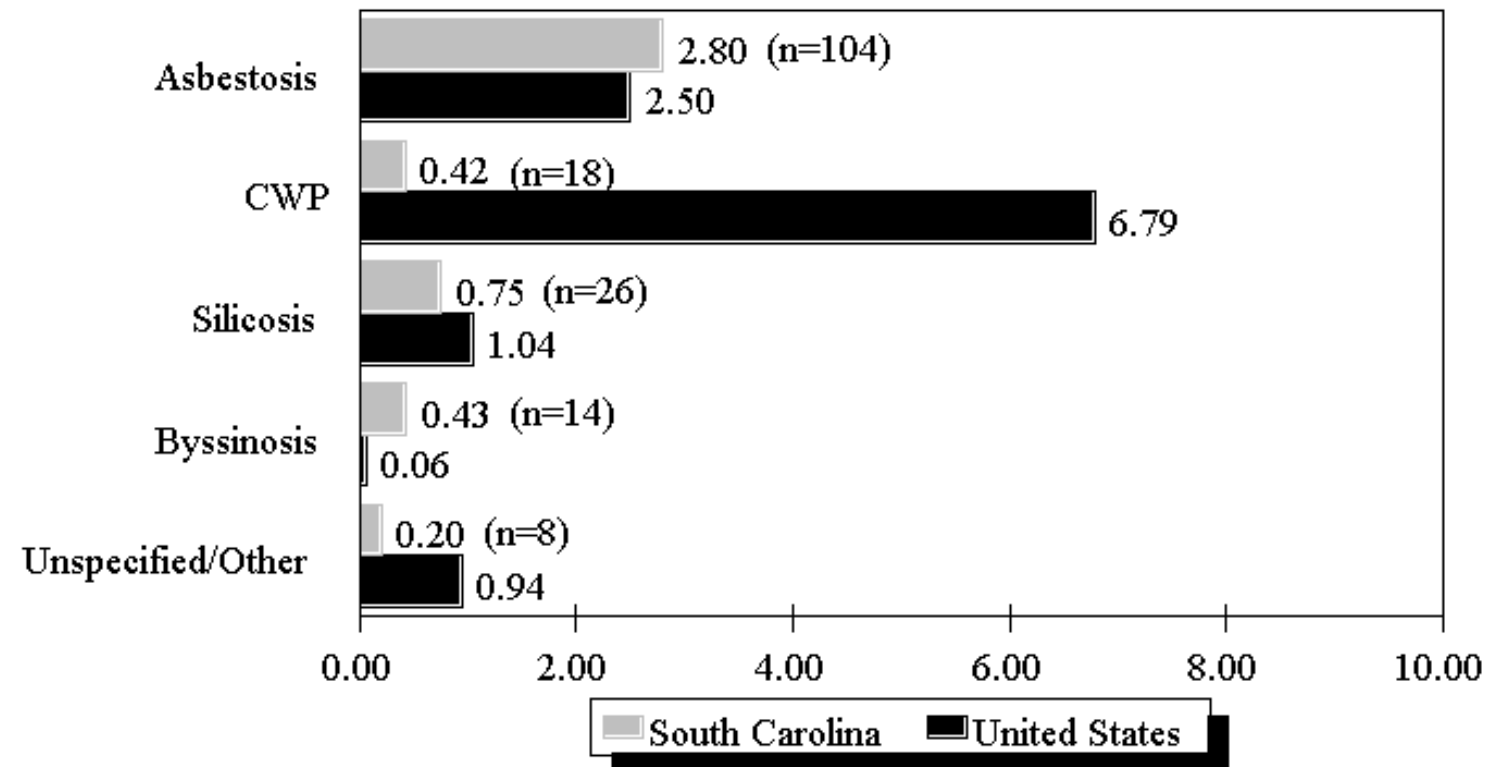

NOTE: Rates based on small numbers may be unstable. See Appendix A for source description and Appendix B for methods and ICD-8 and ICD-9 codes.

SOURCE: National Center for Health Statistics multiple cause of death data. Population estimates from U.S. Bureau of the Census. 
Table SC-3 (page 1 of 2). Number of deaths and age-adjusted mortality rate (per 1,000,000 population) by condition and county, South Carolina residents age 15 and over, 1983-1992

\begin{tabular}{|c|c|c|c|c|c|c|c|c|c|c|c|c|}
\hline \multirow[b]{2}{*}{ County } & \multicolumn{2}{|c|}{ Asbestosis } & \multicolumn{2}{|c|}{$\begin{array}{c}\text { Coal workers' } \\
\text { pneumoconiosis }\end{array}$} & \multicolumn{2}{|c|}{ Silicosis } & \multicolumn{2}{|c|}{ Byssinosis } & \multicolumn{2}{|c|}{$\begin{array}{r}\text { Unspecified/Other } \\
\text { pneumoconioses }\end{array}$} & \multicolumn{2}{|c|}{$\begin{array}{r}\text { All } \\
\text { pneumoconioses }\end{array}$} \\
\hline & $\#$ & Rate & \# & Rate & $\#$ & Rate & \# & Rate & \# & Rate & $\#$ & Rate \\
\hline Abbeville & - & - & 1 & 1.89 & - & - & - & - & - & - & 1 & 1.89 \\
\hline Aiken & 1 & 0.58 & - & - & - & - & 2 & 1.77 & 1 & 0.58 & 4 & 2.93 \\
\hline Anderson & 1 & 0.38 & 1 & 0.38 & 2 & 1.06 & 1 & 0.68 & - & - & 5 & 2.50 \\
\hline Bamberg & 1 & 3.34 & - & - & - & - & - & - & - & - & 1 & 3.34 \\
\hline Barnwell & 1 & 5.34 & - & - & - & - & - & - & - & - & 1 & 5.34 \\
\hline Beaufort & 2 & 1.80 & - & - & - & - & - & - & - & - & 2 & 1.80 \\
\hline Berkeley & 7 & 9.12 & - & - & 1 & 1.41 & - & - & - & - & 8 & 10.53 \\
\hline Charleston & 44 & 15.98 & 1 & 0.43 & 2 & 0.55 & - & - & - & - & 47 & 16.95 \\
\hline Cherokee & 2 & 4.68 & - & - & - & - & - & - & - & - & 2 & 4.68 \\
\hline Chester & - & - & 1 & 1.68 & 1 & 1.68 & 1 & 3.13 & - & - & 3 & 6.49 \\
\hline Clarendon & 1 & 3.53 & - & - & 2 & 10.75 & - & - & 1 & 3.53 & 4 & 17.81 \\
\hline Colleton & 5 & 10.98 & 2 & 4.64 & - & - & - & - & - & - & 7 & 15.62 \\
\hline Darlington & 1 & 2.27 & 1 & 1.00 & 1 & 2.27 & - & - & - & - & 3 & 5.55 \\
\hline Dillon & - & - & - & - & 1 & 5.29 & - & - & - & - & 1 & 5.29 \\
\hline Dorchester & 7 & 11.99 & - & - & - & - & - & - & - & - & 7 & 11.99 \\
\hline Fairfield & - & - & - & - & 1 & 4.72 & - & - & 1 & 4.72 & 2 & 9.44 \\
\hline Florence & 2 & 1.99 & - & - & - & - & - & - & - & - & 2 & 1.99 \\
\hline Georgetown & - & - & 1 & 2.16 & - & - & - & - & - & - & 1 & 2.16 \\
\hline Greenville & 4 & 1.23 & - & - & 2 & 0.40 & 1 & 0.44 & - & - & 7 & 2.07 \\
\hline Greenwood & - & - & - & - & 1 & 2.46 & - & - & - & - & 1 & 2.46 \\
\hline Horry & - & - & 2 & 1.07 & 1 & 0.54 & - & - & 2 & 1.07 & 5 & 2.69 \\
\hline Kershaw & 3 & 7.16 & - & - & 1 & 2.39 & - & - & - & - & 4 & 9.55 \\
\hline Lancaster & - & - & 1 & 1.92 & - & - & 2 & 3.84 & - & - & 3 & 5.76 \\
\hline Laurens & - & - & 2 & 2.63 & - & - & - & - & - & - & 2 & 2.63 \\
\hline Lexington & - & - & - & - & 2 & 1.55 & - & - & 1 & 0.55 & 3 & 2.09 \\
\hline McCormick & - & - & 1 & 6.07 & - & - & - & - & - & - & 1 & 6.07 \\
\hline Marion & 1 & 3.12 & - & - & - & - & 1 & 1.80 & - & - & 2 & 4.92 \\
\hline Marlboro & 1 & 3.50 & - & - & 1 & 3.50 & 2 & 7.00 & - & - & 4 & 14.01 \\
\hline Newberry & 1 & 2.84 & - & - & 1 & 2.84 & - & - & - & - & 2 & 5.68 \\
\hline Oconee & 1 & 1.65 & - & - & - & - & 2 & 4.12 & - & - & 3 & 5.77 \\
\hline Orangeburg & 2 & 2.53 & 1 & 1.27 & - & - & - & - & - & - & 3 & 3.80 \\
\hline
\end{tabular}

See footnotes at end of table. 
Table SC-3 (page 2 of 2). Number of deaths and age-adjusted mortality rate (per 1,000,000 population) by condition and county, South Carolina residents age 15 and over, 1983-1992

\begin{tabular}{|c|c|c|c|c|c|c|c|c|c|c|c|c|}
\hline \multirow[b]{2}{*}{ County } & \multicolumn{2}{|c|}{ Asbestosis } & \multicolumn{2}{|c|}{$\begin{array}{c}\text { Coal workers' } \\
\text { pneumoconiosis }\end{array}$} & \multicolumn{2}{|c|}{ Silicosis } & \multicolumn{2}{|c|}{ Byssinosis } & \multicolumn{2}{|c|}{$\begin{array}{r}\text { Unspecified/Other } \\
\text { pneumoconioses }\end{array}$} & \multicolumn{2}{|c|}{$\begin{array}{r}\text { All } \\
\text { pneumoconioses }\end{array}$} \\
\hline & \# & Rate & $\#$ & Rate & \# & Rate & \# & Rate & $\#$ & Rate & \# & Rate \\
\hline Pickens & - & - & - & - & 2 & 1.38 & - & - & - & - & 2 & 1.38 \\
\hline Richland & 6 & 2.46 & - & - & 1 & 0.29 & - & - & 1 & 0.47 & 8 & 3.22 \\
\hline Spartanburg & 5 & 2.26 & 1 & 0.26 & 2 & 0.87 & 1 & 0.46 & - & - & 9 & 3.86 \\
\hline Sumter & - & - & - & - & - & - & - & - & 1 & 1.33 & 1 & 1.33 \\
\hline York & 5 & 3.85 & 2 & 1.14 & 1 & 0.90 & 1 & 0.90 & - & - & 9 & 6.79 \\
\hline
\end{tabular}

- indicates no deaths listed.

NOTE: Counties with no deaths for all listed conditions are not displayed. Rates based on small numbers may be unstable. Sum of individual conditions may exceed total because of deaths with multiple pneumoconioses. See Appendix A for source description and Appendix B for methods and ICD-8 and ICD-9 codes.

SOURCE: National Center for Health Statistics multiple cause of death data. Population estimates from U.S. Bureau of the Census. 


\section{South Dakota: Selected statistics from the United States section}

All pneumoconioses mortality, 1988-1992

Total deaths

$\underline{\text { Value }}$

$\underline{\text { State rank }}$

Crude mortality rate (per $1,000,000$ population)

Age-adjusted mortality rate (per $1,000,000$ population)

Total years of potential life lost to life expectancy

Years of potential life lost to life expectancy (per death)

Deaths by type of pneumoconiosis, 1988-1992

Asbestosis

Coal workers' pneumoconiosis

7

2.66

1.14

9.9

Silicosis

Deaths*
0
3
3
0
1

Percent**

Byssinosis

Unspecified/Other pneumoconiosis

distribution

$0 \quad 0.0$

3

42.9

3

42.9

0

0.0

* Sum of column may exceed total deaths because of deaths with multiple pneumoconioses.

** Percentages may total more than $100 \%$ due to deaths with multiple pneumoconioses.

NOTE: $\quad$ See Appendix A for source description and Appendix B for methods and ICD-8 and ICD-9 codes.

SOURCE: National Center for Health Statistics multiple cause of death data. Population estimates from U.S. Bureau of the Census.

\section{Figure SD-1. All pneumoconioses: South Dakota and U.S. age-adjusted mortality rates, residents age 15 and over, 1968-1992}

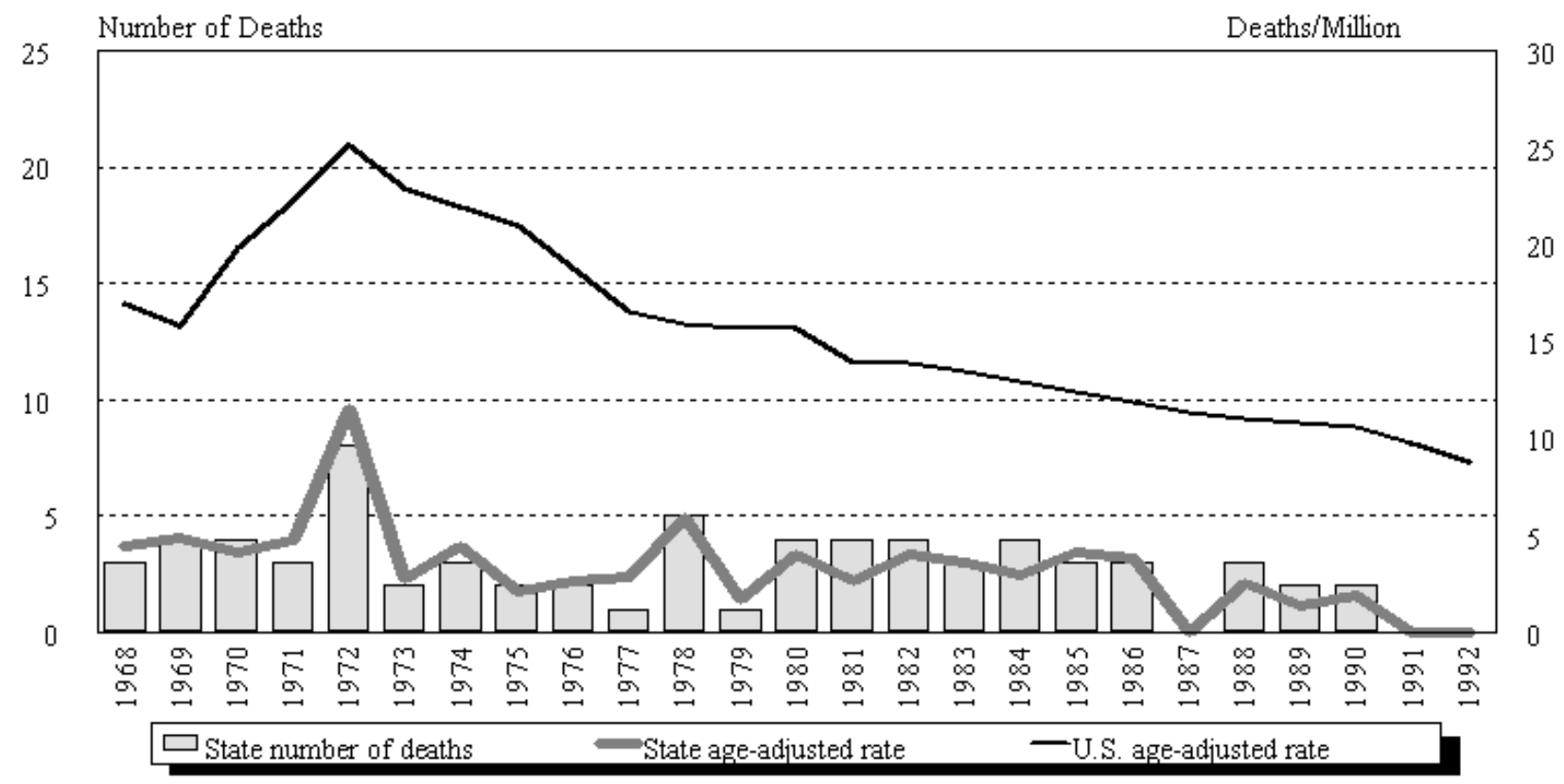

NOTE: $\quad$ See Appendix A for source description and Appendix B for methods and ICD-8 and ICD-9 codes.

SOURCE: $\quad$ National Center for Health Statistics multiple cause of death data. Population estimates from U.S. Bureau of the Census. 


\section{South Dakota}

Table SD-1. Number of deaths and age-adjusted mortality rate (per 1,000,000 population) by condition, South Dakota residents age 15 and over, 1968-1992

\begin{tabular}{|c|c|c|c|c|c|c|c|c|c|c|c|c|}
\hline \multirow[b]{2}{*}{ Year } & \multicolumn{2}{|c|}{ Asbestosis } & \multicolumn{2}{|c|}{$\begin{array}{l}\text { Coal workers' } \\
\text { pneumoconiosis }\end{array}$} & \multicolumn{2}{|c|}{ Silicosis } & \multicolumn{2}{|c|}{ Byssinosis* } & \multicolumn{2}{|c|}{$\begin{array}{c}\text { Unspecified/Other } \\
\text { pneumoconioses }\end{array}$} & \multicolumn{2}{|c|}{ All pneumoconioses } \\
\hline & Number & Rate & Number & Rate & Number & Rate & Number & Rate & Number & Rate & Number & Rate \\
\hline 1968 & - & - & - & - & 3 & 4.48 & & & - & - & 3 & 4.48 \\
\hline 1969 & - & - & - & - & 3 & 3.44 & & & 1 & 1.38 & 4 & 4.82 \\
\hline 1970 & - & - & - & - & 3 & 2.81 & & & 1 & 1.38 & 4 & 4.18 \\
\hline 1971 & - & - & 1 & 1.72 & 1 & 0.84 & & & 1 & 2.20 & 3 & 4.77 \\
\hline 1972 & - & - & - & - & 6 & 7.14 & & & 2 & 4.39 & 8 & 11.53 \\
\hline 1973 & - & - & - & - & 1 & 1.36 & & & 1 & 1.36 & 2 & 2.73 \\
\hline 1974 & - & - & - & - & 3 & 4.41 & & & - & - & 3 & 4.41 \\
\hline 1975 & - & - & - & - & 1 & 1.35 & & & 1 & 0.80 & 2 & 2.15 \\
\hline 1976 & - & - & - & - & 1 & 1.33 & & & 1 & 1.33 & 2 & 2.66 \\
\hline 1977 & - & - & - & - & - & - & & & 1 & 2.90 & 1 & 2.90 \\
\hline 1978 & 1 & 1.67 & 2 & 1.68 & 1 & 1.30 & & & 1 & 1.30 & 5 & 5.95 \\
\hline 1979 & - & - & - & - & - & - & - & - & 1 & 1.67 & 1 & 1.67 \\
\hline 1980 & - & - & 1 & 1.66 & 2 & 1.63 & - & - & 1 & 0.77 & 4 & 4.05 \\
\hline 1981 & - & - & 2 & 1.10 & 2 & 1.52 & - & - & - & - & 4 & 2.62 \\
\hline 1982 & - & - & 1 & 0.75 & 3 & 3.26 & - & - & - & - & 4 & 4.01 \\
\hline 1983 & 1 & 1.24 & 1 & 1.65 & 1 & 0.74 & - & - & - & - & 3 & 3.63 \\
\hline 1984 & - & - & 1 & 0.31 & 1 & 0.72 & - & - & 2 & 1.95 & 4 & 2.99 \\
\hline 1985 & 1 & 1.65 & - & - & 2 & 2.44 & - & - & - & - & 3 & 4.09 \\
\hline 1986 & - & - & 1 & 1.21 & 2 & 2.65 & - & - & - & - & 3 & 3.86 \\
\hline 1987 & - & - & - & - & - & - & - & - & - & - & - & - \\
\hline 1988 & - & - & 2 & 1.37 & 1 & 1.19 & - & - & - & - & 3 & 2.56 \\
\hline 1989 & - & - & 1 & 0.68 & 1 & 0.68 & - & - & - & - & 2 & 1.35 \\
\hline 1990 & - & - & - & - & 1 & 1.19 & - & - & 1 & 0.67 & 2 & 1.85 \\
\hline 1991 & - & - & - & - & - & - & - & - & - & - & - & - \\
\hline 1992 & - & - & - & - & - & - & - & - & - & - & - & - \\
\hline
\end{tabular}

indicates no deaths listed. ${ }^{*}$ no sp ecific code for by ssinosis in ICD-8 (1968-1978)

NOTE: Rates based on small numbers may be unstable. Sum of individual conditions may exceed total because of deaths with multiple pneumoconioses. See Appendix A for source description and Appendix B for methods and ICD-8 and ICD-9 codes.

SOURCE: National Center for Health Statistics multiple cause of death data. Population estimates from U.S. Bureau of the Census. 


\section{Figure SD-2. All pneumoconioses: Distribution of deaths by sex and race, South Dakota residents age 15 and over, 1968-1992}
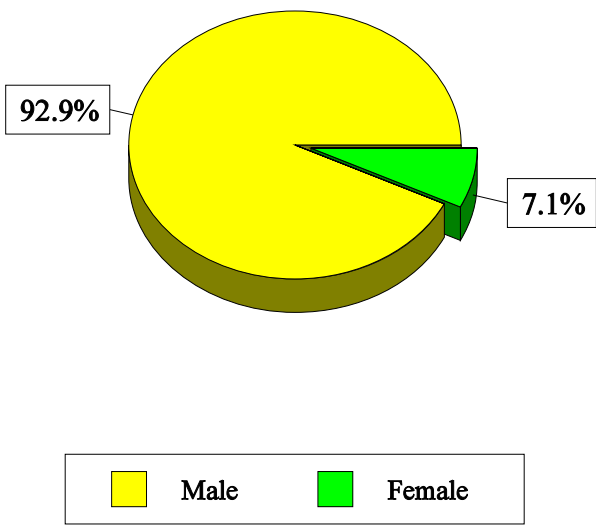

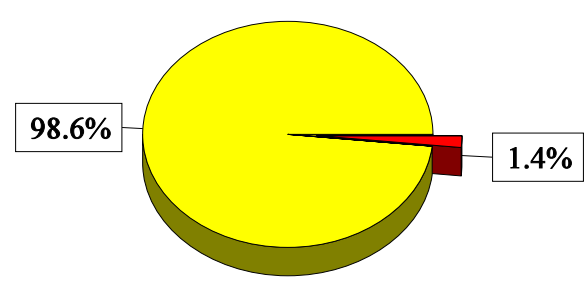

NOTE:

See Appendix A for source description and Appendix B for methods and ICD-8 and ICD-9 codes

SOURCE: National Center for Health Statistics multiple cause of death data.

Table SD-2. All pneumoconioses: Distribution of deaths by sex, race, and age, South Dakota residents age 15 and over, 1968-1992

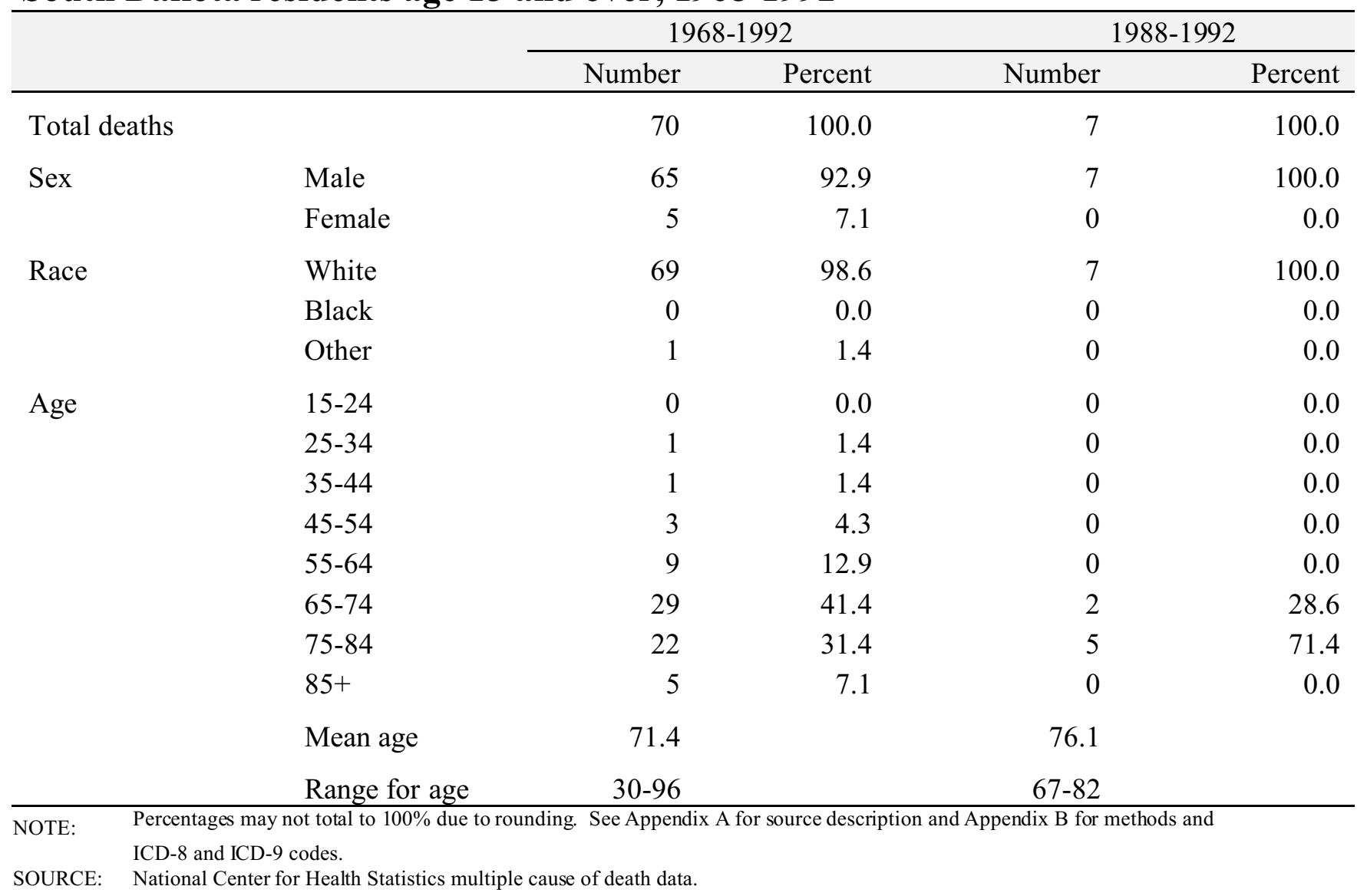


Figure SD-3. Pneumoconiosis deaths by condition, South Dakota residents age 15 and over, 1968-1992

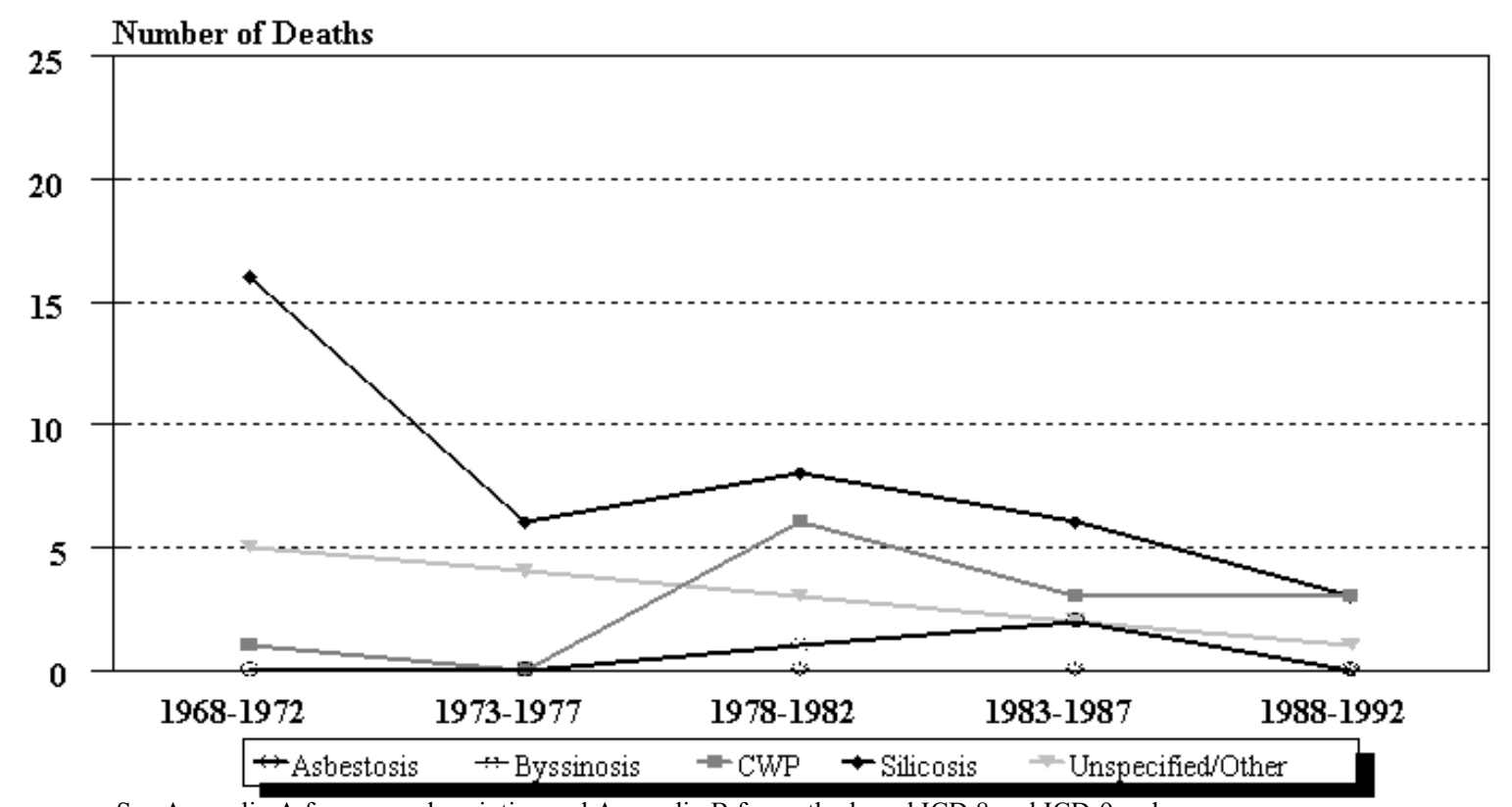

NOTE: See Appendix A for source description and Appendix B for methods and ICD-8 and ICD-9 codes.

SOURCE: National Center for Health Statistics multiple cause of death data.

Figure SD-4. South Dakota and U.S. age-adjusted mortality rates (per 1,000,000 population) by condition, residents age 15 and over, 1983-1992

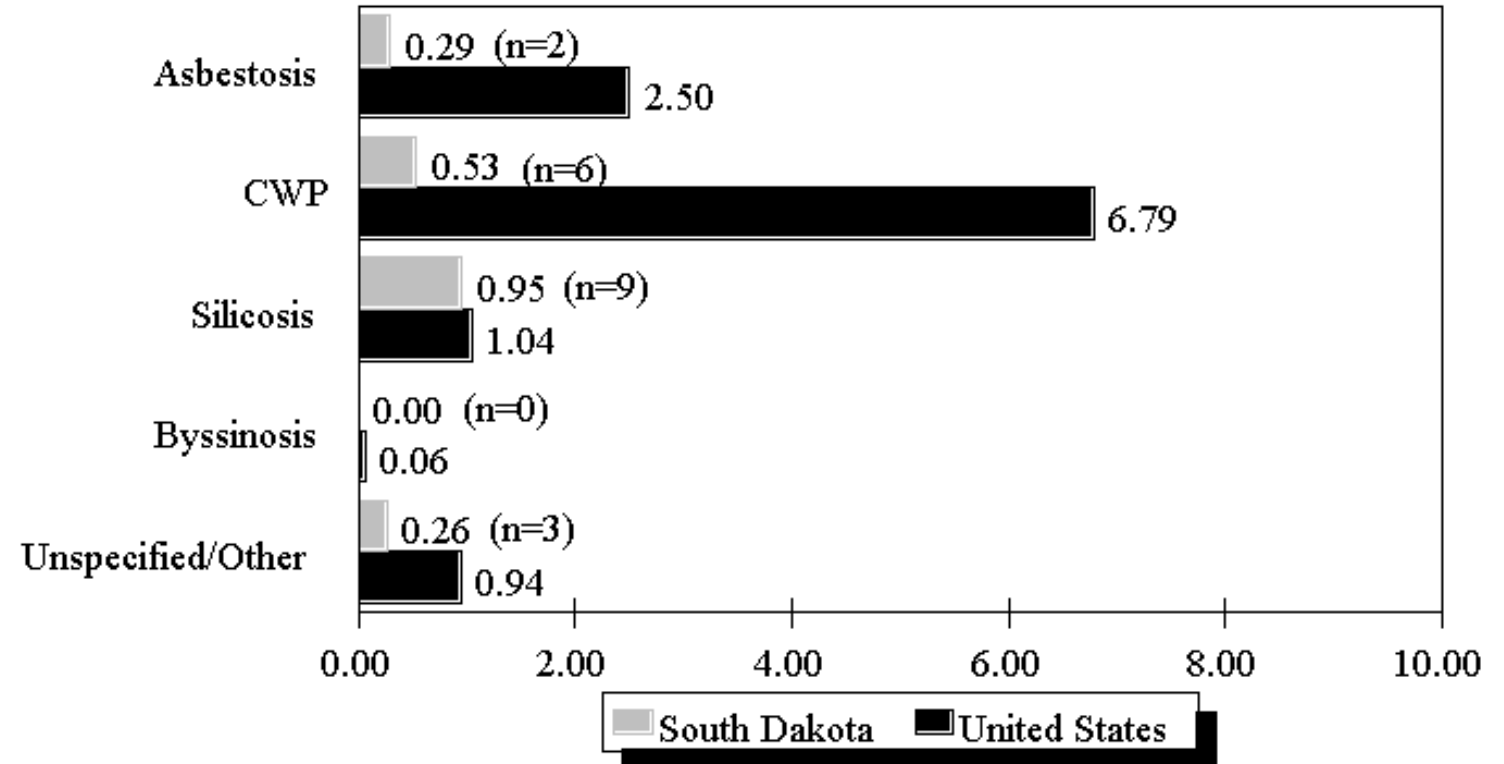

NOTE: Rates based on small numbers may be unstable. See Appendix A for source description and Appendix B for methods and ICD-8 and ICD-9 codes.

SOURCE: National Center for Health Statistics multiple cause of death data. Population estimates from U.S. Bureau of the Census. 
Table SD-3. Number of deaths and age-adjusted mortality rate (per 1,000,000 population) by condition and county, South Dakota residents age 15 and over, 1983-1992

\begin{tabular}{|c|c|c|c|c|c|c|c|c|c|c|c|c|}
\hline \multirow[b]{2}{*}{ County } & \multicolumn{2}{|c|}{ Asbestosis } & \multicolumn{2}{|c|}{$\begin{array}{c}\text { Coal workers' } \\
\text { pneumoconiosis }\end{array}$} & \multicolumn{2}{|c|}{ Silicosis } & \multicolumn{2}{|c|}{ Byssinosis } & \multicolumn{2}{|c|}{$\begin{array}{r}\text { Unspecified/Other } \\
\text { pneumoconioses }\end{array}$} & \multicolumn{2}{|c|}{$\begin{array}{r}\text { All } \\
\text { pneumoconioses } \\
\end{array}$} \\
\hline & \# & Rate & \# & Rate & \# & Rate & \# & Rate & \# & Rate & \# & Rate \\
\hline Brown & 1 & 2.88 & - & - & - & - & - & - & - & - & 1 & 2.88 \\
\hline Butte & - & - & 1 & 11.24 & - & - & - & - & - & - & 1 & 11.24 \\
\hline Custer & - & - & - & - & 1 & 14.53 & - & - & - & - & 1 & 14.53 \\
\hline Fall River & 1 & 9.32 & - & - & - & - & - & - & - & - & 1 & 9.32 \\
\hline Grant & - & - & - & - & 2 & 7.45 & - & - & 1 & 10.72 & 3 & 18.17 \\
\hline Kingsbury & - & - & - & - & 1 & 12.15 & - & - & - & - & 1 & 12.15 \\
\hline Lawrence & - & - & 1 & 5.16 & 3 & 12.45 & - & - & - & - & 4 & 17.61 \\
\hline Meade & - & - & 2 & 6.17 & 1 & 3.09 & - & - & 1 & 3.09 & 4 & 12.34 \\
\hline Miner & - & - & 1 & 6.63 & - & - & - & - & - & - & 1 & 6.63 \\
\hline Minnehaha & - & - & 1 & 0.43 & - & - & - & - & - & - & 1 & 0.43 \\
\hline Pennington & - & - & - & - & 1 & 1.64 & - & - & 1 & 0.84 & 2 & 2.48 \\
\hline
\end{tabular}

- indicates no deaths listed.

NOTE: Counties with no deaths for all listed conditions are not displayed. Rates based on small numbers may be unstable. Sum of individual conditions may exceed total because of deaths with multiple pneumoconioses. See Appendix A for source description and Appendix B for methods and ICD-8 and ICD-9 codes

SOURCE: National Center for Health Statistics multiple cause of death data. Population estimates from U.S. Bureau of the Census. 


\section{Tennessee: Selected statistics from the United States section}

All pneumoconioses mortality, 1988-1992

Total deaths

Crude mortality rate (per $1,000,000$ population)

Age-adjusted mortality rate (per $1,000,000$ population)

Total years of potential life lost to life expectancy

Years of potential life lost to life expectancy (per death)

\begin{tabular}{rr} 
Value & State rank \\
\hline 265 & 13 \\
13.69 & 11 \\
7.76 & 12 \\
3,129 & 13 \\
11.8 & 29
\end{tabular}

Percent** $^{* *}$

Deaths by type of pneumoconiosis, 1988-1992

Deaths*

distribution

Asbestosis

35

13.2

168

63.4

Silicosis

19

7.2

Byssinosis

0

0.0

Unspecified/Other pneumoconiosis

44

16.6

* Sum of column may exceed total deaths because of deaths with multiple pneumoconioses.

** Percentages may total more than $100 \%$ due to deaths with multiple pneumoconioses.

NOTE: $\quad$ See Appendix A for source description and Appendix B for methods and ICD-8 and ICD-9 codes.

SOURCE: National Center for Health Statistics multiple cause of death data. Population estimates from U.S. Bureau of the Census.

Figure TN-1. All pneumoconioses: Tennessee and U.S. age-adjusted mortality rates, residents age 15 and over, 1968-1992

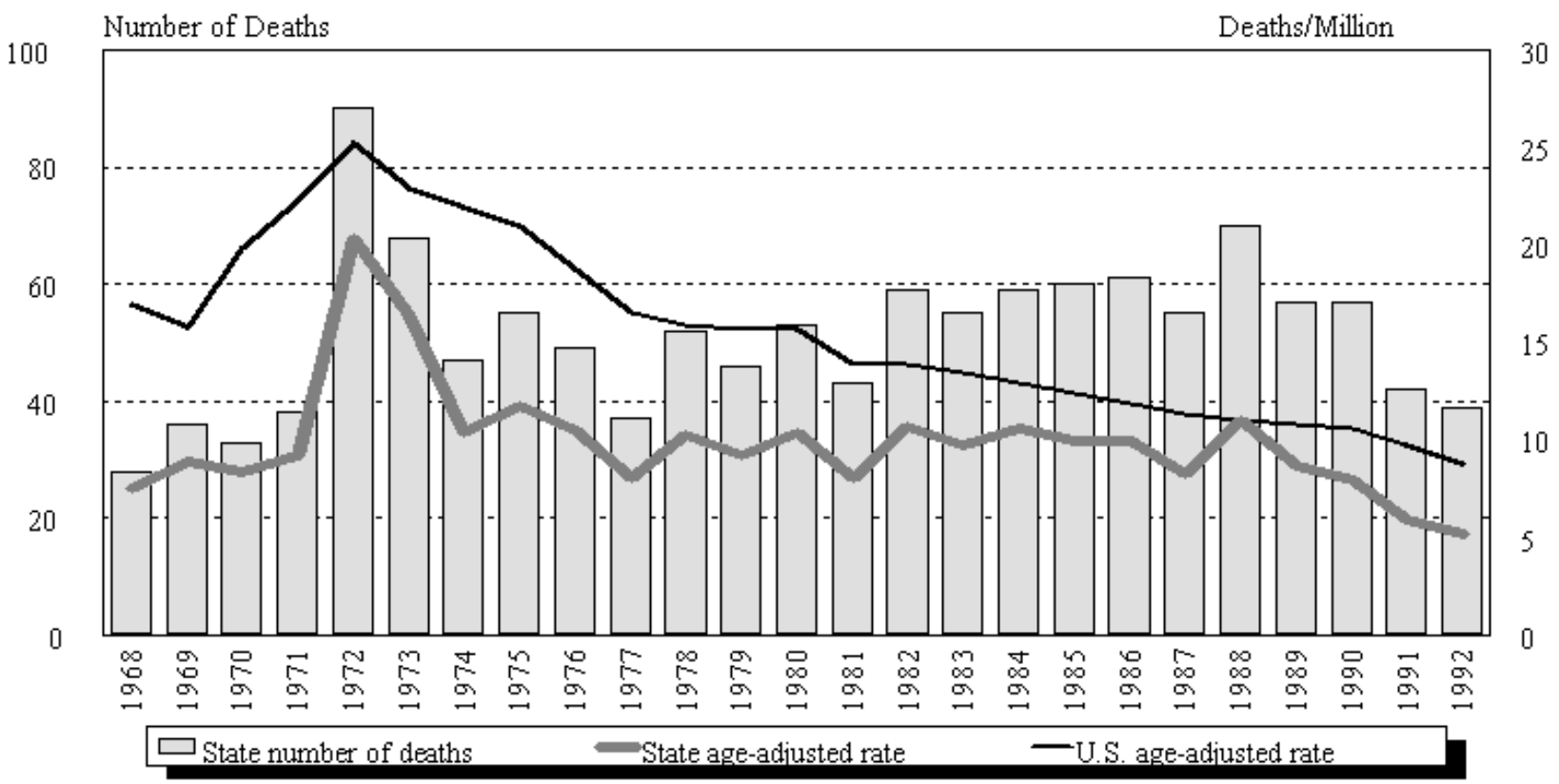

NOTE: $\quad$ See Appendix A for source description and Appendix B for methods and ICD-8 and ICD-9 codes.

SOURCE: National Center for Health Statistics multiple cause of death data. Population estimates from U.S. Bureau of the Census. 
Tennessee

\begin{tabular}{|c|c|c|c|c|c|c|c|c|c|c|c|c|}
\hline \multirow[b]{2}{*}{ Year } & \multicolumn{2}{|c|}{ Asbestosis } & \multicolumn{2}{|c|}{$\begin{array}{c}\text { Coal workers' } \\
\text { pneumoconiosis }\end{array}$} & \multicolumn{2}{|c|}{ Silicosis } & \multicolumn{2}{|c|}{ Byssinosis* } & \multicolumn{2}{|c|}{$\begin{array}{c}\text { Unspecified/Other } \\
\text { pneumoconioses }\end{array}$} & \multicolumn{2}{|c|}{ All pneumoconioses } \\
\hline & Number & Rate & Number & Rate & Number & Rate & Number & Rate & Number & Rate & Number & Rate \\
\hline 1968 & 1 & 0.36 & - & - & 18 & 4.79 & & & 9 & 2.43 & 28 & 7.57 \\
\hline 1969 & - & - & 4 & 0.87 & 22 & 5.44 & & & 11 & 2.69 & 36 & 8.86 \\
\hline 1970 & - & - & 6 & 1.50 & 9 & 2.43 & & & 18 & 4.43 & 33 & 8.37 \\
\hline 1971 & - & - & 8 & 1.90 & 9 & 2.31 & & & 21 & 5.03 & 38 & 9.24 \\
\hline 1972 & 4 & 1.00 & 20 & 4.23 & 16 & 3.77 & & & 50 & 11.33 & 90 & 20.32 \\
\hline 1973 & - & - & 17 & 4.27 & 10 & 2.78 & & & 42 & 9.56 & 68 & 16.33 \\
\hline 1974 & - & - & 17 & 3.70 & 4 & 0.99 & & & 26 & 5.70 & 47 & 10.39 \\
\hline 1975 & 1 & 0.23 & 19 & 4.01 & 13 & 3.07 & & & 24 & 4.94 & 55 & 11.80 \\
\hline 1976 & - & & 13 & 3.11 & 10 & 2.42 & & & 28 & 5.44 & 49 & 10.48 \\
\hline 1977 & 5 & 1.36 & 14 & 2.80 & 6 & 1.45 & & & 13 & 2.57 & 37 & 8.02 \\
\hline 1978 & 1 & 0.16 & 22 & 4.28 & 7 & 1.46 & & & 25 & 5.01 & 52 & 10.24 \\
\hline 1979 & 5 & 1.35 & 16 & 2.95 & 9 & 1.93 & - & - & 17 & 3.29 & 46 & 9.27 \\
\hline 1980 & 3 & 0.60 & 25 & 4.74 & 8 & 1.53 & - & - & 17 & 3.51 & 53 & 10.38 \\
\hline 1981 & - & - & 35 & 6.46 & 6 & 1.27 & - & - & 2 & 0.29 & 43 & 8.02 \\
\hline 1982 & 10 & 1.84 & 33 & 5.80 & 4 & 0.78 & - & - & 12 & 2.32 & 59 & 10.74 \\
\hline 1983 & 5 & 1.37 & 37 & 5.77 & 11 & 2.30 & - & - & 4 & 0.77 & 55 & 9.77 \\
\hline 1984 & 5 & 1.21 & 41 & 7.08 & 9 & 1.83 & - & - & 5 & 0.73 & 59 & 10.62 \\
\hline 1985 & 8 & 1.29 & 40 & 6.62 & 5 & 1.04 & - & - & 7 & 1.07 & 60 & 10.02 \\
\hline 1986 & 10 & 1.89 & 39 & 6.10 & 5 & 0.87 & 1 & 0.13 & 6 & 1.01 & 61 & 9.99 \\
\hline 1987 & 4 & 0.79 & 39 & 5.72 & 6 & 0.75 & - & - & 6 & 0.98 & 55 & 8.24 \\
\hline 1988 & 12 & 2.08 & 45 & 6.96 & 5 & 0.79 & - & - & 9 & 1.33 & 70 & 11.06 \\
\hline 1989 & 5 & 0.91 & 33 & 4.65 & 6 & 1.23 & - & - & 13 & 1.94 & 57 & 8.73 \\
\hline 1990 & 6 & 1.02 & 37 & 4.82 & 3 & 0.61 & - & - & 11 & 1.52 & 57 & 7.97 \\
\hline 1991 & 8 & 1.50 & 23 & 2.77 & 3 & 0.49 & - & - & 8 & 1.20 & 42 & 5.96 \\
\hline 1992 & 4 & 0.78 & 30 & 3.65 & 2 & 0.36 & - & - & 3 & 0.46 & 39 & 5.25 \\
\hline
\end{tabular}

indicates no deaths listed. $\quad *$ no sp ecific code for by ssinosis in ICD-8 (1968-1978).

NOTE: Rates based on small numbers may be unstable. Sum of individual conditions may exceed total because of deaths with multiple pneumoconioses. See Appendix A for source description and Appendix B for methods and ICD-8 and ICD-9 codes.

SOURCE: National Center for Health Statistics multiple cause of death data. Population estimates from U.S. Bureau of the Census. 


\section{Figure TN-2. All pneumoconioses: Distribution of deaths by sex and race,} Tennessee residents age 15 and over, 1968-1992
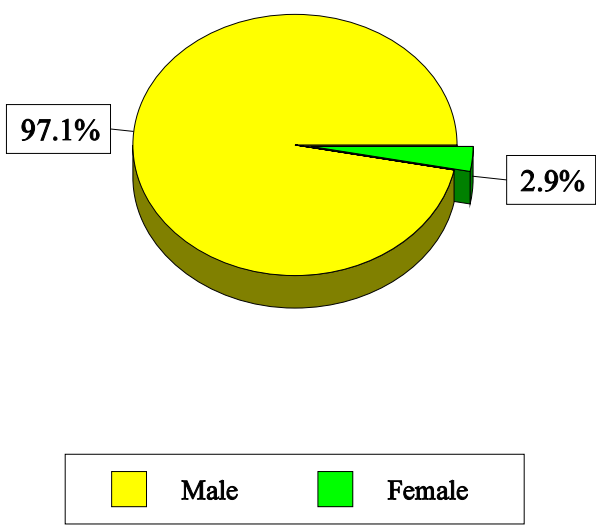
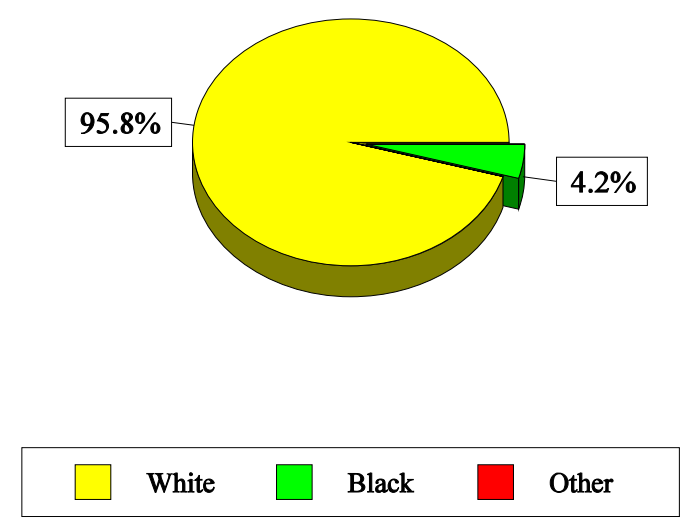

NOTE: $\quad$ See Appendix A for source description and Appendix B for methods and ICD-8 and ICD-9 codes.

SOURCE: National Center for Health Statistics multiple cause of death data.

Table TN-2. All pneumoconioses: Distribution of deaths by sex, race, and age, Tennessee residents age 15 and over, 1968-1992

\begin{tabular}{llrrrr}
\hline & & \multicolumn{2}{c}{$1968-1992$} & \multicolumn{2}{c}{$1988-1992$} \\
\cline { 3 - 6 } Total deaths & & Number & Percent & Number & Percent \\
Sex & Male & 1,289 & 100.0 & 265 & 100.0 \\
\multirow{3}{*}{ Race } & Female & 1,252 & 97.1 & 253 & 95.5 \\
& White & 37 & 2.9 & 12 & 4.5 \\
& Black & 1,235 & 95.8 & 252 & 95.1 \\
\multirow{4}{*}{ Age } & Other & 54 & 4.2 & 13 & 4.9 \\
& $15-24$ & 0 & 0.0 & 0 & 0.0 \\
& $25-34$ & 1 & 0.1 & 1 & 0.4 \\
& $35-44$ & 2 & 0.2 & 0 & 0.0 \\
& $45-54$ & 12 & 0.9 & 2 & 0.8 \\
& $55-64$ & 41 & 3.2 & 5 & 1.9 \\
& $65-74$ & 212 & 16.4 & 27 & 10.2 \\
& $75-84$ & 472 & 36.6 & 85 & 32.1 \\
& $85+$ & 428 & 33.2 & 105 & 39.6 \\
& Mean age & 121 & 9.4 & 40 & 15.1 \\
& Range for age & $21-99$ & & 74.9 & \\
\hline NOTE: & Percentages may not total to 100\% due to rounding. See Appendix A for source description and Appendix B for methods and \\
SOURCE: & ICD-8 and ICD-9 codes. & National Center for Health Statistics multiple cause of death data. & & &
\end{tabular}




\section{Figure TN-3. Pneumoconiosis deaths by condition, Tennessee residents age 15 and over, 1968-1992}

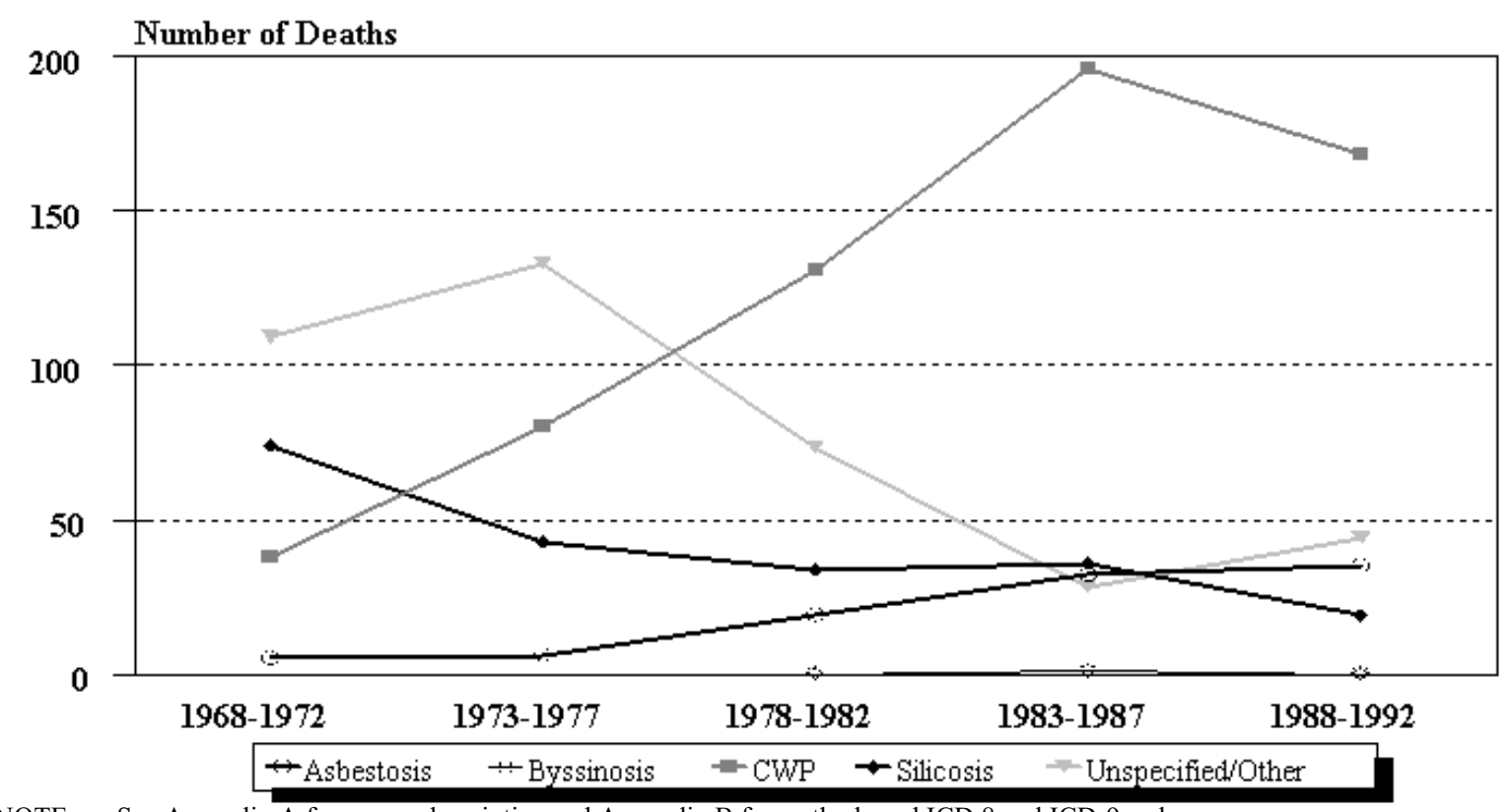

NOTE: See Appendix A for source description and Appendix B for methods and ICD-8 and ICD-9 codes.

SOURCE: National Center for Health Statistics multiple cause of death data.

Figure TN-4. Tennessee and U.S. age-adjusted mortality rates (per 1,000,000 population) by condition, residents age 15 and over, 1983-1992

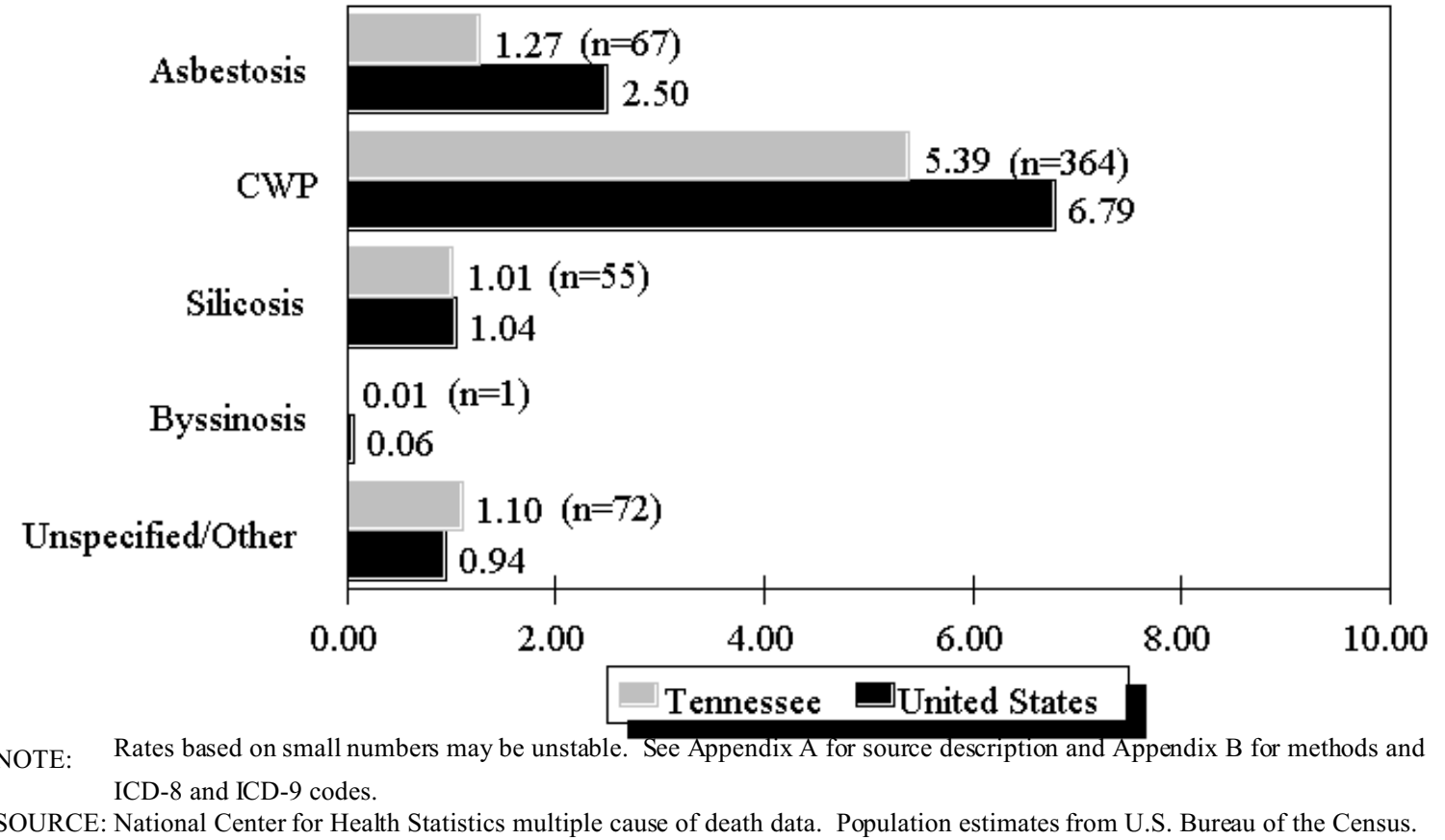


Table TN-3 (page 1 of 2). Number of deaths and age-adjusted mortality rate (per $1,000,000$ population) by condition and county, Tennessee residents age 15 and over, 1983-1992

\begin{tabular}{|c|c|c|c|c|c|c|c|c|c|c|c|c|}
\hline \multirow[b]{2}{*}{ County } & \multicolumn{2}{|c|}{ Asbestosis } & \multicolumn{2}{|c|}{$\begin{array}{c}\text { Coal workers' } \\
\text { pneumoconiosis }\end{array}$} & \multicolumn{2}{|c|}{ Silicosis } & \multicolumn{2}{|c|}{ Byssinosis } & \multicolumn{2}{|c|}{$\begin{array}{r}\text { Unspecified/Other } \\
\text { pneumoconioses }\end{array}$} & \multicolumn{2}{|c|}{$\begin{array}{r}\text { All } \\
\text { pneumoconioses }\end{array}$} \\
\hline & $\#$ & Rate & $\#$ & Rate & $\#$ & Rate & \# & Rate & $\#$ & Rate & $\#$ & Rate \\
\hline Anderson & 3 & 3.85 & 43 & 42.69 & - & - & - & - & 9 & 8.25 & 54 & 53.51 \\
\hline Bedford & 1 & 3.09 & - & - & - & - & - & - & 1 & 3.09 & 2 & 6.19 \\
\hline Benton & 1 & 2.47 & 1 & 2.47 & 1 & 2.47 & - & - & - & - & 3 & 7.42 \\
\hline Bledsoe & - & - & 6 & 51.21 & - & - & - & - & - & - & 6 & 51.21 \\
\hline Blount & 2 & 1.69 & 3 & 2.81 & - & - & 1 & 0.57 & 1 & 1.12 & 7 & 6.20 \\
\hline Bradley & 3 & 4.67 & 1 & 1.56 & 1 & 1.56 & - & - & - & - & 5 & 7.78 \\
\hline Campbell & - & - & 72 & 135.24 & 3 & 6.60 & - & - & 7 & 11.77 & 82 & 153.61 \\
\hline Cannon & - & - & - & - & 1 & 8.61 & - & - & - & - & 1 & 8.61 \\
\hline Carroll & 1 & 1.27 & 1 & 1.27 & - & - & - & - & - & - & 2 & 2.53 \\
\hline Carter & - & - & 3 & 3.51 & 1 & 0.87 & - & - & - & - & 4 & 4.38 \\
\hline Chester & 1 & 7.63 & - & - & - & - & - & - & - & - & 1 & 7.63 \\
\hline Claiborne & 1 & 2.08 & 26 & 84.27 & 3 & 9.89 & - & - & 5 & 15.87 & 33 & 104.30 \\
\hline Cocke & - & - & 1 & 1.80 & - & - & - & - & - & - & 1 & 1.80 \\
\hline Cumberland & - & - & 6 & 9.89 & - & - & - & - & 2 & 2.57 & 8 & 12.46 \\
\hline Davidson & 5 & 0.89 & - & - & 1 & 0.22 & - & - & 2 & 0.33 & 8 & 1.45 \\
\hline Decatur & 1 & 7.44 & - & - & - & - & - & - & - & - & 1 & 7.44 \\
\hline Dickson & 2 & 4.66 & - & - & - & - & - & - & - & - & 2 & 4.66 \\
\hline Fayette & - & - & - & - & - & - & - & - & 1 & 1.88 & 1 & 1.88 \\
\hline Fentress & - & - & 6 & 29.63 & - & - & - & - & 2 & 13.38 & 8 & 43.01 \\
\hline Franklin & - & - & 1 & 1.39 & - & - & - & - & - & - & 1 & 1.39 \\
\hline Grainger & - & - & 5 & 23.56 & - & - & - & - & - & - & 5 & 23.56 \\
\hline Greene & 1 & 0.88 & 5 & 5.16 & 3 & 5.62 & - & - & 1 & 2.33 & 10 & 13.99 \\
\hline Grundy & - & - & 17 & 94.66 & 1 & 7.53 & - & - & 1 & 10.56 & 19 & 112.75 \\
\hline Hamblen & 2 & 3.95 & 9 & 14.13 & - & - & - & - & - & - & 11 & 18.08 \\
\hline Hamilton & 5 & 1.54 & 5 & 1.41 & 4 & 1.23 & - & - & 5 & 1.41 & 19 & 5.59 \\
\hline Hancock & - & - & 2 & 19.33 & - & - & - & - & - & - & 2 & 19.33 \\
\hline Hawkins & 1 & 2.24 & 7 & 15.34 & - & - & - & - & 4 & 5.80 & 12 & 23.38 \\
\hline Henry & 1 & 2.68 & - & - & - & - & - & - & 1 & 1.19 & 2 & 3.87 \\
\hline Jackson & - & - & - & - & 1 & 8.96 & - & - & - & - & 1 & 8.96 \\
\hline Jefferson & - & - & 2 & 4.46 & 1 & 2.88 & - & - & - & - & 3 & 7.34 \\
\hline Johnson & 1 & 2.84 & 4 & 11.35 & - & - & - & - & 1 & 2.84 & 6 & 17.02 \\
\hline Knox & 6 & 1.74 & 12 & 2.79 & 9 & 1.90 & - & - & 1 & 0.16 & 27 & 6.42 \\
\hline Loudon & - & - & 1 & 2.89 & 2 & 4.44 & - & - & 1 & 1.55 & 4 & 8.87 \\
\hline
\end{tabular}

See footnotes at end of table. 
Table TN-3 (page 2 of 2). Number of deaths and age-adjusted mortality rate (per $1,000,000$ population) by condition and county, Tennessee residents age 15 and over, 1983-1992

\begin{tabular}{|c|c|c|c|c|c|c|c|c|c|c|c|c|}
\hline \multirow[b]{2}{*}{ County } & \multicolumn{2}{|c|}{ Asbestosis } & \multicolumn{2}{|c|}{$\begin{array}{c}\text { Coal workers' } \\
\text { pneumoconiosis }\end{array}$} & \multicolumn{2}{|c|}{ Silicosis } & \multicolumn{2}{|c|}{ Byssinosis } & \multicolumn{2}{|c|}{$\begin{array}{r}\text { Unspecified/Other } \\
\text { pneumoconioses }\end{array}$} & \multicolumn{2}{|c|}{$\begin{array}{r}\text { All } \\
\text { pneumoconioses }\end{array}$} \\
\hline & $\#$ & Rate & \# & Rate & $\#$ & Rate & $\#$ & Rate & \# & Rate & $\#$ & Rate \\
\hline McMinn & 1 & 3.24 & - & - & - & - & - & - & - & - & 1 & 3.24 \\
\hline McNairy & 1 & 3.94 & 1 & 1.71 & - & - & - & - & - & - & 2 & 5.65 \\
\hline Marion & - & - & 9 & 29.83 & - & - & - & - & 2 & 6.41 & 11 & 36.24 \\
\hline Maury & 4 & 7.20 & 1 & 1.86 & 2 & 2.75 & - & - & - & - & 7 & 11.81 \\
\hline Meigs & - & - & 1 & 8.02 & - & - & - & - & 1 & 13.11 & 2 & 21.13 \\
\hline Monroe & - & - & - & - & 1 & 3.25 & - & - & - & - & 1 & 3.25 \\
\hline Montgomery & 1 & 1.54 & 6 & 6.72 & 2 & 1.82 & - & - & - & - & 9 & 10.08 \\
\hline Moore & - & - & - & - & 1 & 19.94 & - & - & - & - & 1 & 19.94 \\
\hline Morgan & - & - & 17 & 79.52 & 1 & 6.38 & - & - & - & - & 18 & 85.90 \\
\hline Overton & - & - & 3 & 15.29 & - & - & - & - & - & - & 3 & 15.29 \\
\hline Polk & - & - & 2 & 7.18 & - & - & - & - & - & - & 2 & 7.18 \\
\hline Putnam & 1 & 2.09 & 5 & 7.18 & 1 & 1.00 & - & - & 2 & 4.17 & 9 & 14.45 \\
\hline Rhea & 2 & 8.22 & 4 & 9.93 & 1 & 4.11 & - & - & - & - & 7 & 22.26 \\
\hline Roane & 4 & 8.16 & 15 & 15.99 & - & - & - & - & 3 & 3.94 & 22 & 28.09 \\
\hline Roberton & 1 & 1.28 & - & - & - & - & - & - & - & - & 1 & 1.28 \\
\hline Rutherford & 2 & 2.63 & 1 & 0.72 & - & - & - & - & - & - & 3 & 3.35 \\
\hline Scott & 1 & 5.90 & 7 & 29.44 & - & - & - & - & 2 & 8.84 & 10 & 44.18 \\
\hline Sequatchic & 1 & 12.65 & 5 & 42.29 & - & - & - & - & - & - & 6 & 54.95 \\
\hline Sevier & - & - & 3 & 5.22 & 1 & 2.02 & - & - & - & - & 4 & 7.24 \\
\hline Shelby & 1 & 0.15 & 2 & 0.30 & 7 & 1.06 & - & - & 1 & 0.15 & 11 & 1.66 \\
\hline Smith & - & - & 1 & 6.38 & - & - & - & - & - & - & 1 & 6.38 \\
\hline Sullivan & 3 & 1.36 & 23 & 11.30 & 1 & 0.37 & - & - & 8 & 4.48 & 35 & 17.51 \\
\hline Sumner & 1 & 0.68 & 1 & 1.23 & - & - & - & - & - & - & 2 & 1.91 \\
\hline Unicoi & - & - & 2 & 4.74 & 3 & 9.73 & - & - & 1 & 4.99 & 6 & 19.45 \\
\hline Union & 1 & 8.75 & 4 & 26.46 & - & - & - & - & 1 & 4.48 & 6 & 39.69 \\
\hline Van Buren & - & - & 1 & 11.69 & - & - & - & - & - & - & 1 & 11.69 \\
\hline Warren & - & - & 1 & 1.38 & - & - & - & - & - & - & 1 & 1.38 \\
\hline Washington & 2 & 2.14 & 6 & 4.23 & - & - & - & - & 1 & 0.52 & 9 & 6.89 \\
\hline Wayne & - & - & 1 & 3.25 & - & - & - & - & - & - & 1 & 3.25 \\
\hline Weakley & - & - & - & - & 1 & 2.99 & - & - & 1 & 1.15 & 2 & 4.14 \\
\hline White & - & - & 1 & 2.05 & - & - & - & - & 2 & 4.10 & 3 & 6.15 \\
\hline Williamson & 1 & 1.78 & - & - & 1 & 1.06 & - & - & 1 & 1.06 & 3 & 3.91 \\
\hline Wilson & 1 & 1.90 & 2 & 2.13 & - & - & - & - & 1 & 1.06 & 4 & 5.09 \\
\hline
\end{tabular}

NOTE: Counties with no deaths for all listed conditions are not displayed. Rates based on small numbers may be unstable. Sum of individual conditions may exceed total because of deaths with multiple pneumoconioses. See Appendix A for source description and Appendix B for methods and ICD-8 and ICD-9 codes.

SOURCE: National Center for Health Statistics multiple cause of death data. Population estimates from U.S. Bureau of the Census. 


\section{Texas: Selected statistics from the United States section}

All pneumoconioses mortality, 1988-1992

Total deaths

Crude mortality rate (per $1,000,000$ population)

Age-adjusted mortality rate (per $1,000,000$ population)

Total years of potential life lost to life expectancy

Years of potential life lost to life expectancy (per death)

\begin{tabular}{rr} 
Value & State rank \\
\hline 426 & 10 \\
6.59 & 32 \\
4.81 & 28 \\
5,537 & 10 \\
13.0 & 9
\end{tabular}

Percent** $^{* *}$

Deaths by type of pneumoconiosis, 1988-1992

Deaths*

distribution

Asbestosis

335

78.6

Coal workers' pneumoconiosis

33

7.7

Silicosis

45

10.6

Byssinosis

1

0.2

Unspecified/Other pneumoconiosis

* Sum of column may exceed total deaths because of deaths with multiple pneumoconioses.

** Percentages may total more than $100 \%$ due to deaths with multiple pneumoconioses.

NOTE: $\quad$ See Appendix A for source description and Appendix B for methods and ICD-8 and ICD-9 codes.

SOURCE: National Center for Health Statistics multiple cause of death data. Population estimates from U.S. Bureau of the Census.

\section{Figure TX-1. All pneumoconioses: Texas and U.S. age-adjusted mortality rates, residents age 15 and over, 1968-1992}

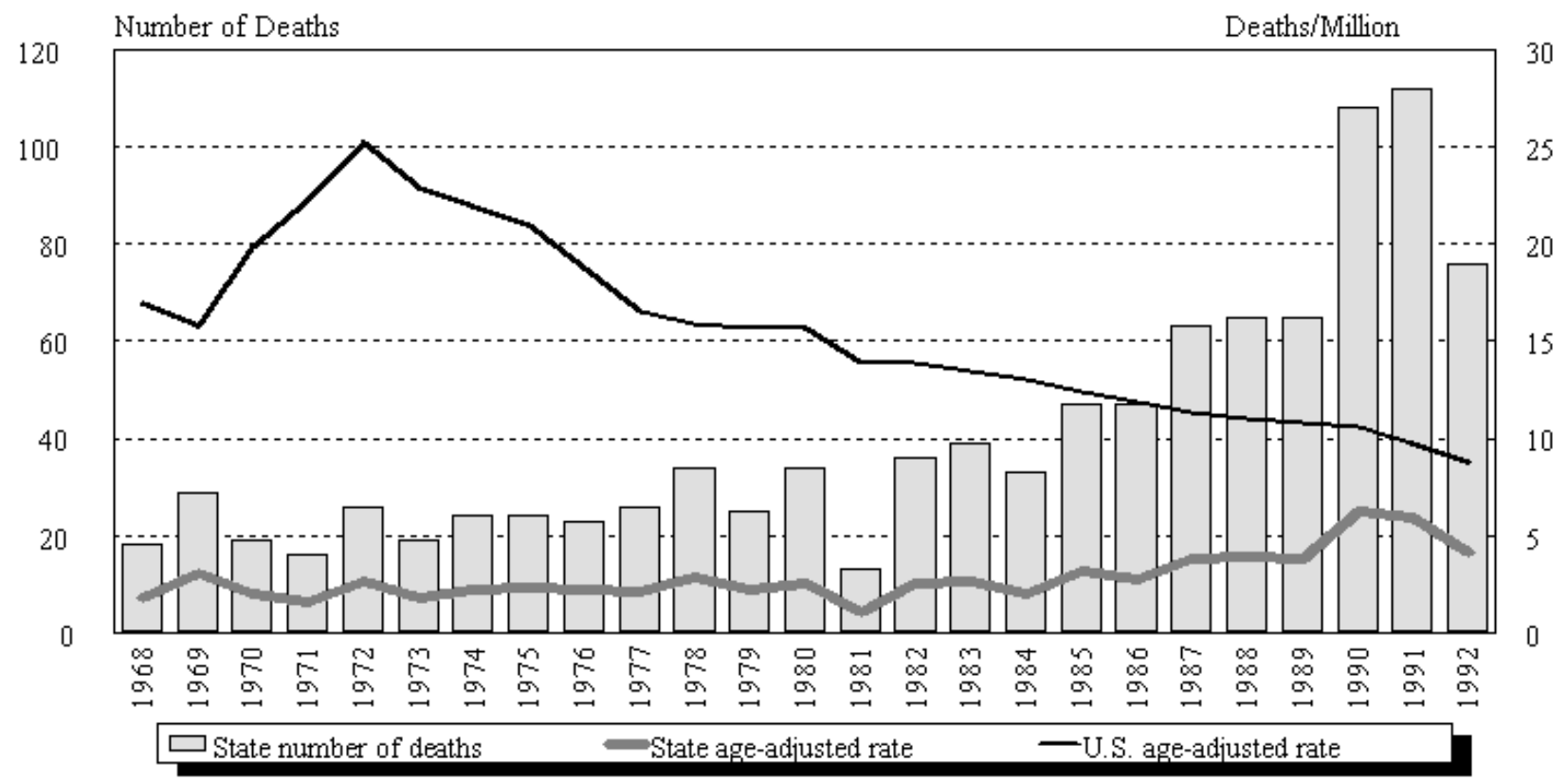

NOTE: $\quad$ See Appendix A for source description and Appendix B for methods and ICD-8 and ICD-9 codes.

SOURCE: National Center for Health Statistics multiple cause of death data. Population estimates from U.S. Bureau of the Census. 


\begin{tabular}{|c|c|c|c|c|c|c|c|c|c|c|c|c|}
\hline \multirow[b]{2}{*}{ Year } & \multicolumn{2}{|c|}{ Asbestosis } & \multicolumn{2}{|c|}{$\begin{array}{c}\text { Coal workers' } \\
\text { pneumoconiosis }\end{array}$} & \multicolumn{2}{|c|}{ Silicosis } & \multicolumn{2}{|c|}{ Byssinosis* } & \multicolumn{2}{|c|}{$\begin{array}{c}\text { Unspecified/Other } \\
\text { pneumoconioses }\end{array}$} & \multicolumn{2}{|c|}{ All pneumoconioses } \\
\hline & Number & Rate & Number & Rate & Number & Rate & Number & Rate & Number & Rate & Number & Rate \\
\hline 1968 & 2 & 0.22 & 2 & 0.20 & 8 & 0.75 & & & 6 & 0.64 & 18 & 1.81 \\
\hline 1969 & 2 & 0.21 & 3 & 0.26 & 15 & 1.58 & & & 10 & 1.04 & 29 & 3.02 \\
\hline 1970 & 2 & 0.22 & 2 & 0.23 & 7 & 0.71 & & & 9 & 0.97 & 19 & 2.01 \\
\hline 1971 & - & - & 1 & 0.05 & 9 & 0.87 & & & 6 & 0.64 & 16 & 1.56 \\
\hline 1972 & 6 & 0.62 & - & - & 12 & 1.17 & & & 10 & 1.08 & 26 & 2.68 \\
\hline 1973 & 1 & 0.11 & 4 & 0.33 & 6 & 0.60 & & & 8 & 0.79 & 19 & 1.82 \\
\hline 1974 & 4 & 0.41 & 5 & 0.39 & 7 & 0.66 & & & 8 & 0.73 & 24 & 2.18 \\
\hline 1975 & 3 & 0.31 & 3 & 0.24 & 11 & 1.13 & & & 8 & 0.71 & 24 & 2.30 \\
\hline 1976 & 6 & 0.55 & 3 & 0.21 & 10 & 1.00 & & & 4 & 0.41 & 23 & 2.17 \\
\hline 1977 & 4 & 0.36 & 6 & 0.38 & 7 & 0.66 & & & 9 & 0.76 & 26 & 2.16 \\
\hline 1978 & 4 & 0.28 & 2 & 0.14 & 13 & 1.20 & & & 16 & 1.27 & 34 & 2.83 \\
\hline 1979 & 12 & 1.10 & 6 & 0.48 & 5 & 0.47 & - & - & 2 & 0.13 & 25 & 2.18 \\
\hline 1980 & 8 & 0.67 & 4 & 0.26 & 16 & 1.23 & - & - & 6 & 0.41 & 34 & 2.57 \\
\hline 1981 & 6 & 0.47 & 5 & 0.43 & - & - & - & - & 2 & 0.15 & 13 & 1.05 \\
\hline 1982 & 12 & 0.94 & 12 & 0.73 & 12 & 0.91 & - & - & 2 & 0.06 & 36 & 2.53 \\
\hline 1983 & 19 & 1.29 & 7 & 0.40 & 8 & 0.63 & - & - & 5 & 0.28 & 39 & 2.60 \\
\hline 1984 & 12 & 0.79 & 8 & 0.45 & 11 & 0.68 & 1 & 0.08 & 1 & 0.07 & 33 & 2.06 \\
\hline 1985 & 26 & 1.73 & 11 & 0.67 & 5 & 0.37 & - & - & 5 & 0.39 & 47 & 3.16 \\
\hline 1986 & 25 & 1.42 & 7 & 0.36 & 14 & 0.87 & 1 & 0.07 & 1 & 0.07 & 47 & 2.73 \\
\hline 1987 & 48 & 2.85 & 1 & 0.04 & 10 & 0.70 & - & - & 4 & 0.18 & 63 & 3.77 \\
\hline 1988 & 46 & 2.68 & 7 & 0.37 & 12 & 0.81 & - & - & 2 & 0.15 & 65 & 3.87 \\
\hline 1989 & 49 & 2.83 & 5 & 0.22 & 4 & 0.33 & 1 & 0.04 & 6 & 0.36 & 65 & 3.79 \\
\hline 1990 & 91 & 5.29 & 5 & 0.17 & 7 & 0.52 & - & - & 6 & 0.36 & 108 & 6.28 \\
\hline 1991 & 95 & 5.04 & 7 & 0.26 & 11 & 0.64 & - & - & 1 & 0.02 & 112 & 5.88 \\
\hline 1992 & 54 & 3.01 & 9 & 0.35 & 11 & 0.62 & - & - & 3 & 0.19 & 76 & 4.08 \\
\hline
\end{tabular}

-indicates no deaths listed. $\quad *$ no specific code for by ssinosis in ICD-8 (1968-1978)

NOTE: Rates based on small numbers may be unstable. Sum of individual conditions may exceed total because of deaths with multiple pneumoconioses. See Appendix A for source description and Appendix B for methods and ICD-8 and ICD-9 codes.

SOURCE: National Center for Health Statistics multiple cause of death data. Population estimates from U.S. Bureau of the Census. 


\section{Figure TX-2. All pneumoconioses: Distribution of deaths by sex and race,} Texas residents age 15 and over, 1968-1992
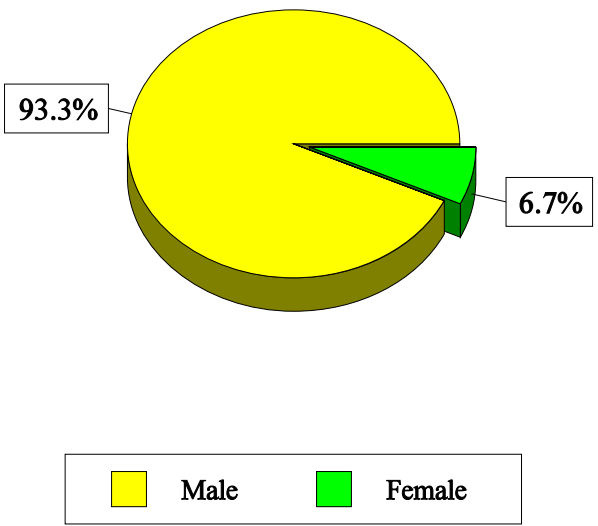
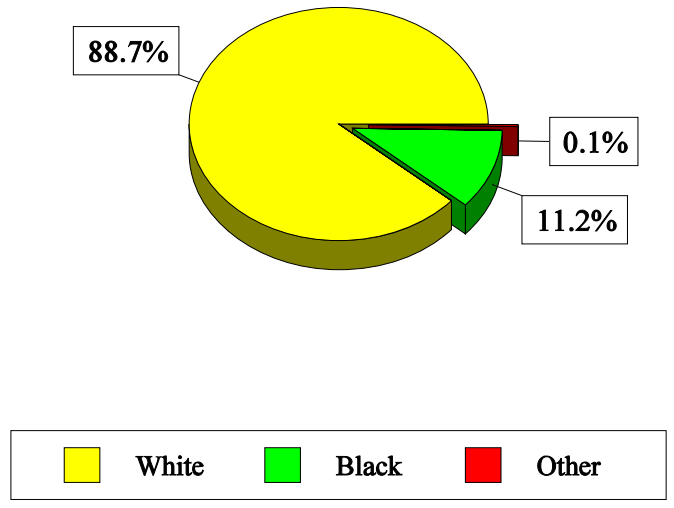

NOTE: $\quad$ See Appendix A for source description and Appendix B for methods and ICD-8 and ICD-9 codes.

SOURCE: National Center for Health Statistics multiple cause of death data.

Table TX-2. All pneumoconioses: Distribution of deaths by sex, race, and age, Texas residents age 15 and over, 1968-1992

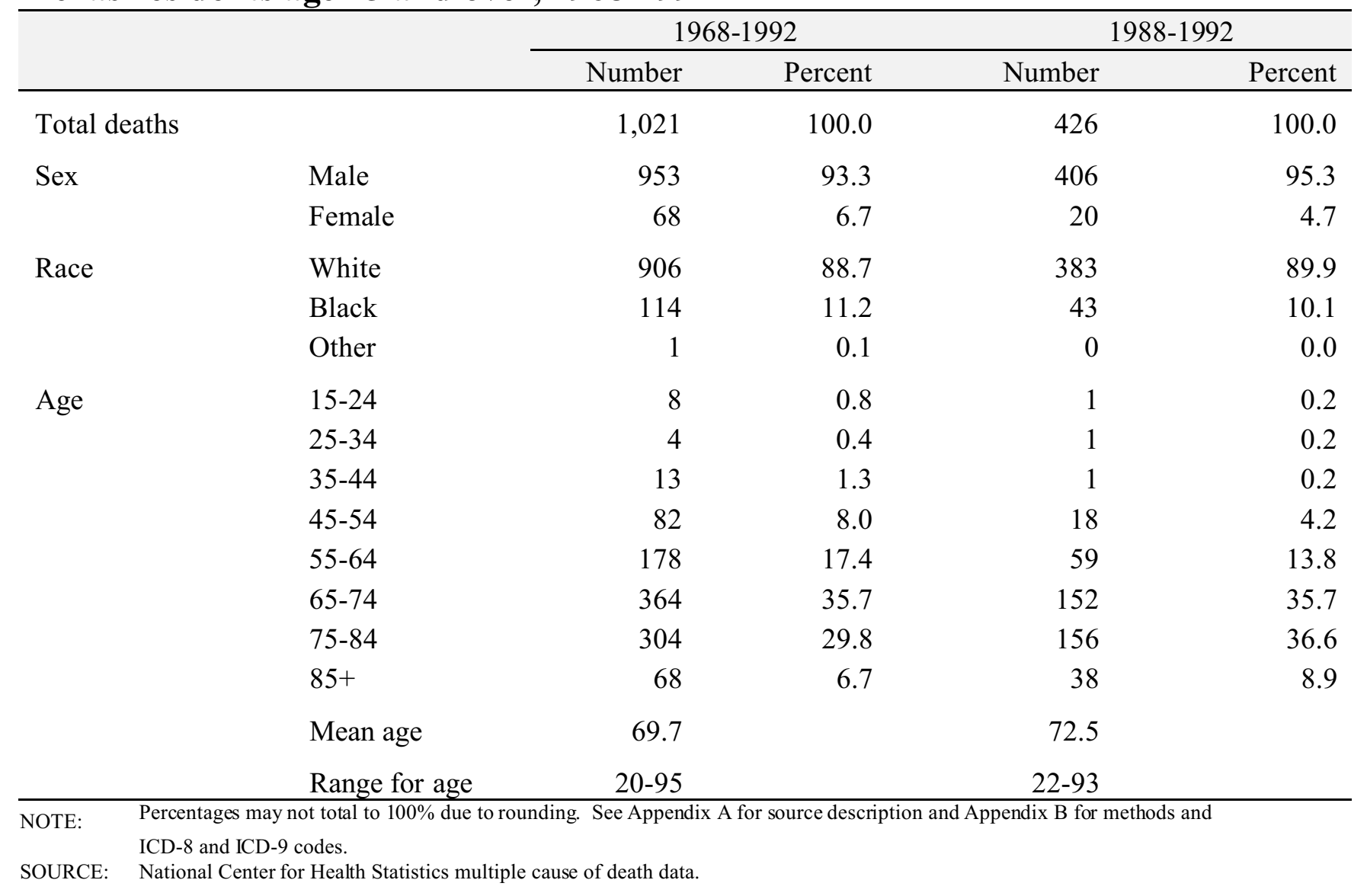


Figure TX-3. Pneumoconiosis deaths by condition, Texas residents age 15 and over, 1968-1992

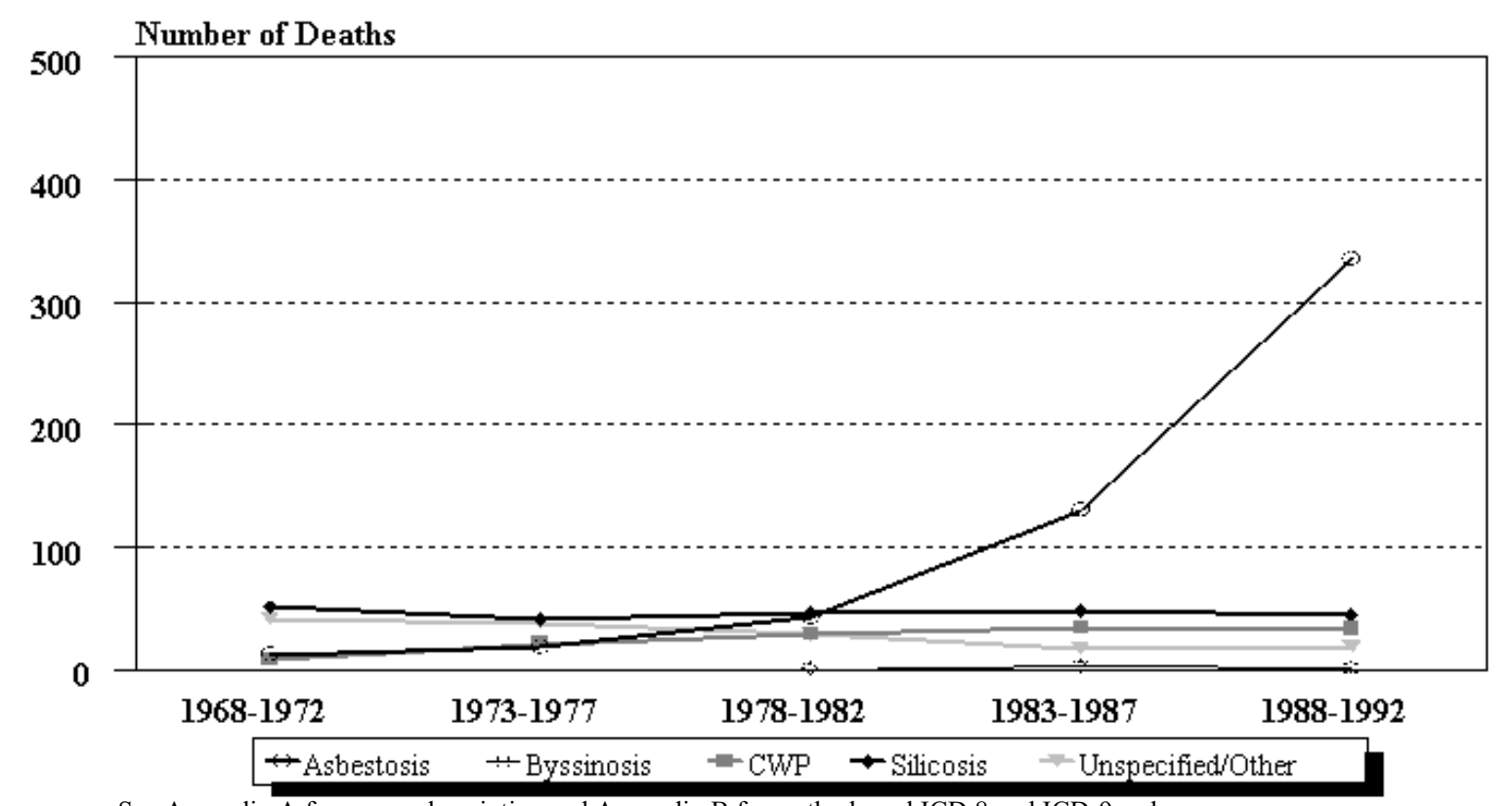

NOTE: See Appendix A for source description and Appendix B for methods and ICD-8 and ICD-9 codes.

SOURCE: National Center for Health Statistics multiple cause of death data.

Figure TX-4. Texas and U.S. age-adjusted mortality rates (per 1,000,000 population) by condition, residents age 15 and over, 1983-1992

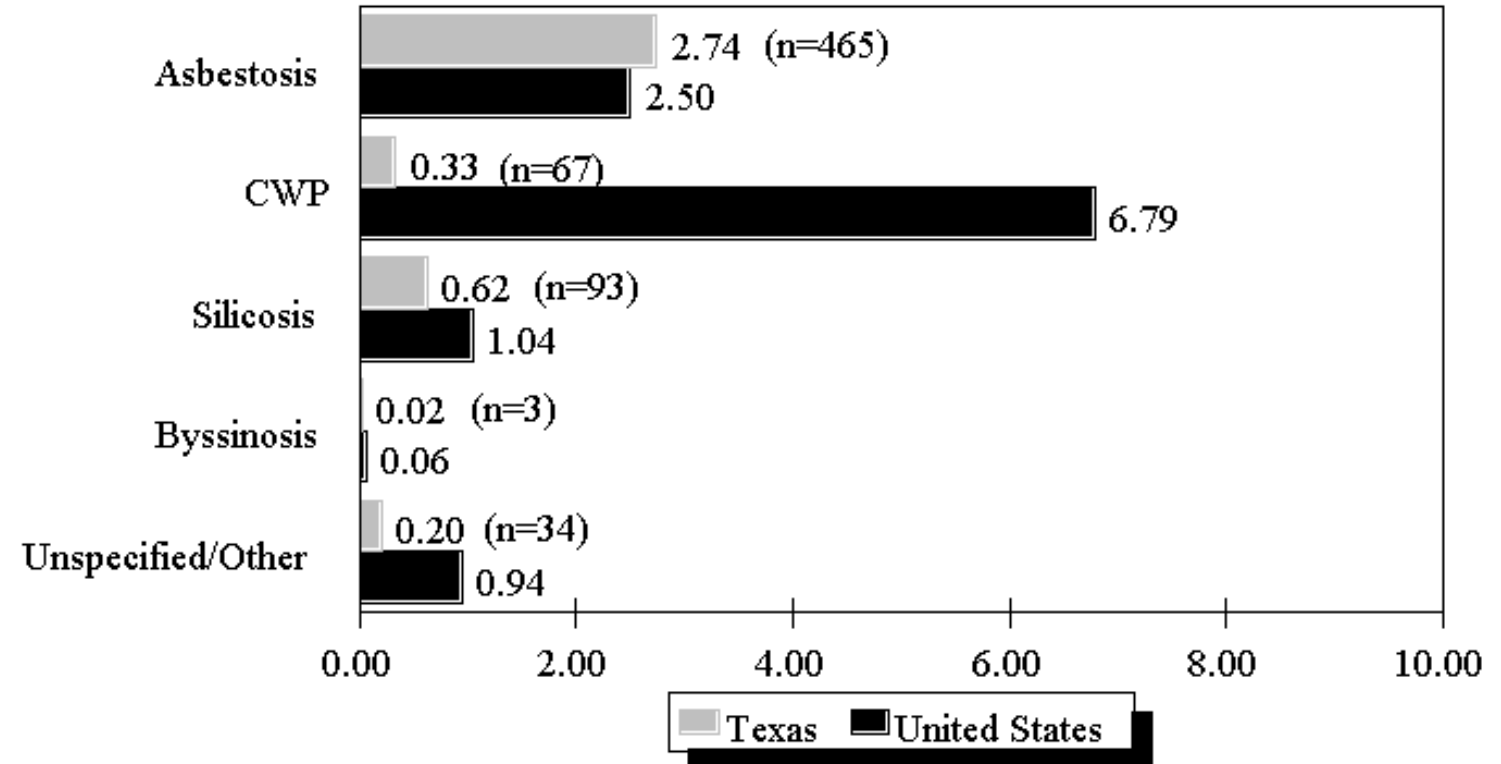

NOTE: Rates based on small numbers may be unstable. See Appendix A for source description and Appendix B for methods and ICD-8 and ICD-9 codes.

SOURCE: National Center for Health Statistics multiple cause of death data. Population estimates from U.S. Bureau of the Census. 
Table TX-3 (page 1 of 4). Number of deaths and age-adjusted mortality rate (per $1,000,000$ population) by condition and county, Texas residents age 15 and over, 1983-1992

\begin{tabular}{|c|c|c|c|c|c|c|c|c|c|c|c|c|}
\hline \multirow[b]{2}{*}{ County } & \multicolumn{2}{|c|}{ Asbestosis } & \multicolumn{2}{|c|}{$\begin{array}{c}\text { Coal workers' } \\
\text { pneumoconiosis }\end{array}$} & \multicolumn{2}{|c|}{ Silicosis } & \multicolumn{2}{|c|}{ Byssinosis } & \multicolumn{2}{|c|}{$\begin{array}{r}\text { Unspecified/Other } \\
\text { pneumoconioses }\end{array}$} & \multicolumn{2}{|c|}{$\begin{array}{r}\text { All } \\
\text { pneumoconioses }\end{array}$} \\
\hline & $\#$ & Rate & $\#$ & Rate & $\#$ & Rate & $\#$ & Rate & $\#$ & Rate & $\#$ & Rate \\
\hline Anderson & 1 & 2.40 & - & - & - & - & - & - & - & - & 1 & 2.40 \\
\hline Angelina & 5 & 6.67 & - & - & 4 & 6.15 & - & - & - & - & 9 & 12.82 \\
\hline Aransas & 1 & 3.96 & - & - & - & - & - & - & - & - & 1 & 3.96 \\
\hline Atascosa & - & - & 1 & 1.85 & - & - & - & - & - & - & 1 & 1.85 \\
\hline Austin & 1 & 4.51 & - & - & - & - & - & - & - & - & 1 & 4.51 \\
\hline Bastrop & 1 & 1.30 & - & - & 3 & 7.10 & - & - & - & - & 4 & 8.40 \\
\hline Bee & 2 & 9.19 & - & - & - & - & - & - & - & - & 2 & 9.19 \\
\hline Bell & 1 & 0.80 & - & - & - & - & - & - & - & - & 1 & 0.80 \\
\hline Bexar & 8 & 0.68 & 2 & 0.17 & 9 & 0.79 & - & - & 1 & 0.06 & 20 & 1.71 \\
\hline Bosque & - & - & - & - & 1 & 4.74 & - & - & - & - & 1 & 4.74 \\
\hline Brazoria & 16 & 10.62 & - & - & - & - & - & - & - & - & 16 & 10.62 \\
\hline Brazos & 3 & 4.95 & - & - & - & - & - & - & - & - & 3 & 4.95 \\
\hline Burleson & 2 & 8.31 & 1 & 2.46 & - & - & - & - & - & - & 3 & 10.77 \\
\hline Burnet & 1 & 1.25 & - & - & - & - & - & - & - & - & 1 & 1.25 \\
\hline Cameron & 1 & 0.49 & 1 & 0.49 & 3 & 1.02 & - & - & - & - & 5 & 2.00 \\
\hline Carson & - & - & 2 & 19.77 & - & - & - & - & - & - & 2 & 19.77 \\
\hline Chambers & 3 & 15.72 & - & - & 1 & 6.31 & - & - & - & - & 4 & 22.04 \\
\hline Cherokee & 4 & 7.38 & - & - & 2 & 6.02 & - & - & - & - & 6 & 13.40 \\
\hline Clay & 1 & 3.42 & - & - & - & - & - & - & - & - & 1 & 3.42 \\
\hline Collin & 1 & 0.51 & - & - & - & - & - & - & - & - & 1 & 0.51 \\
\hline Comal & 3 & 4.22 & - & - & - & - & - & - & 1 & 1.69 & 4 & 5.91 \\
\hline Comanche & 1 & 1.72 & - & - & - & - & - & - & - & - & 1 & 1.72 \\
\hline Cooke & 1 & 3.05 & - & - & - & - & - & - & - & - & 1 & 3.05 \\
\hline Coryell & 1 & 3.14 & - & - & - & - & - & - & - & - & 1 & 3.14 \\
\hline Dallas & 23 & 1.53 & 3 & 0.14 & 4 & 0.25 & 1 & 0.05 & 2 & 0.12 & 32 & 2.04 \\
\hline Denton & 1 & 0.51 & 2 & 1.03 & - & - & - & - & - & - & 3 & 1.54 \\
\hline Duval & 1 & 7.78 & - & - & - & - & - & - & - & - & 1 & 7.78 \\
\hline Ector & 1 & 1.00 & 1 & 1.23 & - & - & - & - & 1 & 1.00 & 3 & 3.22 \\
\hline El Paso & 3 & 0.49 & 1 & 0.16 & 3 & 0.65 & - & - & 3 & 0.65 & 10 & 1.95 \\
\hline Erath & - & - & 1 & 6.54 & 1 & 3.85 & - & - & - & - & 2 & 10.39 \\
\hline Falls & 1 & 1.48 & - & - & 1 & 1.48 & - & - & - & - & 2 & 2.96 \\
\hline Fannin & 1 & 3.06 & - & - & - & - & - & - & - & - & 1 & 3.06 \\
\hline Foard & 1 & 38.68 & - & - & - & - & - & - & - & - & 1 & 38.68 \\
\hline
\end{tabular}

See footnotes at end of table. 
Table TX-3 (page 2 of 4). Number of deaths and age-adjusted mortality rate (per $1,000,000$ population) by condition and county, Texas residents age 15 and over, 1983-1992

\begin{tabular}{|c|c|c|c|c|c|c|c|c|c|c|c|c|}
\hline \multirow[b]{2}{*}{ County } & \multicolumn{2}{|c|}{ Asbestosis } & \multicolumn{2}{|c|}{$\begin{array}{c}\text { Coal workers' } \\
\text { pneumoconiosis }\end{array}$} & \multicolumn{2}{|c|}{ Silicosis } & \multicolumn{2}{|c|}{ Byssinosis } & \multicolumn{2}{|c|}{$\begin{array}{r}\text { Unspecified/Other } \\
\text { pneumoconioses }\end{array}$} & \multicolumn{2}{|c|}{$\begin{array}{r}\text { All } \\
\text { pneumoconioses }\end{array}$} \\
\hline & $\#$ & Rate & \# & Rate & $\#$ & Rate & $\#$ & Rate & $\#$ & Rate & $\#$ & Rate \\
\hline Fort Bend & 2 & 1.69 & - & - & - & - & - & - & - & - & 2 & 1.69 \\
\hline Freestone & 1 & 5.25 & - & - & 1 & 1.75 & - & - & - & - & 2 & 7.00 \\
\hline Galveston & 23 & 9.32 & 1 & 0.51 & 4 & 2.00 & - & - & 2 & 0.84 & 29 & 12.03 \\
\hline Gonzales & - & - & 1 & 1.82 & - & - & - & - & 1 & 1.82 & 2 & 3.64 \\
\hline Grayson & 1 & 0.39 & 1 & 0.96 & - & - & - & - & 1 & 0.39 & 3 & 1.73 \\
\hline Gregg & 7 & 5.92 & - & - & - & - & - & - & - & - & 7 & 5.92 \\
\hline Grimes & - & - & - & - & 1 & 5.33 & - & - & - & - & 1 & 5.33 \\
\hline Guadalupe & 2 & 2.68 & - & - & 1 & 1.75 & - & - & - & - & 3 & 4.42 \\
\hline Hale & - & - & 1 & 1.41 & 1 & 3.10 & - & - & - & - & 2 & 4.51 \\
\hline Hall & - & - & - & - & 1 & 16.20 & - & - & - & - & 1 & 16.20 \\
\hline Hardin & 5 & 8.15 & - & - & - & - & - & - & - & - & 5 & 8.15 \\
\hline Harris & 74 & 3.52 & 12 & 0.52 & 21 & 1.08 & - & - & 2 & 0.08 & 107 & 5.09 \\
\hline Harrison & 2 & 3.38 & 1 & 1.79 & 1 & 1.79 & - & - & - & - & 4 & 6.96 \\
\hline Hemphill & - & - & - & - & - & - & - & - & 1 & 10.93 & 1 & 10.93 \\
\hline Henderson & 5 & 4.55 & - & - & 1 & 1.22 & - & - & - & - & 6 & 5.78 \\
\hline Hidalgo & 1 & 0.19 & - & - & 1 & 0.36 & - & - & - & - & 2 & 0.56 \\
\hline Hill & 1 & 1.02 & - & - & 1 & 1.02 & - & - & - & - & 2 & 2.05 \\
\hline Hockley & - & - & 1 & 5.03 & - & - & - & - & - & - & 1 & 5.03 \\
\hline Hood & 1 & 2.85 & - & - & - & - & - & - & - & - & 1 & 2.85 \\
\hline Houston & 1 & 1.39 & - & - & 1 & 3.97 & - & - & - & - & 2 & 5.36 \\
\hline Howard & - & - & - & - & - & - & - & - & 1 & 1.41 & 1 & 1.41 \\
\hline Hunt & - & - & 1 & 0.68 & - & - & - & - & - & - & 1 & 0.68 \\
\hline Hutchinson & 3 & 8.36 & - & - & - & - & - & - & - & - & 3 & 8.36 \\
\hline Jasper & 11 & 28.75 & 1 & 1.30 & - & - & - & - & - & - & 12 & 30.05 \\
\hline Jefferson & 73 & 22.14 & 1 & 0.20 & 9 & 3.58 & - & - & 1 & 0.59 & 83 & 26.12 \\
\hline Jim Wells & - & - & 1 & 1.48 & - & - & - & - & - & - & 1 & 1.48 \\
\hline Johnson & 3 & 3.38 & - & - & - & - & - & - & 1 & 1.45 & 4 & 4.83 \\
\hline Kerr & 4 & 2.83 & - & - & - & - & - & - & - & - & 4 & 2.83 \\
\hline Lavaca & 1 & 3.94 & - & - & - & - & - & - & - & - & 1 & 3.94 \\
\hline Leon & 2 & 11.39 & - & - & - & - & - & - & 1 & 13.03 & 3 & 24.43 \\
\hline Liberty & 3 & 5.94 & - & - & - & - & - & - & 1 & 1.98 & 4 & 7.92 \\
\hline Limestone & 1 & 1.38 & - & - & - & - & - & - & 1 & 1.38 & 2 & 2.77 \\
\hline
\end{tabular}

See footnotes at end of table. 
Table TX-3 (page 3 of 4). Number of deaths and age-adjusted mortality rate (per $1,000,000$ population) by condition and county, Texas residents age 15 and over, 1983-1992

\begin{tabular}{|c|c|c|c|c|c|c|c|c|c|c|c|c|}
\hline \multirow[b]{2}{*}{ County } & \multicolumn{2}{|c|}{ Asbestosis } & \multicolumn{2}{|c|}{$\begin{array}{c}\text { Coal workers' } \\
\text { pneumoconiosis }\end{array}$} & \multicolumn{2}{|c|}{ Silicosis } & \multicolumn{2}{|c|}{ Byssinosis } & \multicolumn{2}{|c|}{$\begin{array}{r}\text { Unspecified/Other } \\
\text { pneumoconioses }\end{array}$} & \multicolumn{2}{|c|}{$\begin{array}{r}\text { All } \\
\text { pneumoconioses }\end{array}$} \\
\hline & $\#$ & Rate & $\#$ & Rate & $\#$ & Rate & $\#$ & Rate & \# & Rate & $\#$ & Rate \\
\hline Live Oak & 1 & 4.32 & - & - & - & - & - & - & - & - & 1 & 4.32 \\
\hline Llano & - & - & 3 & 7.35 & - & - & - & - & - & - & 3 & 7.35 \\
\hline Lubbock & 2 & 1.18 & - & - & 1 & 0.31 & 1 & 0.59 & 1 & 0.59 & 5 & 2.68 \\
\hline McCulloch & 1 & 9.28 & - & - & - & - & 1 & 9.28 & - & - & 2 & 18.56 \\
\hline McLennan & 9 & 3.13 & - & - & 1 & 0.56 & - & - & - & - & 10 & 3.69 \\
\hline Madison & 1 & 9.40 & 1 & 3.08 & 1 & 3.08 & - & - & - & - & 2 & 12.48 \\
\hline Mason & 1 & 19.60 & - & - & - & - & - & - & - & - & 1 & 19.60 \\
\hline Matagorda & 1 & 2.91 & - & - & - & - & - & - & - & - & 1 & 2.91 \\
\hline Maverick & 1 & 2.51 & - & - & - & - & - & - & - & - & 1 & 2.51 \\
\hline Midland & 1 & 0.77 & 1 & 1.16 & - & - & - & - & - & - & 2 & 1.93 \\
\hline Mills & - & - & 1 & 4.47 & - & - & - & - & - & - & 1 & 4.47 \\
\hline Mitchell & - & - & - & - & - & - & - & - & 1 & 9.32 & 1 & 9.32 \\
\hline Montgomery & 5 & 3.59 & 3 & 1.79 & 2 & 1.28 & - & - & - & - & 10 & 6.66 \\
\hline Morris & - & - & 1 & 2.71 & - & - & - & - & - & - & 1 & 2.71 \\
\hline Nacogdoches & - & - & - & - & 1 & 2.30 & - & - & 1 & 2.30 & 2 & 4.60 \\
\hline Navarro & - & - & 1 & 0.79 & 1 & 0.79 & - & - & 1 & 0.79 & 3 & 2.36 \\
\hline Newton & 2 & 10.53 & - & - & - & - & - & - & 1 & 6.88 & 3 & 17.41 \\
\hline Nueces & 10 & 3.92 & 4 & 0.99 & - & - & - & - & - & - & 14 & 4.91 \\
\hline Orange & 50 & 56.05 & 2 & 2.23 & 1 & 0.91 & - & - & - & - & 52 & 58.28 \\
\hline Palo Pinto & 2 & 2.87 & - & - & - & - & - & - & - & - & 2 & 2.87 \\
\hline Panola & 1 & 1.73 & 1 & 4.16 & - & - & - & - & - & - & 2 & 5.89 \\
\hline Parker & 1 & 1.79 & - & - & - & - & - & - & - & - & 1 & 1.79 \\
\hline Reeves & - & - & - & - & 1 & 7.33 & - & - & - & - & 1 & 7.33 \\
\hline Rockwall & - & - & 1 & 5.90 & - & - & - & - & - & - & 1 & 5.90 \\
\hline Rusk & 3 & 3.59 & - & - & - & - & - & - & - & - & 3 & 3.59 \\
\hline Sabine & 2 & 11.59 & - & - & 1 & 3.07 & - & - & - & - & 3 & 14.66 \\
\hline San Jacinto & 1 & 4.91 & - & - & - & - & - & - & - & - & 1 & 4.91 \\
\hline Shelby & 2 & 2.77 & 1 & 3.61 & - & - & - & - & - & - & 3 & 6.38 \\
\hline Smith & 11 & 7.28 & - & - & - & - & - & - & 1 & 0.67 & 12 & 7.95 \\
\hline Starr & - & - & 1 & 4.19 & - & - & - & - & - & - & 1 & 4.19 \\
\hline Tarrant & 8 & 0.79 & 6 & 0.54 & 5 & 0.57 & - & - & 2 & 0.20 & 21 & 2.09 \\
\hline Taylor & 1 & 0.97 & - & - & - & - & - & - & 1 & 0.97 & 2 & 1.94 \\
\hline
\end{tabular}

See footnotes at end of table.

\section{Texas}




\begin{tabular}{|c|c|c|c|c|c|c|c|c|c|c|c|c|}
\hline \multirow[b]{2}{*}{ County } & \multicolumn{2}{|c|}{ Asbestosis } & \multicolumn{2}{|c|}{$\begin{array}{c}\text { Coal workers' } \\
\text { pneumoconiosis }\end{array}$} & \multicolumn{2}{|c|}{ Silicosis } & \multicolumn{2}{|c|}{ Byssinosis } & \multicolumn{2}{|c|}{$\begin{array}{l}\text { Unspecified/Other } \\
\text { pneumoconioses }\end{array}$} & \multicolumn{2}{|c|}{$\begin{array}{r}\text { All } \\
\text { pneumoconioses }\end{array}$} \\
\hline & $\#$ & Rate & $\#$ & Rate & \# & Rate & $\#$ & Rate & \# & Rate & $\#$ & Rate \\
\hline Tom Green & - & - & - & - & - & - & - & - & 1 & 1.15 & 1 & 1.15 \\
\hline Travis & 5 & 1.35 & - & - & - & - & - & - & 1 & 0.26 & 6 & 1.61 \\
\hline Trinity & 1 & 2.72 & - & - & - & - & - & - & - & - & 1 & 2.72 \\
\hline Tyler & 3 & 8.16 & - & - & - & - & - & - & - & - & 3 & 8.16 \\
\hline Upshur & 2 & 6.04 & 1 & 1.31 & - & - & - & - & - & - & 3 & 7.35 \\
\hline Val Verde & - & - & - & - & - & - & - & - & 1 & 2.03 & 1 & 2.03 \\
\hline Van Zandt & 2 & 4.20 & - & - & - & - & - & - & - & - & 2 & 4.20 \\
\hline Victoria & 6 & 8.39 & - & - & - & - & - & - & - & - & 6 & 8.39 \\
\hline Waller & - & - & - & - & 1 & 2.40 & - & - & - & - & 1 & 2.40 \\
\hline Washington & 2 & 7.61 & - & - & - & - & - & - & - & - & 2 & 7.61 \\
\hline Wharton & 1 & 2.51 & - & - & - & - & - & - & - & - & 1 & 2.51 \\
\hline Wichita & 3 & 1.66 & - & - & - & - & - & - & 1 & 0.40 & 4 & 2.06 \\
\hline Wilbarger & 1 & 5.49 & - & - & - & - & - & - & - & - & 1 & 5.49 \\
\hline Wilson & 1 & 5.09 & - & - & - & - & - & - & - & - & 1 & 5.09 \\
\hline Wise & 1 & 3.11 & - & - & - & - & - & - & - & - & 1 & 3.11 \\
\hline Wood & 2 & 4.98 & 1 & 1.02 & - & - & - & - & - & - & 3 & 6.00 \\
\hline Yoakum & - & - & - & - & 1 & 14.71 & - & - & - & - & 1 & 14.71 \\
\hline Young & - & - & 1 & 1.61 & - & - & - & - & - & - & 1 & 1.61 \\
\hline Zapata & 1 & 5.35 & - & - & - & - & - & - & - & - & 1 & 5.35 \\
\hline
\end{tabular}

- indicates no deaths listed.

NOTE: Counties with no deaths for all listed conditions are not displayed. Rates based on small numbers may be unstable. Sum of individual conditions may exceed total because of deaths with multiple pneumoconioses. See Appendix A for source description and Appendix B for methods and ICD-8 and ICD-9 codes.

SOURCE: National Center for Health Statistics multiple cause of death data. Population estimates from U.S. Bureau of the Census. 


\section{Utah: Selected statistics from the United States section}

All pneumoconioses mortality, 1988-1992

Total deaths

Crude mortality rate (per $1,000,000$ population)

Age-adjusted mortality rate (per $1,000,000$ population)

Total years of potential life lost to life expectancy

Years of potential life lost to life expectancy (per death)

\begin{tabular}{rr} 
Value & State rank \\
\hline 111 & 26 \\
18.67 & 10 \\
11.88 & 7 \\
1,119 & 29 \\
10.1 & 48
\end{tabular}

Percent**

Deaths by type of pneumoconiosis, 1988-1992

Asbestosis

Coal workers' pneumoconiosis

Deaths*

distribution

10

9.0

73

65.8

Silicosis

18

16.2

Byssinosis

Unspecified/Other pneumoconiosis

0

0.0

11

* Sum of column may exceed total deaths because of deaths with multiple pneumoconioses.

** Percentages may total more than $100 \%$ due to deaths with multiple pneumoconioses.

NOTE: $\quad$ See Appendix A for source description and Appendix B for methods and ICD-8 and ICD-9 codes.

SOURCE: National Center for Health Statistics multiple cause of death data. Population estimates from U.S. Bureau of the Census.

Figure UT-1. All pneumoconioses: Utah and U.S. age-adjusted mortality rates, residents age 15 and over, 1968-1992

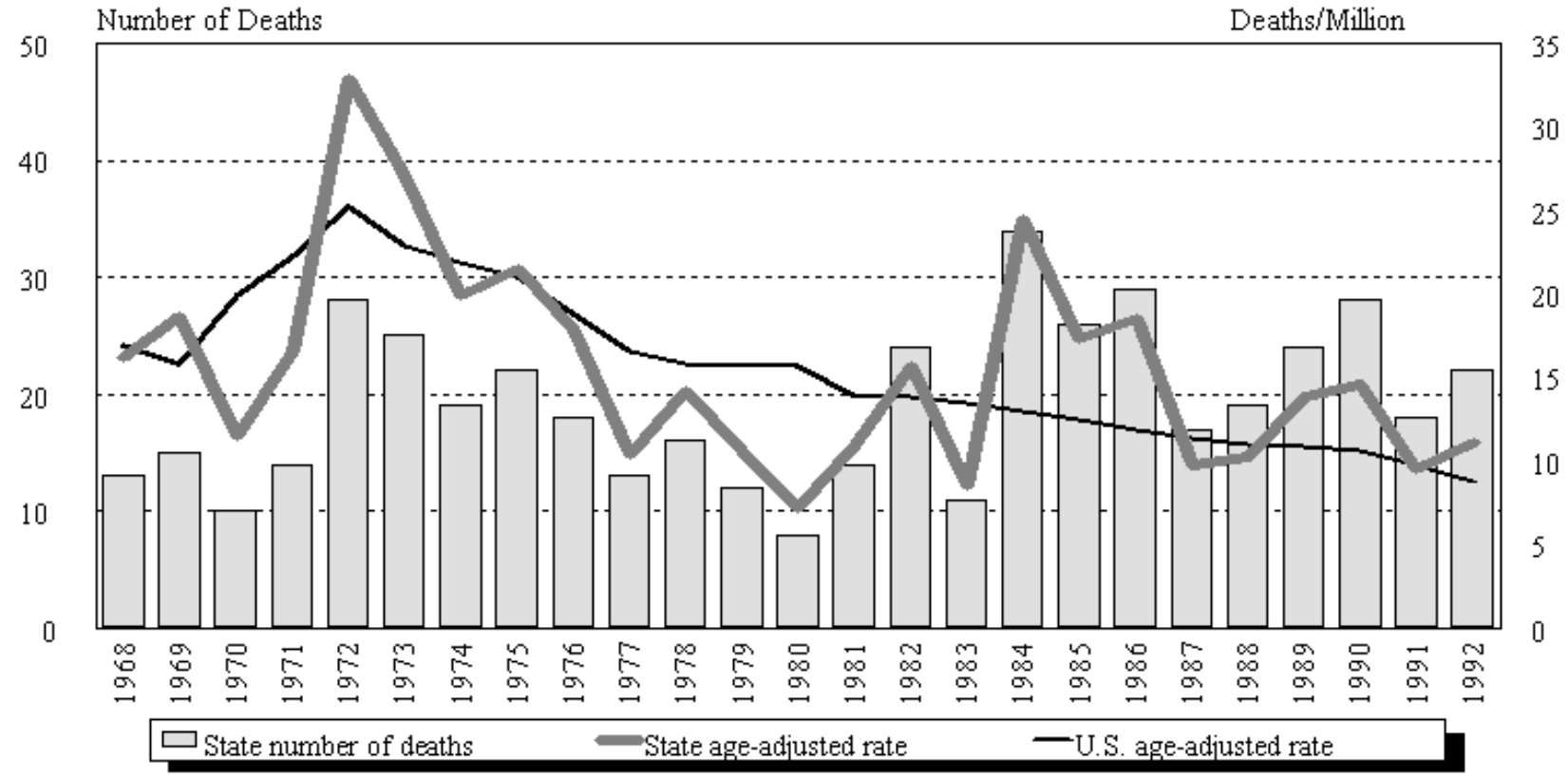

NOTE: $\quad$ See Appendix A for source description and Appendix B for methods and ICD-8 and ICD-9 codes.

SOURCE: National Center for Health Statistics multiple cause of death data. Population estimates from U.S. Bureau of the Census. 


\begin{tabular}{|c|c|c|c|c|c|c|c|c|c|c|c|c|}
\hline \multirow[b]{2}{*}{ Year } & \multicolumn{2}{|c|}{ Asbestosis } & \multicolumn{2}{|c|}{$\begin{array}{l}\text { Coal workers' } \\
\text { pneumoconiosis }\end{array}$} & \multicolumn{2}{|c|}{ Silicosis } & \multicolumn{2}{|c|}{ Byssinosis* } & \multicolumn{2}{|c|}{$\begin{array}{c}\text { Unspecified/Other } \\
\text { pneumoconioses }\end{array}$} & \multicolumn{2}{|c|}{ All pneumoconioses } \\
\hline & Number & Rate & Number & Rate & Number & Rate & Number & Rate & Number & Rate & Number & Rate \\
\hline 1968 & - & - & - & - & 10 & 12.61 & & & 4 & 4.97 & 13 & 16.25 \\
\hline 1969 & - & - & 3 & 3.56 & 10 & 13.22 & & & 2 & 1.96 & 15 & 18.73 \\
\hline 1970 & - & - & - & - & 5 & 5.65 & & & 5 & 5.87 & 10 & 11.51 \\
\hline 1971 & - & - & - & - & 11 & 13.68 & & & 5 & 5.43 & 14 & 16.53 \\
\hline 1972 & - & - & - & - & 12 & 13.20 & & & 16 & 19.65 & 28 & 32.84 \\
\hline 1973 & - & - & 4 & 4.30 & 13 & 13.61 & & & 8 & 9.13 & 25 & 27.04 \\
\hline 1974 & - & - & 3 & 3.72 & 8 & 7.73 & & & 8 & 8.44 & 19 & 19.90 \\
\hline 1975 & - & - & 5 & 4.16 & 8 & 8.29 & & & 10 & 10.15 & 22 & 21.47 \\
\hline 1976 & - & - & 2 & 2.18 & 11 & 10.31 & & & 5 & 5.34 & 18 & 17.82 \\
\hline 1977 & - & - & 3 & 3.02 & 9 & 6.83 & & & 1 & 0.48 & 13 & 10.33 \\
\hline 1978 & 1 & 1.01 & 4 & 4.04 & 5 & 4.44 & & & 6 & 4.76 & 16 & 14.26 \\
\hline 1979 & - & - & 3 & 2.68 & 3 & 2.68 & - & - & 6 & 5.26 & 12 & 10.63 \\
\hline 1980 & 1 & 0.95 & 1 & 0.95 & 5 & 4.62 & - & - & 1 & 0.70 & 8 & 7.22 \\
\hline 1981 & 2 & 1.57 & 6 & 4.72 & 3 & 2.24 & - & - & 3 & 2.48 & 14 & 11.01 \\
\hline 1982 & 1 & 0.36 & 14 & 9.89 & 6 & 3.90 & - & - & 4 & 2.23 & 24 & 15.75 \\
\hline 1983 & 1 & 0.84 & 4 & 3.04 & 4 & 2.63 & - & - & 2 & 1.99 & 11 & 8.50 \\
\hline 1984 & 1 & 0.58 & 17 & 12.80 & 9 & 6.72 & - & - & 7 & 4.43 & 34 & 24.52 \\
\hline 1985 & - & - & 14 & 8.54 & 9 & 6.40 & - & - & 3 & 2.33 & 26 & 17.26 \\
\hline 1986 & 2 & 0.84 & 16 & 10.02 & 4 & 2.40 & - & - & 7 & 5.31 & 29 & 18.57 \\
\hline 1987 & 2 & 1.28 & 12 & 6.98 & 2 & 1.04 & - & - & 1 & 0.52 & 17 & 9.82 \\
\hline 1988 & 1 & 0.76 & 16 & 8.44 & 1 & 0.50 & - & - & 1 & 0.50 & 19 & 10.21 \\
\hline 1989 & 2 & 1.74 & 14 & 7.17 & 6 & 3.24 & - & - & 2 & 1.66 & 24 & 13.81 \\
\hline 1990 & 3 & 1.68 & 18 & 9.21 & 2 & 1.27 & - & - & 5 & 2.48 & 28 & 14.64 \\
\hline 1991 & - & - & 12 & 6.84 & 5 & 2.21 & - & - & 1 & 0.46 & 18 & 9.50 \\
\hline 1992 & 4 & 2.02 & 13 & 6.29 & 4 & 2.57 & - & - & 2 & 0.68 & 22 & 11.11 \\
\hline
\end{tabular}

NOTE: Rates based on small numbers may be unstable. Sum of individual conditions may exceed total because of deaths with multiple pneumoconioses. See Appendix A for source description and Appendix B for methods and ICD-8 and ICD-9 codes.

SOURCE: National Center for Health Statistics multiple cause of death data. Population estimates from U.S. Bureau of the Census. 
Figure UT-2. All pneumoconioses: Distribution of deaths by sex and race, Utah residents age 15 and over, 1968-1992
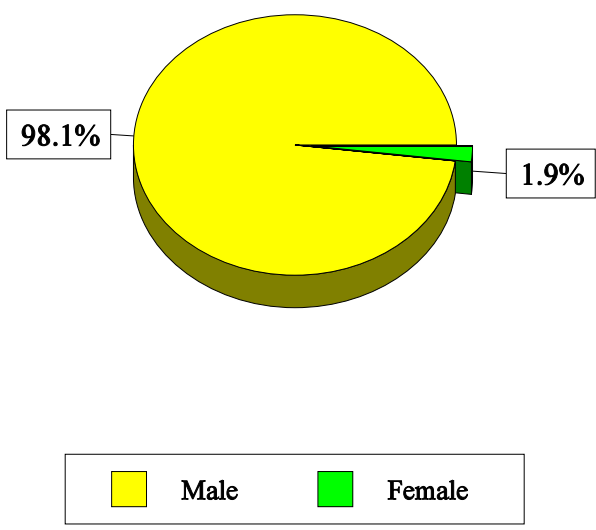
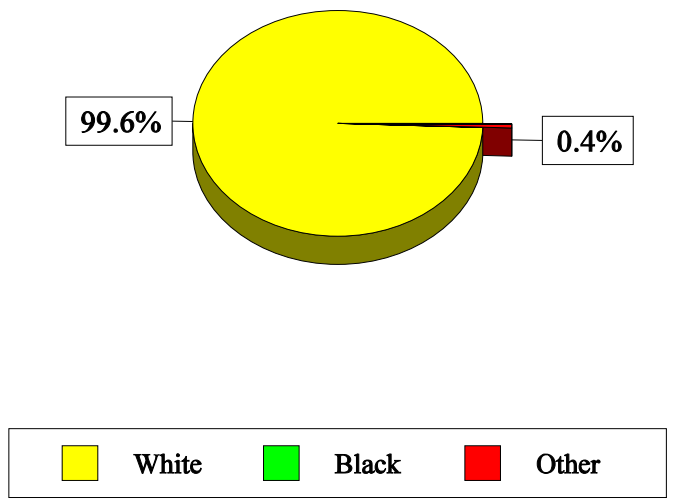

NOTE: $\quad$ See Appendix A for source description and Appendix B for methods and ICD-8 and ICD-9 codes.

SOURCE: National Center for Health Statistics multiple cause of death data.

Table UT-2. All pneumoconioses: Distribution of deaths by sex, race, and age, Utah residents age 15 and over, 1968-1992

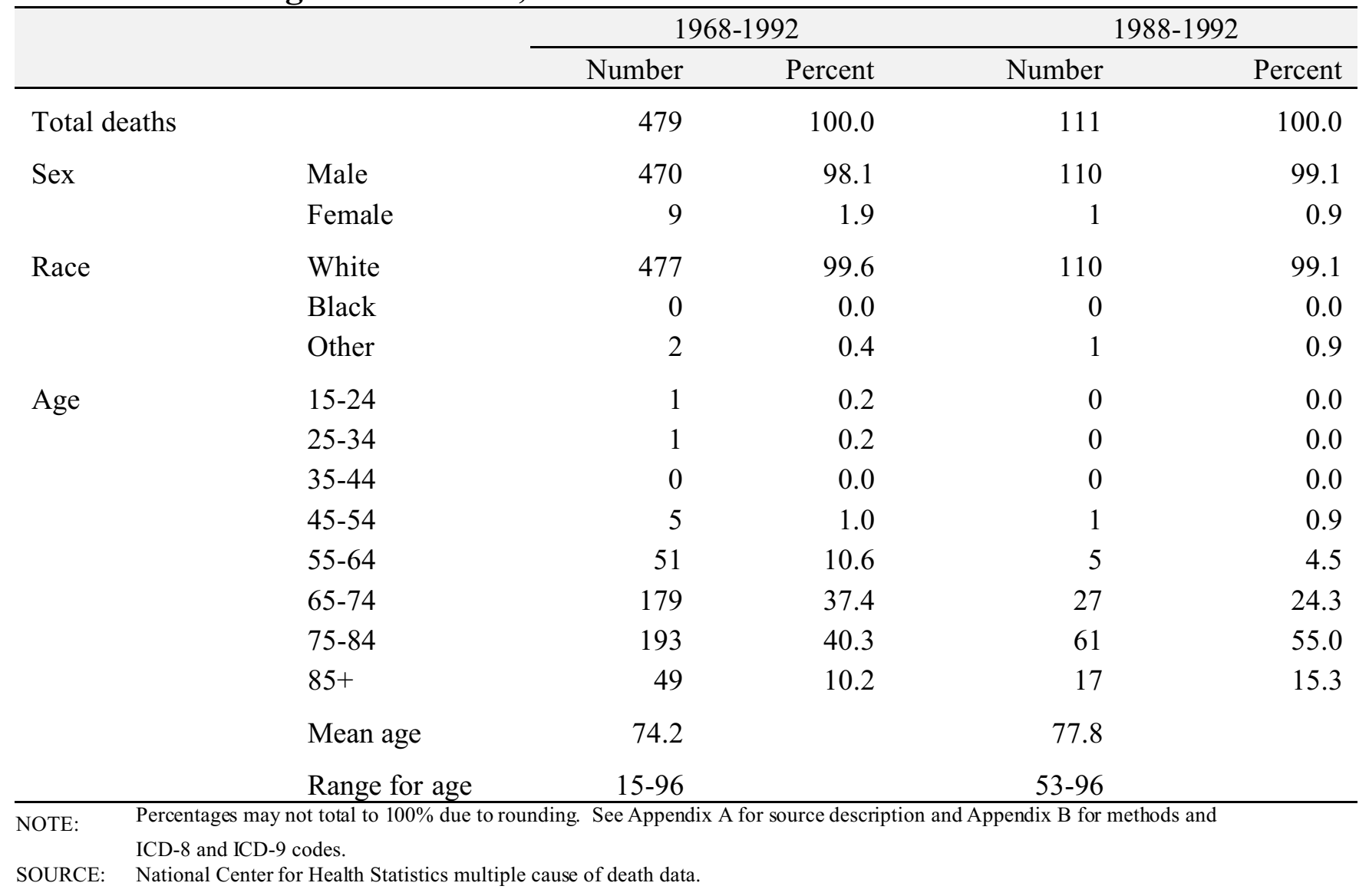


Figure UT-3. Pneumoconiosis deaths by condition, Utah residents age 15 and over, 1968-1992

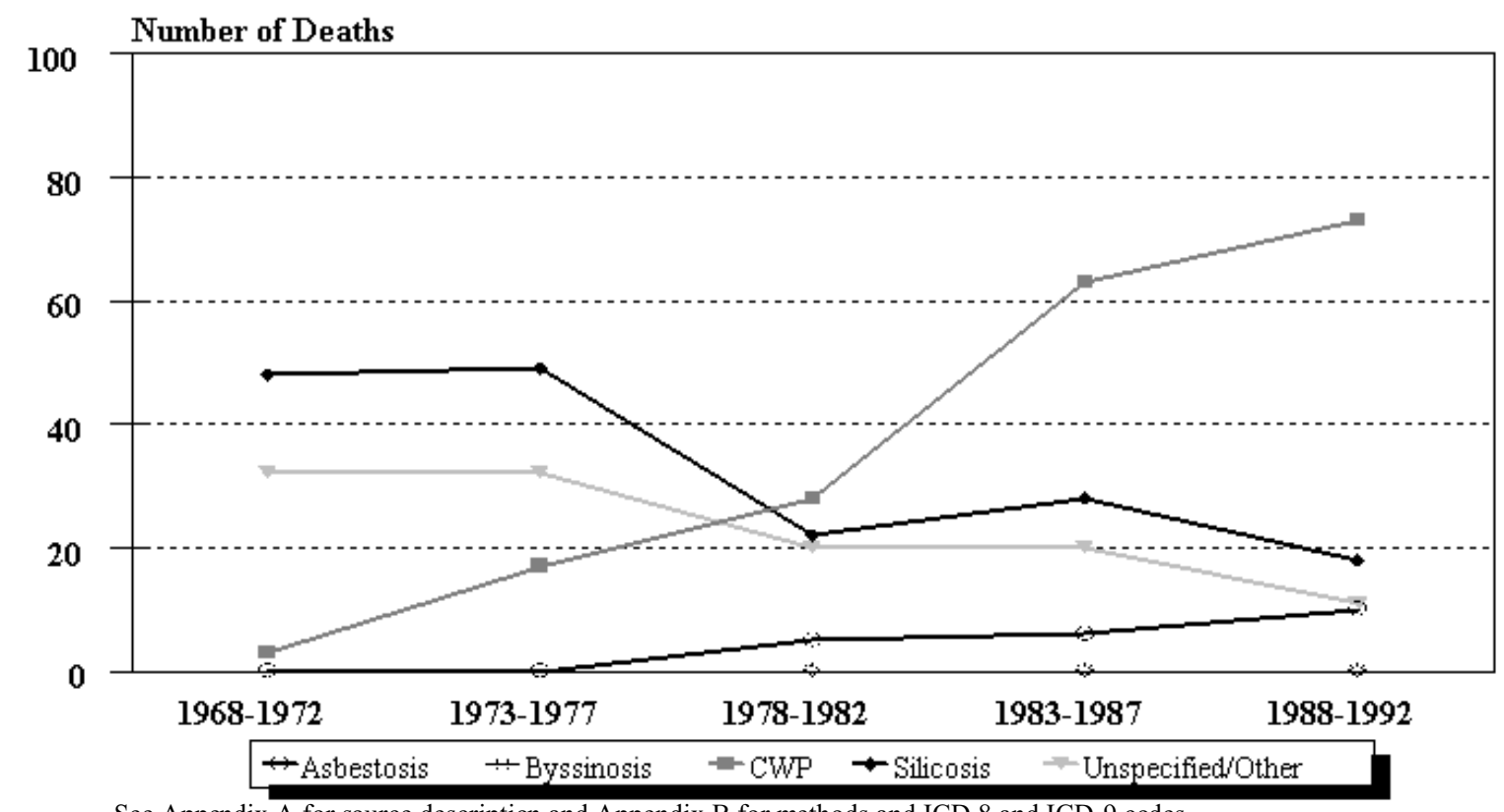

NOTE: See Appendix A for source description and Appendix B for methods and ICD-8 and ICD-9 codes.

SOURCE: National Center for Health Statistics multiple cause of death data.

Figure UT-4. Utah and U.S. age-adjusted mortality rates (per 1,000,000 population) by condition, residents age 15 and over, 1983-1992

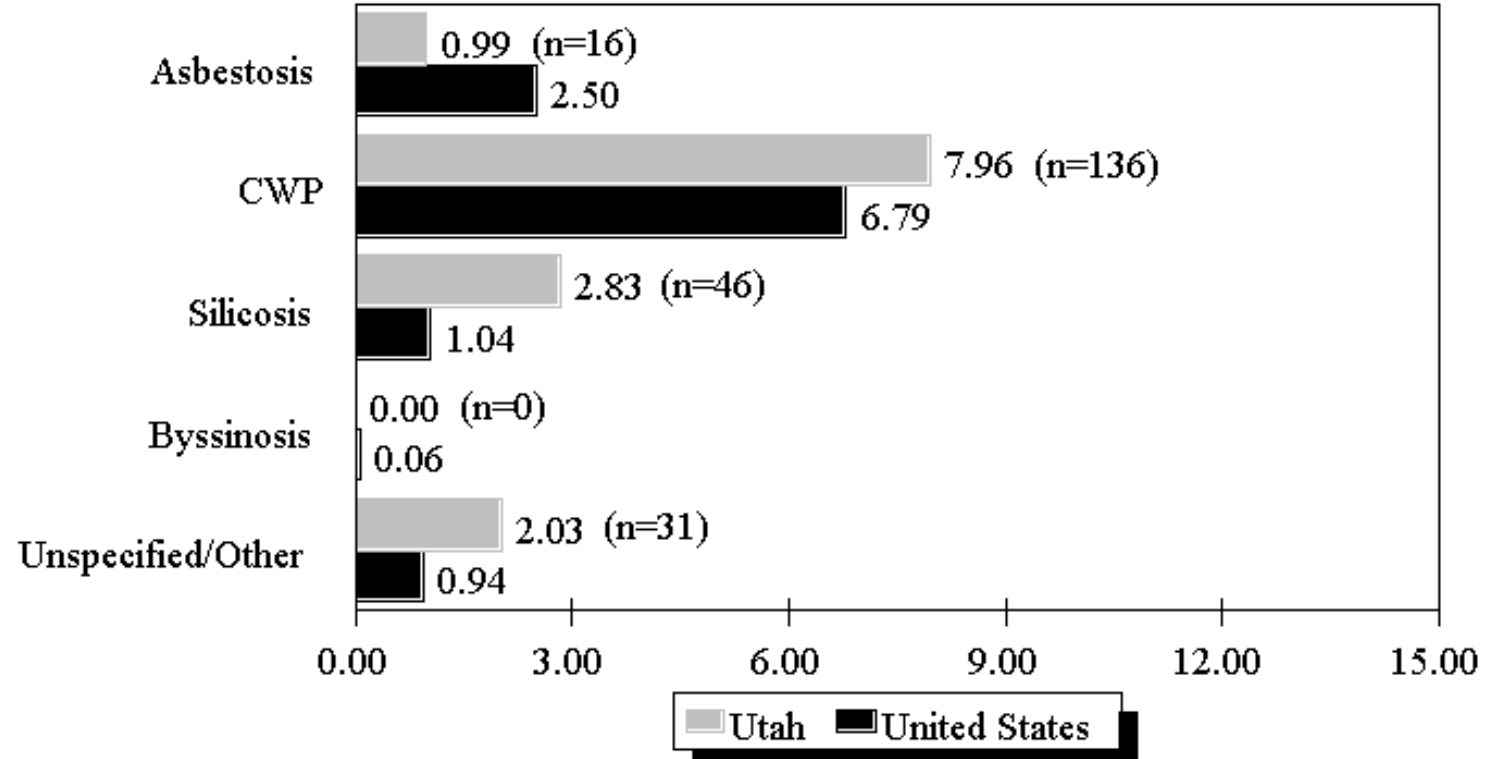

NOTE: Rates based on small numbers may be unstable. See Appendix A for source description and Appendix B for methods and ICD-8 and ICD-9 codes.

SOURCE: National Center for Health Statistics multiple cause of death data. Population estimates from U.S. Bureau of the Census. 
Table UT-3. Number of deaths and age-adjusted mortality rate (per 1,000,000 population) by condition and county, Utah residents age 15 and over, 1983-1992

\begin{tabular}{|c|c|c|c|c|c|c|c|c|c|c|c|c|}
\hline \multirow[b]{2}{*}{ County } & \multicolumn{2}{|c|}{ Asbestosis } & \multicolumn{2}{|c|}{$\begin{array}{c}\text { Coal workers' } \\
\text { pneumoconiosis }\end{array}$} & \multicolumn{2}{|c|}{ Silicosis } & \multicolumn{2}{|c|}{ Byssinosis } & \multicolumn{2}{|c|}{$\begin{array}{r}\text { Unspecified/Other } \\
\text { pneumoconioses }\end{array}$} & \multicolumn{2}{|c|}{$\begin{array}{r}\text { All } \\
\text { pneumoconioses }\end{array}$} \\
\hline & $\#$ & Rate & \# & Rate & $\#$ & Rate & $\#$ & Rate & $\#$ & Rate & \# & Rate \\
\hline Box Elder & - & - & - & - & 1 & 3.61 & - & - & - & - & 1 & 3.61 \\
\hline Cache & - & - & - & - & 1 & 1.03 & - & - & - & - & 1 & 1.03 \\
\hline Carbon & - & - & 74 & 270.72 & 1 & 5.16 & - & - & 22 & 87.02 & 97 & 362.90 \\
\hline Davis & - & - & 3 & 2.41 & - & - & - & - & - & - & 3 & 2.41 \\
\hline Emery & - & - & 15 & 149.91 & - & - & - & - & 1 & 15.06 & 16 & 164.97 \\
\hline Grand & - & - & 1 & 10.34 & 2 & 24.87 & - & - & - & - & 3 & 35.21 \\
\hline Iron & 1 & 3.61 & - & - & - & - & - & - & - & - & 1 & 3.61 \\
\hline Juab & - & - & - & - & 1 & 7.22 & - & - & - & - & 1 & 7.22 \\
\hline Salt Lake & 12 & 1.76 & 18 & 2.33 & 22 & 3.40 & - & - & 4 & 0.55 & 56 & 8.04 \\
\hline San Juan & - & - & 1 & 14.13 & - & - & - & - & - & - & 1 & 14.13 \\
\hline Sanpete & - & - & 2 & 5.60 & - & - & - & - & - & - & 2 & 5.60 \\
\hline Sevier & - & - & 1 & 3.00 & - & - & - & - & - & - & 1 & 3.00 \\
\hline Summit & - & - & 1 & 13.20 & 3 & 34.59 & - & - & 1 & 8.19 & 5 & 55.98 \\
\hline Tooele & - & - & 1 & 3.41 & 2 & 8.30 & - & - & 1 & 4.89 & 4 & 16.61 \\
\hline Uintah & - & - & 1 & 6.52 & - & - & - & - & - & - & 1 & 6.52 \\
\hline Utah & 3 & 2.22 & 8 & 3.72 & 8 & 4.45 & - & - & 2 & 0.82 & 21 & 11.21 \\
\hline Washington & - & - & 6 & 5.87 & 2 & 1.96 & - & - & - & - & 7 & 6.85 \\
\hline Weber & - & - & 4 & 2.69 & 3 & 1.56 & - & - & - & - & 7 & 4.25 \\
\hline
\end{tabular}




\section{Vermont: Selected statistics from the United States section}

All pneumoconioses mortality, 1988-1992

Total deaths

Crude mortality rate (per $1,000,000$ population)

Age-adjusted mortality rate (per $1,000,000$ population)

Total years of potential life lost to life expectancy

Years of potential life lost to life expectancy (per death)

\begin{tabular}{rr} 
Value & State rank \\
\hline 28 & 43 \\
12.67 & 15 \\
6.73 & 18 \\
274 & 44 \\
9.8 & 50
\end{tabular}

Percent**

Deaths by type of pneumoconiosis, 1988-1992

Asbestosis

Coal workers' pneumoconiosis

$\underline{\text { Deaths* }}$

distribution

5

17.9

14.3

Silicosis

4

57.1

Byssinosis

16

0.0

Unspecified/Other pneumoconiosis

* Sum of column may exceed total deaths because of deaths with multiple pneumoconioses.

** Percentages may total more than $100 \%$ due to deaths with multiple pneumoconioses.

NOTE: $\quad$ See Appendix A for source description and Appendix B for methods and ICD-8 and ICD-9 codes.

SOURCE: National Center for Health Statistics multiple cause of death data. Population estimates from U.S. Bureau of the Census.

Figure VT-1. All pneumoconioses: Vermont and U.S. age-adjusted mortality rates, residents age 15 and over, 1968-1992

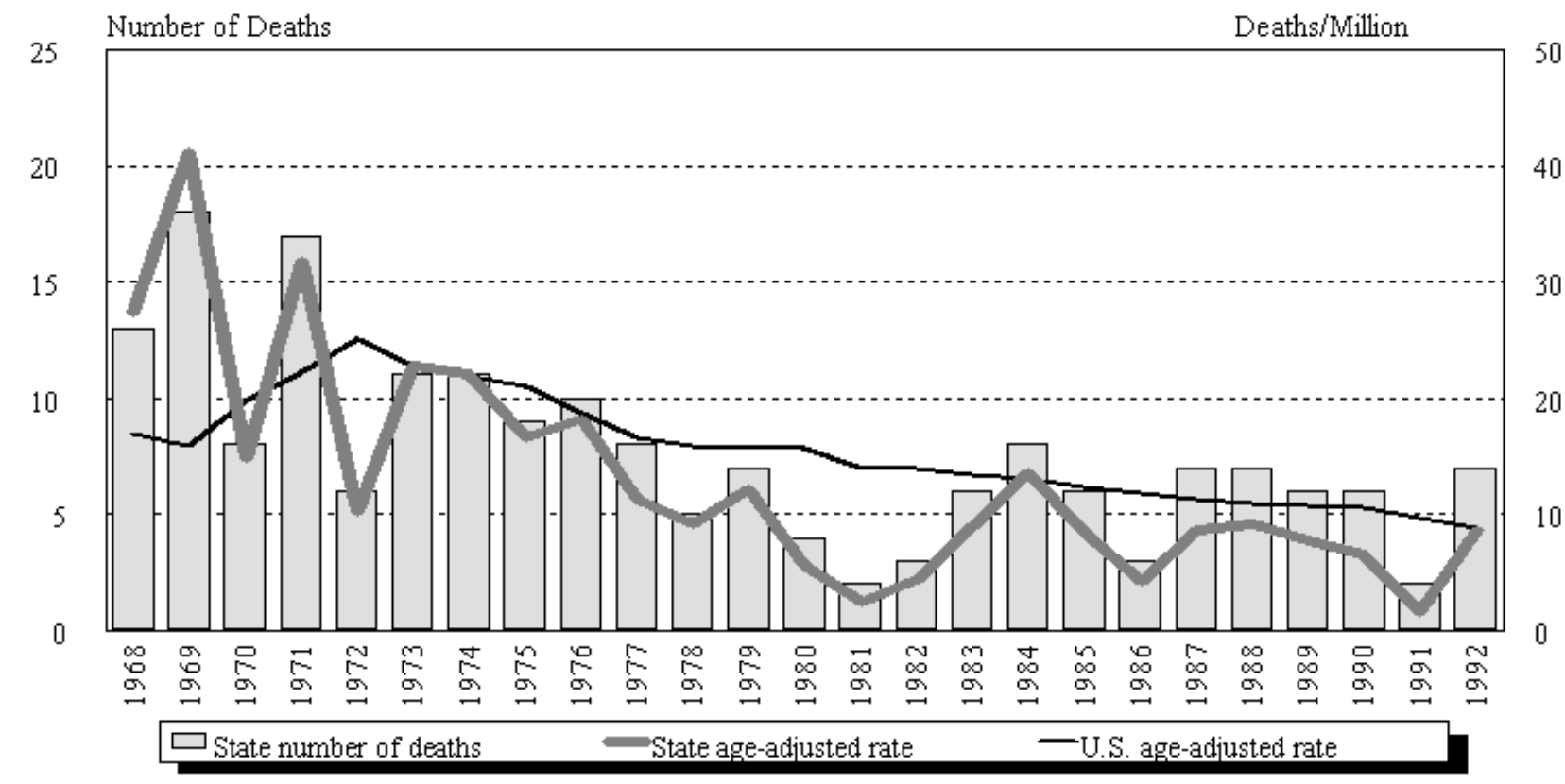

NOTE: $\quad$ See Appendix A for source description and Appendix B for methods and ICD-8 and ICD-9 codes.

SOURCE: National Center for Health Statistics multiple cause of death data. Population estimates from U.S. Bureau of the Census. 
Vermont

\begin{tabular}{|c|c|c|c|c|c|c|c|c|c|c|c|c|}
\hline \multirow[b]{2}{*}{ Year } & \multicolumn{2}{|c|}{ Asbestosis } & \multicolumn{2}{|c|}{$\begin{array}{l}\text { Coal workers' } \\
\text { pneumoconiosis }\end{array}$} & \multicolumn{2}{|c|}{ Silicosis } & \multicolumn{2}{|c|}{ Byssinosis* } & \multicolumn{2}{|c|}{$\begin{array}{c}\text { Unsp ecified/Other } \\
\text { pneumoconioses }\end{array}$} & \multicolumn{2}{|c|}{ All pneumoconioses } \\
\hline & Number & Rate & Number & Rate & Number & Rate & Number & Rate & Number & Rate & Number & Rate \\
\hline 1968 & - & - & - & - & 11 & 22.06 & & & 2 & 5.39 & 13 & 27.45 \\
\hline 1969 & 2 & 4.96 & - & - & 16 & 36.16 & & & 1 & 2.69 & 18 & 41.12 \\
\hline 1970 & - & - & - & - & 6 & 11.84 & & & 2 & 3.08 & 8 & 14.92 \\
\hline 1971 & - & - & 1 & 0.88 & 10 & 17.87 & & & 7 & 15.32 & 17 & 31.84 \\
\hline 1972 & - & - & - & - & 2 & 4.39 & & & 4 & 5.89 & 6 & 10.28 \\
\hline 1973 & - & - & - & - & 9 & 17.99 & & & 2 & 4.78 & 11 & 22.77 \\
\hline 1974 & - & - & - & - & 11 & 22.13 & & & - & - & 11 & 22.13 \\
\hline 1975 & - & - & - & - & 7 & 12.42 & & & 2 & 4.15 & 9 & 16.57 \\
\hline 1976 & - & - & - & - & 8 & 14.14 & & & 2 & 4.07 & 10 & 18.22 \\
\hline 1977 & - & - & - & - & 6 & 8.69 & & & 3 & 4.01 & 8 & 11.36 \\
\hline 1978 & - & - & - & - & 2 & 3.27 & & & 3 & 5.91 & 5 & 9.18 \\
\hline 1979 & 2 & 3.88 & - & - & 5 & 8.38 & - & - & - & - & 7 & 12.26 \\
\hline 1980 & - & - & - & - & 4 & 5.66 & - & - & - & - & 4 & 5.66 \\
\hline 1981 & - & - & - & - & 2 & 2.50 & - & - & - & - & 2 & 2.50 \\
\hline 1982 & 1 & 1.89 & - & - & 2 & 2.45 & - & - & - & - & 3 & 4.34 \\
\hline 1983 & 1 & 2.35 & 1 & 1.21 & 3 & 3.63 & - & - & 1 & 1.87 & 6 & 9.05 \\
\hline 1984 & 1 & 1.86 & - & - & 5 & 6.52 & - & - & 2 & 5.14 & 8 & 13.52 \\
\hline 1985 & 1 & 1.85 & 1 & 0.55 & 3 & 4.88 & 1 & 1.18 & - & - & 6 & 8.46 \\
\hline 1986 & 1 & 1.83 & - & - & - & - & - & - & 2 & 2.32 & 3 & 4.16 \\
\hline 1987 & 2 & 2.89 & 1 & 1.81 & 3 & 2.87 & 1 & 1.14 & - & - & 7 & 8.70 \\
\hline 1988 & 1 & 1.11 & 2 & 2.30 & 2 & 2.22 & - & - & 2 & 3.56 & 7 & 9.20 \\
\hline 1989 & - & - & 1 & 1.09 & 4 & 4.35 & - & - & 1 & 2.37 & 6 & 7.81 \\
\hline 1990 & 1 & 1.07 & - & - & 5 & 5.44 & - & - & - & - & 6 & 6.51 \\
\hline 1991 & - & - & 1 & 0.48 & 1 & 1.04 & - & - & - & - & 2 & 1.52 \\
\hline 1992 & 3 & 3.91 & - & & 4 & 4.78 & - & & - & - & 7 & 8.69 \\
\hline
\end{tabular}

indicates no deaths listed. ${ }^{*}$ no specific code for by ssinosis in ICD-8 (1968-1978)

NOTE: Rates based on small numbers may be unstable. Sum of individual conditions may exceed total because of deaths with multiple pneumoconioses. See Appendix A for source description and Appendix B for methods and ICD-8 and ICD-9 codes.

SOURCE: National Center for Health Statistics multiple cause of death data. Population estimates from U.S. Bureau of the Census. 


\section{Figure VT-2. All pneumoconioses: Distribution of deaths by sex and race,} Vermont residents age 15 and over, 1968-1992
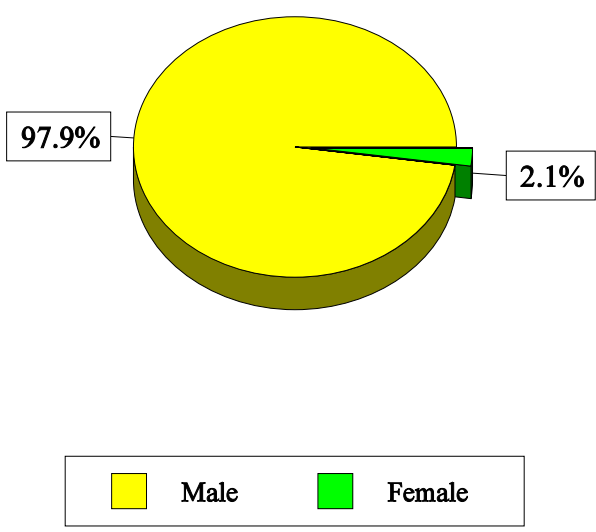
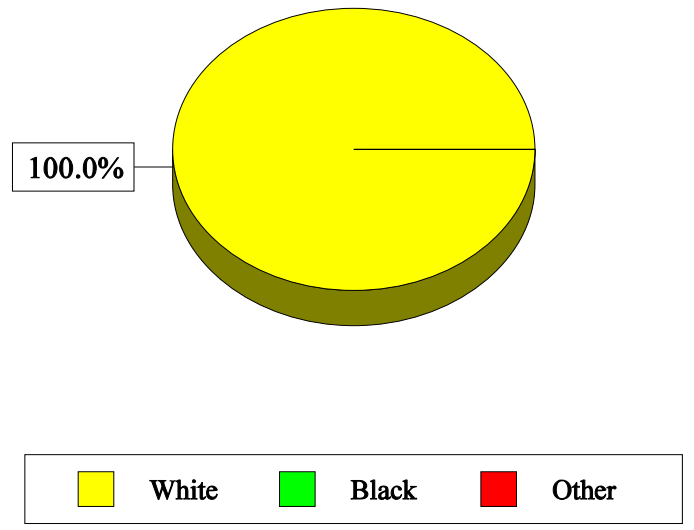

NOTE: $\quad$ See Appendix A for source description and Appendix B for methods and ICD-8 and ICD-9 codes.

SOURCE: National Center for Health Statistics multiple cause of death data.

Table VT-2. All pneumoconioses: Distribution of deaths by sex, race, and age, Vermont residents age 15 and over, 1968-1992

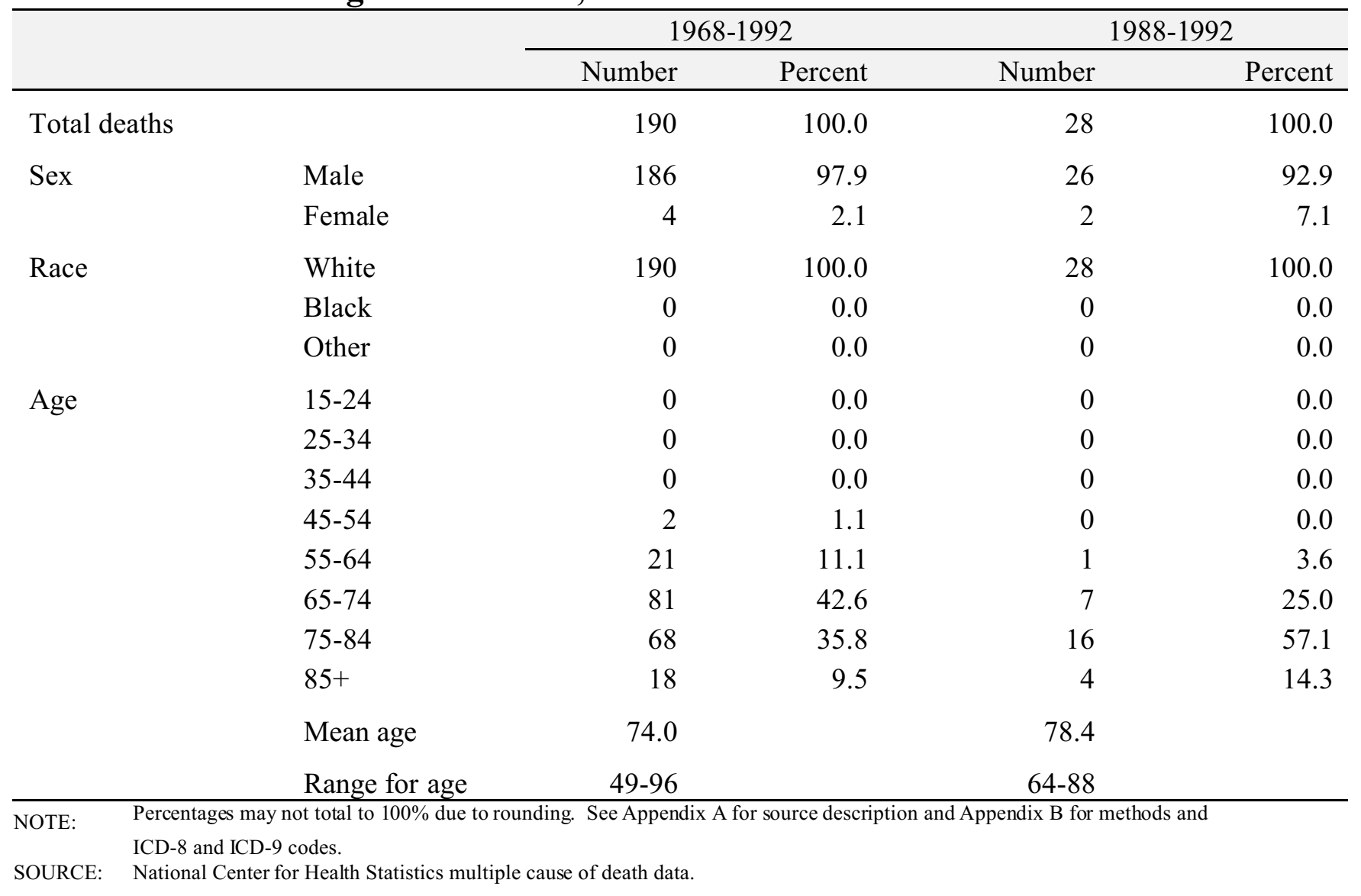




\section{Figure VT-3. Pneumoconiosis deaths by condition, Vermont residents age 15 and over, 1968-1992}

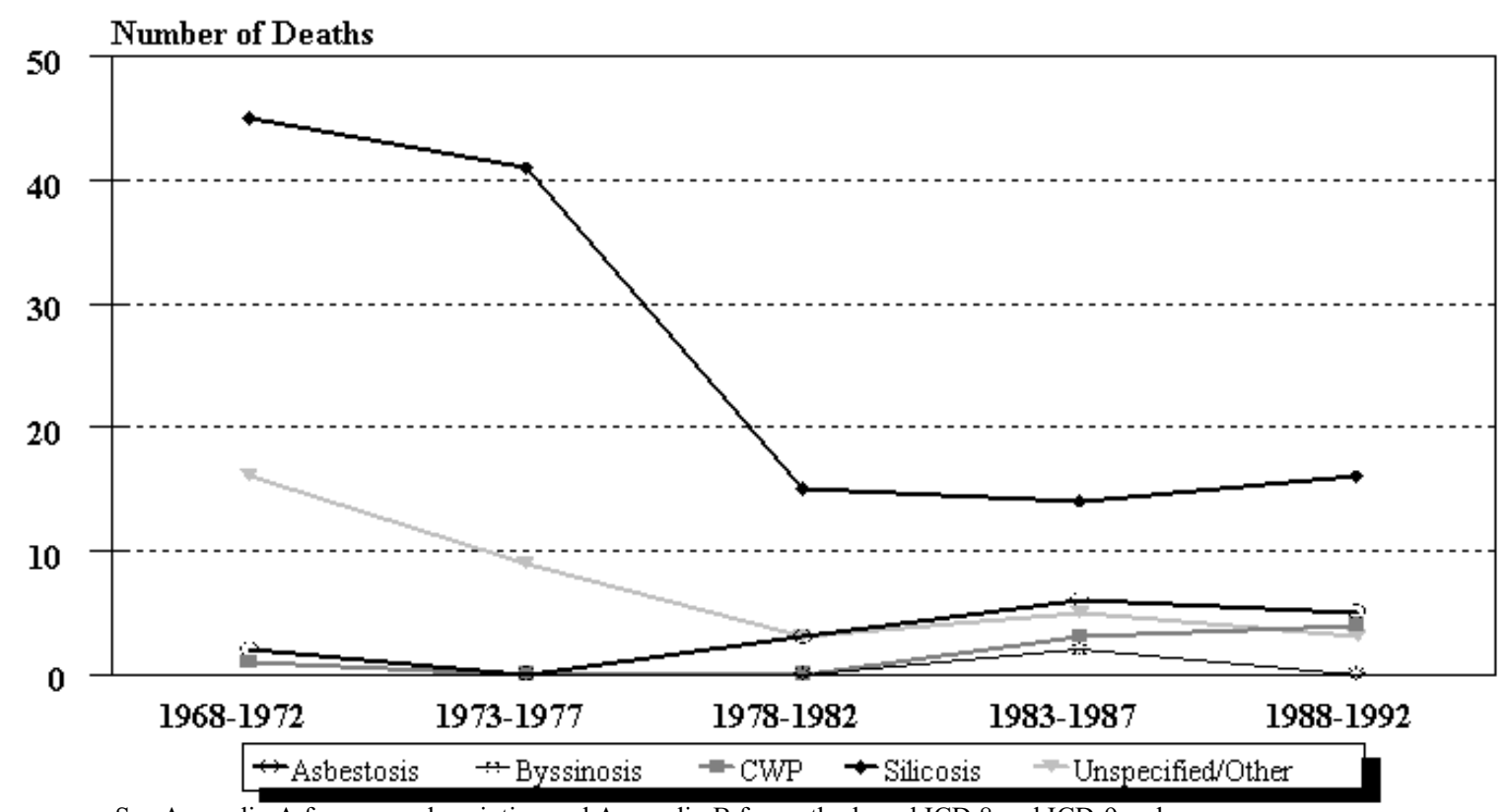

NOTE: See Appendix A for source description and Appendix B for methods and ICD-8 and ICD-9 codes.

SOURCE: National Center for Health Statistics multiple cause of death data.

Figure VT-4. Vermont and U.S. age-adjusted mortality rates (per 1,000,000 population) by condition, residents age 15 and over, 1983-1992

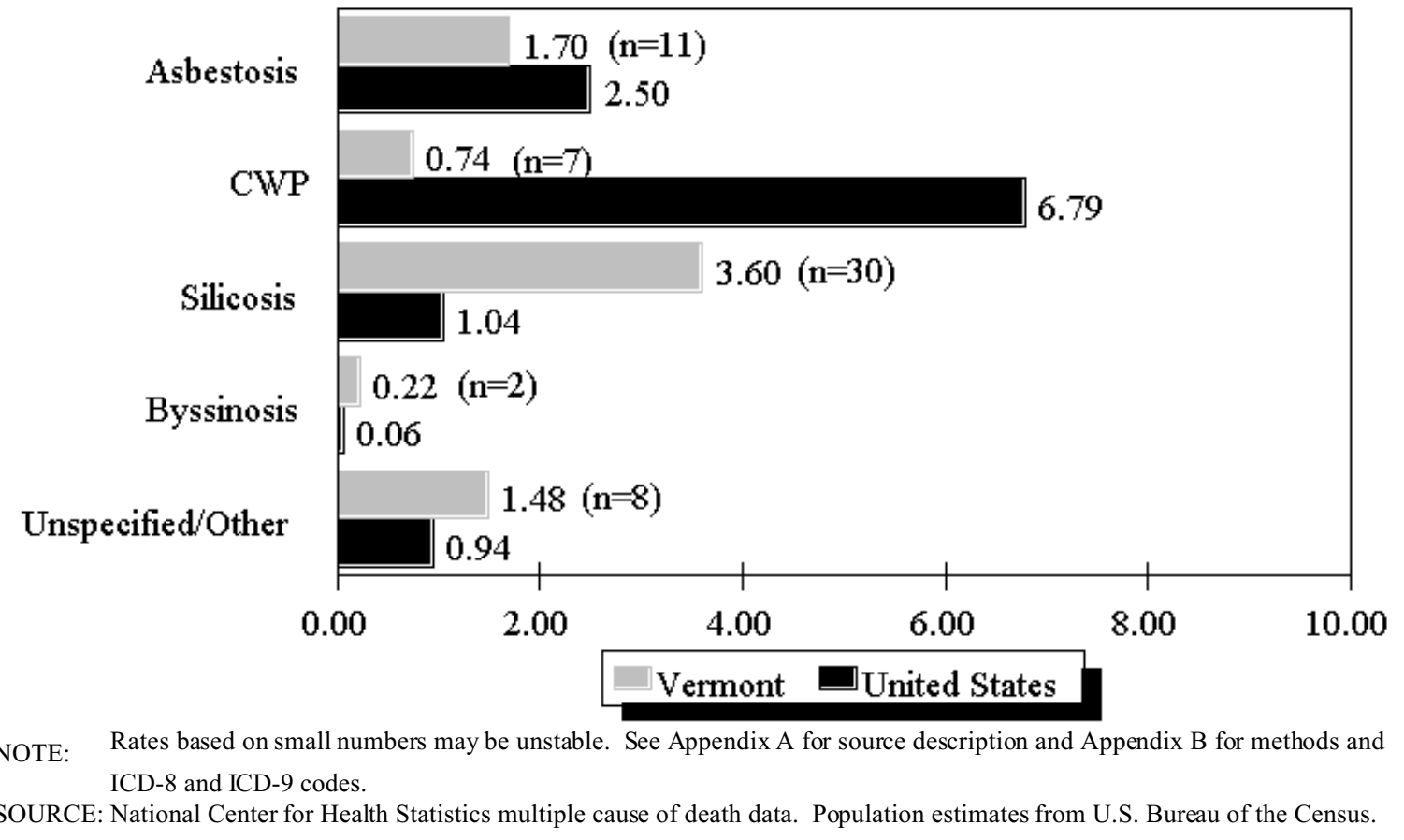


Table VT-3. Number of deaths and age-adjusted mortality rate (per 1,000,000 population) by condition and county, Vermont residents age 15 and over, 1983-1992

\begin{tabular}{|c|c|c|c|c|c|c|c|c|c|c|c|c|}
\hline \multirow[b]{2}{*}{ County } & \multicolumn{2}{|c|}{ Asbestosis } & \multicolumn{2}{|c|}{$\begin{array}{c}\text { Coal workers' } \\
\text { pneumoconiosis }\end{array}$} & \multicolumn{2}{|c|}{ Silicosis } & \multicolumn{2}{|c|}{ Byssinosis } & \multicolumn{2}{|c|}{$\begin{array}{r}\text { Unspecified/Other } \\
\text { pneumoconioses }\end{array}$} & \multicolumn{2}{|c|}{$\begin{array}{r}\text { All } \\
\text { pneumoconioses }\end{array}$} \\
\hline & \# & Rate & \# & Rate & \# & Rate & $\#$ & Rate & \# & Rate & \# & Rate \\
\hline Bennington & 1 & 2.81 & 1 & 1.13 & - & - & - & - & - & - & 2 & 3.94 \\
\hline Caledonia & 1 & 3.89 & - & - & 1 & 1.78 & - & - & 1 & 3.89 & 3 & 9.56 \\
\hline Chittenden & 2 & 2.39 & - & - & 1 & 0.62 & - & - & - & - & 3 & 3.01 \\
\hline Essex & - & - & 1 & 7.92 & - & - & - & - & - & - & 1 & 7.92 \\
\hline Franklin & 2 & 6.27 & - & - & - & - & - & - & 1 & 3.13 & 3 & 9.40 \\
\hline Grand Isle & - & - & - & - & - & - & 1 & 12.28 & - & - & 1 & 12.28 \\
\hline Lamoille & 3 & 12.78 & 1 & 3.11 & 1 & 3.11 & - & - & 1 & 6.56 & 6 & 25.56 \\
\hline Orleans & - & - & - & - & 1 & 2.04 & - & - & - & - & 1 & 2.04 \\
\hline Rutland & - & - & 2 & 1.49 & 10 & 12.18 & - & - & 3 & 4.74 & 15 & 18.40 \\
\hline Washington & - & - & 1 & 2.12 & 15 & 15.40 & 1 & 0.86 & - & - & 17 & 18.38 \\
\hline Windham & 2 & 2.26 & - & - & 1 & 1.13 & - & - & - & - & 3 & 3.39 \\
\hline Windsor & - & - & 1 & 1.81 & - & - & - & - & 2 & 2.65 & 3 & 4.46 \\
\hline
\end{tabular}




\section{Virginia: Selected statistics from the United States section}

All pneumoconioses mortality, 1988-1992

Total deaths

$\underline{\text { Value }}$

980

39.76

28.00

12,282

12.5

Years of potential life lost to life expectancy (per death)
Crude mortality rate (per $1,000,000$ population)

Age-adjusted mortality rate (per $1,000,000$ population)

Total years of potential life lost to life expectancy
Deaths by type of pneumoconiosis, 1988-1992

Asbestosis

Coal workers' pneumoconiosis

Silicosis

Byssinosis

Unspecified/Other pneumoconiosis $\underline{\text { State rank }}$

3

4

4

Percent** distribution

20.0

73.0

1.4

0.2

5.5

* Sum of column may exceed total deaths because of deaths with multiple pneumoconioses.

** Percentages may total more than $100 \%$ due to deaths with multiple pneumoconioses.

NOTE: $\quad$ See Appendix A for source description and Appendix B for methods and ICD-8 and ICD-9 codes.

SOURCE: National Center for Health Statistics multiple cause of death data. Population estimates from U.S. Bureau of the Census.

Figure VA-1. All pneumoconioses: Virginia and U.S. age-adjusted mortality rates, residents age 15 and over, 1968-1992

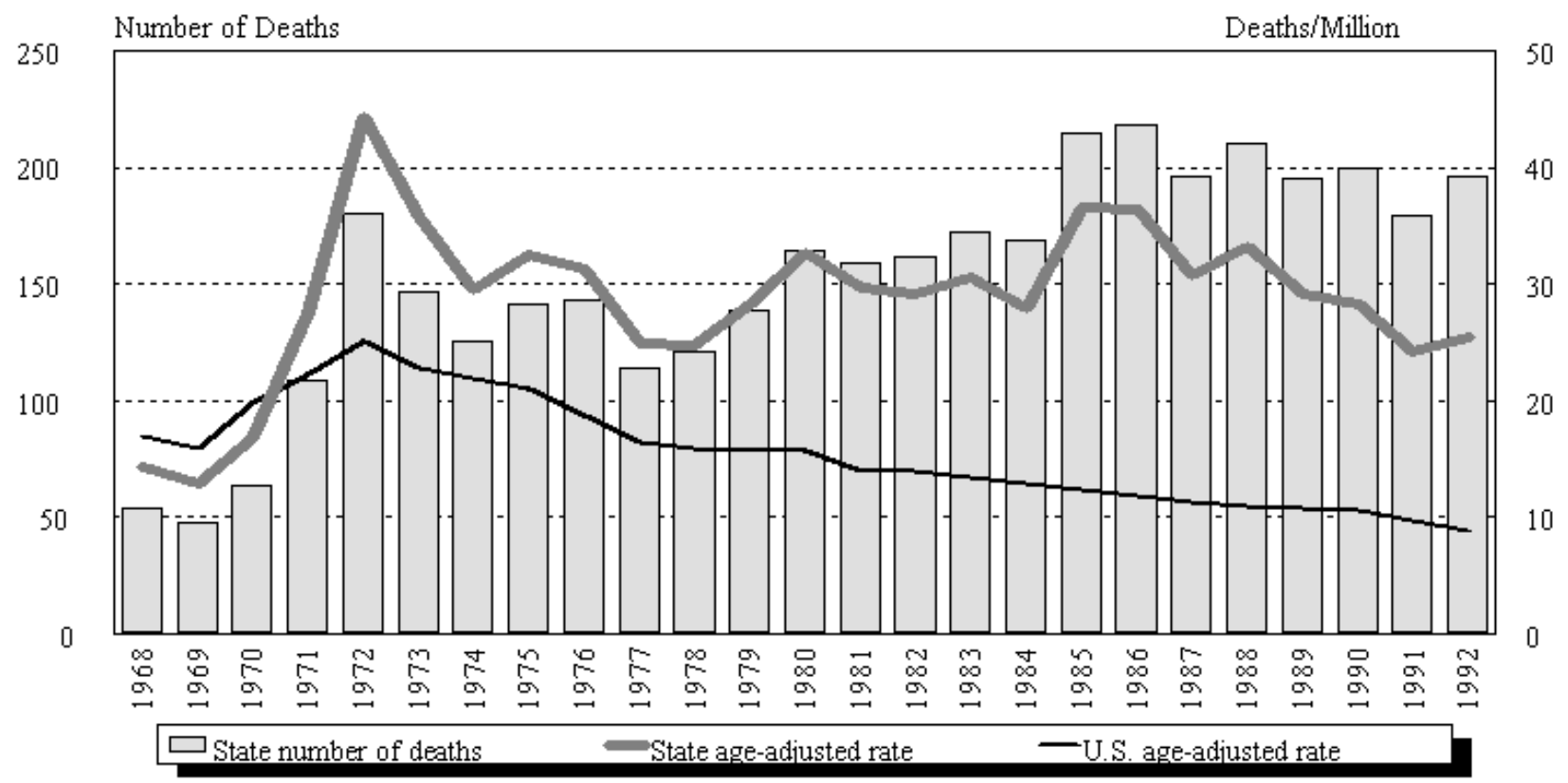

NOTE: $\quad$ See Appendix A for source description and Appendix B for methods and ICD-8 and ICD-9 codes.

SOURCE: National Center for Health Statistics multiple cause of death data. Population estimates from U.S. Bureau of the Census. 
Virginia

\begin{tabular}{|c|c|c|c|c|c|c|c|c|c|c|c|c|}
\hline \multirow[b]{2}{*}{ Year } & \multicolumn{2}{|c|}{ Asb estosis } & \multicolumn{2}{|c|}{$\begin{array}{l}\text { Coal workers' } \\
\text { pneumoconiosis }\end{array}$} & \multicolumn{2}{|c|}{ Silicosis } & \multicolumn{2}{|c|}{ Byssinosis* } & \multicolumn{2}{|c|}{$\begin{array}{l}\text { Unspecified/Other } \\
\text { pneumoconioses }\end{array}$} & \multicolumn{2}{|c|}{ All pneu moconioses } \\
\hline & Number & Rate & Number & Rate & Number & Rate & Number & Rate & Number & Rate & Number & Rate \\
\hline 1968 & 2 & 0.57 & 3 & 0.77 & 38 & 10.27 & & & 11 & 2.78 & 54 & 14.38 \\
\hline 1969 & 1 & 0.28 & 4 & 1.04 & 35 & 9.44 & & & 8 & 2.08 & 48 & 12.84 \\
\hline 1970 & 4 & 1.12 & 10 & 2.58 & 15 & 3.91 & & & 37 & 9.68 & 64 & 16.81 \\
\hline 1971 & 1 & 0.27 & 46 & 11.65 & 15 & 3.82 & & & 50 & 12.67 & 109 & 27.64 \\
\hline 1972 & 2 & 0.58 & 48 & 11.77 & 38 & 9.26 & & & 94 & 23.20 & 180 & 44.29 \\
\hline 1973 & 2 & 0.59 & 45 & 10.94 & 22 & 5.21 & & & 80 & 19.40 & 147 & 35.69 \\
\hline 1974 & 2 & 0.49 & 43 & 9.72 & 22 & 5.26 & & & 59 & 14.16 & 125 & 29.45 \\
\hline 1975 & 5 & 1.19 & 55 & 12.96 & 17 & 3.84 & & & 65 & 14.73 & 141 & 32.49 \\
\hline 1976 & 3 & 0.69 & 55 & 12.11 & 20 & 4.33 & & & 68 & 14.74 & 143 & 31.20 \\
\hline 1977 & 9 & 1.88 & 40 & 8.75 & 13 & 2.87 & & & 56 & 12.24 & 114 & 24.89 \\
\hline 1978 & 8 & 1.65 & 49 & 9.99 & 14 & 3.08 & & & 54 & 10.84 & 121 & 24.74 \\
\hline 1979 & 13 & 2.75 & 46 & 9.57 & 7 & 1.34 & - & - & 73 & 14.63 & 139 & 28.28 \\
\hline 1980 & 14 & 2.94 & 56 & 11.36 & 8 & 1.70 & - & - & 86 & 16.70 & 164 & 32.70 \\
\hline 1981 & 11 & 2.14 & 66 & 12.40 & 10 & 1.87 & - & - & 72 & 13.35 & 159 & 29.76 \\
\hline 1982 & 11 & 2.08 & 110 & 19.68 & 7 & 1.26 & - & - & 35 & 6.20 & 162 & 29.08 \\
\hline 1983 & 18 & 3.26 & 129 & 22.98 & 7 & 1.22 & - & - & 19 & 3.11 & 172 & 30.49 \\
\hline 1984 & 14 & 2.43 & 133 & 22.03 & 7 & 1.30 & - & - & 16 & 2.31 & 169 & 27.93 \\
\hline 1985 & 27 & 4.81 & 167 & 28.20 & 5 & 0.70 & 1 & 0.21 & 16 & 2.69 & 215 & 36.54 \\
\hline 1986 & 24 & 4.13 & 169 & 28.22 & 8 & 1.16 & - & - & 17 & 2.93 & 218 & 36.44 \\
\hline 1987 & 22 & 3.46 & 150 & 23.55 & 11 & 1.67 & 1 & 0.21 & 14 & 2.14 & 196 & 30.73 \\
\hline 1988 & 35 & 5.58 & 157 & 24.81 & 2 & 0.19 & - & - & 16 & 2.56 & 210 & 33.13 \\
\hline 1989 & 35 & 5.37 & 146 & 21.85 & 4 & 0.56 & - & - & 10 & 1.45 & 195 & 29.23 \\
\hline 1990 & 47 & 6.71 & 131 & 18.48 & 3 & 0.44 & 2 & 0.27 & 17 & 2.38 & 200 & 28.28 \\
\hline 1991 & 36 & 5.20 & 134 & 18.07 & 2 & 0.32 & - & - & 7 & 0.69 & 179 & 24.28 \\
\hline 1992 & 43 & 6.19 & 147 & 18.57 & 3 & 0.38 & - & - & 4 & 0.43 & 196 & 25.46 \\
\hline
\end{tabular}

- indicates no deaths listed. * * no sp ecific code for by ssinosis in ICD-8 (1968-1978).

NOTE: Rates based on small numbers may be unstable. Sum of individual conditions may exceed total because of deaths with multiple pneumoconioses. See Appendix A for source description and Appendix B for methods and ICD-8 and ICD-9 codes.

SOURCE: National Center for Health Statistics multiple cause of death data. Population estimates from U.S. Bureau of the Census. 
Figure VA-2. All pneumoconioses: Distribution of deaths by sex and race, Virginia residents age 15 and over, 1968-1992
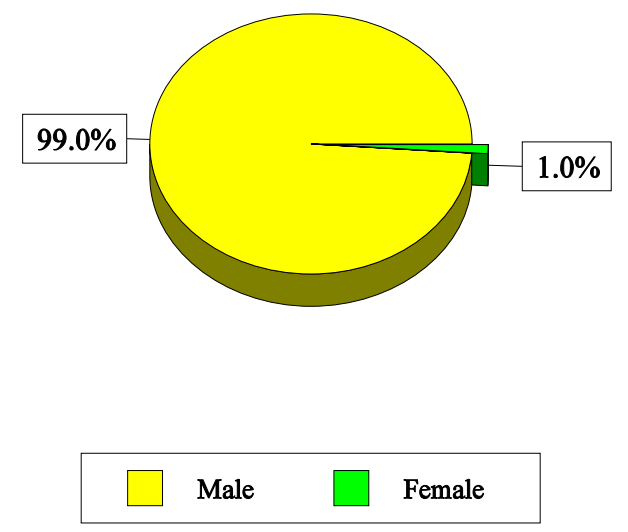
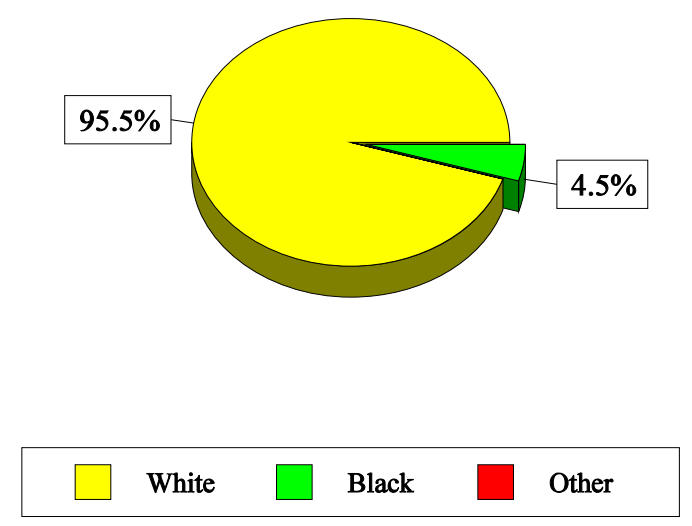

NOTE: See Appendix A for source description and Appendix B for methods and ICD-8 and ICD-9 codes.

SOURCE: National Center for Health Statistics multiple cause of death data.

Table VA-2. All pneumoconioses: Distribution of deaths by sex, race, and age, Virginia residents age 15 and over, 1968-1992

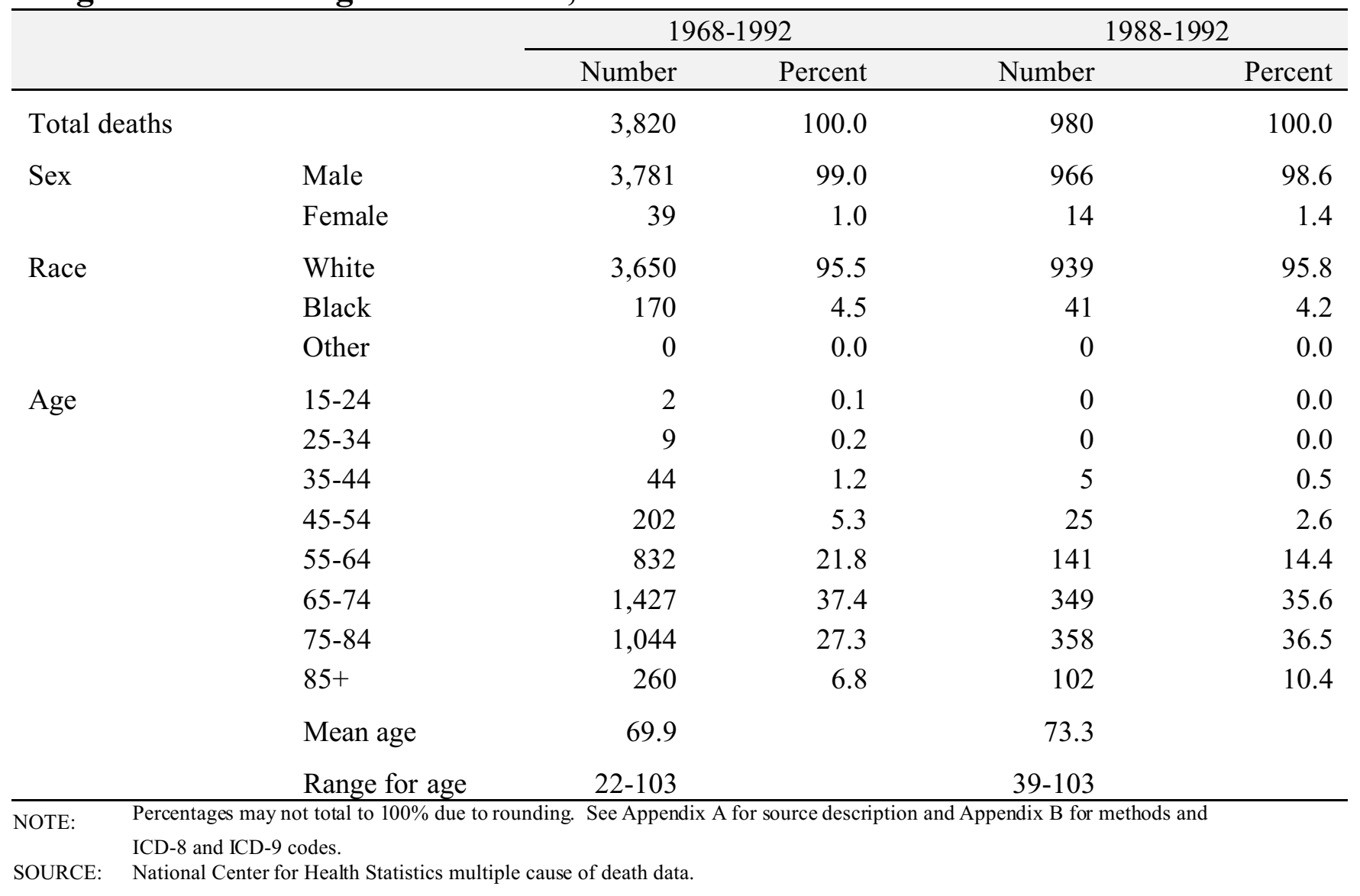




\section{Figure VA-3. Pneumoconiosis deaths by condition, Virginia residents age 15 and over, 1968-1992}

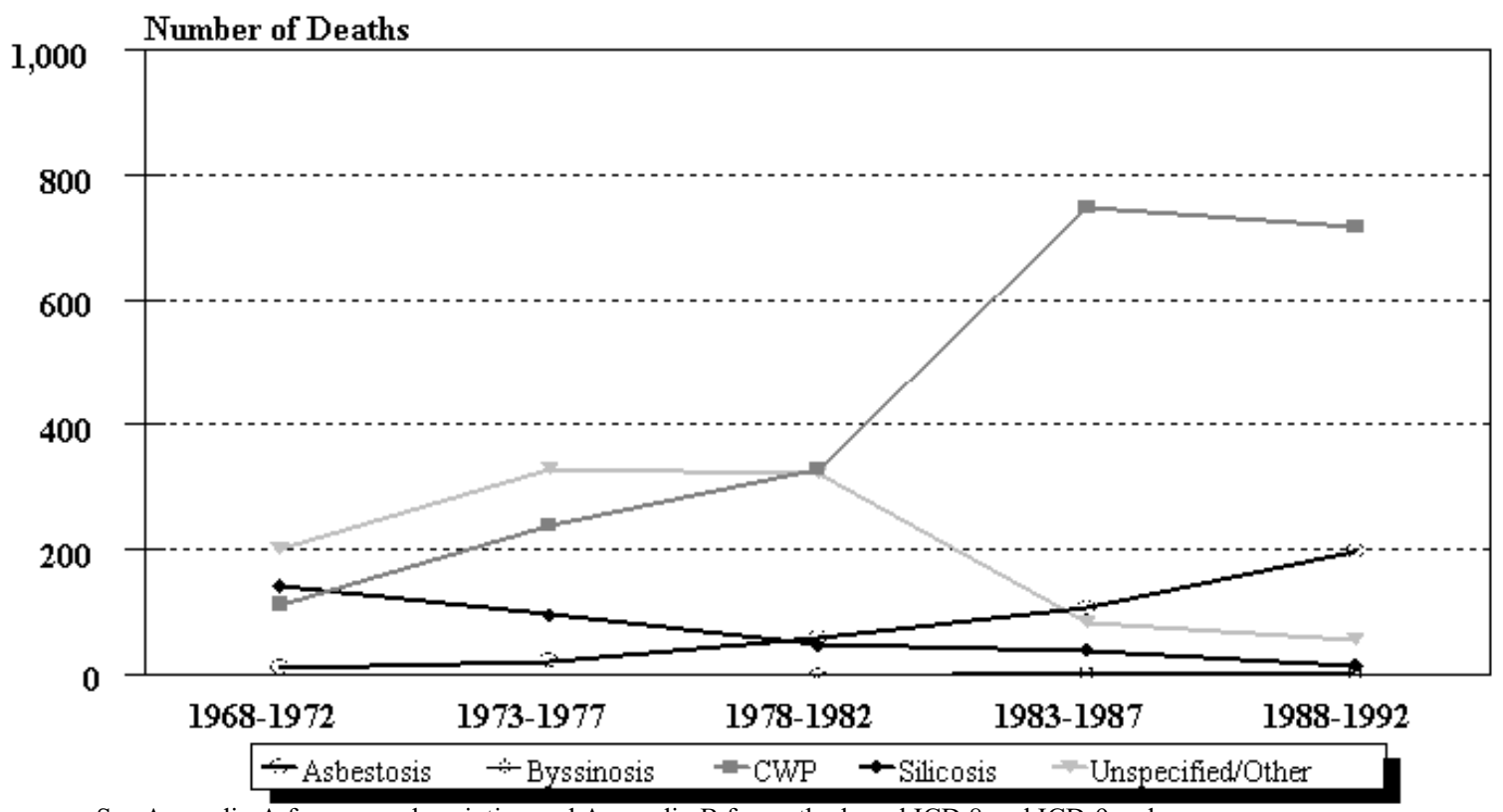

NOTE: See Appendix A for source description and Appendix B for methods and ICD-8 and ICD-9 codes.

SOURCE: National Center for Health Statistics multiple cause of death data.

Figure VA-4. Virginia and U.S. age-adjusted mortality rates (per 1,000,000 population) by condition, residents age 15 and over, 1983-1992

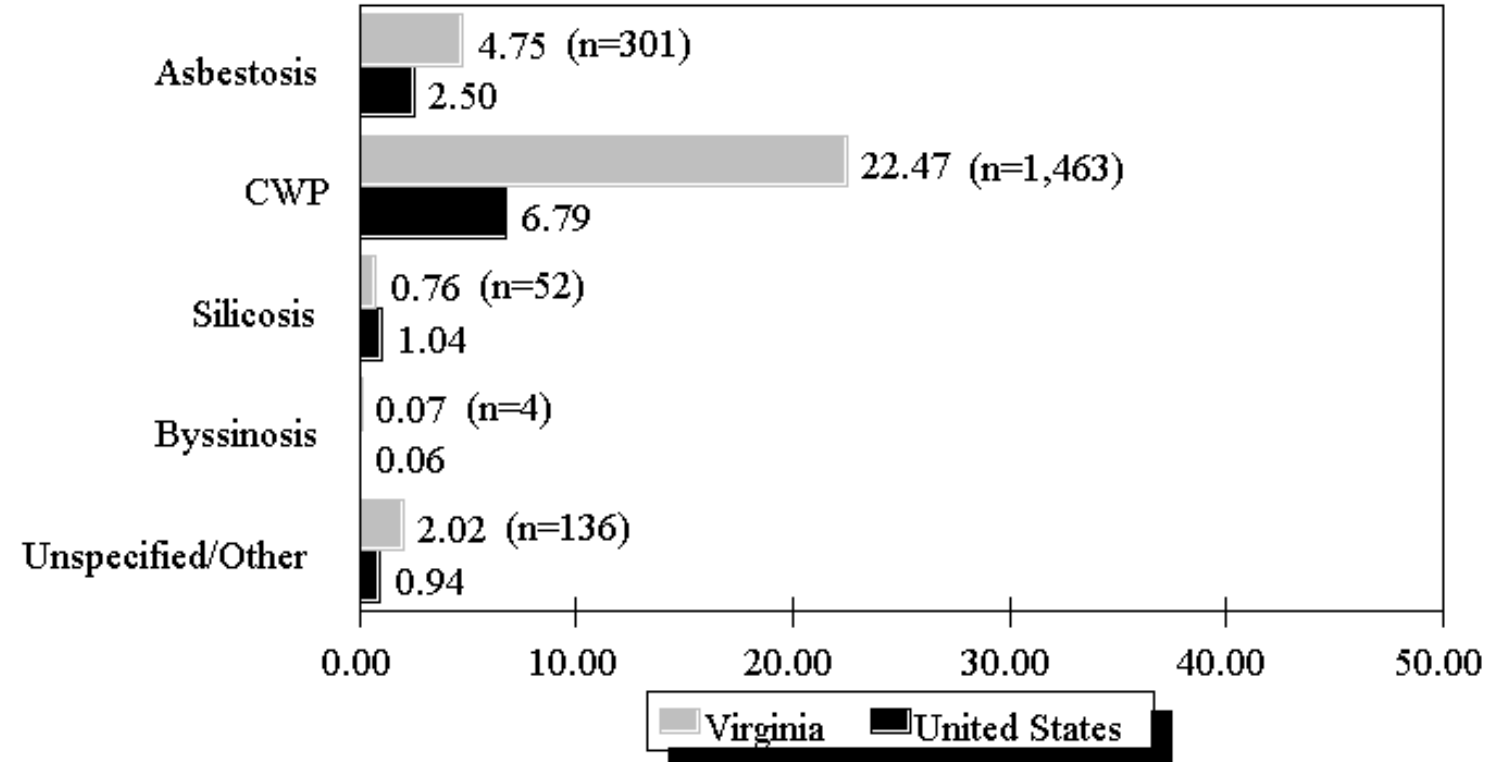

NOTE: Rates based on small numbers may be unstable. See Appendix A for source description and Appendix B for methods and ICD-8 and ICD-9 codes.

SOURCE: National Center for Health Statistics multiple cause of death data. Population estimates from U.S. Bureau of the Census. 
Table VA-3 (page 1 of 4). Number of deaths and age-adjusted mortality rate (per $1,000,000$ population) by condition and county, Virginia residents age 15 and over, 1983-1992

\begin{tabular}{|c|c|c|c|c|c|c|c|c|c|c|c|c|}
\hline \multirow[b]{2}{*}{ County } & \multicolumn{2}{|c|}{ Asbestosis } & \multicolumn{2}{|c|}{$\begin{array}{c}\text { Coal workers' } \\
\text { pneumoconiosis }\end{array}$} & \multicolumn{2}{|c|}{ Silicosis } & \multicolumn{2}{|c|}{ Byssinosis } & \multicolumn{2}{|c|}{$\begin{array}{r}\text { Unspecified/Other } \\
\text { pneumoconioses }\end{array}$} & \multicolumn{2}{|c|}{$\begin{array}{r}\text { All } \\
\text { pneumoconioses }\end{array}$} \\
\hline & $\#$ & Rate & $\#$ & Rate & $\#$ & Rate & $\#$ & Rate & $\#$ & Rate & \# & Rate \\
\hline Accomack & 2 & 4.93 & 1 & 1.11 & - & - & - & - & - & - & 3 & 6.04 \\
\hline Albemarle & - & - & 3 & 3.37 & - & - & - & - & 1 & 1.94 & 4 & 5.31 \\
\hline Alleghany & - & - & 1 & 4.15 & - & - & - & - & - & - & 1 & 4.15 \\
\hline Amherst & - & - & - & - & 3 & 7.52 & - & - & 2 & 5.52 & 5 & 13.03 \\
\hline Arlington & 2 & 0.68 & 4 & 2.08 & - & - & - & - & - & - & 6 & 2.76 \\
\hline Augusta & 4 & 6.27 & 3 & 4.32 & - & - & - & - & - & - & 7 & 10.60 \\
\hline Bedford & - & - & 5 & 10.48 & - & - & - & - & - & - & 5 & 10.48 \\
\hline Bland & - & - & 5 & 59.66 & - & - & - & - & 1 & 7.59 & 6 & 67.25 \\
\hline Botetourt & 2 & 7.86 & - & - & 1 & 2.48 & - & - & - & - & 3 & 10.34 \\
\hline Buchanan & 1 & 3.95 & 292 & 1036.86 & - & - & - & - & 7 & 26.49 & 300 & 1067.29 \\
\hline Buckingham & 2 & 11.02 & 1 & 3.60 & - & - & - & - & 1 & 3.60 & 4 & 18.22 \\
\hline Campbell & - & - & - & - & 1 & 2.20 & - & - & - & - & 1 & 2.20 \\
\hline Caroline & - & - & 2 & 8.81 & - & - & - & - & - & - & 2 & 8.81 \\
\hline Carroll & 3 & 4.63 & 6 & 12.50 & 1 & 3.16 & - & - & 2 & 4.70 & 12 & 24.99 \\
\hline Chesterfield & 5 & 3.93 & 2 & 1.47 & - & - & - & - & - & - & 7 & 5.40 \\
\hline Clarke & - & - & 1 & 8.14 & 1 & 8.14 & - & - & - & - & 2 & 16.28 \\
\hline Craig & 1 & 21.36 & - & - & - & - & - & - & - & - & 1 & 21.36 \\
\hline Culpeper & - & - & 2 & 8.38 & - & - & - & - & - & - & 2 & 8.38 \\
\hline Dickinson & - & - & 128 & 613.86 & - & - & - & - & 9 & 36.07 & 137 & 649.94 \\
\hline Dinwiddie & 1 & 4.65 & - & - & - & - & - & - & - & - & 1 & 4.65 \\
\hline Fairfax & 1 & 0.14 & 7 & 1.27 & 2 & 0.35 & - & - & 2 & 0.36 & 11 & 1.95 \\
\hline Fauquier & - & - & 1 & 2.73 & - & - & - & - & 1 & 2.73 & 2 & 5.45 \\
\hline Floyd & - & - & 1 & 3.16 & - & - & - & - & 1 & 7.38 & 2 & 10.54 \\
\hline Fluvanna & 1 & 7.99 & - & - & 1 & 4.96 & - & - & - & - & 2 & 12.95 \\
\hline Franklin & 1 & 1.38 & 4 & 9.84 & - & - & - & - & - & - & 5 & 11.21 \\
\hline Frederick & - & - & - & - & 1 & 3.01 & - & - & - & - & 1 & 3.01 \\
\hline Giles & 2 & 5.47 & 4 & 13.07 & 1 & 2.73 & - & - & 2 & 7.60 & 9 & 28.87 \\
\hline Gloucester & 2 & 7.90 & 1 & 2.08 & - & - & - & - & - & - & 3 & 9.98 \\
\hline Goochland & - & - & 1 & 4.40 & - & - & - & - & - & - & 1 & 4.40 \\
\hline Grayson & - & - & 1 & 5.00 & - & - & - & - & - & - & 1 & 5.00 \\
\hline Greensville & - & - & 1 & 8.20 & 1 & 8.20 & - & - & 3 & 30.40 & 4 & 38.59 \\
\hline Halifax & 1 & 4.69 & 1 & 2.96 & 2 & 4.40 & - & - & - & - & 4 & 12.04 \\
\hline Hanover & 4 & 6.12 & - & - & - & - & - & - & - & - & 4 & 6.12 \\
\hline
\end{tabular}

See footnotes at end of table. 
Table VA-3 (page 2 of 4). Number of deaths and age-adjusted mortality rate (per 1,000,000 population) by condition and county, Virginia residents age 15 and over, 1983-1992

\begin{tabular}{|c|c|c|c|c|c|c|c|c|c|c|c|c|}
\hline \multirow[b]{2}{*}{ County } & \multicolumn{2}{|c|}{ Asbestosis } & \multicolumn{2}{|c|}{$\begin{array}{c}\text { Coal workers' } \\
\text { pneumoconiosis }\end{array}$} & \multicolumn{2}{|c|}{ Silicosis } & \multicolumn{2}{|c|}{ Byssinosis } & \multicolumn{2}{|c|}{$\begin{array}{r}\text { Unspecified/Other } \\
\text { pneumoconioses }\end{array}$} & \multicolumn{2}{|c|}{$\begin{array}{r}\text { All } \\
\text { pneumoconioses }\end{array}$} \\
\hline & \# & Rate & $\#$ & Rate & \# & Rate & $\#$ & Rate & $\#$ & Rate & $\#$ & Rate \\
\hline Henrico & 6 & 2.36 & - & - & - & - & - & - & - & - & 6 & 2.36 \\
\hline Henry & - & - & 6 & 7.34 & 1 & 1.12 & - & - & - & - & 7 & 8.46 \\
\hline Highland & - & - & - & - & 1 & 12.63 & - & - & - & - & 1 & 12.63 \\
\hline Isle of Wight & 10 & 41.83 & - & - & - & - & - & - & - & - & 10 & 41.83 \\
\hline James City & - & - & 1 & 3.23 & - & - & - & - & - & - & 1 & 3.23 \\
\hline $\begin{array}{l}\text { King and } \\
\text { Queen }\end{array}$ & 1 & 13.53 & - & - & - & - & - & - & - & - & 1 & 13.53 \\
\hline Lancaster & 1 & 5.47 & - & - & - & - & - & - & - & - & 1 & 5.47 \\
\hline Lee & - & - & 80 & 217.51 & - & - & - & - & 10 & 28.43 & 90 & 245.94 \\
\hline Loudoun & - & - & 2 & 2.75 & - & - & - & - & 1 & 2.20 & 3 & 4.95 \\
\hline Louisa & - & - & - & - & 1 & 4.77 & - & - & - & - & 1 & 4.77 \\
\hline Mathews & 1 & 7.98 & - & - & - & - & - & - & - & - & 1 & 7.98 \\
\hline Mecklenburg & 3 & 7.94 & - & - & 1 & 2.65 & - & - & - & - & 4 & 10.59 \\
\hline Middlesex & 1 & 7.70 & 1 & 7.70 & - & - & - & - & 1 & 7.70 & 3 & 23.09 \\
\hline Montgomery & - & - & 1 & 1.14 & 1 & 1.14 & - & - & - & - & 2 & 2.29 \\
\hline Nelson & - & - & 1 & 3.39 & - & - & - & - & - & - & 1 & 3.39 \\
\hline Northampton & 1 & 2.44 & - & - & 1 & 5.44 & - & - & - & - & 2 & 7.89 \\
\hline $\begin{array}{l}\text { North- } \\
\text { umberland }\end{array}$ & 1 & 5.46 & - & - & - & - & - & - & - & - & 1 & 5.46 \\
\hline Nottoway & 1 & 2.34 & 1 & 2.34 & - & - & - & - & - & - & 2 & 4.67 \\
\hline Orange & - & - & 2 & 4.49 & - & - & - & - & - & - & 2 & 4.49 \\
\hline Page & - & - & 1 & 4.16 & - & - & - & - & - & - & 1 & 4.16 \\
\hline Patrick & 1 & 2.26 & 3 & 9.47 & - & - & - & - & - & - & 4 & 11.73 \\
\hline Pittsylvania & 1 & 1.25 & 1 & 0.73 & 2 & 1.98 & - & - & 1 & 0.73 & 5 & 4.69 \\
\hline Powhatan & 1 & 6.42 & 1 & 9.01 & - & - & - & - & - & - & 2 & 15.43 \\
\hline Prince Edward & - & - & 1 & 2.48 & - & - & - & - & - & - & 1 & 2.48 \\
\hline Prince George & 1 & 5.49 & 1 & 5.49 & - & - & - & - & 1 & 5.49 & 2 & 10.98 \\
\hline Prince William & 2 & 2.64 & 4 & 5.49 & - & - & - & - & 1 & 1.42 & 7 & 9.55 \\
\hline Pulaski & 2 & 5.38 & 10 & 21.69 & 1 & 2.69 & - & - & 1 & 1.39 & 14 & 31.15 \\
\hline Roanoke & 2 & 1.29 & 3 & 2.54 & - & - & - & - & - & - & 5 & 3.83 \\
\hline Rockbridge & - & - & 1 & 3.22 & 1 & 4.64 & - & - & 1 & 4.64 & 3 & 12.50 \\
\hline Rockingham & 2 & 1.78 & - & - & 1 & 0.89 & - & - & - & - & 3 & 2.67 \\
\hline Russell & - & - & 114 & 335.83 & 1 & 3.47 & - & - & 10 & 27.13 & 125 & 366.43 \\
\hline
\end{tabular}

See footnotes at end of table. 
Table VA-3 (page 3 of 4). Number of deaths and age-adjusted mortality rate (per $1,000,000$ population) by condition and county, Virginia residents age 15 and over, 1983-1992

\begin{tabular}{|c|c|c|c|c|c|c|c|c|c|c|c|c|}
\hline \multirow[b]{2}{*}{ County } & \multicolumn{2}{|c|}{ Asbestosis } & \multicolumn{2}{|c|}{$\begin{array}{c}\text { Coal workers' } \\
\text { pneumoconiosis }\end{array}$} & \multicolumn{2}{|c|}{ Silicosis } & \multicolumn{2}{|c|}{ Byssinosis } & \multicolumn{2}{|c|}{$\begin{array}{c}\text { Unspecified/Other } \\
\text { pneumoconioses }\end{array}$} & \multicolumn{2}{|c|}{$\begin{array}{r}\text { All } \\
\text { pneumoconioses }\end{array}$} \\
\hline & $\#$ & Rate & $\#$ & Rate & $\#$ & Rate & $\#$ & Rate & $\#$ & Rate & $\#$ & Rate \\
\hline Scott & 1 & 3.55 & 29 & 69.42 & - & - & - & - & 5 & 10.30 & 35 & 83.27 \\
\hline Smyth & - & - & 14 & 27.06 & 1 & 1.39 & - & - & - & - & 15 & 28.45 \\
\hline Southampton & - & - & 1 & 3.00 & 1 & 3.00 & - & - & - & - & 2 & 6.00 \\
\hline Spotsylvania & 3 & 7.16 & 2 & 5.08 & - & - & - & - & - & - & 5 & 12.24 \\
\hline Stafford & - & - & 1 & 2.31 & 1 & 2.31 & - & - & - & - & 1 & 2.31 \\
\hline Surry & 2 & 30.47 & - & - & - & - & - & - & 1 & 15.24 & 3 & 45.71 \\
\hline Tazewell & - & - & 285 & 488.14 & 3 & 4.30 & 2 & 3.16 & 23 & 35.94 & 313 & 531.55 \\
\hline Warren & 3 & 11.80 & - & - & - & - & - & - & - & - & 3 & 11.80 \\
\hline Washington & 2 & 3.98 & 49 & 73.70 & 1 & 1.99 & - & - & 2 & 2.15 & 54 & 81.82 \\
\hline Westmoreland & - & - & 1 & 2.58 & - & - & - & - & 1 & 4.83 & 2 & 7.41 \\
\hline Wise & - & - & 291 & 575.96 & - & - & - & - & 24 & 44.95 & 315 & 620.92 \\
\hline Wythe & - & - & 3 & 6.96 & 1 & 1.71 & - & - & 3 & 10.61 & 6 & 17.57 \\
\hline York & 4 & 12.18 & 1 & 3.35 & - & - & - & - & - & - & 5 & 15.53 \\
\hline \multicolumn{13}{|c|}{ Independent cities: } \\
\hline Alexandria & 3 & 2.90 & 2 & 1.15 & 1 & 1.17 & - & - & 1 & 1.16 & 7 & 6.38 \\
\hline Bedford & - & - & 1 & 3.39 & 1 & 3.39 & - & - & - & - & 1 & 3.39 \\
\hline Bristol & 1 & 1.86 & 4 & 9.86 & - & - & - & - & 1 & 4.28 & 6 & 16.00 \\
\hline Buena Vista & - & - & - & - & - & - & - & - & 1 & 6.48 & 1 & 6.48 \\
\hline Charlottesville & 1 & 1.23 & - & - & 1 & 2.42 & - & - & - & - & 2 & 3.65 \\
\hline Chesapeake & 29 & 24.79 & - & - & - & - & - & - & - & - & 29 & 24.79 \\
\hline $\begin{array}{l}\text { Colonial } \\
\text { Heights }\end{array}$ & 1 & 3.46 & - & - & - & - & - & - & - & - & 1 & 3.46 \\
\hline Covington & - & - & - & - & - & - & - & - & 1 & 10.06 & 1 & 10.06 \\
\hline Danville & 1 & 0.99 & - & - & - & - & 1 & 2.14 & 1 & 0.99 & 3 & 4.12 \\
\hline Fairfax & - & - & 1 & 5.44 & - & - & - & - & - & - & 1 & 5.44 \\
\hline Fredericksburg & 1 & 2.47 & - & - & - & - & - & - & - & - & 1 & 2.47 \\
\hline Galax & 1 & 4.22 & - & - & - & - & - & - & - & - & 1 & 4.22 \\
\hline Hampton & 31 & 25.34 & 6 & 4.79 & - & - & - & - & 1 & 0.67 & 38 & 30.80 \\
\hline Harrisonburg & 2 & 6.84 & - & - & - & - & - & - & - & - & 2 & 6.84 \\
\hline Lynchburg & - & - & 2 & 1.99 & 5 & 8.20 & - & - & 1 & 0.54 & 8 & 10.73 \\
\hline Manassas & - & - & 1 & 9.39 & 1 & 5.06 & - & - & - & - & 2 & 14.45 \\
\hline Manassas Park & 1 & 30.89 & 1 & 30.89 & - & - & - & - & - & - & 2 & 61.77 \\
\hline
\end{tabular}

See footnotes at end of table.

\section{Virginia}


Table VA-3 (page 4 of 4). Number of deaths and age-adjusted mortality rate (per $1,000,000$ population) by condition and county, Virginia residents age 15 and over, 1983-1992

\begin{tabular}{|c|c|c|c|c|c|c|c|c|c|c|c|c|}
\hline & \multicolumn{2}{|c|}{ Asbestosis } & \multicolumn{2}{|c|}{$\begin{array}{r}\text { Coal workers' } \\
\text { pneumoconiosis }\end{array}$} & \multicolumn{2}{|c|}{ Silicosis } & \multicolumn{2}{|c|}{ Byssinosis } & \multicolumn{2}{|c|}{$\begin{array}{r}\text { Unspecified/Other } \\
\text { pneumoconioses }\end{array}$} & \multicolumn{2}{|c|}{$\begin{array}{r}\text { All } \\
\text { pneumoconioses }\end{array}$} \\
\hline & \# & Rate & \# & Rate & \# & Rate & \# & Rate & $\#$ & Rate & $\#$ & Rate \\
\hline \multicolumn{13}{|c|}{ Independent cities: } \\
\hline Martinsville & 1 & 4.51 & 2 & 6.62 & - & - & - & - & - & - & 3 & 11.13 \\
\hline Newport News & 34 & 20.80 & 4 & 1.98 & 1 & 0.49 & - & - & 1 & 0.79 & 40 & 24.05 \\
\hline Norfolk & 25 & 11.25 & 3 & 1.46 & 1 & 0.26 & - & - & 2 & 0.97 & 31 & 13.94 \\
\hline Norton & - & - & 28 & 445.35 & - & - & - & - & 3 & 53.04 & 31 & 498.39 \\
\hline Petersburg & - & - & 1 & 2.38 & - & - & - & - & - & - & 1 & 2.38 \\
\hline Poquoson & 8 & 84.92 & 1 & 12.66 & - & - & - & - & - & - & 9 & 97.58 \\
\hline Portsmouth & 36 & 28.90 & - & - & 2 & 2.09 & - & - & - & - & 38 & 30.99 \\
\hline Radford & - & - & 1 & 5.23 & - & - & - & - & - & - & 1 & 5.23 \\
\hline Richmond & 7 & 3.04 & - & - & 1 & 0.47 & - & - & 1 & 0.19 & 9 & 3.70 \\
\hline Roanoke & 5 & 2.89 & 9 & 4.89 & 2 & 1.26 & - & - & 2 & 0.74 & 18 & 9.79 \\
\hline Salem & - & - & 1 & 1.75 & - & - & - & - & 1 & 5.90 & 2 & 7.65 \\
\hline Suffolk & 12 & 17.95 & 1 & 1.09 & - & - & - & - & 1 & 1.09 & 14 & 20.12 \\
\hline Virginia Beach & 8 & 3.46 & 3 & 1.15 & 1 & 0.38 & 1 & 0.48 & - & - & 13 & 5.47 \\
\hline Waynesboro & 2 & 4.85 & 1 & 4.52 & - & - & - & - & - & - & 3 & 9.36 \\
\hline Winchester & 3 & 13.05 & - & - & - & - & - & - & - & - & 3 & 13.05 \\
\hline
\end{tabular}




\section{Washington: Selected statistics from the United States section}

All pneumoconioses mortality, 1988-1992

Total deaths

Crude mortality rate (per $1,000,000$ population)

Age-adjusted mortality rate (per $1,000,000$ population)

Total years of potential life lost to life expectancy

Years of potential life lost to life expectancy (per death) $\underline{\text { Value }}$

243

12.77

7.54

2,755

11.3

Deaths by type of pneumoconiosis, 1988-1992

Asbestosis

Coal workers' pneumoconiosis

Silicosis

Byssinosis

Unspecified/Other pneumoconiosis $\underline{\text { Deaths* }}$

195

distribution

15

22

0

13
Percent**

$\underline{\text { State rank }}$

16

14

14

16

37

80.2

6.2

9.1

0.0

5.3

* Sum of column may exceed total deaths because of deaths with multiple pneumoconioses.

** Percentages may total more than $100 \%$ due to deaths with multiple pneumoconioses.

NOTE: $\quad$ See Appendix A for source description and Appendix B for methods and ICD-8 and ICD-9 codes.

SOURCE: National Center for Health Statistics multiple cause of death data. Population estimates from U.S. Bureau of the Census.

Figure WA-1. All pneumoconioses: Washington and U.S. age-adjusted mortality rates, residents age 15 and over, 1968-1992

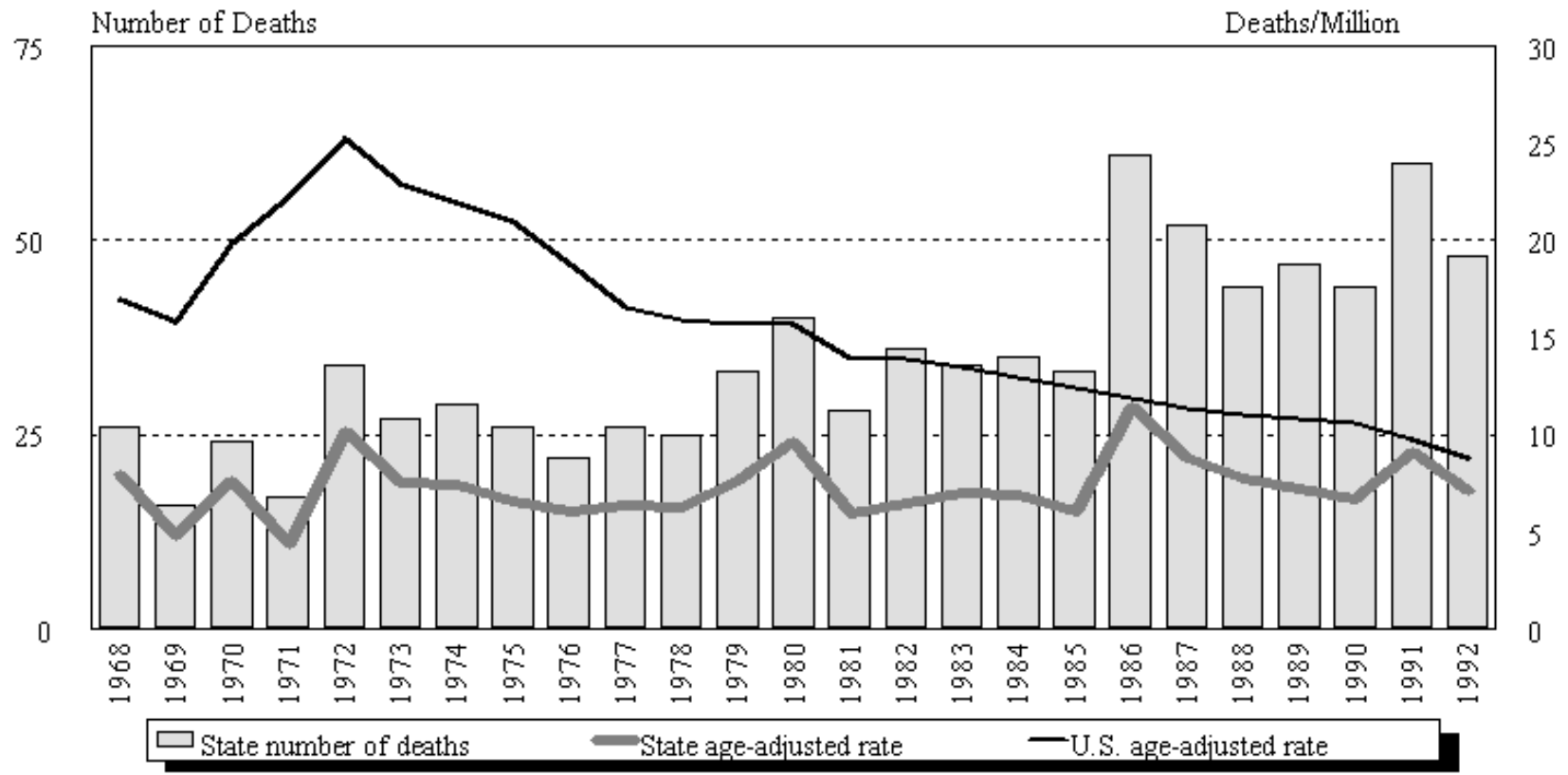

NOTE: $\quad$ See Appendix A for source description and Appendix B for methods and ICD-8 and ICD-9 codes.

SOURCE: National Center for Health Statistics multiple cause of death data. Population estimates from U.S. Bureau of the Census. 


\section{Washington}

Table WA-1. Number of deaths and age-adjusted mortality rate (per 1,000,000 population) by condition, Washington residents age 15 and over, 1968-1992

\begin{tabular}{|c|c|c|c|c|c|c|c|c|c|c|c|c|}
\hline \multirow[b]{2}{*}{ Year } & \multicolumn{2}{|c|}{ Asbestosis } & \multicolumn{2}{|c|}{$\begin{array}{l}\text { Coal workers' } \\
\text { pneumoconiosis }\end{array}$} & \multicolumn{2}{|c|}{ Silicosis } & \multicolumn{2}{|c|}{ Byssinosis* } & \multicolumn{2}{|c|}{$\begin{array}{c}\text { Unspecified/Other } \\
\text { pneumoconioses }\end{array}$} & \multicolumn{2}{|c|}{ All pneumoconioses } \\
\hline & Number & Rate & Number & Rate & Number & Rate & Number & Rate & Number & Rate & Number & Rate \\
\hline 1968 & 6 & 2.28 & 1 & 0.34 & 17 & 4.97 & & & 2 & 0.37 & 26 & 7.95 \\
\hline 1969 & - & - & 1 & 0.14 & 15 & 4.59 & & & - & - & 16 & 4.73 \\
\hline 1970 & 4 & 1.43 & 3 & 0.90 & 11 & 3.31 & & & 6 & 1.99 & 24 & 7.64 \\
\hline 1971 & 6 & 1.70 & - & - & 9 & 2.12 & & & 2 & 0.55 & 17 & 4.36 \\
\hline 1972 & 8 & 2.86 & 2 & 0.68 & 14 & 3.87 & & & 10 & 2.74 & 34 & 10.15 \\
\hline 1973 & 10 & 3.00 & 3 & 0.86 & 12 & 2.94 & & & 2 & 0.71 & 27 & 7.52 \\
\hline 1974 & 7 & 2.07 & 4 & 0.55 & 9 & 2.25 & & & 10 & 2.75 & 29 & 7.41 \\
\hline 1975 & 8 & 2.03 & 2 & 0.50 & 12 & 2.98 & & & 4 & 1.08 & 26 & 6.58 \\
\hline 1976 & 12 & 3.56 & - & - & 6 & 1.45 & & & 4 & 1.00 & 22 & 6.01 \\
\hline 1977 & 10 & 2.56 & 1 & 0.10 & 10 & 2.35 & & & 7 & 1.83 & 26 & 6.37 \\
\hline 1978 & 10 & 2.76 & 2 & 0.49 & 10 & 2.22 & & & 3 & 0.78 & 25 & 6.25 \\
\hline 1979 & 16 & 3.67 & 3 & 0.73 & 14 & 3.39 & 1 & 0.09 & 1 & 0.19 & 33 & 7.62 \\
\hline 1980 & 23 & 6.05 & 7 & 1.40 & 10 & 2.20 & - & - & 1 & 0.09 & 40 & 9.65 \\
\hline 1981 & 18 & 4.04 & 2 & 0.17 & 2 & 0.35 & - & - & 6 & 1.37 & 28 & 5.93 \\
\hline 1982 & 20 & 4.14 & 6 & 0.84 & 10 & 1.51 & - & - & - & - & 36 & 6.48 \\
\hline 1983 & 24 & 5.18 & 4 & 0.72 & 6 & 1.14 & - & - & - & - & 34 & 7.04 \\
\hline 1984 & 28 & 5.60 & 4 & 0.77 & 1 & 0.08 & - & - & 2 & 0.44 & 35 & 6.88 \\
\hline 1985 & 21 & 4.08 & 3 & 0.23 & 8 & 1.54 & - & - & 1 & 0.22 & 33 & 6.08 \\
\hline 1986 & 47 & 9.00 & 7 & 1.09 & 6 & 1.16 & - & - & 1 & 0.22 & 61 & 11.46 \\
\hline 1987 & 40 & 6.92 & 6 & 0.97 & 6 & 0.92 & - & - & - & - & 52 & 8.81 \\
\hline 1988 & 34 & 6.04 & 4 & 0.55 & 6 & 1.14 & - & - & - & - & 44 & 7.73 \\
\hline 1989 & 37 & 5.57 & 3 & 0.46 & 4 & 0.60 & - & - & 3 & 0.53 & 47 & 7.16 \\
\hline 1990 & 34 & 5.30 & 6 & 0.70 & - & - & - & - & 4 & 0.67 & 44 & 6.68 \\
\hline 1991 & 50 & 7.54 & 1 & 0.12 & 7 & 0.99 & - & - & 3 & 0.59 & 60 & 9.12 \\
\hline 1992 & 40 & 6.02 & 1 & 0.12 & 5 & 0.63 & - & - & 3 & 0.42 & 48 & 7.06 \\
\hline
\end{tabular}

indicates no deaths listed. $\quad$ * no sp ecific code for by ssinosis in ICD-8 (1968-1978).

NOTE: Rates based on small numbers may be unstable. Sum of individual conditions may exceed total because of deaths with multiple pneumoconioses. See Appendix A for source description and Appendix B for methods and ICD-8 and ICD-9

SOURCE: National Center for Health Statistics multiple cause of death data. Population estimates from U.S. Bureau of the Census. 


\section{Figure WA-2. All pneumoconioses: Distribution of deaths by sex and race,} Washington residents age 15 and over, 1968-1992
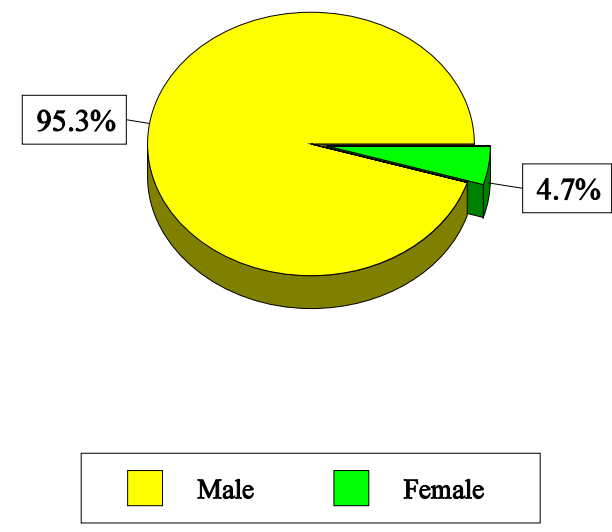
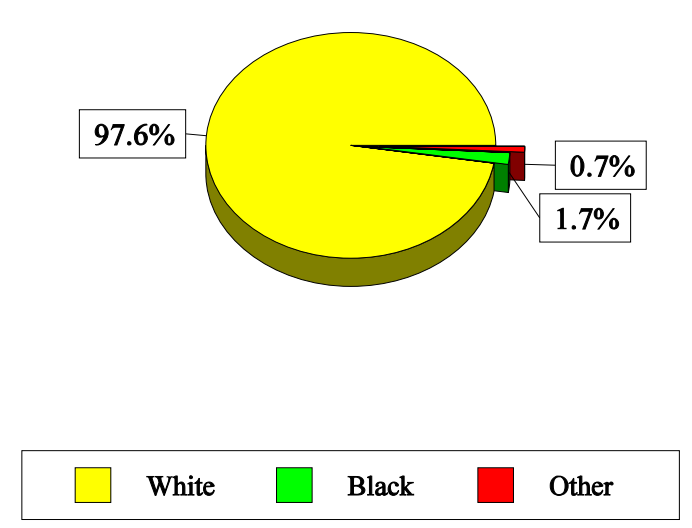

NOTE: $\quad$ See Appendix A for source description and Appendix B for methods and ICD-8 and ICD-9 codes.

SOURCE: National Center for Health Statistics multiple cause of death data.

Table WA-2. All pneumoconioses: Distribution of deaths by sex, race, and age, Washington residents age 15 and over, 1968-1992

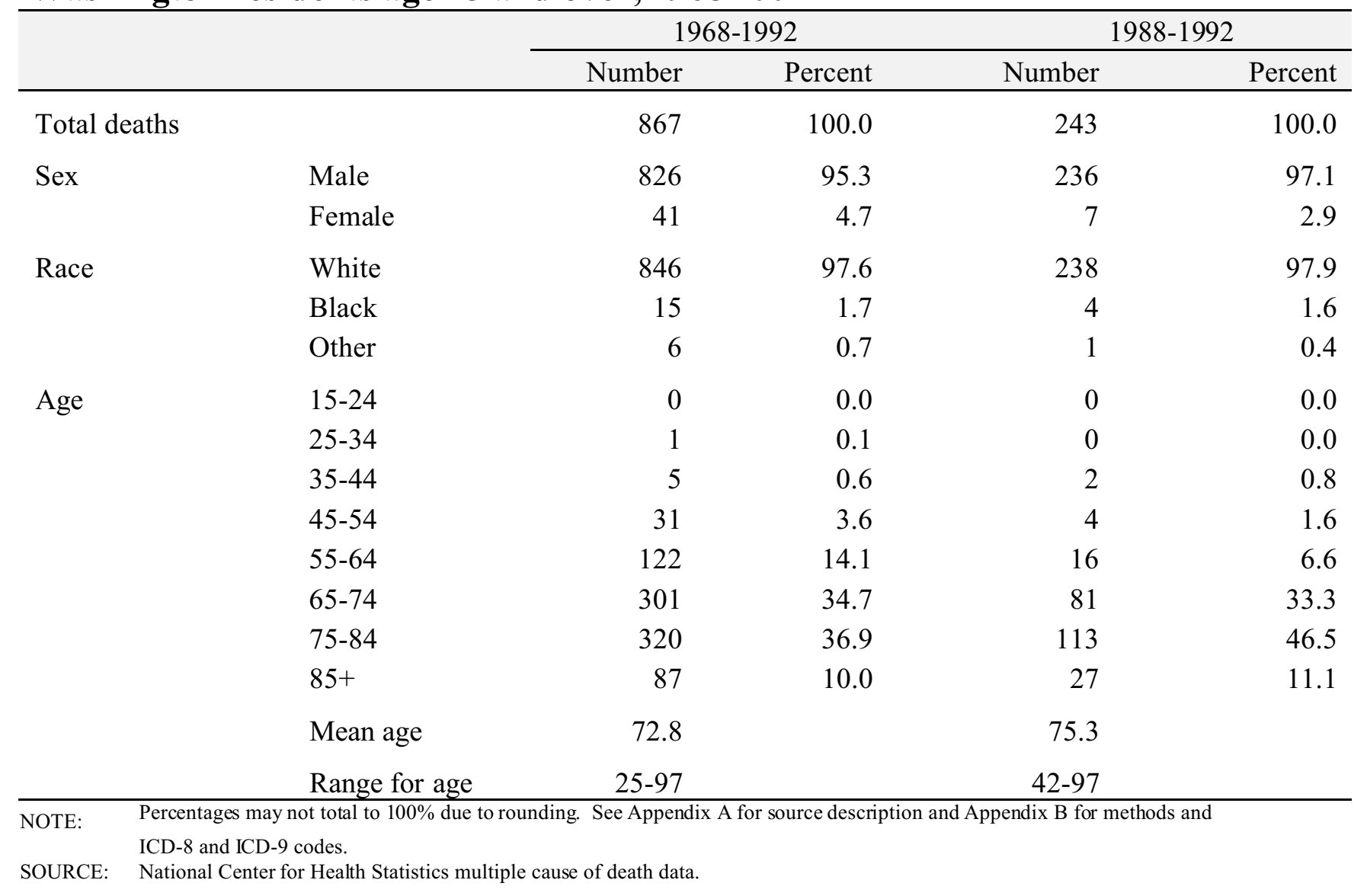


Figure WA-3. Pneumoconiosis deaths by condition, Washington residents age 15 and over, 1968-1992

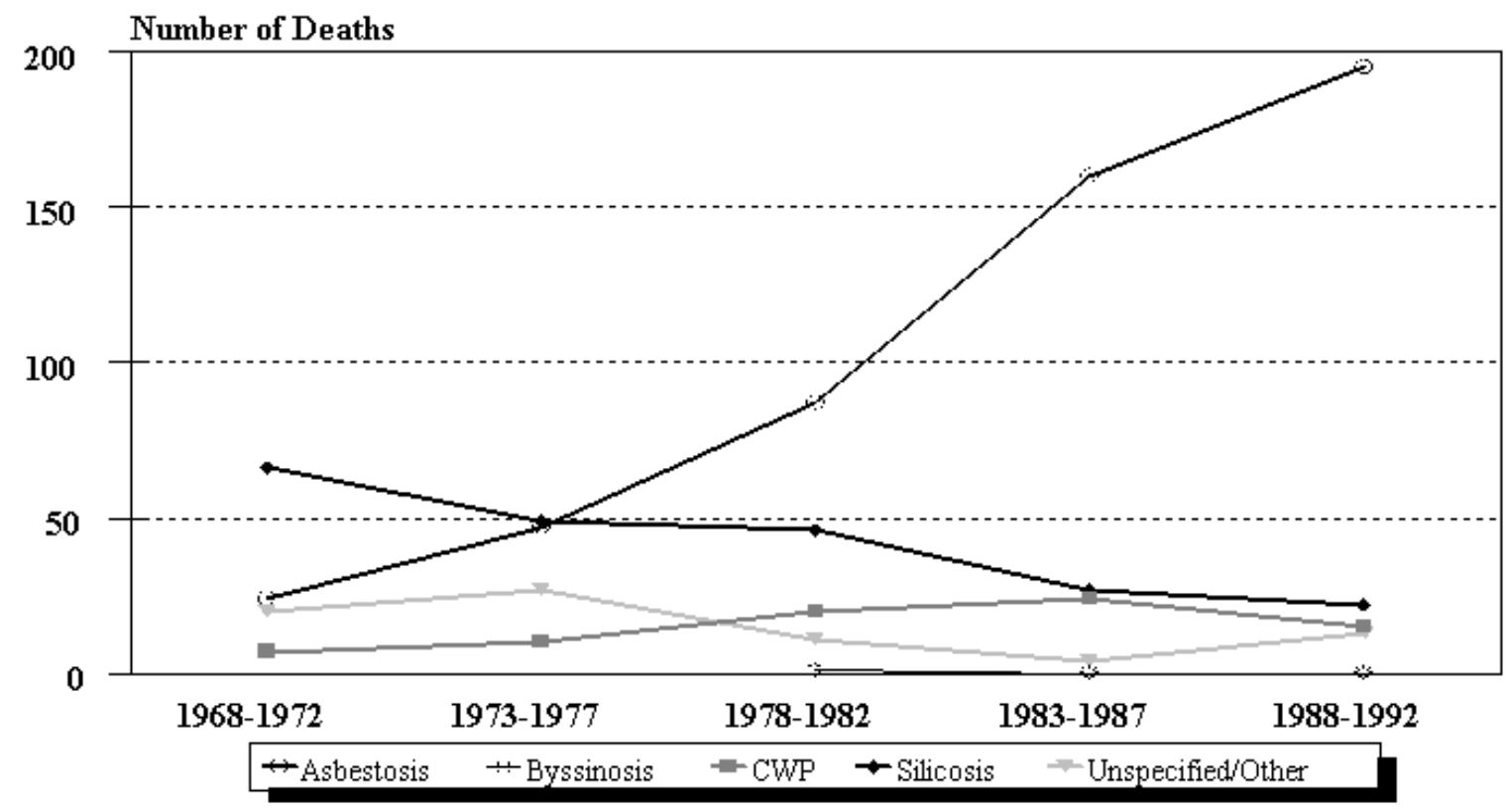

NOTE: See Appendix A for source description and Appendix B for methods and ICD-8 and ICD-9 codes.

SOURCE: National Center for Health Statistics multiple cause of death data.

Figure WA-4. Washington and U.S. age-adjusted mortality rates (per 1,000,000 population) by condition, residents age 15 and over, 1983-1992

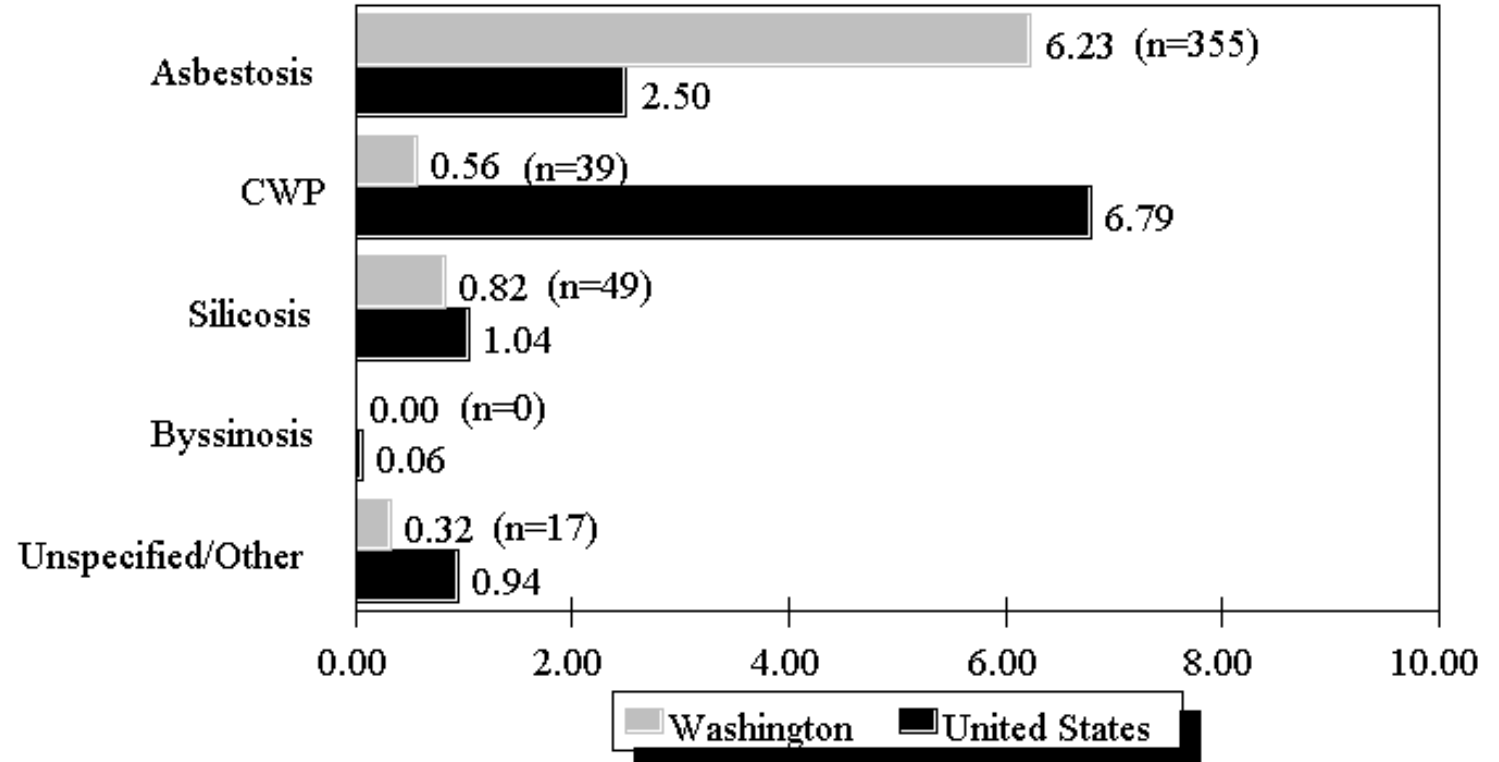

NOTE: Rates based on small numbers may be unstable. See Appendix A for source description and Appendix B for methods and ICD-8 and ICD-9 codes.

SOURCE: National Center for Health Statistics multiple cause of death data. Population estimates from U.S. Bureau of the Census. 
Table WA-3. Number of deaths and age-adjusted mortality rate (per 1,000,000 population) by condition and county, Washington residents age 15 and over, 1983-1992

\begin{tabular}{|c|c|c|c|c|c|c|c|c|c|c|c|c|}
\hline \multirow[b]{2}{*}{ County } & \multicolumn{2}{|c|}{ Asbestosis } & \multicolumn{2}{|c|}{$\begin{array}{r}\text { Coal workers' } \\
\text { pneumoconiosis }\end{array}$} & \multicolumn{2}{|c|}{ Silicosis } & \multicolumn{2}{|c|}{ Byssinosis } & \multicolumn{2}{|c|}{$\begin{array}{r}\text { Unspecified/Other } \\
\text { pneumoconioses }\end{array}$} & \multicolumn{2}{|c|}{$\begin{array}{r}\text { All } \\
\text { pneumoconioses }\end{array}$} \\
\hline & $\#$ & Rate & \# & Rate & \# & Rate & $\#$ & Rate & \# & Rate & \# & Rate \\
\hline Benton & 12 & 11.17 & 2 & 1.79 & - & - & - & - & 1 & 0.69 & 15 & 13.65 \\
\hline Clallam & 5 & 5.61 & 1 & 1.43 & 1 & 1.43 & - & - & 1 & 0.66 & 8 & 9.13 \\
\hline Clark & 20 & 8.83 & 1 & 0.30 & 1 & 0.56 & - & - & - & - & 22 & 9.68 \\
\hline Columbia & - & - & 1 & 8.21 & 1 & 19.47 & - & - & - & - & 2 & 27.68 \\
\hline Cowlitz & 7 & 7.11 & 2 & 1.26 & 2 & 1.26 & - & - & - & - & 11 & 9.62 \\
\hline Douglas & - & - & - & - & 2 & 6.65 & - & - & - & - & 2 & 6.65 \\
\hline Ferry & - & - & - & - & 1 & 19.27 & - & - & - & - & 1 & 19.27 \\
\hline Franklin & 1 & 3.30 & - & - & 1 & 2.10 & - & - & - & - & 2 & 5.40 \\
\hline Grant & 2 & 2.26 & - & - & 1 & 1.13 & - & - & - & - & 3 & 3.39 \\
\hline Grays Harbor & 3 & 2.84 & 1 & 0.69 & - & - & - & - & - & - & 4 & 3.53 \\
\hline Island & 7 & 9.84 & - & - & 2 & 2.24 & - & - & - & - & 9 & 12.08 \\
\hline King & 104 & 6.12 & 11 & 0.63 & 8 & 0.46 & - & - & 4 & 0.25 & 126 & 7.43 \\
\hline Kitsap & 61 & 33.85 & 3 & 1.78 & - & - & - & - & - & - & 64 & 35.63 \\
\hline Kittitas & 3 & 8.12 & 6 & 13.76 & 1 & 1.88 & - & - & - & - & 10 & 23.77 \\
\hline Lewis & - & - & - & - & - & - & - & - & 2 & 2.35 & 2 & 2.35 \\
\hline Mason & 6 & 11.56 & 1 & 1.39 & - & - & - & - & - & - & 7 & 12.95 \\
\hline Okanogan & 2 & 4.44 & - & - & 1 & 1.42 & - & - & - & - & 3 & 5.87 \\
\hline Pacific & 1 & 3.73 & - & - & - & - & - & - & - & - & 1 & 3.73 \\
\hline Pend Oreille & - & - & - & - & 3 & 31.45 & - & - & - & - & 3 & 31.45 \\
\hline Pierce & 41 & 6.52 & 4 & 0.47 & 6 & 1.02 & - & - & 2 & 0.49 & 53 & 8.51 \\
\hline San Juan & 1 & 4.02 & - & - & - & - & - & - & 1 & 7.14 & 2 & 11.16 \\
\hline Skagit & 4 & 2.32 & - & - & 1 & 0.58 & - & - & 2 & 1.83 & 7 & 4.72 \\
\hline Snohomish & 26 & 6.17 & 2 & 0.35 & 4 & 0.83 & - & - & 3 & 0.80 & 35 & 8.14 \\
\hline Spokane & 22 & 5.89 & 1 & 0.39 & 9 & 2.19 & - & - & 1 & 0.31 & 33 & 8.78 \\
\hline Stevens & 1 & 1.77 & 1 & 3.66 & 1 & 3.66 & - & - & - & - & 3 & 9.10 \\
\hline Thurston & 12 & 6.21 & - & - & - & - & - & - & - & - & 12 & 6.21 \\
\hline Wahkiakum & 1 & 23.49 & - & - & - & - & - & - & - & - & 1 & 23.49 \\
\hline Walla Walla & 1 & 2.08 & - & - & - & - & - & - & - & - & 1 & 2.08 \\
\hline Whatcom & 3 & 2.35 & - & - & 1 & 0.96 & - & - & - & - & 4 & 3.31 \\
\hline Whitman & 1 & 3.98 & - & - & 1 & 1.70 & - & - & - & - & 2 & 5.68 \\
\hline Yakima & 8 & 3.47 & 2 & 0.53 & 1 & 0.26 & - & - & - & - & 10 & 4.00 \\
\hline
\end{tabular}




\section{West Virginia: Selected statistics from the United States section}

All pneumoconioses mortality, 1988-1992

Total deaths

$\frac{\text { Value }}{1,878}$

$\underline{\text { State rank }}$

Crude mortality rate (per $1,000,000$ population)

262.60

Age-adjusted mortality rate (per $1,000,000$ population)

132.76

Total years of potential life lost to life expectancy

22,378

Years of potential life lost to life expectancy (per death)

11.9

Deaths by type of pneumoconiosis, 1988-1992 $\underline{\text { Deaths* }}$

86

1,458

29

1

309
Percent**

distribution

Coal workers' pneumoconiosis

Silicosis

Byssinosis

Unspecified/Other pneumoconiosis

* Sum of column may exceed total deaths because of deaths with multiple pneumoconioses.

** Percentages may total more than $100 \%$ due to deaths with multiple pneumoconioses.

NOTE: $\quad$ See Appendix A for source description and Appendix B for methods and ICD-8 and ICD-9 codes.

SOURCE: National Center for Health Statistics multiple cause of death data. Population estimates from U.S. Bureau of the Census.

Figure WV-1. All pneumoconioses: West Virginia and U.S. age-adjusted mortality rates, residents age 15 and over, 1968-1992

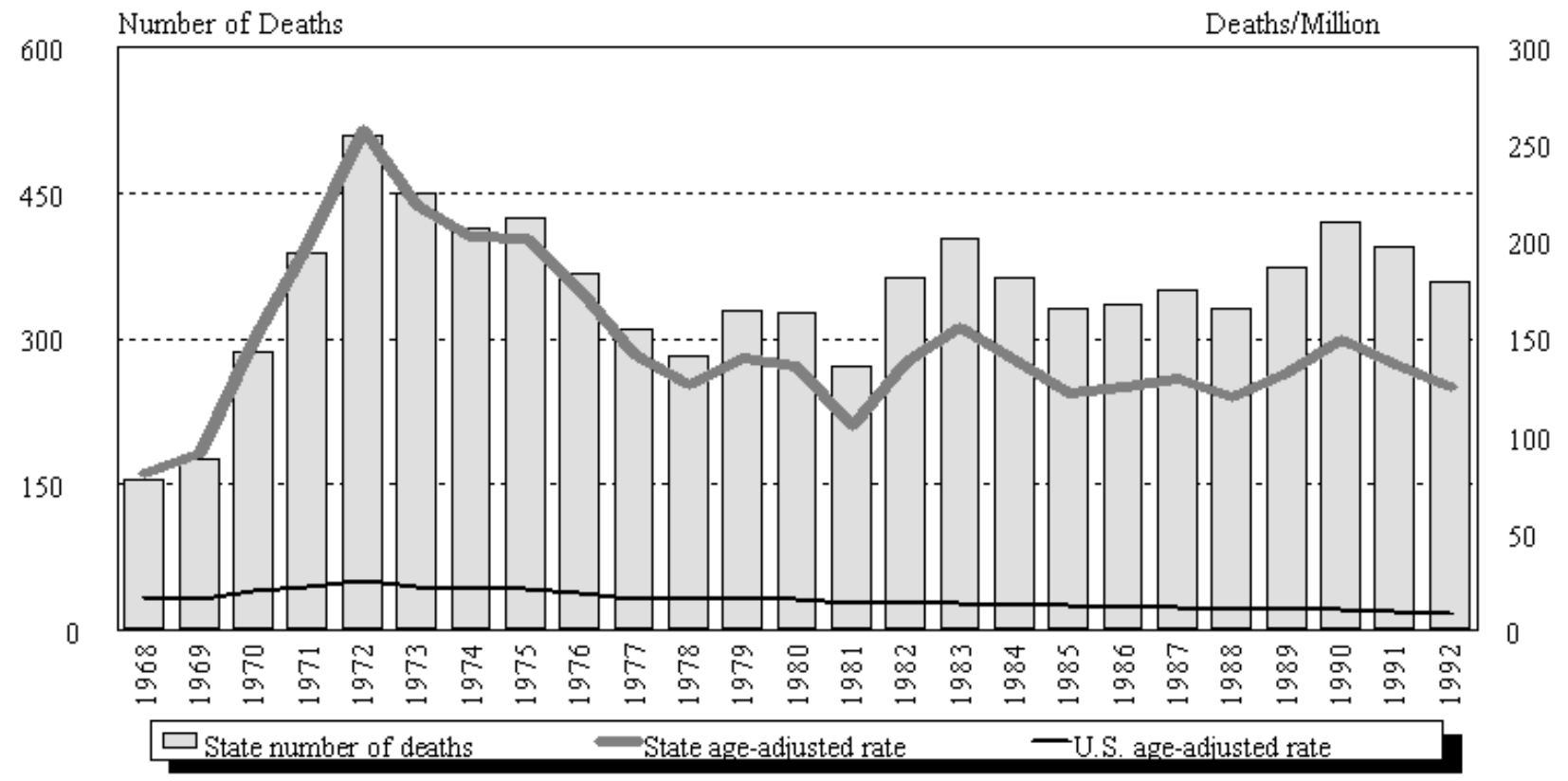

NOTE: $\quad$ See Appendix A for source description and Appendix B for methods and ICD-8 and ICD-9 codes.

SOURCE: National Center for Health Statistics multiple cause of death data. Population estimates from U.S. Bureau of the Census. 
West Virginia

Table WV-1. Number of deaths and age-adjusted mortality rate (per 1,000,000 population) by condition, West Virginia residents age 15 and over, 1968-1992

\begin{tabular}{|c|c|c|c|c|c|c|c|c|c|c|c|c|}
\hline \multirow[b]{2}{*}{ Year } & \multicolumn{2}{|c|}{ Asbestosis } & \multicolumn{2}{|c|}{$\begin{array}{l}\text { Coal workers' } \\
\text { pneumoconiosis }\end{array}$} & \multicolumn{2}{|c|}{ Silicosis } & \multicolumn{2}{|c|}{ Byssinosis* } & \multicolumn{2}{|c|}{$\begin{array}{c}\text { Unspecified/Other } \\
\text { pneu moconioses }\end{array}$} & \multicolumn{2}{|c|}{ All pneumoconioses } \\
\hline & Number & Rate & Number & Rate & Number & Rate & Number & Rate & Number & Rate & Number & Rate \\
\hline 1968 & - & - & 30 & 15.42 & 75 & 38.96 & & & 52 & 27.35 & 156 & 80.98 \\
\hline 1969 & - & - & 37 & 18.75 & 87 & 44.50 & & & 53 & 27.93 & 177 & 91.17 \\
\hline 1970 & 1 & 0.59 & 70 & 37.10 & 71 & 36.74 & & & 150 & 77.68 & 287 & 149.51 \\
\hline 1971 & - & - & 109 & 55.69 & 55 & 26.99 & & & 231 & 119.19 & 388 & 197.85 \\
\hline 1972 & - & - & 190 & 98.39 & 58 & 27.35 & & & 270 & 136.98 & 508 & 257.40 \\
\hline 1973 & - & - & 166 & 82.93 & 47 & 23.65 & & & 241 & 114.89 & 449 & 218.87 \\
\hline 1974 & 1 & 0.58 & 156 & 76.84 & 44 & 21.96 & & & 224 & 108.88 & 413 & 202.07 \\
\hline 1975 & 1 & 0.75 & 159 & 77.85 & 32 & 16.11 & & & 236 & 109.52 & 423 & 201.41 \\
\hline 1976 & - & - & 148 & 71.89 & 32 & 14.11 & & & 190 & 90.17 & 366 & 175.17 \\
\hline 1977 & 1 & 0.75 & 125 & 57.89 & 17 & 7.86 & & & 169 & 77.47 & 309 & 142.53 \\
\hline 1978 & 2 & 1.22 & 115 & 52.44 & 24 & 11.11 & & & 144 & 62.81 & 283 & 126.68 \\
\hline 1979 & 2 & 0.78 & 153 & 65.95 & 10 & 4.34 & - & - & 164 & 69.17 & 329 & 140.23 \\
\hline 1980 & 4 & 1.27 & 262 & 109.60 & 7 & 2.93 & 1 & 0.32 & 54 & 22.58 & 326 & 135.73 \\
\hline 1981 & - & - & 204 & 79.21 & 9 & 3.84 & - & - & 59 & 22.05 & 272 & 105.10 \\
\hline 1982 & 4 & 1.22 & 315 & 120.52 & 7 & 2.50 & - & - & 37 & 14.05 & 363 & 138.28 \\
\hline 1983 & 6 & 2.92 & 345 & 133.53 & 4 & 1.05 & - & - & 48 & 18.64 & 403 & 156.14 \\
\hline 1984 & 3 & 0.87 & 326 & 125.56 & 12 & 3.87 & - & - & 25 & 9.48 & 363 & 138.78 \\
\hline 1985 & 5 & 2.52 & 281 & 103.06 & 5 & 1.56 & - & - & 39 & 14.88 & 330 & 122.03 \\
\hline 1986 & 11 & 4.62 & 302 & 111.52 & 3 & 0.84 & - & - & 20 & 8.23 & 336 & 125.21 \\
\hline 1987 & 15 & 6.14 & 284 & 105.23 & 7 & 1.98 & - & - & 46 & 16.82 & 350 & 129.33 \\
\hline 1988 & 15 & 6.51 & 255 & 93.26 & 5 & 1.50 & - & - & 56 & 19.30 & 330 & 120.15 \\
\hline 1989 & 6 & 2.23 & 333 & 116.01 & 2 & 0.68 & - & - & 33 & 13.20 & 374 & 132.13 \\
\hline 1990 & 17 & 6.42 & 279 & 99.02 & 6 & 2.42 & - & - & 118 & 41.15 & 420 & 149.01 \\
\hline 1991 & 20 & 7.29 & 308 & 104.42 & 12 & 5.75 & 1 & 0.62 & 58 & 20.03 & 395 & 136.44 \\
\hline 1992 & 28 & 10.02 & 283 & 96.58 & 4 & 1.76 & - & - & 44 & 16.49 & 359 & 124.85 \\
\hline
\end{tabular}

- indicates no deaths listed. $\quad *$ no specific code for byssinosis in ICD-8 (1968-1978)

NOTE: Rates based on small numbers may be unstable. Sum of individual conditions may exceed total because of deaths with multiple pneumoconioses. See Appendix A for source description and Appendix B for methods and ICD-8 and ICD-9 codes.

SOURCE: National Center for Health Statistics multiple cause of death data. Population estimates from U.S. Bureau of the Census. 
Figure WV-2. All pneumoconioses: Distribution of deaths by sex and race, West Virginia residents age 15 and over, 1968-1992
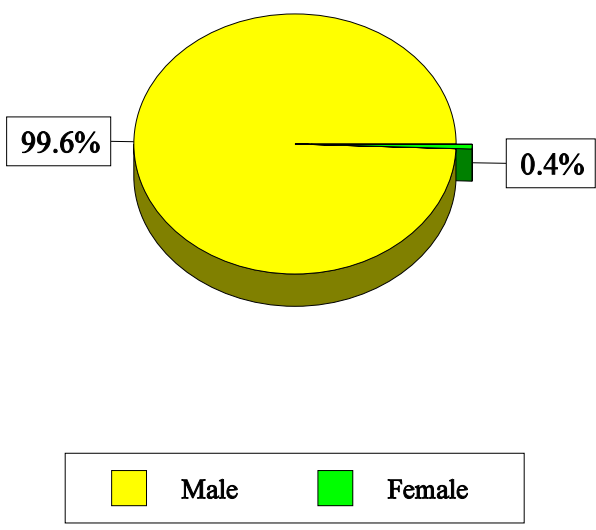
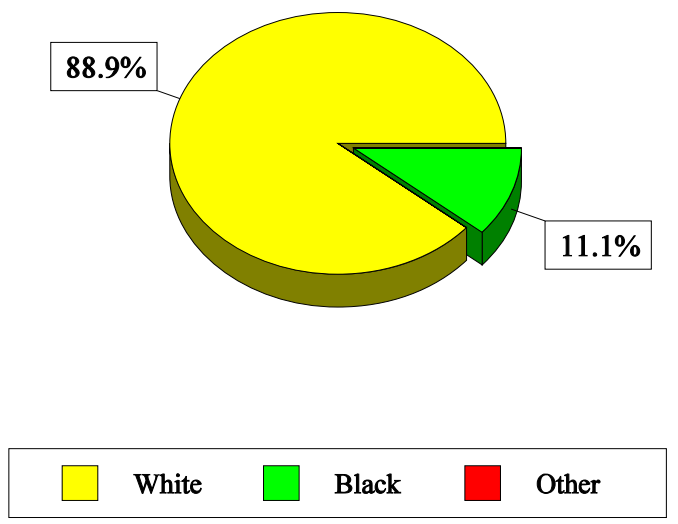

NOTE: See Appendix A for source description and Appendix B for methods and ICD-8 and ICD-9 codes.

SOURCE: National Center for Health Statistics multiple cause of death data.

Table WV-2. All pneumoconioses: Distribution of deaths by sex, race, and age, West Virginia residents age 15 and over, 1968-1992

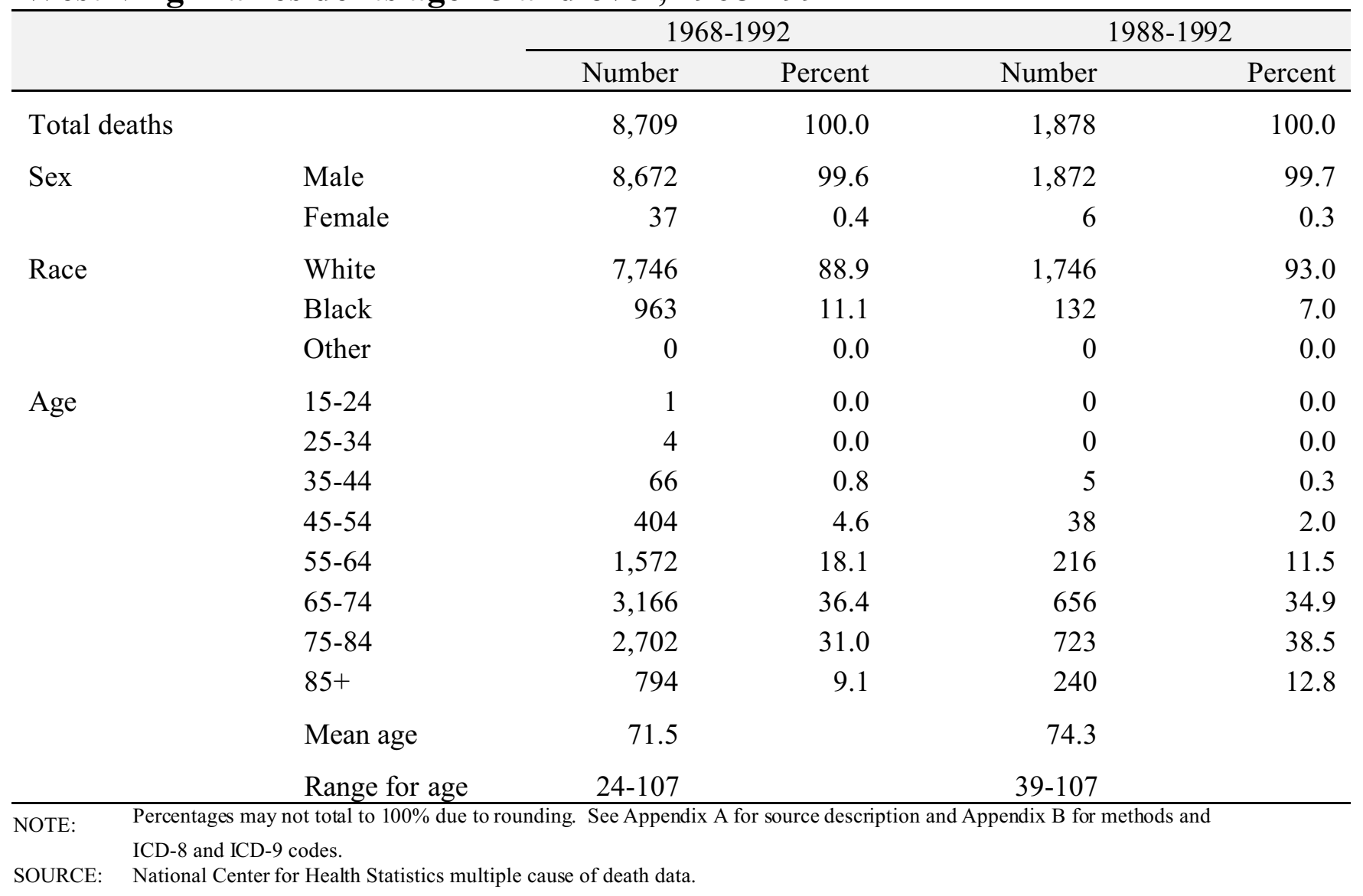


Figure WV-3. Pneumoconiosis deaths by condition, West Virginia residents age 15 and over, 1968-1992

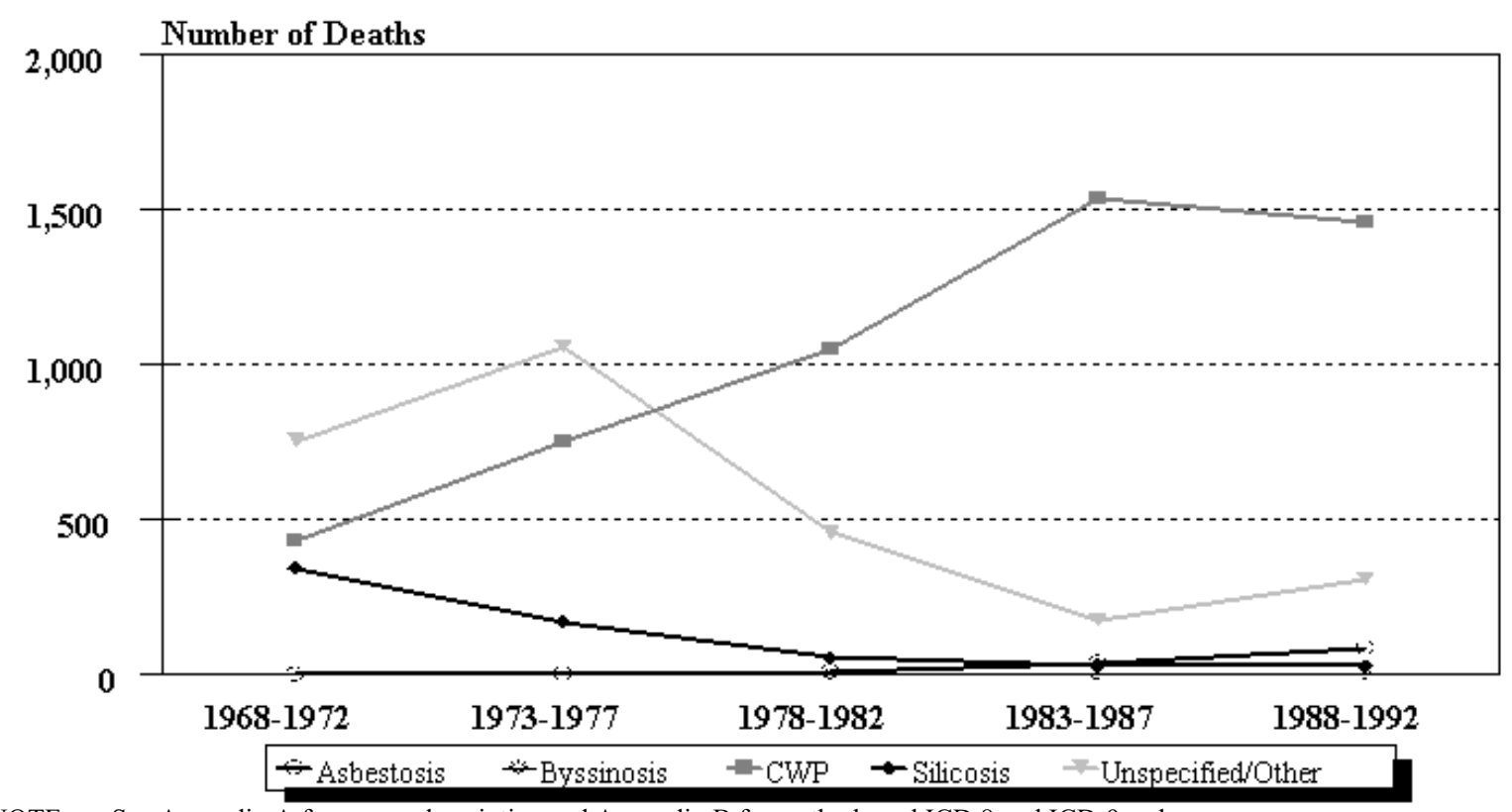

NOTE: See Appendix A for source description and Appendix B for methods and ICD-8 and ICD-9 codes.

SOURCE: National Center for Health Statistics multiple cause of death data.

Figure WV-4. West Virginia and U.S. age-adjusted mortality rates (per $1,000,000$ population) by condition, residents age 15 and over, 1983-1992

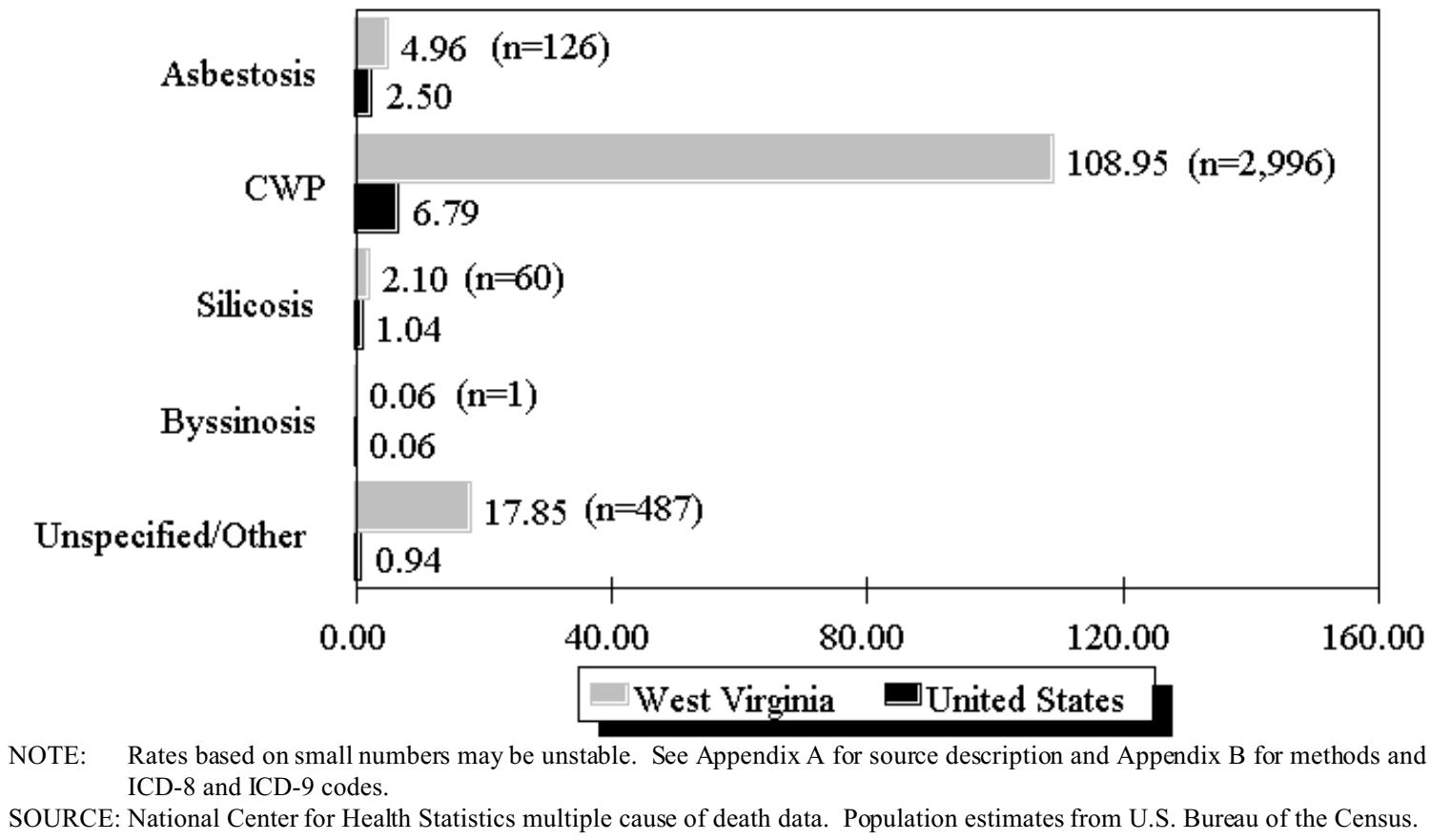


Table WV-3 (page 1 of 2). Number of deaths and age-adjusted mortality rate (per 1,000,000 population) by condition and county, West Virginia residents age 15 and over, 1983-1992

\begin{tabular}{|c|c|c|c|c|c|c|c|c|c|c|c|c|}
\hline \multirow[b]{2}{*}{ County } & \multicolumn{2}{|c|}{ Asbestosis } & \multicolumn{2}{|c|}{$\begin{array}{c}\text { Coal workers' } \\
\text { pneumoconiosis }\end{array}$} & \multicolumn{2}{|c|}{ Silicosis } & \multicolumn{2}{|c|}{ Byssinosis } & \multicolumn{2}{|c|}{$\begin{array}{r}\text { Unspecified/Other } \\
\text { pneumoconioses }\end{array}$} & \multicolumn{2}{|c|}{$\begin{array}{r}\text { All } \\
\text { pneumoconioses }\end{array}$} \\
\hline & $\#$ & Rate & \# & Rate & \# & Rate & $\#$ & Rate & \# & Rate & $\#$ & Rate \\
\hline Barbour & - & - & 16 & 61.88 & - & - & - & - & 1 & 2.35 & 17 & 64.23 \\
\hline Berkeley & - & - & 1 & 1.84 & - & - & - & - & - & - & 1 & 1.84 \\
\hline Boone & 1 & 2.21 & 163 & 522.99 & 4 & 11.86 & - & - & 32 & 96.38 & 200 & 633.45 \\
\hline Braxton & - & - & 6 & 23.65 & - & - & - & - & 2 & 13.48 & 8 & 37.13 \\
\hline Brooke & 2 & 5.99 & 9 & 23.92 & - & - & - & - & 1 & 1.61 & 12 & 31.52 \\
\hline Cabell & 8 & 4.14 & 13 & 7.56 & - & - & - & - & 4 & 3.61 & 25 & 15.31 \\
\hline Calhoun & - & - & 1 & 4.74 & - & - & - & - & - & - & 1 & 4.74 \\
\hline Clay & - & - & 16 & 132.62 & - & - & - & - & 2 & 14.73 & 18 & 147.35 \\
\hline Doddridge & - & - & 1 & 12.02 & - & - & - & - & 1 & 12.02 & 2 & 24.03 \\
\hline Fayette & 1 & 1.68 & 279 & 350.83 & 1 & 0.78 & - & - & 60 & 77.35 & 341 & 430.64 \\
\hline Gilmer & - & - & 1 & 4.32 & 1 & 12.27 & - & - & - & - & 2 & 16.59 \\
\hline Grant & - & - & 4 & 22.17 & - & - & - & - & - & - & 4 & 22.17 \\
\hline Greenbrier & 1 & 1.02 & 70 & 119.25 & - & - & - & - & 10 & 19.75 & 81 & 140.02 \\
\hline Hampshire & 3 & 20.18 & - & - & 2 & 11.35 & - & - & - & - & 5 & 31.53 \\
\hline Hancock & 1 & 2.20 & - & - & 1 & 1.31 & - & - & 1 & 1.31 & 3 & 4.82 \\
\hline Hardy & - & - & - & - & - & - & - & - & 1 & 13.39 & 1 & 13.39 \\
\hline Harrison & 7 & 6.88 & 58 & 45.87 & 2 & 2.34 & - & - & 17 & 13.36 & 83 & 67.28 \\
\hline Jackson & 1 & 5.12 & 3 & 9.11 & 1 & 1.84 & - & - & 1 & 3.63 & 6 & 19.70 \\
\hline Jefferson & 1 & 1.90 & - & - & - & - & - & - & - & - & 1 & 1.90 \\
\hline Kanawha & 49 & 17.14 & 127 & 38.92 & 3 & 1.02 & - & - & 24 & 6.83 & 202 & 63.50 \\
\hline Lewis & - & - & 8 & 30.49 & 1 & 2.02 & - & - & - & - & 9 & 32.51 \\
\hline Lincoln & 1 & 4.90 & 17 & 61.75 & 1 & 4.90 & - & - & 2 & 9.80 & 21 & 81.35 \\
\hline Logan & 1 & 2.20 & 168 & 319.60 & 3 & 4.88 & - & - & 48 & 91.15 & 220 & 417.83 \\
\hline McDowell & - & - & 333 & 635.19 & 6 & 8.96 & 1 & 2.37 & 18 & 32.13 & 356 & 676.02 \\
\hline Marion & 1 & 0.60 & 139 & 133.41 & 2 & 2.00 & - & - & 26 & 26.28 & 168 & 162.29 \\
\hline Marshall & 3 & 7.14 & 9 & 16.61 & 1 & 1.25 & - & - & 1 & 1.25 & 14 & 26.25 \\
\hline Mason & 4 & 12.86 & 3 & 9.20 & 1 & 1.87 & - & - & 1 & 3.66 & 9 & 27.59 \\
\hline Mercer & 2 & 1.25 & 168 & 170.47 & 3 & 1.87 & - & - & 29 & 28.16 & 200 & 199.82 \\
\hline Mineral & 4 & 10.48 & 2 & 3.55 & - & - & - & - & 1 & 3.46 & 7 & 17.49 \\
\hline Mingo & - & - & 59 & 169.26 & 3 & 8.71 & - & - & 26 & 70.28 & 87 & 244.95 \\
\hline Monongalia & 6 & 6.51 & 55 & 68.36 & 1 & 1.65 & - & - & 7 & 8.16 & 69 & 84.69 \\
\hline Monroe & - & - & 9 & 54.68 & - & - & - & - & 1 & 3.26 & 10 & 57.94 \\
\hline
\end{tabular}

See footnotes at end of table. 
Table WV-3 (page 2 of 2). Number of deaths and age-adjusted mortality rate (per 1,000,000 population) by condition and county, West Virginia residents age 15 and over, 1983-1992

\begin{tabular}{|c|c|c|c|c|c|c|c|c|c|c|c|c|}
\hline \multirow[b]{2}{*}{ County } & \multicolumn{2}{|c|}{ Asbestosis } & \multicolumn{2}{|c|}{$\begin{array}{r}\text { Coal workers' } \\
\text { pneumoconiosis }\end{array}$} & \multicolumn{2}{|c|}{ Silicosis } & \multicolumn{2}{|c|}{ Byssinosis } & \multicolumn{2}{|c|}{$\begin{array}{r}\text { Unspecified/Other } \\
\text { pneumoconioses }\end{array}$} & \multicolumn{2}{|c|}{$\begin{array}{r}\text { All } \\
\text { pneumoconioses }\end{array}$} \\
\hline & $\#$ & Rate & $\#$ & Rate & $\#$ & Rate & $\#$ & Rate & \# & Rate & $\#$ & Rate \\
\hline Morgan & - & - & 1 & 3.76 & - & - & - & - & 1 & 6.98 & 2 & 10.74 \\
\hline Nicholas & - & - & 70 & 210.26 & - & - & - & - & 18 & 55.12 & 88 & 265.38 \\
\hline Ohio & 2 & 1.33 & 12 & 12.10 & 4 & 3.49 & - & - & 6 & 7.29 & 24 & 24.21 \\
\hline Pendleton & - & - & - & - & - & - & - & - & 1 & 10.75 & 1 & 10.75 \\
\hline Pleasants & 2 & 11.85 & - & - & - & - & - & - & - & - & 2 & 11.85 \\
\hline Pocahontas & 1 & 8.89 & 1 & 8.89 & - & - & - & - & 2 & 7.01 & 4 & 24.80 \\
\hline Preston & - & - & 47 & 123.40 & 1 & 4.81 & - & - & 1 & 3.29 & 49 & 131.50 \\
\hline Putnam & 10 & 23.05 & 7 & 14.06 & 1 & 1.54 & - & - & 7 & 15.15 & 25 & 53.80 \\
\hline Raleigh & 3 & 2.82 & 784 & 664.06 & 3 & 1.71 & - & - & 75 & 65.17 & 865 & 733.76 \\
\hline Randolph & 1 & 1.44 & 22 & 56.69 & - & - & - & - & - & - & 23 & 58.12 \\
\hline Ritchie & - & - & 1 & 8.53 & 1 & 8.53 & - & - & - & - & 2 & 17.05 \\
\hline Roane & - & - & 2 & 8.64 & - & - & - & - & 2 & 8.64 & 4 & 17.27 \\
\hline Summers & - & - & 14 & 59.18 & - & - & - & - & 3 & 11.15 & 17 & 70.32 \\
\hline Taylor & - & - & 16 & 66.40 & - & - & - & - & 8 & 33.20 & 24 & 99.60 \\
\hline Tucker & - & - & 8 & 48.93 & - & - & - & - & - & - & 8 & 48.93 \\
\hline Upshur & - & - & 28 & 75.72 & 1 & 1.73 & - & - & 8 & 13.80 & 37 & 91.25 \\
\hline Wayne & 2 & 4.46 & 22 & 37.06 & 3 & 6.69 & - & - & 1 & 2.23 & 27 & 48.22 \\
\hline Webster & 1 & 8.47 & 41 & 258.65 & - & - & - & - & 5 & 42.36 & 47 & 309.48 \\
\hline Wetzel & - & - & 2 & 6.71 & 2 & 4.17 & - & - & - & - & 4 & 10.89 \\
\hline Wirt & 1 & 9.02 & - & - & - & - & - & - & - & - & 1 & 9.02 \\
\hline Wood & 6 & 4.72 & 3 & 2.65 & 4 & 2.57 & - & - & 4 & 3.73 & 17 & 13.67 \\
\hline Wyoming & - & - & 177 & 546.97 & 3 & 10.33 & - & - & 28 & 91.71 & 206 & 642.12 \\
\hline
\end{tabular}




\section{Wisconsin: Selected statistics from the United States section}

All pneumoconioses mortality, 1988-1992

Total deaths

$\underline{\text { Value }}$

Years of potential life lost to life expectancy (per death)
Age-adjusted mortality rate (per $1,000,000$ population)

Total years of potential life lost to life expectancy
Crude mortality rate (per $1,000,000$ population)
Deaths by type of pneumoconiosis, 1988-1992

Asbestosis

Coal workers' pneumoconiosis

Silicosis

Byssinosis

Unspecified/Other pneumoconiosis
109

5.73

3.64

1,513

13.9

$\underline{\text { State rank }}$

27

39

36

23

5

Percent**

Deaths*

distribution

37

33.9

7

6.4

58

53.2

1

0.9

8

* Sum of column may exceed total deaths because of deaths with multiple pneumoconioses.

** Percentages may total more than $100 \%$ due to deaths with multiple pneumoconioses.

NOTE: $\quad$ See Appendix A for source description and Appendix B for methods and ICD-8 and ICD-9 codes.

SOURCE: National Center for Health Statistics multiple cause of death data. Population estimates from U.S. Bureau of the Census.

Figure WI-1. All pneumoconioses: Wisconsin and U.S. age-adjusted mortality rates, residents age 15 and over, 1968-1992

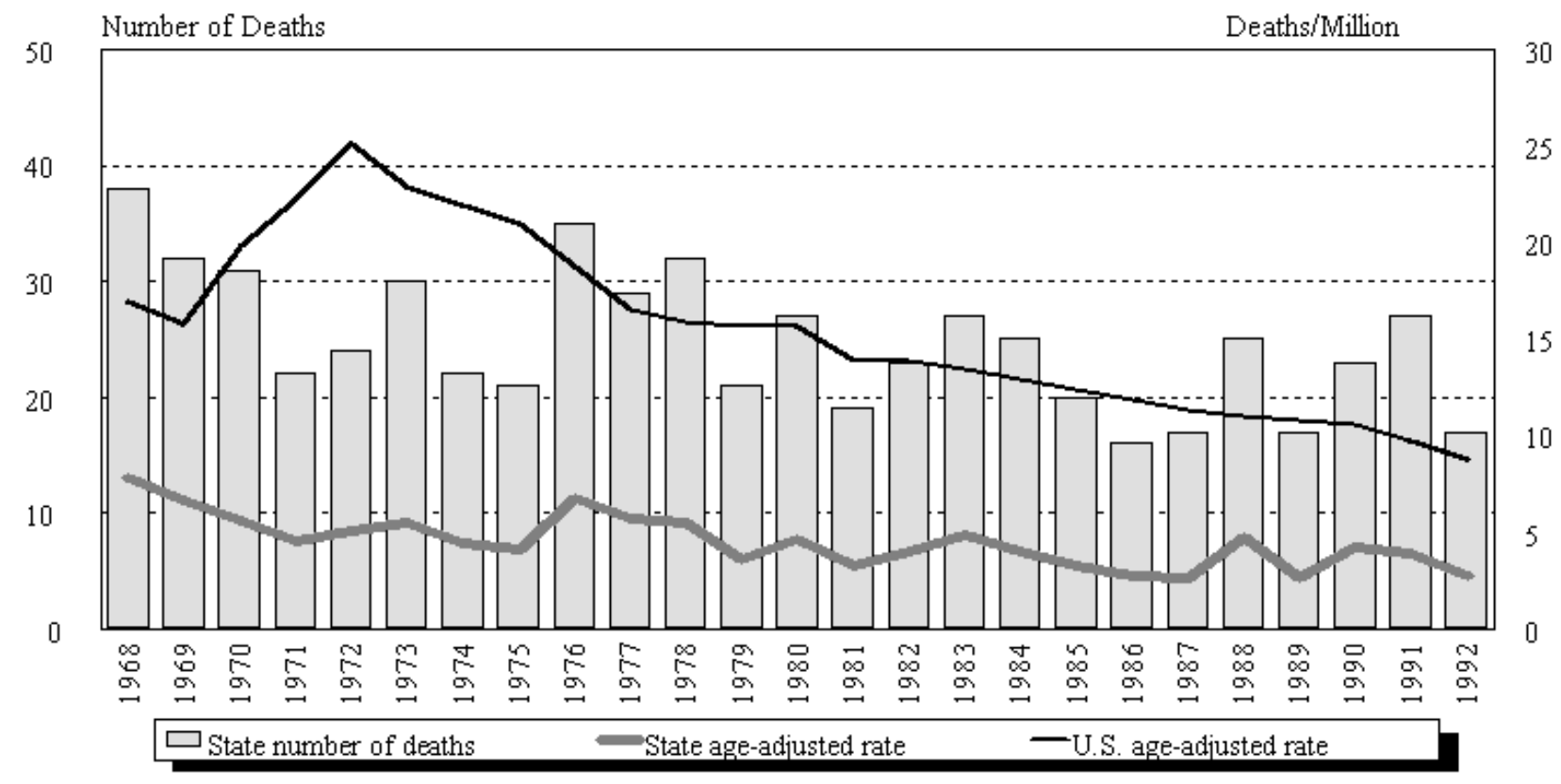

NOTE: $\quad$ See Appendix A for source description and Appendix B for methods and ICD-8 and ICD-9 codes.

SOURCE: National Center for Health Statistics multiple cause of death data. Population estimates from U.S. Bureau of the Census. 
Wisconsin

\begin{tabular}{|c|c|c|c|c|c|c|c|c|c|c|c|c|}
\hline \multirow[b]{2}{*}{ Year } & \multicolumn{2}{|c|}{ Asbestosis } & \multicolumn{2}{|c|}{$\begin{array}{c}\text { Coal workers' } \\
\text { pneumoconiosis }\end{array}$} & \multicolumn{2}{|c|}{ Silicosis } & \multicolumn{2}{|c|}{ Byssinosis* } & \multicolumn{2}{|c|}{$\begin{array}{c}\text { Unspecified/Other } \\
\text { pneumoconioses }\end{array}$} & \multicolumn{2}{|c|}{ All pneumoconioses } \\
\hline & Number & Rate & Number & Rate & Number & Rate & Number & Rate & Number & Rate & Number & Rate \\
\hline 1968 & 1 & 0.26 & 2 & 0.49 & 30 & 6.29 & & & 5 & 0.84 & 38 & 7.88 \\
\hline 1969 & - & - & 1 & 0.23 & 20 & 4.19 & & & 11 & 2.23 & 32 & 6.65 \\
\hline 1970 & - & - & 4 & 0.92 & 22 & 3.87 & & & 7 & 1.09 & 31 & 5.57 \\
\hline 1971 & - & - & 1 & 0.26 & 14 & 2.82 & & & 7 & 1.45 & 22 & 4.53 \\
\hline 1972 & 2 & 0.52 & - & - & 14 & 2.88 & & & 8 & 1.73 & 24 & 5.13 \\
\hline 1973 & 3 & 0.58 & 1 & 0.26 & 19 & 3.56 & & & 9 & 1.47 & 30 & 5.51 \\
\hline 1974 & - & - & 3 & 0.52 & 15 & 3.02 & & & 7 & 1.39 & 22 & 4.41 \\
\hline 1975 & 1 & 0.33 & 1 & 0.26 & 12 & 1.90 & & & 7 & 1.64 & 21 & 4.12 \\
\hline 1976 & 2 & 0.28 & 2 & 0.29 & 22 & 4.62 & & & 10 & 1.88 & 35 & 6.82 \\
\hline 1977 & 2 & 0.46 & 3 & 0.59 & 17 & 3.24 & & & 7 & 1.41 & 29 & 5.71 \\
\hline 1978 & 5 & 0.91 & 1 & 0.25 & 14 & 2.34 & & & 13 & 2.05 & 32 & 5.48 \\
\hline 1979 & - & - & 1 & 0.20 & 15 & 2.71 & - & - & 5 & 0.71 & 21 & 3.62 \\
\hline 1980 & 4 & 0.71 & 2 & 0.26 & 17 & 2.93 & - & - & 5 & 0.85 & 27 & 4.62 \\
\hline 1981 & 2 & 0.44 & 3 & 0.43 & 13 & 2.26 & - & - & 1 & 0.13 & 19 & 3.25 \\
\hline 1982 & 6 & 1.41 & 3 & 0.38 & 12 & 1.87 & - & - & 2 & 0.39 & 23 & 4.05 \\
\hline 1983 & 6 & 1.00 & 3 & 0.76 & 18 & 3.06 & - & - & - & - & 27 & 4.82 \\
\hline 1984 & 6 & 1.10 & 1 & 0.19 & 17 & 2.51 & - & - & 2 & 0.44 & 25 & 4.05 \\
\hline 1985 & 3 & 0.55 & 1 & 0.19 & 12 & 1.95 & - & - & 4 & 0.61 & 20 & 3.31 \\
\hline 1986 & 5 & 1.11 & - & - & 10 & 1.56 & - & - & 1 & 0.12 & 16 & 2.78 \\
\hline 1987 & 4 & 0.60 & 1 & 0.19 & 10 & 1.59 & - & - & 2 & 0.24 & 17 & 2.61 \\
\hline 1988 & 4 & 0.75 & 1 & 0.18 & 16 & 2.79 & 1 & 0.25 & 3 & 0.78 & 25 & 4.76 \\
\hline 1989 & 7 & 0.98 & 2 & 0.16 & 7 & 1.28 & - & - & 1 & 0.18 & 17 & 2.60 \\
\hline 1990 & 9 & 1.66 & 3 & 0.70 & 10 & 1.67 & - & - & 2 & 0.43 & 23 & 4.20 \\
\hline 1991 & 11 & 2.04 & 1 & 0.10 & 16 & 2.09 & - & - & - & - & 27 & 3.92 \\
\hline 1992 & 6 & 0.94 & - & & 9 & 1.58 & - & - & 2 & 0.20 & 17 & 2.72 \\
\hline
\end{tabular}

indicates no deaths listed. $\quad *$ no specific code for by ssinosis in ICD-8 (1968-1978).

NOTE: Rates based on small numbers may be unstable. Sum of individual conditions may exceed total because of deaths with multiple pneumoconioses. See Appendix A for source description and Appendix B for methods and ICD-8 and ICD-9 codes.

SOURCE: National Center for Health Statistics multiple cause of death data. Population estimates from U.S. Bureau of the Census. 


\section{Figure WI-2. All pneumoconioses: Distribution of deaths by sex and race,} Wisconsin residents age 15 and over, 1968-1992
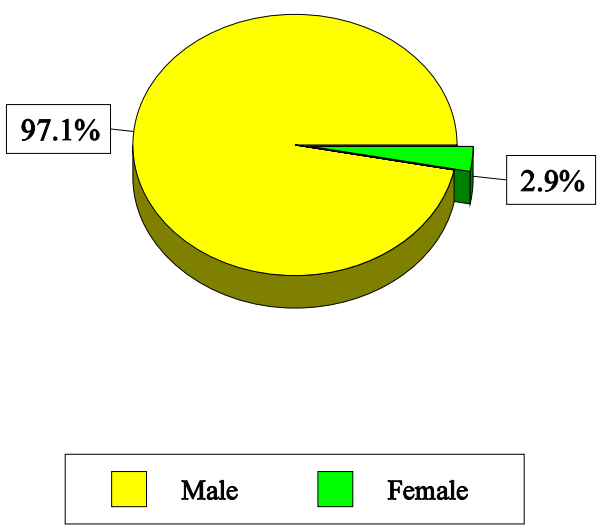

\section{$90.8 \%$}
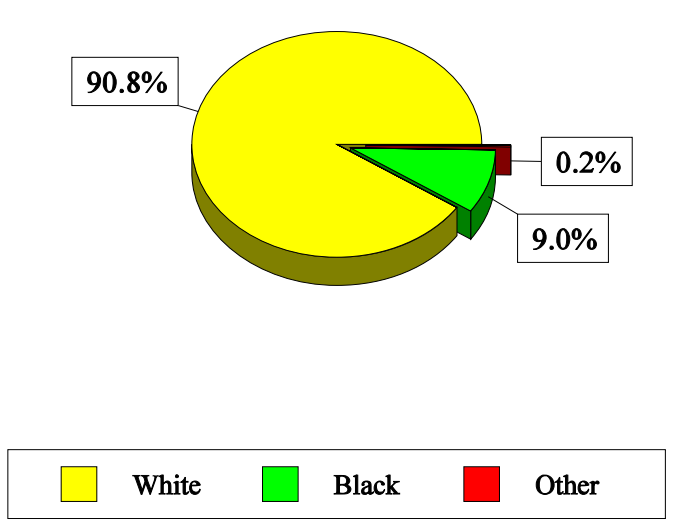

NOTE:

See Appendix A for source description and Appendix B for methods and ICD-8 and ICD-9 codes.

SOURCE: National Center for Health Statistics multiple cause of death data.

Table WI-2. All pneumoconioses: Distribution of deaths by sex, race, and age, Wisconsin residents age 15 and over, 1968-1992

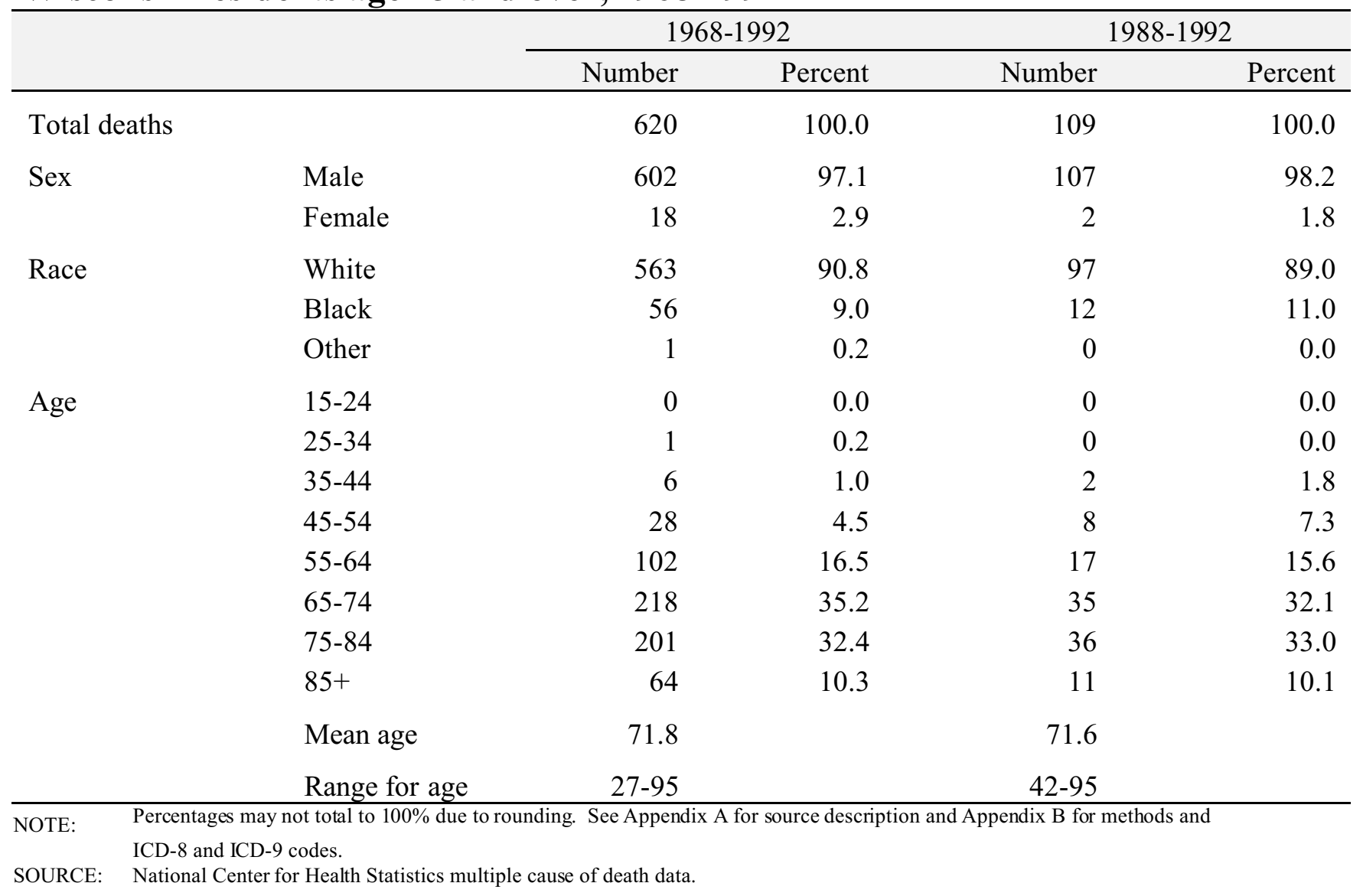




\section{Figure WI-3. Pneumoconiosis deaths by condition, Wisconsin residents age 15 and over, 1968-1992}

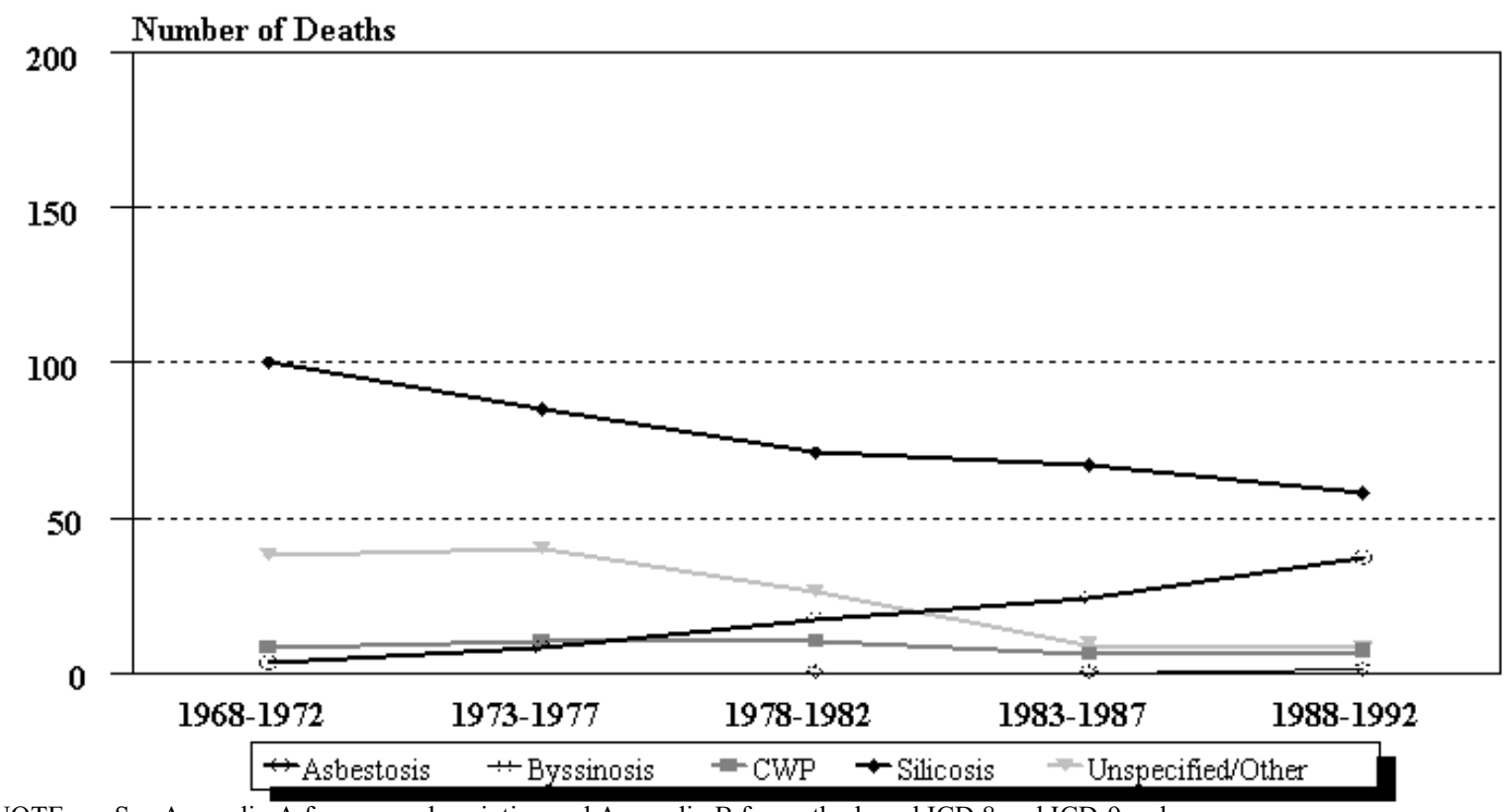

NOTE: See Appendix A for source description and Appendix B for methods and ICD-8 and ICD-9 codes.

SOURCE: National Center for Health Statistics multiple cause of death data.

Figure WI-4. Wisconsin and U.S. age-adjusted mortality rates (per 1,000,000 population) by condition, residents age 15 and over, 1983-1992

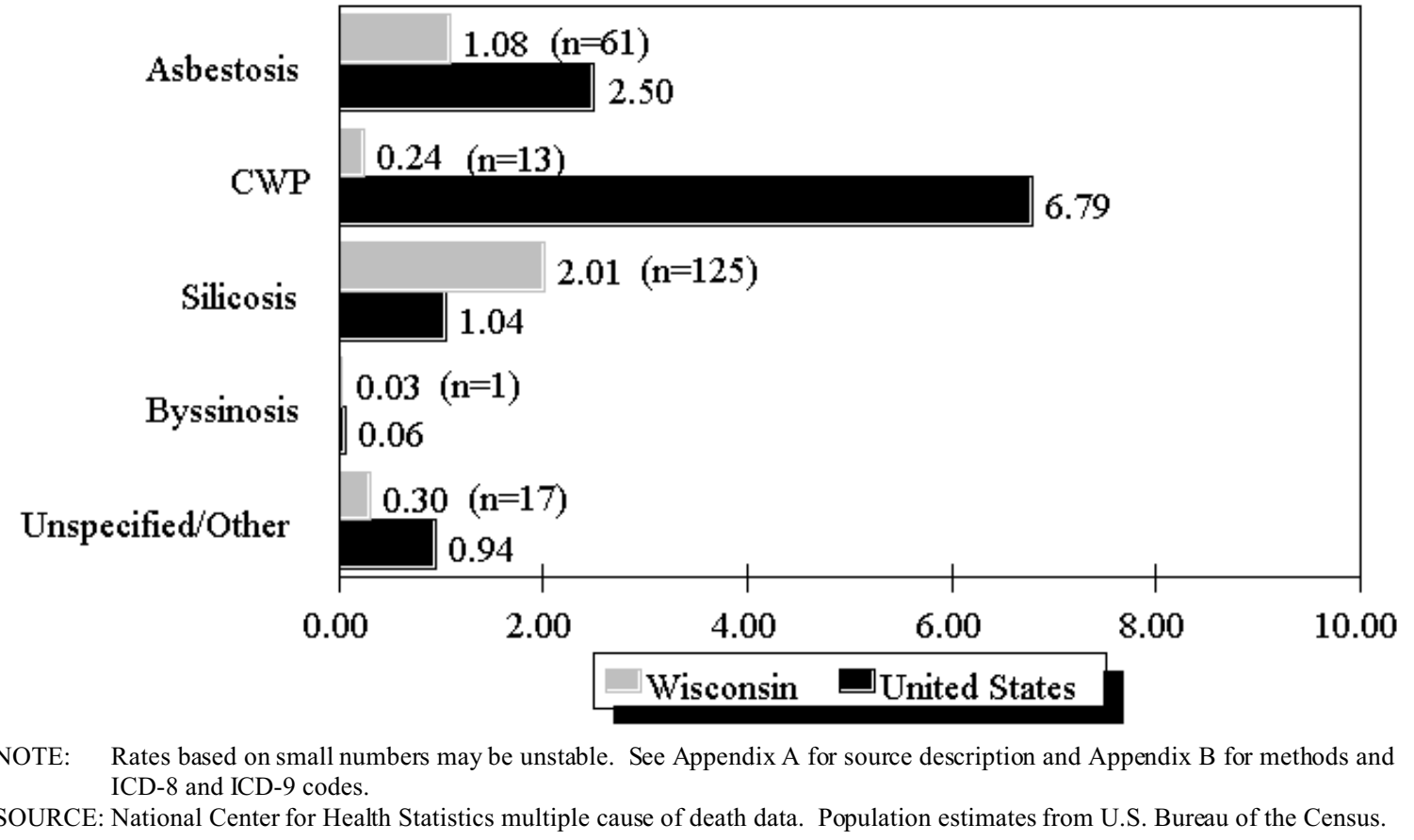


Table WI-3 (page 1 of 2). Number of deaths and age-adjusted mortality rate (per $1,000,000$ population) by condition and county, Wisconsin residents age 15 and over, 1983-1992

\begin{tabular}{|c|c|c|c|c|c|c|c|c|c|c|c|c|}
\hline \multirow[b]{2}{*}{ County } & \multicolumn{2}{|c|}{ Asbestosis } & \multicolumn{2}{|c|}{$\begin{array}{c}\text { Coal workers' } \\
\text { pneumoconiosis }\end{array}$} & \multicolumn{2}{|c|}{ Silicosis } & \multicolumn{2}{|c|}{ Byssinosis } & \multicolumn{2}{|c|}{$\begin{array}{r}\text { Unspecified/Other } \\
\text { pneumoconioses }\end{array}$} & \multicolumn{2}{|c|}{$\begin{array}{r}\text { All } \\
\text { pneumoconioses }\end{array}$} \\
\hline & \# & Rate & \# & Rate & $\#$ & Rate & $\#$ & Rate & $\#$ & Rate & $\#$ & Rate \\
\hline Barron & 1 & 2.34 & - & - & - & - & - & - & - & - & 1 & 2.34 \\
\hline Bayfield & 2 & 8.56 & - & - & - & - & - & - & - & - & 2 & 8.56 \\
\hline Brown & 3 & 1.75 & - & - & 2 & 0.98 & - & - & - & - & 5 & 2.73 \\
\hline Buffalo & 2 & 9.19 & - & - & - & - & - & - & - & - & 2 & 9.19 \\
\hline Burnett & 3 & 19.76 & - & - & 1 & 11.72 & - & - & - & - & 3 & 19.76 \\
\hline Calumet & - & - & - & - & 1 & 3.51 & - & - & - & - & 1 & 3.51 \\
\hline Chippewa & - & - & - & - & 1 & 0.81 & - & - & - & - & 1 & 0.81 \\
\hline Columbia & - & - & - & - & 2 & 4.27 & - & - & - & - & 2 & 4.27 \\
\hline Crawford & - & - & - & - & 1 & 9.70 & - & - & - & - & 1 & 9.70 \\
\hline Dane & - & - & - & - & 4 & 1.19 & - & - & 2 & 0.59 & 6 & 1.79 \\
\hline Door & 2 & 6.91 & - & - & 1 & 1.32 & - & - & - & - & 3 & 8.23 \\
\hline Douglas & 3 & 4.11 & - & - & - & - & - & - & - & - & 3 & 4.11 \\
\hline Eau Claire & 1 & 1.90 & - & - & - & - & - & - & - & - & 1 & 1.90 \\
\hline Fond du Lac & - & - & 1 & 0.48 & - & - & - & - & 2 & 0.96 & 3 & 1.44 \\
\hline Green Lake & 1 & 4.48 & - & - & - & - & - & - & - & - & 1 & 4.48 \\
\hline Jefferson & 2 & 2.33 & - & - & 2 & 2.33 & - & - & 1 & 1.63 & 5 & 6.29 \\
\hline Juneau & - & - & - & - & 1 & 3.94 & - & - & - & - & 1 & 3.94 \\
\hline Kenosha & 4 & 2.59 & 1 & 0.88 & 2 & 1.75 & - & - & 1 & 0.88 & 8 & 6.09 \\
\hline Kewaunee & - & - & - & - & 1 & 5.08 & - & - & - & - & 1 & 5.08 \\
\hline Langlade & - & - & - & - & 1 & 1.71 & - & - & - & - & 1 & 1.71 \\
\hline Lincoln & 1 & 1.38 & - & - & 1 & 3.40 & - & - & - & - & 2 & 4.78 \\
\hline Manitowoc & 3 & 2.84 & - & - & 2 & 3.04 & - & - & - & - & 5 & 5.88 \\
\hline Marathon & 2 & 1.95 & - & - & 7 & 4.73 & - & - & 1 & 0.98 & 10 & 7.65 \\
\hline Marinette & - & - & - & - & 1 & 0.86 & - & - & - & - & 1 & 0.86 \\
\hline Marquette & - & - & 1 & 5.64 & 2 & 8.44 & - & - & - & - & 3 & 14.08 \\
\hline Milwaukee & 15 & 1.27 & 3 & 0.32 & 29 & 3.03 & - & - & 5 & 0.54 & 51 & 5.06 \\
\hline Oneida & - & - & 1 & 1.22 & - & - & - & - & - & - & 1 & 1.22 \\
\hline Outagamie & 1 & 0.90 & - & - & 3 & 2.40 & - & - & - & - & 4 & 3.30 \\
\hline Ozaukee & 1 & 0.90 & - & - & 2 & 1.81 & - & - & - & - & 3 & 2.71 \\
\hline Pierce & - & - & - & - & 1 & 4.11 & - & - & - & - & 1 & 4.11 \\
\hline Polk & 1 & 2.86 & - & - & - & - & - & - & - & - & 1 & 2.86 \\
\hline Portage & 1 & 2.20 & - & - & - & - & - & - & - & - & 1 & 2.20 \\
\hline
\end{tabular}

See footnotes at end of table. 
Table WI-3 (page 2 of 2). Number of deaths and age-adjusted mortality rate (per $1,000,000$ population) by condition and county, Wisconsin residents age 15 and over, 1983-1992

\begin{tabular}{|c|c|c|c|c|c|c|c|c|c|c|c|c|}
\hline \multirow[b]{2}{*}{ County } & \multicolumn{2}{|c|}{ Asbestosis } & \multicolumn{2}{|c|}{$\begin{array}{c}\text { Coal workers' } \\
\text { pneumoconiosis }\end{array}$} & \multicolumn{2}{|c|}{ Silicosis } & \multicolumn{2}{|c|}{ Byssinosis } & \multicolumn{2}{|c|}{$\begin{array}{r}\text { Unspecified/Other } \\
\text { pneumoconioses }\end{array}$} & \multicolumn{2}{|c|}{$\begin{array}{r}\text { All } \\
\text { pneumoconioses }\end{array}$} \\
\hline & $\#$ & Rate & \# & Rate & \# & Rate & \# & Rate & $\#$ & Rate & \# & Rate \\
\hline Racine & 1 & 0.32 & 1 & 0.64 & 6 & 3.05 & - & - & - & - & 8 & 4.00 \\
\hline Rock & 1 & 0.79 & 2 & 1.57 & 5 & 2.22 & - & - & - & - & 8 & 4.58 \\
\hline Rusk & - & - & - & - & 1 & 2.27 & - & - & - & - & 1 & 2.27 \\
\hline Sauk & - & - & - & - & 1 & 0.82 & - & - & - & - & 1 & 0.82 \\
\hline Shawano & - & - & - & - & 1 & 2.34 & - & - & - & - & 1 & 2.34 \\
\hline Sheboygan & - & - & - & - & 31 & 14.93 & - & - & 1 & 0.41 & 32 & 15.33 \\
\hline Trempealeau & - & - & - & - & - & - & 1 & 3.65 & - & - & 1 & 3.65 \\
\hline Vernon & - & - & - & - & - & - & - & - & 1 & 1.23 & 1 & 1.23 \\
\hline Walworth & 1 & 1.43 & - & - & 1 & 1.43 & - & - & - & - & 2 & 2.86 \\
\hline Washington & 3 & 4.17 & - & - & 1 & 0.67 & - & - & - & - & 4 & 4.84 \\
\hline Waukesha & 1 & 0.24 & 1 & 0.40 & 5 & 1.70 & - & - & 2 & 0.64 & 9 & 2.99 \\
\hline Waupaca & 1 & 0.70 & - & - & 1 & 2.07 & - & - & - & - & 2 & 2.77 \\
\hline Waushara & 1 & 1.74 & - & - & - & - & - & - & - & - & 1 & 1.74 \\
\hline Winnebago & - & - & 2 & 1.61 & 2 & 1.15 & - & - & 1 & 0.81 & 5 & 3.56 \\
\hline Wood & 3 & 4.31 & - & - & 2 & 2.87 & - & - & - & - & 4 & 5.74 \\
\hline
\end{tabular}




\section{Wyoming: Selected statistics from the United States section}

All pneumoconioses mortality, 1988-1992

Total deaths

Crude mortality rate (per $1,000,000$ population)

Age-adjusted mortality rate (per $1,000,000$ population)

Total years of potential life lost to life expectancy

Years of potential life lost to life expectancy (per death) $\underline{\text { Value }}$

41

24.17

12.77

382

9.3

$\underline{\text { State rank }}$

38

\section{6}

6

42

51

Percent**

Deaths by type of pneumoconiosis, 1988-1992

Deaths*

distribution

Asbestosis

Coal workers' pneumoconiosis

Silicosis

26

19.5

63.4

2.4

0.0

Byssinosis

Unspecified/Other pneumoconiosis

* Sum of column may exceed total deaths because of deaths with multiple pneumoconioses.

** Percentages may total more than $100 \%$ due to deaths with multiple pneumoconioses.

NOTE: $\quad$ See Appendix A for source description and Appendix B for methods and ICD-8 and ICD-9 codes.

SOURCE: National Center for Health Statistics multiple cause of death data. Population estimates from U.S. Bureau of the Census.

\section{Figure WY-1. All pneumoconioses: Wyoming and U.S. age-adjusted mortality rates, residents age 15 and over, 1968-1992}

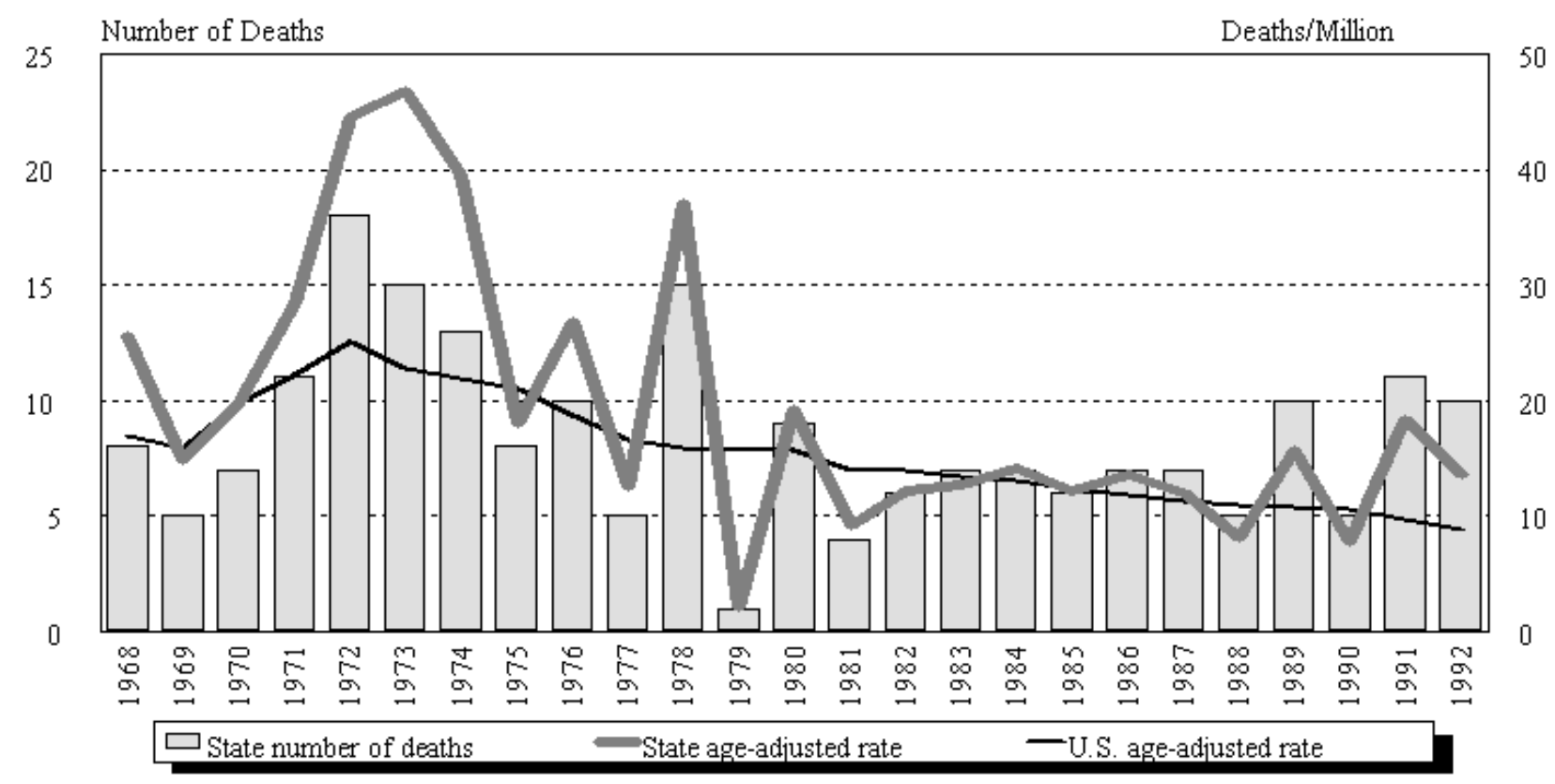

NOTE: 
Wyoming

Table WY-1. Number of deaths and age-adjusted mortality rate (per 1,000,000 population) by condition, Wyoming residents age 15 and over, 1968-1992

\begin{tabular}{|c|c|c|c|c|c|c|c|c|c|c|c|c|}
\hline \multirow[b]{2}{*}{ Year } & \multicolumn{2}{|c|}{ Asbestosis } & \multicolumn{2}{|c|}{$\begin{array}{l}\text { Coal workers' } \\
\text { pneumoconiosis }\end{array}$} & \multicolumn{2}{|c|}{ Silicosis } & \multicolumn{2}{|c|}{ Byssinosis* } & \multicolumn{2}{|c|}{$\begin{array}{c}\text { Unspecified/Other } \\
\text { pneumoconioses }\end{array}$} & \multicolumn{2}{|c|}{ All pneumoconioses } \\
\hline & Number & Rate & Number & Rate & Number & Rate & Number & Rate & Number & Rate & Number & Rate \\
\hline 1968 & - & - & - & - & 2 & 7.23 & & & 6 & 18.34 & 8 & 25.57 \\
\hline 1969 & - & - & 1 & 3.51 & 1 & 3.61 & & & 3 & 7.66 & 5 & 14.77 \\
\hline 1970 & - & - & 1 & 3.51 & 1 & 2.37 & & & 5 & 14.12 & 7 & 20.00 \\
\hline 1971 & - & - & 2 & 7.24 & 3 & 5.65 & & & 6 & 15.58 & 11 & 28.47 \\
\hline 1972 & 2 & 7.23 & 8 & 15.47 & - & - & & & 8 & 21.78 & 18 & 44.49 \\
\hline 1973 & - & - & 6 & 20.41 & - & - & & & 9 & 26.44 & 15 & 46.85 \\
\hline 1974 & - & - & 7 & 20.58 & 3 & 9.83 & & & 3 & 9.09 & 13 & 39.50 \\
\hline 1975 & - & - & 2 & 4.42 & 1 & 2.21 & & & 5 & 11.37 & 8 & 18.00 \\
\hline 1976 & - & - & 4 & 10.88 & 1 & 3.10 & & & 6 & 15.94 & 10 & 26.81 \\
\hline 1977 & - & - & 2 & 5.22 & - & - & & & 3 & 7.26 & 5 & 12.48 \\
\hline 1978 & - & - & 4 & 9.81 & 2 & 5.15 & & & 9 & 22.12 & 15 & 37.08 \\
\hline 1979 & - & - & - & & - & - & - & - & 1 & 2.16 & 1 & 2.16 \\
\hline 1980 & - & - & 6 & 11.95 & - & - & - & - & 3 & 7.32 & 9 & 19.27 \\
\hline 1981 & - & - & 2 & 4.61 & - & - & - & - & 2 & 4.61 & 4 & 9.21 \\
\hline 1982 & - & - & 2 & 5.04 & 1 & 0.93 & - & - & 3 & 6.22 & 6 & 12.18 \\
\hline 1983 & - & - & 4 & 6.87 & 1 & 2.44 & - & - & 2 & 3.33 & 7 & 12.63 \\
\hline 1984 & - & - & 5 & 9.28 & 1 & 2.41 & - & - & 1 & 2.41 & 7 & 14.09 \\
\hline 1985 & - & - & 6 & 12.17 & 1 & 1.68 & - & - & - & - & 6 & 12.17 \\
\hline 1986 & - & - & 4 & 7.23 & 3 & 6.30 & - & - & - & - & 7 & 13.53 \\
\hline 1987 & 2 & 3.20 & 2 & 3.98 & 1 & 2.35 & - & - & 2 & 2.47 & 7 & 12.00 \\
\hline 1988 & 1 & 1.61 & 4 & 6.42 & - & - & - & - & - & - & 5 & 8.03 \\
\hline 1989 & 1 & 2.99 & 5 & 7.17 & 1 & 1.58 & - & - & 3 & 3.99 & 10 & 15.73 \\
\hline 1990 & 1 & 1.55 & 4 & 6.22 & - & - & - & - & - & - & 5 & 7.77 \\
\hline 1991 & 4 & 7.64 & 7 & 10.64 & - & - & - & - & - & - & 11 & 18.28 \\
\hline 1992 & 1 & 0.76 & 6 & 8.37 & - & - & - & - & 3 & 4.42 & 10 & 13.56 \\
\hline
\end{tabular}

indicates no deaths listed. * no specific code for byssinosis in ICD-8 (1968-1978).

NOTE: Rates based on small numbers may be unstable. Sum of individual conditions may exceed total because of deaths with multiple pneumo conioses. See Appendix A for source description and Appendix B for methods and ICD-8 and ICD-9 codes.

SOURCE: National Center for Health Statistics multiple cause of death data. Population estimates from U.S. Bureau of the Census. 
Figure WY-2. All pneumoconioses: Distribution of deaths by sex and race, Wyoming residents age 15 and over, 1968-1992
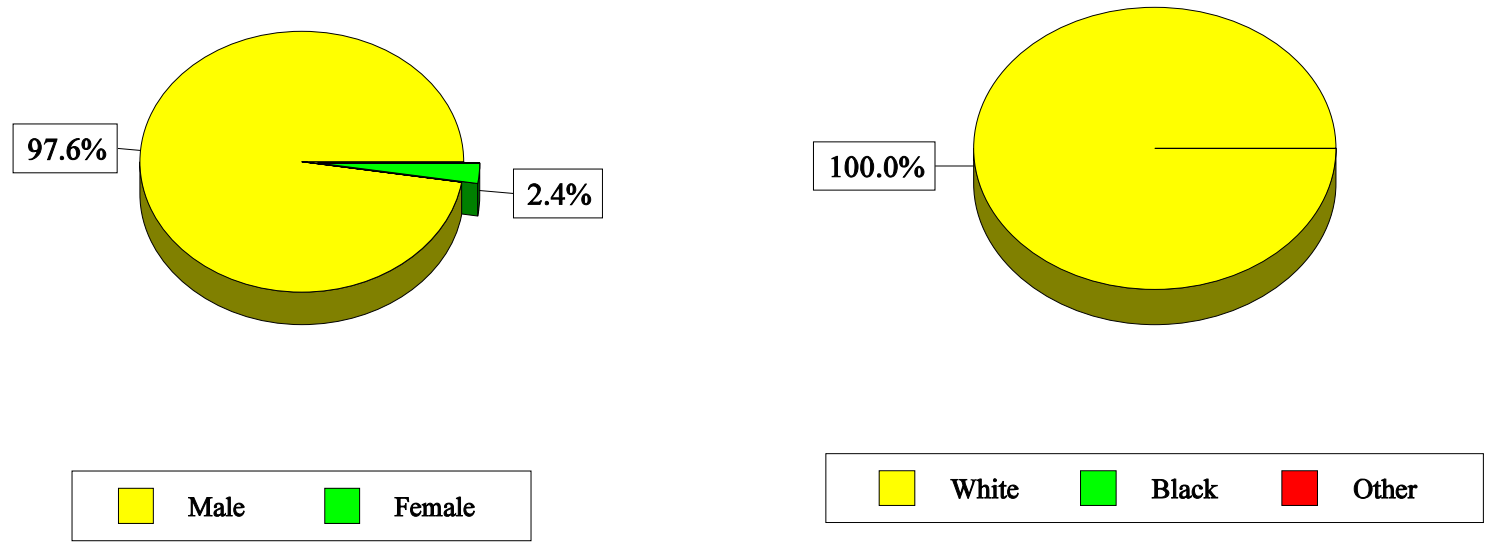

NOTE:

See Appendix A for source description and Appendix B for methods and ICD-8 and ICD-9 codes.

SOURCE: National Center for Health Statistics multiple cause of death data.

Table WY-2. All pneumoconioses: Distribution of deaths by sex, race, and age, Wyoming residents age 15 and over, 1968-1992

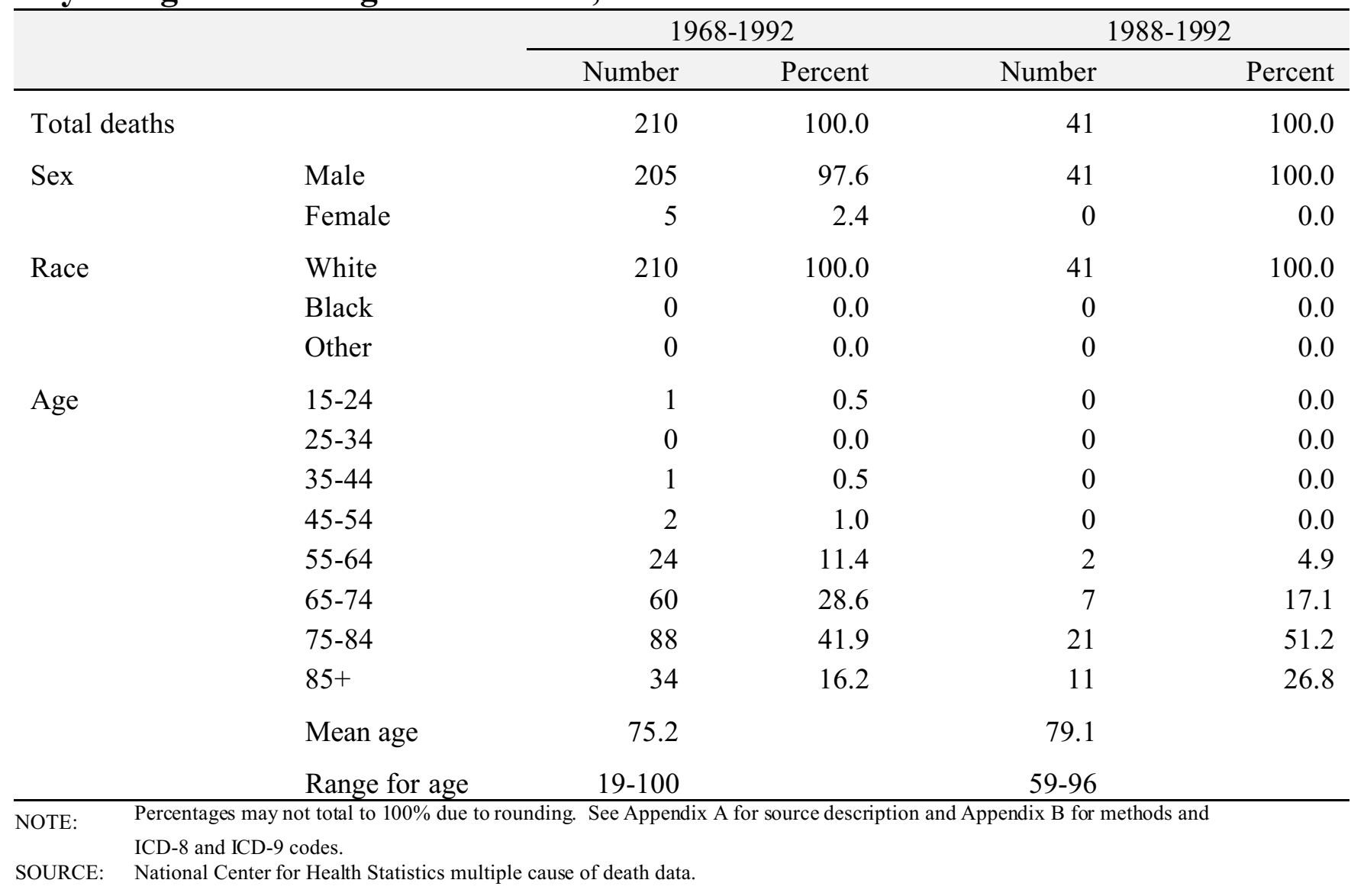


Figure WY-3. Pneumoconiosis deaths by condition, Wyoming residents age 15 and over, 1968-1992

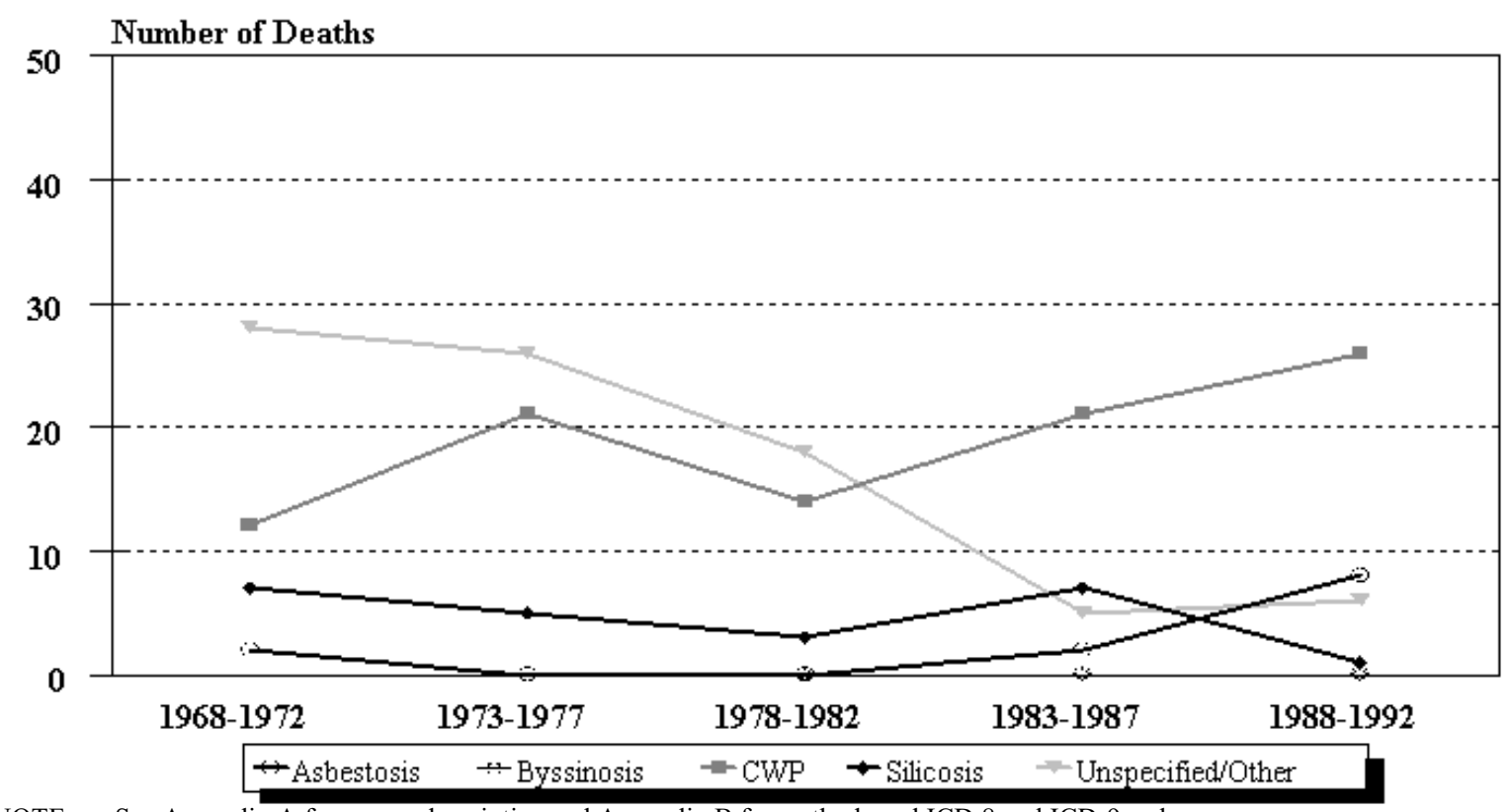

NOTE: See Appendix A for source description and Appendix B for methods and ICD-8 and ICD-9 codes.

SOURCE: National Center for Health Statistics multiple cause of death data.

Figure WY-4. Wyoming and U.S. age-adjusted mortality rates (per 1,000,000 population) by condition, residents age 15 and over, 1983-1992

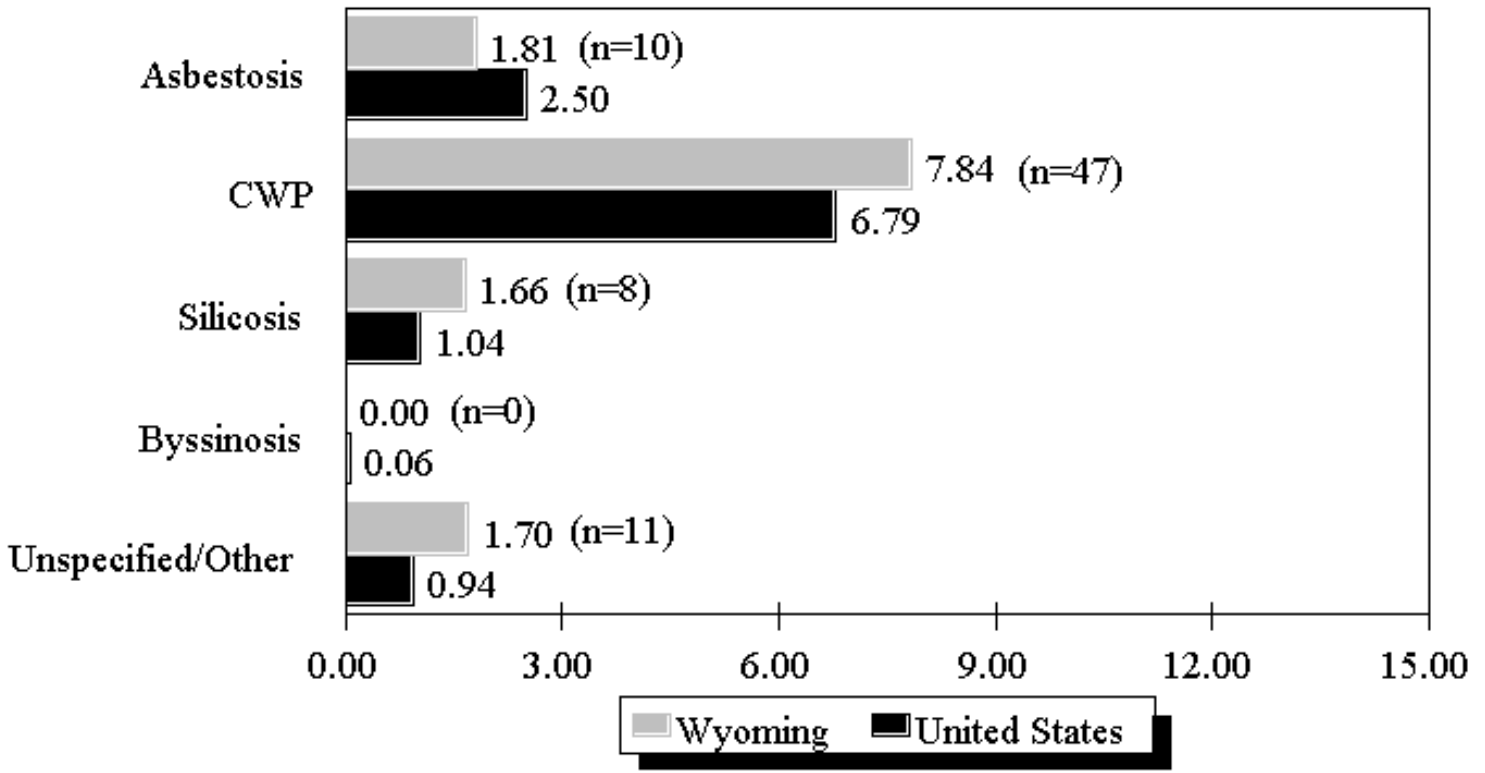

NOTE: $\quad$ Rates based on small numbers may be unstable. See Appendix A for source description and Appendix B for methods and ICD-8 and ICD-9 codes.

SOURCE: National Center for Health Statistics multiple cause of death data. Population estimates from U.S. Bureau of the Census. 
Table WY-3. Number of deaths and age-adjusted mortality rate (per 1,000,000 population) by condition and county, Wyoming residents age 15 and over, 1983-1992

\begin{tabular}{|c|c|c|c|c|c|c|c|c|c|c|c|c|}
\hline \multirow[b]{2}{*}{ County } & \multicolumn{2}{|c|}{ Asbestosis } & \multicolumn{2}{|c|}{$\begin{array}{c}\text { Coal workers' } \\
\text { pneumoconiosis }\end{array}$} & \multicolumn{2}{|c|}{ Silicosis } & \multicolumn{2}{|c|}{ Byssinosis } & \multicolumn{2}{|c|}{$\begin{array}{r}\text { Unspecified/Other } \\
\text { pneumoconioses }\end{array}$} & \multicolumn{2}{|c|}{$\begin{array}{r}\text { All } \\
\text { pneumoconioses }\end{array}$} \\
\hline & $\#$ & Rate & $\#$ & Rate & $\#$ & Rate & $\#$ & Rate & \# & Rate & $\#$ & Rate \\
\hline Albany & - & - & 2 & 8.35 & 2 & 5.70 & - & - & - & - & 3 & 11.19 \\
\hline Campbell & 1 & 7.80 & 1 & 6.03 & - & - & - & - & - & - & 2 & 13.83 \\
\hline Carbon & - & - & 3 & 15.13 & - & - & - & - & - & - & 3 & 15.13 \\
\hline Converse & - & - & 1 & 6.64 & - & - & - & - & - & - & 1 & 6.64 \\
\hline Fremont & - & - & 2 & 3.83 & - & - & - & - & - & - & 2 & 3.83 \\
\hline Goshen & - & - & 1 & 3.12 & - & - & - & - & 1 & 3.12 & 2 & 6.23 \\
\hline Hot Springs & - & - & 3 & 17.46 & - & - & - & - & - & - & 3 & 17.46 \\
\hline Johnson & - & - & 3 & 33.57 & 1 & 13.84 & - & - & - & - & 4 & 47.41 \\
\hline Laramie & 6 & 6.98 & 1 & 0.92 & - & - & - & - & - & - & 7 & 7.90 \\
\hline Lincoln & 1 & 5.94 & 6 & 46.06 & - & - & - & - & - & - & 7 & 51.99 \\
\hline Natrona & 2 & 2.98 & 3 & 3.56 & 1 & 1.19 & - & - & 1 & 1.79 & 7 & 9.52 \\
\hline Platte & - & - & - & - & 1 & 11.79 & - & - & - & - & 1 & 11.79 \\
\hline Sublette & - & - & - & - & - & - & - & - & 1 & 11.07 & 1 & 11.07 \\
\hline Sweetwater & - & - & 20 & 57.07 & 1 & 4.10 & - & - & 8 & 21.16 & 29 & 82.33 \\
\hline Uinta & - & - & - & - & 2 & 20.16 & - & - & - & - & 2 & 20.16 \\
\hline Washakie & - & - & 1 & 11.22 & - & - & - & - & - & - & 1 & 11.22 \\
\hline
\end{tabular}

- indicates no deaths listed.

NOTE: Counties with no deaths for all listed conditions are not displayed. Rates based on small numbers may be unstable. Sum of individual conditions may exceed total because of deaths with multiple pneumoconioses. See Appendix A for source description and Appendix B for methods and ICD- 8 and ICD-9 codes.

SOURCE: National Center for Health Statistics multiple cause of death data. Population estimates from U.S. Bureau of the Census. 
APPENDICES 


\section{Multiple Cause of Death Data, NCHS}

The National Center for Health Statistics (NCHS) makes available multiple cause of death data files for the years 1968 to 1992 . The files contain records of all deaths in the United States (approximately two million annually) that are reported to state vital statistics offices. All conditions listed on the death certificate, both underlying and contributing, are coded for each decedent. Additional data include age, race, sex, and state and county of residence at time of death. The usual occupation and industry of each decedent are available for some states since 1985 (see Appendix C).

Since 1989, the NCHS public use data files have not included data for counties reporting less than 3 deaths, for any given year. Files containing data not available for public use have been obtained directly from NCHS for use in generating mortality maps by county.

Potential limitations of multiple cause of death data include: under- or over-reporting of conditions on the death certificate by certifying physicians; incomplete or unclassified reporting of usual occupation and industry; and non-specificity of codes.

For more information refer to: Vital Statistics of the United States, 1992, Vol. 1, DHHS Pub. No. (PHS) 96-1100 and Vol. 11, Part A, DHHS Pub. No. (PHS) 97-1101, Public Health Service, National Center for Health Statistics. U.S. Government Printing Office, Washington, D.C. 20402.

\section{Population Estimates, Bureau of the Census}

National population estimates used in this report are based on data from the United States Bureau of the Census for national, state, and county levels. A census of the United States population has been taken every 10 years since 1790 . After the decennial population censuses are completed, intercensal population estimates are prepared for the preceding decade to replace postcensal estimates. Intercensal estimates take into account the census of the population at the beginning and end of each decade. In the current report, intercensal estimates have been used for all years preceding 1990. United States postcensal (Demo-Detail) files were used to compute rates for 1990 to 1992. As of June 1996, the census data may be found on the Wide-Ranging Online Data for Epidemiological Research (WONDER) computerized information system, maintained by the Centers for Disease Control and Prevention.

For information on the 1990 census, refer to: U.S. Bureau of the Census, 1990 Census of the Population, General Population Characteristics, Series 1900, CP-1.

\section{Respirable Coal Mine Dust Data, MSHA}

These data were obtained from MSHA and represent respirable coal mine dust levels measured by MSHA inspectors at surface and underground coal mines beginning in 1970 . The data include the sample collection date, dust concentration, occupation associated with the sample, an MSHA designator as to the validity of the sample, and the mine at which the sample was obtained.

The MSHA respirable coal mine dust samples are obtained by drawing mine air through a filter at the rate of two liters per minute, with a cyclone used to extract non-respirable particles prior to the filter. The dust weight collected on the filter is multiplied by 1.38 to complete the conversion to Mines Research Establishment (MRE) units. The "MRE" designation indicates that measurements obtained by MSHA were converted so that they would be equivalent to those obtained with an instrument on which the British standards have been based (Isleworth Type 113A Gravimetric Dust Sampler).

For more information, contact: Mine Safety and Health Administration, Information Resource Center, P.O. Box 25367, Denver, CO 80225-0367.

\section{Respirable Coal Mine Quartz Dust Data, MSHA} These data were obtained from MSHA and represent respirable quartz levels derived from respirable coal mine dust samples collected by MSHA inspectors at 
surface and underground coal mines beginning in 1982. The data include the sampling date, sampling time, initial and final weights, percent quartz, production level during sampling, the occupation associated with the sample, and the mine at which the sample was obtained.

For more information, contact: Mine Safety and Health Administration, Safety and Health Technology Center, Dust Division, PO Box 18233, Pittsburgh, PA 15236.

\section{Mine Inspection Data Analysis System, BoM}

The Mine Inspection Data Analysis System (MIDAS) was developed by the Bureau of Mines (BoM) to analyze the records of industrial hygiene samples collected by Mine Safety and Health Administration (MSHA) inspectors in non-coal (metal/non-metal) surface and underground mines beginning in 1974. Data in MIDAS, for both personal exposure samples and area samples, include the sampling date, contaminant code, airborne concentration, occupation, PEL, percent silica and silica concentration (where applicable), standard industrial classification, and the mine at which the sample was obtained. The MIDAS data were previously edited by the Bureau of Mines staff. This report uses all the MIDAS data reported for the agents listed in Appendix D.
For more information contact: W. F. Watts, Jr. at the University of Minnesota, Center for Diesel Research, Department of Mechanical Engineering, $125 \mathrm{ME}, 111$ Church St. SE, Minneapolis, MN 55455 or E-mail Watts@012.tc.umn.edu .

Note: The quartz reference standard used for MIDAS samples changed in 1988. For information regarding the quartz reference standard used for the MIDAS samples, contact: Laboratory Division, Denver Technical Support Center, Mine Safety and Health Administration, PO Box 25367, Denver, CO 802250367.

\section{Integrated Management Information System, OSHA}

The Integrated Management Information System (IMIS) includes most of the industrial hygiene sampling data from Occupational Safety and Health Administration (OSHA) compliance inspections beginning in May 1979. The data are reported by OSHA field compliance officers. OSHA provides IMIS data to NIOSH on an annual basis. The IMIS data include the sampling date, substance code, airborne concentration, sample and exposure type (see Methods, Appendix B), occupation, PEL, and standard industrial classification.

For more information contact: OSHA, Office of Management Data Systems, 200 Constitution Avenue, NW, Washington, D.C. 20210. 
International Classification of Disease (ICD) Codes

\begin{tabular}{|c|c|c|c|c|}
\hline \multirow{2}{*}{$\begin{array}{l}\text { Condition } \\
\text { (as defined for } \\
\text { this report) }\end{array}$} & \multicolumn{2}{|l|}{ ICD-8 (1968-1978) } & \multicolumn{2}{|l|}{ ICD-9 (1979-1992) } \\
\hline & Rubrics & Codes & Rubrics & Codes \\
\hline Asbestosis & Asbestosis & 515.2 & Asbestosis & 501 \\
\hline $\begin{array}{l}\text { Coal Workers' } \\
\text { Pneumoconiosis }\end{array}$ & $\begin{array}{l}\text { Anthracosilicosis } \\
\text { Anthracosis } \\
\text { Coal miners' lung }\end{array}$ & 515.1 & $\begin{array}{l}\text { Coal Workers' Pn eum oco niosis } \\
\text { Anthracosilicosis } \\
\text { Anthracosis } \\
\text { Black lung disease } \\
\text { Coal workers' lung } \\
\text { Miners' asthma }\end{array}$ & 500 \\
\hline \multirow{2}{*}{ Silicosis } & $\begin{array}{l}\text { Silicosis } \\
\text { Calcicosis } \\
\text { Chalicosis }\end{array}$ & 515.0 & $\begin{array}{l}\text { Pneumoconiosis due to other silica } \\
\text { or silicates } \\
\text { Pneumoconiosis due to talc } \\
\text { Silicotic fibrosis (massive) } \\
\text { Silicosis } \\
\text { (simple)/(complicated) }\end{array}$ & 502 \\
\hline & $\begin{array}{l}\text { Silicotuberculosis } \\
\text { Colliers' phthisis } \\
\text { Grinders' phthisis } \\
\text { Miners' phthisis } \\
\text { Stonemasons' phthisis }\end{array}$ & 010 & No equivalent ICD-9 code & \\
\hline Byssinosis & $\begin{array}{l}\text { No specific ICD- } 8 \text { code for } \\
\text { byssinosis }\end{array}$ & & $\begin{array}{l}\text { Pneumonopathy due to inhalation } \\
\text { of other dust } \\
\text { Byssinosis } \\
\text { Cannabinosis } \\
\text { Flax-dressers' disease }\end{array}$ & 504 \\
\hline \multirow{2}{*}{$\begin{array}{l}\text { Unspecified/ } \\
\text { Other } \\
\text { Pneumoconioses }\end{array}$} & $\begin{array}{l}\text { Pneumoconiosis due to } \\
\text { inhalation of other } \\
\text { inorganic dust } \\
\text { Aluminosis (of lung) } \\
\text { Bauxite fibrosis (of lung) } \\
\text { Berylliosis } \\
\text { Graphite fibrosis (of lung) }\end{array}$ & 516.0 & $\begin{array}{l}\text { Pneumoconiosis due to } \\
\text { other inorganic dust } \\
\text { Aluminosis (of lung) } \\
\text { Bauxite fibrosis (of lung) } \\
\text { Berylliosis } \\
\text { Graphite fibrosis (of lung) } \\
\text { Siderosis } \\
\text { Stannosis }\end{array}$ & 503 \\
\hline & $\begin{array}{l}\text { Other pneumoconiosis, including } \\
\text { unspecified } \\
\text { Pneumoconiosis: NOS; } \\
\text { due to: } \\
\text { silicates NEC } \\
\text { talc }\end{array}$ & 515.9 & $\begin{array}{l}\text { Pneumoconiosis, } \\
\text { unspecified }\end{array}$ & 505 \\
\hline
\end{tabular}




\section{MORTALITY}

\section{Number of Deaths}

The number of deaths for each occupational respiratory condition is the number of deaths in which the condition was coded as either underlying or contributing cause of death. These numbers were tabulated from the record axis of the NCHS multiple cause of death data files. Cause of death codes were defined as shown in the previous table [International Classification of Disease (ICD) Codes]. In the current report, the number of deaths by condition are reported both annually and for selected time periods. Where numbers of deaths are presented in this report, the total for the specific period is reported. Reported deaths are restricted to United States residents, 15 years or older, based on state of residence at death. For this report, race was classified as white, black, and all others.

\section{Crude Mortality Rates}

Cause-specific crude mortality rates for occupational respiratory conditions were computed for all United States decedents 15 years and older, from 1968 to 1992. To compute the annual rates, the total number of deaths with a specific condition mentioned as either underlying or contributing cause was divided by the comparable United States population, in the same year. Additional restrictions were placed on the data to compute race- and sex-specific rates.

State-specific crude mortality rates for 1991-1992 were computed by dividing the average number of reported deaths in each state by the average state population, 15 years and older, in that time period. State-specific crude mortality rates for other multiyear periods were computed by dividing the average annual number of deaths for the period by the midyear population for that period.

\section{Age-adjusted Mortality Rates}

Age-adjusted mortality rates presented in this report were based on deaths with the condition of interest mentioned as either underlying or contributing cause of death. Age-adjusted rates were computed by the direct method. Rates were calculated annually for each specified condition from 1968 through 1992, as well as for selected periods. The age-adjusted rates, for a given year, represent the rates that would have been observed if the age-specific rates for specified age groups had occurred in a population with the same age distribution as that of the standard population. For this report, the 1940 United States population was used as the standard. The specific age intervals used were 15-24, 25-34, 35-44, 45-54, 55-64, 65-74, 75-84, and 85 years and older. Rates for the entire U.S. population, and for each sex-race group were adjusted separately, each using the same standard population.

The method of calculation first computed the annual age-specific rates for the population of interest. The product of the age-specific rates and the number in the comparable age-specific group in the standard population equals the expected number of deaths per million population for each age group. The total expected numbers of deaths were then summed over all age groups. The sum of the expected number of deaths was divided by the sum of the standard population and the resulting quotient was multiplied by $1,000,000$ to produce the age-adjusted rate.

Age-adjusted mortality rates were computed at the national, state, and county level for multi-year periods. The method of calculation first computed age-specific rates by dividing the average annual number of deaths for each age group by the corresponding age-grouped mid-year population for the comparable geographic unit. Age-adjusted rates were then computed as described above.

Age-adjusted rates computed for multi-year periods, by county, were based upon wider age intervals in order to aggregate a larger number of cause-specific deaths within each age group. Specific intervals used were $15-34,35-54,55-74$, and 75 years and older.

\section{Years of Potential Life Lost}

Years of potential life lost (YPLL) were based on deaths with the condition of interest mentioned as 
either underlying or contributing cause of death. They were calculated using the method described by the Centers for Disease Control (CDC) (MMWR, Vol 34/2S: December 19,1986). YPLL were calculated both to age 65 and to life expectancy. YPLL to age 65 may be considered as a loss of years from a normal working life, while YPLL until life expectancy may be considered as a loss of years from the overall life span. For YPLL to age 65, the number of deaths with a mention of a specific condition of interest were classified into the ten-year age groups mentioned in the section on age-adjusted mortality rates. The number of deaths in each age group was then multiplied by the difference between 65 years and the mid-point of the age group. Thus for the 15-24 year age group, the number of deaths would be multiplied by 45 (i.e., 65 minus 20 years). The age-specific YPLL were summed over all age groups for the total value.

For YPLL to life expectancy, the single difference was that the calculation was based on the number of deaths in the age-specific group multiplied by the difference between the mid-point of the age group and the life expectancy at that age in the year of death. Life tables published annually by NCHS were used to determine life expectancy. State-specific YPLL (to life expectancy) per death were calculated for only one period, 1988-1992. To calculate this index, YPLL (to life expectancy) were divided by the total number of deaths in the time period.

\section{Proportionate Mortality Ratio (PMR)}

The data for PMR analyses were a subset of the NCHS multiple cause of death files for which usual occupation and industry are available (see Appendix $\mathrm{C}$ for a list of states and years reporting).

The PMR is defined as the observed number of deaths with the condition of interest (mentioned as either underlying or contributing cause) in a specified occupation or industry divided by the expected number of deaths with that condition. The expected number of deaths is the total number of deaths in the occupation or industry of interest multiplied by the proportion, defined as the number of cause-specific deaths for the condition of interest, divided by the total number of deaths in the population. The PMRs in the report have been internally age-adjusted (i.e., 15-34, 35-54, 55-74, and 75 years and over). Confidence intervals were calculated assuming Poisson distribution of the data.

A PMR over 1.00 indicates that there were more deaths with the condition in a specified occupation or industry than expected. PMRs with the lower 95\% confidence limit exceeding 1.0 have been listed for occupations or industries with at least 5 deaths from the condition of interest.

\section{Rank Order}

For each state, a rank order is presented for each of several mortality measures. Depending on the specific mortality measures, a rank order of " 1 " indicates the greatest number of deaths, highest mortality rate, or highest YPLL among all states in the U.S.

\section{EXPOSURE}

\section{Data Selection}

MSHA respirable coal mine dust samples selected for analysis were restricted to those samples which met all three of the following criteria:

1) samples obtained in the 50 U.S. states or Washington, D.C. (i.e., the Virgin Islands and Puerto Rico were excluded); and

2) samples designated by MSHA as valid; and

3) samples coded as "designated occupation," "non-designated occupation," "designated work position," "non-designated work position" with valid occupation codes, or "designated area" other than "intake air."

MSHA coal mine quartz samples selected for analysis are those samples which met all five of the following criteria: 
1) samples obtained in the 50 U.S. states or Washington, D.C. (i.e., the Virgin Islands and Puerto Rico were excluded); and

2) samples designated by MSHA as valid; and

3) samples with sampling time greater than zero; and

4) samples with quartz concentration greater than zero; and

5) samples coded as "designated occupation," "non-designated occupation," "designated work position," "non-designated work position" with valid occupation codes, or "designated area" other than "intake air."

MIDAS data selected for analysis are all sample records for agents listed in Appendix D that were collected in the 50 U.S. states or Washington, D.C. (i.e., the Virgin Islands and Puerto Rico were excluded).

IMIS data selected for analysis were a subset of all sample records for agents listed in Appendix D. The subset for analysis resulted from selecting records which met all four of the following criteria:

1) records with the state code of one of the 50 U.S. states or Washington, D.C. (i.e., the Virgin Islands and Puerto Rico were excluded); and

2) records with sample type "area" or "personal" (i.e., "bulk," "wipe," "screen," "blood," and "urine samples were excluded); and

3) records with exposure type "time-weighted average," "ceiling," "peak," or "not detected" (i.e., "dose," "sound reading," "not analyzed," and "not valid" were excluded); and

4) records for which the indicated PEL and units were applicable to the contaminant indicated by the substance code for the recorded date of sampling.

\section{Data Analysis}

The number of samples within an exposure category of interest was the total number of samples selected by the above criteria for the agents in that exposure category (see Appendix D for agents and exposure categories).

The severity level for any sample was calculated as the measured exposure level divided by either the enforced PEL or a surrogate for the enforced PEL (see the last two paragraphs of Data Analysis).

The percent of samples exceeding the PEL (or its surrogate) for an exposure category was calculated as the number of samples in that category with measured exposure exceeding the corresponding PEL (or its surrogate), divided by the total number of samples, and finally multiplying by 100 .

The average severity level for an exposure category was calculated by determining the severity level for each sample in that category, then summing the severity levels, and finally dividing the sum by the number of samples. The average severity level for samples exceeding the PEL (or its surrogate) was calculated in the same manner, after restricting the data to samples for which measured exposure exceeded the PEL (or its surrogate).

With two exceptions (see below), the average severity levels for categories of pneumoconiotic agents were calculated using the enforced PEL as the denominator. Due to complexities of determining the PEL for respirable coal mine dust samples and for respirable coal mine quartz samples, the denominators used for calculating average severity levels for this report were always $2 \mathrm{mg} / \mathrm{m}^{3}$ MRE for respirable coal mine dust and $0.1 \mathrm{mg} / \mathrm{m}^{3}$ MRE for respirable coal mine quartz. By using these surrogate PELs in the denominators, the severity levels have consistent comparison points which are distinct for respirable coal mine dust and respirable coal mine quartz. The following paragraph explains the method of determining PELs for coal mine dust and provides a rationale for selecting the denominator used for determining severity levels of quartz samples from coal mines.

Since December 1972, the maximum PEL for respirable coal mine dust has been $2 \mathrm{mg} / \mathrm{m}^{3}$ MRE 
unless the quartz concentration at the particular mine has been found in excess of 5\%. MSHA has no specific PEL for quartz in coal mines. MSHA's respirable coal mine quartz data are based on their analysis of respirable coal mine dust samples. However, for the period covered by this report (through 1994 for exposure data), inspector samples with less than $0.45 \mathrm{mg}$ net weight gain were not analyzed for quartz. When the quartz content has been found to be in excess of $5 \%$ in dust from a particular mine, the $2 \mathrm{mg} / \mathrm{m}^{3}$ MRE PEL is reduced based on the following formula:

$$
\mathrm{PEL}=\frac{10 \mathrm{mg} / \mathrm{m}^{3} \mathrm{MRE}}{\% \text { quartz }}
$$

Using this formula, one sees that at $100 \%$ quartz, the PEL would be $0.1 \mathrm{mg} / \mathrm{m}^{3}$ MRE.

\section{Permissible Exposure Limits}

OSHA and MSHA each enforce regulations which establish the legal limits on workplace exposures to pneumoconiotic agents. These legal limits are described in this report as permissible exposure limits
(PELs), although the regulations sometimes use the term "standard" or "exposure limit." The current legal limits may be found in the U.S. Code of Federal Regulations (CFR), as follows:

\section{OSHA}

general industry: $\quad 29$ CFR 1910.1000

29 CFR 1910.1001

29 CFR 1910.1043

construction industry: 29 CFR 1926.55

29 CFR 1926.1001

maritime industry: $\quad 29$ CFR 1915.1000

29 CFR 1915.1101

MSHA

coal mine industry: $\quad 30$ CFR 70.100-.101

30 CFR 71.100-.101

30 CFR 71.700

30 CFR 75.321

non-coal industry: $\quad 30$ CFR 56.5001

30 CFR 57.5001

This report uses the PELs which were enforced at the time of the sample, unless a surrogate PEL was being used (see preceding section, Data Analysis). 
States reporting industry and occupation codes from death certificates to NCHS, 1985-1992

\begin{tabular}{l|c|c|c|c|c|c|c|c}
\hline State & 1985 & 1986 & 1987 & 1988 & 1989 & 1990 & 1991 & 1992 \\
\hline Alaska & & & $\mathrm{X}$ & $\mathrm{X}$ & & & & \\
\hline Colorado & $\mathrm{X}$ & $\mathrm{X}$ & $\mathrm{X}$ & $\mathrm{X}$ & $\mathrm{X}$ & $\mathrm{X}$ & $\mathrm{X}$ & $\mathrm{X}$ \\
\hline Georgia & $\mathrm{X}$ & $\mathrm{X}$ & $\mathrm{X}$ & $\mathrm{X}$ & $\mathrm{X}$ & $\mathrm{X}$ & $\mathrm{X}$ & $\mathrm{X}$ \\
\hline Idaho & & & & $\mathrm{X}$ & $\mathrm{X}$ & $\mathrm{X}$ & $\mathrm{X}$ & $\mathrm{X}$ \\
\hline Indiana & & $\mathrm{X}$ & $\mathrm{X}$ & $\mathrm{X}$ & $\mathrm{X}$ & $\mathrm{X}$ & $\mathrm{X}$ & $\mathrm{X}$ \\
\hline Kansas & $\mathrm{X}$ & $\mathrm{X}$ & $\mathrm{X}$ & $\mathrm{X}$ & $\mathrm{X}$ & $\mathrm{X}$ & $\mathrm{X}$ & $\mathrm{X}$ \\
\hline Kentucky & $\mathrm{X}$ & $\mathrm{X}$ & $\mathrm{X}$ & $\mathrm{X}$ & & & & \\
\hline Maine & $\mathrm{X}$ & $\mathrm{X}$ & $\mathrm{X}$ & $\mathrm{X}$ & $\mathrm{X}$ & $\mathrm{X}$ & $\mathrm{X}$ & $\mathrm{X}$ \\
\hline Missouri & $\mathrm{X}$ & $\mathrm{X}$ & & & & & & \\
\hline Nebraska & $\mathrm{X}$ & & & & & & & \\
\hline Nevada & $\mathrm{X}$ & $\mathrm{X}$ & $\mathrm{X}$ & $\mathrm{X}$ & $\mathrm{X}$ & $\mathrm{X}$ & $\mathrm{X}$ & $\mathrm{X}$ \\
\hline New Hampshire & $\mathrm{X}$ & $\mathrm{X}$ & $\mathrm{X}$ & $\mathrm{X}$ & $\mathrm{X}$ & $\mathrm{X}$ & $\mathrm{X}$ & $\mathrm{X}$ \\
\hline New Jersey & & & & $\mathrm{X}$ & $\mathrm{X}$ & $\mathrm{X}$ & $\mathrm{X}$ & $\mathrm{X}$ \\
\hline New Mexico & & $\mathrm{X}$ & $\mathrm{X}$ & $\mathrm{X}$ & $\mathrm{X}$ & $\mathrm{X}$ & $\mathrm{X}$ & $\mathrm{X}$ \\
\hline North Carolina & & & $\mathrm{X}$ & $\mathrm{X}$ & $\mathrm{X}$ & $\mathrm{X}$ & $\mathrm{X}$ & $\mathrm{X}$ \\
\hline Ohio & $\mathrm{X}$ & $\mathrm{X}$ & $\mathrm{X}$ & $\mathrm{X}$ & $\mathrm{X}$ & $\mathrm{X}$ & $\mathrm{X}$ & $\mathrm{X}$ \\
\hline Oklahoma & $\mathrm{X}$ & $\mathrm{X}$ & $\mathrm{X}$ & $\mathrm{X}$ & $\mathrm{X}$ & $\mathrm{X}$ & $\mathrm{X}$ & $\mathrm{X}$ \\
\hline Rhode Island & $\mathrm{X}$ & $\mathrm{X}$ & $\mathrm{X}$ & $\mathrm{X}$ & $\mathrm{X}$ & $\mathrm{X}$ & $\mathrm{X}$ & $\mathrm{X}$ \\
\hline South Carolina & $\mathrm{X}$ & $\mathrm{X}$ & $\mathrm{X}$ & $\mathrm{X}$ & $\mathrm{X}$ & $\mathrm{X}$ & $\mathrm{X}$ & $\mathrm{X}$ \\
\hline Tennessee & $\mathrm{X}$ & $\mathrm{X}$ & $\mathrm{X}$ & $\mathrm{X}$ & & & & \\
\hline Utah & $\mathrm{X}$ & $\mathrm{X}$ & $\mathrm{X}$ & $\mathrm{X}$ & $\mathrm{X}$ & $\mathrm{X}$ & $\mathrm{X}$ & $\mathrm{X}$ \\
\hline Vermont & & $\mathrm{X}$ & $\mathrm{X}$ & $\mathrm{X}$ & $\mathrm{X}$ & $\mathrm{X}$ & $\mathrm{X}$ & $\mathrm{X}$ \\
\hline Washington & & & & $\mathrm{X}$ & $\mathrm{X}$ & $\mathrm{X}$ & $\mathrm{X}$ \\
\hline West Virginia & & & $\mathrm{X}$ & $\mathrm{X}$ & $\mathrm{X}$ & $\mathrm{X}$ & $\mathrm{X}$ \\
\hline Wisconsin & & $\mathrm{X}$ & $\mathrm{X}$ & $\mathrm{X}$ & $\mathrm{X}$ & $\mathrm{X}$ & $\mathrm{X}$ \\
\hline
\end{tabular}


Pneumoconiotic Agent Categories for MSHA and OSHA Inspector Data

\begin{tabular}{|c|c|c|}
\hline $\begin{array}{l}\text { Pneumoconiotic } \\
\text { Agent Category } \\
\text { (as defined for } \\
\text { this report) }\end{array}$ & OSHA Agents in Category & MSHA Agents in Category \\
\hline $\begin{array}{c}\text { Coal and } \\
\text { Coal Mine Dust }\end{array}$ & $\begin{array}{l}\text { Coal dust } \\
\text { Coal dust }\left(\leq 5 \% \mathrm{SiO}_{2}\right) \text { (respirable quartz fraction) }\end{array}$ & $\begin{array}{l}\text { Coal dust, respirable fraction, }<5 \% \text { quartz } \\
\text { Coal mine respirable dust }\end{array}$ \\
\hline Silica & $\begin{array}{l}\text { Silica (quartz, non-respirable) } \\
\text { Silica crystalline quartz (as quartz), respirable dust } \\
\text { Silica, amorphous, diatomaceous earth ( }>1 \% \\
\quad \text { crystalline silica) } \\
\text { Silica, crystalline cristobalite respirable dust } \\
\text { Silica, crystalline tridymite respirable dust } \\
\text { Silica, crystalline tripoli (as quartz)(respirable dust) }\end{array}$ & $\begin{array}{l}\text { Coal mine quartz } \\
\text { Cristobalite, respirable fraction } \\
\text { Nuisance dust, respirable fraction, }<1 \% \text { quartz }^{* *} \\
\text { Quartz, respirable fraction, }>1 \% \text { quartz } \\
\text { Respirable dust (not analyzed or below detection limit)** } \\
\text { Tridymite, respirable fraction } \\
\text { Unlisted particulate, respirable fraction, }<1 \% \text { quartz** }\end{array}$ \\
\hline Other & $\begin{array}{l}\text { Alpha-alumina (total dust)* } \\
\text { Aluminum (as Al), metal (respirable fraction) } \\
\text { Aluminum(as Al), metal (total dust) } \\
\text { Aluminum (as Al), welding fumes } \\
\text { Aluminum oxide } \\
\text { Aluminum silicate } \\
\text { Antimony and compounds (as Sb) } \\
\text { Barium (insoluble compounds) } \\
\text { Barium sulfate (total dust) } \\
\text { Beryllium and compounds } \\
\text { Cadmium dust (as Cd) } \\
\text { Cadmium fume (as Cd) } \\
\text { Carbon black } \\
\text { Cobalt, metal, fume and dust (as Co) } \\
\text { Emery } \\
\text { Emery (respirable fraction) } \\
\text { Graphite (natural) } \\
\text { Iron oxide fume } \\
\text { Kaolin } \\
\text { Kaolin (respirable fraction) } \\
\text { Magnesite* } \\
\text { Magnesite (respirable fraction) } \\
\text { Mica (less than 1\% crystalline silica) } \\
\text { Portland cement (less than 1\% quartz) } \\
\text { Portland cement (respirable fraction) } \\
\text { Rouge } \\
\text { Rouge (respirable fraction) } \\
\text { Talc (containing no asbestos) } \\
\text { Talc, fibrous non-tremolite } \\
\text { Tin oxide } \\
\text { Titanium dioxide } \\
\text { Tungsten and compounds (insoluble) (as W) } \\
\text { Welding fumes (total particulate) } \\
\text { Wollastonite* }\end{array}$ & $\begin{array}{l}\text { Aluminum oxide dust, as } \mathrm{Al}_{2} \mathrm{O}_{3} \\
\text { Aluminum oxide fume, as } \mathrm{Al}_{2} \mathrm{O}_{3} \\
\text { Antimony dusts, as } \mathrm{Sb} \\
\text { Beryllium dusts, as } \mathrm{Be} \\
\text { Beryllium fumes, as } \mathrm{Be} \\
\text { Cadmium oxide fume, as } \mathrm{Cd} \\
\text { Cadmium, metal dusts and soluble salts, as Cd } \\
\text { Carbon black } \\
\text { Cobalt dusts, as Co } \\
\text { Cobalt fumes, as Co } \\
\text { Graphite (natural) } \\
\text { Iron oxide fume, as } \mathrm{Fe}_{2} \mathrm{O}_{3} \\
\text { Mica* } \\
\text { Talc, fibers }>5 \text { m in length }\left(\mathrm{Mg}_{3} \mathrm{Si}_{4} \mathrm{O}_{10}(\mathrm{OH})_{2}\right) \\
\text { Talc, nonfibrous, }<1 \% \text { quartz } \\
\text { Tin oxide dust, as } \mathrm{SnO}_{2} \\
\text { Tin oxide fume, as } \mathrm{SnO}_{2} \\
\text { Titanium dioxide dust, as } \mathrm{TiO}_{2} \\
\text { Titanium dioxide fume, as } \mathrm{TiO}_{2} \\
\text { Tungsten fumes, as } \mathrm{W}^{*} \\
\text { Tungsten, insoluble dusts, as } \mathrm{W}^{*} \\
\text { Welding fumes, total particulate }\end{array}$ \\
\hline
\end{tabular}

\footnotetext{
* No data reported for these agents.
}

** See Selected Limitations, page 6. 


\section{Summary Description of Previous Work-Related Lung Disease (WoRLD) Surveillance Reports}

See page ii of this report for information on how to order copies of the previous Work-Related Lung Disease Surveillance Reports described below.

\begin{abstract}
Work-Related Lung Disease Surveillance Report (original report issued in 1991)

The 1991 Work-Related Lung Disease Surveillance Report was the first in a series of occupational respiratory disease surveillance reports produced by the Division of Respiratory Disease Studies (DRDS), National Institute for Occupational Safety and Health (NIOSH). The report represents a summary of surveillance data for various occupational respiratory diseases. The majority of data presented in the report is for the time period 1968-1987, however, the time period varies for different data sources.
\end{abstract}

Data presented in the report originated from programs administered by NIOSH (e.g., the Coal Workers' X-ray Surveillance Program, the National Coal Workers' Autopsy Study), the National Center for Health Statistics, the Bureau of Labor Statistics, the Mine Safety and Health Administration, the Occupational Safety and Health Administration, the Health Care Financing Administration, and the Social Security Administration.

The 1991 report is organized into two major sections: Figures and Tables. Section I contains 21 figures and Section II contains 59 tables. Within the figures and tables sections, data are provided under the following subheadings: asbestosis, coal workers' pneumoconiosis, silicosis, exposure to cotton dust, pneumonopathy due to inhalation of other dust, hypersensitivity pneumonitis, toxic agents, dust diseases of the lung, and compensation.

\section{Work-Related Lung Disease Surveillance Report Supplement, 1992 \\ The 1992 Work-Related Lung Disease Surveillance Report, Supplement, presents updated data for many of the figures and tables presented in the 1991 report. Data from the 1991 report are updated to include 1988 mortality data.}

In addition to updated data, this supplement includes data not previously presented. These data include: (1) sex, race, geographic distribution, industry and occupation from the multiple cause of death data for deaths with mention of asbestosis, malignant neoplasms of the pleura, malignant neoplasms of the peritoneum, coal workers' pneumoconiosis, silicosis, byssinosis, or hypersensitivity pneumonitis; (2) number of discharges with silicosis or asbestosis from the National Hospital Discharge Survey; and (3) reports of occupational asthma and silicosis from the Sentinel Event Notification Systems for Occupational Risks (SENSOR) program.

Work-Related Lung Disease Surveillance Report, 1994 The majority of data presented in the 1994 Work-Related Lung Disease Surveillance Report is for the time period 1968-1990. However, the time period covered varies for some of the data sources.

A portion of the data contained in the 1994 WoRLD Surveillance Report originates from programs and activities administered by NIOSH (e.g., the Coal Workers' X-ray Surveillance Program, the National Occupational Health Survey of Mining, and the SENSOR program). Other data were obtained from the $\mathrm{N}$ ational Center for Health Statistics, the Department of Labor, the Social Security Administration, the Mine Safety and Health Administration, the Occupational Safety and Health Administration, the Bureau of Mines, and the Association of Occupational and Environmental Clinics.

The organization of the 1994 WoRLD Surveillance Report differs from earlier editions. It is divided into 11 major sections. The first ten sections are specific to selected occupational lung diseases or conditions, summarizing mortality and morbidity data, and other available information, such as occupational exposures or numbers of workers at risk. These sections include: asbestosis, malignant neoplasms of the pleura, coal workers' pneumoconiosis, silicosis, pneumoconiosis due to other inorganic dust, unspecified pneumoconiosis, byssinosis, hypersensitivity pneumonitis, occupational asthma, and other lung conditions. The remaining section provides data from the Association of Occupational and Environmental Clinics database. Most sections contain an initial group of figures, followed by data tables.

The 1994 WoRLD Surveillance Report contains major additions, both in the addition of previously unreported data such as that from the National Health Interview Survey and the Association of Occupational and Environmental Clinics, and in supplementing the data with selected statistical measures, such as proportionate mortality ratios, both crude and age-adjusted rates at national and state levels, and years of potential life lost to age 65 and to life expectancy. 
The following is a revision to Table 1-6 of the 1994 Work-Related Lung Disease(WoRLD) Surveillance Report. The overall rate reported in the 1994 WoRLD Surveillance Report included only whites and blacks. The revised overall rates presented below are based on all races. U.S. population data files have also been revised and updated for the tables presented below.

Table 1-6. Asbestosis: crude mortality rates (per $1,000,000$ population), U.S. residents age 15 and over, by race and sex, 1968-1990

\begin{tabular}{|c|c|c|c|c|c|}
\hline \multirow[b]{2}{*}{ Year } & \multirow[b]{2}{*}{ Overall rate } & \multicolumn{2}{|c|}{ White } & \multicolumn{2}{|c|}{ Black } \\
\hline & & Males & Females & Males & Females \\
\hline 1968 & 0.53 & 1.08 & 0.09 & 0.44 & - \\
\hline 1969 & 0.49 & 1.03 & 0.07 & 0.15 & - \\
\hline 1970 & 0.60 & 1.30 & 0.03 & 0.59 & - \\
\hline 1971 & 0.56 & 1.15 & 0.07 & 0.72 & - \\
\hline 1972 & 0.91 & 2.04 & 0.03 & 0.56 & - \\
\hline 1973 & 0.75 & 1.67 & 0.06 & 0.41 & - \\
\hline 1974 & 0.72 & 1.51 & 0.11 & 0.66 & - \\
\hline 1975 & 0.78 & 1.62 & 0.15 & 0.65 & - \\
\hline 1976 & 0.87 & 1.89 & 0.04 & 1.13 & - \\
\hline 1977 & 0.98 & 2.15 & 0.05 & 0.98 & - \\
\hline 1978 & 1.41 & 3.01 & 0.13 & 1.68 & 0.10 \\
\hline 1979 & 1.79 & 3.90 & 0.11 & 1.76 & - \\
\hline 1980 & 1.93 & 4.09 & 0.19 & 2.62 & - \\
\hline 1981 & 1.78 & 3.67 & 0.31 & 2.12 & - \\
\hline 1982 & 2.37 & 5.03 & 0.25 & 2.96 & - \\
\hline 1983 & 2.61 & 5.49 & 0.33 & 3.23 & - \\
\hline 1984 & 2.41 & 5.20 & 0.18 & 3.18 & 0.09 \\
\hline 1985 & 2.86 & 6.11 & 0.27 & 3.97 & - \\
\hline 1986 & 3.72 & 7.96 & 0.32 & 4.92 & 0.09 \\
\hline 1987 & 3.73 & 8.27 & 0.31 & 3.03 & 0.09 \\
\hline 1988 & 4.00 & 8.76 & 0.28 & 4.49 & 0.26 \\
\hline 1989 & 4.54 & 9.83 & 0.29 & 5.72 & 0.34 \\
\hline 1990 & 4.85 & 10.23 & 0.51 & 7.40 & 0.17 \\
\hline
\end{tabular}

- indicates no deaths listed.

SOURCE: National Center for Health Statistics multiple cause of death data.

U.S. Bureau of the Census: 1970-1990 population estimates of the U.S. 
The following is a revision to Table 1-7 of the 1994 Work-Related Lung Disease(WoRLD) Surveillance Report. The overall rate reported in the 1994 WoRLD Surveillance Report included only whites and blacks. The revised overall rates presented below are based on all races. U.S. population data files have also been revised and updated for the tables presented below.

Table 1-7. Asbestosis: age-adjusted mortality rates (per 1,000,000 population), U.S. residents age 15 and over, by race and sex, 1968-1990

\begin{tabular}{|c|c|c|c|c|c|}
\hline \multirow[b]{2}{*}{ Year } & \multirow[b]{2}{*}{ Overall rate } & \multicolumn{2}{|c|}{ White } & \multicolumn{2}{|c|}{ Black } \\
\hline & & Males & Females & Males & Females \\
\hline 1968 & 0.44 & 0.92 & 0.07 & 0.43 & - \\
\hline 1969 & 0.39 & 0.85 & 0.05 & 0.14 & - \\
\hline 1970 & 0.49 & 1.09 & 0.02 & 0.65 & - \\
\hline 1971 & 0.44 & 0.94 & 0.06 & 0.69 & - \\
\hline 1972 & 0.73 & 1.69 & 0.03 & 0.56 & - \\
\hline 1973 & 0.58 & 1.37 & 0.03 & 0.42 & - \\
\hline 1974 & 0.58 & 1.26 & 0.09 & 0.70 & - \\
\hline 1975 & 0.61 & 1.33 & 0.10 & 0.62 & - \\
\hline 1976 & 0.66 & 1.53 & 0.02 & 1.18 & - \\
\hline 1977 & 0.74 & 1.73 & 0.03 & 0.99 & - \\
\hline 1978 & 1.08 & 2.42 & 0.08 & 1.87 & 0.07 \\
\hline 1979 & 1.34 & 3.09 & 0.08 & 1.92 & - \\
\hline 1980 & 1.43 & 3.24 & 0.12 & 2.71 & - \\
\hline 1981 & 1.30 & 2.88 & 0.20 & 2.10 & - \\
\hline 1982 & 1.75 & 3.94 & 0.15 & 3.18 & - \\
\hline 1983 & 1.86 & 4.20 & 0.18 & 3.28 & - \\
\hline 1984 & 1.67 & 3.86 & 0.12 & 3.31 & 0.08 \\
\hline 1985 & 2.00 & 4.58 & 0.14 & 4.02 & - \\
\hline 1986 & 2.51 & 5.78 & 0.18 & 5.05 & 0.08 \\
\hline 1987 & 2.49 & 5.95 & 0.17 & 3.13 & 0.05 \\
\hline 1988 & 2.62 & 6.21 & 0.14 & 4.56 & 0.24 \\
\hline 1989 & 2.84 & 6.70 & 0.14 & 5.75 & 0.21 \\
\hline 1990 & 3.01 & 6.90 & 0.25 & 7.41 & 0.15 \\
\hline
\end{tabular}

- indicated no deaths listed.

SOURCE: National Center for Health Statistics multiple cause of death data.

U.S. Bureau of the Census: 1970-1990 population estimates of the U.S. 
The following is a revision to Table 2-6 of the 1994 Work-Related Lung Disease(WoRLD) Surveillance Report. The overall rate reported in the 1994 WoRLD Surveillance Report included only whites and blacks. The revised overall rates presented below are based on all races. U.S. population data files have also been revised and updated for the tables presented below.

Table 2-6. Malignant neoplasm of the pleura: crude mortality rates (per 1,000,000 population), U.S. residents age 15 and over, by race and sex, 1968-1990

\begin{tabular}{|c|c|c|c|c|c|}
\hline \multirow[b]{2}{*}{ Year } & \multirow[b]{2}{*}{ Overall rate } & \multicolumn{2}{|c|}{ White } & \multicolumn{2}{|c|}{ Black } \\
\hline & & Males & Females & Males & Females \\
\hline 1968 & 2.67 & 3.51 & 2.00 & 3.39 & 1.65 \\
\hline 1969 & 2.51 & 3.37 & 1.99 & 2.06 & 1.01 \\
\hline 1970 & 2.90 & 4.25 & 1.88 & 3.24 & 1.39 \\
\hline 1971 & 2.89 & 4.43 & 1.75 & 2.72 & 1.23 \\
\hline 1972 & 2.80 & 4.05 & 1.57 & 5.00 & 2.15 \\
\hline 1973 & 2.54 & 3.42 & 1.91 & 2.71 & 1.17 \\
\hline 1974 & 2.60 & 3.78 & 1.77 & 2.12 & 1.47 \\
\hline 1975 & 2.66 & 3.79 & 1.85 & 2.71 & 1.33 \\
\hline 1976 & 2.72 & 3.90 & 1.91 & 3.14 & 0.75 \\
\hline 1977 & 2.60 & 4.09 & 1.54 & 1.72 & 1.15 \\
\hline 1978 & 2.77 & 4.31 & 1.79 & 1.32 & 1.33 \\
\hline 1979 & 2.59 & 4.21 & 1.45 & 1.88 & 0.90 \\
\hline 1980 & 2.60 & 4.20 & 1.44 & 2.05 & 1.46 \\
\hline 1981 & 2.54 & 4.30 & 1.23 & 2.68 & 0.76 \\
\hline 1982 & 2.79 & 4.21 & 1.99 & 2.08 & 0.47 \\
\hline 1983 & 2.69 & 4.76 & 1.31 & 1.29 & 0.92 \\
\hline 1984 & 2.89 & 4.68 & 1.57 & 2.65 & 1.18 \\
\hline 1985 & 2.51 & 4.33 & 1.24 & 1.57 & 0.98 \\
\hline 1986 & 2.62 & 4.33 & 1.43 & 2.26 & 0.88 \\
\hline 1987 & 2.65 & 4.71 & 1.22 & 1.82 & 0.86 \\
\hline 1988 & 2.56 & 4.34 & 1.39 & 2.10 & 0.43 \\
\hline 1989 & 2.80 & 4.75 & 1.43 & 2.17 & 1.26 \\
\hline 1990 & 2.83 & 5.13 & 1.31 & 1.95 & 0.58 \\
\hline
\end{tabular}


The following is a revision to Table 2-7 of the 1994 Work-Related Lung Disease(WoRLD) Surveillance Report. The overall rate reported in the 1994 WoRLD Surveillance Report included only whites and blacks. The revised overall rates presented below are based on all races. U.S. population data files have also been revised and updated for the tables presented below.

Table 2-7. Malignant neoplasm of the pleura: age-adjusted mortality rates (per 1,000,000 population), U.S. residents age 15 and over, by race and sex, 1968-1990

\begin{tabular}{|c|c|c|c|c|c|}
\hline \multirow[b]{2}{*}{ Year } & \multirow[b]{2}{*}{ Overall rate } & \multicolumn{2}{|c|}{ White } & \multicolumn{2}{|c|}{ Black } \\
\hline & & Males & Females & Males & Females \\
\hline 1968 & 2.10 & 2.90 & 1.44 & 3.42 & 1.34 \\
\hline 1969 & 2.02 & 2.80 & 1.52 & 2.01 & 0.95 \\
\hline 1970 & 2.29 & 3.49 & 1.34 & 3.27 & 1.34 \\
\hline 1971 & 2.17 & 3.57 & 1.14 & 2.62 & 1.09 \\
\hline 1972 & 2.18 & 3.32 & 1.04 & 5.11 & 2.10 \\
\hline 1973 & 1.94 & 2.73 & 1.37 & 2.87 & 1.03 \\
\hline 1974 & 2.02 & 3.10 & 1.21 & 2.25 & 1.39 \\
\hline 1975 & 2.05 & 3.11 & 1.26 & 2.87 & 1.15 \\
\hline 1976 & 2.05 & 3.17 & 1.24 & 3.22 & 0.73 \\
\hline 1977 & 2.00 & 3.35 & 1.03 & 1.85 & 1.22 \\
\hline 1978 & 2.08 & 3.50 & 1.14 & 1.55 & 1.10 \\
\hline 1979 & 1.96 & 3.45 & 0.94 & 1.97 & 0.74 \\
\hline 1980 & 1.91 & 3.37 & 0.84 & 2.27 & 1.20 \\
\hline 1981 & 1.83 & 3.36 & 0.70 & 2.96 & 0.55 \\
\hline 1982 & 2.06 & 3.36 & 1.20 & 2.35 & 0.44 \\
\hline 1983 & 1.90 & 3.63 & 0.82 & 1.36 & 0.82 \\
\hline 1984 & 2.09 & 3.61 & 0.97 & 2.96 & 1.03 \\
\hline 1985 & 1.77 & 3.30 & 0.75 & 1.67 & 0.88 \\
\hline 1986 & 1.83 & 3.25 & 0.84 & 2.46 & 0.70 \\
\hline 1987 & 1.89 & 3.61 & 0.70 & 1.93 & 0.73 \\
\hline 1988 & 1.78 & 3.24 & 0.82 & 2.26 & 0.47 \\
\hline 1989 & 1.92 & 3.47 & 0.87 & 2.28 & 1.01 \\
\hline 1990 & 1.91 & 3.72 & 0.73 & 2.02 & 0.40 \\
\hline
\end{tabular}

SOURCE: National Center for Health Statistics multiple cause of death data.

U.S. Bureau of the Census: 1970-1990 population estimates of the U.S. 
The following is a revision to Table 3-6 of the 1994 Work-Related Lung Disease(WoRLD) Surveillance Report. The overall rate reported in the 1994 WoRL D Surveillance Report included only whites and blacks. The revised overall rates presented below are based on all races. U.S. population data files have also been revised and updated for the tables presented below.

Table 3-6. Coal workers' pneumoconiosis: crude mortality rates (per 1,000,000 population), U.S. residents age 15 and over, by race and sex, 1968-1990

\begin{tabular}{|c|c|c|c|c|c|}
\hline \multirow[b]{2}{*}{ Year } & \multirow[b]{2}{*}{ Overall rate } & \multicolumn{2}{|c|}{ White } & \multicolumn{2}{|c|}{ Black } \\
\hline & & Males & Females & Males & Females \\
\hline 1968 & 12.15 & 27.87 & 0.19 & 4.42 & 0.13 \\
\hline 1969 & 10.50 & 24.13 & 0.06 & 4.57 & - \\
\hline 1970 & 14.99 & 34.29 & 0.15 & 7.07 & 0.13 \\
\hline 1971 & 17.06 & 38.96 & 0.31 & 7.30 & 0.12 \\
\hline 1972 & 18.85 & 43.01 & 0.26 & 9.73 & - \\
\hline 1973 & 17.20 & 39.00 & 0.27 & 10.98 & 0.23 \\
\hline 1974 & 17.27 & 39.23 & 0.32 & 10.58 & 0.11 \\
\hline 1975 & 16.81 & 38.34 & 0.34 & 9.29 & - \\
\hline 1976 & 15.11 & 34.32 & 0.28 & 10.44 & 0.11 \\
\hline 1977 & 14.00 & 31.89 & 0.25 & 9.22 & 0.21 \\
\hline 1978 & 13.40 & 30.80 & 0.21 & 7.46 & - \\
\hline 1979 & 13.96 & 32.16 & 0.17 & 7.76 & 0.10 \\
\hline 1980 & 14.65 & 33.84 & 0.14 & 8.43 & 0.10 \\
\hline 1981 & 14.33 & 33.06 & 0.17 & 8.59 & 0.38 \\
\hline 1982 & 15.27 & 35.04 & 0.26 & 11.07 & - \\
\hline 1983 & 14.81 & 33.93 & 0.16 & 12.08 & 0.37 \\
\hline 1984 & 14.55 & 33.86 & 0.16 & 7.85 & 0.36 \\
\hline 1985 & 14.03 & 32.61 & 0.14 & 8.25 & 0.27 \\
\hline 1986 & 12.95 & 29.79 & 0.25 & 9.75 & 0.26 \\
\hline 1987 & 11.85 & 27.46 & 0.13 & 8.49 & 0.26 \\
\hline 1988 & 11.55 & 26.94 & 0.17 & 6.98 & 0.26 \\
\hline 1989 & 11.03 & 25.79 & 0.09 & 7.20 & 0.08 \\
\hline 1990 & 10.19 & 23.71 & 0.14 & 6.82 & 0.17 \\
\hline
\end{tabular}

- indicates no deaths listed.

SOURCE: National Center for Health Statistics multiple cause of death data.

U.S. Bureau of the Census: 1970-1990 population estimates of the U.S. 
The following is a revision to Table 3-7 of the 1994 Work-Related Lung Disease(WoRLD) Surveillance Report. The overall rate reported in the 1994 WoRLD Surveillance Report included only whites and blacks. The revised overall rates presented below are based on all races. U.S. population data files have also been revised and updated for the tables presented below.

Table 3-7. Coal workers' pneumoconiosis: age-adjusted mortality rates (per 1,000,000 population), U.S. residents age 15 and over, by race and sex, 1968-1990

\begin{tabular}{|c|c|c|c|c|c|}
\hline \multirow[b]{2}{*}{ Year } & \multirow[b]{2}{*}{ Overall rate } & \multicolumn{2}{|c|}{ White } & \multicolumn{2}{|c|}{ Black } \\
\hline & & Males & Females & Males & Females \\
\hline 1968 & 8.53 & 21.03 & 0.12 & 4.17 & 0.12 \\
\hline 1969 & 7.31 & 18.10 & 0.05 & 4.34 & - \\
\hline 1970 & 10.47 & 25.80 & 0.09 & 6.60 & 0.11 \\
\hline 1971 & 11.92 & 29.41 & 0.17 & 6.95 & 0.14 \\
\hline 1972 & 13.32 & 32.92 & 0.11 & 9.24 & - \\
\hline 1973 & 12.10 & 29.89 & 0.15 & 10.19 & 0.19 \\
\hline 1974 & 12.11 & 30.08 & 0.17 & 9.71 & 0.13 \\
\hline 1975 & 11.58 & 29.07 & 0.22 & 8.71 & - \\
\hline 1976 & 10.38 & 26.02 & 0.12 & 9.75 & 0.09 \\
\hline 1977 & 9.44 & 23.81 & 0.17 & 8.62 & 0.17 \\
\hline 1978 & 8.89 & 22.71 & 0.10 & 7.24 & - \\
\hline 1979 & 9.23 & 23.61 & 0.09 & 7.24 & 0.08 \\
\hline 1980 & 9.61 & 24.64 & 0.10 & 7.85 & 0.06 \\
\hline 1981 & 9.05 & 23.43 & 0.11 & 7.99 & 0.37 \\
\hline 1982 & 9.45 & 24.48 & 0.12 & 10.05 & - \\
\hline 1983 & 9.16 & 23.55 & 0.09 & 11.24 & 0.31 \\
\hline 1984 & 8.94 & 23.35 & 0.07 & 7.23 & 0.31 \\
\hline 1985 & 8.32 & 21.95 & 0.07 & 6.98 & 0.18 \\
\hline 1986 & 7.61 & 19.82 & 0.09 & 8.69 & 0.24 \\
\hline 1987 & 6.86 & 17.97 & 0.05 & 7.52 & 0.22 \\
\hline 1988 & 6.48 & 17.15 & 0.08 & 6.35 & 0.19 \\
\hline 1989 & 6.11 & 16.16 & 0.04 & 6.50 & 0.02 \\
\hline 1990 & 5.44 & 14.43 & 0.05 & 5.91 & 0.14 \\
\hline
\end{tabular}

- indicates no deaths listed.

SOURCE: National Center for Health Statistics multiple cause of death data.

U.S. Bureau of the Census: 1970-1990 population estimates of the U.S. 
The following is a revision to Table 4-6 of the 1994 Work-Related Lung Disease(WoRLD) Surveillance Report. The overall rate reported in the 1994 WoRLD Surveillance Report included only whites and blacks. The revised overall rates presented below are based on all races. U.S. population data files have also been revised and updated for the tables presented below.

Table 4-6. Silicosis: crude mortality rates (per $1,000,000$ population), U.S. residents age 15 and over, by race and sex, 1968-1990

\begin{tabular}{|c|c|c|c|c|c|}
\hline \multirow[b]{2}{*}{ Year } & \multirow[b]{2}{*}{ Overall rate } & \multicolumn{2}{|c|}{ White } & \multicolumn{2}{|c|}{ Black } \\
\hline & & Males & Females & Males & Females \\
\hline 1968 & 7.92 & 16.52 & 0.19 & 16.94 & 0.38 \\
\hline 1969 & 7.83 & 16.26 & 0.18 & 17.38 & 0.13 \\
\hline 1970 & 7.44 & 15.70 & 0.19 & 13.70 & 0.13 \\
\hline 1971 & 6.20 & 13.30 & 0.15 & 10.02 & 0.12 \\
\hline 1972 & 6.45 & 13.33 & 0.17 & 14.45 & - \\
\hline 1973 & 5.47 & 11.41 & 0.15 & 11.25 & 0.23 \\
\hline 1974 & 4.99 & 10.56 & 0.11 & 9.39 & 0.23 \\
\hline 1975 & 4.52 & 9.43 & 0.16 & 9.29 & 0.11 \\
\hline 1976 & 4.09 & 8.54 & 0.12 & 8.80 & - \\
\hline 1977 & 3.36 & 7.02 & 0.07 & 7.50 & - \\
\hline 1978 & 3.16 & 6.41 & 0.19 & 7.58 & 0.10 \\
\hline 1979 & 2.61 & 5.25 & 0.06 & 7.52 & 0.10 \\
\hline 1980 & 2.55 & 4.99 & 0.18 & 7.52 & - \\
\hline 1981 & 2.12 & 4.37 & 0.22 & 3.46 & 0.10 \\
\hline 1982 & 2.16 & 4.36 & 0.04 & 6.14 & - \\
\hline 1983 & 1.97 & 4.04 & 0.06 & 4.85 & - \\
\hline 1984 & 2.07 & 4.09 & 0.11 & 6.15 & 0.09 \\
\hline 1985 & 1.79 & 3.52 & 0.10 & 5.43 & 0.09 \\
\hline 1986 & 1.69 & 3.32 & 0.10 & 4.82 & - \\
\hline 1987 & 1.80 & 3.50 & 0.18 & 4.95 & 0.09 \\
\hline 1988 & 1.57 & 3.00 & 0.11 & 5.29 & 0.17 \\
\hline 1989 & 1.56 & 3.03 & 0.14 & 4.34 & 0.25 \\
\hline 1990 & 1.58 & 3.14 & 0.11 & 4.38 & 0.08 \\
\hline
\end{tabular}

- indicates no deaths listed.

SOURCE: National Center for Health Statistics multiple cause of death data.

U.S. Bureau of the Census: 1970-1990 population estimates of the U.S. 
The following is a revision to Table 4-7 of the 1994 Work-Related Lung Disease(WoRLD) Surveillance Report. The overall rate reported in the 1994 WoRLD Surveillance Report included only whites and blacks. The revised overall rates presented below are based on all races. U.S. population data files have also been revised and updated for the tables presented below.

Table 4-7. Silicosis: age-adjusted mortality rates (per 1,000,000 population), U.S. residents age 15 and over, by race and sex, 1968-1990

\begin{tabular}{|c|c|c|c|c|c|}
\hline \multirow[b]{2}{*}{ Year } & \multirow[b]{2}{*}{ Overall rate } & \multicolumn{2}{|c|}{ White } & \multicolumn{2}{|c|}{ Black } \\
\hline & & Males & Females & Males & Females \\
\hline 1968 & 5.64 & 12.52 & 0.13 & 16.26 & 0.39 \\
\hline 1969 & 5.51 & 12.22 & 0.12 & 16.75 & 0.12 \\
\hline 1970 & 5.21 & 11.75 & 0.14 & 13.03 & 0.14 \\
\hline 1971 & 4.39 & 10.06 & 0.10 & 9.87 & 0.05 \\
\hline 1972 & 4.58 & 10.10 & 0.13 & 14.36 & - \\
\hline 1973 & 3.78 & 8.59 & 0.10 & 10.73 & 0.23 \\
\hline 1974 & 3.48 & 7.99 & 0.07 & 9.25 & 0.22 \\
\hline 1975 & 3.09 & 7.05 & 0.11 & 9.27 & 0.10 \\
\hline 1976 & 2.86 & 6.49 & 0.08 & 8.79 & - \\
\hline 1977 & 2.29 & 5.22 & 0.04 & 7.56 & - \\
\hline 1978 & 2.19 & 4.80 & 0.14 & 7.66 & 0.07 \\
\hline 1979 & 1.79 & 3.88 & 0.04 & 7.71 & 0.04 \\
\hline 1980 & 1.70 & 3.60 & 0.12 & 7.72 & - \\
\hline 1981 & 1.40 & 3.17 & 0.11 & 3.44 & 0.08 \\
\hline 1982 & 1.42 & 3.10 & 0.02 & 6.36 & - \\
\hline 1983 & 1.28 & 2.85 & 0.05 & 4.85 & - \\
\hline 1984 & 1.31 & 2.80 & 0.06 & 6.50 & 0.12 \\
\hline 1985 & 1.15 & 2.44 & 0.05 & 5.60 & 0.10 \\
\hline 1986 & 1.03 & 2.26 & 0.05 & 4.63 & - \\
\hline 1987 & 1.12 & 2.38 & 0.08 & 5.19 & 0.03 \\
\hline 1988 & 0.94 & 1.96 & 0.05 & 5.27 & 0.14 \\
\hline 1989 & 0.94 & 1.98 & 0.07 & 4.31 & 0.26 \\
\hline 1990 & 0.96 & 2.06 & 0.04 & 4.54 & 0.08 \\
\hline
\end{tabular}

- indicates no deaths listed.

SOURCE: National Center for Health Statistics multiple cause of death data.

U.S. Bureau of the Census: 1970-1990 population estimates of the U.S. 
The following is a revision to Table 5-6 of the 1994 Work-Related Lung Disease(WoRLD) Surveillance Report. The overall rate reported in the 1994 WoRLD Surveillance Report included only whites and blacks. The revised overall rates presented below are based on all races. U.S. population data files have also been revised and updated for the tables presented below.

Table 5-6. Pneumoconiosis due to other inorganic dust: crude mortality rates (per $1,000,000$ population), U.S. residents age 15 and over, by race and sex, 1968-1990

\begin{tabular}{|c|c|c|c|c|c|}
\hline \multirow[b]{2}{*}{ Year } & \multirow[b]{2}{*}{ Overall rate } & \multicolumn{2}{|c|}{ White } & \multicolumn{2}{|c|}{ Black } \\
\hline & & Males & Females & Males & Females \\
\hline 1968 & 0.09 & 0.18 & 0.01 & - & 0.13 \\
\hline 1969 & 0.10 & 0.10 & 0.10 & 0.15 & - \\
\hline 1970 & 0.12 & 0.18 & 0.09 & - & - \\
\hline 1971 & 0.08 & 0.11 & 0.04 & 0.14 & 0.12 \\
\hline 1972 & 0.11 & 0.12 & 0.09 & 0.28 & - \\
\hline 1973 & 0.06 & 0.11 & 0.03 & - & - \\
\hline 1974 & 0.09 & 0.15 & 0.03 & 0.13 & - \\
\hline 1975 & 0.06 & 0.09 & 0.04 & 0.13 & - \\
\hline 1976 & 0.08 & 0.10 & 0.07 & 0.13 & - \\
\hline 1977 & 0.08 & 0.13 & 0.05 & - & - \\
\hline 1978 & 0.08 & 0.06 & 0.08 & 0.36 & - \\
\hline 1979 & 0.09 & 0.08 & 0.06 & 0.59 & - \\
\hline 1980 & 0.06 & 0.08 & 0.05 & 0.11 & - \\
\hline 1981 & 0.08 & 0.11 & 0.07 & - & - \\
\hline 1982 & 0.07 & 0.15 & - & 0.22 & - \\
\hline 1983 & 0.08 & 0.12 & 0.06 & 0.11 & - \\
\hline 1984 & 0.11 & 0.13 & 0.10 & 0.32 & - \\
\hline 1985 & 0.09 & 0.14 & 0.05 & 0.10 & - \\
\hline 1986 & 0.05 & 0.09 & 0.04 & - & - \\
\hline 1987 & 0.04 & 0.05 & 0.04 & - & - \\
\hline 1988 & 0.07 & 0.06 & 0.07 & 0.30 & - \\
\hline 1989 & 0.09 & 0.14 & 0.06 & 0.10 & - \\
\hline 1990 & 0.07 & 0.15 & 0.02 & - & - \\
\hline
\end{tabular}

- indicates no deaths listed.

SOURCE: National Center for Health Statistics multiple cause of death data.

U.S. Bureau of the Census: 1970-1990 population estimates of the U.S. 
The following is a revision to Table 5-7 of the 1994 Work-Related Lung Disease(WoRLD) Surveillance Report. The overall rate reported in the 1994 WoRLD Surveillance Report included only whites and blacks. The revised overall rates presented below are based on all races. U.S. population data files have also been revised and updated for the tables presented below.

Table 5-7. Pneumoconiosis due to other inorganic dust: age-adjusted mortality rates (per 1,000,000 population), U.S. residents age 15 and over, by race and sex, 1968-1990

\begin{tabular}{|c|c|c|c|c|c|}
\hline \multirow[b]{2}{*}{ Year } & \multirow[b]{2}{*}{ Overall rate } & \multicolumn{2}{|c|}{ White } & \multicolumn{2}{|c|}{ Black } \\
\hline & & Males & Females & Males & Females \\
\hline 1968 & 0.07 & 0.15 & 0.01 & - & 0.12 \\
\hline 1969 & 0.09 & 0.09 & 0.10 & 0.17 & - \\
\hline 1970 & 0.10 & 0.16 & 0.07 & - & - \\
\hline 1971 & 0.06 & 0.08 & 0.04 & 0.11 & 0.14 \\
\hline 1972 & 0.10 & 0.12 & 0.08 & 0.31 & - \\
\hline 1973 & 0.05 & 0.10 & 0.02 & - & - \\
\hline 1974 & 0.07 & 0.13 & 0.02 & 0.12 & - \\
\hline 1975 & 0.05 & 0.08 & 0.03 & 0.15 & - \\
\hline 1976 & 0.07 & 0.09 & 0.06 & 0.11 & - \\
\hline 1977 & 0.06 & 0.11 & 0.04 & - & - \\
\hline 1978 & 0.06 & 0.05 & 0.06 & 0.37 & - \\
\hline 1979 & 0.08 & 0.07 & 0.04 & 0.64 & - \\
\hline 1980 & 0.05 & 0.08 & 0.04 & 0.12 & - \\
\hline 1981 & 0.06 & 0.08 & 0.04 & - & - \\
\hline 1982 & 0.05 & 0.11 & - & 0.23 & - \\
\hline 1983 & 0.07 & 0.10 & 0.05 & 0.09 & - \\
\hline 1984 & 0.09 & 0.10 & 0.08 & 0.36 & - \\
\hline 1985 & 0.07 & 0.11 & 0.04 & 0.12 & - \\
\hline 1986 & 0.04 & 0.06 & 0.03 & - & - \\
\hline 1987 & 0.02 & 0.04 & 0.02 & - & - \\
\hline 1988 & 0.06 & 0.05 & 0.05 & 0.33 & - \\
\hline 1989 & 0.06 & 0.11 & 0.03 & 0.10 & - \\
\hline 1990 & 0.06 & 0.13 & 0.01 & - & - \\
\hline
\end{tabular}

- indicates no deaths listed.

SOURCE: National Center for Health Statistics multiple cause of death data.

U.S. Bureau of the Census: 1970-1990 population estimates of the U.S. 
The following is a revision to Table 6-6 of the 1994 Work-Related Lung Disease(WoRLD) Surveillance Report. The overall rate reported in the 1994 WoRLD Surveillance Report included only whites and blacks. The revised overall rates presented below are based on all races. U.S. population data files have also been revised and updated for the tables presented below.

Table 6-6. Unspecified pneumoconiosis: crude mortality rates (per 1,000,000 population), U.S. residents age 15 and over, by race and sex, 1968-1990

\begin{tabular}{|c|c|c|c|c|c|}
\hline \multirow[b]{2}{*}{ Year } & \multirow[b]{2}{*}{ Overall rate } & \multicolumn{2}{|c|}{ White } & \multicolumn{2}{|c|}{ Black } \\
\hline & & Males & Females & Males & Females \\
\hline 1968 & 3.74 & 7.79 & 0.22 & 6.78 & 0.13 \\
\hline 1969 & 3.90 & 7.99 & 0.19 & 8.54 & 0.38 \\
\hline 1970 & 5.67 & 11.90 & 0.27 & 9.87 & 0.51 \\
\hline 1971 & 8.54 & 18.21 & 0.36 & 13.31 & - \\
\hline 1972 & 9.90 & 21.02 & 0.49 & 15.84 & - \\
\hline 1973 & 9.52 & 20.53 & 0.58 & 10.85 & 0.58 \\
\hline 1974 & 8.66 & 18.28 & 0.88 & 9.79 & 0.79 \\
\hline 1975 & 8.63 & 17.80 & 1.06 & 11.74 & 0.88 \\
\hline 1976 & 7.30 & 15.02 & 0.90 & 10.69 & 0.54 \\
\hline 1977 & 6.16 & 12.63 & 0.74 & 9.59 & 0.52 \\
\hline 1978 & 5.80 & 12.10 & 0.83 & 5.77 & 0.72 \\
\hline 1979 & 5.04 & 11.19 & 0.05 & 6.23 & 0.20 \\
\hline 1980 & 4.52 & 9.99 & 0.06 & 6.15 & - \\
\hline 1981 & 3.34 & 7.42 & 0.02 & 4.46 & - \\
\hline 1982 & 2.02 & 4.33 & 0.11 & 3.29 & - \\
\hline 1983 & 1.80 & 3.87 & 0.15 & 2.48 & - \\
\hline 1984 & 1.69 & 3.66 & 0.04 & 2.87 & 0.09 \\
\hline 1985 & 1.56 & 3.46 & 0.04 & 1.98 & - \\
\hline 1986 & 1.24 & 2.68 & 0.06 & 1.74 & - \\
\hline 1987 & 1.50 & 3.26 & 0.07 & 2.22 & 0.09 \\
\hline 1988 & 1.52 & 3.35 & 0.04 & 2.29 & - \\
\hline 1989 & 1.48 & 3.12 & 0.14 & 2.47 & - \\
\hline 1990 & 1.95 & 4.32 & 0.08 & 2.43 & 0.08 \\
\hline
\end{tabular}

- indicates no deaths listed.

SOURCE: National Center for Health Statistics multiple cause of death data.

U.S. Bureau of the Census: 1970-1990 population estimates of the U.S. 
The following is a revision to Table 6-7 of the 1994 Work-Related Lung Disease(WoRLD) Surveillance Report. The overall rate reported in the 1994 WoRLD Surveillance Report included only whites and blacks. The revised overall rates presented below are based on all races. U.S. population data files have also been revised and updated for the tables presented below.

Table 6-7. Unspecified pneumoconiosis: age-adjusted mortality rates (per 1,000,000 population), U.S. residents age 15 and over, by race and sex, 1968-1990

\begin{tabular}{|c|c|c|c|c|c|}
\hline \multirow[b]{2}{*}{ Year } & \multirow[b]{2}{*}{ Overall rate } & \multicolumn{2}{|c|}{ White } & \multicolumn{2}{|c|}{ Black } \\
\hline & & Males & Females & Males & Females \\
\hline 1968 & 2.57 & 5.75 & 0.14 & 6.45 & 0.14 \\
\hline 1969 & 2.72 & 5.96 & 0.14 & 8.11 & 0.36 \\
\hline 1970 & 3.96 & 8.86 & 0.21 & 9.38 & 0.49 \\
\hline 1971 & 5.91 & 13.62 & 0.26 & 12.39 & - \\
\hline 1972 & 6.85 & 15.80 & 0.34 & 15.03 & - \\
\hline 1973 & 6.60 & 15.52 & 0.42 & 10.17 & 0.61 \\
\hline 1974 & 6.02 & 13.84 & 0.57 & 9.40 & 0.74 \\
\hline 1975 & 5.94 & 13.38 & 0.73 & 11.27 & 0.90 \\
\hline 1976 & 5.03 & 11.36 & 0.59 & 10.05 & 0.49 \\
\hline 1977 & 4.18 & 9.42 & 0.49 & 8.96 & 0.59 \\
\hline 1978 & 3.86 & 8.86 & 0.55 & 5.41 & 0.75 \\
\hline 1979 & 3.28 & 8.16 & 0.03 & 5.62 & 0.13 \\
\hline 1980 & 2.91 & 7.21 & 0.04 & 5.85 & - \\
\hline 1981 & 2.08 & 5.22 & 0.01 & 4.15 & - \\
\hline 1982 & 1.28 & 3.07 & 0.05 & 3.15 & - \\
\hline 1983 & 1.12 & 2.67 & 0.06 & 2.57 & - \\
\hline 1984 & 0.99 & 2.45 & 0.02 & 2.67 & 0.06 \\
\hline 1985 & 0.92 & 2.31 & 0.02 & 1.92 & - \\
\hline 1986 & 0.73 & 1.80 & 0.02 & 1.57 & - \\
\hline 1987 & 0.86 & 2.12 & 0.04 & 2.11 & 0.05 \\
\hline 1988 & 0.89 & 2.19 & 0.02 & 2.17 & - \\
\hline 1989 & 0.86 & 1.99 & 0.07 & 2.55 & - \\
\hline 1990 & 1.09 & 2.70 & 0.03 & 2.28 & 0.07 \\
\hline
\end{tabular}

- indicates no deaths listed.

SOURCE: National Center for Health Statistics multiple cause of death data.

U.S. Bureau of the Census: 1970-1990 population estimates of the U.S. 
The following is a revision to Table 7-5 of the 1994 Work-Related Lung Disease(WoRLD) Surveillance Report. The overall rate reported in the 1994 WoRLD Surveillance Report included only whites and blacks. The revised overall rates presented below are based on all races. U.S. population data files have also been revised and updated for the tables presented below.

Table 7-5. Byssinosis: crude mortality rates (per 1,000,000 population), U.S. residents age 15 and over, by race and sex, 1979-1990

\begin{tabular}{lccccc} 
& & \multicolumn{3}{c}{ White } & \multicolumn{2}{c}{ Black } \\
\cline { 2 - 5 } Year & Overall rate & Males & Females & Males & Females \\
\hline 1979 & 0.06 & 0.08 & 0.05 & - & - \\
1980 & 0.05 & 0.10 & 0.03 & - & - \\
1981 & 0.07 & 0.15 & 0.01 & 0.11 & - \\
1982 & 0.02 & 0.04 & 0.01 & - & - \\
1983 & 0.09 & 0.08 & 0.11 & 0.11 & - \\
1984 & 0.10 & 0.13 & 0.09 & 0.11 & - \\
1985 & 0.08 & 0.10 & 0.06 & 0.10 & - \\
1986 & 0.08 & 0.12 & 0.07 & 0.10 & - \\
1987 & 0.08 & 0.17 & 0.01 & 0.20 & - \\
1988 & 0.07 & 0.15 & 0.02 & - & - \\
1989 & 0.04 & 0.06 & 0.01 & 0.20 & - \\
1990 & 0.09 & 0.15 & 0.06 & 0.10 & - \\
\hline
\end{tabular}

- indicates no deaths listed.

SOURCE: National Center for Health Statistics multiple cause of death data.

U.S. Bureau of the Census: 1970-1990 population estimates of the U.S. 
The following is a revision to Table 7-6 of the 1994 Work-Related Lung Disease(WoRLD) Surveillance Report. The overall rate reported in the 1994 WoRLD Surveillance Report included only whites and blacks. The revised overall rates presented below are based on all races. U.S. population data files have also been revised and updated for the tables presented below.

Table 7-6. Byssinosis: age-adjusted mortality rates (per 1,000,000 population), U.S. residents age 15 and over, by race and sex, 1979-1990

\begin{tabular}{lccccc}
\hline & & \multicolumn{3}{c}{ White } & \multicolumn{3}{c}{ Black } \\
\cline { 2 - 5 } Year & Overall rate & Males & Females & Males & Females \\
\hline 1979 & 0.04 & 0.05 & 0.04 & - & - \\
1980 & 0.03 & 0.07 & 0.01 & - & - \\
1981 & 0.05 & 0.11 & 0.01 & 0.12 & - \\
1982 & 0.01 & 0.03 & 0.00 & - & - \\
1983 & 0.07 & 0.06 & 0.07 & 0.13 & - \\
1984 & 0.07 & 0.09 & 0.06 & 0.12 & - \\
1985 & 0.06 & 0.09 & 0.04 & 0.12 & - \\
1986 & 0.07 & 0.09 & 0.06 & 0.11 & - \\
1987 & 0.06 & 0.12 & 0.01 & 0.21 & - \\
1988 & 0.05 & 0.11 & 0.02 & - & - \\
1989 & 0.03 & 0.06 & 0.01 & 0.18 & - \\
1990 & 0.06 & 0.12 & 0.02 & 0.10 & - \\
\hline
\end{tabular}

- indicates no deaths listed.

SOURCE: National Center for Health Statistics multiple cause of death data

U.S. Bureau of the Census: 1970-1990 population estimates of the U.S. 
The following is a revision to Table 8-5 of the 1994 Work-Related Lung Disease(WoRLD) Surveillance Report. The overall rate reported in the $1994 \mathrm{WoRLD}$ Surveillance Report included only whites and blacks. The revised overall rates presented below are based on all races. U.S. population data files have also been revised and updated for the tables presented below.

Table 8-5. Hypersensitivity pneumonitis: crude mortality rates (per 1,000,000 population), U.S. residents age 15 and over, by race and sex, 1979-1990

\begin{tabular}{lccccc}
\hline & & \multicolumn{2}{c}{ White } & \multicolumn{3}{c}{ Black } \\
\cline { 2 - 5 } Year & Overall rate & Males & Females & Males & Females \\
\hline 1979 & 0.09 & 0.14 & 0.05 & 0.12 & - \\
1980 & 0.09 & 0.12 & 0.06 & - & 0.10 \\
1981 & 0.07 & 0.12 & 0.04 & - & - \\
1982 & 0.08 & 0.17 & 0.02 & - & - \\
1983 & 0.11 & 0.20 & 0.06 & - & - \\
1984 & 0.20 & 0.39 & 0.06 & - & 0.09 \\
1985 & 0.19 & 0.28 & 0.13 & 0.10 & 0.09 \\
1986 & 0.13 & 0.23 & 0.05 & - & 0.18 \\
1987 & 0.14 & 0.23 & 0.08 & - & 0.09 \\
1988 & 0.13 & 0.20 & 0.08 & - & 0.09 \\
1989 & 0.14 & 0.20 & 0.13 & 0.10 & - \\
1990 & 0.21 & 0.36 & 0.11 & 0.10 & - \\
\hline
\end{tabular}

- indicates no deaths listed.

SOURCE: National Center for Health Statistics multiple cause of death data.

U.S. Bureau of the Census: 1970-1990 population estimates of the U.S. 
The following is a revision to Table 8-6 of the 1994 Work-Related Lung Disease(WoRLD) Surveillance Report. The overall rate reported in the 1994 WoRLD Surveillance Report included only whites and blacks. The revised overall rates presented below are based on all races. U.S. population data files have also been revised and updated for the tables presented below.

Table 8-6. Hypersensitivity pneumonitis: age-adjusted mortality rates (per 1,000,000 population), U.S. residents age 15 and over, by race and sex, 1979-1990

White

Black

\begin{tabular}{lccccc}
\cline { 2 - 5 } Year & Overall rate & Males & Females & Males & Females \\
\hline 1979 & 0.06 & 0.11 & 0.03 & 0.11 & - \\
1980 & 0.07 & 0.10 & 0.05 & - & 0.10 \\
1981 & 0.04 & 0.08 & 0.03 & - & - \\
1982 & 0.06 & 0.13 & 0.02 & - & - \\
1983 & 0.07 & 0.14 & 0.04 & - & - \\
1984 & 0.15 & 0.32 & 0.03 & - & 0.12 \\
1985 & 0.12 & 0.21 & 0.05 & 0.15 & 0.12 \\
1986 & 0.09 & 0.18 & 0.03 & - & 0.15 \\
1987 & 0.08 & 0.16 & 0.03 & - & 0.12 \\
1988 & 0.08 & 0.14 & 0.05 & - & 0.08 \\
1989 & 0.10 & 0.15 & 0.06 & 0.12 & - \\
1990 & 0.15 & 0.27 & 0.05 & 0.08 & - \\
\hline
\end{tabular}

- indicates no deaths listed.

SOURCE: National Center for Health Statistics multiple cause of death data.

U.S. Bureau of the Census: 1970-1990 population estimates of the U.S. 
The following is a correction to Figure 2-4. in the 1994 Work-Related Lung Disease Surveillance Report. The state of Pennsylvania was shaded incorrectly. Pennsylvania should have been shaded to be included in the 0 to 2.4 range.

Figure 2-4. Malignant neoplasm of the pleura: crude mortality rates, U.S. residents age 15 and over, by state, 1989-1990

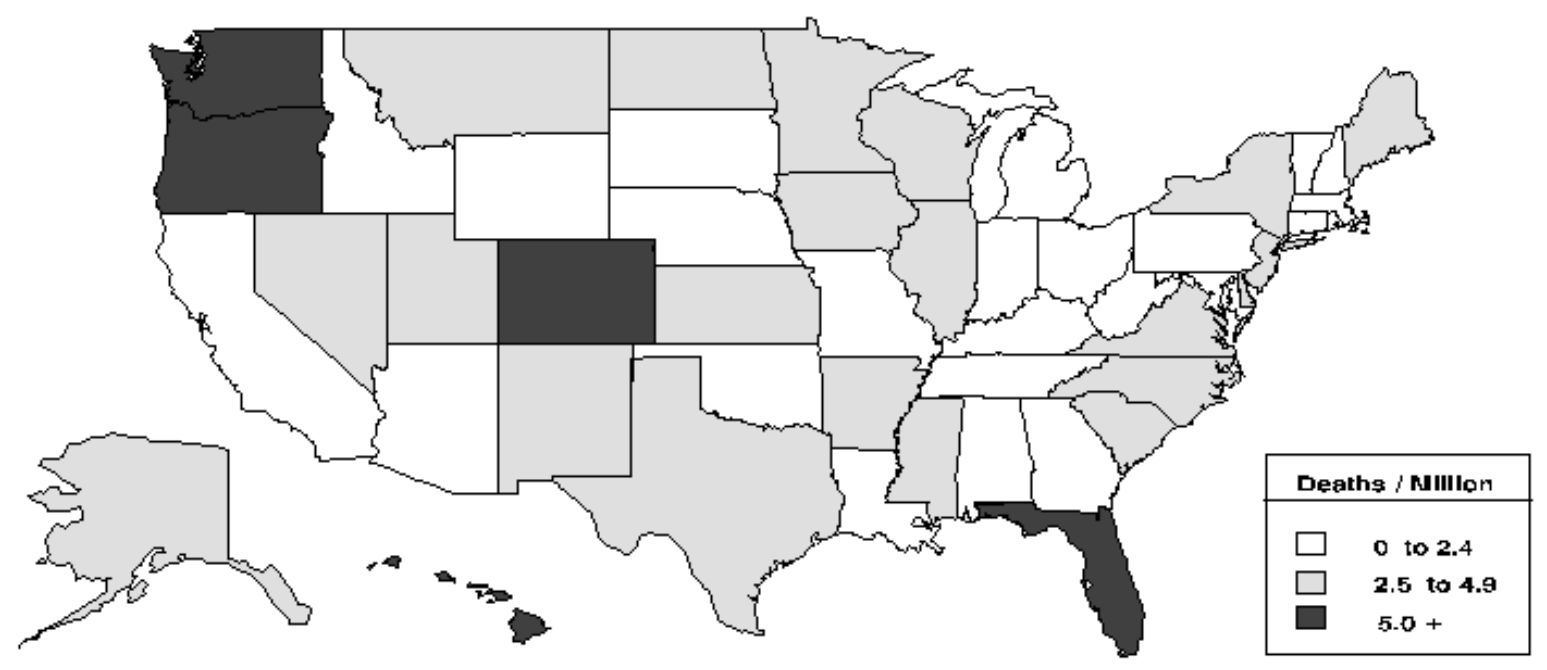

The following is a correction to Figure 5-2 in the 1994 Work-Related Lung Disease Surveillance Report. The state of Wisconsin was shaded incorrectly. Wisconsin should have been shaded to be included in the 0 to 5 range.

Figure 5-2 Pneumoconiosis due to other inorganic dust: number of deaths, U.S. residents age 15 and over, 1968-1990

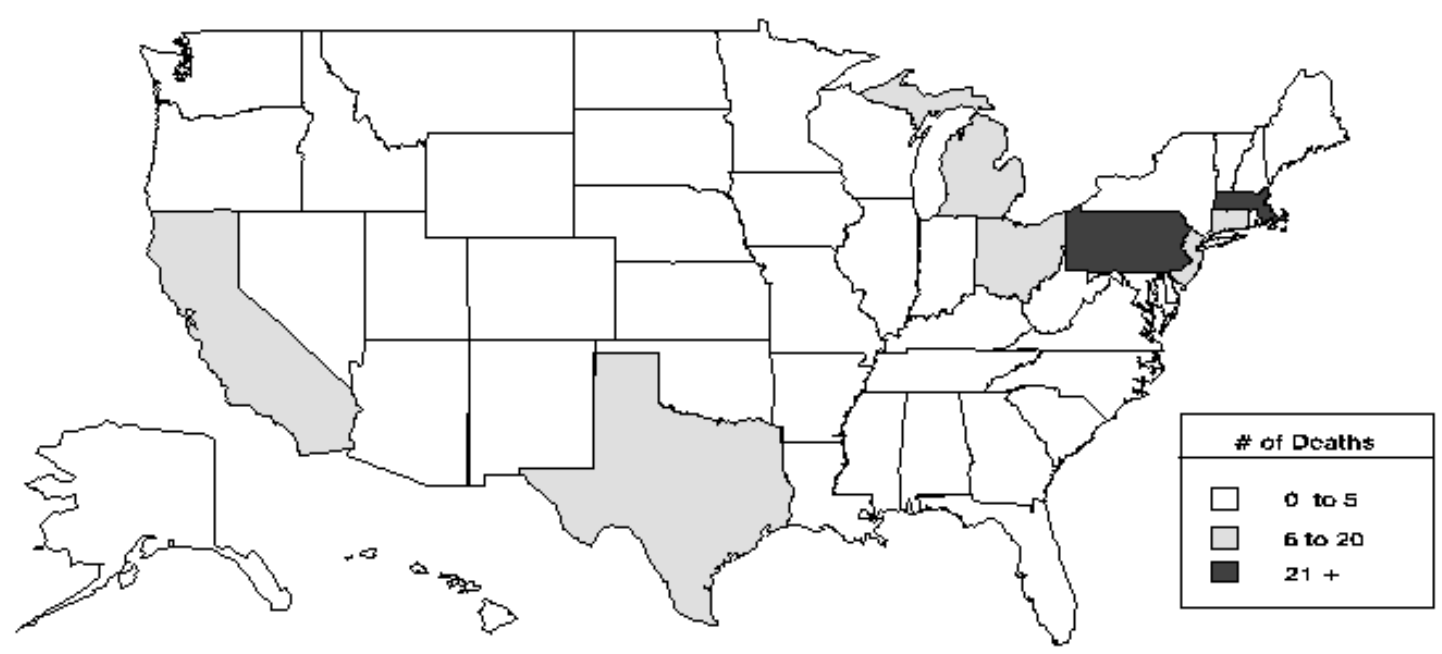


The following is a correction to Figure 8-4 in the 1994 Work-Related Lung Disease Surveillance Report. The state of Mississippi was shaded incorrectly. Mississippi should have been shaded to be included in the 0 to 0.4 range.

Figure 8-4. Hypersensitivity Pneumonitis: crude mortality rates, U.S. residents age 15 and over, by state, 1989-1990

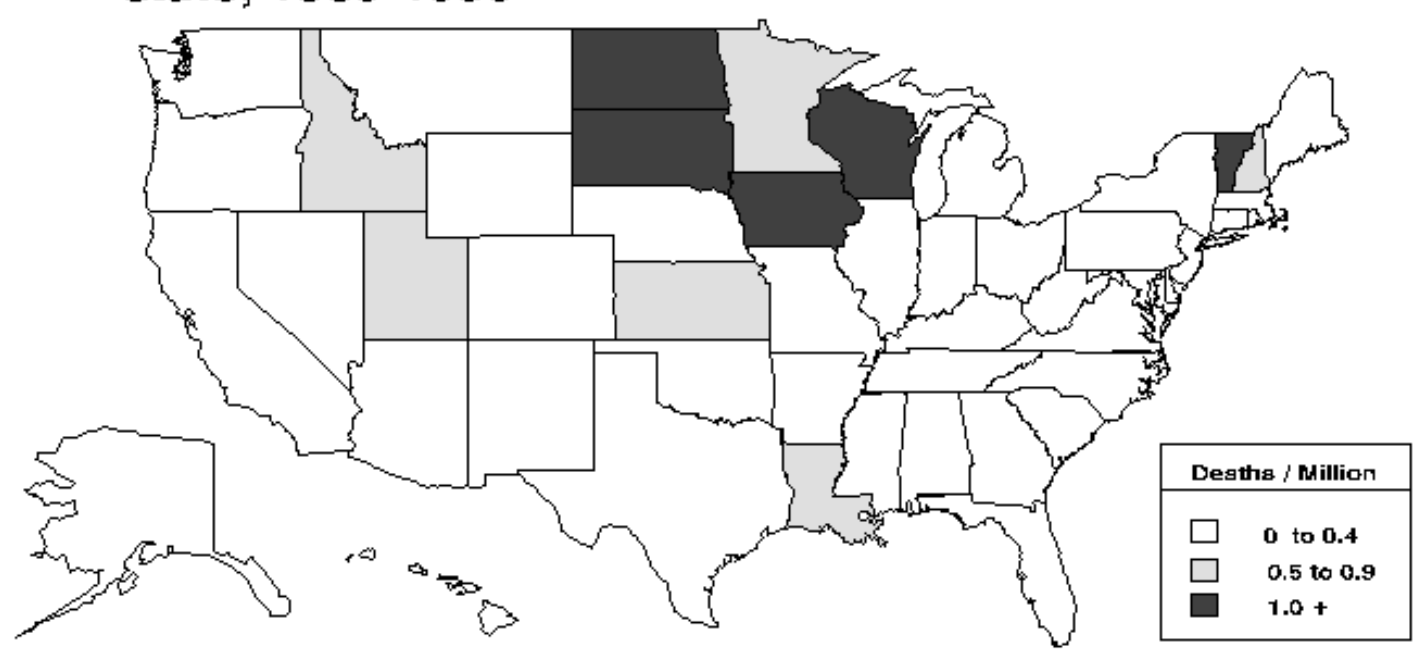

The following is a correction to Figure 9-1 in the 1994 Work-Related Lung Disease Surveillance Report. The legend printed in the 1994 was incorrect. The correct graph and legend is presented below.

Figure 9-1. States with SENSOR cccupational asthma programs

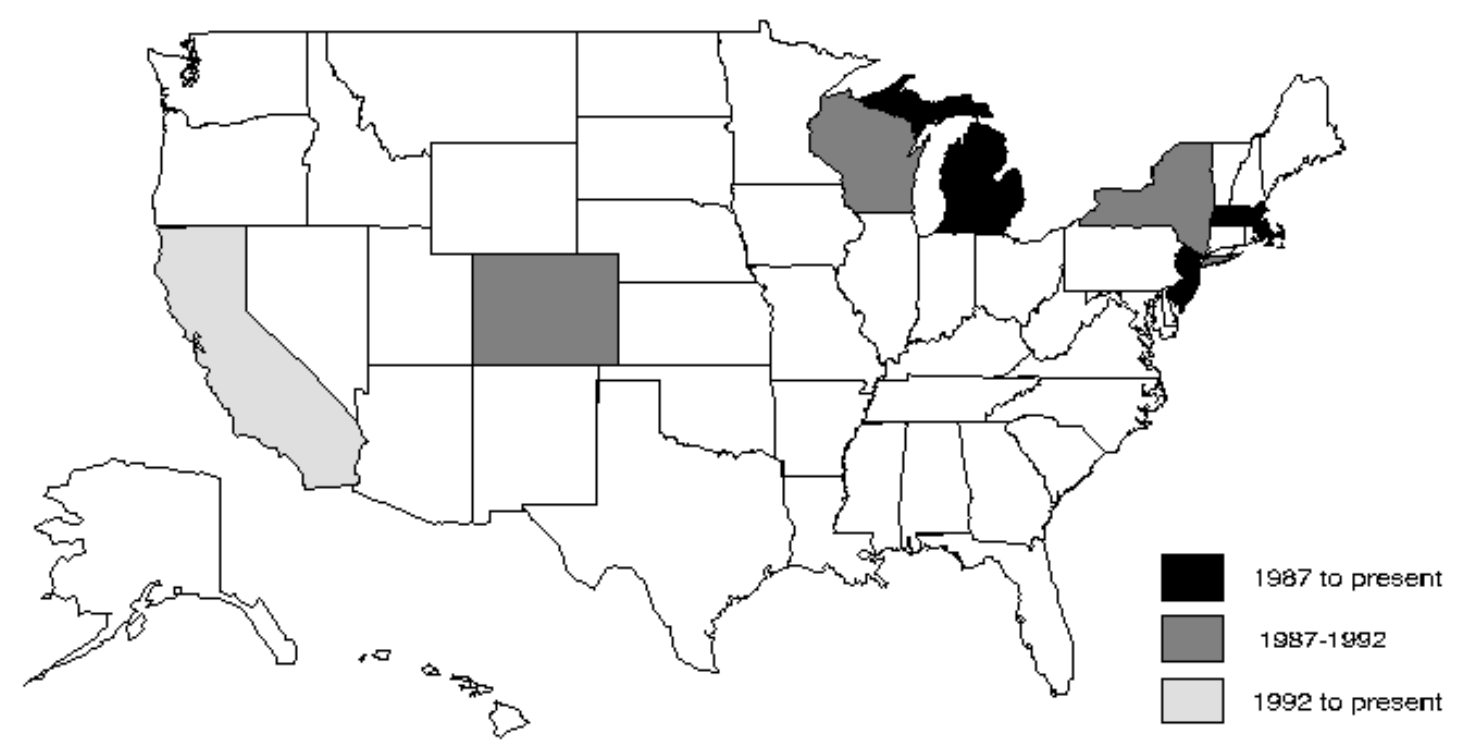

